SERI/TR-215-3513B

UC Categories: 233,234

DE89009448

\title{
Spectral Solar Radiation Data Base Documentation
}

\section{Volume II}

Carol J. Riordan

Daryl R. Myers

Roland L. Hulstrom

January 1990

Prepared under Task No. RA910201

Solar Energy Research Institute A Division of Midwest Research Institute

1617 Cole Boulevard

Golden, Colorado 80401-3393

Prepared for the U.S. Department of Energy Contract No. DE-AC02-83CH10093 



\section{PREFACE}

This report documents the Solar Energy Research Institute's spectral solar radiation data base. Volume I describes the history, approach, content, and format of the data, and Volume II contains graphs of each of the spectral solar radiation curves and associated field notes.

This is the first public release of these data. Our intent is to make the data available as soon as possible so that others can use it. However, we also intend to create additional products and data subsets in the future to illustrate applications of the data for special purposes or analyses.

Comments or questions about these data should be directed to the Solar Energy Research Institute, Solar Electric Research Division, Resource Assessment and Instrumentation Branch, in Golden, Colorado, 80401.

\section{ACKNOWLEDGMENTS}

SERI would like to acknowledge the work of the Florida Solar Energy Center and the Pacific Gas and Electric Company in building this spectral solar radiation data base. Without their help, this data base would not have been possible. 


\section{SUMMARY}

The objective of this report is to provide documentation for the spectral solar radiation data base at the Solar Energy Research Institute. The data reside on magnetic tape.

\section{Discussion}

Volume I of this report documents the history, approach, content, and format of the data base. Volume II contains graphs and field notes for each of the spectral data sets. Together, these two volumes should allow users to find and access any particular field of data or desired data subset contained on the magnetic tape.

\section{Conclusions}

These data are being released at this point to make the information available to those who have immediate uses or applications. We also intend to create other products and data subsets in the future to illustrate particular applications and analyses. 
TABLE OF CONTENTS

Page

1.0 Introduction $\ldots \ldots \ldots \ldots \ldots \ldots \ldots \ldots \ldots \ldots \ldots \ldots \ldots \ldots \ldots \ldots \ldots \ldots \ldots, 1$

2.0 Contents $. . \ldots \ldots \ldots \ldots \ldots \ldots \ldots \ldots \ldots \ldots \ldots \ldots \ldots \ldots \ldots \ldots \ldots, 2$

3.0 Daily Notes and Graphs $\ldots \ldots \ldots \ldots \ldots \ldots \ldots \ldots \ldots \ldots \ldots \ldots \ldots \ldots \ldots, 5$

3.1 Florida Solar Energy Center, Cape Canaveral, Fla. ............... 5

3.2 Pacific Gas and Electric Company, San Ramon, Calif. ............ 342

3.3 Solar Energy Research Institute, Golden, Colo. ................ 377 


\subsection{INTRODUCTION}

This second volume of the Spectral Solar Radiation Data Base Documentation and User's Manual contains copies of the daily field notes and graphs of broadband and spectral solar radiation data that were created during the quality-control processing. All of these data are contained on the data-base magnetic tape. Volume I of this series should be consulted for detailed information about the data. 


\subsection{CONIENTS}

This volume can be used as a catalog for the information contained in the data base. Each page of graphs represents the data measured on one day of the year, from October 1986 through April 1988, at one of three locations: the Florida Solar Energy Center (FSEC), Cape Canaveral, Fla.; the Pacific Gas and Electric Company (PG\&E), San Ramon, Calif.; and the Solar Energy Research Institute (SERI), Golden, Colo.

The following information is contained on each page:

1. Field notes. The first line of the field notes begins with an " $\mathrm{N}^{n}$ followed by the site, year, and day number. The second line contains a log sheet number (FSEC only), day number, date, serial number of the LI-COR spectroradiometer(s), and measurement mode(s). The measurement modes are direct normal DN (irradiance from the solar disk in about a 5-deg field of view), global normal GN (flat plate tracking the sun), global horizontal GH (flat plate on a horizontal surface), global tilt GT (flat plate tilted at or near the latitude angle), and diffuse horizontal DF. Next are operator observations, such as weather or instrument operations, observations about the data, and notes on the existence of all-sky pictures. (The pictures are labeled as good G, marginal M, or poor $\mathrm{P}_{\text {.) }}$

2. Broadband solar radiation graph. The broadband solar radiation values (in watts per square meter) recorded by Eppley Precision Spectral Pyranometers and a Normal Incidence Pyrheliometer, before the spectral data were acquired, are plotted versus standard time. The site and day number are printed at the top of the graph. The four measurement modes (if available) include global normal GN, direct normal DN, global tilt GT, and global horizontal GH.

3. Spectral solar radiation graph(s). The spectral solar radiation data acquired on this day are graphed for each measurement mode. The titles of the graphs include site, day number, and measurement mode (including a symbol for the spectroradiometer foreoptics). The measurement modes are:

- DNT Direct normal measured with a view-limiting tube receiver

- GNS Global normal measured with an integrating sphere receiver

- GND Global normal measured with a Teflon dome receiver

- GHS Global horizontal measured with an integrating sphere

- GHD Global horizontal measured with a Teflon dome (for comparison with GHS only; GHS data should be used)

- GTS Global tilt measured with an integrating sphere

- DFS Diffuse horizontal measured with an integrating sphere

- DFD Diffuse horizontal measured with a Teflon dome (for comparison with DFS data only; DFS data should be used). 
Spectral irradiance (in watts per square meter per nanometer) is graphed versus wavelength in nanometers. The legends to the right of the spectral graphs give the standard time and air mass when the spectra were acquired. PG\&E and SERI operated one spectroradiometer, rotating through measurement modes. FSEC generally operated two spectroradiometers simultaneously, with one in the direct-normal mode and the other in one of the global modes. 


\subsection{DAILY NOTES AND GRAPHS}

3.1 Florida Solar Energy Center, Cape Canaveral, Fla. 
N 33586296

$00121023 \quad 86 \quad 296 \quad 218$

No $\sigma_{I}$ - being callbrated

No albedo measurenent

reap controller $40 \mathrm{C}$ at 1510

Partly clouds

No photos

Fsec 2as

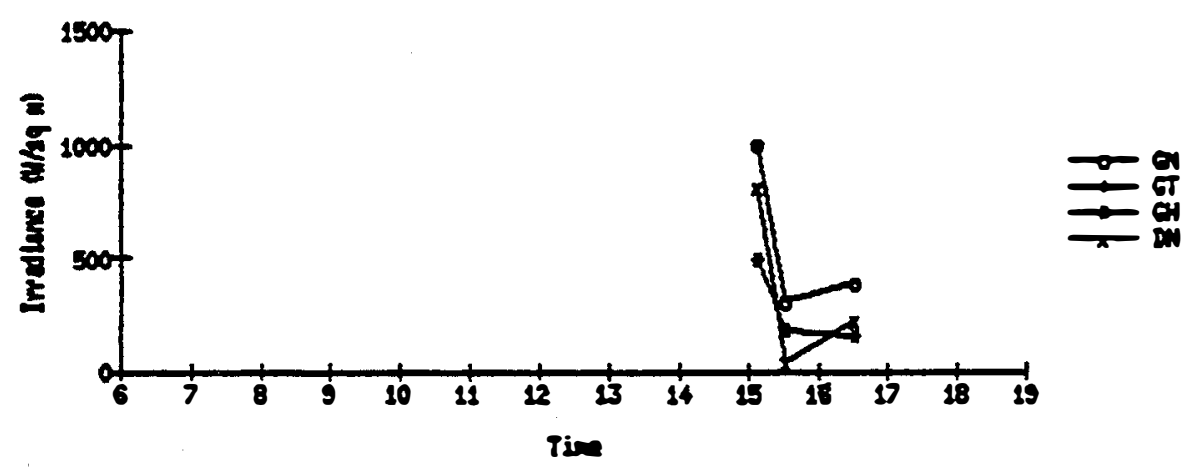

Fax as en

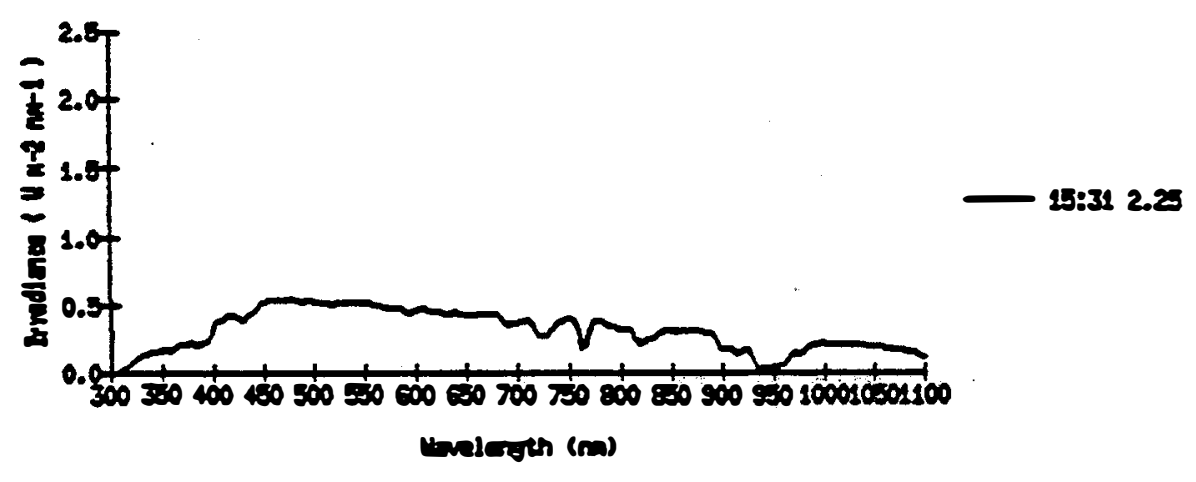


N) 72586297

$002 \quad 1024 \quad 86 \quad 297 \quad 218$

Ho GI - being callbrated

Ho ulbedo measurenent

Teap contzoller $41 \mathrm{C}$ at 1030 ; $41 \mathrm{C}$ at 1430

Partly cloudy

No photos

Fsec 297

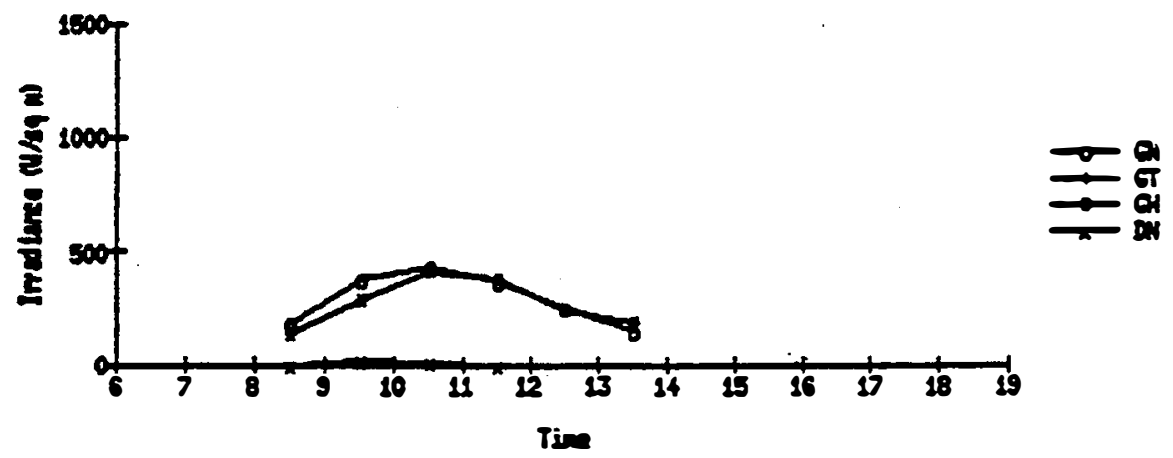

Fasc 29700

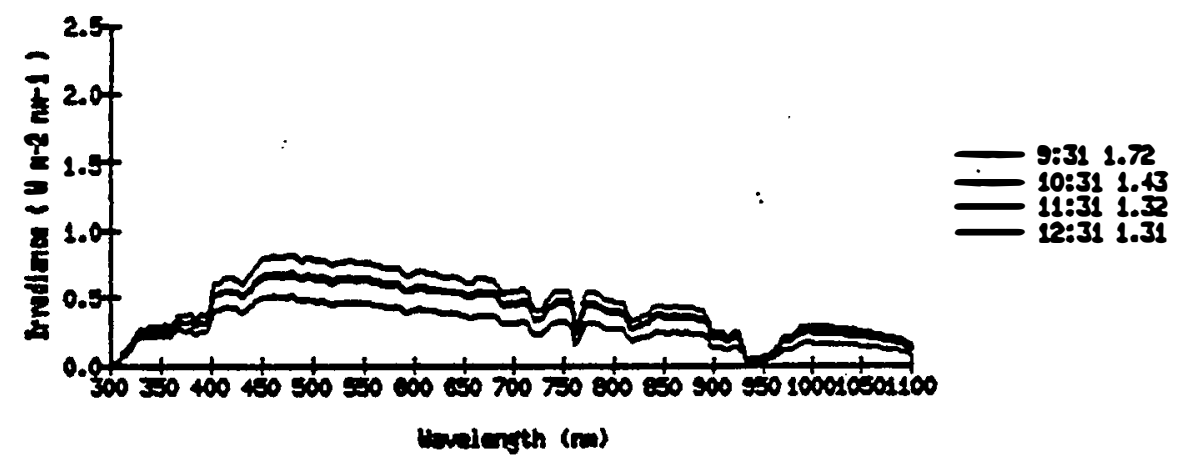


A 752586298

$003 \quad 1025 \quad 86298 \quad 218$

Ho GI - belng callbrated

No albado zeasurement

Pertly cloudy

No photos

Fsec 208

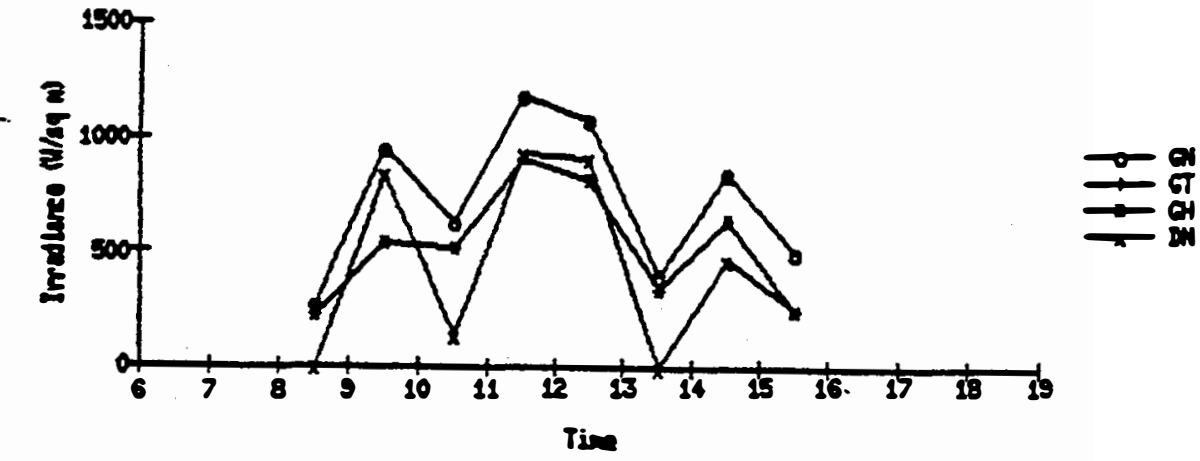

FEx 20800

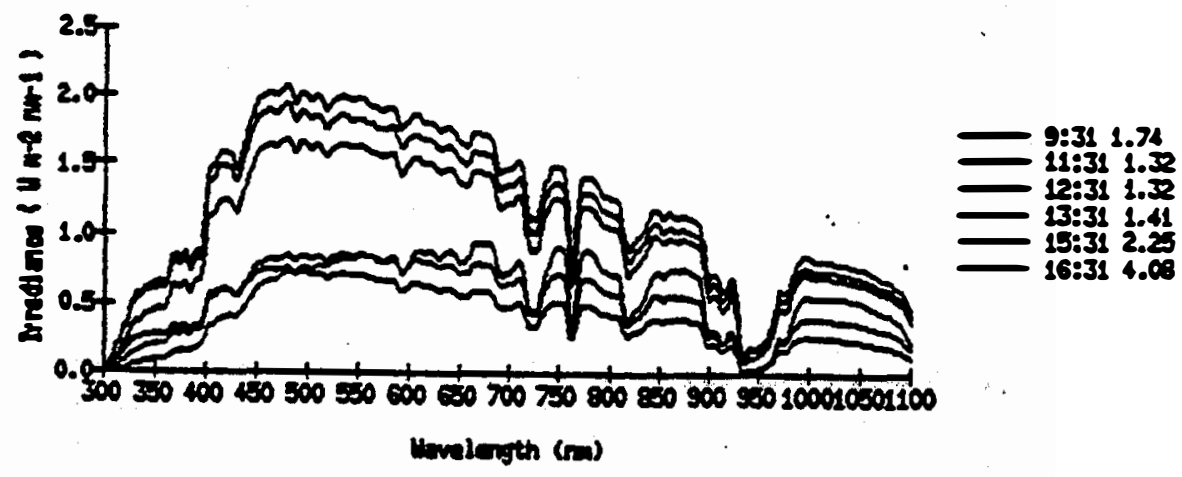


I. Jiय286299

$\begin{array}{llllll}004 & 10 & 26 & 86 & 299 & 218\end{array}$

Ho GI - belng ealibrated

Ho ardo vanurent

Partly elouds

wo potos

frac 29

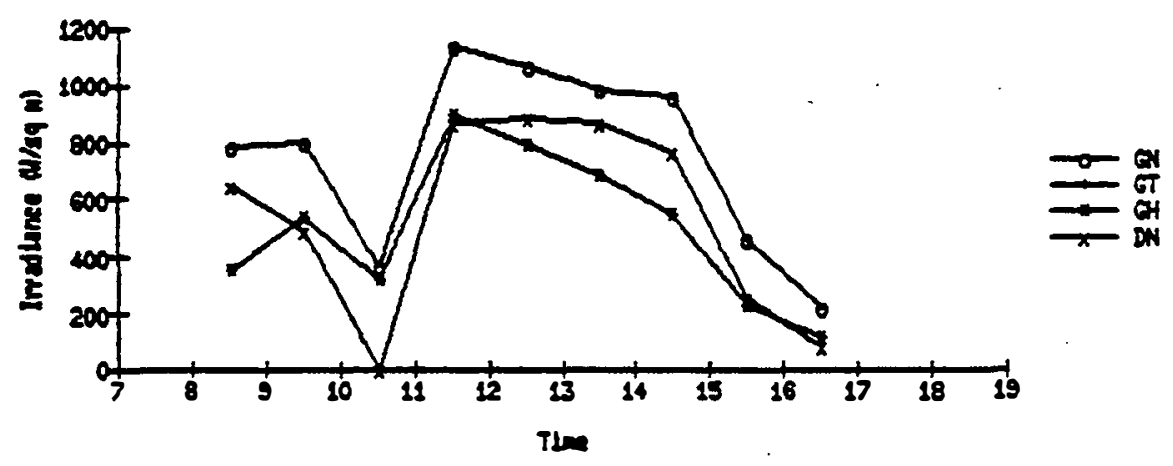

Pas 200

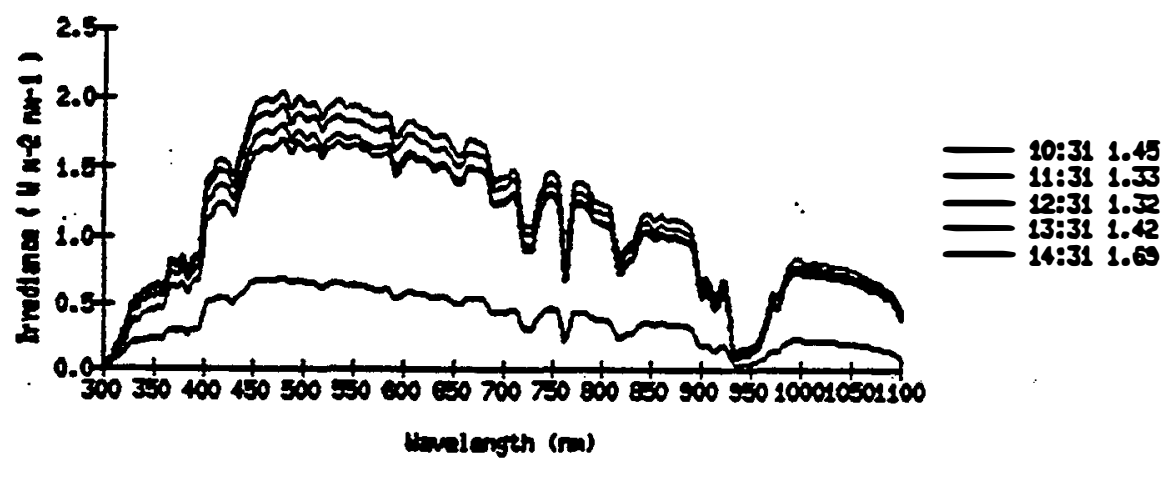


A हSPC86300

$005102786300 \quad 218$

Do $\sigma_{L}$ - besng eallbrated

Ho albedo seasurement

Teap controller 39 C at 0830: $40 \mathrm{C}$ at 1330

overcast. light rain/esst for part of afternoon

Vo photos

PISE 300

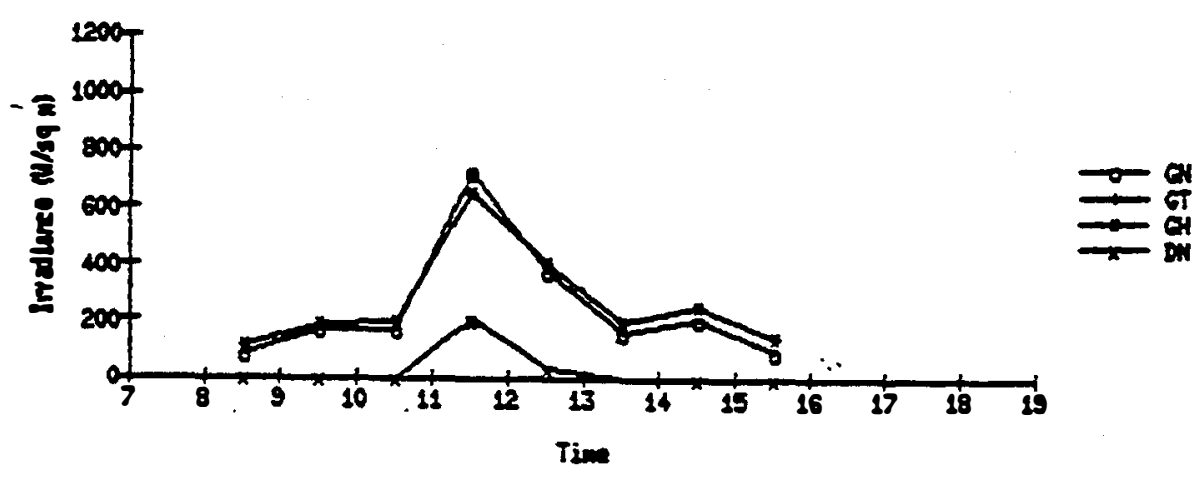

$\operatorname{Psec} 30000$

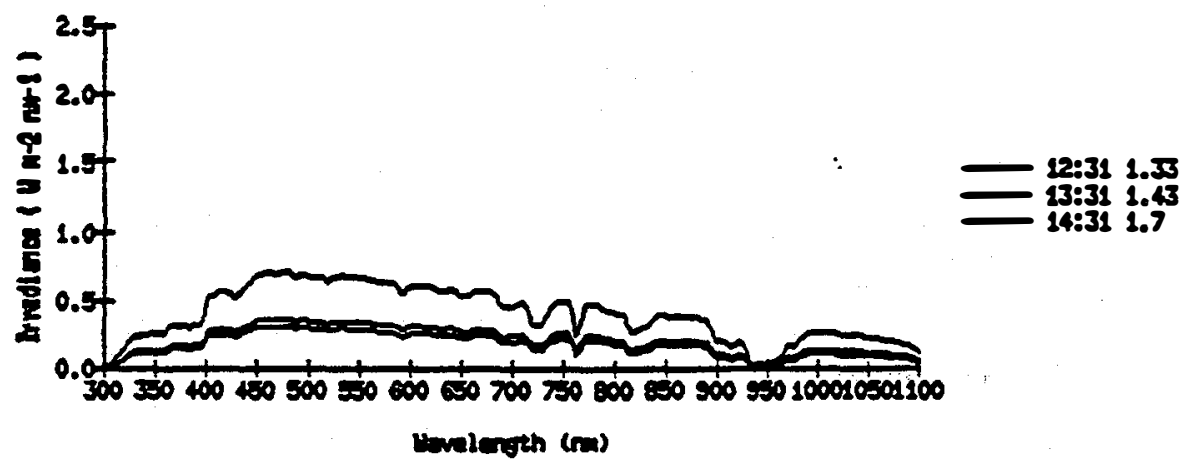


4. F्यु 86301

$0061028 \quad 86301 \quad 218$

Ho GI - being callbrated

Ho clbedo sesurement

Temp controller $40 \mathrm{C}$ at 1030

Pertly cloudy to orercast

Ho photos

$\operatorname{PSEC} 301$

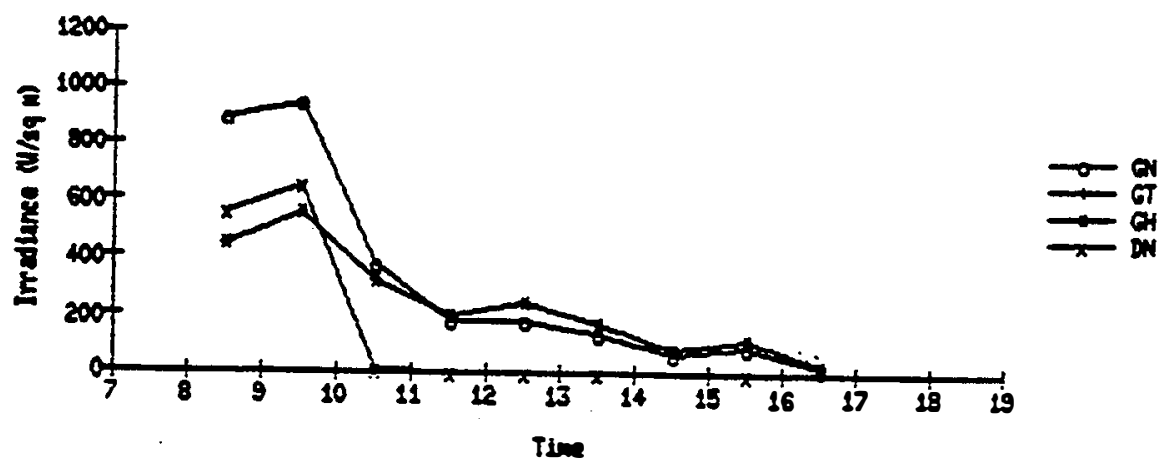

Fasc 30100

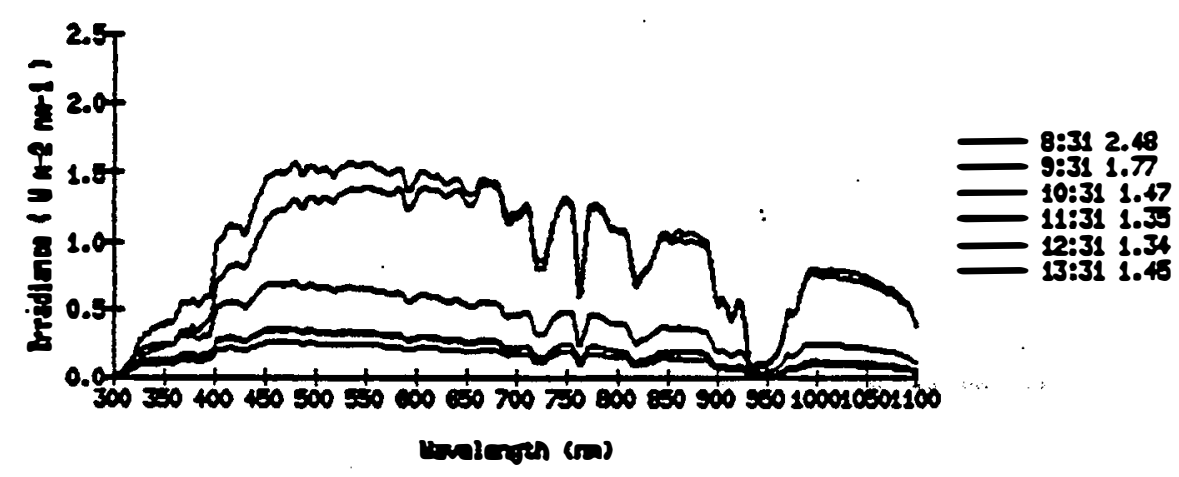


N FS1086302

$007 \quad 10 \quad 2986 \quad 302 \quad 218$

Wo GI - belng callbrated

No albedo measurencre

Teap controller $39 \mathrm{C}$ at 0830; $40 \mathrm{C}$ at 1500

Orercast. 110ht rats 1000-1100

No date at 1430 - tracker alfunction

No photos

$\operatorname{Psec} 302$

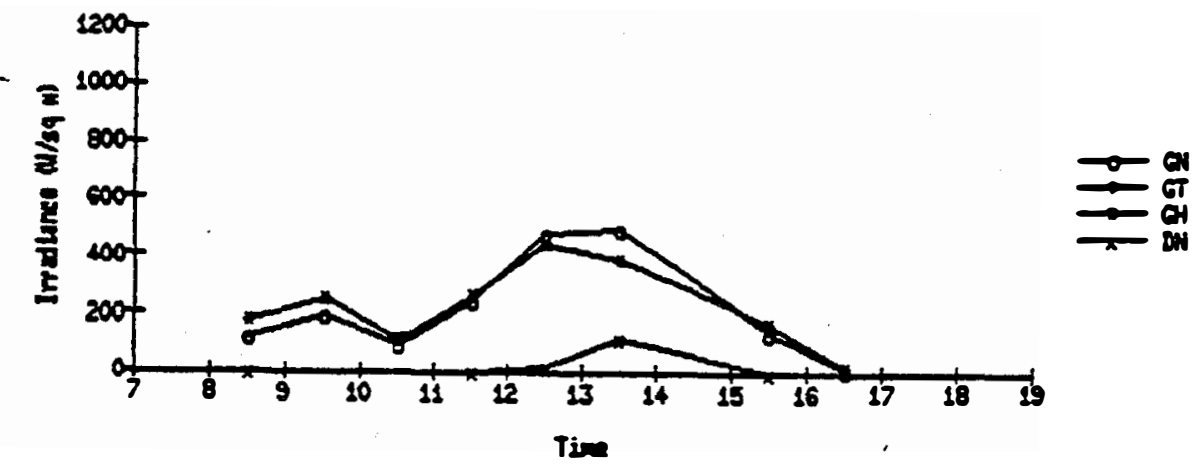

Fas 3000

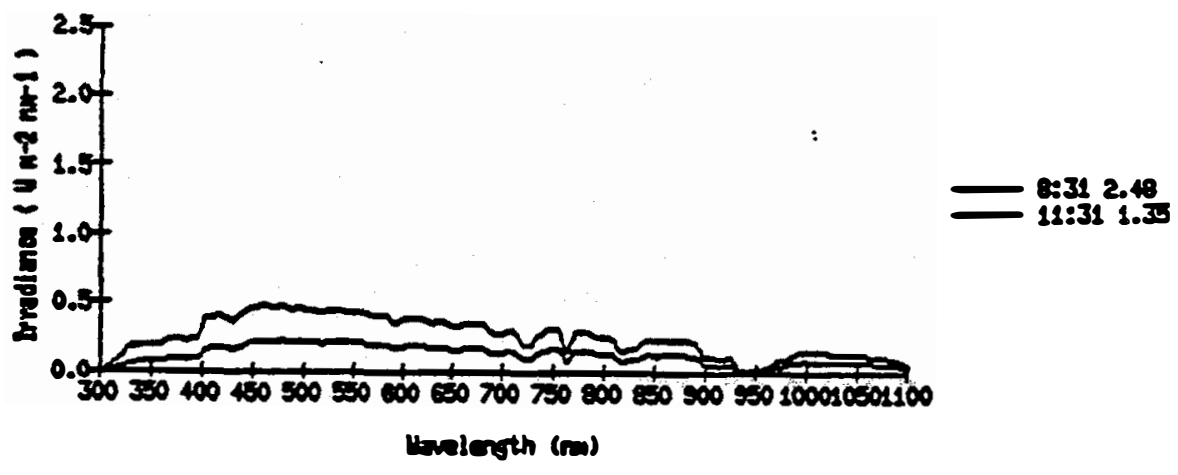


FST्C86303

$008 \cdot 10.3086303218$

No $G I$ - being callibrated

Bo abedo sessurenent

Ien controller $40 \mathrm{C}$ at 1030: $40 \mathrm{C}$ it 1530

Partis cloudy

Ho photos

Fasc 303

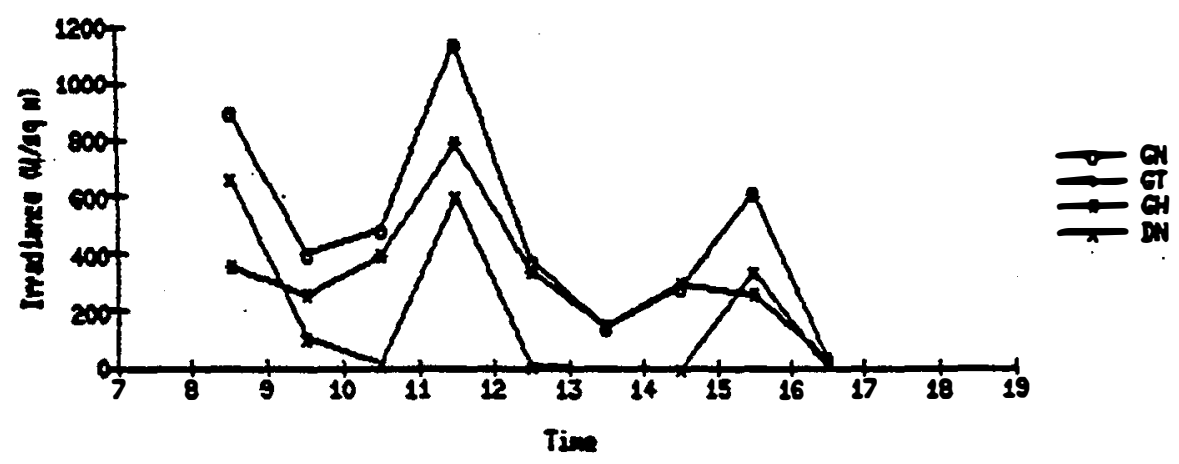

$\operatorname{Fecs} 30300$

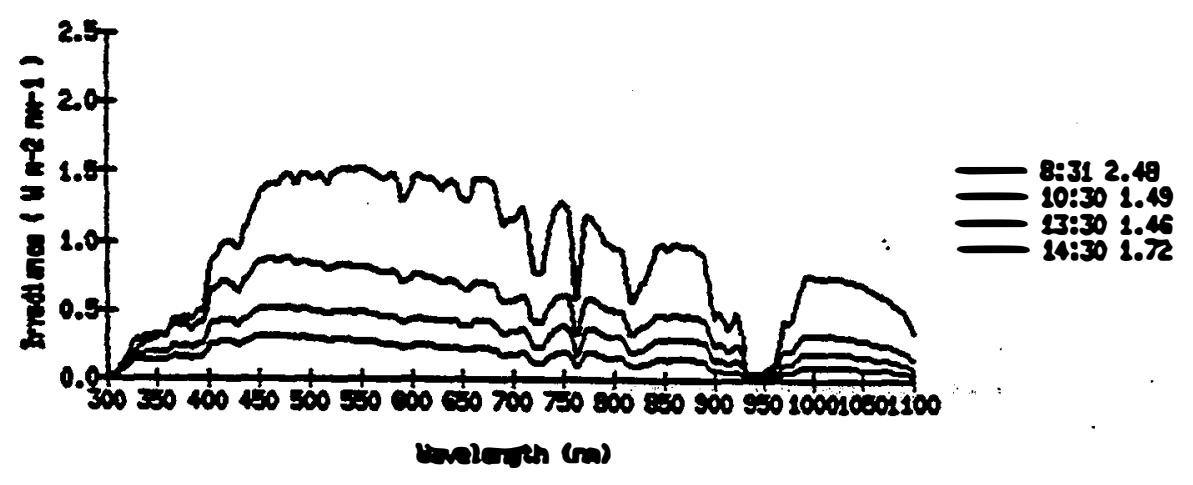


A

$009 \quad 10 \quad 3186 \cdot 304 \quad 218$

No Gr

Ho albedo neasurenent

Terp controller $41 \mathrm{C}$ at 1130 ; $41 \mathrm{C}$ at 1430

Partly clouds

No photos

spectrin at 1530 an be eloud enhneenent

Pesc 304

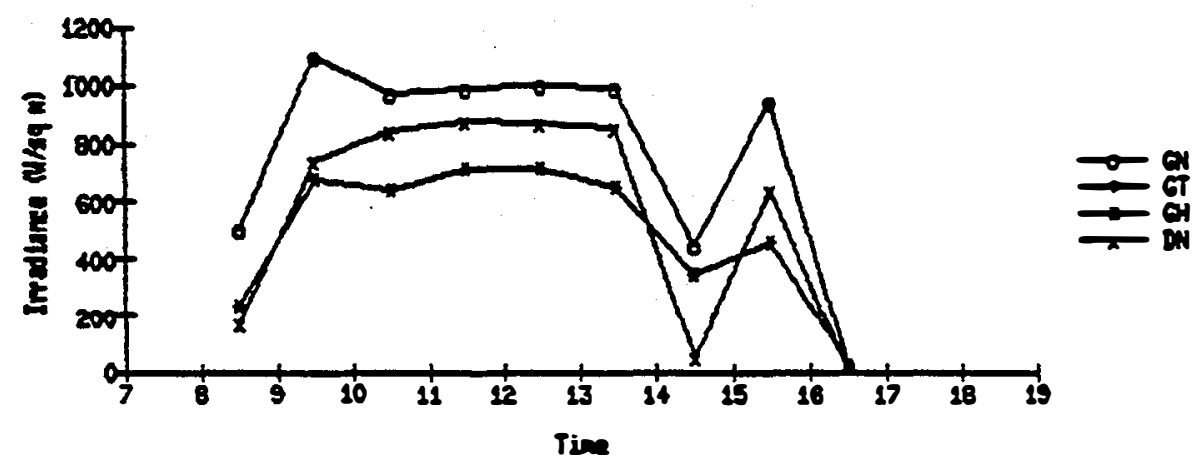

rac 3040

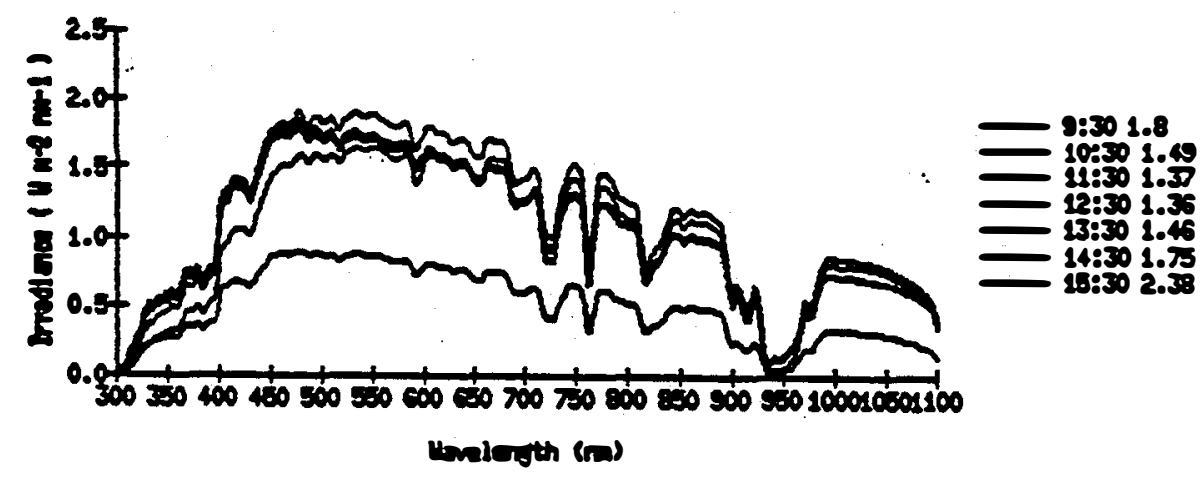


4 Tiयe8s305

$010 \quad 1101.86305 \quad 218$

Mo $\sigma$

Ho abado enarurcoent

vostly clear Nis partly cloudJ PH

No pobeos

Spectirl at 1430 eng be cloud enbnneenent

$\operatorname{Fesc} 305$

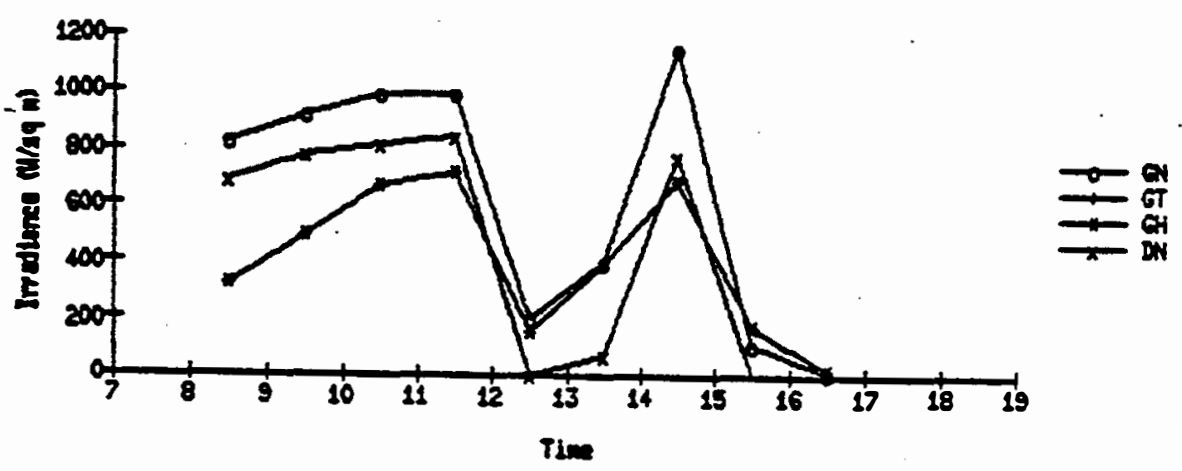

$\operatorname{sese} 3000$

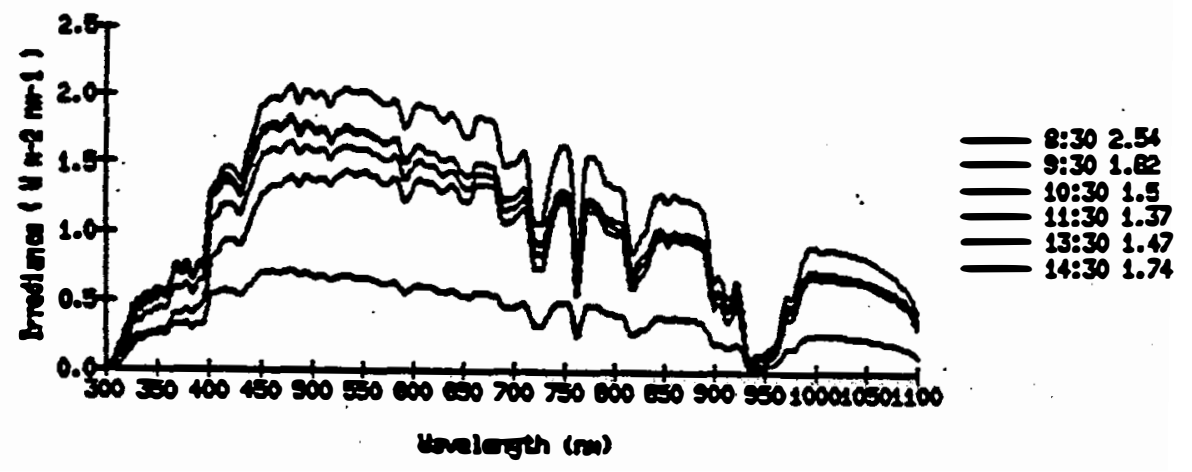




\section{SEN *}

4 FS13836306

$01121 \quad 02 \quad 86 \quad 306 \quad 218$

Bo $\sigma_{1}$

Do albado marrement

Overeast to partif eloudy

ilo photos

$\operatorname{rex} 306$

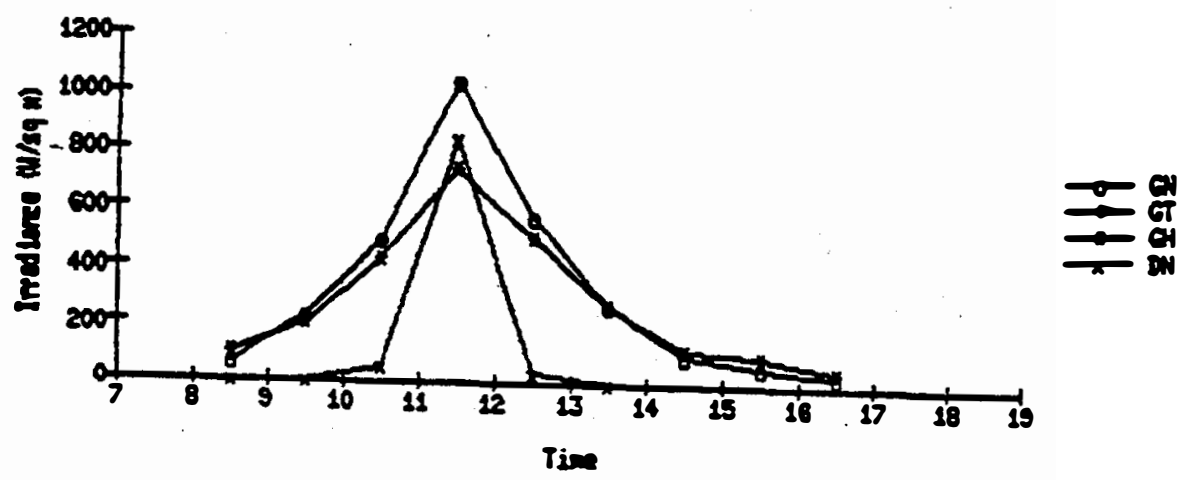

PEx 3000

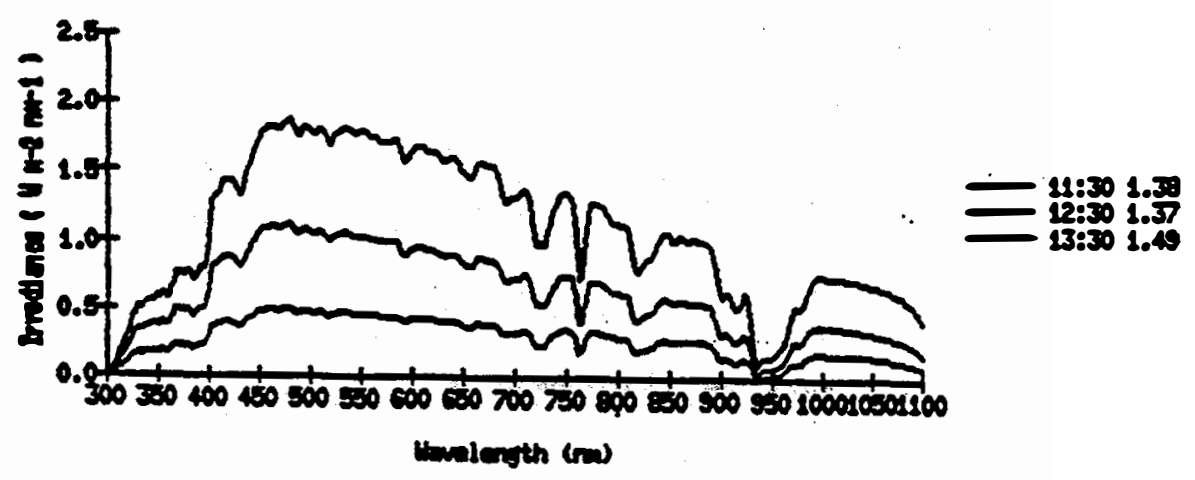




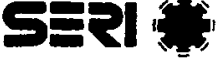

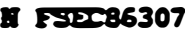

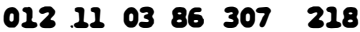

עo

Ho aibedo sasurenent

Temp controller $40 \mathrm{C}$ at 0830 , $40 \mathrm{C}$ at 1430

Partlr elouds

Do photos

PSEC 309

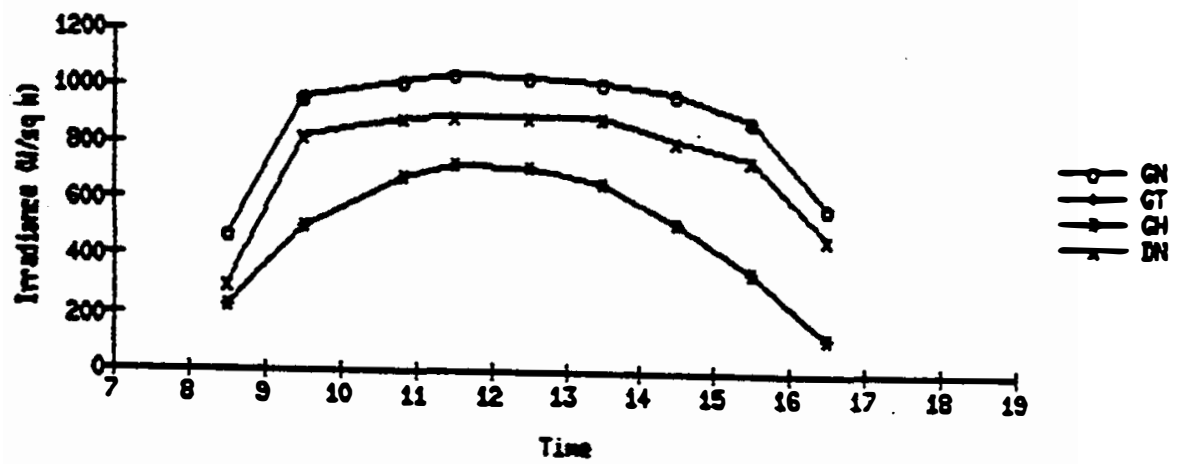

$\operatorname{Psx} 3070$

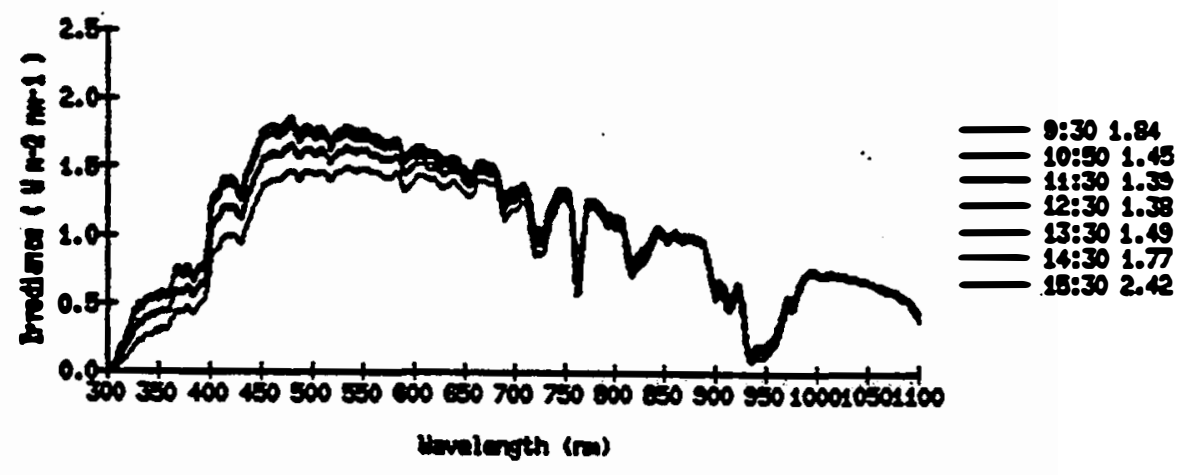


A FSPC86308

$013 \quad 1104 \quad 86 \quad 308 \quad 218$

Ho $\mathbf{F r}$

Ho albedo aessureaent

Texp controller $40 \mathrm{C}$ at 083.08 il C at 1410

Partly cloudy

Do photos

FEeC 300

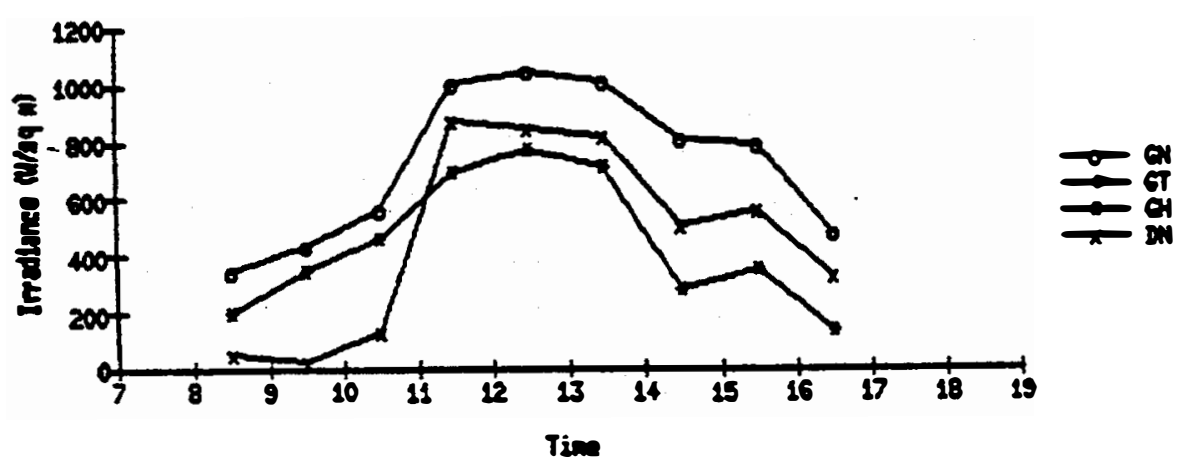

FEב 30000

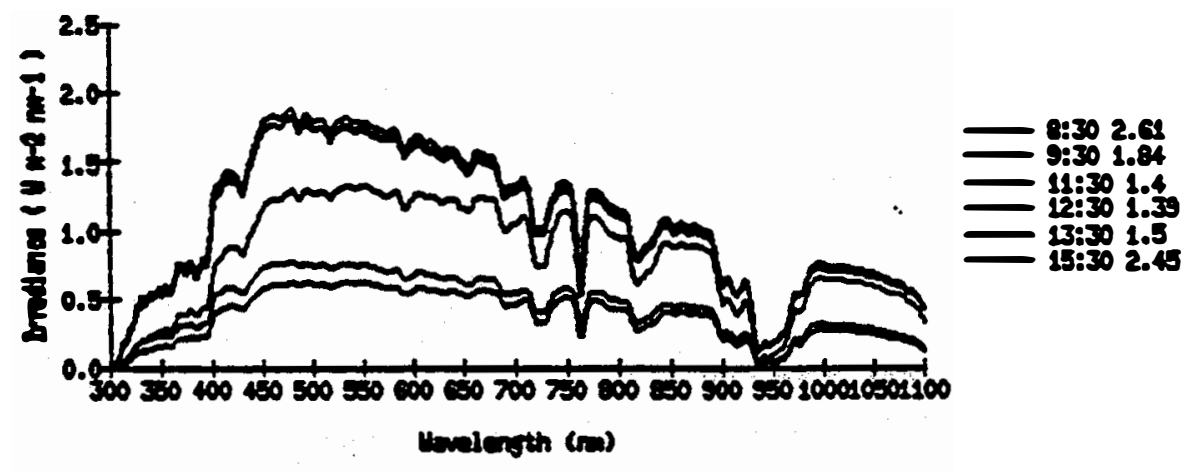


SEP *

I) Pi20:6309

0141105.86309218

Ho 01

Ho alsodo enserroest

Tenp controller $40 \mathrm{C}$ at 0830 s $40 \mathrm{C}$ at 1415

Partly elouds light elrrus elouds

Ho photos

$\operatorname{Fax} 300$

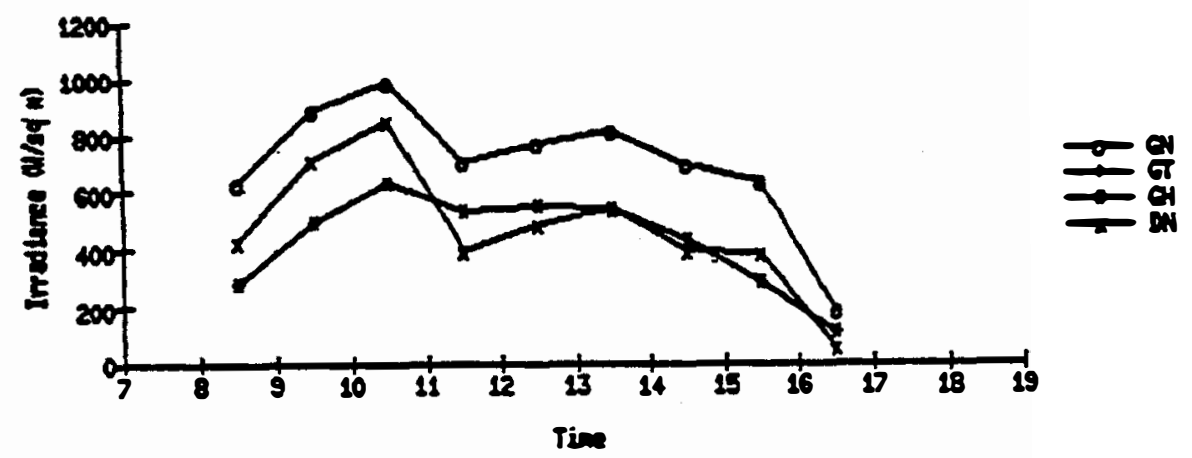

Psex 30000

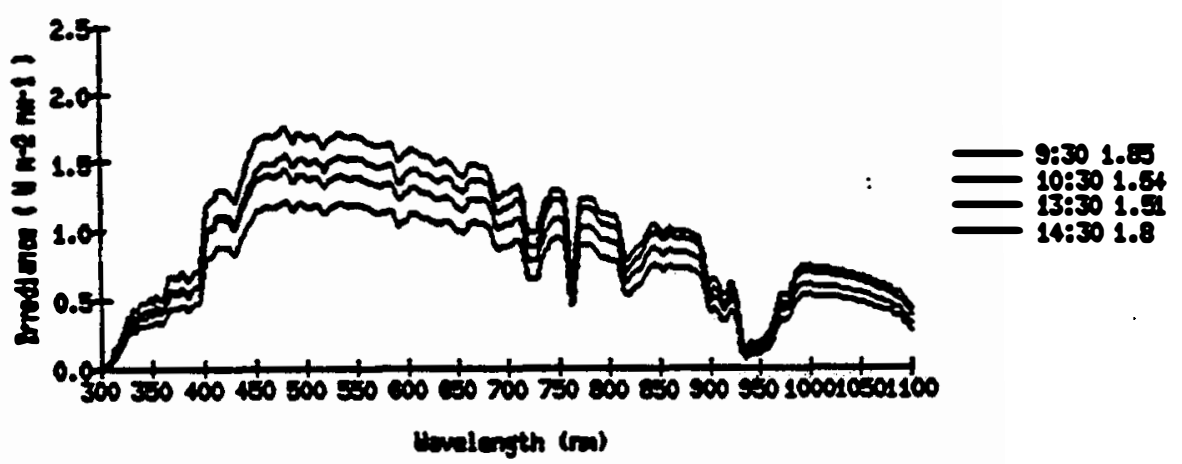


A $7 \$ 3086311$

$\begin{array}{llllll}015 & 11 & 07 & 86 & 311 & 218\end{array}$

to

No uludo neasurgent

Ten sontroller $40 \mathrm{C}$ at 0830

Partly eloudy

Do photos

Fase 311

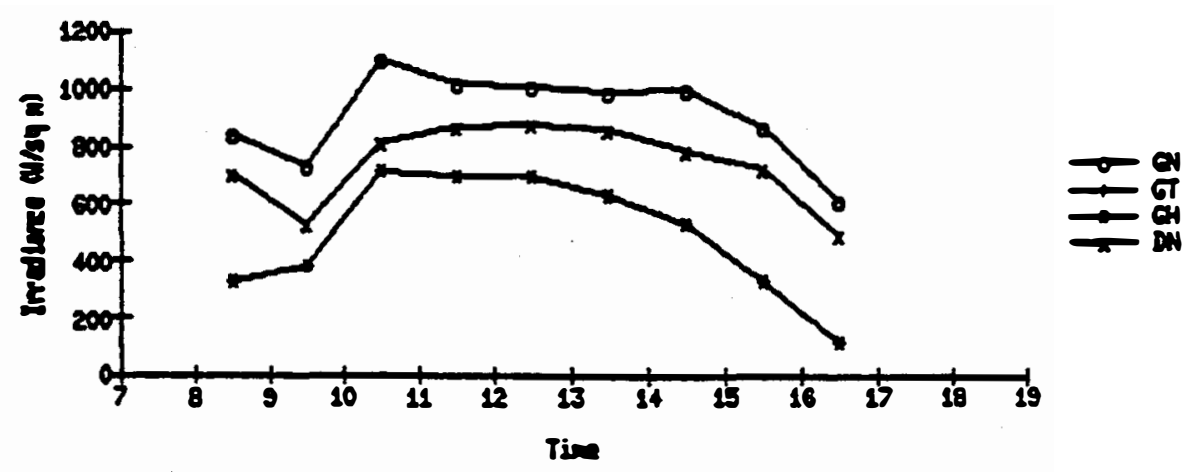

FEE 3120

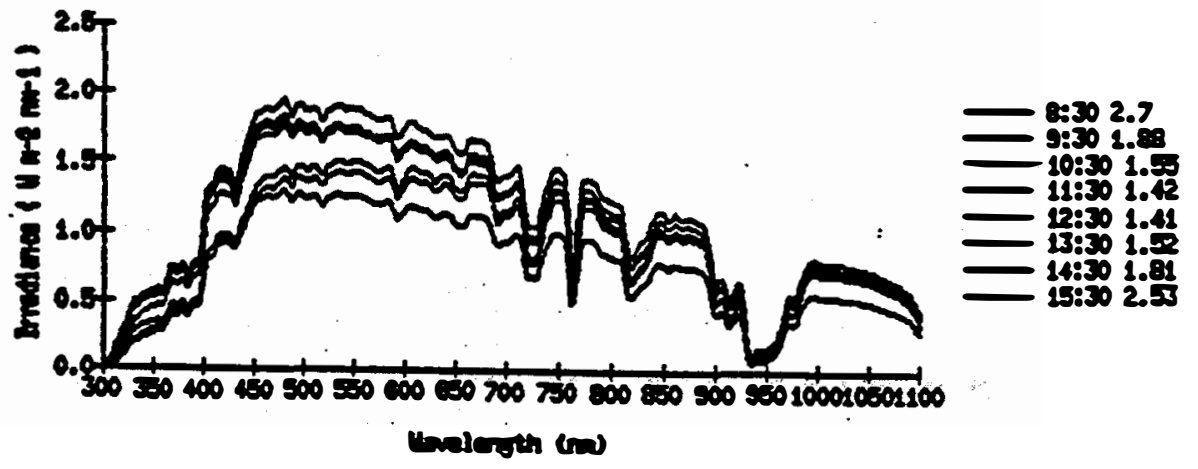


SEP *

A 252086312

016110886312218

Bo $\sigma_{1}$

Ho albado seasureacnt

partly eloudy

Do photos

Fsec 312

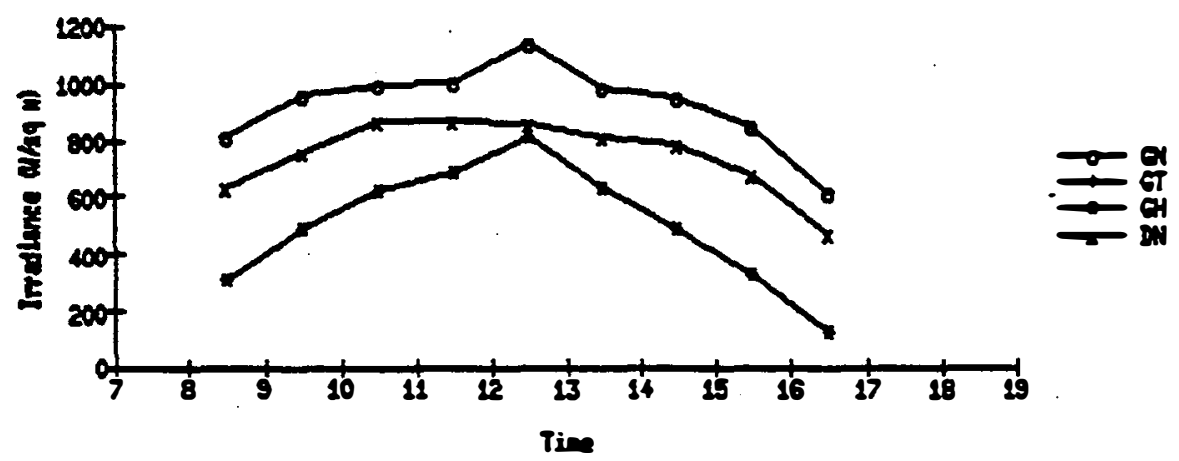

Prex 31200

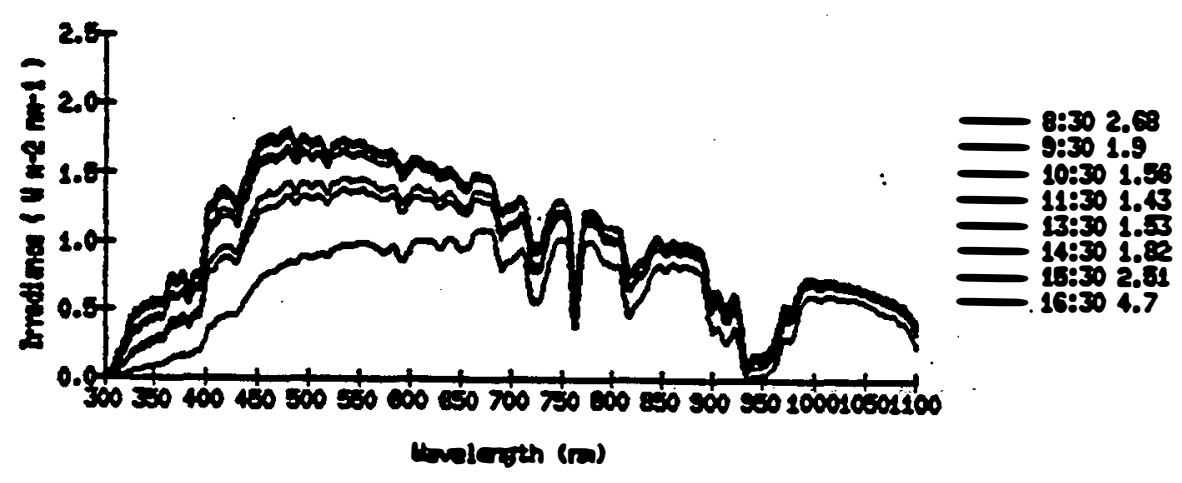




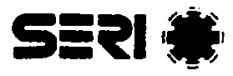

1. Fند286313

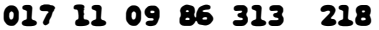

Bo $G$

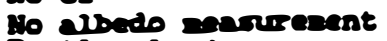

Pertls clouds

Ho pootos

Fsec 313

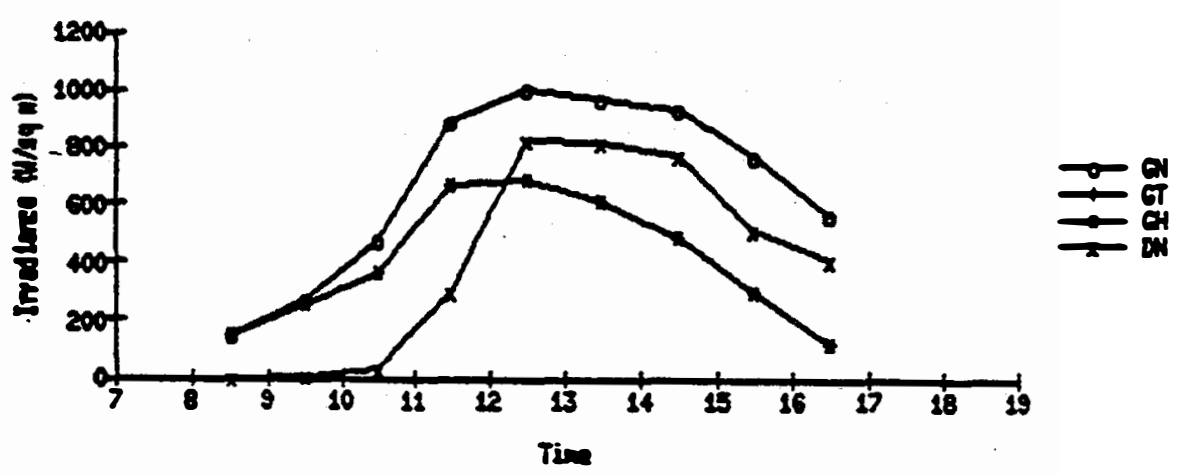

$\operatorname{FES} 31300$

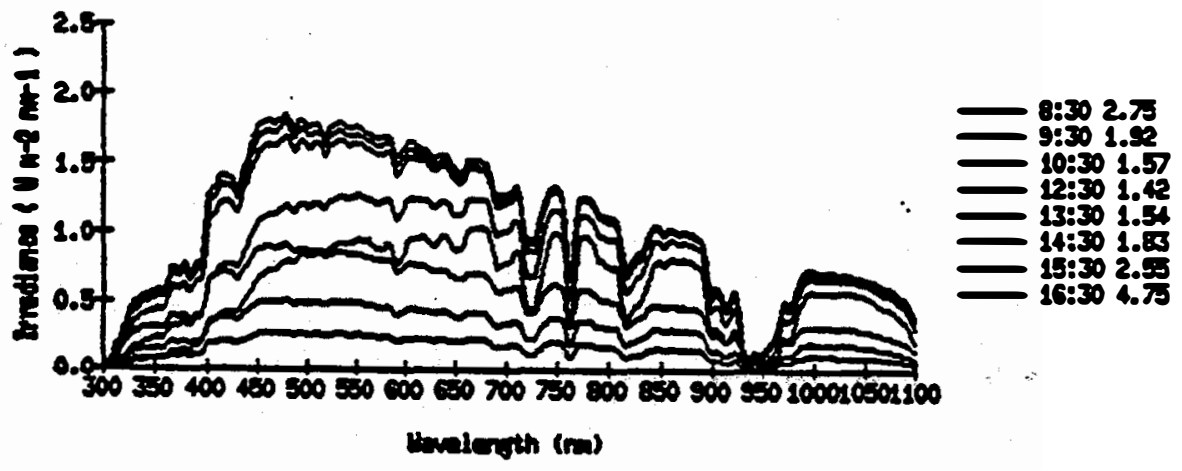




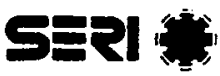

1. ह52086314

$018121086314 \quad 218$

Io $\boldsymbol{F T}^{2}$

Ho elbedo sasturement

Teap controller $40 \mathrm{C}$ at $0810,40 \mathrm{C}$ at 1630

Partly cloudy at 0830; aostly clear at 1630

No photos

Readlings onls at 0830 and 1630

(no spectra)

$\operatorname{Paxc} 314$

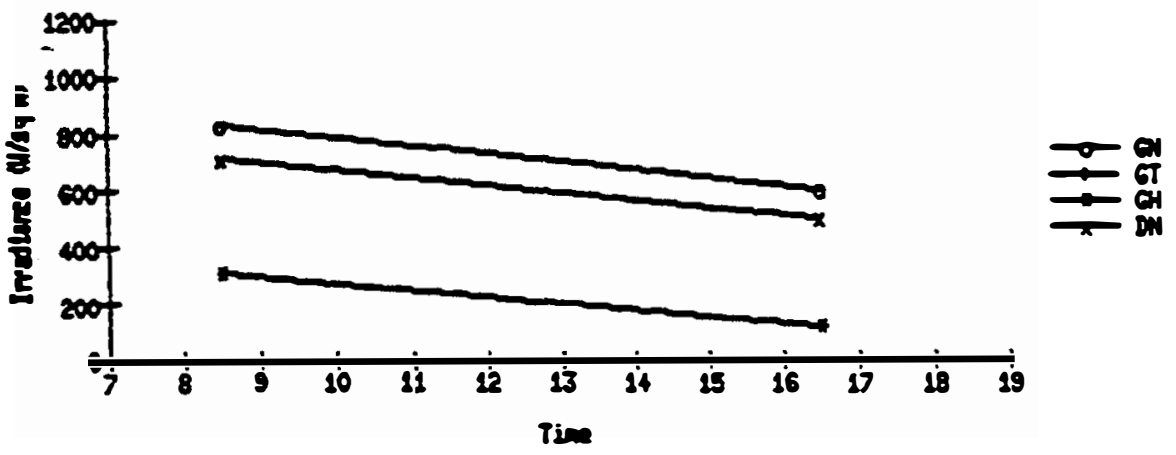


4 Fiग286315

$\begin{array}{lllll}019 & 11 \quad 11.86 \quad 315 \quad 218\end{array}$

Ho $G L$

Ho elsedo Eeasurement

Partis elouds

lio photos

Fise 315

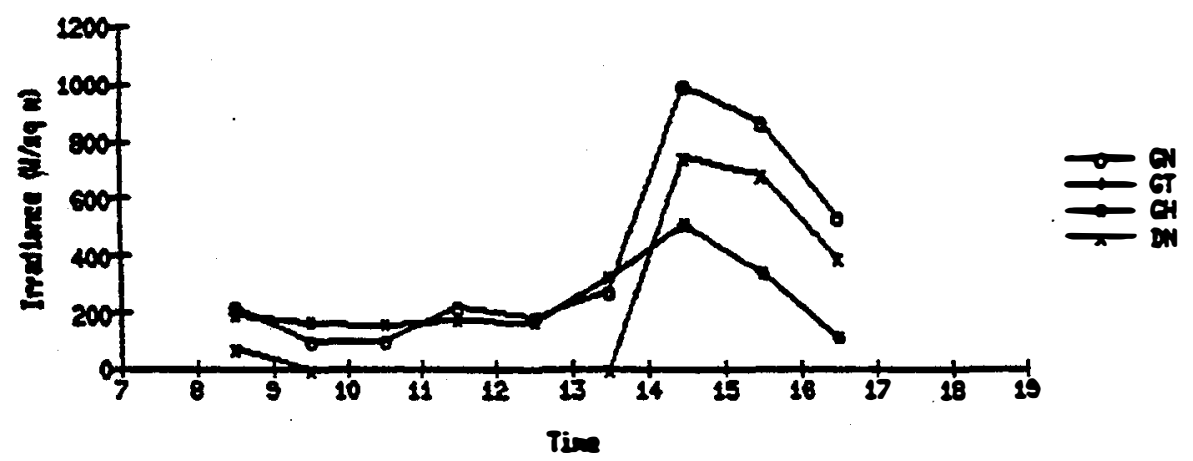

FEx 3150

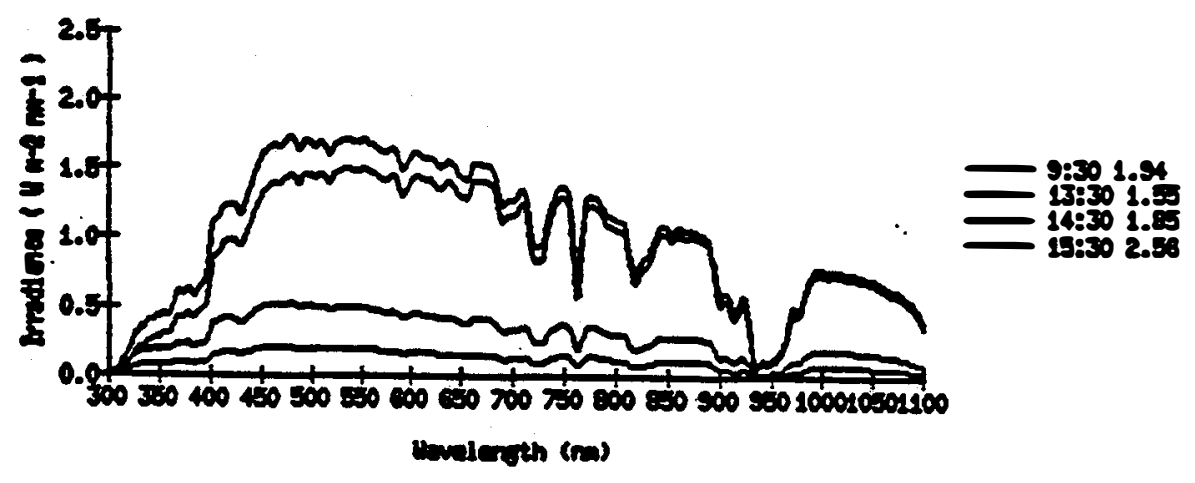




\section{SEP *}

F51286316

$020111286 \quad 316 \quad 218$

No $\sigma^{2}$

Bo albado nensurement

Terp controller $40 \mathrm{C}$ at 0830

Parely clouds

Ho photos

FIs 316

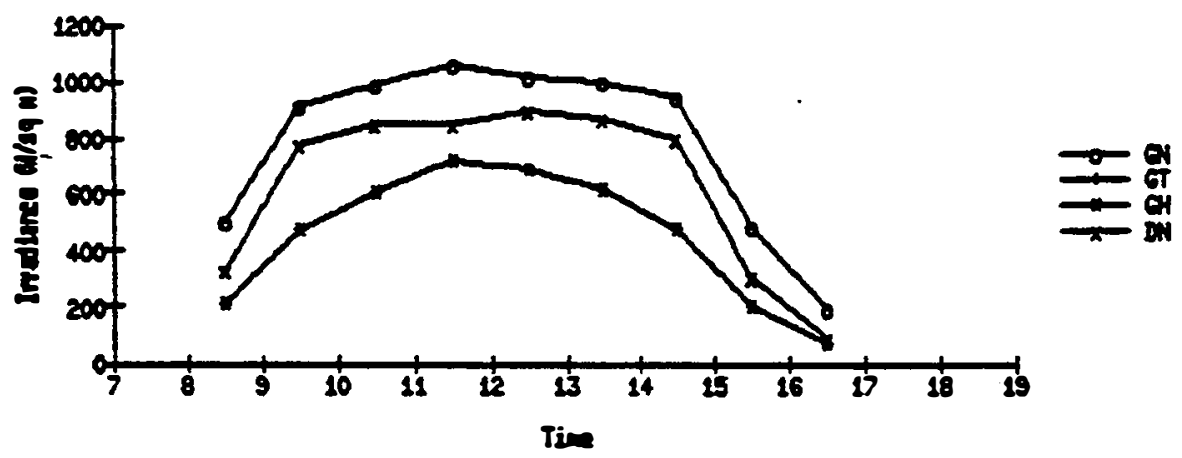

Fas 3460

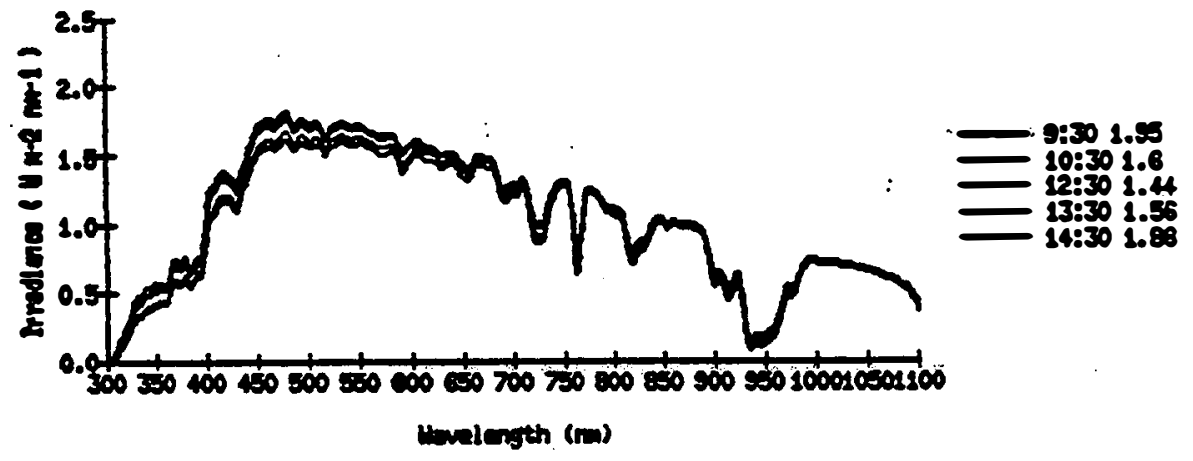


a FS1286317

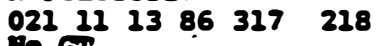

Vio $G$

Do albado cantureacent

Tenp controller $40 \mathrm{C}$ at 0830 ; $40 \mathrm{C}$ at 1430

Eurly norming fogi nontly clear

Pmotos? $1530 \mathrm{G}$

Fase 317

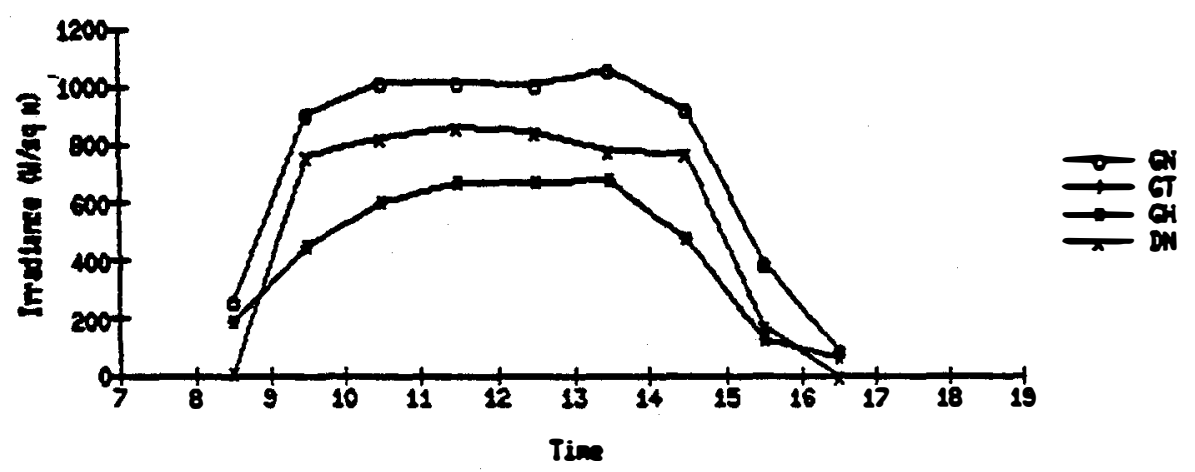

F=36 31700

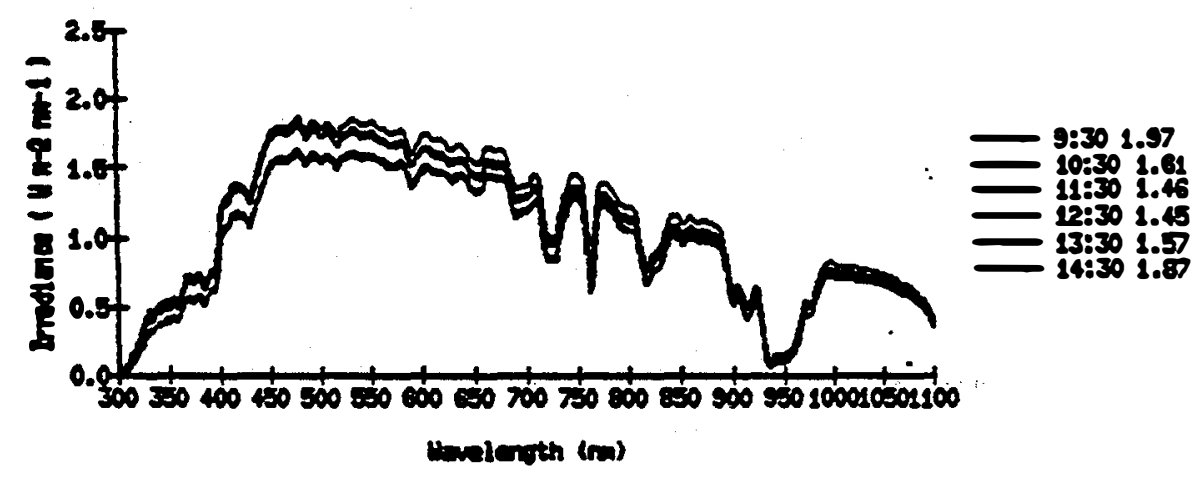




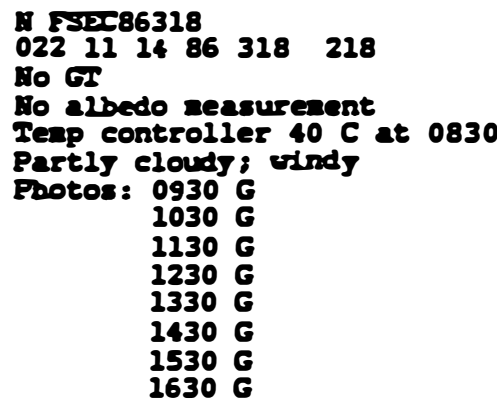

PSEC 318

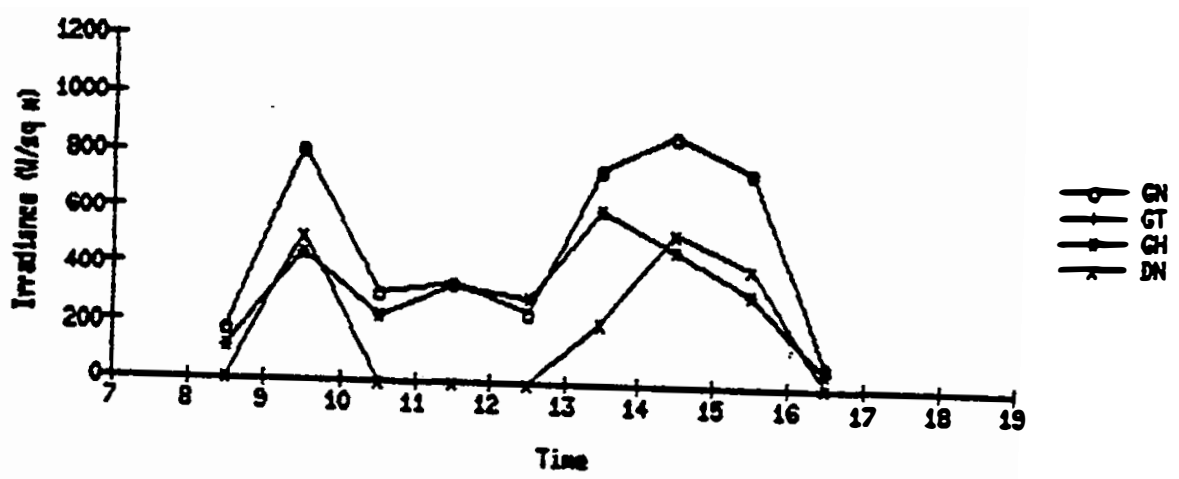

$\operatorname{Pssc} 3400$

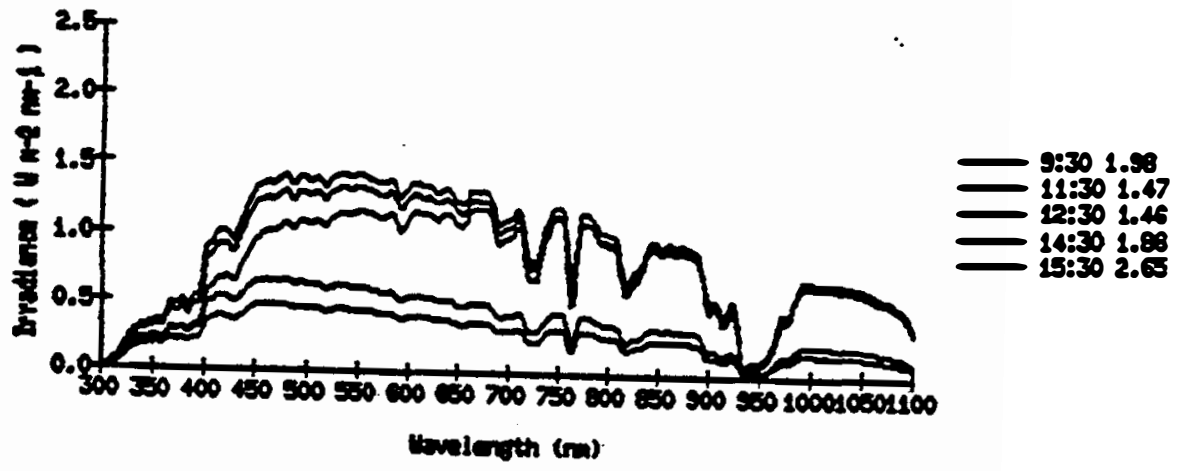


i. ए512086319

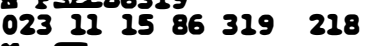

Mo GI

Ho albodo mensurceent

Pertly elouds Mis overenst Fy

Protos: $0830 \mathrm{G}$

$0930 \mathrm{G}$

$1030 \mathrm{G}$

$1130 \mathrm{G}$

$1230 \mathrm{G}$

$1330 \mathrm{G}$

$1430 \mathrm{G}$

$2530 \mathrm{G}$

$1630 \mathrm{G}$

$F=2319$

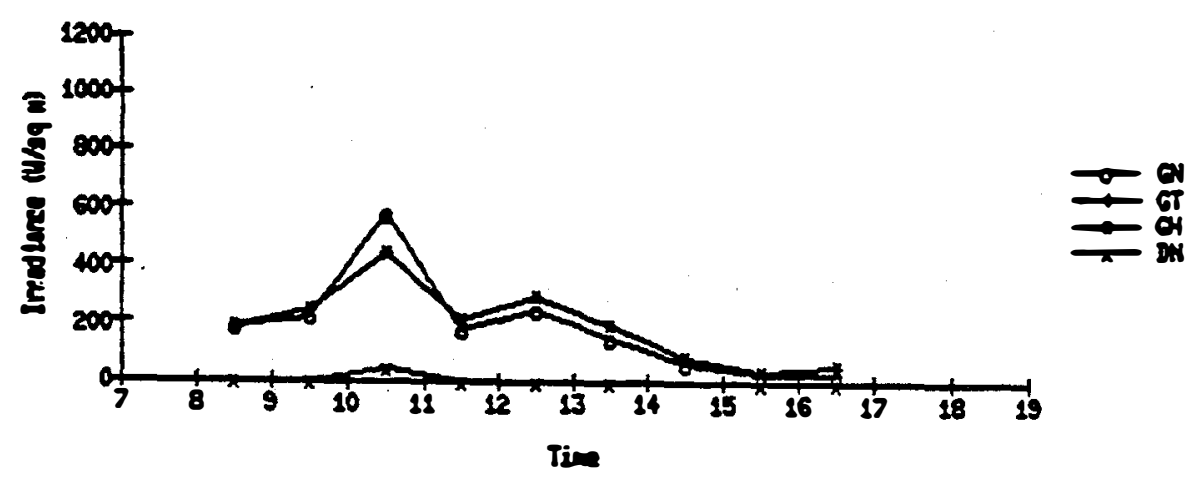

pise 31900

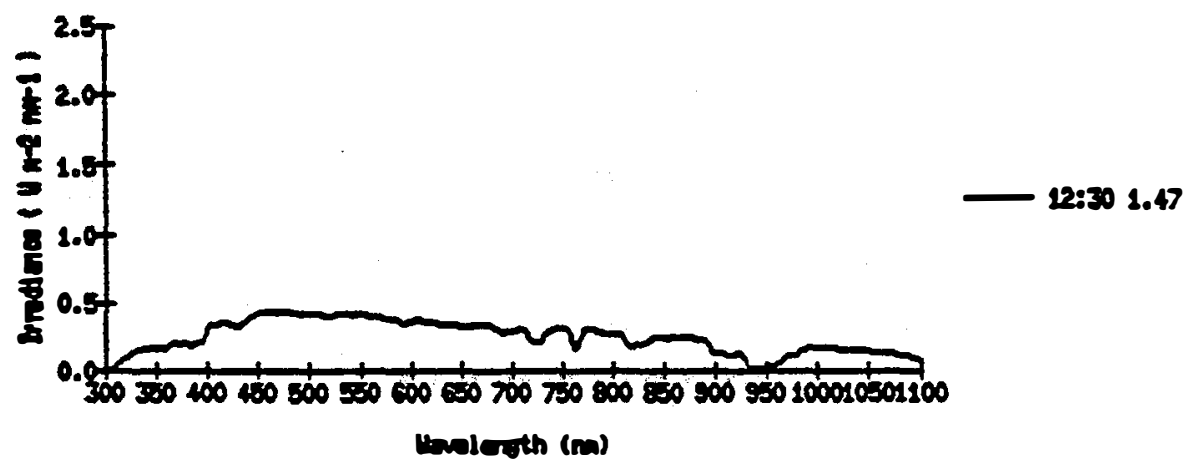




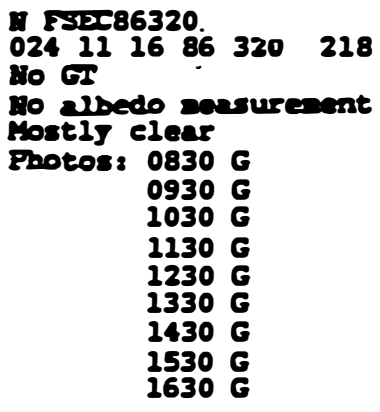

Pase

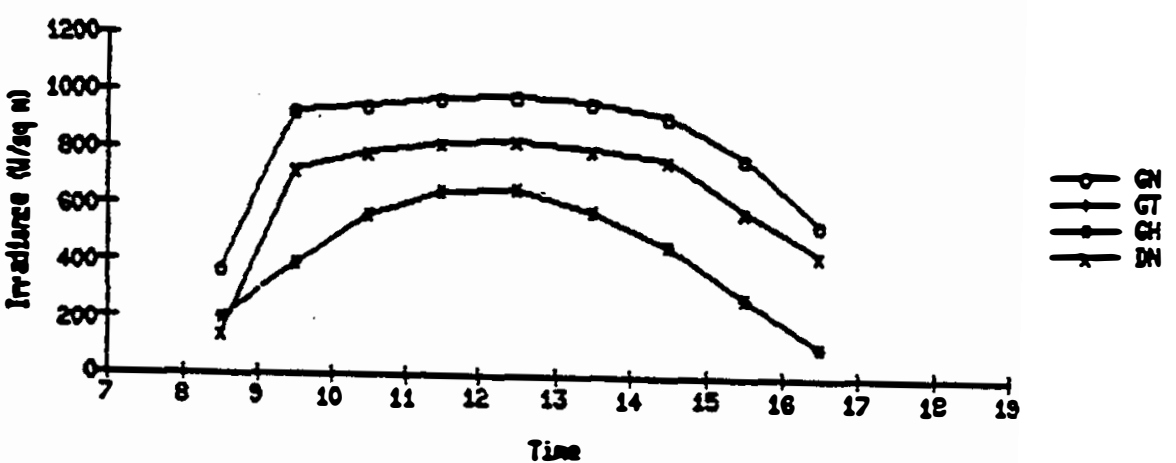

rix $=000$

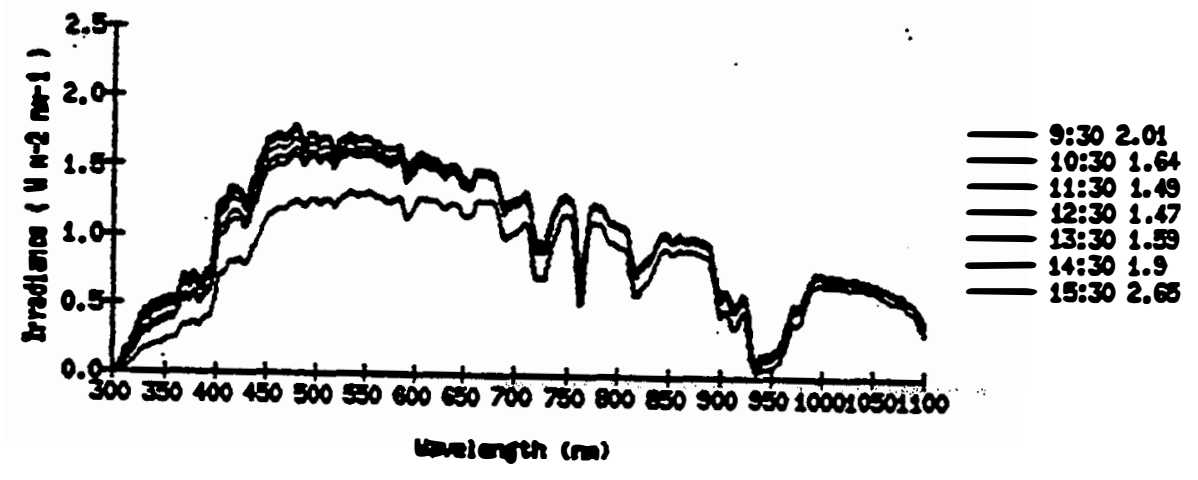




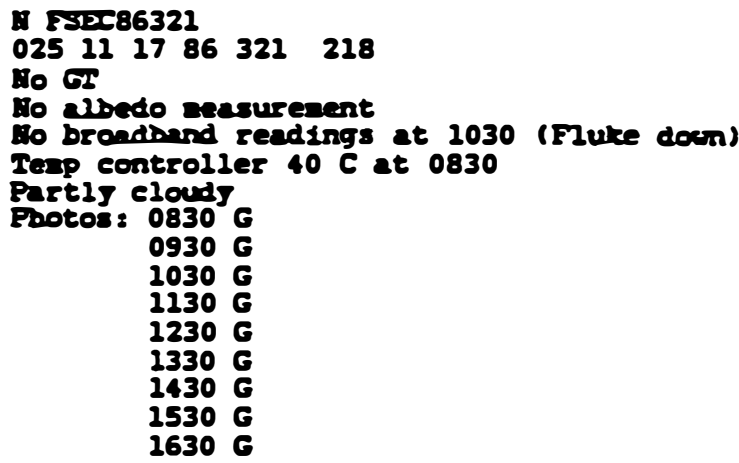

$\operatorname{Fsec} 321$

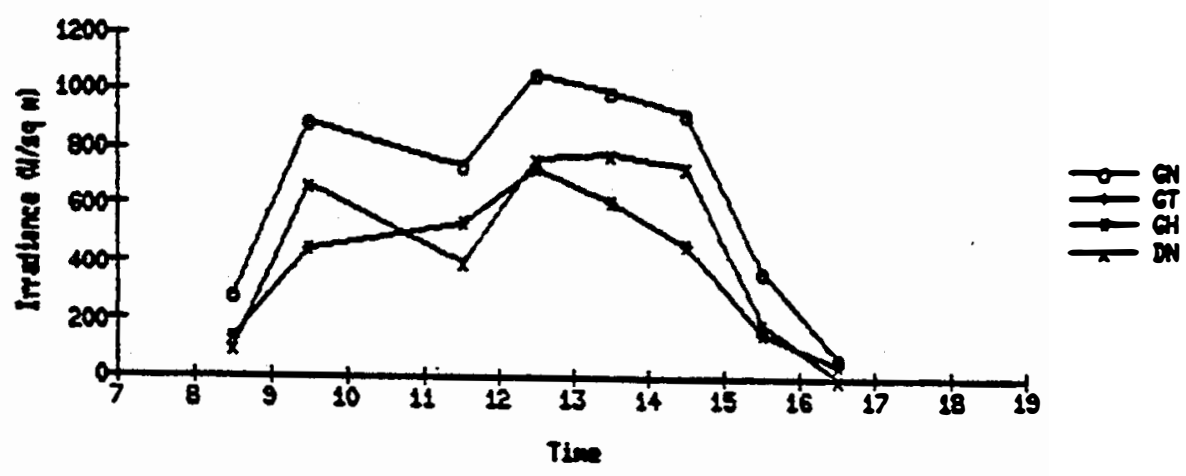

Fex $3 x$ on

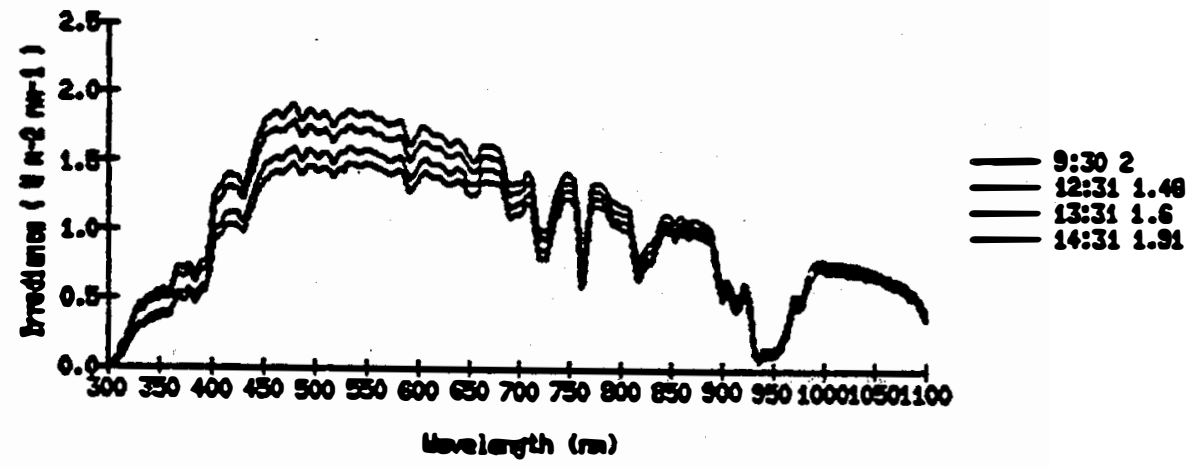


एड्रत86322

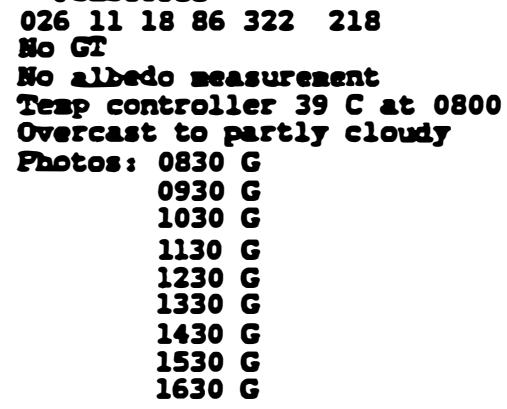

$1330 \mathrm{G}$

$1430 \mathrm{G}$

$1530 \mathrm{G}$

$1630 \mathrm{G}$

0831 bef/aft rendinge different

PEST

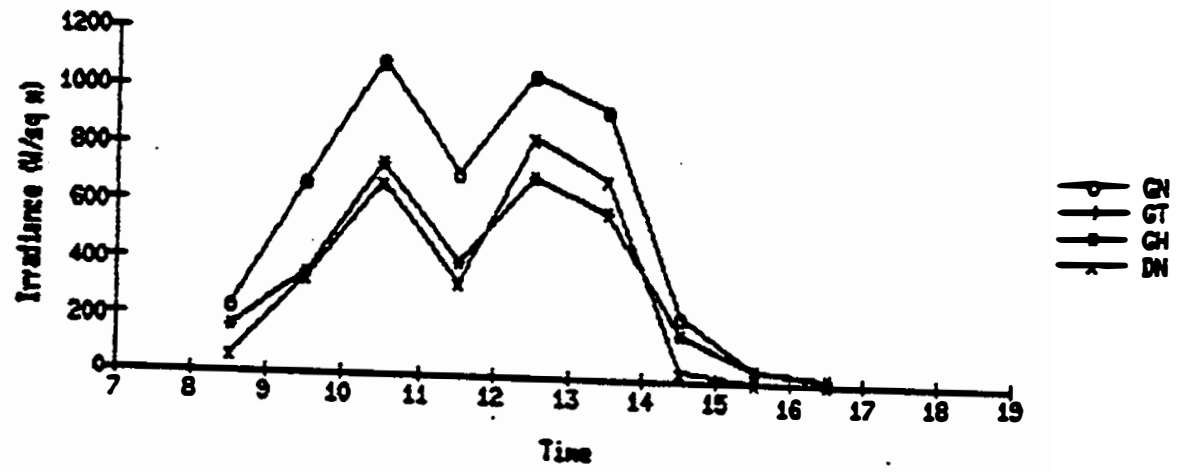

$\sin =0$

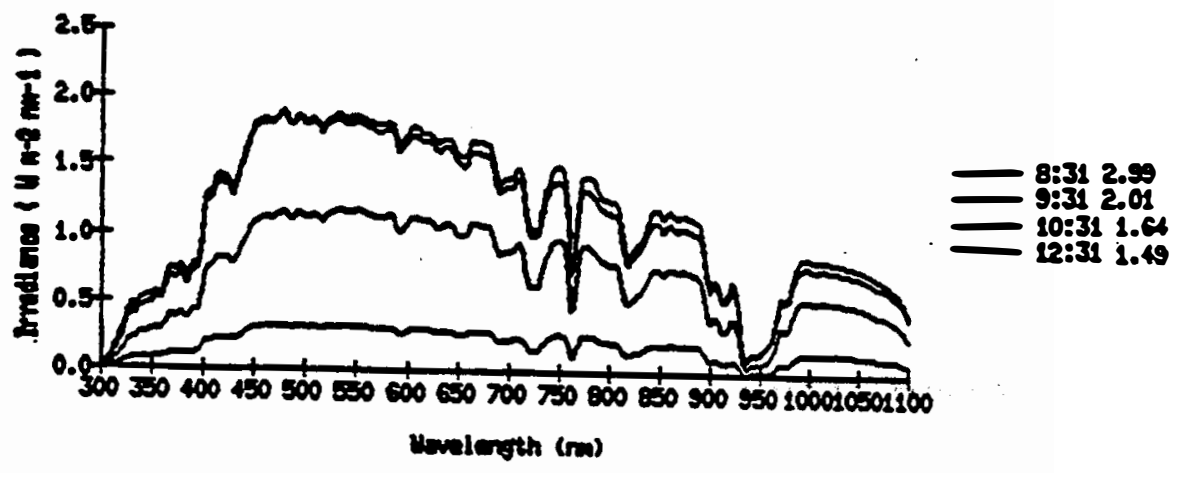




\section{SEP}

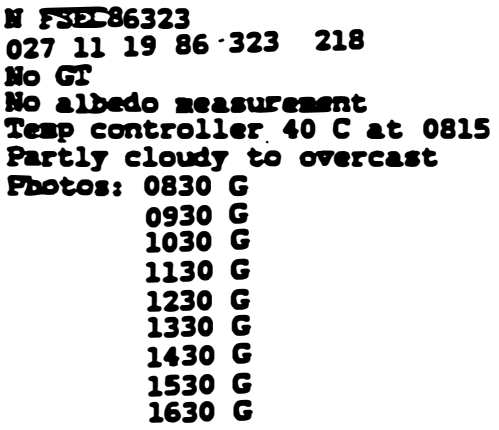

$\operatorname{Fsec} 323$

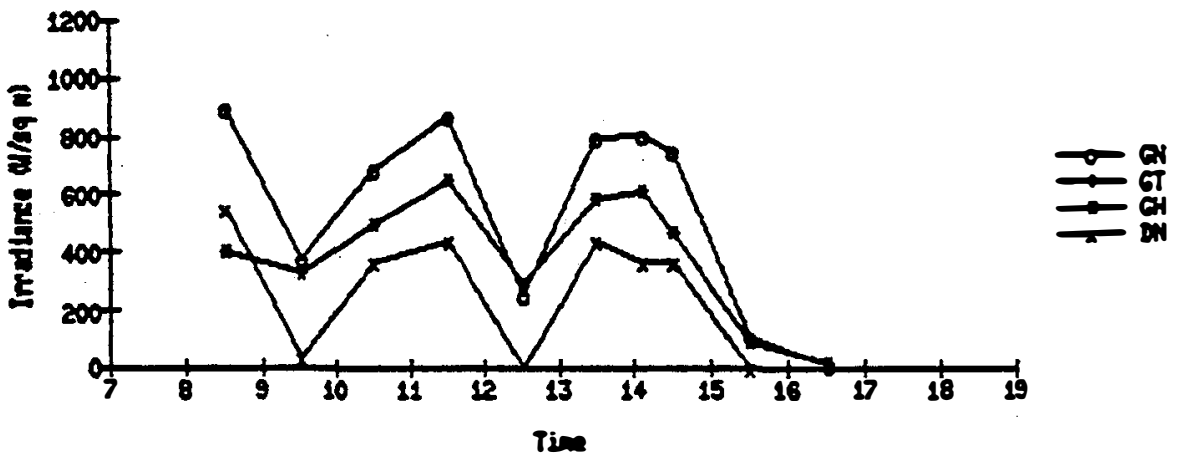

Fist 20300

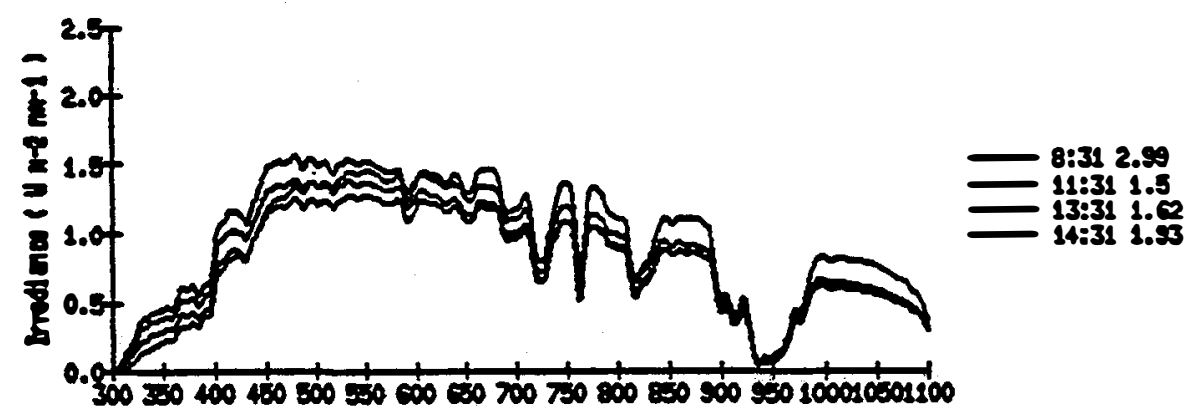

boviergth (m) 


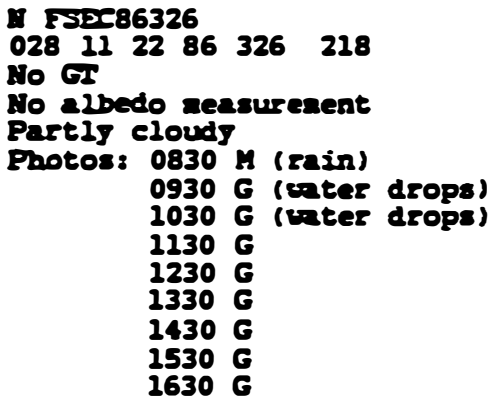

Fsec 325

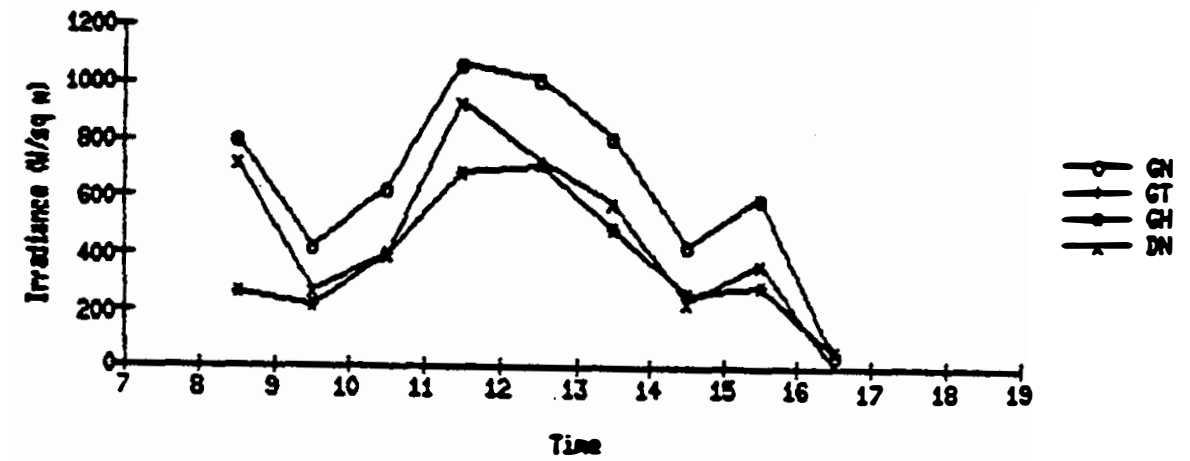

Fix $=5000$

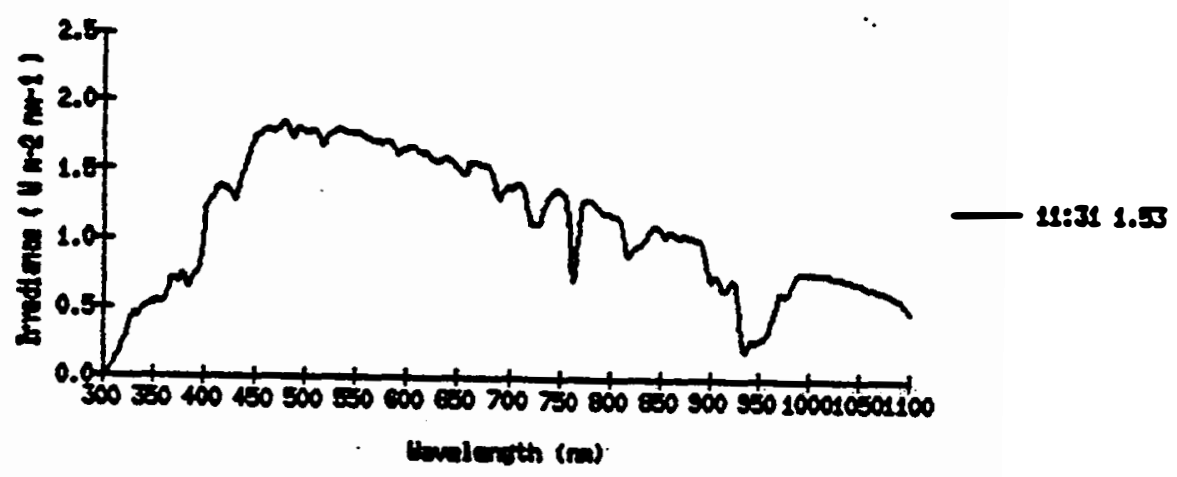


1. एक्य 86327

$\begin{array}{lllll}029 & 23 & 86 & 327 & 218\end{array}$

Bo $\boldsymbol{G r}$

Ho albedo sensurement

clear to partis clouds

Frotos: $0830 \mathrm{G}$

$0930 \mathrm{G}$

$1030 \mathrm{G}$

$1130 \mathrm{G}$

$1230 \mathrm{G}$

$1330 \mathrm{G}$

$1430 \mathrm{G}$

$1530 \mathrm{G}$

$1630 \mathrm{G}$

Fsec 30

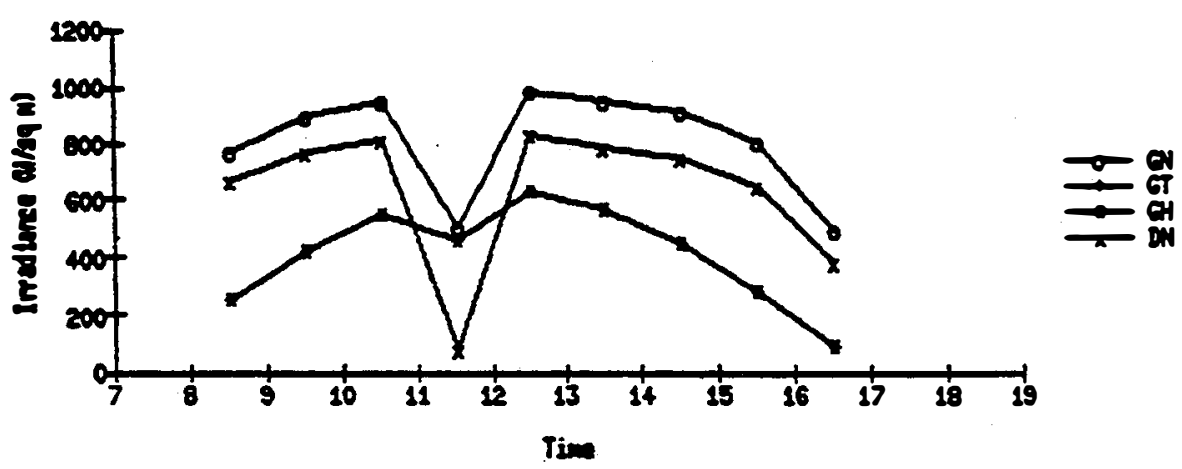

$\operatorname{Fsec} \pi 0$

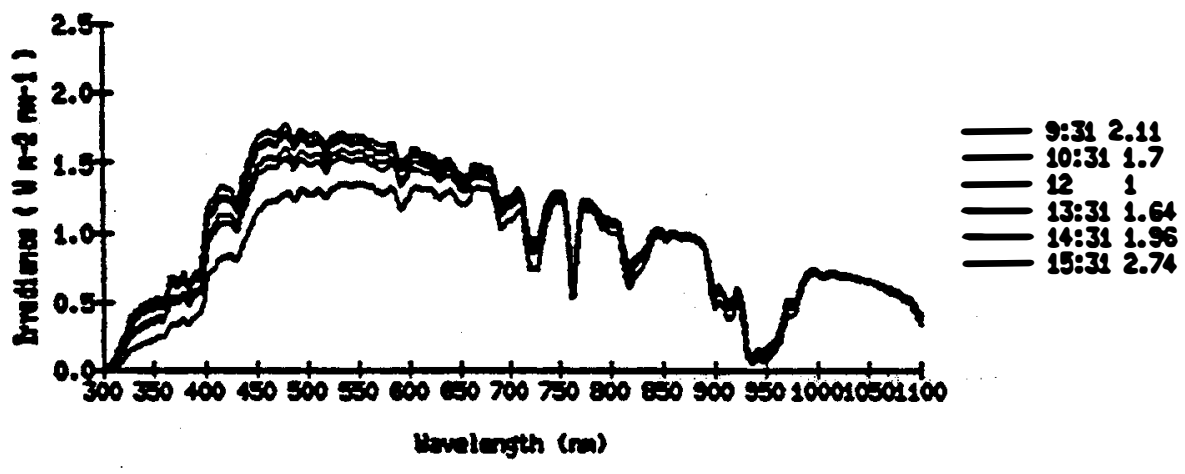




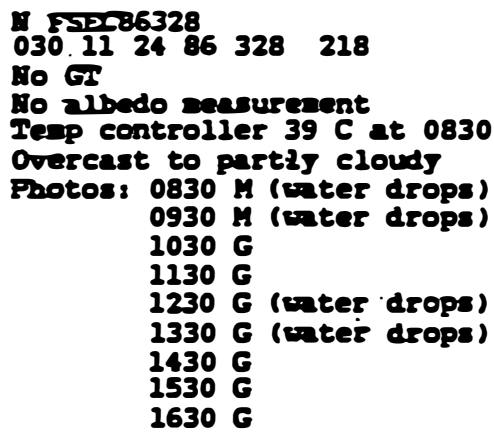

Fase 200

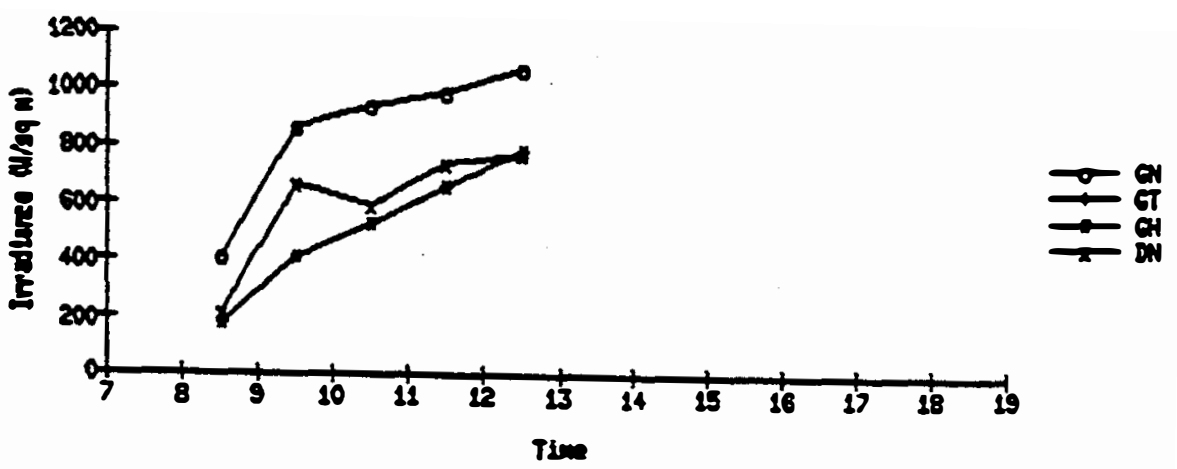

Fise 3000

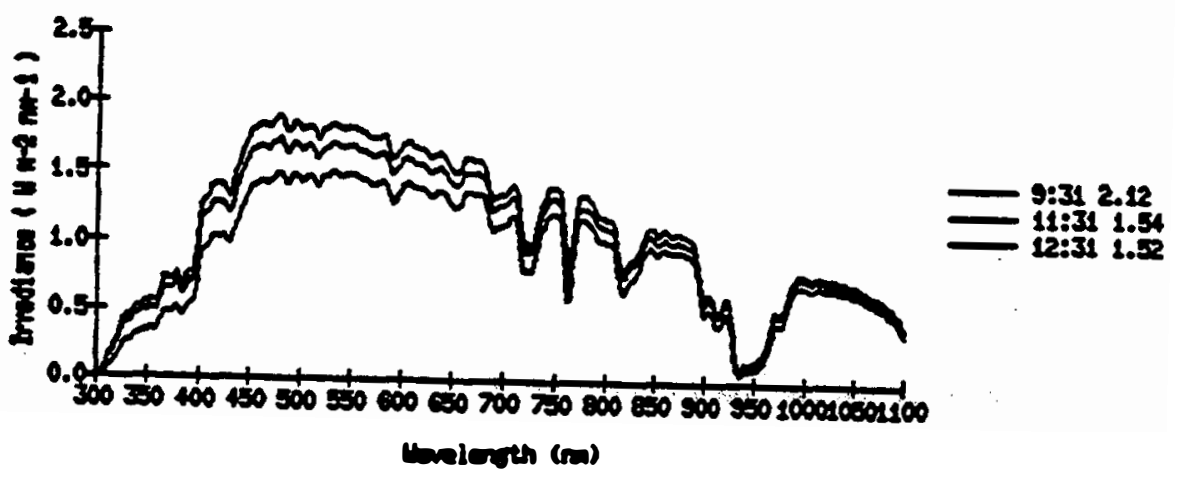


A PSग्य 86329

031112586329218

GI and Muedo PSP installed

Mes eal constant for GS PSP (ves 9.0; now 8.85)

Tenp controller $40 \mathrm{C}$ at 1030

Clear to partly cloudy

Fbotos? $0830 \mathrm{G}$

$0930 \mathrm{C}$

$1030 \mathrm{G}$

$1130 \mathrm{G}$

$1230 \mathrm{G}$

$1330 \mathrm{G}$

$1430 \mathrm{G}$

$1530 \mathrm{G}$

$1630 \mathrm{G}$

1231 SA. SH, Eis are -99 (before)

Fsec 320

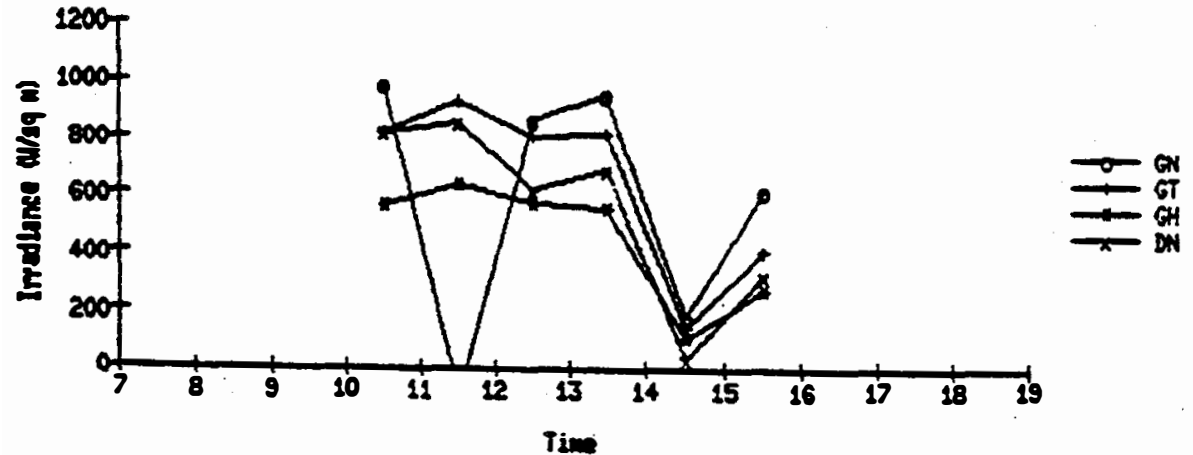

pas 200

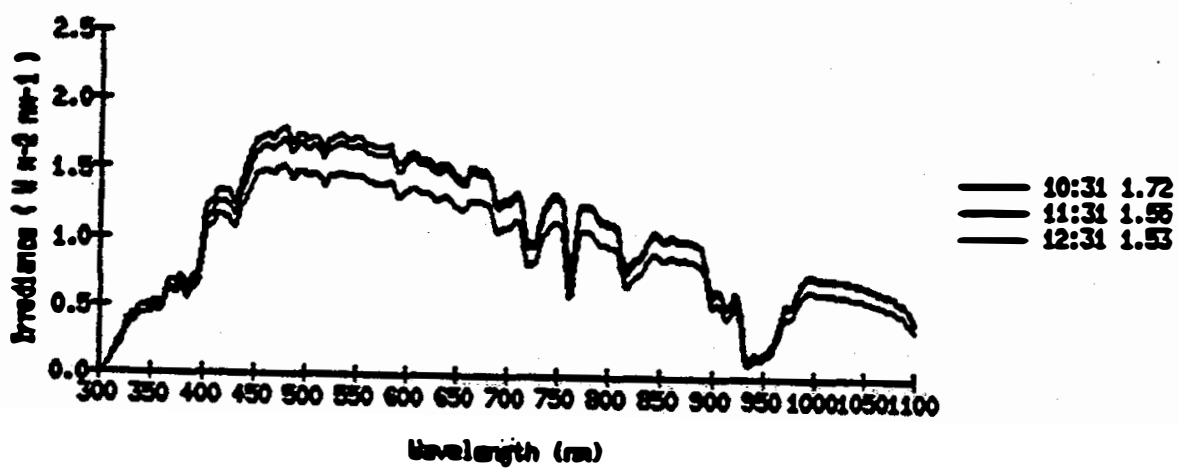


P Fi2C86330

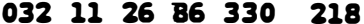

Most IJ orercest

Fhotos: $0830 \mathrm{G}$

$0930 \mathrm{G}$

$1030 \mathrm{G}$

$1130 \mathrm{G}$

$1230 \mathrm{G}$

$1330 \mathrm{G}$

$1430 \mathrm{G}$

$1530 \mathrm{G}$

$1630 \mathrm{G}$

Fise 300

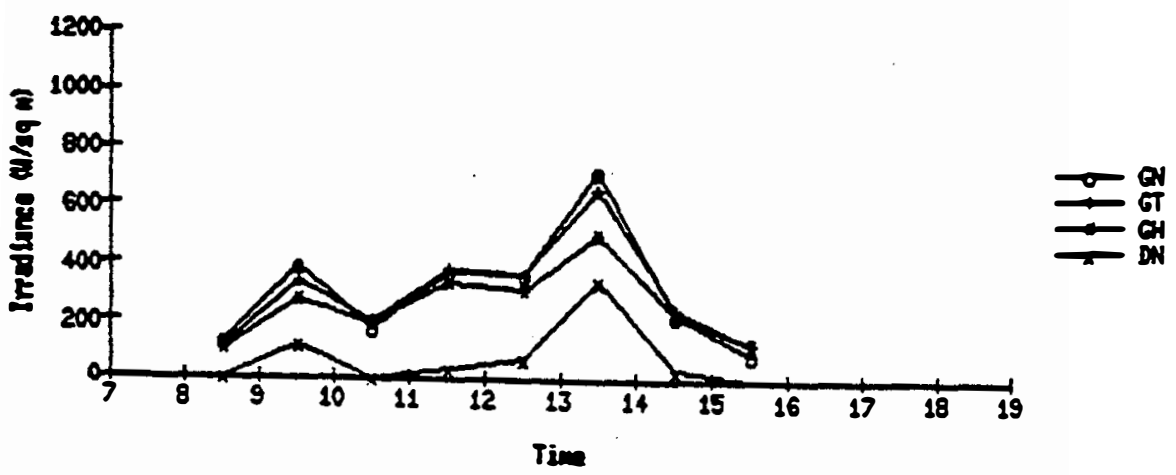

PSEC 35000

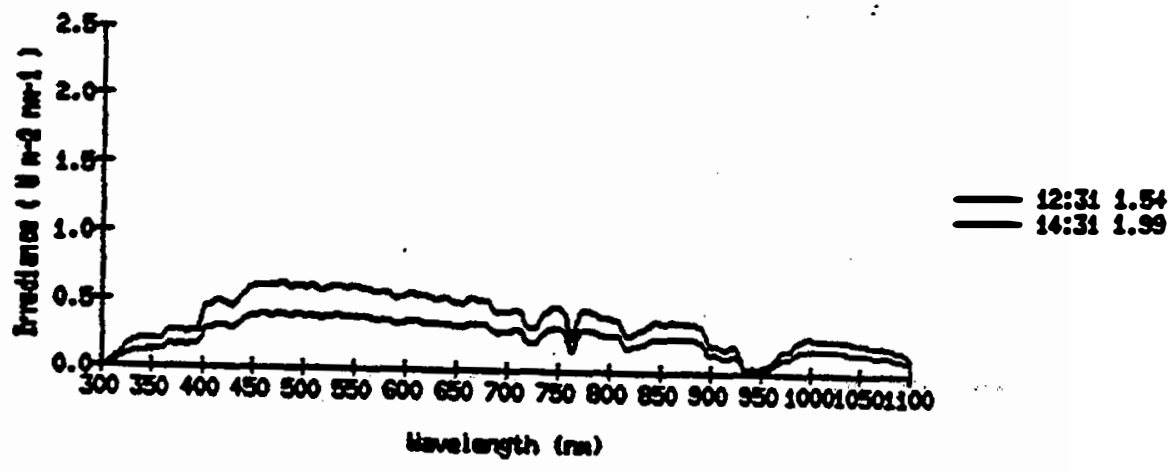




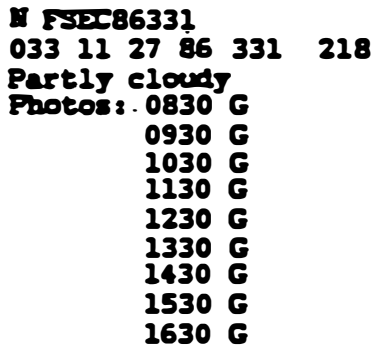

Faxe 331

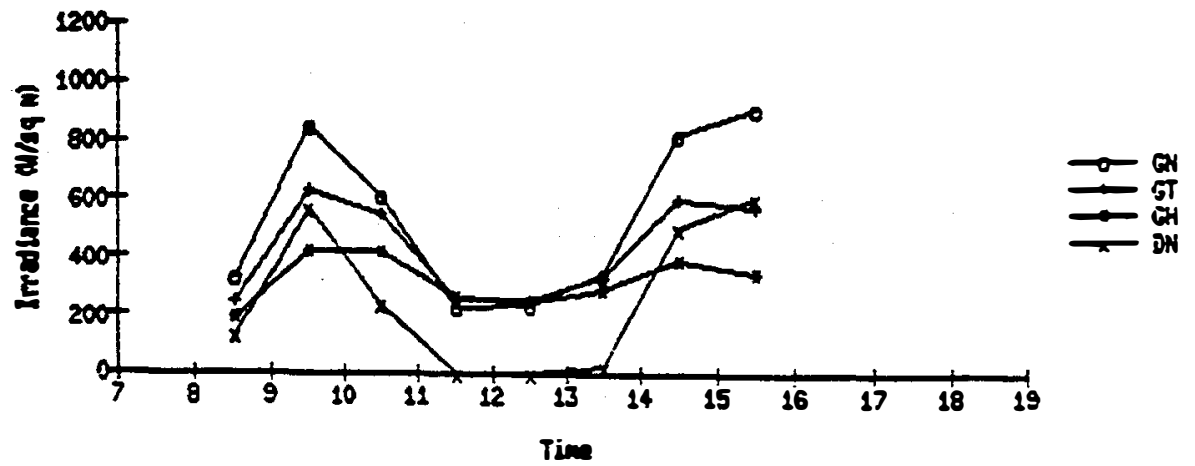

Fiss 320

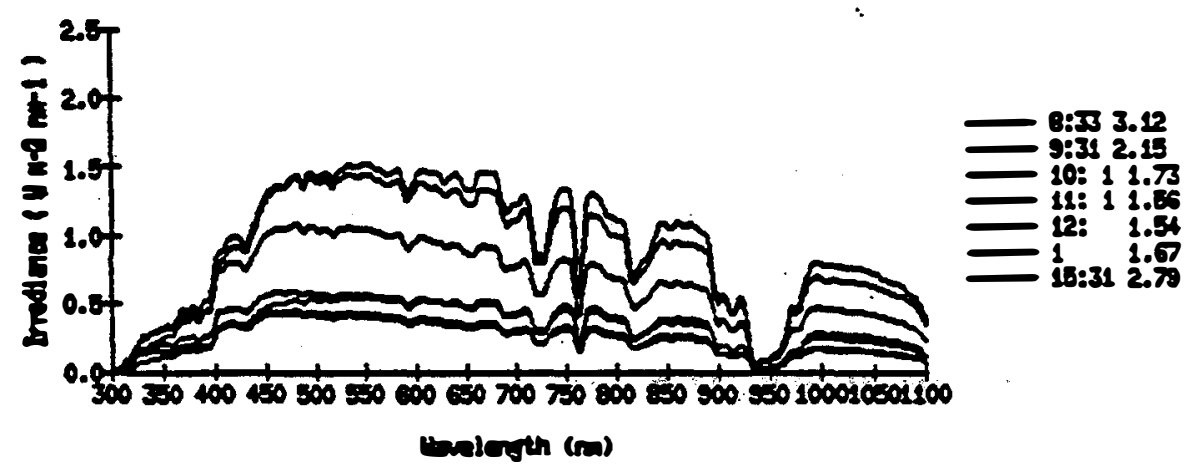


1. ए52086332

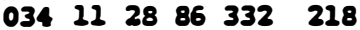

Partls eloidy

Protos: $0830 \mathrm{G}$

0930

$1030 \mathrm{G}$

$1230 \mathrm{G}$

$1230 \mathrm{G}$

$1330 \mathrm{G}$

$1430 \mathrm{G}$

(yliseing 11-28 1530 through

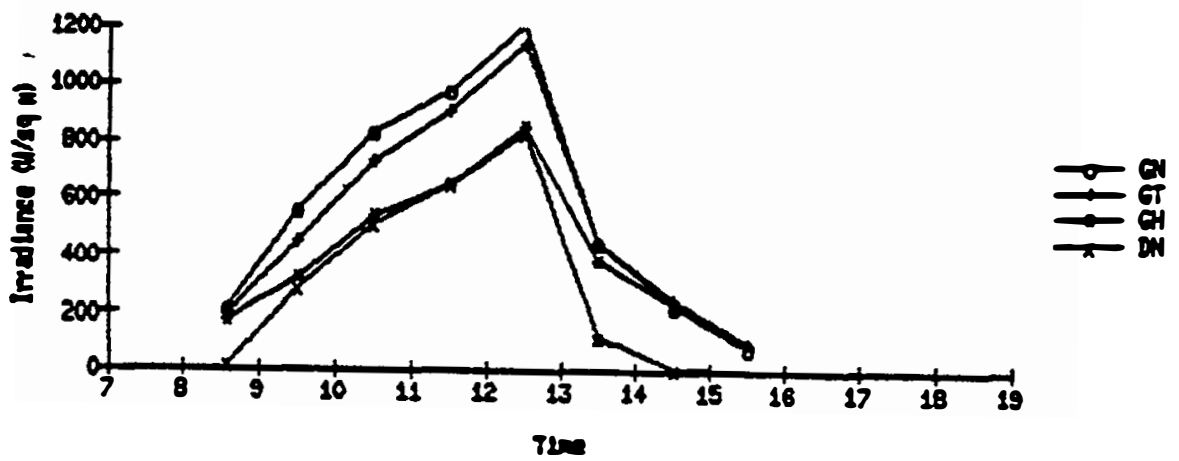

Fises or

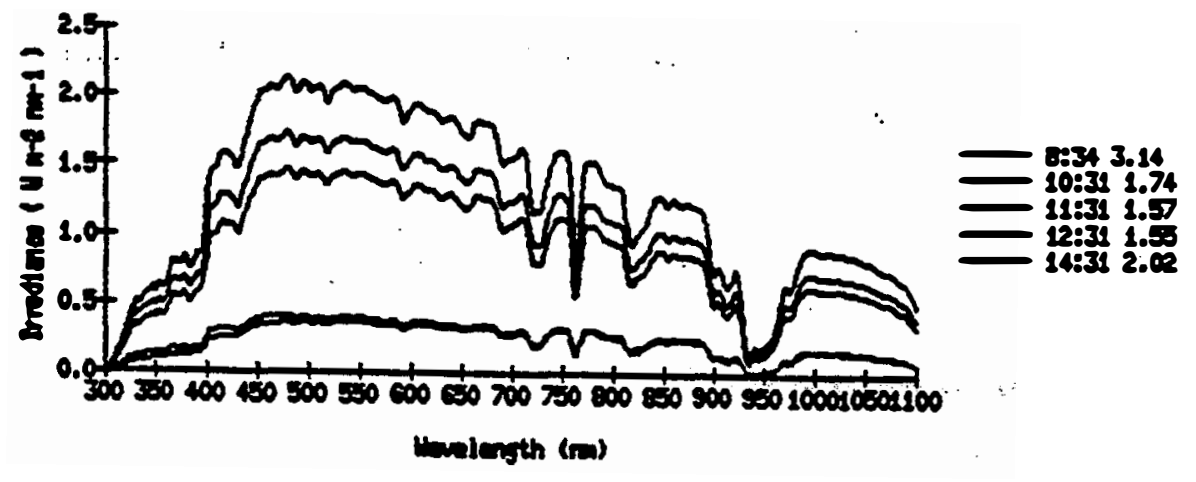


D F51286333

$\begin{array}{llllll}035 & 11 & 29 & 86 & 333 & 218\end{array}$

Partis cloudy

ino photos

$\operatorname{Psec} 353$

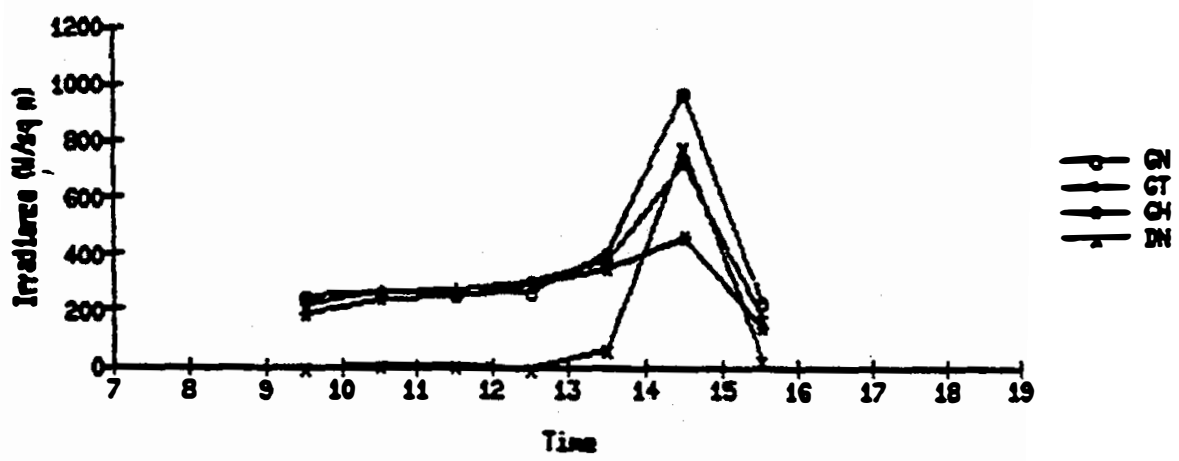

pase 300

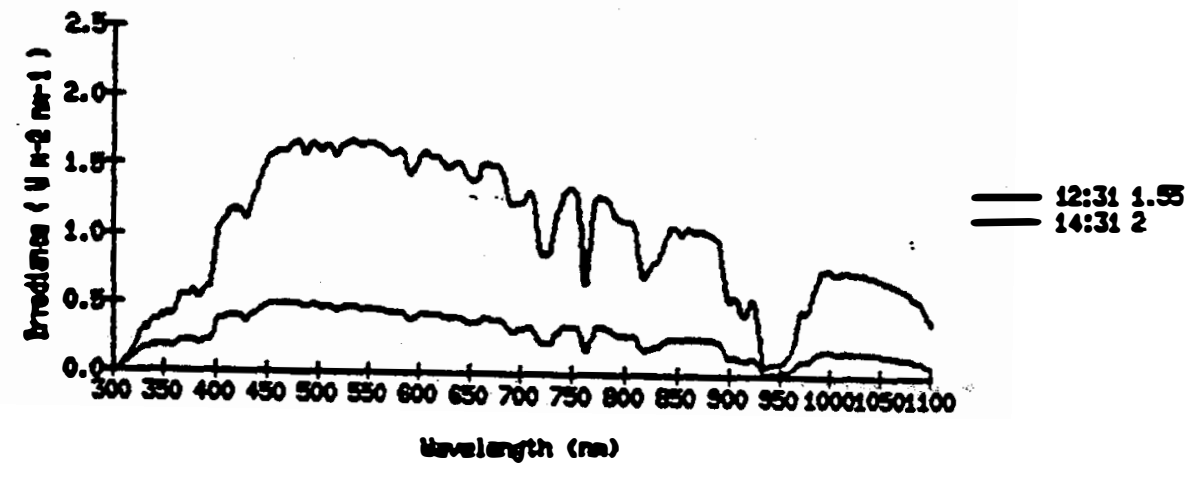




\section{FSP86334}

$\begin{array}{lllll}036 \quad 1130 \quad 86 & 334 & 218\end{array}$

Pertls cloudy to clear

Ppotos: Bone in norming

$1330 \mathrm{G}$ (unter drops)

$1430 \mathrm{G}$ (enter drops)

$1530 \mathrm{G}$ (enter drops)

$1630 \mathrm{G}$ (leter drops)

Fase 33

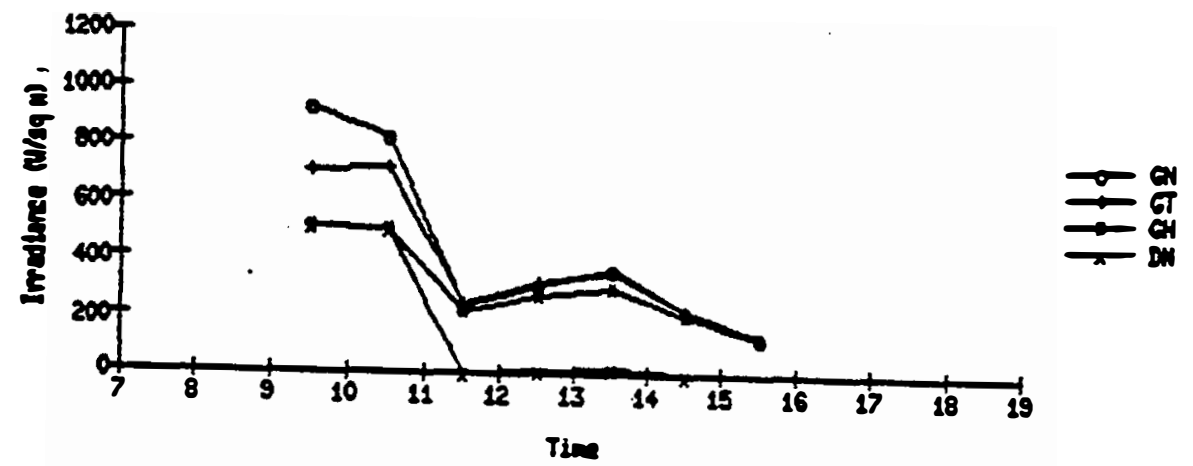

Fax 300

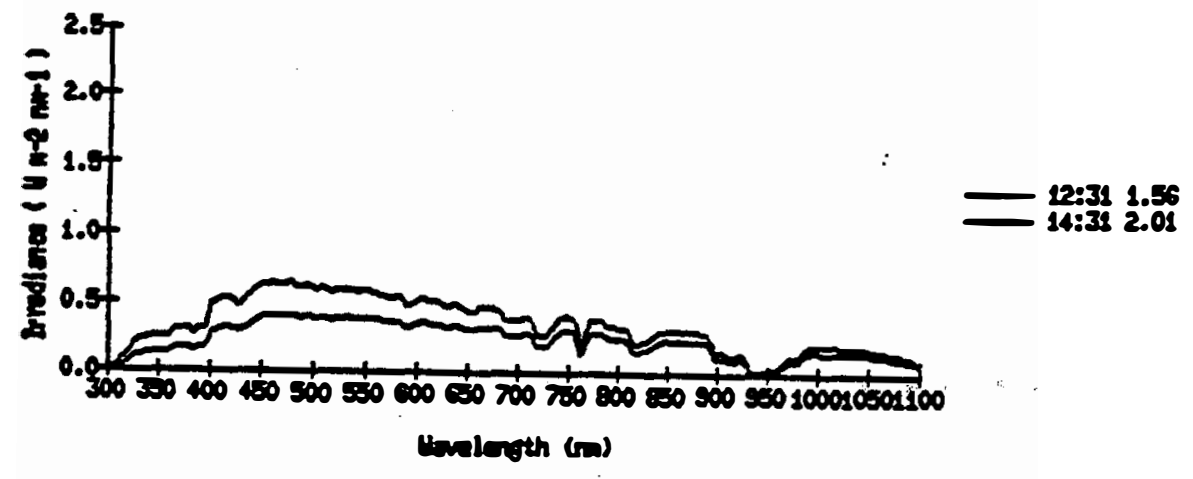




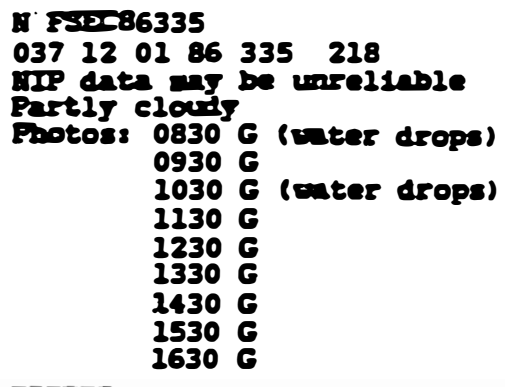

HIP date at 2131 is bad

FSEC 35

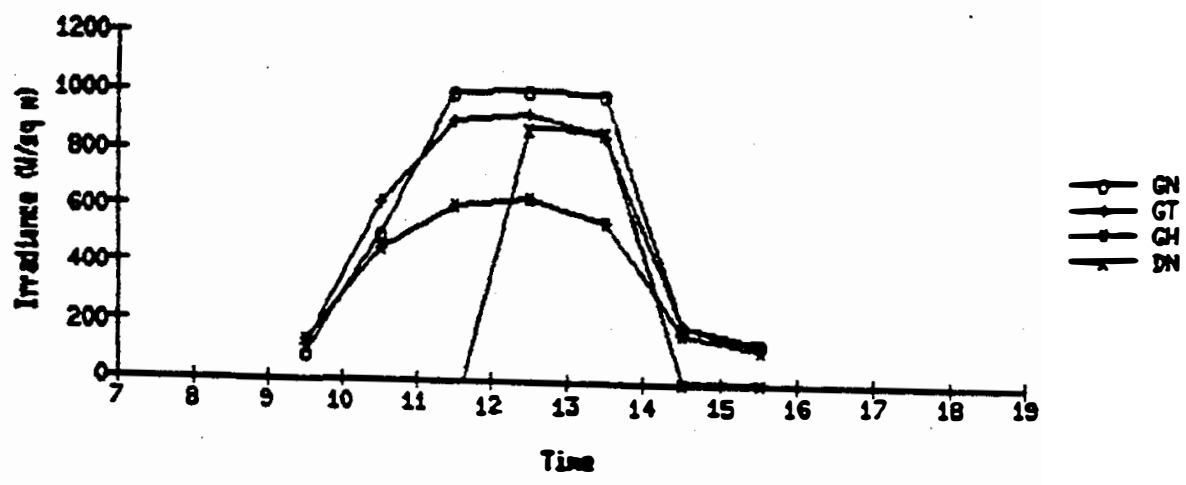

PSEC 3BS OD

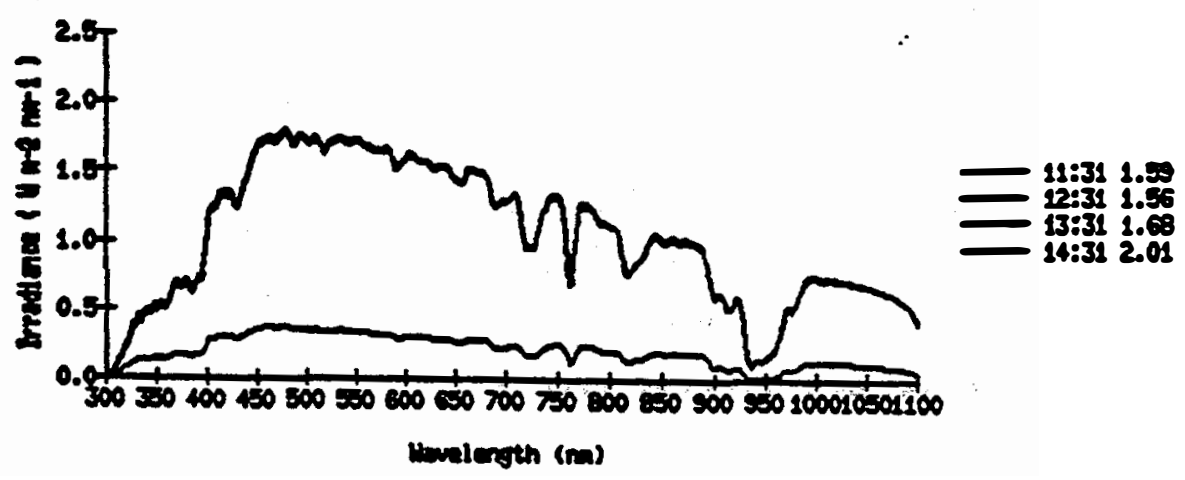




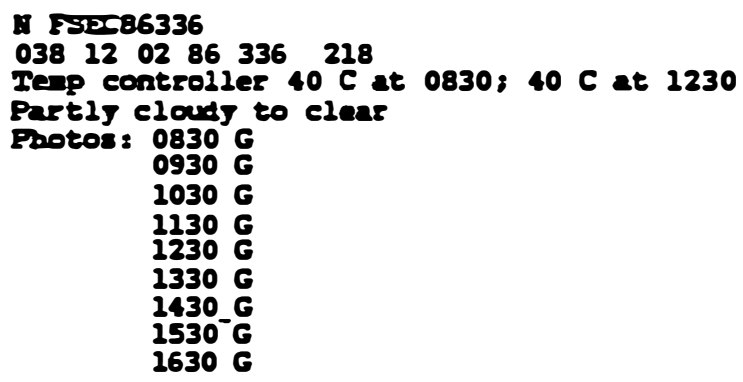

FSEC 356

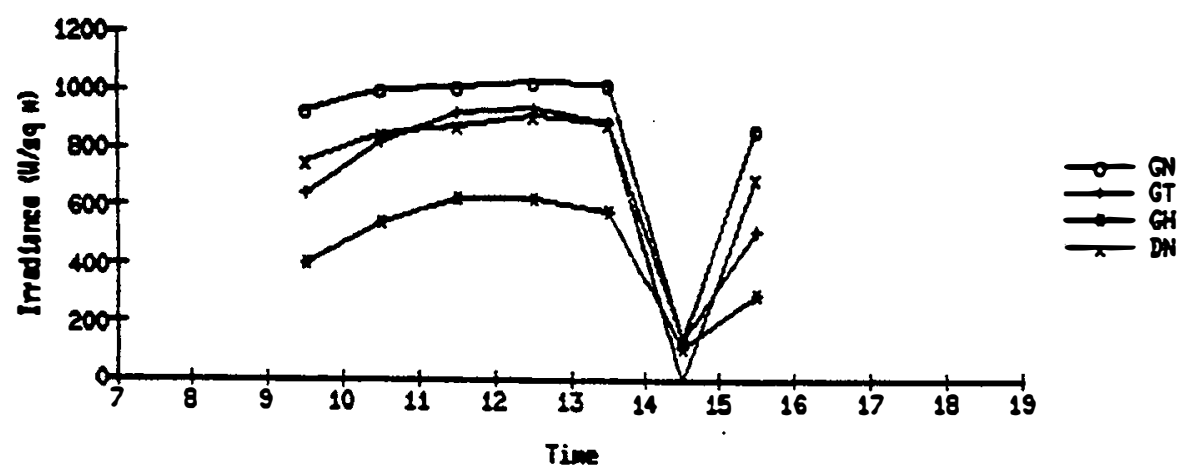

Fsac 3500

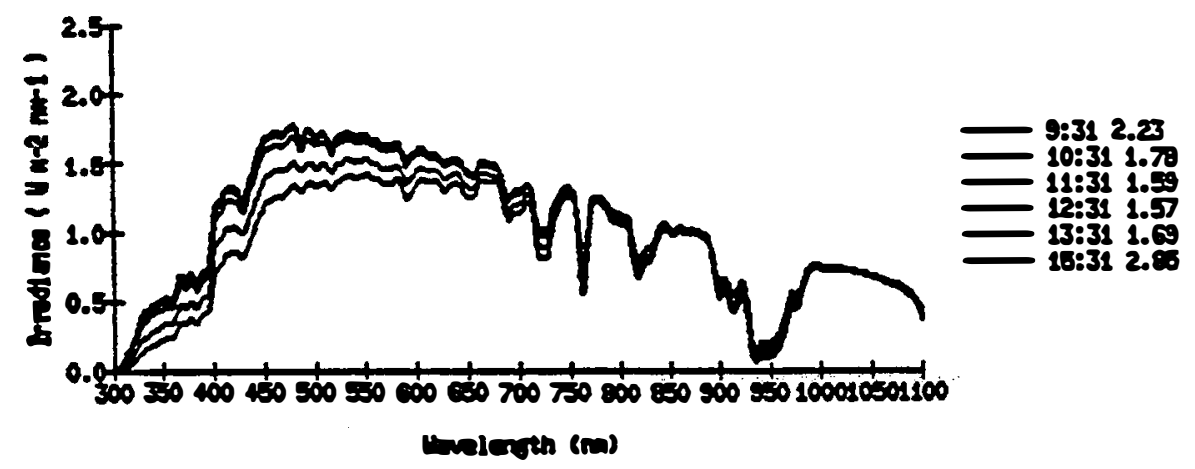


P

$\begin{array}{llllll}039 & 12 & 03 & 86 & 337 & 218\end{array}$

Iep controlizer $40 \mathrm{C}$ at 1130

Mostly elent als becondrig hazy Pu

Frotos: $0830 \mathrm{G}$

$0930 \mathrm{G}$

$1030 \mathrm{G}$

$1130 \mathrm{G}$

$1230 \mathrm{G}$

$1330 \mathrm{G}$

$1430 \mathrm{G}$

$1530 \mathrm{G}$

$1630 \mathrm{G}$

$\operatorname{raxc} 35$

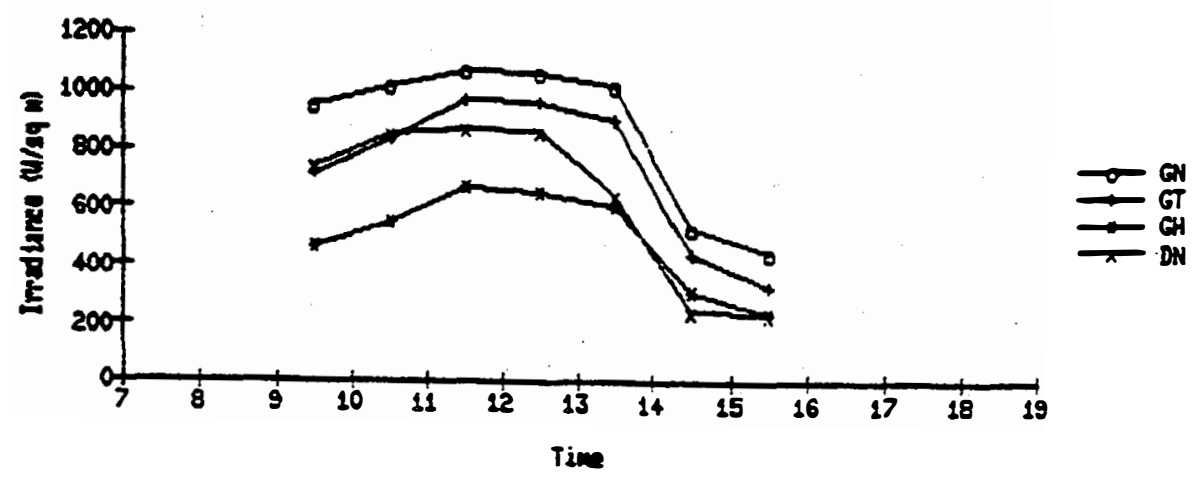

Pax 300

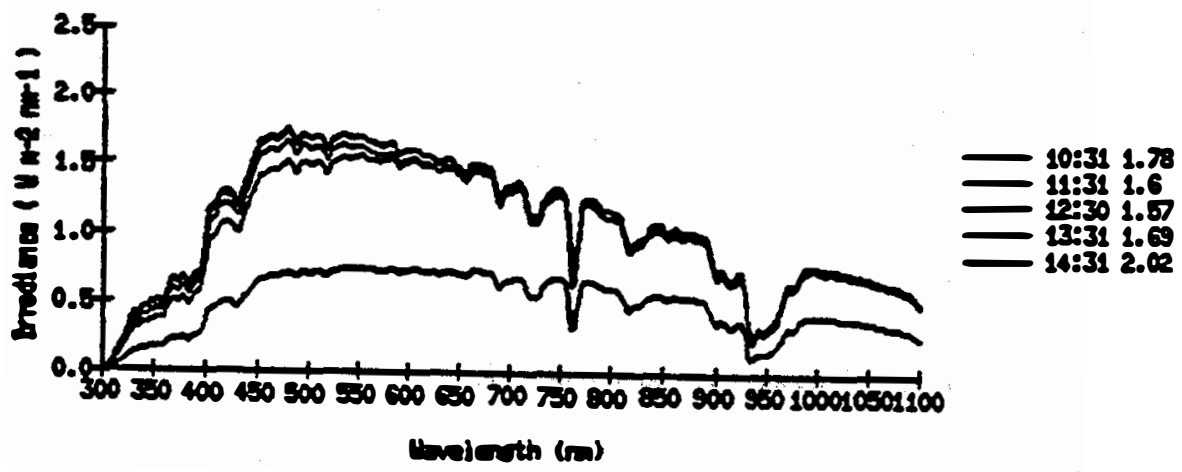




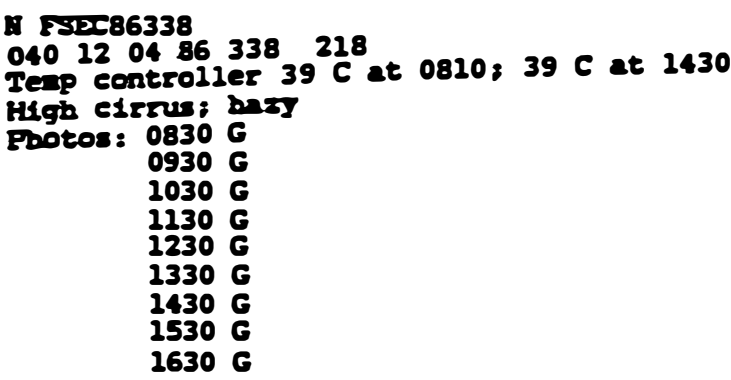

$1330 \mathrm{G}$

$1430 \mathrm{G}$

$1530 \mathrm{G}$

$1630 \mathrm{G}$

PIST 38

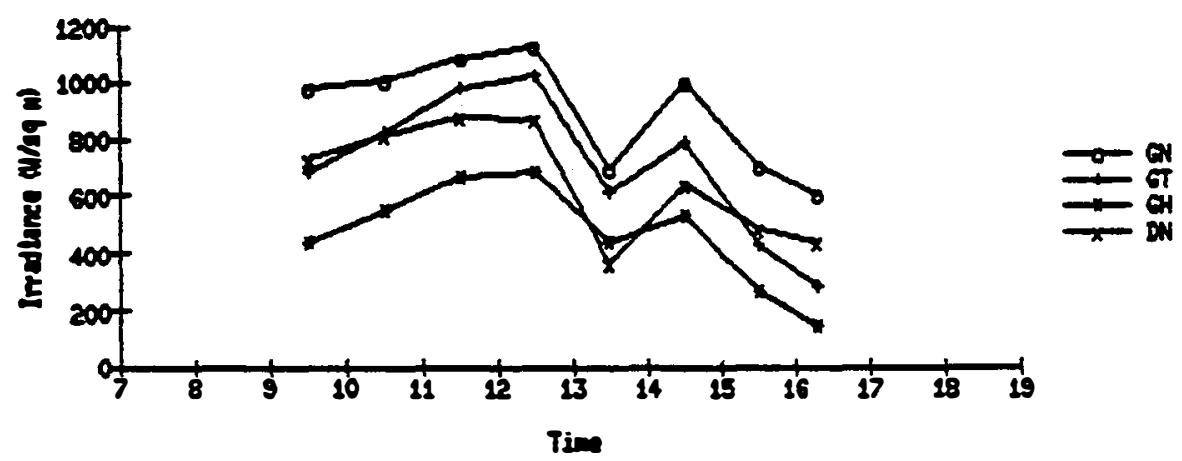

Fise 3000

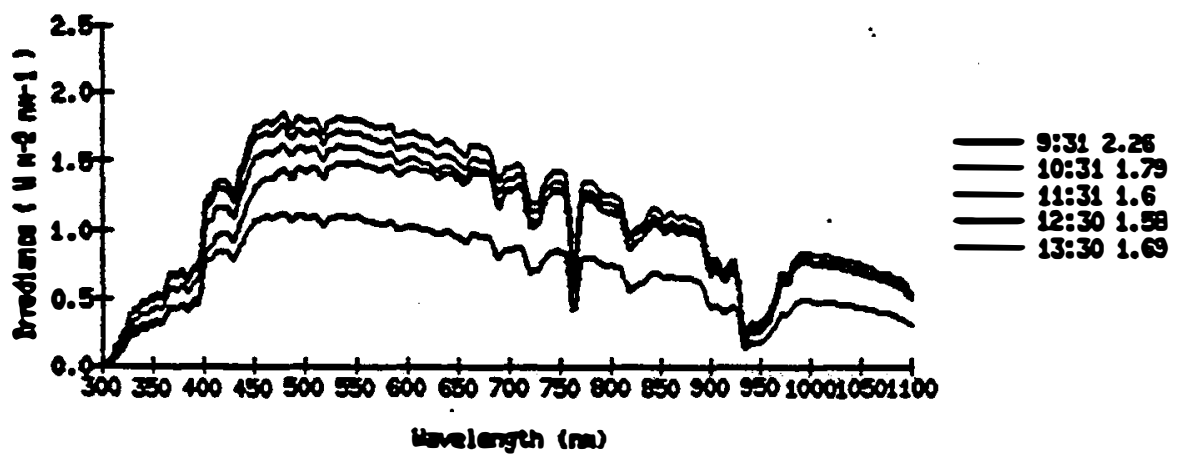


月.

$041 \quad 120586339218$

HIP nufunction: readings are negetive

Teap cortroller 39 C at 0900: 39 C at 1500

Overcast

Pbotos: 0830 Y (processing prableas)

$1630 \mathrm{G}$ (itsessog $12-50930$ througt

10 spects

12-5 1530)

PEEC 300

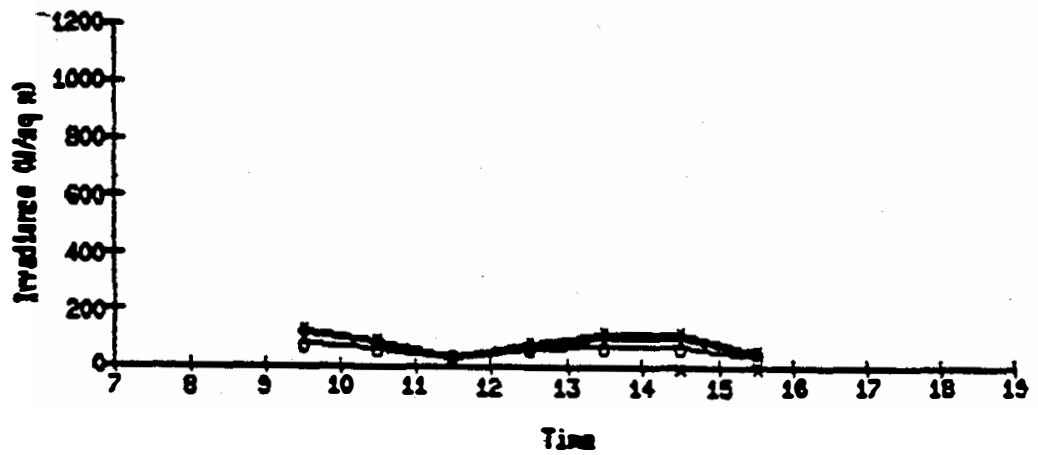


4 गयग286340

$042 \quad 1206 \quad 86340 \quad 218$

Clent to partly elouds

Protos: $0830 \mathrm{G}$

$1030 \mathrm{G}$

$1130 \mathrm{G}$
$1230 \mathrm{G}$

$1330 \mathrm{G}$

$1430 \mathrm{C}$

$1530 \mathrm{G}$

$1630 \mathrm{G}$

$F \leq x=310$

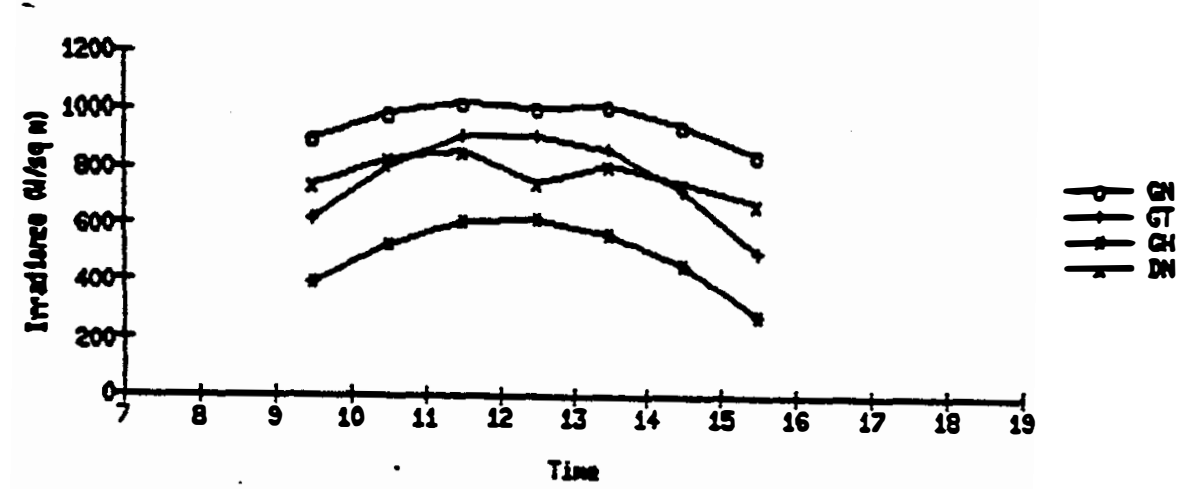

PIE 3400

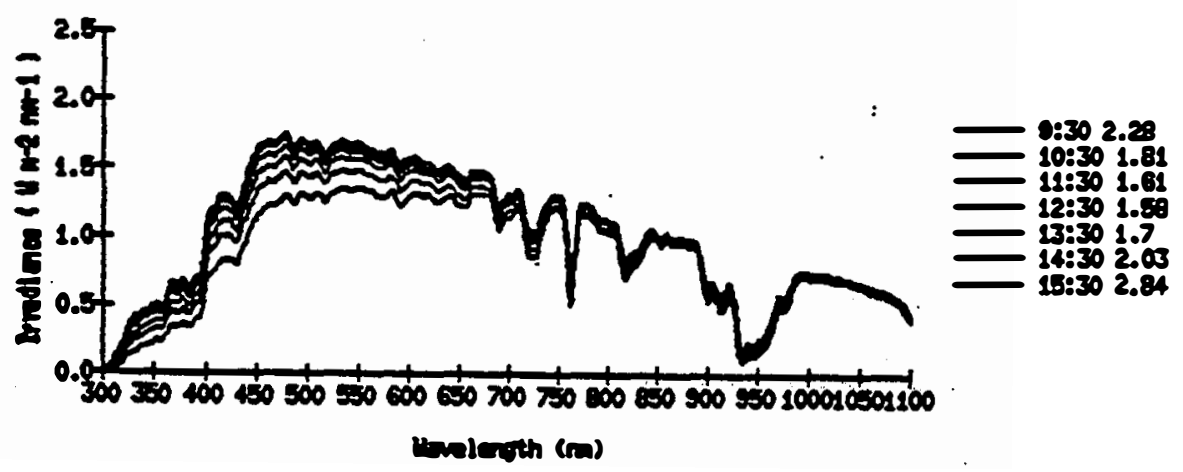


1. 25086342

$\begin{array}{llllll}04322 & 07 & 86 & 342 & 228\end{array}$

NIP elfunctions readings negative (internstent) during nom

Partls elouds

Photos 0830 G (eater drops)

$0930 \mathrm{G}$ (ereter drops)

$1030 \mathrm{G}$

$1230 \mathrm{G}$

$1230 \mathrm{G}$

$1330 \mathrm{G}$

$2430 \mathrm{G}$ (sereteh)

$2530 \mathrm{G}$

$2630 \mathrm{G}$

uPP rendung -99 at 0930

Fase 341

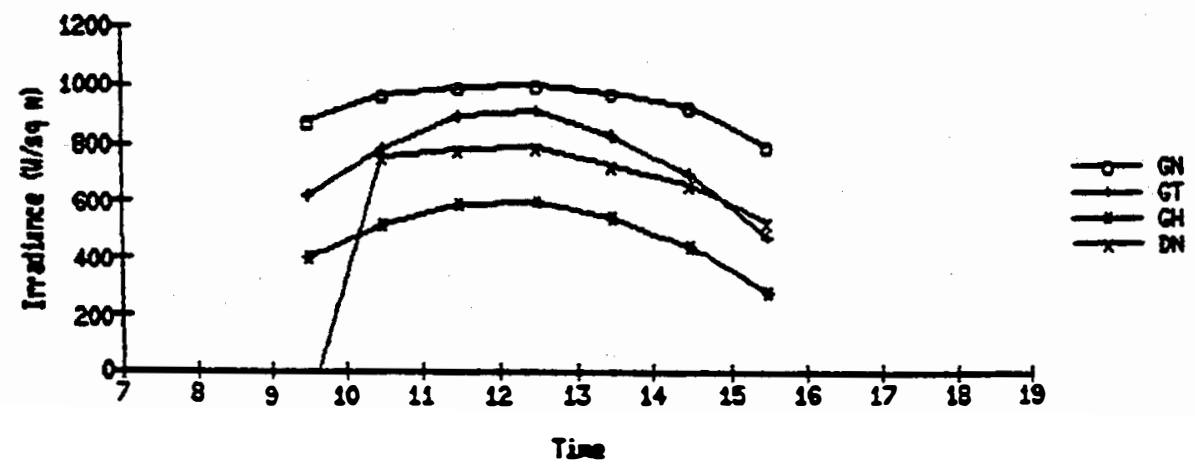

$\operatorname{Fax} 340$

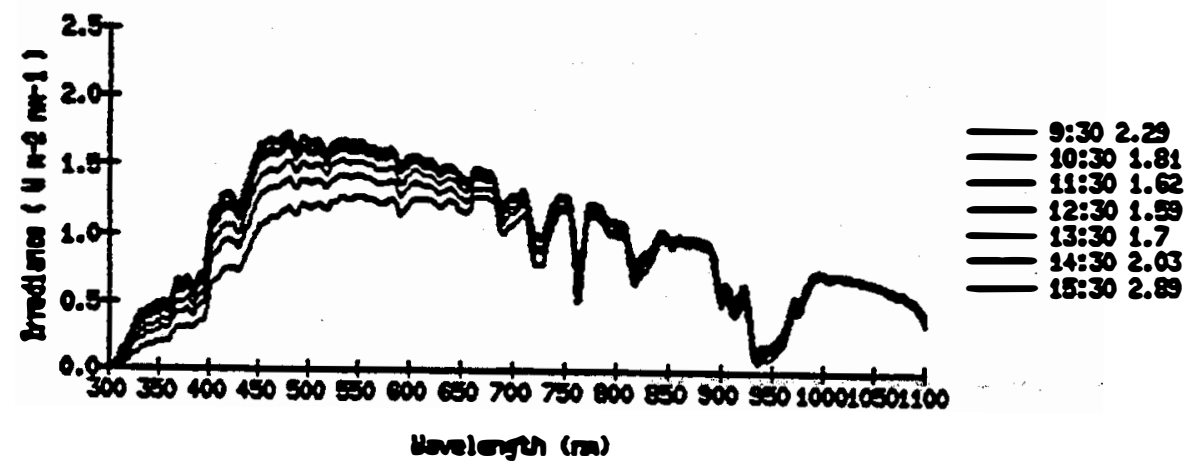




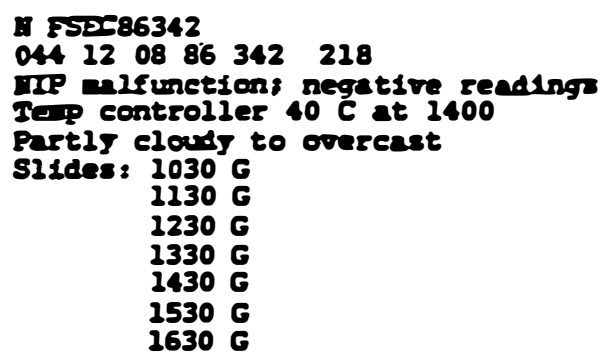

FSEC 342

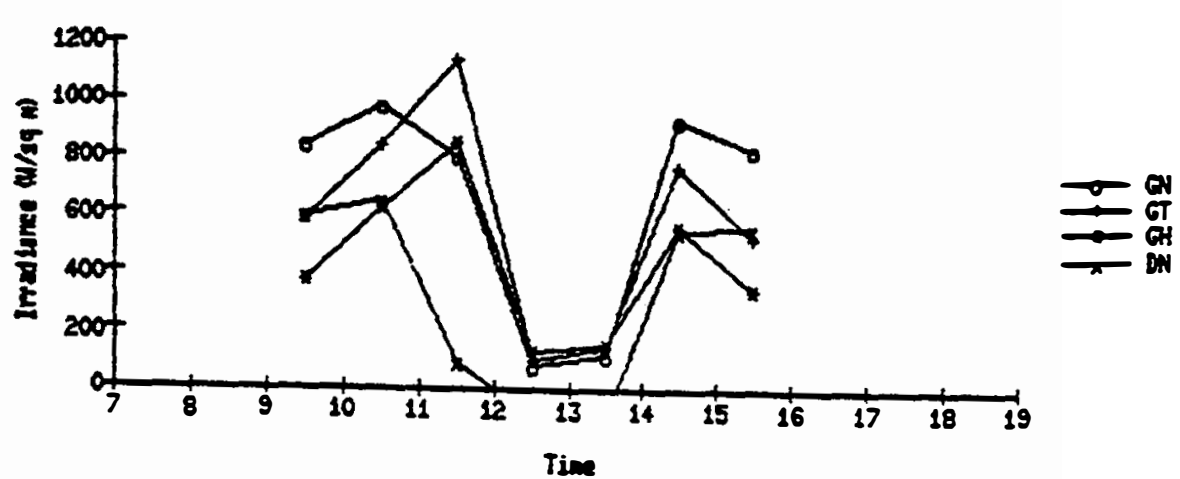

$\cos 200$

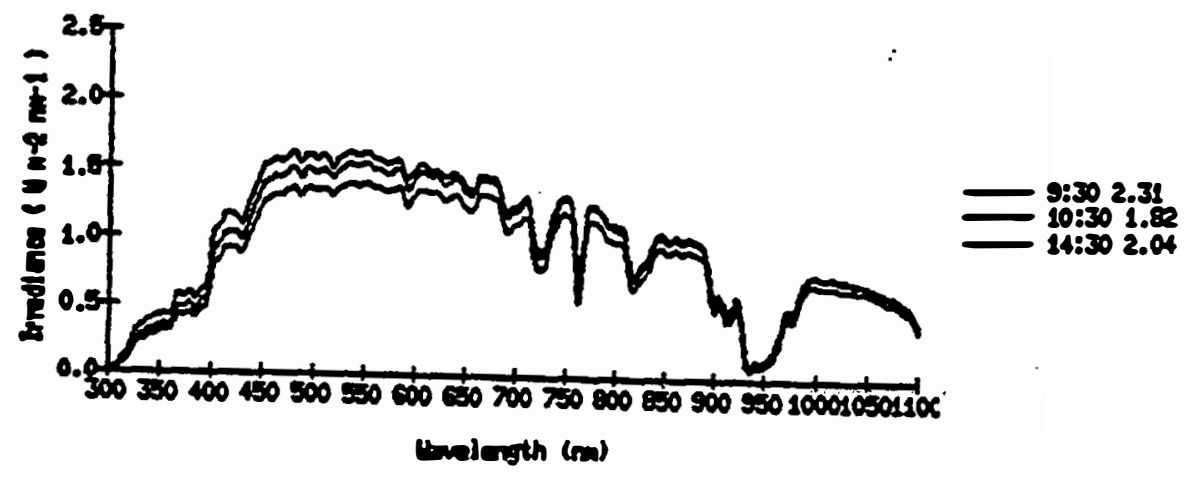




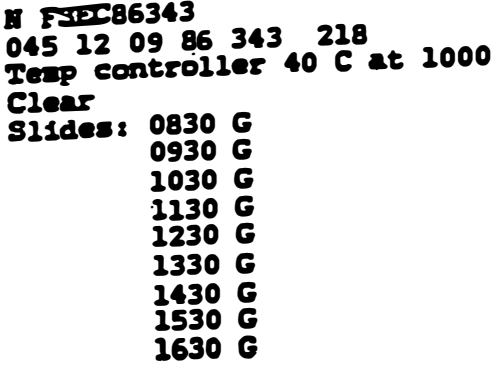

PISC 343

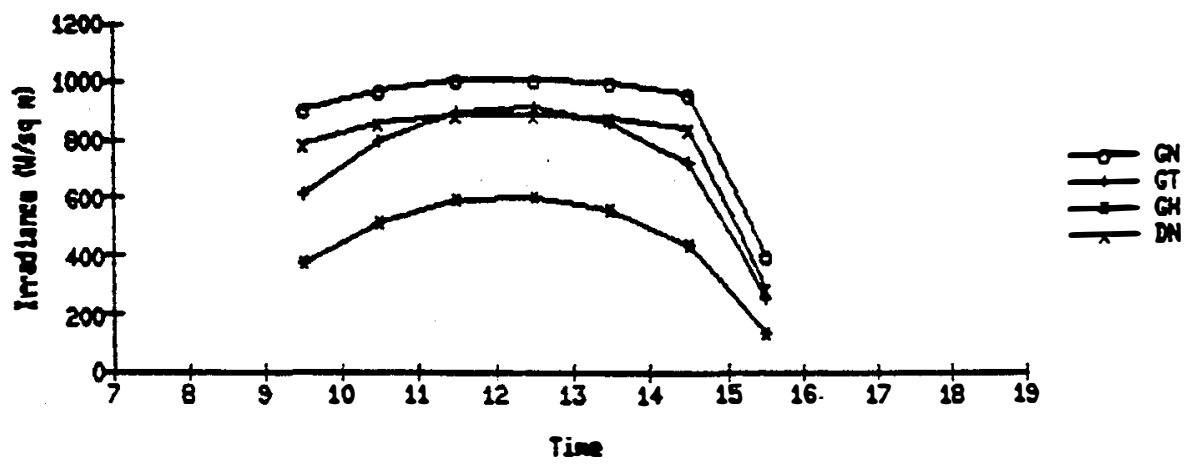

Fביב 31300

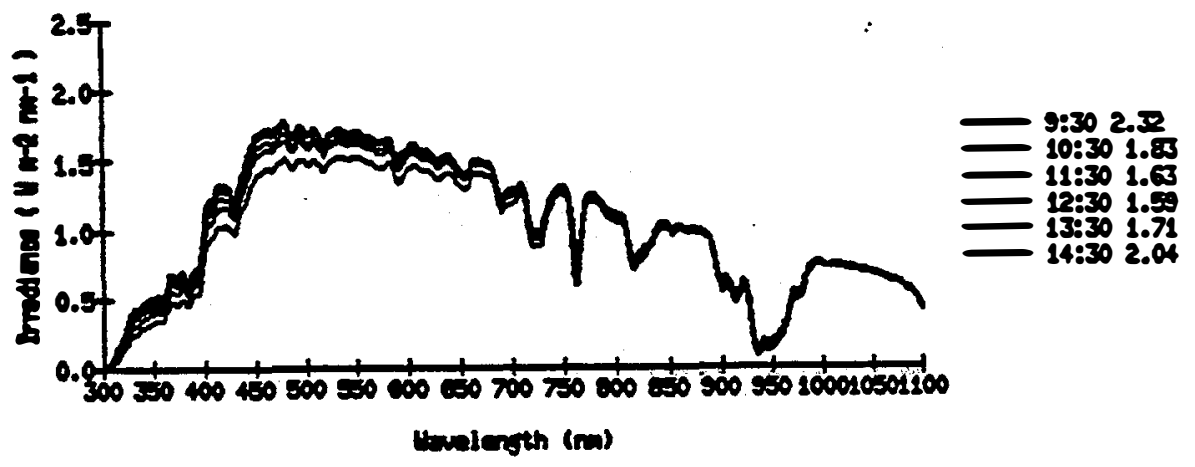




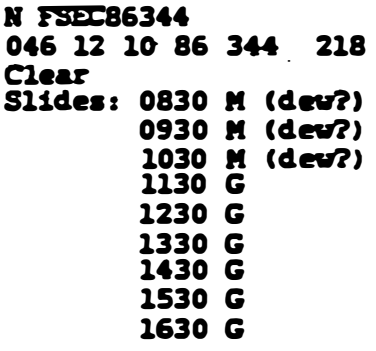

Fsec 34

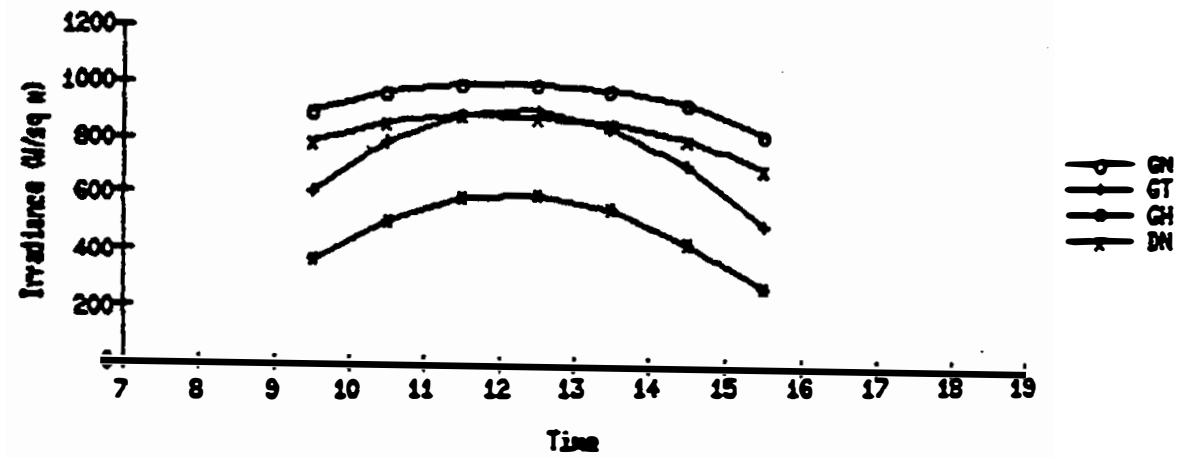

Fase 340

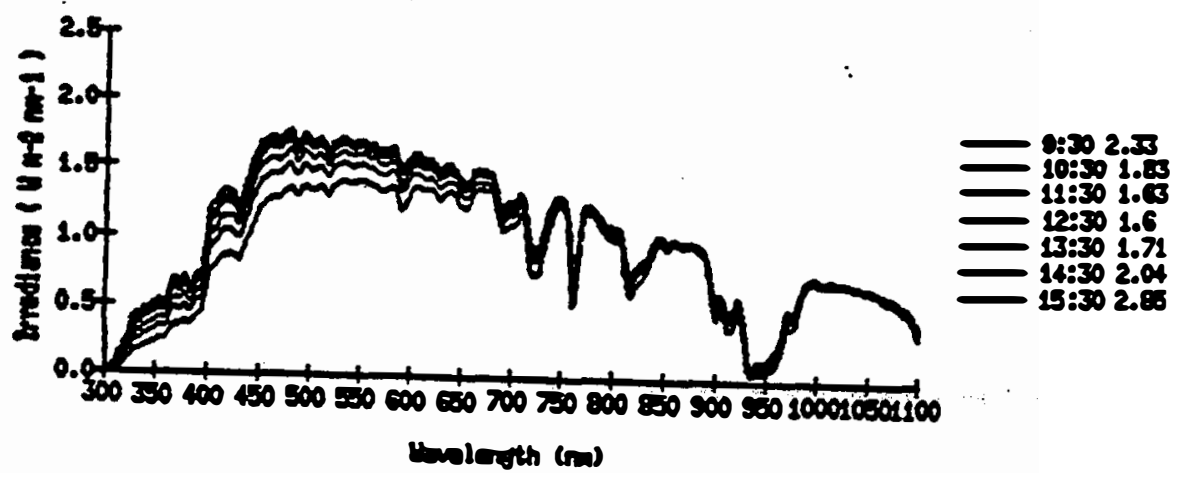




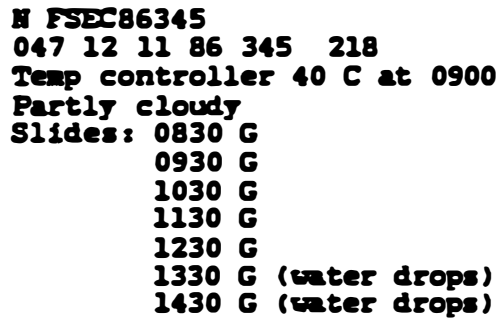

$1130 \mathrm{G}$

$1230 \mathrm{G}$

$1330 \mathrm{G}$ (erter drops)

$1430 \mathrm{G}$ (weter drops)

FSEC 345

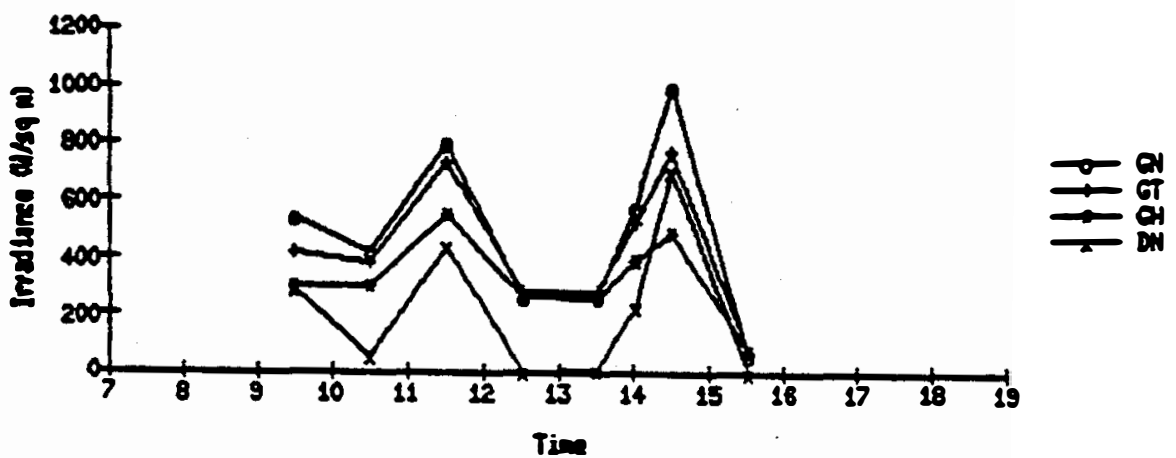

$\operatorname{Fin} 310$

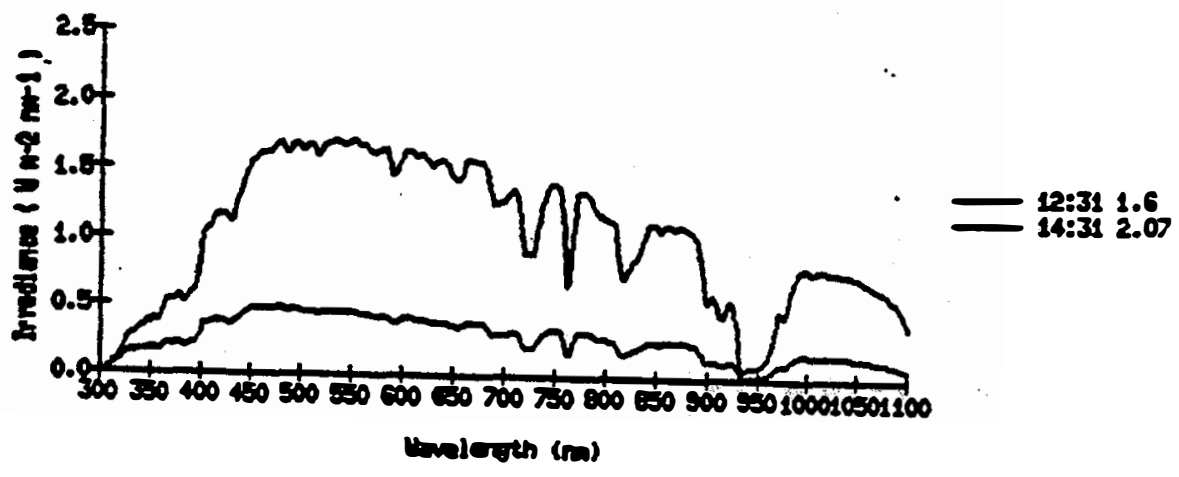


$048121286346 \quad 218$

Teap controller $39 \mathrm{C}$ at 0810 ; $40 \mathrm{C}$ at 1230

Ho photos

PISC 346

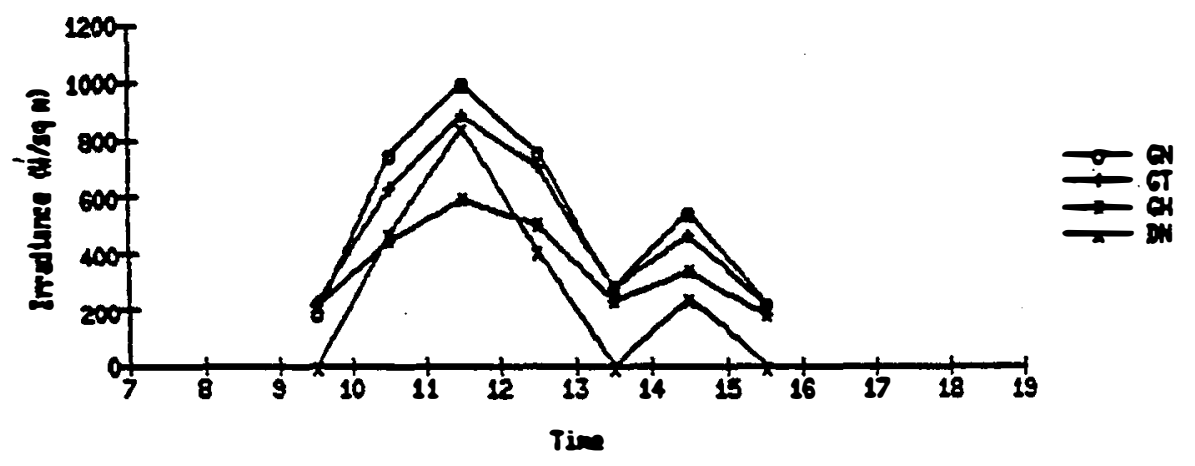

$\operatorname{Fin} 3400$

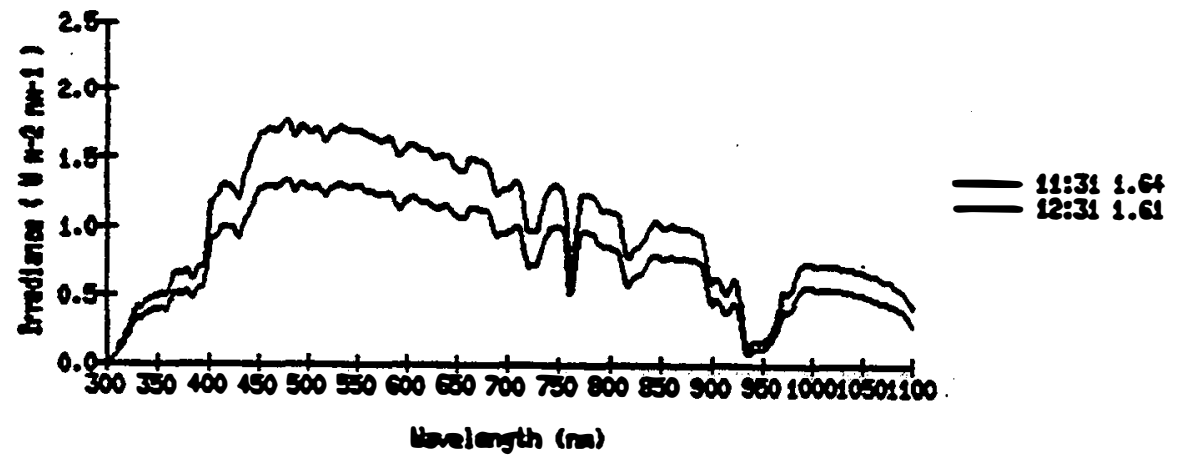


A P51286347.

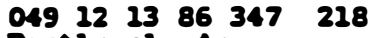

Partis cloudy

Do phoots

F्25 367

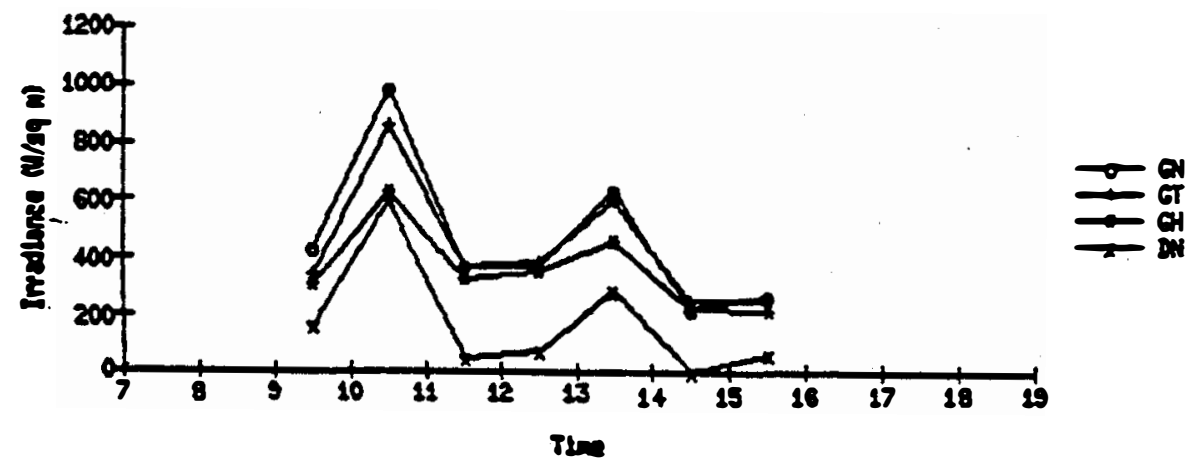

Pax 31700

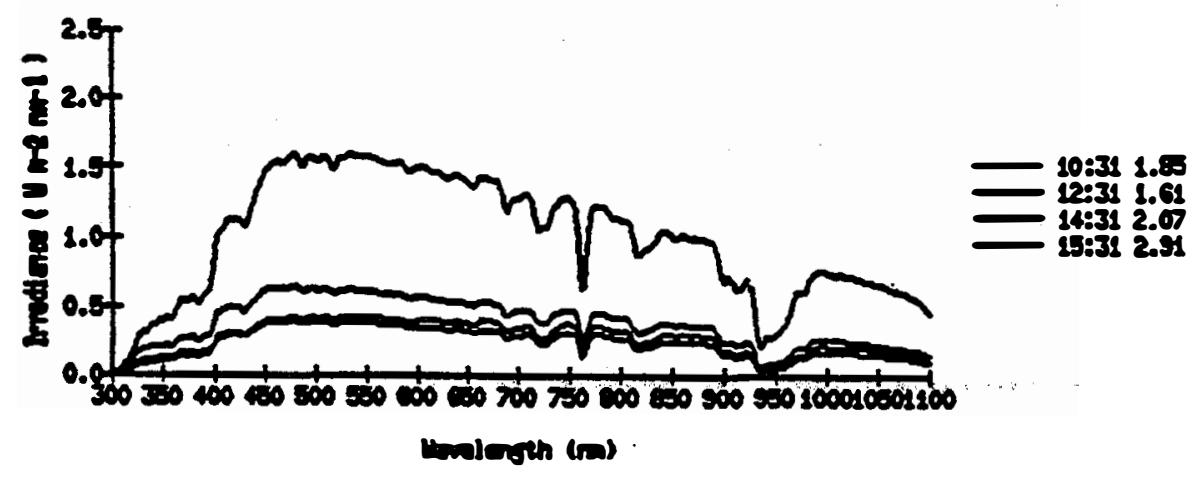




\section{SEP *}

TR-3513B

1. PSPC86348

$0501214 \quad 86 \quad 348 \quad 218$

Partly elouds

Bo photos

Fise 360

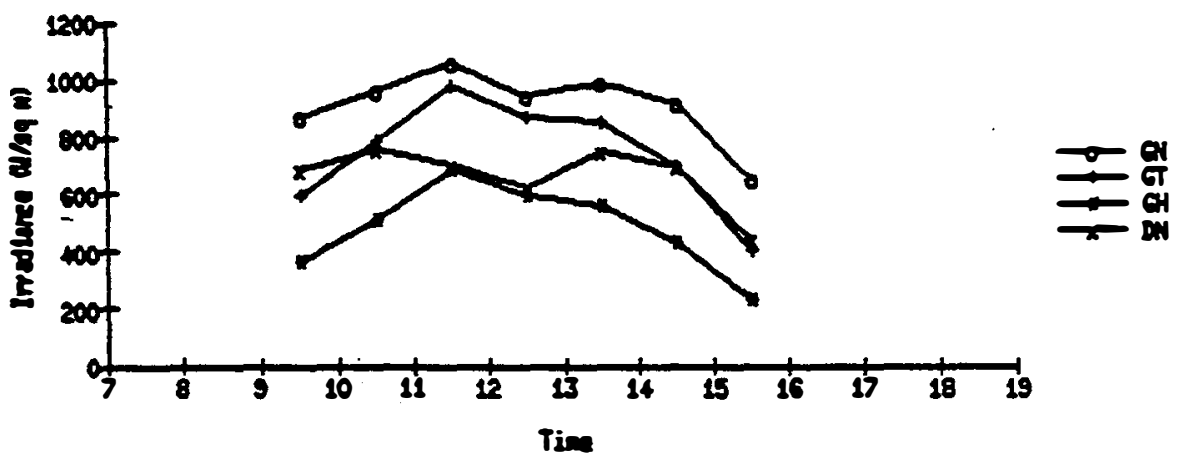

P:30 3480

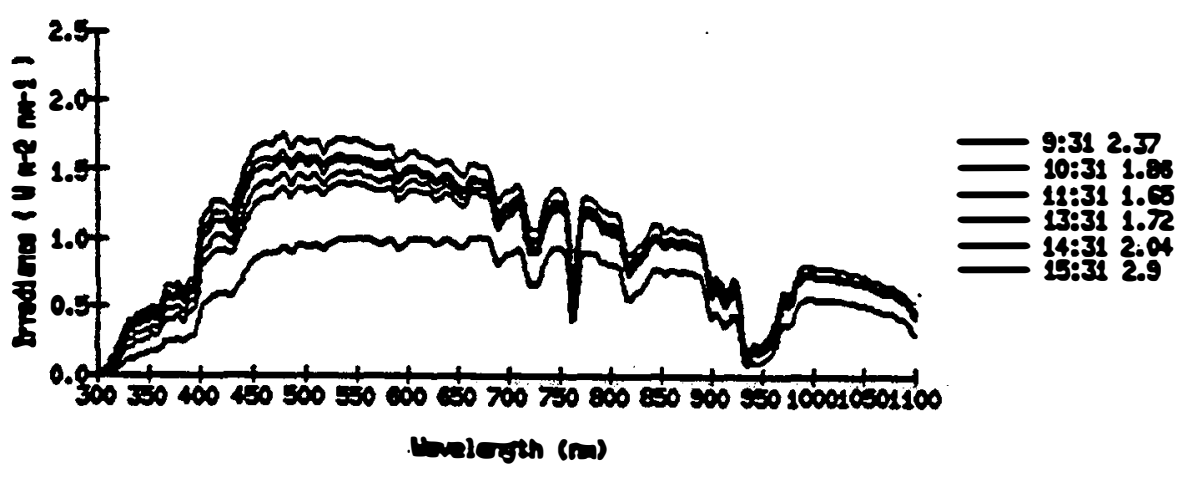


1. T20086349

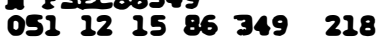

Itep controller $40 \mathrm{C}$ at 1100

Pertly elouds

sideles $1030 \mathrm{G}$

$1130 \mathrm{G}$

$1230 \mathrm{G}$

$1330 \mathrm{G}$

$1430 \mathrm{G}$

$1530 \mathrm{G}$

$1630 \mathrm{G}$

$\operatorname{PSEC} 340$

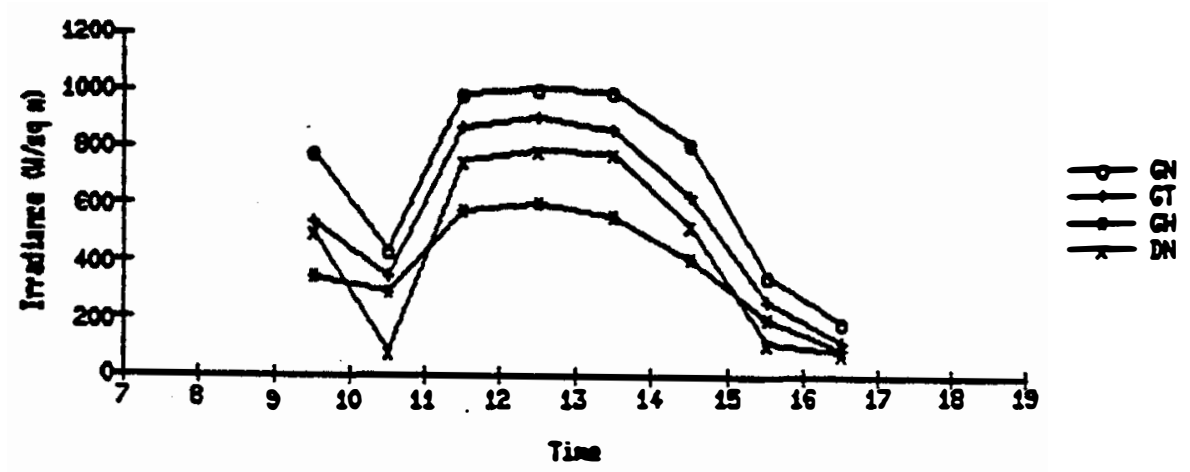

FSEC 340 a0

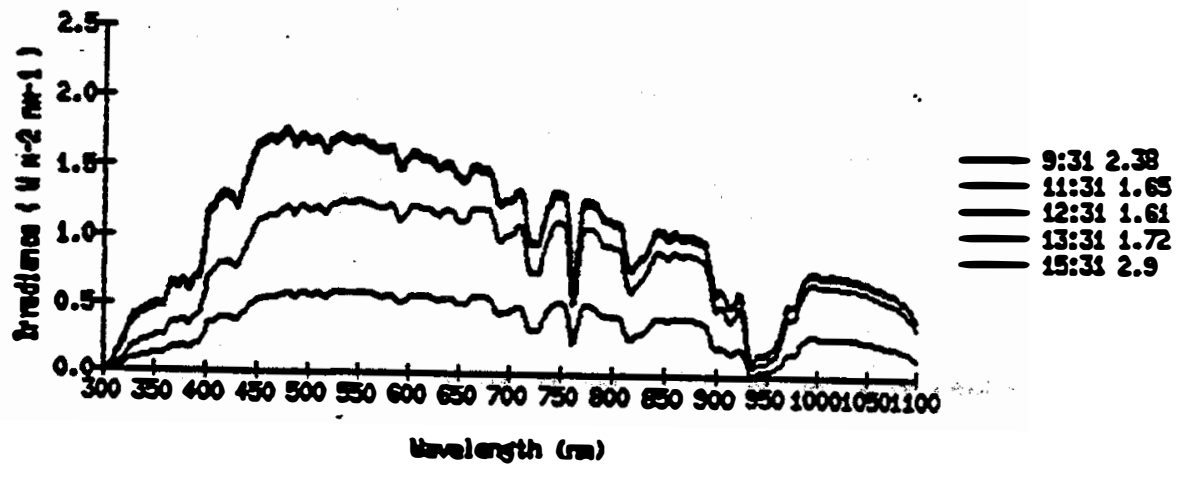


PID 86350

$052121686350 \quad 218$

Teap controller $39 \mathrm{C}$ at 0815; $39 \mathrm{C}$ at 1610

Partly eloudy to overcast. Uhes becondog clear

Sildes: $0830 \mathrm{G}$

$0930 \mathrm{G}$

$1030 \mathrm{G}$

$1130 \mathrm{G}$

$1230 \mathrm{G}$

$1330 \mathrm{G}$

$1430 \mathrm{G}$

$2530 \mathrm{G}$

$2630 \mathrm{G}$

FEES 350

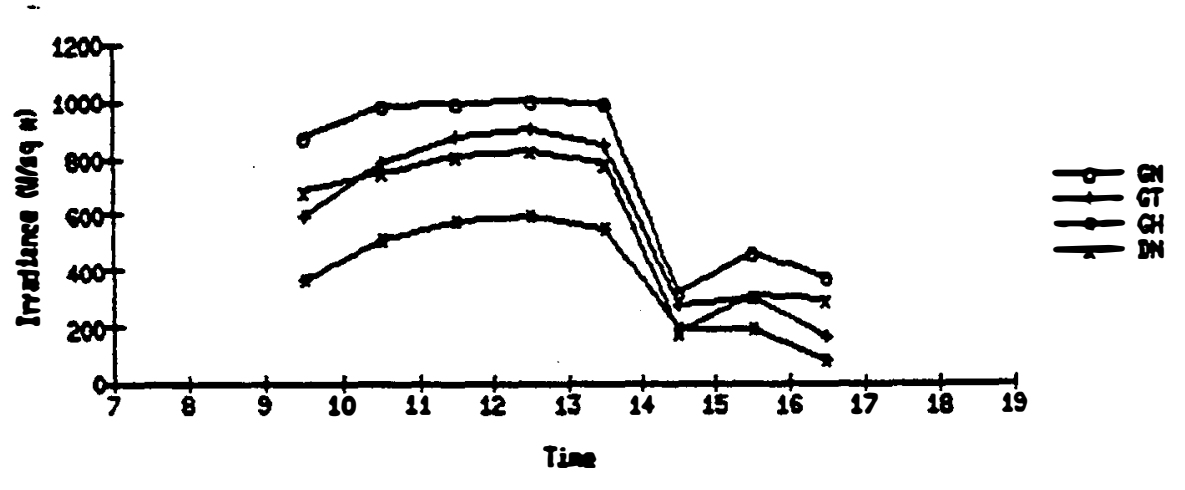

$\operatorname{Pase} 3000$

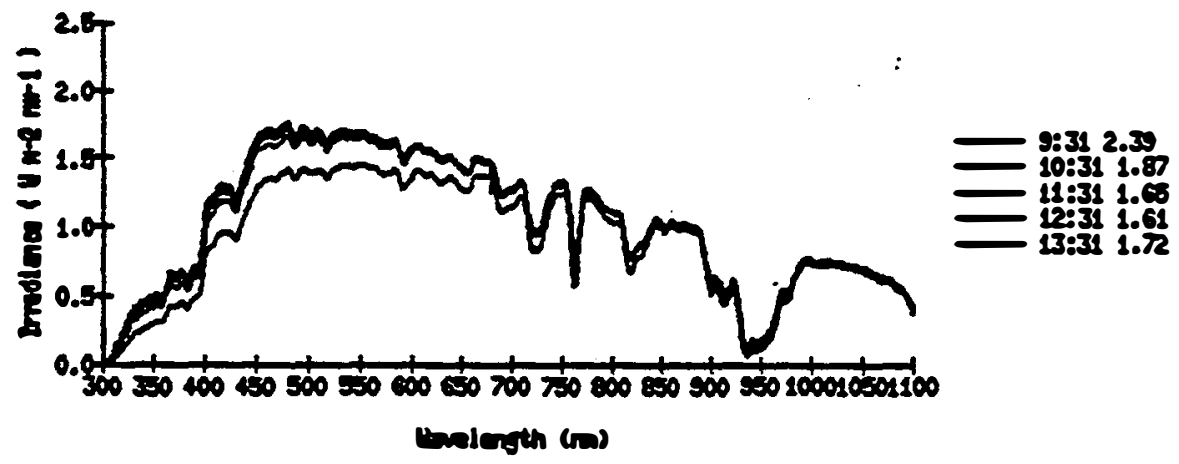


A PSDC86352

$053121886,352 \quad 218$

Replaced IIP; old = 1430656, cal 8.43 × 10-6 V/ln-2

Do net date $1230-1430$

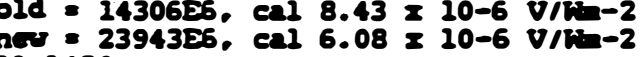

TerP controller $40 \mathrm{C}$ at $0830840 \mathrm{C}$ at 1315

Partls clovd

Photos: 0830 P (processing problen)

$0930 \mathrm{P}$

$1030 P$

$2130 \mathrm{M}$

$1230 \mathrm{H}$

$1330 \mathrm{G}$

$1430 \mathrm{G}$

$1530 \mathrm{M}$

$1630 \mathrm{H}$

IOR Integral dind at 1631 ; nissing date in spectron in near-IR

Fexe 362

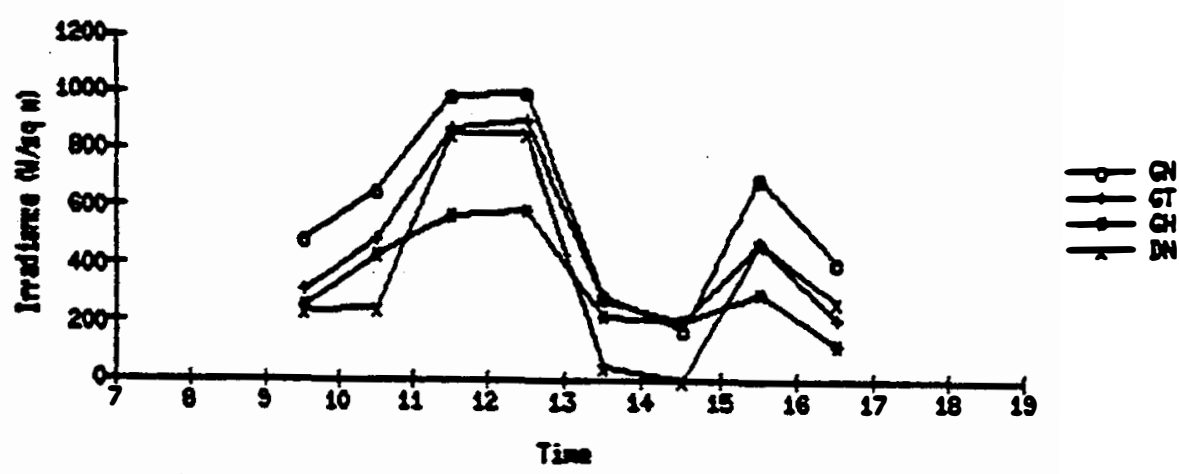

Pac 3000

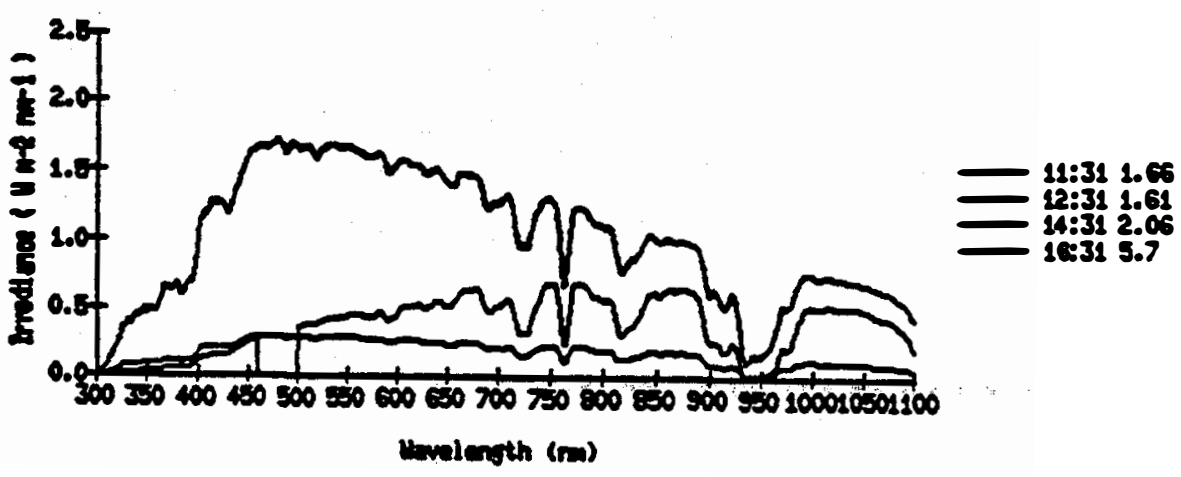


1.

$054121986353 \quad 218$

Tenp cortroiler $39 \mathrm{C}$ at $0830 ; 40 \mathrm{C}$ at 2320

Partis clouds to overent

Ho pbotos

ISR integral at 1631 bad; alssing data in spectron in Fisible

Pase 35

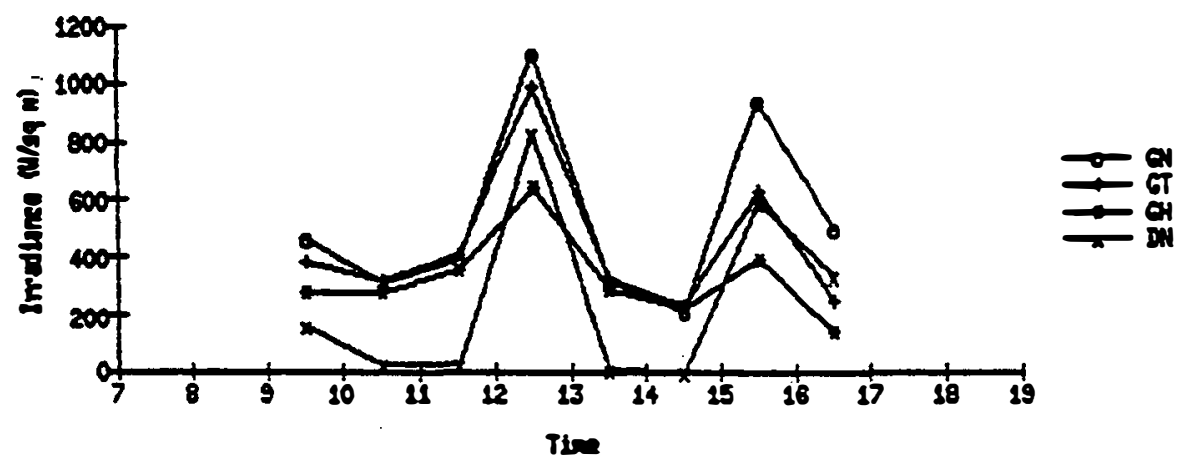

pise $=300$

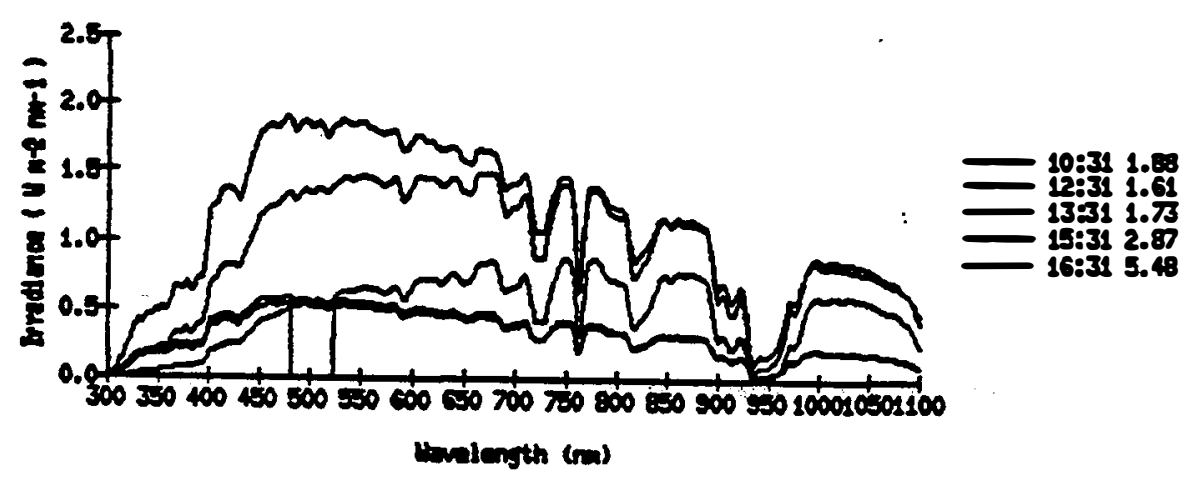


1) 351586354

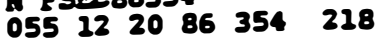

Clouds

Fmotos: $0830 \mathrm{G}$

$0930 \mathrm{G}$

$1130 \mathrm{G}$

$1230 \mathrm{G}$

$1330 \mathrm{G}$

$1430 \mathrm{G}$

$2530 \mathrm{G}$

$1630 \mathrm{G}$

Fsec 364

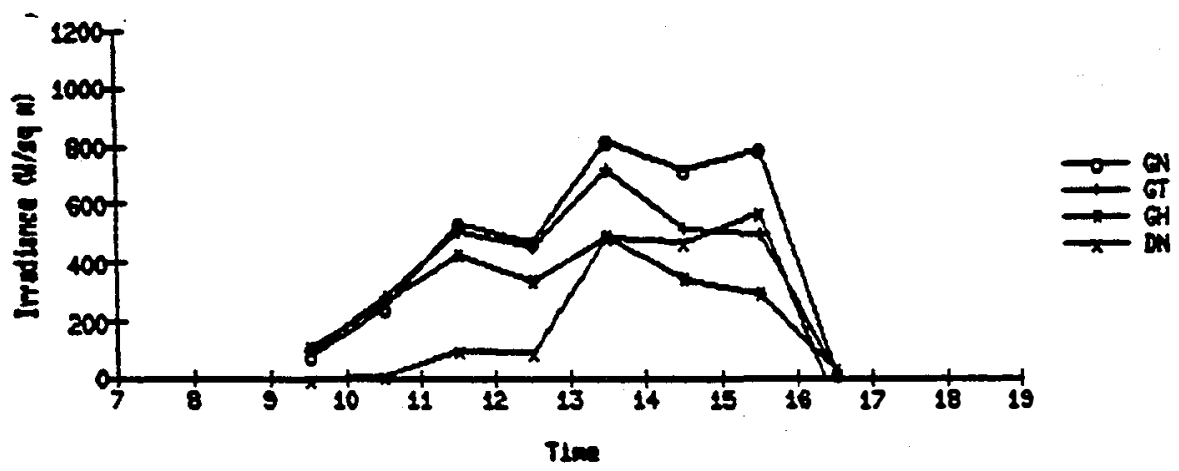

$P=x 300$

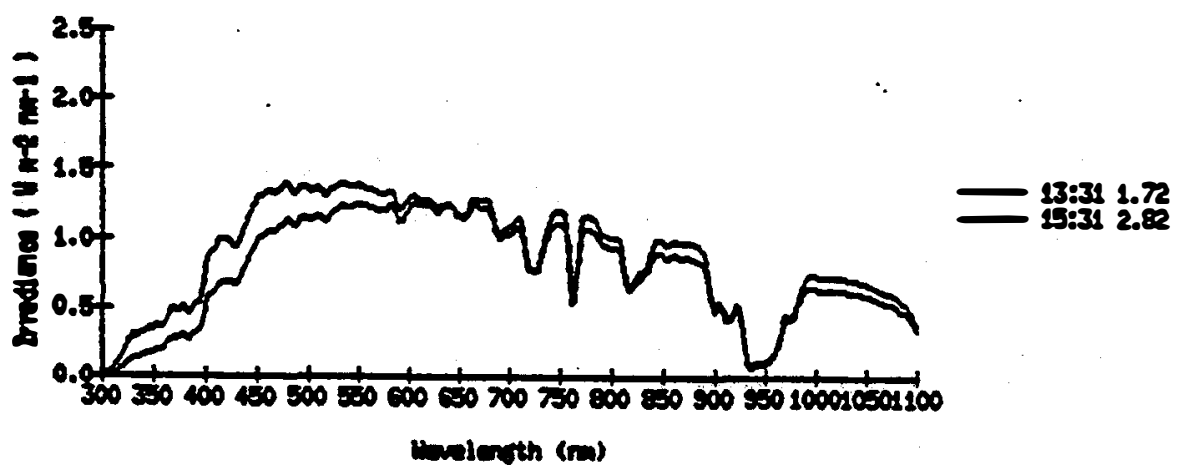




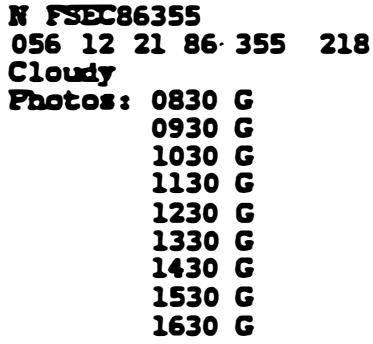

FESC

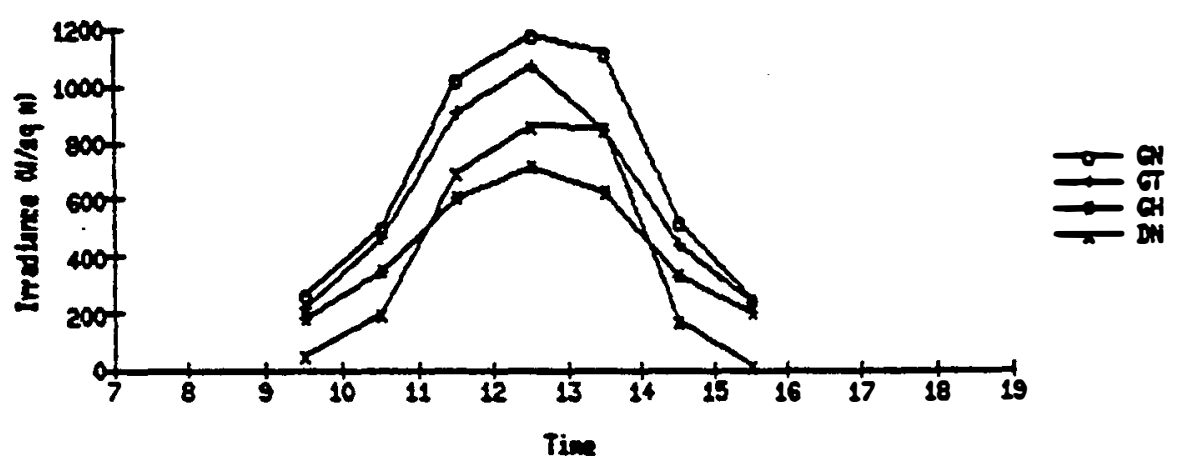

$\begin{array}{lll}- & 1 & \text { PISE }\end{array}$

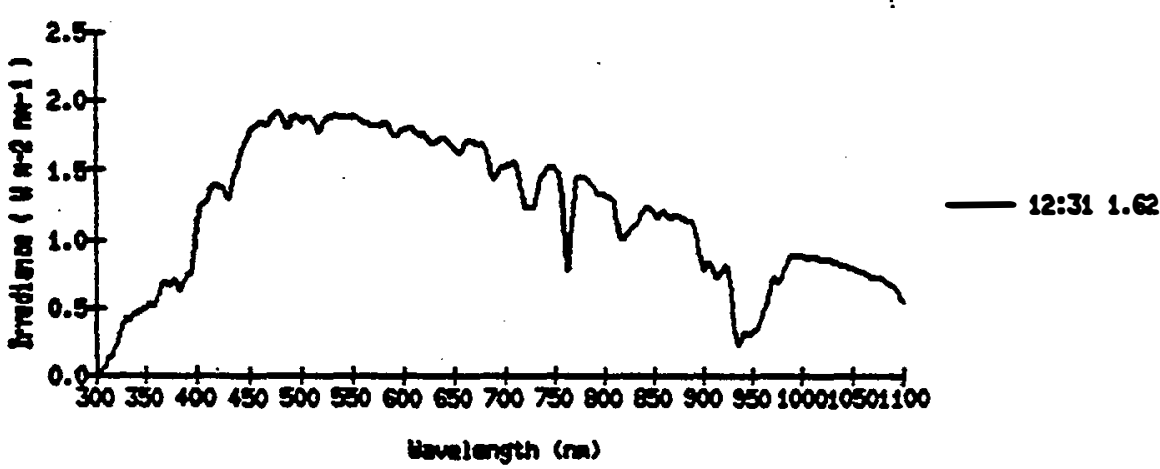


Paxe 368

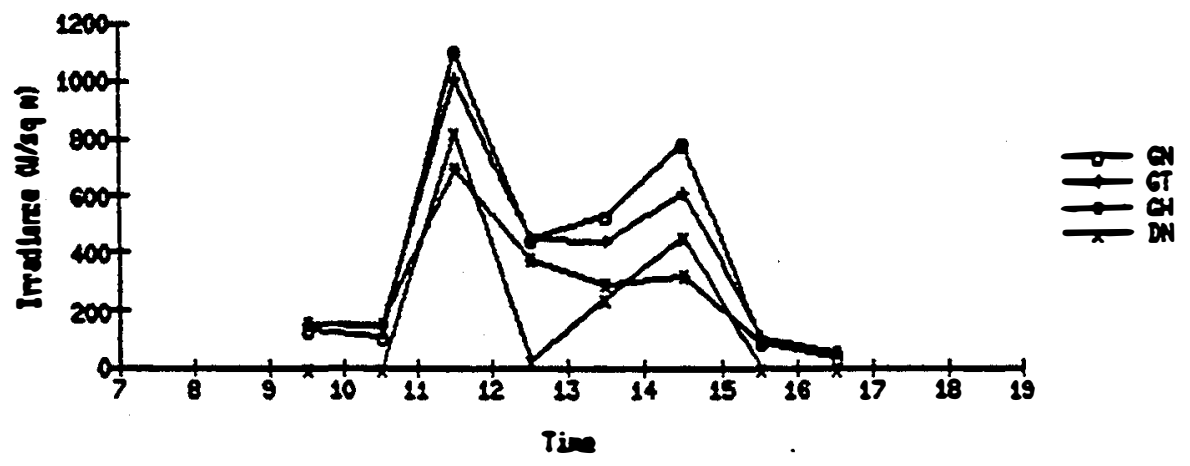

Fase 300

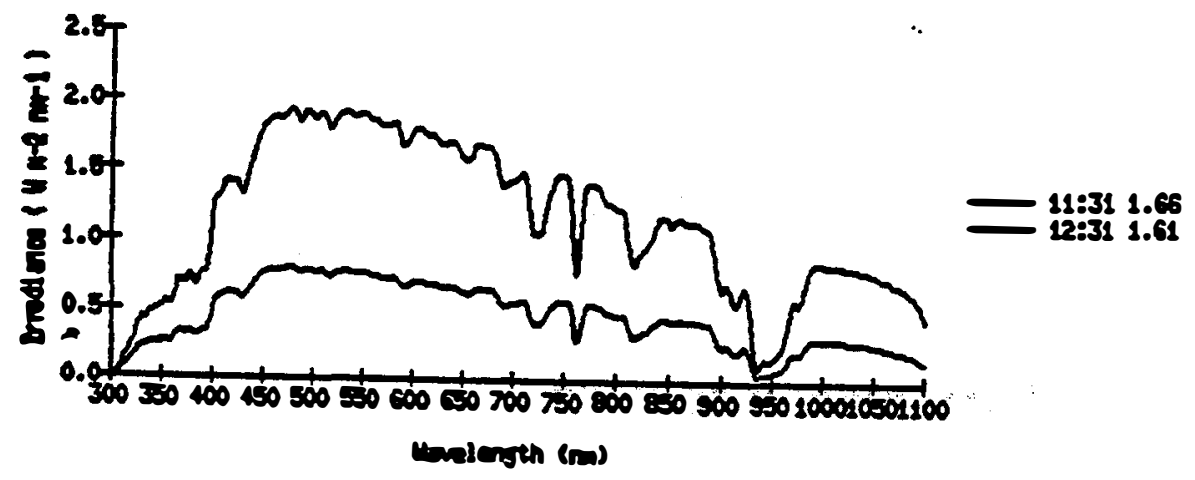



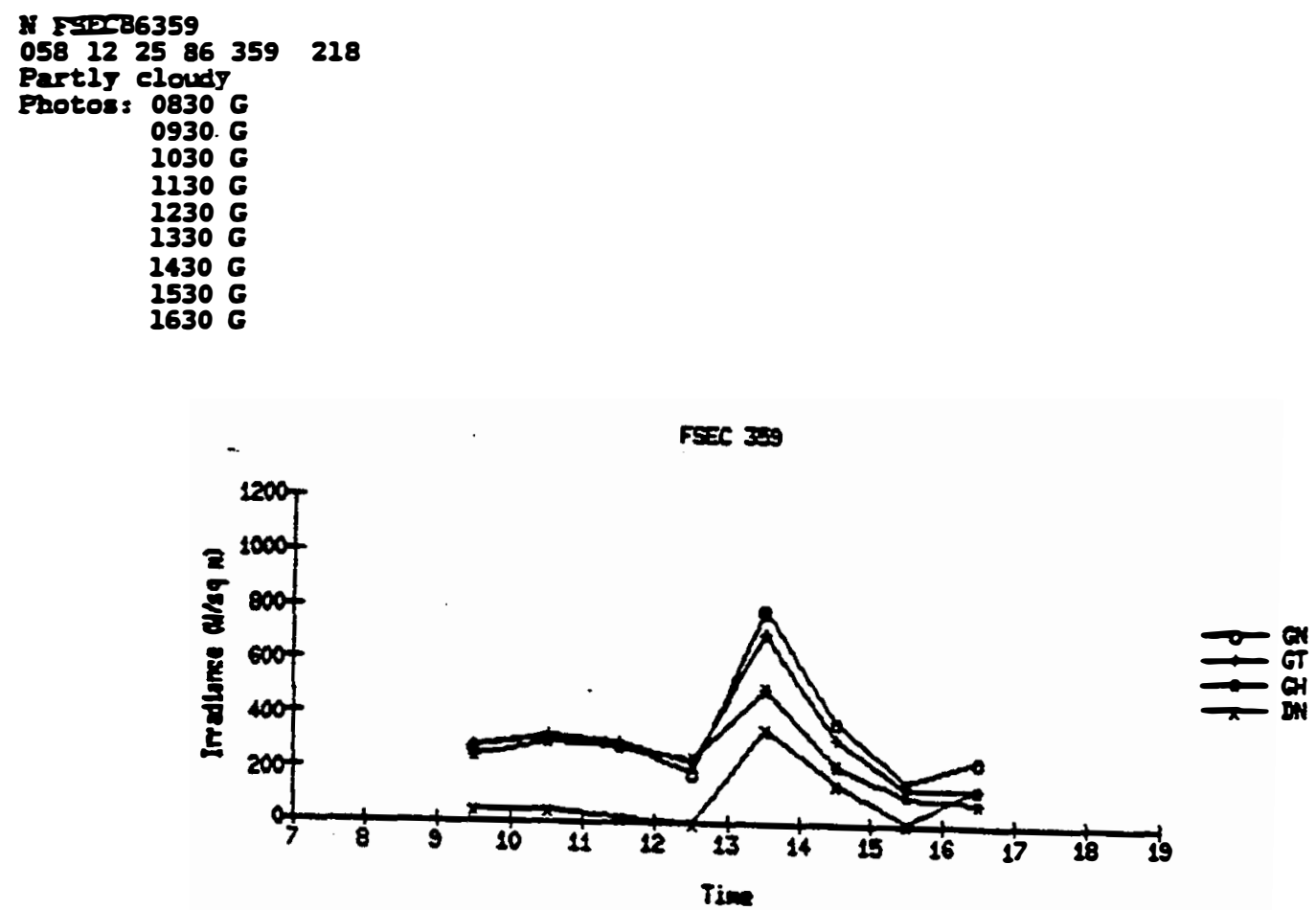

PIIC

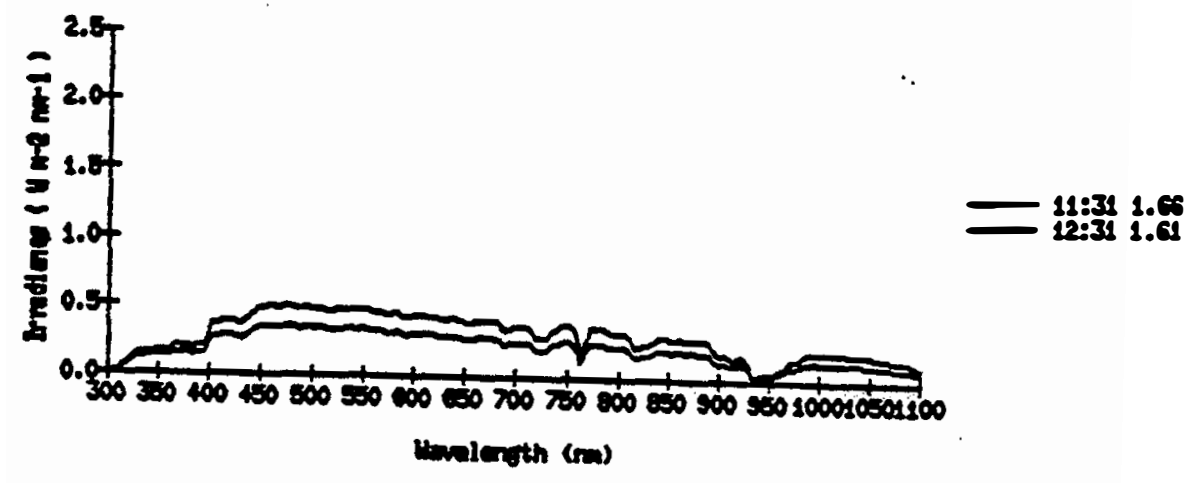




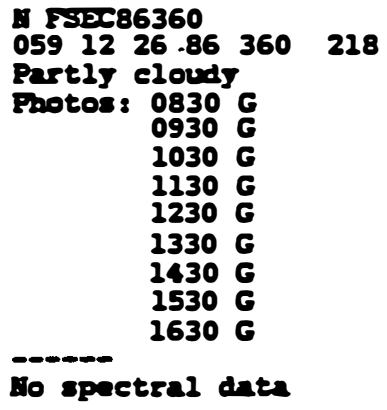

io spectral datea

F्בC 360

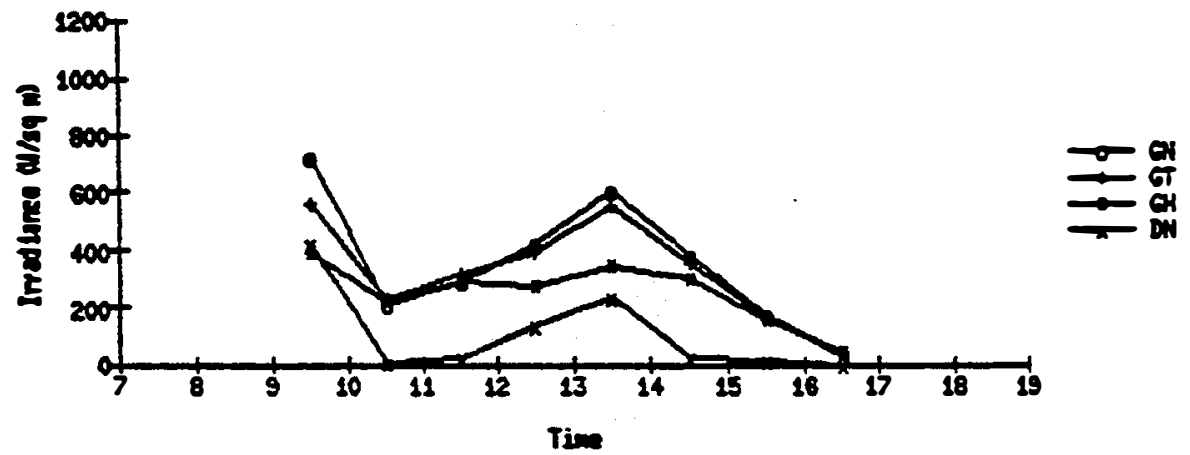


A PSPCB6361

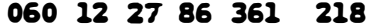

Cloudr

Photos: $0830 \mathrm{G}$

$0930 \mathrm{G}$ (enter drops)

$1030 \mathrm{G}$

$1130 \mathrm{G}$

1230 G (enter drops)

1330 G (enter drops)

$1430 \mathrm{G}$

$1530 \mathrm{G}$

$1630 \mathrm{G}$

Appears to be slat spot in spectron in near-IR at 1231 and 1230

Fase 361

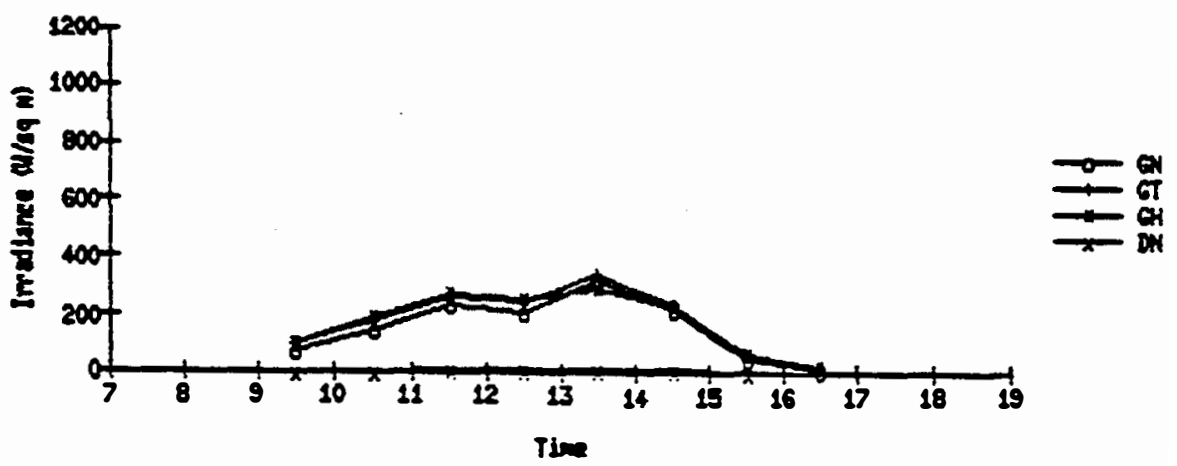

Fase 39100

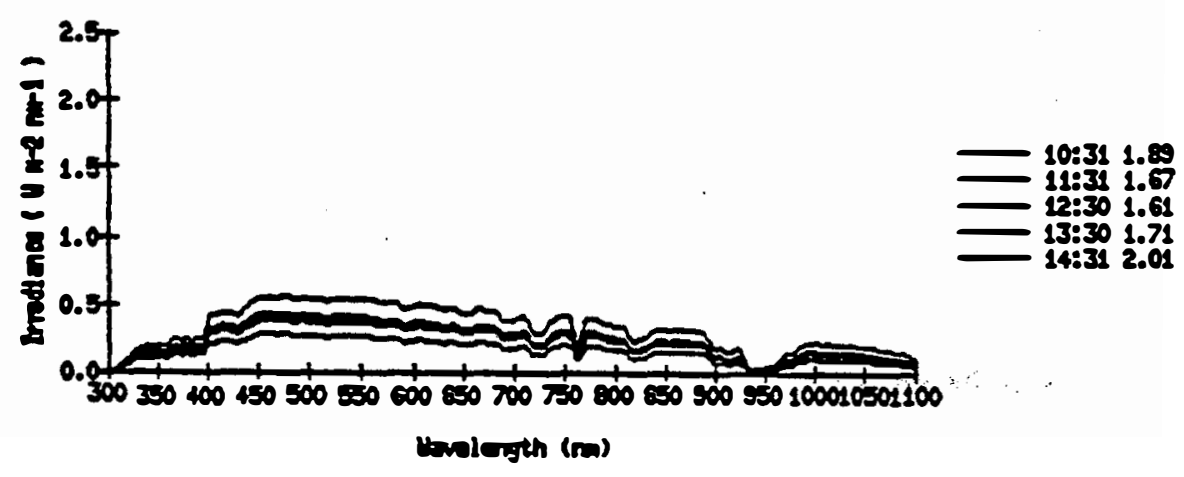




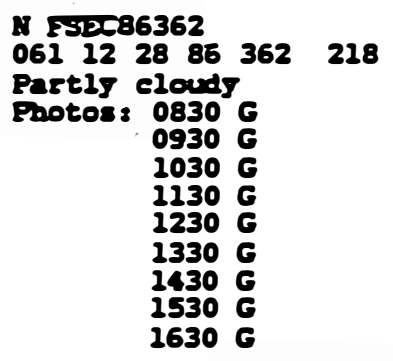

Fsec 30

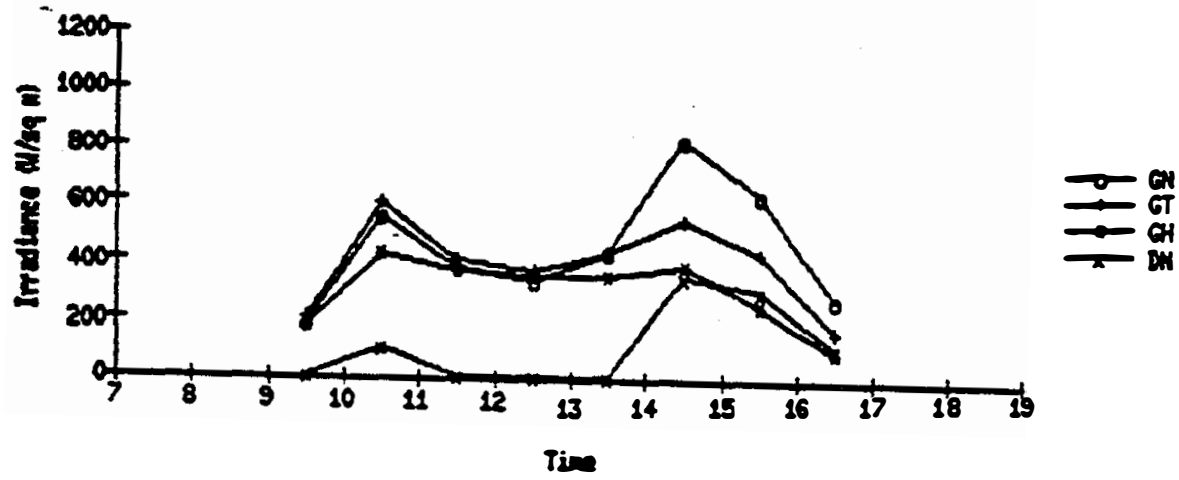

Psex 3020

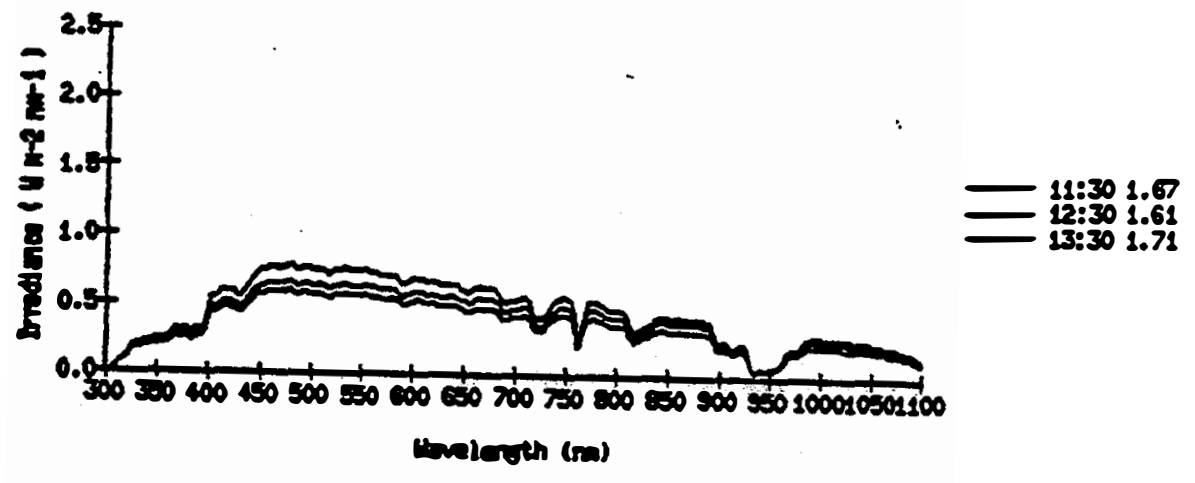


N FSP8 86363

0621229.86363218

Temp controller $39 \mathrm{C}$ at $0820 ; 40 \mathrm{C}$ at 1430

seattered clouds

Enotos: $0830 \mathrm{G}$

$0930 \mathrm{G}$

$1030 \mathrm{G}$

$1230 \mathrm{G}$

$1230 \mathrm{G}$

$1330 \mathrm{G}$

$1430 \mathrm{G}$

$2530 \mathrm{G}$

$1630 \mathrm{G}$

Paxe 33

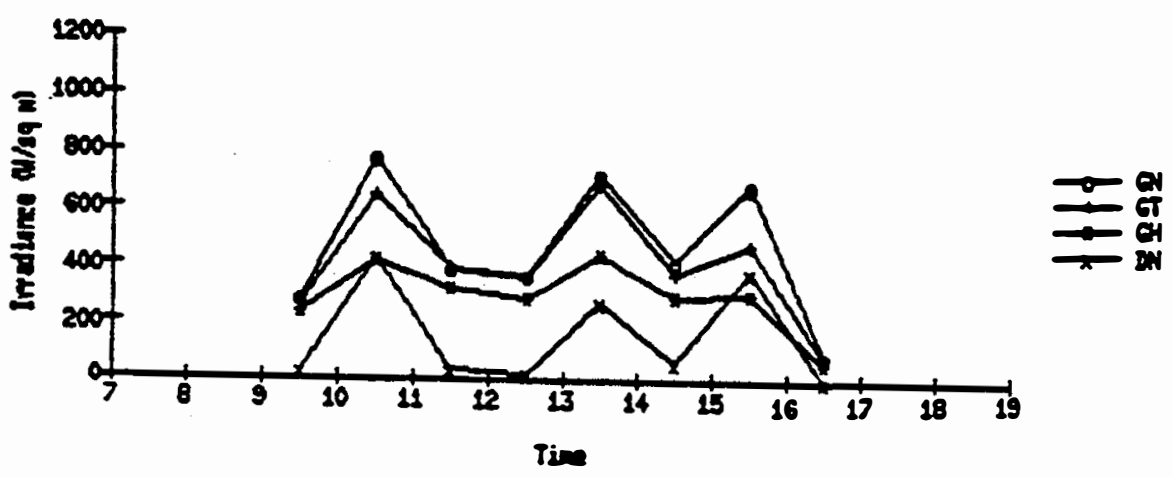

Fex 300

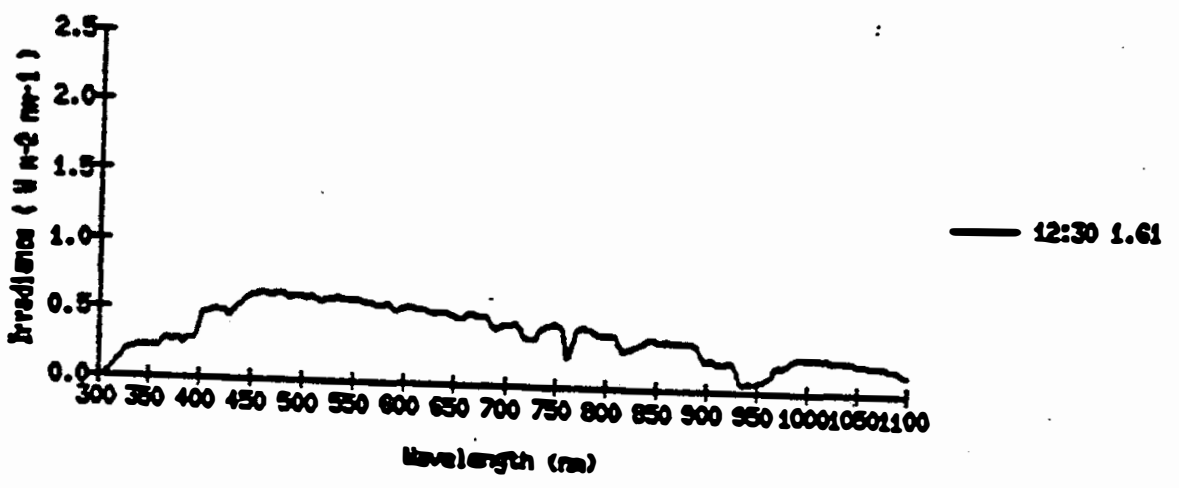




\section{SEF * *}

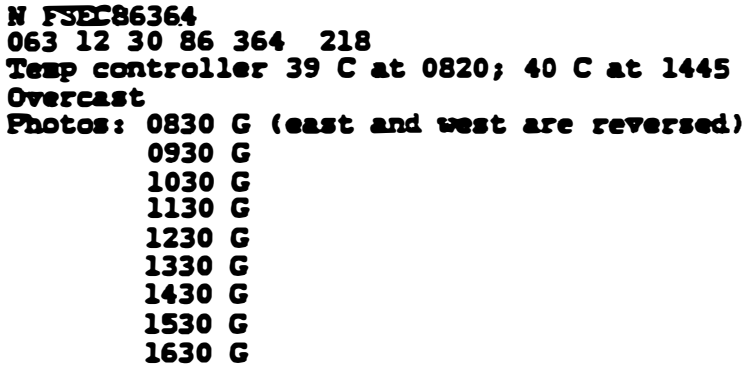

$1430 \mathrm{G}$

$1530 \mathrm{G}$

$1630 \mathrm{G}$

Fec 3e4

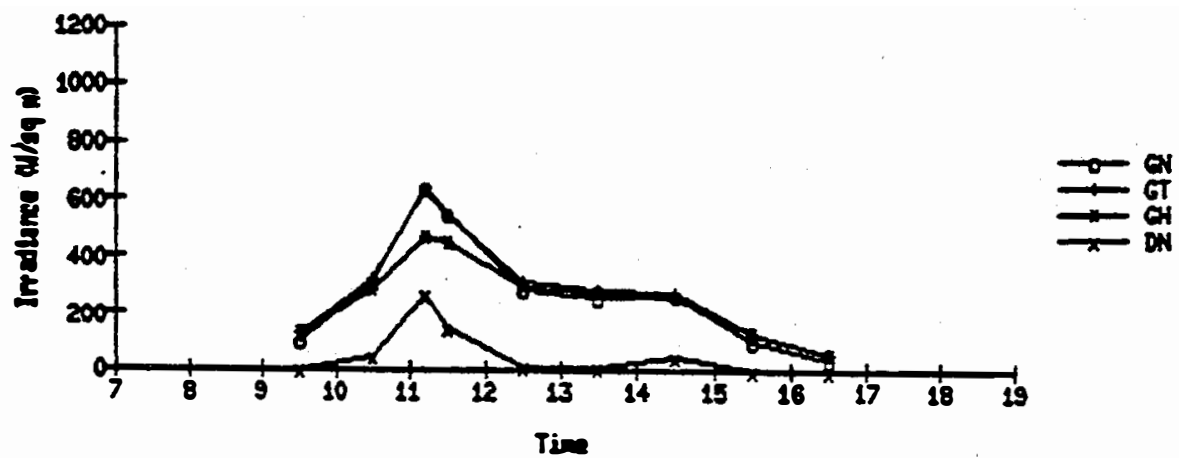

Fisc 3et on

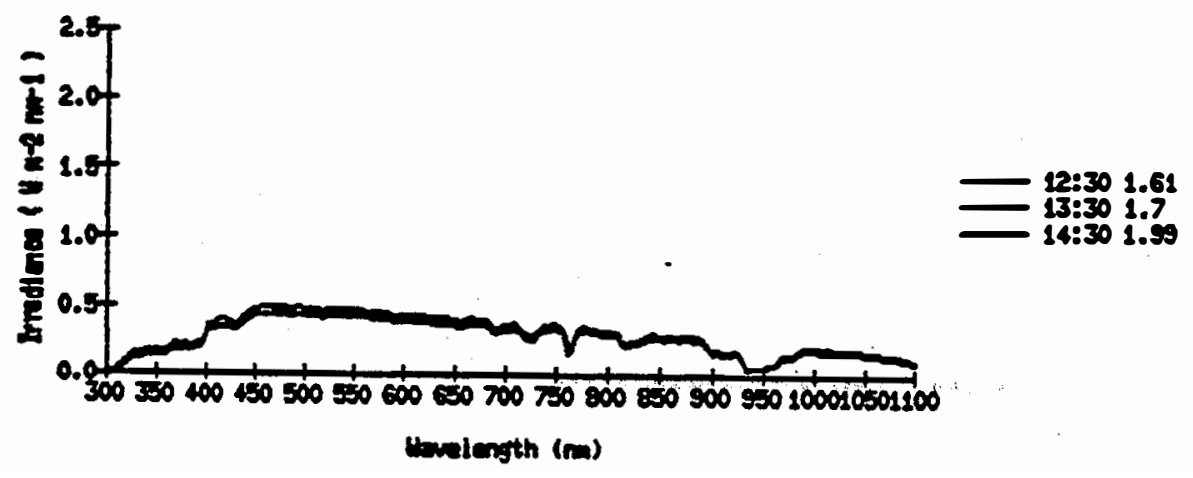


म.

$064 \quad 12 \quad 31 \quad 86 \quad 365 \quad 218$

Tenp controller 39 C at 0820

Clouds

Fhotos: $0830 \mathrm{G}$ (east and rest are reversed)

$0930 \mathrm{G}$

$1030 \mathrm{G}$

$1130 \mathrm{G}$

$1230 \mathrm{G}$

$1330 \mathrm{G}$

$1430 \mathrm{G}$

$1530 \mathrm{G}$

$1630 \mathrm{G}$

Spectral 1ntegral = broadband value at 1130

$=$

Fsec 365

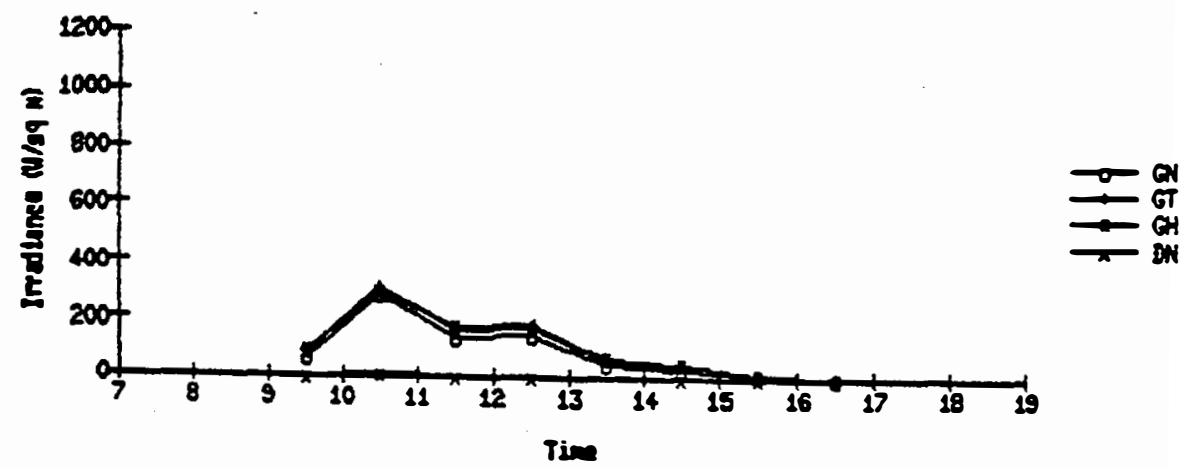

FEer 32000

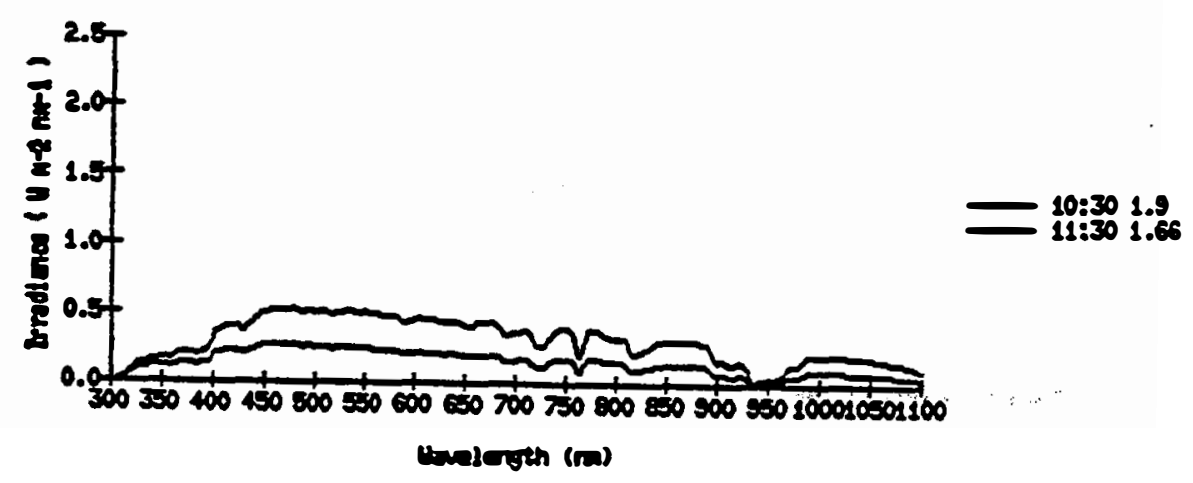


PST287001

$\begin{array}{llllll}065 & 01 & 01 & 87 & 002 & 218\end{array}$

Cloudy

Fwotos: $0830 \mathrm{G}$ (east and enest are reversed)

$1030 \mathrm{G}$

$2130 \mathrm{G}$

$1230 \mathrm{G}$

$1330 \mathrm{G}$

$1430 \mathrm{G}$

$1530 \mathrm{G}$

$1630 \mathrm{G}$

PIEC 1

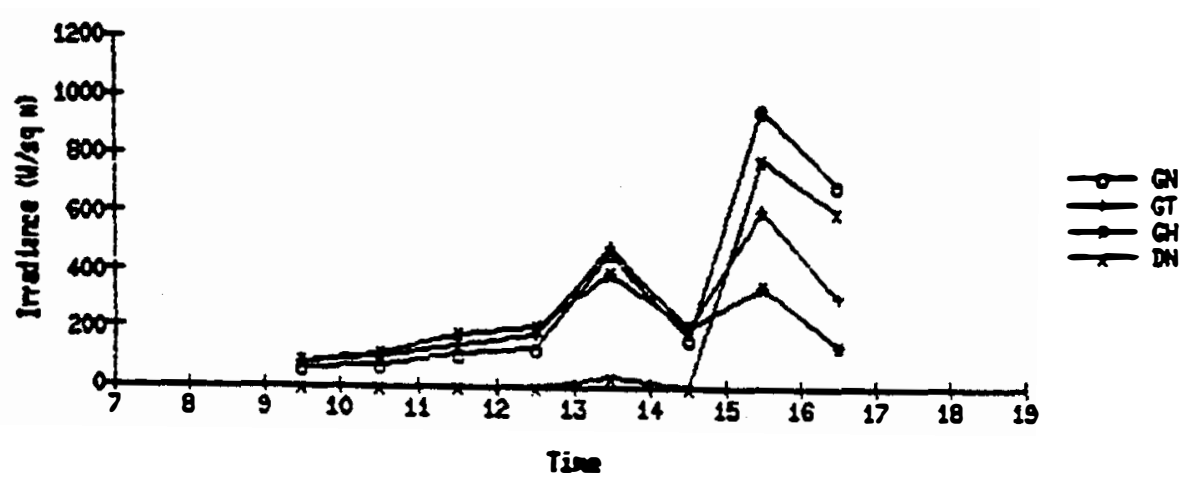

Fis 100

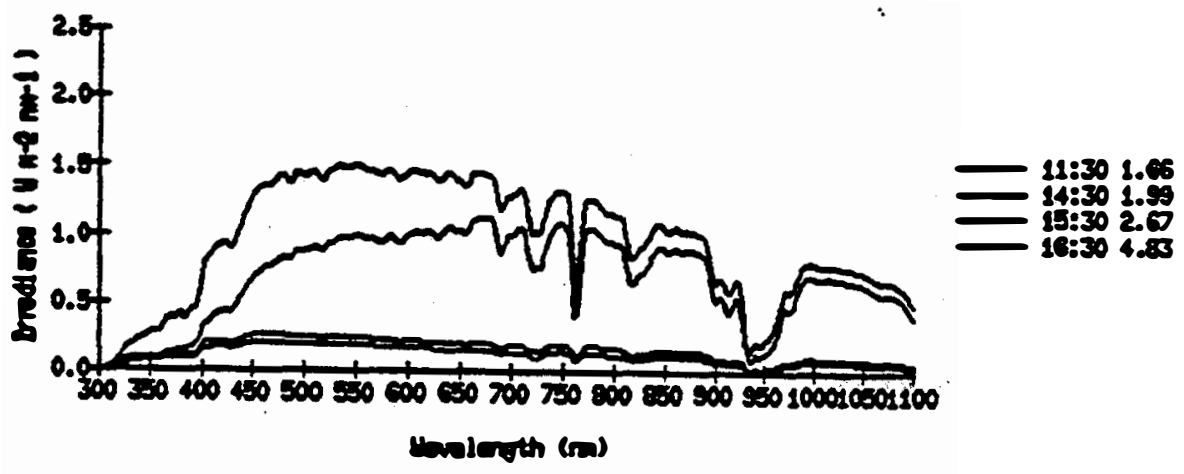




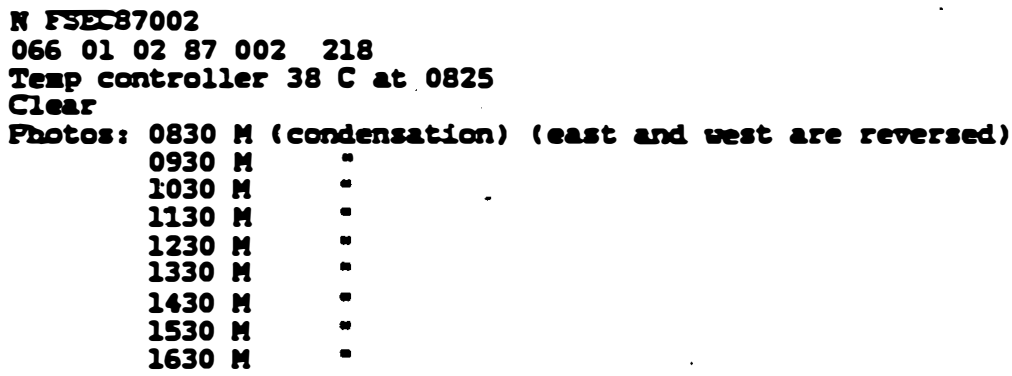

PIS 2

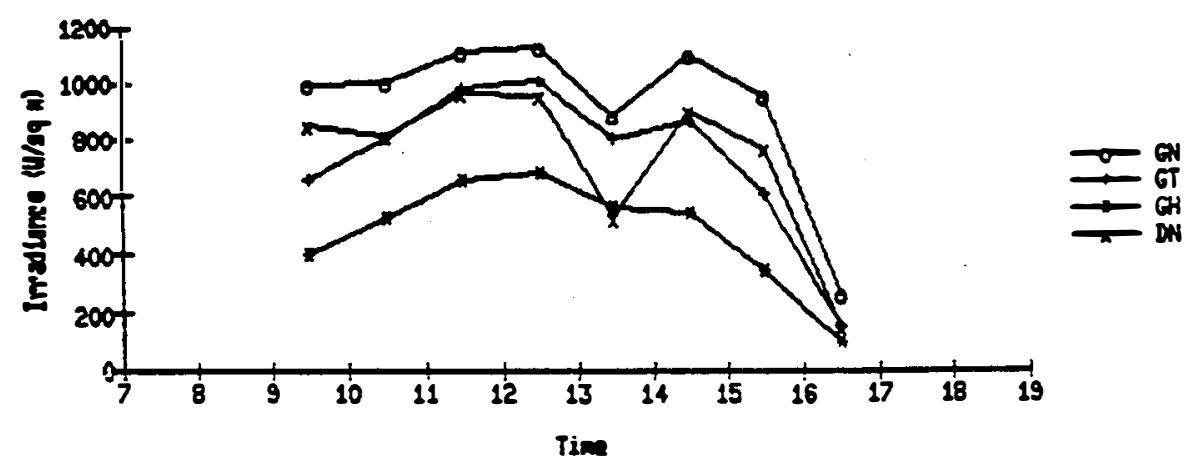

Fise 200

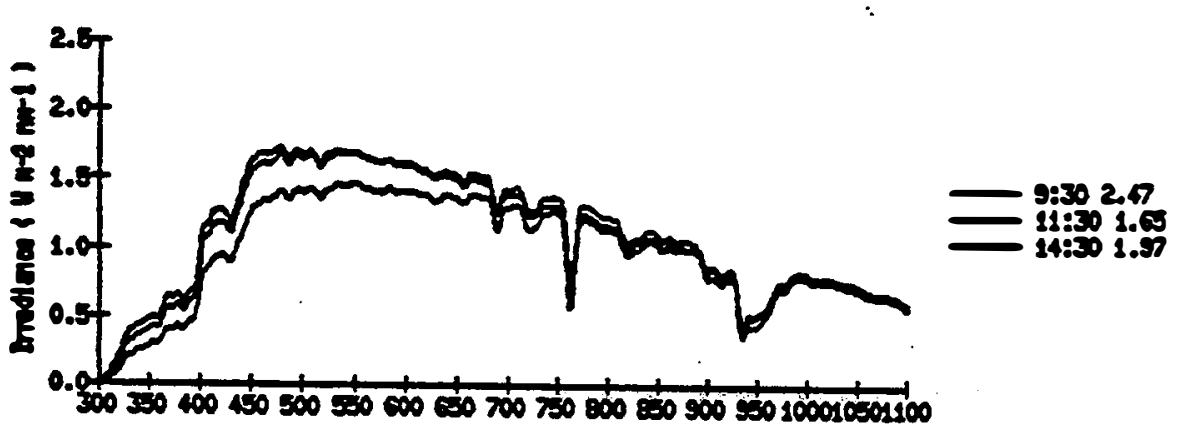

croletith (m) 


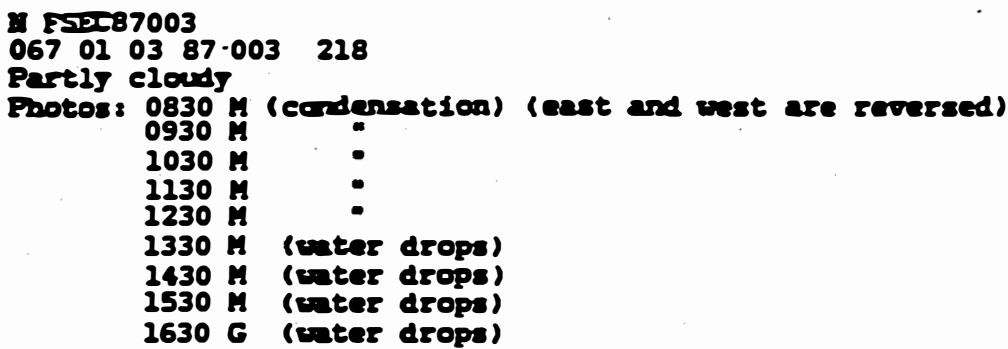

FEx 3

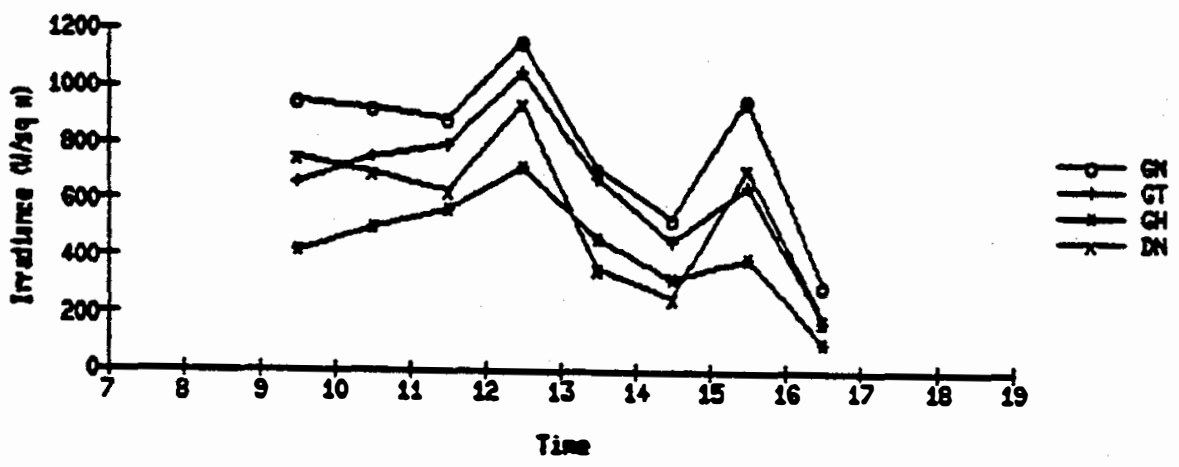

Fise 300

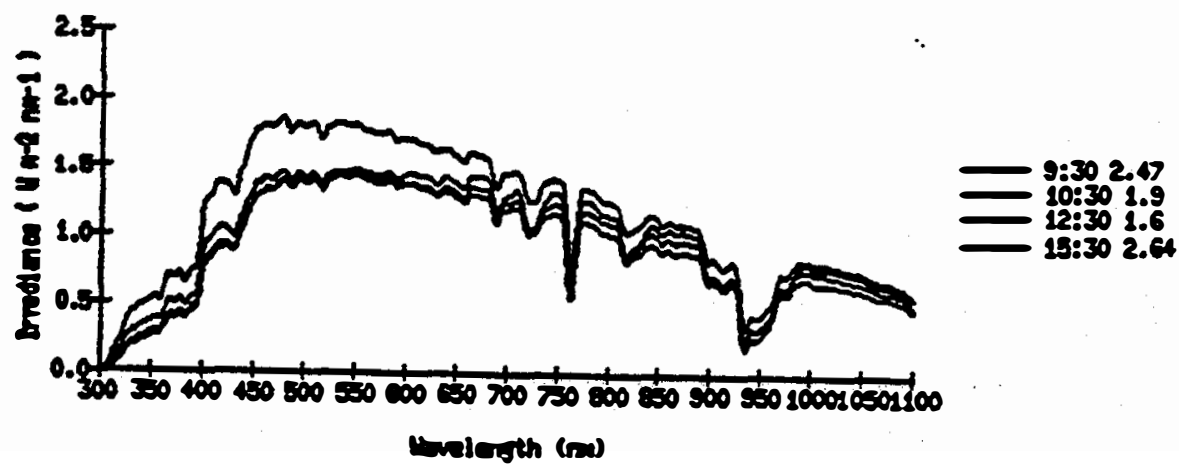


IV FSEC87004

$0680104 \quad 87004 \quad 818$

Overeast; zaisn

Fpotos: 0830 G (east and vest are reversed)

0930 P (processing problens)

1030
1130

$1230 \mathrm{P}$

$1330 \mathrm{P}$

$1430 \mathrm{P}$

Fese 4

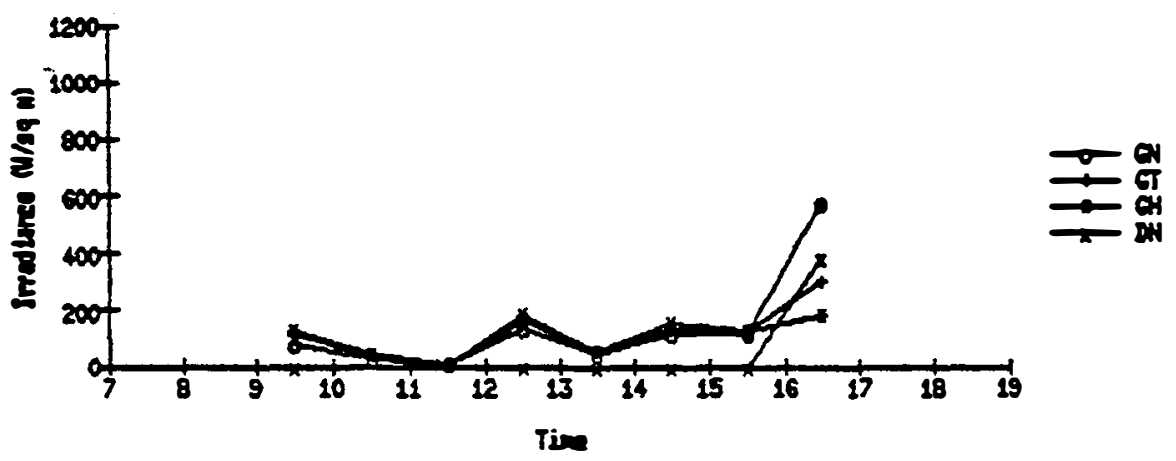

$\operatorname{Psec} 40$

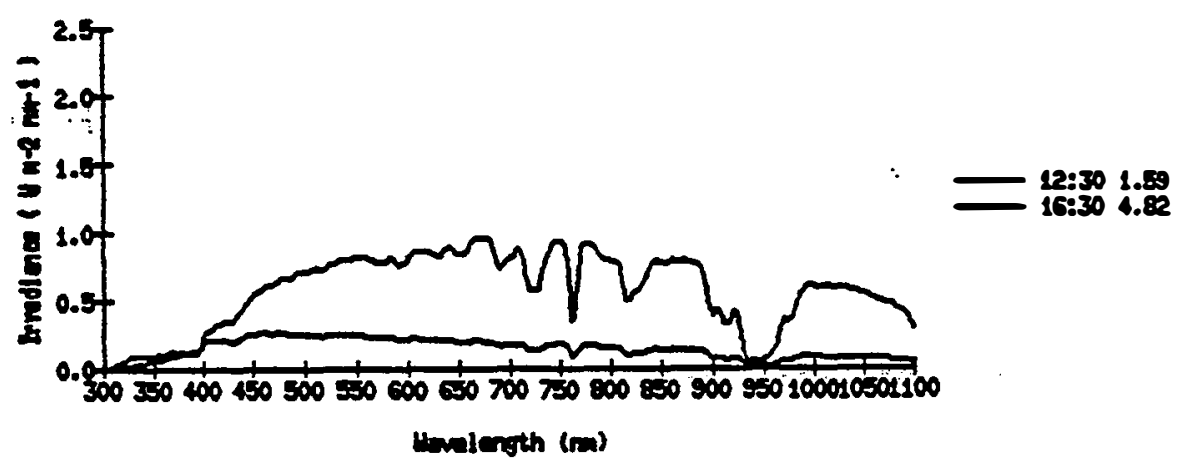


A FSPC87005

$069010687005 \quad 218$

Tep controller $38 \mathrm{C}$ at 1100

$180 \mathrm{deg}$ less lostealled on all-sk canera

overcast, windy

Photos: $1030 \mathrm{G}$

$1130 \mathrm{G}$

$1230 \mathrm{G}$

$1330 \mathrm{G}$

$1430 \mathrm{G}$

$1530 \mathrm{G}$

$1630 \mathrm{G}$

io spectral dint

PIES 5

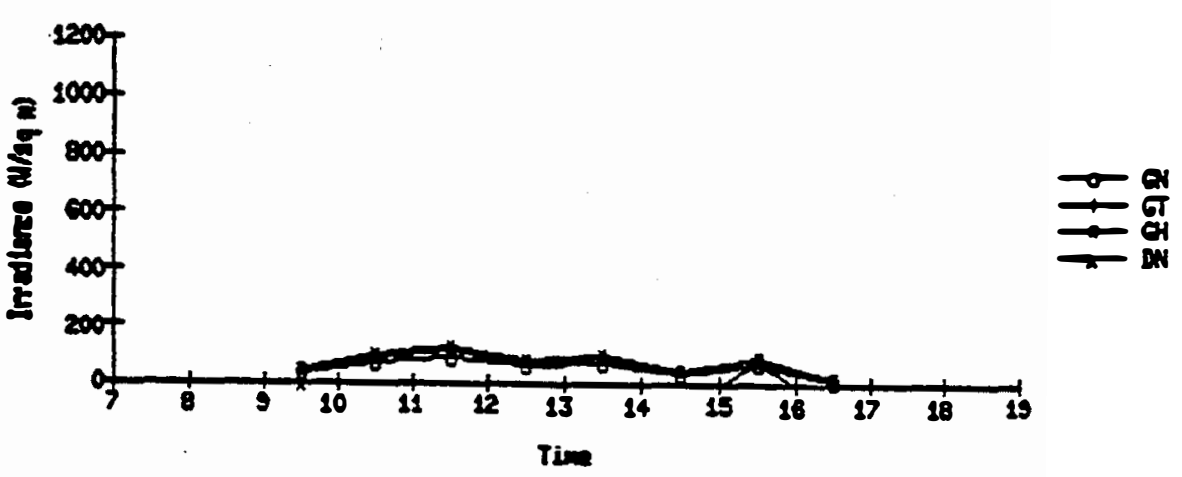




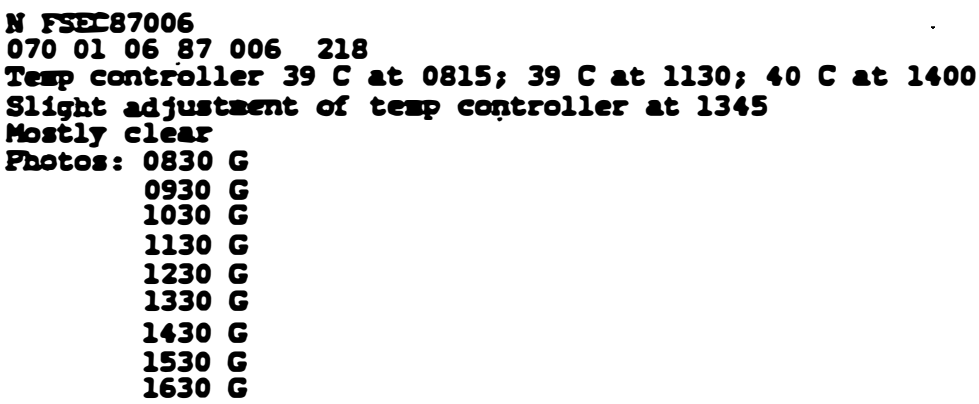

Fax 6

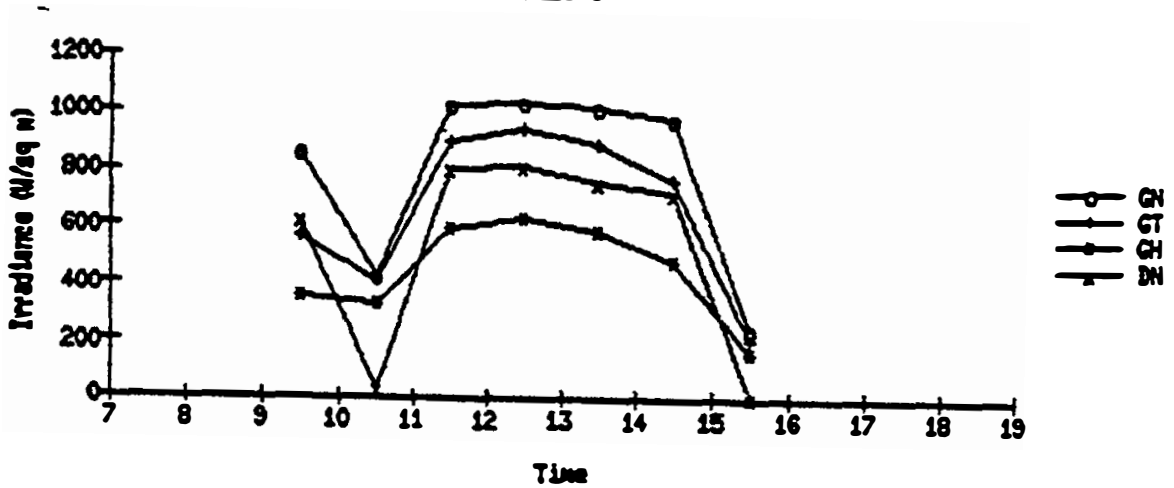

Fis 600

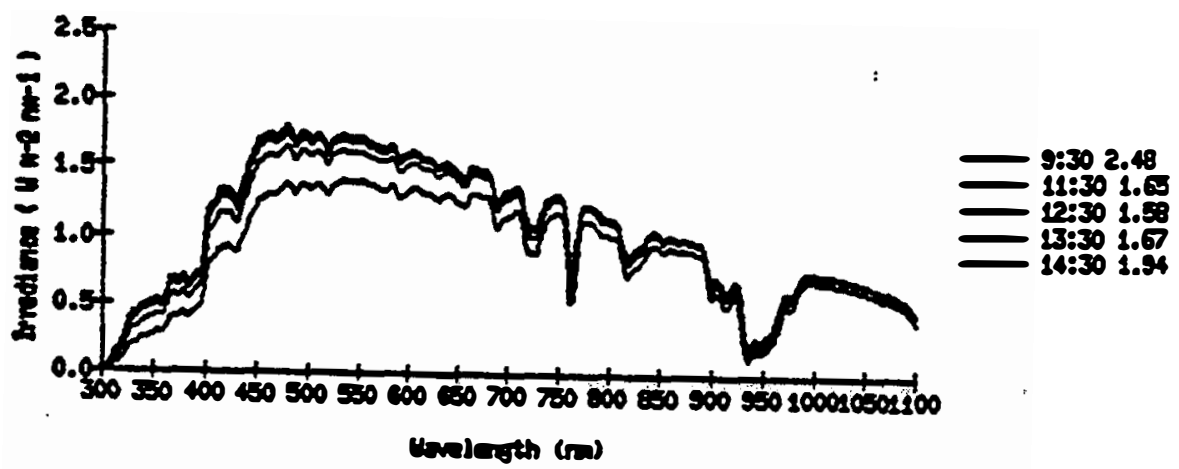


FSPC87007

$071010787007 \quad 218$

Tep controller $39 \mathrm{C}$ at 0930

overcast, beconing partly cloudy.

Fwotos: Poine ourege 0830-0930

$1030 \mathrm{G}$

$1230 \mathrm{G}$

$1230 \mathrm{G}$

$1330 \mathrm{G}$

$1430 \mathrm{G}$

$1530 \mathrm{G}$

$1630 \mathrm{G}$

Fax 7

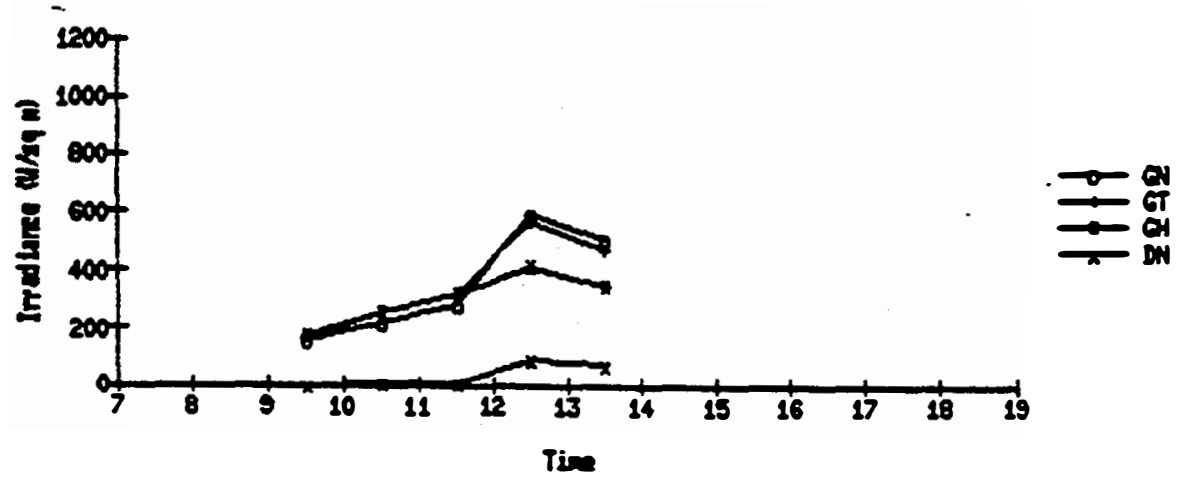

Fax6 70

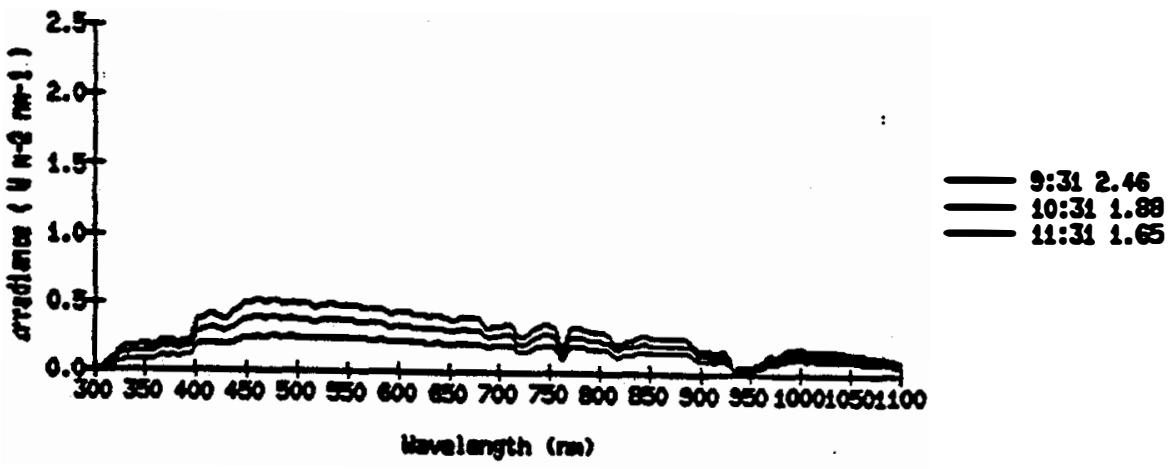


A FSPC87008

$\begin{array}{llll}07201 & 08 \quad 87.008 \quad 218\end{array}$

Tep controller $39 \mathrm{C}$ at 0900 ; $40 \mathrm{C}$ at 1330

Mostly elear

Photos: 0830 M (condermtson)

$0930 \mathrm{M}$

$1030 \mathrm{~K}$

$1130 \mathrm{M}$

$1230 \mathrm{M}$

$1330 \mathrm{M}$

$1430 M$

$1530 M$

$1630 \mathrm{M}$

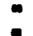

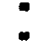

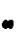

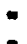

$\operatorname{Fsec} 8$

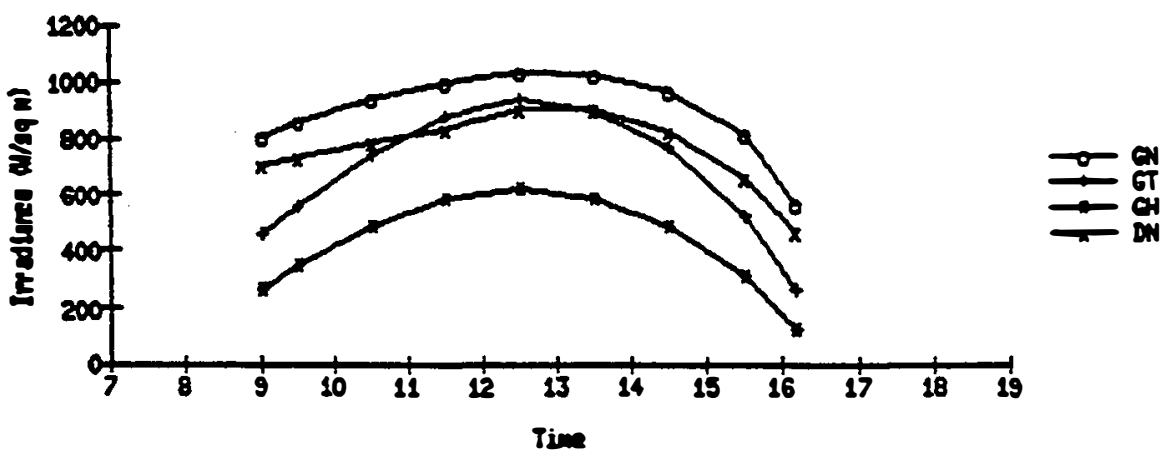

$\operatorname{FES} 80$

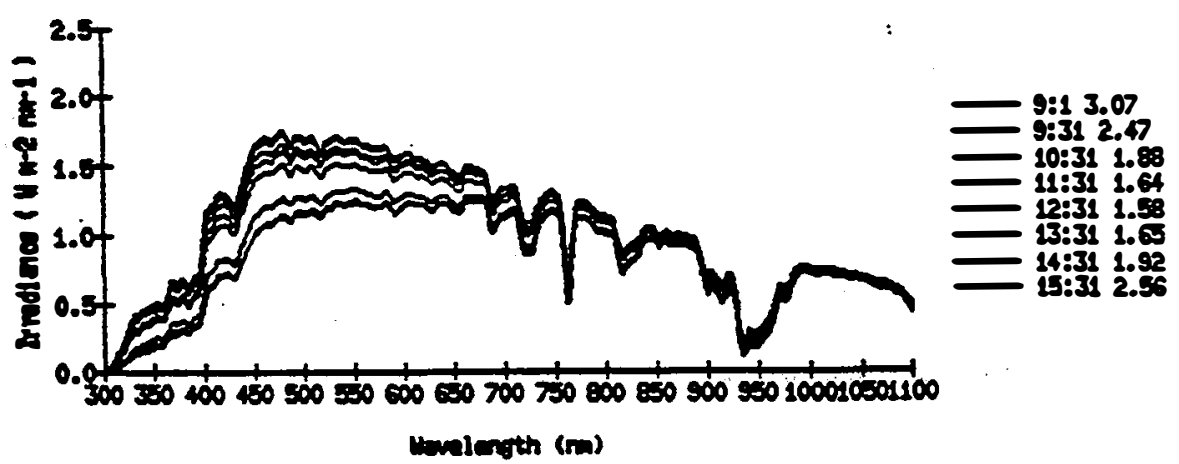


1. FSTC87009

$\begin{array}{llllll}073 & 01 & 09 & 87 & 009 & 218\end{array}$

Tep controller $39 \mathrm{C}$ at $1000 ; 38 \mathrm{C}$ at 1500

Partly elouds. beconing overenst

Photos: 0830 M (condenentles)

$0930 \mathrm{M}$

$1030 \mathrm{G}$

$1130 \mathrm{G}$

$1230 \mathrm{G}$

$1330 \mathrm{G}$

$1430 \mathrm{G}$

$1530 \mathrm{G}$

$1630 \mathrm{G}$

Fese 9

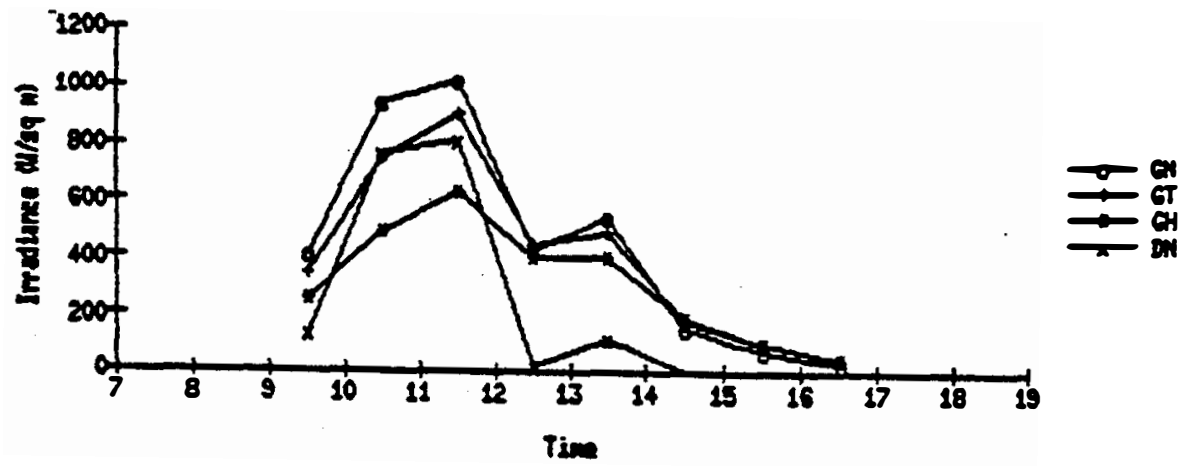

Fexc 90

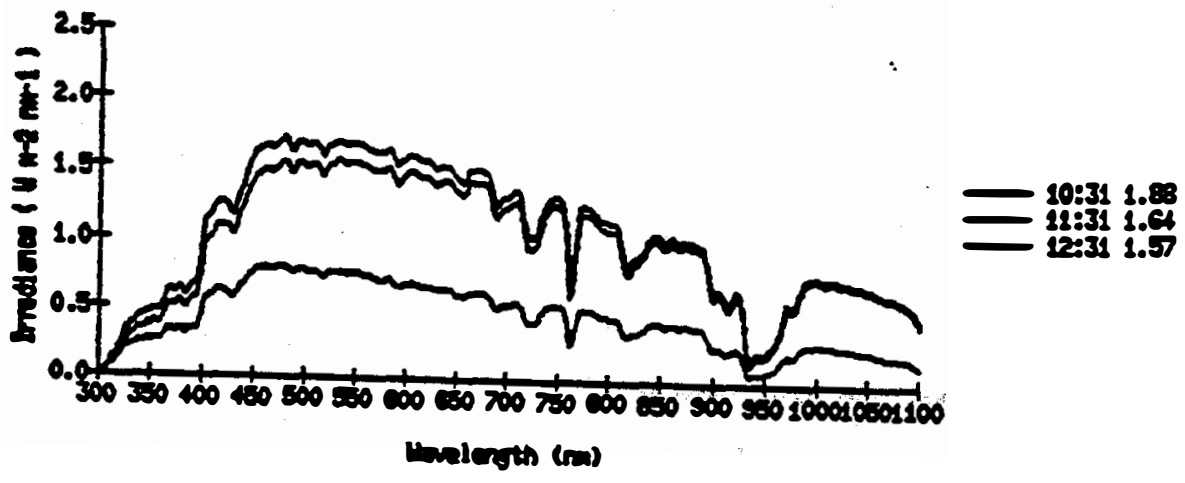


घ

$\begin{array}{llllll}074 & 01 & 10.87 & 010 & 218\end{array}$

Partly elouds

Fhotos2 $0830 \mathrm{G}$

$0930 \mathrm{G}$

$1030 \mathrm{G}$

$1130 \mathrm{G}$

$1230 \mathrm{G}$

$1330 \mathrm{G}$

$1430 \mathrm{G}$

$1530 \mathrm{G}$

$1630 \mathrm{G}$

Senll glat spot in specting about $600 \mathrm{~m}$ (?) at 1631

FEבC 10

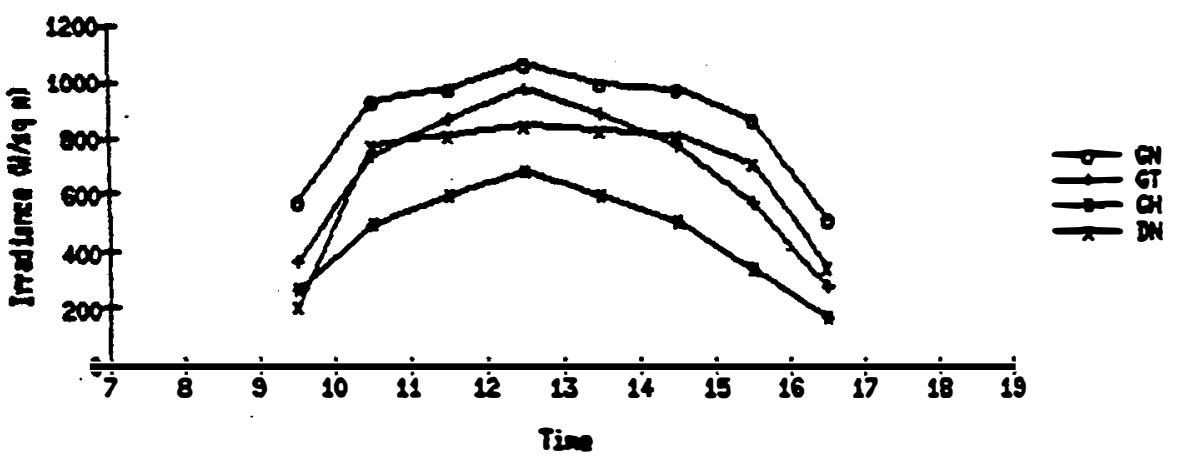

$P=21000$

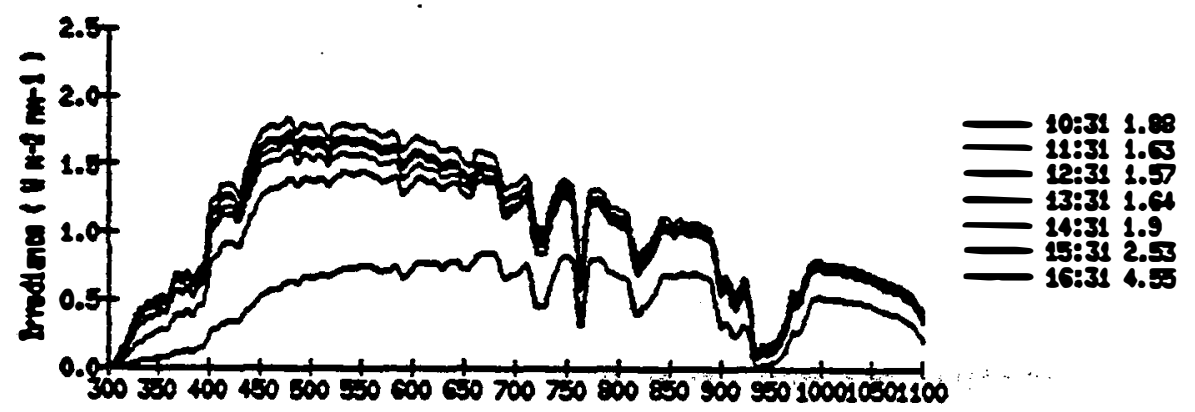

Uinelerth (m) 


\section{FSPC87011}

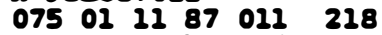

overcast, becoling nostly clear at 1100

Photos: $0830 \mathrm{G}$

$0930 \mathrm{G}$

$1030 \mathrm{G}$

$1130 \mathrm{G}$

$1230 \mathrm{G}$

$1330 \mathrm{G}$

$1430 \mathrm{G}$

$1530 \mathrm{G}$

$1630 \mathrm{G}$

Faxe 11

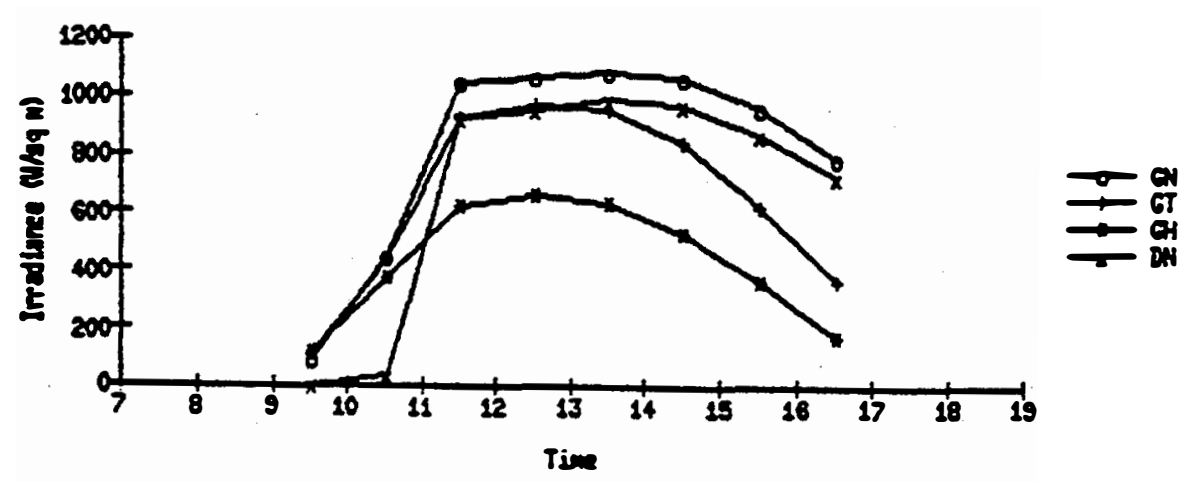

Fese 1100

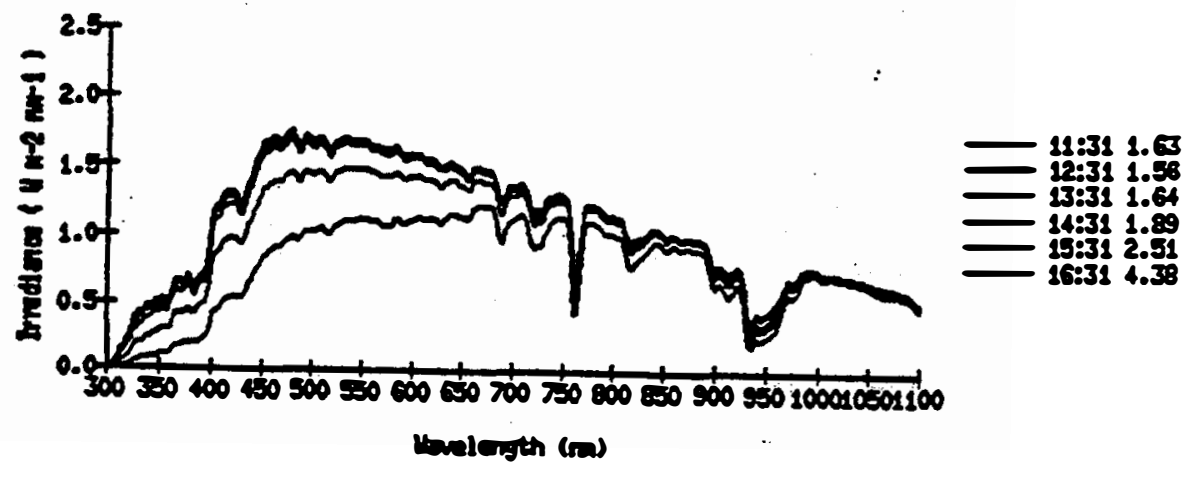




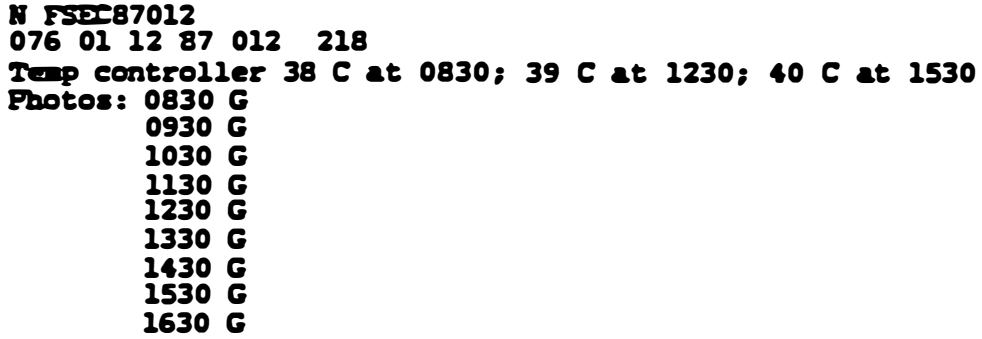

$1230 \mathrm{G}$

$1330 \mathrm{G}$

$1430 \mathrm{G}$

$1530 \mathrm{G}$

$1630 \mathrm{G}$

FSEC 12

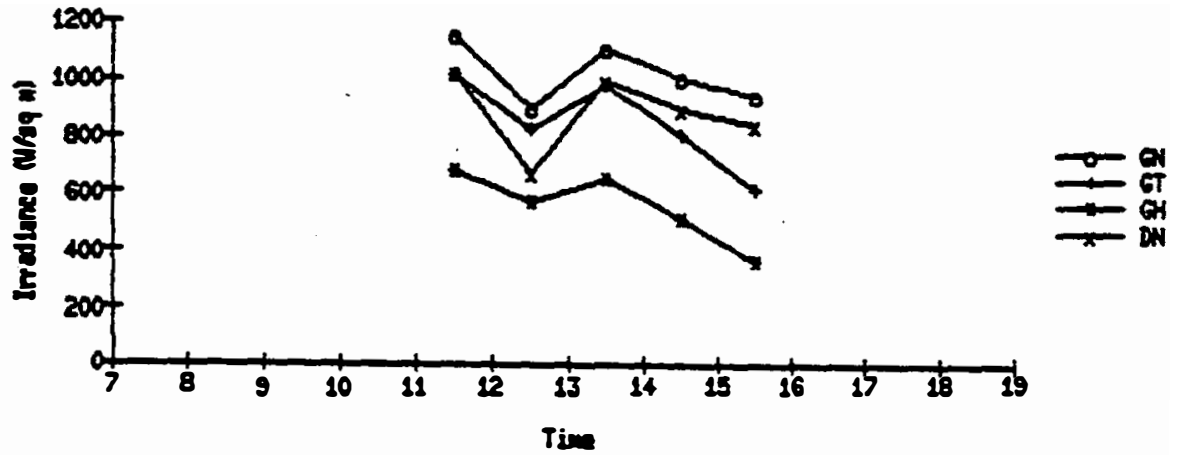

Fix 120

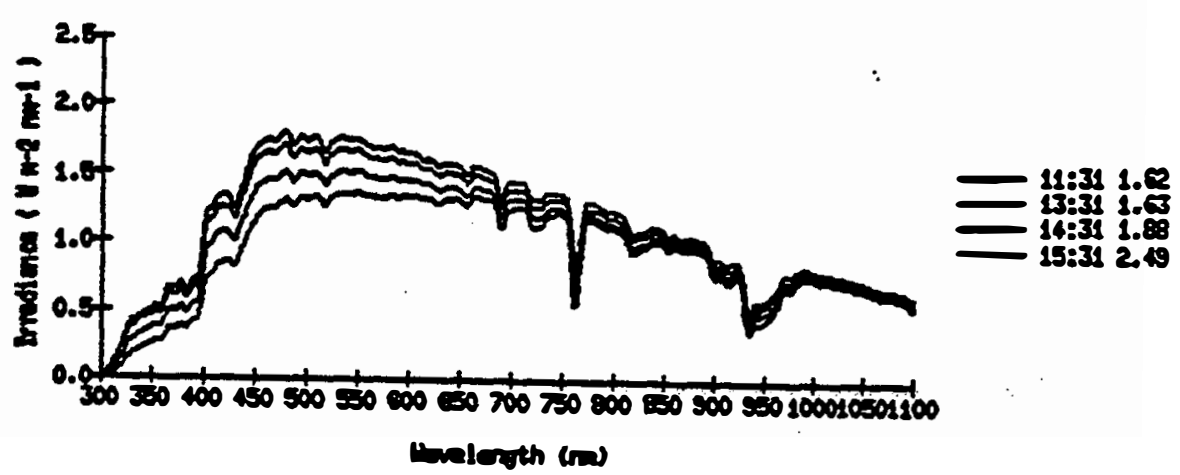




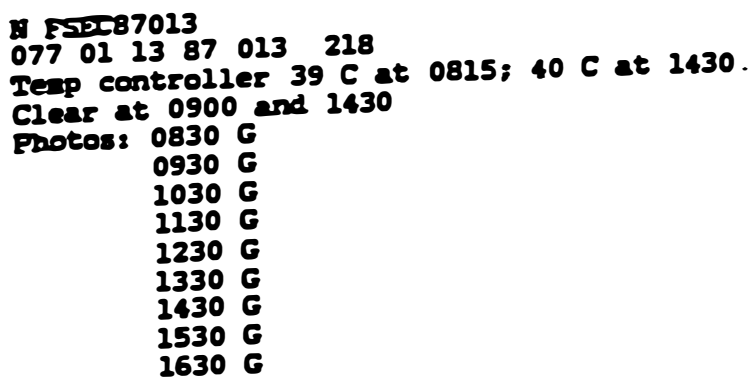

Fase 23

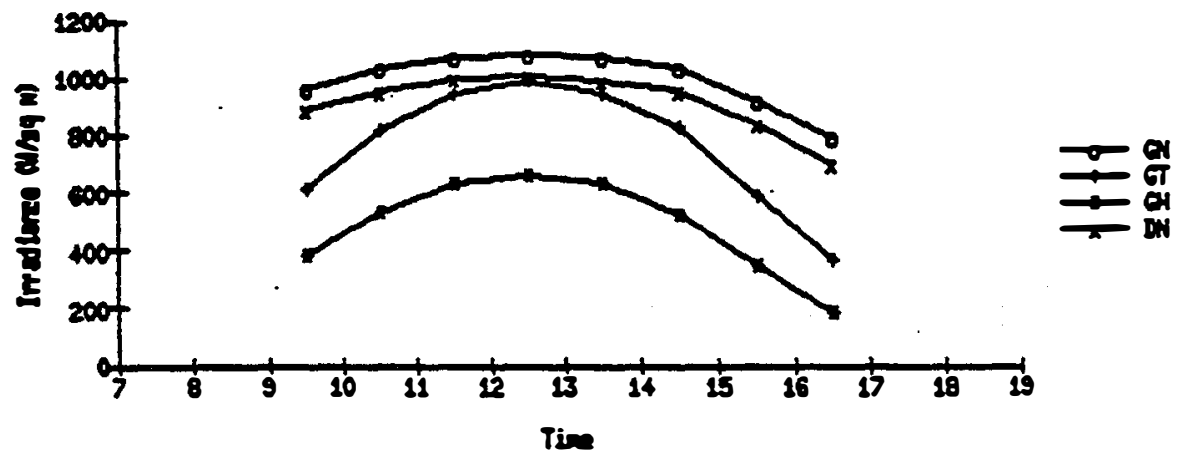

$\operatorname{cise} 2300$

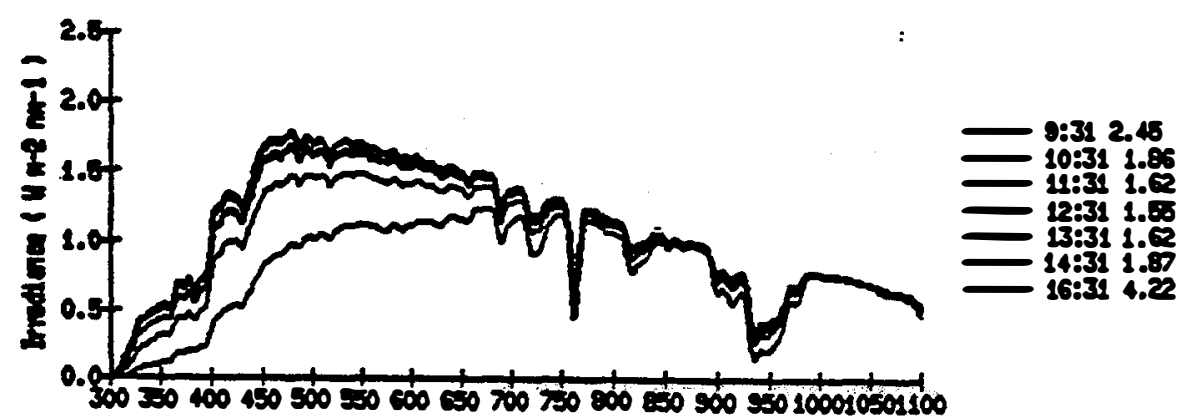

Canlarith (m) 
IN FSPC87014

$\begin{array}{llllll}078 & 01 & 14 & 87 & 014 \quad 218\end{array}$

Tenp controller $39 \mathrm{C}$ at 0815; il C at 1230; il C at 1530

Protos? $0830 \mathrm{G}$

$0930 \mathrm{G}$

$1030 \mathrm{G}$

$1130 \mathrm{G}$

$1230 \mathrm{G}$

$1330 \mathrm{G}$

$1430 \mathrm{G}$

$1530 \mathrm{G}$

Hote tine stap differences 1211 ve. 1231

FSEC 14

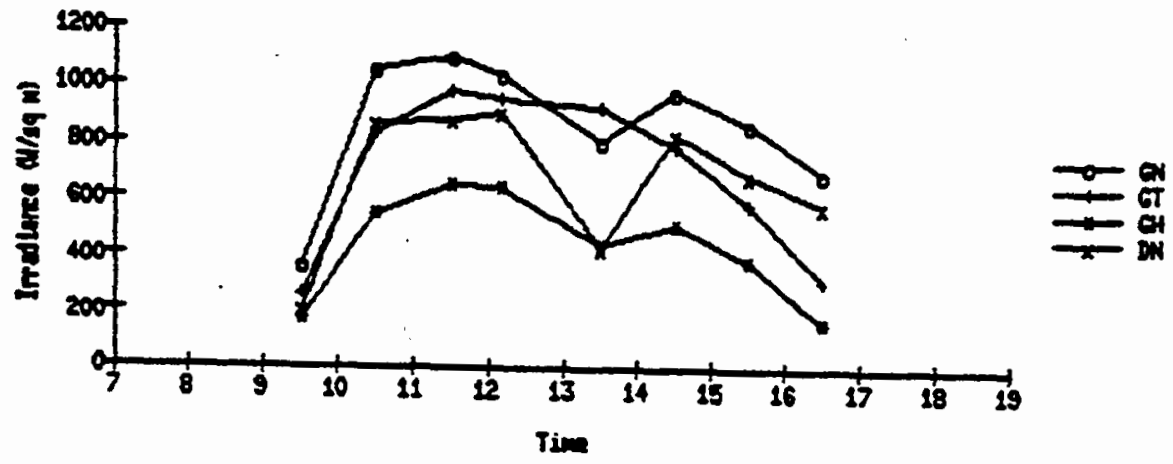

Fsxc 1400

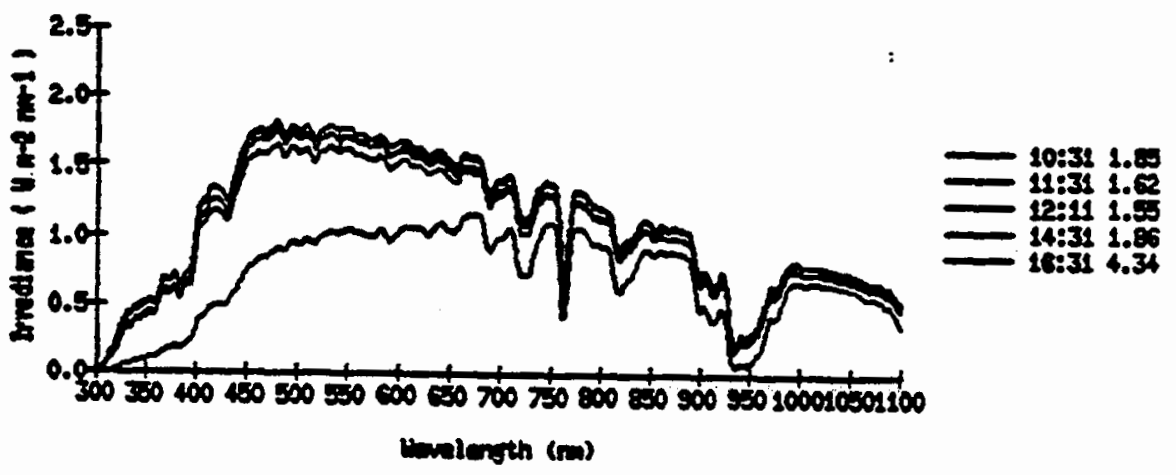


4 FSTC87015

$079012587015 \quad 218$

Temp controller $40 \mathrm{C}$ at 1030

Clear at $1030,0.1$ eloud cover at 1530

Frotos: 0830 M (condensation)

$0930 \mathrm{~A}$ (condensetion)

1030 P (processing probleas)

Fesc 25

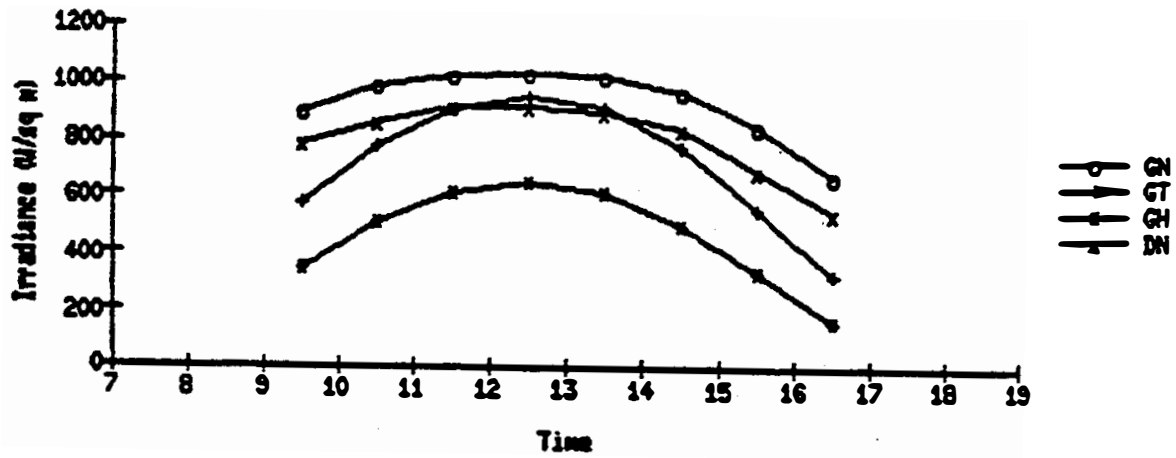

Fic 2800

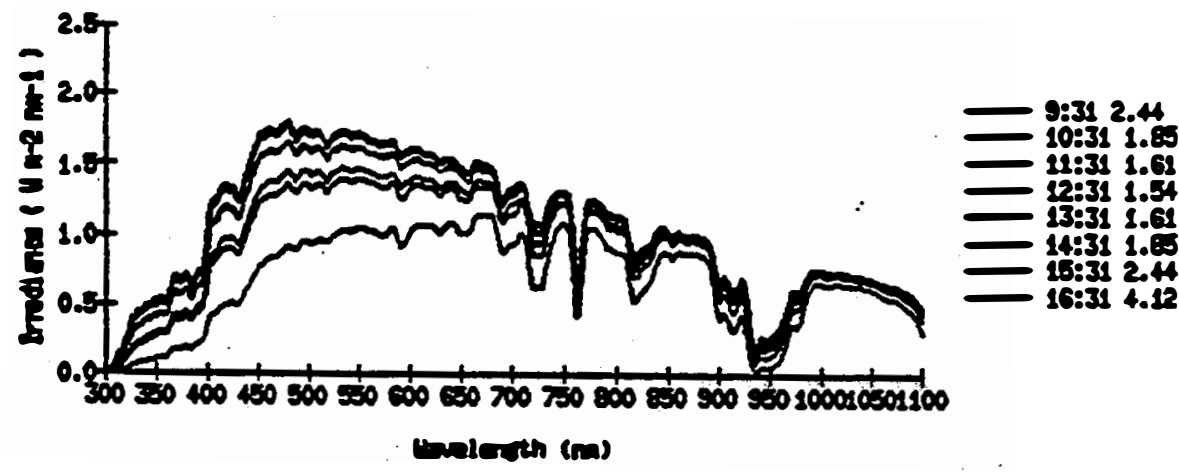


N FSEC87016

$08001 \quad 1687016 \quad 218$

Temp controller $40 \mathrm{C}$ at $0815 ; 41 \mathrm{C}$ at 1430

0.1 cloud cover at 0830 ; clear at 1430

No photos

FSEC 16

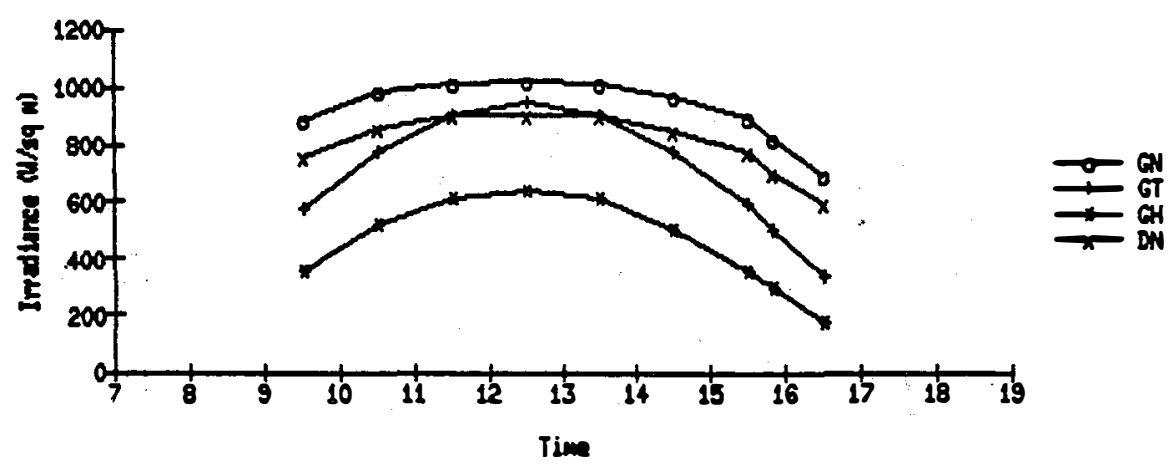

FSEC 16 aNo

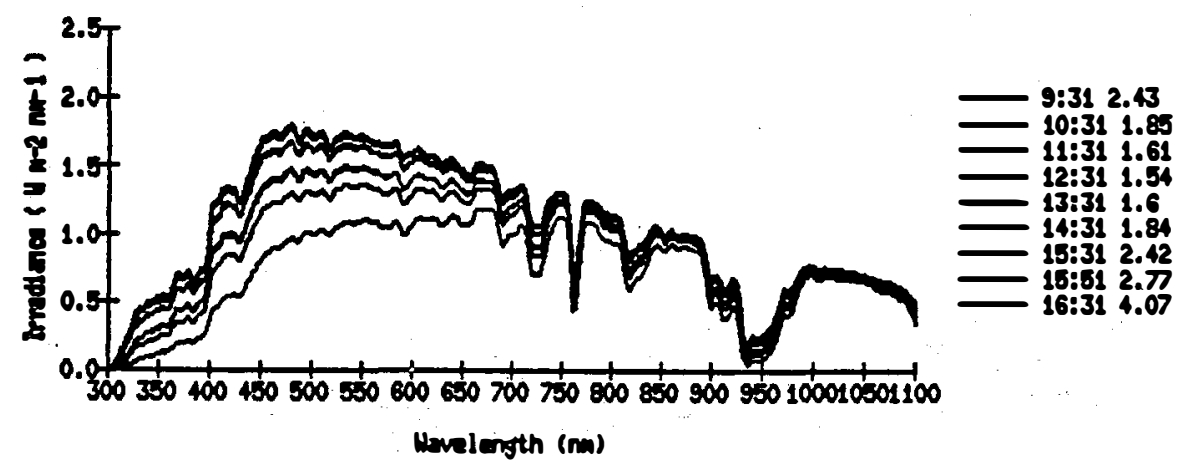


N FSEC87017

$\begin{array}{lllllll}081 & 01 & 17 & 87 & 017 & 218\end{array}$

Power interrupt; trackers guit at 1400

Partly eloudy

No photos

FSEC 17

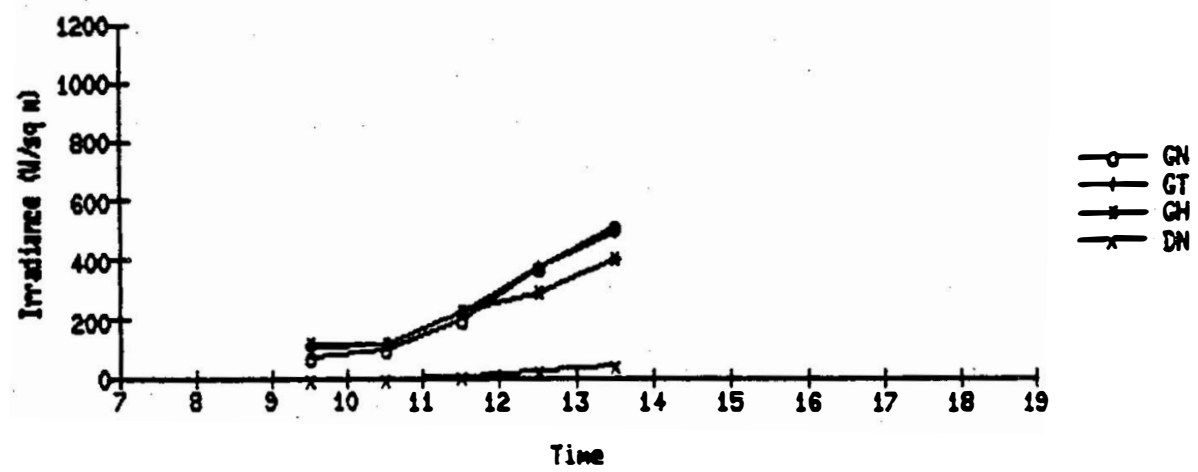

FSEC 17 OND

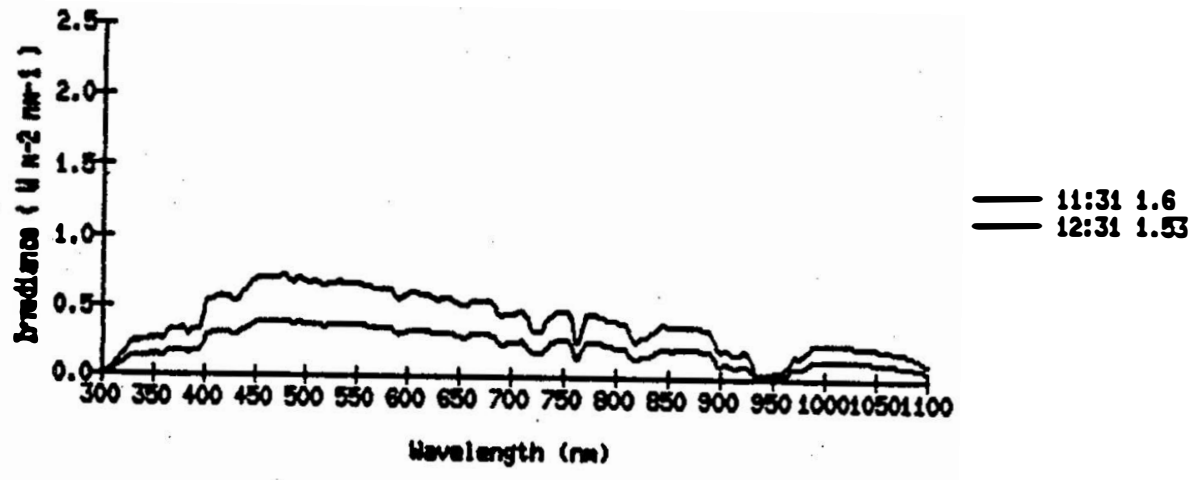


A. FS2587020

$082012087020 \quad 218$

Terp controller $40 \mathrm{C}$ at 1300

Partly cloud's; 0.8 cloud cover at 1300

Ho photos

CI bef/after 18 bad at 1530

FSEC 20

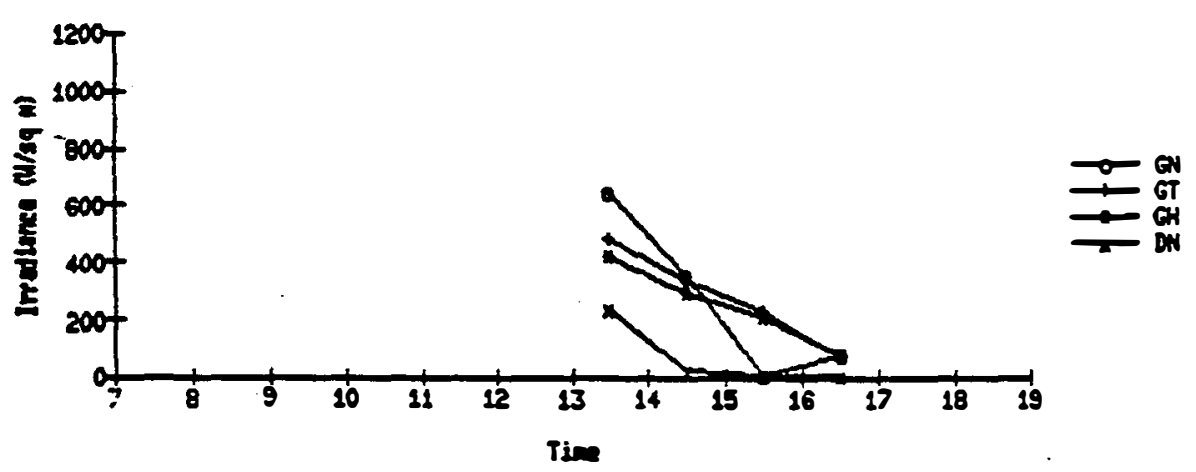

$\operatorname{cosec} 200$

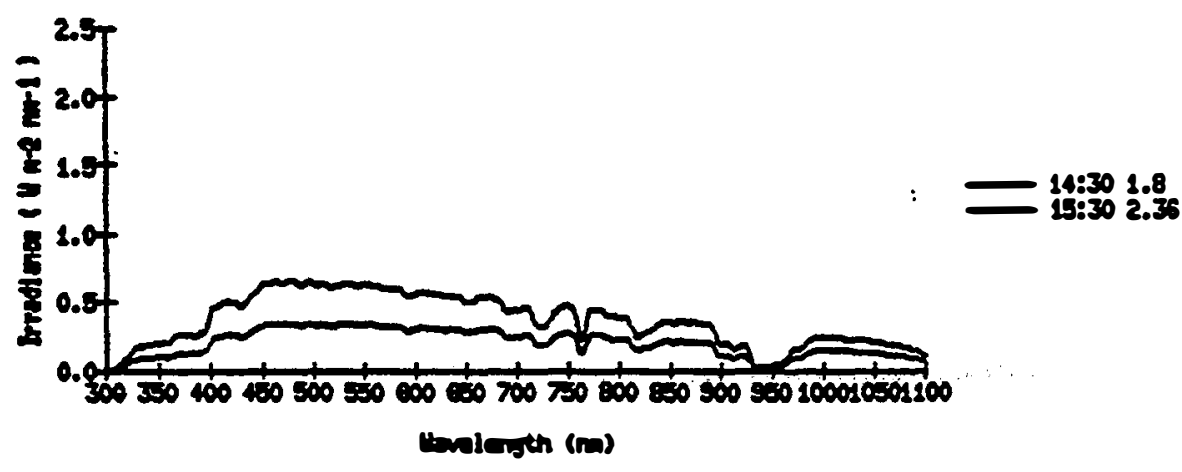


4. FSDC87021

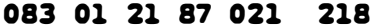

Tenp controlier $39 \mathrm{C}$ at 0830

Opercast at $0830 ; 0.9$ eloud cover at 1530

Ho photos

FEEC 21

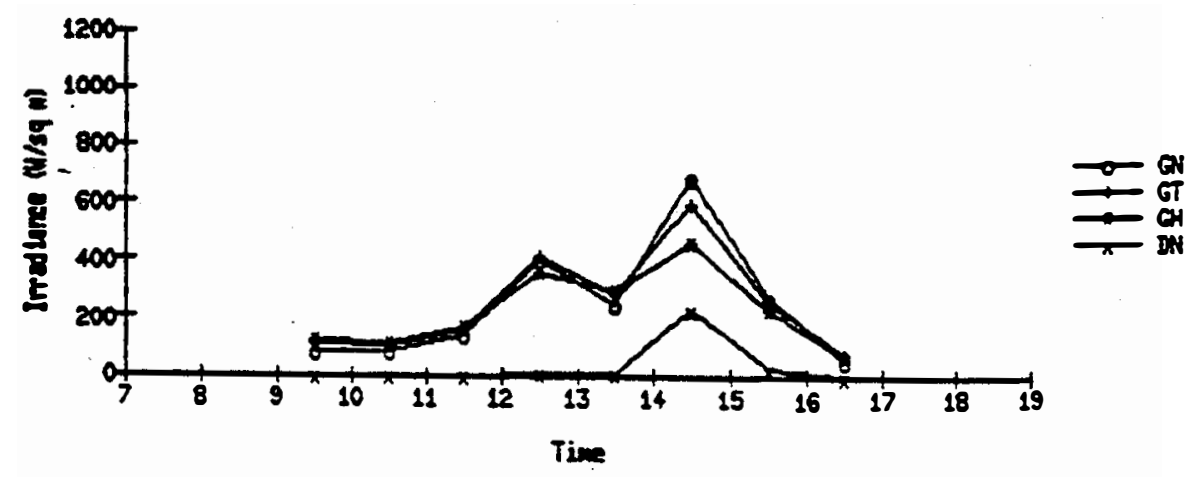

Fise 2100

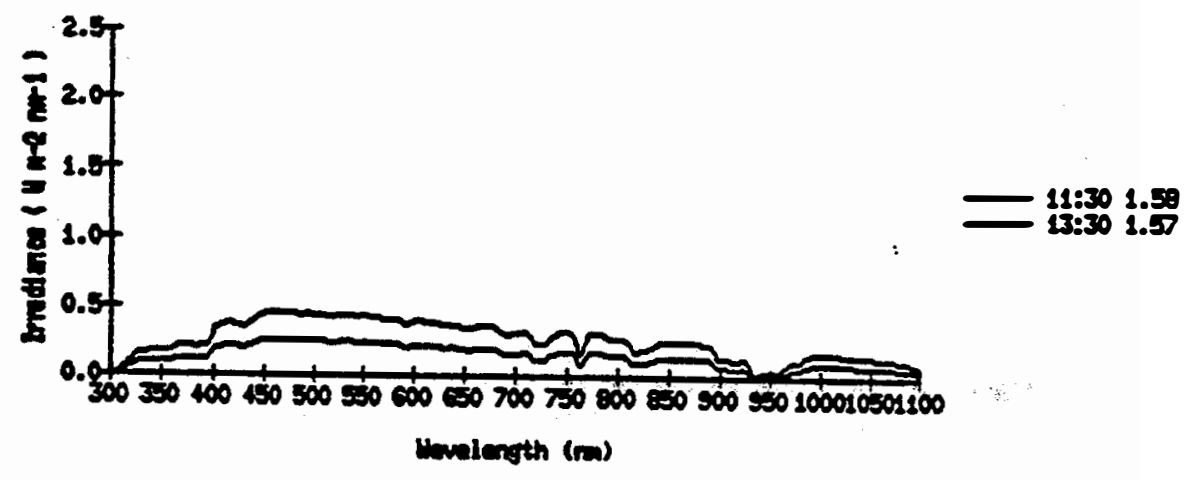


4 FST्87022

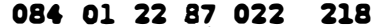

Tenp controiler $39 \mathrm{C}$ at 0830

Overcast at 0830; rain 1000-1200, 1.0 eloud cover at 1530

No photos

No spectral data

Fsec 22

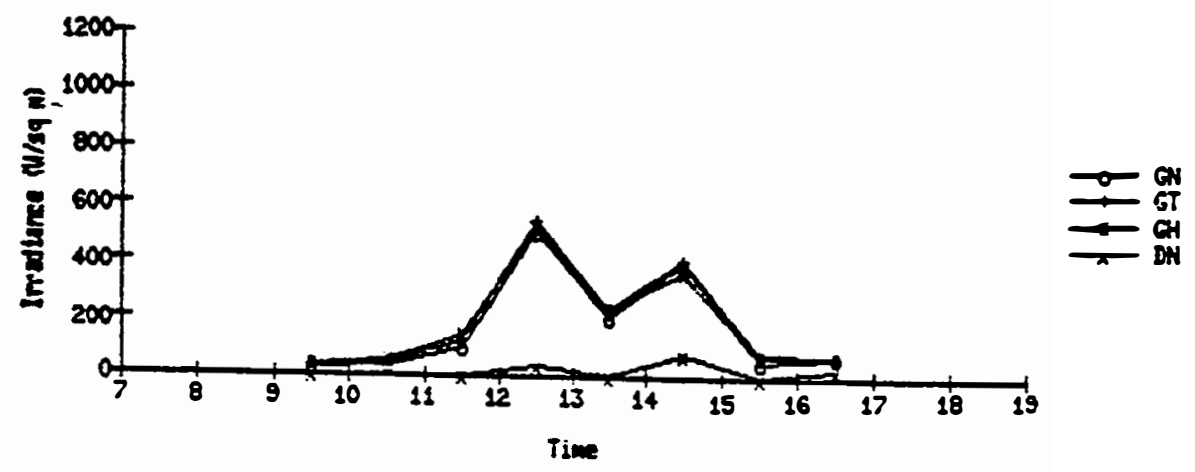

$\operatorname{Fsec} 2200$

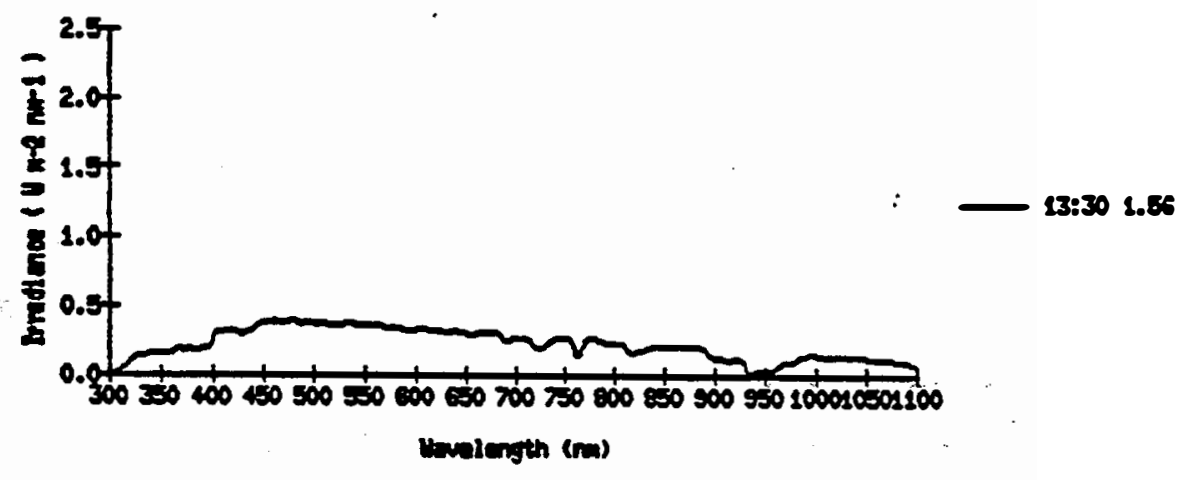




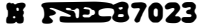

$085012387.023 \quad 218$

Teap controller $38 \mathrm{C}$ at 1030 ; $38 \mathrm{C}$ at 1330

High D.

Clear at 0815 and 1630 ; rindy

I. photos

Fifst dffferent at 1030, 1130, 1230

Fise 23

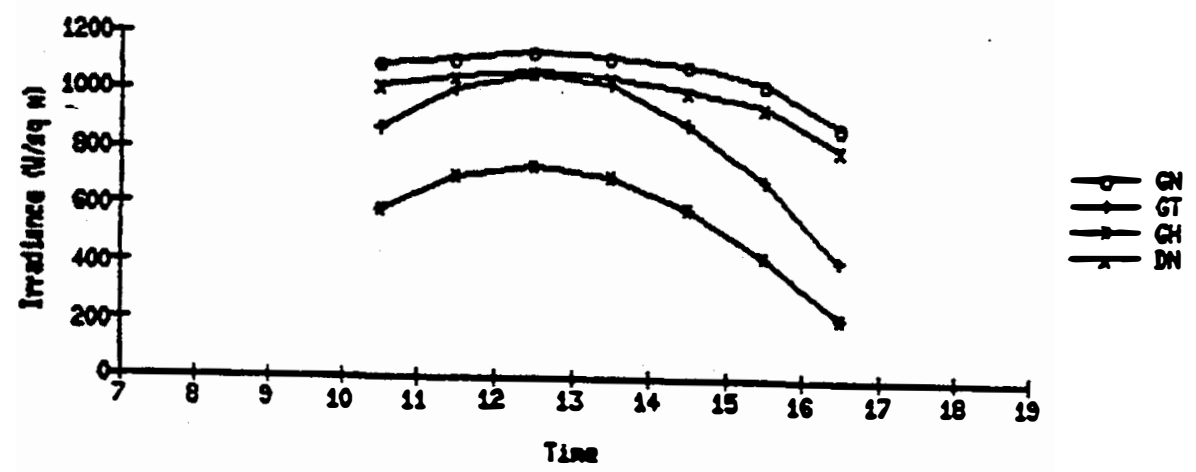

Fise $2 x 00$

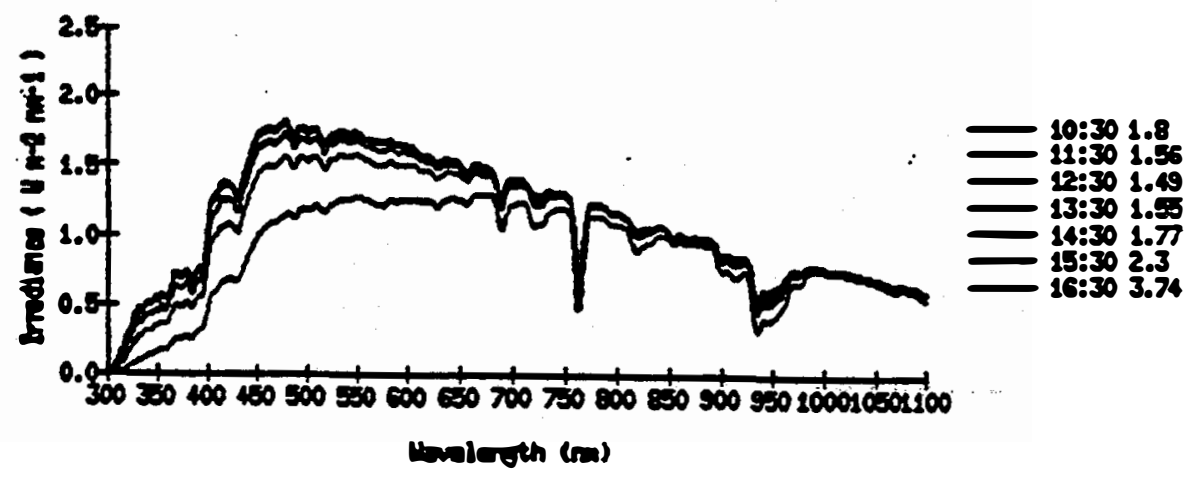


a F्Sग्य7024

$\begin{array}{llll}08601 & 24 \quad 87024 \quad 228\end{array}$

Partis eloudy

Photos: $0830 \mathrm{G}$

$0930 \mathrm{G}$

$1030 \mathrm{G}$

$1230 \mathrm{G}$

$2230 \mathrm{G}$

$1330 \mathrm{G}$

$1430 \mathrm{G}$

$2530 \mathrm{G}$

PSEC 24

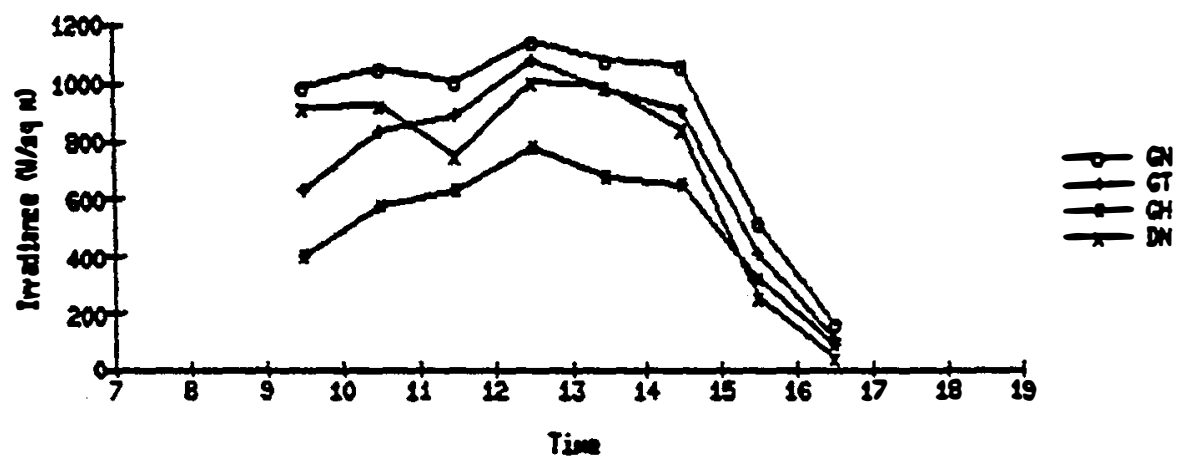

$\operatorname{Fesc} 2400$

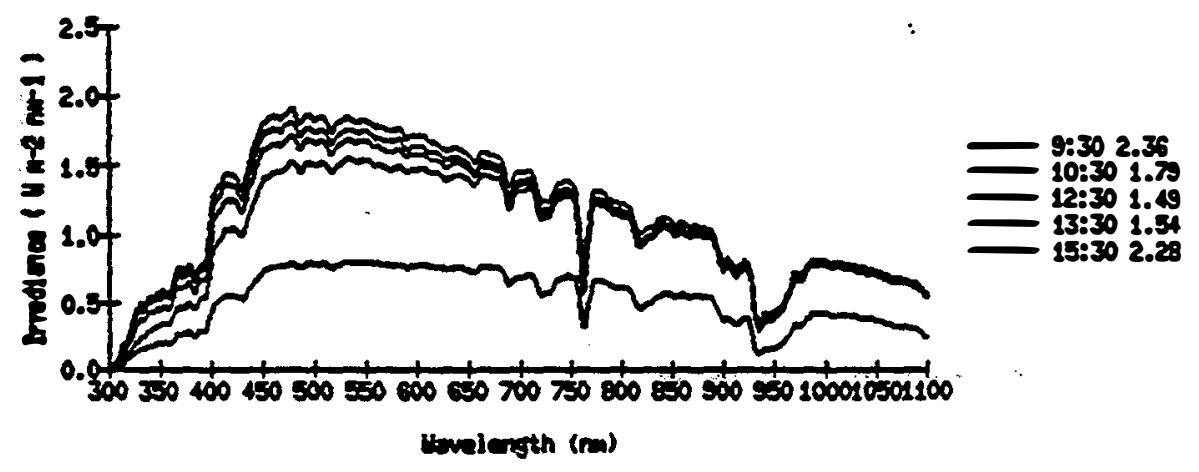


SEN * *

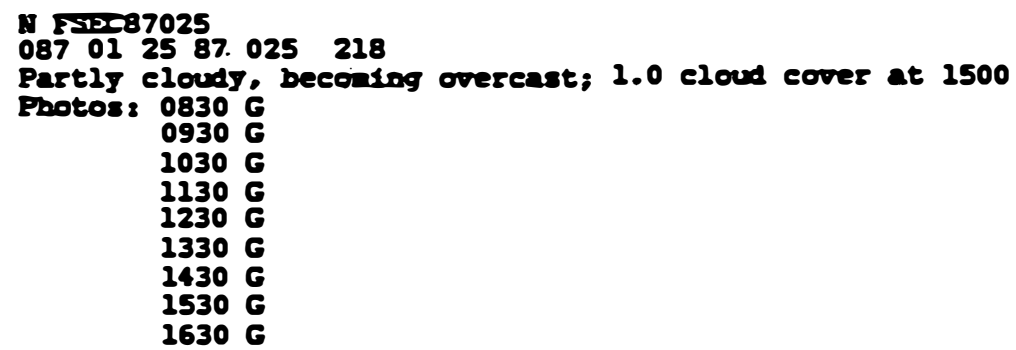

Fsac 28

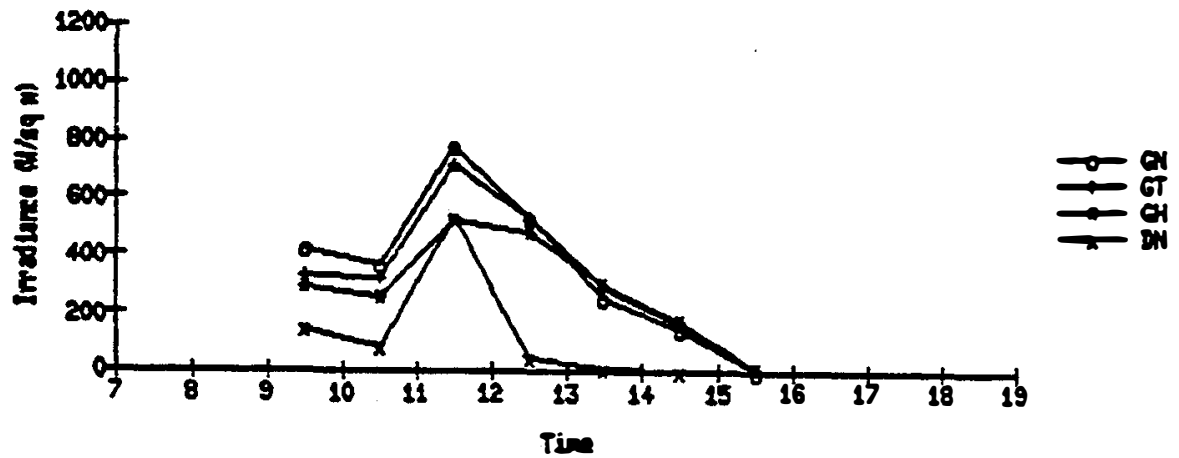

Fsex 200

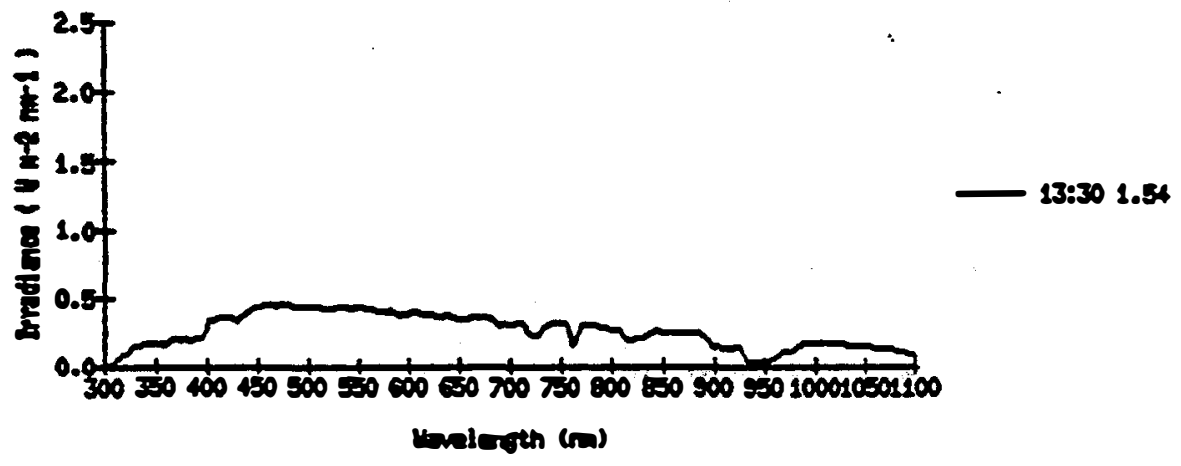




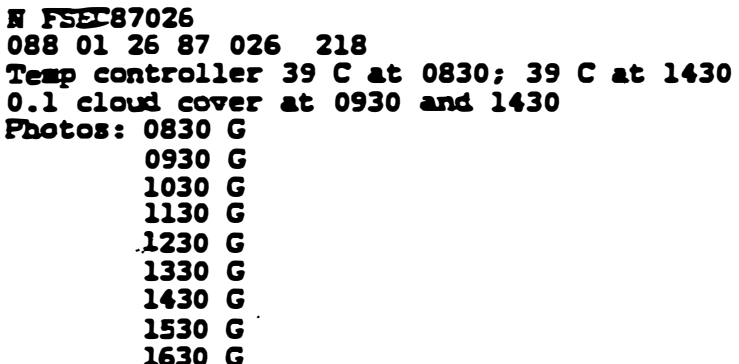

FEs 26

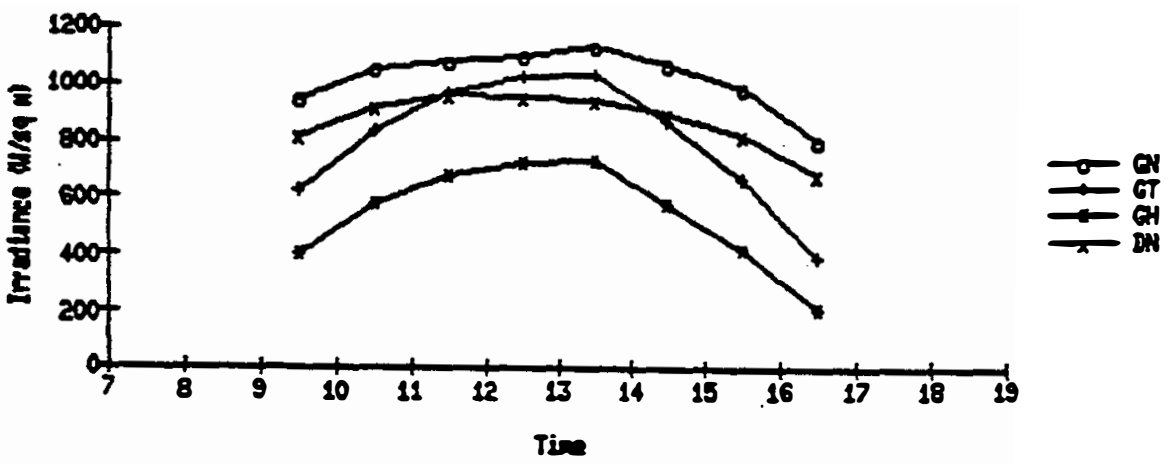

Fase 2800

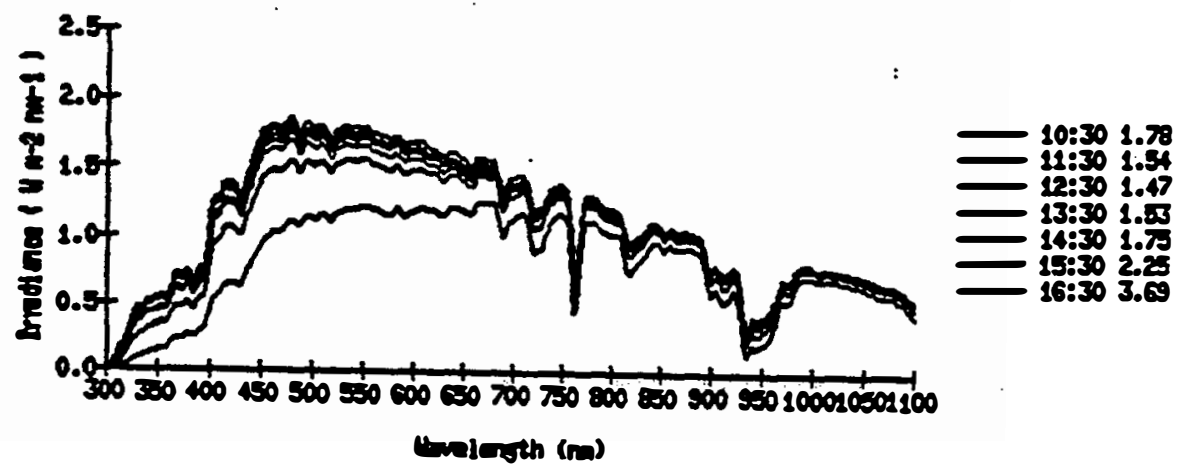


F5T587093

$\begin{array}{lllllll}089 & 04 & 03 & 87 & 093 & 172 & \text { DW }\end{array}$

Congged 다 2-17-87; new = 1432253, eal 6.38

0.5 cloud cover

sildes: $0826 \mathrm{G}$

$0926 \mathrm{G}$

$1026 \mathrm{G}$

$1126 \mathrm{G}$

$1226 \mathrm{G}$

$1326 \mathrm{G}$

$1426 \mathrm{G}$

$1526 \mathrm{G}$

$1625 \mathrm{G}$

$1725 \mathrm{G}$

Spectral sean 0 (no direct noral) at 1631

$\operatorname{rsec} 93$

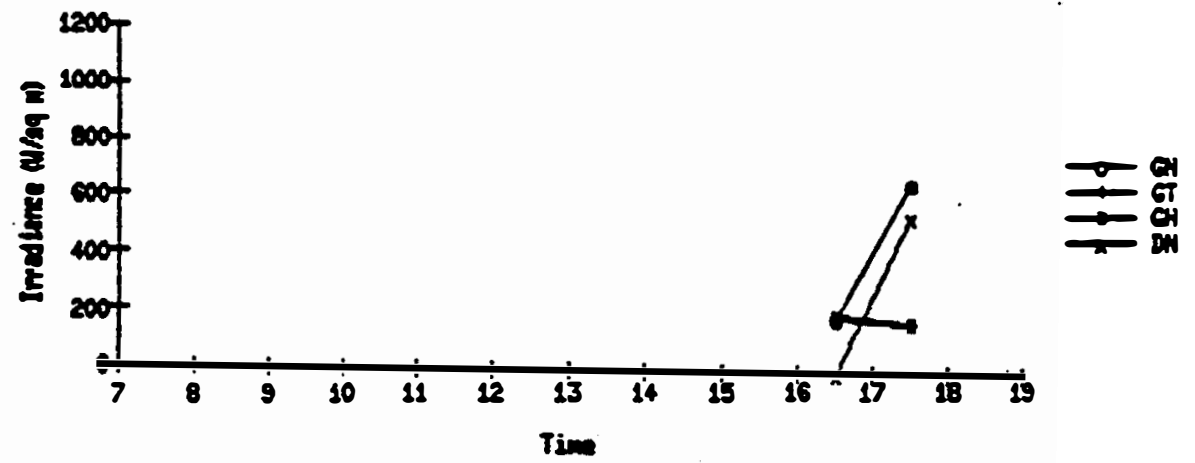

FEב 93 BN

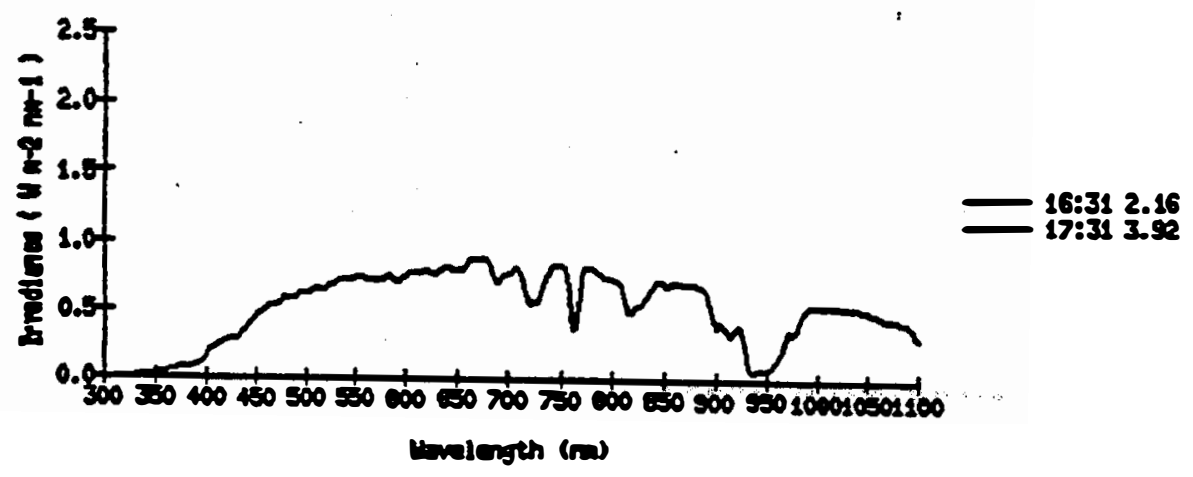




\section{SEN * *}

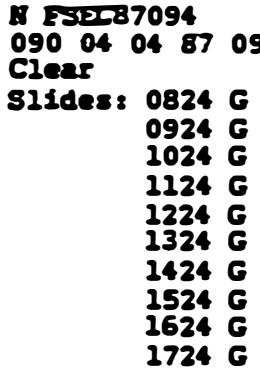

GISH different

Spectru at 1631 appears cut off in plot

Fase as

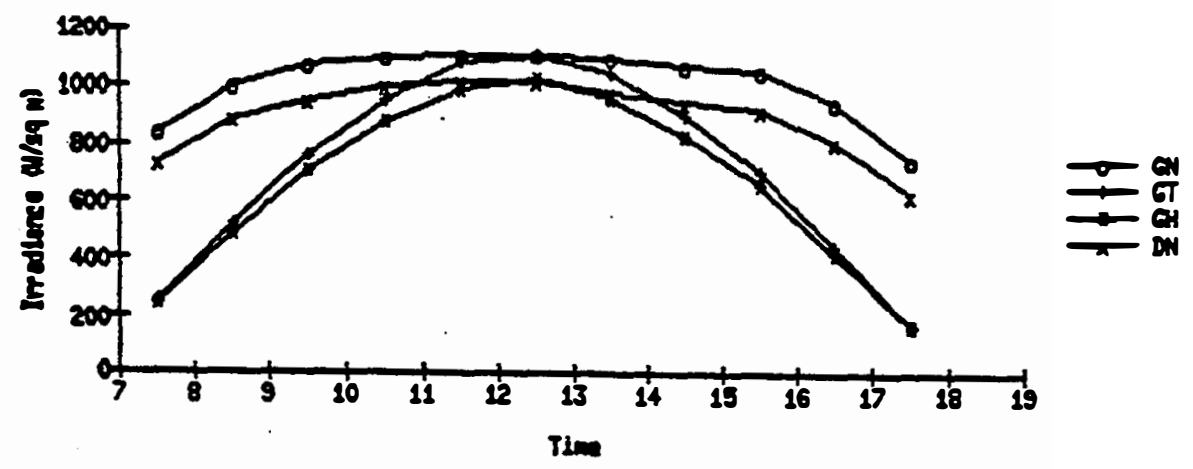

Fesc as but

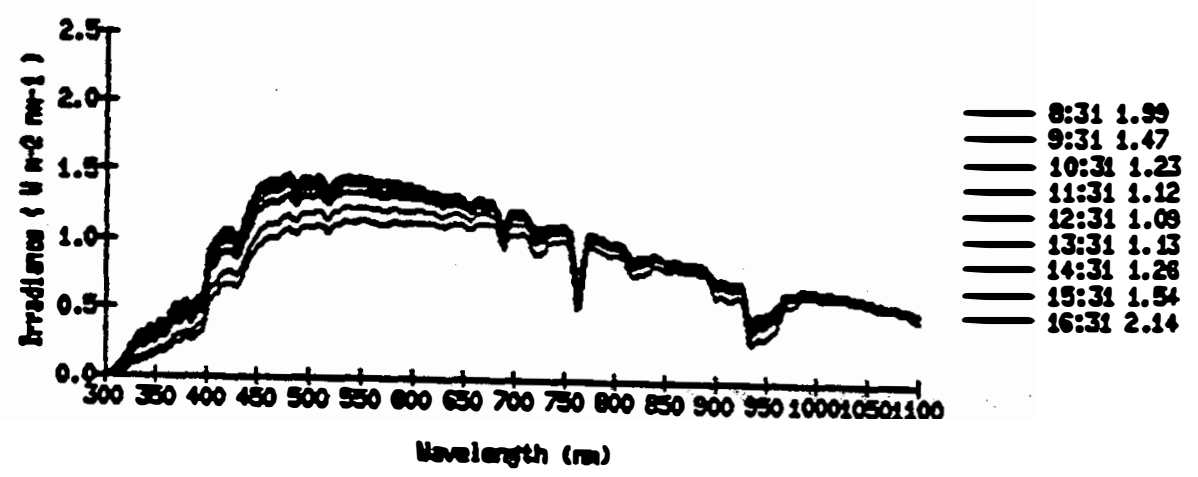


A FSP्P87095

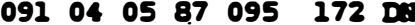

Clear

Slides: $0825 \mathrm{G}$

$0925 \mathrm{G}$

$1026 \mathrm{G}$

$1126 \mathrm{G}$

$1226 \mathrm{G}$

$1326 \mathrm{G}$

$1426 \mathrm{G}$

$1526 \mathrm{G}$

$1627 \mathrm{G}$

$1727 \mathrm{G}$

DA before is bad at 1131

Dil after is bad at 1231

Dis before is bad ad 1531

pote time stip differences 1731 ve. 1711

FIEC 95

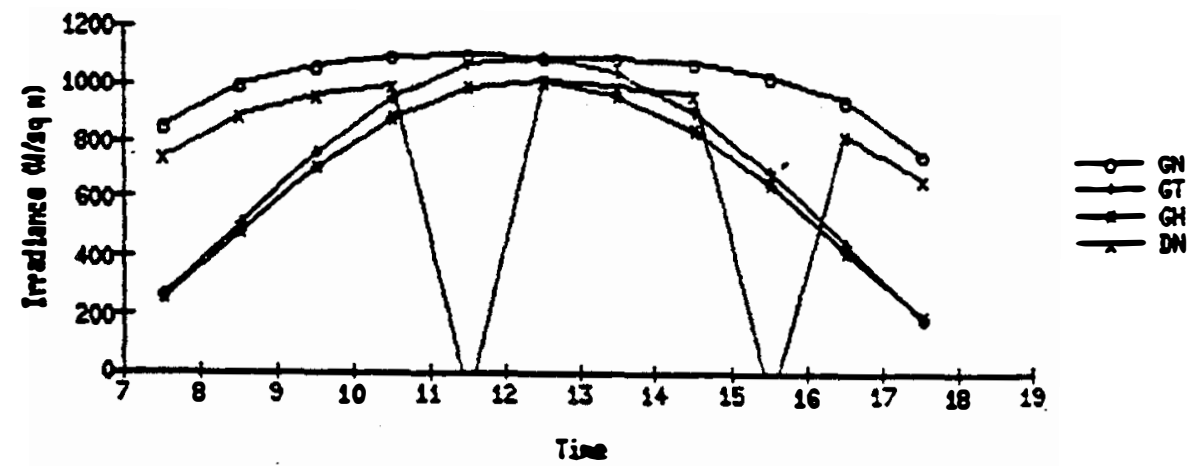

FSEC SS BT

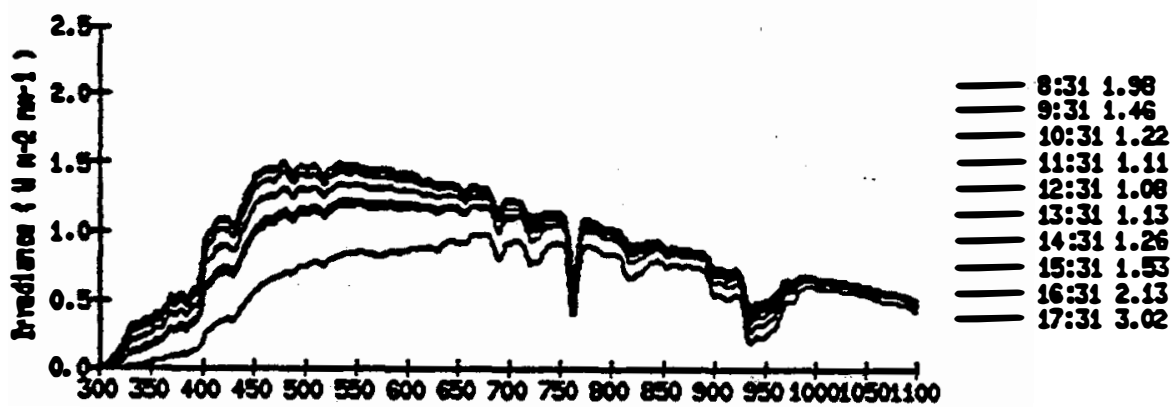

Uolength (m) 
A FSTC87096

$\begin{array}{lllll}09204 & 06.87 & 096 \quad 172 \quad \mathrm{DI}\end{array}$

Clear

Slides: $0828 \mathrm{G}$

$0928 \mathrm{G}$

$1028 \mathrm{G}$

$1128 \mathrm{G}$

$1228 \mathrm{G}$

$1328 \mathrm{C}$

$1432 \mathrm{G}$

1532 G

$1632 \mathrm{G}$

$1732 \mathrm{G}$

S12 before is bad at 0831

D. before is bad at 1331

FSEC 96

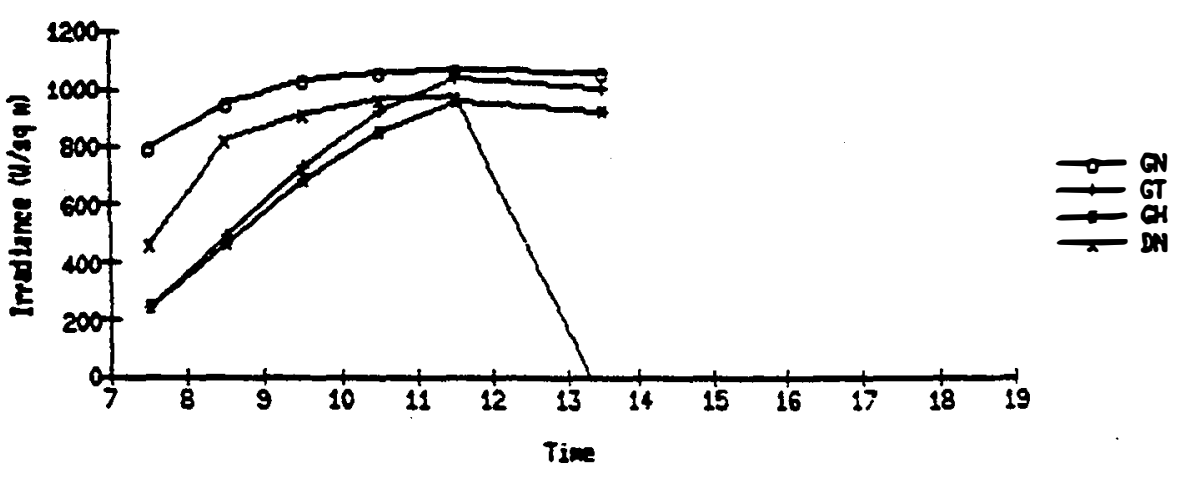

FEEC SS ant

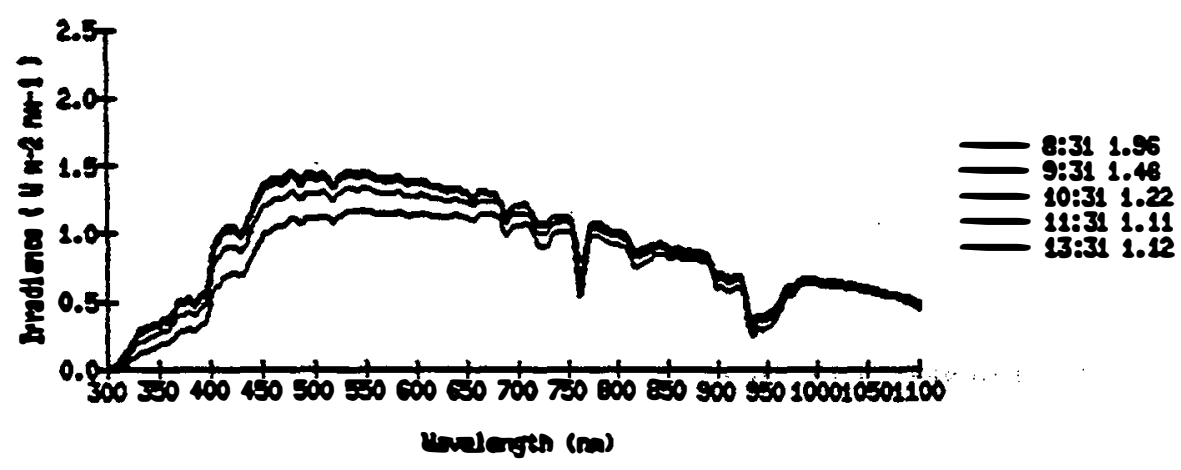




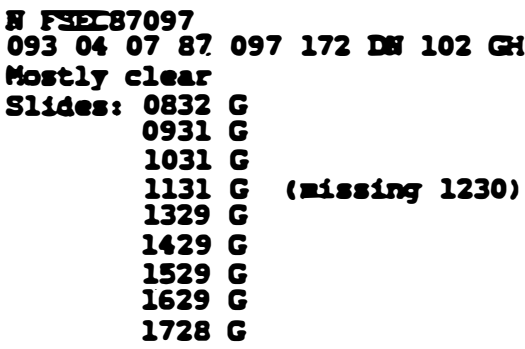

Mssing dinta in Dil spectron at 1531

Fsec 97

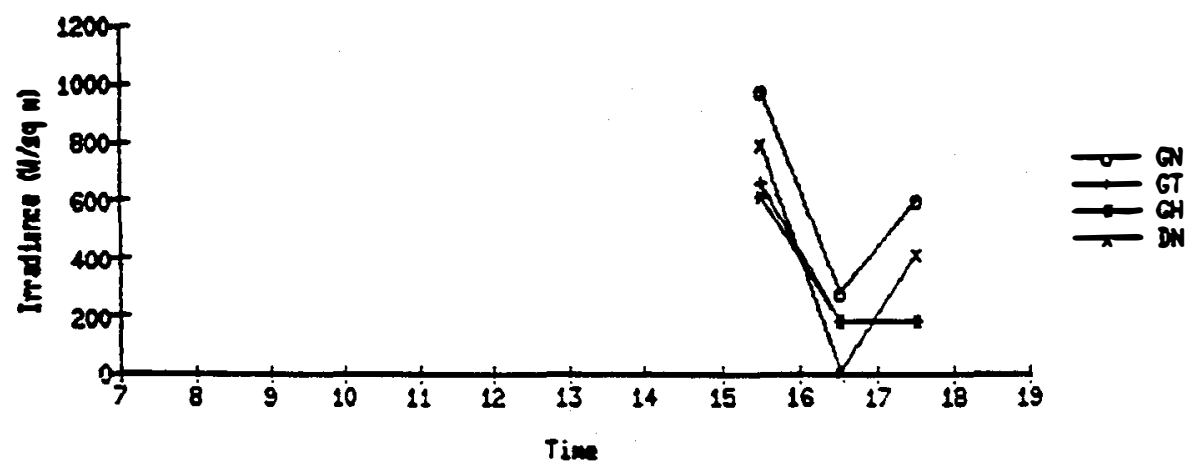

FSEC 97 DNT

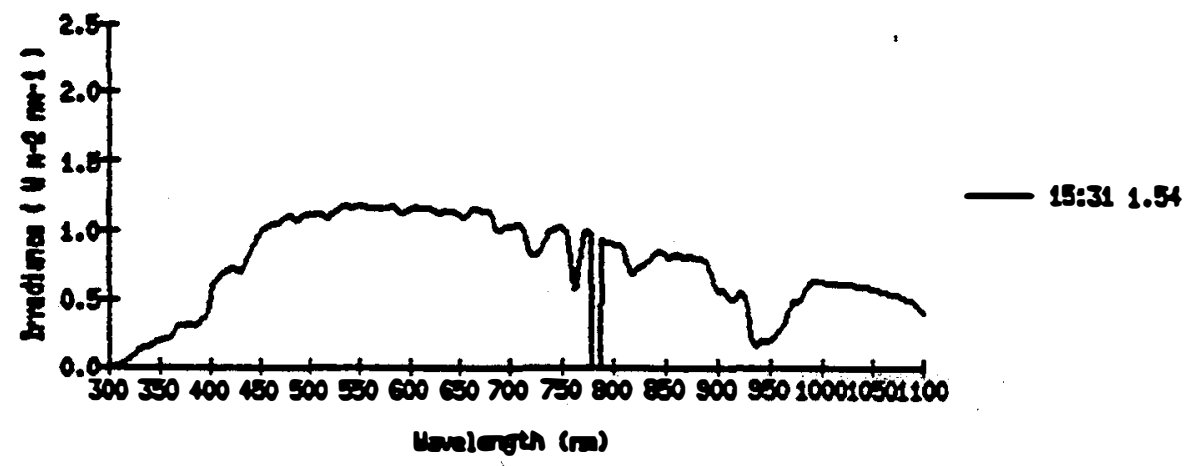

Fsec 97 as

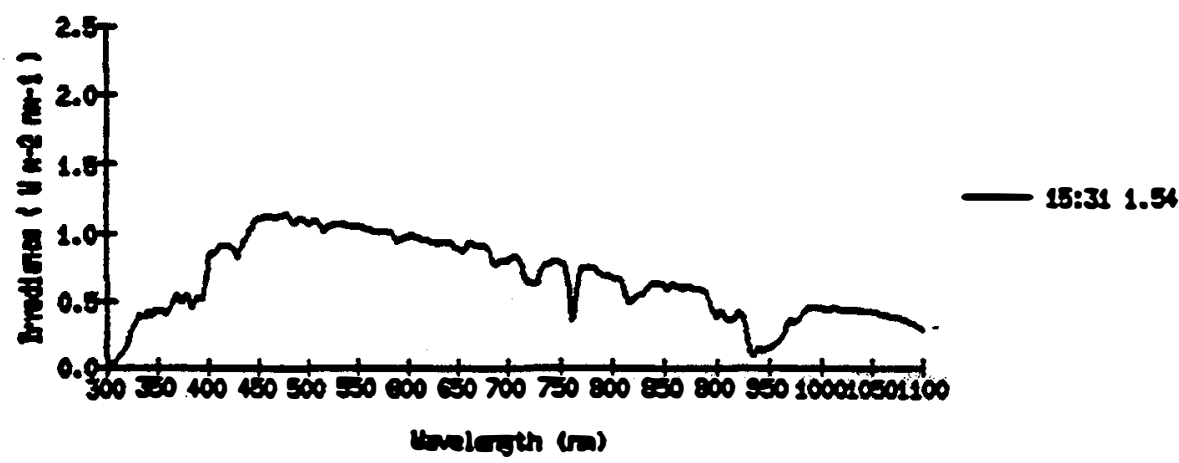


4 P512587098

$\begin{array}{lllllllllll}094 & 04 & 08 & 87 & 098 & 172 & 02 & 102 & \text { CF }\end{array}$

Most ly clear: 0.1 cloud corer at $0800 ; 0.6$ at 1230

Slides: $0826 \mathrm{G}$

0926 G

$1026 \mathrm{G}$

$1126 \mathrm{G}$

$1226 \mathrm{G}$

$1326 \mathrm{G}$

$1426 \mathrm{G}$

$1526 \mathrm{G}$

$1626 \mathrm{G}$

$1726 \mathrm{G}$

Fsec 58

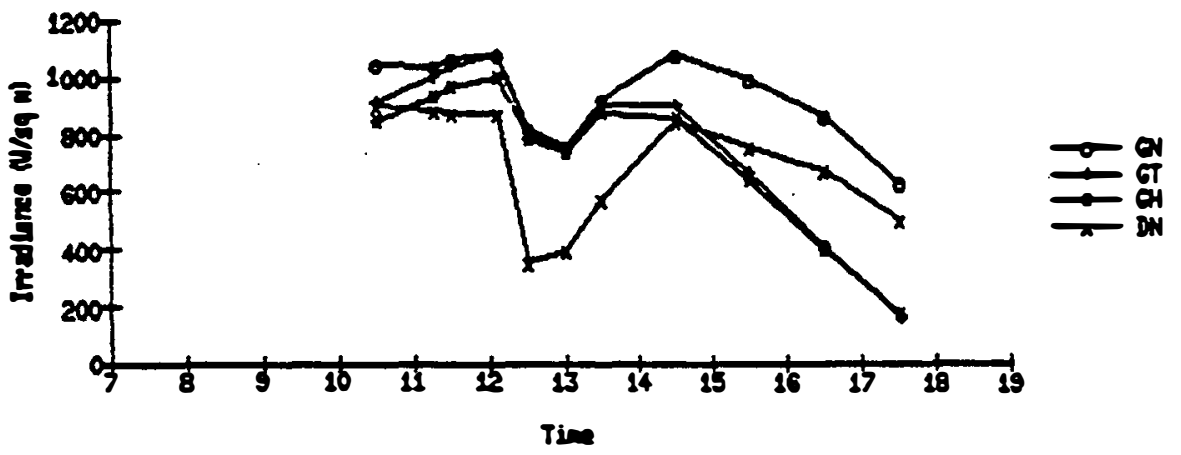

FEC $98 \mathrm{BT}$

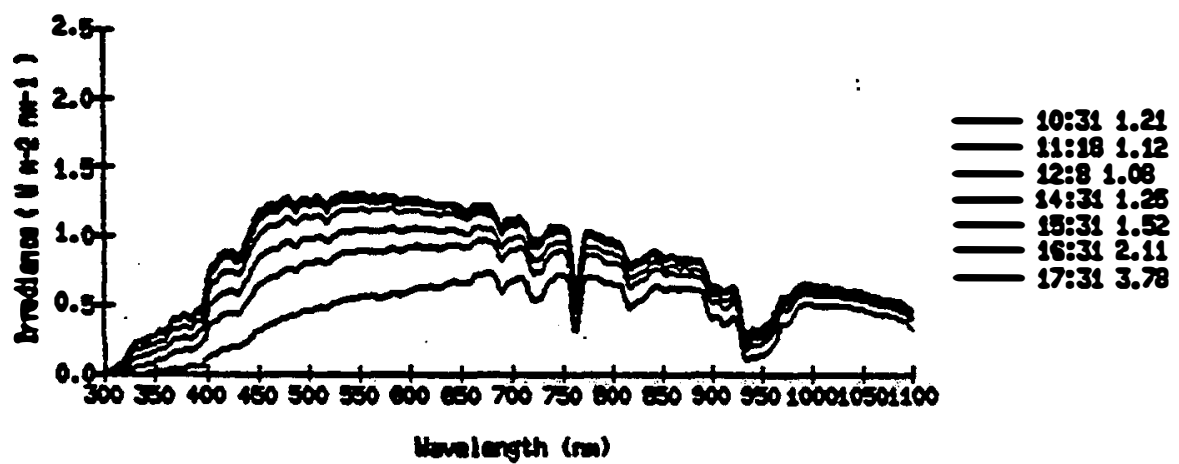

PEx 58 as

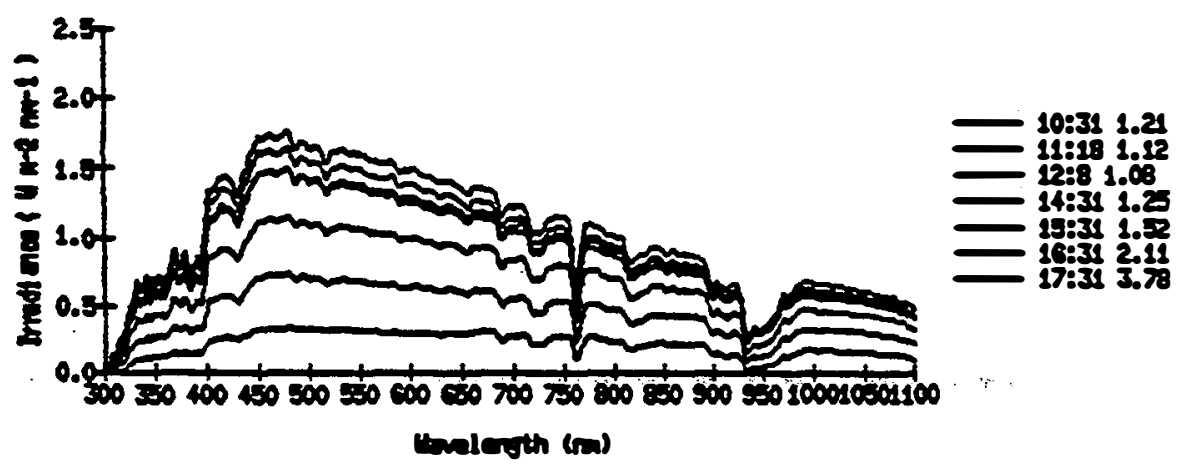


4 ह5ग्587099

$09504 \quad 0987099 \quad 172$ D표 102 뎌

Hing: 0.5 cloud cover at 0730

Slides: $0825 \mathrm{G}$

0925 G

$1025 \mathrm{G}$

$1125 \mathrm{G}$

$1225 \mathrm{G}$

$1325 \mathrm{G}$

$1425 \mathrm{G}$

1525 G

1625

$1724 \mathrm{G}$

GH spectron appears noisy in ON and near-IR at 1131

FEEC 90

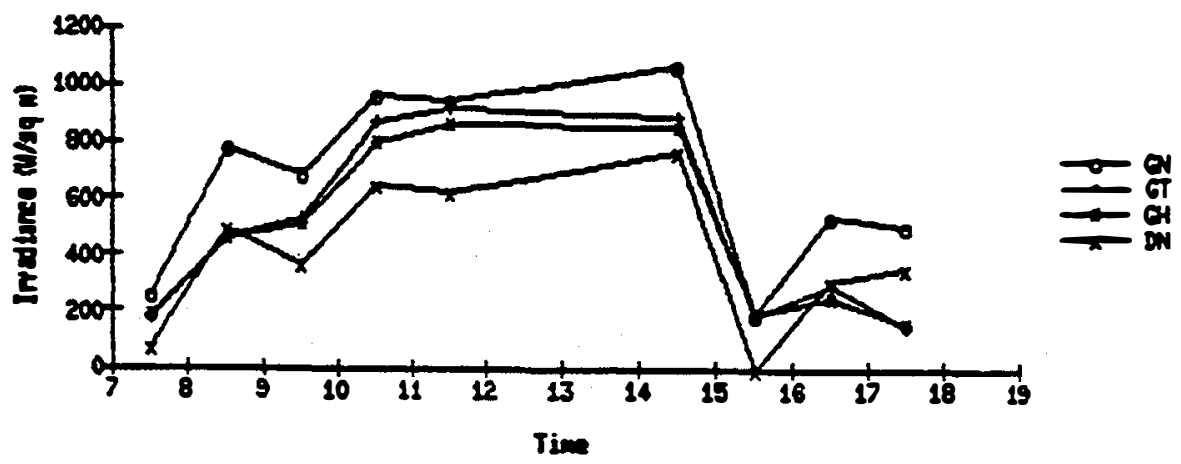

Fesces

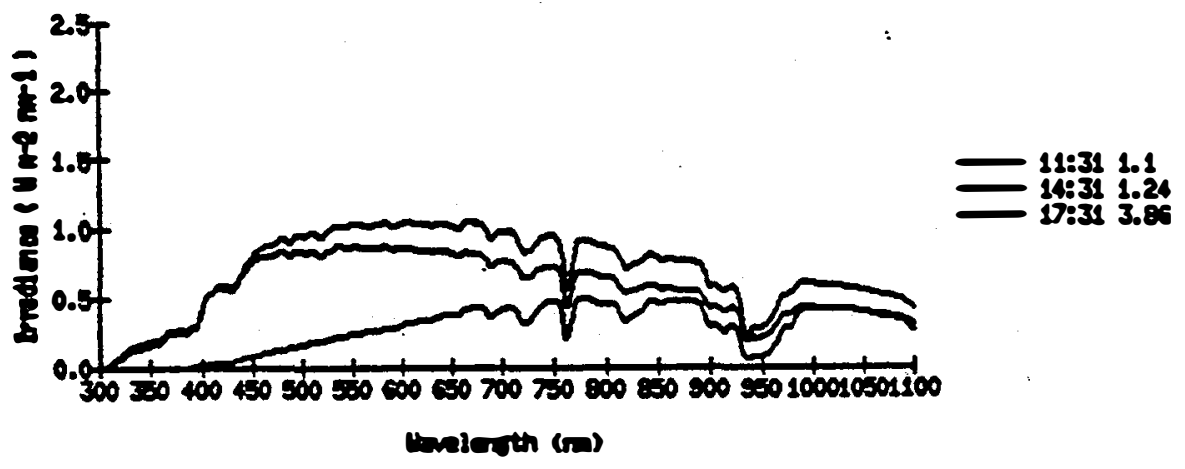

pisese as

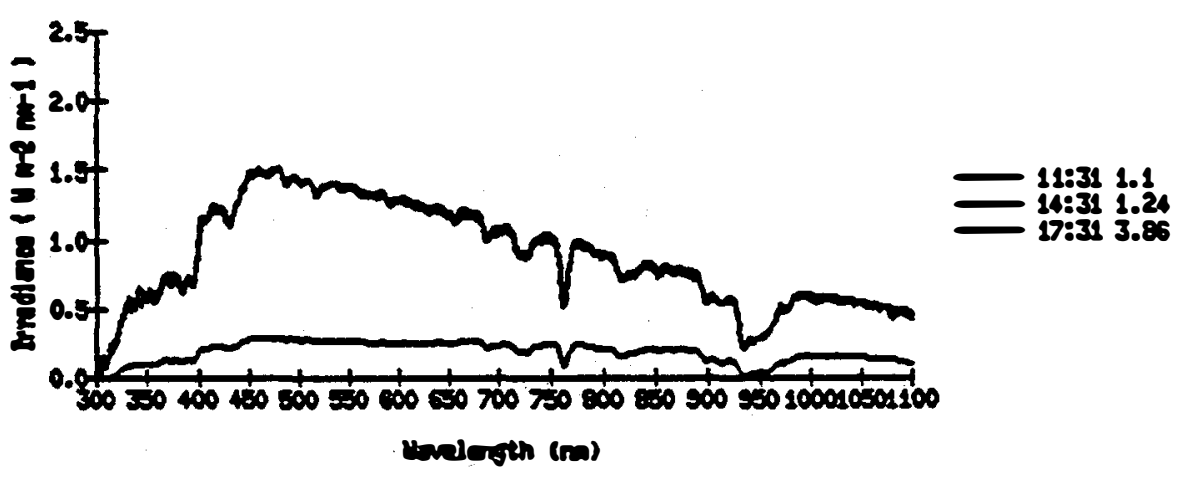


1. 252087100

$\begin{array}{llllllll}096 & 04 & 10 & 87 & 100 & 172 \mathrm{DP} & 102 \mathrm{FH}\end{array}$

S1yders $0823 \mathrm{G}$

$0923 \mathrm{G}$

$1023 \mathrm{G}$

11236

$1230 \mathrm{G}$

$1330 \mathrm{G}$

$1430 \mathrm{G}$

$1530 \mathrm{G}$

$1630 \mathrm{G}$

$1730 \mathrm{G}$

GH spectron bad in near-IR at 1531 (pegged)

Hote t1ae stanp differences 1531 v8. 1634

PISP 100

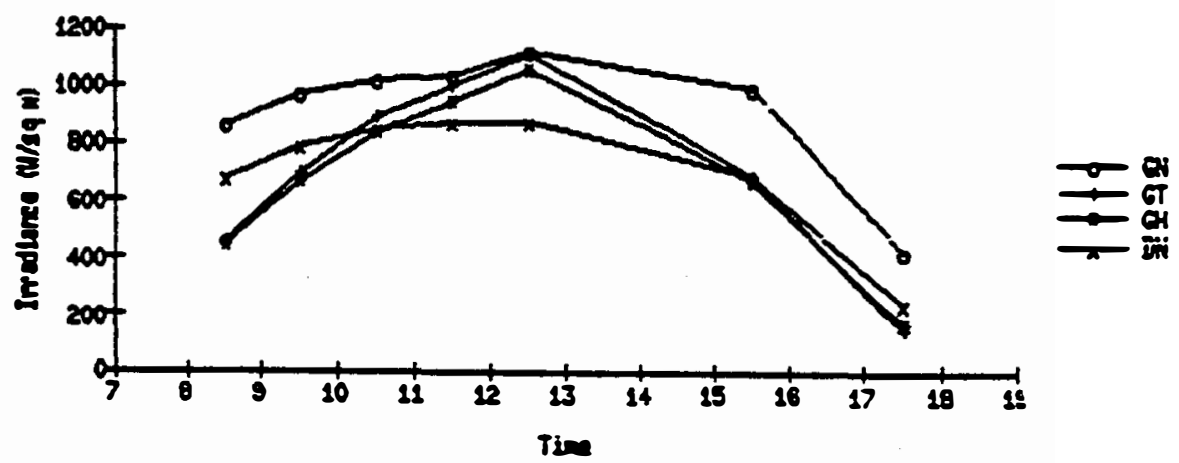

F=x $100 \mathrm{dm}$

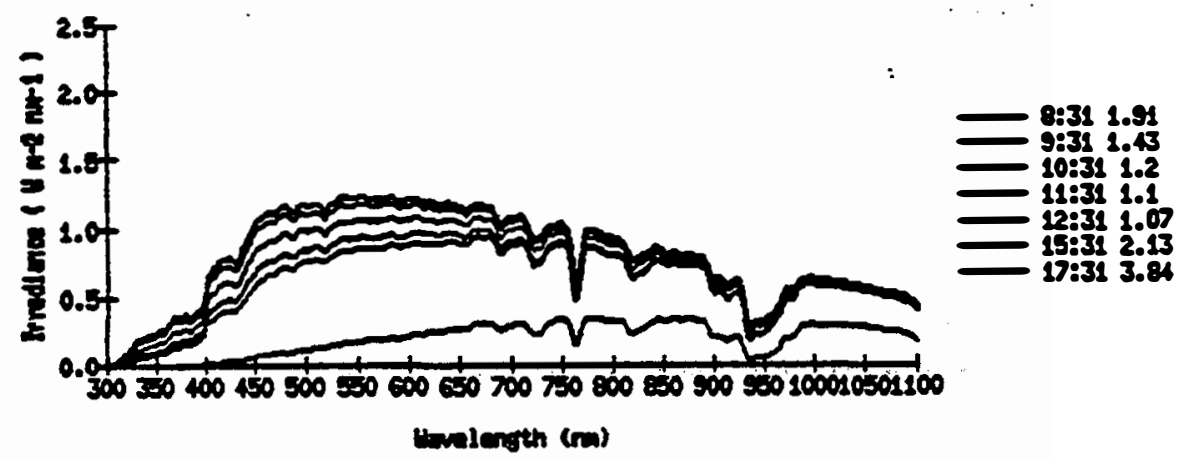

F 200 es

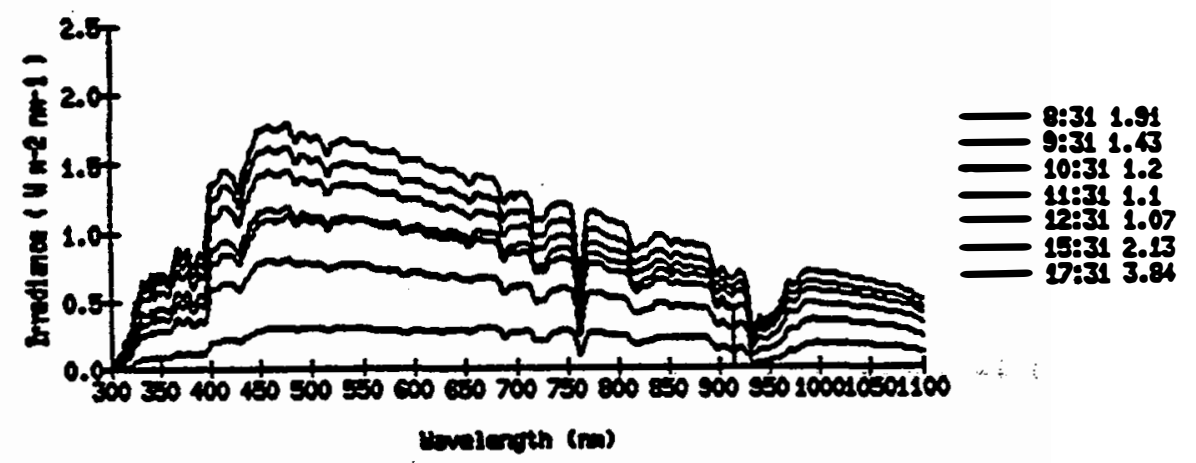


4 FSX287101

097 of $1287 \quad 101 \quad 172$ DA 102 G

slides: $0830 \mathrm{G}$

$0930 \mathrm{G}$

$1030 \mathrm{G}$

$1130 \mathrm{G}$

$1230 \mathrm{G}$

$1330 \mathrm{G}$

$1430 \mathrm{G}$

$1530 \mathrm{G}$

$1630 \mathrm{G}$

$1730 \mathrm{G}$

FSEC 101

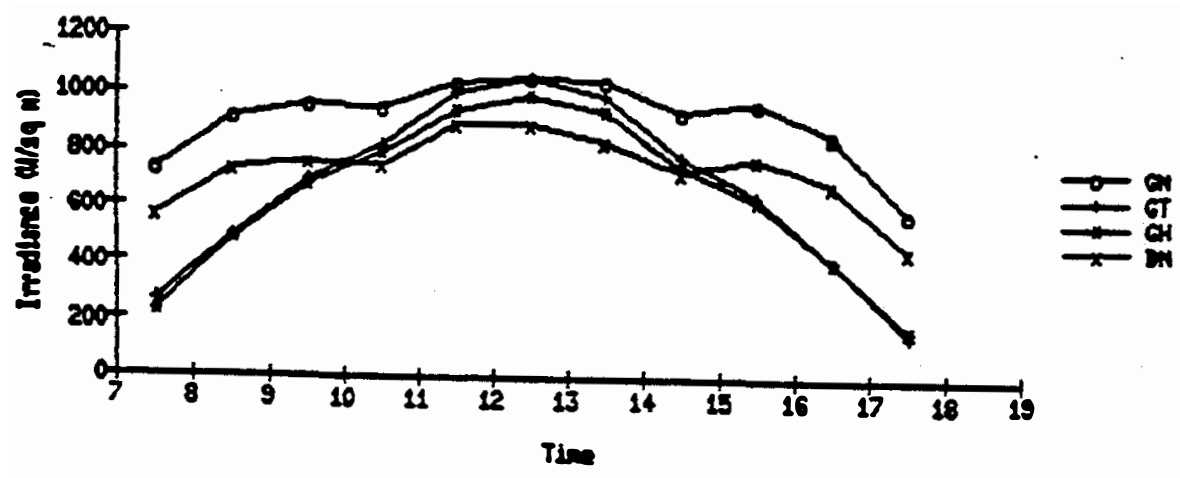

FIST 202 ENT

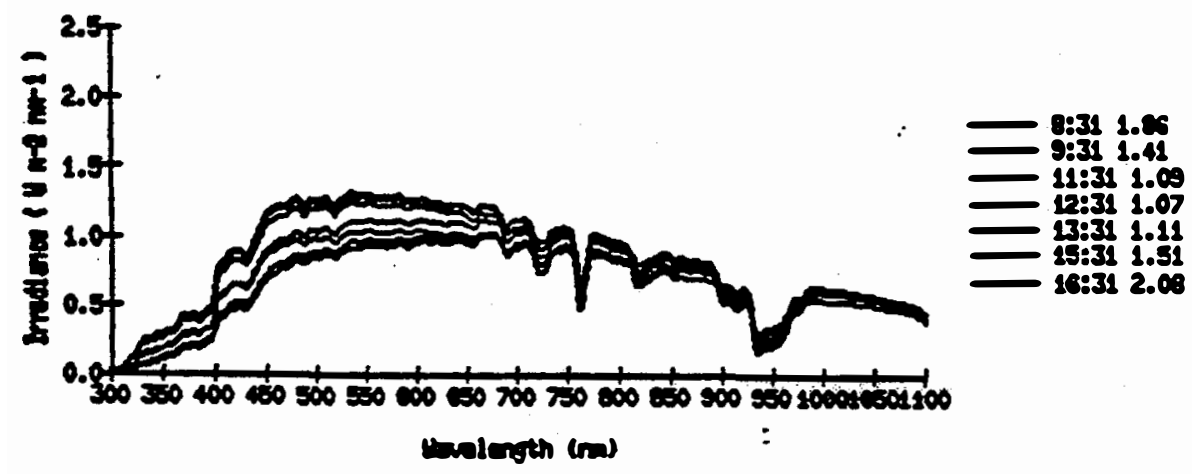

Fis 102 os

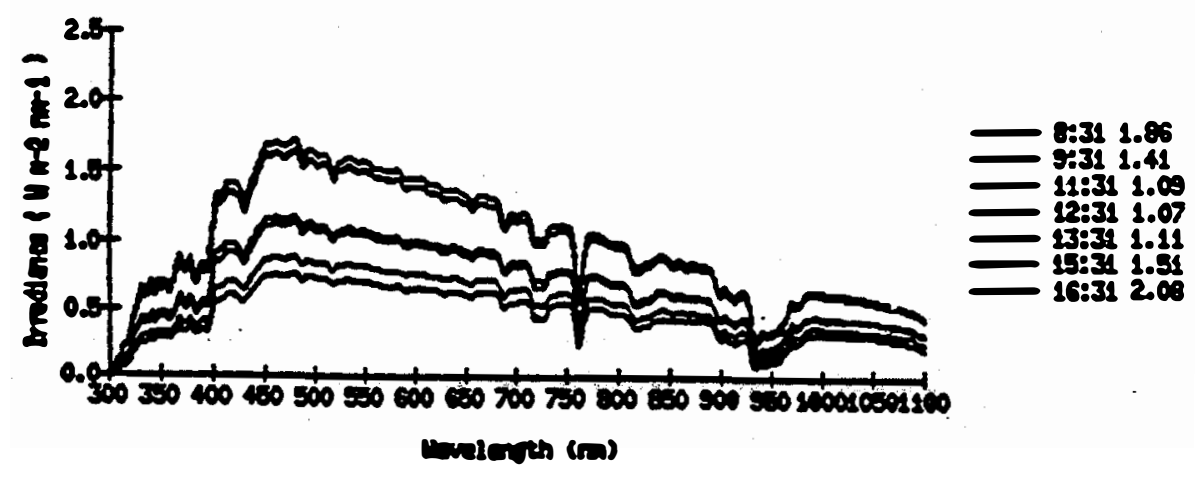


P.2087104

098 क $1487104 \quad 172 \quad 102$ F

Ihts has. 0.8 cloud cover at 1000 : verg 11 ght overenst, 1.0 at 1530

S1108: $0630 \mathrm{G}$ $0730 \mathrm{G}$

$0831 \mathrm{G}$

$0931 \mathrm{G}$

$1031 \mathrm{G}$

$1131 \mathrm{H}$ (dark)

$1231 \mathrm{G}$

1331 in (dark)

$1431 \mathrm{G}$

$1531 \mathrm{G}$

$1631 \mathrm{G}$

$1731 \mathrm{G}$

Fise 104

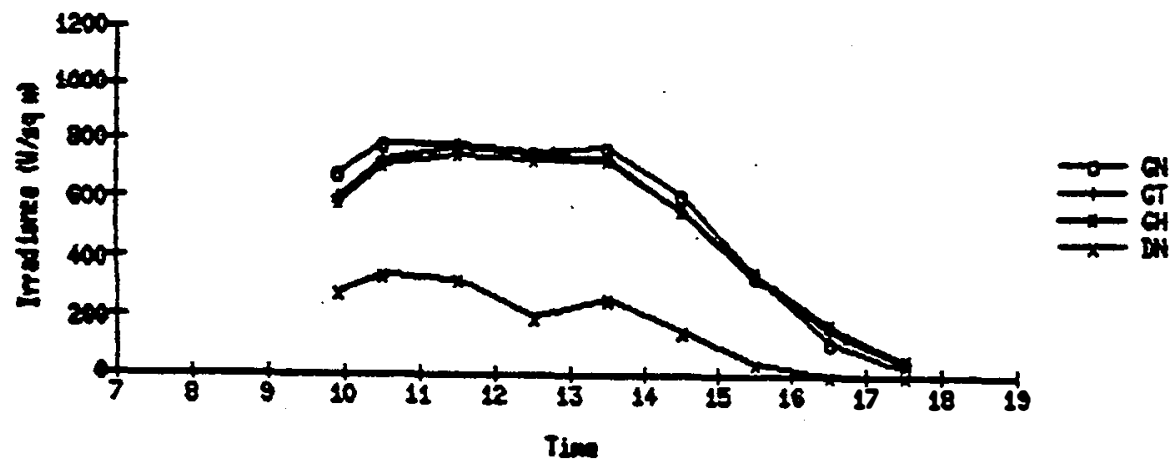

FEx 2048

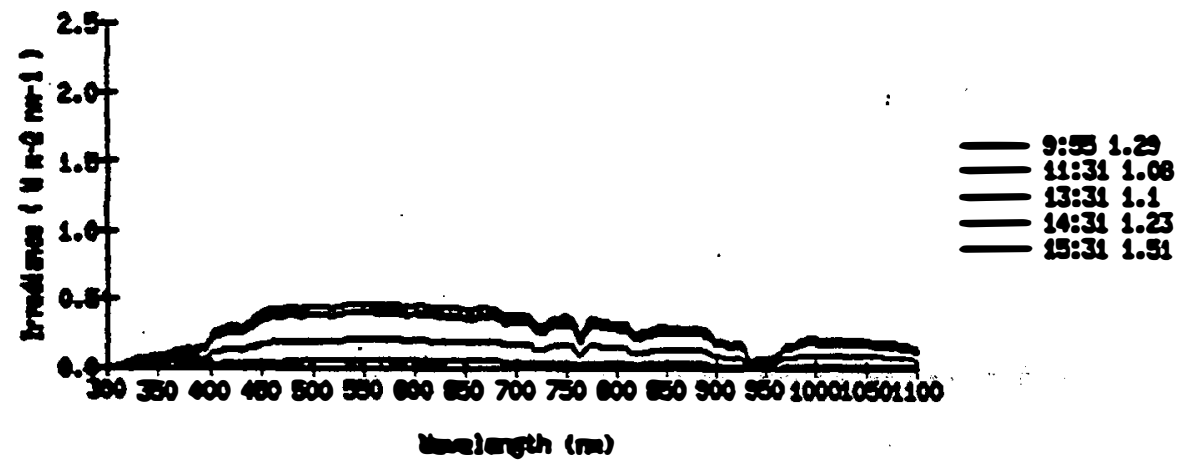

Pus 20465

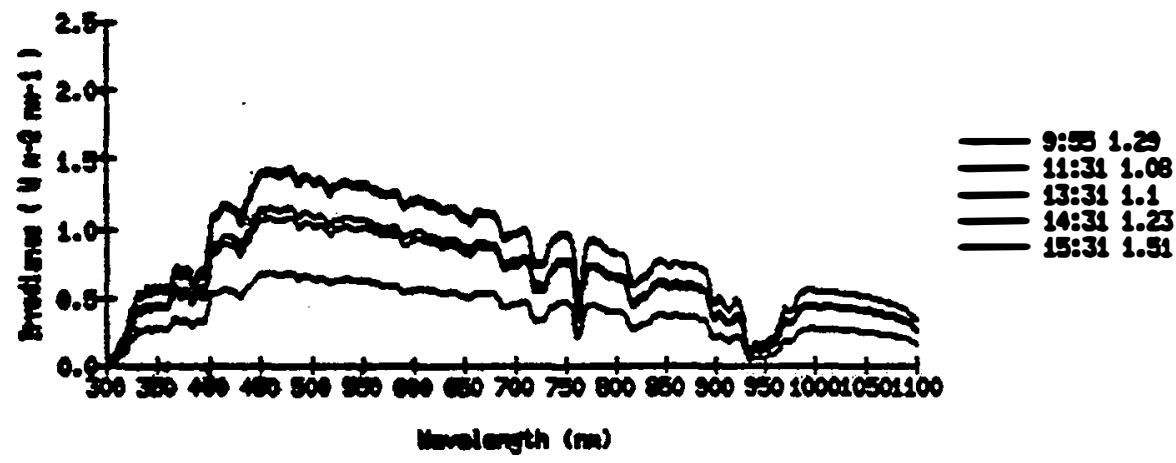


8. PSTC87105

099 O4 $1587 \quad 105 \quad 172$ DI 102 GI

Mostly overcast: 0.9 cloud cover at 0730 and 1130

Rist at 1430 .

Observed solsture indside dowe on sphere; will reseal after erjout

Slides: $0631 \mathrm{G}$

$0731 \mathrm{G}$

$0831 \mathrm{G}$

$0932 \mathrm{G}$

1032 A (dnsk)

$1132 \mathrm{G}$

$1232 \mathrm{G}$

$1332 \mathrm{G}$

$1432 \mathrm{G}$

$1532 \mathrm{G}$

$1632 \mathrm{G}$

$1732 \mathrm{G}$

One ISR startifig 1509, no spectrw (no direct bea) at 1509

$\begin{array}{lllll}100 & 04 & 15 \quad 87 & 2.05 \quad 172 \quad \mathrm{DP}\end{array}$

One ISR

Cloudsi 1.0 cloud cover at 1530, then clearing

slides: see above
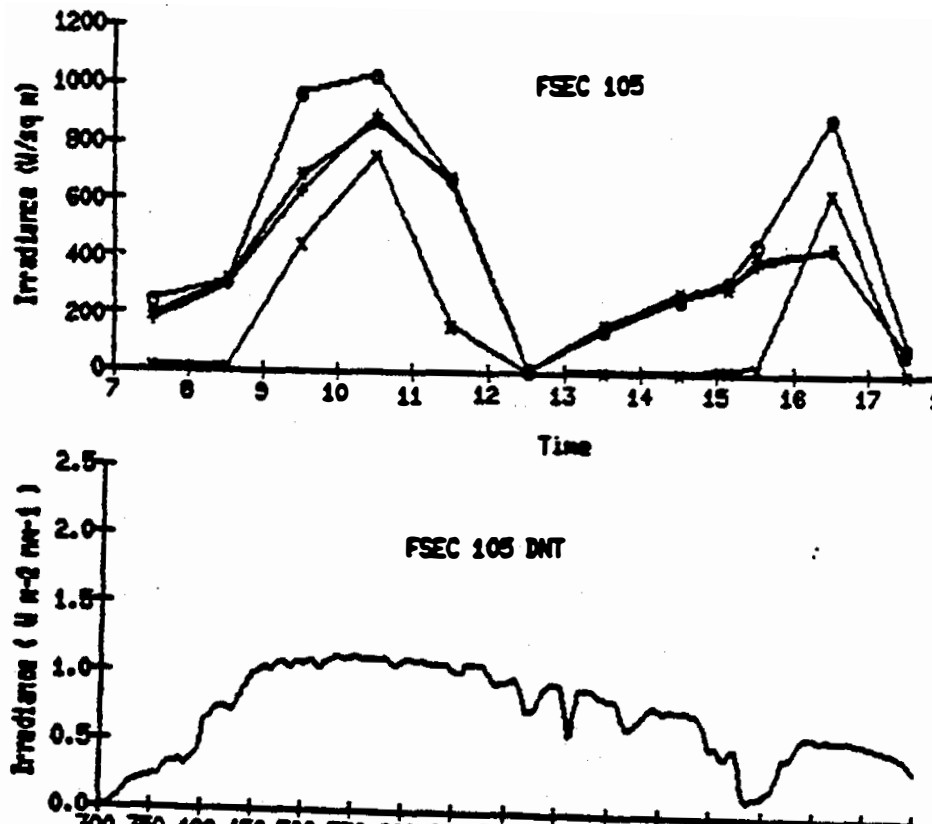

$10: 311.18$

$14: 31$ 1.23
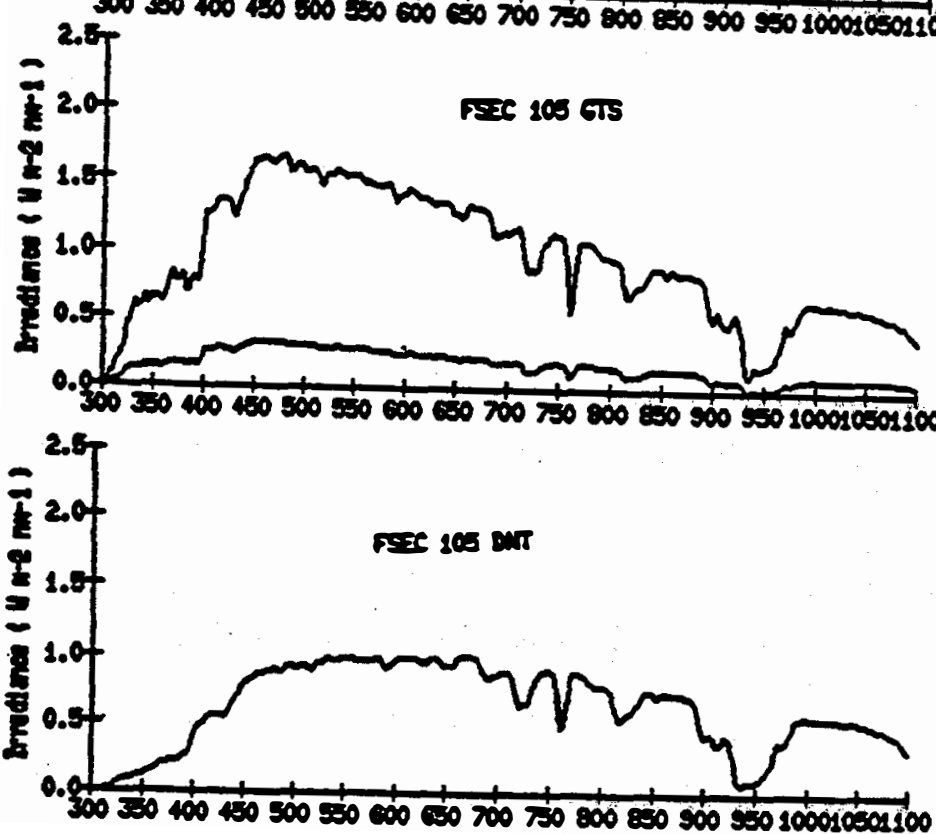
1. TS12887106

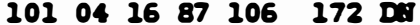

rostly elear: 0.1 eloud cover at 0930: 0.3 at 1530 partly eloudy

Sl1des: $0632 \mathrm{G}$

$0732 \mathrm{G}$

$0832 \mathrm{G}$

0932 A (dnsk)

$1032 \mathrm{M}$

$1132 M$

$1232 \mathrm{G}$

1332 M (dark)

$1432 \mathrm{G}$

1532 G

$1632 G$

$1732 \mathrm{G}$

Hote time stanp differences 1431 vs. 1531

Fsec 100

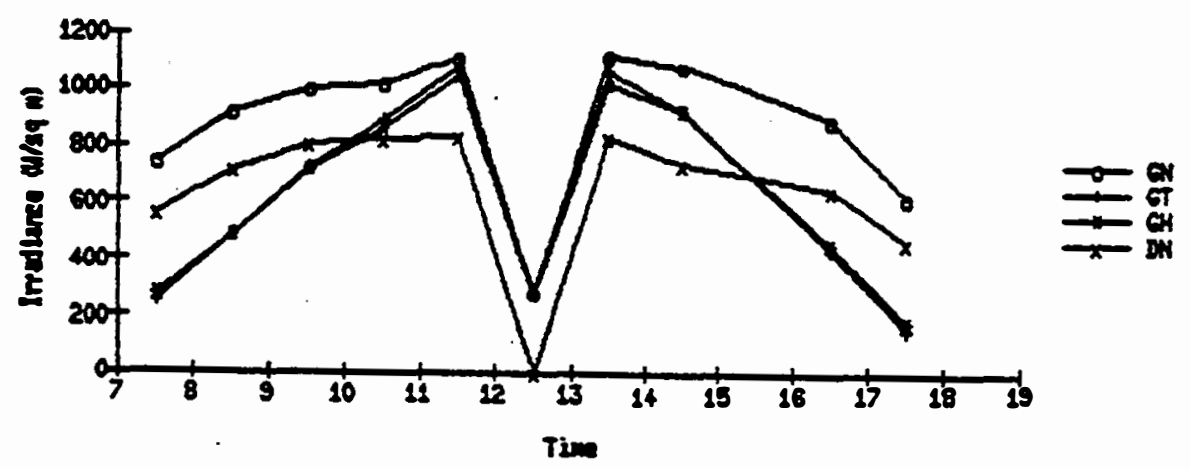

FESC 100 ant

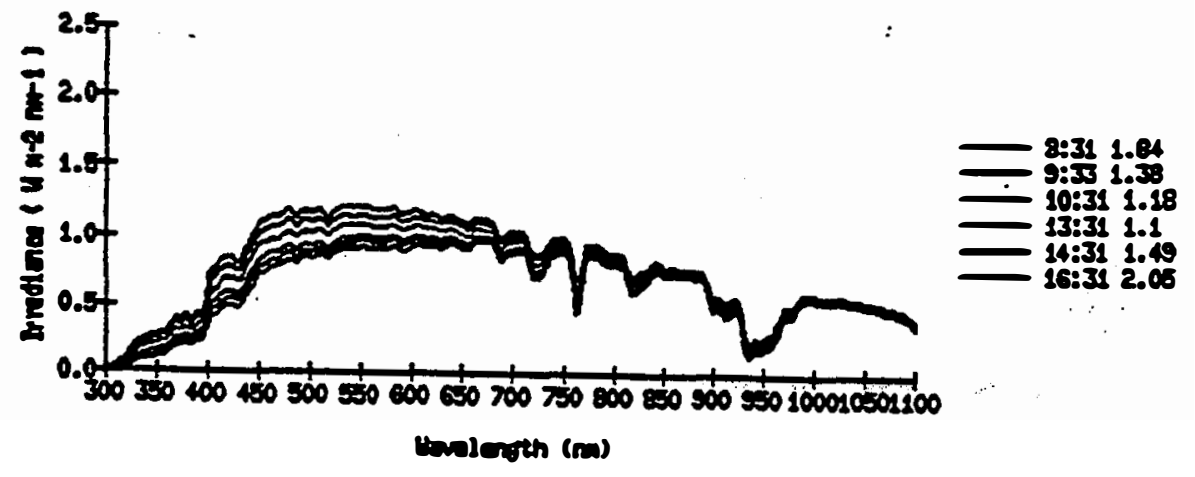



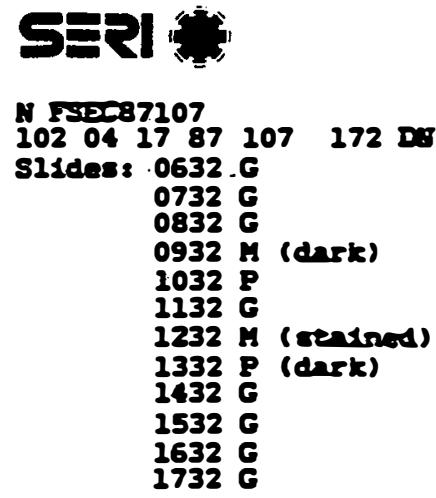

F्בx 107

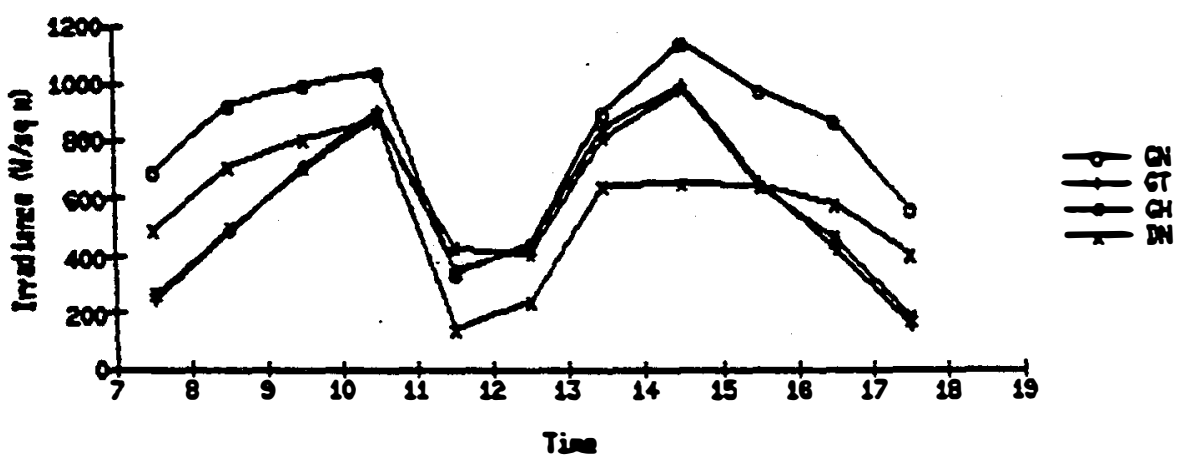

FE⿰ $107 \mathrm{Bm}$

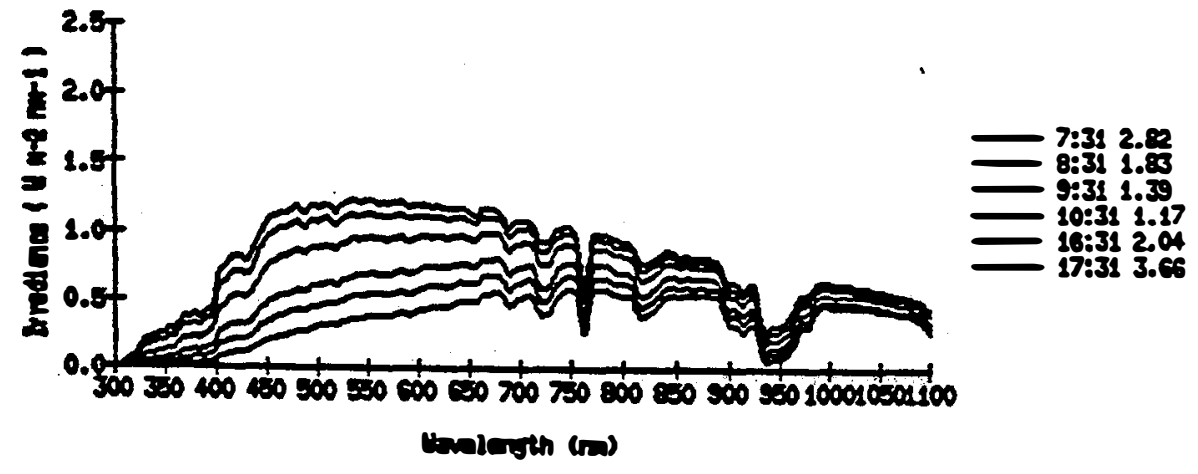


1. FSPC87108

$\begin{array}{lllllllll}103 & 04 & 18 & 87 & 108 & 172 & \mathrm{DP}\end{array}$

Aftermoor rasin

Sl1des: $0632 \mathrm{G}$

$0732 \mathrm{G}$

$0832 \mathrm{G}$

0932 H (dnzk)

$1032 \mathrm{P}$

$1233 \mathrm{G}$

$2233 \mathrm{P}$ (dnsk)

$1333 \mathrm{G}$

$1433 \mathrm{G}$ (enter drops)

1533 G (veter drops)

1633 G (enter drops)

$1733 \mathrm{G}$ (evter drops)

$\operatorname{PSEC} 108$

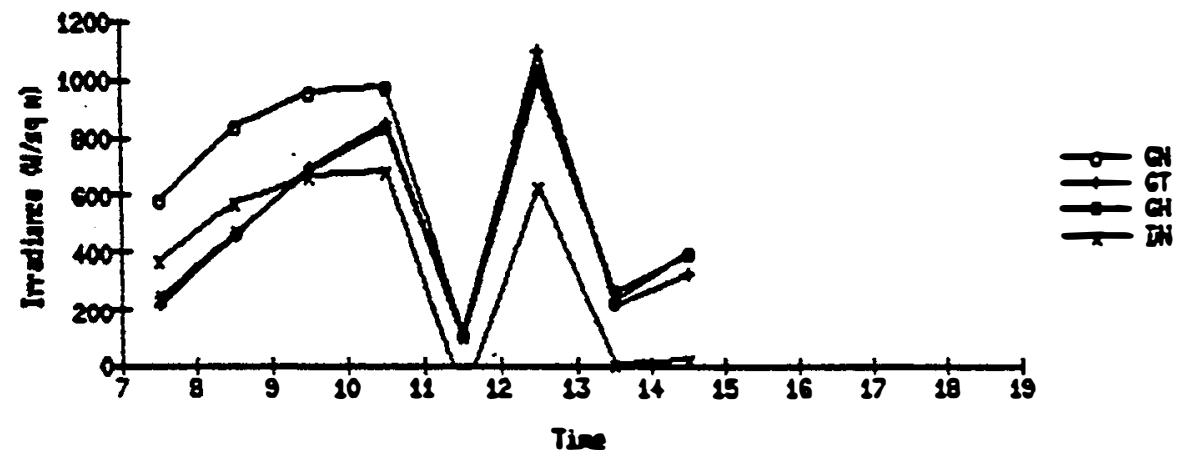

Fesc $108 \mathrm{BN}$

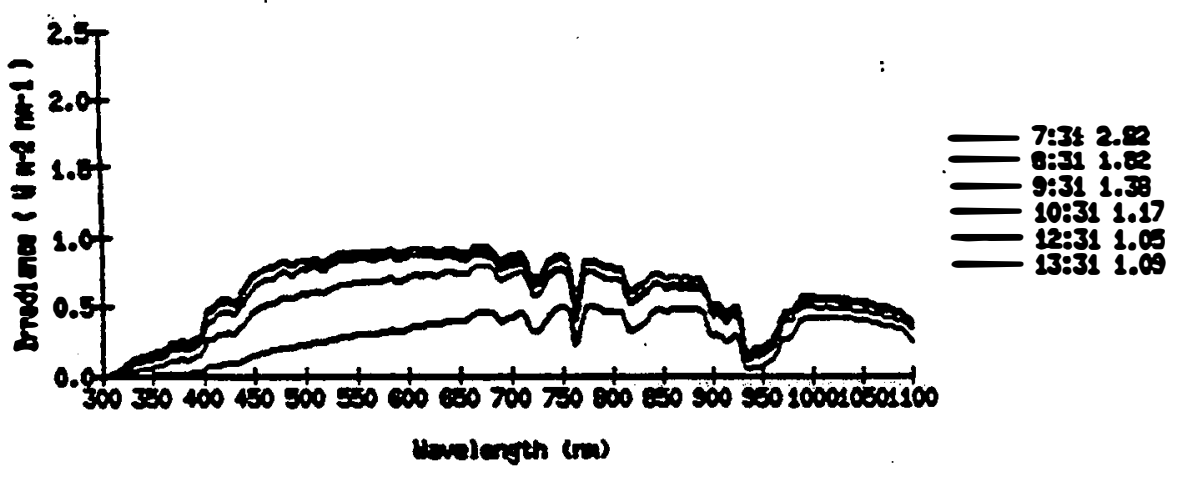




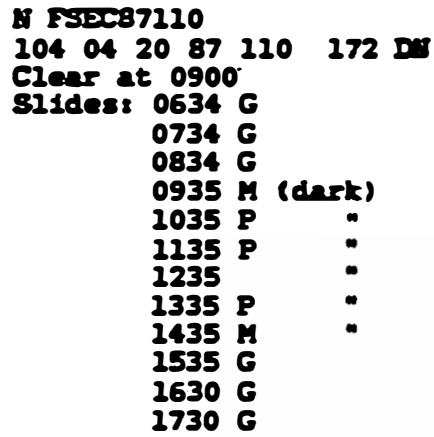

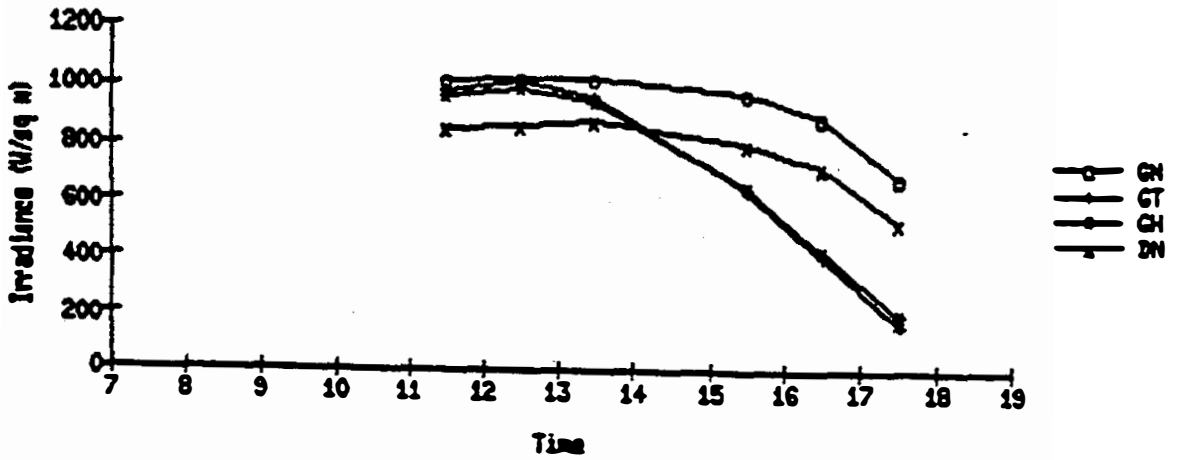

FEבC 110 ANT

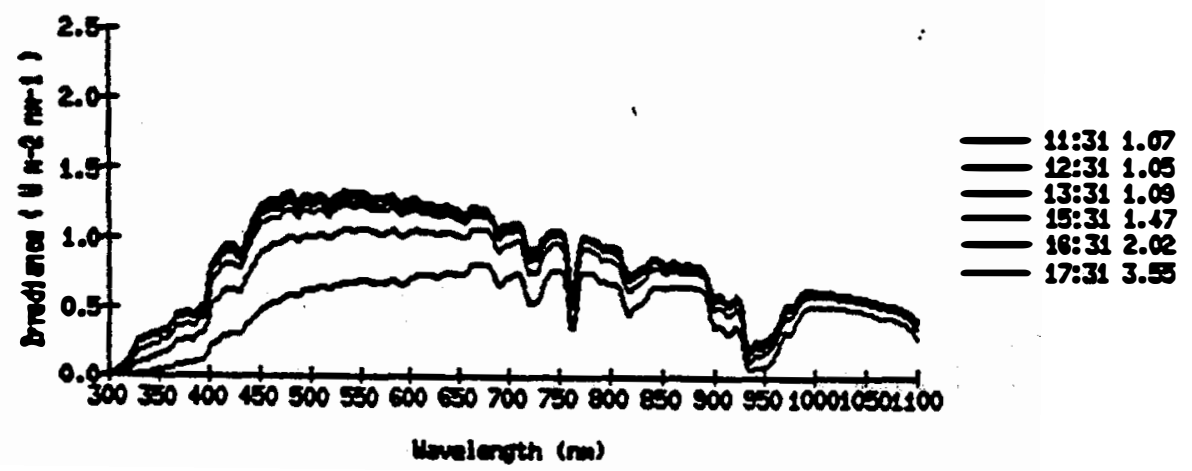




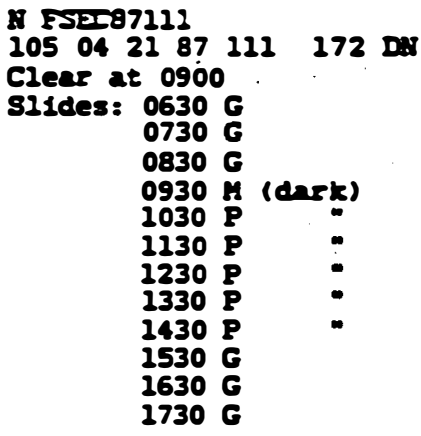

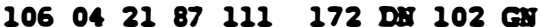

TVO ISRs

ERs 102 installed after sphere dryout and reference f1le check

0.3 cloud cover at 133080.1 at 1500

Sildes: See above
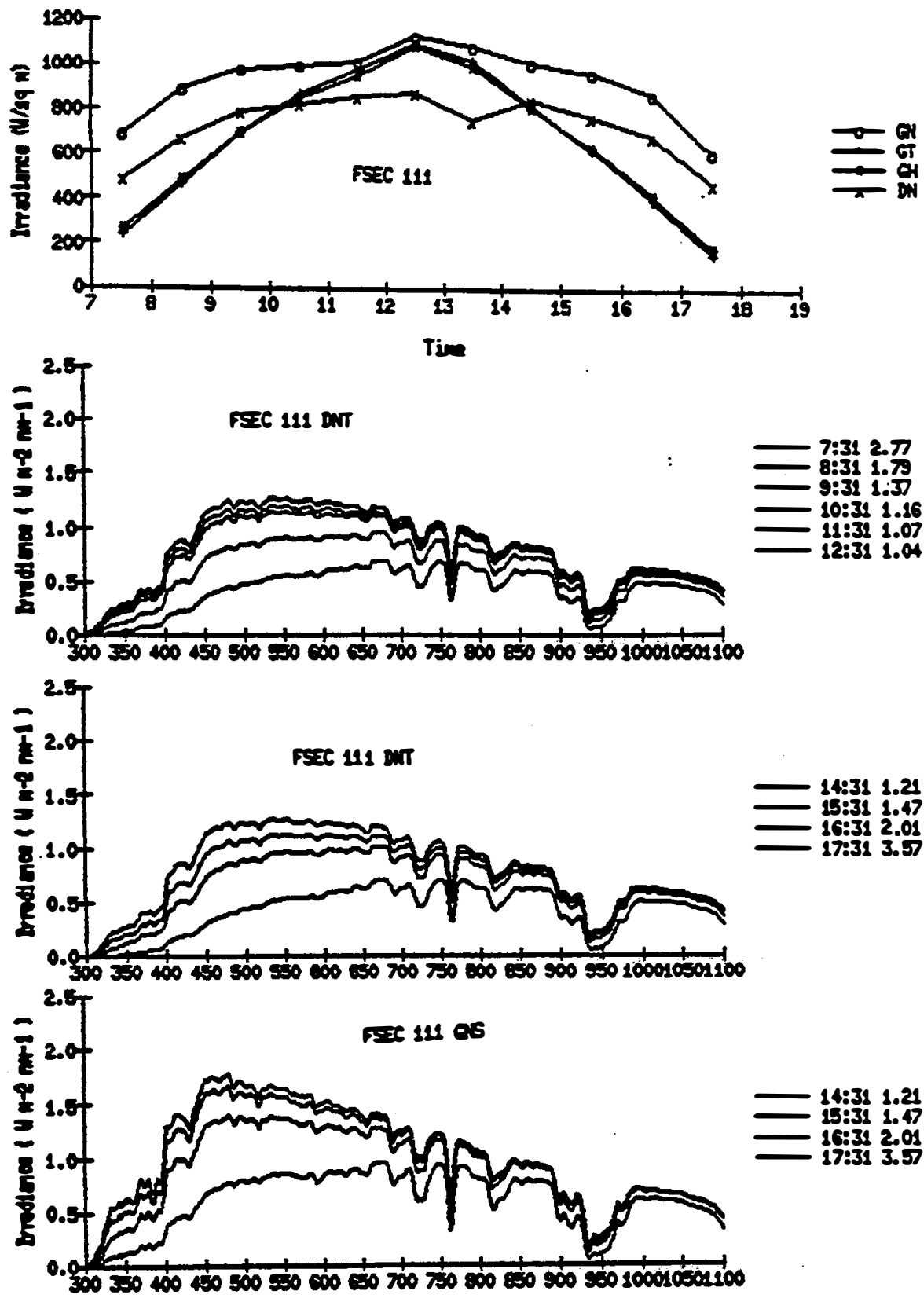

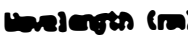


1. F51587112

107. O4 $2287 \quad 112 \quad 172 \quad \mathrm{D} \quad 102 \mathrm{G}$

0.1 eloud cover at 07308 at $1200 ; 0.1$ at 1500

sildes $0629 \mathrm{G}$

0729 G

0829 G

$0930 \mathrm{M}$ (dnsk)

$1030 \mathrm{P}$

$1130 \mathrm{P}$

$1230 \mathrm{P}$

$1329 P$

$1429 P$

$1529 \mathrm{G}$

$1629 \mathrm{G}$

1729 G

Fist 112

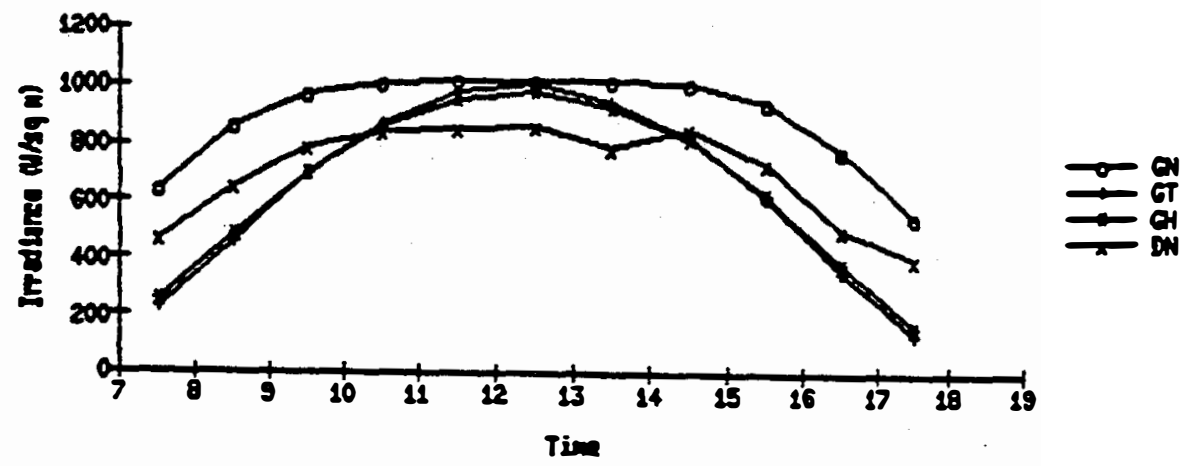

FEבC 212 BTा

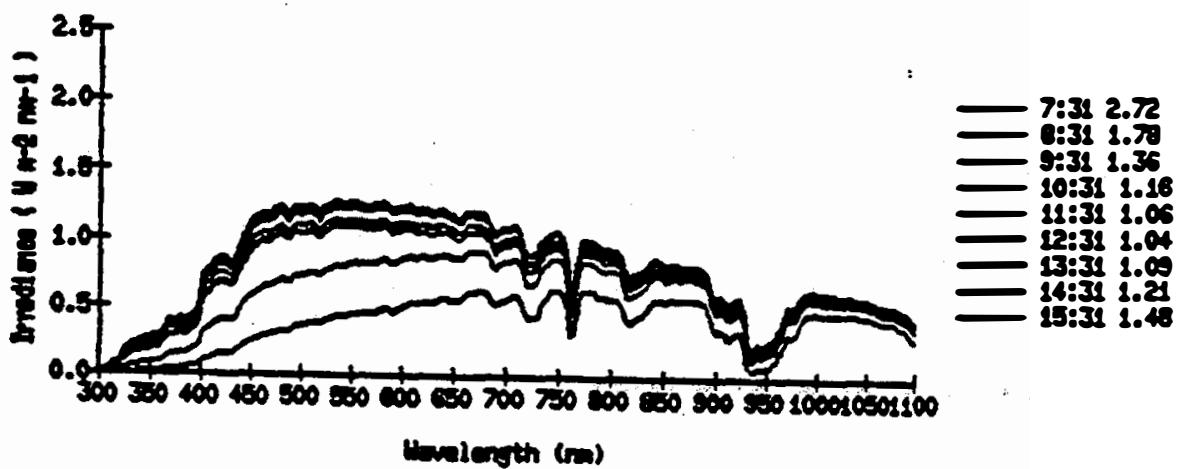

PIXY 212 as

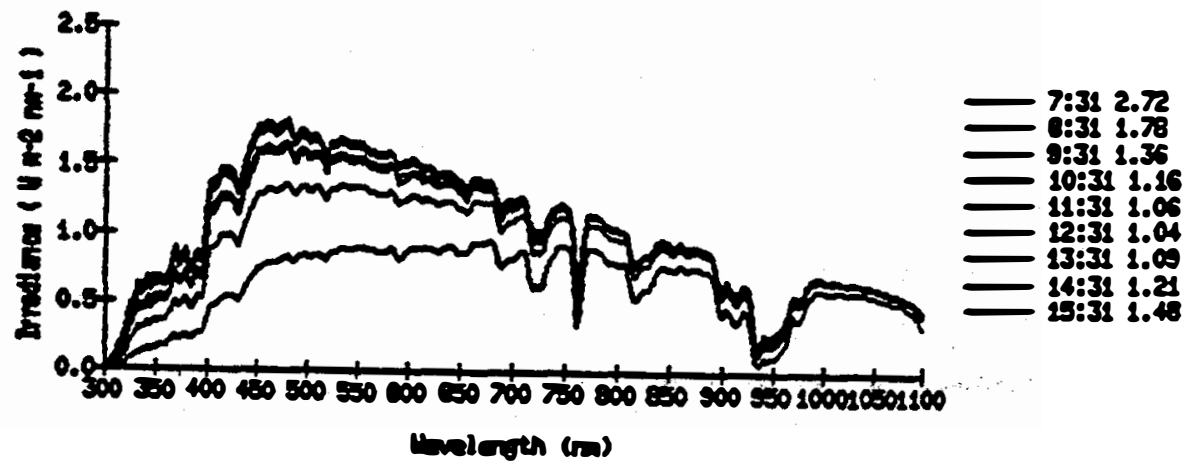




\section{SEN *}

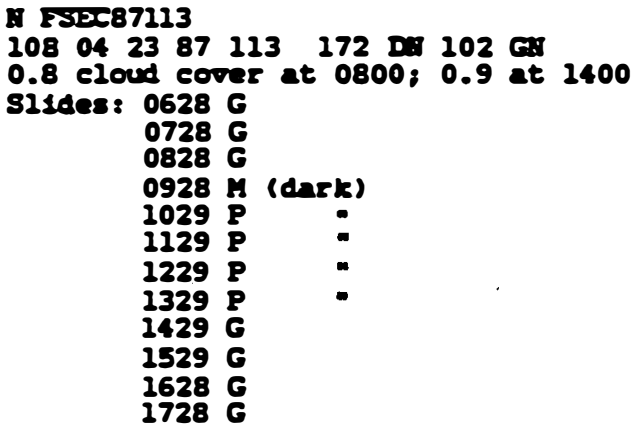

$\operatorname{Fose} 143$

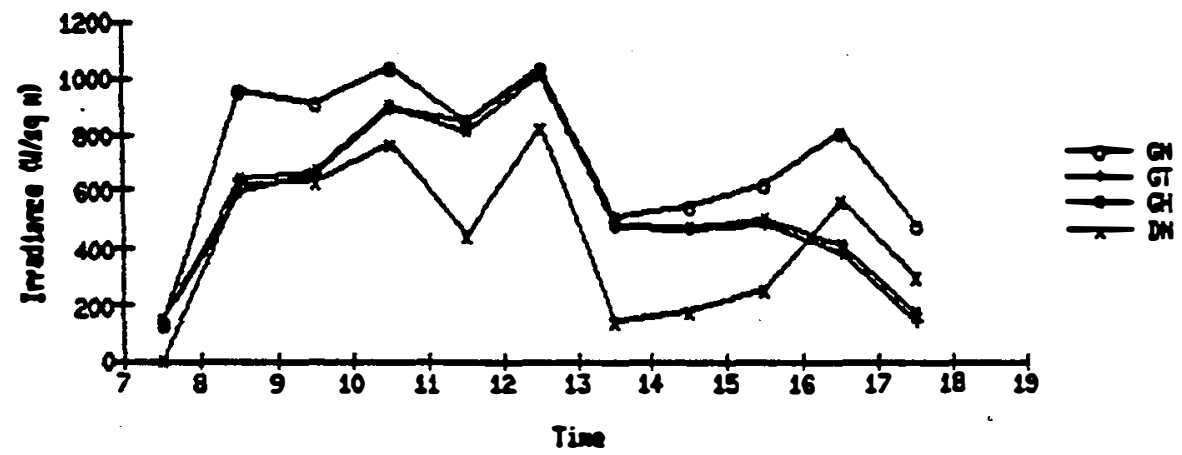

FIse 113 BTT

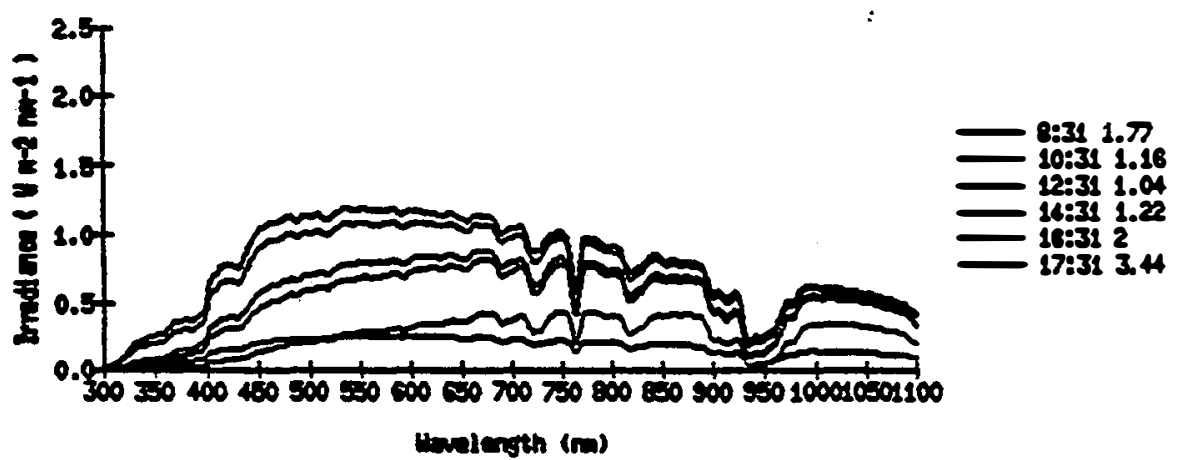

Fext 113 Os

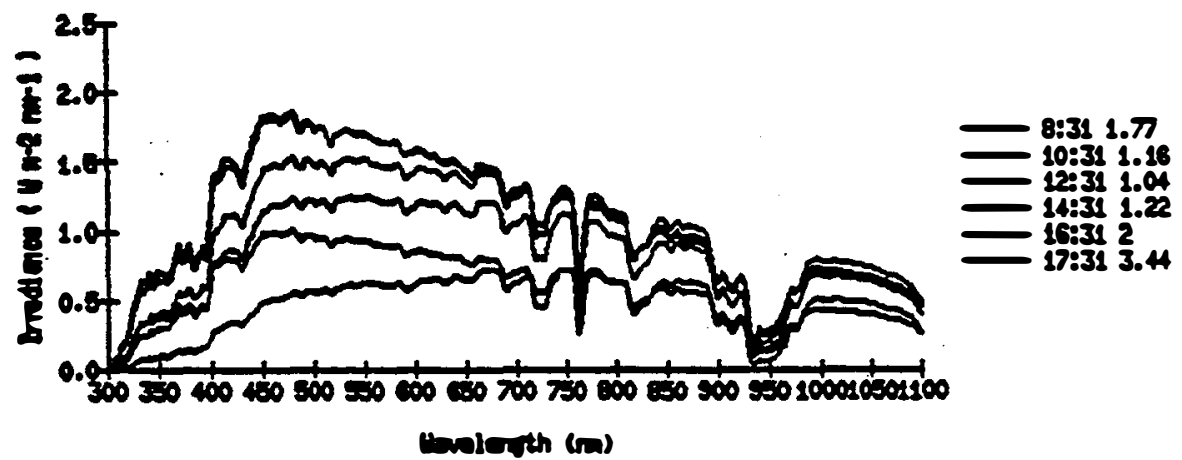




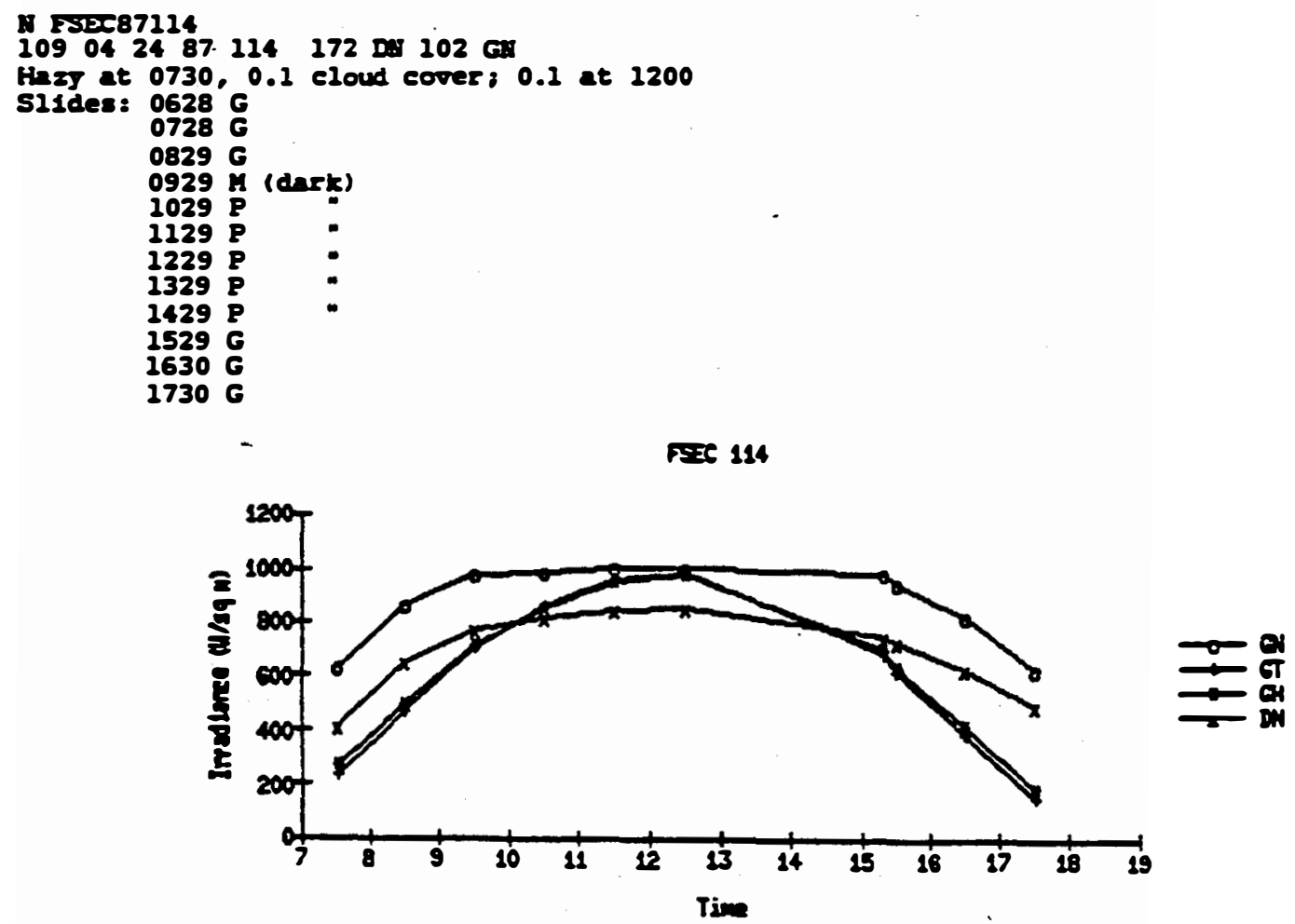

FEx 114 81T

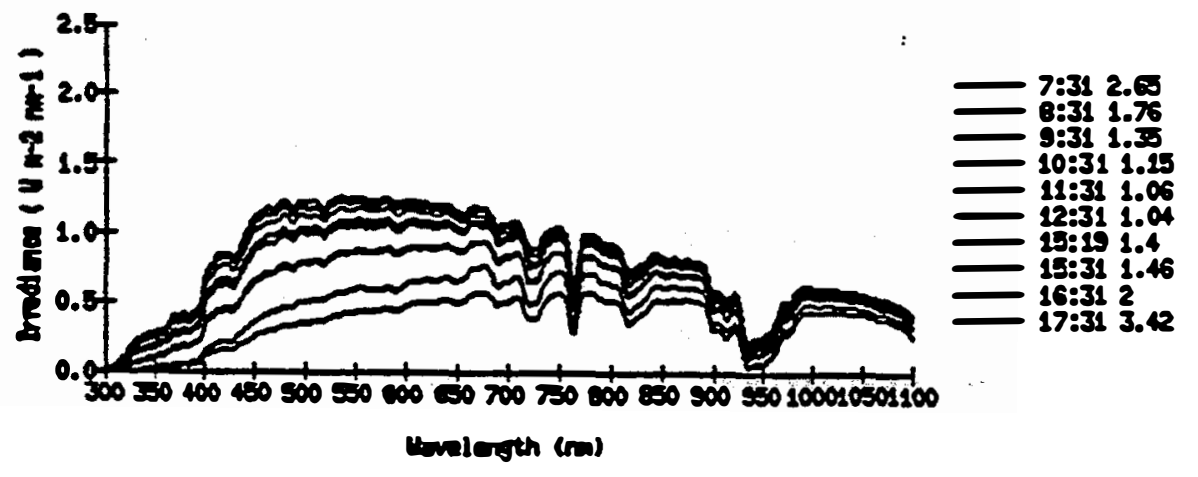

paxs 114 OS

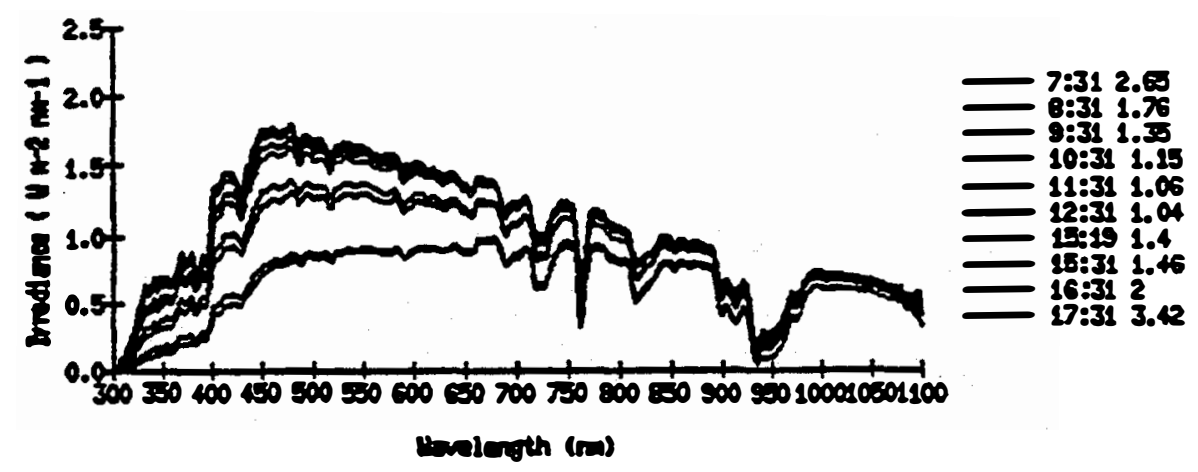




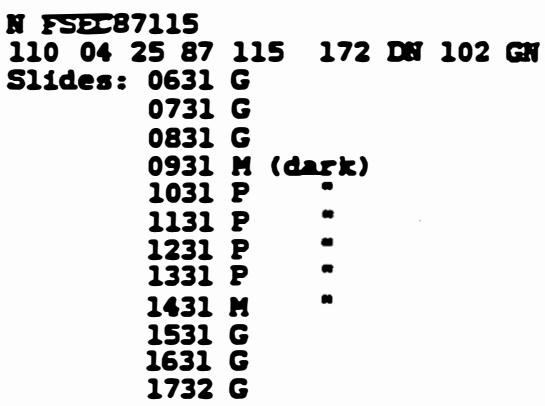

$\operatorname{Fsec} 115$

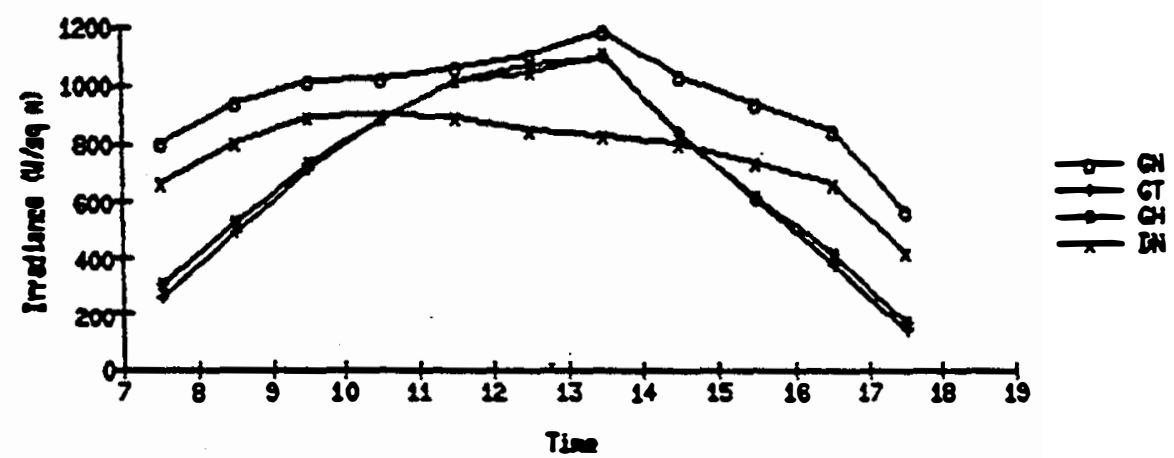

FEבC 115 anT

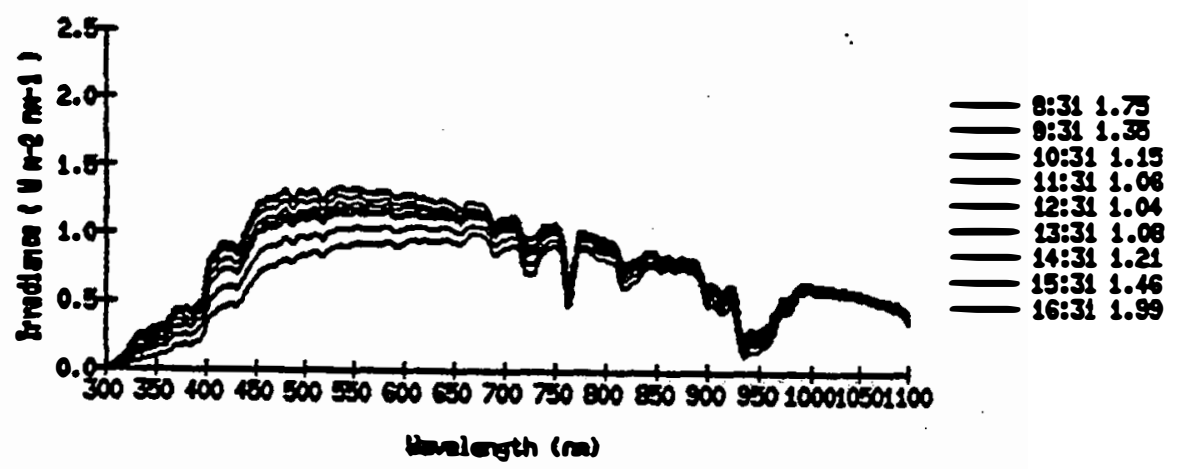

FIE 125 os

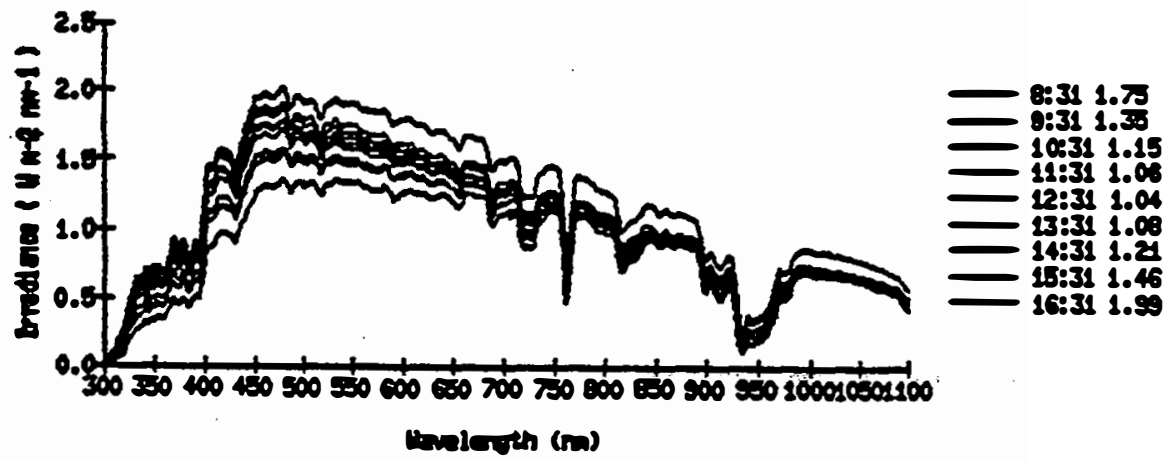



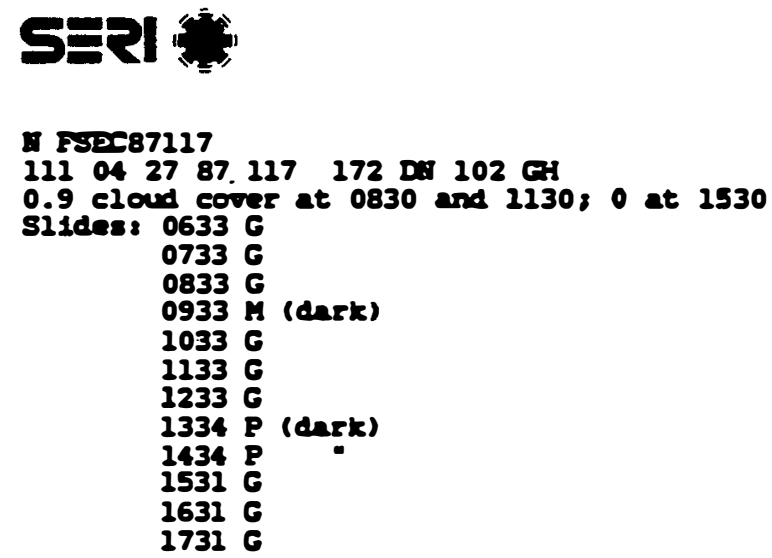

$1631 \mathrm{G}$

$1731 \mathrm{G}$

FESC 217

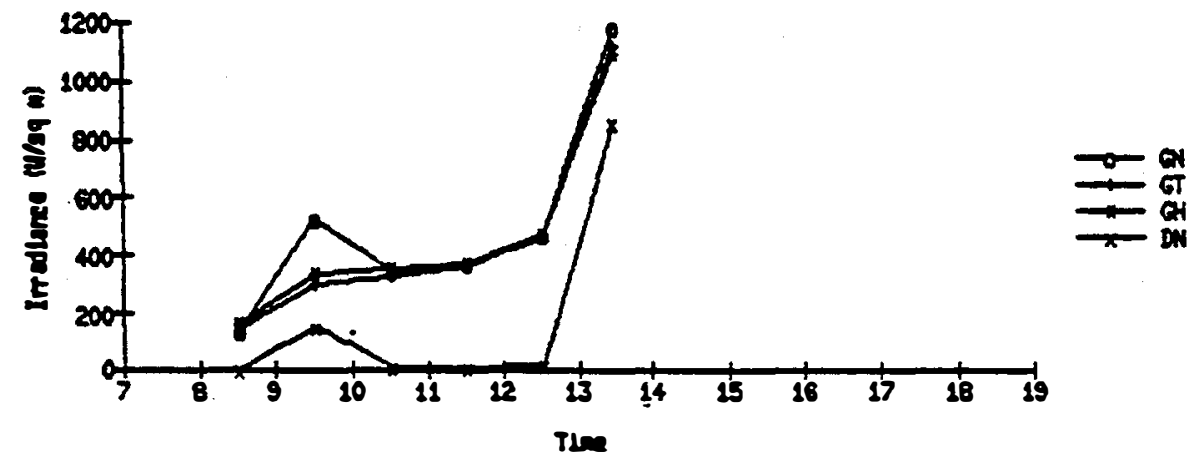

FIיב 127 ont

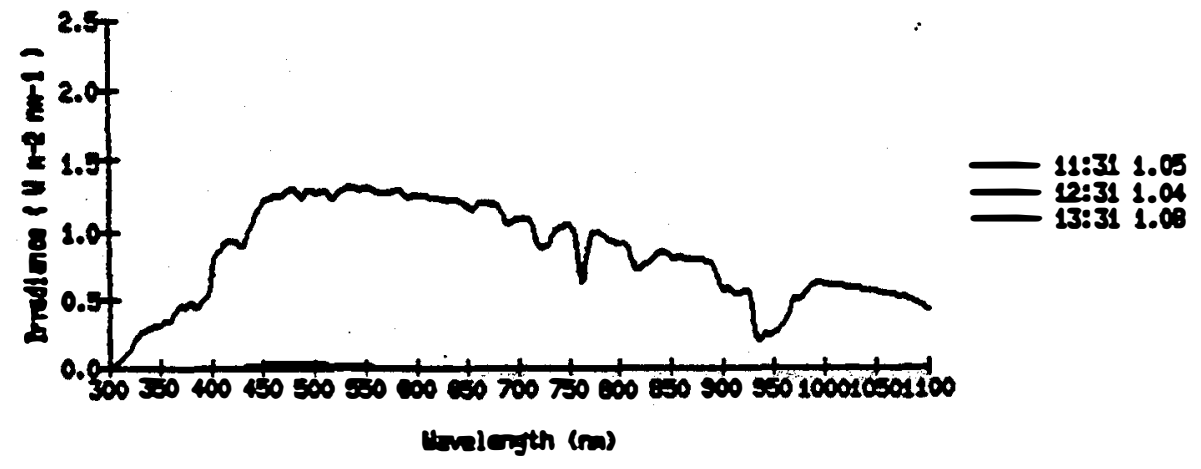

FIXC 117 as

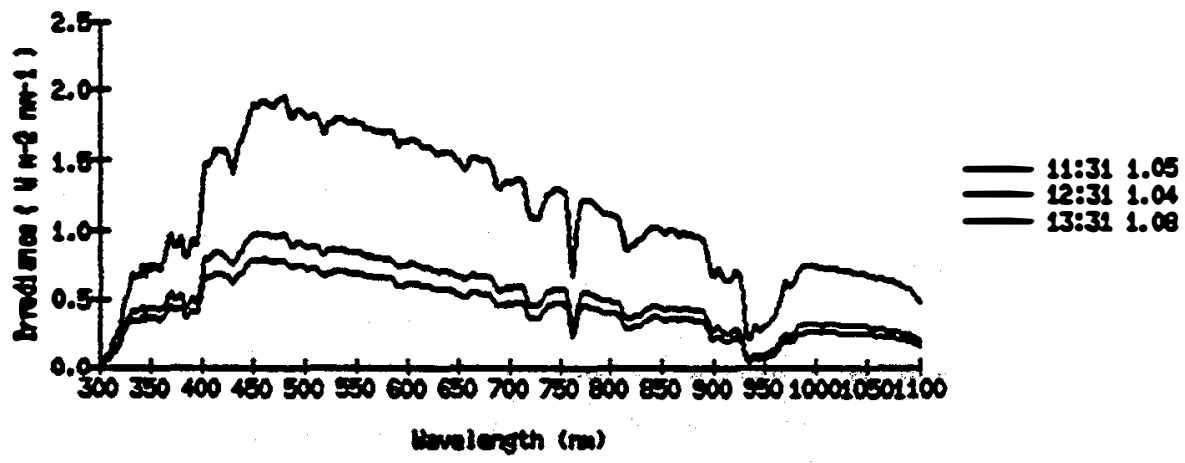


A. FS2C87118

$\begin{array}{llllllll}12 & 04 & 28.87 & 118 & 172 & 102 & \text { G }\end{array}$

Clear at 0900 and 1530

$80631 \mathrm{G}$

$0731 \mathrm{G}$

$0831 \mathrm{G}$

0931 M (dnst)

$1031 P$

$1231 \mathrm{P}$.

$1231 \mathrm{P}$

$1331 P$

$1431 \mathrm{H}$

$1531 \mathrm{G}$

$1631 \mathrm{G}$

$1731 \mathrm{G}$

MSssing date in DS spoction at 0931 and 1531

Fise 118

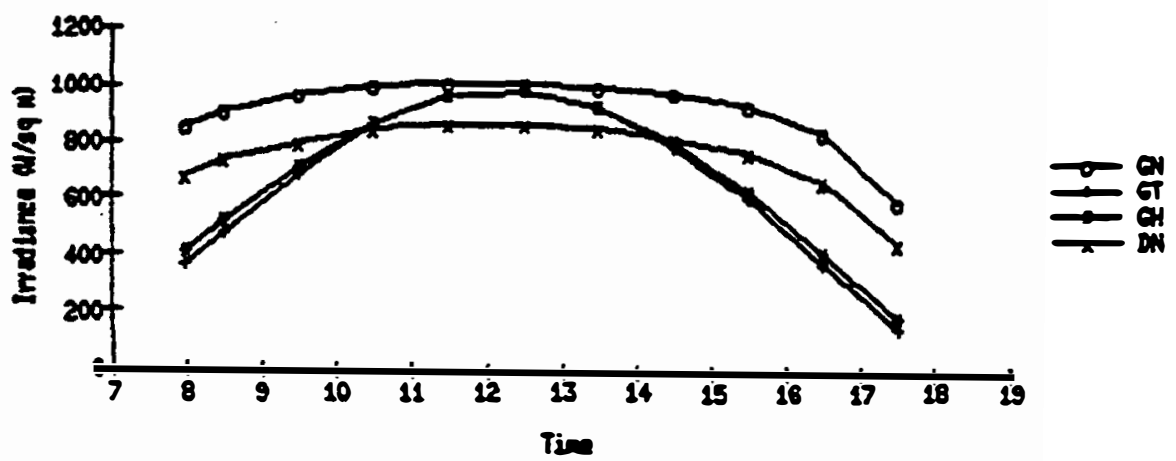

FSבE 218 हnT
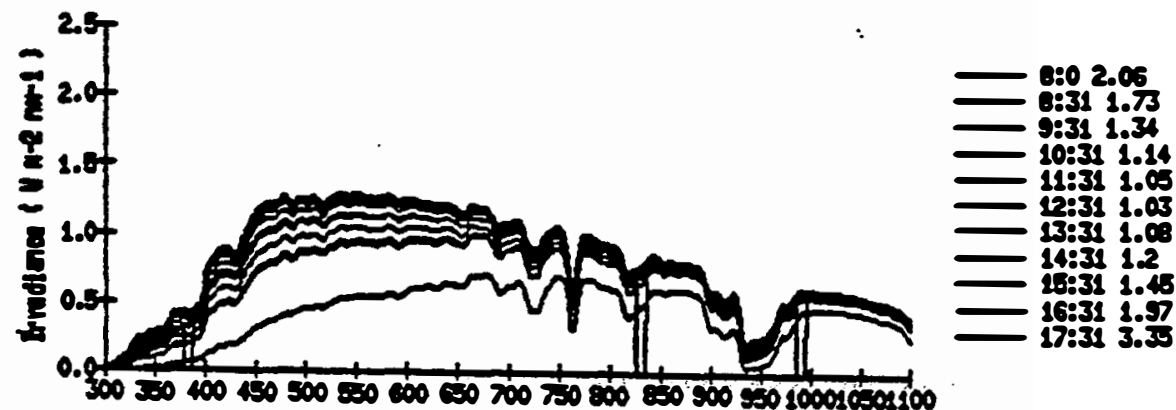

lableth (m)

FIST 118 95

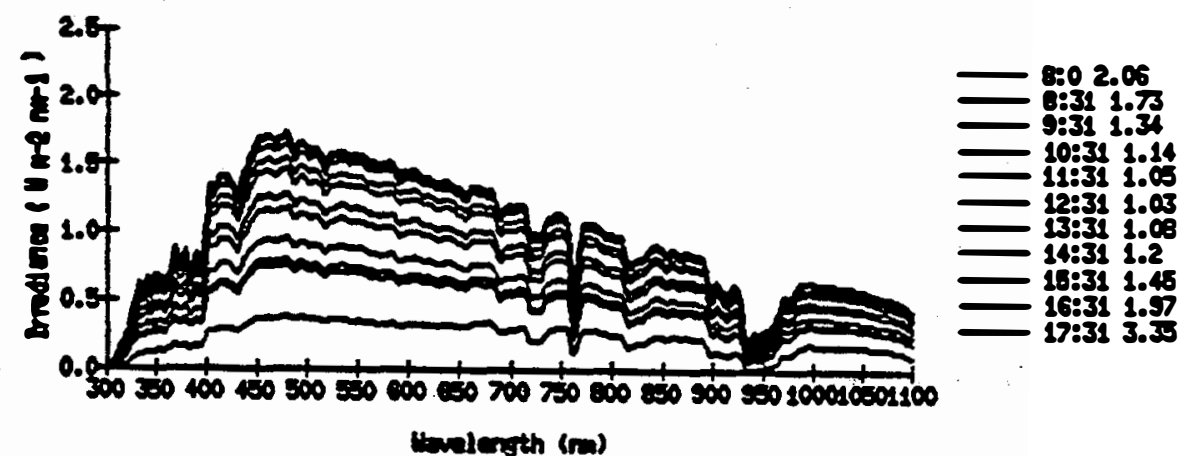


N FS2587119

113 O4 $2987 \quad 119 \quad 172$ DA 102 GH

Clear at $0800 ; 0.1$ eloud corer at 1230

Slides: 0629 G

0829

0929 \& (dark)

1129

1229

1329

Fix 149

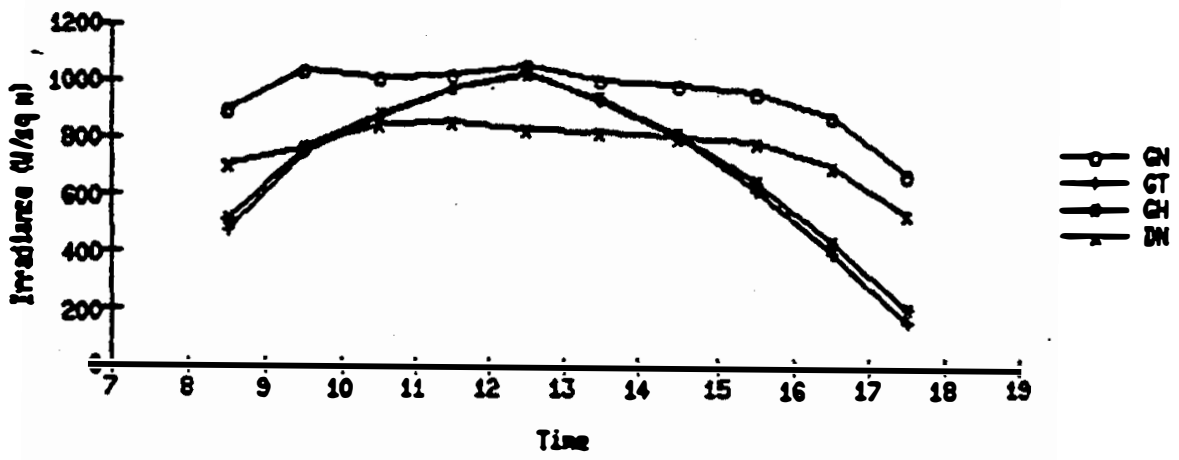

Fa्य $219 \mathrm{BnT}$

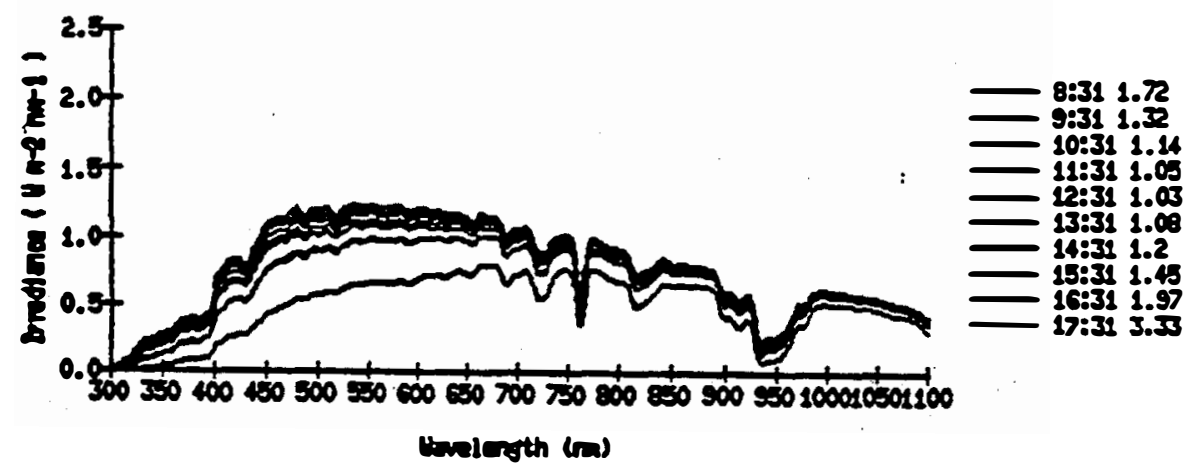

Fs36 129 as

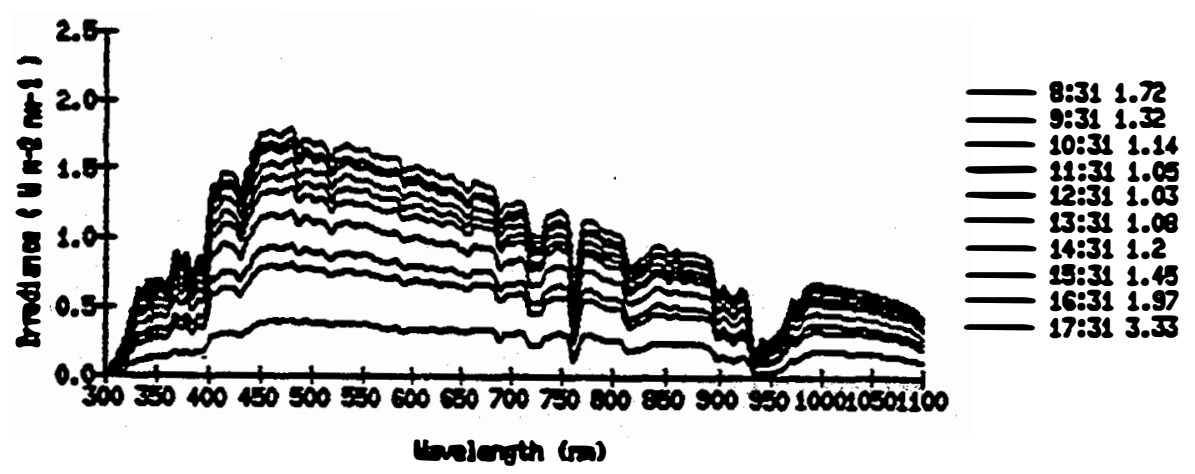




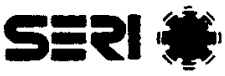

PSPC87120

114 O4 $30 \quad 87120 \quad 172$ DA 102 GH

Clear at 070080.9 cloud cover at 1130 (hasj) 8.8 at 1600 (hazy)

SIIdes. $0626 \mathrm{G}$

$0726 \mathrm{G}$

$0832 \mathrm{G}$

0932 I (dark)

$1032 \mathrm{P}$

1132

$1332 \mathrm{P}=$

$1432 \mathrm{M}$

$1532 \mathrm{G}$

$1632 \mathrm{G}$

$1732 \mathrm{G}$

Sall spike in DA spectin in Fisible at 0731

Appenss to be spike in $\mathrm{G}$ spectron in ov at 0931

FIse 280

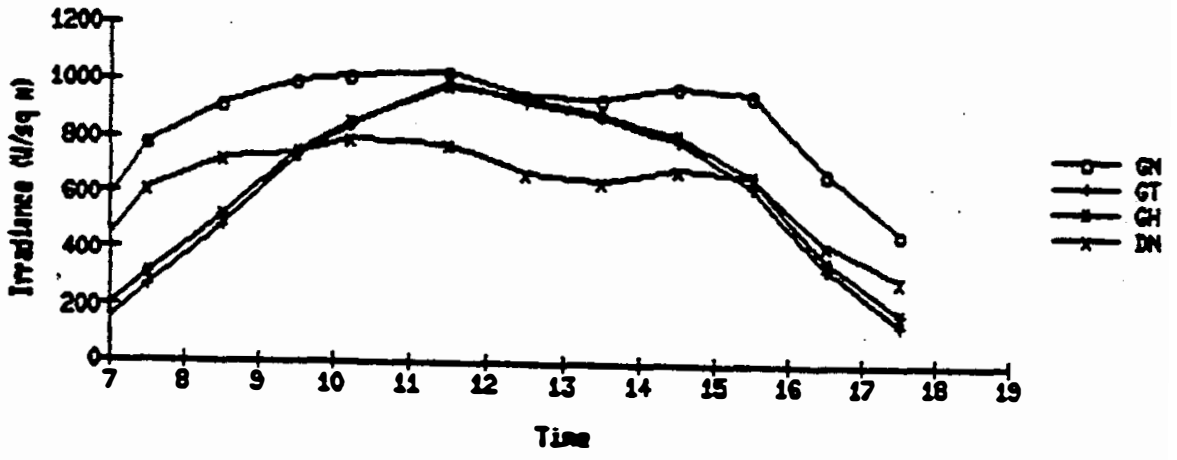

FISE $200 \mathrm{Bm}$

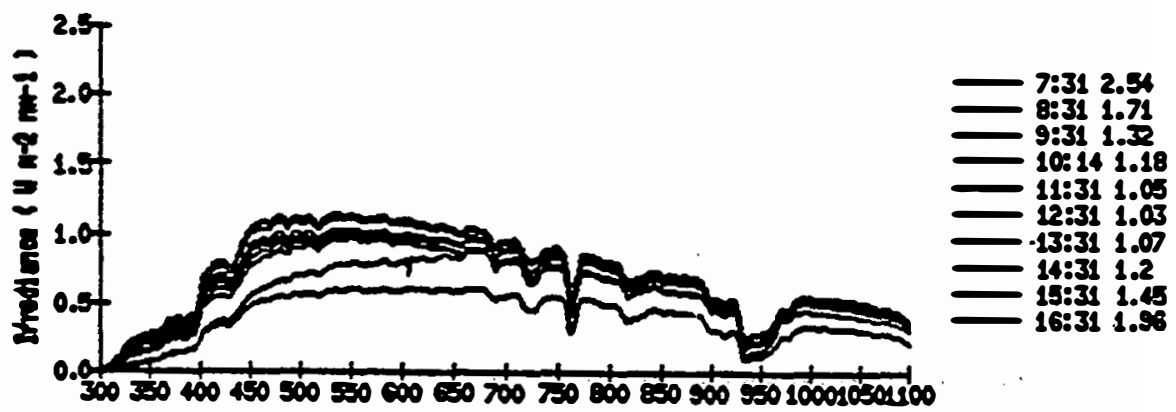

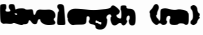

Fsec 220 ats

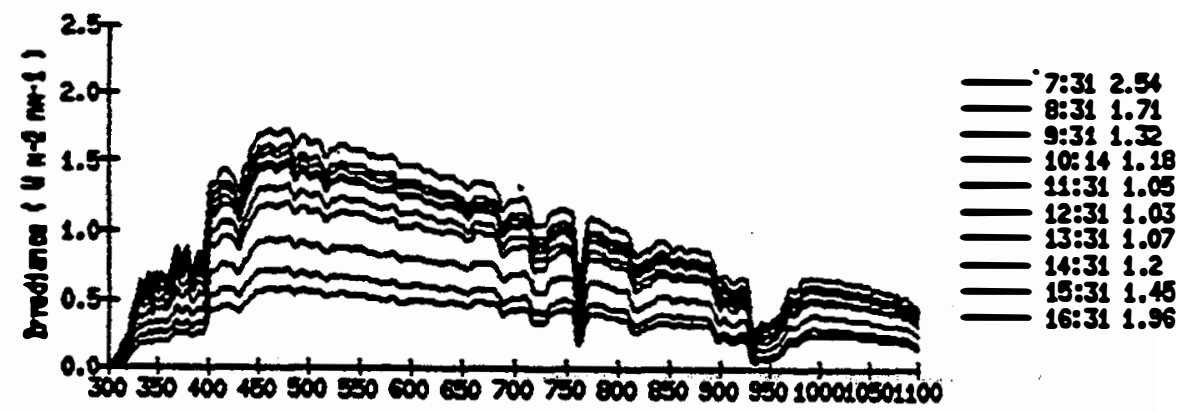

underath (rm) 


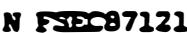

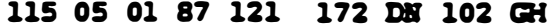

Clear at 0730; 0.1 eloud cover at 1130; 0 at 1600

S11des: $0632 \mathrm{G}$

$0732 \mathrm{G}$

$0832 \mathrm{G}$

0932 A (dnrk)

$1032 \mathrm{~F}$

$1233 \mathrm{P}$

$1333 \mathrm{P}$

1433

$1533 \mathrm{G}$

$1633 \mathrm{G}$

$1733 \mathrm{G}$

Spikes in GH pectra in ov at 0731 and 0830

Hote tins stap differences 0830 v8. 0935

FEeC 121

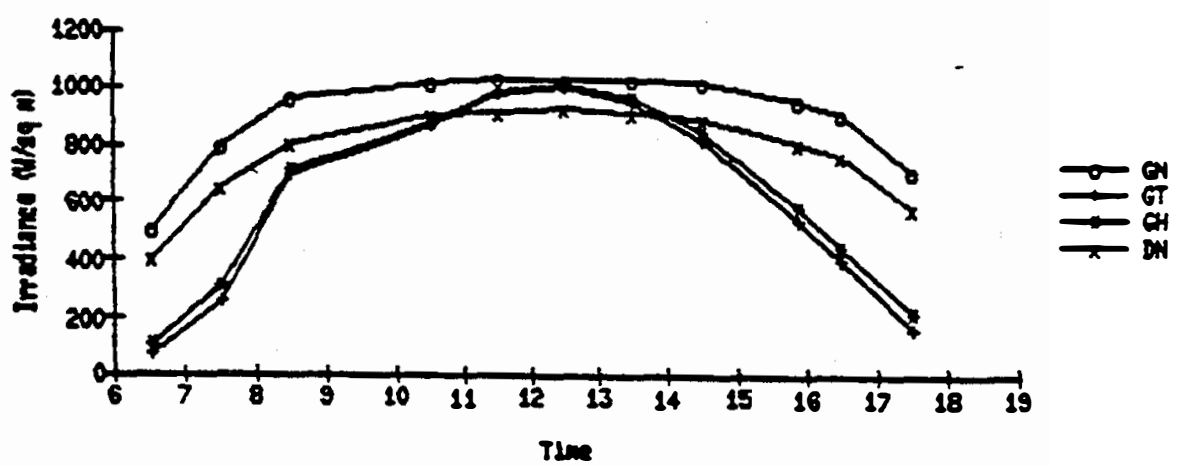

FSEC $228 \mathrm{BNT}$

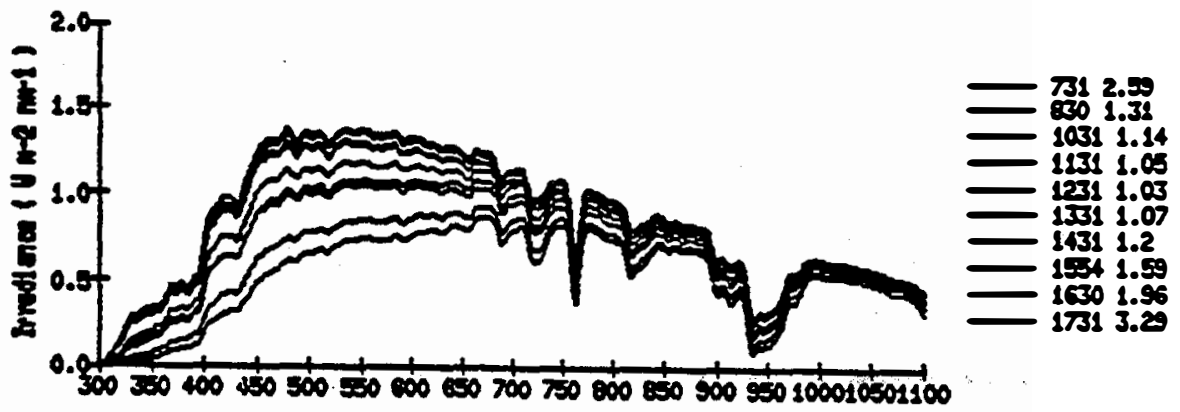

taviergth (n)

Fis 2909

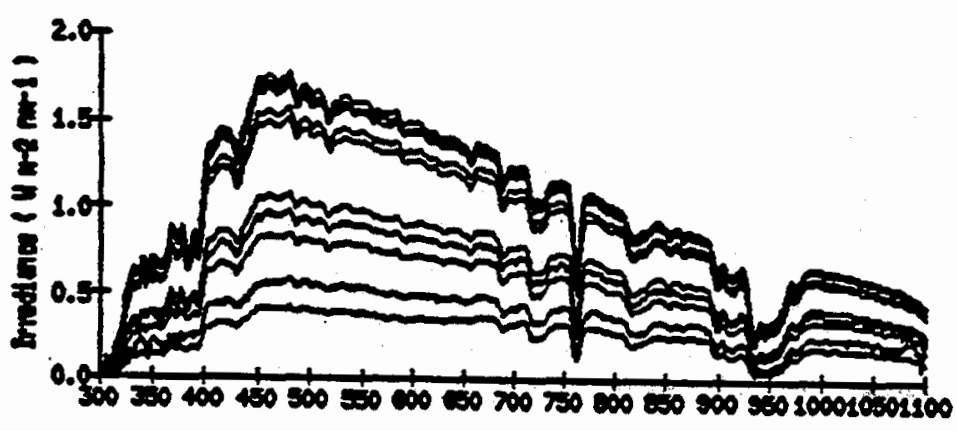

Unelorgth (n) 
I) FSTC87122

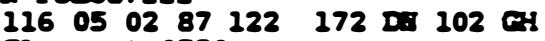

Clear at 0730

GH for first two scans, then GI

S1sdes: $0632 \mathrm{G}$

$0733 \mathrm{G}$

$0833 \mathrm{C}$

$0933 \mathrm{H}$ (dark)

1033

$1133 \mathrm{P}$

$1233 \mathrm{P}$

$1333 \mathrm{P}$

$1433 \mathrm{M}$

$1533 \mathrm{G}$

$1633 \mathrm{G}$

$1733 \mathrm{G}$

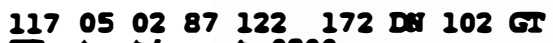
GI starting at 0800

Clear at 0800

Fise 122

Slides see above

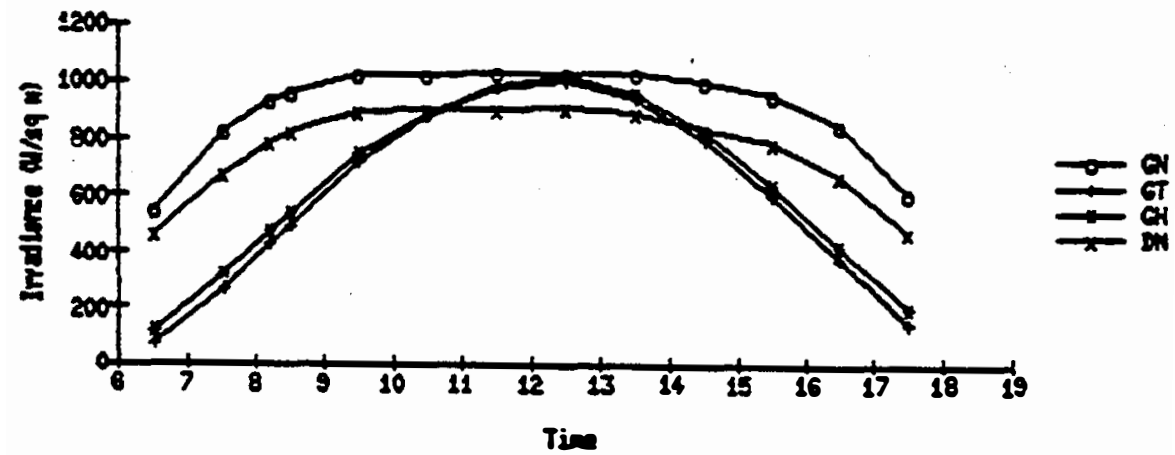

FSEC 222 BT

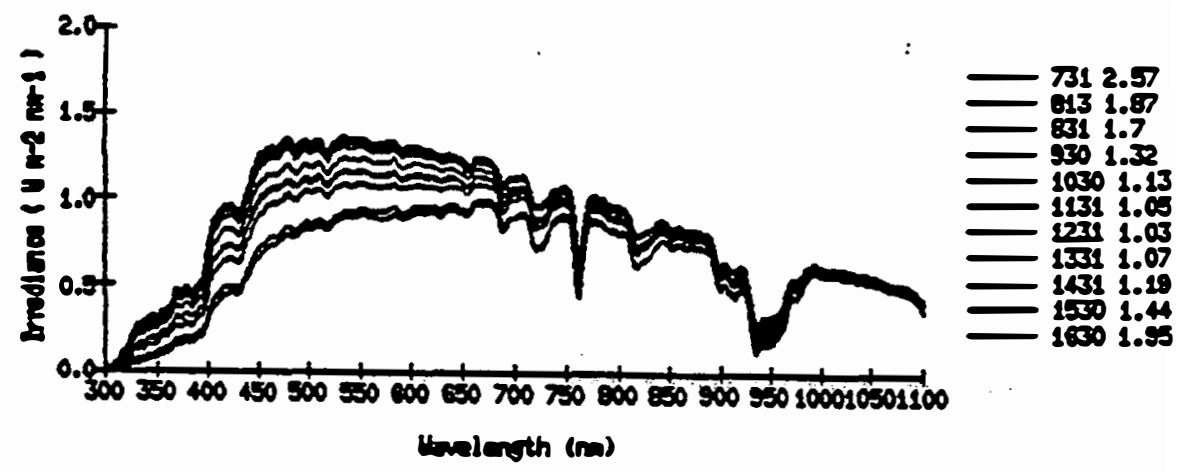

$\operatorname{Psx} 122 \mathrm{sT}$

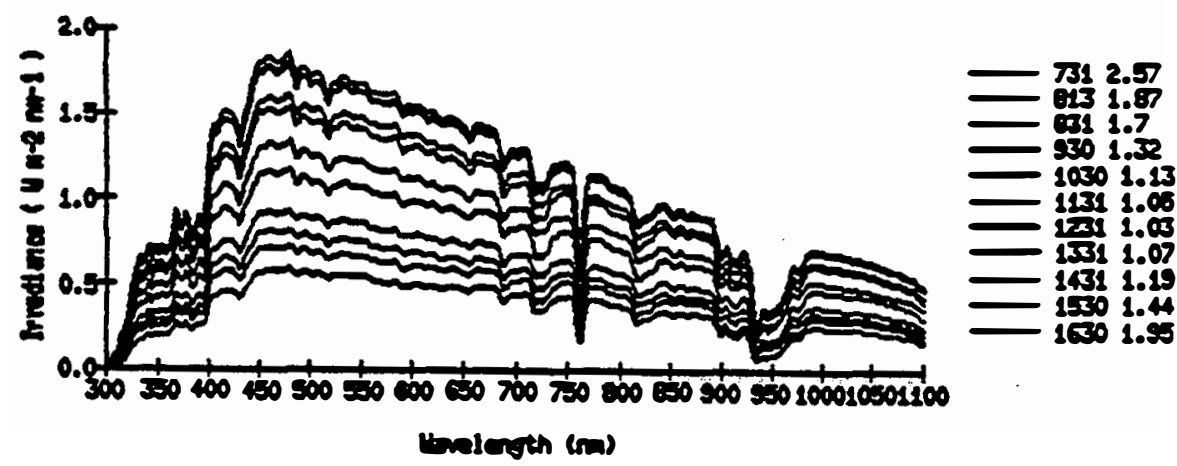




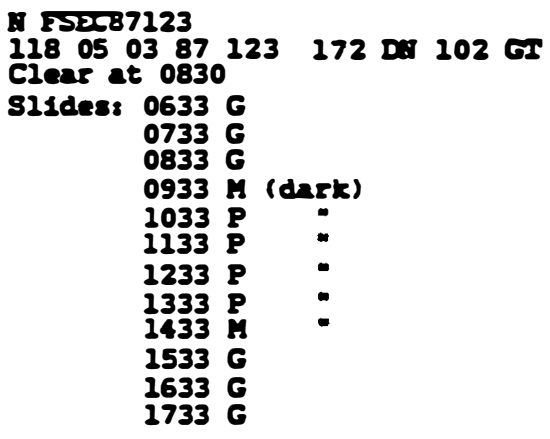

Fise 123

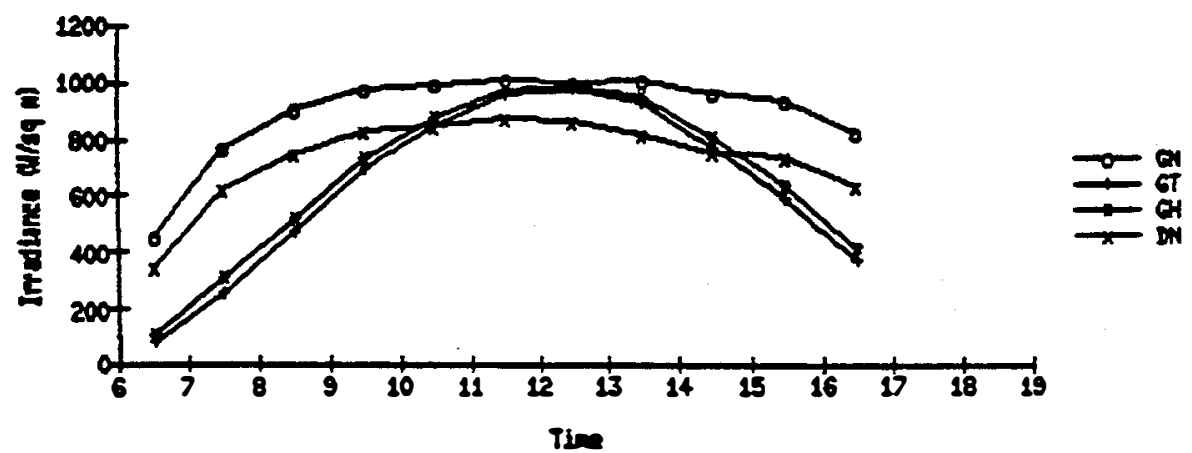

Fase 123 an

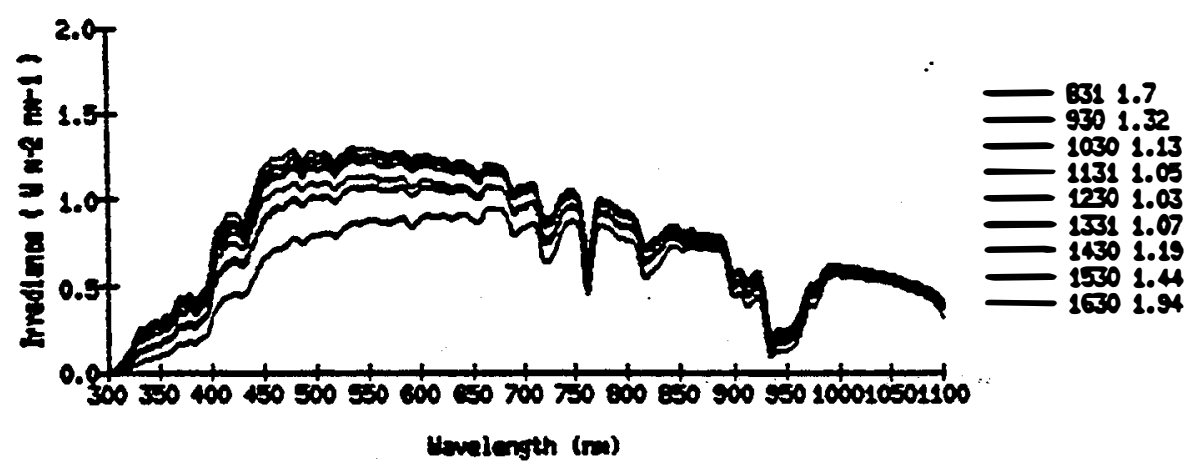

FEsC 223 GT

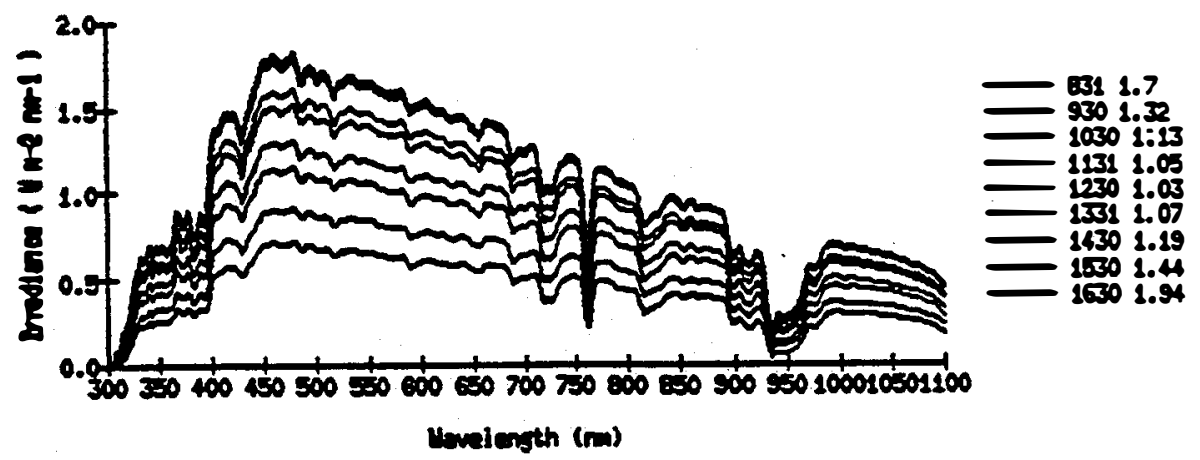


A.

$\begin{array}{lllllllll}119 & 05 \quad 04 & 87 & 124 & 172 & \text { DN } & 102 & G T\end{array}$

Clear at 0850 and 1600

S11des: $0633 \mathrm{G}$

$0733 \mathrm{G}$

$0833 \mathrm{G}$

$0933 \mathrm{G}$

1033 P (dark)

$1134 \mathrm{P}$

$1234 \mathrm{P}$

$1334 \mathrm{P}$

1434

$1534 \mathrm{G}$

1634 G

$1735 \mathrm{G}$

FSEC 224

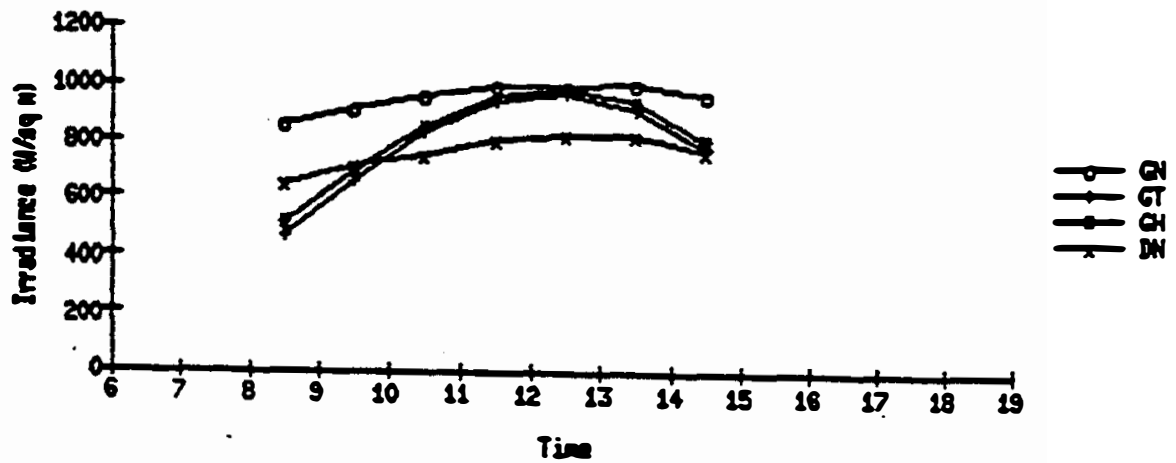

Fsex 124 ant

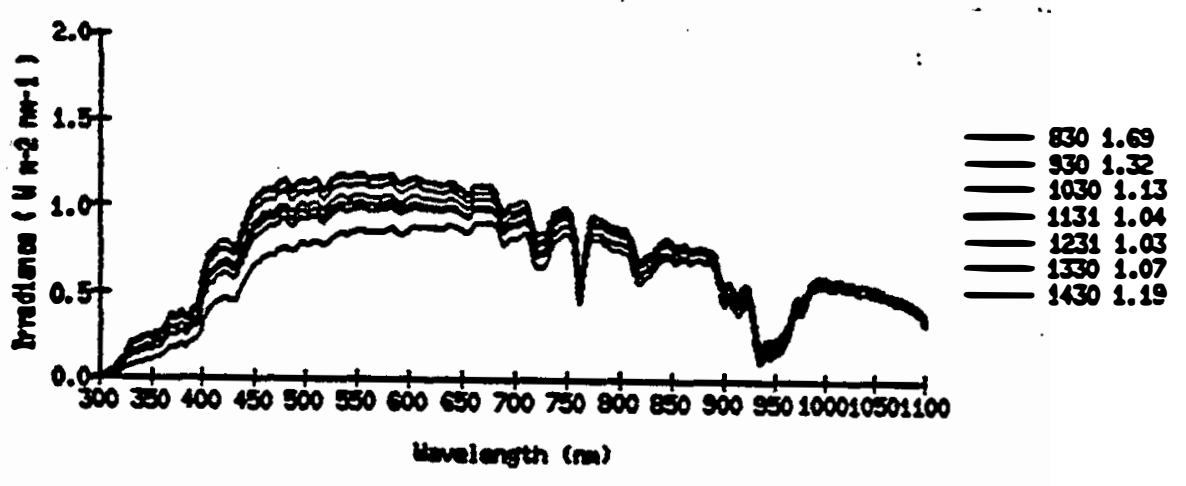

FSES 124 CTS

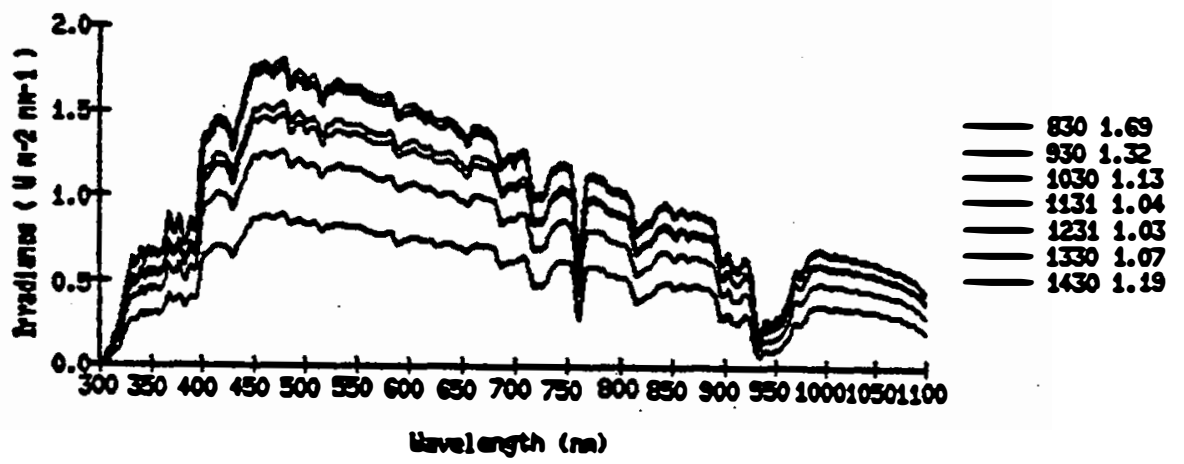


FST287125

$\begin{array}{llllll}120 & 05 & 0587 & 125 & 172 & 02\end{array}$

0.05 cloud cover with light hase at 0830 ; then 0.4

Irackers quit: reset at 0800

Slides: $0635 \mathrm{G}$

$0735 \mathrm{G}$

$0835 \mathrm{G}$

$0935 \mathrm{G}$

$1035 \mathrm{M}$ (dnzk)

$1135 \mathrm{P}$

$1235 \mathrm{P}$ -

$1335 \mathrm{P}:$

$1535 \mathrm{G}$

1635 G

$1735 \mathrm{G}$

Fise

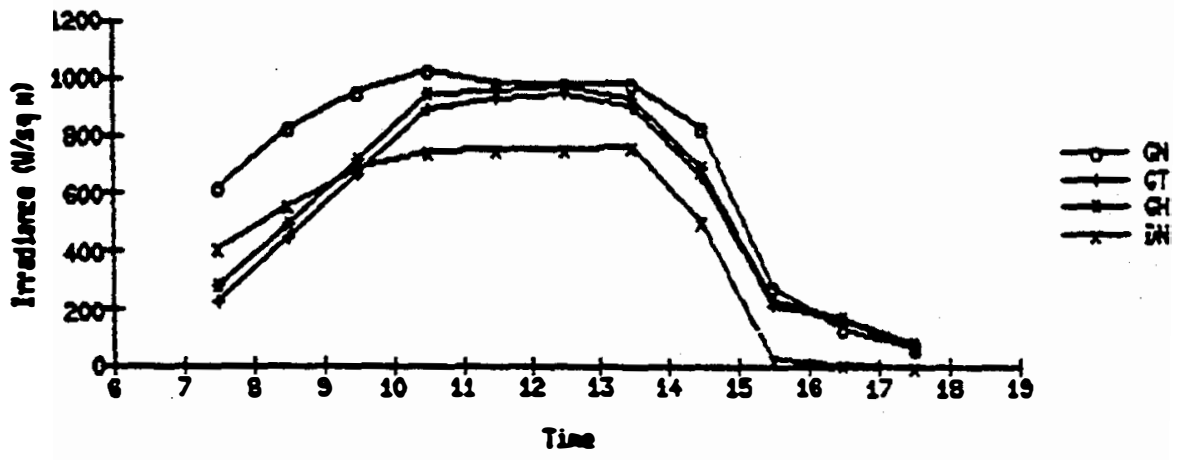

FSEC 125 BT

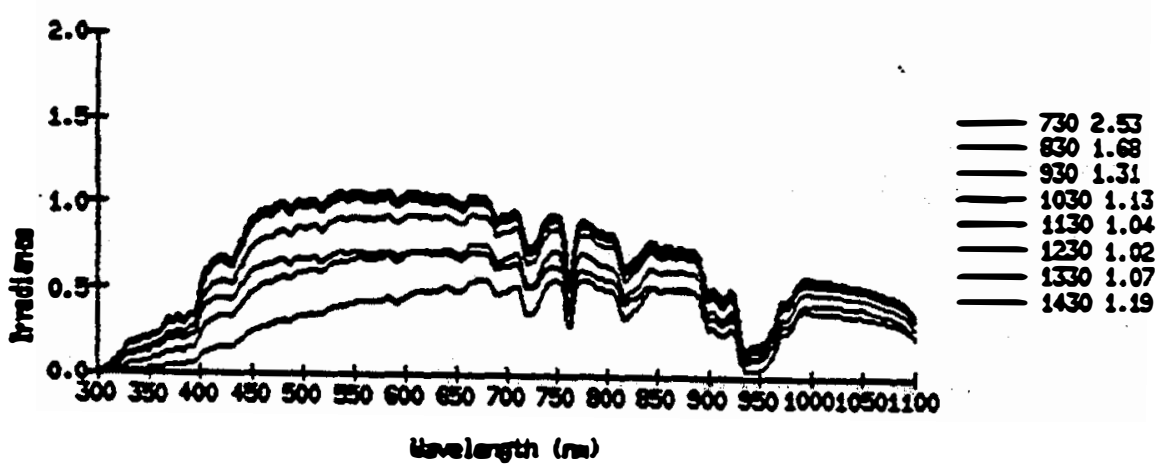

PIST 250 GTS

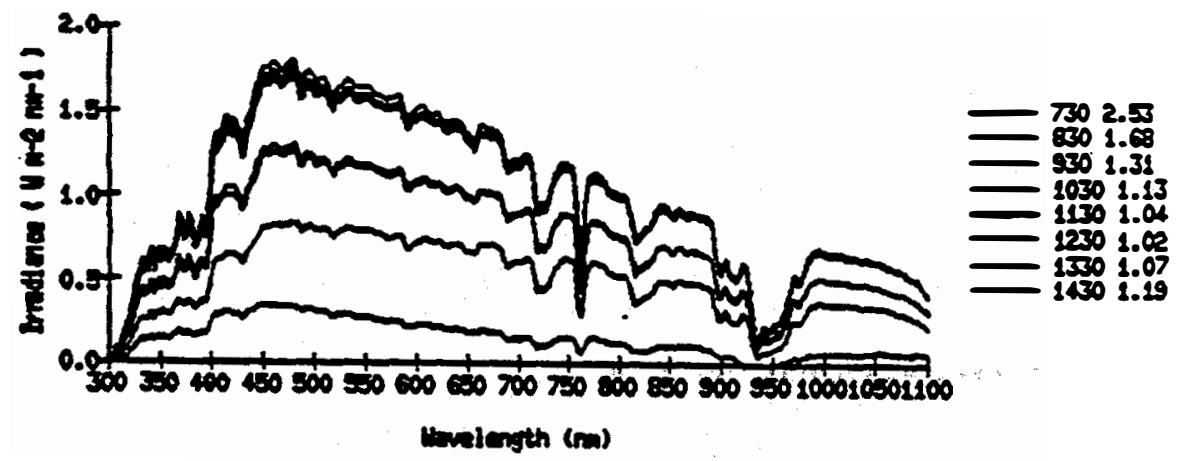




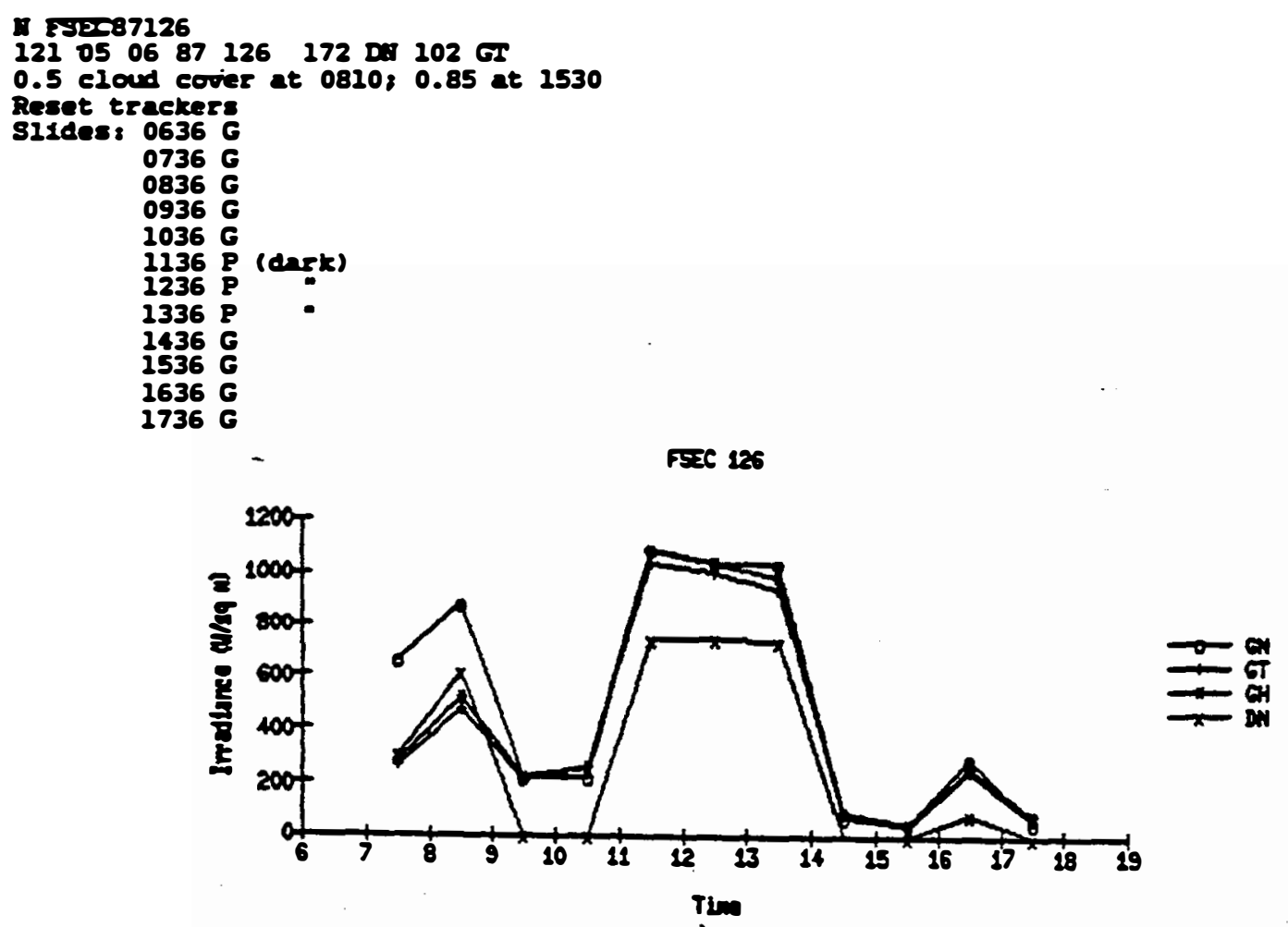

FSEC 225 anT

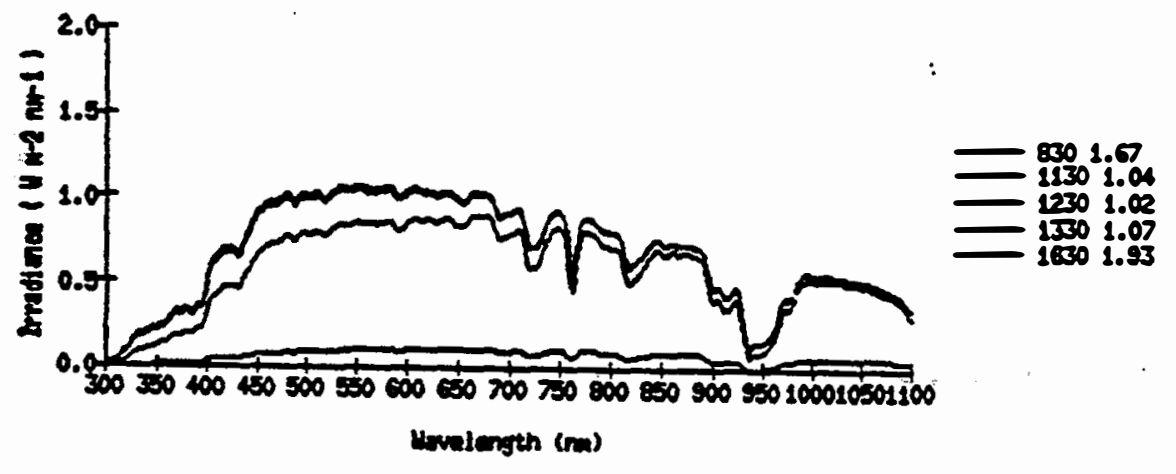

FISC 126 GTS

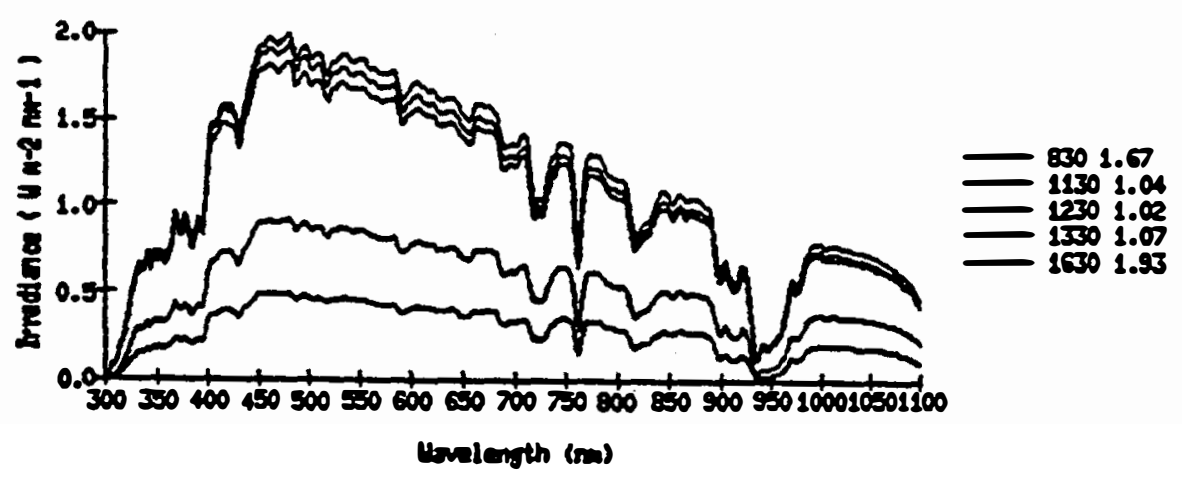




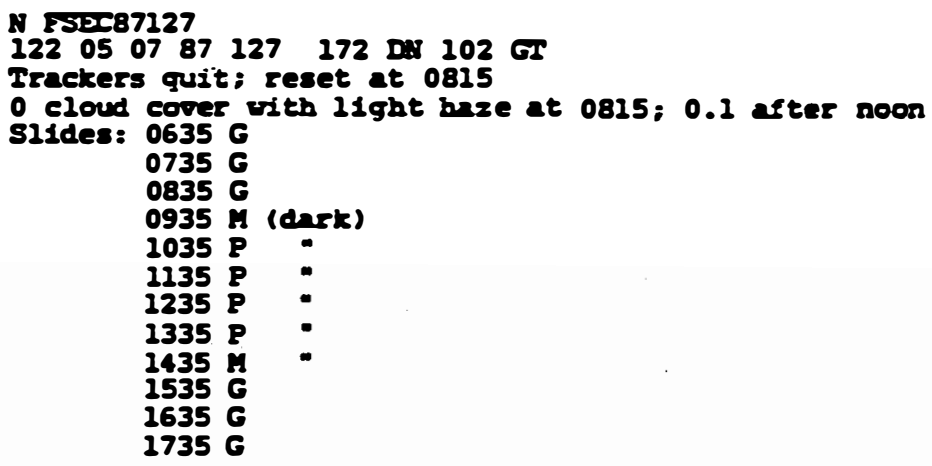

Spike in DA spêctren at 0830

Onstuble condtitions at 0930

FIEC 120

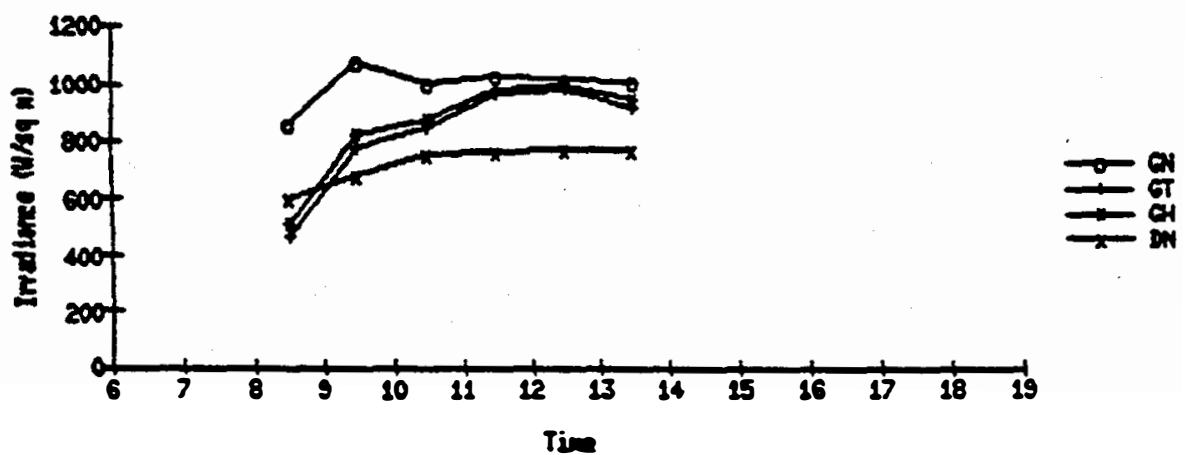

FSEC $225 \mathrm{DNT}$

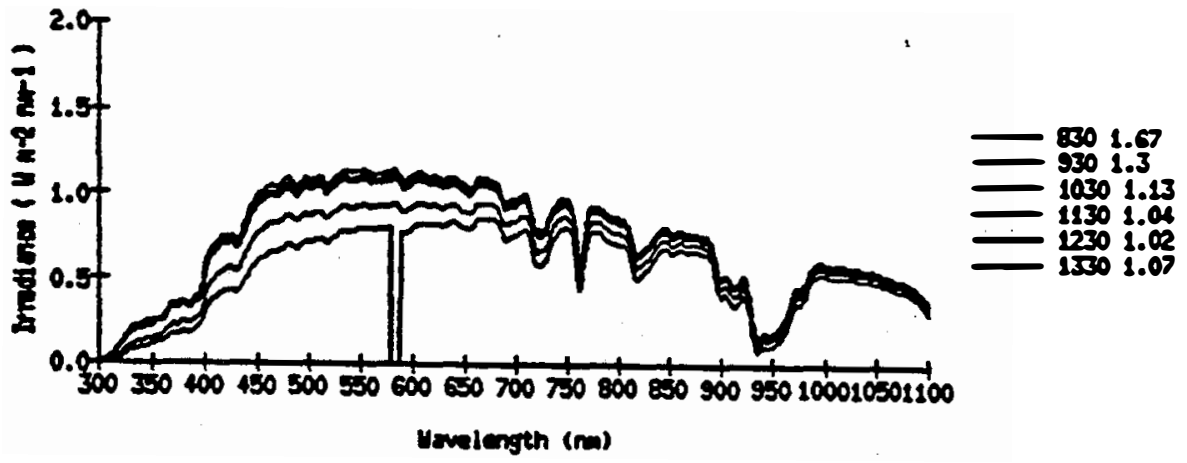

FSEC 227 GTS

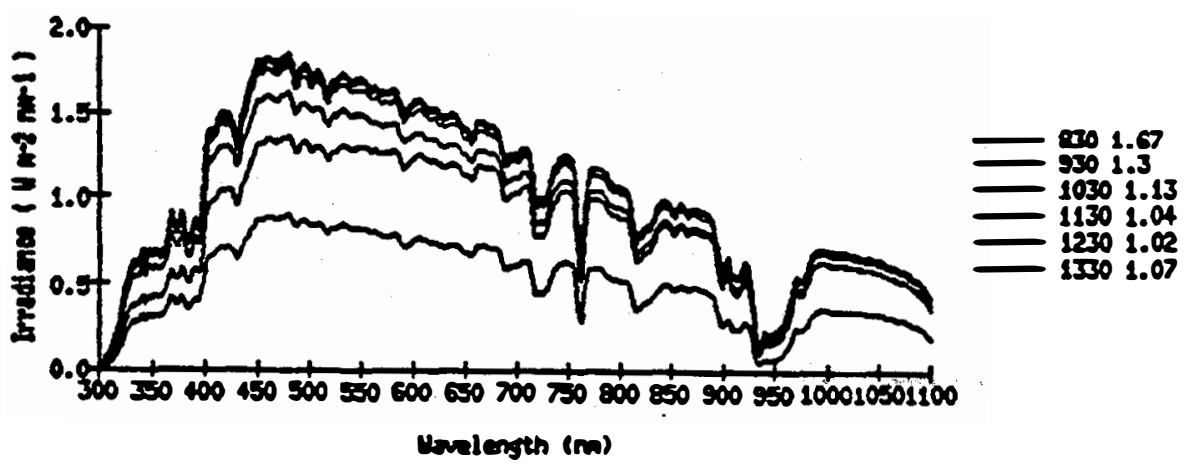


4.

$\begin{array}{lllllllll}123 & 05 & 08 & 87 & 128 & 172 & 09 & 102 & G\end{array}$

0.05 eloud cover with sore has

Reset trackers at 0820

Sl1desz $0634 \mathrm{G}$

$0734 \mathrm{G}$

$0834 G$

0934 A (dnrk)

1034

$1134 \mathrm{~F}$

$1234 \mathrm{G}$

$1334 \mathrm{G}$

$1434 G$

$1534 \mathrm{G}$

Hote tine strp differences 0830 ve 0931

FISE 198

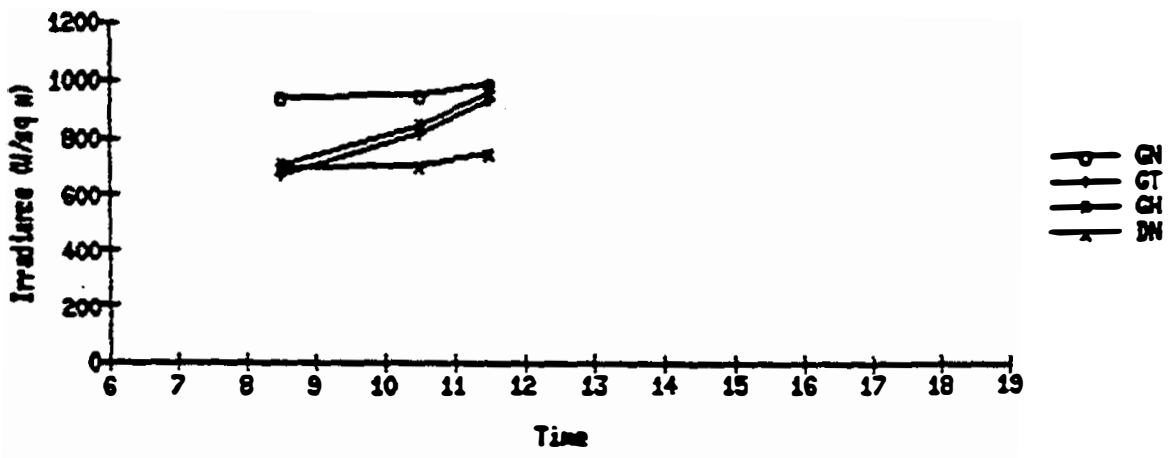

FEC 128 MIT

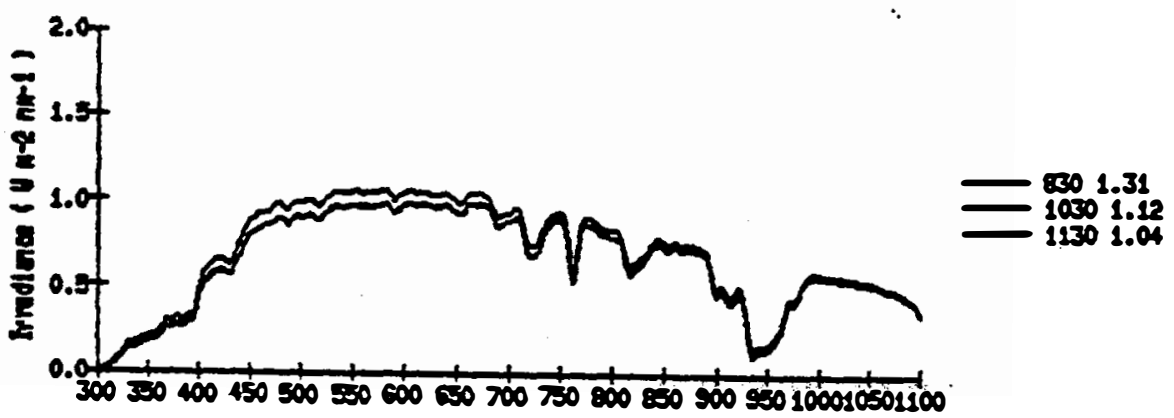

Unelgoth (m)

F्236 128 G5

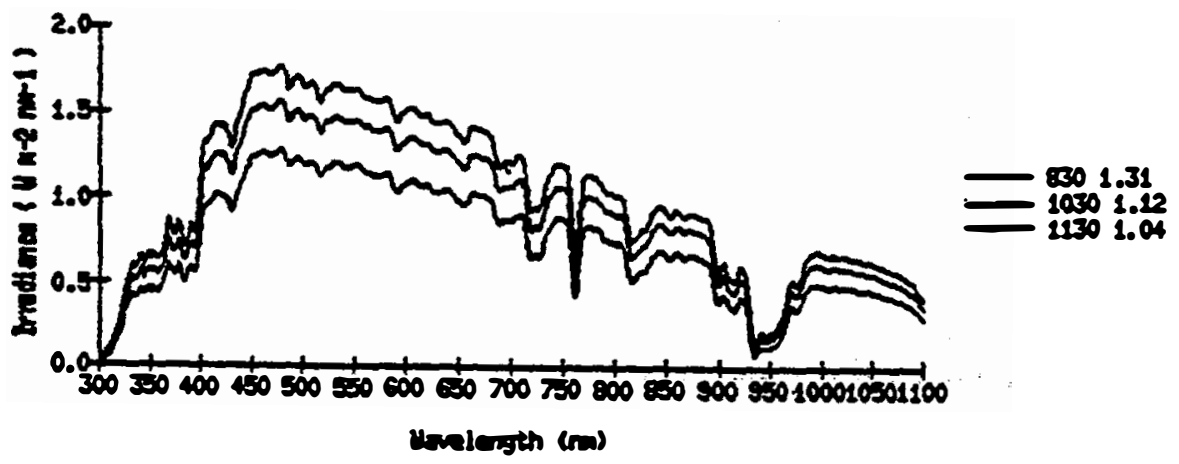



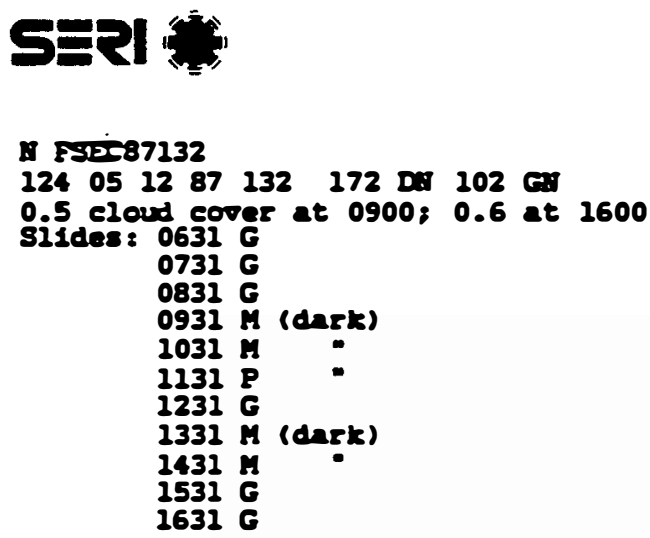

$1031 \mathrm{H}$

$1131 \mathrm{P}$

$1231 \mathrm{G}$

$1331 \mathrm{M}$ (dark)

$1431 \mathrm{H}$

$1531 \mathrm{G}$

$1631 G$

FSEC 132

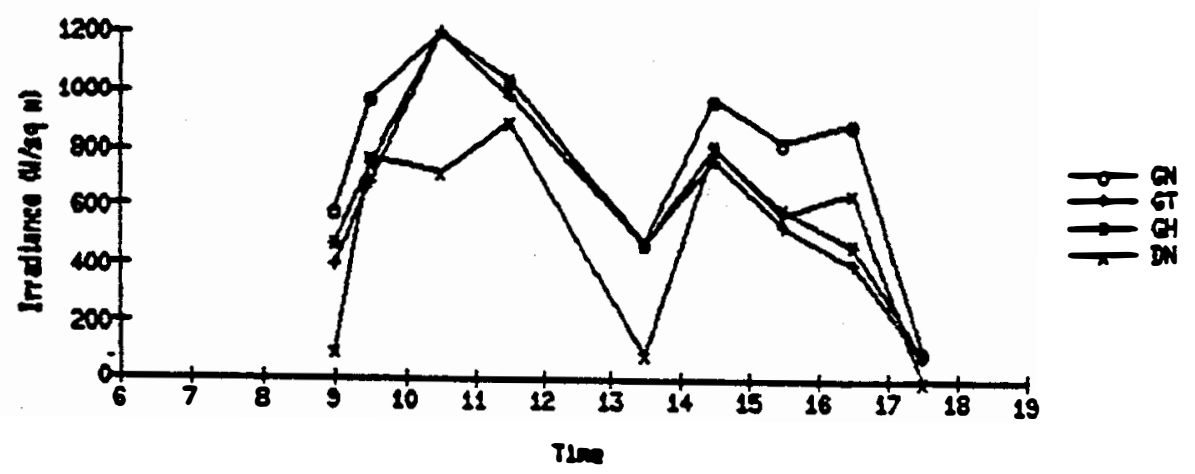

Fsx 252 net

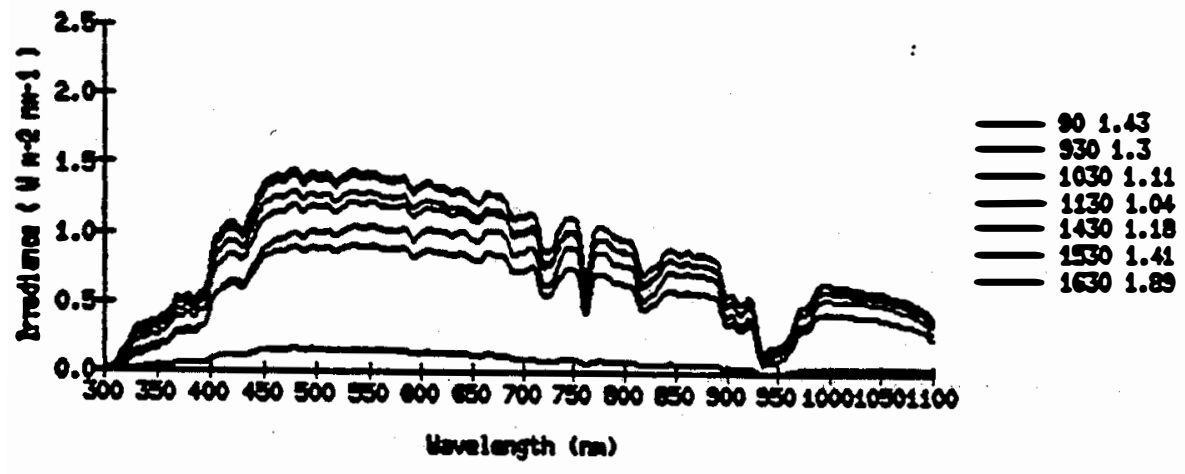

Fise 13205

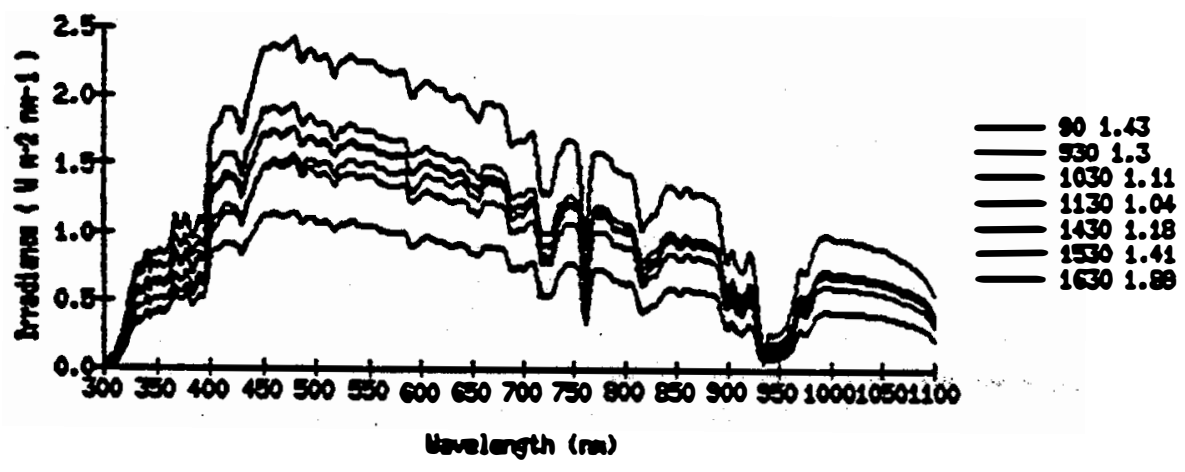




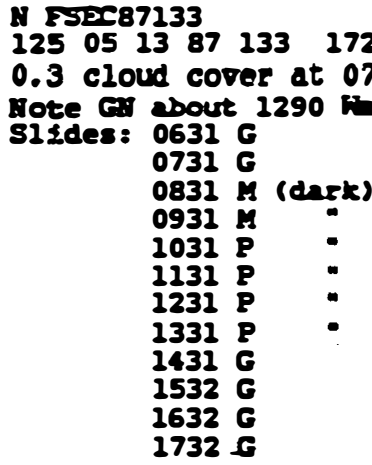

PIIC 233

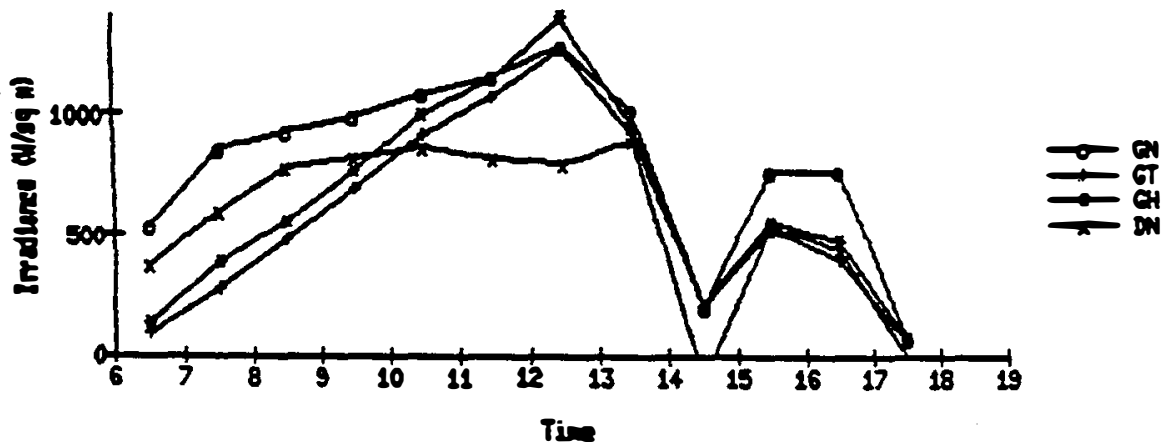

FSEC 133 ant

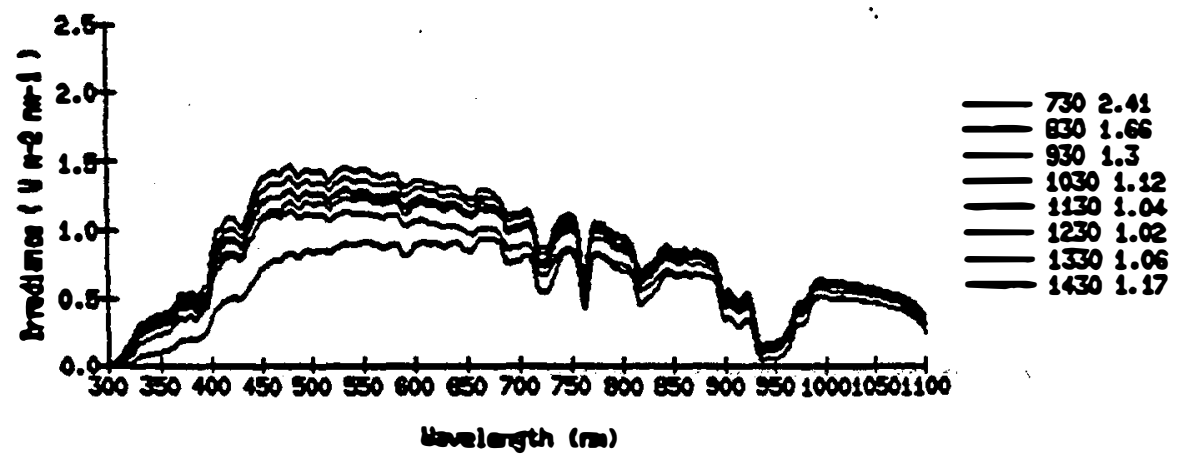

Psec 133 as

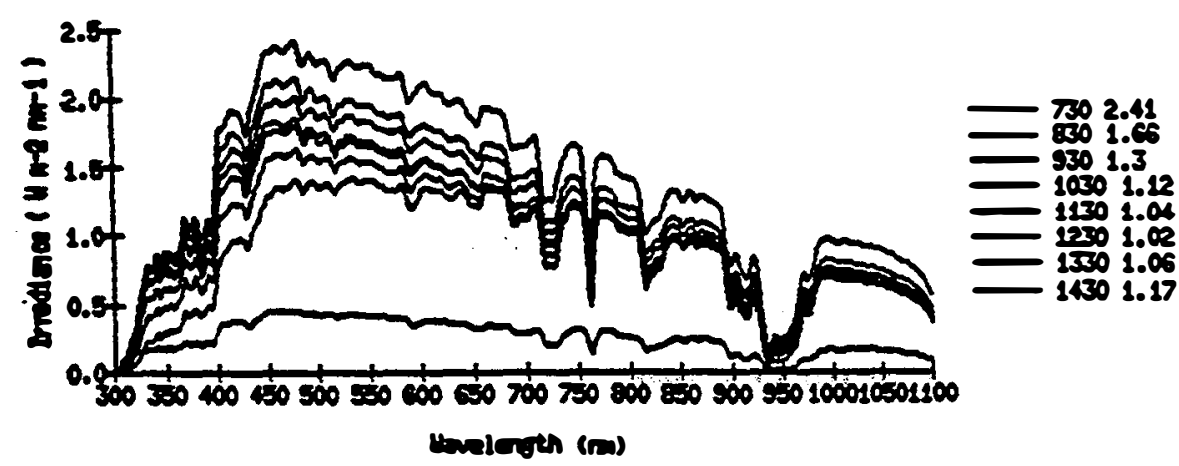


N PSTEC87134

$\begin{array}{llllllllll}126 & 05 & 14 & 87 & 134 & 172 & \mathrm{DS} & 102 & \mathrm{GS}\end{array}$

0.2 cloud cover at 0730

Slides: $0632 \mathrm{G}$

$0732 \mathrm{C}$

0832 \& (dark)

0932

1032

1132

$1232 \mathrm{P}$ -

$1332 P$

1432

$1532 \mathrm{G}$

$1632 \mathrm{G}$

$1732 \mathrm{G}$

FIS 134

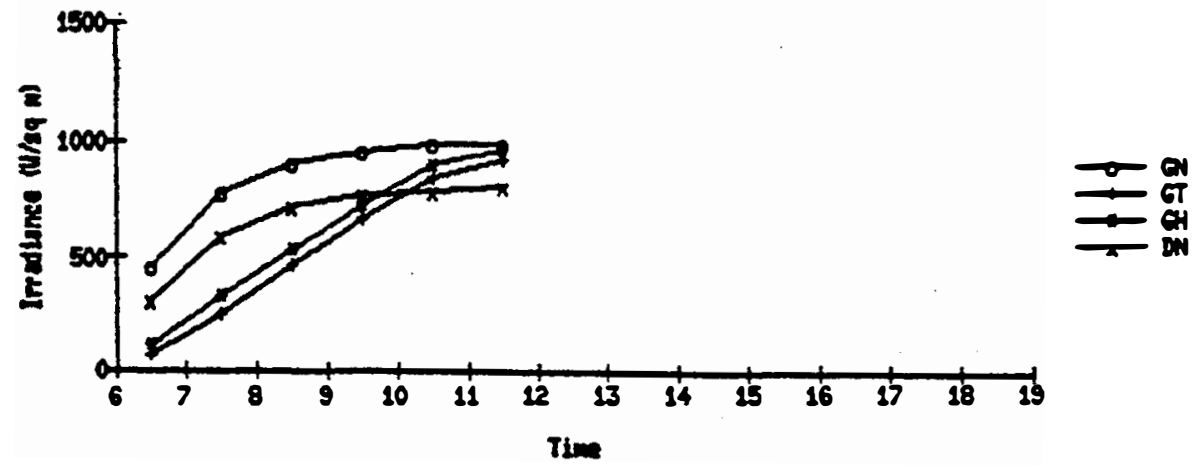

PSEC 234 04T

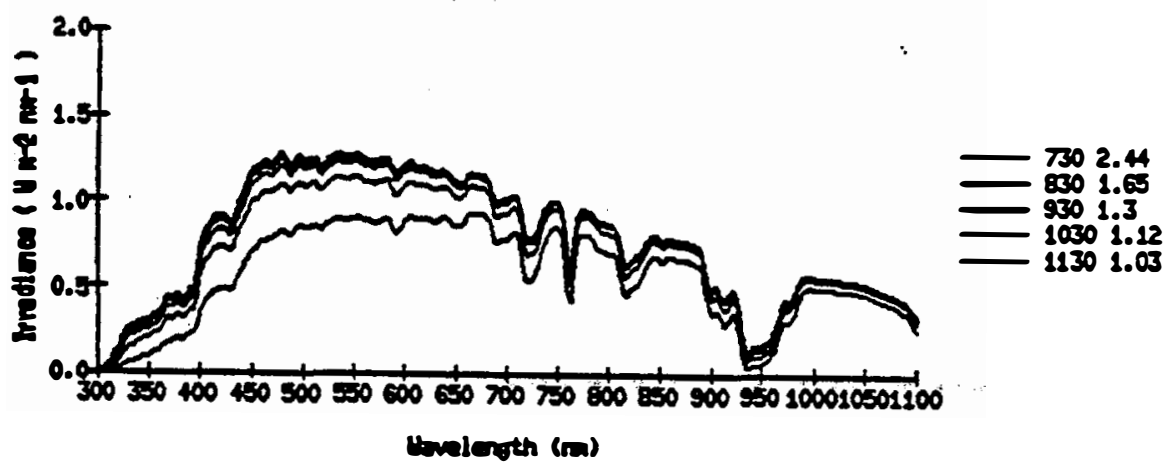

FEsC 234 OS

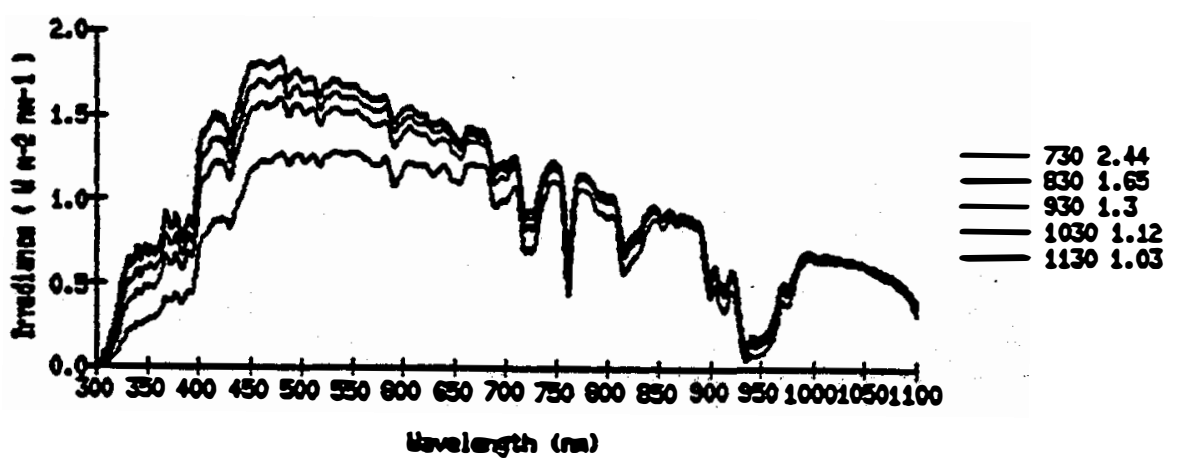


A

$\begin{array}{llllll}127 & 051587 & 135 & 172 & 02\end{array}$

0.7 cloud cover at 1230 ; 0.2 at 1515

Han rain in morms aren under abedo llooded

slides: $0631 \mathrm{G}$ (veter drops)

0732 G

$0832 \mathrm{G}$

$0931 \mathrm{G}-$

$1031 \mathrm{G}$

$1131 \mathrm{G}$

$1231 \mathrm{G}$

1331 G

1431 i (dark)

$1532 \mathrm{G}$

1632 G

$1732 \mathrm{G}$

क्या⿰㇒⿻二丨冂

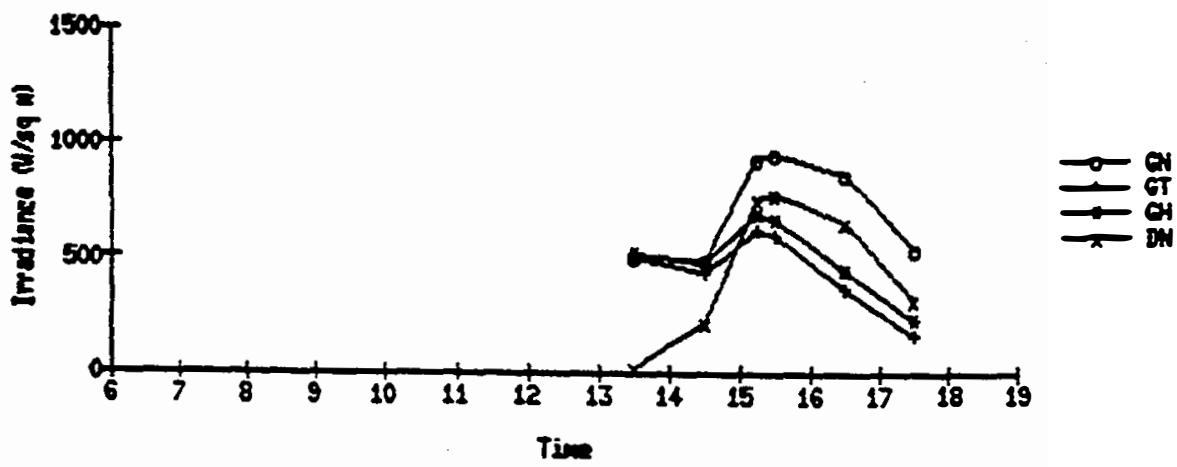

Fisx 15 and

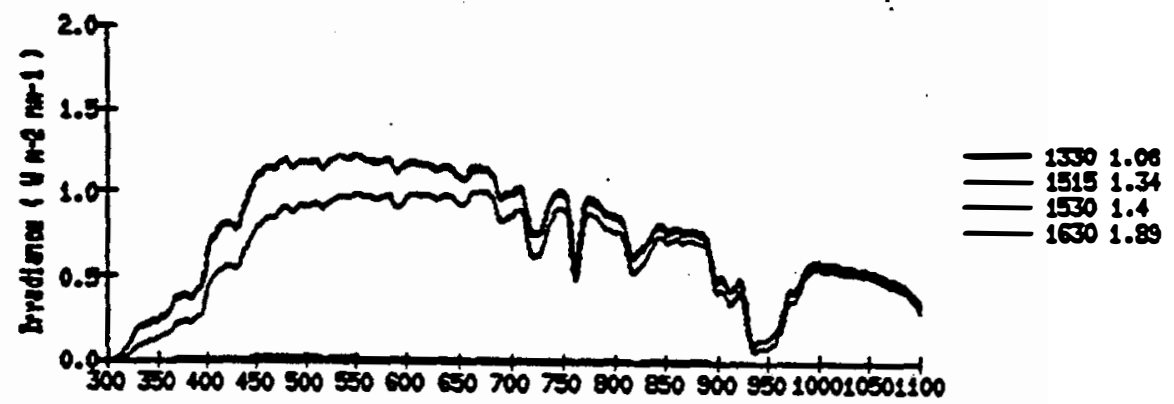

bovelength (ra)

PSEC 135 as

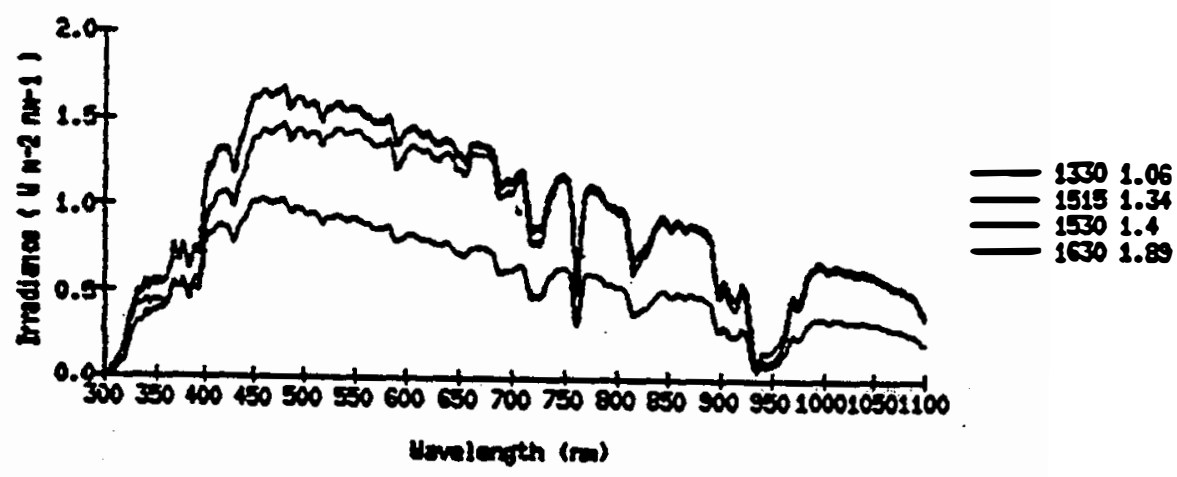


IST287136

$\begin{array}{llllllllll}128 & 05 & 16 & 87 & 136 & 172 & \text { DA } & 102 & \text { Cas }\end{array}$

Part15 clouds

Slides: $0631 \mathrm{G}$

$0731 G$

$0831 \mathrm{G}$

$\operatorname{senc} 275$

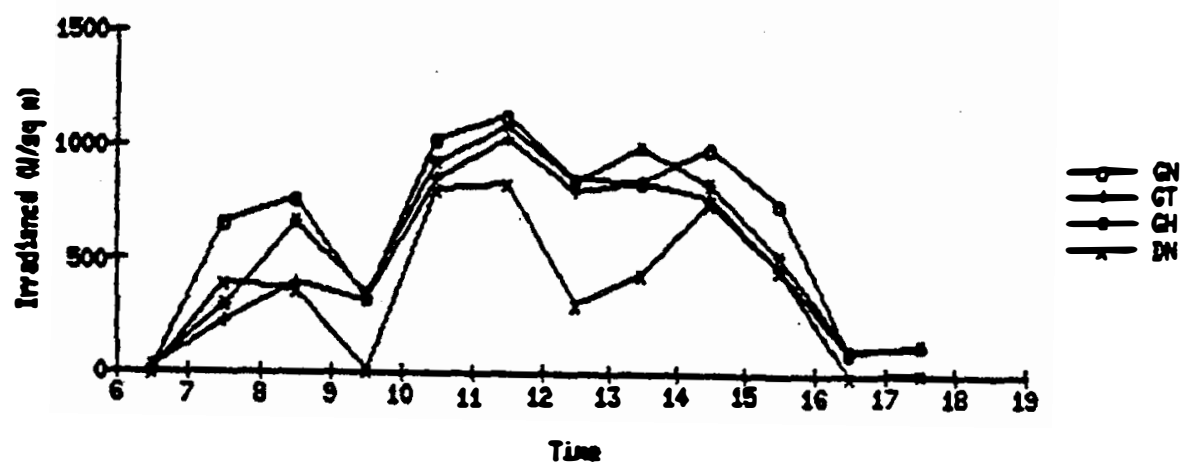

FEST $135 \mathrm{ANT}$

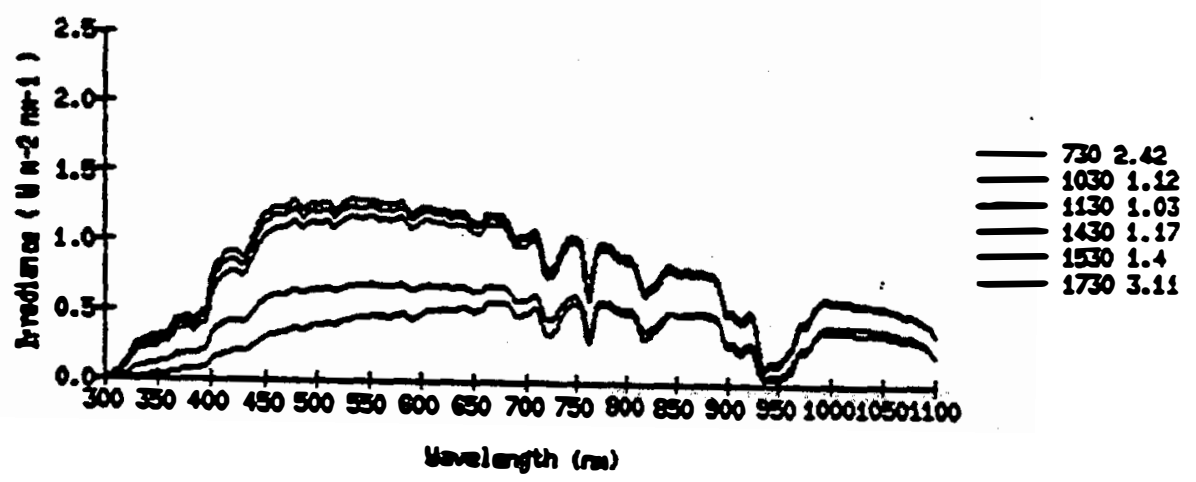

fise 235 as

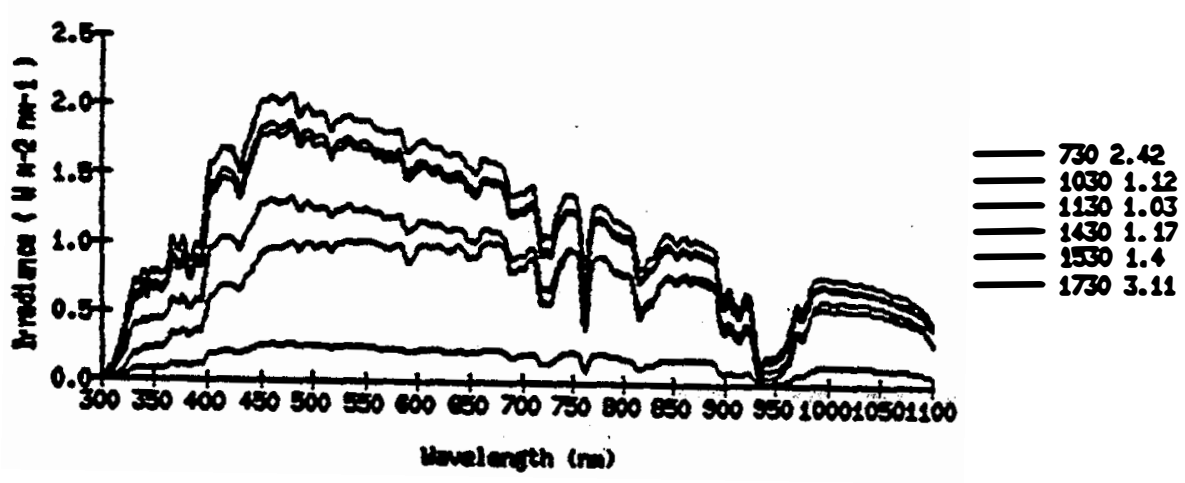


A 552887137

$\begin{array}{llllllll}129 & 05 & 17 & 87 & 137 & 172 & \text { DE } & 02\end{array}$

Partly cloudy

Ho slides

ussing dates in DA spectro at 1430

F्25 137

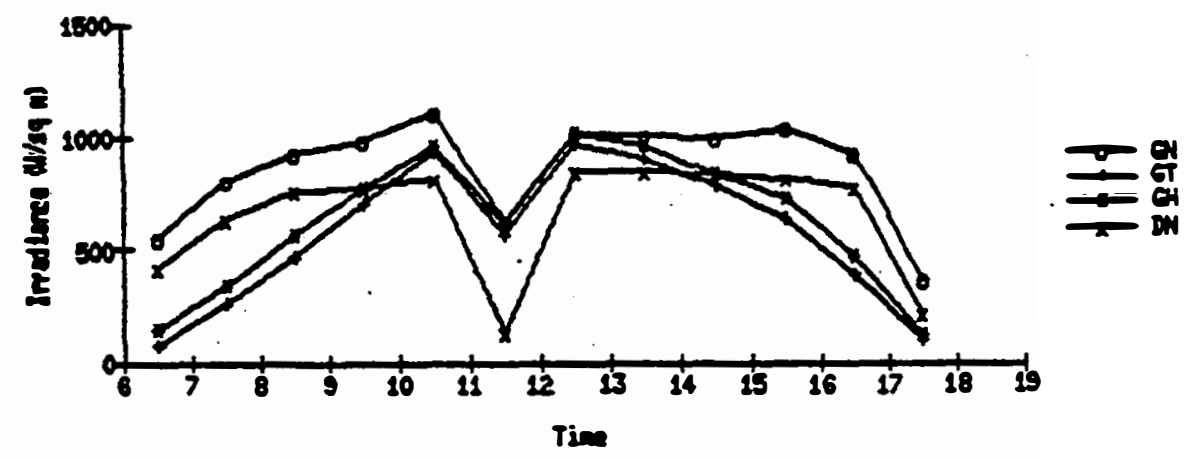

FSEC 137 ow

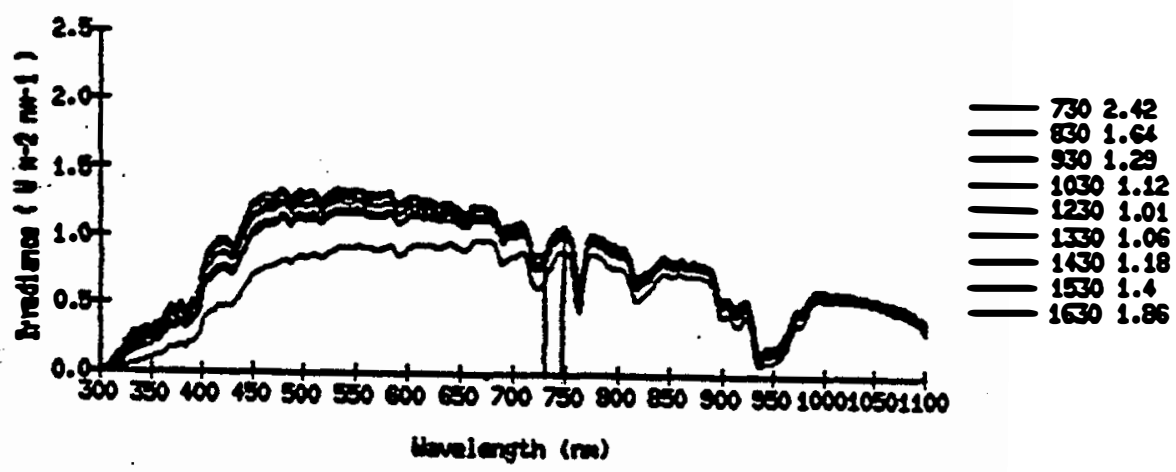

Fex 280 as

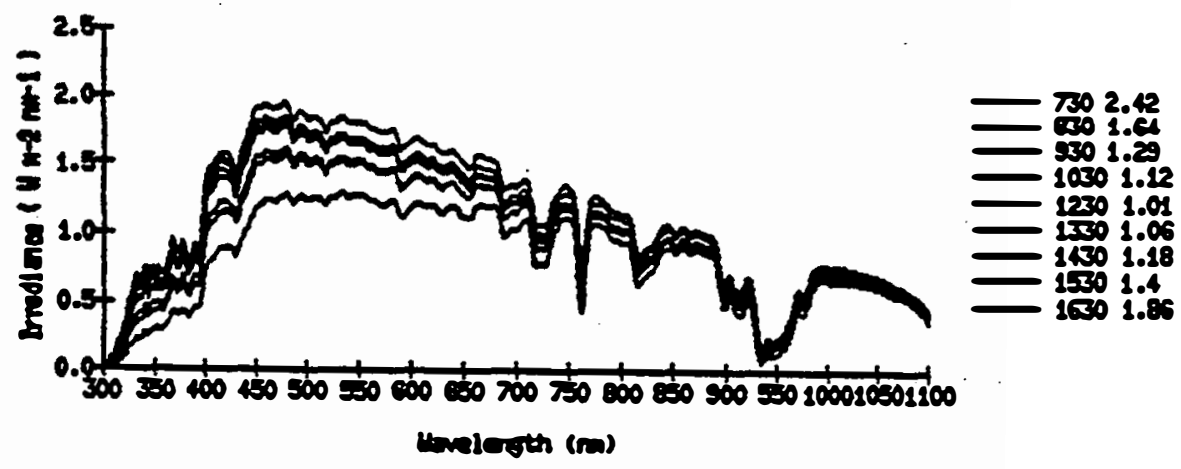


I. TSP 87138

$\begin{array}{lllllllll}130 & 05 & 18 & 87.138 & 172 & \mathrm{DA} & 102 & \mathrm{Gd}\end{array}$

1.0 cloud cover at 0730; 0.5 at $1200,0.8$ at 1500

ISO photos

FSEC 138

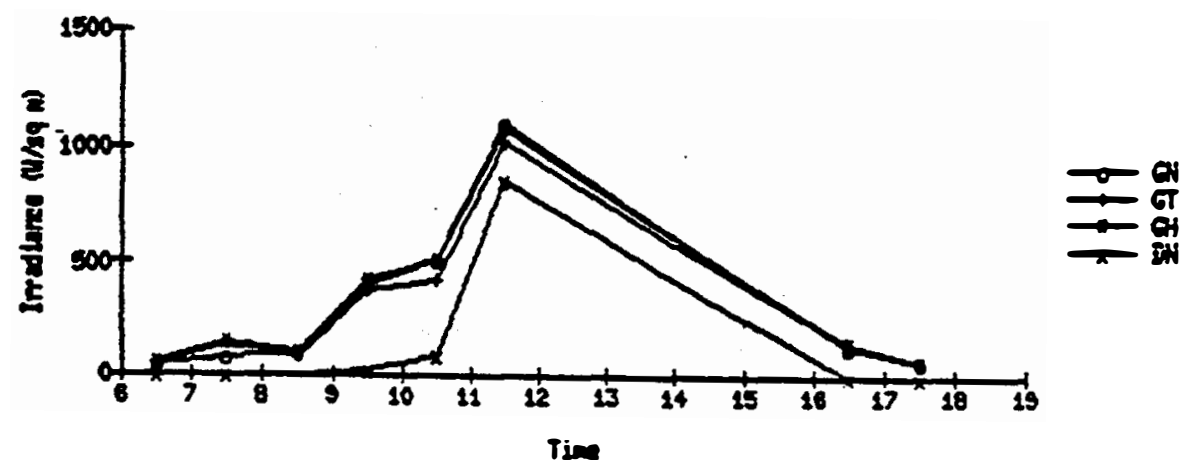

Fax 2018

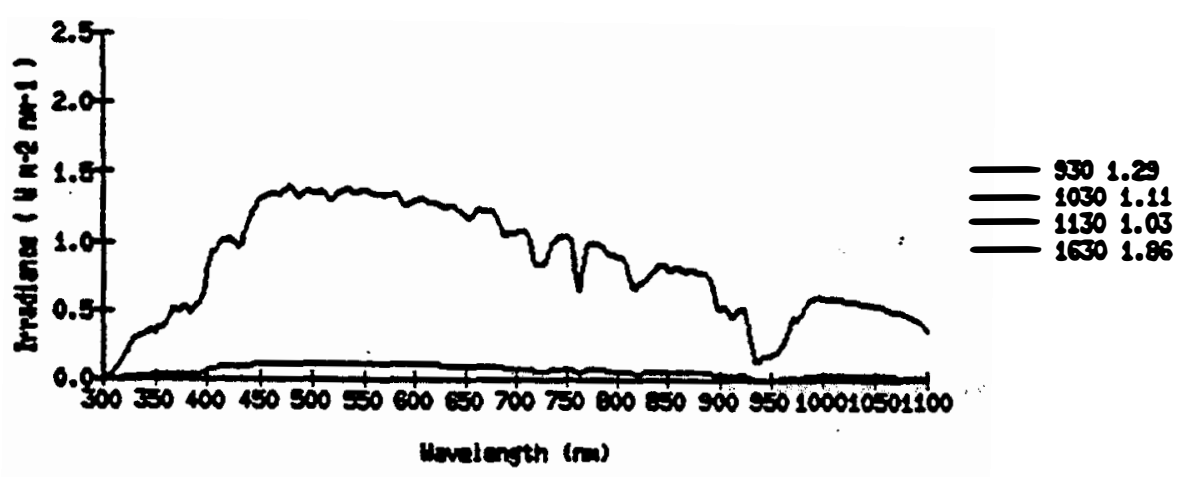

FISE 138 as

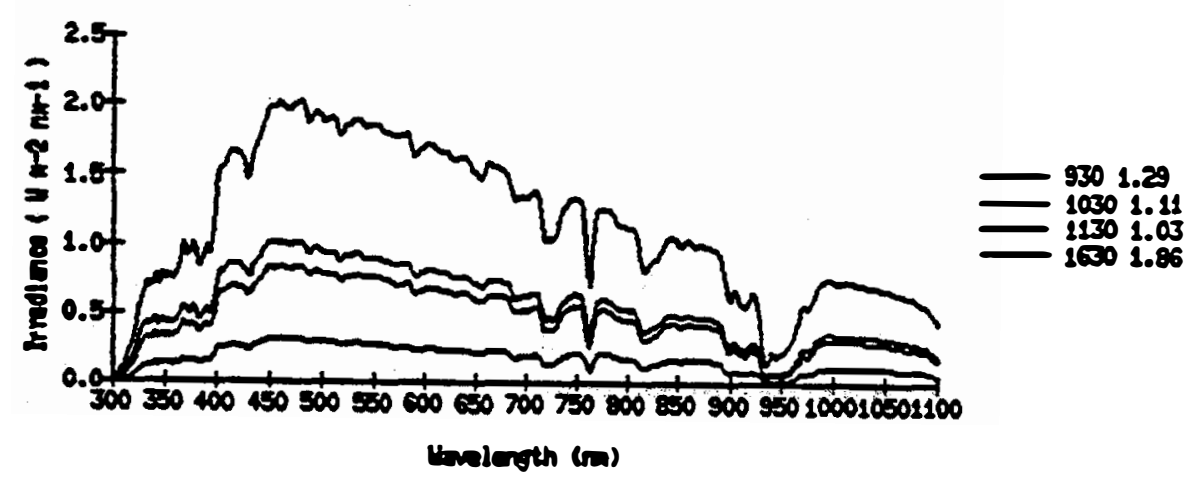


FST87148

$\begin{array}{lllll}131 & 05 \quad 88 & 848 & 102 & \mathrm{GH}\end{array}$

ISR 172 reooved for repair - lost neeors

Replaced SI pyranoneter: old = PYZ276, Cal 8.45 × 10-6 V/H-2

0.9 cloud coorer

Mo slides

Appenrs to be epike in spectru in UV at 2431

FSEC 148

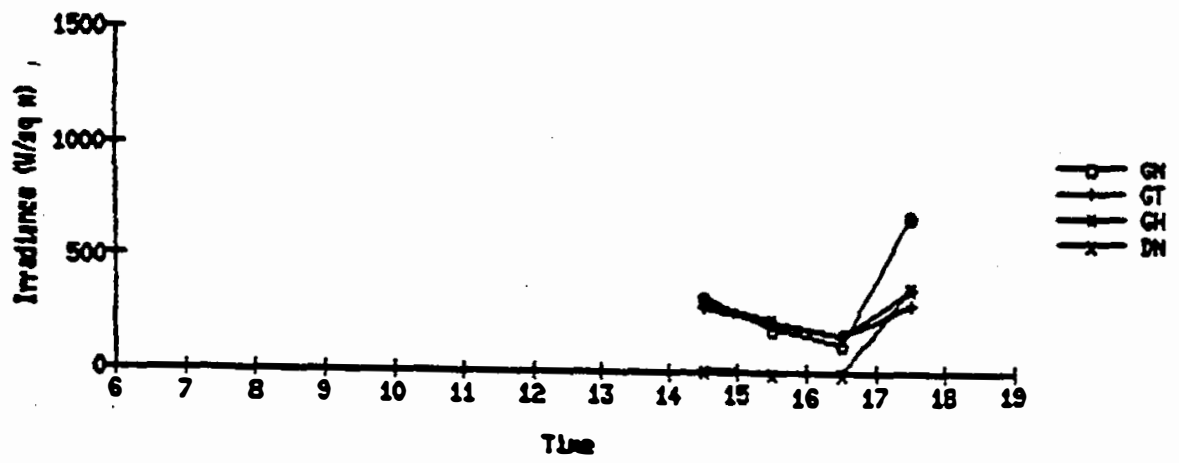

FSEC 149 as

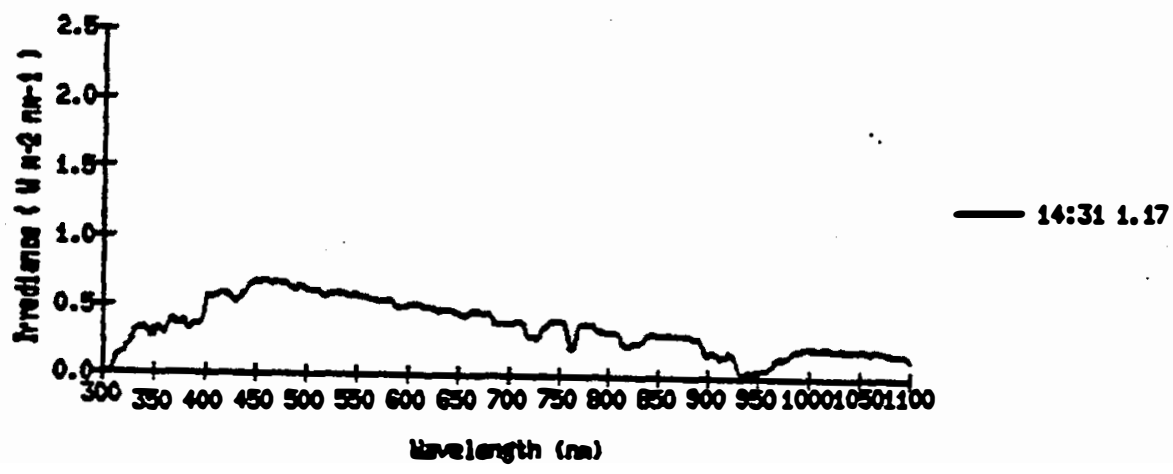


A PSTC87149

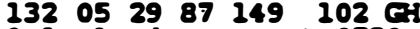

0.1 cloud cover at 0730 and $1230: 1.0$ at 1430

sildes: all blaet

spikes in spectra in UV at 0731, 0831, 0931, 1131, 1231, 1431

Fis 140

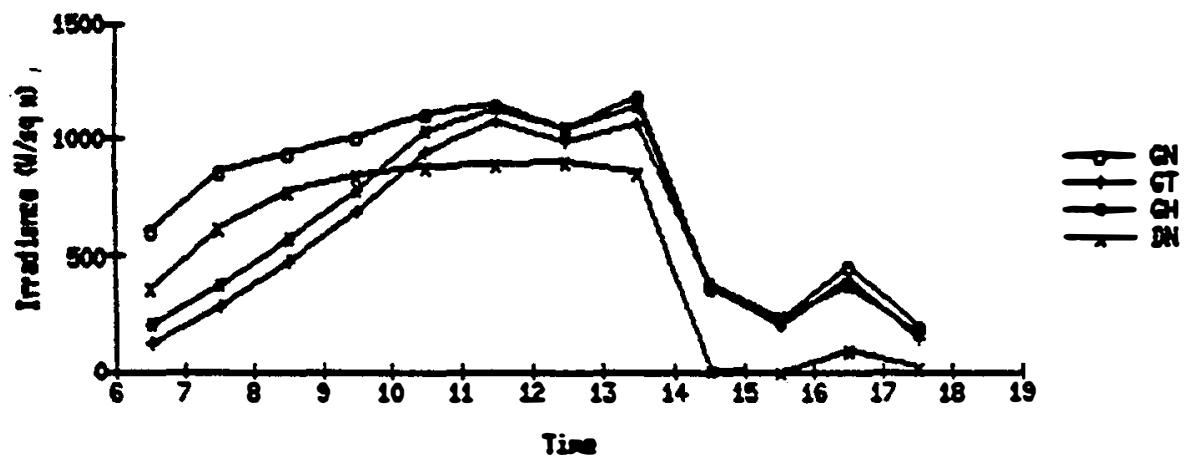

Fas 1900

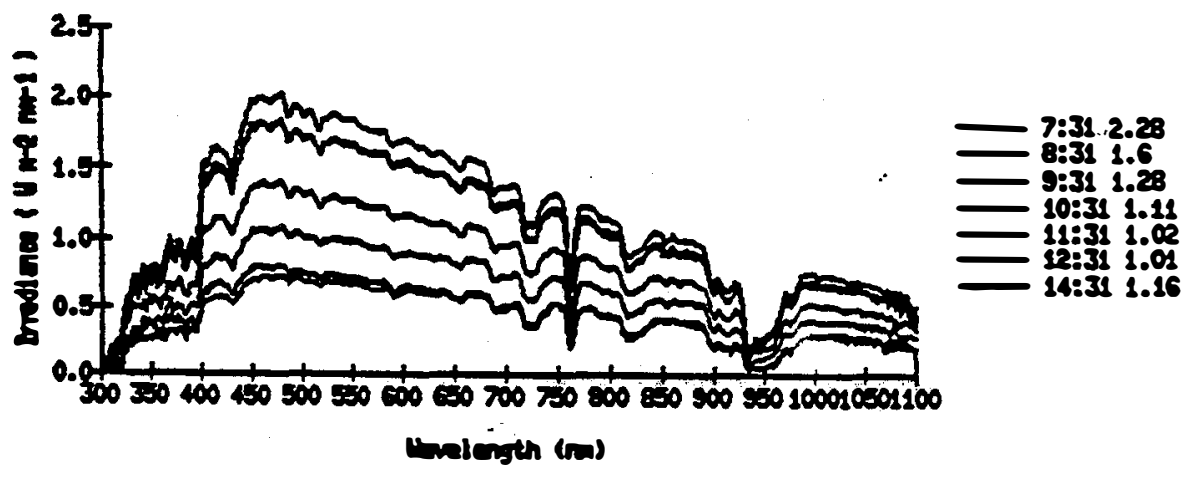


4. PSIDC87152

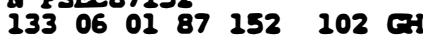

0.1 cloud cover at $0730 ; 0.2$ at 1130

Slides $\mathrm{Nl}$ blaek

FSEC 2LP

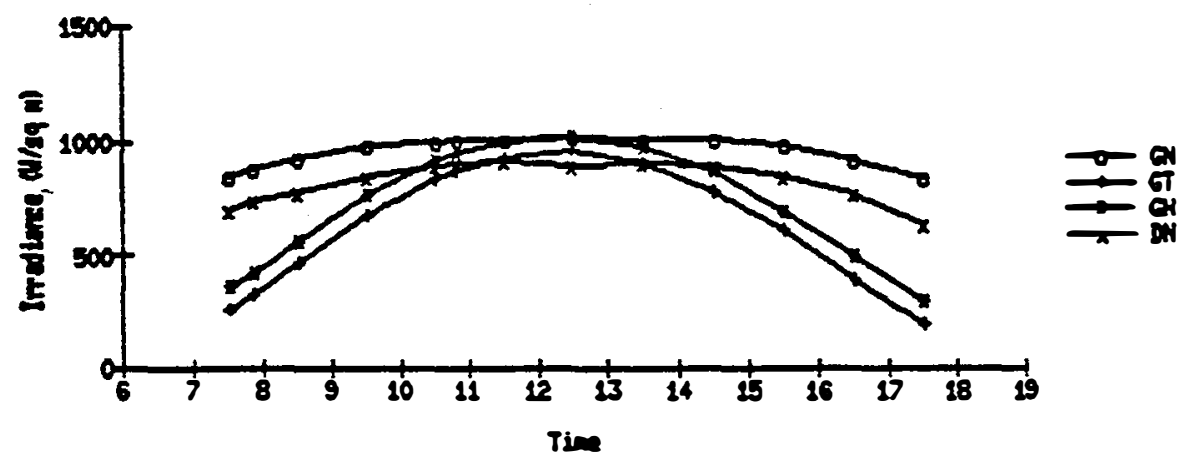

FSEC 250 ars

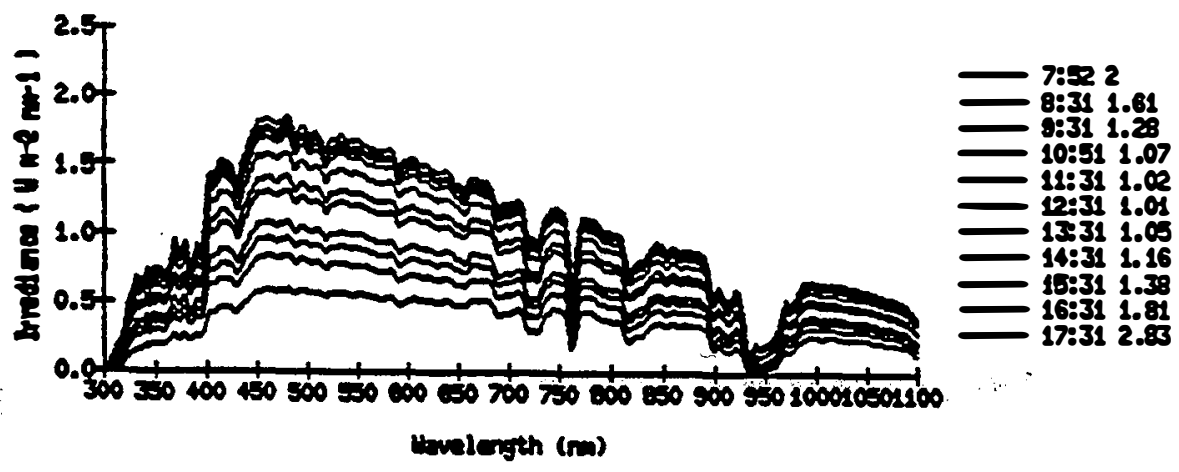


FST287153

$\begin{array}{lllllll}134 & 06 & 02 & 87 & 153 & 102 & \text { GH }\end{array}$

0.3 cloud cover at $0830 ; 0.2$ at 1200

slides: NIl black

FSEC 153

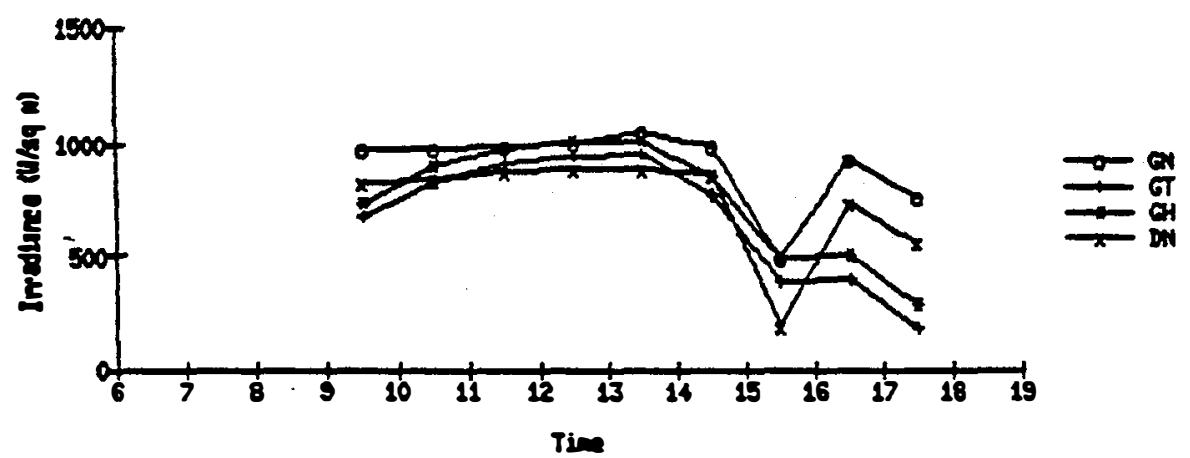

Fux 250 As

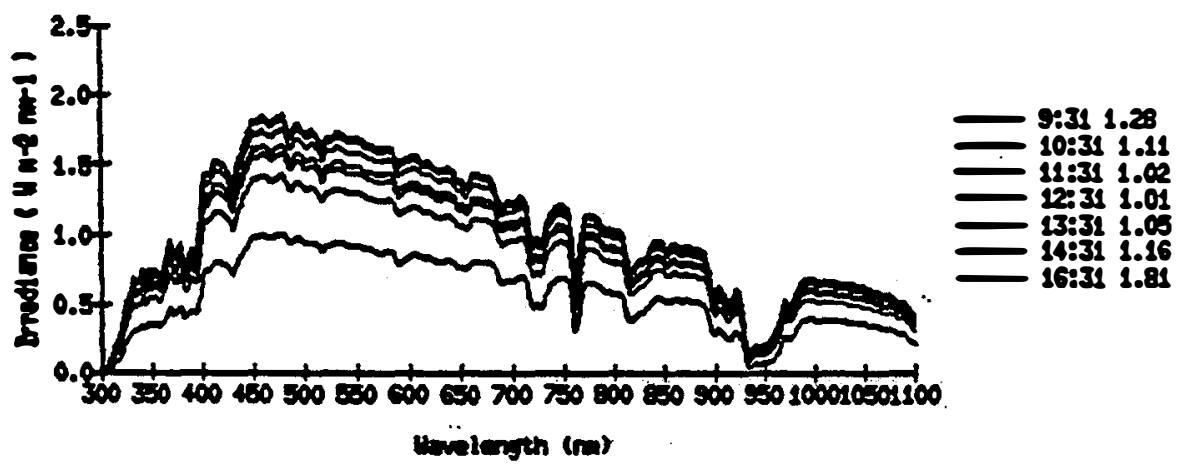


4 PST्287154

$\begin{array}{llllll}135 & 06 & 03 & 87 & 154 & 102 \\ \mathrm{FH}\end{array}$

0.4 cloud cover at 0700

sildes al black

FSEC 254

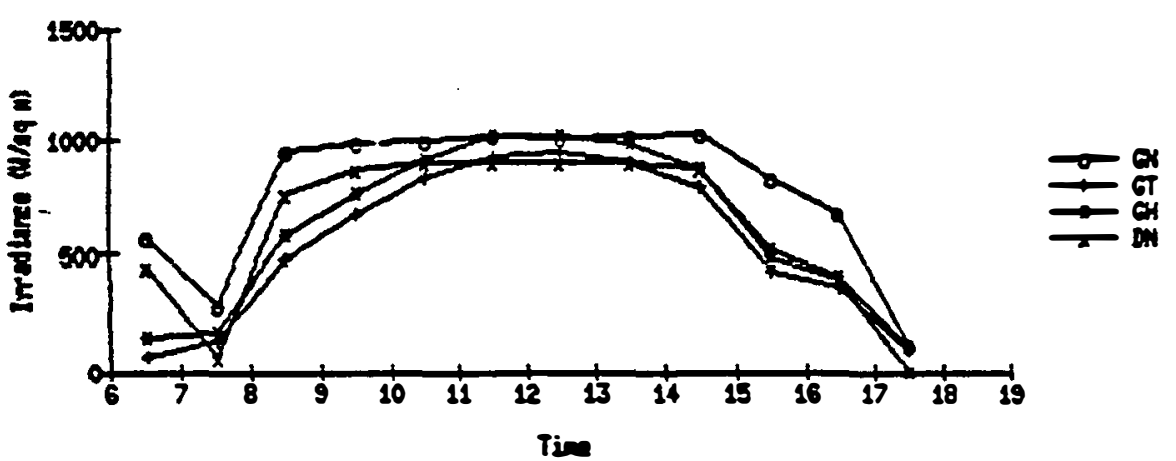

Fast ets ats

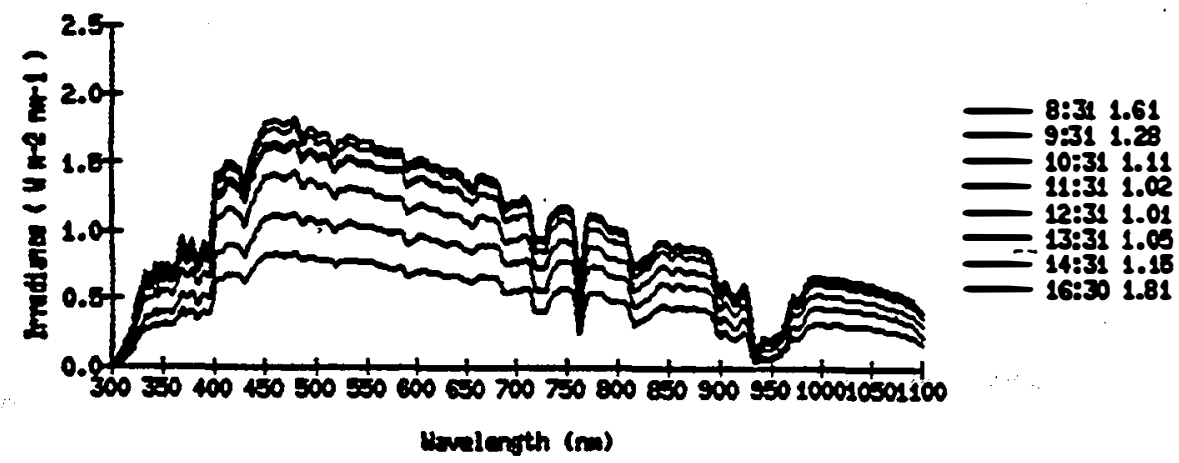




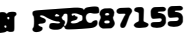

$136060487 \quad 155 \quad 102$ EH

ef in norning

0.5 cloud corer at 0730

sildes: Ali black

no spectra

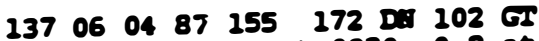

0.6 cloud cover at $0930 ; 0.2$ at $2130,0.5$ and hasy at 1600

sildes All black

FSE LS

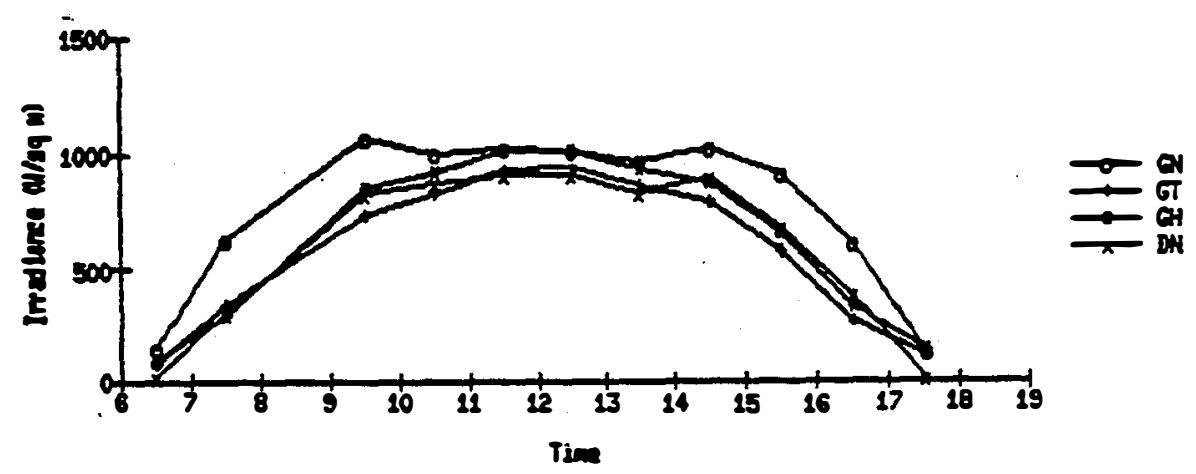

FSEC 150 an

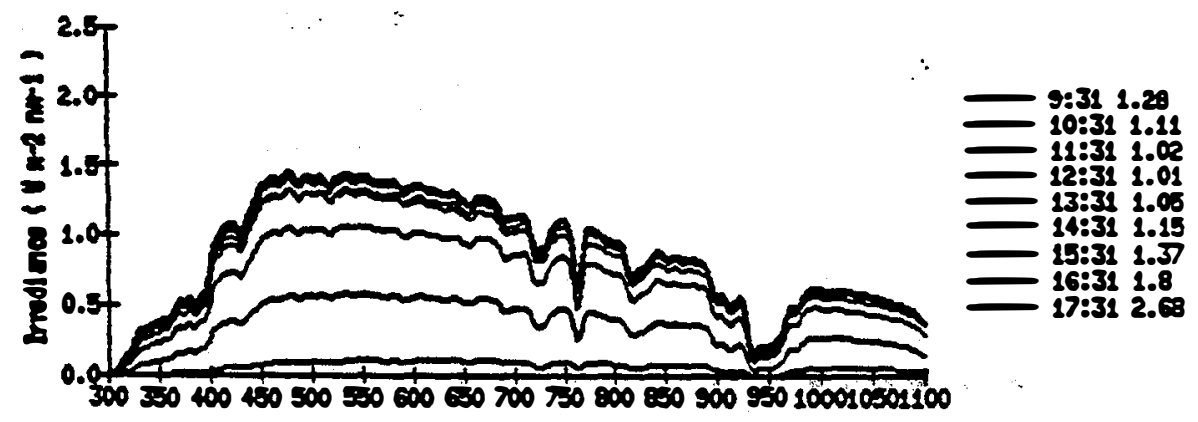

thelergth (m)

FSEC 250675

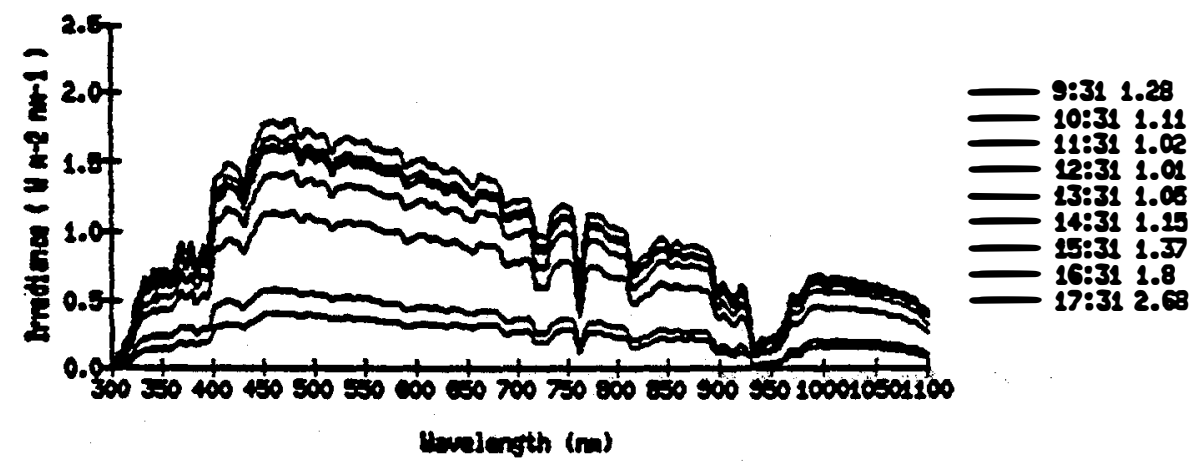


1.

$\begin{array}{llllll}138 & 06 & 0587 & 156 \quad 172 \text { DA } & 102 \mathrm{GT}\end{array}$

0.1 cloud corer at 0730 and 1400 (mazy)

Slides: All black

Dil before bad at 1401

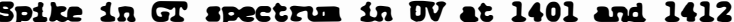

Spectral integral, $\sigma$ brandinnd at 0634

Fasc 185

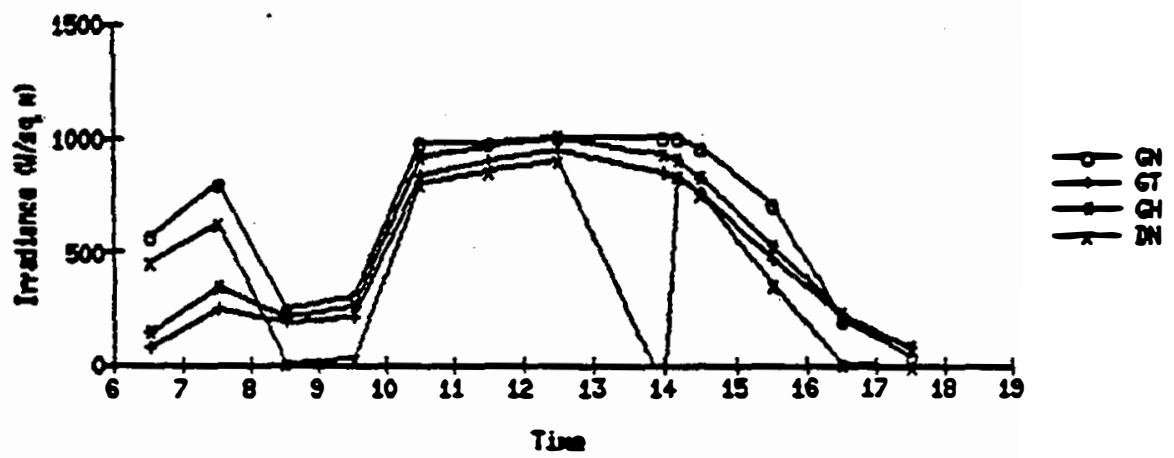

FSEC 156 BNT

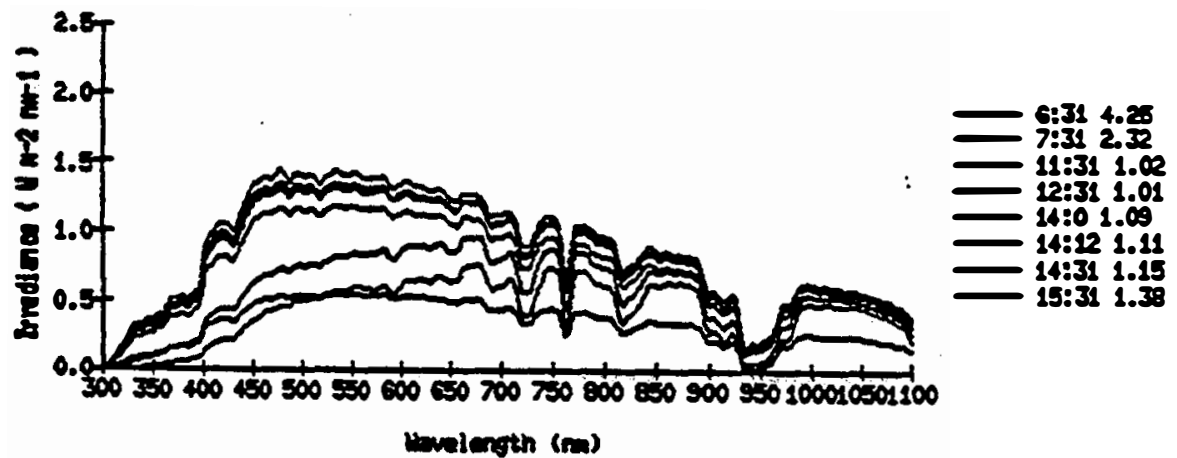

Prex IES GTS

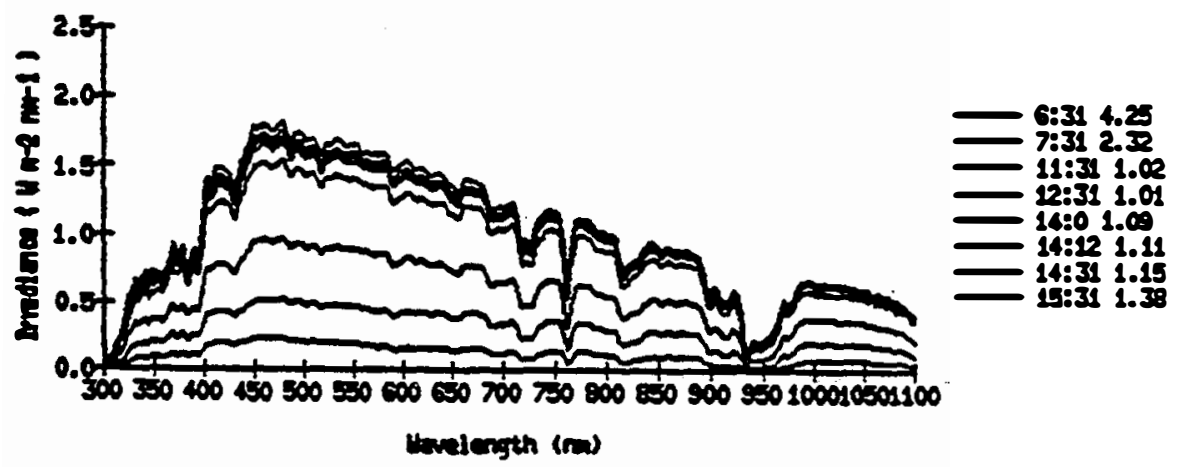


A 752887257

$\begin{array}{llllllll}139 & 06 & 06 & 87 & 157 & 172 & 0 . & 02\end{array}$

Partly clouds:

glsdes $\mathrm{Ml}$ black.

FEEC 157

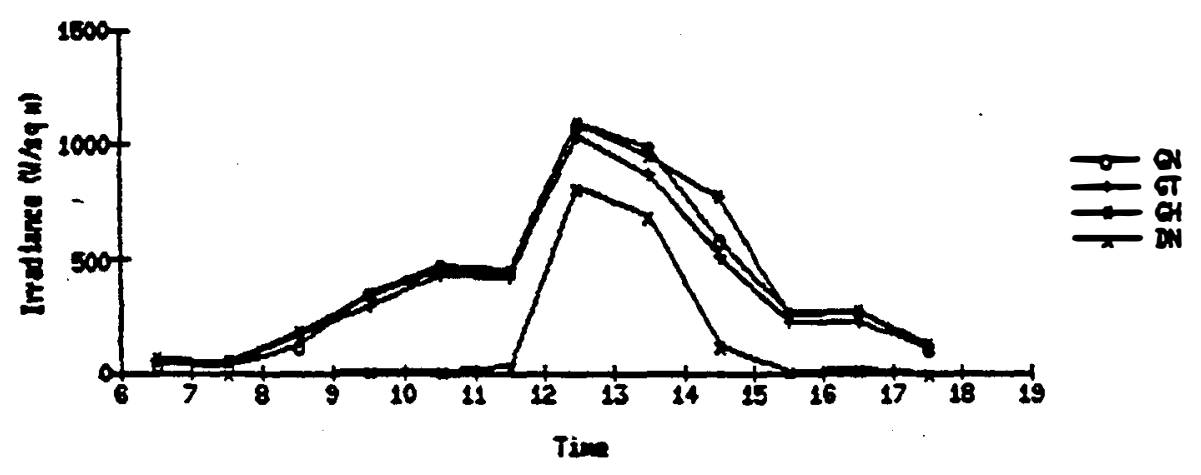

FEבC 257 ANT

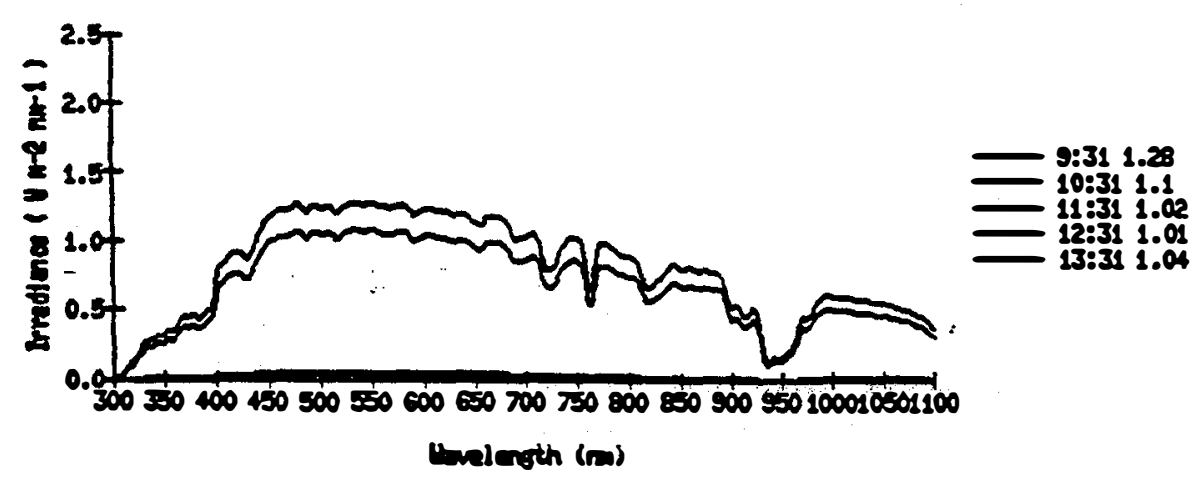

PSEC 25 GTS

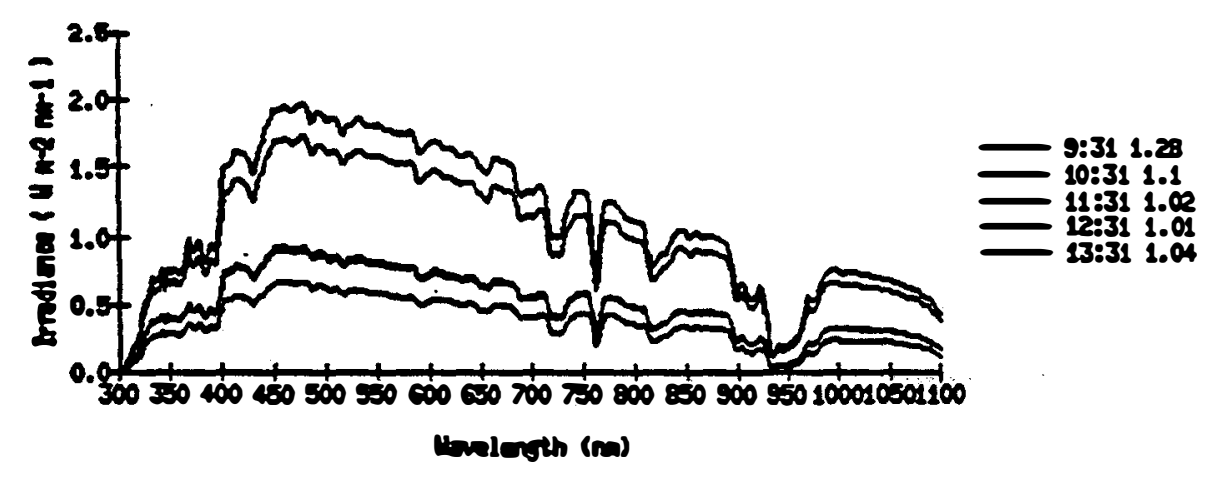


I FS1087158

$\begin{array}{lllllllll}140 & 06 & 07 & 87 & 158 & 172 & \text { DA } & 202 & \text { Gr }\end{array}$

Partly cloudy

Slides: Al black

FIST 258

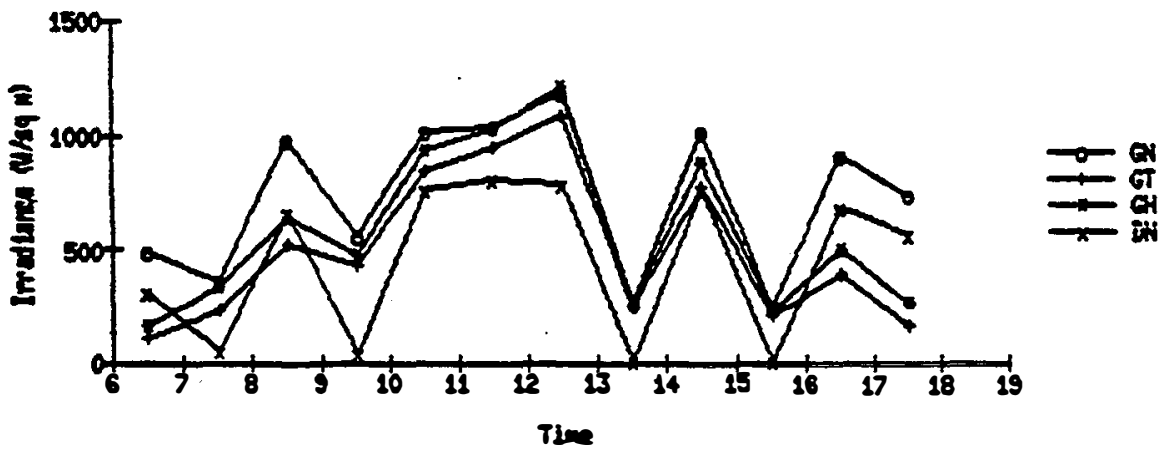

FSEC 258 DNT

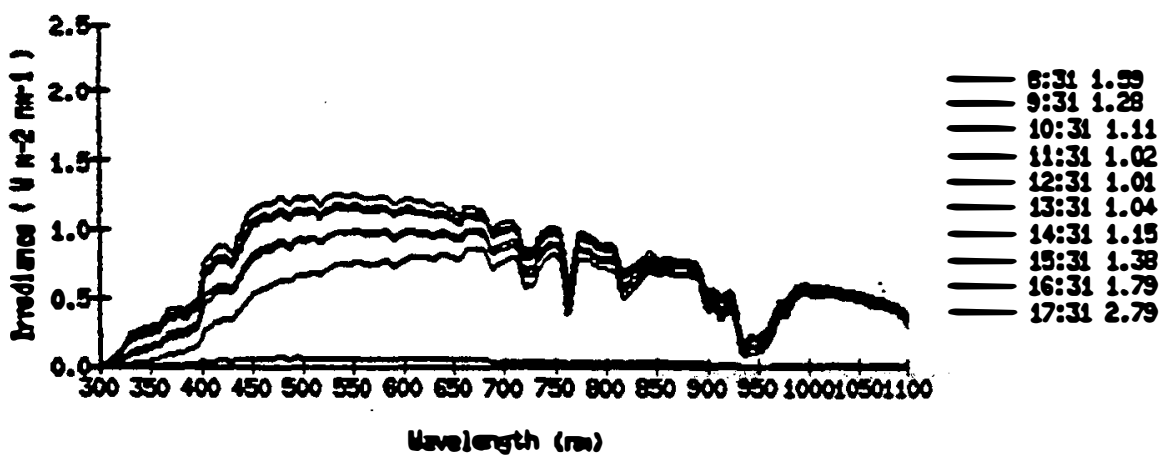

FSIC 258 GTS

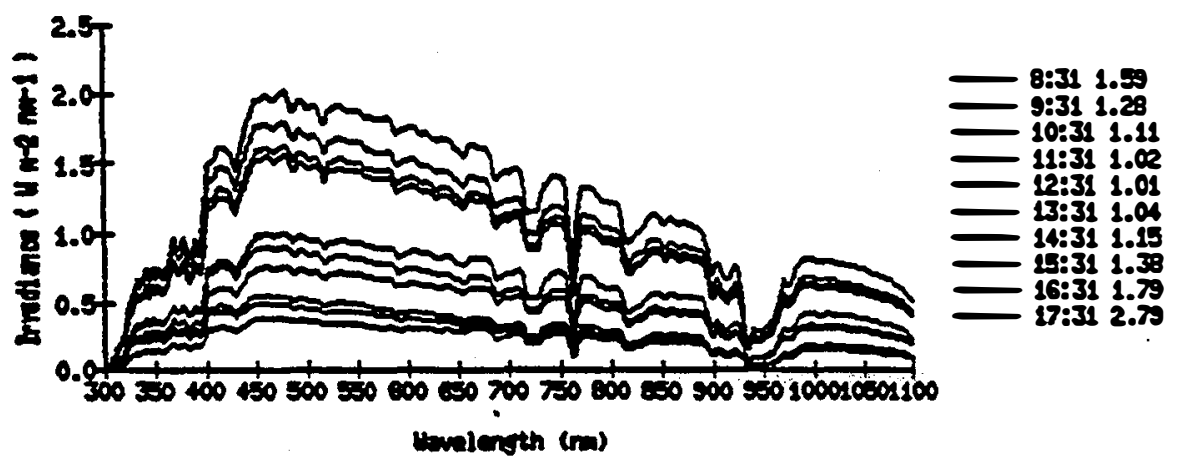


F5T287159

$\begin{array}{llllllllll}141 & 06 & 08 & 87 & 159 & 172 & 0 . & 102 & G 1\end{array}$

Clear at 0730; 0.2 cloud cover at 1030

slides: Al black

Did spectru at 1331 his an odd shape

$\operatorname{Fese} 150$

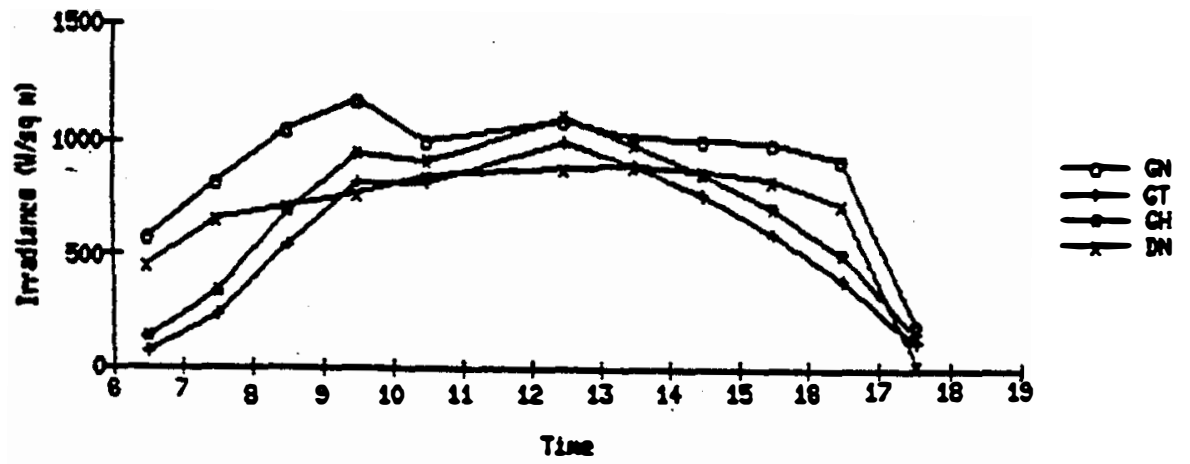

Fase 103 ivt

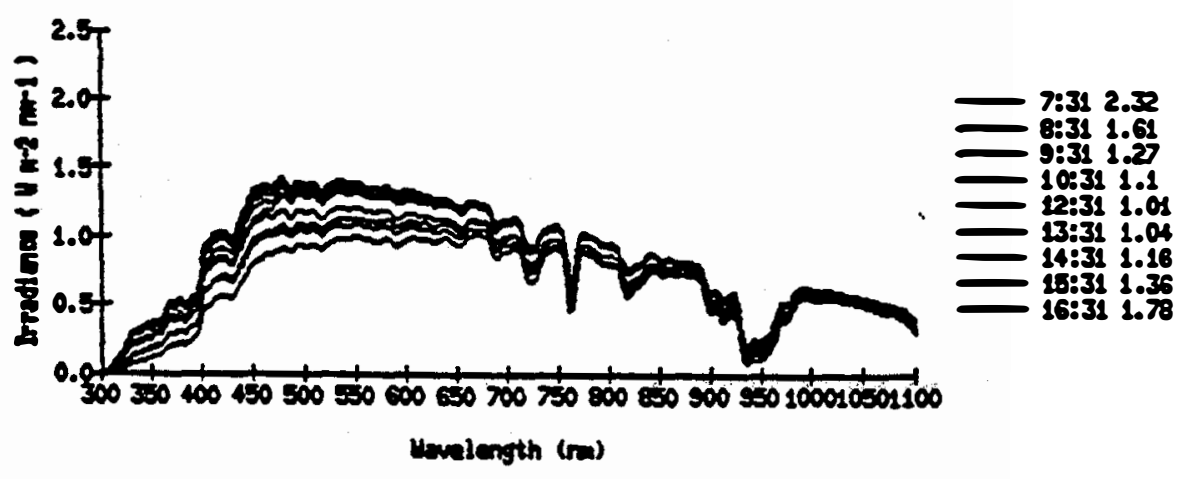

FSEC 150 GTS

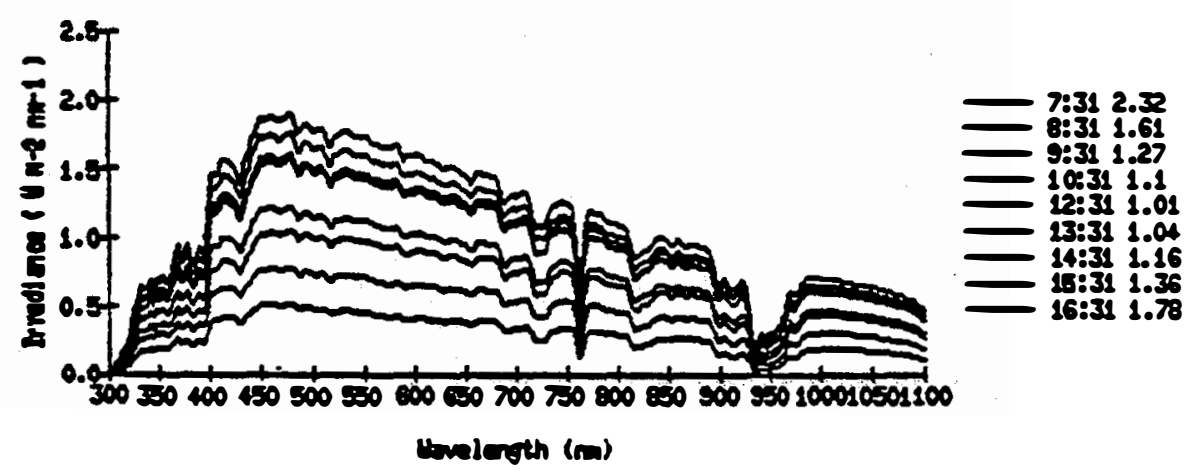




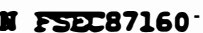

$\begin{array}{llllllllll}142 & 06 & 09 & 87 & 160 & 172 & 102 & \text { Fr }\end{array}$

0.3 cloud cooter at $0730 ; 0.2$ at 1600

ISR 172 lost nesors about 1300

Slides: All black

$\operatorname{cosec} 100$

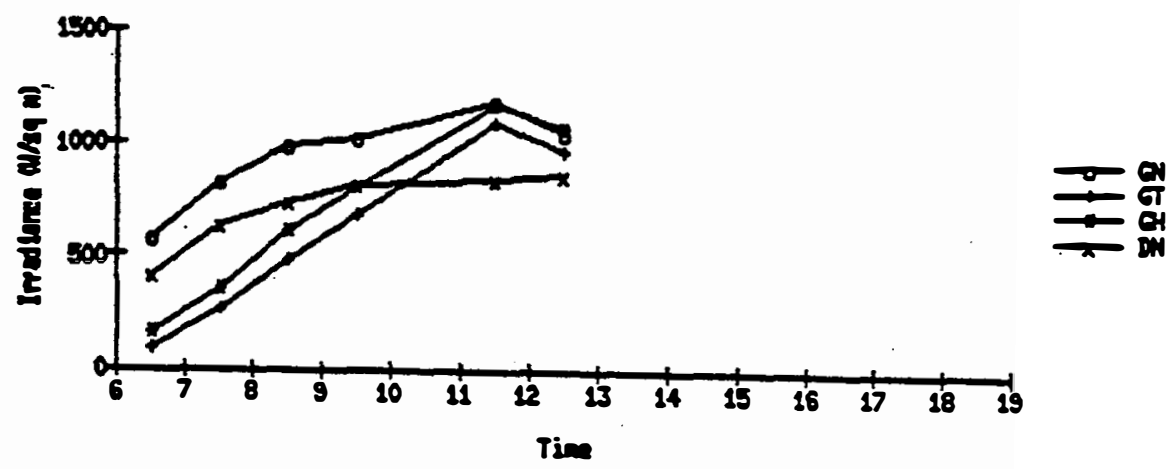

PSEC 200 Dit

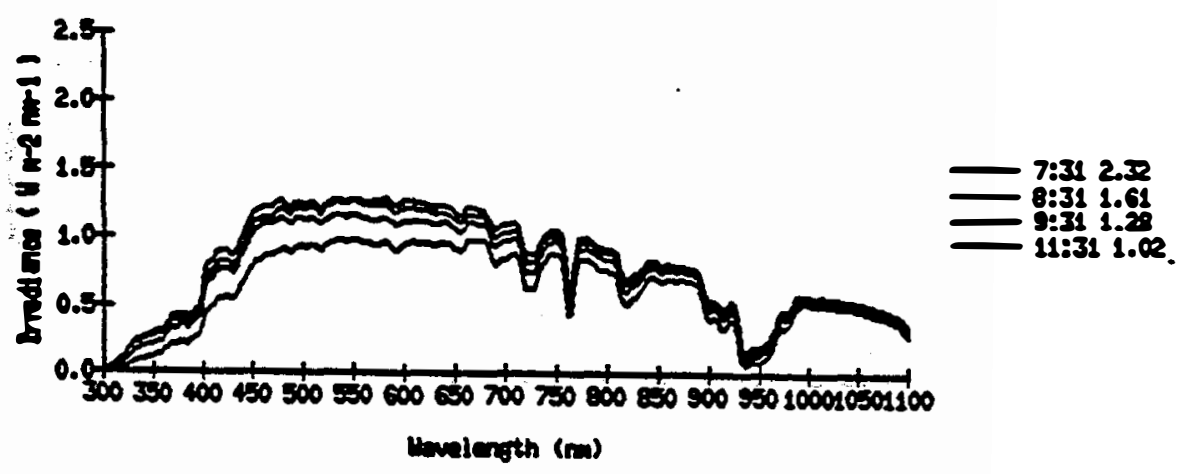

FEse 100 TIS

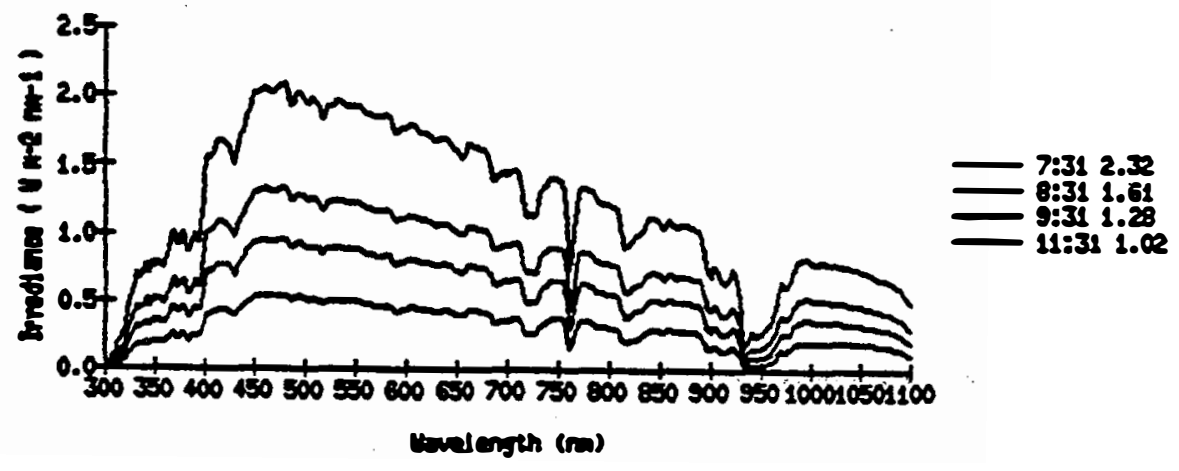


I FS2087161

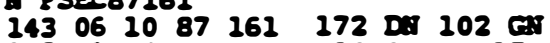

0.2 cloud cover at 1000 and 1500

ISR 172 lost nenory for second tine; reloaded cal files

Slides: Al black

FEC 161

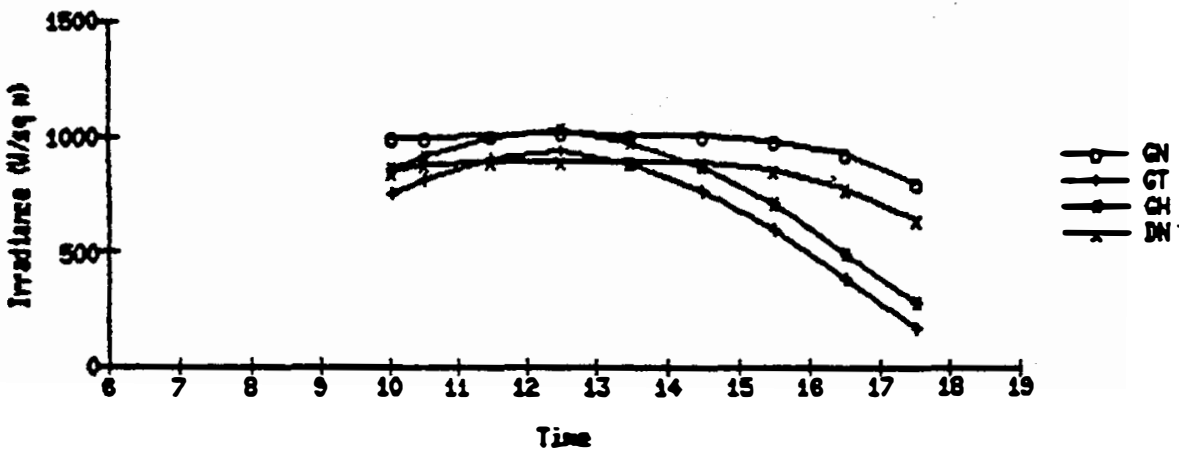

FISC 161 DNT

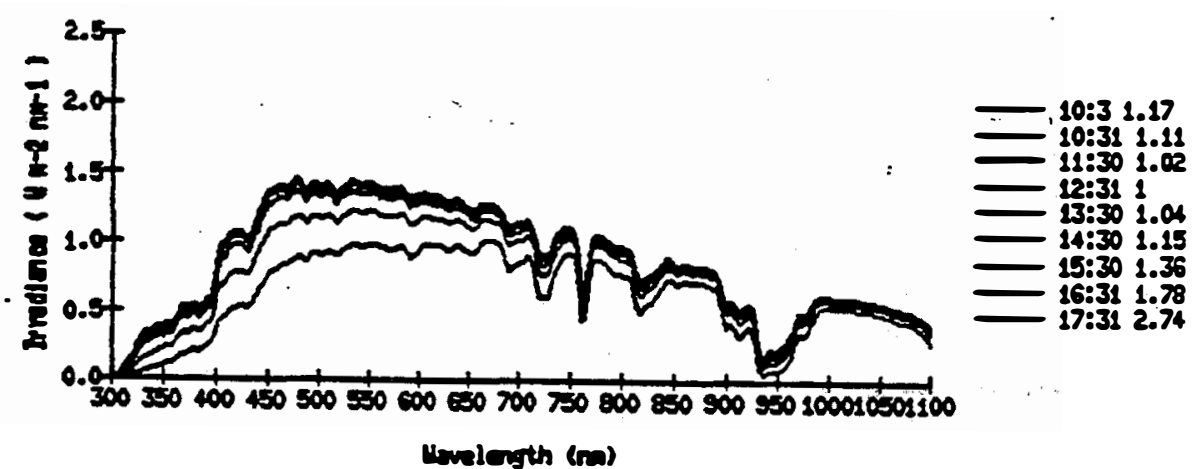

Fis 201 as

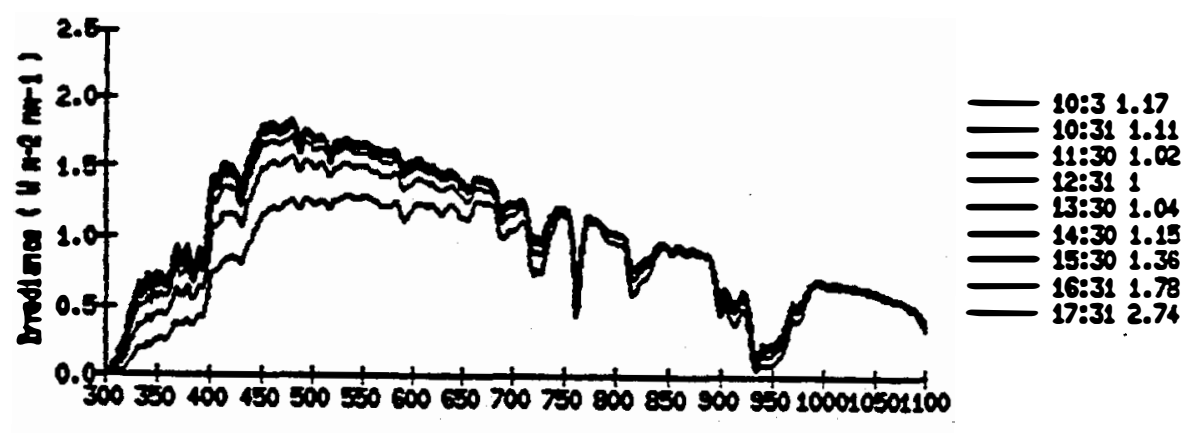

Hevilength (ros) 
4. T52887162

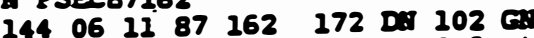

0.4 cloud cover at 0730; 0.9 at 1130

sildes: Al black

$\operatorname{Fsec} 10$

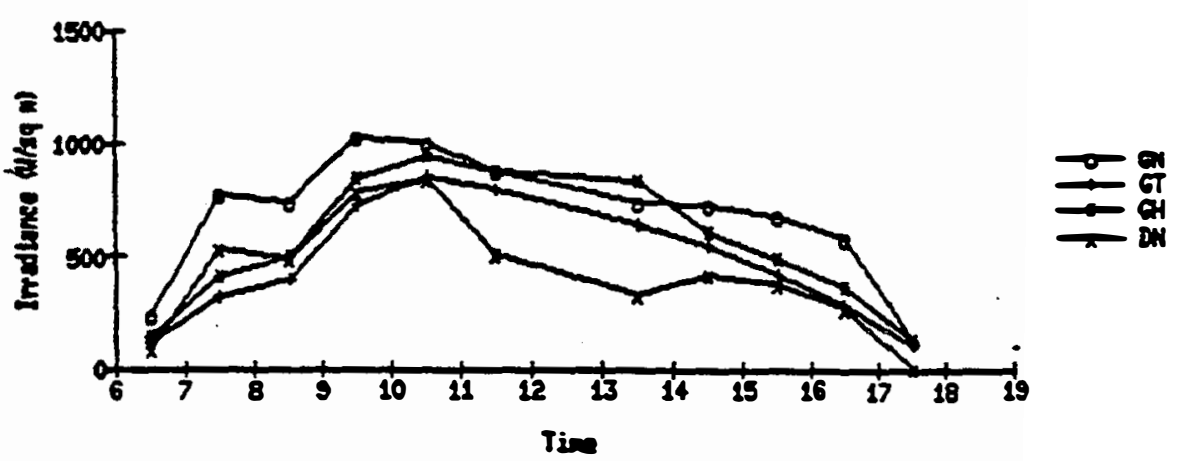

FES 102 AT

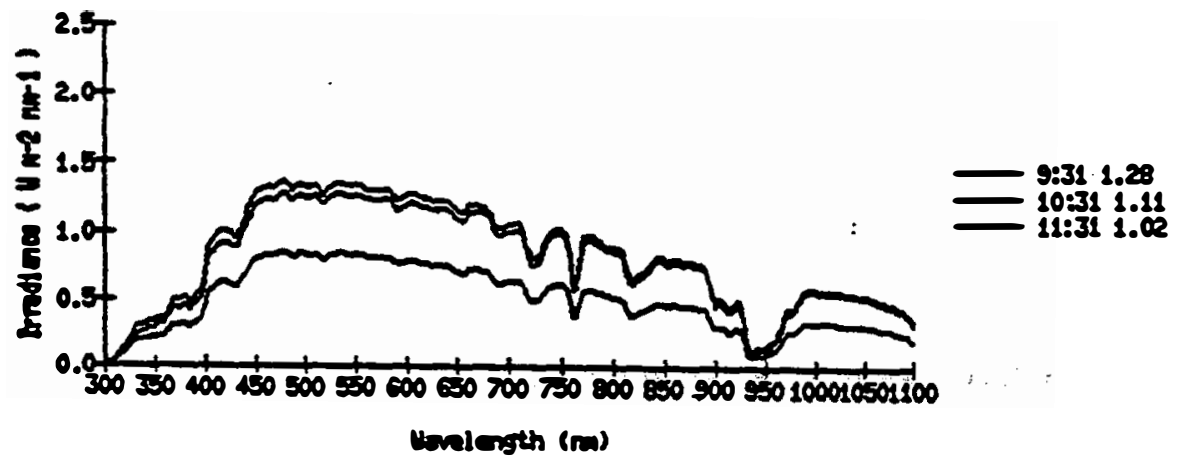

FEב 102 OS

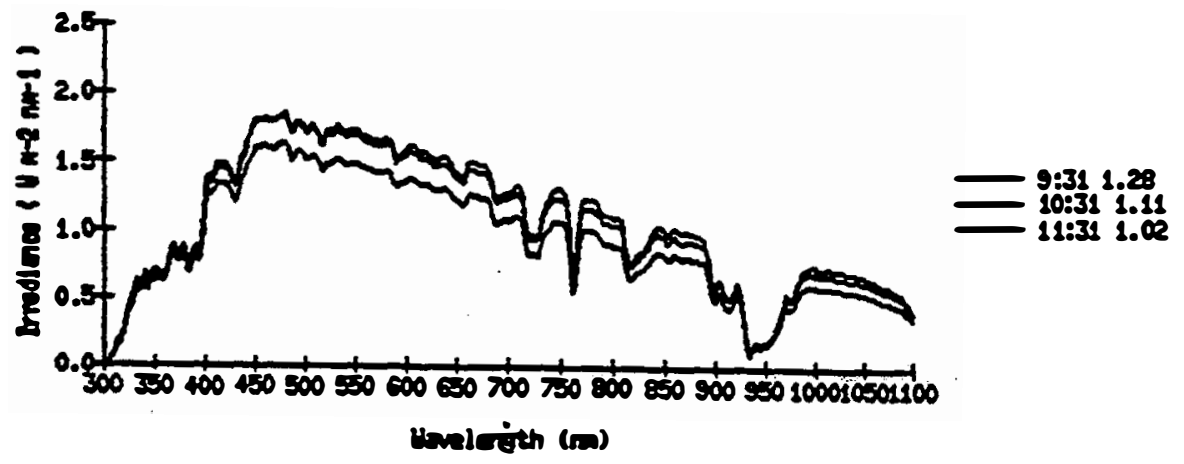


852087163

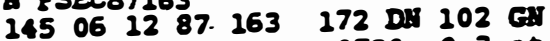

0.9 cloud cover at 0730; 0.3 at $1030 ; 0.2$ at 1430

sildes: All black

PSEC 103

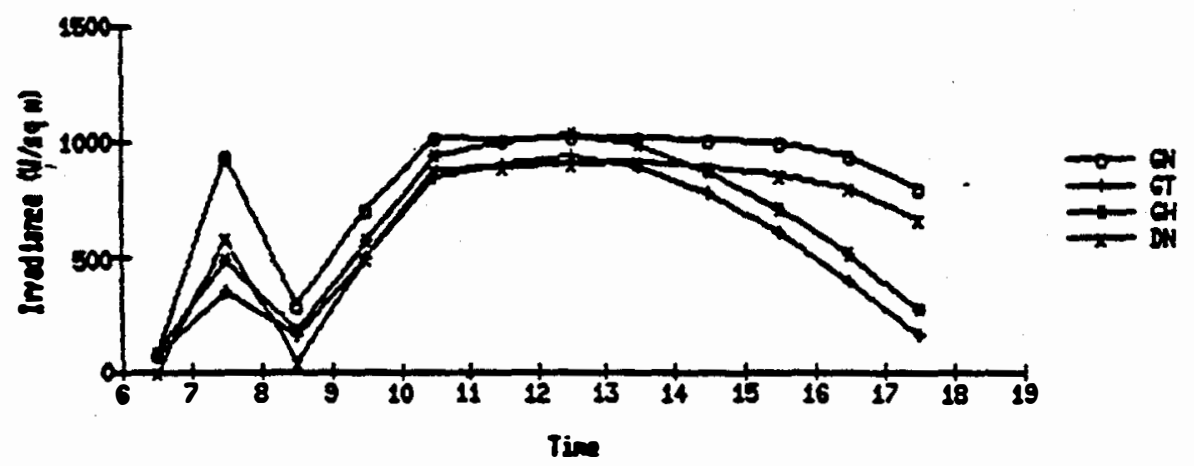

FES 183 ENT

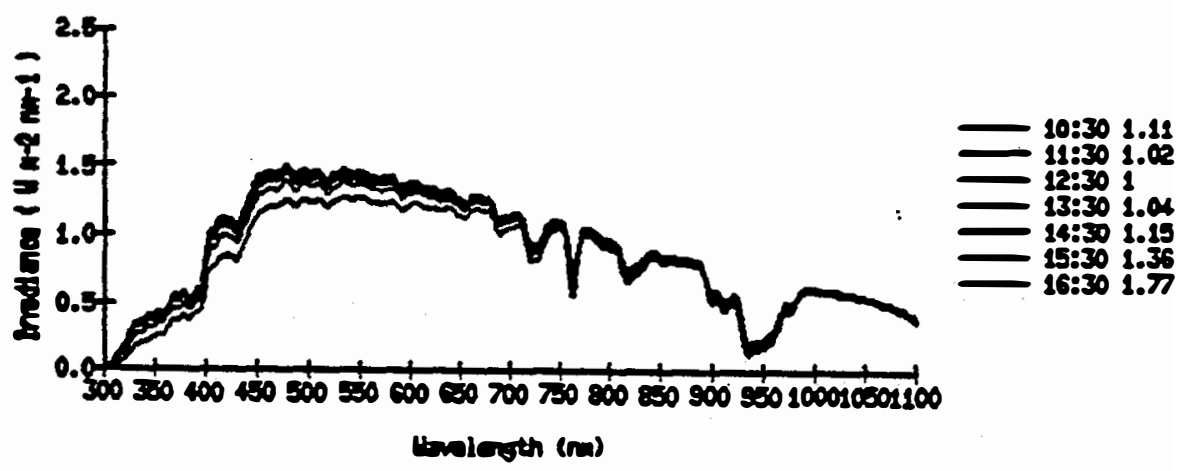

Fax 183 os

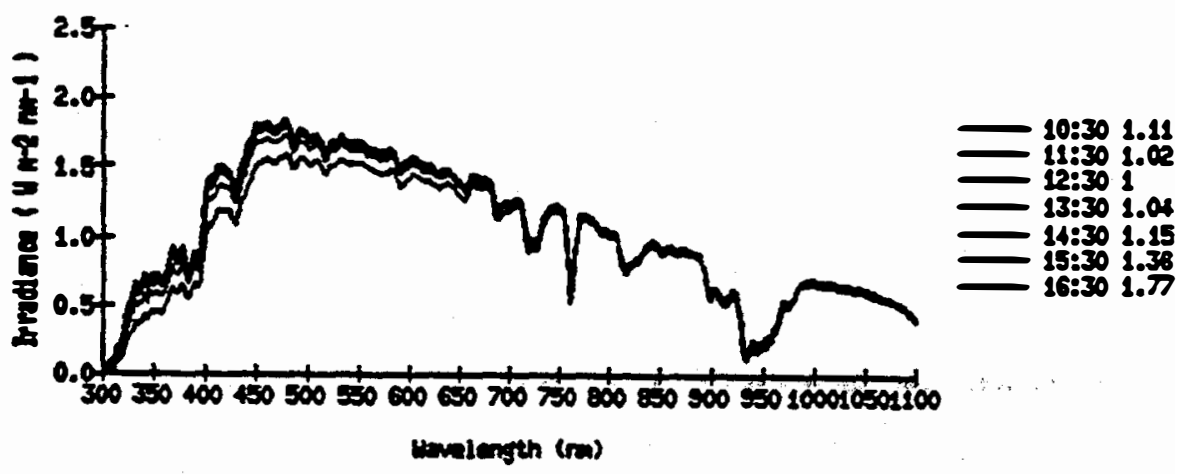


1. एक्ड887164

$\begin{array}{llllllllll}146 & 06 & 13 & 87 & 164 & 172 & \mathrm{IN} & 102 & \mathrm{FH}\end{array}$

Partly eloudy

S11des: 0631 G

$0731 \mathrm{G}$

0831 if (dnsk)

$0931 K$

$1031 \mathrm{~B}$

$1131 \mathrm{P}$

$1231 P$

$1331 \mathrm{P}$

$1432 P$

$1532 \mathrm{M}$

$1632 G$

$1731 \mathrm{G}$

$1832 \mathrm{G}$

Fesc 164

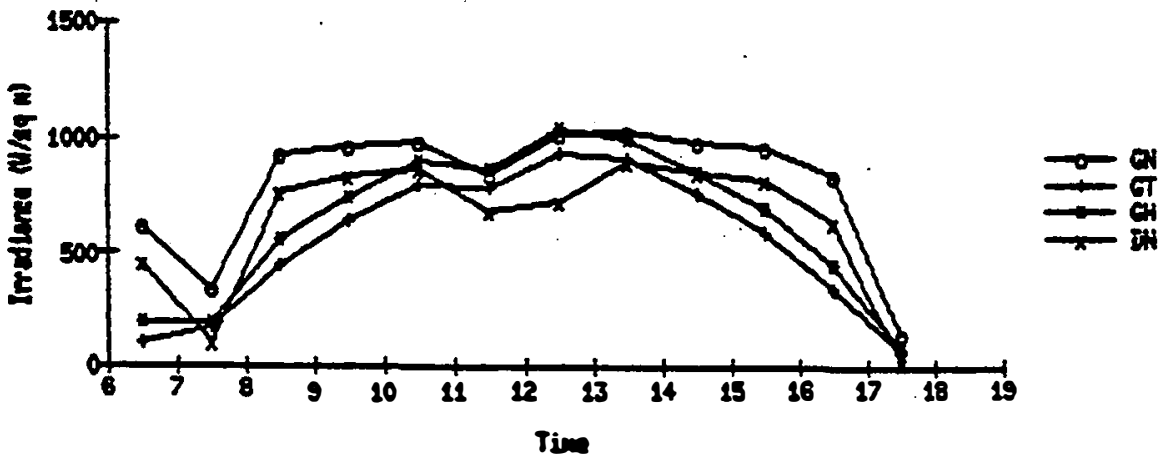

FEC 1C4 INT

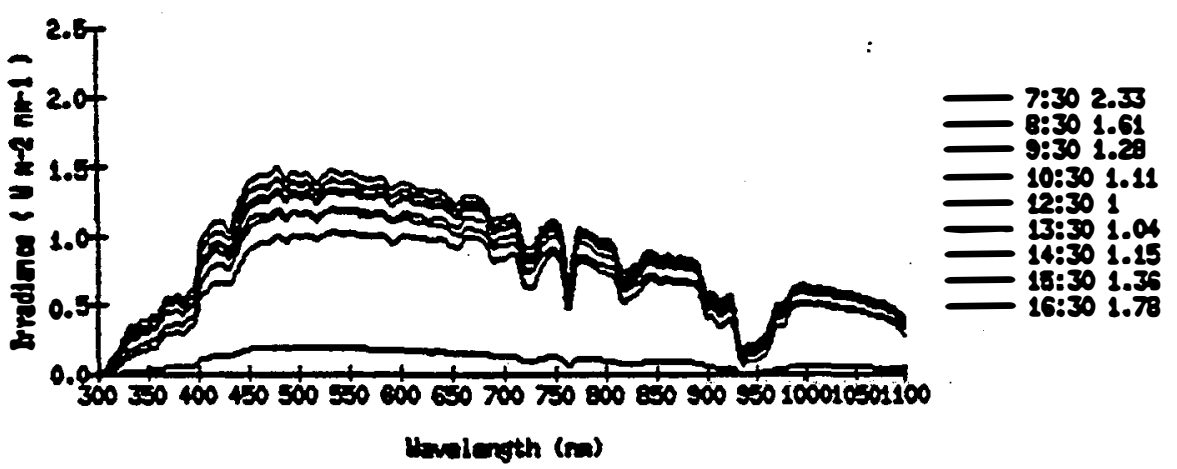

PIIS les ers

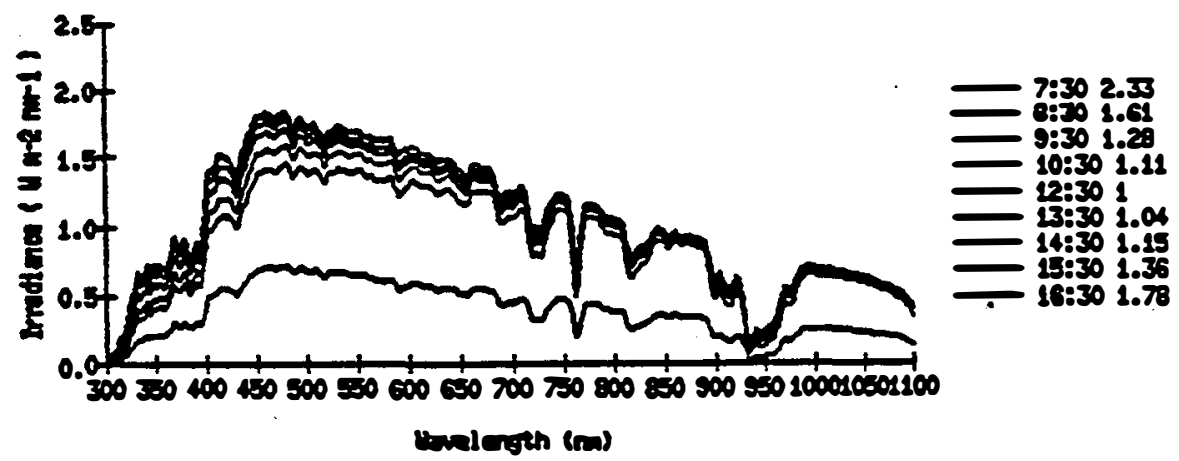




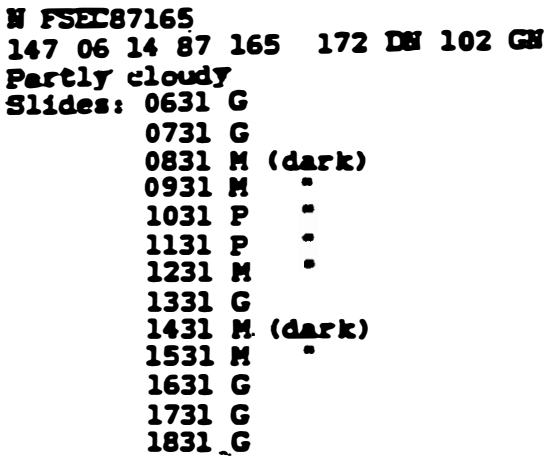

$1631 G$

$1731 \mathrm{G}$

$1832 \mathrm{G}$

Fac 105

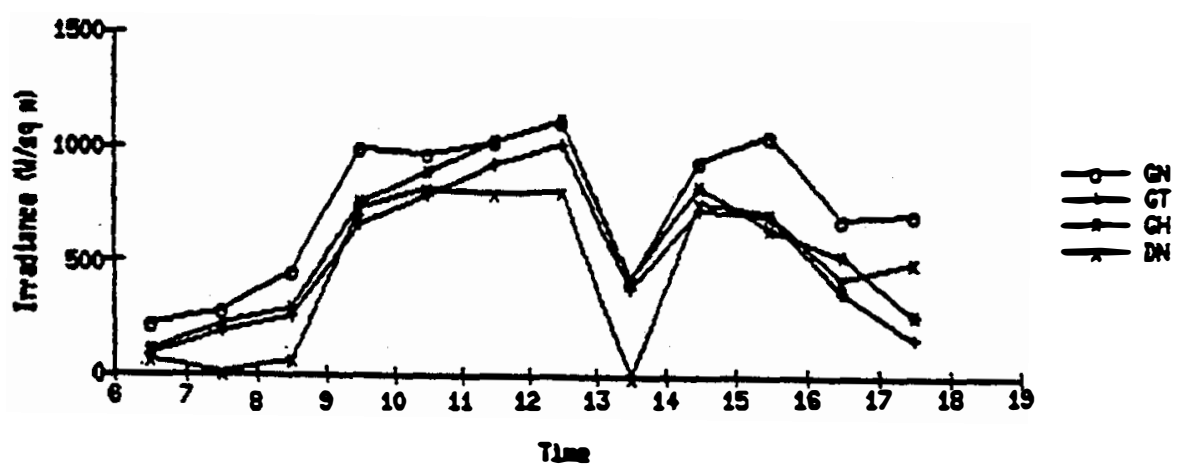

Fese $205 \mathrm{~m}$
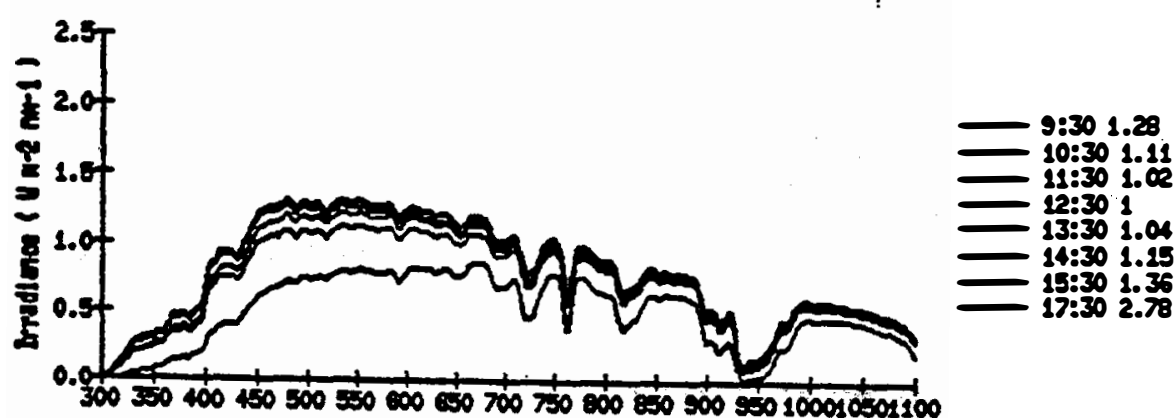

bevelength (ra)

PEEC 195 as

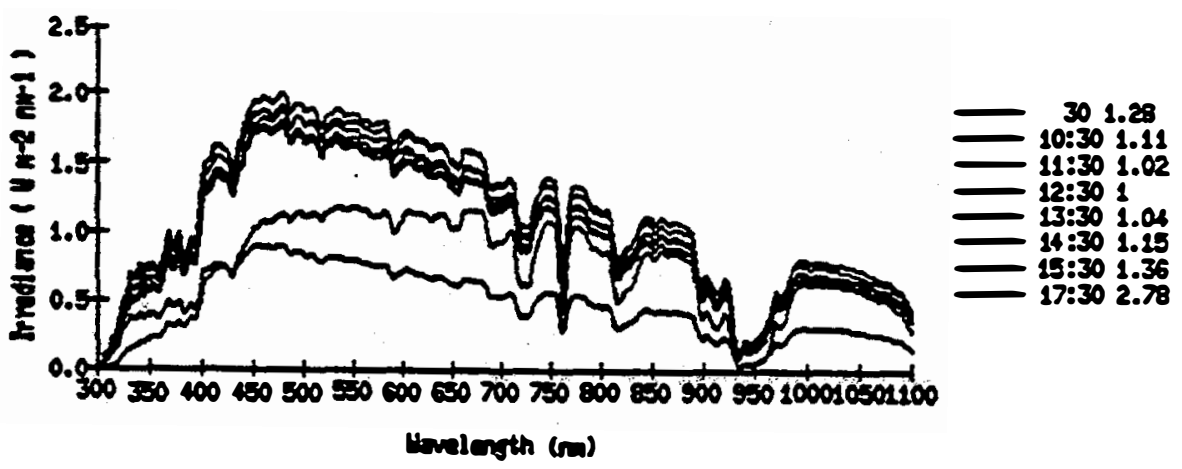




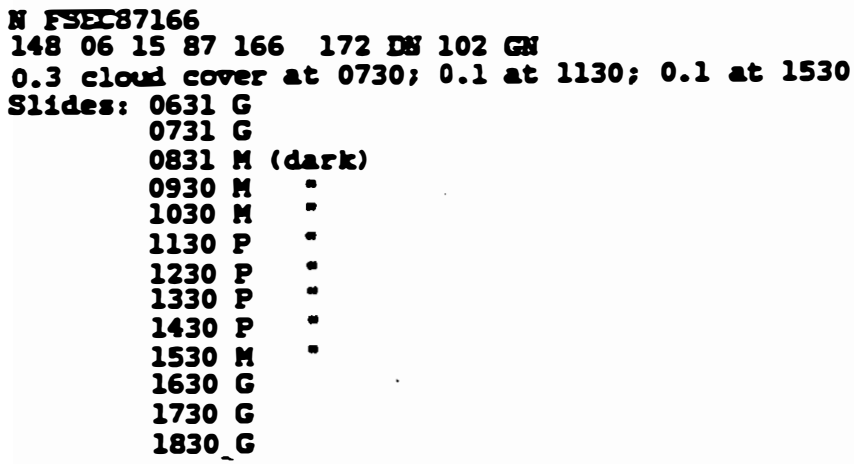

Fese 260

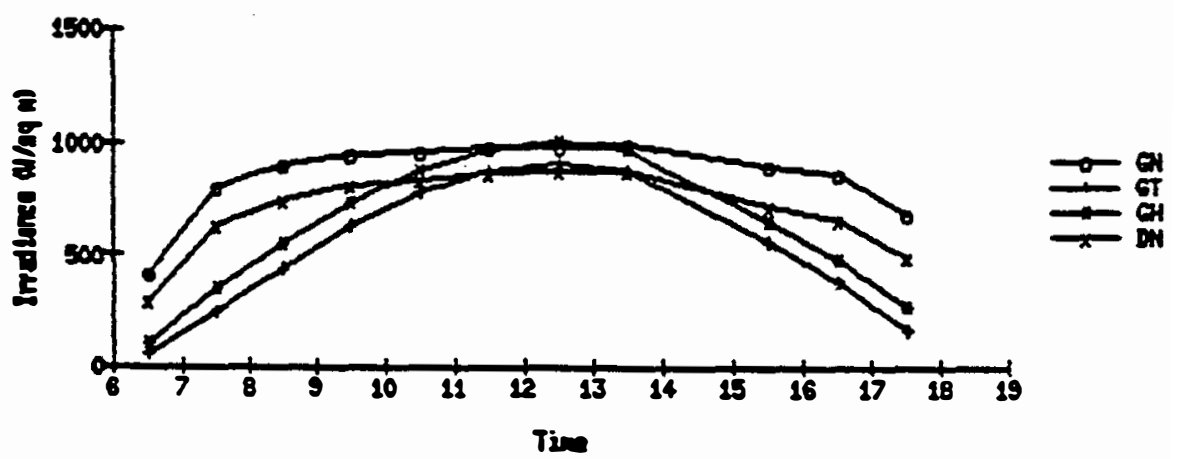

P्בت 195 BN

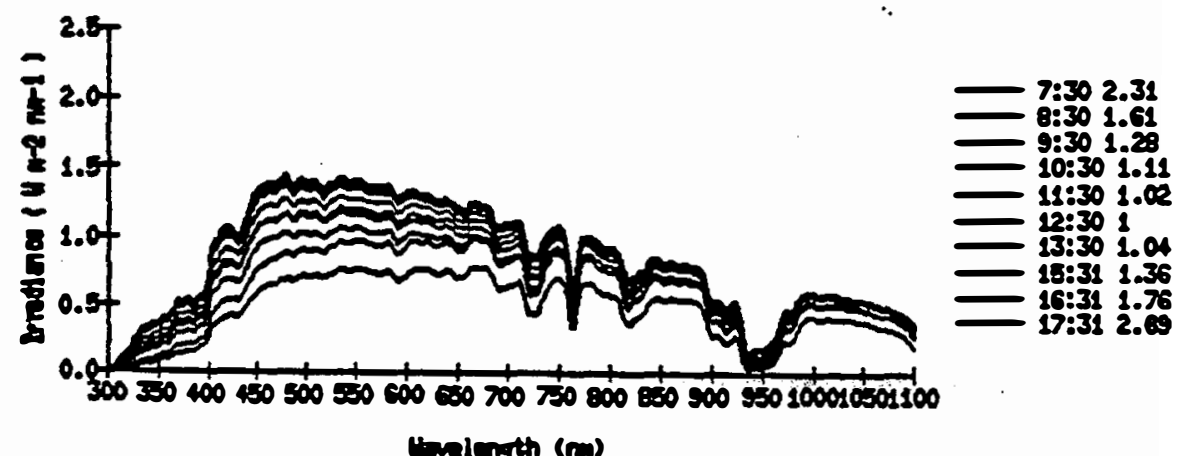

pess acs es

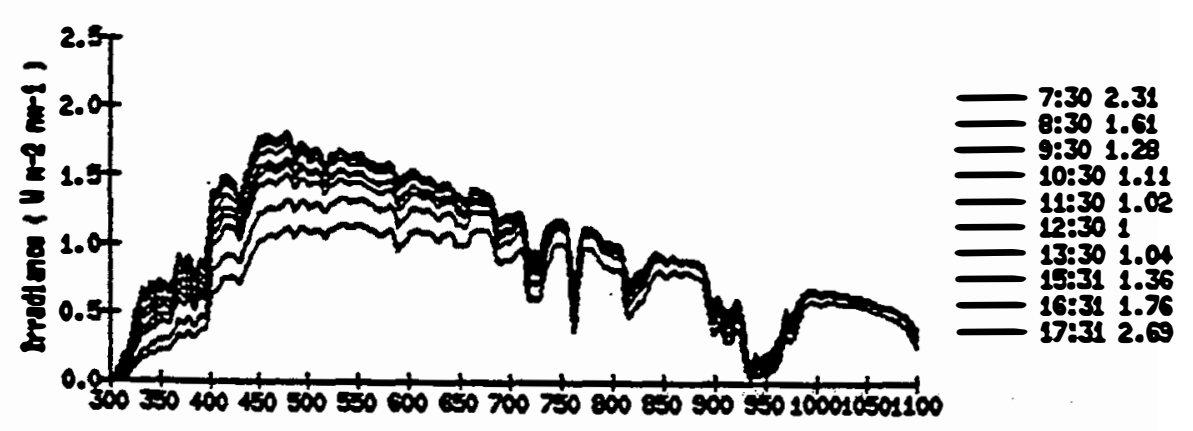

venlength (m) 


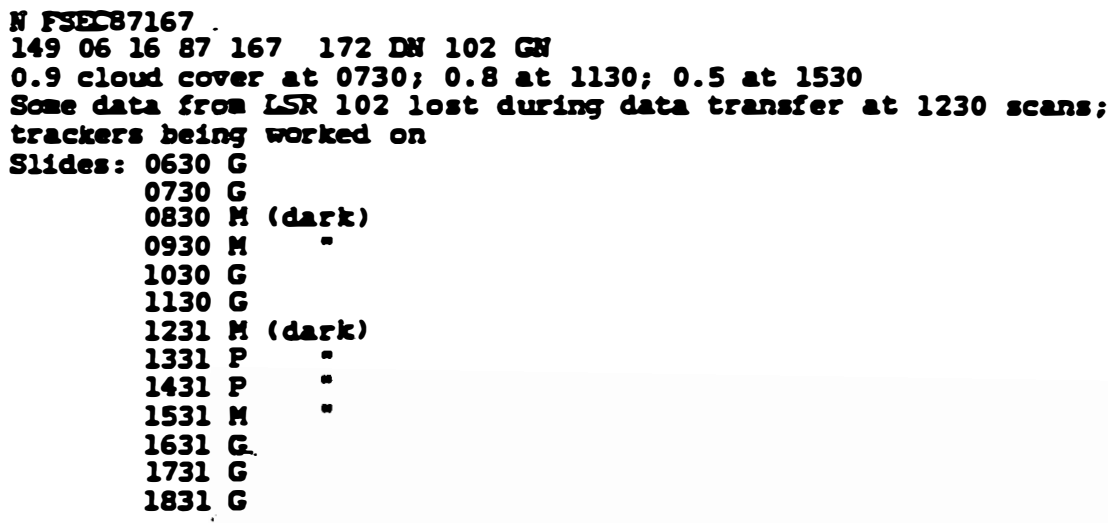

FEx 16

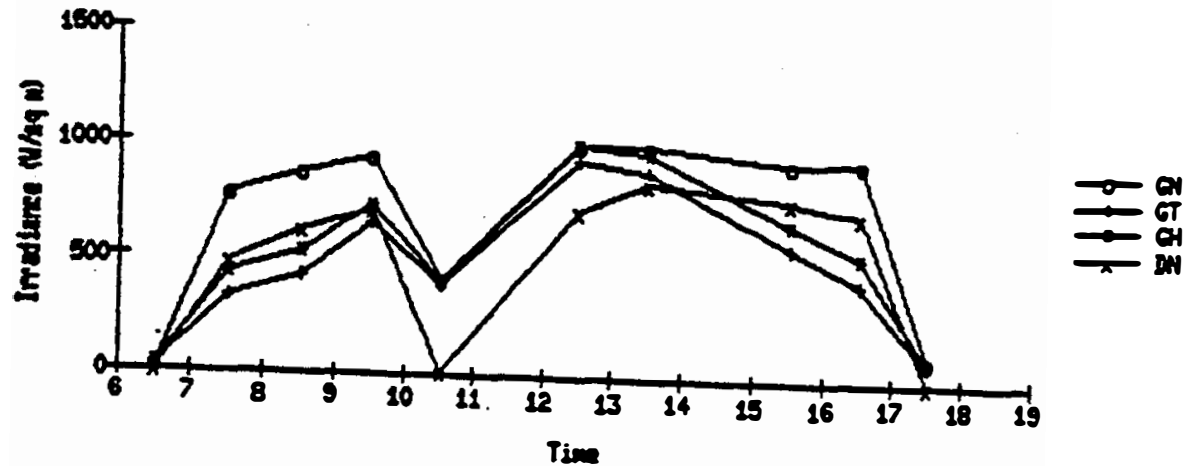

Fasc 107 on

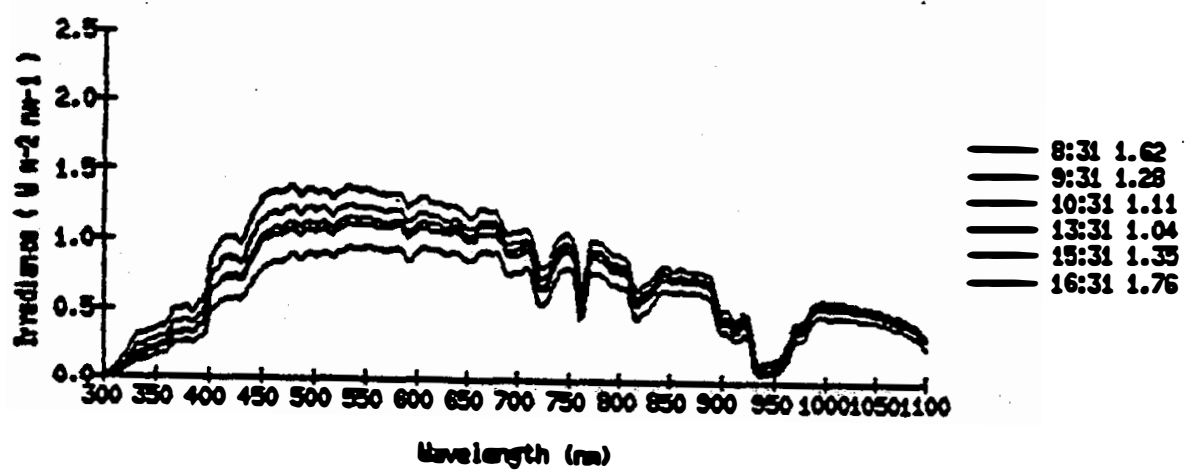

F्य 1070

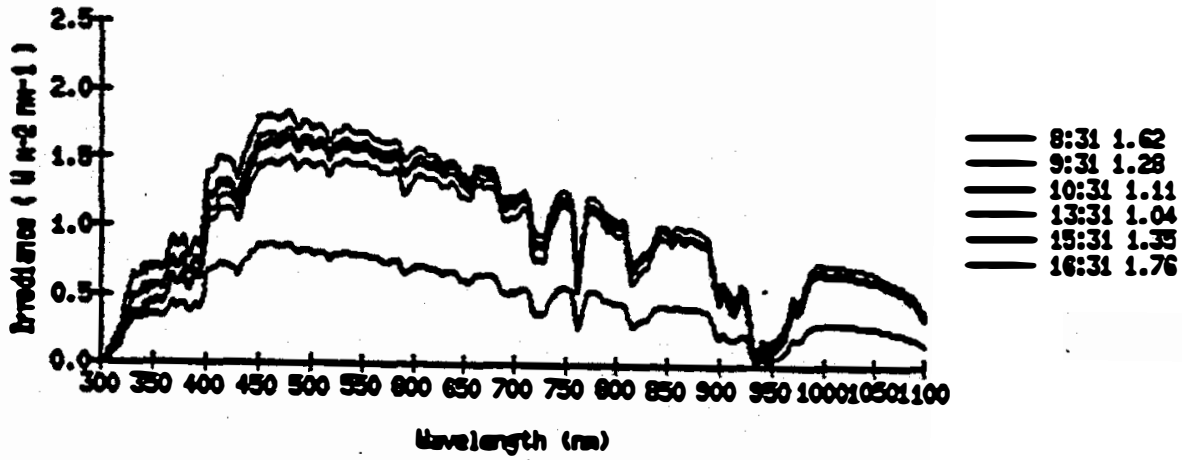


A F्रT87168

$\begin{array}{llllllllllll}150 & 06 & 17 & 87 & 168 & 172 & \text { DI } & 102 & \text { GH }\end{array}$

0 eloud cover at $0750,0.2$ at $2200,0.7$ at 1600

slides: $0631 \mathrm{G}$

$0731 \mathrm{G}$

0831 A (dnrk)

0932 M

$2031 P$ -

$1231 \stackrel{P}{P}$

$2232 \mathrm{P}$

$1331 P$

$1431 \mathrm{P}$

$1529 \mathrm{G}$

1629 G

$1729 \mathrm{G}$

$1829 \mathrm{G}$

Appears to be a sandl spike in OV in GH spectrin at 1231

FEC 208

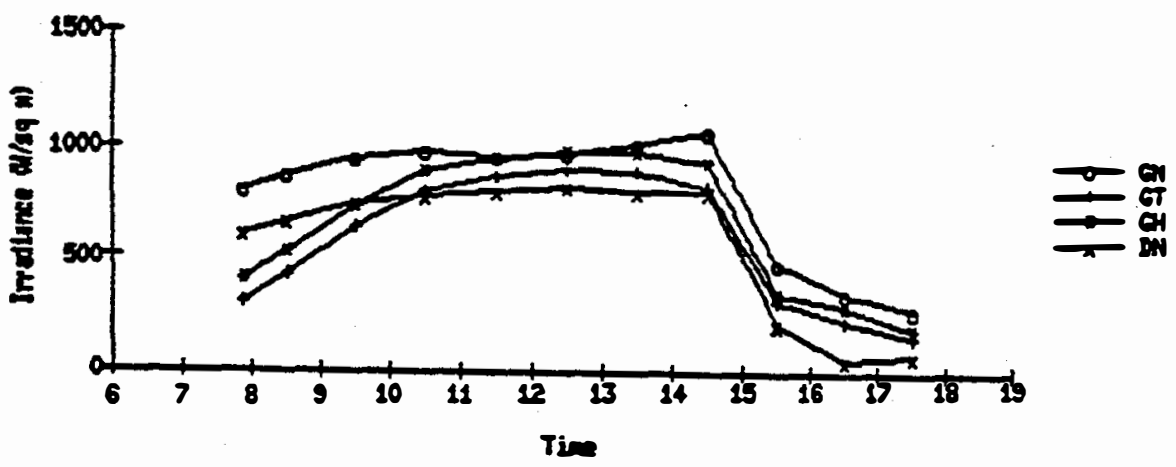

Frate $100 \mathrm{mt}$

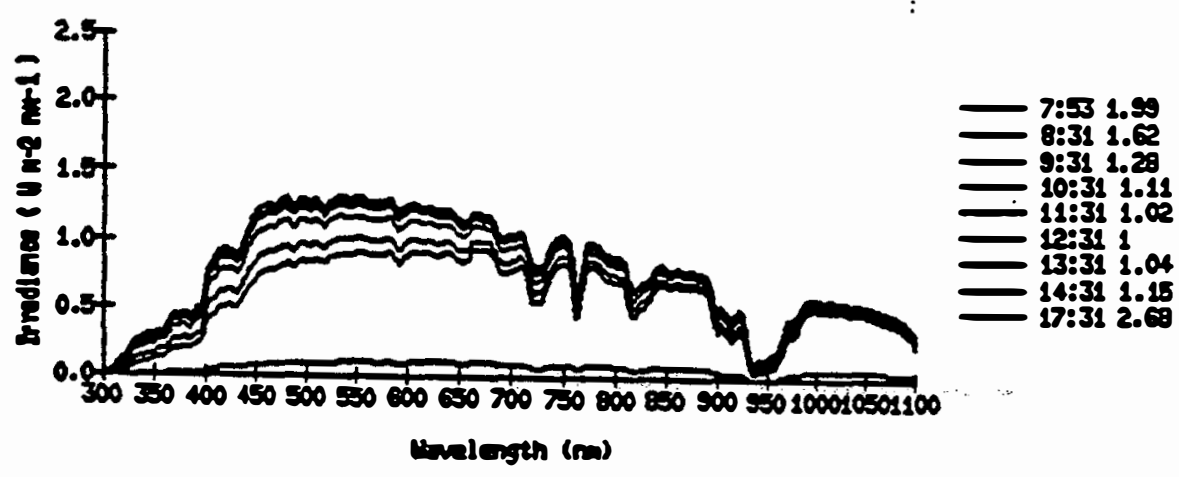

PSEC 190 06

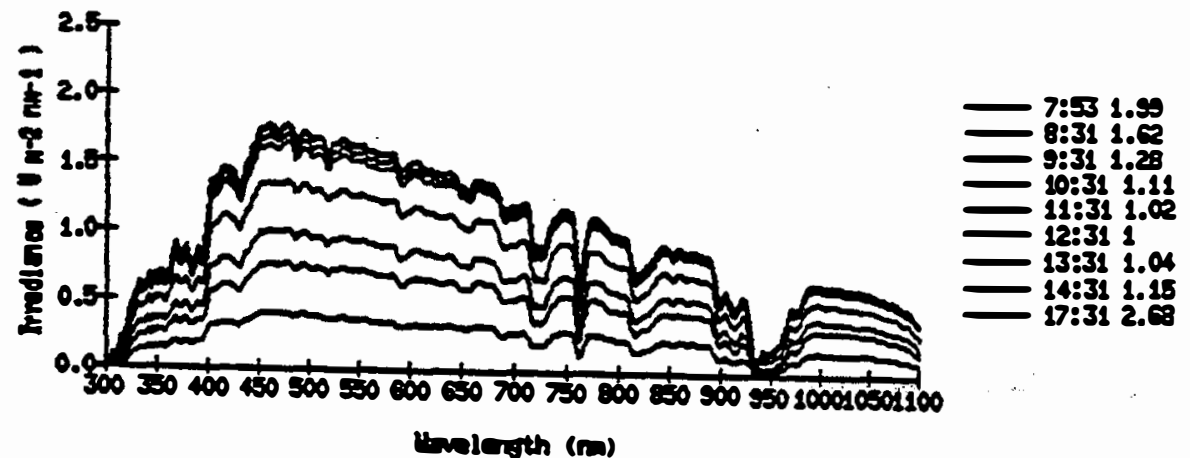




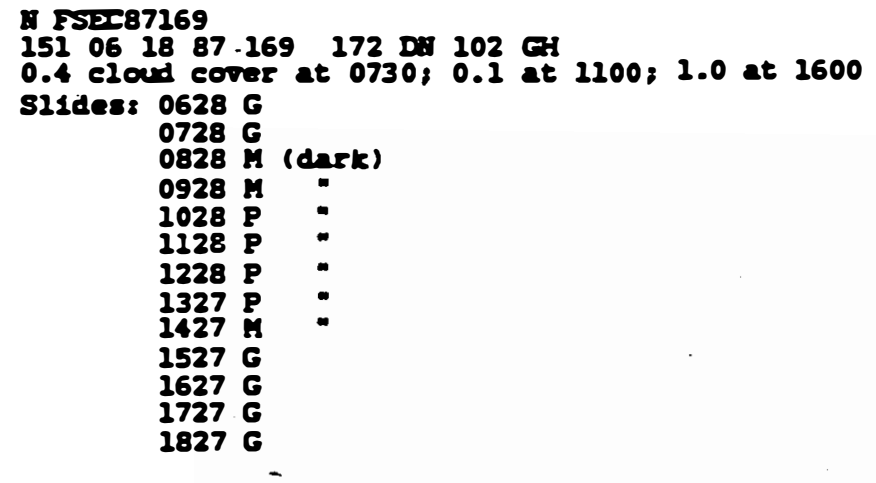

$\operatorname{Psec} 100$

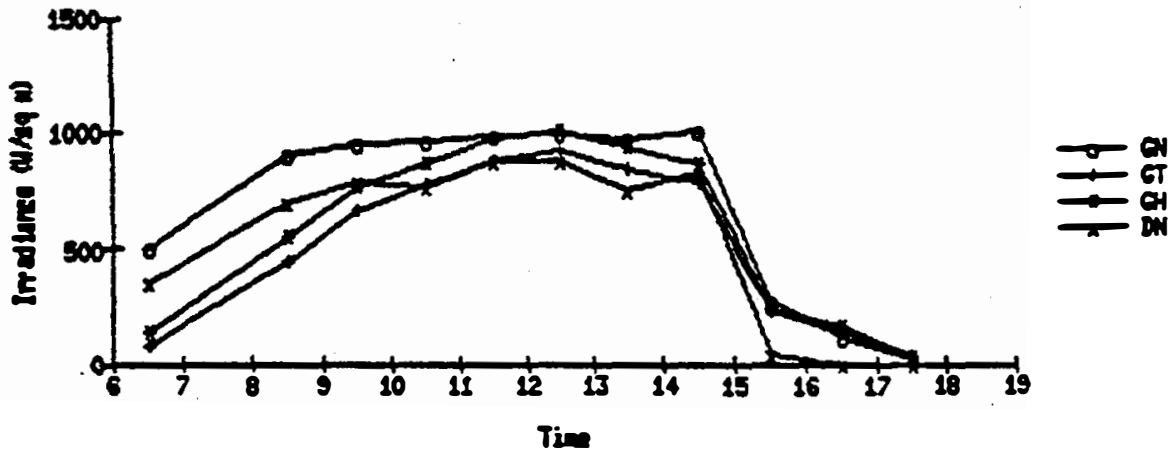

FSEC $100 \mathrm{mT}$

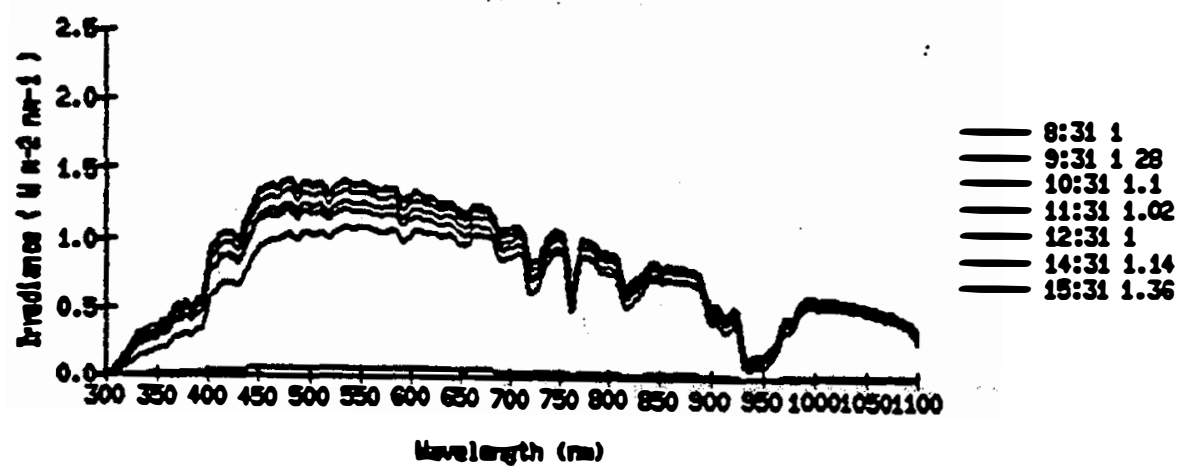

FEx 100 as

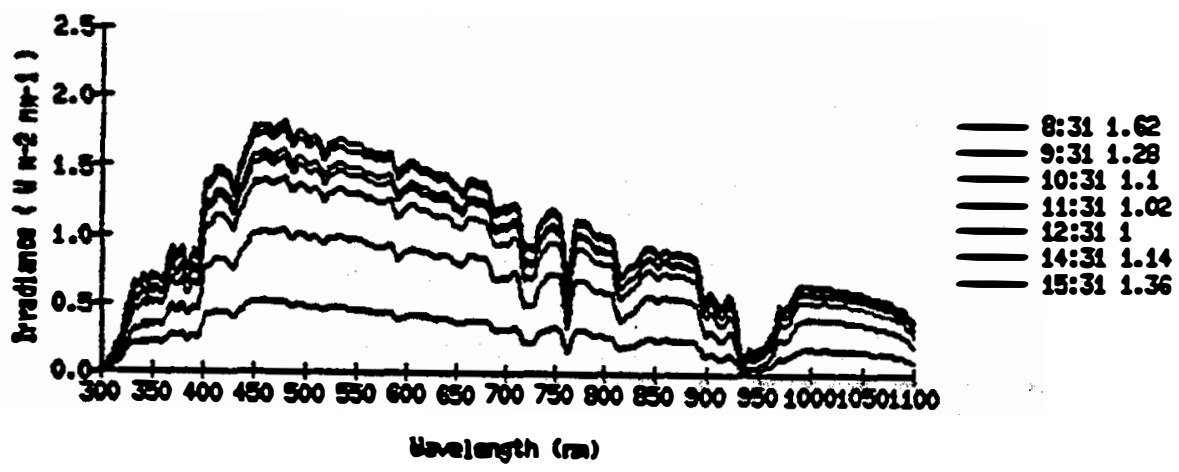


B.258710

$152061987170 \quad 172$ DN $102 \mathrm{GH}$

0.3 cloud cover at $0730 ; 0.3$ at $1130 ; 0.6$ at 1520

Hightened 4 set screvs securing direct bean tube to base: tube

ins silghtig loose (ISR 172); bird sitting on tube just prior to

check; belfeve no effect on data

Sl1des: 0630 A (condensation)

$0730 \mathrm{M}$

0830 H (dark)

$0930 \mathrm{M}$

$1030 \mathrm{P}$

$1130 \mathrm{P}$

$1230 \mathrm{P}$

$1330 \mathrm{P}$

$1430 \mathrm{M}$

$1530 \mathrm{H}$

$1630 \mathrm{G}$

$1730 \mathrm{G}$

$1830 \mathrm{G}$

Appears to be onall spike in of in Gi spectron at 1531

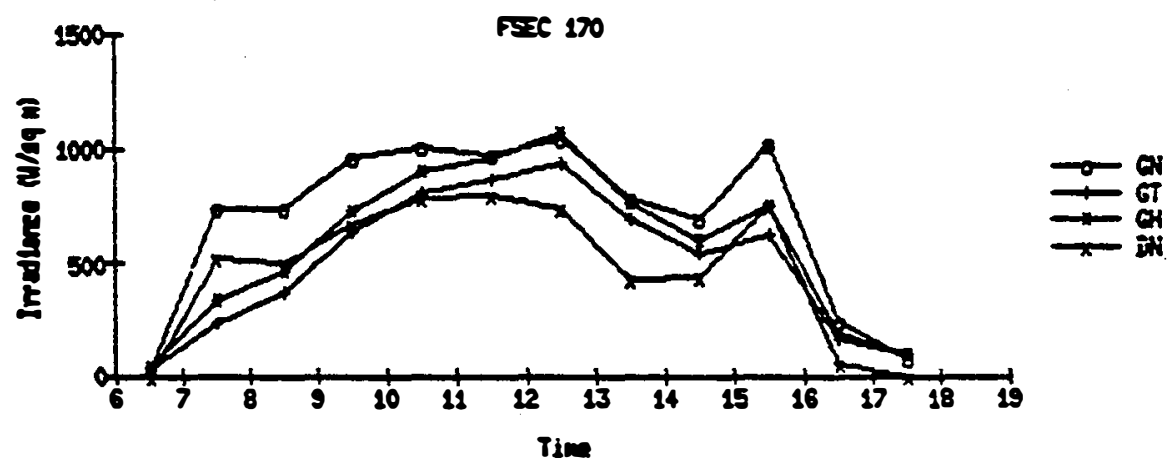

Pasc 170 on

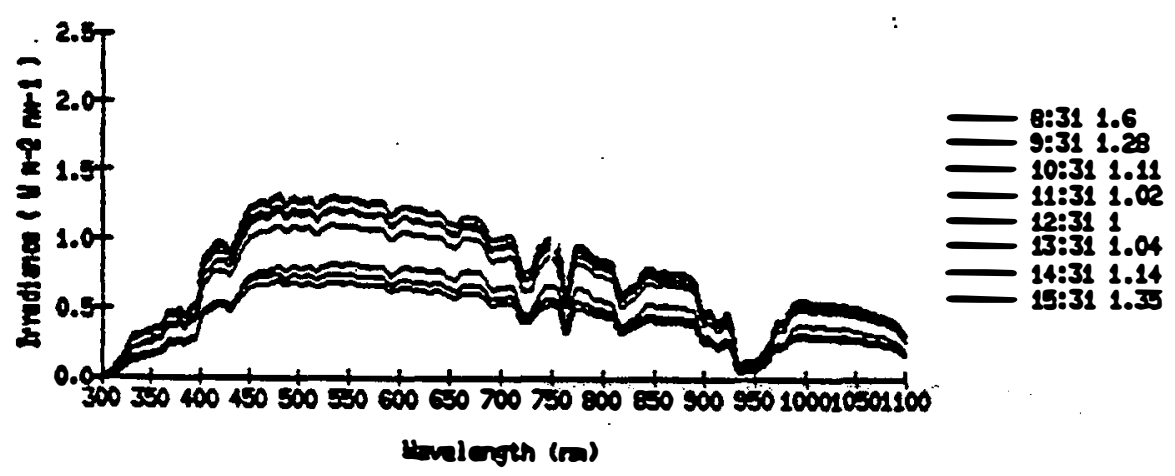

Paxe 170 as

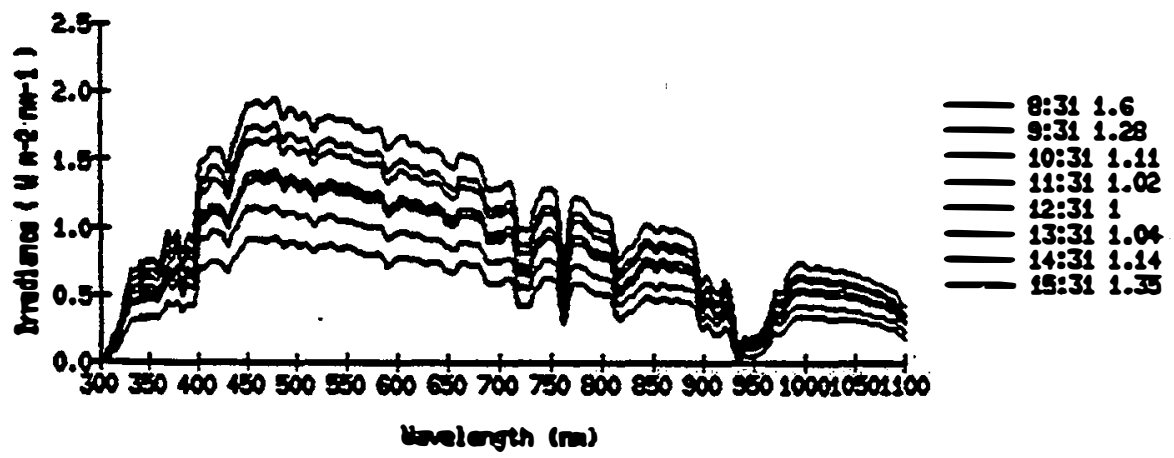




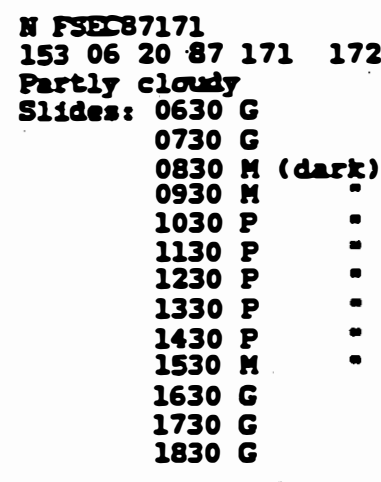

Fsec 171

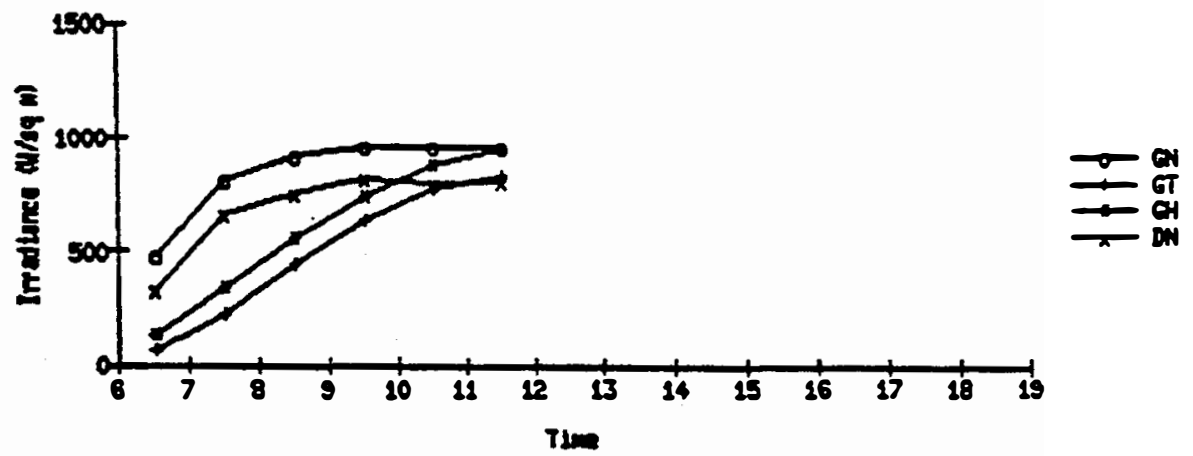

FEC 171 BT

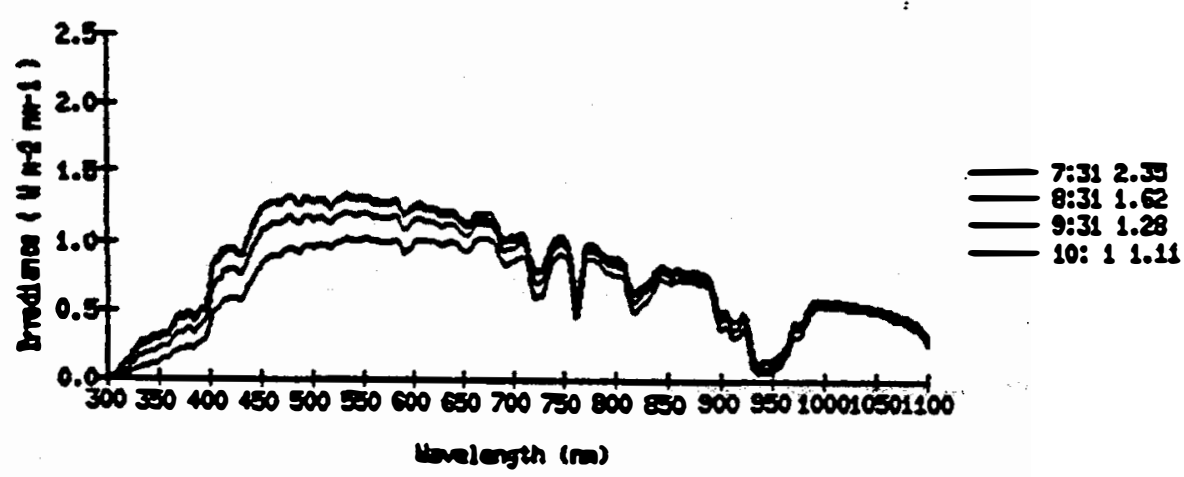

FEבC 171 o5

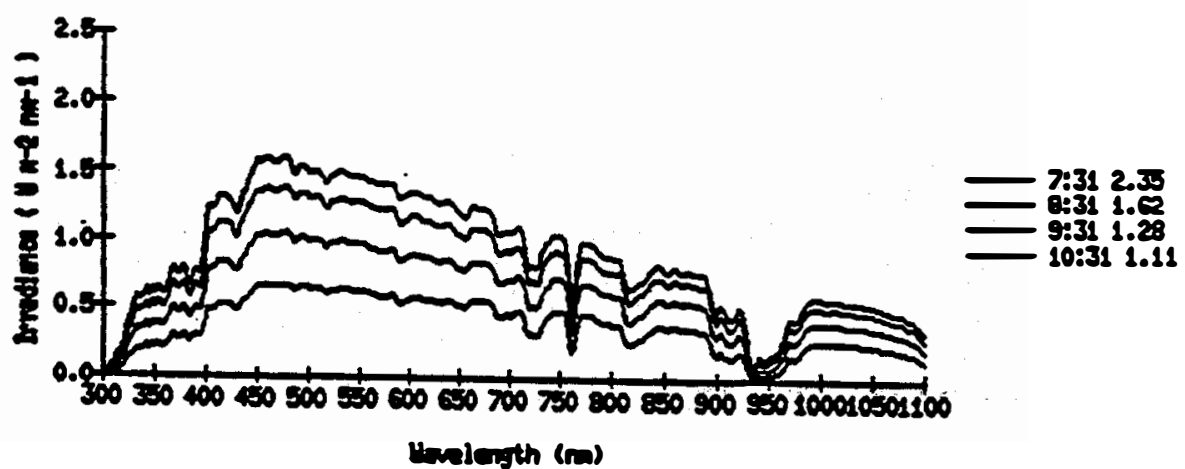




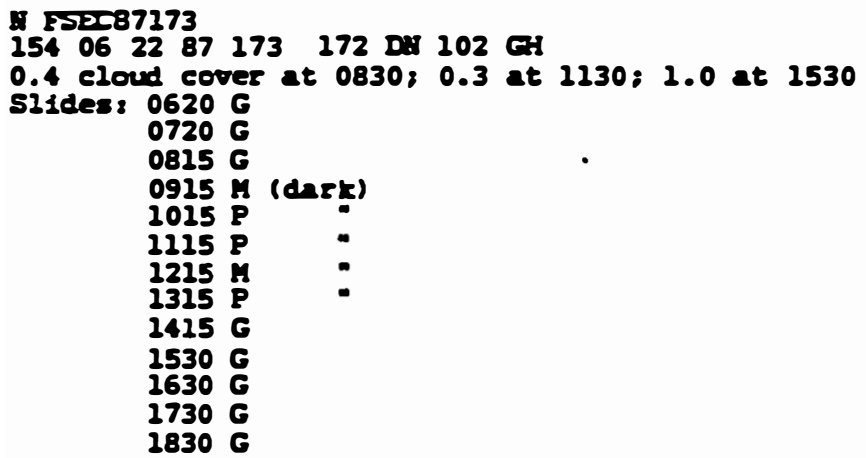

FEC 173

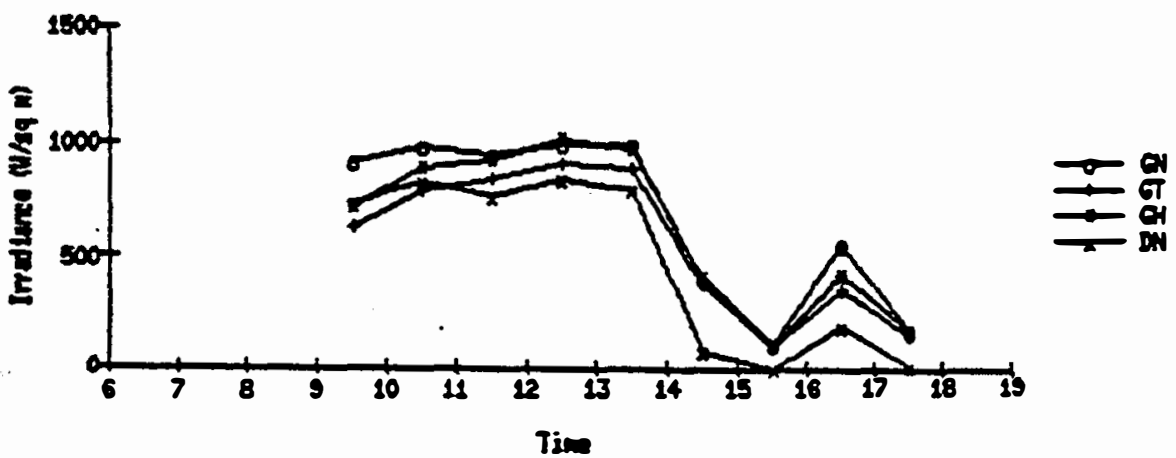

F्בS $173 \mathrm{mT}$

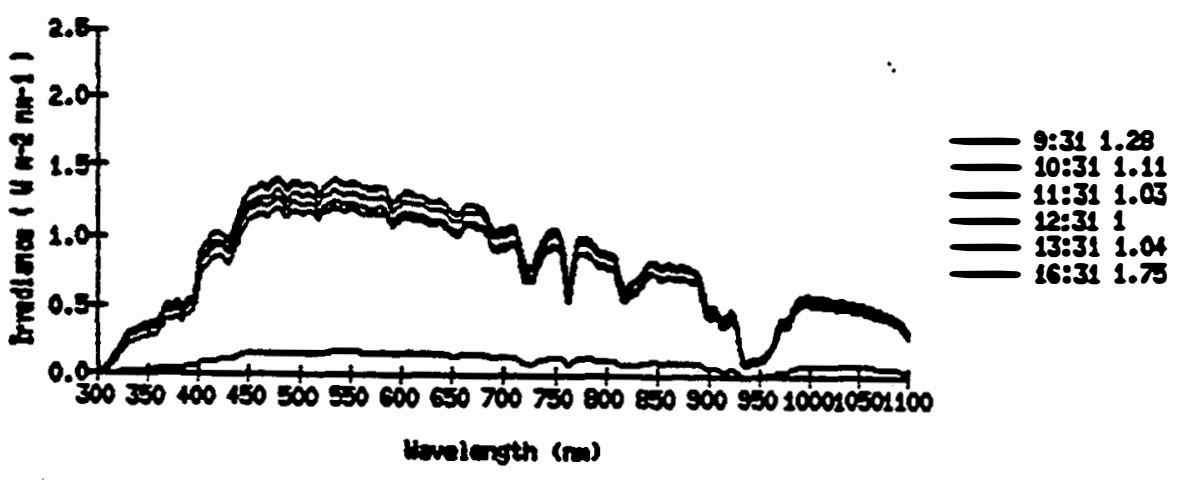

$\operatorname{Psec} 173$ as

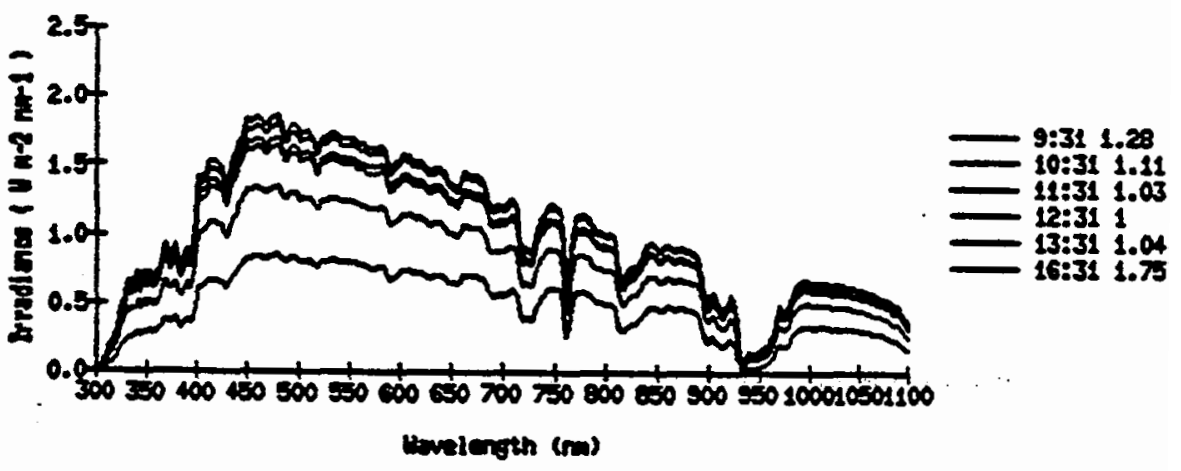


म52587174

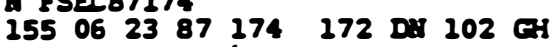

0.8 cloud cover at $0730 ; 0.3$ at 1230 and 1500

ISR 102 teap controller reading high

Tenp controller $44 \mathrm{C}$ at $1130: 40 \mathrm{C}$ at 1300

Believe caused by east side of case not shielded by mplar

East side vill be covered with uglar foil

Sildes: $0630 \mathrm{G}$ (veter drops)

$0730 \mathrm{G}$ (veter drops)

0830 H (dark)

$0930 \mathrm{H}$

$1030 \mathrm{P}$

$2130 \mathrm{P}$

$1230 \mathrm{G}$

$1330 \mathrm{H}$ (dark)

$1430 \mathrm{H}$

$1530 \mathrm{G}$

$1630 \mathrm{G}$

$1730 \mathrm{G}$

$2830 \mathrm{G}$

-c--co

Spiken in OV in GH spectru at 0931

$\operatorname{Fsec} 274$

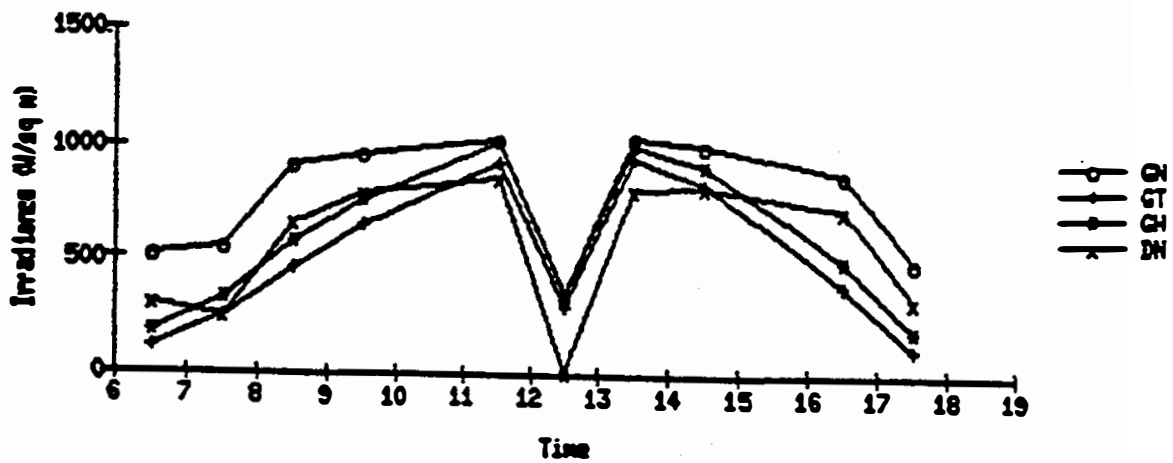

FSEC 274 DNT

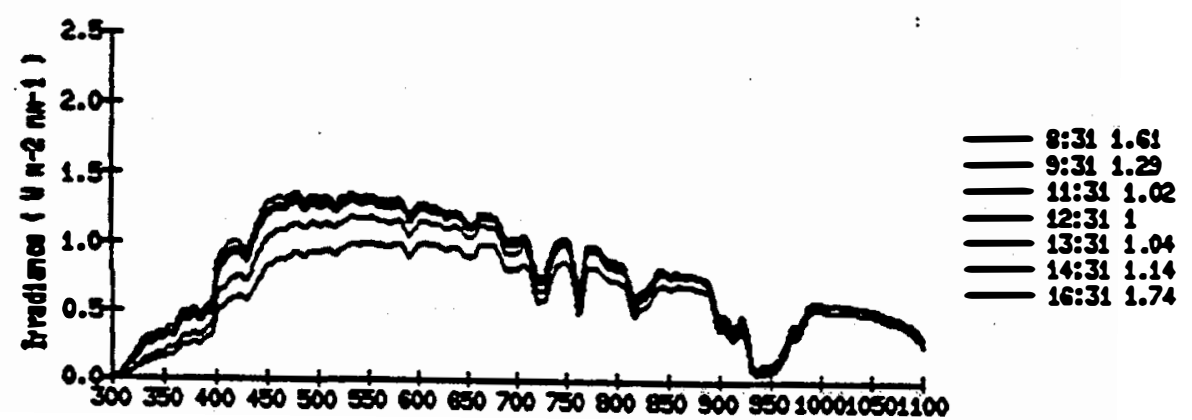

Vinlerjth (ma)

Fas 174 as

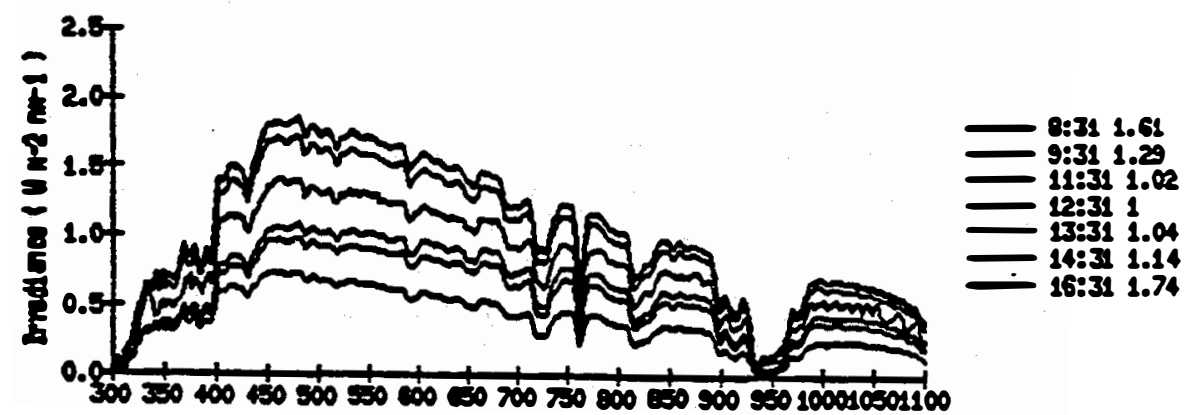

Holergth (ma) 
IST287175

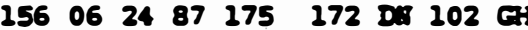

0 cloud cover at 075080.2 at 1200

Corered east side of LSR 102 with fo1 to nininize solar heat gain

Tracker out of al1grment $1.5-2 \mathrm{deg}$ at 1145 ; reset

Slides: $0630 \mathrm{G}$

$0730 \mathrm{G}$

$0830 \mathrm{H}$ (derz)

$0930 \mathrm{H}$

$1030 \mathrm{P}$

$2130 \mathrm{P}$

$1230 \mathrm{P}$

$1330 \mathrm{P}$

$2430 P$

$1530 \mathrm{G}$

$1630 \mathrm{G}$

1730 G (veter drops)

$2830 \mathrm{G}$ - (weter drops)

FEC 175

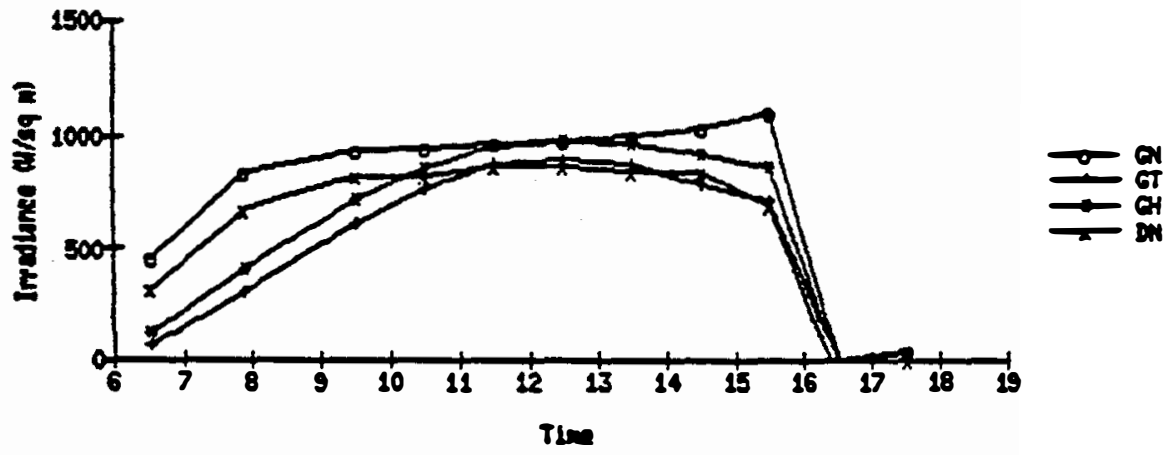

Fis 175 or

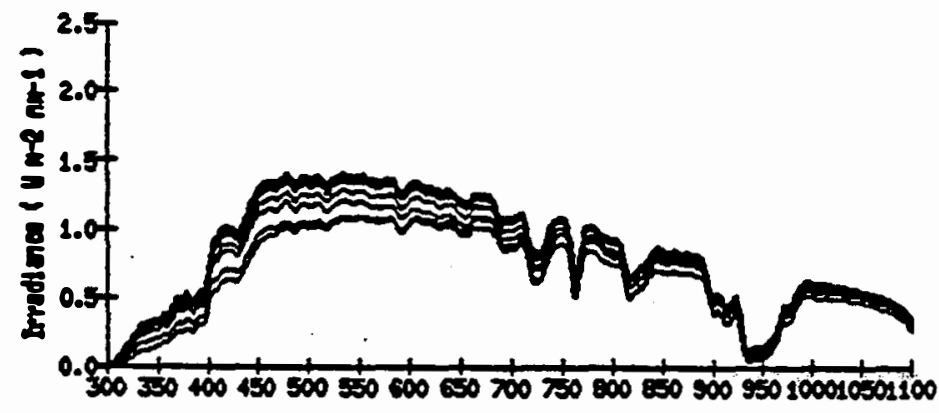

$7: 541.99$

9.311 .29

$9: 331.20$

$11: 311.03$

$12: 311$

13:31 1.0

$14: 311.14$

15:31 1.30

Uvelergth (m)

FEEC 275 of

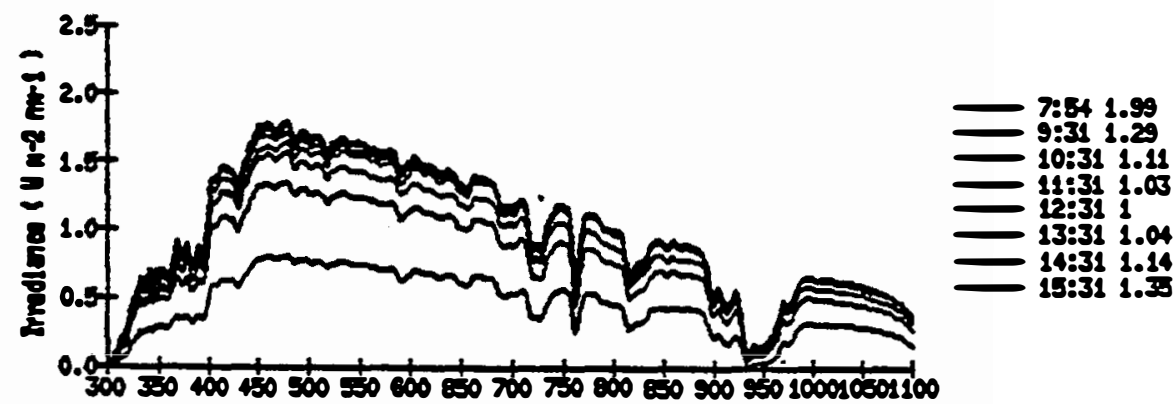

Henelength (m) 
P.2087182

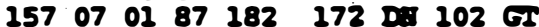

0.1 cloud cover at $0830 ; 0.5$ at $1130 ; 0.4$ at 1530

First day date collection since 6-26-87 lightening strike (VxX out)

Protos: $0627 \mathrm{G}$

$0727 \mathrm{G}$

$.0827 \mathrm{G}$

$0926 \mathrm{H}$ (dark)

s11des $\begin{aligned} & 1026 \mathrm{M} \\ & 1630 \mathrm{G}\end{aligned}$

$1730 \mathrm{G}$

$1830 \mathrm{G}$

Fise 182

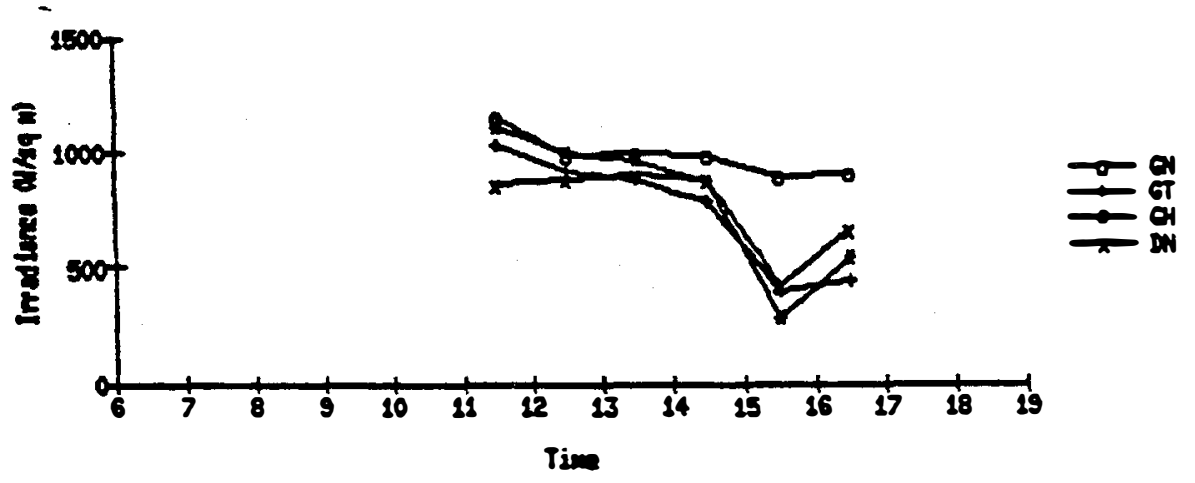

F्בי 182 INT

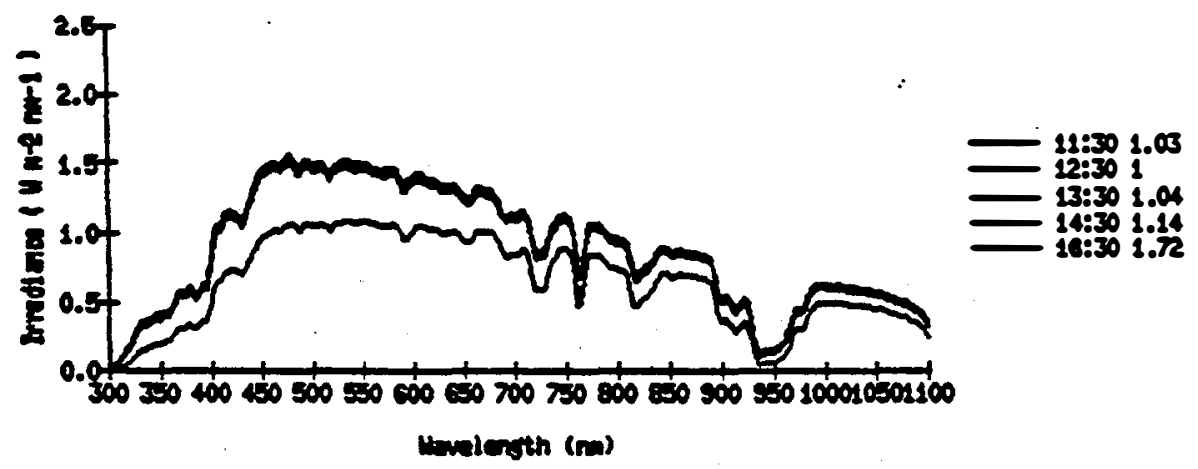

FEIS IRE GTS

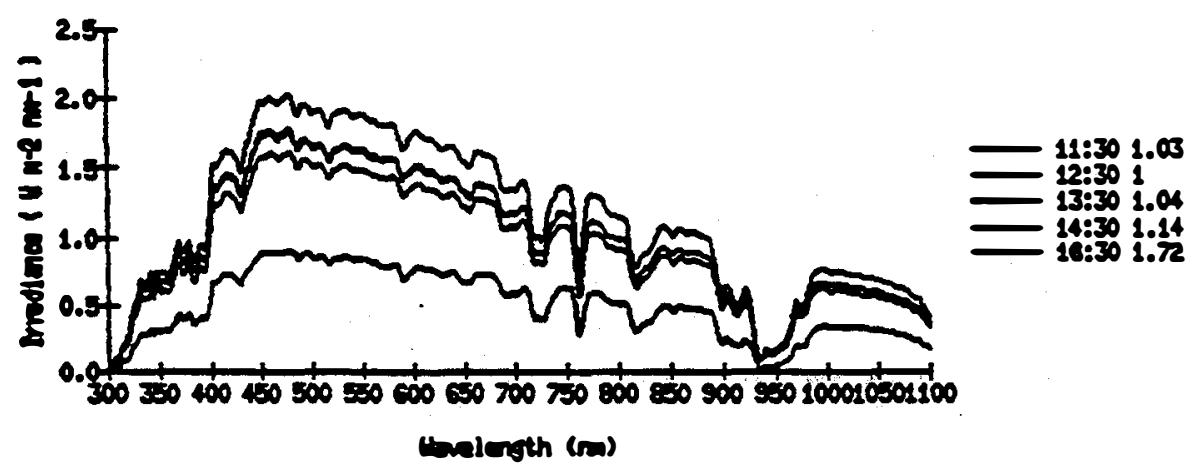




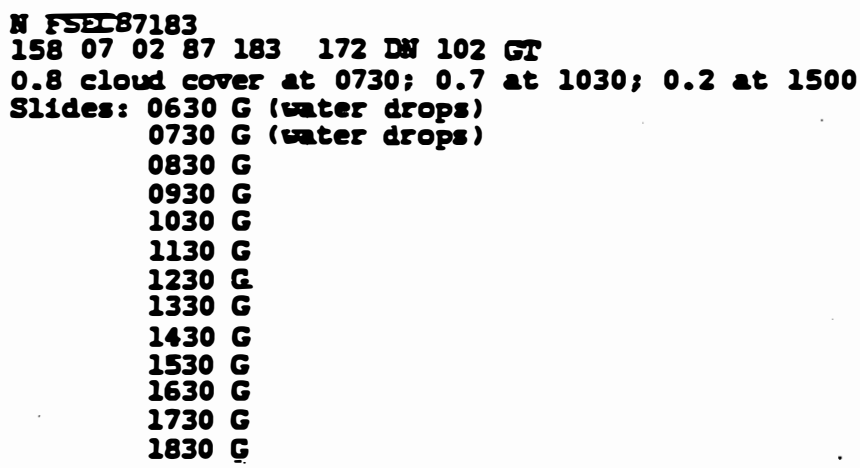

FEEC 183

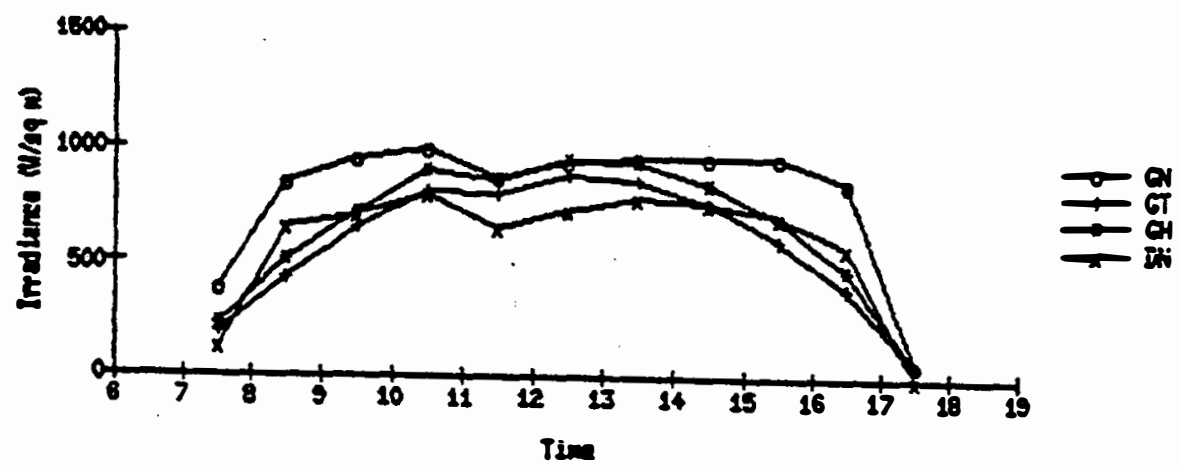

FIEC $183 \mathrm{BNT}$

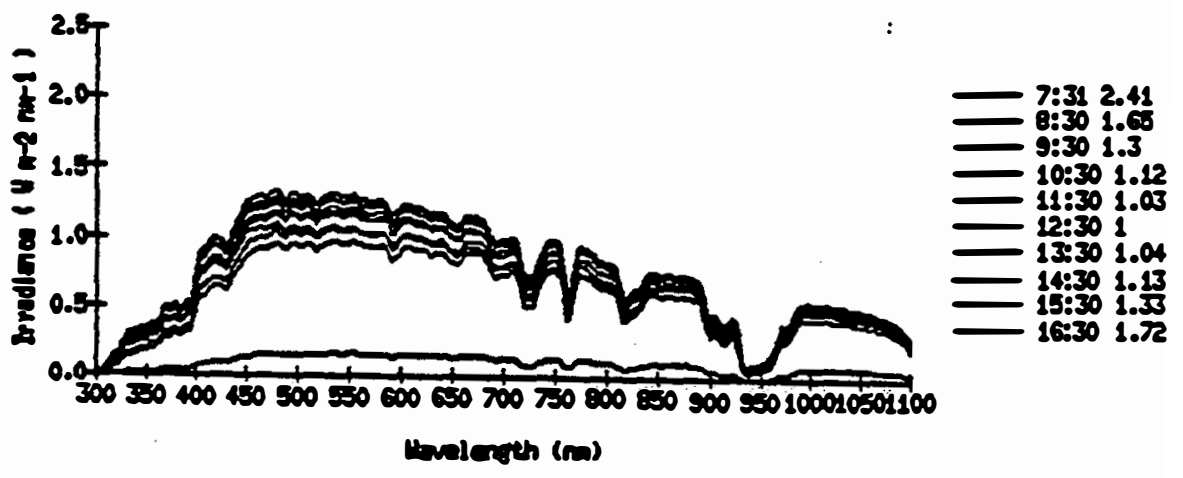

FIES 183 GTS

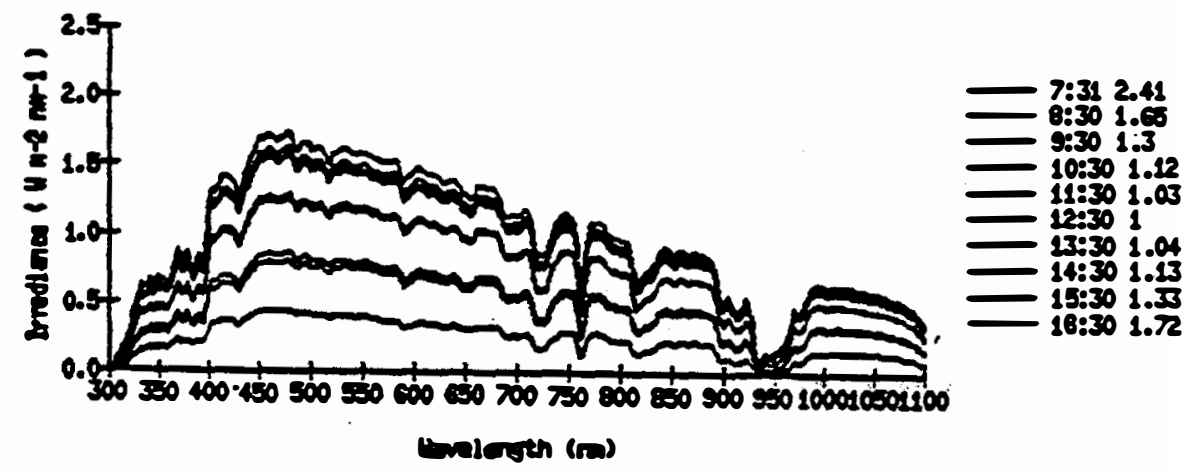


10 F587184

$\begin{array}{llllll}15907 & 0387 & 184 & 172 \text { DN } & 102 \text { GS }\end{array}$

Partls clouds

slides: $0630 \mathrm{G}$

$0730 \mathrm{G}$

$0830 \mathrm{G}$

$0930 \mathrm{G}$

$1030 \mathrm{G}$

$1130 \mathrm{G}$

$1230 \mathrm{G}$

$1330 \mathrm{G}$

$1430 \mathrm{G}$

$1530 \mathrm{G}$

$1630 \mathrm{G}$

$1730 \mathrm{G}$

$1830 \mathrm{G}$

spike in 00 in GI spectirn at 1230

Fose 204

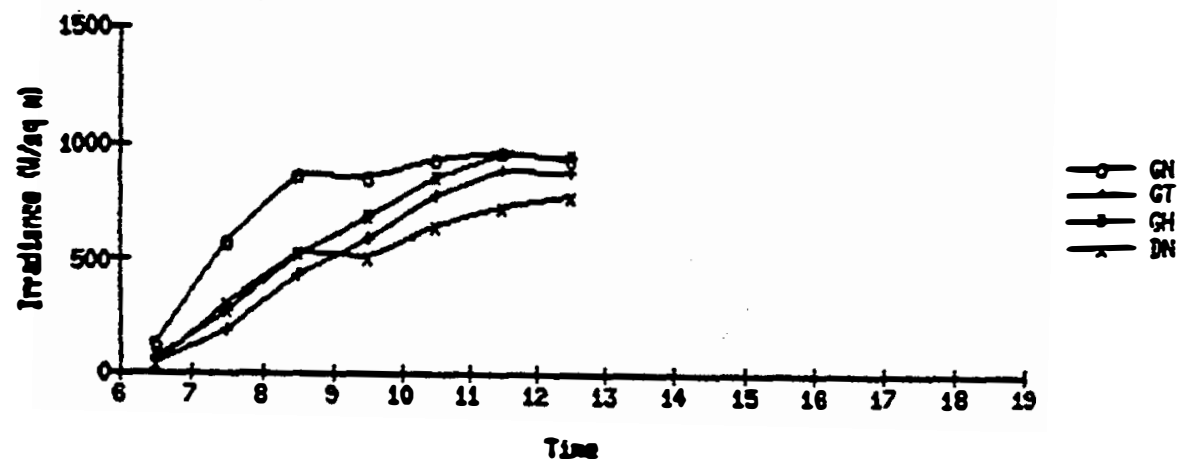

PE्S 184 जा

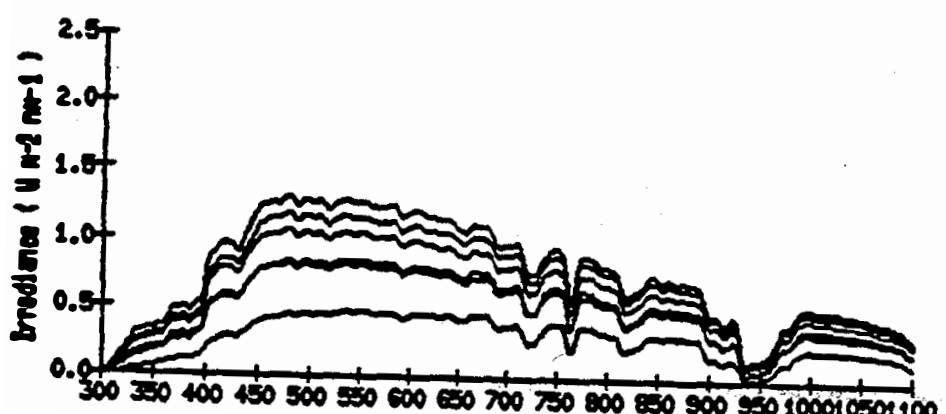

$300=0400450500500000=0700750800800500500400010501100$

Honlorish (m)

FEEC 184 GTS

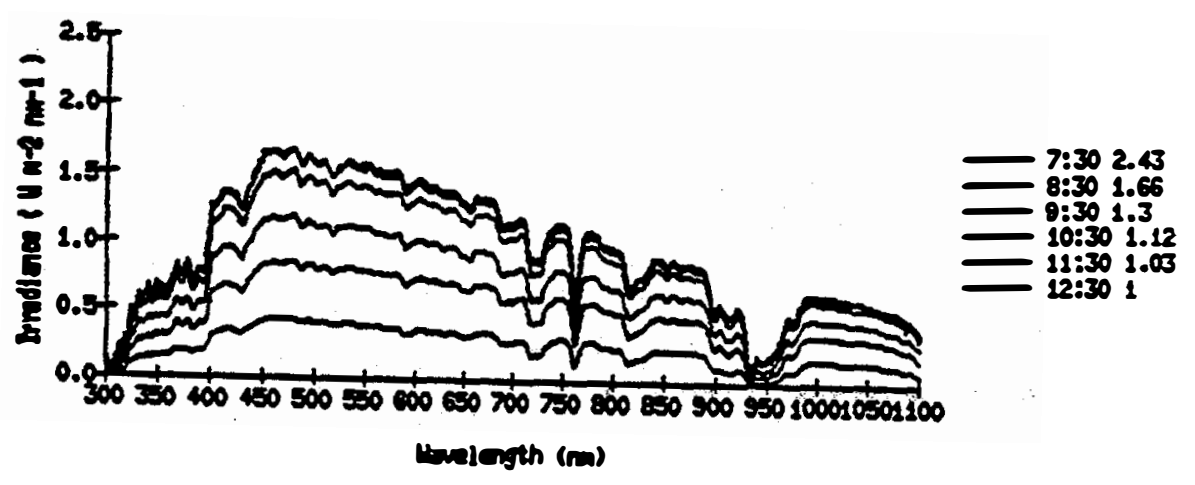


1. PS1287186

$\begin{array}{lllllllllll}160 & 07 & 05 & 87 & 186 & 172 & 012 & \text { OS }\end{array}$

Pertly clouds

s11des: 0630 G (enter dropes)

$0730 \mathrm{G}$ (enter drops)

$0830 \mathrm{G}$

$0930 \mathrm{G}$

$1030 \mathrm{G}$

Spike in Fisible in Dis spectrin at 1430

FEC 186

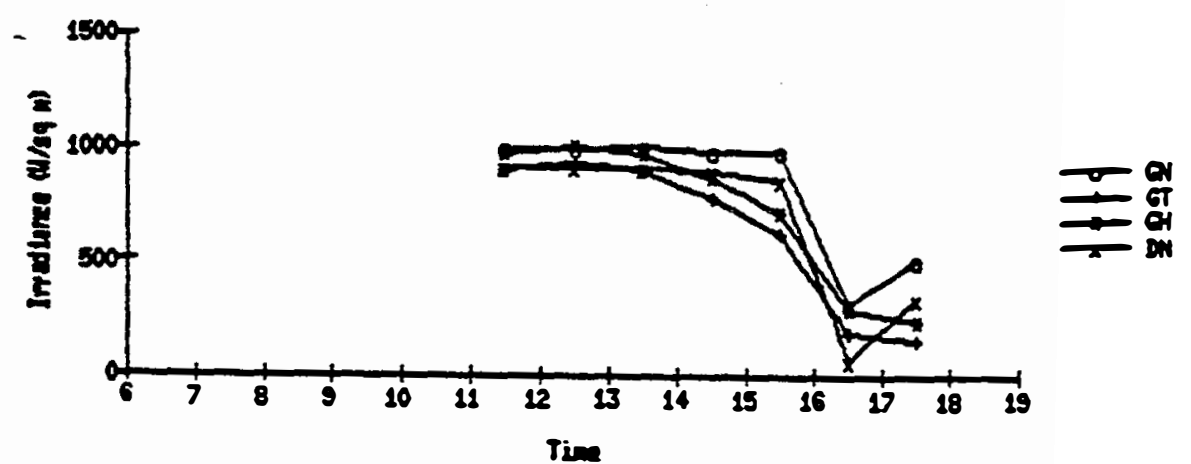

Fax 180 DNT

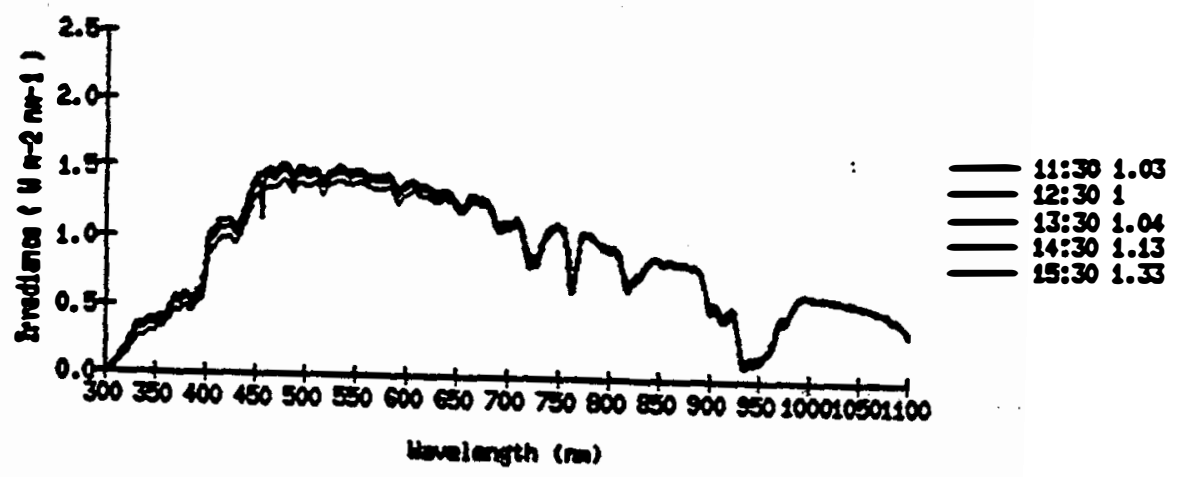

FEEC 200 GTS

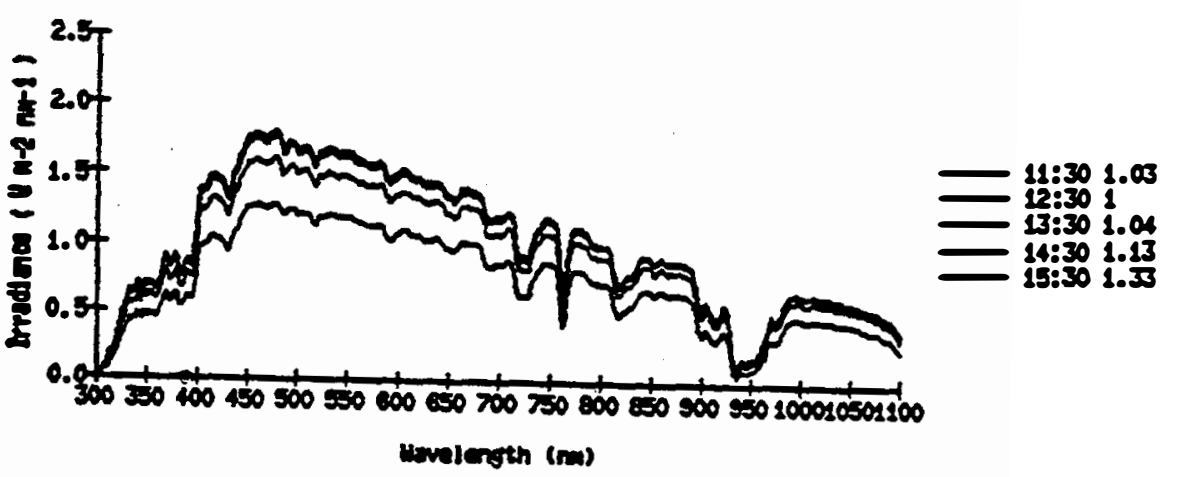




\section{SEPI}

स 752087187

$\begin{array}{llllll}161 & 07 \quad 6 \quad 87 \quad 187 \quad 172 \quad D M & 102 \quad G P\end{array}$

0.4 cloud cover at 0800 ; 0.2 at 0930 and 1130: 0.3 at 1530

ISR 172 teap controller $42 \mathrm{C}$ at 1330; $41 \mathrm{C}$ at 1530

S11des: $0830 \mathrm{G}$

$0930 \mathrm{G}$

$1030 \mathrm{G}$

$1130 \mathrm{G}$

$1230 \mathrm{G}$

$1330 \mathrm{G}$

$1430 \mathrm{G}$

$1530 \mathrm{G}$

$1630 \mathrm{G}$

$1730 \mathrm{G}$

$1830 \mathrm{G}$

spikes

in UV th GI apectern at 0730

FEx 280

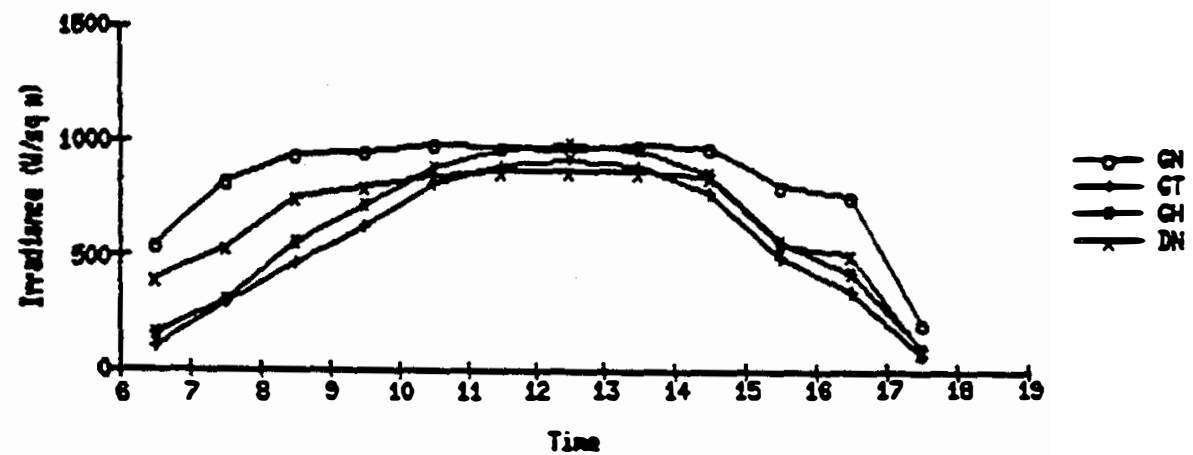

FEC 187 IN

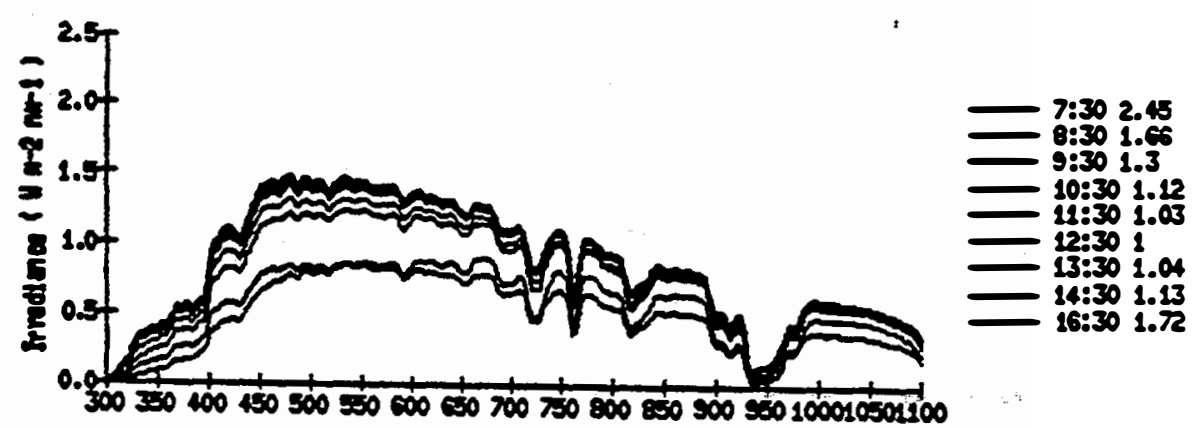

uniesth (m)

FIE 180 TIS

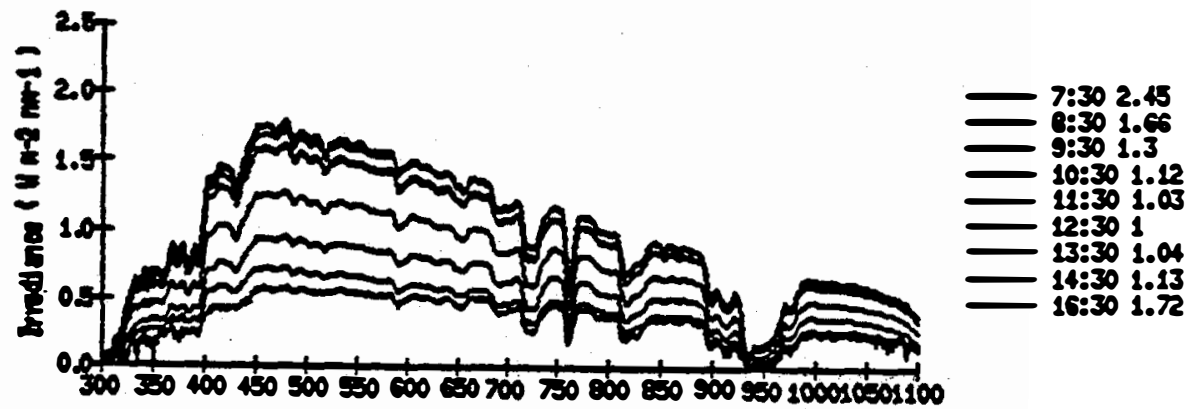

(anderith (m) 
म.

$\begin{array}{lllllllll}162 & 07 & 07 & 87 & 188 & 172 & \mathrm{DP} & 102 & \mathrm{GT}\end{array}$

0 eloud cover at 0730; 0.2 at 1200 and 1530

Ho sldes

arier opikes in ov in Gr spectra at 1030 and 1230

FIS 188

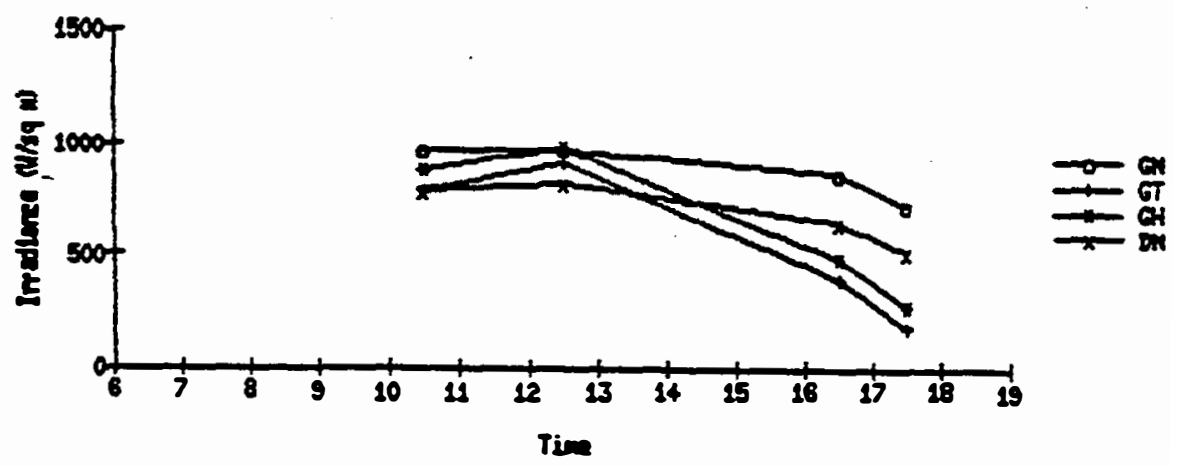

Fres 488 an

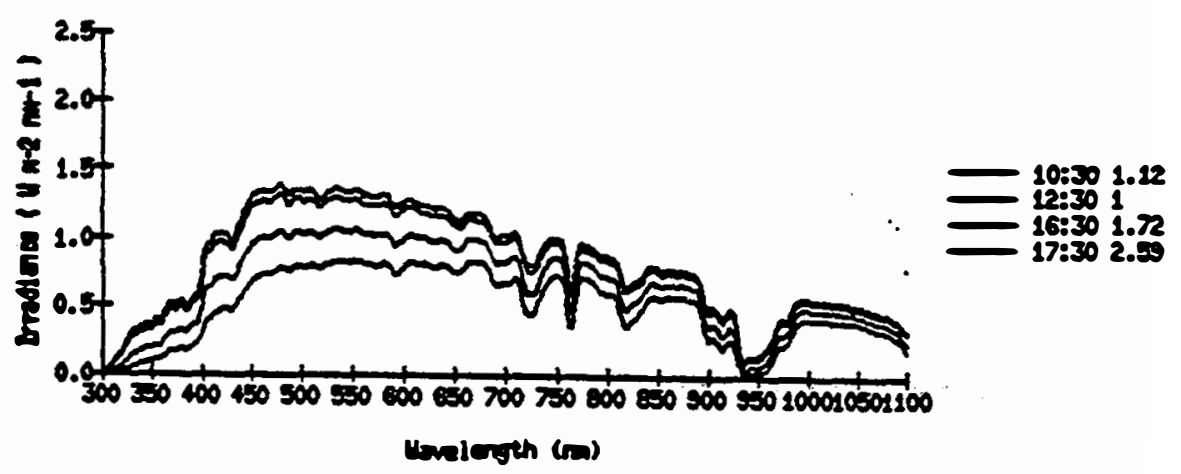

F⿻36 $183 \mathrm{as}$

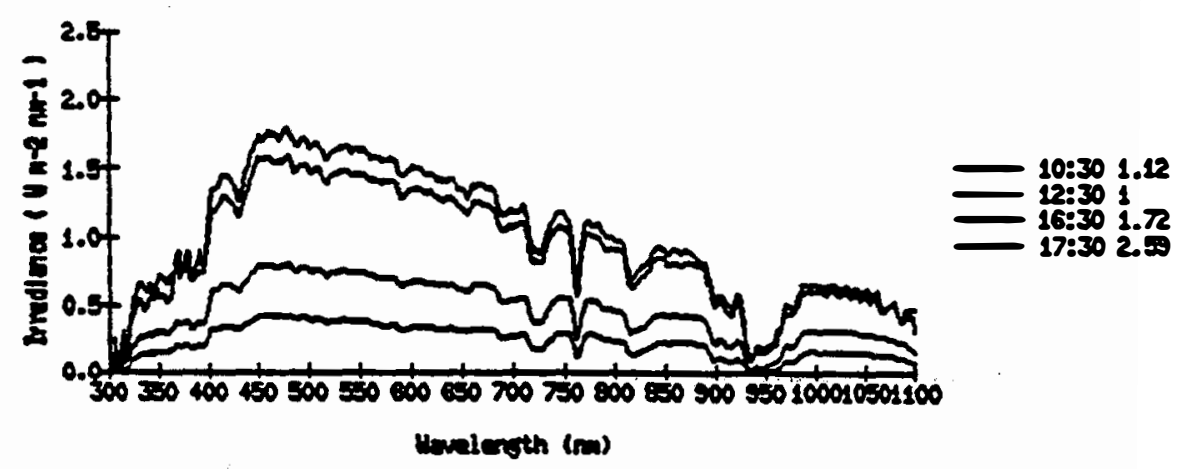




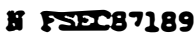

$\begin{array}{llllllllll}16307 & 08 & 87 & 189 & 172 & 09 & 102 & G\end{array}$

Clear at 0630

vo slides

Spike in ov in G spectron at 0630

FISC 189

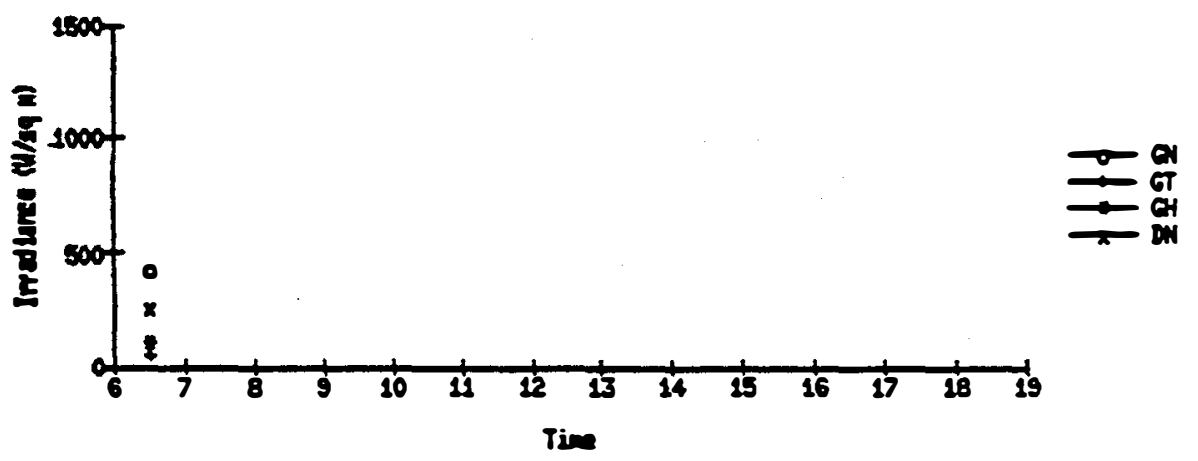

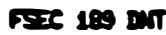

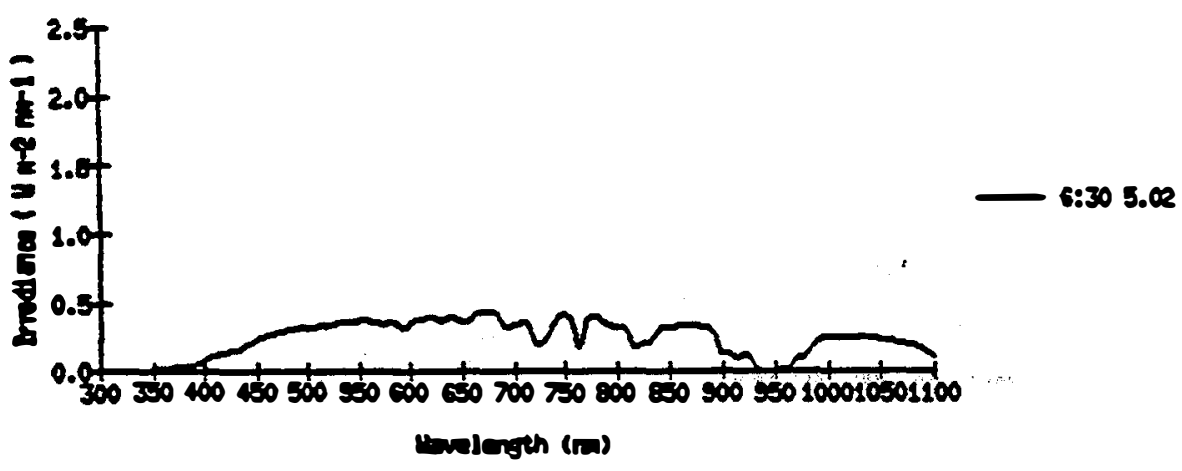

Pac is ats

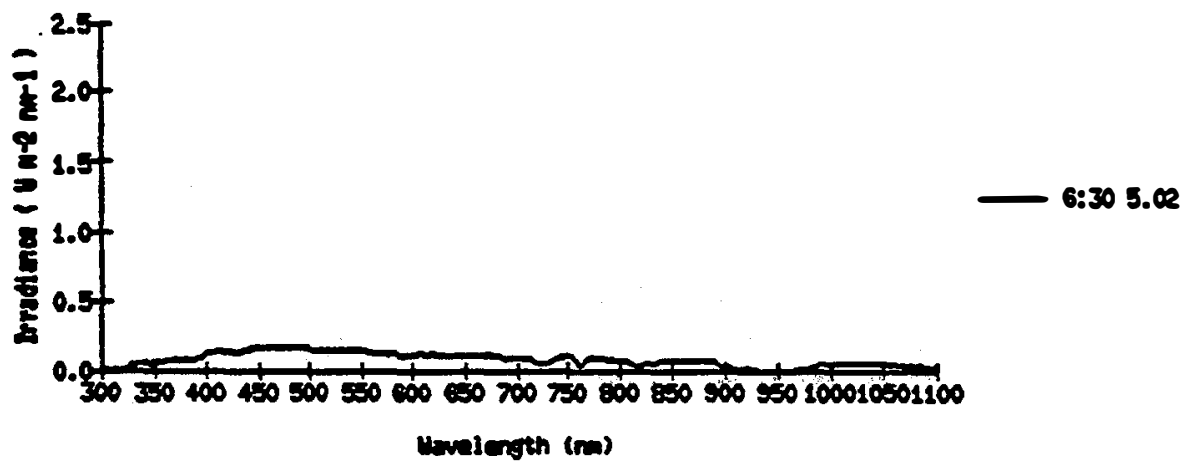


a $\overline{512587190}$

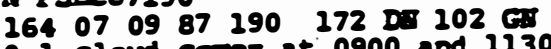

0.1 cloud cover at 0900 and 1130 at $1230,45 \mathrm{C}$ at 13308 44 C at 1430.

LSR 172 teap

$43 \mathrm{C}$ at 1530

Bo slides

Fase 190

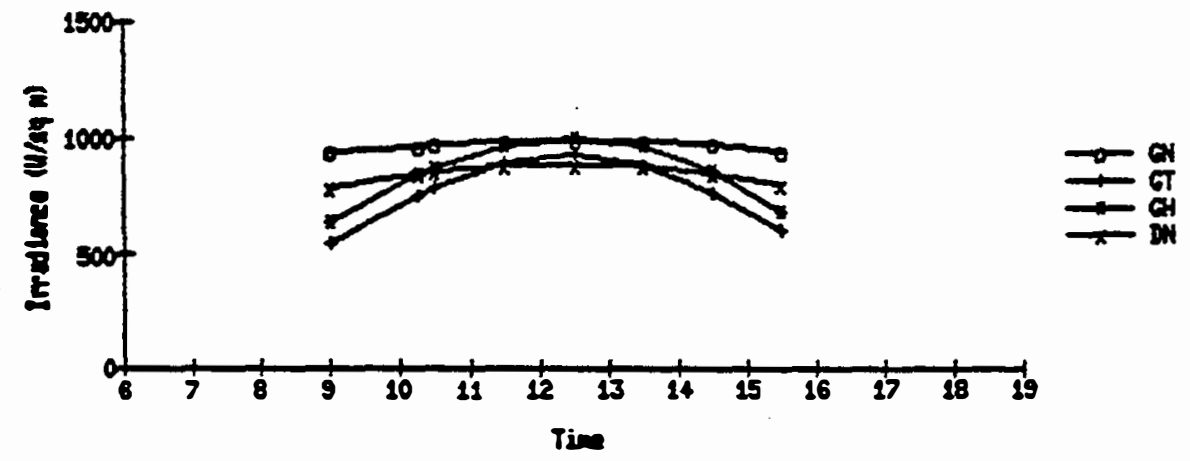

FSEC 190 BNT

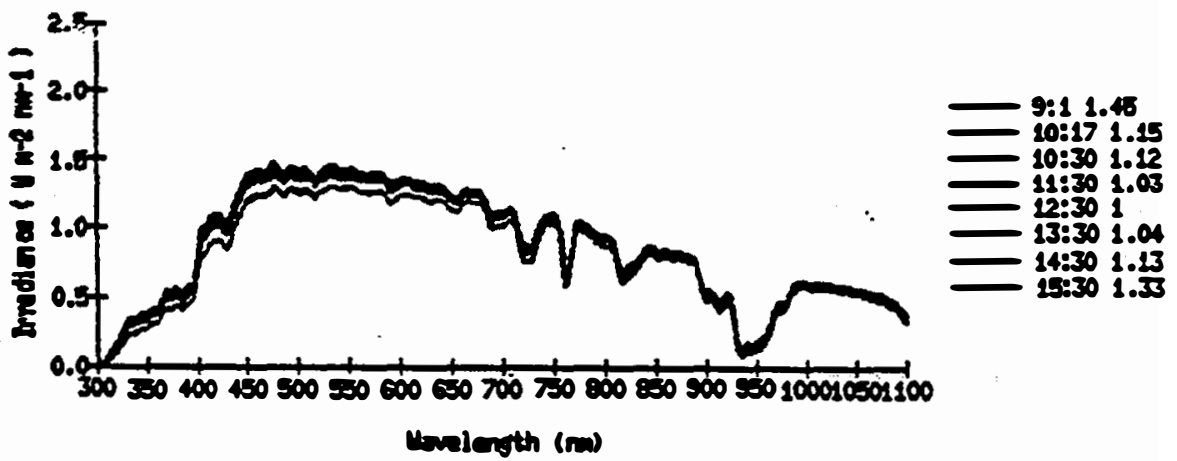

Fas 200 os

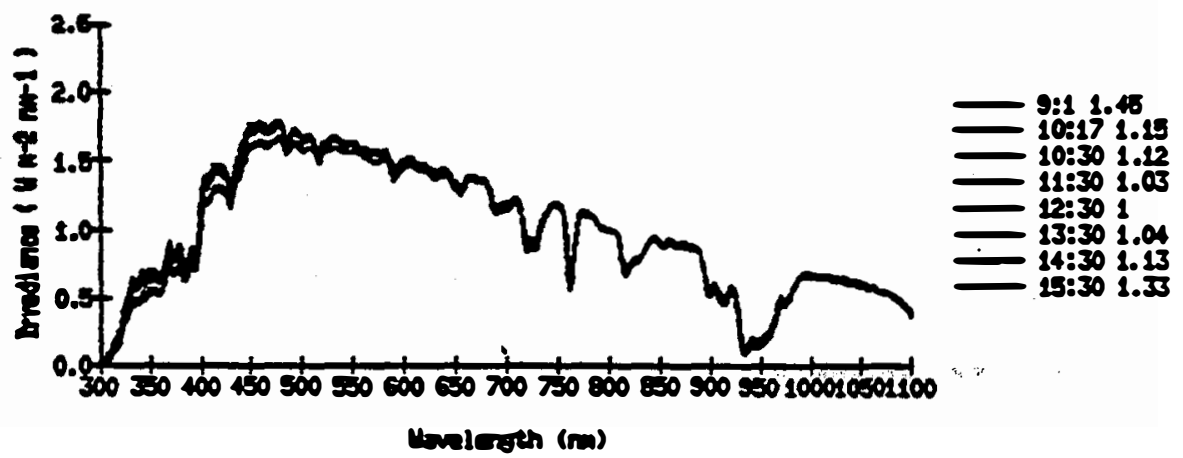


855x87191

$\begin{array}{lllll}16507 & 1087 & 191 & 172 \quad 02 & 0 .\end{array}$

0.2 cloud cover at 0850. 1130. and 1520

IST 172 tenp controller $42 \mathrm{C}$ at 1130 and 1330 , $40 \mathrm{C}$ at 1520

Eo slides

Fsec 291

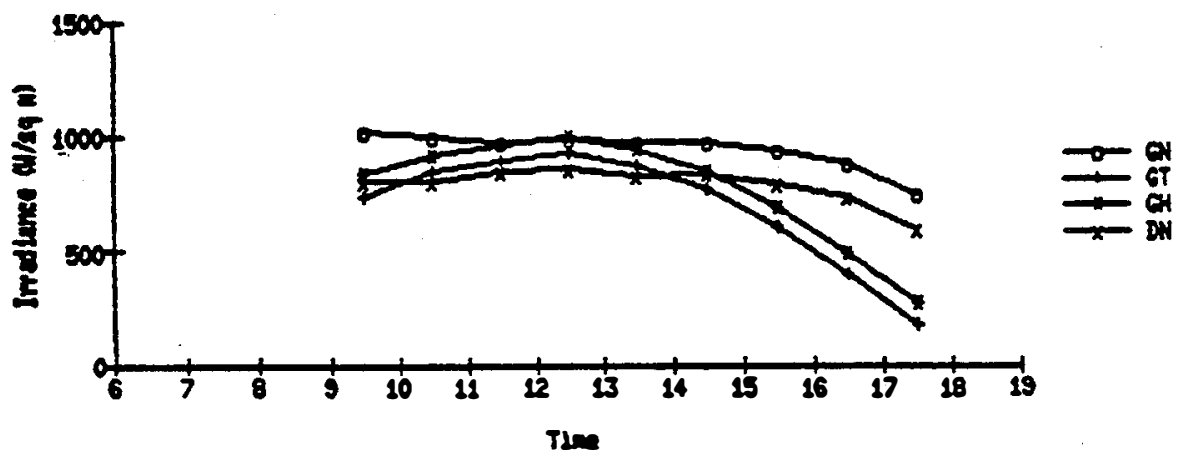

FSEC $191 \mathrm{MT}$

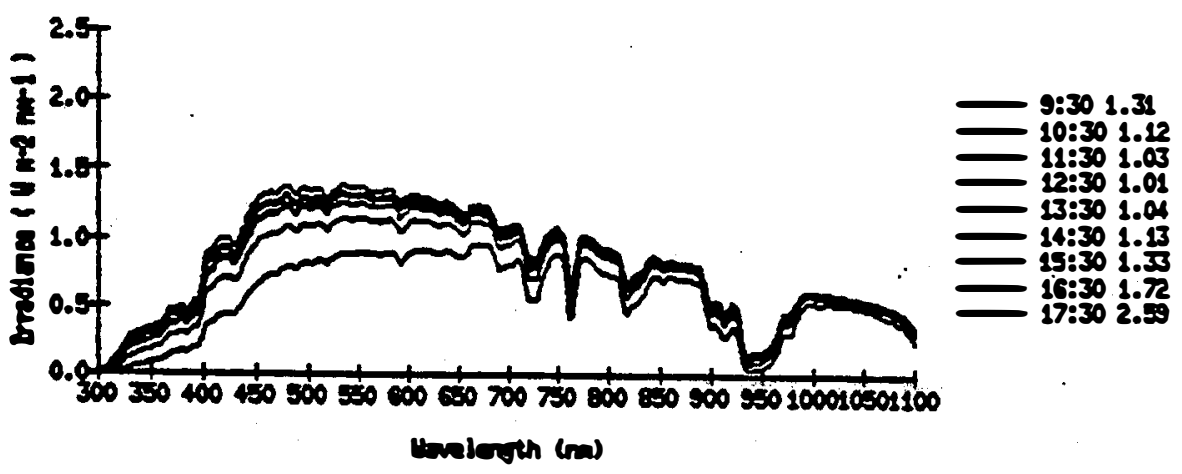

Fase 201 as

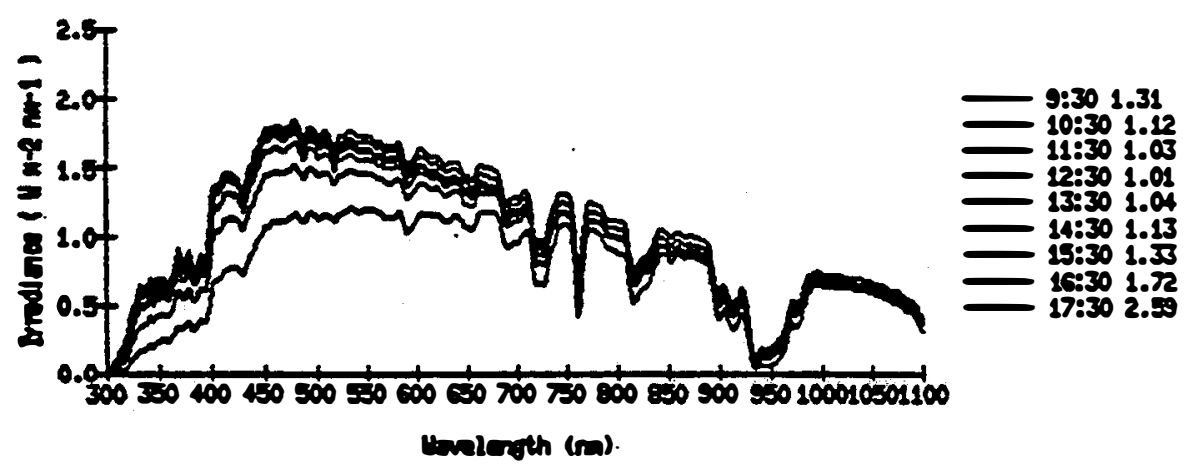


(155087192

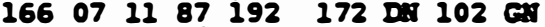

Partly cloudy AY; nostly clear PH

No slides

Brief spike in oO in Gi spectrun at $1230,1330,1430,1530$

FSeC 192

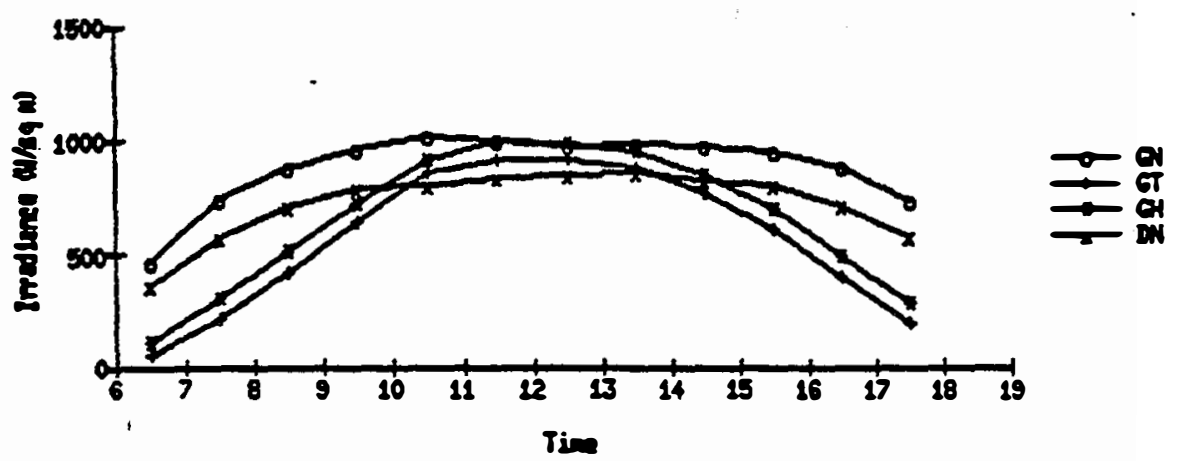

FSEC LSP DIT

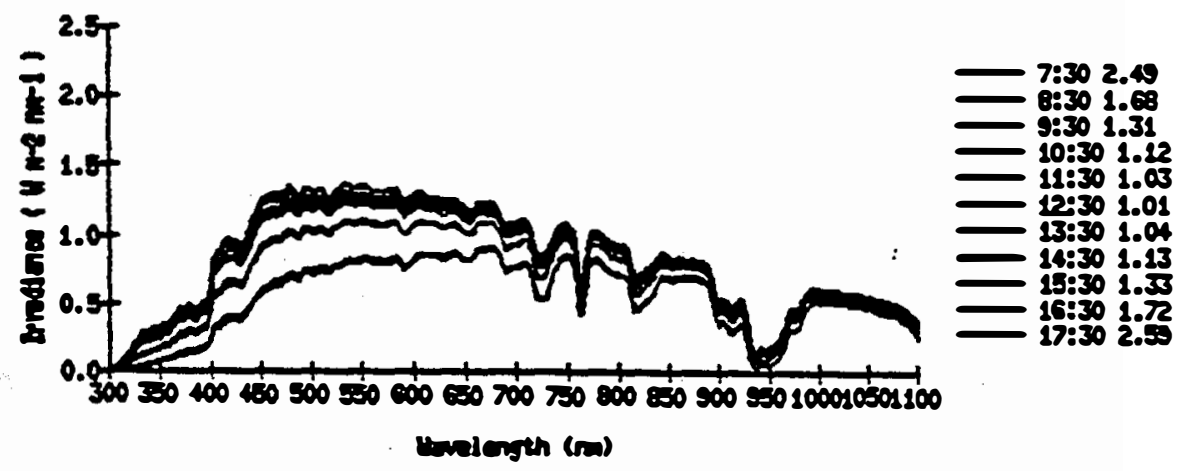

Fase 202 as

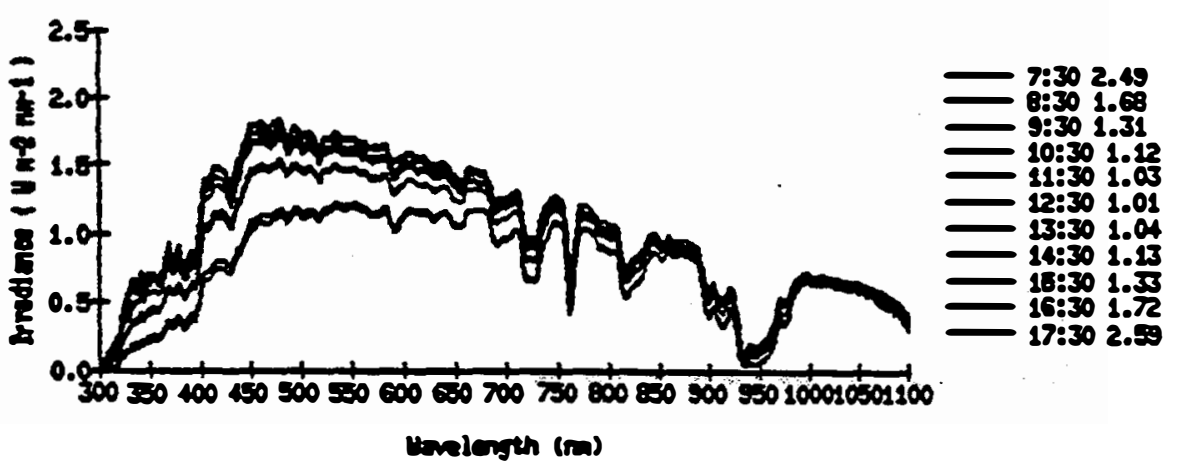


म F52587193

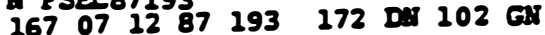

Mostly clear AM; partly cloudy olth rain in PH

Ho slides

Fsec 193

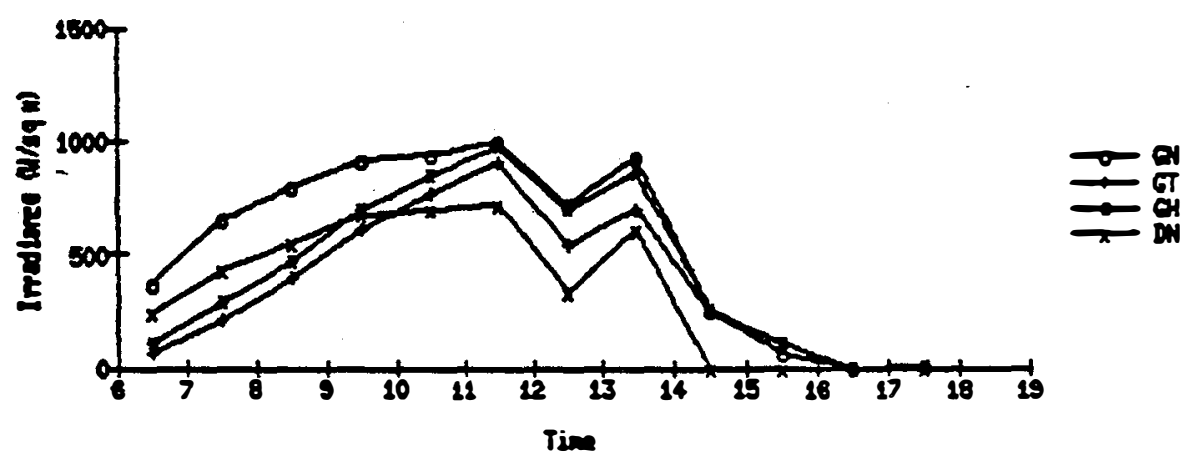

Fesc 193 ont

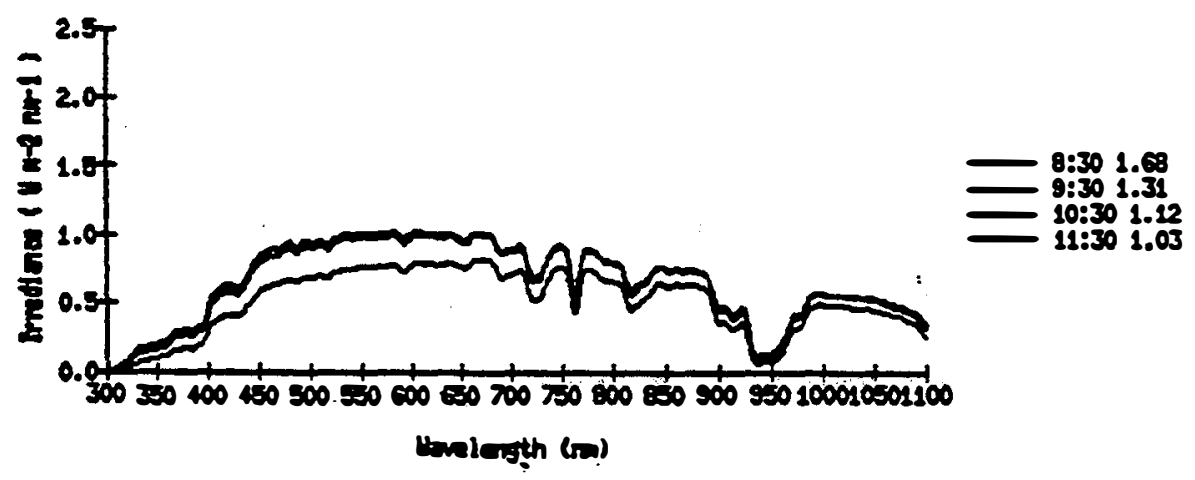

Fasc 293 ax

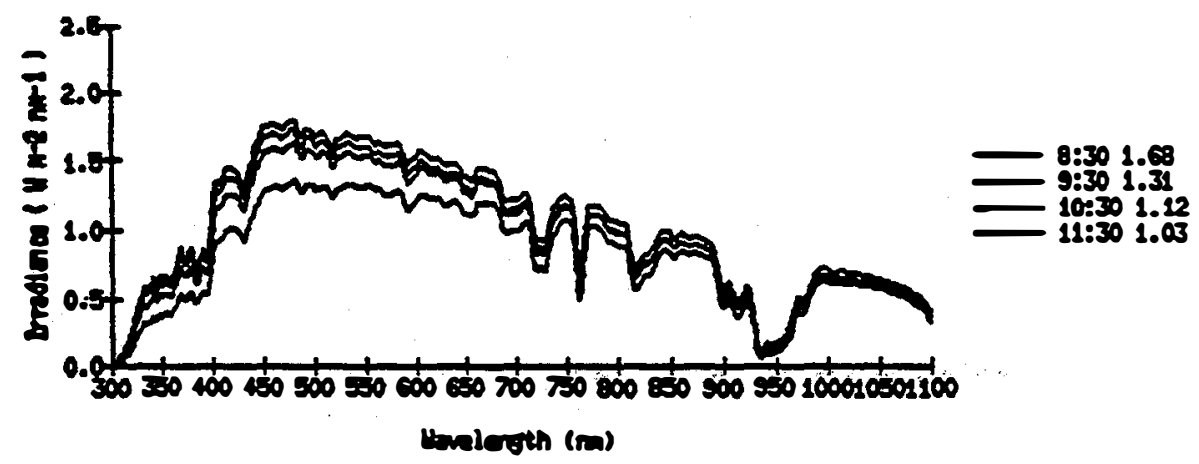


B.5X87194

$168071387194 \quad 172$ DE $102 \mathrm{GM}$

0 cloud cover erith baze at $0730,1.0$ at 1130 , sain at $1300,1.0$ at 1530

Added 5/16" fon "standoffs" to ISR 172 case to separate mlar fros

case surface; pernits afflov betreen mlar and case to prosote better

teap control

Bo slides

FEC 294

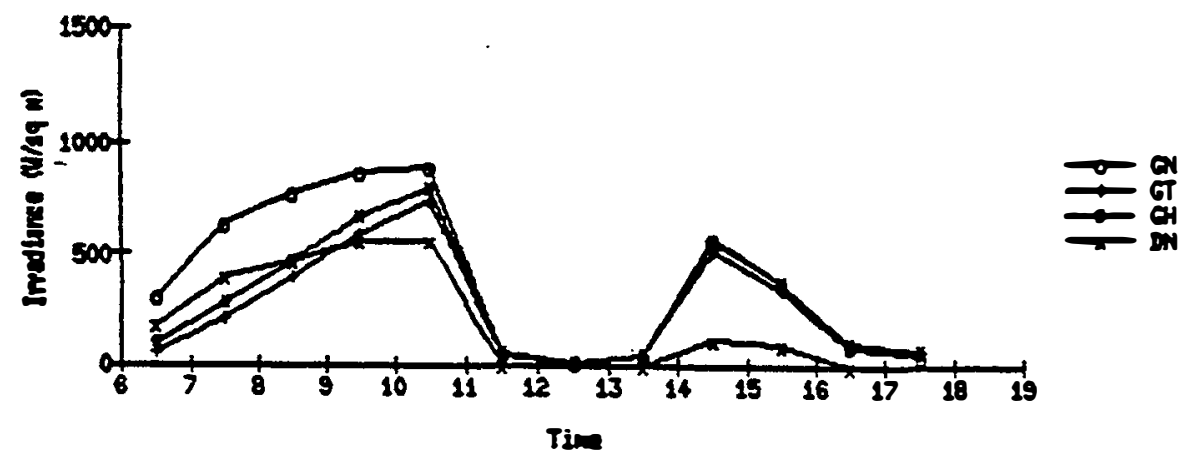

Face 294 ant

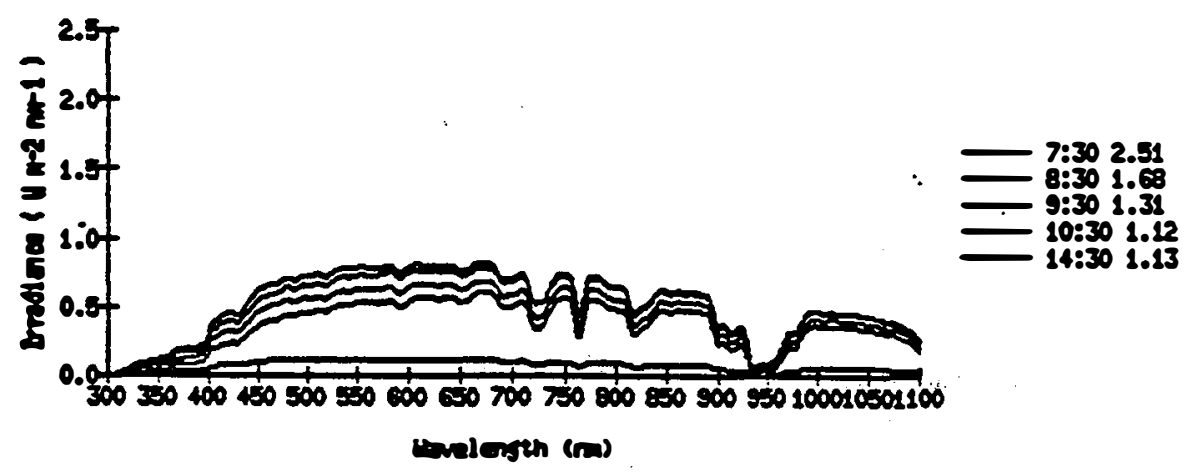

PSEC 194 as

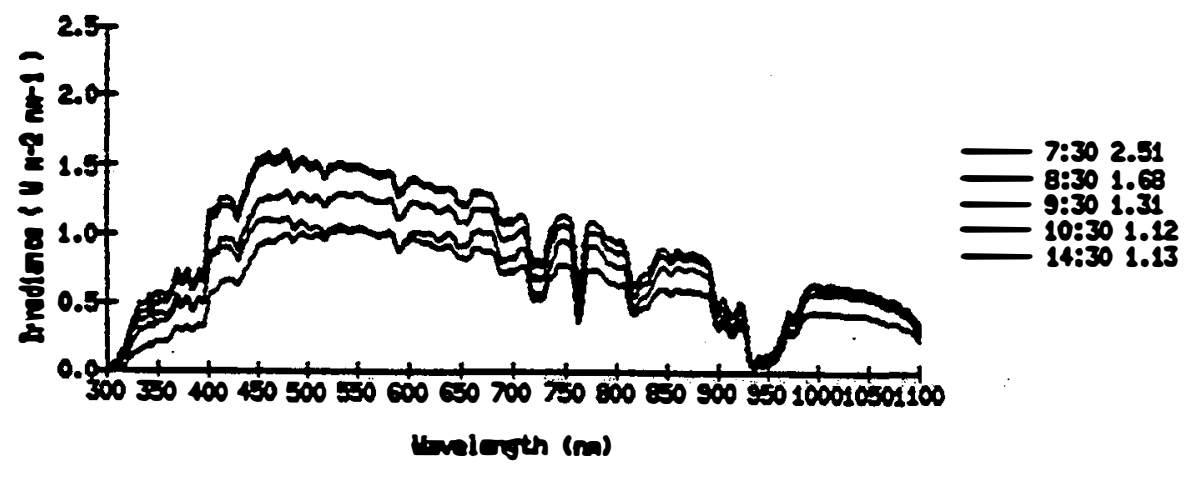


A 55287195

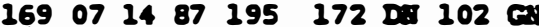

0.3 cloud cover erth baze at 0730; 0.9 at $1130 ; 1.0$ at 1530 , rain

Do slides

Fesc 295

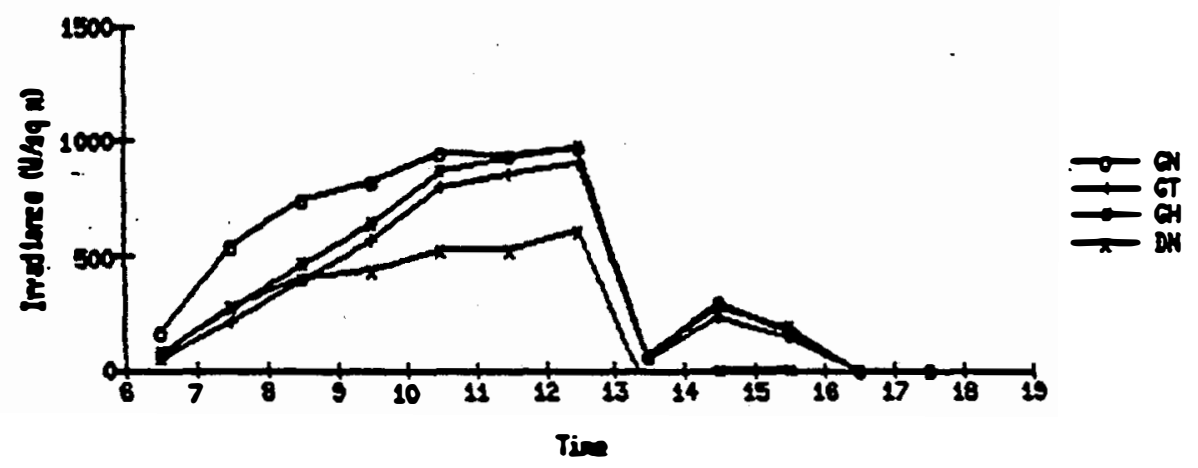

F=ב5 255

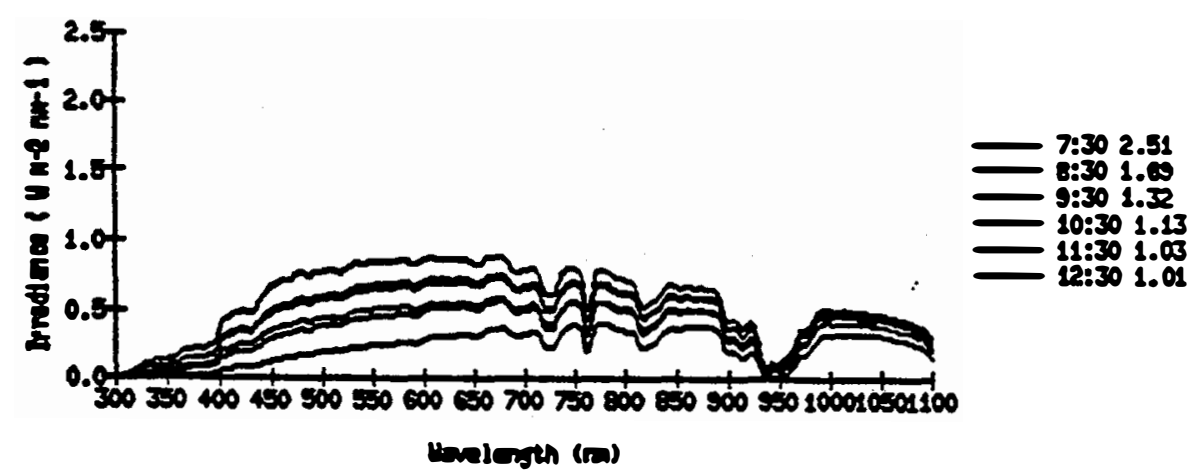

FIS 250 OS

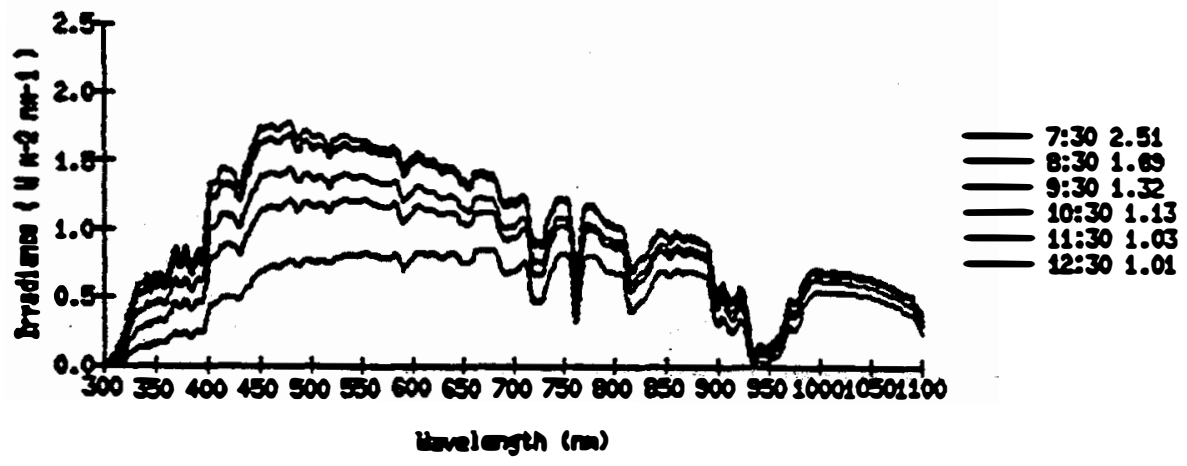


A EST्ट87296

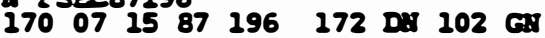

0.8 cloud cover exth haze at $0830 ; 1.0$ at 1130 ; rash at $1230 ; 1.0$ at 1530

No slides

PSEC 296

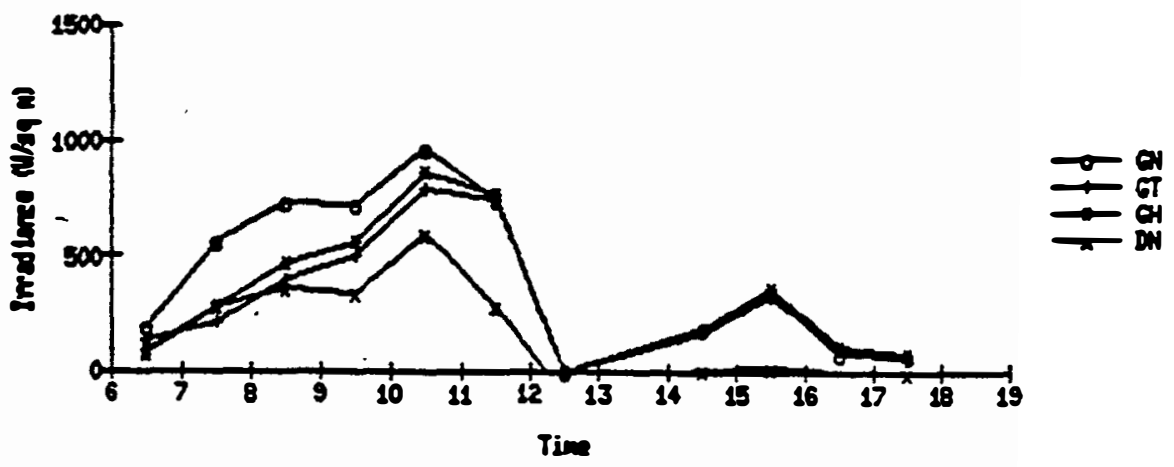

FSEC 296 BNT

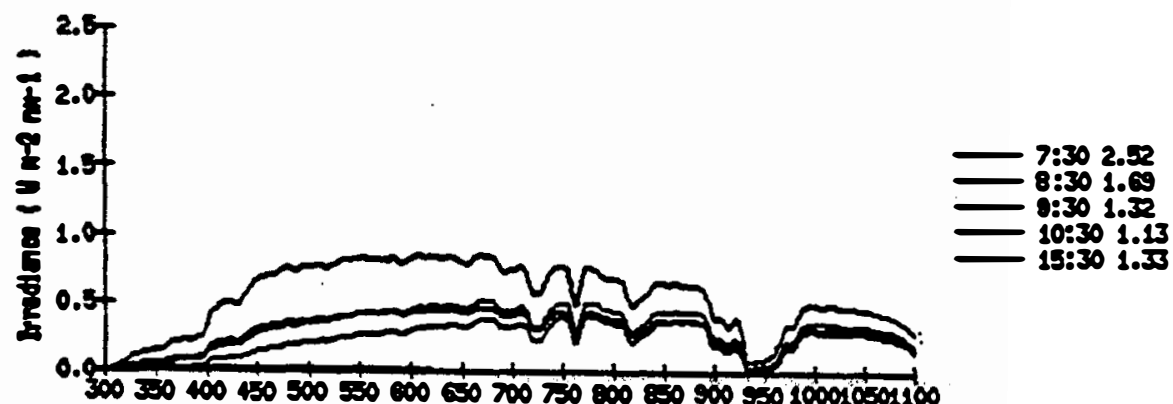

umlagen (m)

Fise 290 os

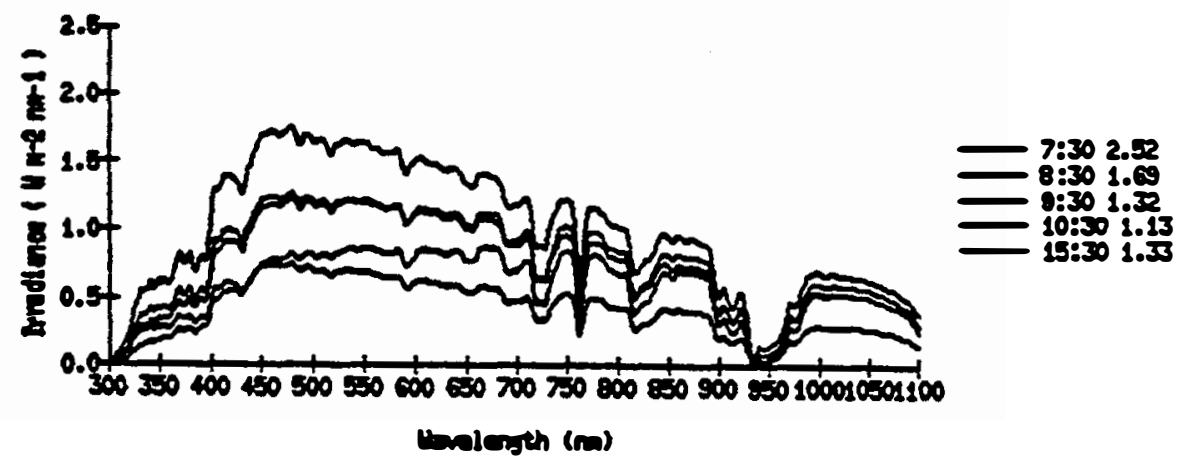


A.

$\begin{array}{lllllllll}171 & 07 & 16 & 87 & 197 & 172 & 09 & 102 & \text { FH }\end{array}$

0.9 cloud cover at 080081.0 at 12008 ras at 1300

Slides: $0830 \mathrm{G}$

$0930 \mathrm{G}$

$1030 \mathrm{G}$

$1130 \mathrm{G}$

1229 M (dnsk)

1329 G (eater drops)

$1429 \mathrm{G}$

15296

$1628 \mathrm{G}$

$1728 \mathrm{G}$

$1828 \mathrm{G}$

Fase 197

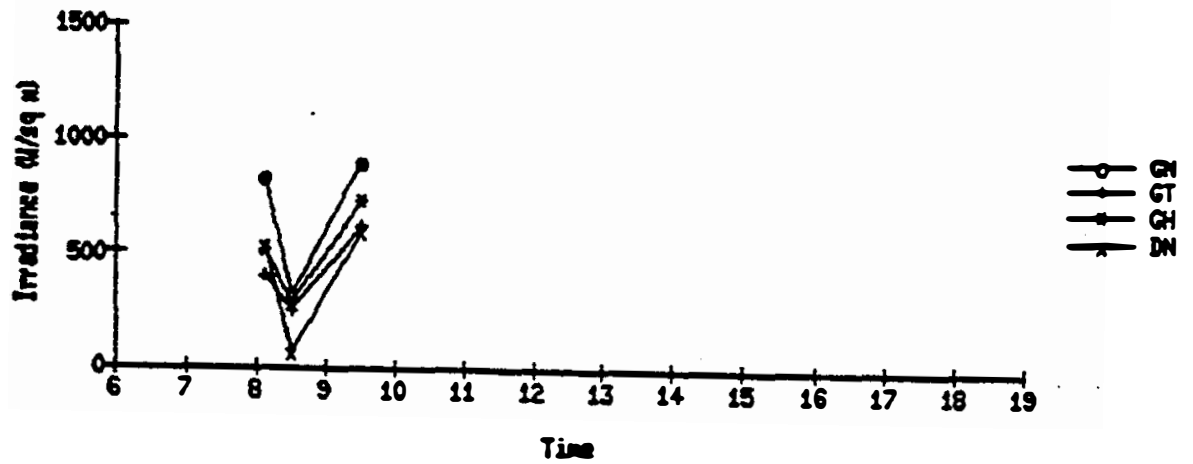

ए5x 297 at

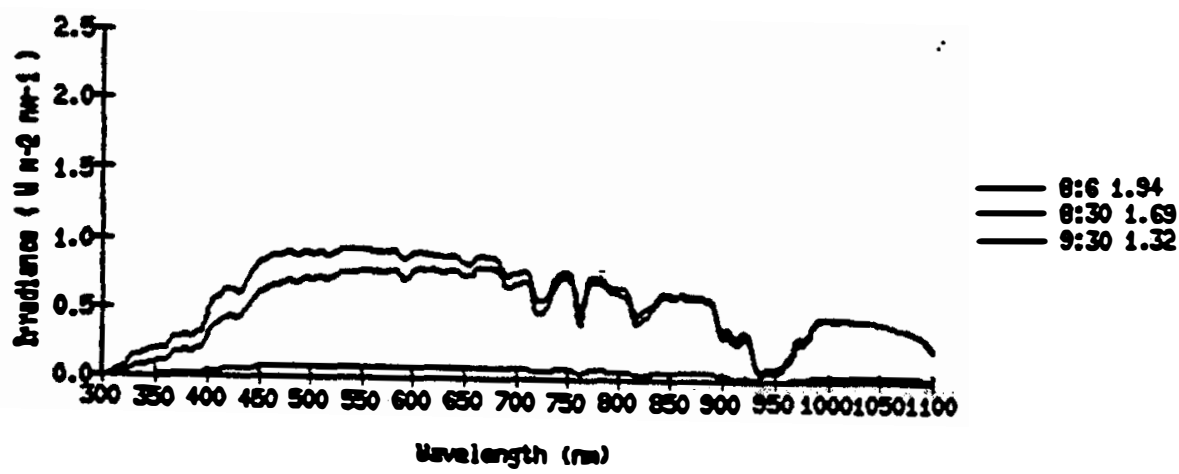

Fase 197 as

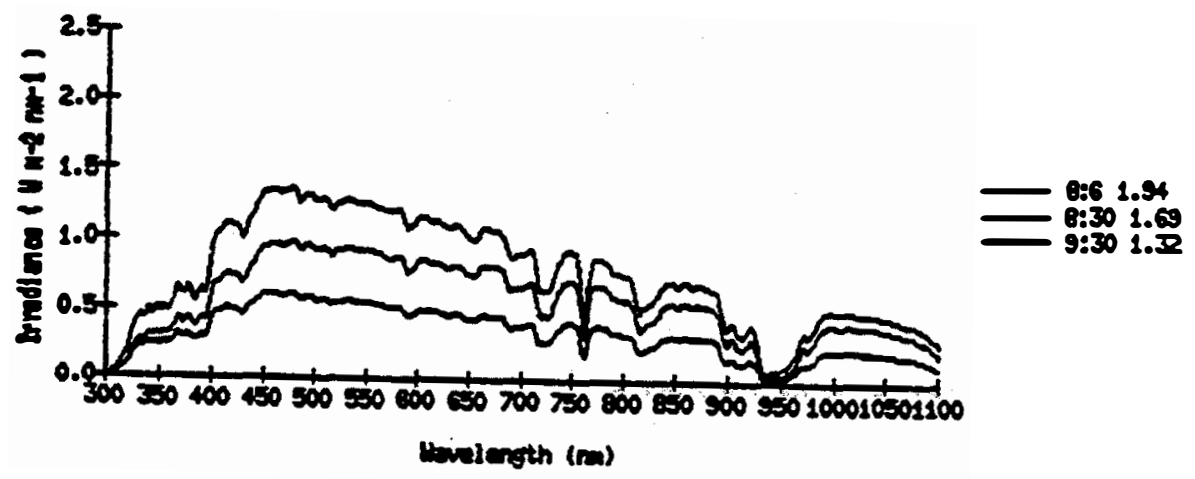




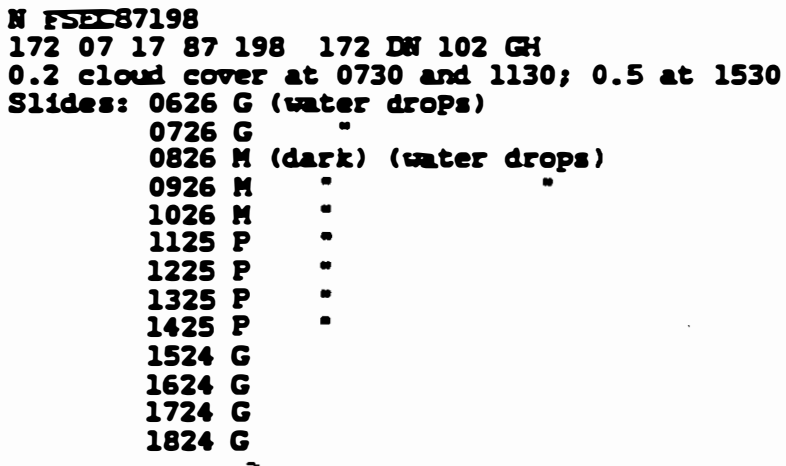

Fase 290

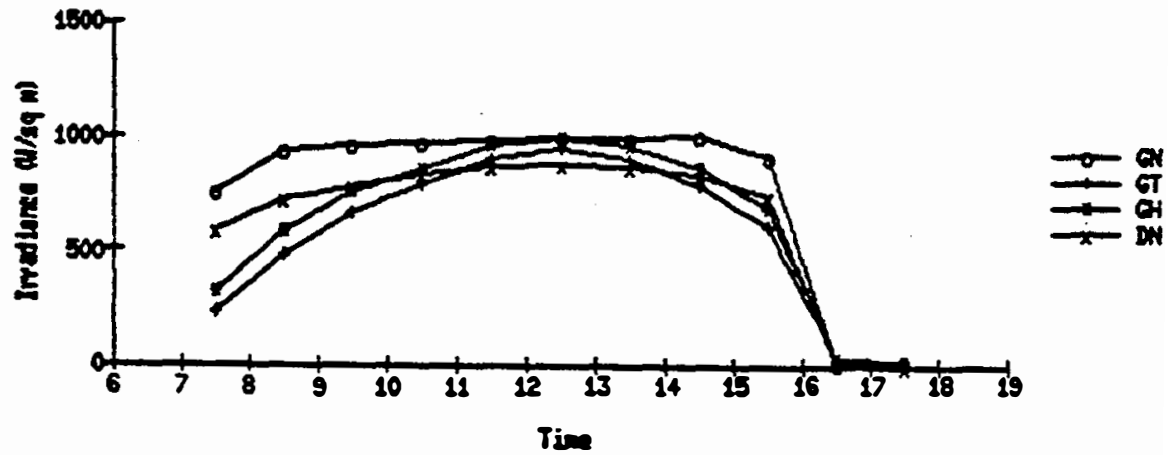

Fsxe 198 ant

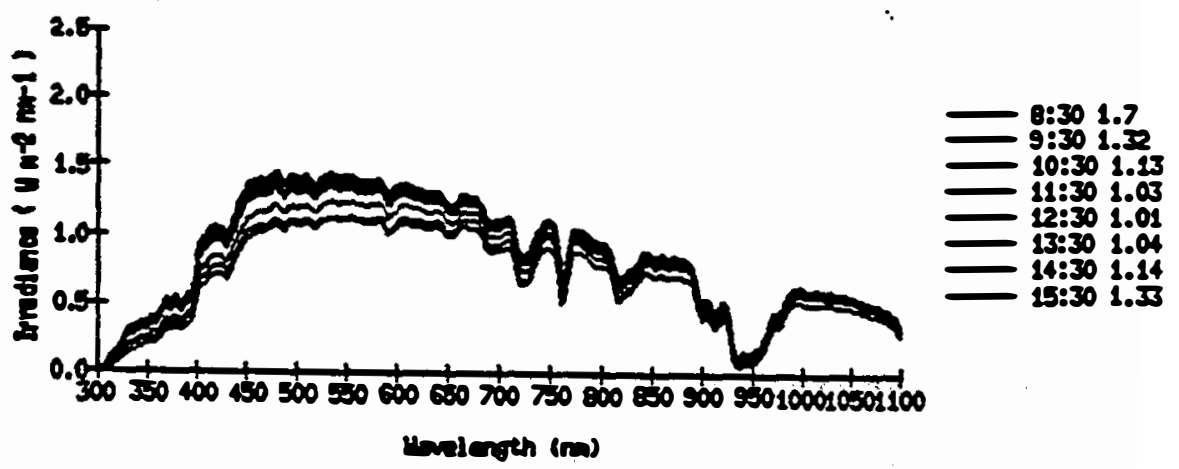

Fist 298 os

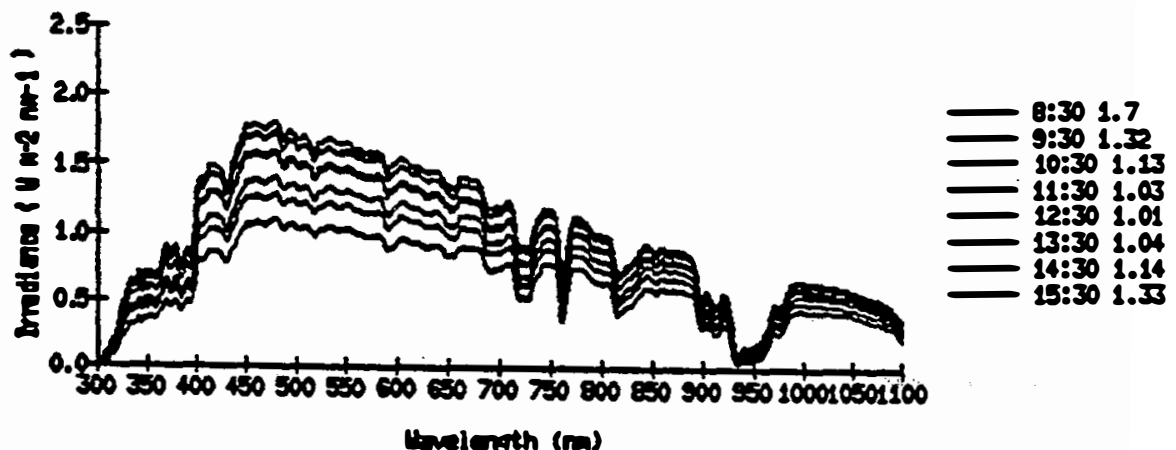


8. FS287201

$\begin{array}{llllllll}173 \quad 07 & 2087 & 801 & 172 & \text { DA } & 102 & \mathrm{GH}\end{array}$

0.1 eloud corer at 0800 ; 0.2 at $1130 ; 0.6$ at 1530

$18 \mathrm{mV}$ drift error on ISR 102 at 0800

slides: $0610 \mathrm{G}$ (rater drops)

$0709 \mathrm{G}$

$0809 \mathrm{G}$

0909 M (dark)

$1009 \mathrm{P}$

108 P

$1208 \mathrm{P}$

$1308 \mathrm{P}$

$1408 P$

$1507 \mathrm{G}$

$1607 \mathrm{G}$

$1707 \mathrm{G}$

$1807 \mathrm{G}$

Ho1se in 00 and near-IR in GH spectron at 0757 and 1130

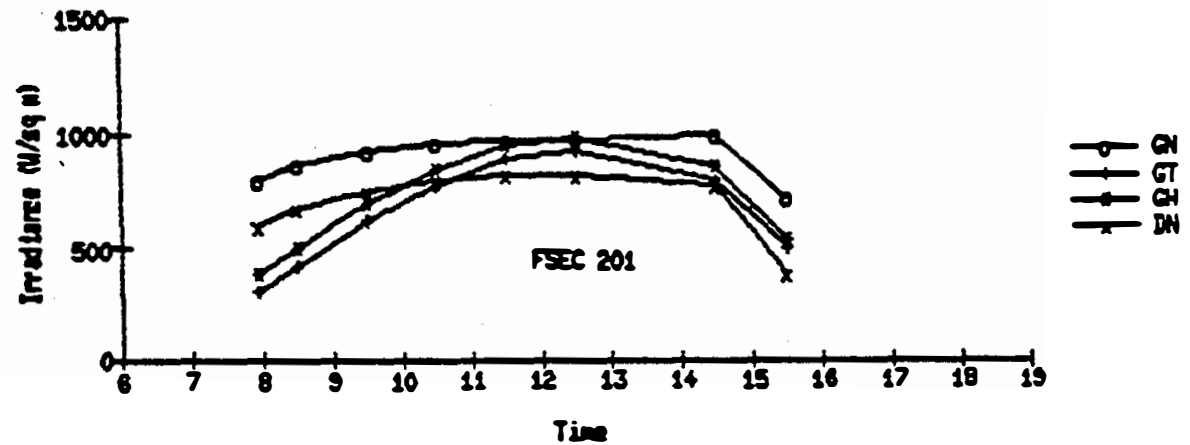

FSEב 201 on

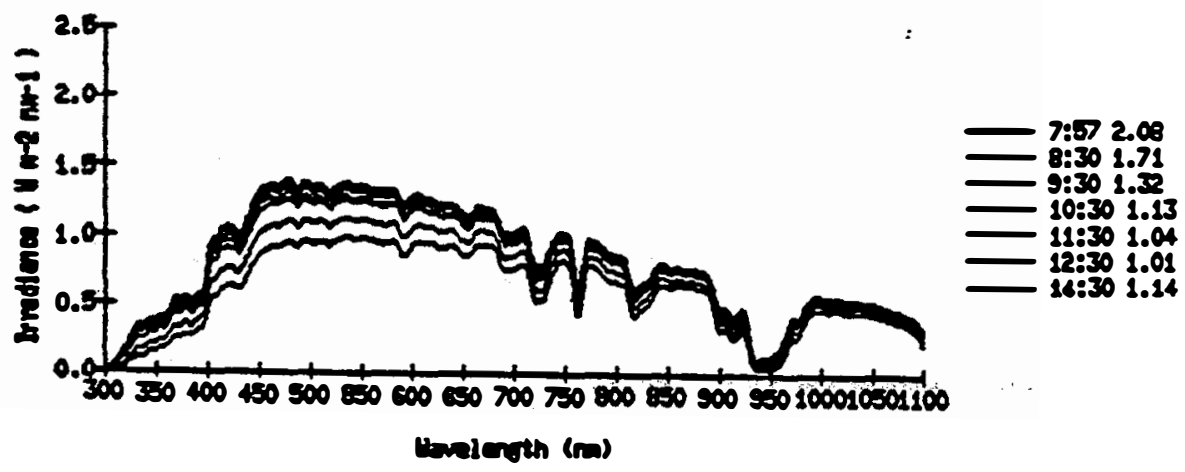

Pase 201 as

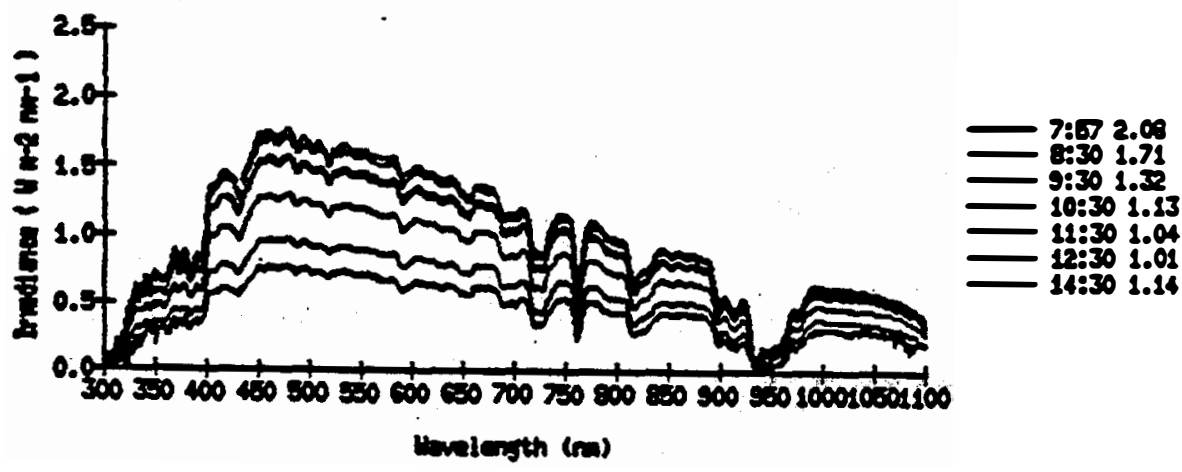


1. F5P 87202

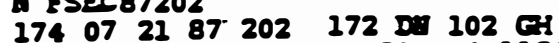

174 cloud cover at 0730 and $1130 ; 0.3$ at 1530

$14 \mathrm{av}$ drift error on ISR 102 at 1130

II aV drift error on ISR 102 at 1530

sildess 0606 G (reter drops)

$0705 \mathrm{G}$

$0805 \mathrm{G}$

0905 i (dark)

$1005 \mathrm{M}$ -

$1105 \mathrm{P}$

$1205 \mathrm{M}:$

G

$1504 \mathrm{G}$

$1604 \mathrm{G}$

$1704 \mathrm{G}$

$1804 \mathrm{G}$

Bolse in 0 and near-IR in GH spectra throughout day

FSeC 200

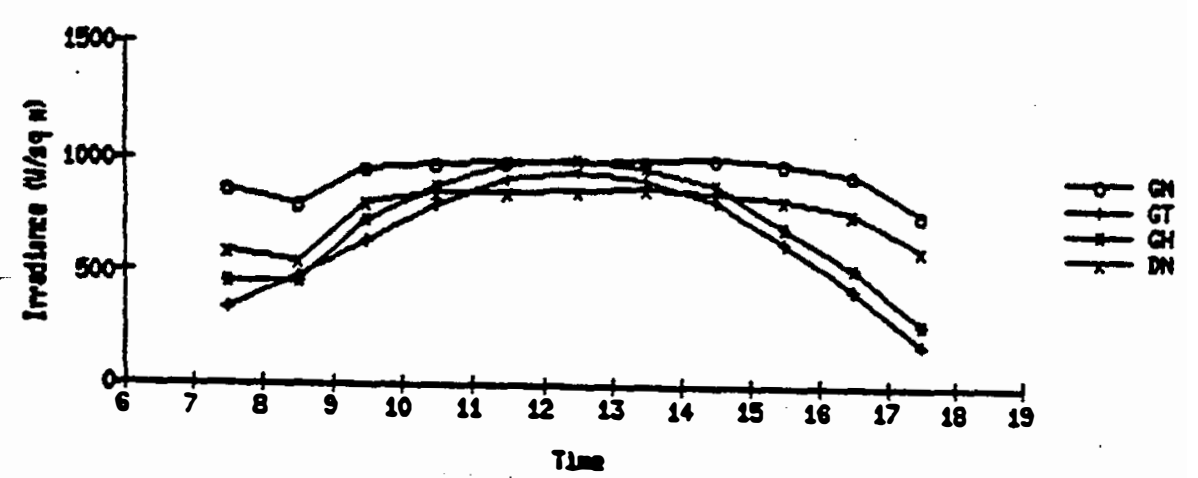

$505202 \mathrm{kr}$

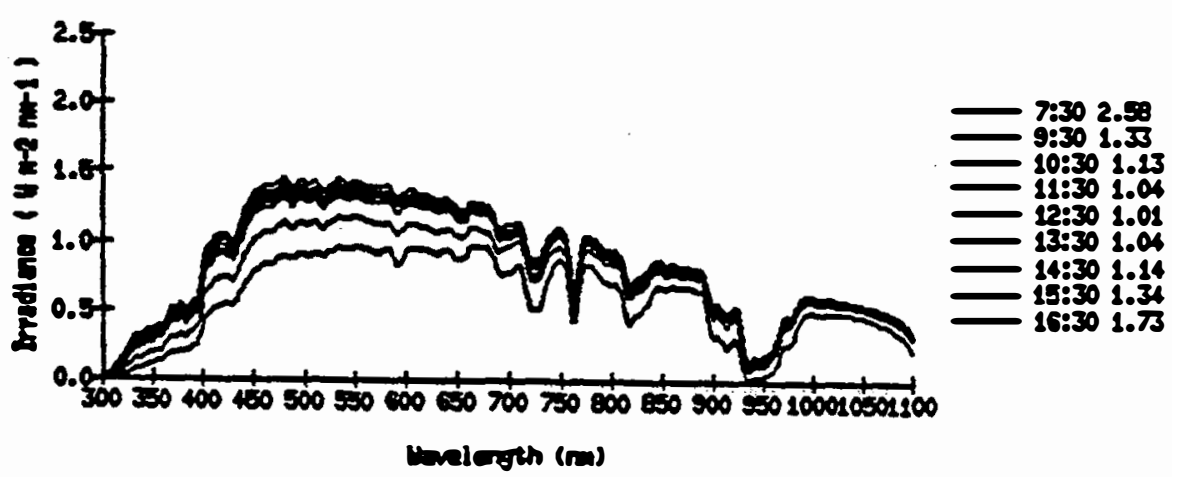

rsac 202 as

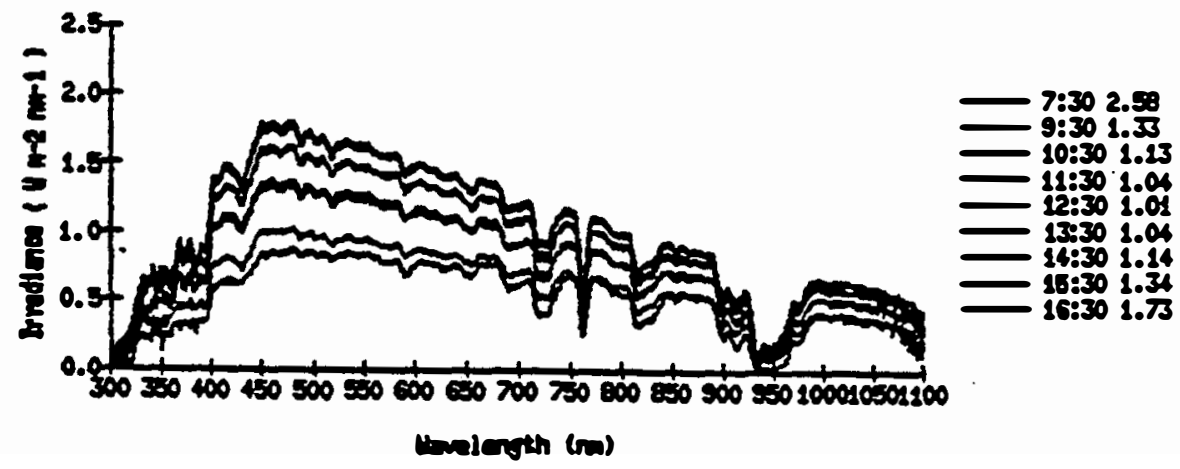




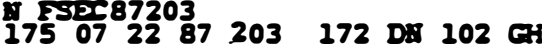

0.2 cloud cover at 0730: 0.4 at $1130 ; 0.2$ at 1530

17 av drift error on ISR 102 at 1130

S11des: $0602 \mathrm{G}$

$0701 \mathrm{G}$

$0801 \mathrm{G}$

$0901 \mathrm{G}$

$1001 \mathrm{G}$

$1101 \mathrm{P}$ (dark)

$1201 \mathrm{P}$

$1301 P$

1400 P

$1500 \mathrm{H}$.

$1600 \mathrm{G}$

$1700 \mathrm{G}$

$1800 \mathrm{G}$.

Woise in 0 and near-IR in GH spectra throughout day

Pase 203

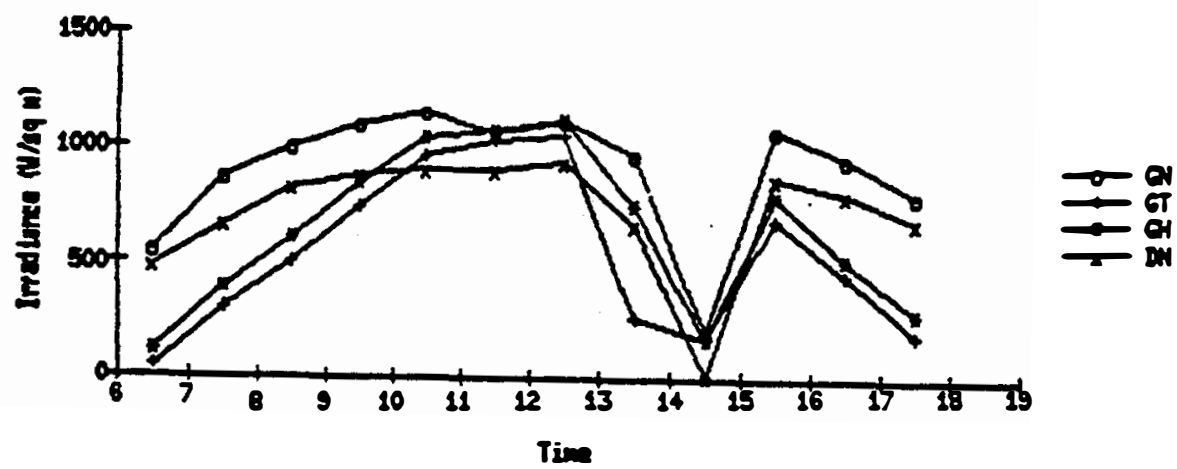

Fise 203 in

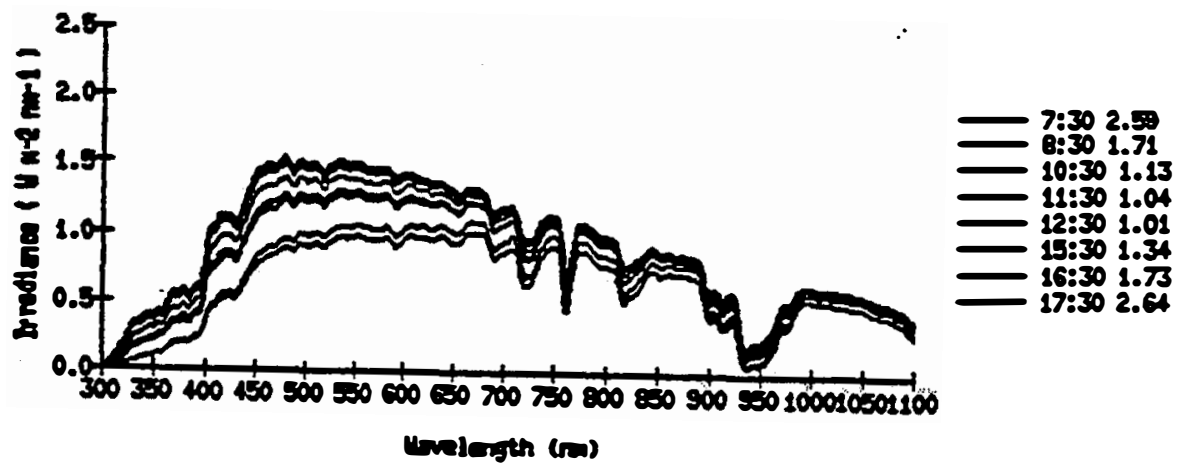

Fere 2003015

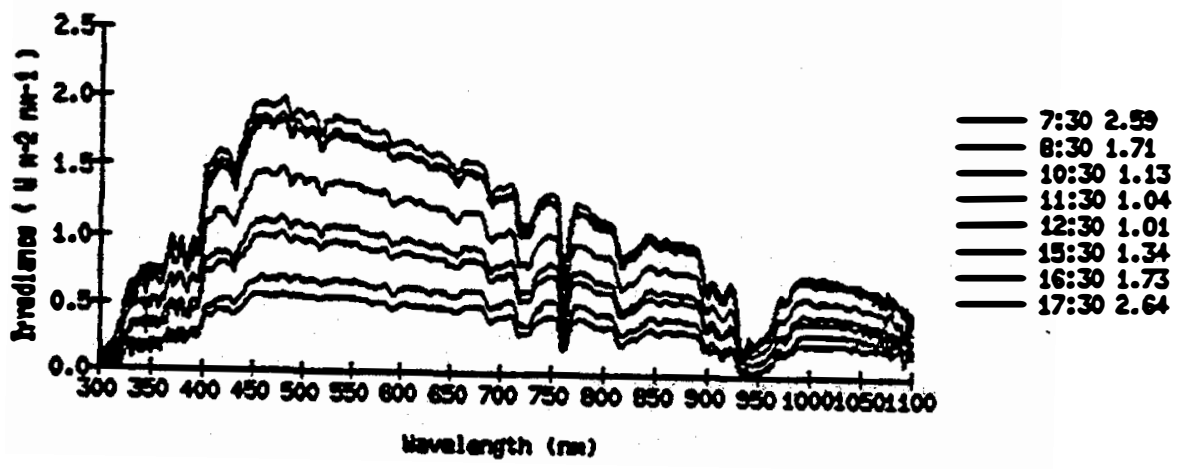


A. FSיC87204

$\begin{array}{llllll}176 & 07 \quad 3 \quad 87 & 204 & 172 \quad 02 & 102\end{array}$

0.4 cloud corer (11ght baze): 0.7 at 1130

Temp controllers: 172102

$\begin{array}{llll}1130 & 44 \mathrm{C} & 43 \mathrm{C} \text {. T(a-b) } 30.8 \mathrm{C} \text {, wad speed } 2.4 \mathrm{als} \\ 1330 & 44 \mathrm{C} & 45 \mathrm{C} \text {. } \\ 31.6 \mathrm{C} \text {. } & \end{array}$

$153043 \mathrm{C} 45 \mathrm{C}$. $31.4 \mathrm{C}, \quad 3.0$

(tenp controller turned off for 45 minutes at 1530)

Sl1desz $0558 \mathrm{G}$

$0658 \mathrm{G}$

$0758 \mathrm{G}$

$0858 \mathrm{M}$ (dnrk)

$1024 \mathrm{G}$

$1124 \mathrm{H}$ (dingk)

$1229 \mathrm{G}$

$1328 \mathrm{G}$

$1428 \mathrm{G}$

$1528 \mathrm{G}$

$1628 \mathrm{G}$

$1728 \mathrm{G}$

$1827 \mathrm{G}$

Fsec 204

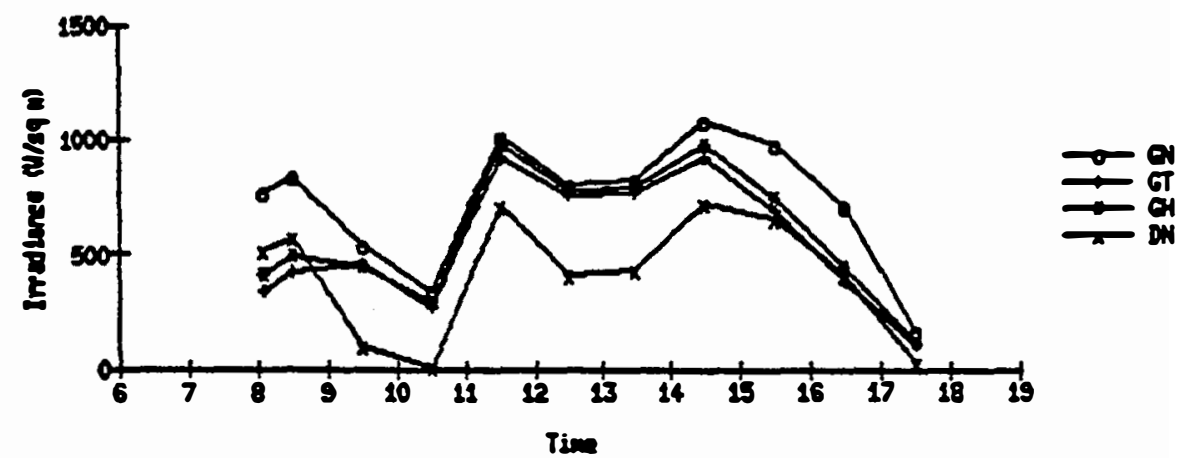

PES 204 DT

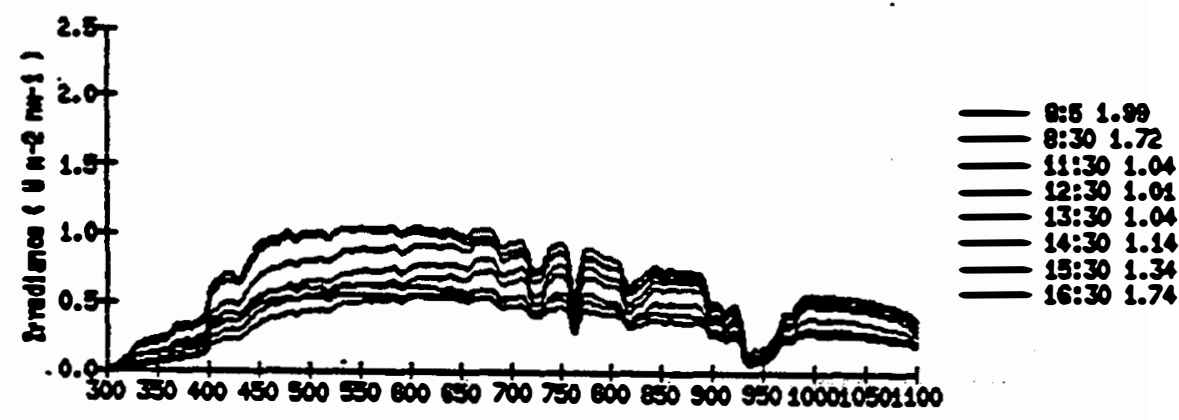

Hovelardh (m)

Pax 204 GTS

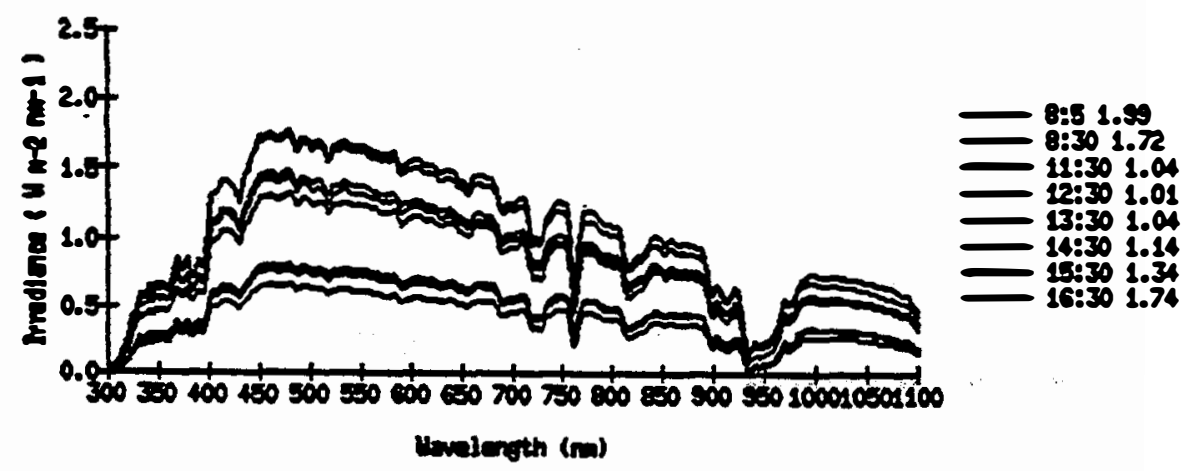


1.

$177072487205 \quad 172$ DN 102 GI

o.l cloud cover wtb baze at 0730; 0.4 at 1230 and 1530

Teap controller: $172 \quad 102$

0830 40 C $40 \mathrm{C}$. I(amb) $28.2 \mathrm{C}$, wind speed $1.7 \mathrm{~m} / \mathrm{s}$

29.7 C. 2.4

$\begin{array}{lllll}0940 & 41 \mathrm{C} & 40 \mathrm{C} & 30.7 & 2.7 \\ 1230 & 40 \mathrm{C} & 40 \mathrm{C} & 30.7 & 4.3 \\ 1500 & 40 \mathrm{C} & 40 \mathrm{C} & 30.7 & \end{array}$

2500

$0724 G$

$0824 G$

$0924 \mathrm{H}$ (dark)

$2023 \mathrm{M}$.

$2123 \mathrm{M}$

$2223 \mathrm{M}$

$1323 \mathrm{G}$

1422 i dark)

$1522 G$

$2622 G$

$1722 \mathrm{G}$

$2822 \mathrm{G}$

Dis spectron at 1233 is bad

Broadiand unserble

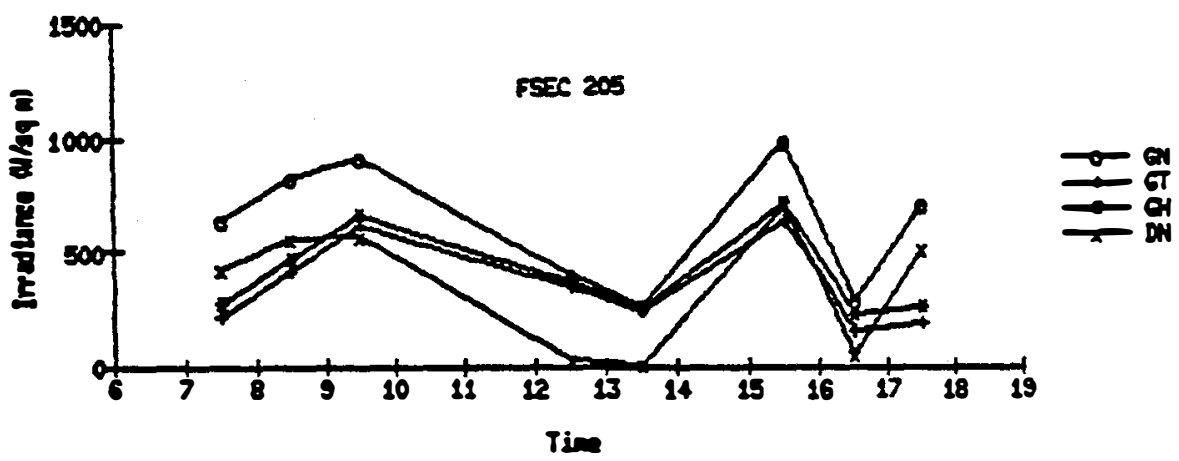

Fax 205 ont

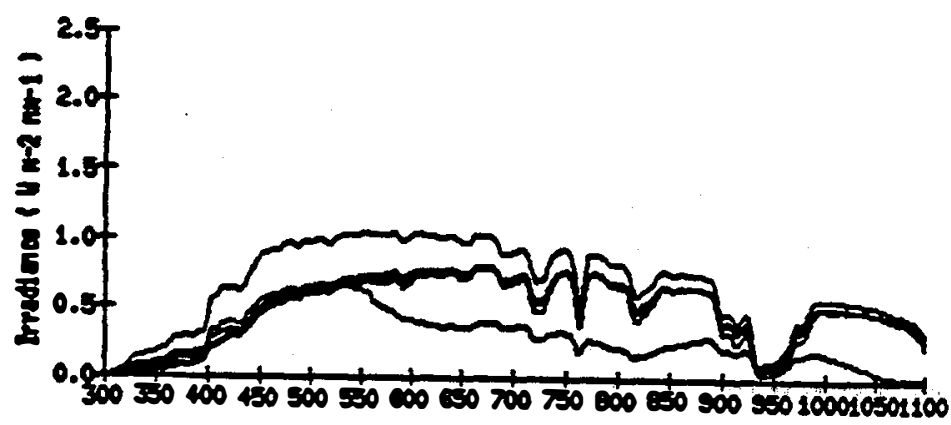

$8: 301.78$ $9: 301.33$ $12: 311.01$

$13: 311.04$

25:31 1.35

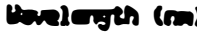

PIE 200 GTS

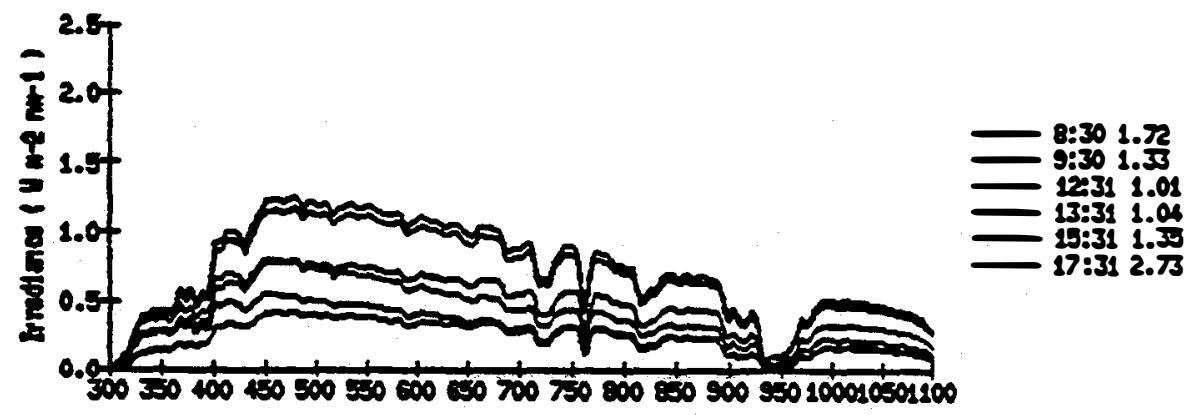

Unvilergth (m) 
\& 252087208

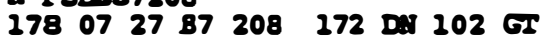

0 eloud cover at $0800,0.3$ at 1130 and 1530

Ien controllers $172 \quad 102$

$\begin{array}{lll}1230 & 12 \mathrm{C} & 40 \mathrm{C}\end{array}$

$1300 \quad 42 \mathrm{C} 40 \mathrm{C}$

$\begin{array}{lll}2430 & 40 \mathrm{C} & 40 \mathrm{C}\end{array}$

a drste errors on ISR 102 at 1130

Slides: $0612 \mathrm{G}$.

$0722 G$

08226

0922 \& (dnzt)

$2012 \mathrm{M}$.

$2120 \mathrm{P}$

$1212 \mathrm{P}$

$2320-2$

$2420 \mathrm{M}$

26096

$2709 \mathrm{G}$

28096

Spike in of in GI spetrun at 1131
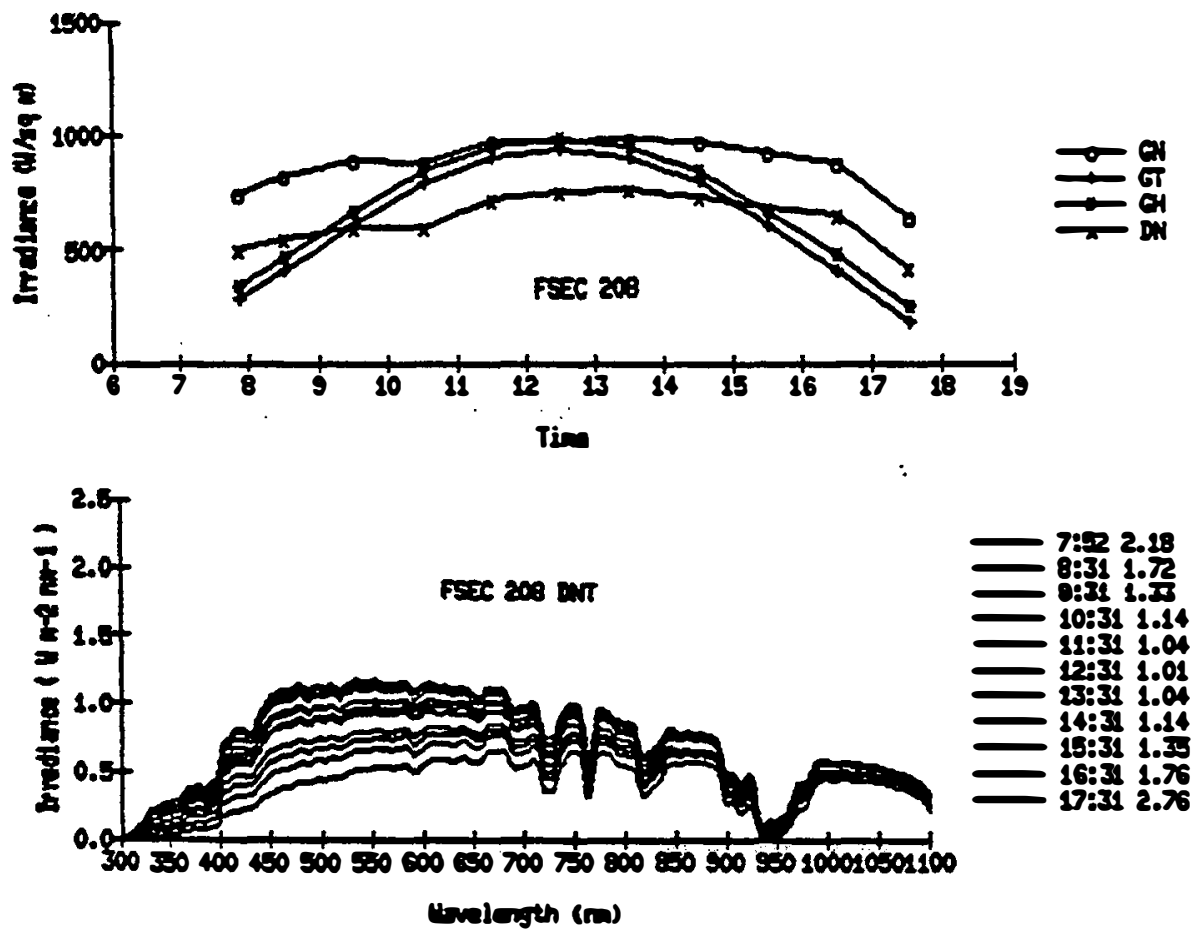

PEx 208 615

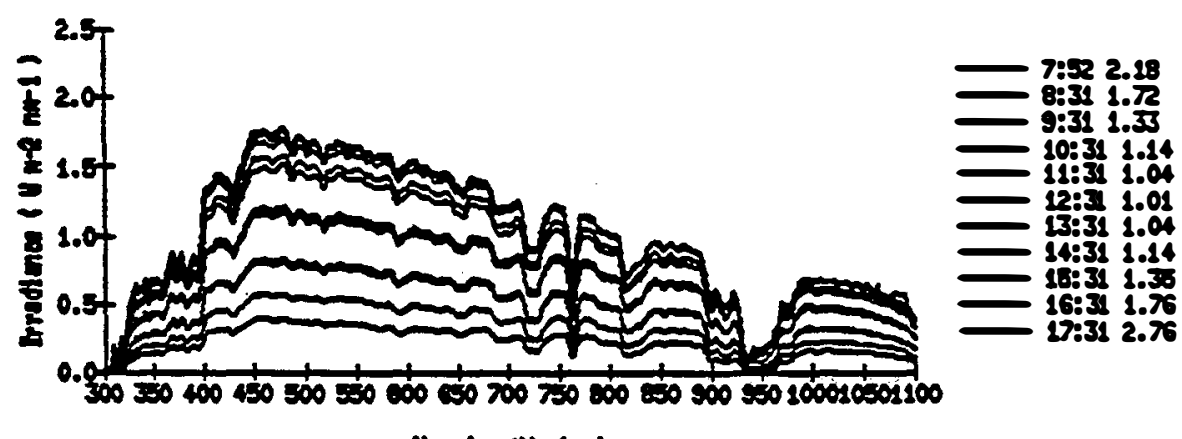

Utulerght (r) 


\section{$\mathrm{SEP} *$}

N P्ST 97209

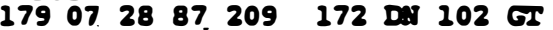

0.4 cloud cover at $0730,0.1$ at $1130 ; 0.2$ at 1530

Teap controller: $172 \quad 102$

$1230 \quad 45 \mathrm{C} \quad 42 \mathrm{C}$

$123043 \mathrm{C} \quad 41 \mathrm{C}$

$\begin{array}{llll}2430 & 42 \mathrm{C} & 40 \mathrm{C} \\ 2530 & 41 \mathrm{C} & 40 \mathrm{C}\end{array}$

av drift error on ISR 102 at 1230, 1330, 1430, and 1530

S1Ldes: $0606 \mathrm{G}$

$0706 \mathrm{G}$

$0805 \mathrm{G}$

$0905 \mathrm{M}$ (dark)

$2005 \mathrm{H}:$

$2105 \mathrm{P}$

$2204 \mathrm{P}$

$2304 \mathrm{P}$

$1503 \mathrm{M}$.

$2603 \mathrm{G}$

$1703 \mathrm{G}$

$1803 \mathrm{G}$

Spikes in OV and near-IR in GI spectra at 0831, 1231, 1331

Vers noiss Gr spectra at 2431 and 1531
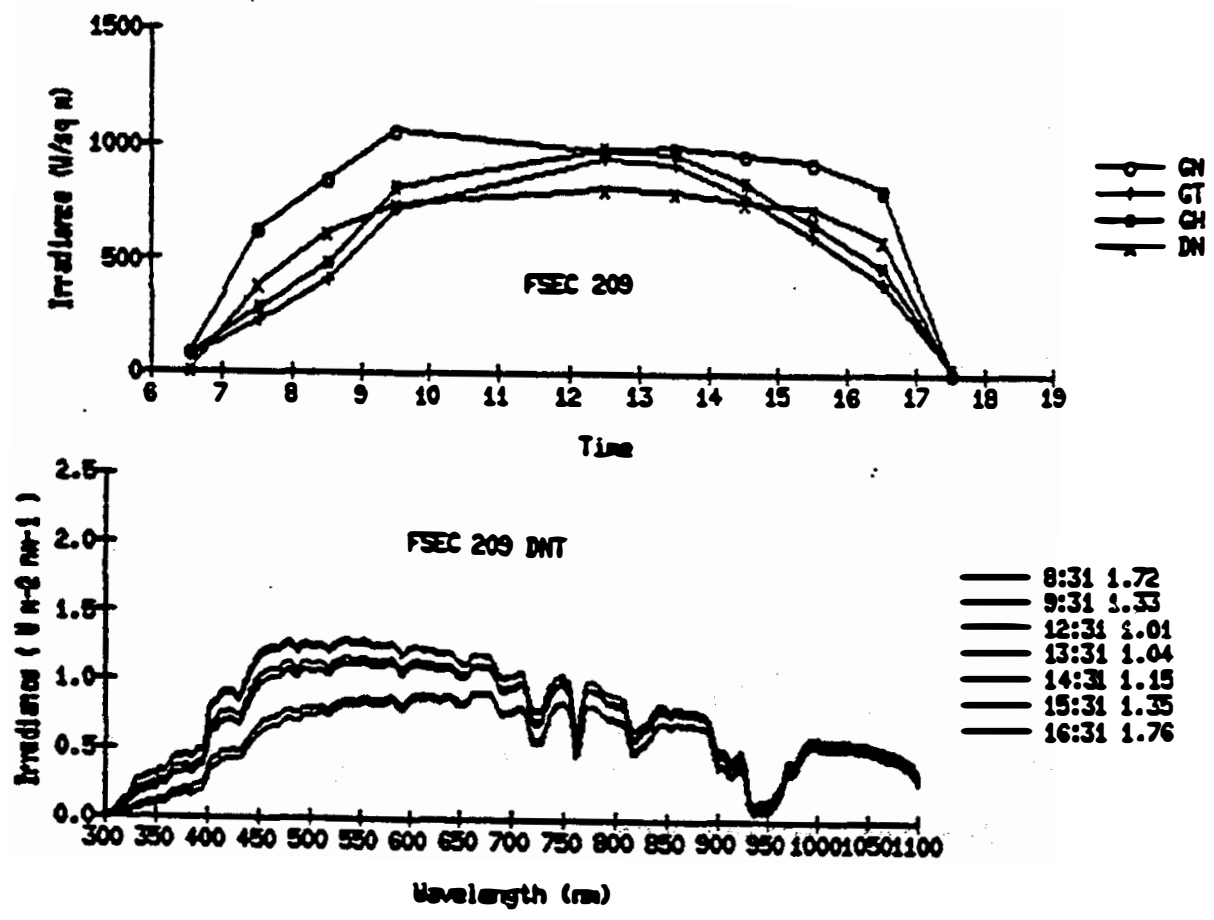

Fis 200 GTS

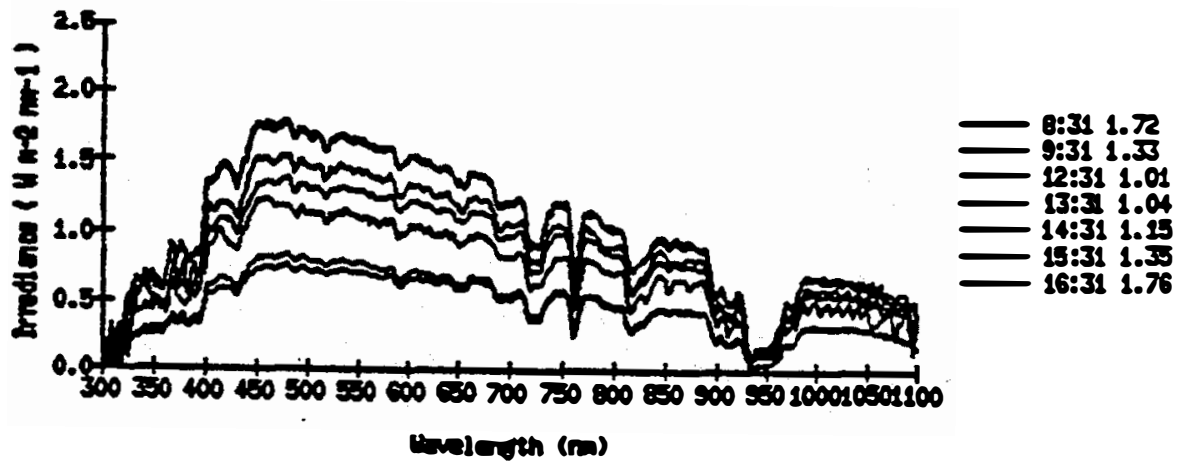




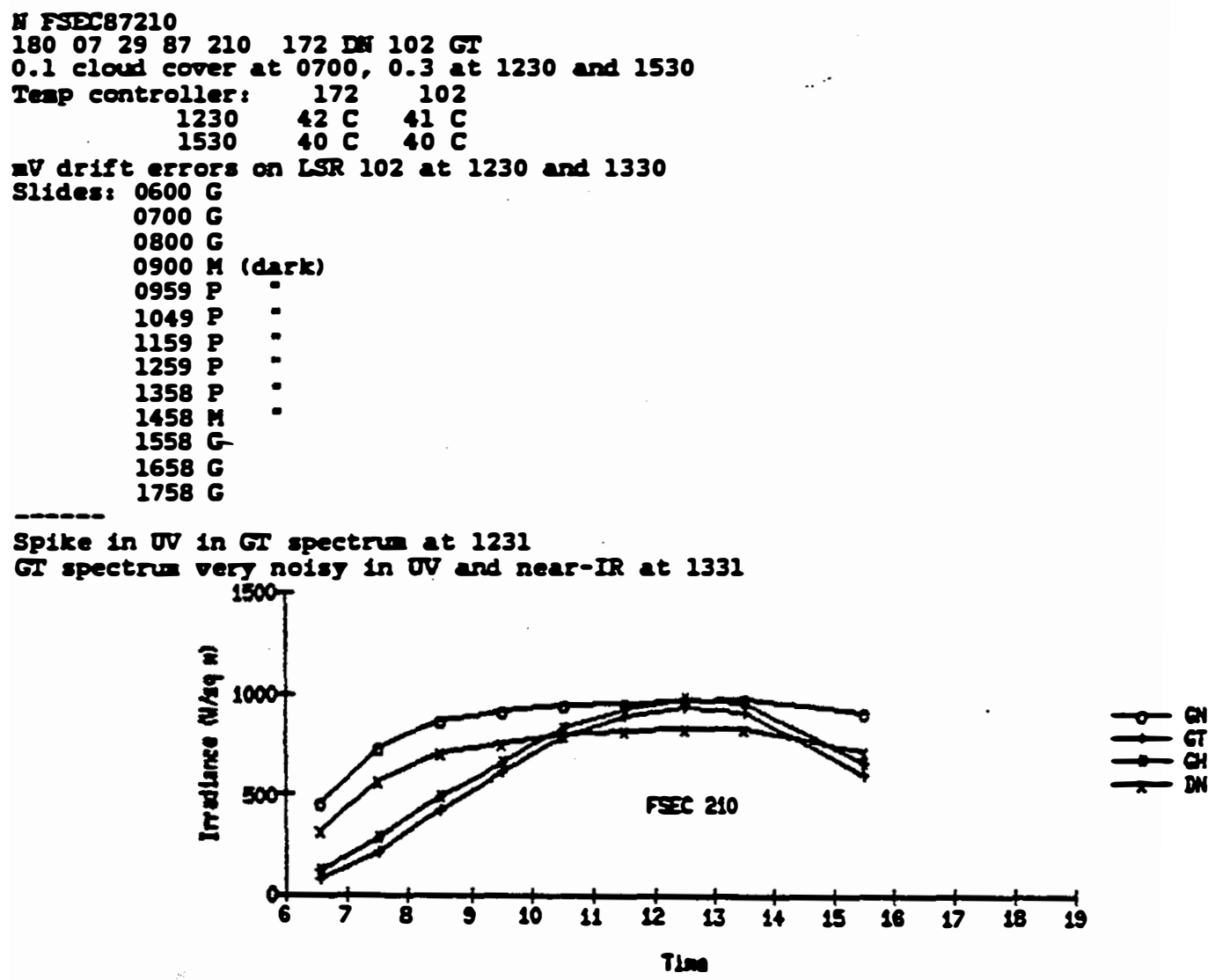

Fتב $220 \mathrm{BT}$
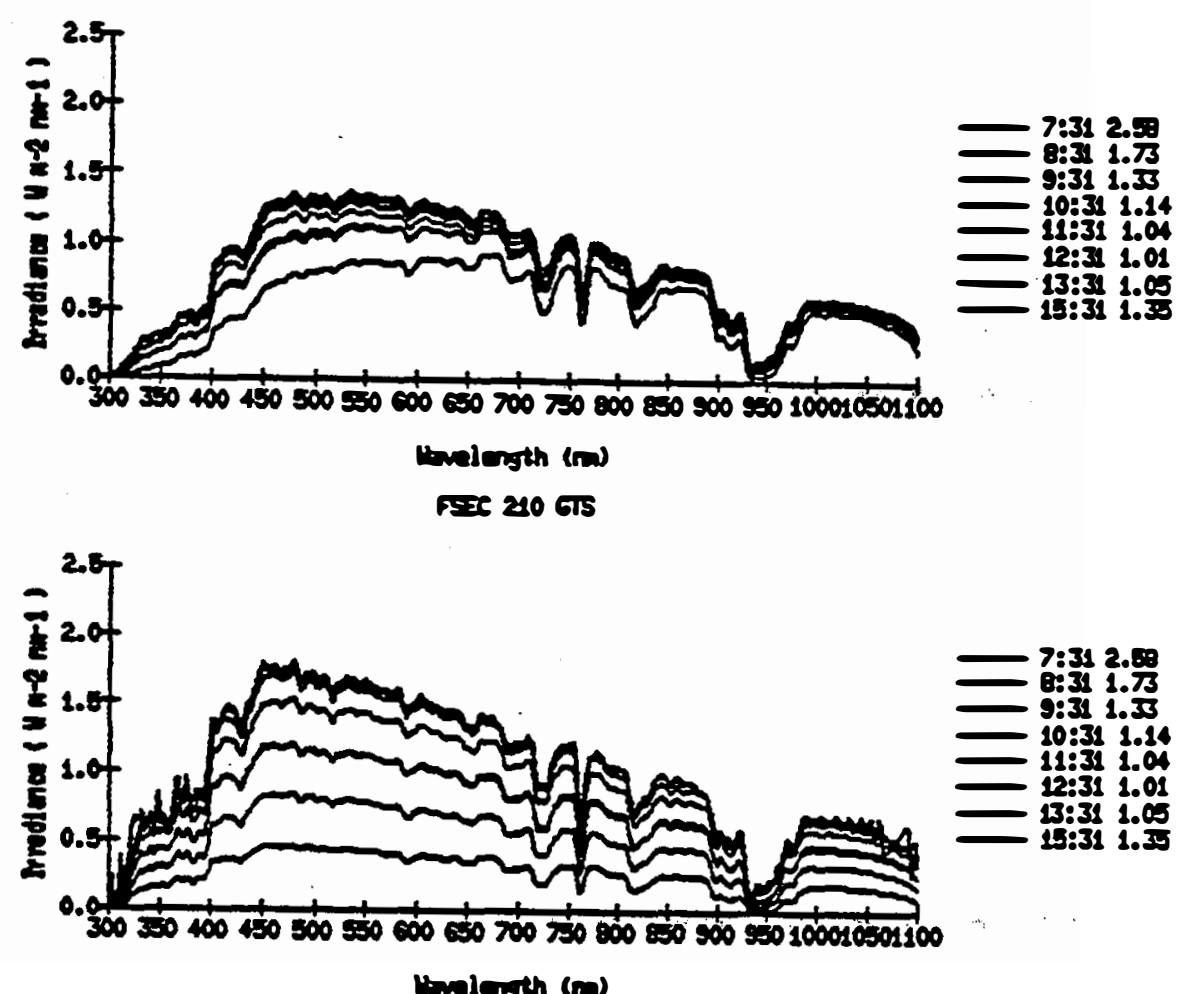


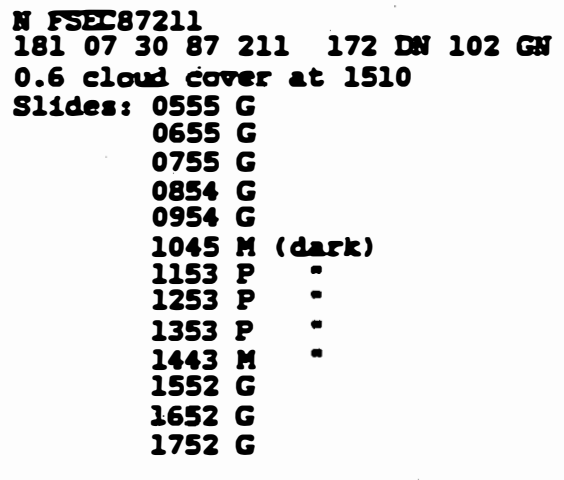

Fase 211

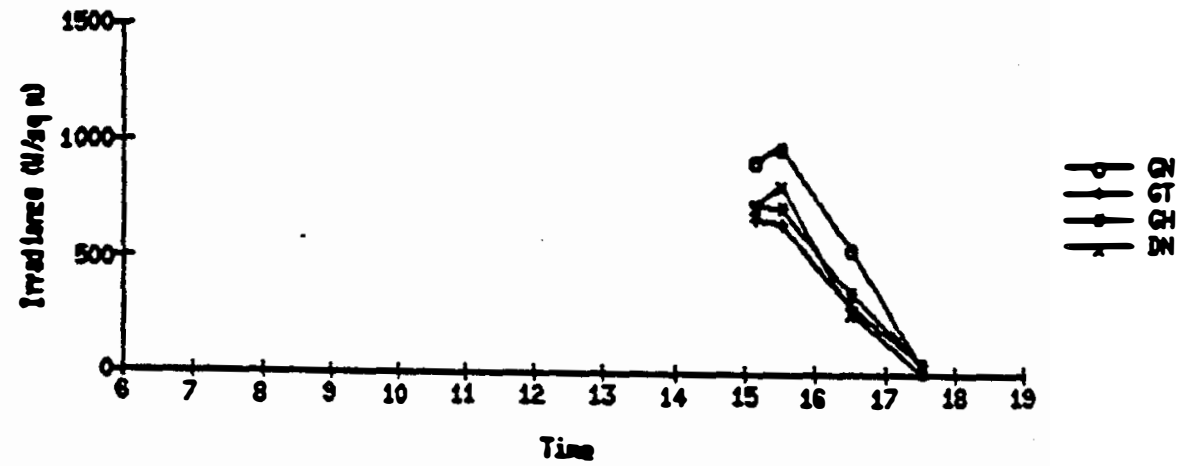

T25 $241 \mathrm{DT}$

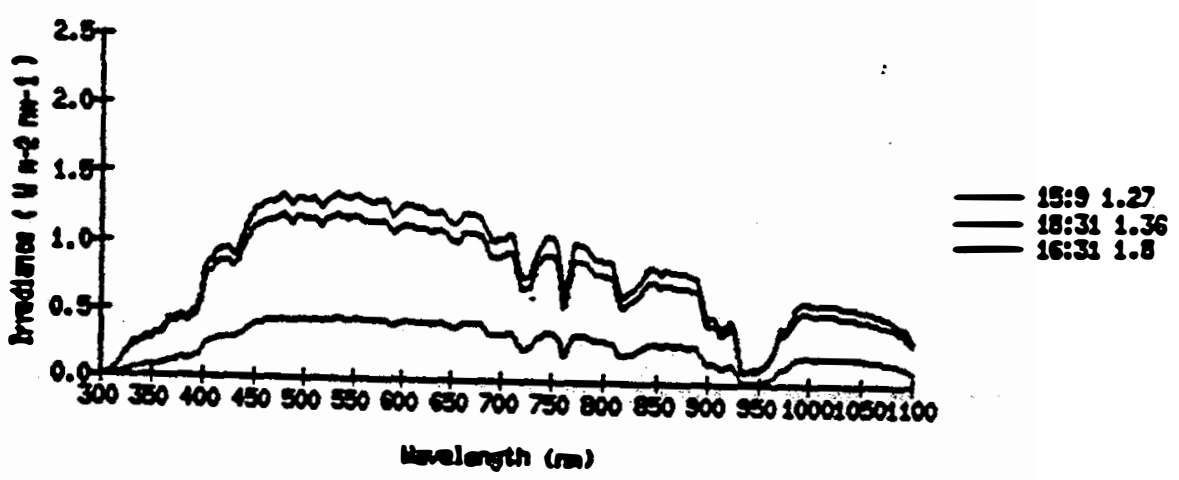

Fase ald os

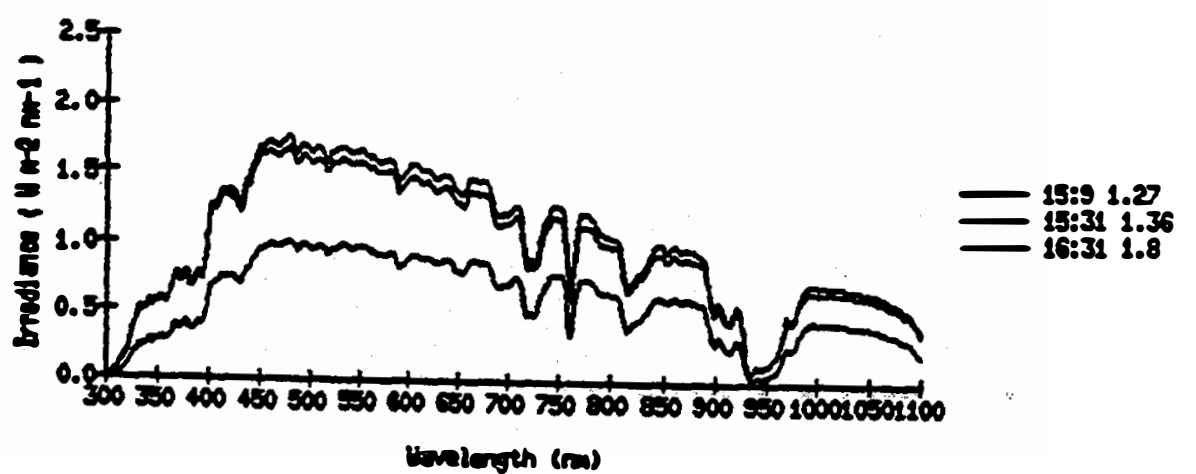


A FS2C87212

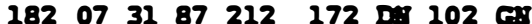

0.5 cloud cover at 0730; raln at 1330

slides: $0552 \mathrm{G}$

$0649 \mathrm{G}$

$0749 \mathrm{G}$

$0849 \mathrm{G}$

$0948 \mathrm{G}$

1048 B (dngk)

1148 A (color chnnge)

$1248 \mathrm{G}$ (unter drops)

$1347 \mathrm{G}$

$1547 \mathrm{G}:$ (a1881ng 2447)

$1628 \mathrm{G}$

$1728 \mathrm{G}$

$1827 \mathrm{G}$

$\operatorname{Fsx} 212$

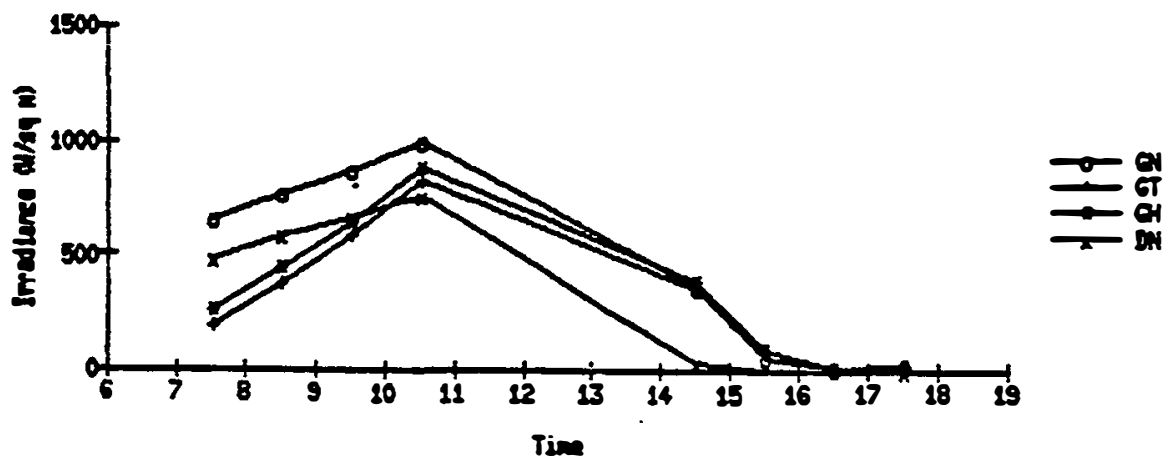

Fsec 212 DNT

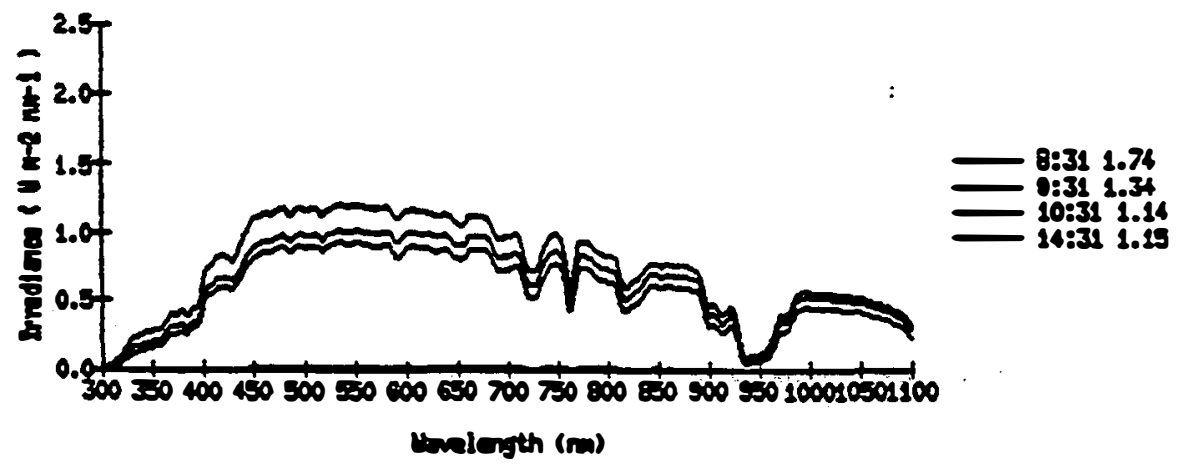

Fis 212 os

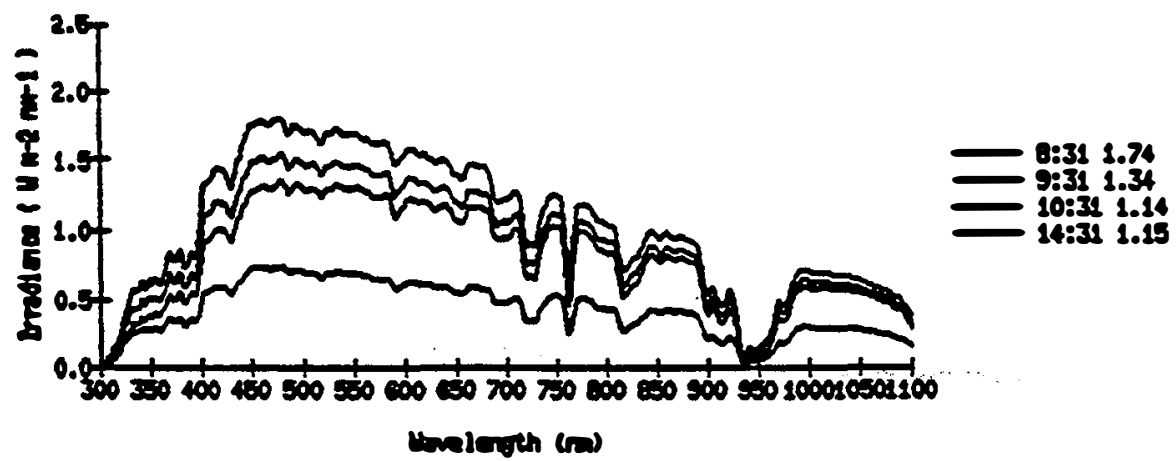


A FSES87213

$\begin{array}{llllll}18308 & 01 & 87.213 \quad 172 \text { D8 } 102 \mathrm{GS}\end{array}$

Hostly clear AMs partly cloudJ PH

slides: $0625 \mathrm{G}$

$0724 \mathrm{G}$

0824 M (danzk)

$0924 P$ P

$1024 P$

$1224 \mathrm{P}$

$1324 \mathrm{P}$

$1423 \mathrm{G}$

$1523 \mathrm{G}$

$1623 \mathrm{G}$

$1723 \mathrm{G}$

$1829 \mathrm{G}$

Beflaft nessurenents show instability at 1333

$\operatorname{Fsec} 213$

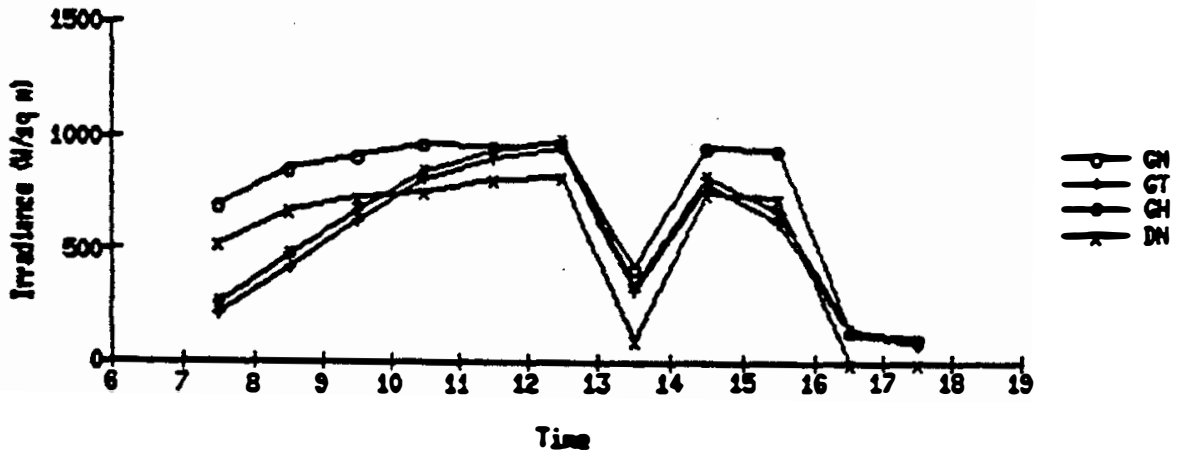

FSEC 243 DT

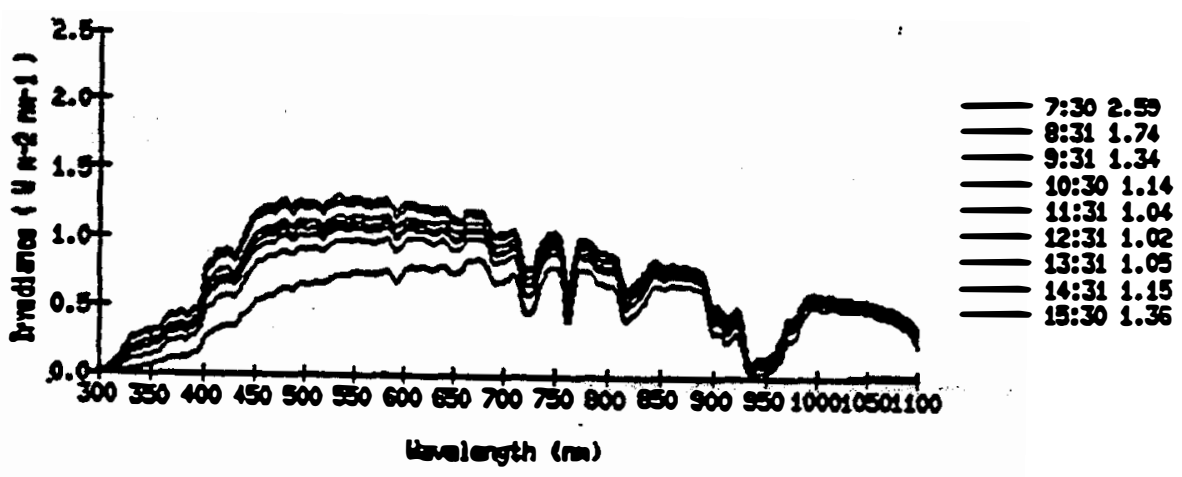

Fste 243 os

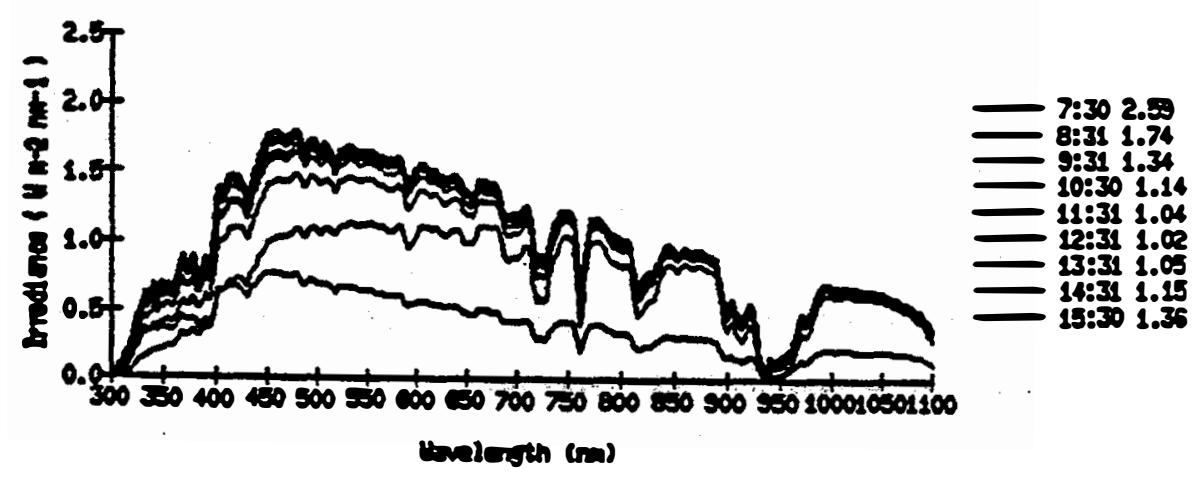




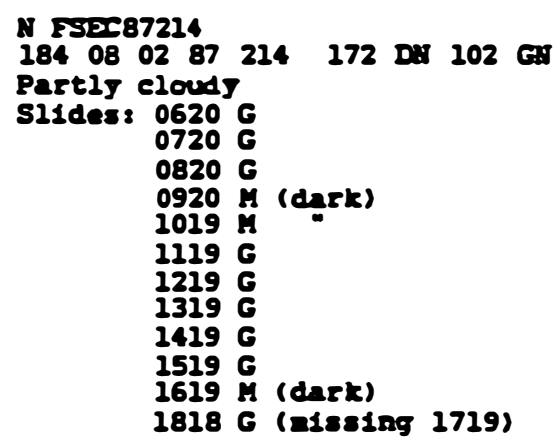

FSEC 214

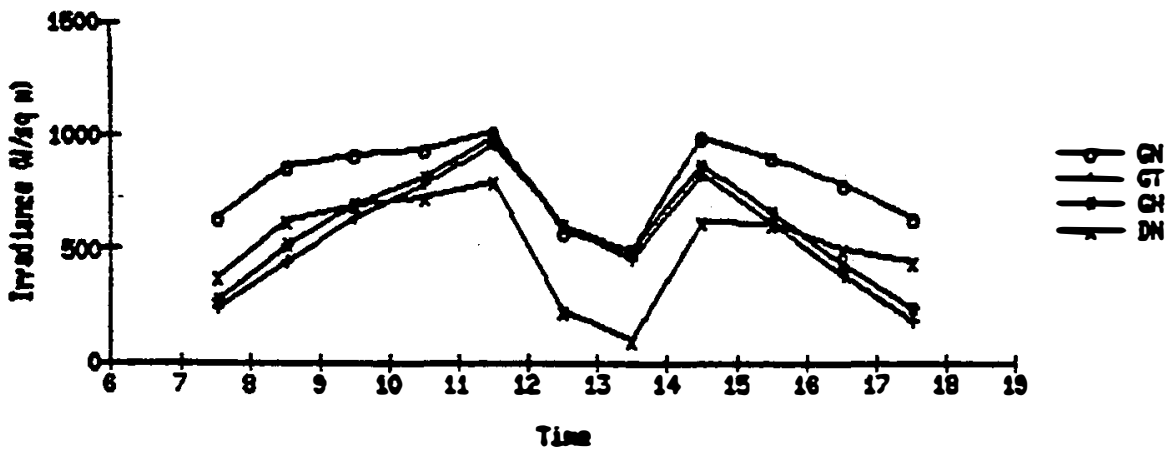

Fext $214 \mathrm{BT}$

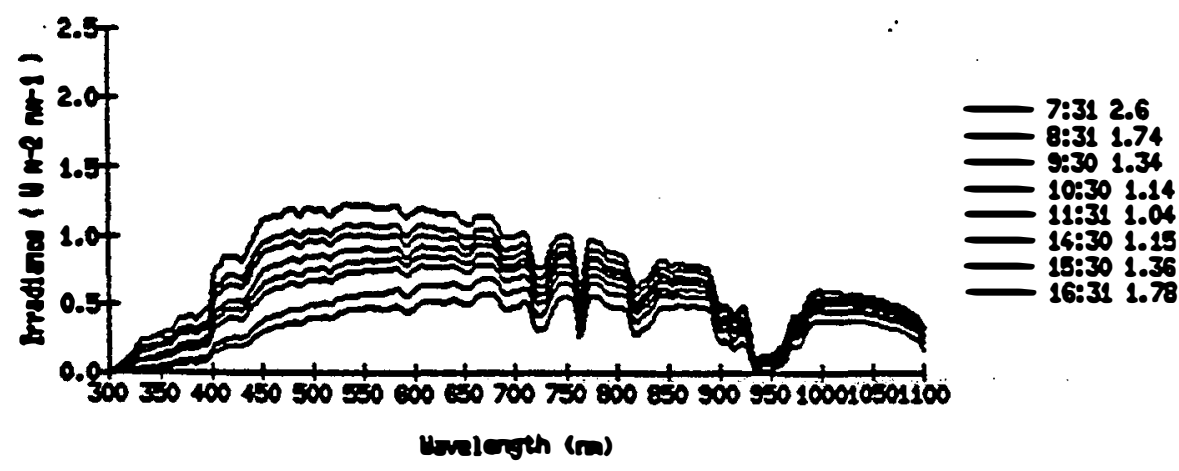

PIIS 214 os

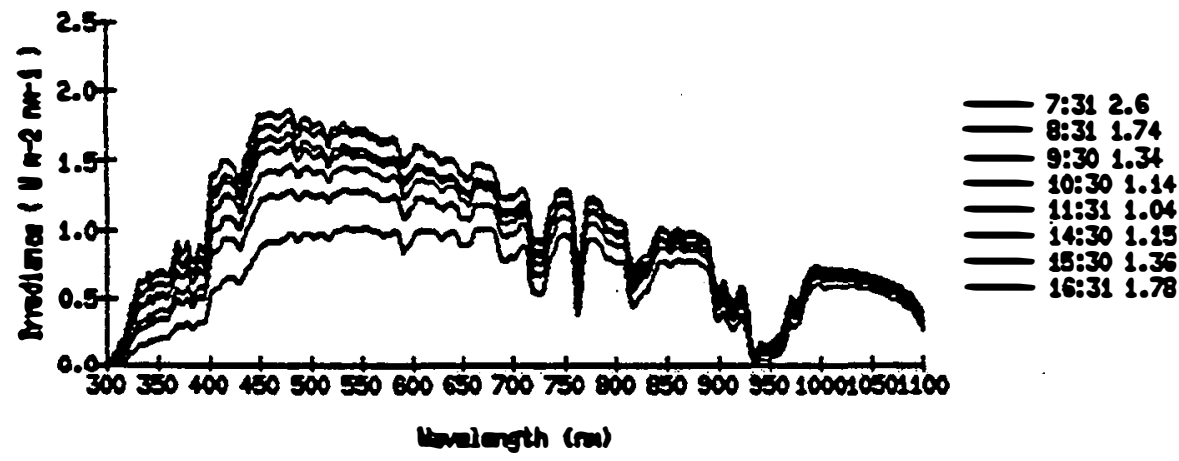




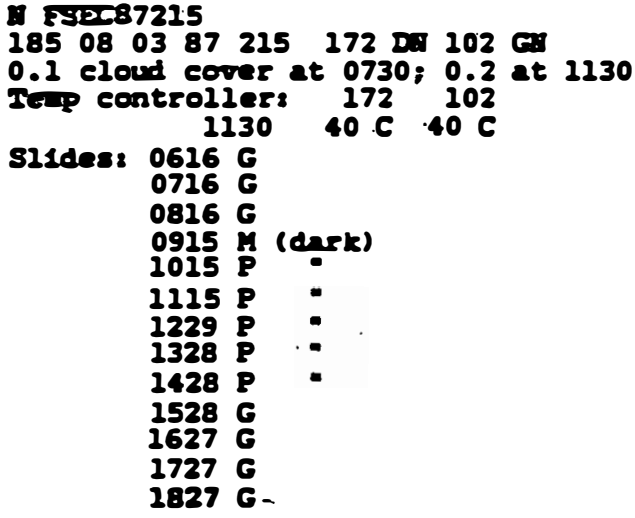

Fise 225

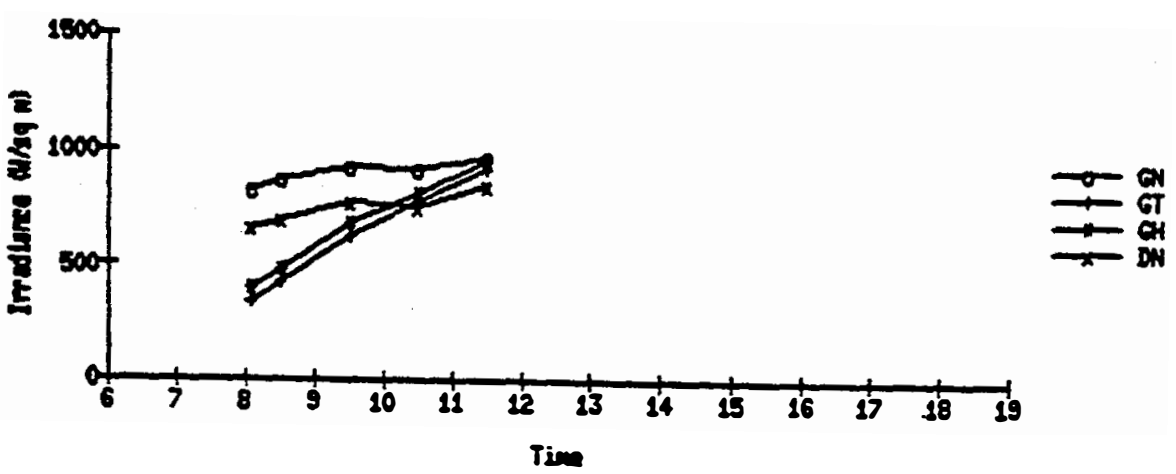

FIי 225 an

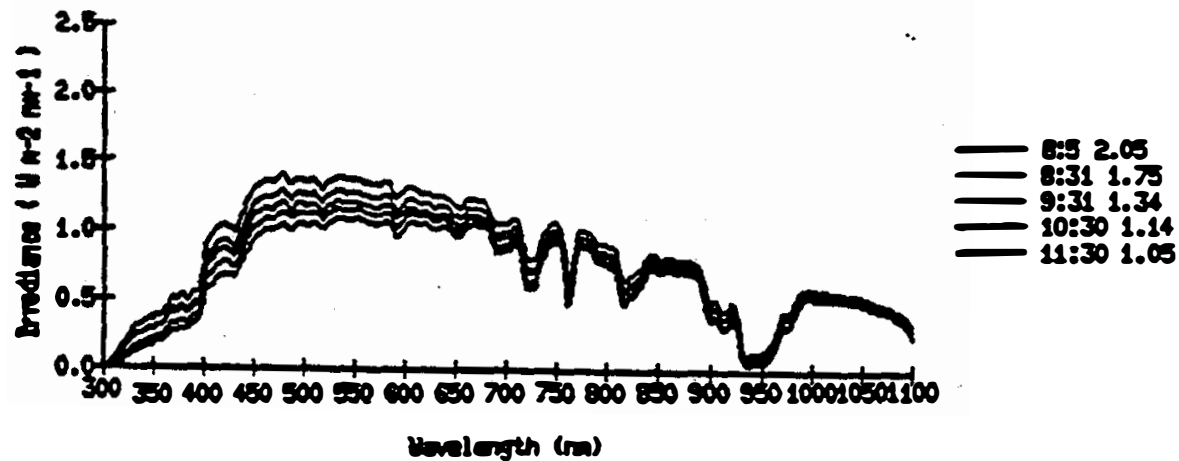

Fser 245 ens

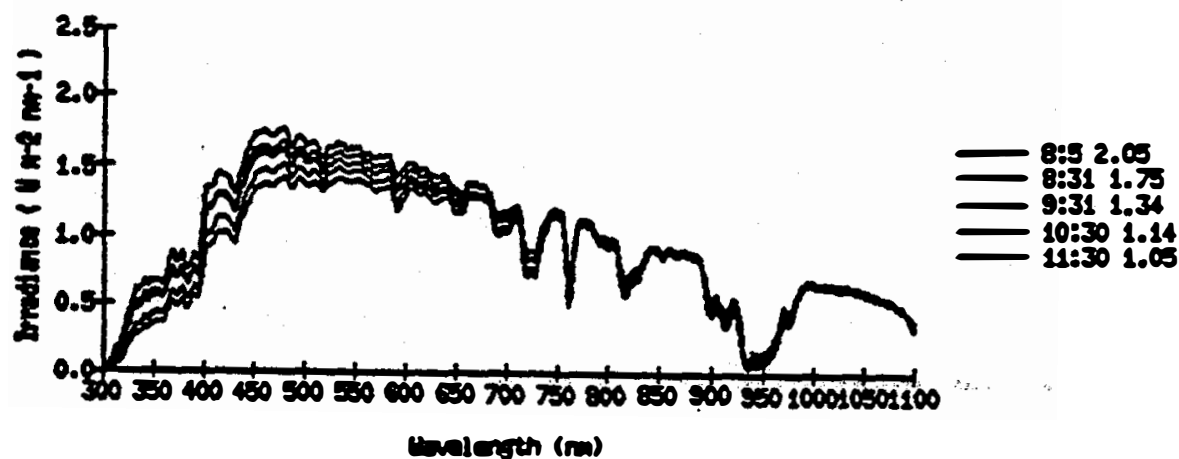




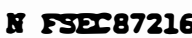

$\begin{array}{llllllllll}186 & 08 & 04 & 87 & 216 & 172 & 0 . & 102 & 6:\end{array}$

0.1 cloud cover at $0730 ; 0.4$ at $1130 ; 0.3$ at 1530

Iep controllers $172 \quad 102$

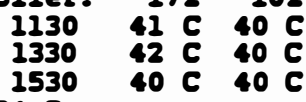

sl1des: $0624 \mathrm{G}$

$0724 \mathrm{G}$

$0824 G$

0924 A (dark)

$1024 P$ P

$1124 P$ -

$\begin{array}{lll}1224 & P \\ 1324 & P & -\end{array}$

$1524 \mathrm{G}$

$1724 \mathrm{G}$

$1824 \mathrm{G}$

Gil spectro noles in 00 and neap-IR at 1434 and 1531

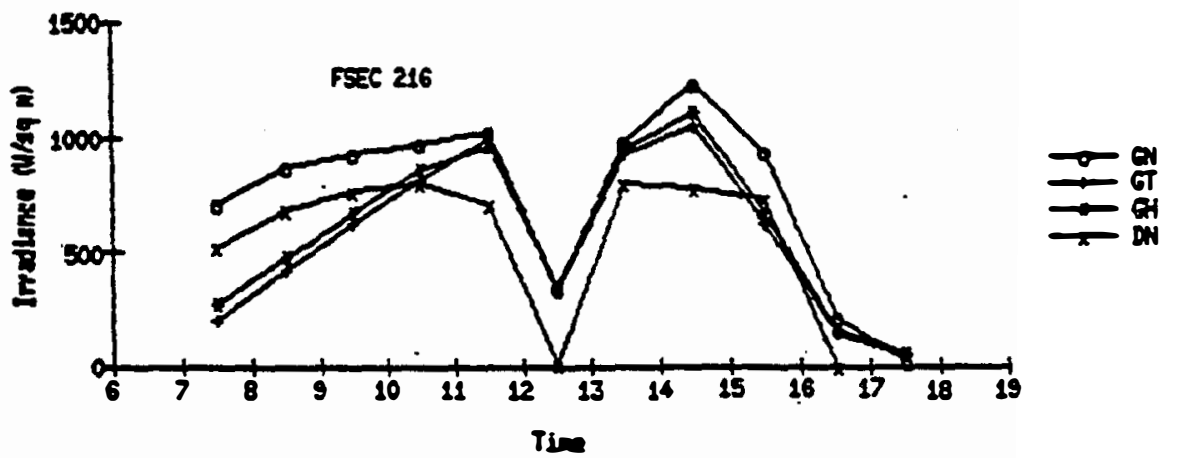

PISC $246 \mathrm{MT}$

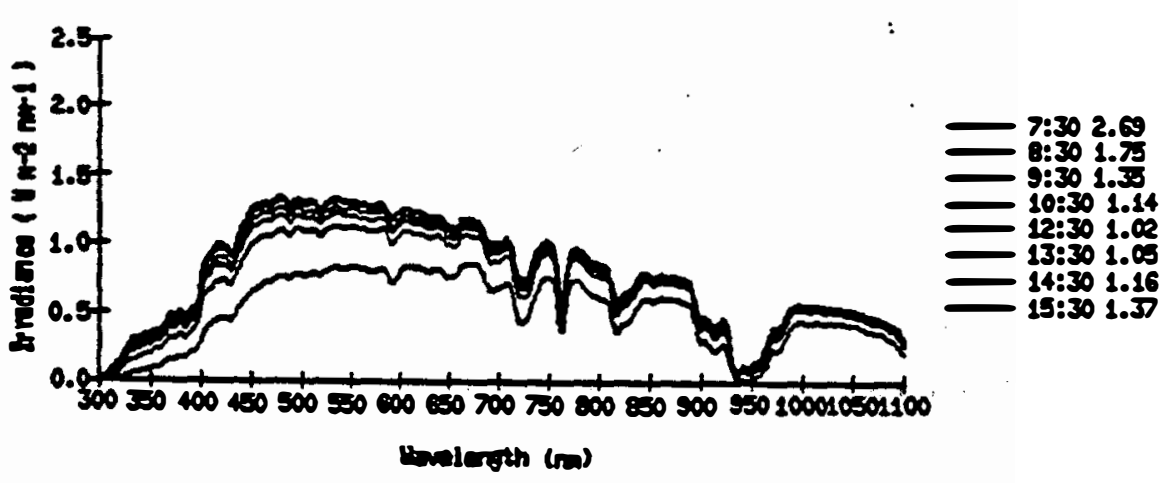

xax 216 as

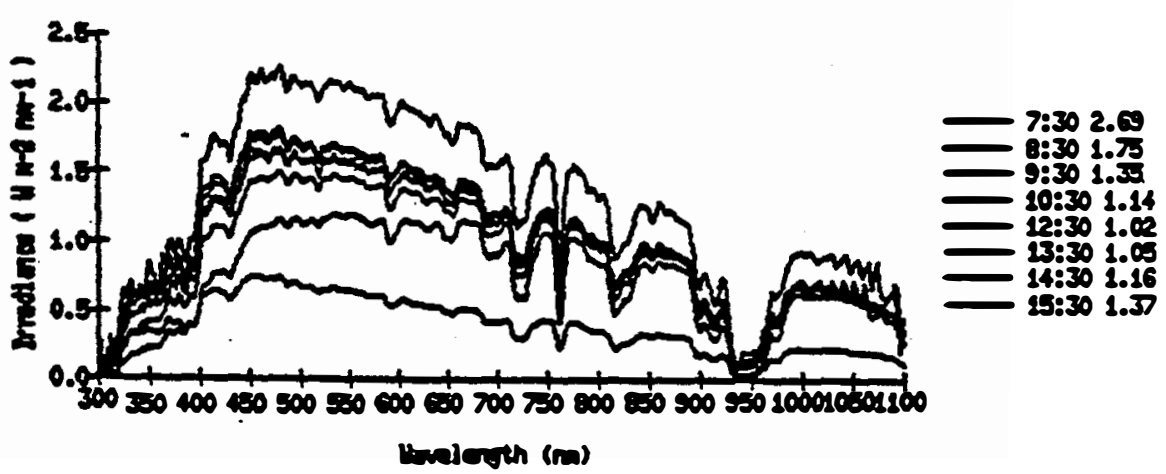




\section{을}
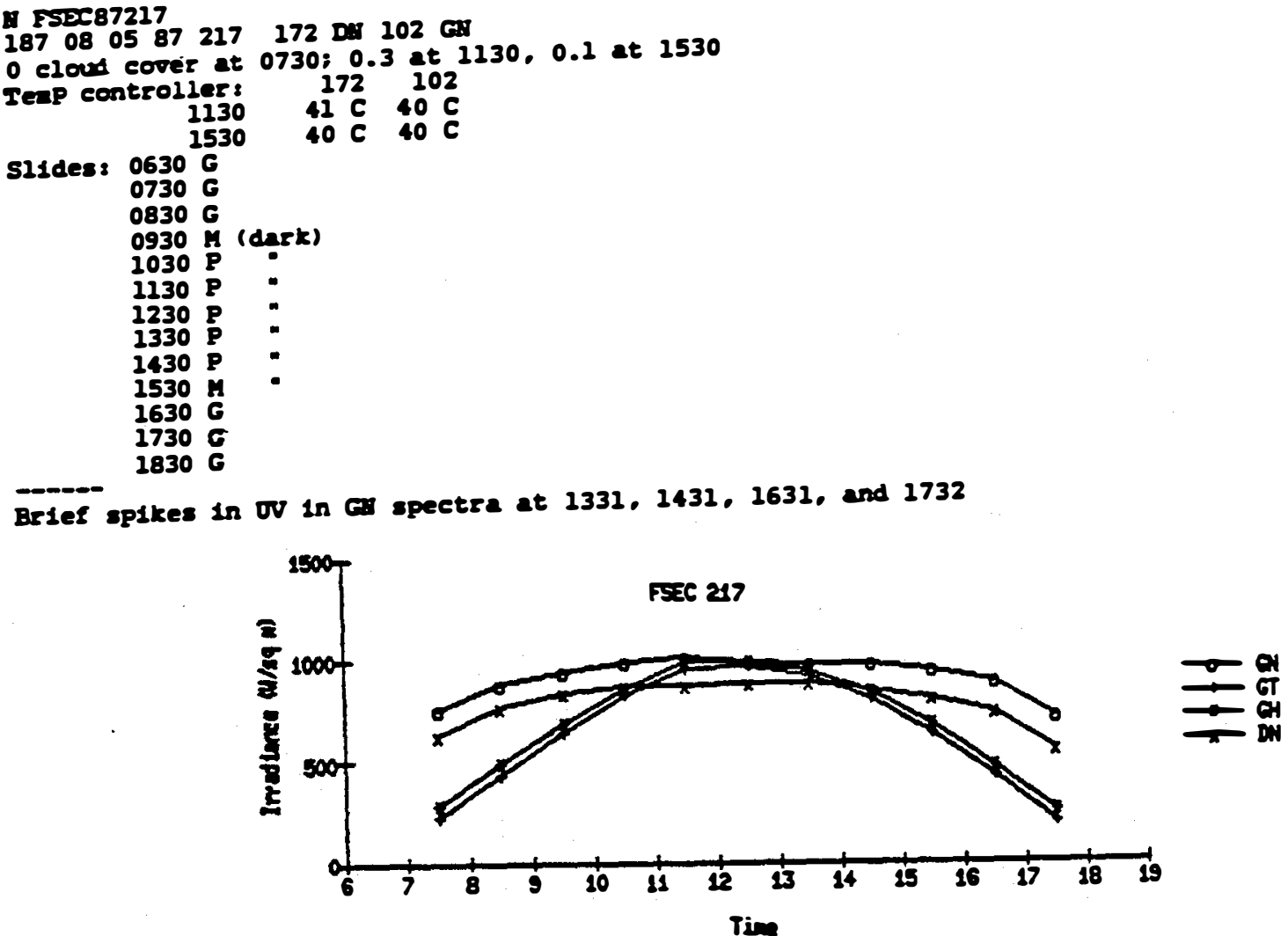

Fise 247 ant

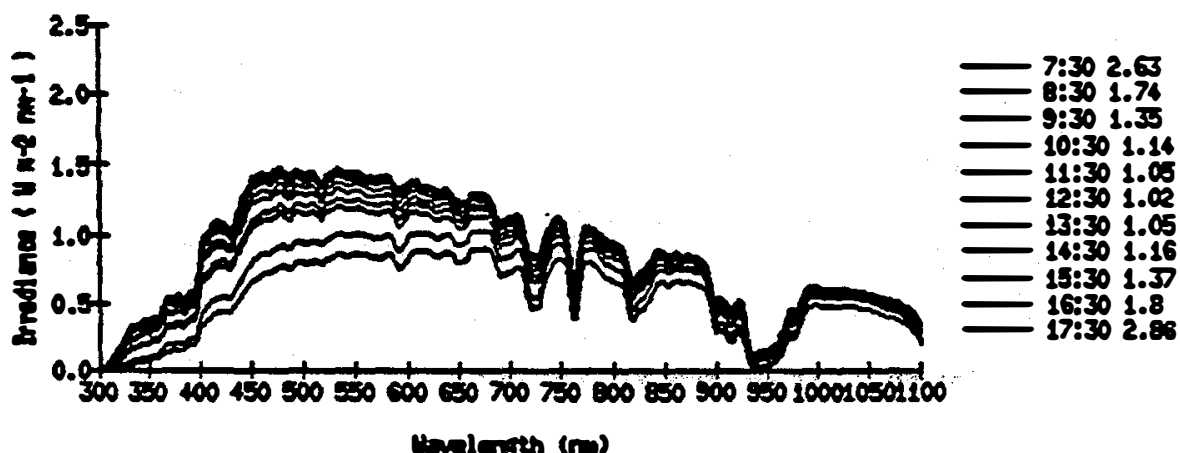

Fise 247 os

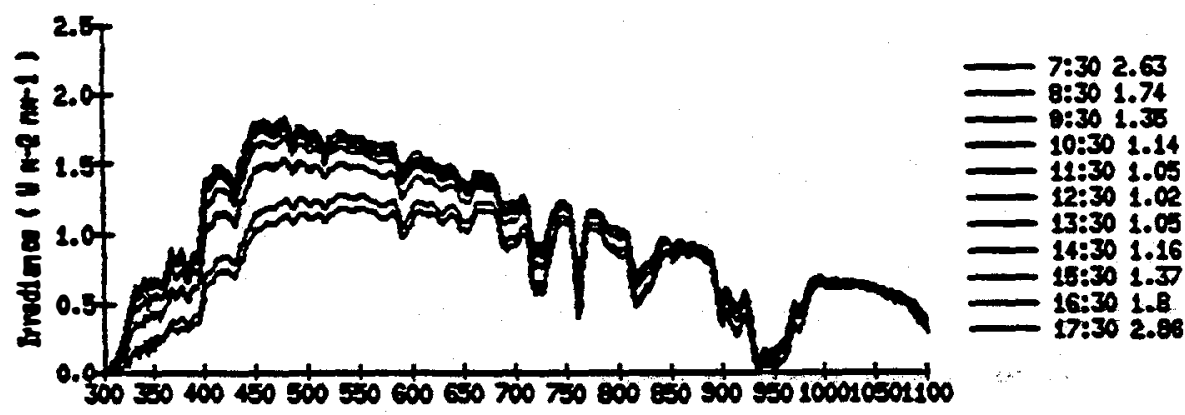

unalersth (n) 


\section{SEP : ثै}

N TS2987218

$\begin{array}{llllllllll}188 & 08 & 06 & 87 & 218 & 172 & \mathrm{DAl} & 102 \mathrm{FH}\end{array}$

0 clowd cover at 080080.1 at 1130 and 1530

Tenp controllers $172 \quad 102$

Sl1des: $0630^{1530} \mathrm{G}$

$0730 \mathrm{G}$

$0830 \mathrm{G}$

0930 A (dnek)

$1030 \mathrm{P}$

$1130 P$ -

$1230 \mathrm{P}$

$1330 \mathrm{P}$

$1430 P$

1530

$1630 \mathrm{G}$

$1730 \mathrm{G}$

Appears to be vers senil spike in WV in Gi spectrun a 0831

Note tine stap differences $1400 \mathrm{Ve} .1434$

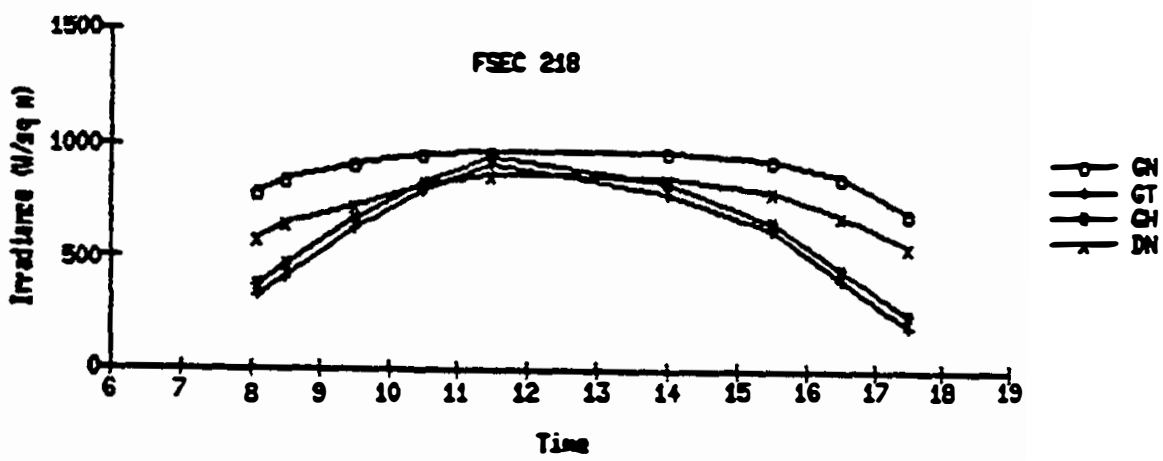

F=E $248 \mathrm{mT}$

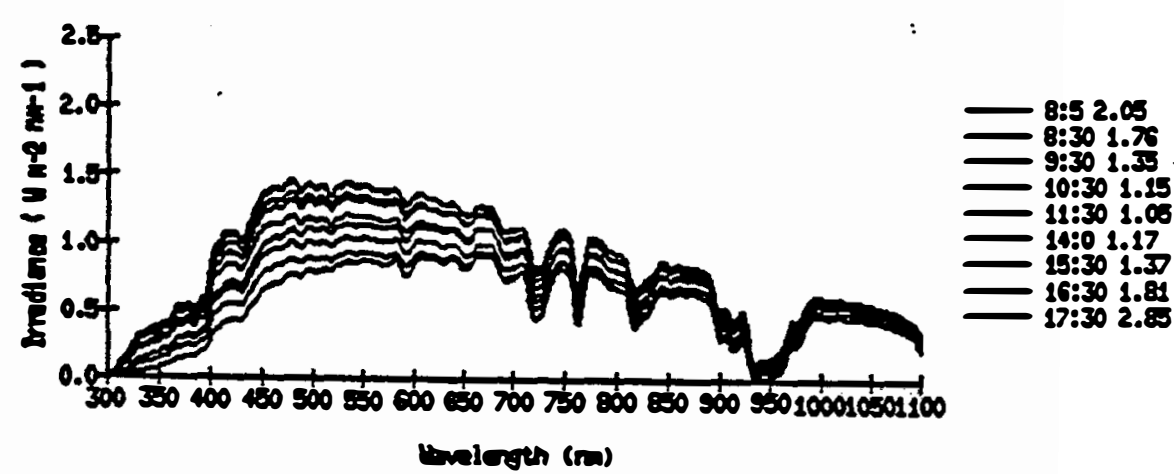

PISE 248 as

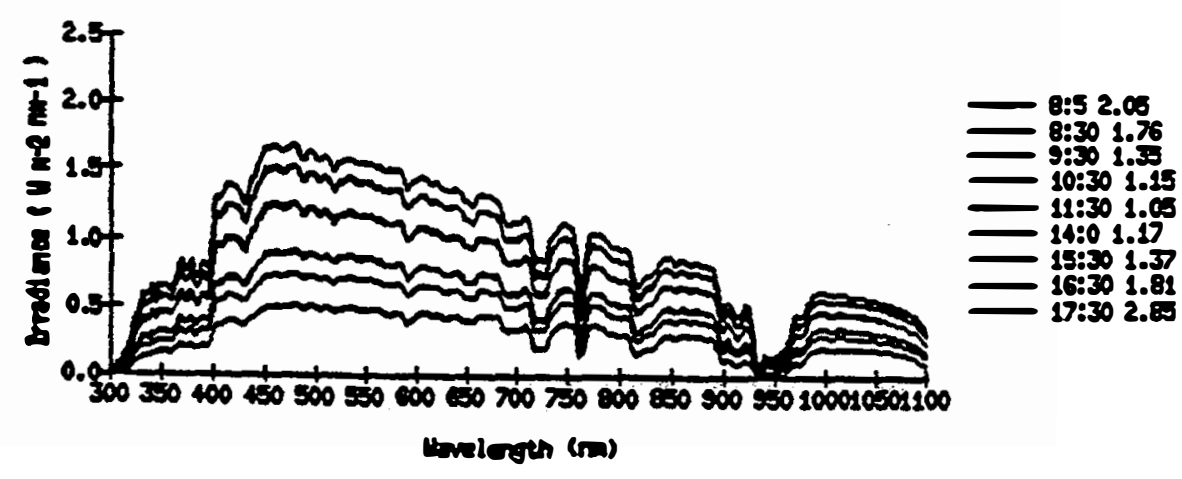


N FPI587219

$\begin{array}{lllll}18908 & 0787 \quad 219 \quad 172 \quad \mathrm{DN} & 102 \mathrm{GH}\end{array}$

0 cloud cover at $0730 ; 0.1$ at 1130 and 1530

Tracker error of about $1.5 \mathrm{deg}$ at 1400: reset

(Iracking 0.X. at 1130 check)

S11dess $0630 \mathrm{G}$

$0730 \mathrm{G}$

$0830 \mathrm{G}$

0930 M (dark)

$1030 \mathrm{P}$

$1130 \mathrm{P}$

$1230 \mathrm{P}$

$1330 \mathrm{P}$

$1430 \mathrm{P}$

1530

$1630 \mathrm{G}$

$1730 \mathrm{G}$

$1830 \mathrm{G}$

Brief spike in or in GH spectrm at 1231 and 1331

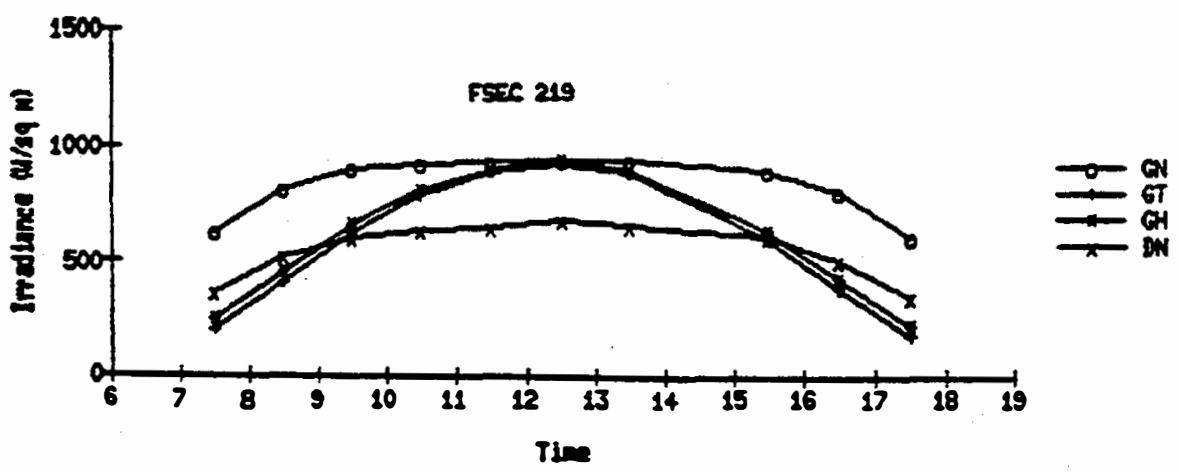

PSEC 219 BNT
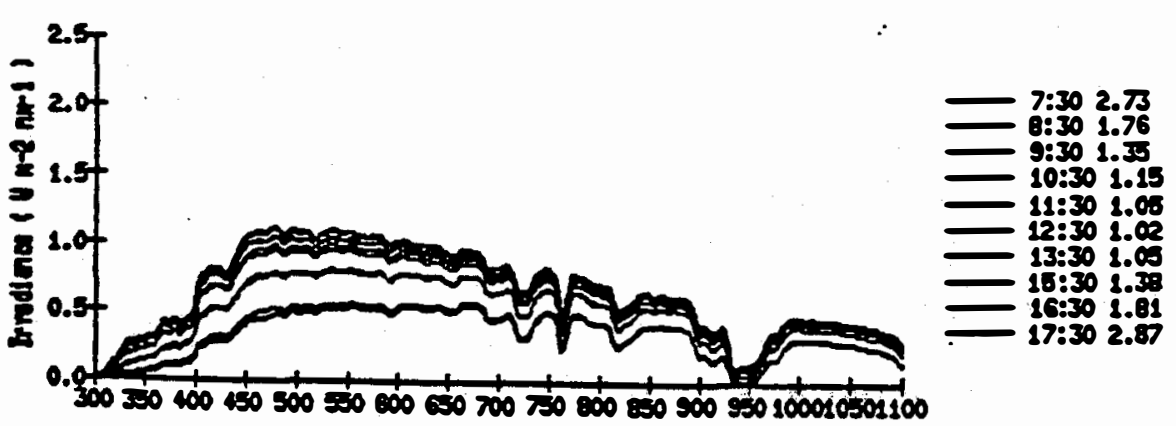

Holength (m)

Fsec 21906

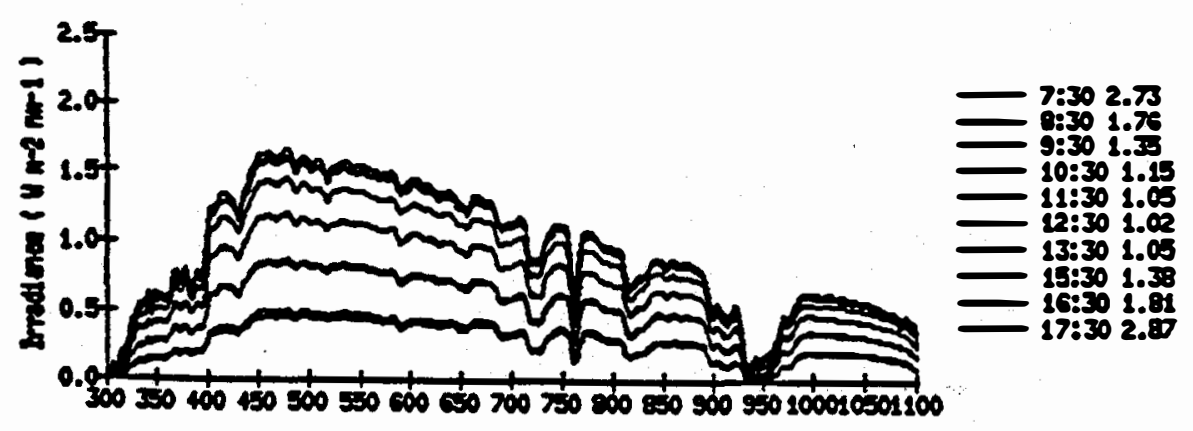

vavilowth (ra) 


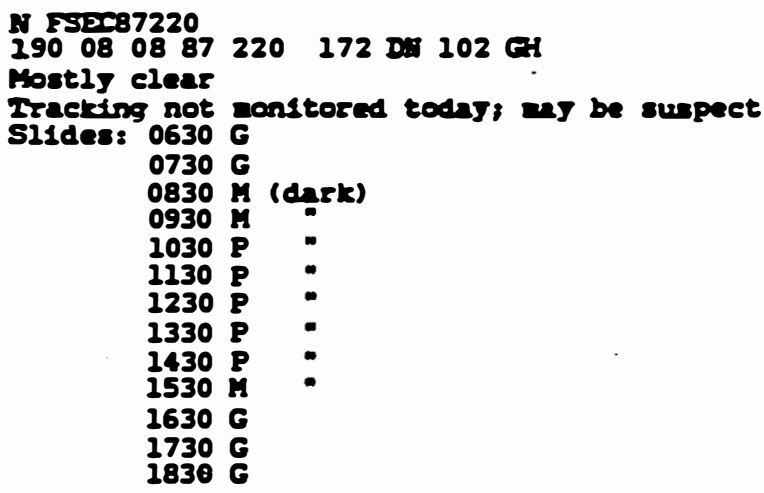

Ver brief spike in ov in GH spectron at 1431

Spike in ov in Gi spectiv at 1734

Di: spectral Integral appears to be 100 conpared to broadband this day

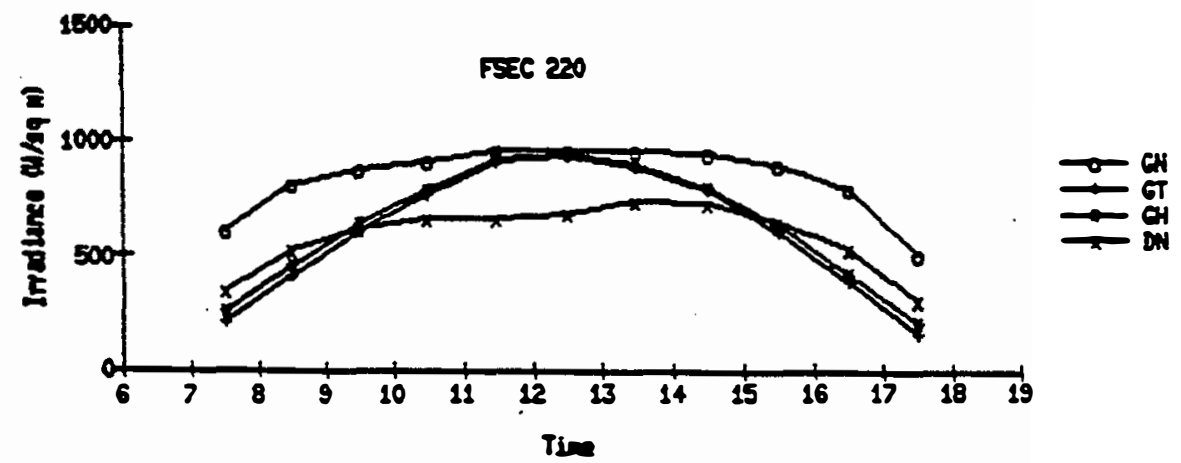

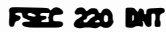

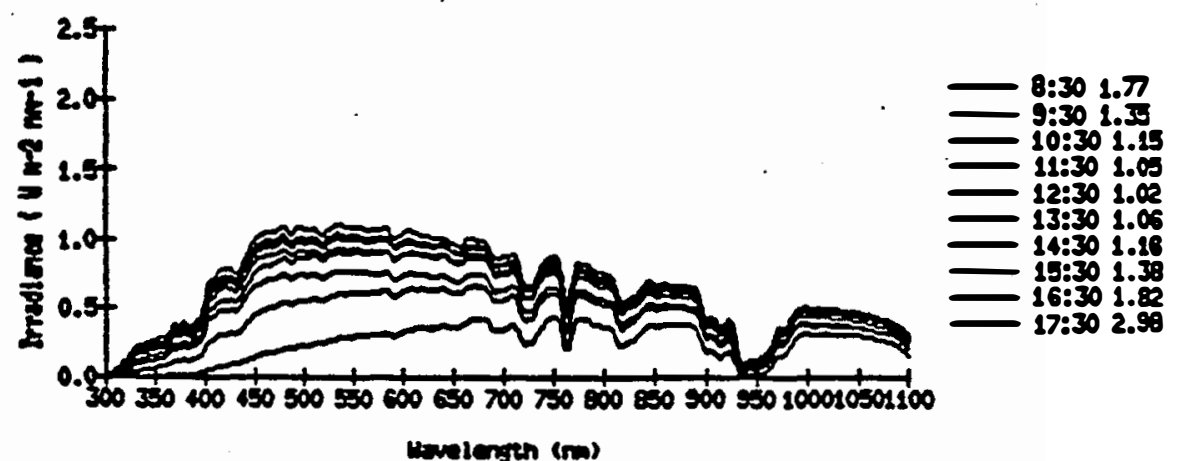

Fis 2000

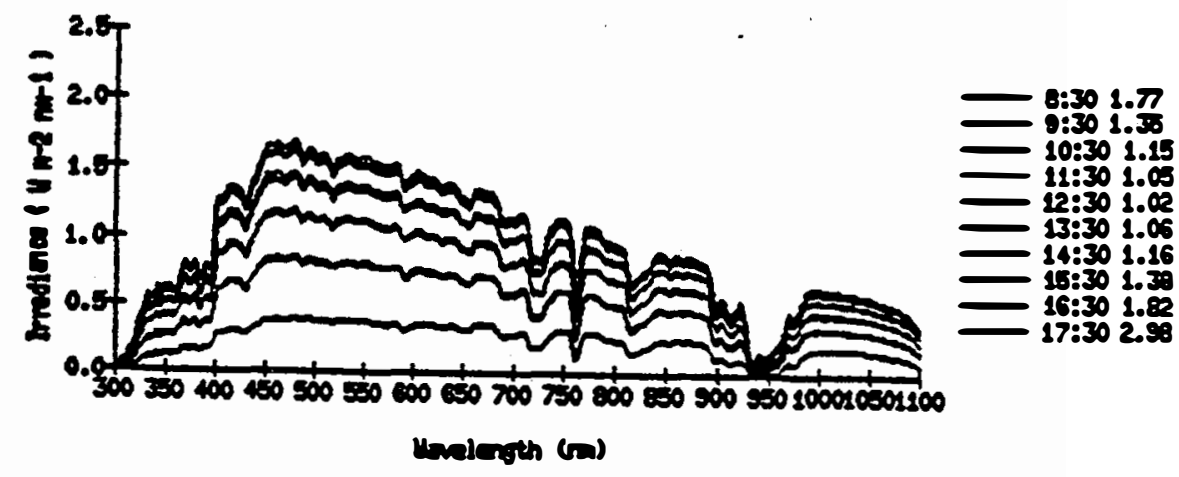




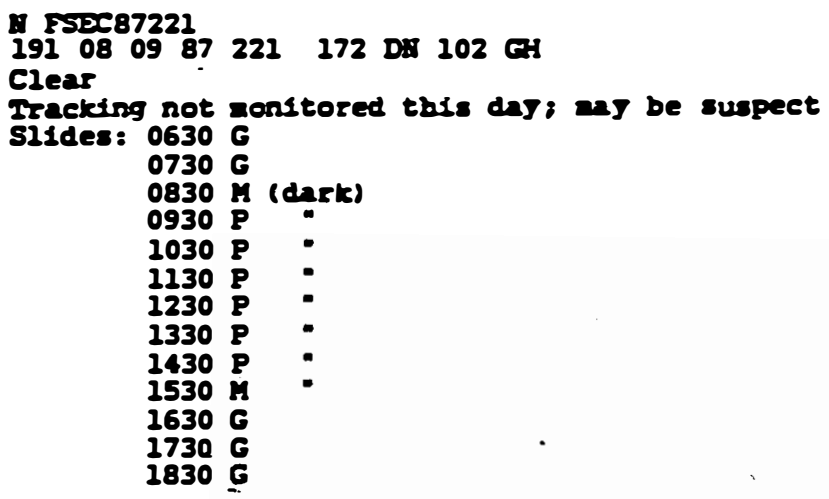

FSEC 221

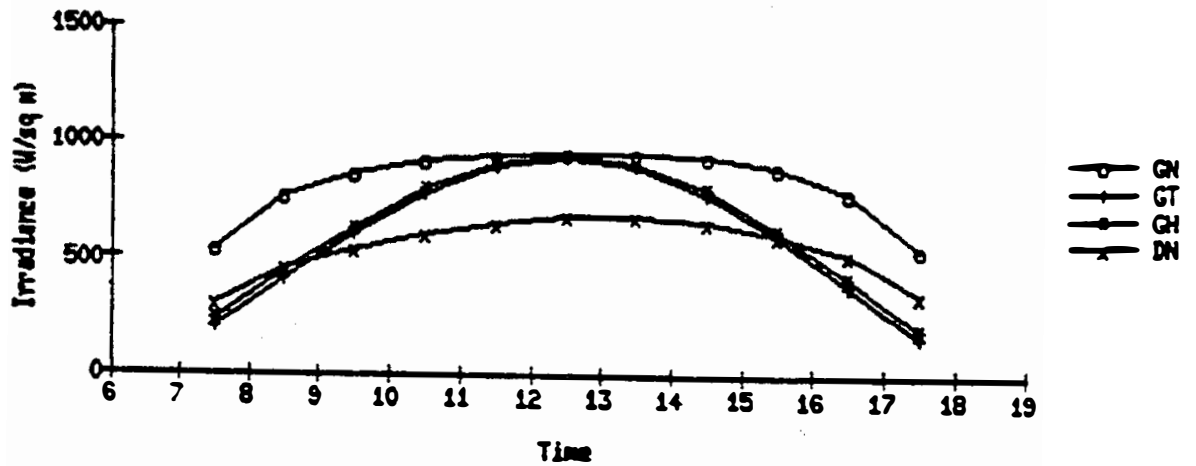

Fise 221

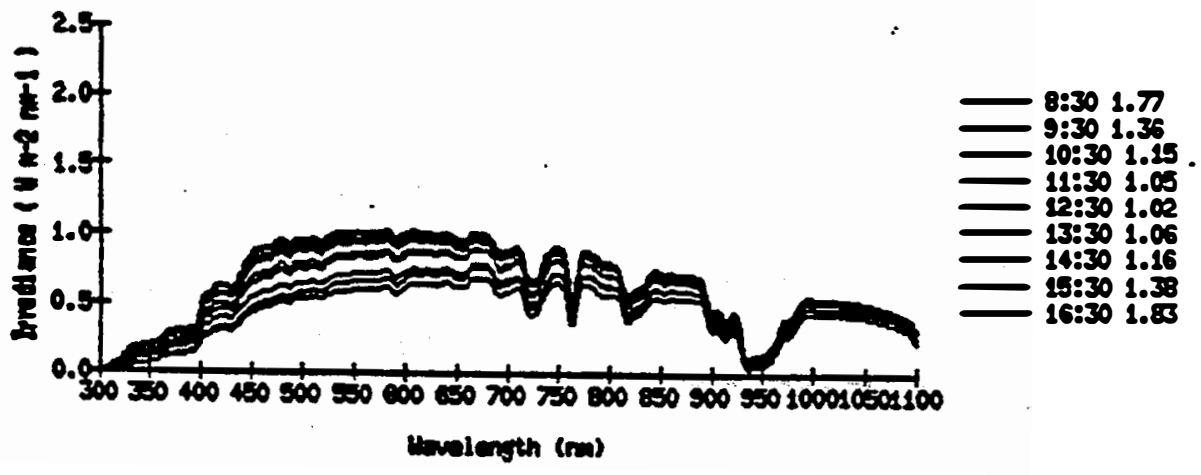

pise as as

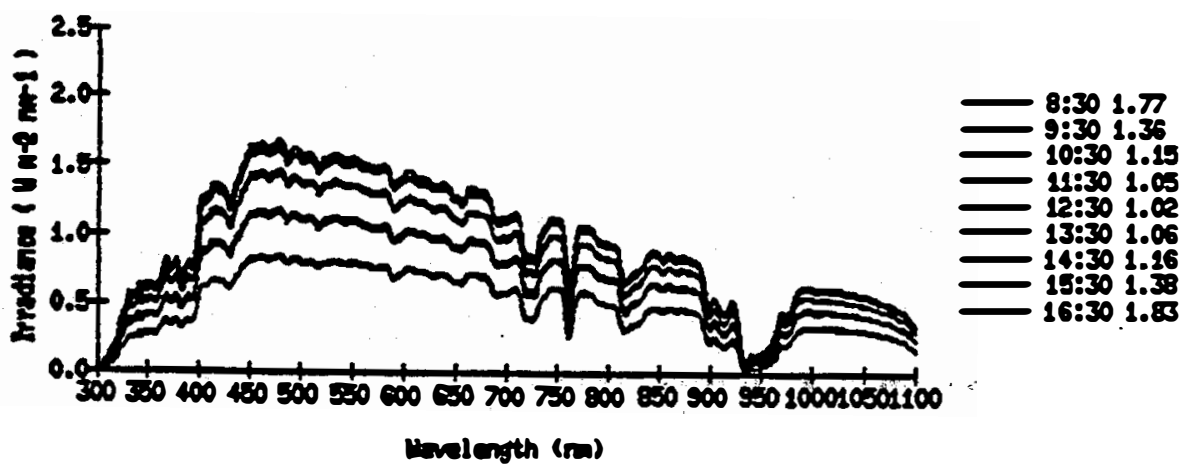


18 FST्287222

$\begin{array}{lllllll}19208 & 1087 & 222 & 172 & \text { DH } & 102 & \mathrm{GH}\end{array}$

Partly cloudy

Iraeksing not monitored todnj: an be suspect

Slides: $0630 \mathrm{G}$

$0730 \mathrm{G}$

$0830 \mathrm{H}$ (dark)

$0930 \mathrm{G}$

$1030 \mathrm{G}$

$1130 \mathrm{P}$ (dnsk)

$1230 \mathrm{P}$

$1330 \mathrm{P}$

$\begin{array}{ll}1430 & \mathrm{P} \\ 1530 & \mathrm{P}\end{array}$

$1630 \mathrm{M}$

$1730 \mathrm{G}$

$1830 \mathrm{G}$

GH spectra noisy in OV and near-IR at 1231, 1331, 1431, and 1531

FSEC 220

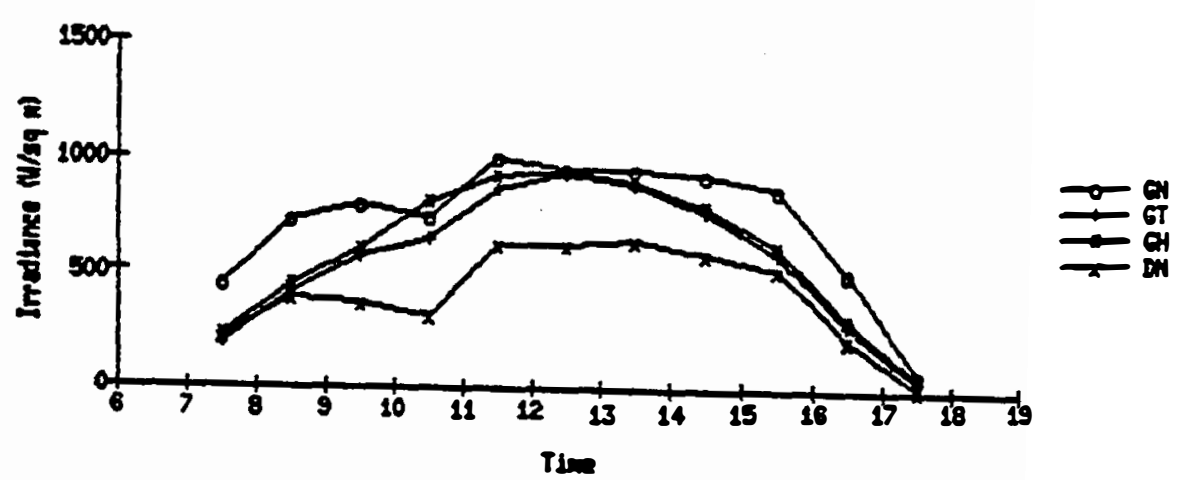

FICE 202 ant

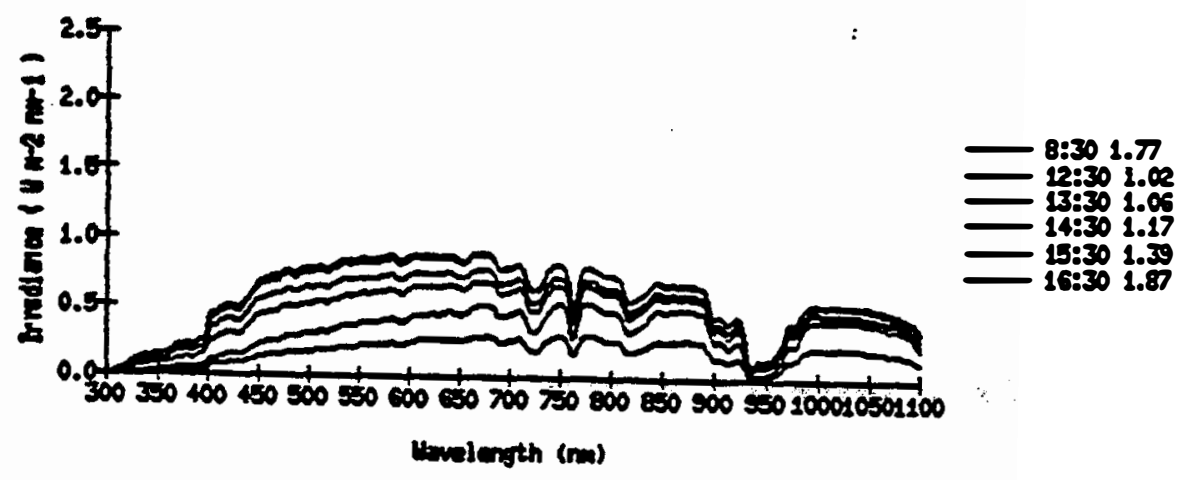

rese 202

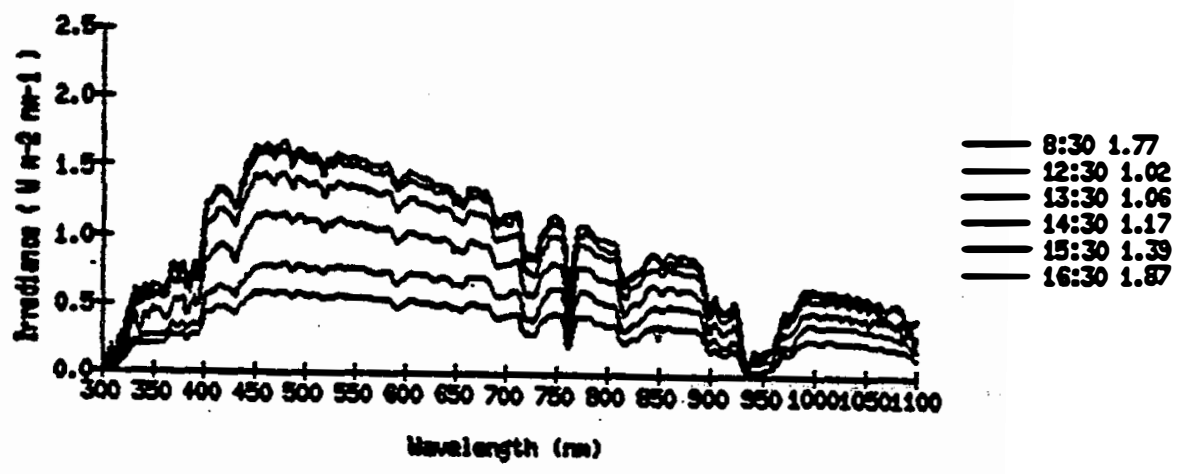


ASP587267

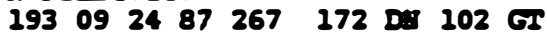

0.9 cloud cover at 1230

Iep controllers $40 \mathrm{C}$ at 1330

S11des: $0723 \mathrm{G}$

$0823 \mathrm{G}$

$0923 \mathrm{G}$

$1023 P$ (dnsk)

$1123 \mathrm{G}$

1222 A (dark)

1322 G

$1422 \mathrm{G}$

1522 G

$1628 \mathrm{G}$

Fsec 257

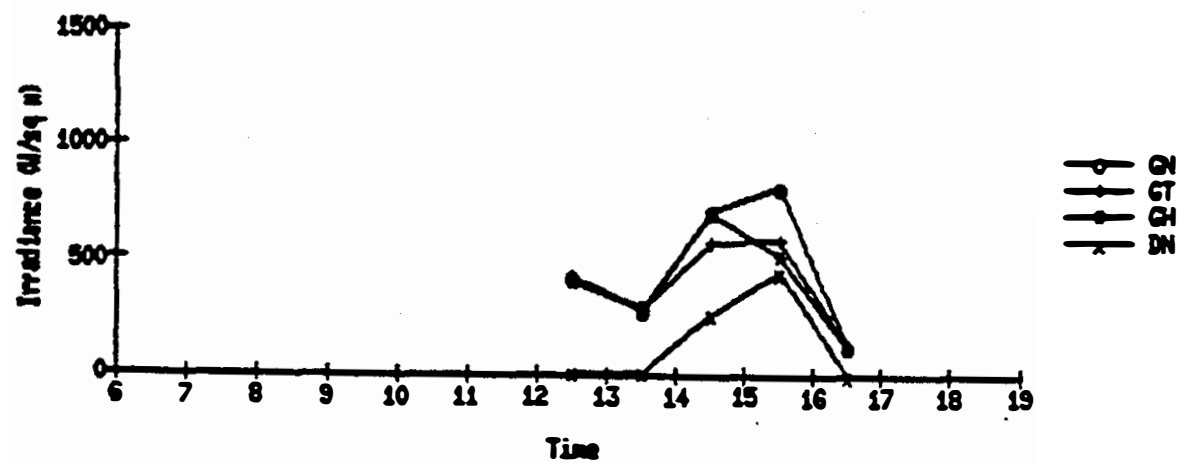

FSEC 257 Dit

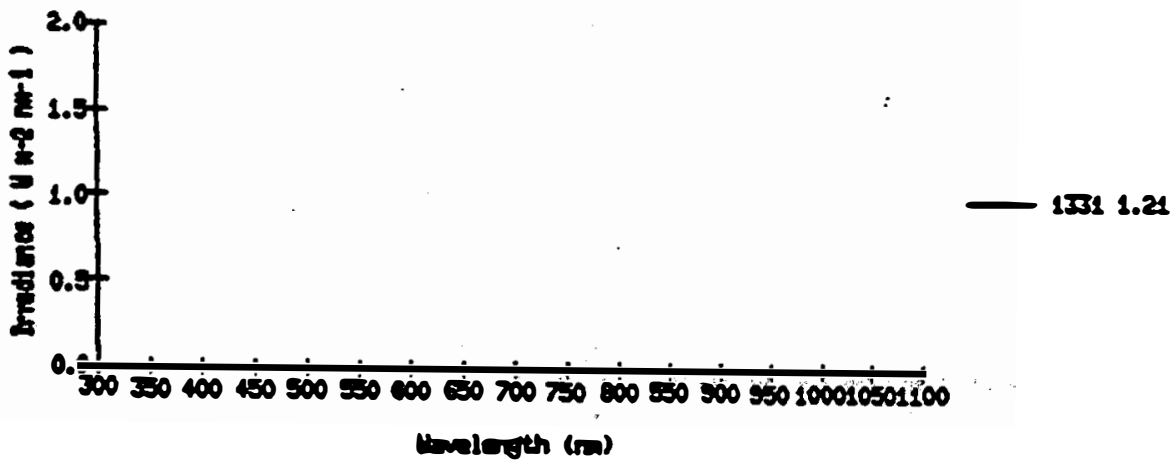

FIE 2707

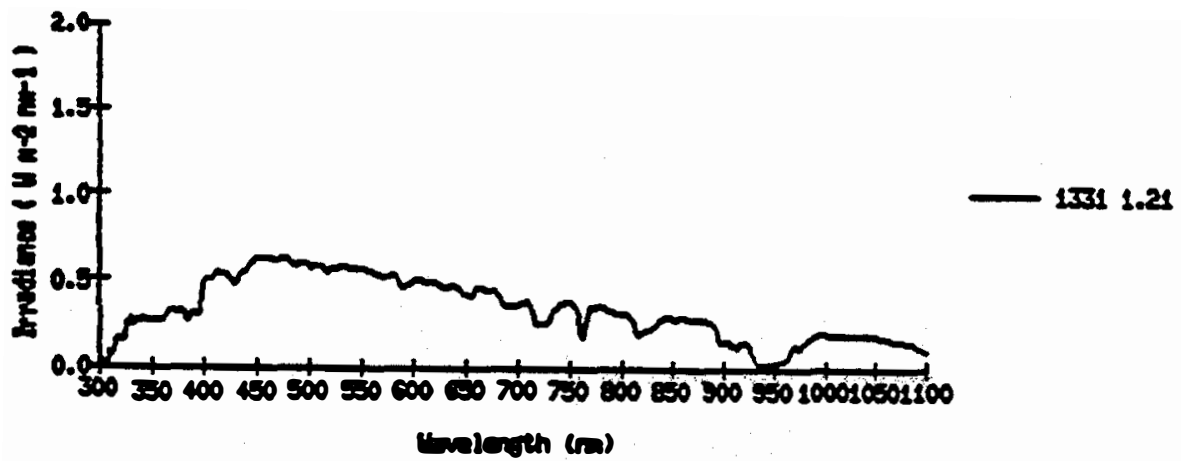


4 ESTC87268

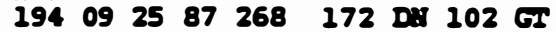

0 cloud cover at 0730

Tenp controllerss $17240 \mathrm{C}$ and $10241 \mathrm{C}$ at 1130

sildes: $0725 \mathrm{G}$

$0825 \mathrm{G}$

$0925 \mathrm{H}$ (dark)

$1025 P$.

$1125 \mathrm{P}$

$1224 \mathrm{P}:$

$1424 G$

$1524 \mathrm{G}$
$1624 \mathrm{G}$

F5EC 258

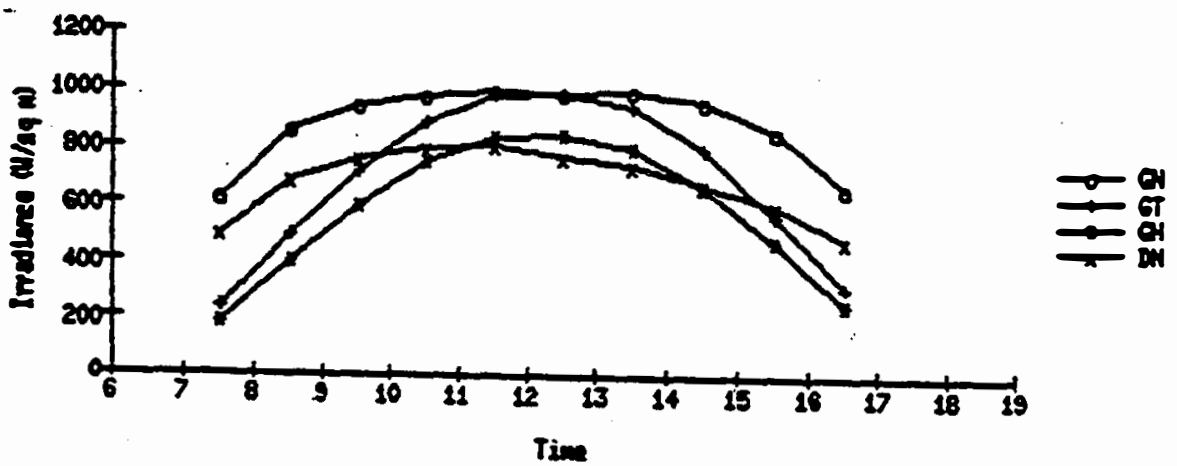

FSEC $248 \mathrm{un}$

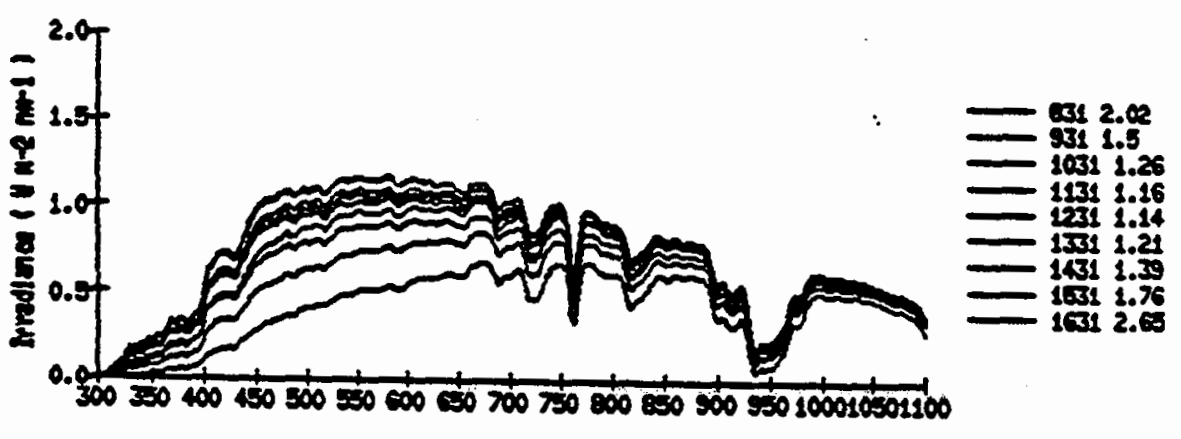

bevilugth (ra)

FISE $298 \mathrm{GT}$

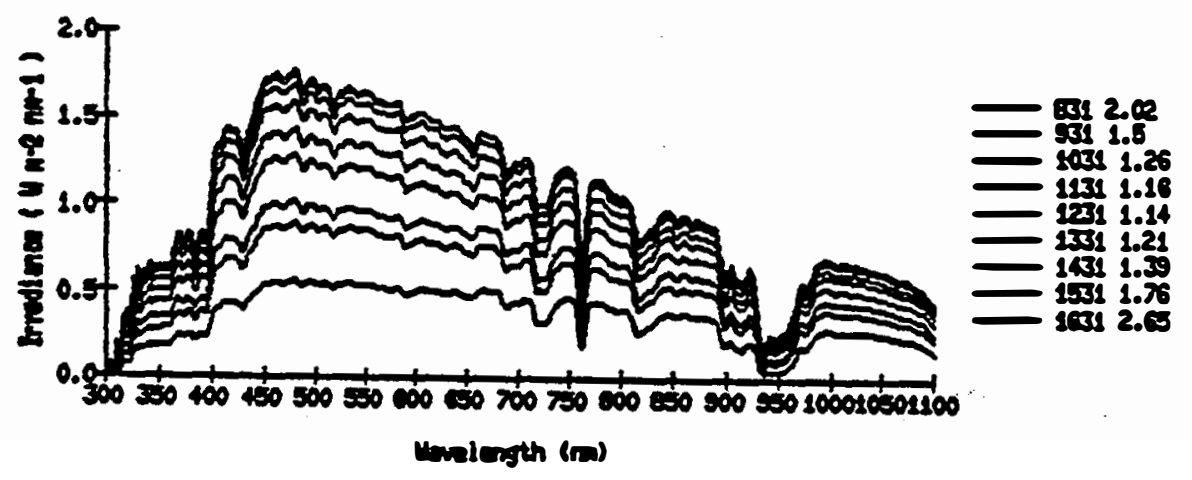




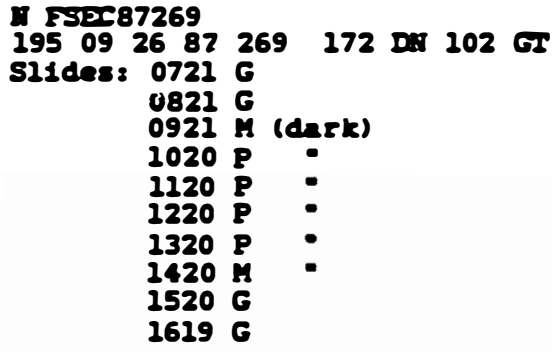

Brief spike in oV in Dis spectrm at 0832

Fase 290

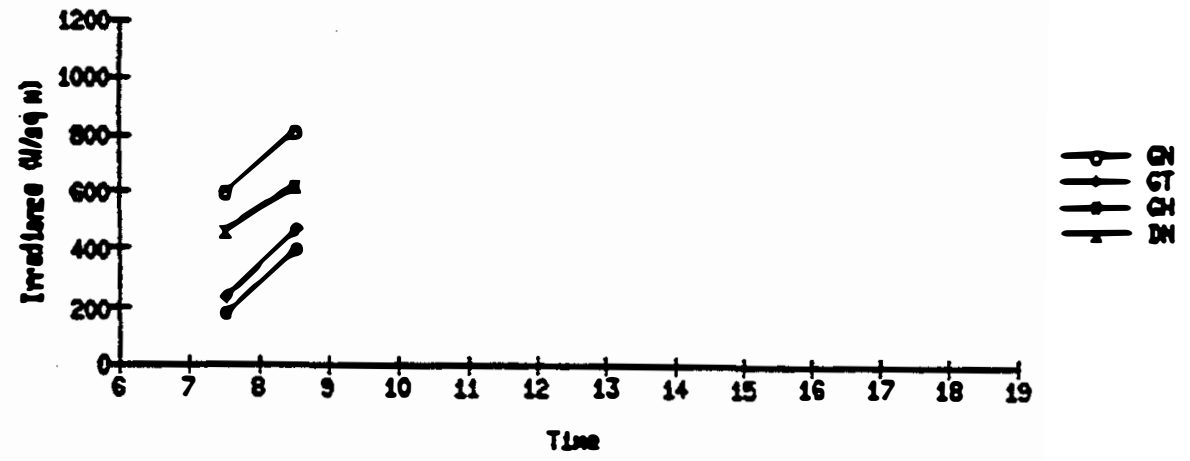

FEeC 200 DIT

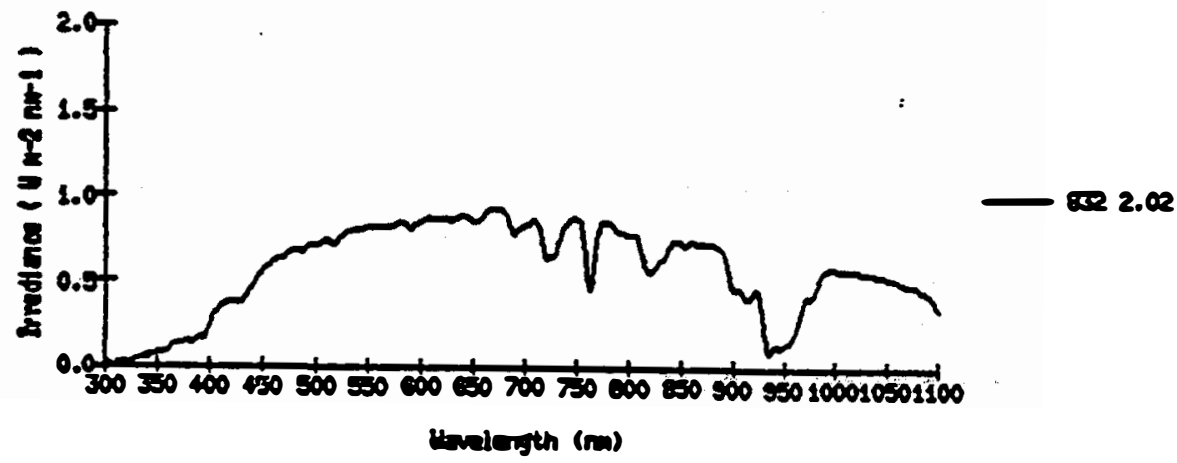

FIE 200 CTS

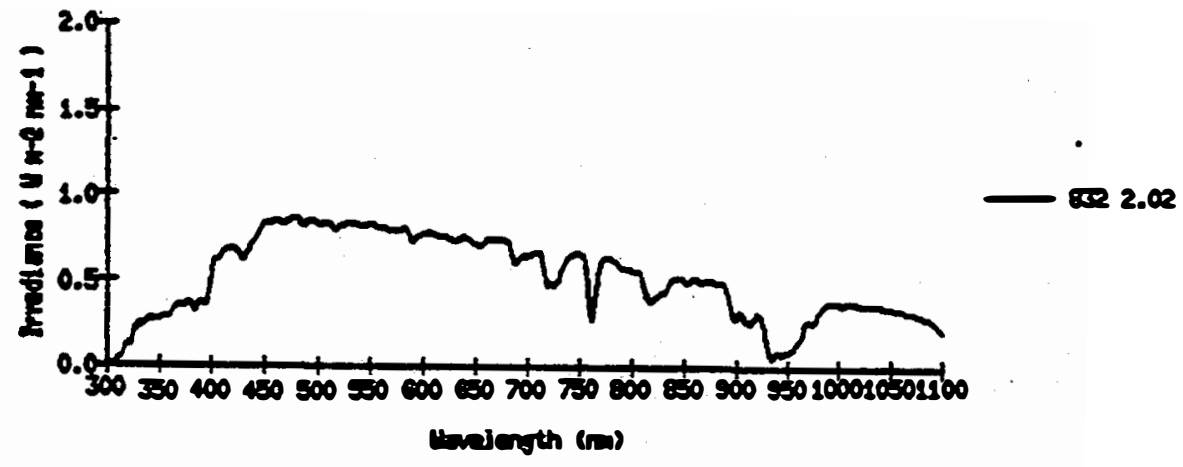




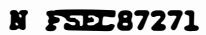

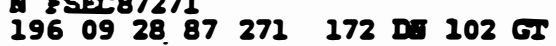

0.9 cloud cover at 0710 and 1130

$\begin{array}{rrrr}0 & \text { controllersi } & 172 & 102 \\ 0710 & 40 \mathrm{C} & 40 \mathrm{C}\end{array}$

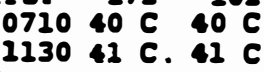

slides: $0712 \mathrm{G}$

$0812 G$

$0911 G$

1011 M (dark)

$1111 P$.

1211

$1311 \mathrm{G}$

$1611 \mathrm{G}$

$1510 \mathrm{G}$

$1610 \mathrm{G}$

FIC 271

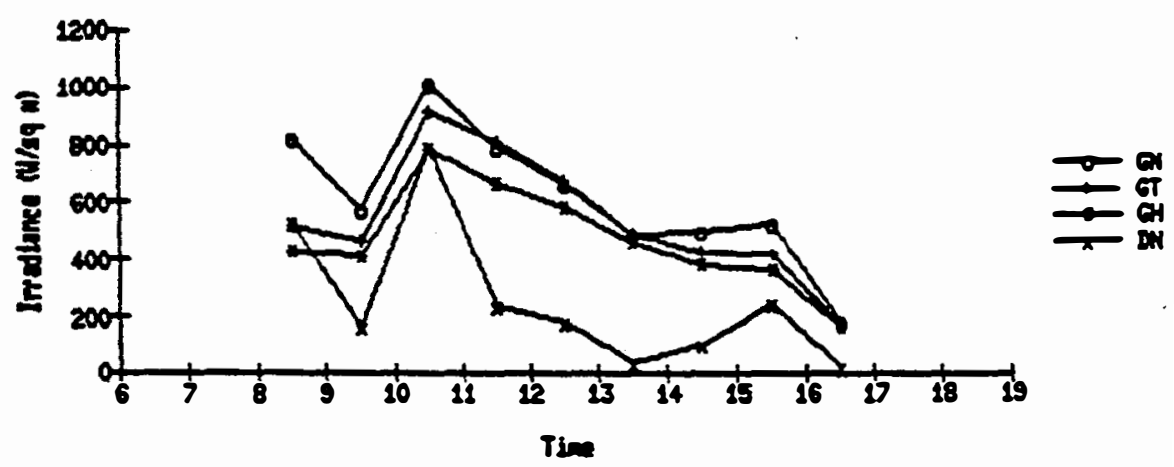

FבC 271 DT

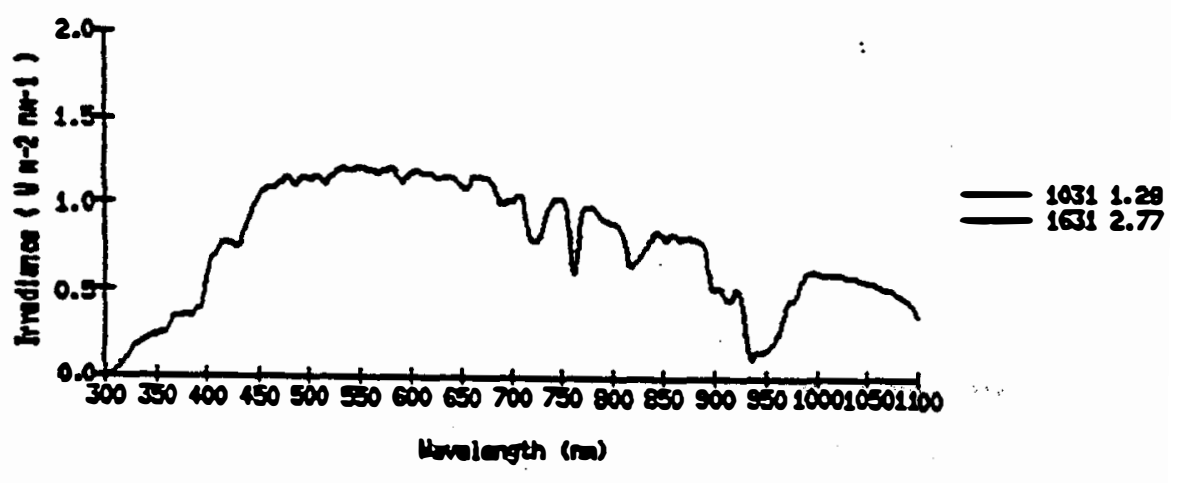

FIS 291 GS

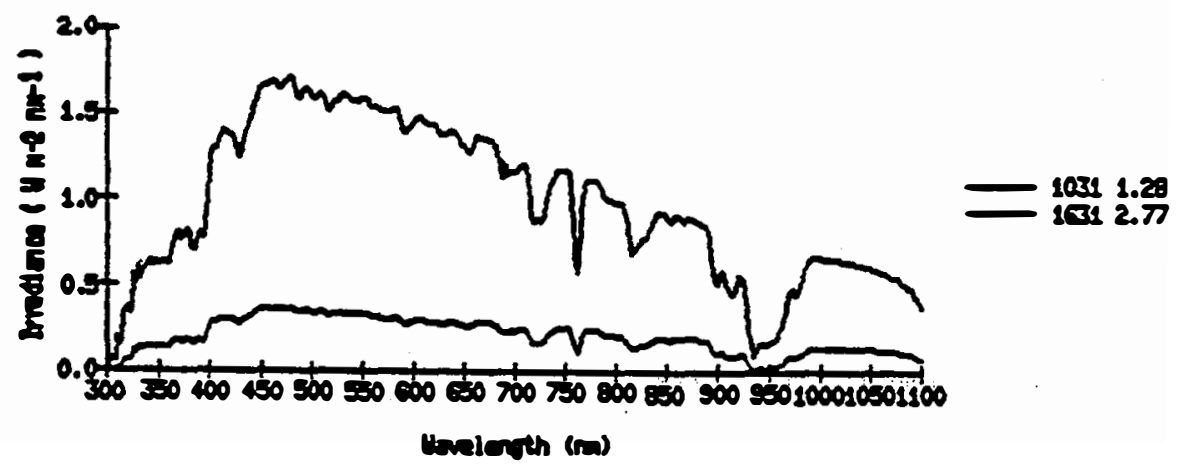


PST2C87272

$\begin{array}{llll}19709 & 29.87 & 872 \quad 172 \quad \mathrm{DP} & 102 \mathrm{GT}\end{array}$

0.9 cloud cover at $0715 ; 0.4$ at $1130 ; 0.2$ at 1530

Ieap controller: 172102

071539 C 39 C

$113044 \mathrm{C} 40 \mathrm{C}$

sl1des $0706 \mathrm{G}$ $42 C 41 C$

$0806 \mathrm{G}$

$0906 \mathrm{G}$

1006 \& (dnsk)

$1106 \mathrm{P}$

$1223 P$ :

$1333 \mathrm{P}$

$1433 \mathrm{G}$

$1532 \mathrm{G}$

$1632 \mathrm{G}$

Spike in of in Da spectren at 0831

Fis $2 \pi$

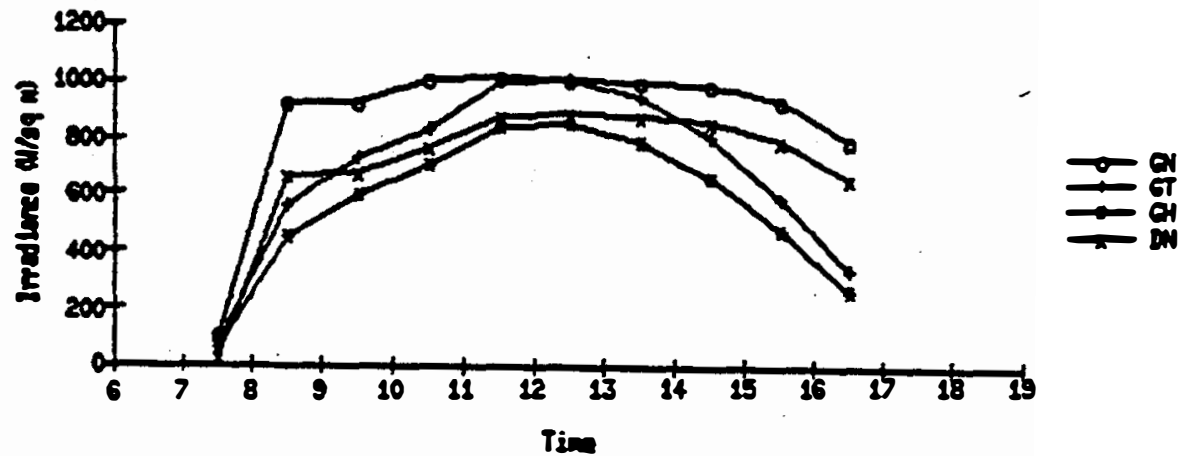

एड्यद $220 \mathrm{DT}$

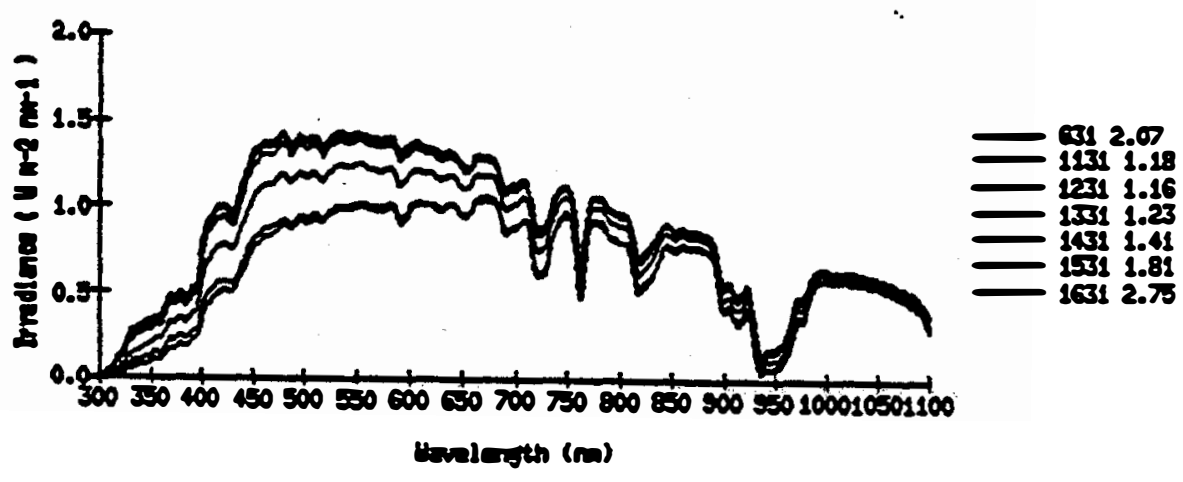

FIי $2 \pi 2 \mathrm{GT}$

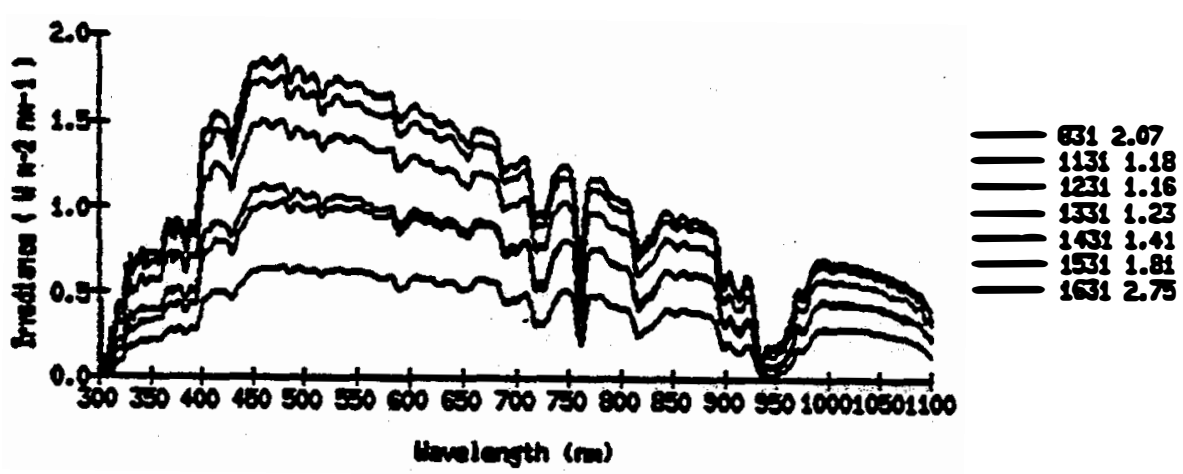




\section{SEP1 *}

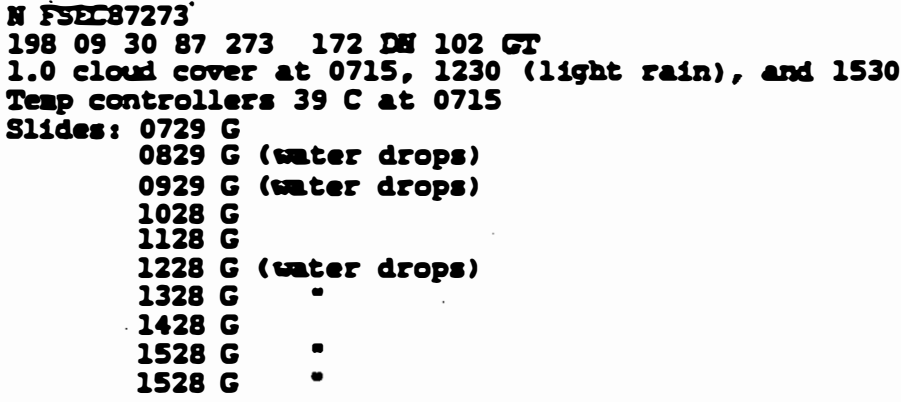

spectral integral a brondsund at 1231

F=ב 23

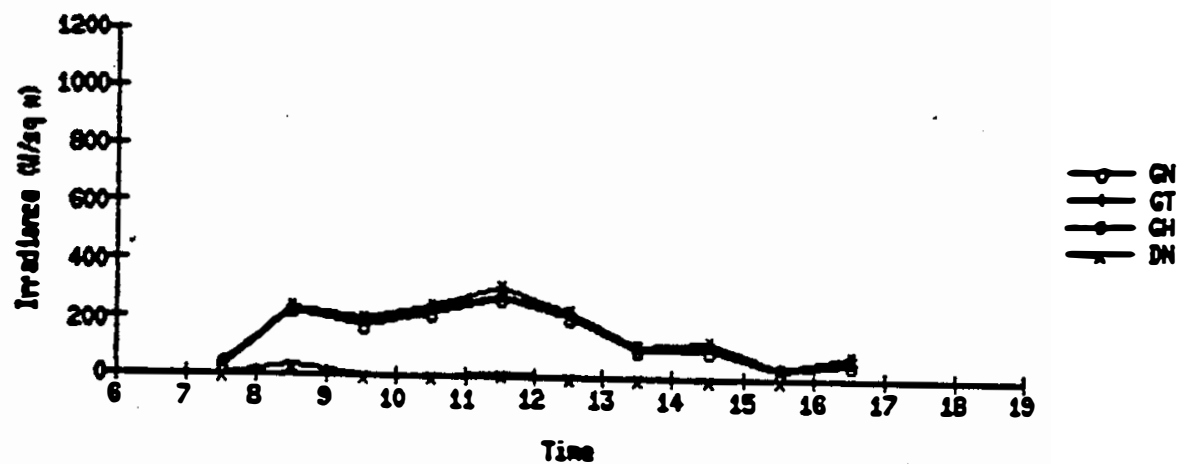

FESC 2OS NT

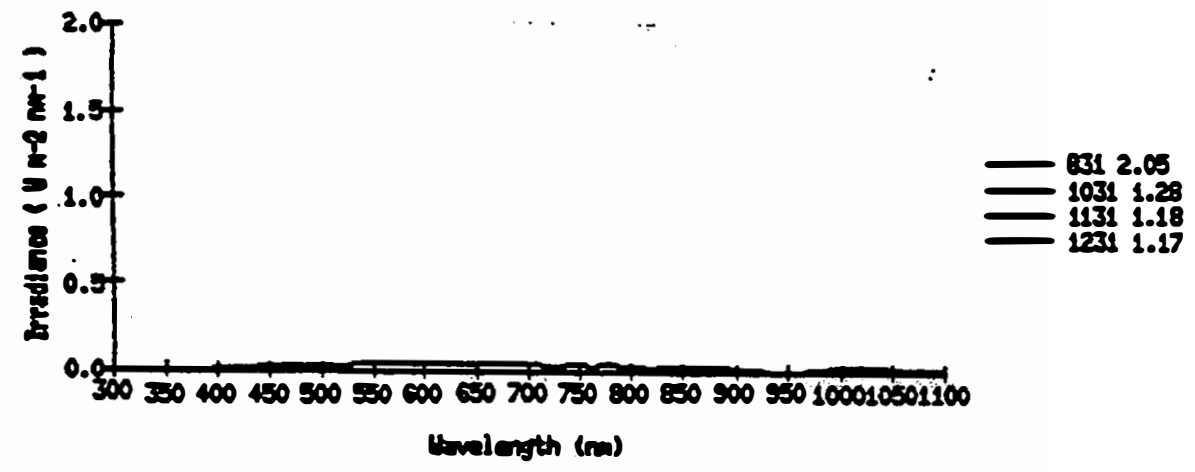

FIs $273 \mathrm{GT}$

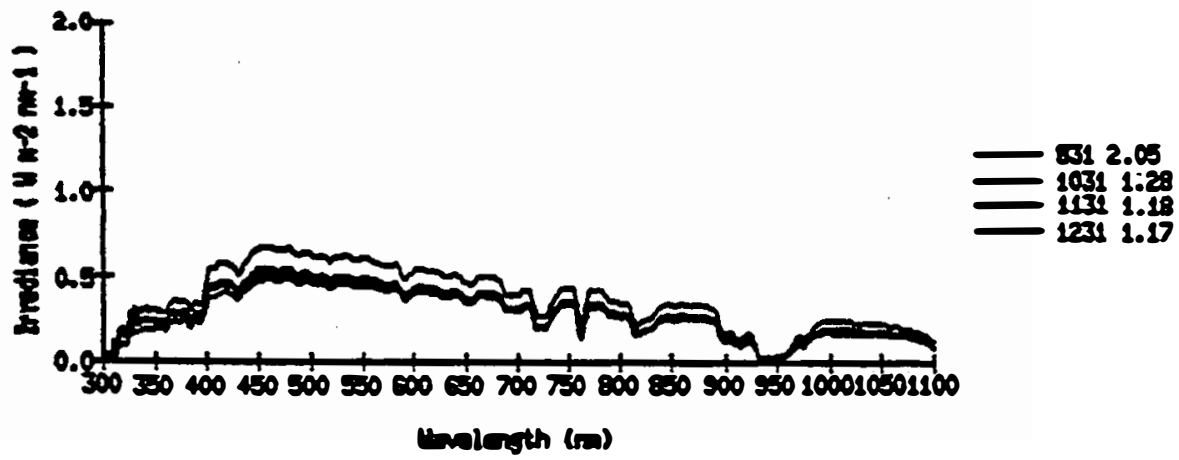


月. 532087274

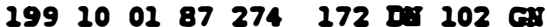

0.4 cloud cover at 2030 and winds: 0.4 at 2600

Teap controllers: $17240 \mathrm{C}$ and $10241 \mathrm{C}$ at 1600

Sl1des? $0725 \mathrm{G}$ (enter drops)

$0825 \mathrm{G}$

$0925 \mathrm{G}$

$2025 \mathrm{G}$

$2125 \mathrm{G}$

1225 (dnsk)

$1325 \mathrm{G}$

$1425 \mathrm{G}$

$2524 G$

$1637 \mathrm{G}$

Spikes in oV in Gi spectra at 1533 and 2631

P्या⿰ 224

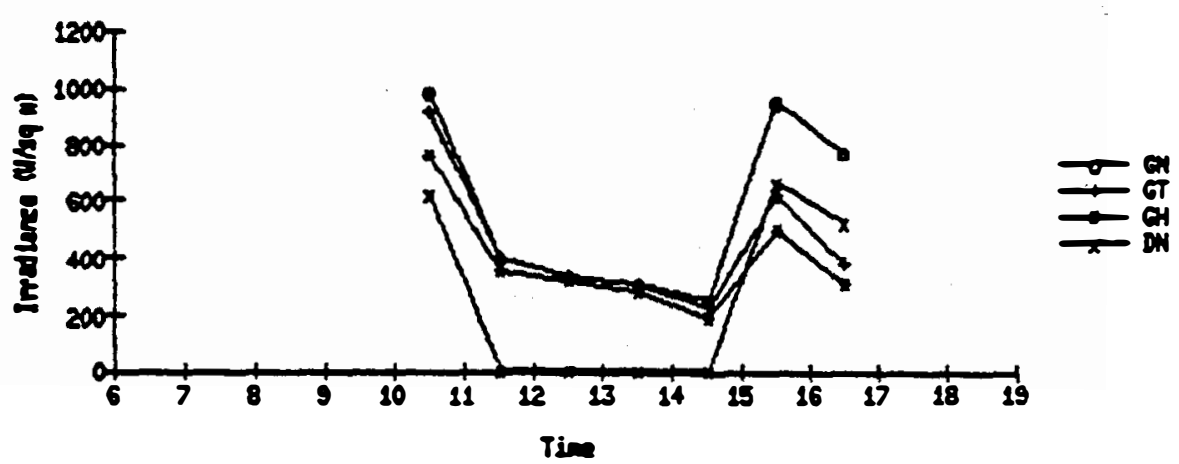

FEस 204

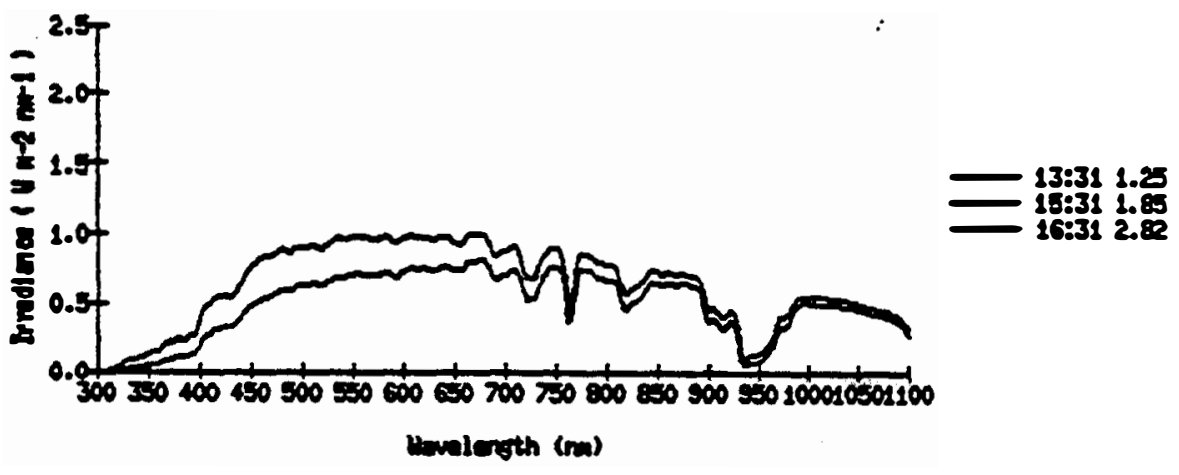

$F=20405$

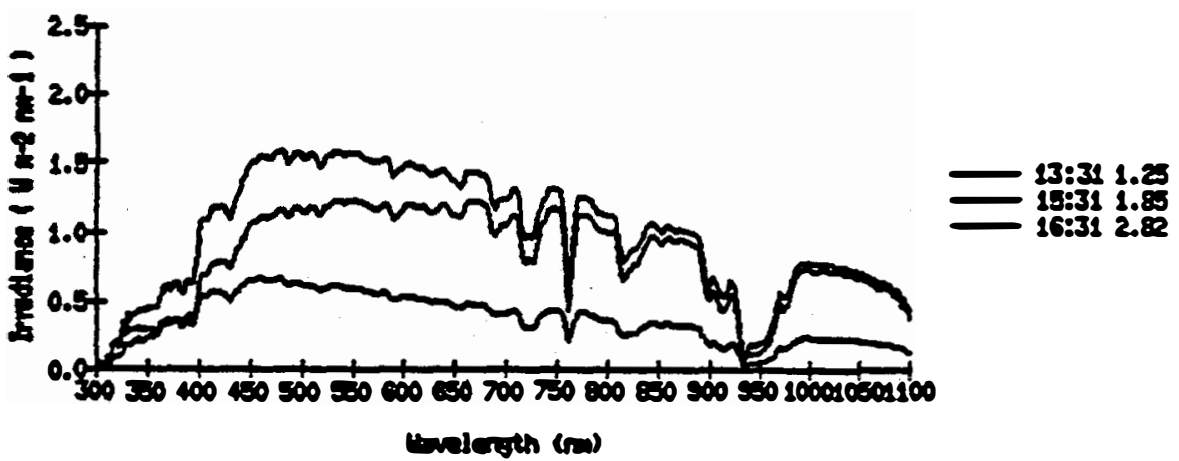




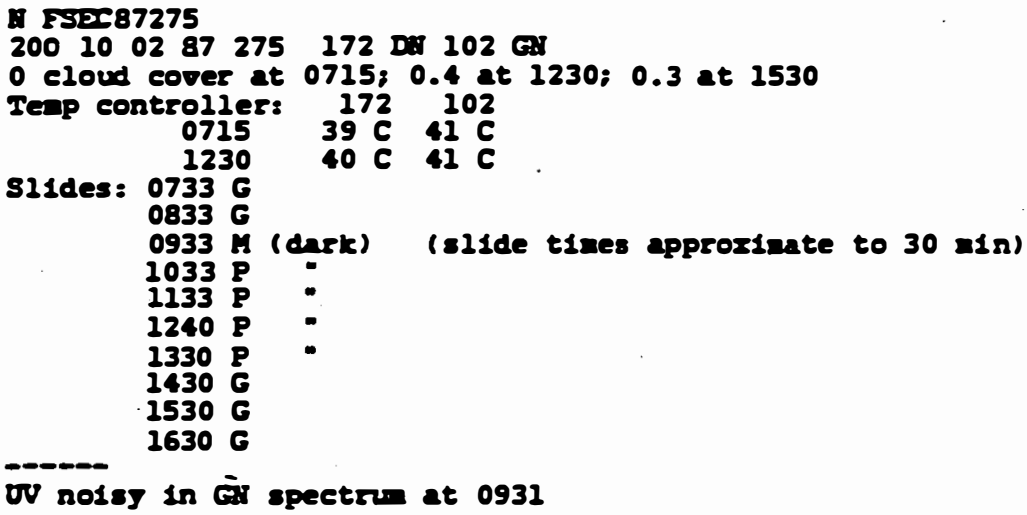

OV noiss in Gil spectrr at 0931

Fase 25
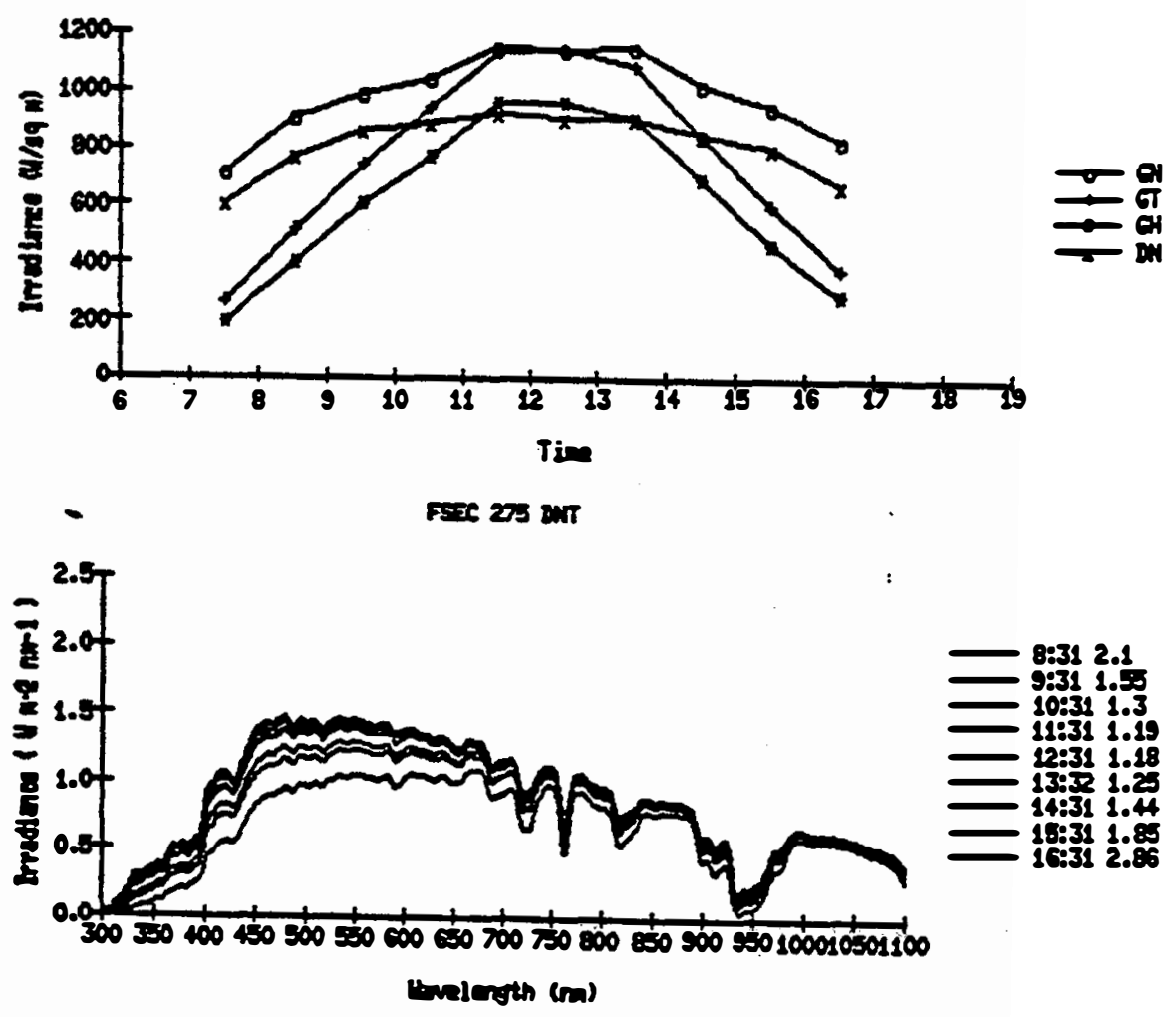

$\operatorname{Fsc} 273$ as

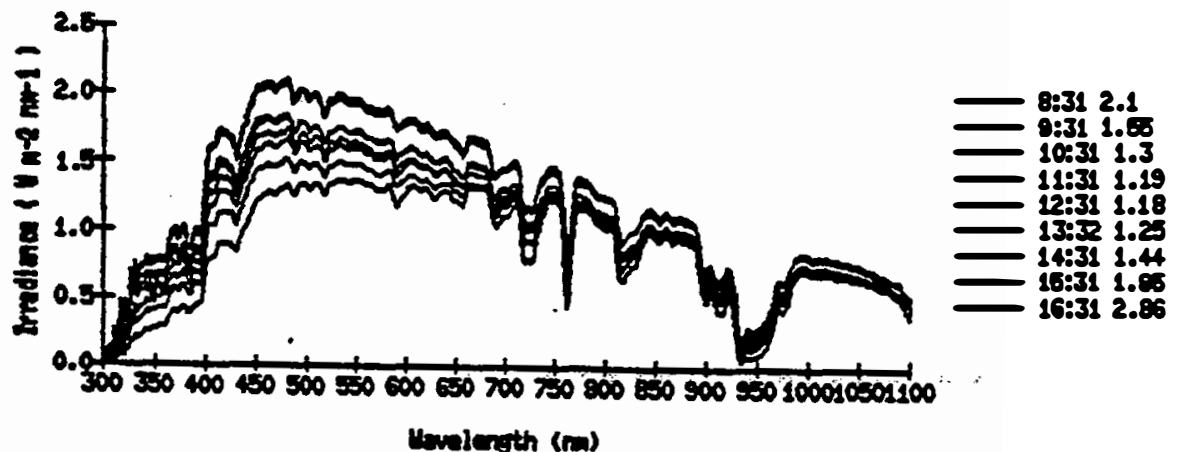


A

$201 \quad 100387276 \quad 172 \quad 102 \quad \mathrm{GP}$

Whekend datas possible tracking errors

Sildes: $0730 \mathrm{G}$ $0830 \mathrm{G}$

$0930 \mathrm{H}$ (dark) (slide tines approrinate to $30 \mathrm{aln}$. )

1030

$1130 \mathrm{P}$

1230

1330

$1430 \mathrm{G}$

$1530 \mathrm{G}$

$1630 \mathrm{G}$

$\operatorname{rasc} 270$

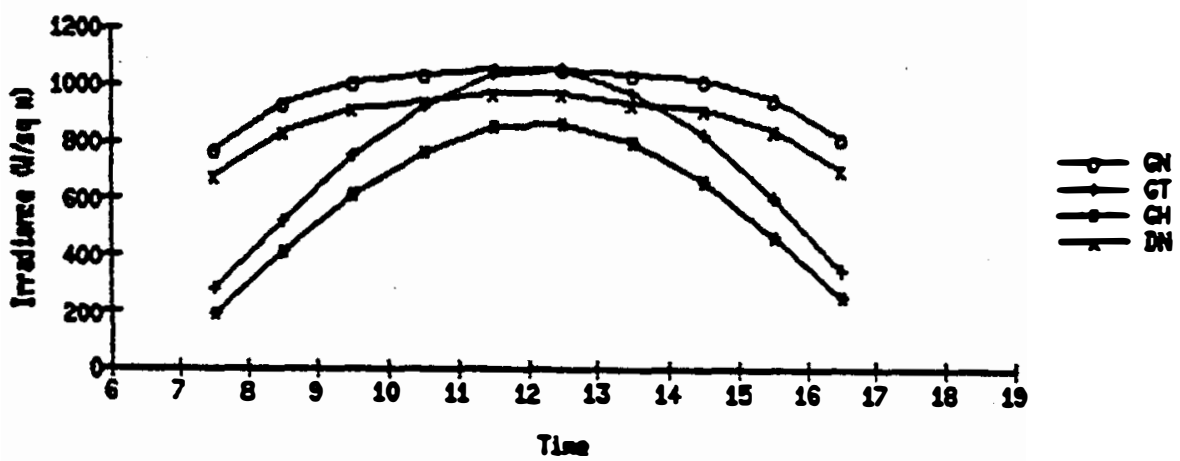

FISC 205 DT

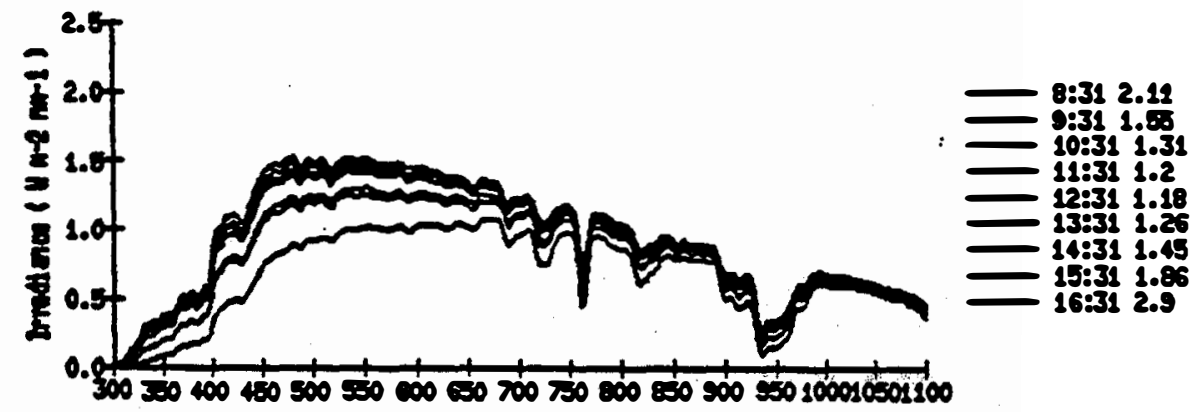

biolerith (m)

pace $2 x 6$ es

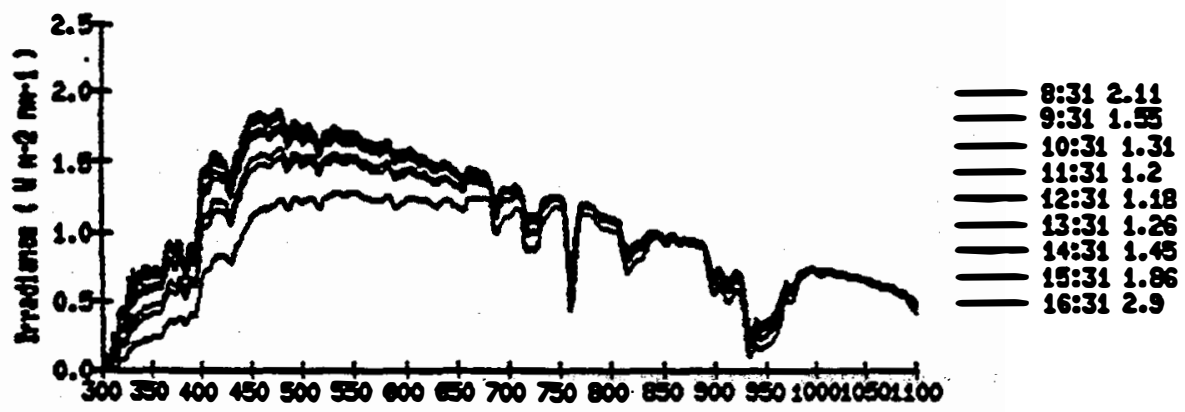

(bolergith (ra) 


\section{DE}

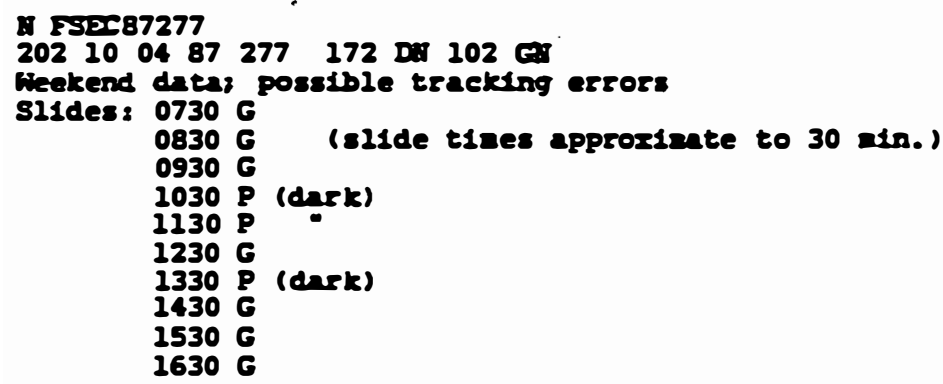

$\operatorname{rsx} 2 \pi$

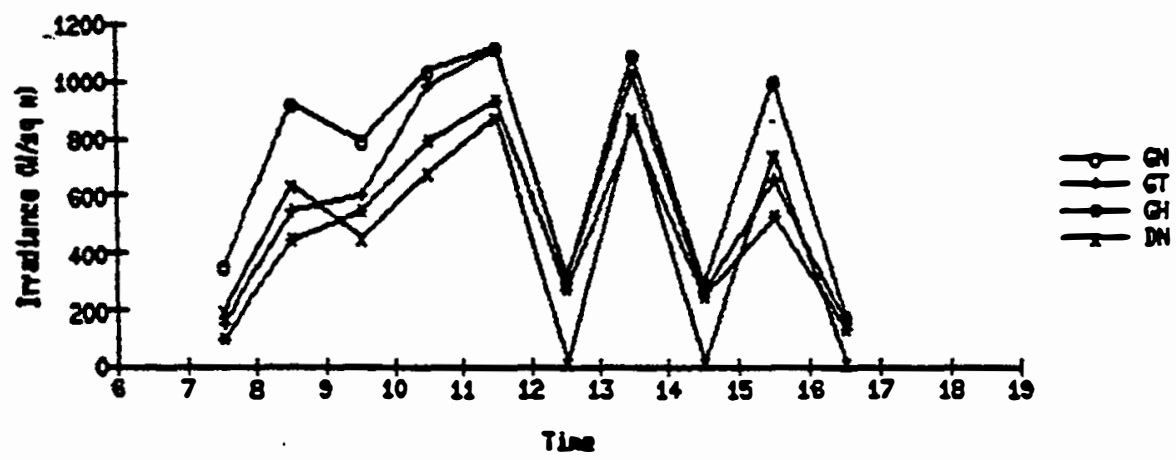

FIE $278 \mathrm{dT}$

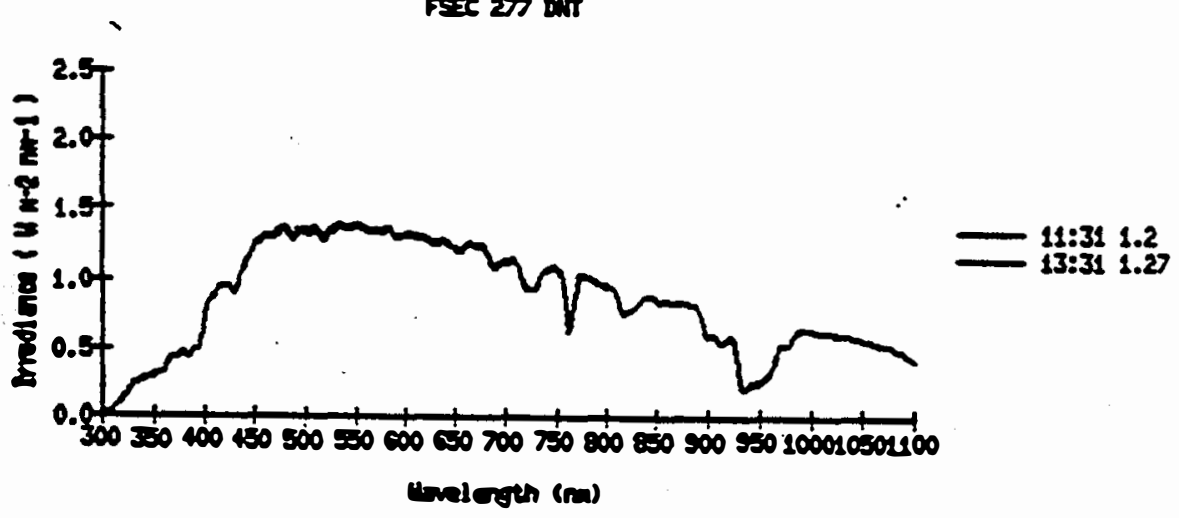

$2=250$

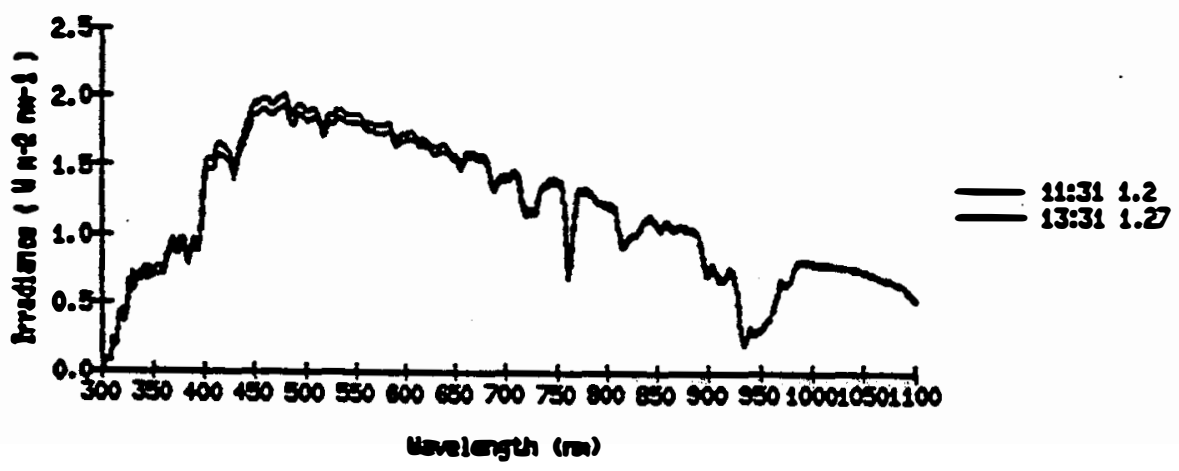


म. PSP 87278

$\begin{array}{lllllllll}203 & 10 & 05 & 87 & 278 & 172 & \mathrm{DM} & 102 \mathrm{GS}\end{array}$

1.0 cloud cover at $0730 ; 0.4$ at $1030 ; 0.5$ at 1530

Tenp controller: 172102

$0730 \quad 39 \mathrm{C} \cdot 40 \mathrm{C}$

$1030 \quad 40 \mathrm{C} \quad 40 \mathrm{C}$

Tracking error of about 3 deg prior to 1030 seans reset

Slides: $0730 \mathrm{G}$

$0830 \mathrm{G}$

$0930 \mathrm{G}$

$1030 \mathrm{M}$ (dark)

$1130 \mathrm{P}$

$1230 \mathrm{P}:$

$1430 \mathrm{G}$

$1630 \mathrm{G}^{-}$

Spectral integral = brondond et 0831

Fise 278

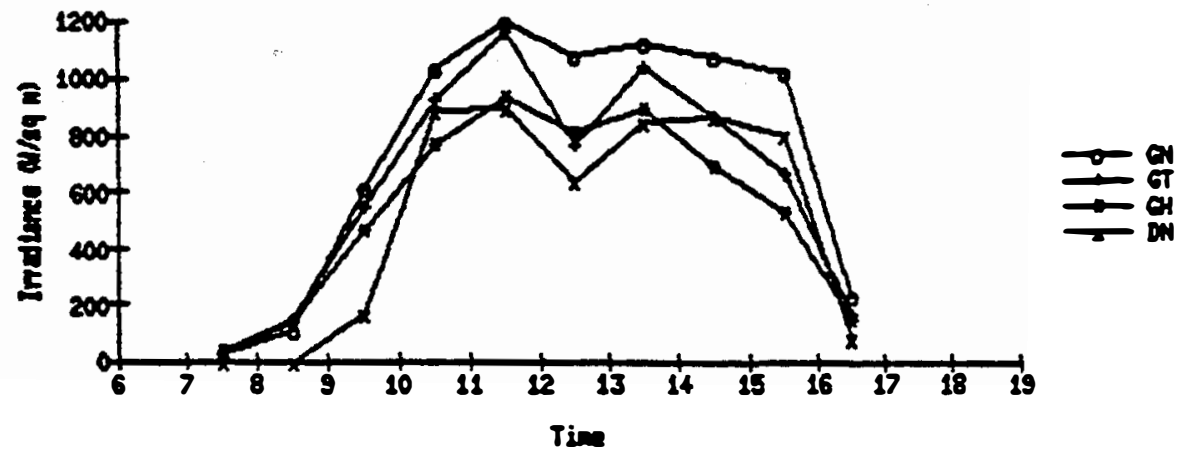

Fise $278 \mathrm{Br}$

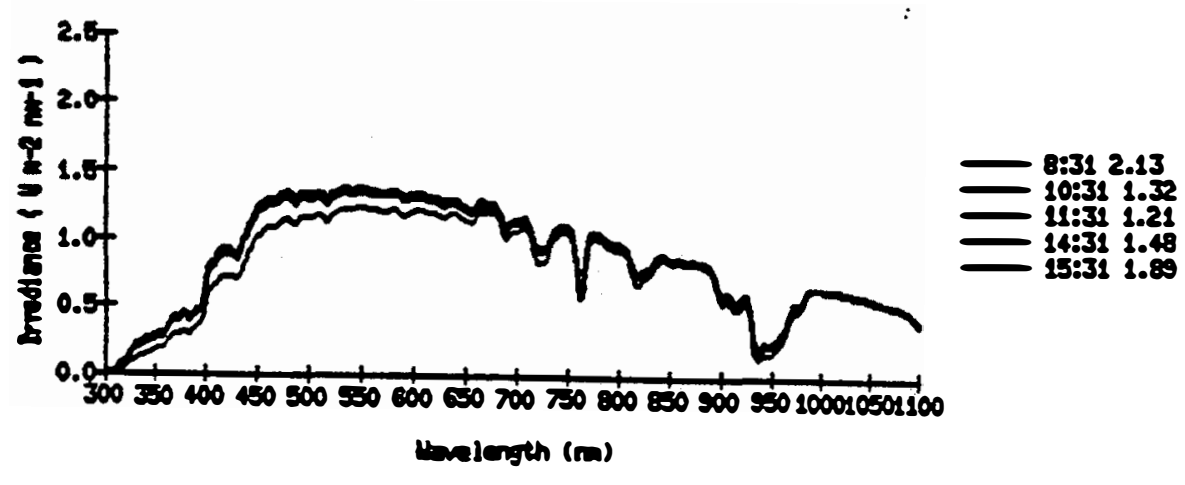

Fise 278 os

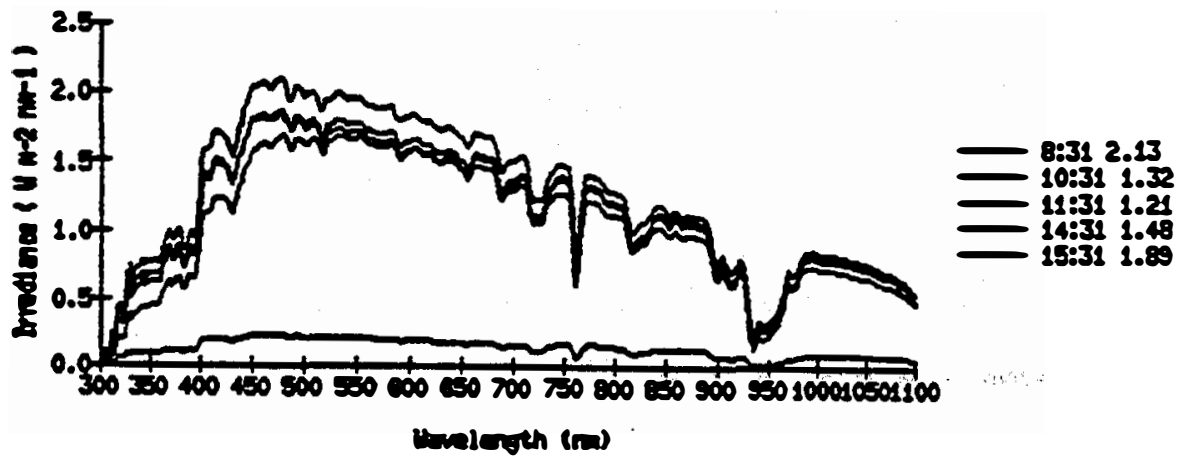




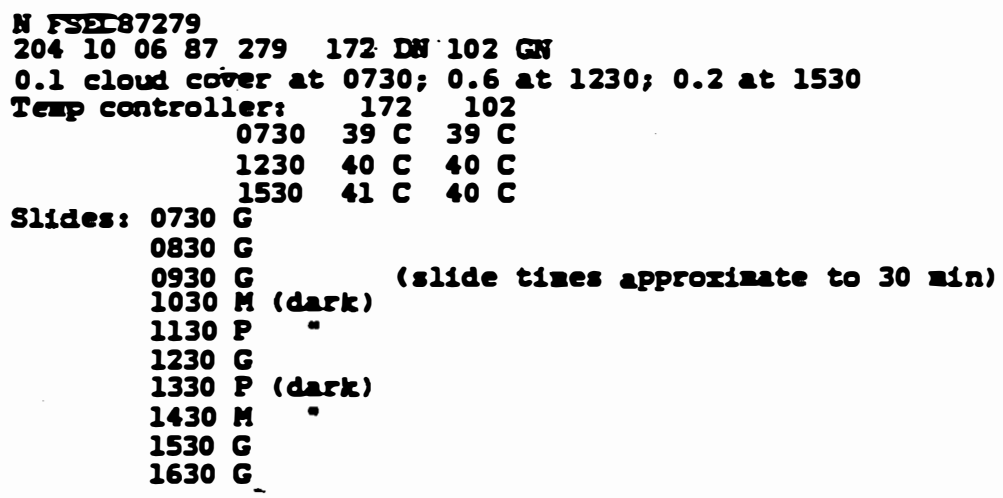

Fsec 279

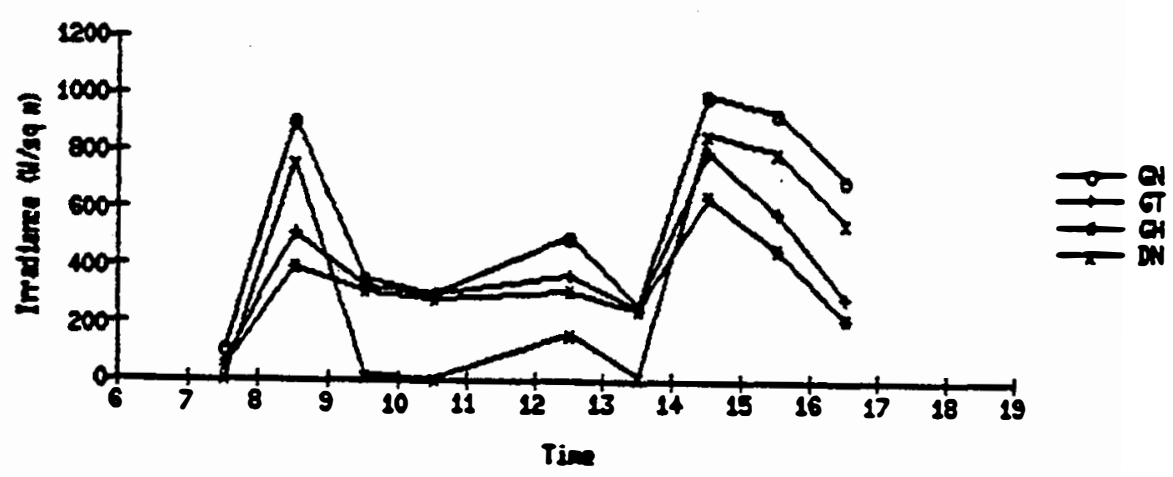

Pase 209

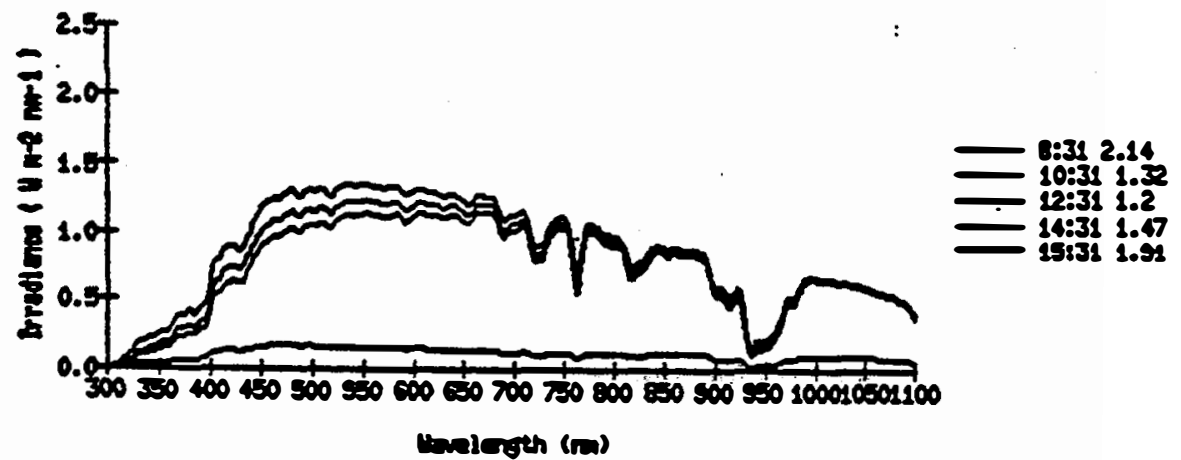

PST6 205

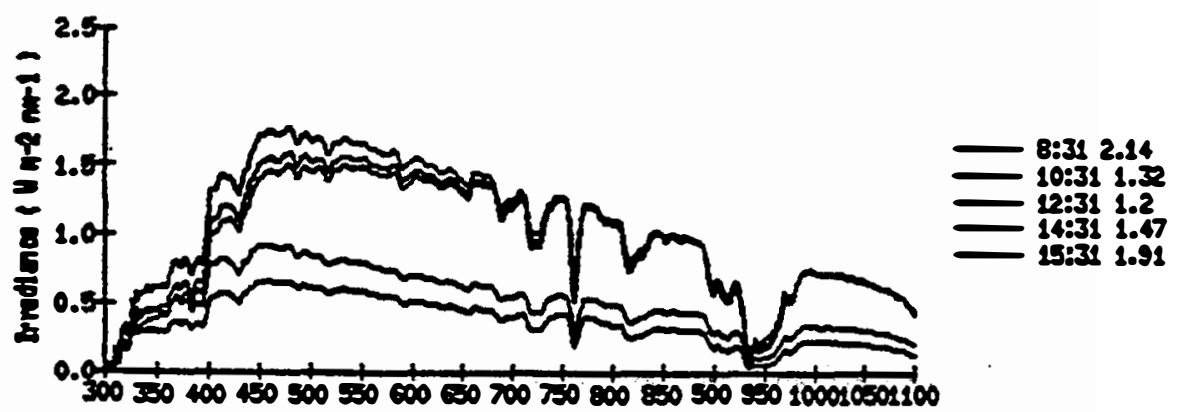

lonigres (m) 


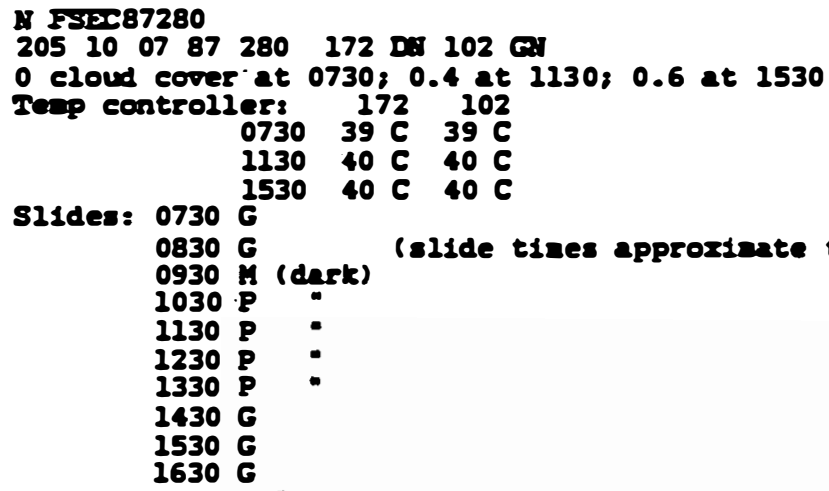

FIC 200

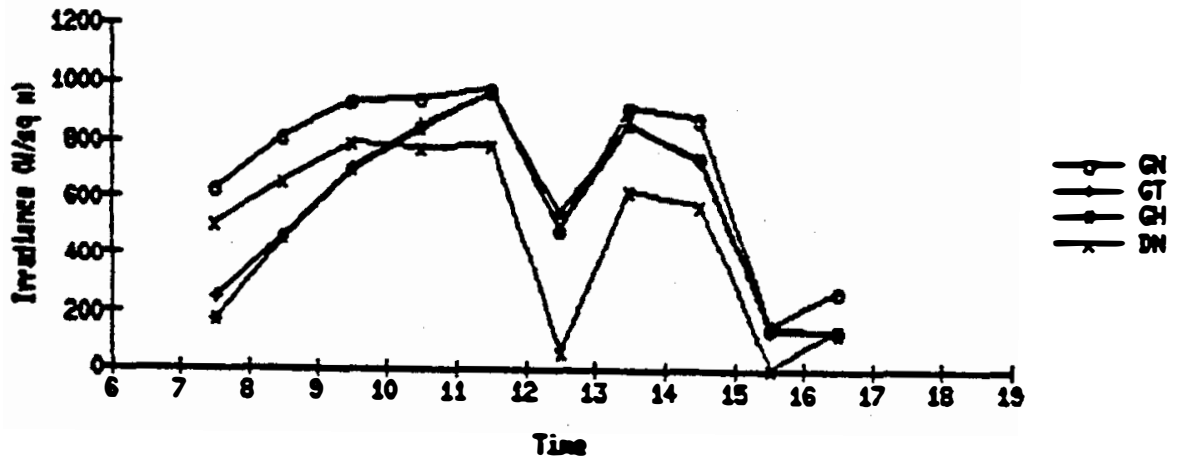

PIE $200 \mathrm{dm}$

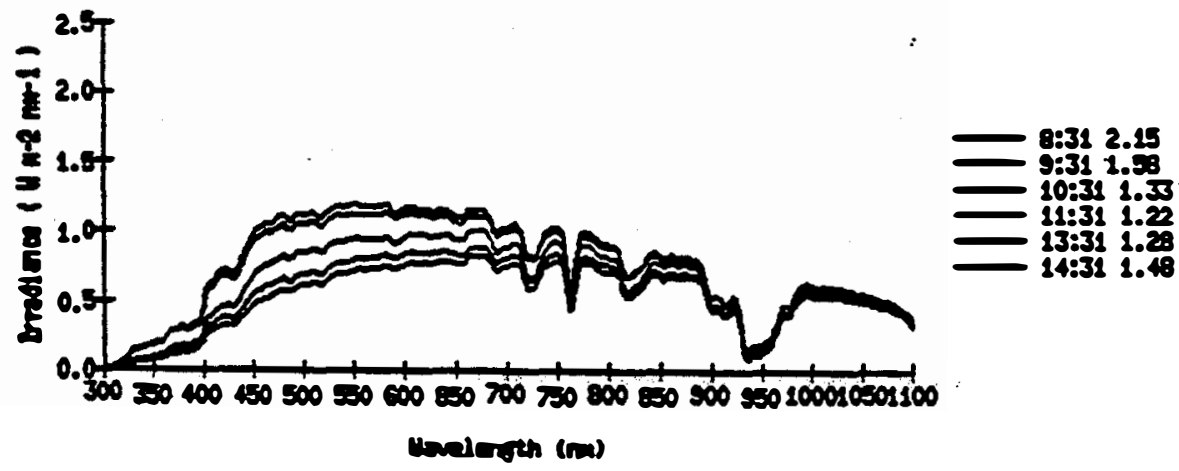

Wre 2000

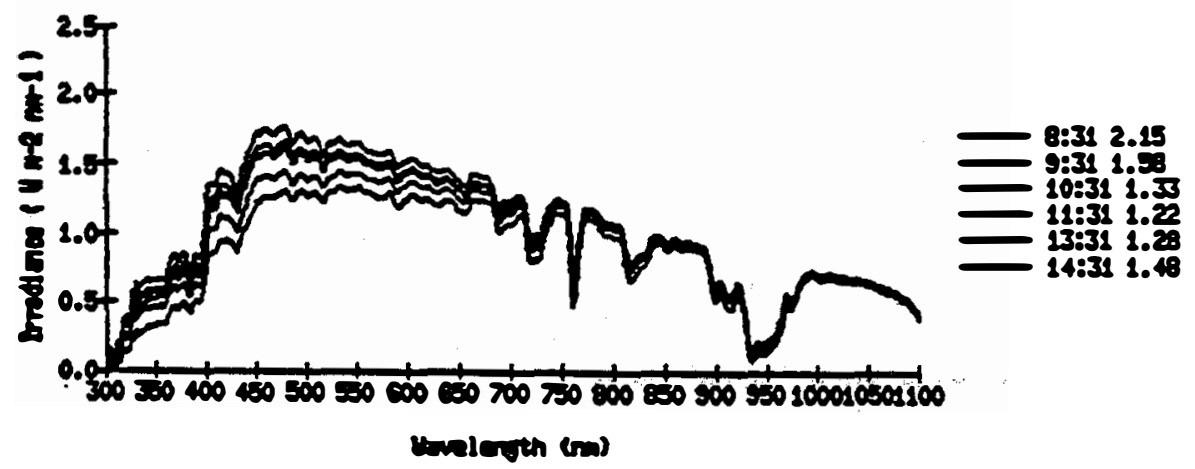




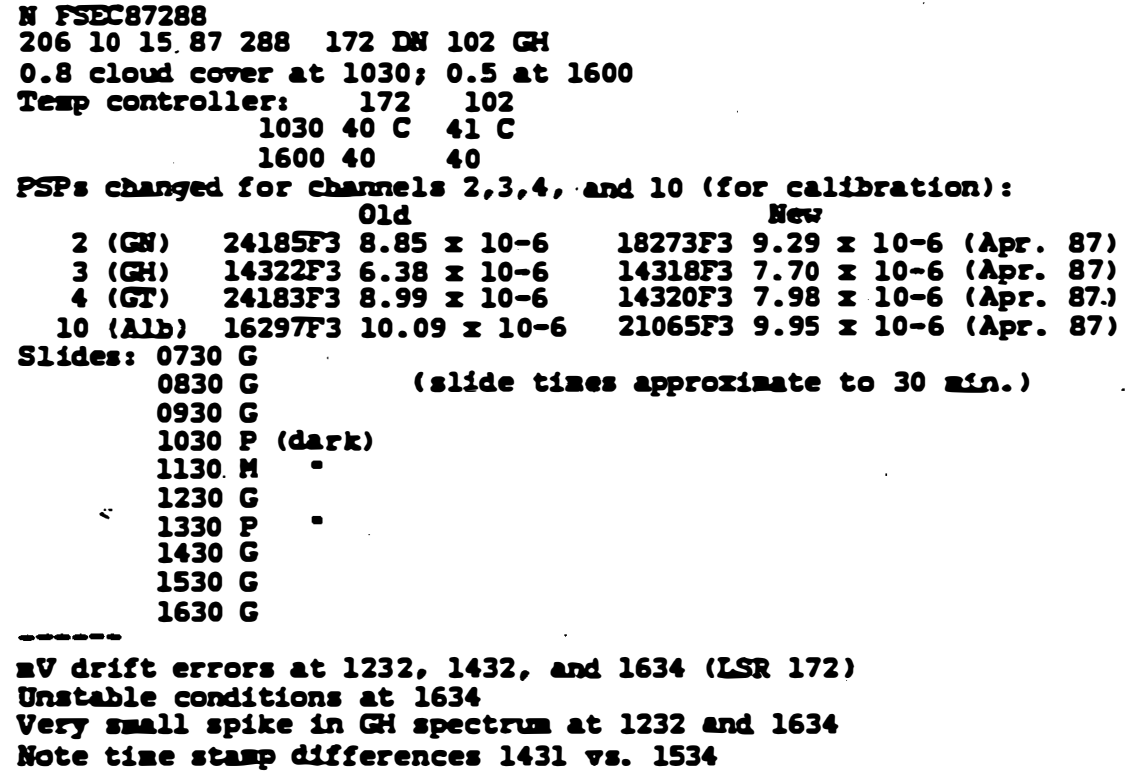

a drift errors at 1232, 1432, and 1634 (ISR 172)

Onatable conditions at 1634

Vers sall spike in GH spectru at 1232 and 1634

Hote tine stam differences 1431 vs. 1534
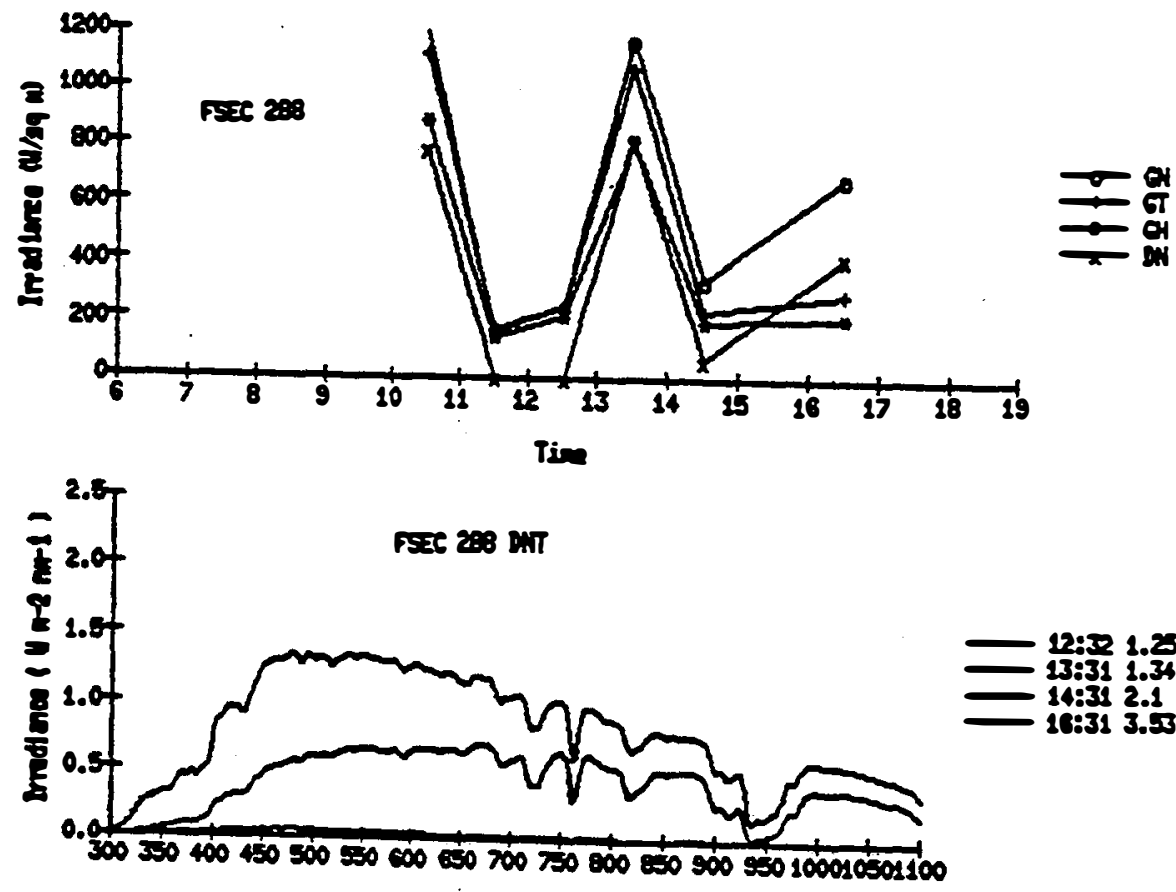

teviongth (m)

Fax 2890 Os

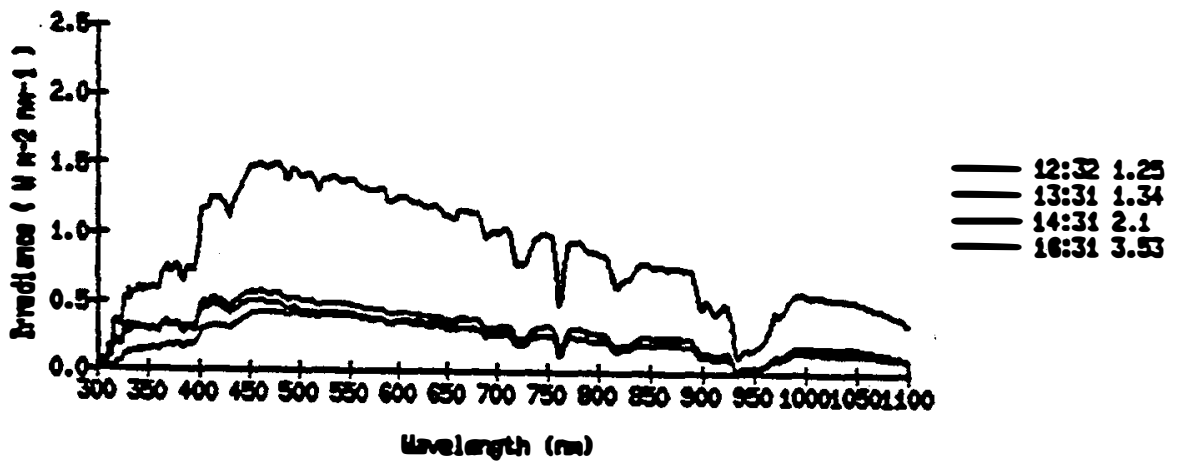



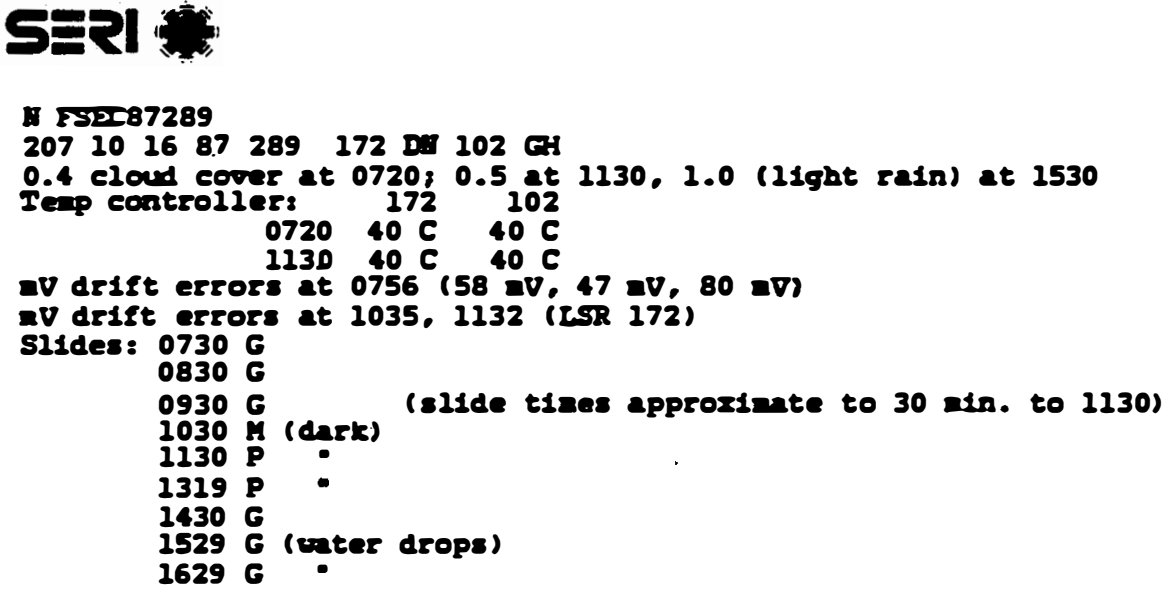

Fise 280

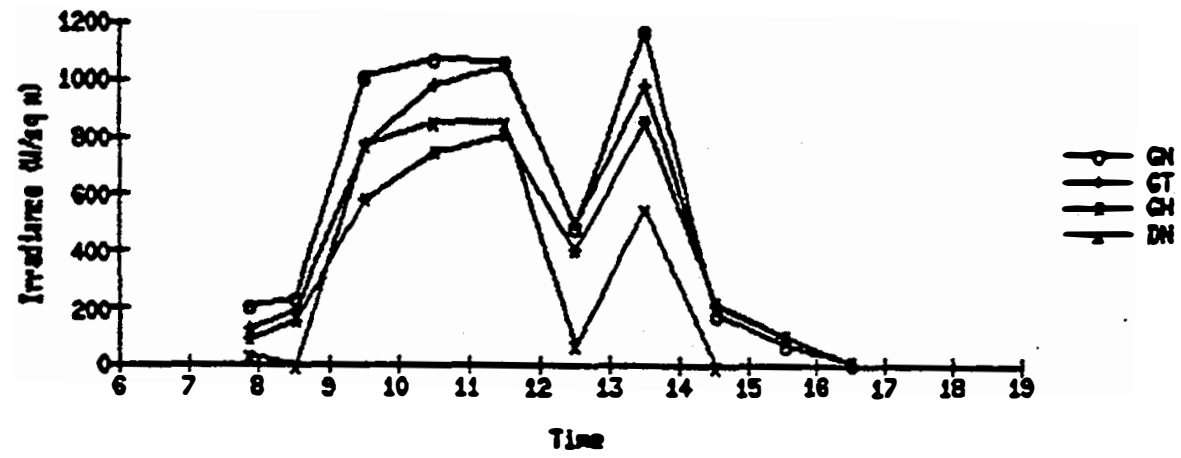

Fase 209 int

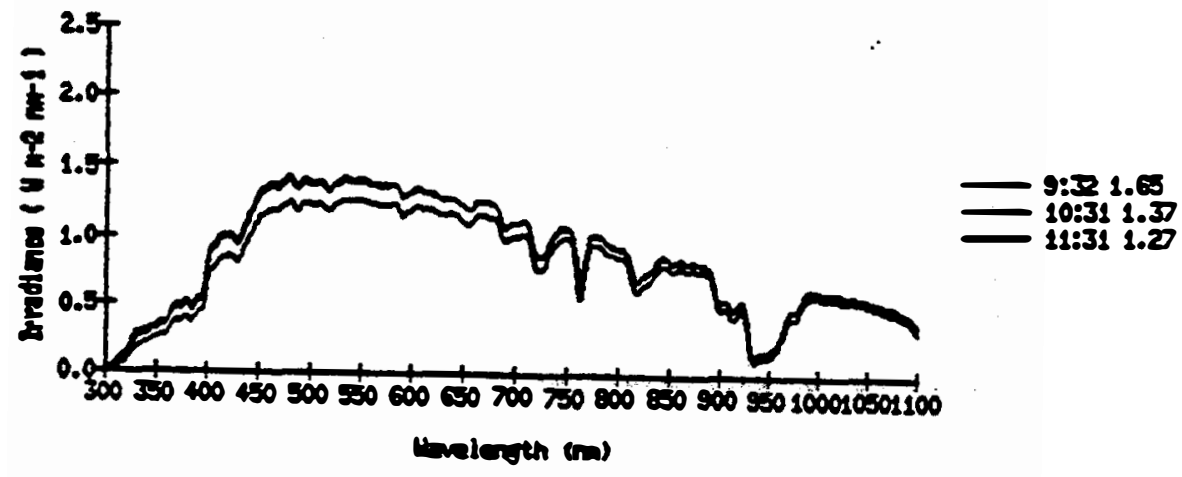

Fise 280 as

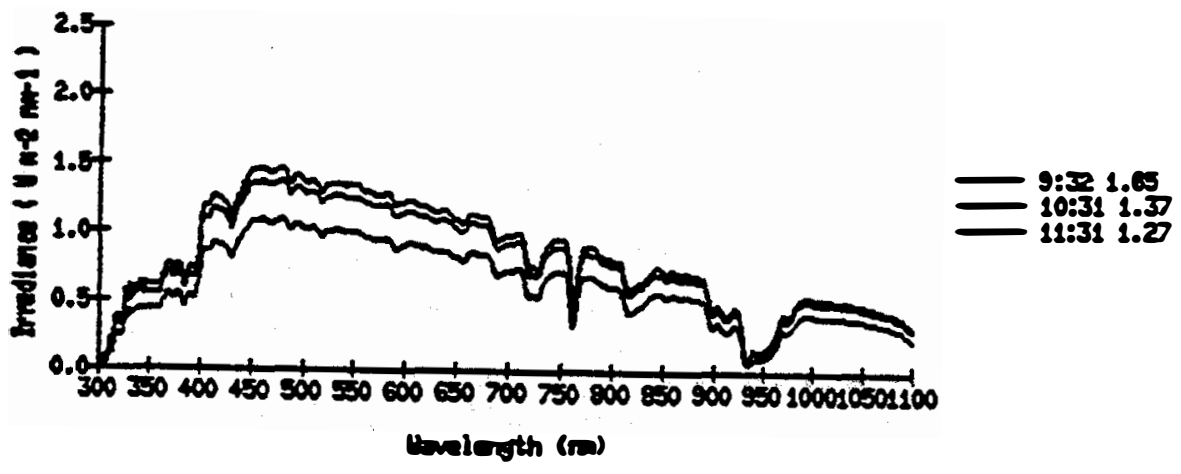




\section{SEPl 눈}

H T52887290

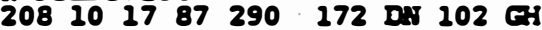

Cloudy: veekend dater

Slides: $0722 \mathrm{G}$

0822 G (rater drops)

0922

$1121 \mathrm{G}$

$1220 \mathrm{G}$

$1320 \mathrm{G}$

$1420 \mathrm{G}$

$1519 \mathrm{G}$

$1619 \mathrm{G}$

No spectra

Fase 20

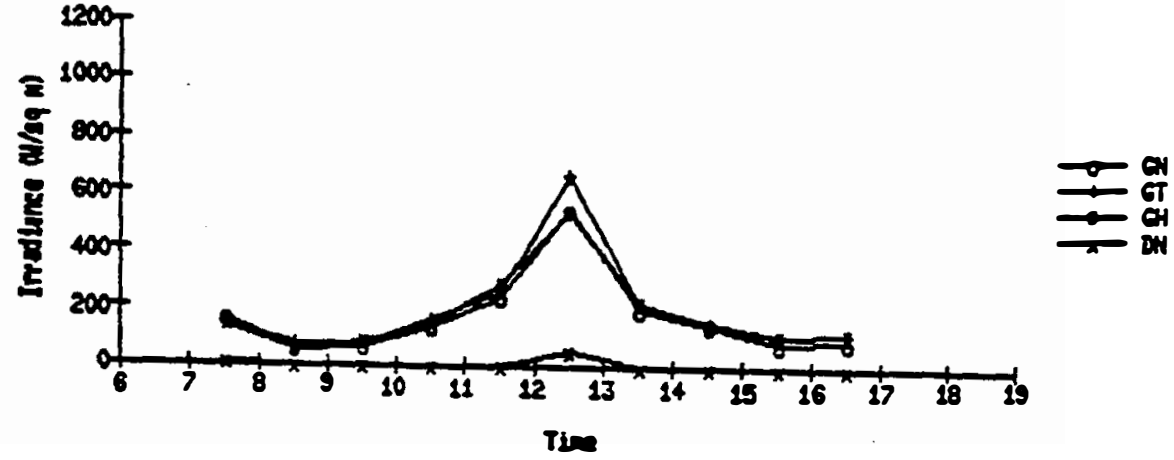


1. TSPE87291

$\begin{array}{lllllllllllll}209 & 10 & 18 & 87 & 291 & 172 & \text { D. } & 102 & \text { GH }\end{array}$

Cloud5: coekerd dater

av drift error at 1133

SILdes $0713 \mathrm{G}$ (weter drops)

$0813 \mathrm{G}$

$0913 \mathrm{G}$

$1012 \mathrm{G}$

1212

$1311 \mathrm{~K}$

$1611 \mathrm{G}$

$1511 \mathrm{G}$

$1610 \mathrm{G}$

Fax zas

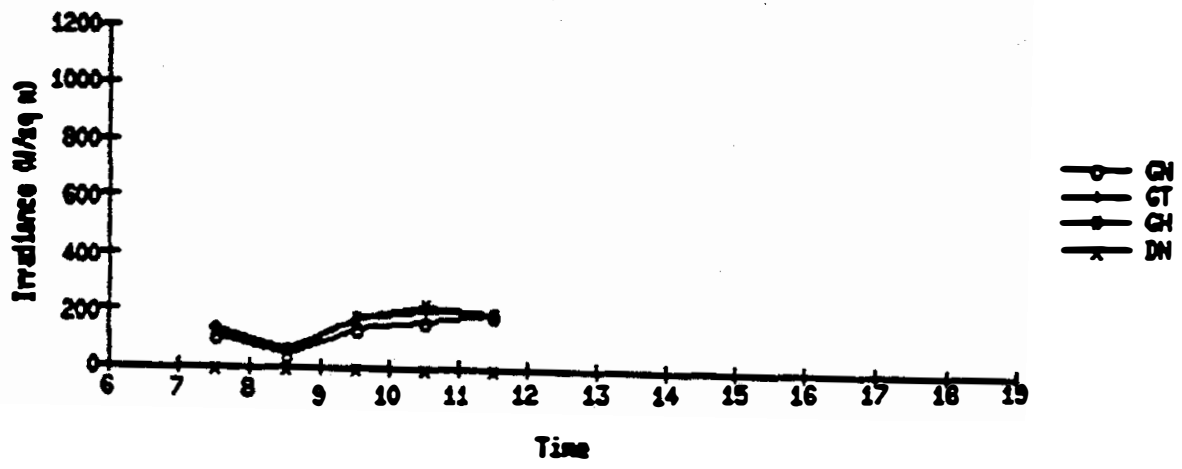

Pax 291 DIT

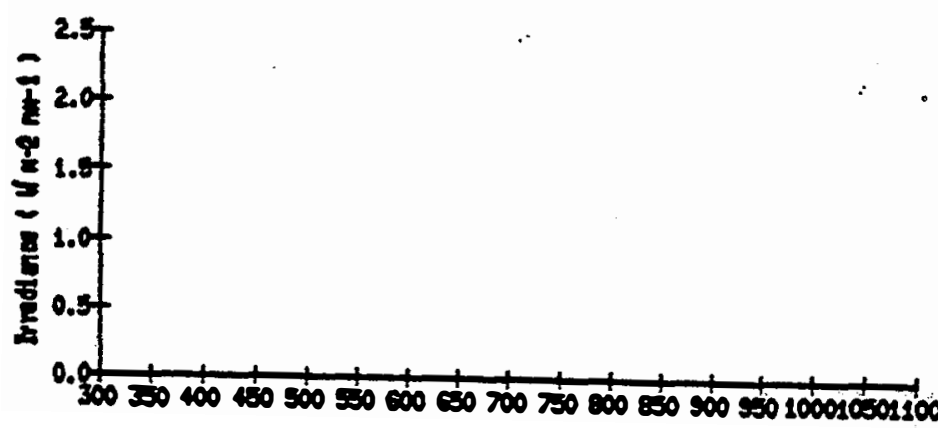

colorien (n)

Fser 291 ars

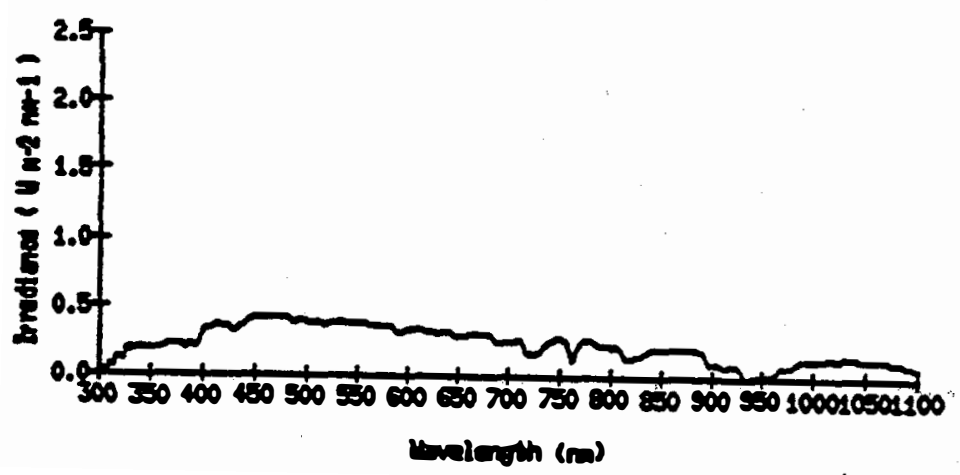


A Fi1587292

$\begin{array}{llllllll}210 & 10 & 1987 & 292 & 172 & \mathrm{Dm} & 102 & \mathrm{G}\end{array}$

210101987292 at 103080.9 at 1530

Iew controller: $172 \quad 102$

$\begin{array}{lll}1030 & 40 \mathrm{C} & 40 \mathrm{C} \\ 1130 & 40 \mathrm{C} & 41 \mathrm{C}\end{array}$

ISR 1725 DV drift error at 1130

TGR 172 dreft errors at 1033 and 1131

slides: 0704 G (wiater drops)

$0803 \mathrm{G}:$

$0903 \mathrm{G}$

$1003 \mathrm{G}$

$1102 \mathrm{P}$ (dark)

$1202 \mathrm{G}$

1301 A (dark)

$1401 \mathrm{G}$

$1501 \cdot G$

$1600 \mathrm{G}$

$1700 \mathrm{G}$

artor in of spectru at 1033, unseable conditions

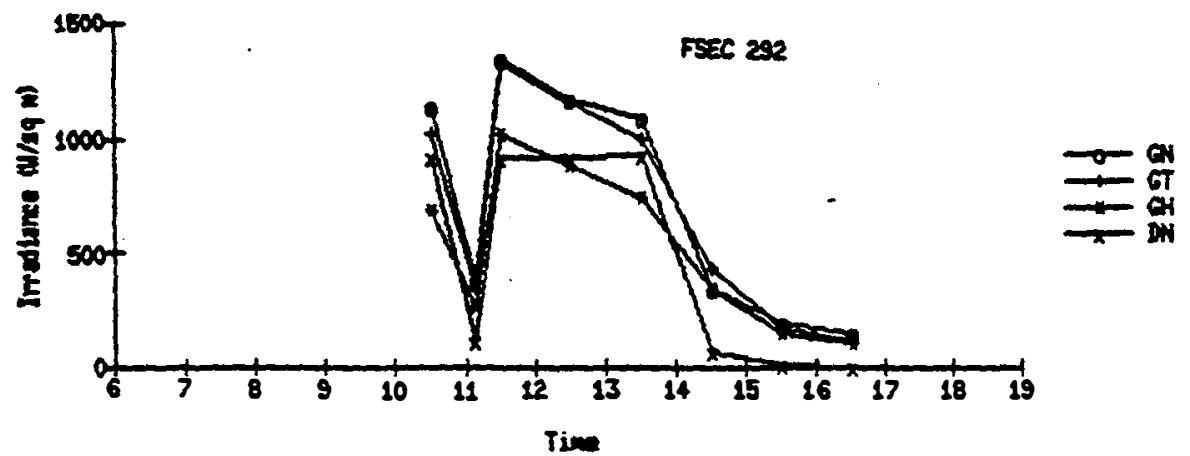

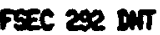

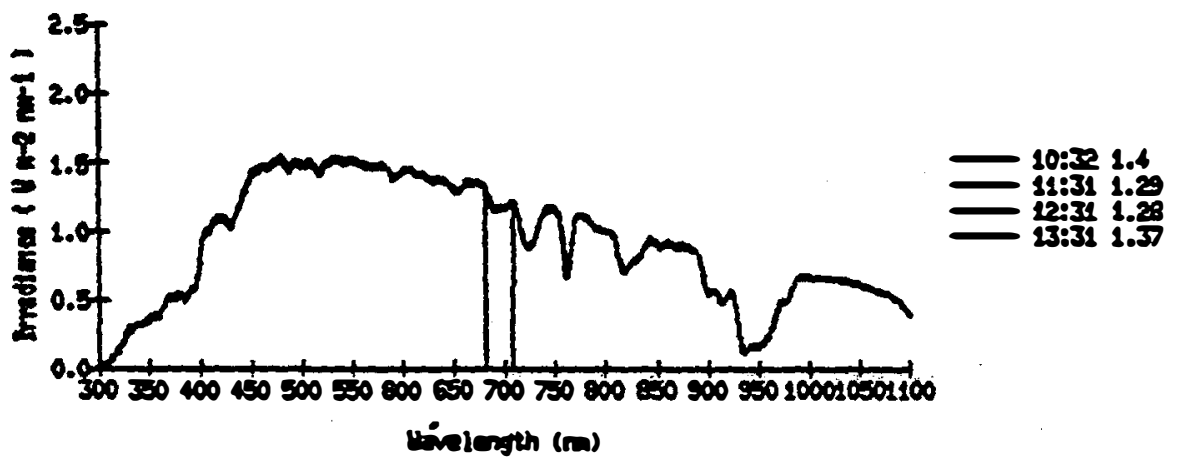

FET Zer OS

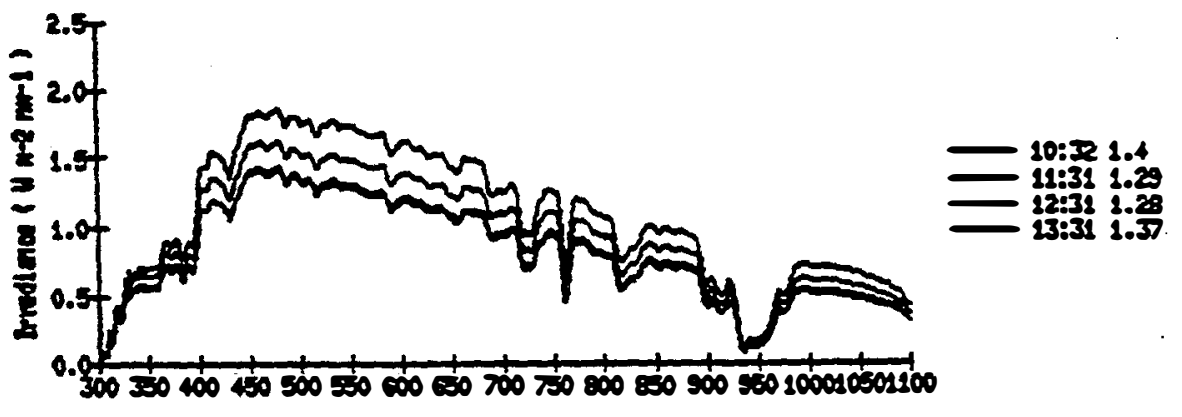

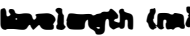




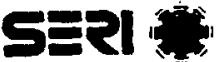

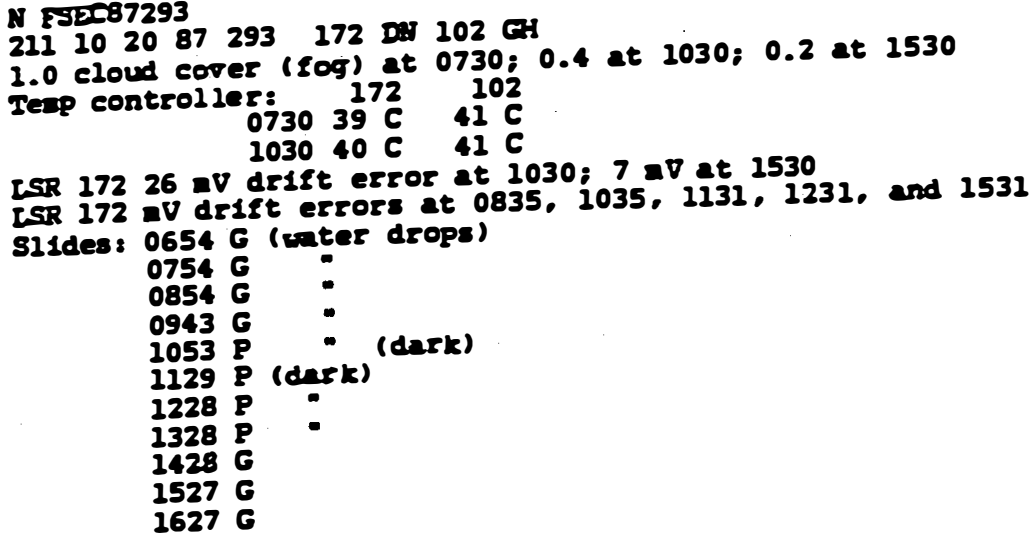

Spikes in of in Dis spectrun at 0831 Fsec 293

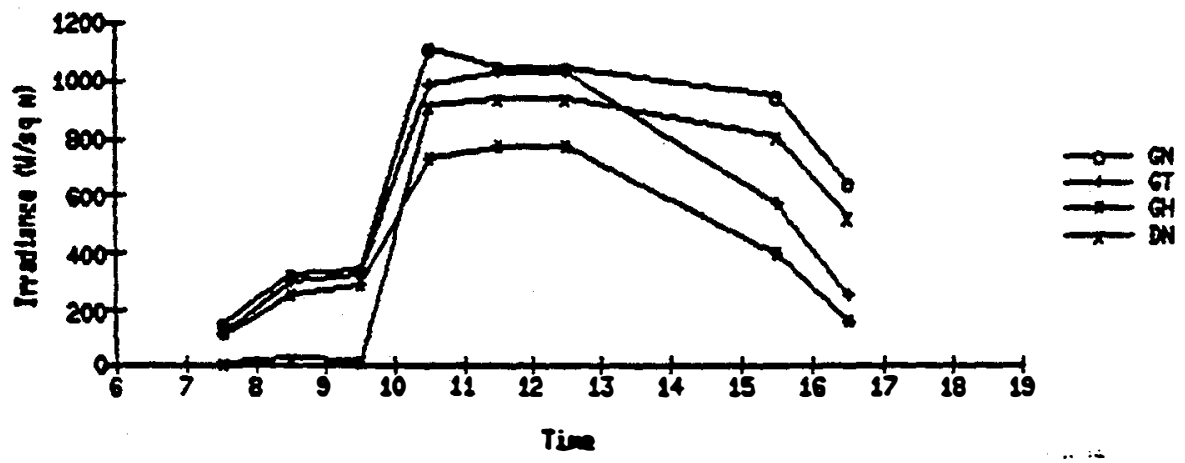

מתד 293 8x

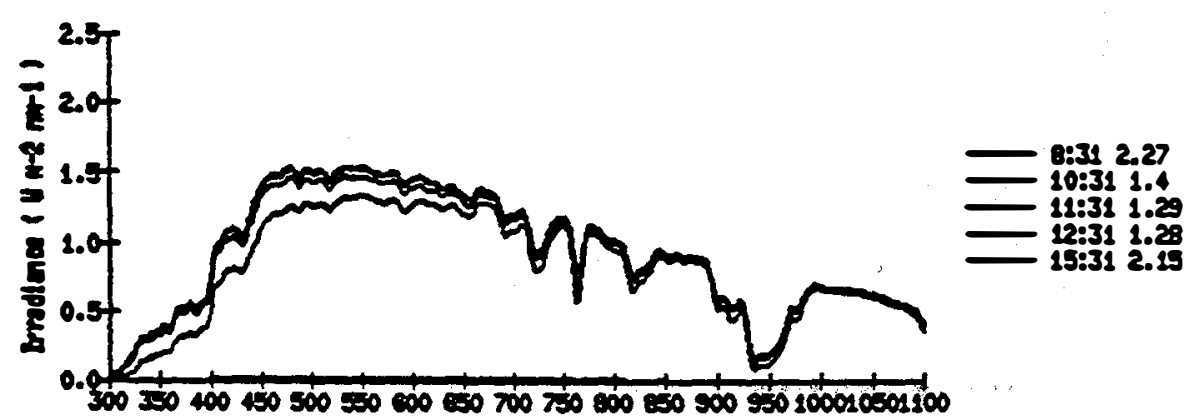

toviergen ton

Fsec 2az Cos

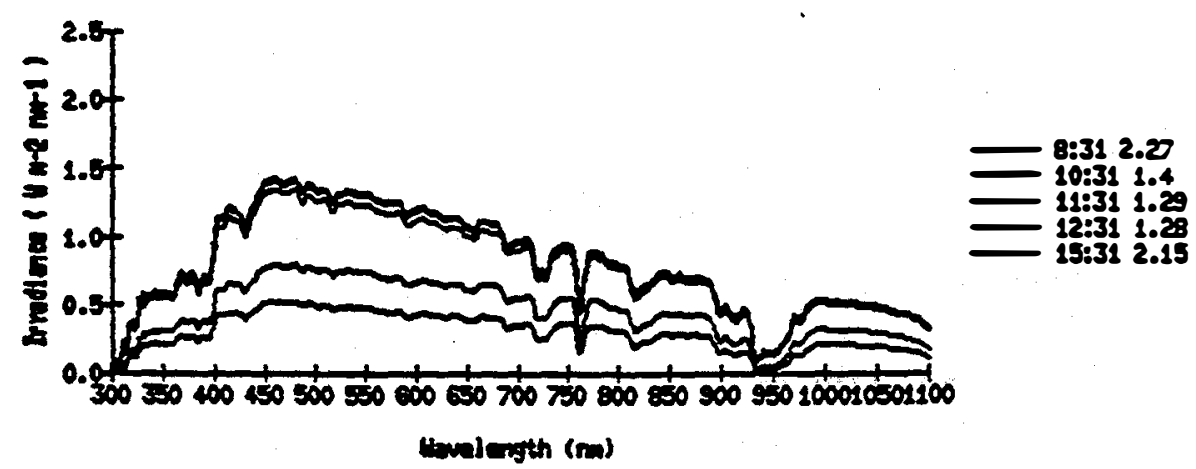


ESTE87294

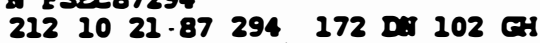

0.9 cloud cover at 073080.4 at 1130

Texp controller: $172 \quad 102$

$\begin{array}{lll}0730 & 39 \mathrm{C} & 40 \mathrm{C}\end{array}$

ISR 172 av drift errors at 0931, 1133, 1231, 1333, and 1631

s11des: $0720 \mathrm{G}$ (reter drops)

$0820 \mathrm{G}:$

$1020 \mathrm{H}$

$1119 ?$

$1219 \mathrm{P}$

$1319 P$

$1418 \mathrm{G}$

$1518 \mathrm{G}$

$1617 \mathrm{G}$

- (dngk)

!

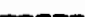

Dis spectru vers noiss in of at 1631

rase zas.

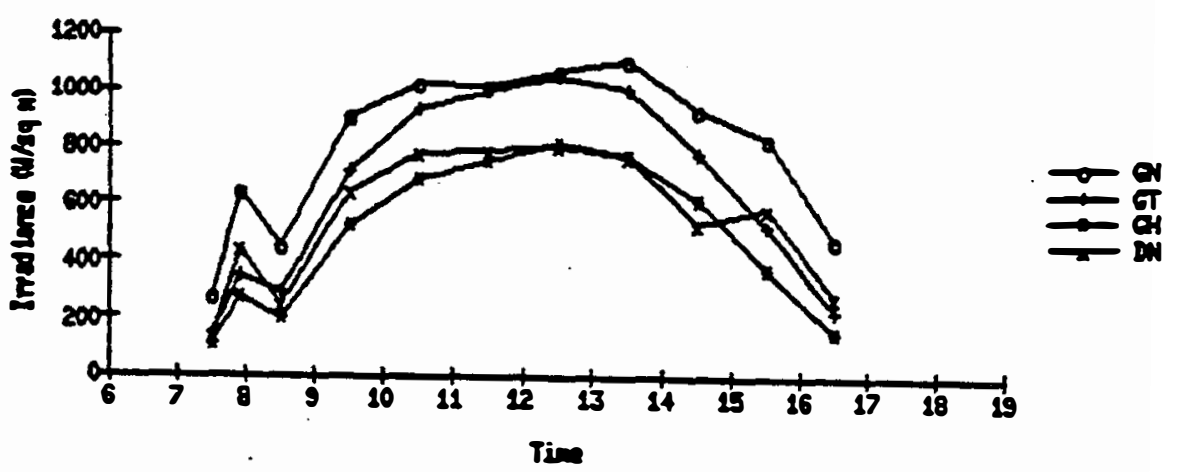

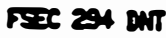

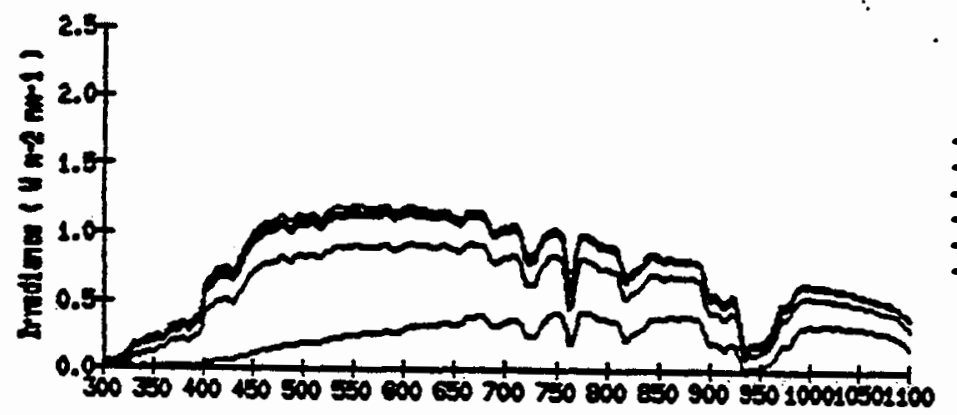

tioloreth (m)

pisc at os

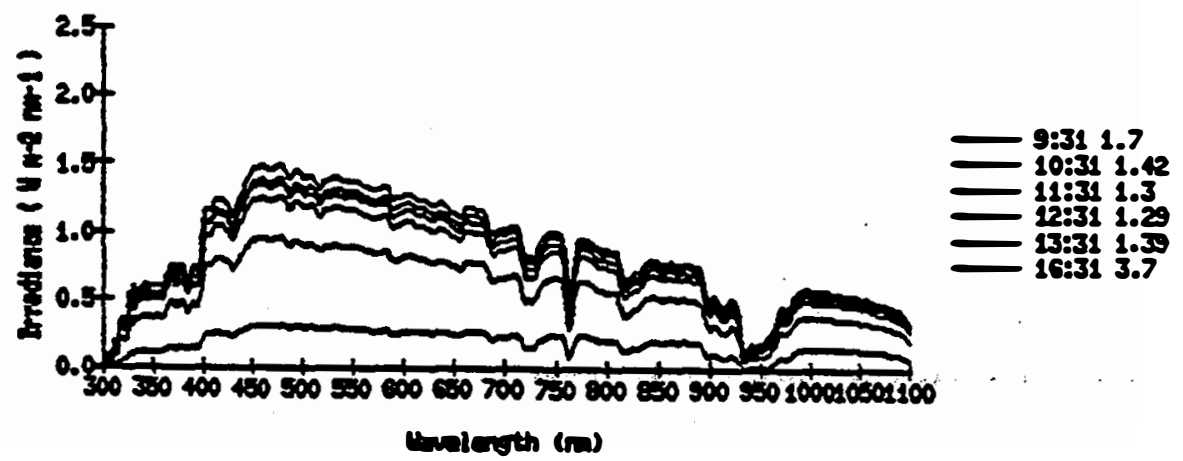


स Pडयद87295

$\begin{array}{llllllll}213 & 10 & 22 & 87 & 295 & 172 & \mathrm{DA} & 102\end{array}$

Gl then Gi on this daf

S11des: $0711 \mathrm{G}$

$0821 \mathrm{G}$

09116

$2010 \mathrm{G}$

$1110 \%$ (dnek)

$1210 \mathrm{G}$

$1309 \mathrm{~g}$ (dnek)

$1409 \mathrm{G}$

1509 G

$1608 \mathrm{G}$

$\begin{array}{lllllllll}214 & 22 & 87 & 295 & 172 & \mathrm{DP} & 102 & \mathrm{G} & \end{array}$

0.3 cloud cover at 0930 and 113080.9 at 1530

Tep controller: $172 \quad 102$

slides: See arove

Fist 205

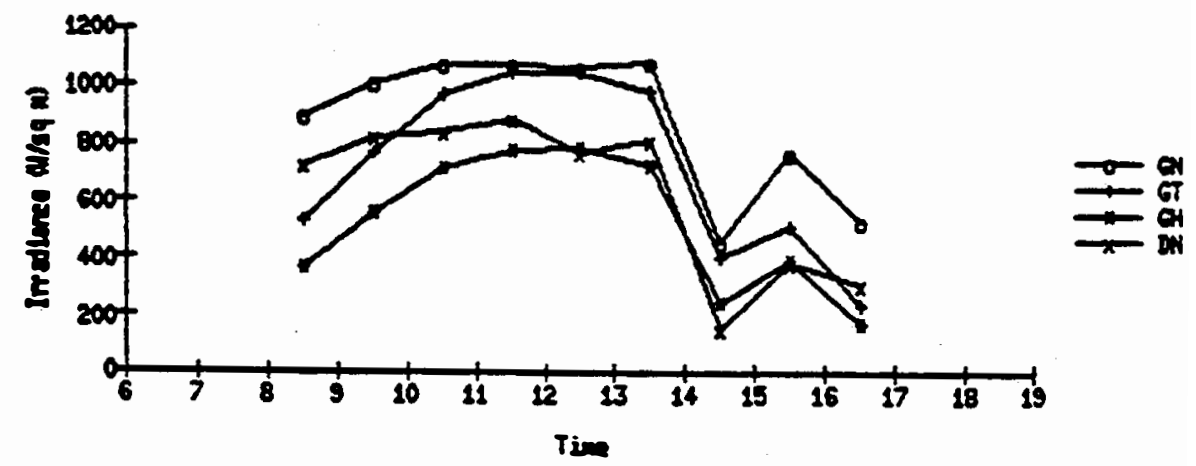

FEx 208 int

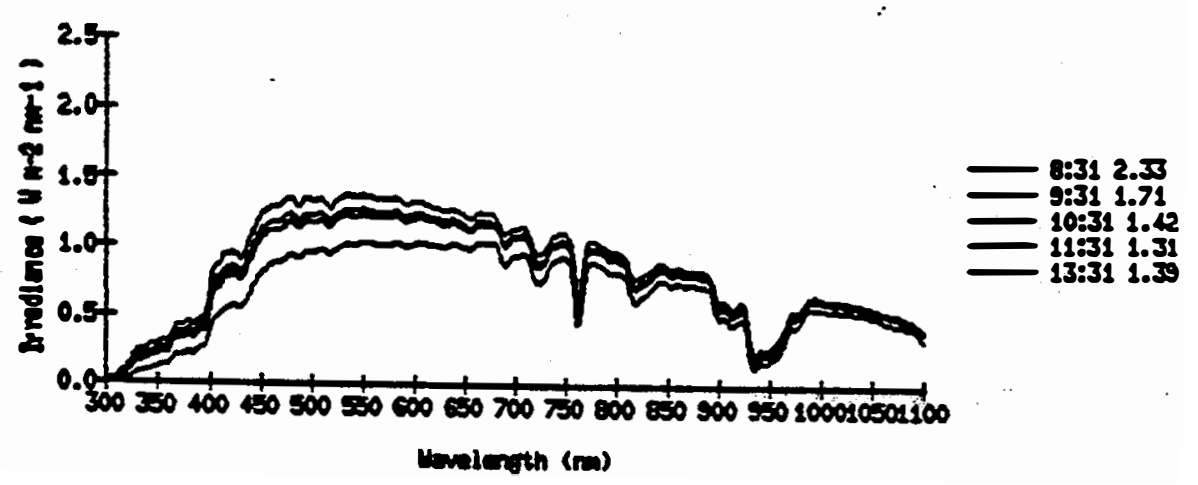

Dite $2: 3$ GIS

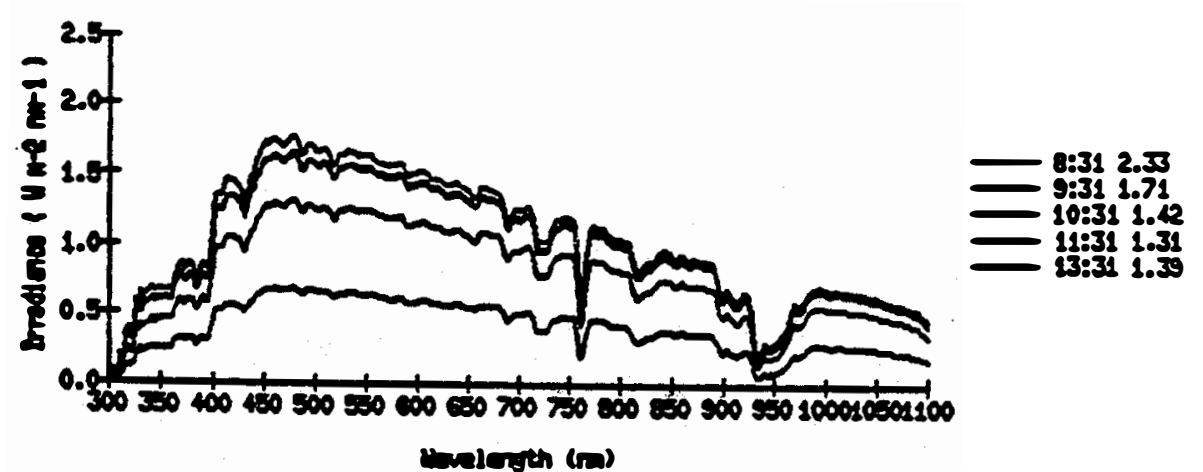

rench (n) 
1. एक्ये87296

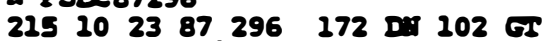

0.4 eloud coires at 0730

$\begin{array}{rrrr}0.972 & 102 \\ 0730 & 39 & \mathrm{C} & 40 \mathrm{C}\end{array}$

ISR 17250 D drsft error at 1030

Slides: $0703 \mathrm{G}$

$0802 \mathrm{G}$

$0930 \mathrm{G}$

$1030 \mathrm{~A}$ (dark)

$1229 \mathrm{G}$

$1229 \mathrm{P}$ (dnels)

$1328 \mathrm{G}$

$1428 \mathrm{G}$

1528 G

$2628 \mathrm{G}$

$\operatorname{Psc} 206$

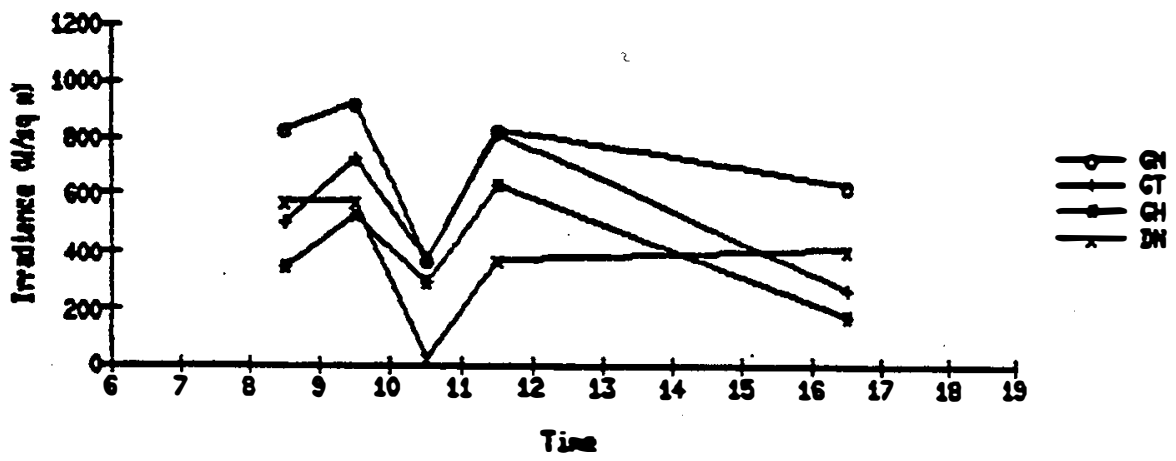

Fise 206 8nT

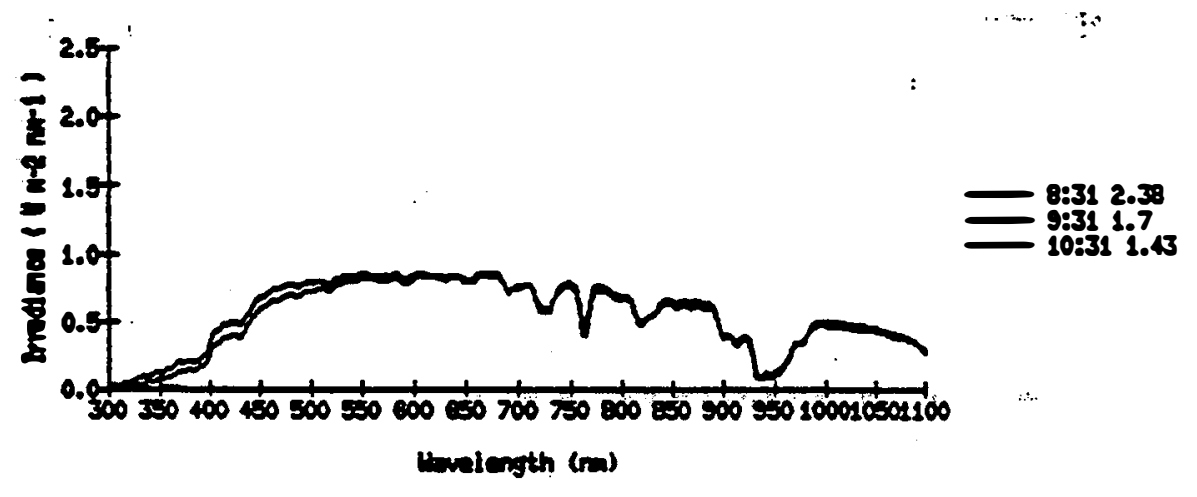

Fise 290 GTS

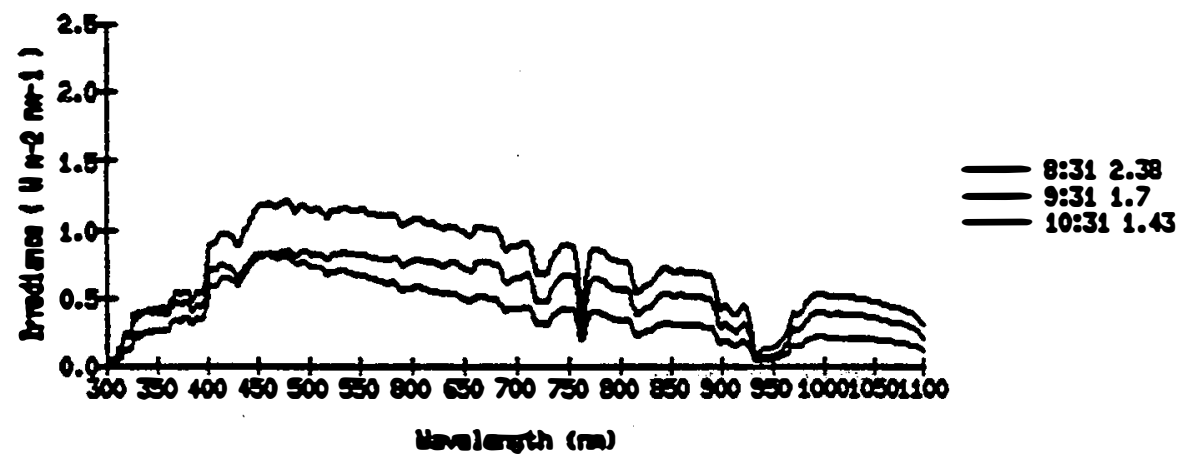


II FSTC87297

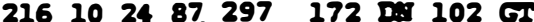

Partly eloudy, mostly elear ay

heokend data: possible tracking error

slides: $0722 \mathrm{G}$

$0821 G$

$0921 \mathrm{G}$

$1027 \mathrm{P}$ (derk)

$1120 \mathrm{P}:$

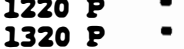

$1419 \mathrm{G}$

$1519 \mathrm{G}$.

$1618 \mathrm{G}$

Fร26 297

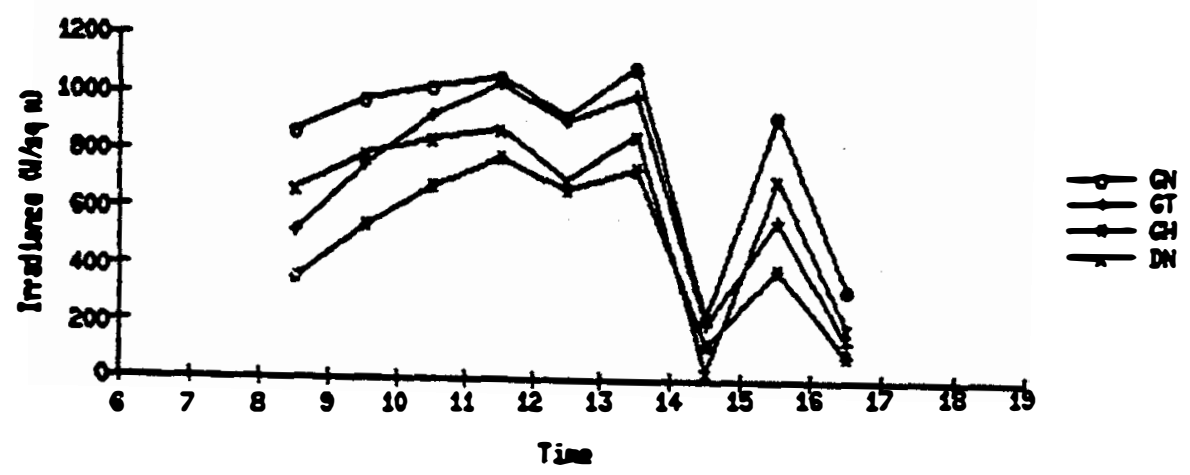

rax 297 m

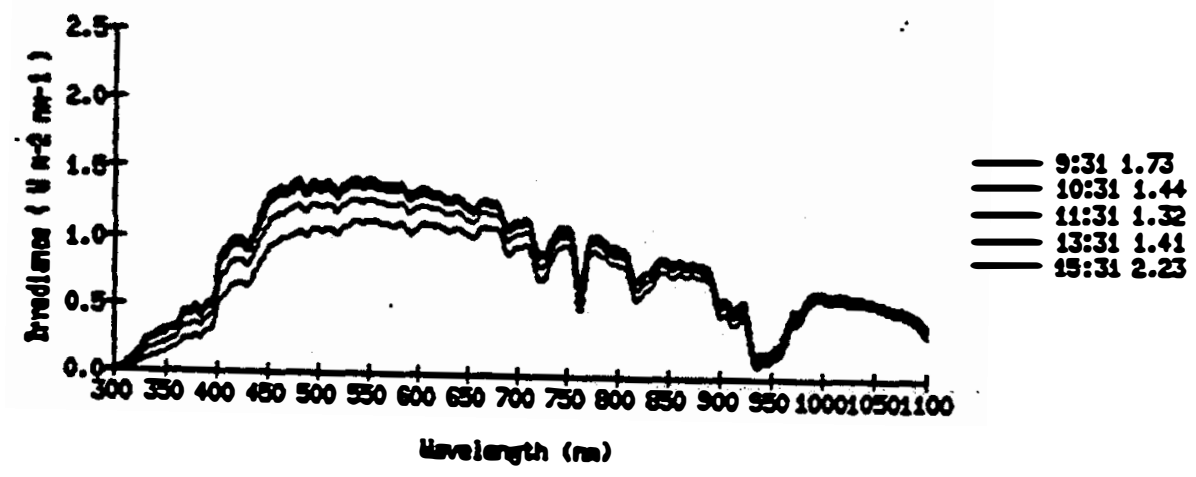

PFe 297 G5

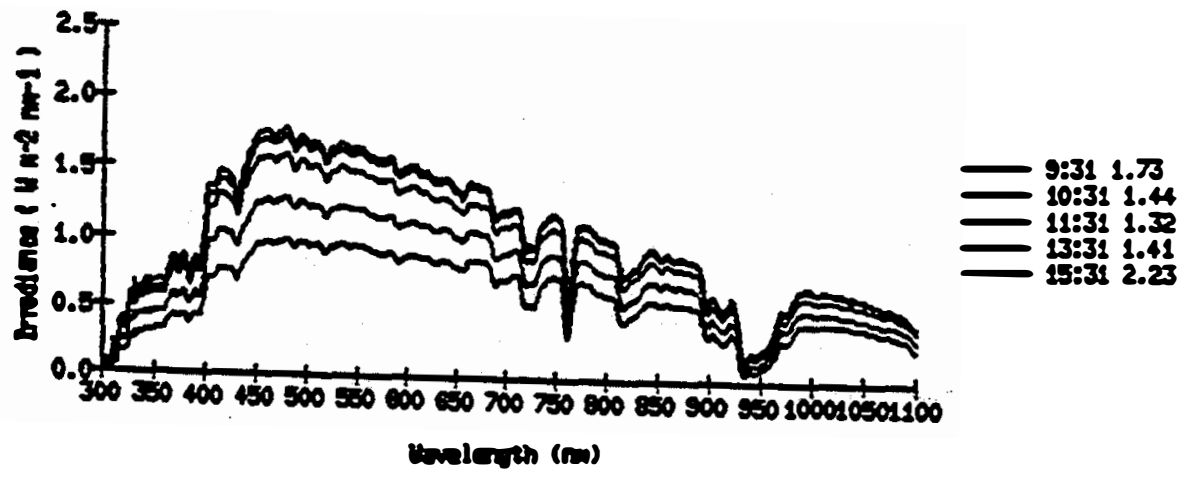




\section{SEP}

A FSP 87298

$217 \quad 102587298 \quad 172$ DI 102 GT

Partly cloudy

Weekend data; possible tracking error

ISR $172 \mathrm{aV}$ drift error at 1031, 1131, 1233, 1331, 1431, and 1531

Slides: $0712 \mathrm{G}$ (reter drops)

$0812 \mathrm{G}:$

$0911 \mathrm{G}$

1011 M (dark)

$1111 P$.

$1210 \mathrm{P}$

$1310 \mathrm{G}$

$1410 \mathrm{G}$

$1509 \mathrm{G}$

$1609 \mathrm{G}$

$1708 \mathrm{G}$

Minor noise in ov in Di spectra at 1031 and 2131

Spikes in ov in Dis speetra at 1431, 1531, and 1631

Fis 28

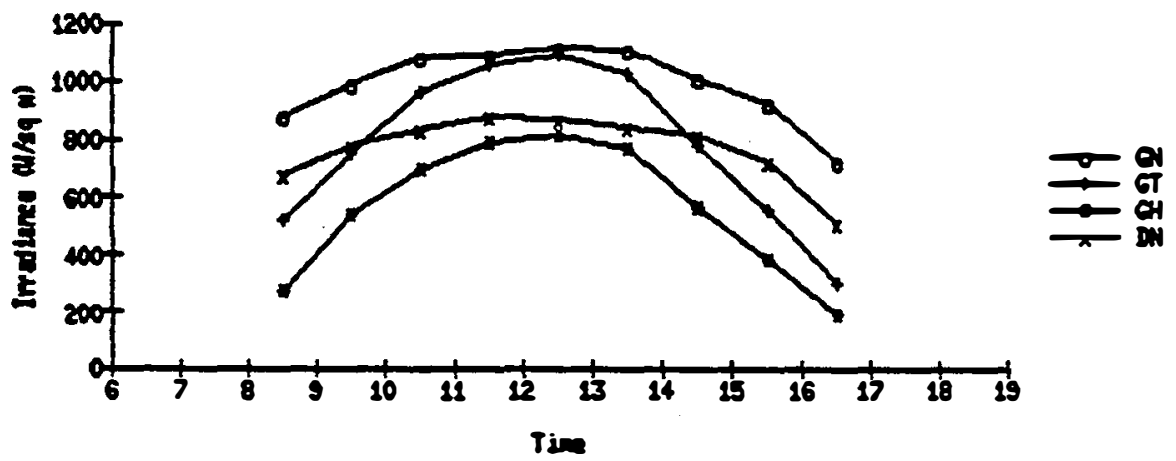

2075

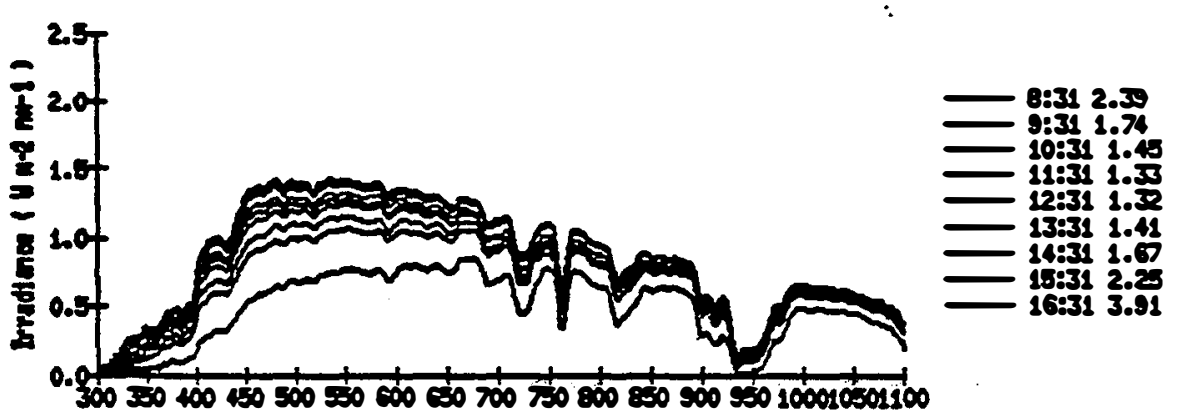

benlongth (n)

Fiš 298 GTS

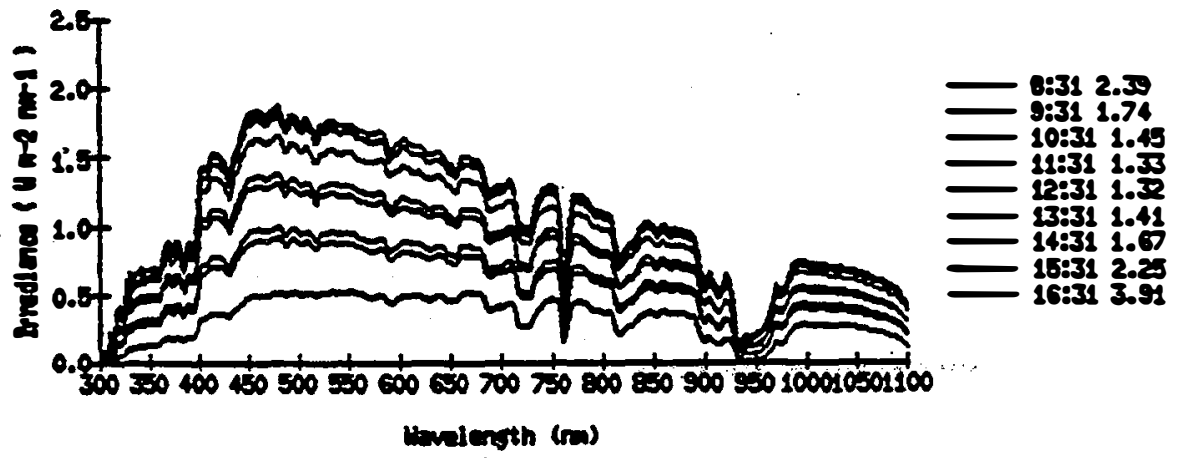




\section{$\Rightarrow=0$}

P Fi2587299

$218 \quad 102687299 \quad 172$ DS 102 GI

1.0 cloud cover at 083080.8 at 103080.4 at $1230 ; 0.1$ at 1530

I.P controller: $172 \quad 102$

$103040 \mathrm{C} \quad 40 \mathrm{C}$

$123041 \mathrm{C} \quad 41 \mathrm{C}$

$153041 \mathrm{C} 41 \mathrm{C}$

sildes $0702 \mathrm{G}$ (condensetion)

0802 H Corde

$0901 \mathrm{H}$

1001 M (dnzk)

$1100 \mathrm{P}$

$1130 \mathrm{P}$

$1229 \mathrm{P}$

$1329 \mathrm{P}$

$1429 \mathrm{G}$

$1528 \mathrm{G}$

$1628 \mathrm{G}$

Spectral integrals and broadsend values different at 0847; bad tine gentp

Hote. spectrun 1s out of sequence - after 1531- could be for 10-27

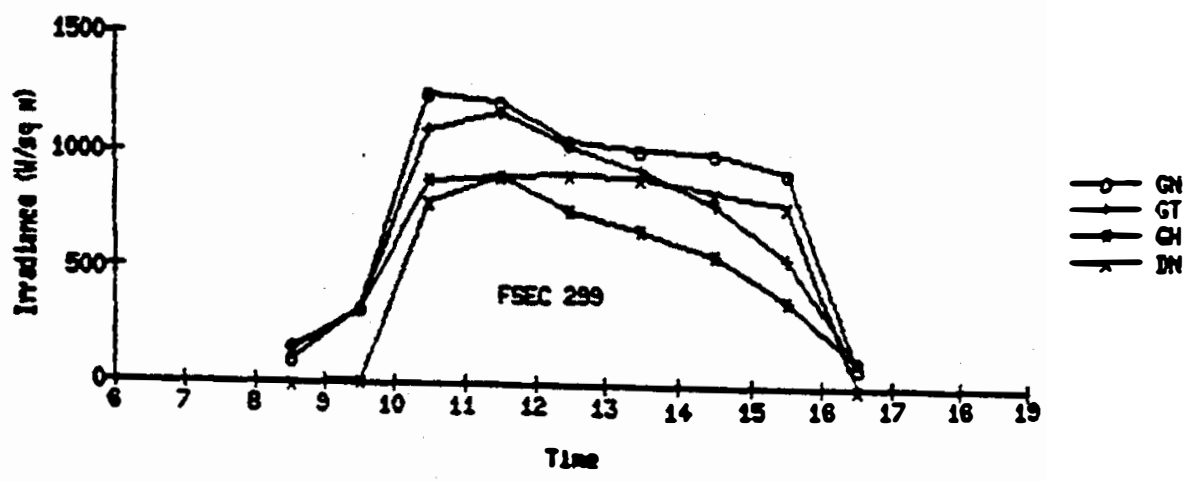

PISE 290
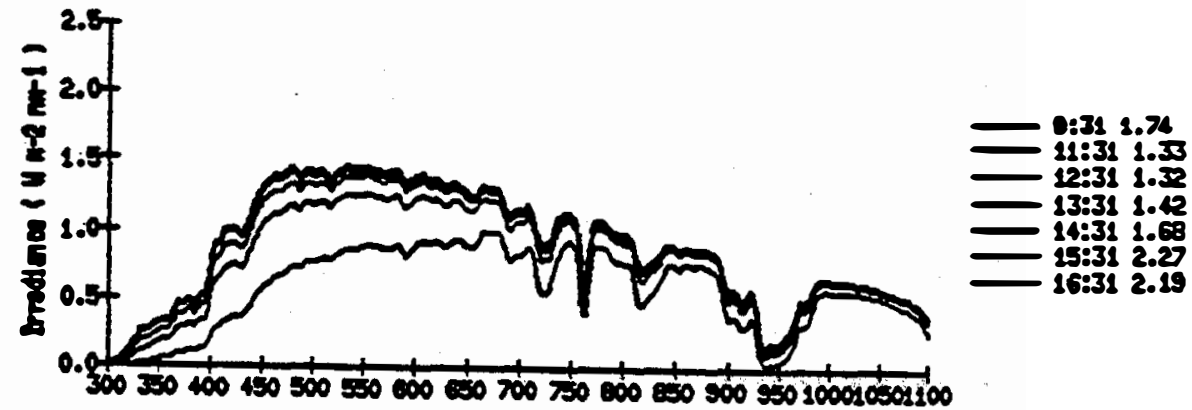

raderen (n)

PISE 290 NTS

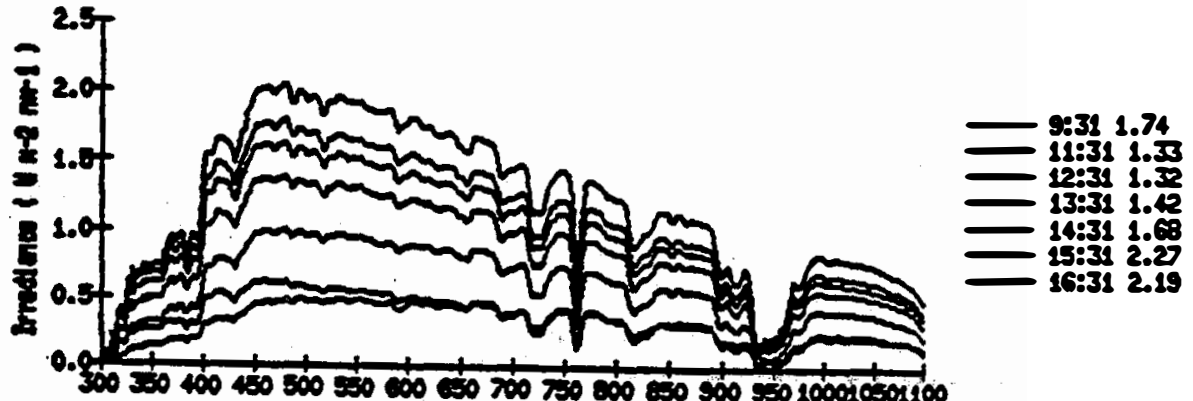

vevierith (ro) 


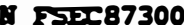

$\begin{array}{lllll}219 & 10 & 2787300 \quad 172 \quad \text { DI } 102 \quad G P\end{array}$

ISR $172 \mathrm{aV}$ drift errors at 1231, 1331, and 1433

slides: $0721 \mathrm{G}$

$0820 \mathrm{G}$

0920 A (durk)

$1119 \mathrm{P}$

$1318 \mathrm{P}$.

$1418 \mathrm{G}$

$1518 \mathrm{G}$

$1617 \mathrm{G}$

Spikes in of in Dis spectru at 1433

Fase 300

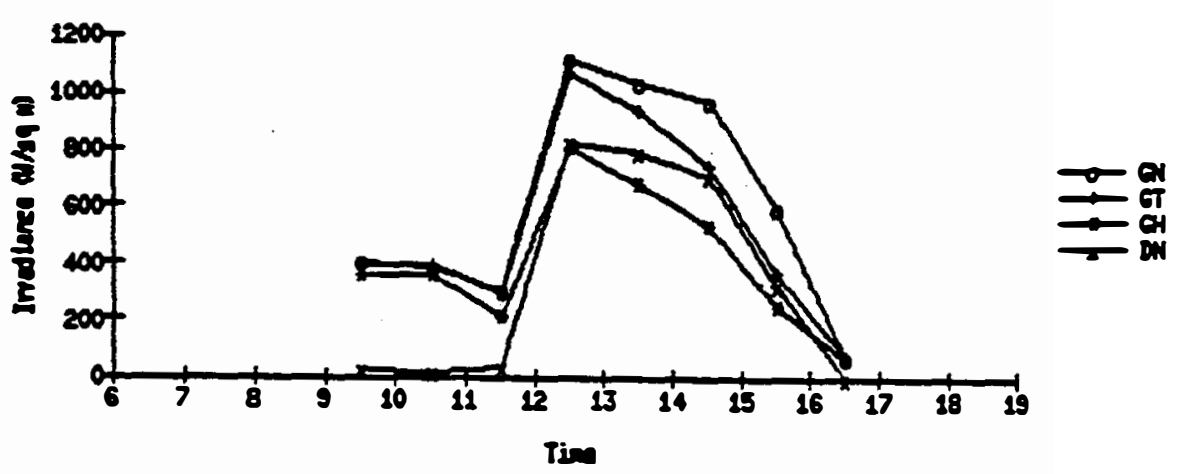

$\operatorname{Fex} 300$ ant

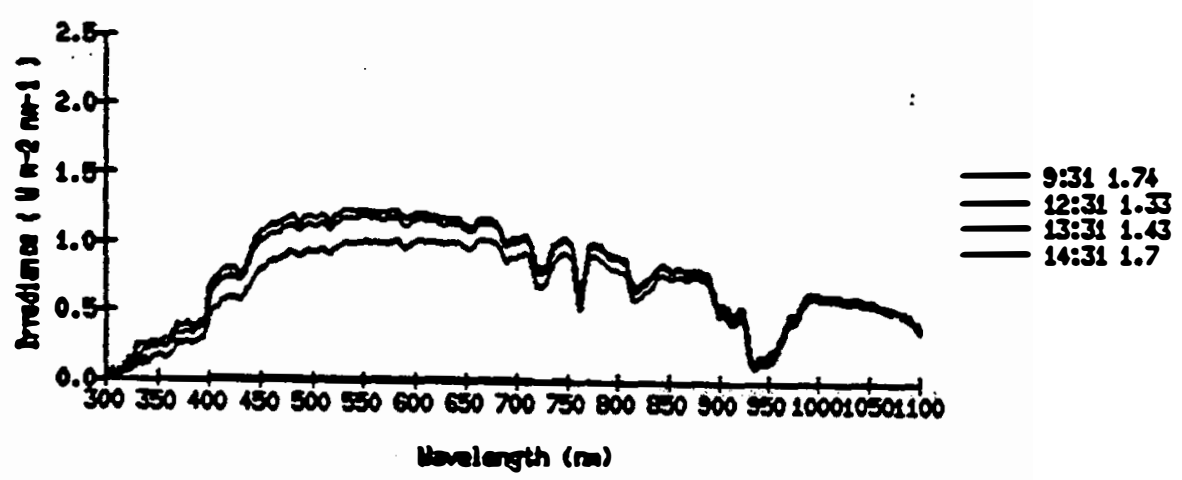

FIE 300 GTS

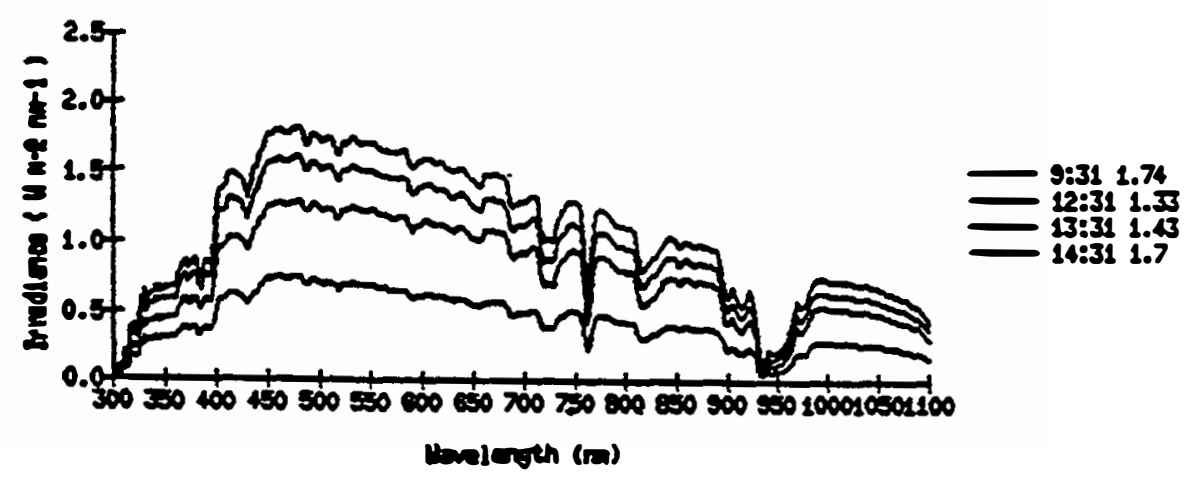




\section{SEN *}

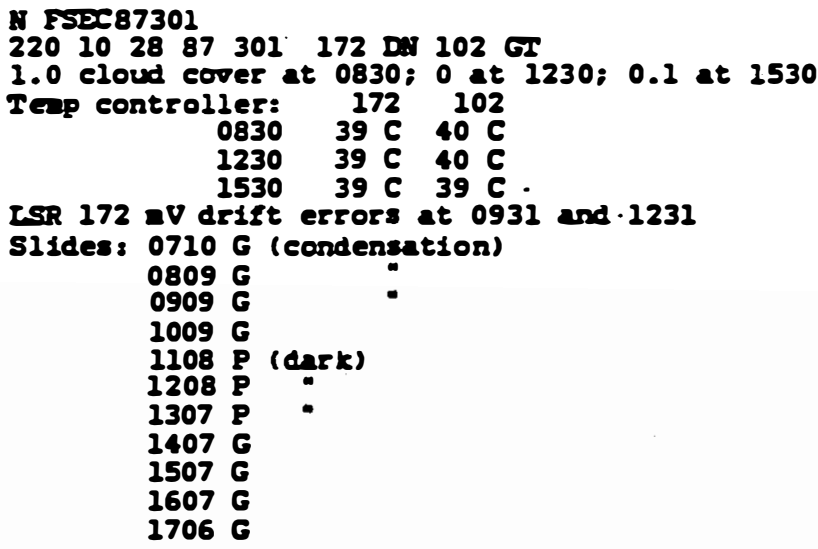

Spikes in ov in Di spectron at 0931

FEse 301

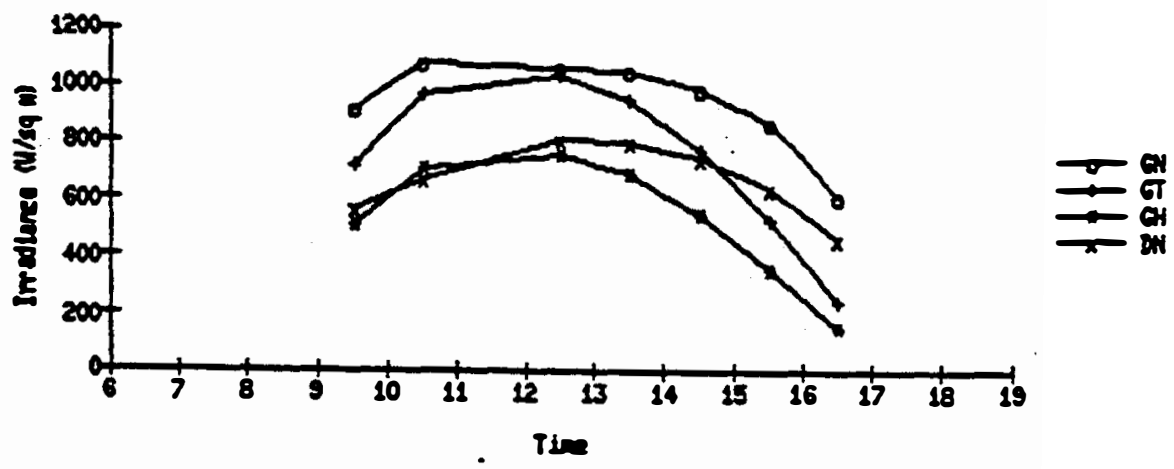

Fise IOS ET

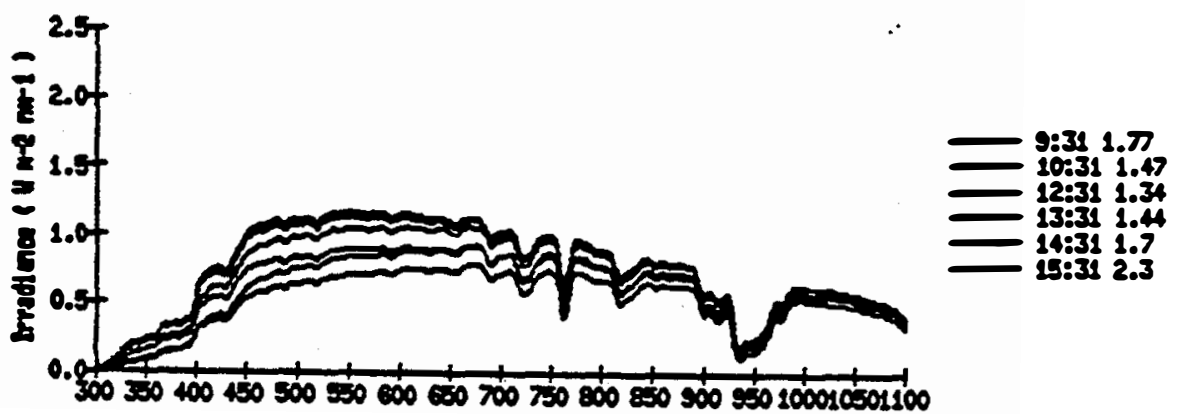

tangergth (m)

PEEC 301 GTS

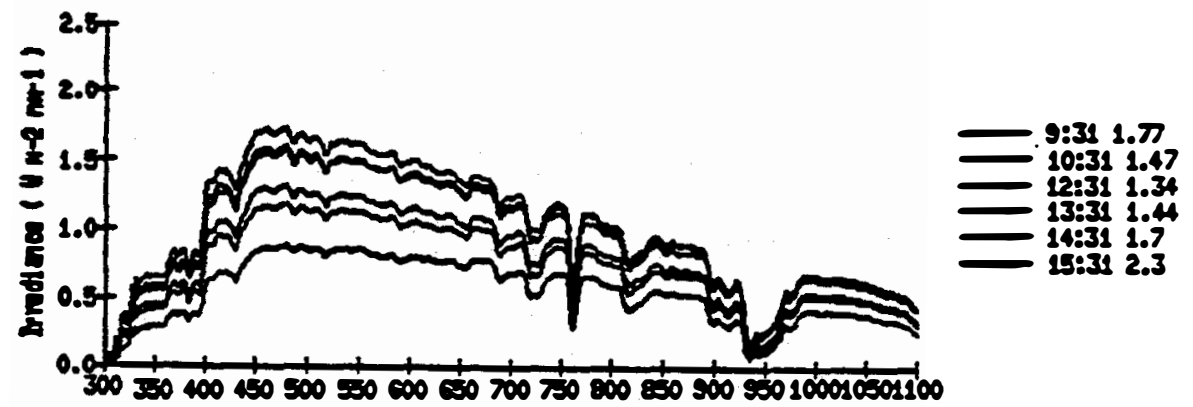

crelerth (m) 


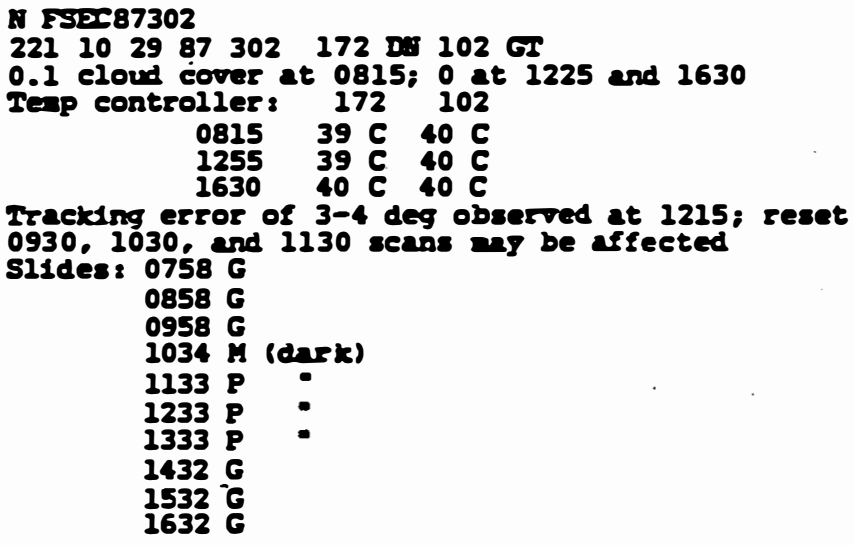

PSEC 300

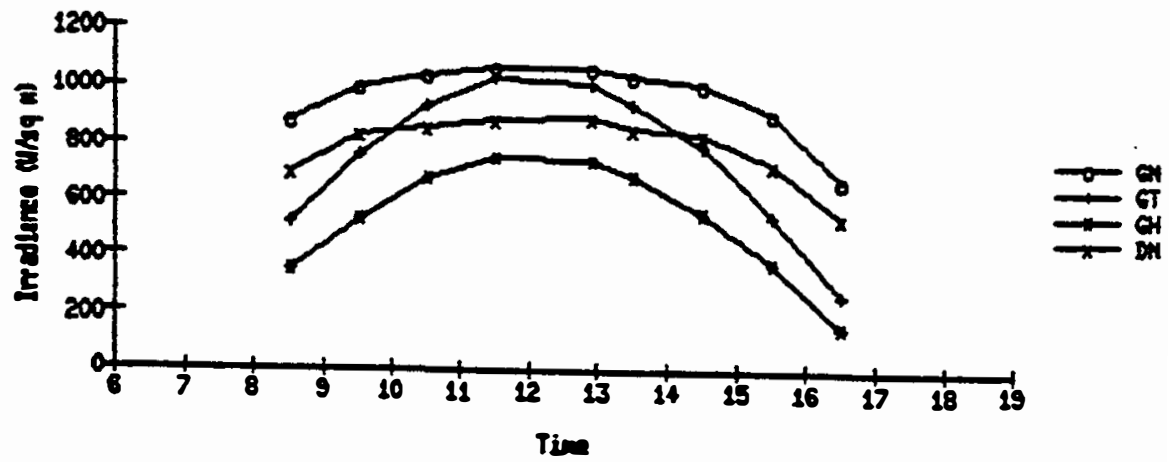

FEC 302 an

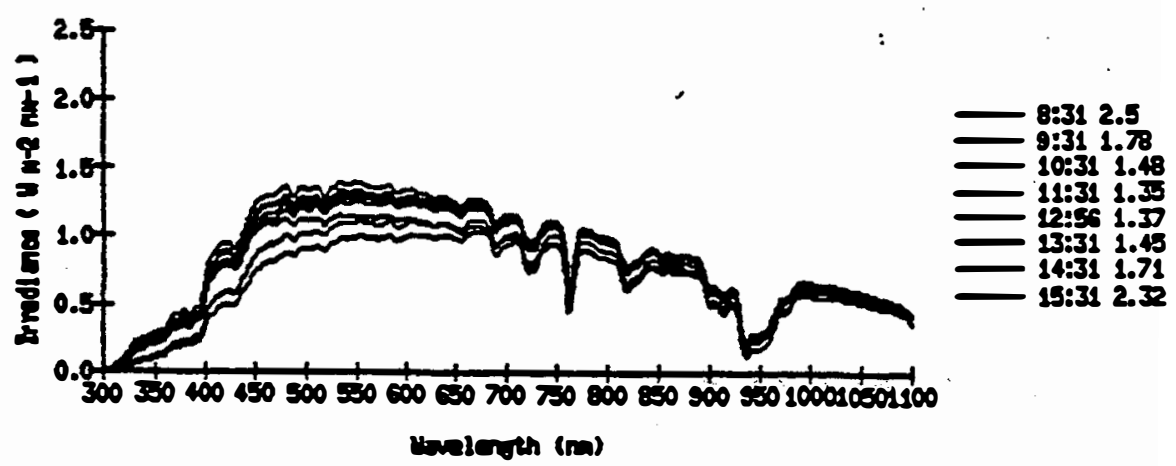

Fisc $392 \mathrm{dT}$

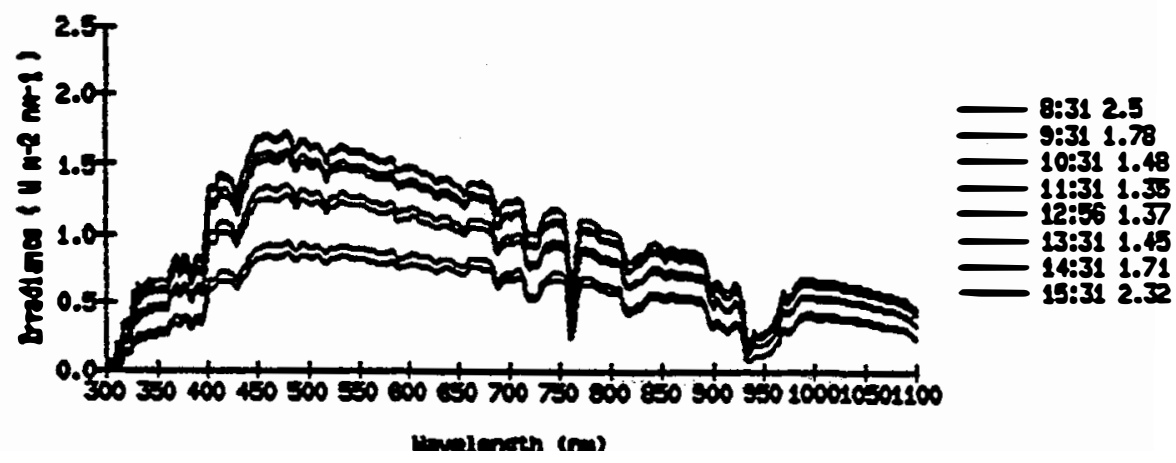




\section{SEN}

\section{9}

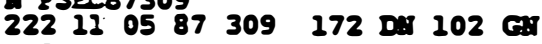

0.8 eloud cover at 1530

Iep controllers $172 \quad 102$

Ho sl1des

$\operatorname{ssc} 300$

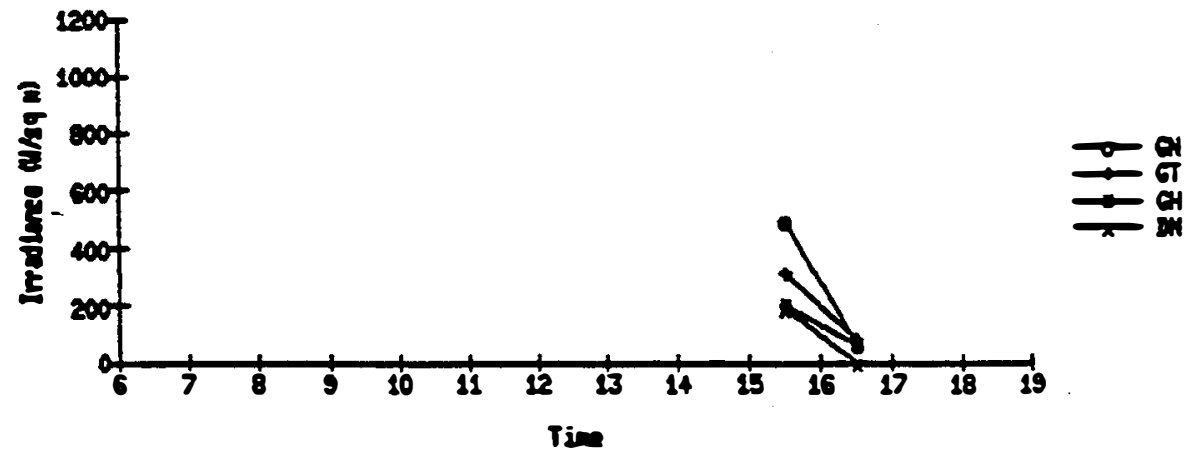




\section{SEP1 *}

A F5ग87310

$\begin{array}{lllllllll}223 & 11 & 06 & 87 & 310 & 172 & \text { od } & 102 & \text { G. }\end{array}$

0.9 cloud cover at 0805; then 1.0

Tepp controller: $172 \quad 102$

$080539 \mathrm{C} 40 \mathrm{C}$

Ho sldes

Fase 310

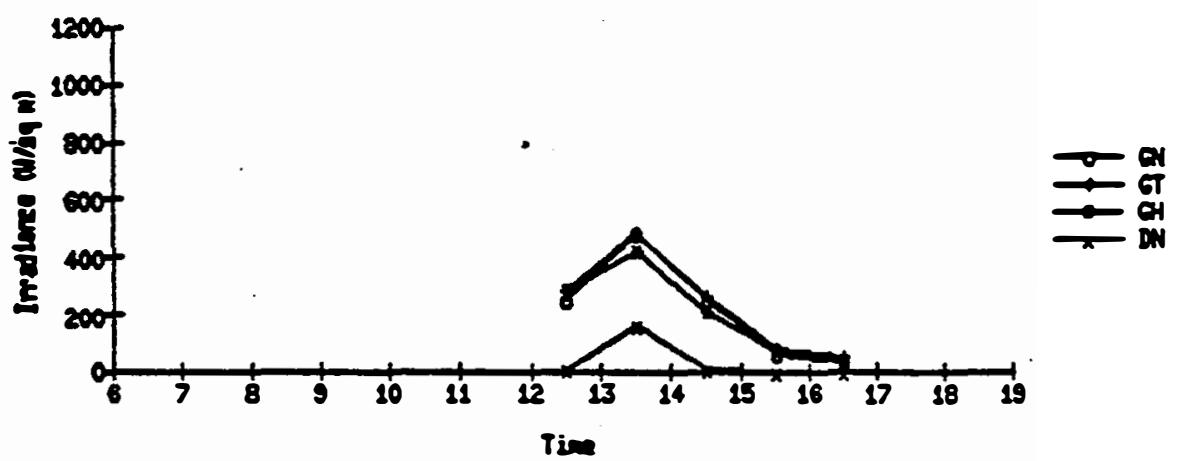

FESC $310 \mathrm{BNT}$

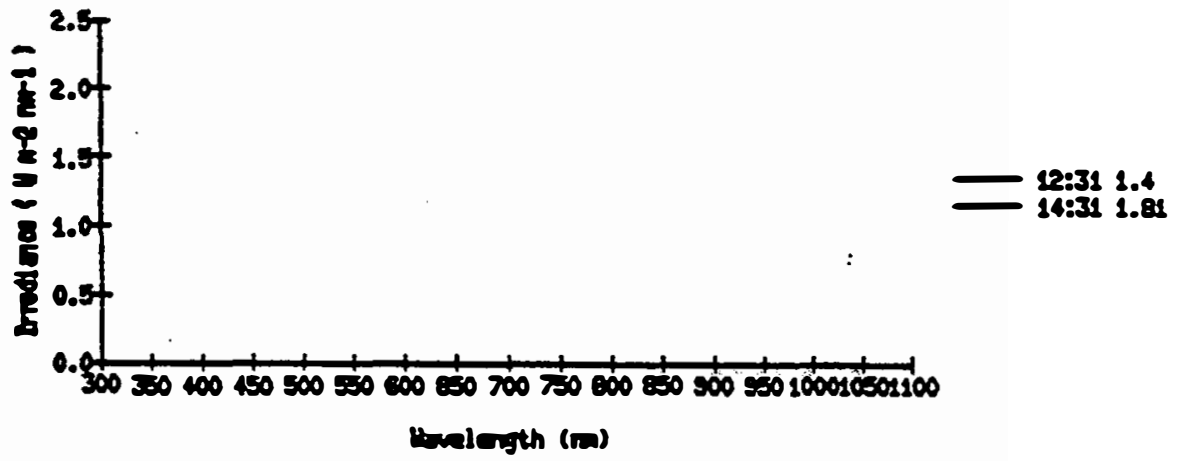

Pase 310 Os

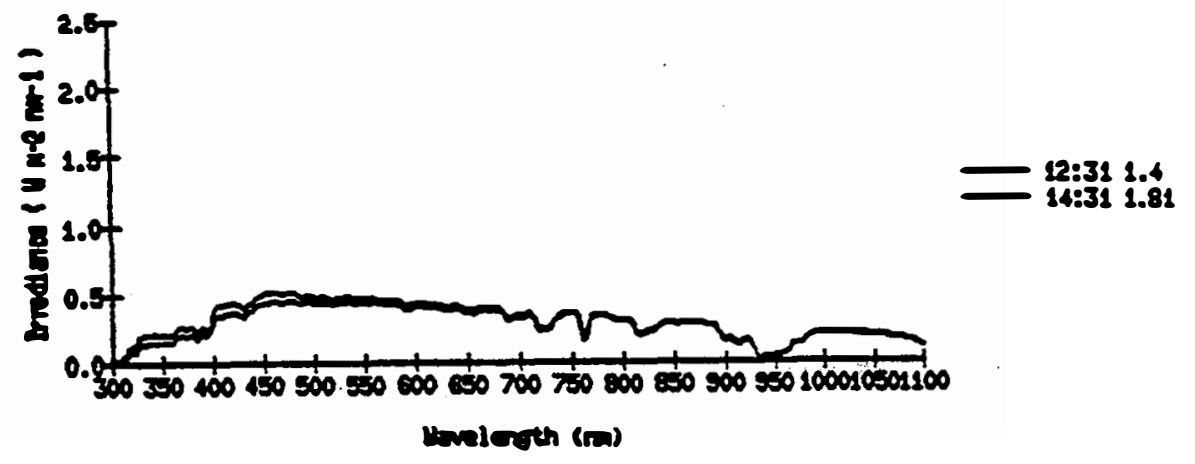




\section{SENI *}

4. FSDC87311

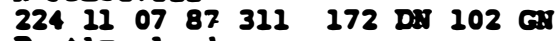

Partly eloudy

No slides

Lost date fron 11-8-87 ebrough 11-13-87 due to vax problens

pasc 311

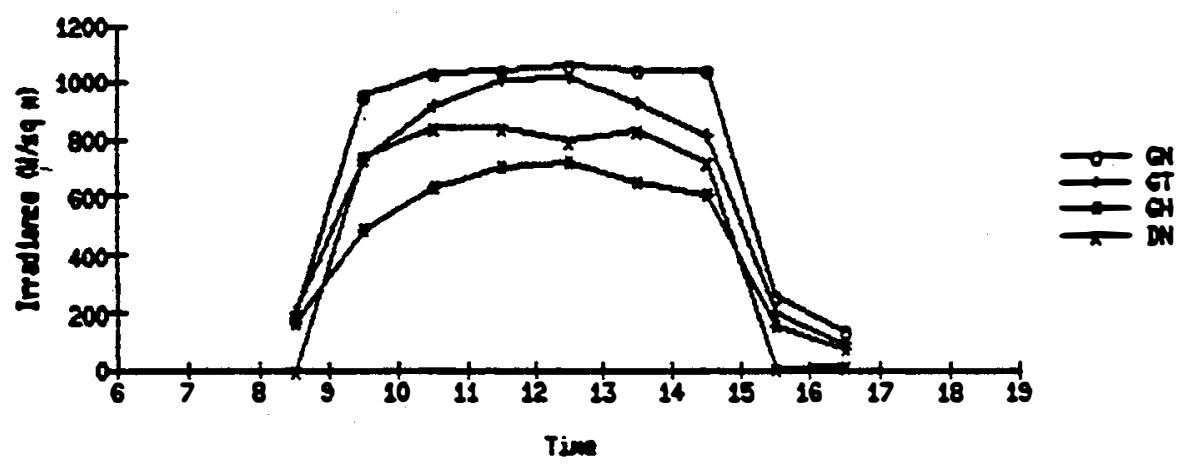

$\operatorname{psec} \operatorname{Int}$ ant

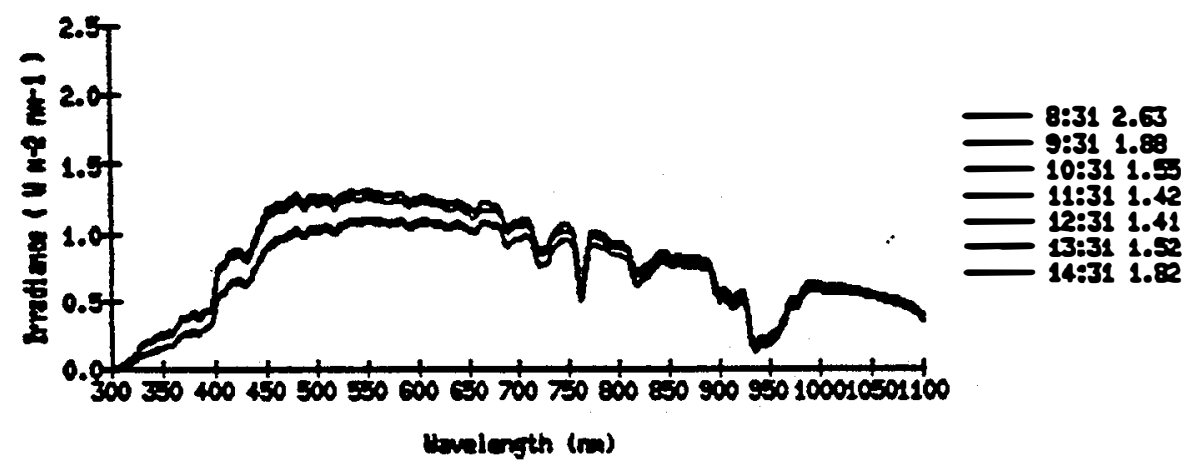

FEC 31100

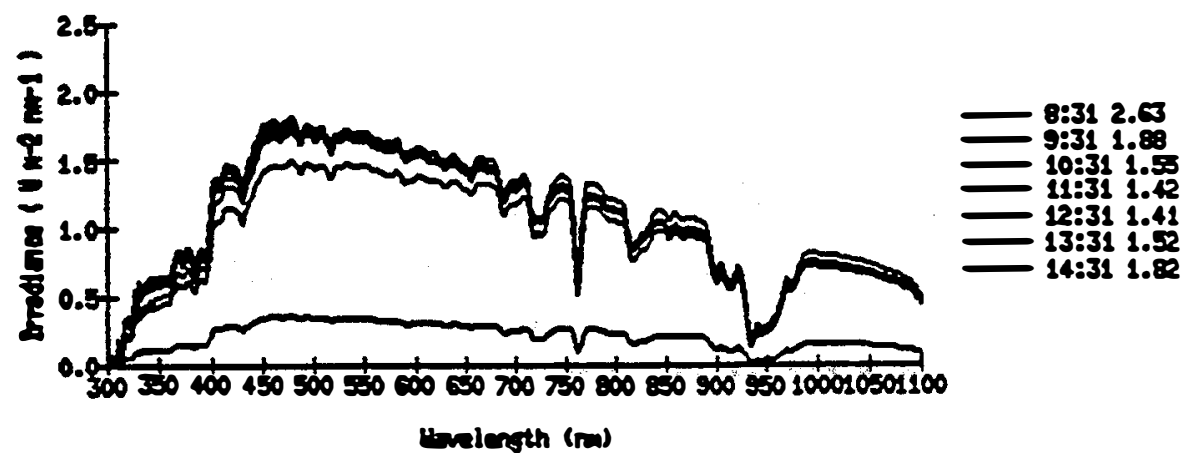


N FSP 87318

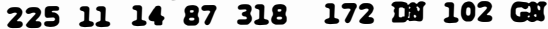

Partis cloudys crekend

No slides

Spikes in of in Dis spectrin at 1031

FESC 318

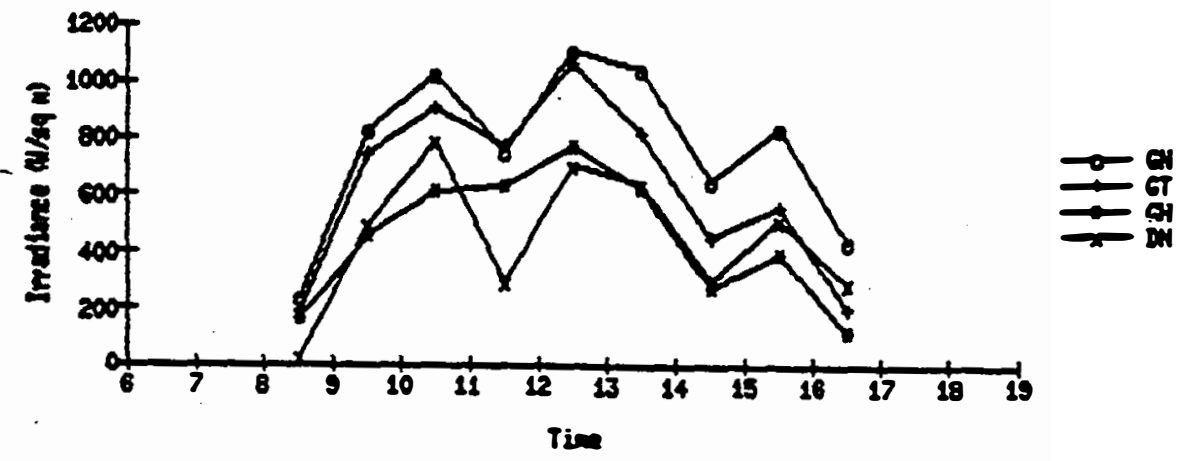

PEבC 318 :

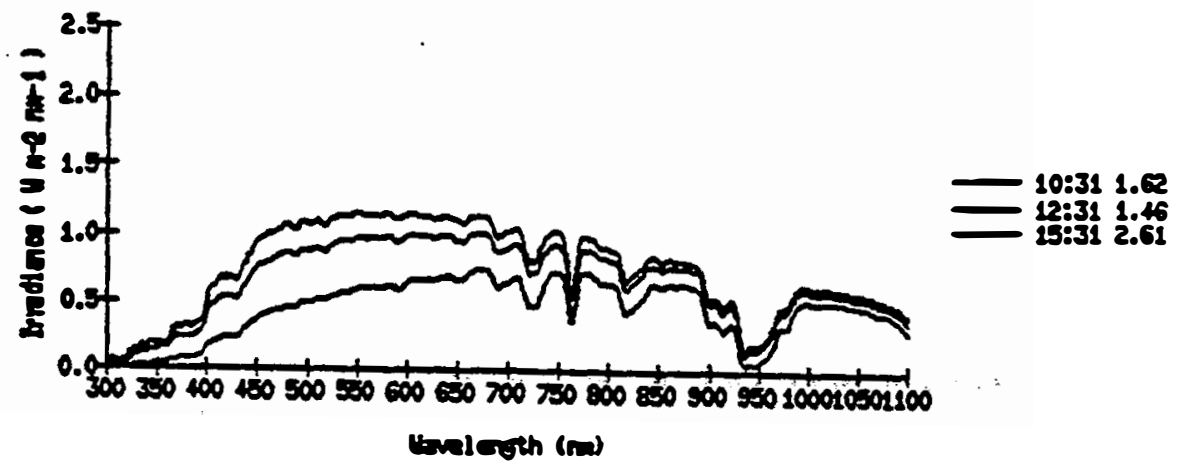

Fase 318 Os

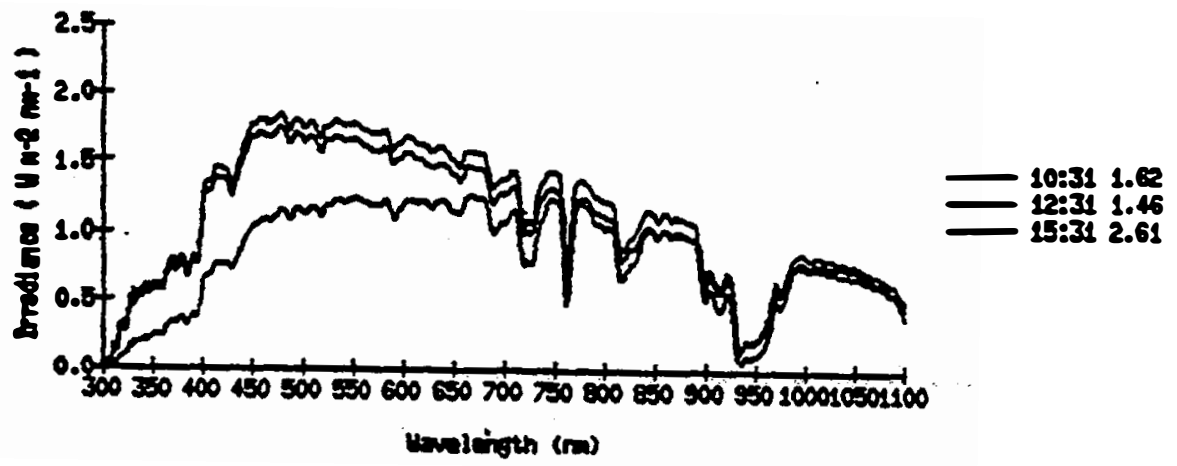




\section{SEP1 $*$}

TR-3513B

4 PSx 87319

$\begin{array}{lllllll}226 & 11 & 15 \quad 87 & 319 & 172 & 102 & \text { G }\end{array}$

Partly clouds' wekend

Ho slides

Spikes in io in Dapecte at 0835, 0931. 1431, and 1531

Dif and Gi spectra at 1033 are bad

$\operatorname{rsex} 349$

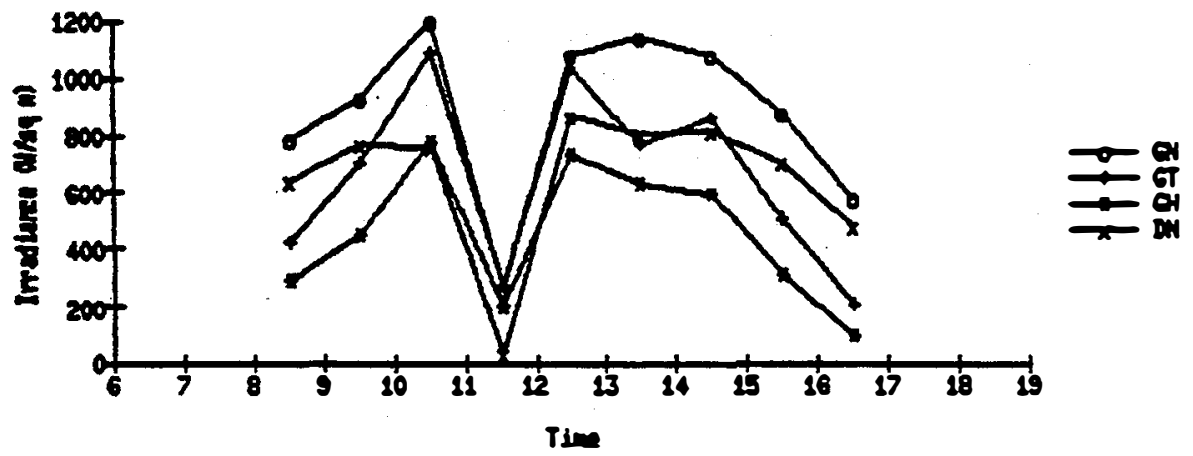

FICX 329 BT

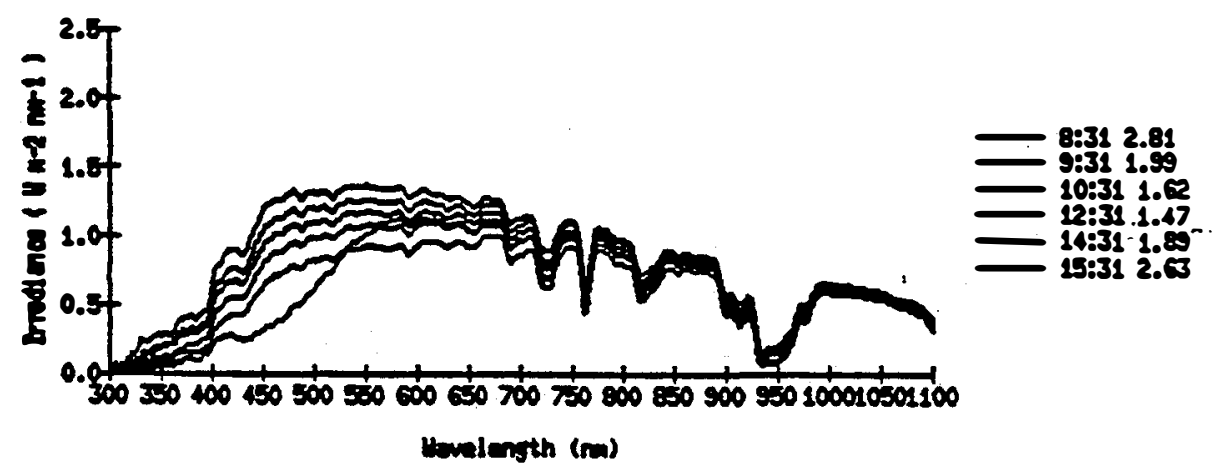

Fix 329 OS

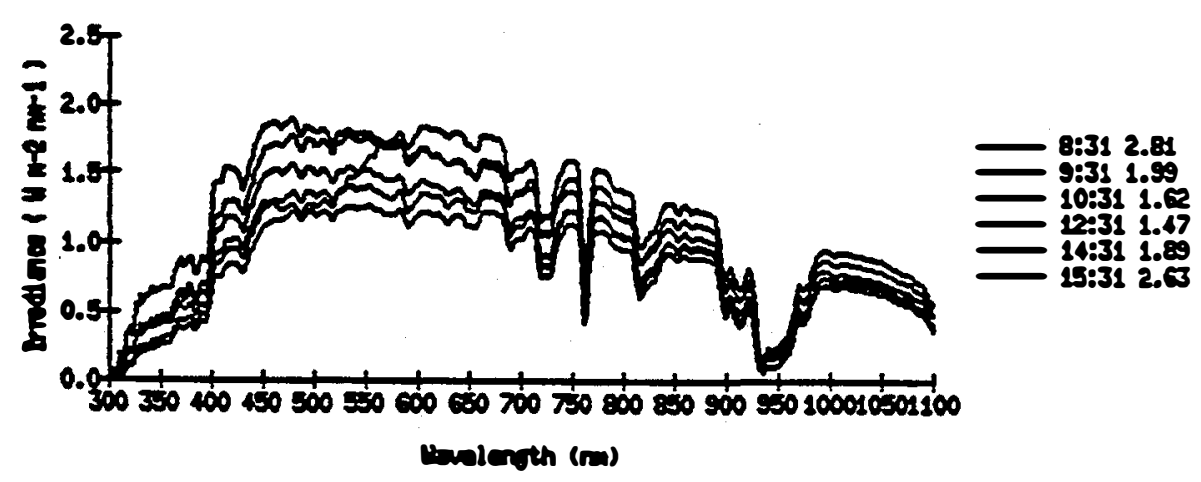


I. P्डग्र 87320

$\begin{array}{lllllll}227 & 11 & 16 & 87 & 320 & 172 & \text { DA } 102\end{array}$

0 cloud cover at 1130 and 1630

Telp controller: $172 \quad 102$

$163040 \mathrm{C} \quad 40 \mathrm{C}$

ISR $17297 \mathrm{av}$ drift error at 1130

ISR 172 av drift error at 1231

S11des: 1230 P (dark)

$1330 \mathrm{M}$.

$1430 \mathrm{G}$

$1529 \mathrm{G}$

$1629 \mathrm{G}$

Appears to be sall spike in Wo in Dis spectra at 1131 and 1331

Fese 320

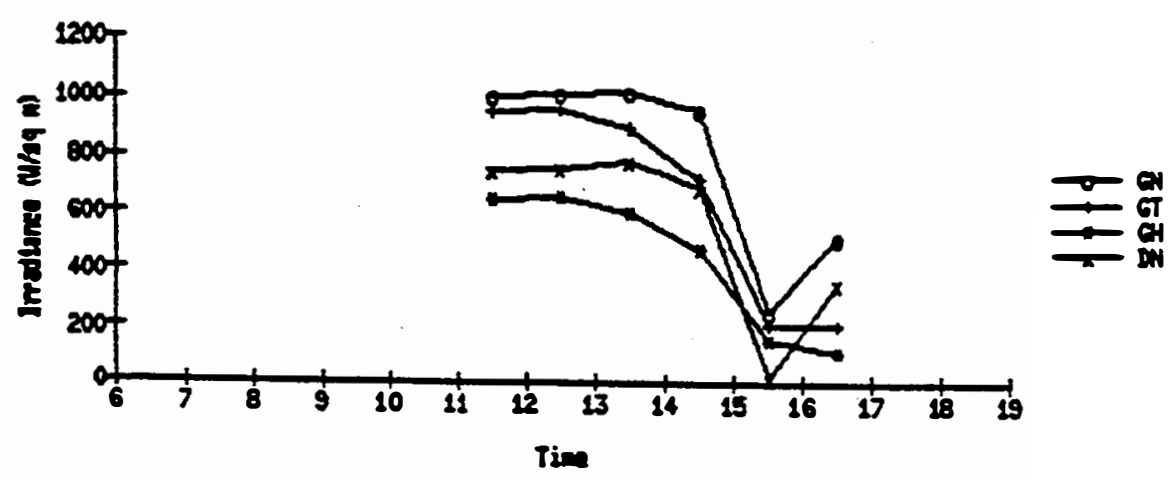

T29 $200 \mathrm{arT}$

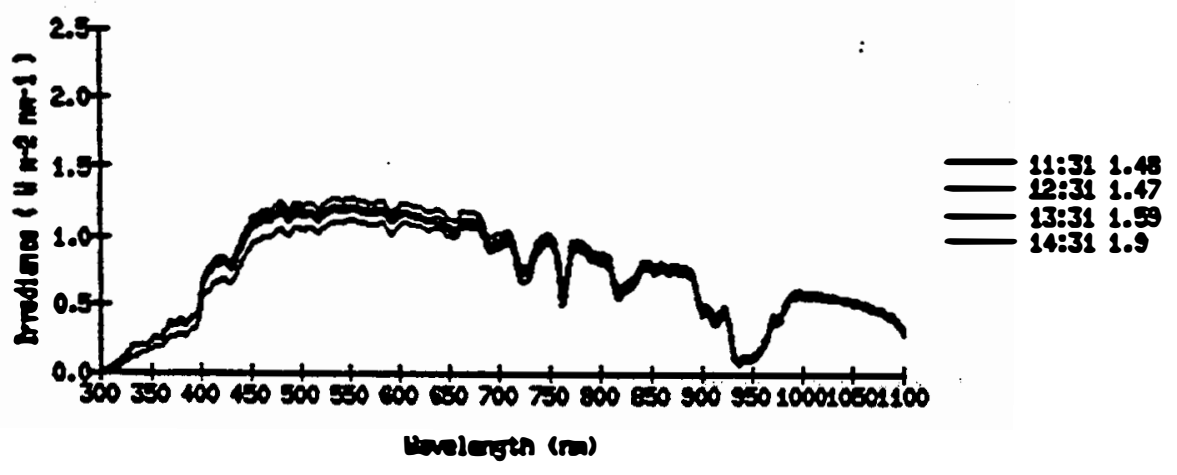

Fise 300 as

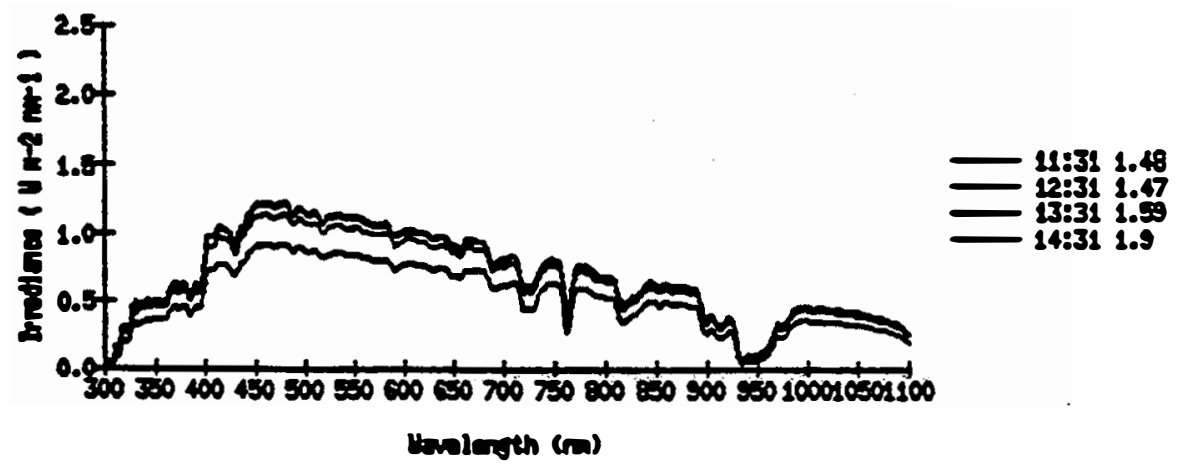


PSTE87321

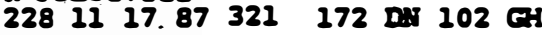

0.8 cloud cover at 0830: 1.0 at 1230 and 1630

ISR 17230 NV drift error at 1230

ISR $172 \mathrm{aV}$ drift errors at 0933, 1031, and 1233

עo slides

Holss ov in Dis spectren at 0933

OV In Dis spectra is bed at 1031 and 1233

Fase 301

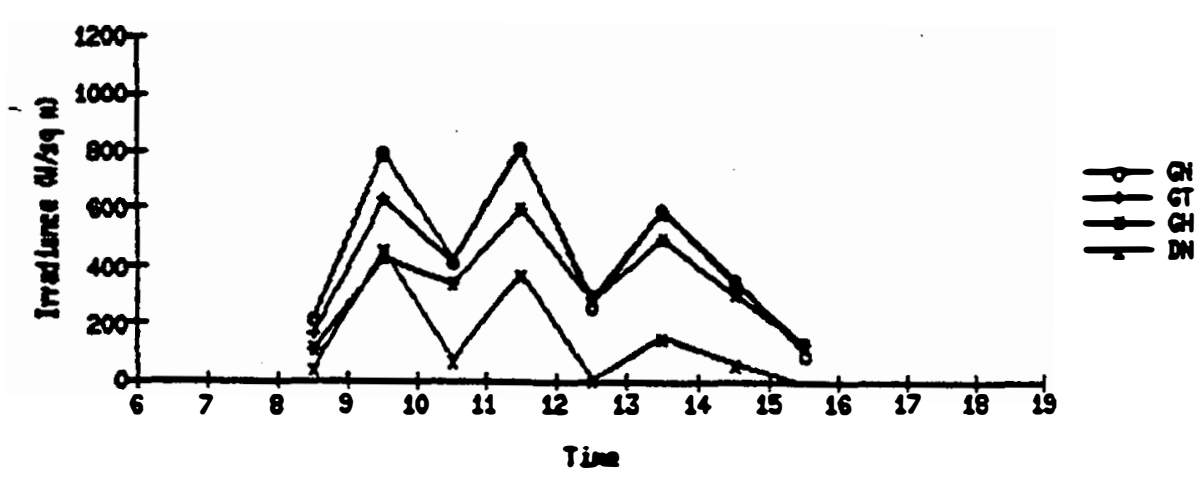

Fisc at at

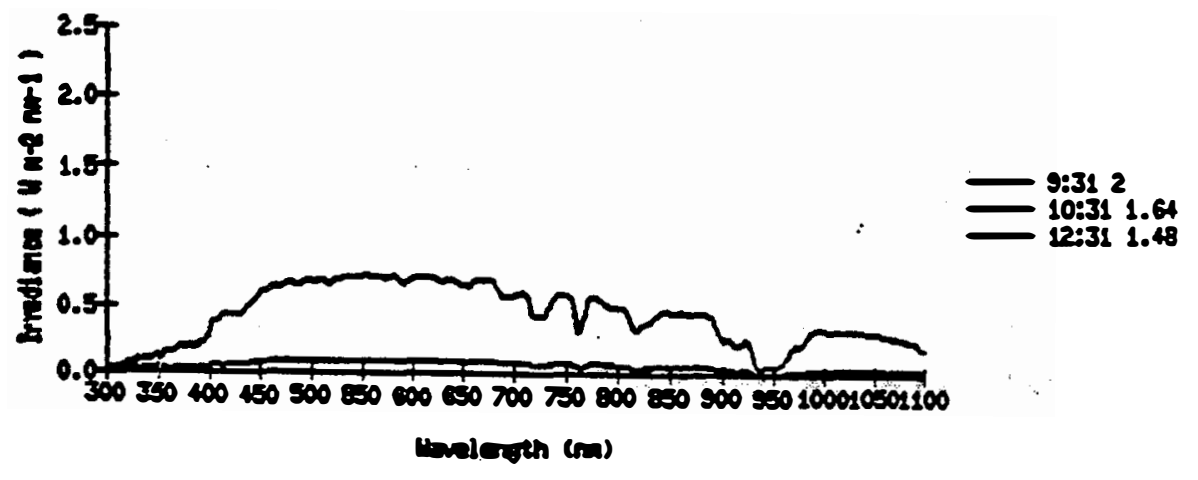

Fsac 321016

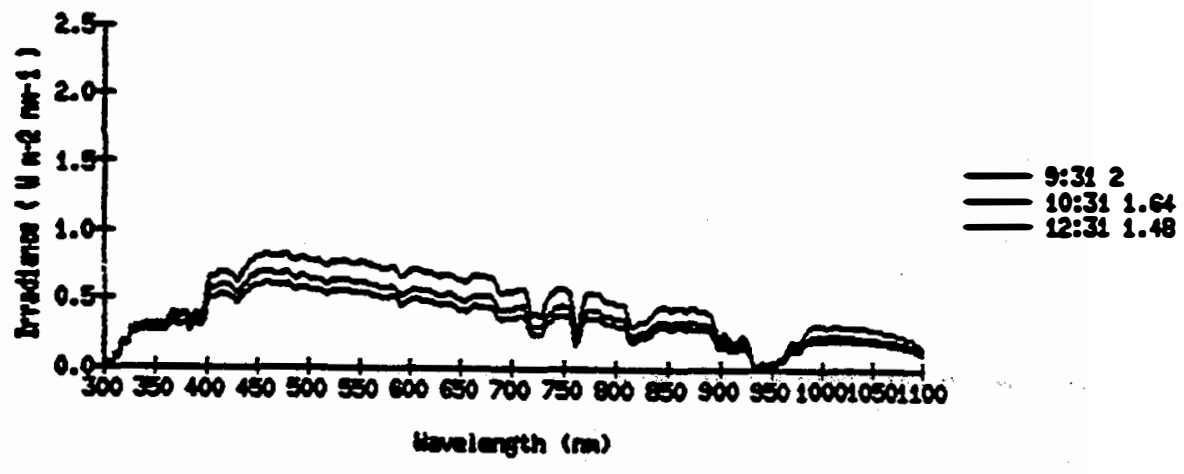


A FSTC87322

$\begin{array}{llllllllll}229 & 11 & 18 & 87 & 322 & 172 & \text { DN } & 102 & \mathrm{GH}\end{array}$

0.9 cloud cover at 0810 ; 1.0 at 1630

Teup controller: $172 \quad 102$

$081039 \mathrm{C} 40 \mathrm{C}$

IFR 172 a drift error at 1233

Bo slides

Da spectin at 1233 is bad (no direct bean)

Fase 302

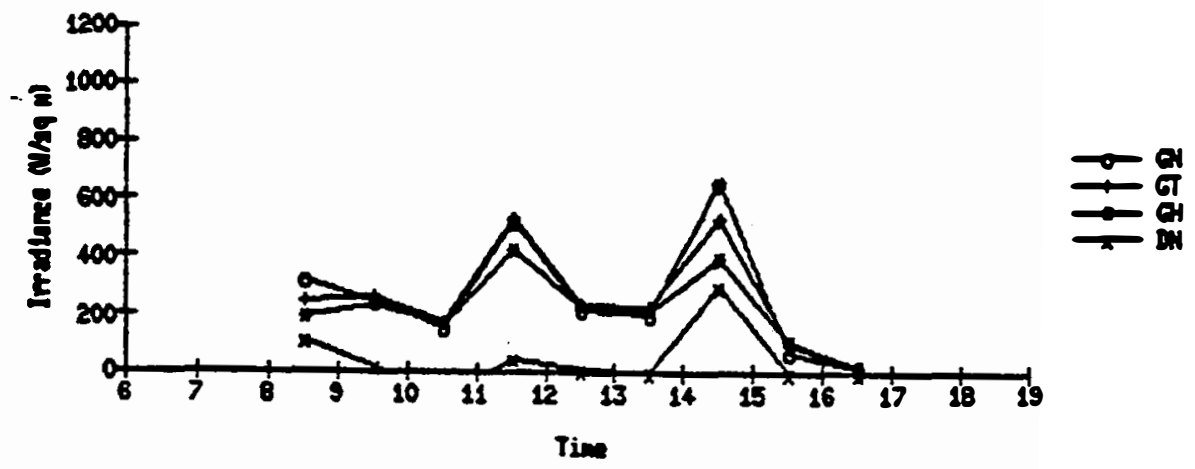

Fase 302 ant

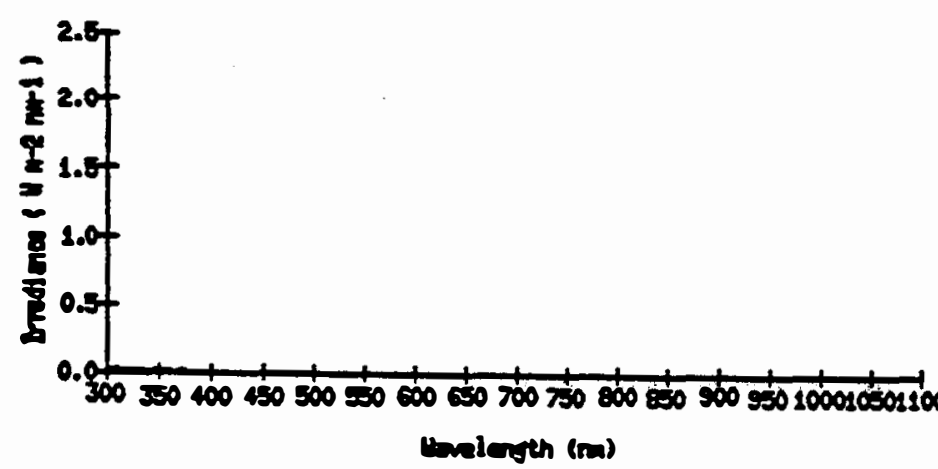

$12: 312.49$

rase sen as

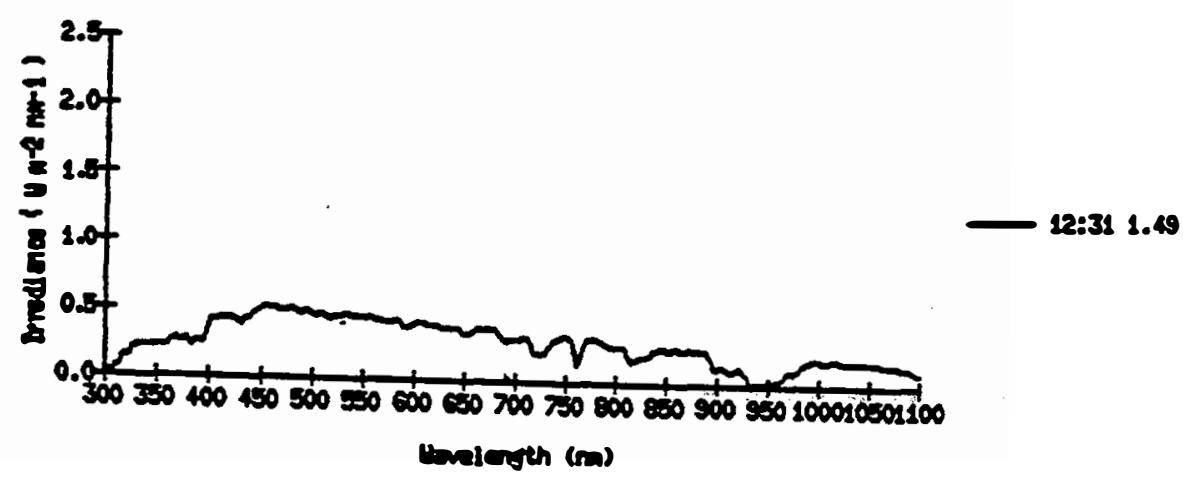




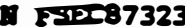

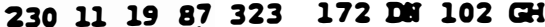

0.9 cloud cover at 081581.0 ulth rain at 1230

ISR 172 av drift errors at 0934 and 1033

Bo slides

OO in Dil spectra at 0831 and 0934 is bad (no direct bean at 0934)

Pras 323

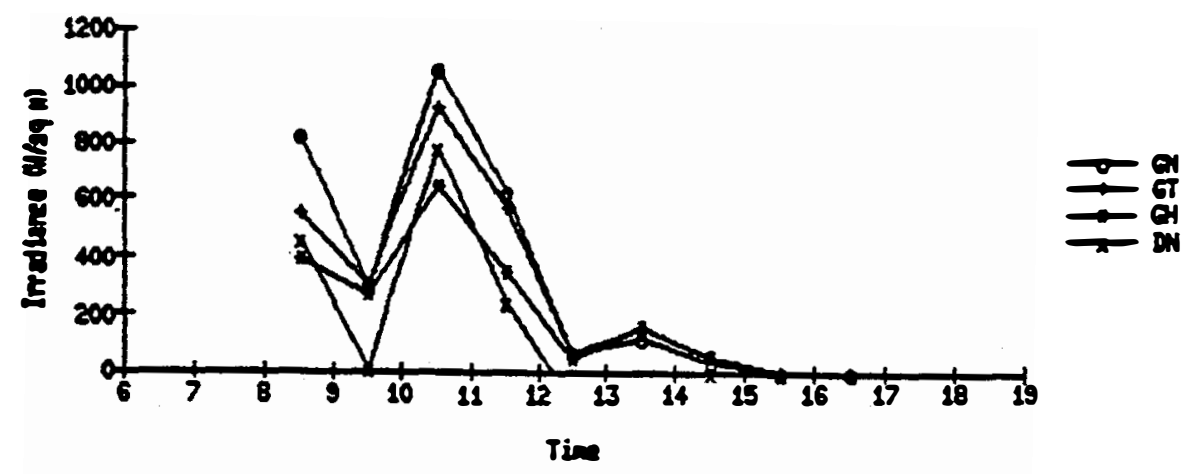

$\operatorname{crax} 203$ ant

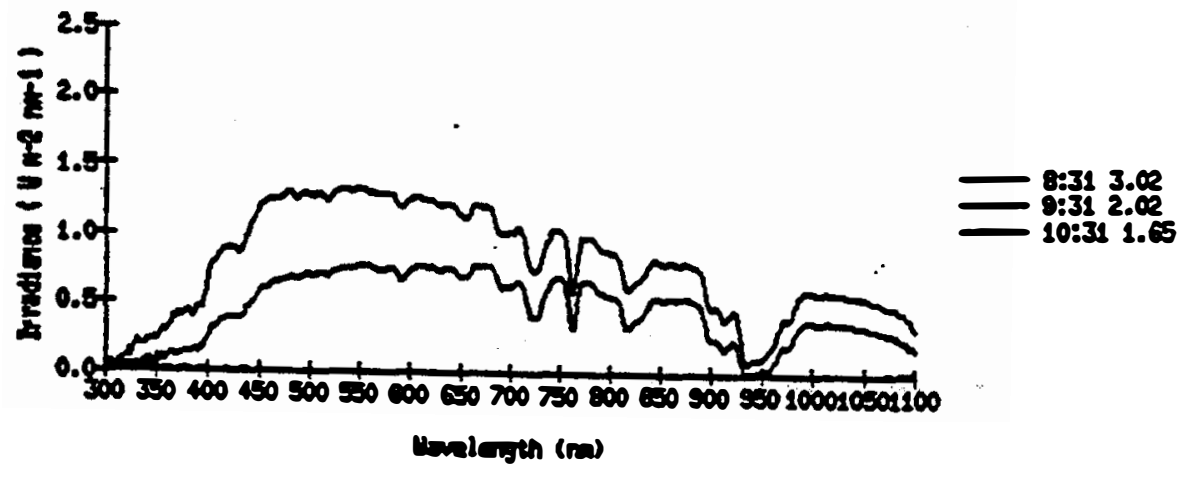

pase 303 as

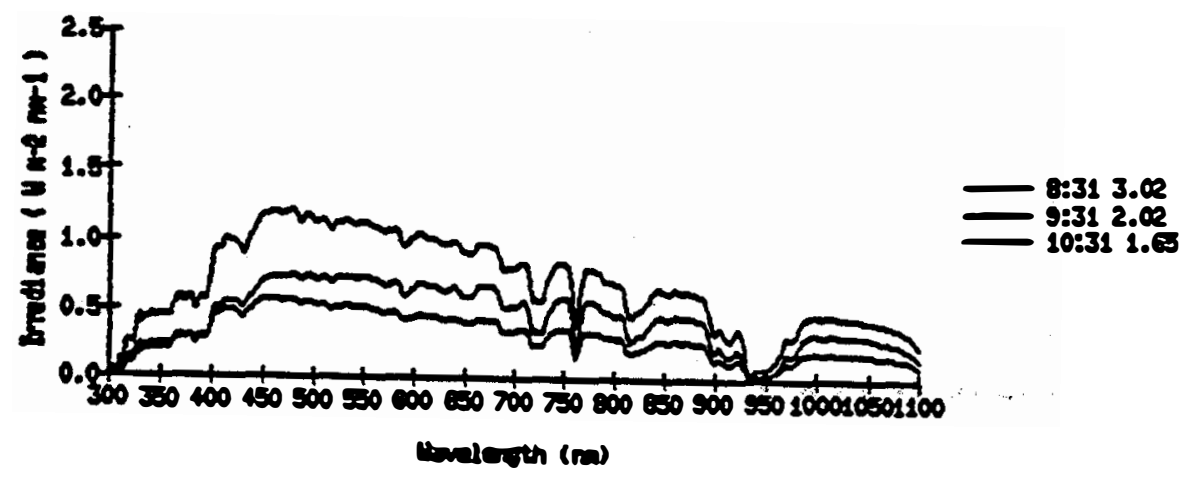




\section{SEN1 *}

P52887324

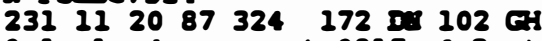

0.1 cloud cover at 081580.3 at 123080.1 at 1530

Iep controllers: $17239 \mathrm{C}$ and $10240 \mathrm{C}$ at 0815

To sildes

FEC 324

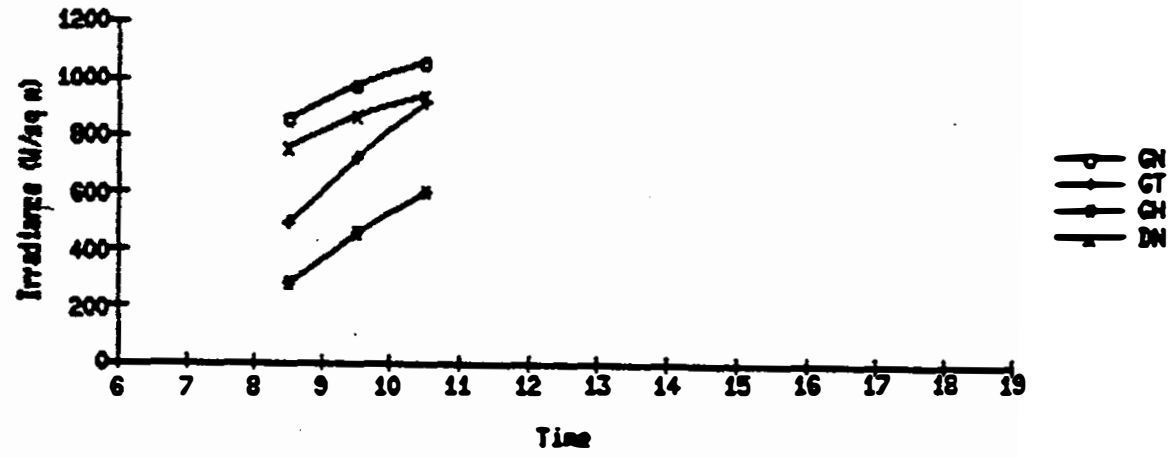

Fise 34 ant

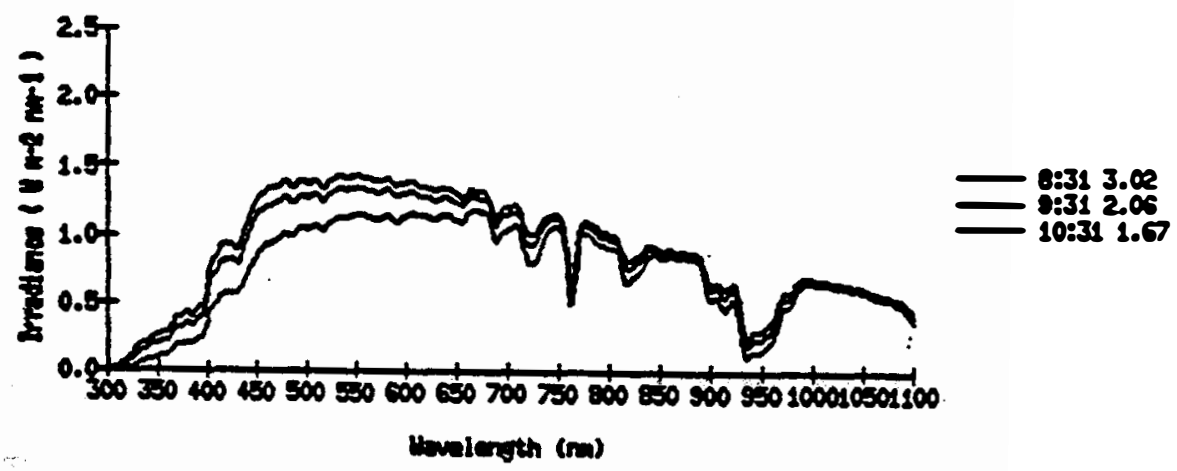

Pix 324 ars

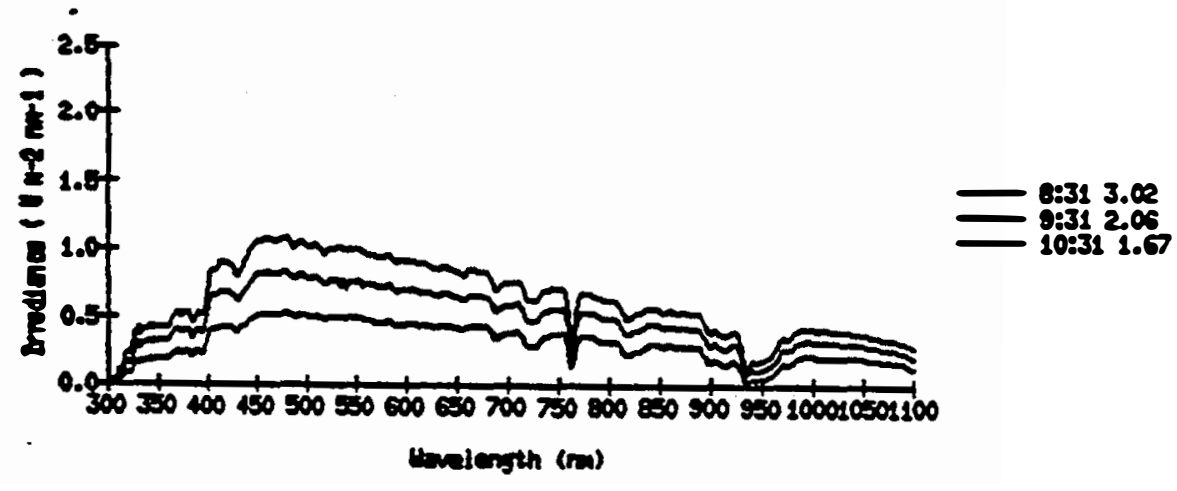


1. Tंग्287334

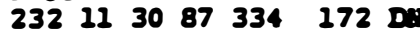

Ieq 102 retumed to ster for eal check

Teap controller $40 \mathrm{C}$ at 1330

ISR 172 av drift error at 1232

SIIdes: $0925 \mathrm{G}$ (comdensation)

$1024 \mathrm{H}$ (dark)

$1225 \mathrm{M}$

$1225 \mathrm{M}$

$1324 G$

$1424 G$

$1524 G$

Fose 334

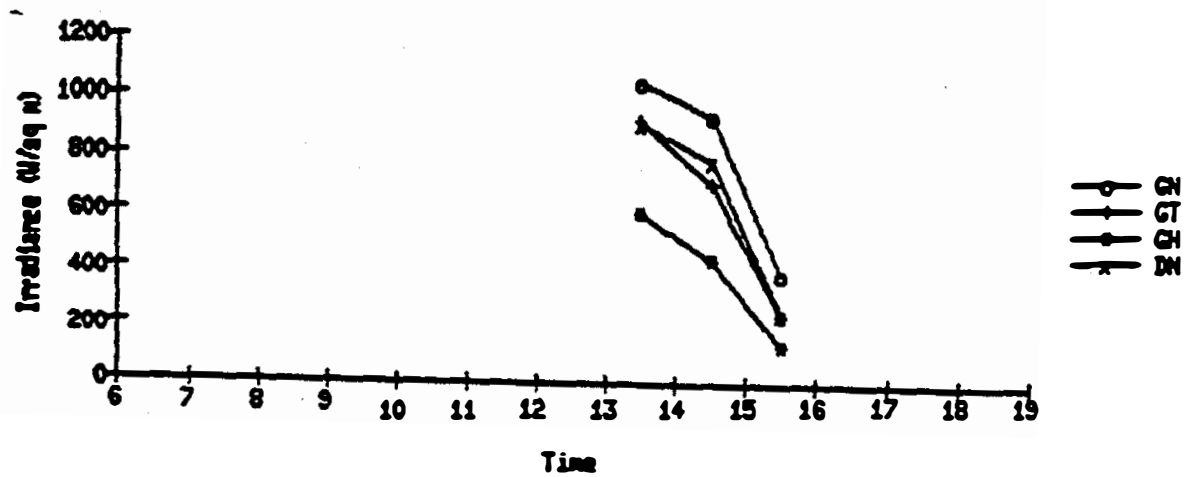

Fase 3a din

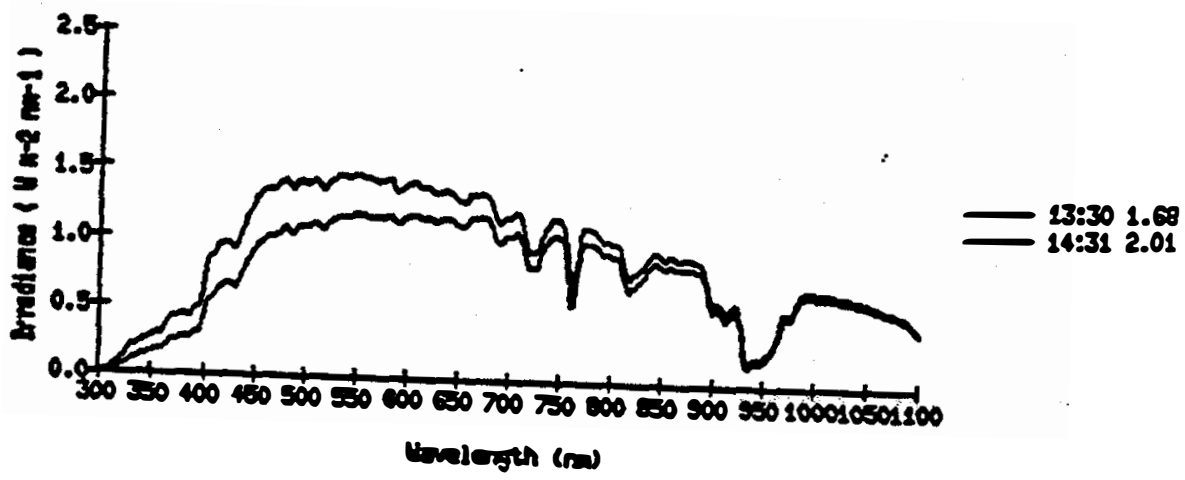


N FSPX87335

$\begin{array}{llllll}233 & 12 & 0187 & 835 & 172 & \mathrm{DH}\end{array}$

0.9 cloud coier at $0810 ; 0.1$ at $1200 ; 0.9$ at 1630

Teap controller $39 \mathrm{C}$ at $0810 ; 40 \mathrm{C}$ at 1200

Slides: 0920 G (condensation)

1128 M (11ght leak)

$1227 \mathrm{G}$

$1327 \mathrm{G}$

$1426 \mathrm{G}$

$1526 \mathrm{G}$

$\operatorname{resc} 30$

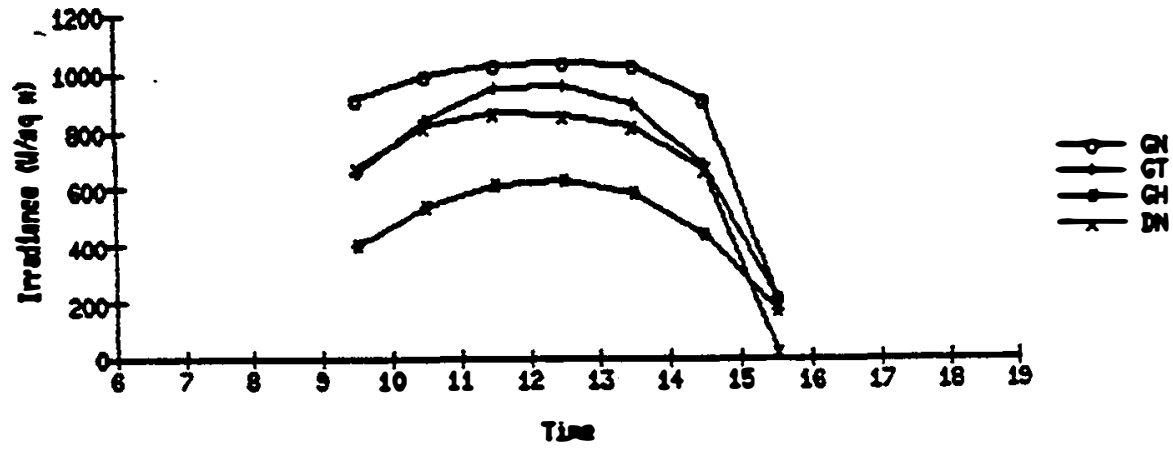

Fise 300 int

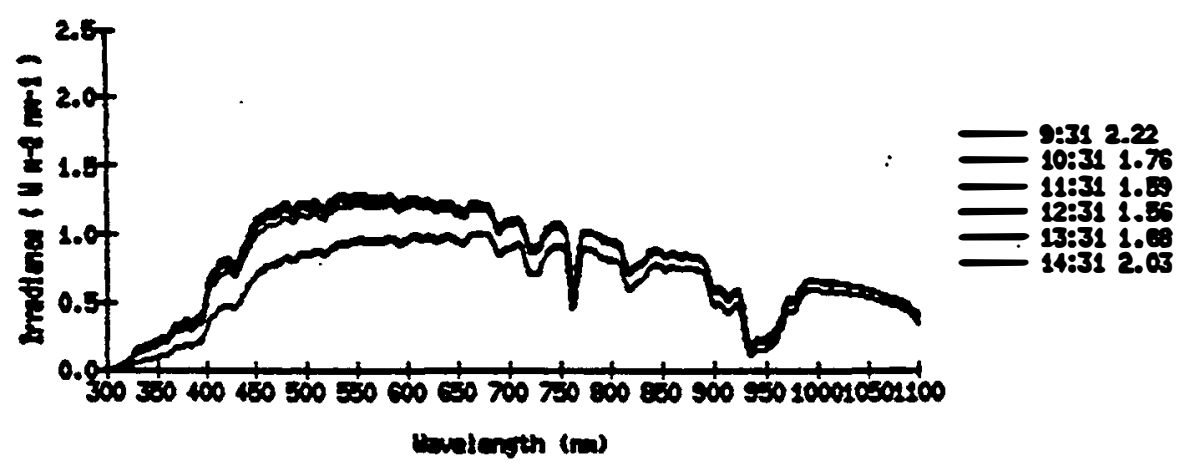




\section{SEP * *}

F5x 87336

$\begin{array}{llllll}234 & 12 \quad 02 \quad 87 & 336 & 172 & \mathrm{DH}\end{array}$

1.0 cloud cover at 0830; 0.1 at 1230 (cold front passed)

Teap controller $39 \mathrm{C}$ at 1230

slides: 0919 H (11ght leak)

$1019 \mathrm{M}$ - (condensation)

$1118 \mathrm{H}$

$1218 \mathrm{P}$

1218

$1417 \%$

$1517 \mathrm{G}$

$\operatorname{Psx} 325$

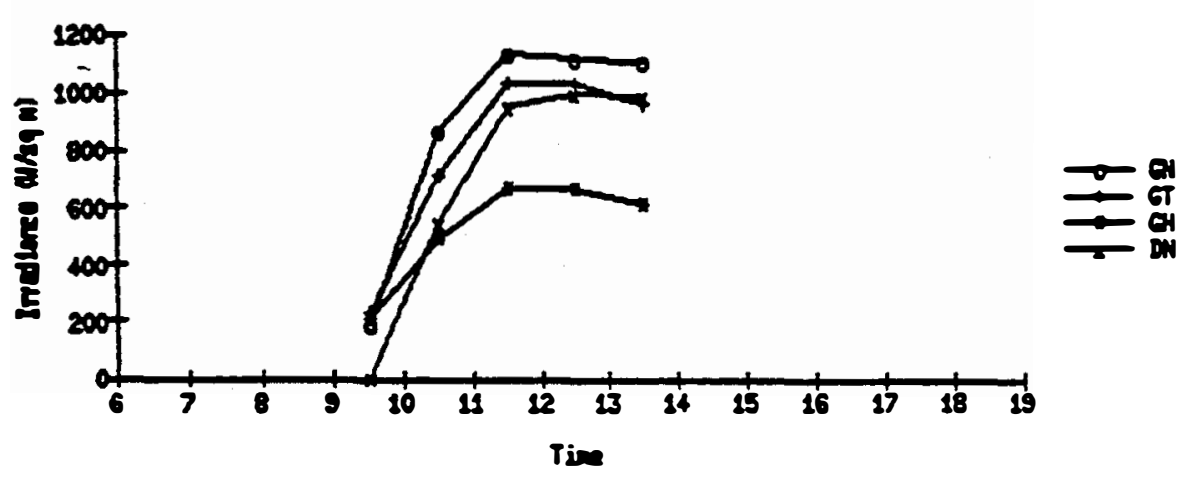

FISC 306 BT

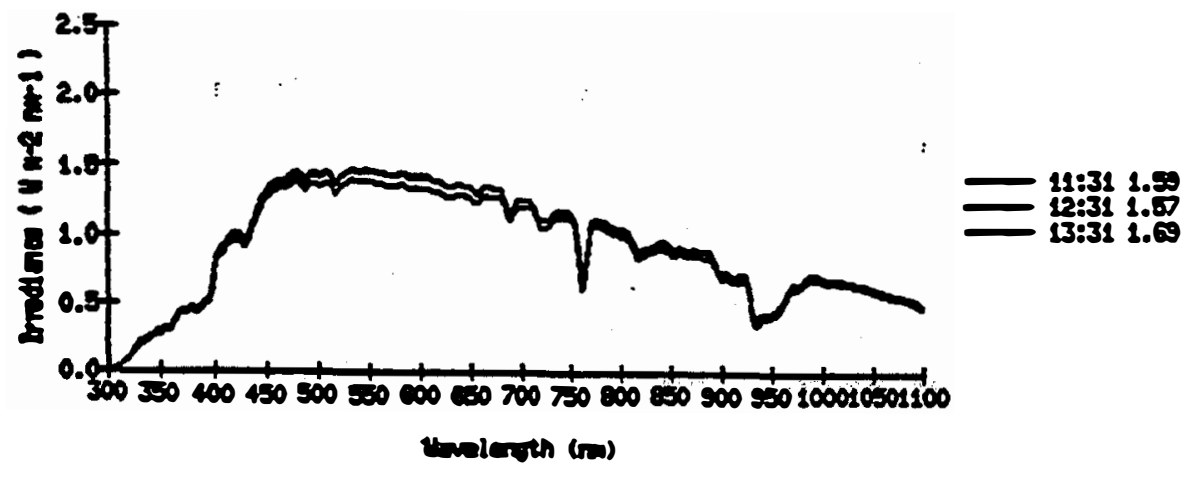


FSEC 35

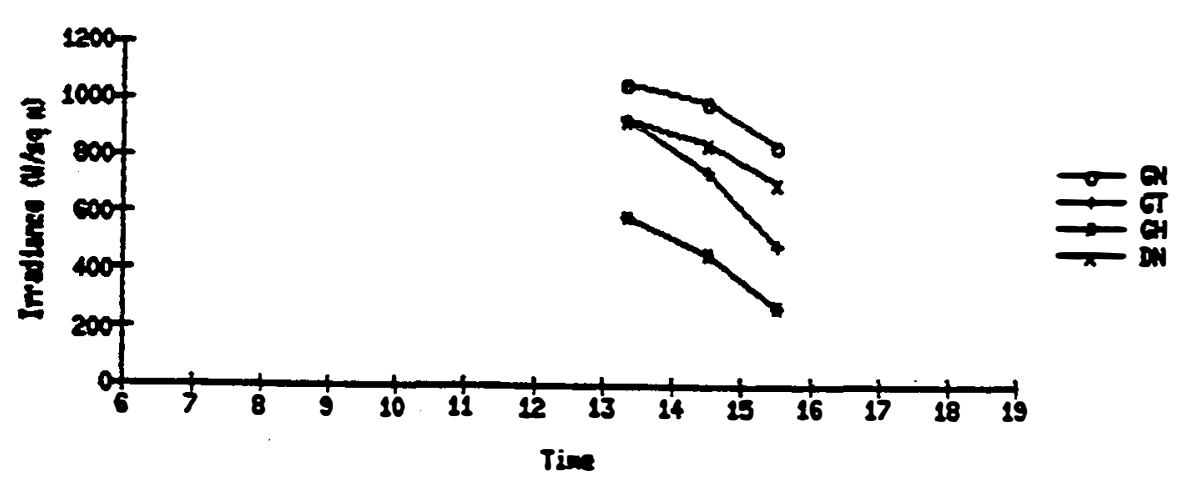

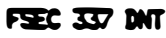

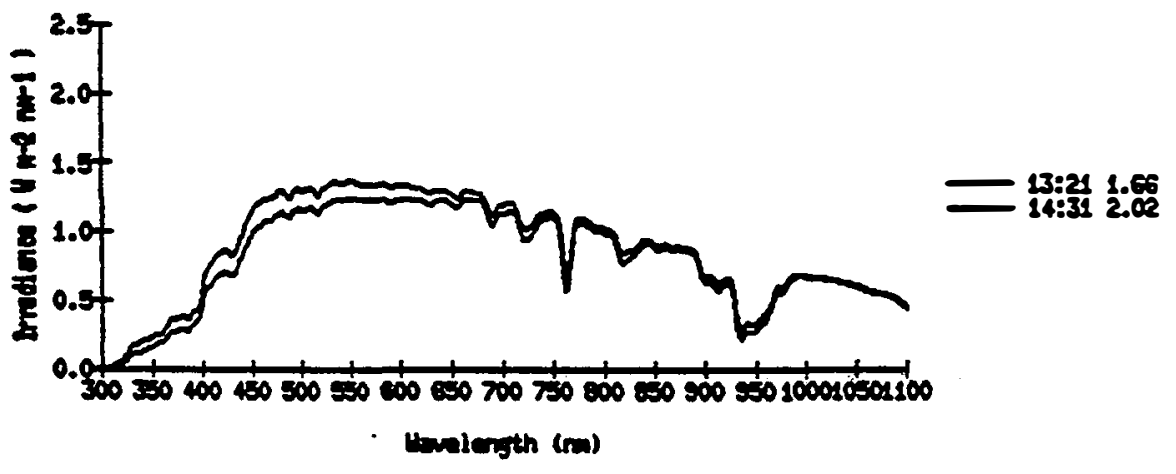


Fose 358

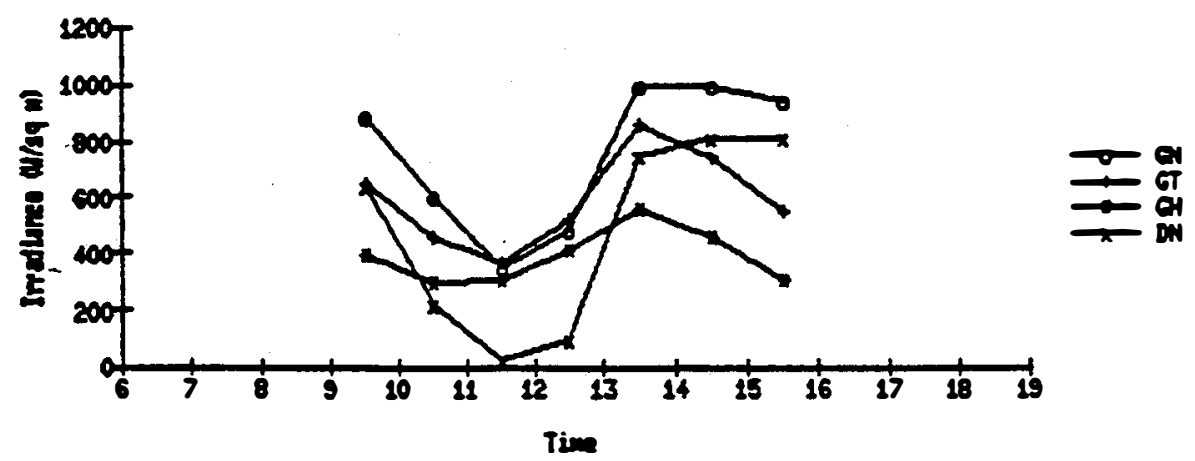

Fבי 358 onT

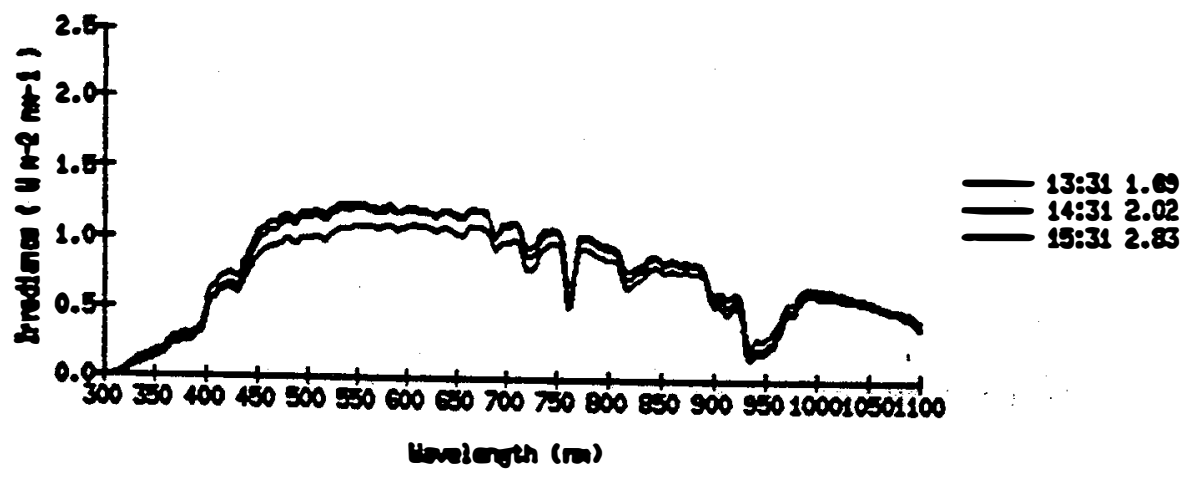




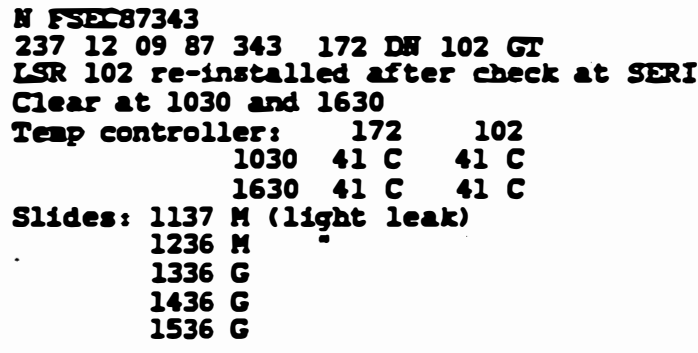

FESC 34

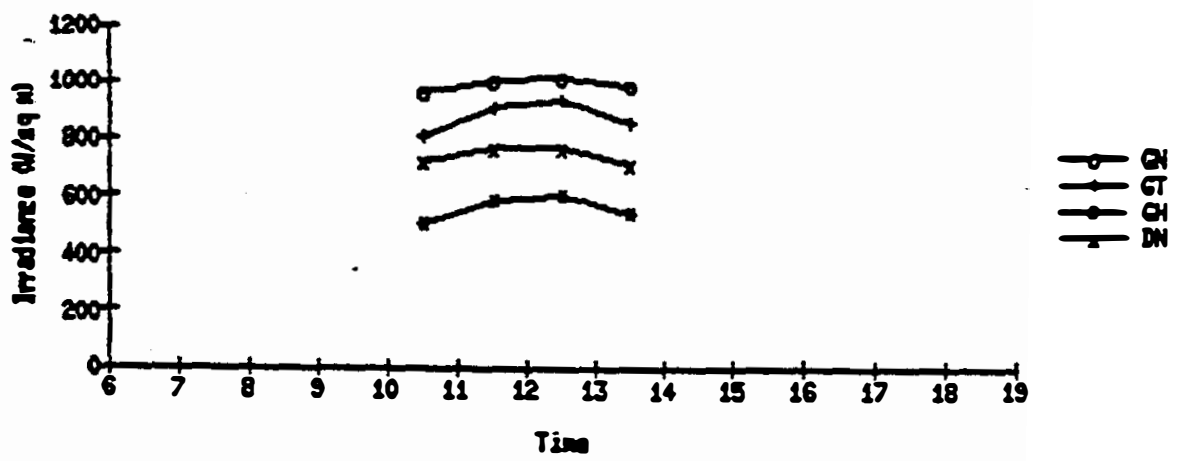

$\operatorname{TSEC} 313$ ant

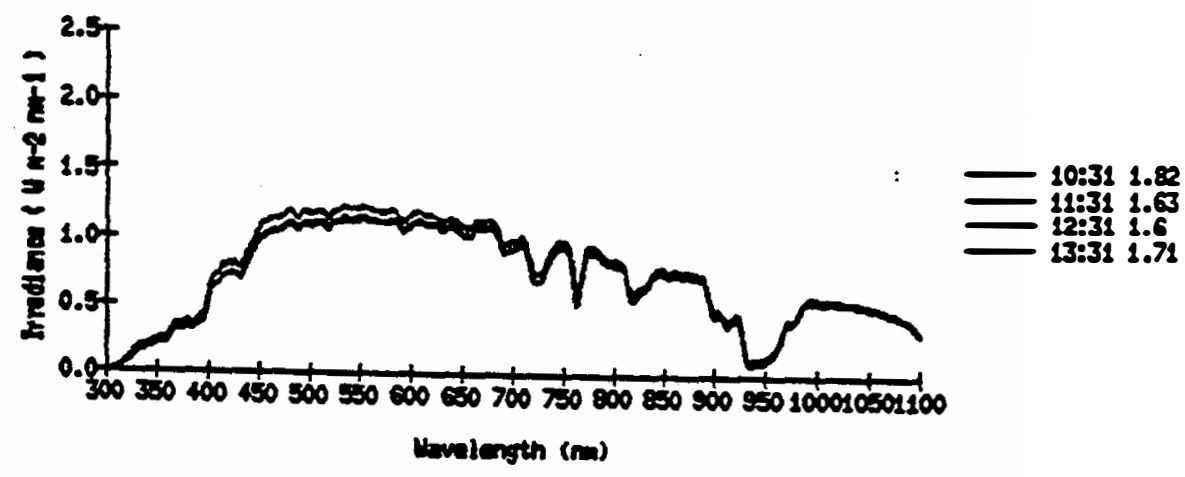

Fsesc 343 GTS

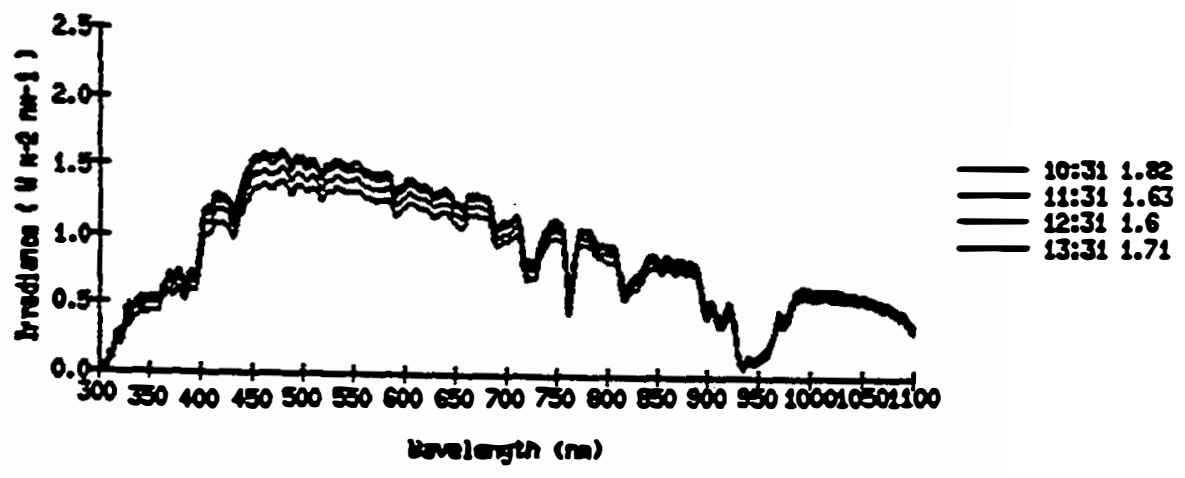


1. PST्287344

$\begin{array}{llllllllll}238 & 12 & 10 & 87 & 344 & 172 & \text { DN } & 102 & \text { GI }\end{array}$

1.0 eloud cover at $0930 ; 0.6$ at $1300 ; 0.9$ at 1630

Temp controller: $172 \quad 102$

$0930 \quad 41 \mathrm{C} 41 \mathrm{C}$

slides: $0930 \mathrm{H}$ (119ht leati condensetion)

$1130 \mathrm{G}$

$1230 \mathrm{G}$

1329 A (light leak)

$1429 \mathrm{M}$ :

$1529 M$

Ho spectra

FEse 344

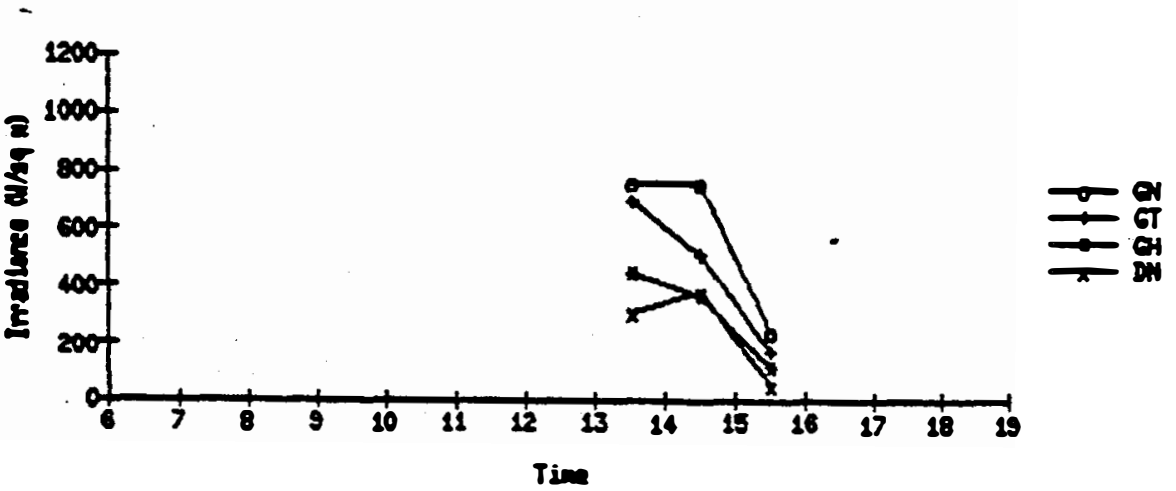


P 2587345

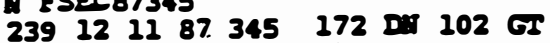

Partly eloudy discovered on lens of direct bean tube on nezt daj:

ing more affected Di spectra enis das

slides $0939 \mathrm{G}$

$1038 \mathrm{G}$

$1138 \mathrm{G}$

$1238 \mathrm{G}$

$1337 \mathrm{G}$

$1437 \mathrm{M}$ (1sght leak)
$1532 \mathrm{~A}$.

Fsec 345

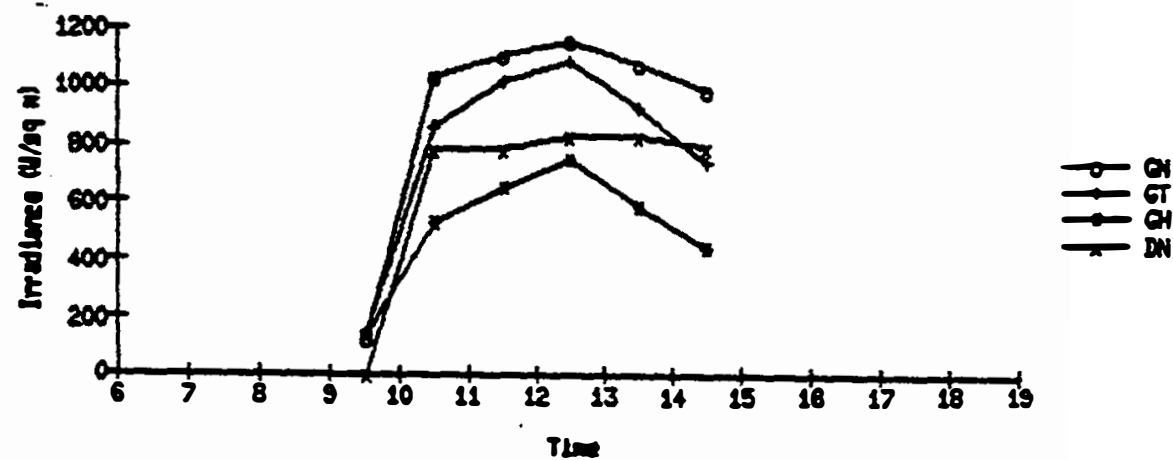

PSEC 346 an

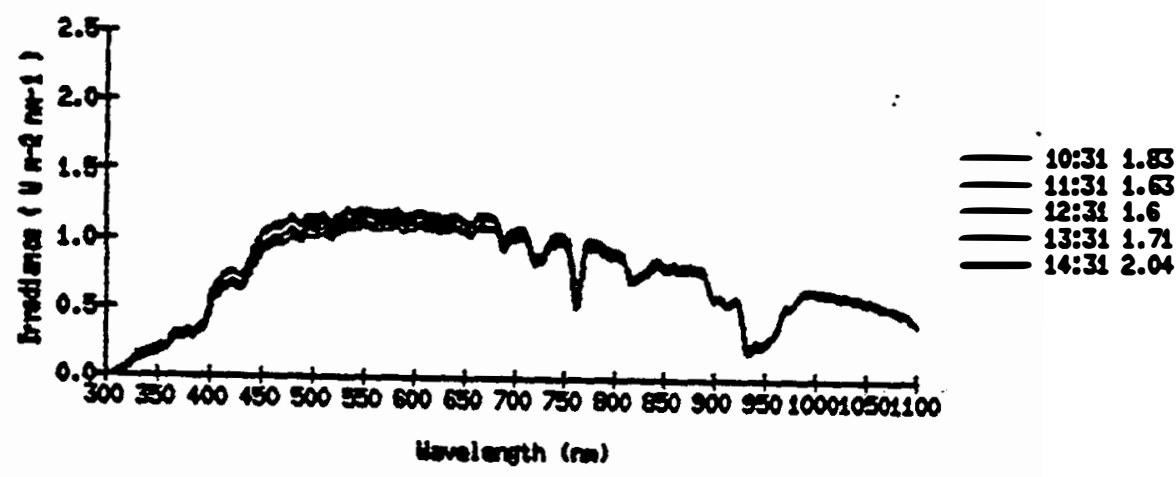

$\operatorname{Fscc} 316 \mathrm{GT}$

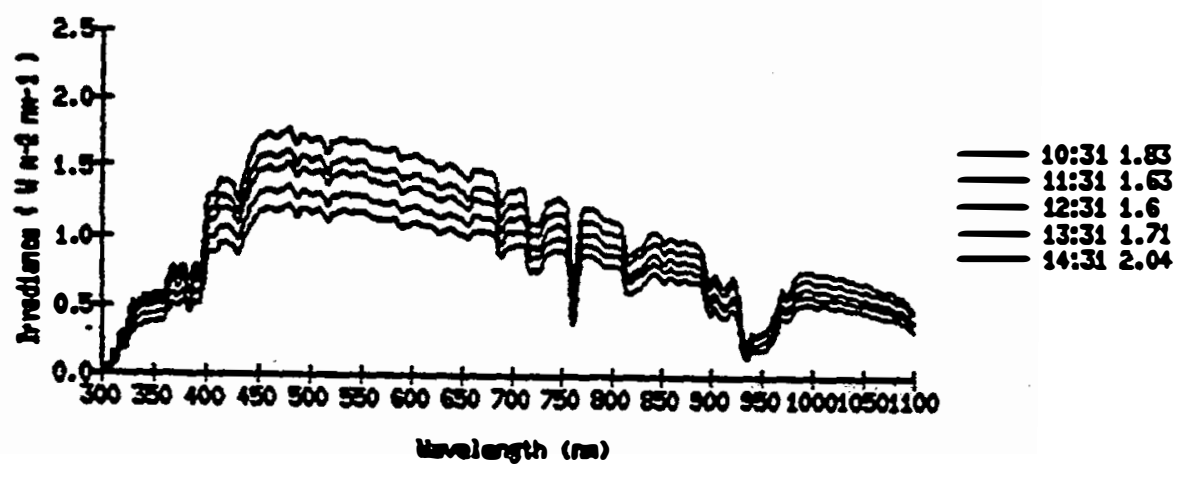


1. 752587348

$\begin{array}{lllllllll}240 & 12 & 14 & 87 & 348 & 172 & 0: & 102 & G 1\end{array}$

0.5 cloud cover at 123080.9 at 1630

Tem controllers $172 \quad 102$

$1230 \quad 40 \mathrm{C} 40 \mathrm{C}$

Bird droppings renoond fron direct been tube

No slides

so spectra

FEC 348

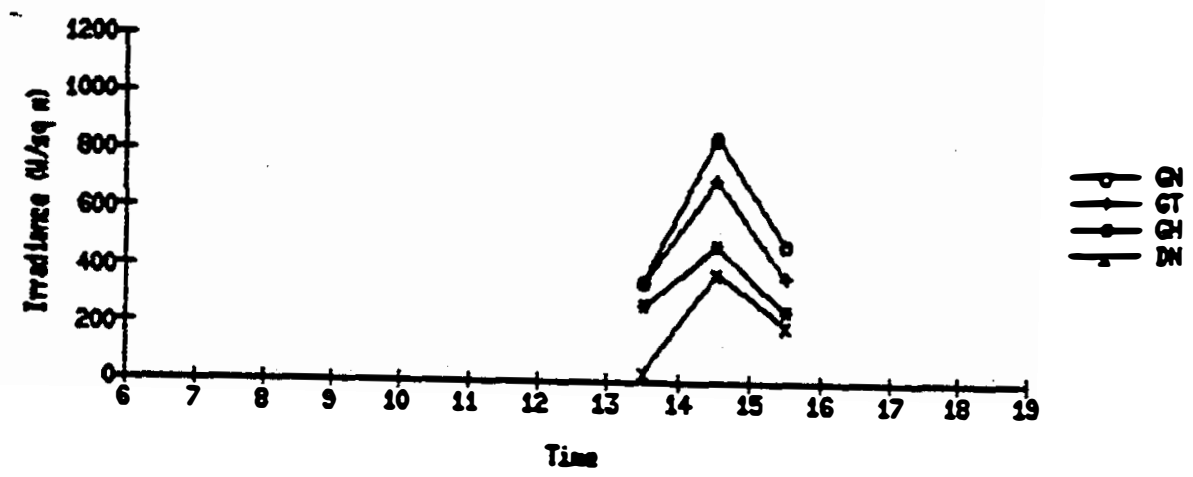


PSTS87350

$\begin{array}{lllllll}241 & 12 \quad 16 \quad 87350 \quad 172 \quad \text { DA } & 102 \text { GP }\end{array}$

0.5 cloud cover at $1130 ; 0.9$ at 1630

Tenp controllers: 172 and $10239 \mathrm{C}$ at 1130

Slides: 0933 A (condensention)

1035

$1135 \mathrm{M}$

$1234 M$

$1334 \mathrm{G}$

$1434 G$

$1534 \mathrm{G}$

$1633 \mathrm{G}$

$\operatorname{Psec} \pi 0$

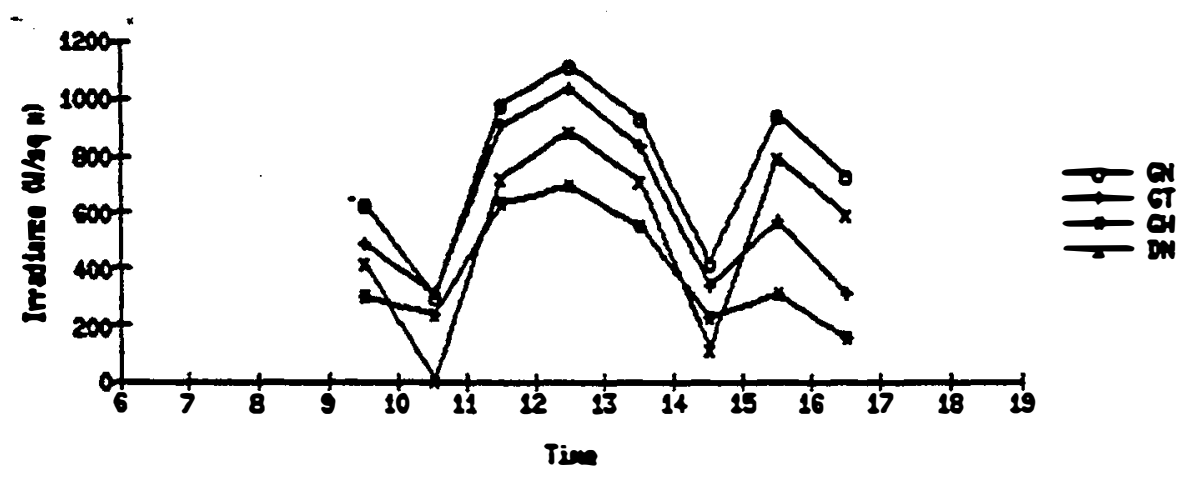

Paxe $\geq 00 \mathrm{mt}$

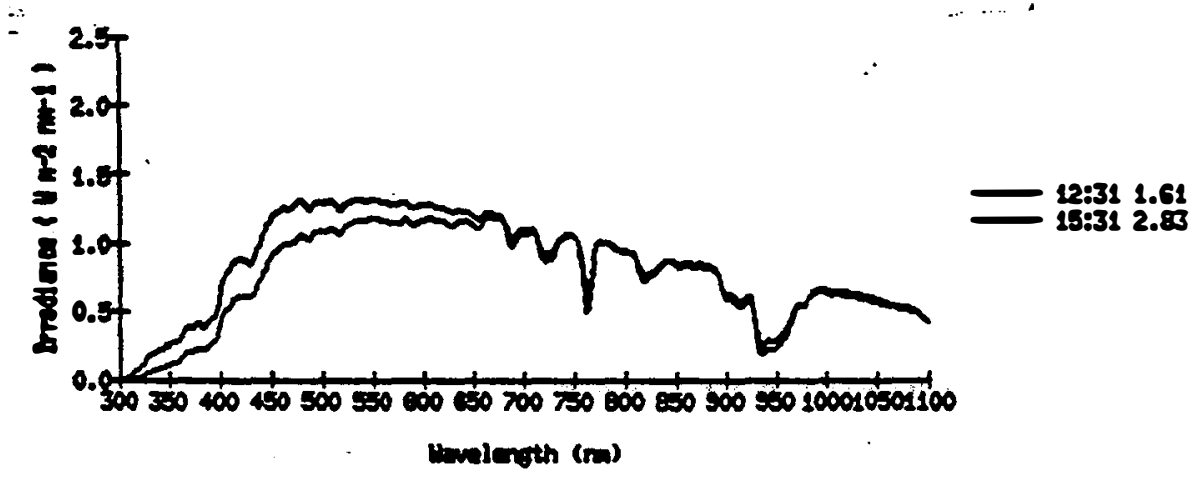

PES $3=0$ GTS

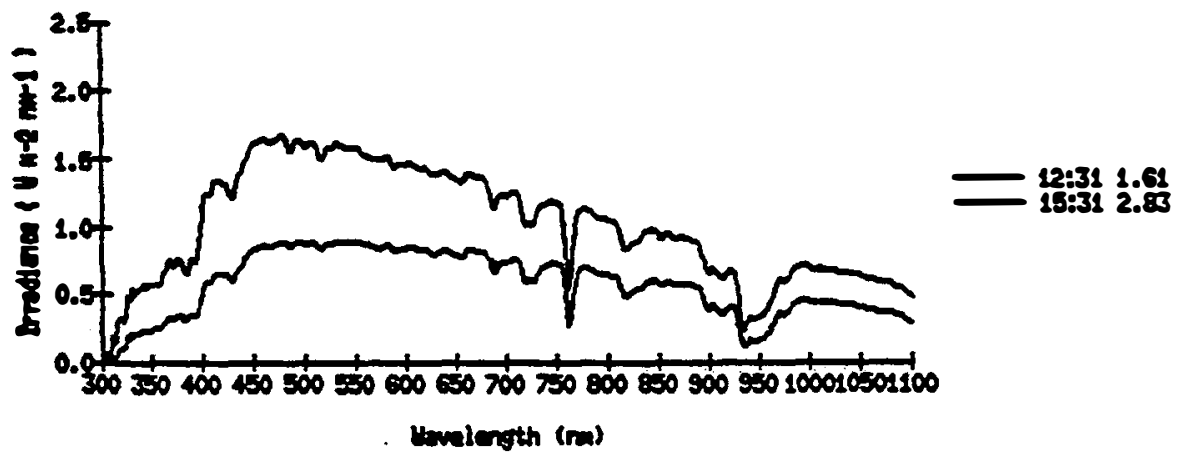




\section{SEP1 *}

TR-3513B

A PST्287351.

$\begin{array}{lllllllll}242 & 12 & 17 & 87 & 351 & 172 & \text { DI } & 102 & G 1\end{array}$

clear at 0830, 1200, and 1530

TeDp controller: $172 \quad 102$

$083038 \mathrm{C} 39 \mathrm{C}$

$220038 \mathrm{C} 40 \mathrm{C}$

Slides: 0929 M (condenantson)

$2029 \mathrm{H}$ (congeriatson)
(dingk)

$1129 \mathrm{P}$ -

$\begin{array}{llll}1229 & \mathrm{P} & - & - \\ 1329 & \mathrm{P} & - & -\end{array}$

$1429 M$

$1529 \mathrm{M}=$

$1528 M$

GI spectri noles in WV and near-IR at 1131. 1231. and 1331

spikes in of in GS spectron at 1531

Fis 201

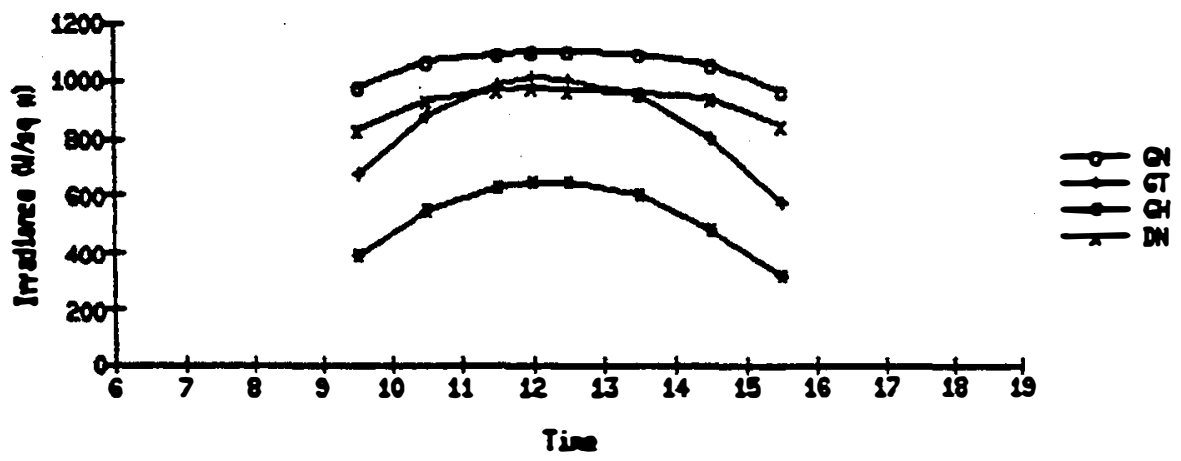

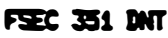

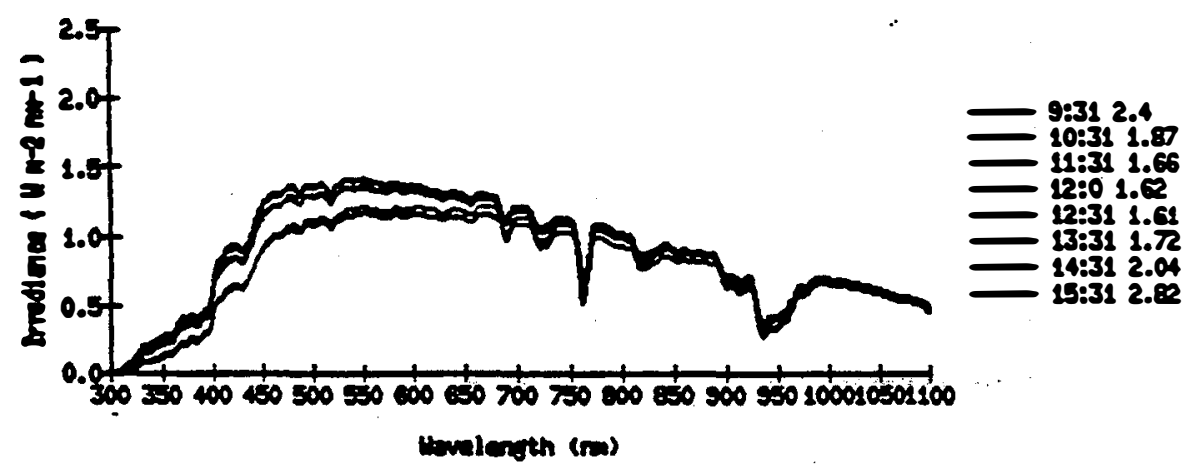

FEx ISB GTS

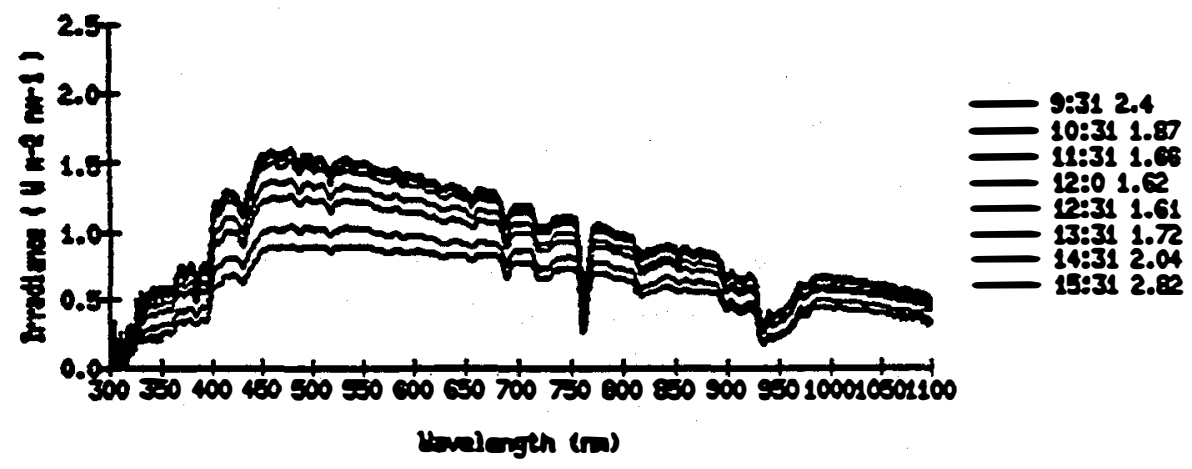


1. 752987352

$\begin{array}{lllllllll}243 & 12 & 18 & 87 & 352 & 172 & \mathrm{DH} & 102 & \mathrm{FH}\end{array}$

0 eloud cover at 100080.2 at 140080.1 at 1630

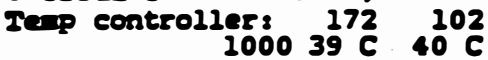

$140040 \mathrm{C} 40 \mathrm{C}$

Slides 0923 M (condencetion)

$1023 \mathrm{H}$

$1123 \mathrm{M}$

$1223 \mathrm{M}$

$1323 \mathrm{M}$

$1423 \mathrm{M}$

$1533 \mathrm{G}$

$1633 \mathrm{G}$

FsEC 3:2

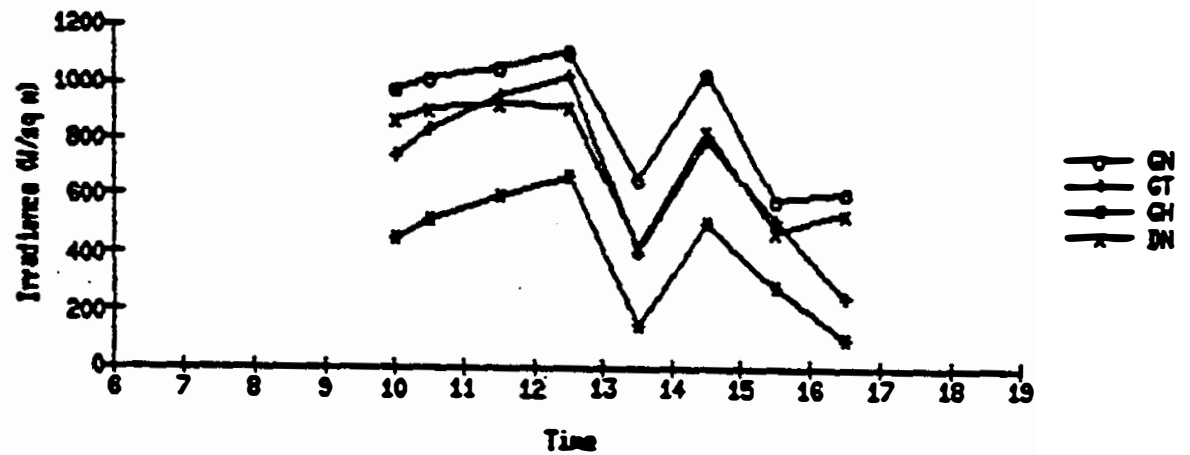

Fsec $3=2$ dit

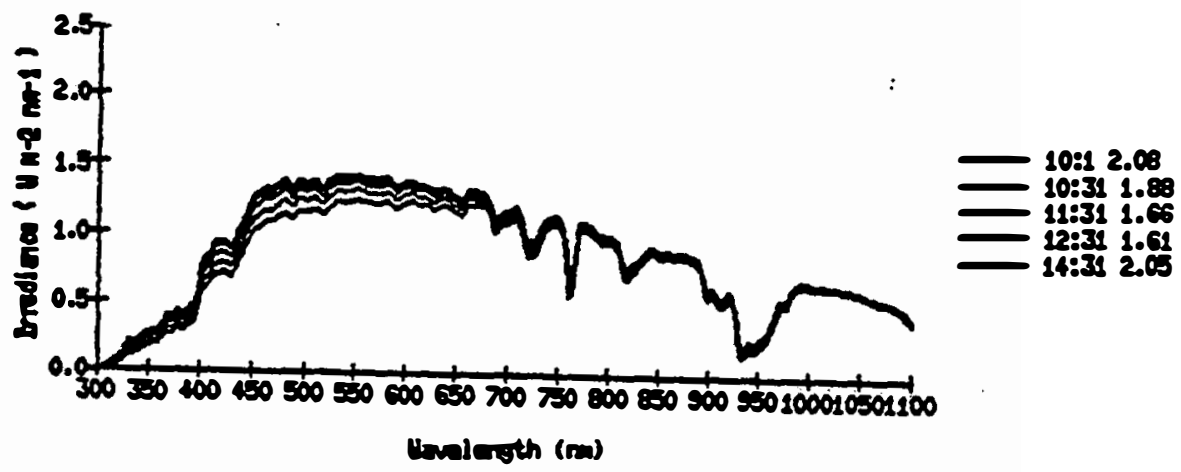

Fsec $3=2$ os

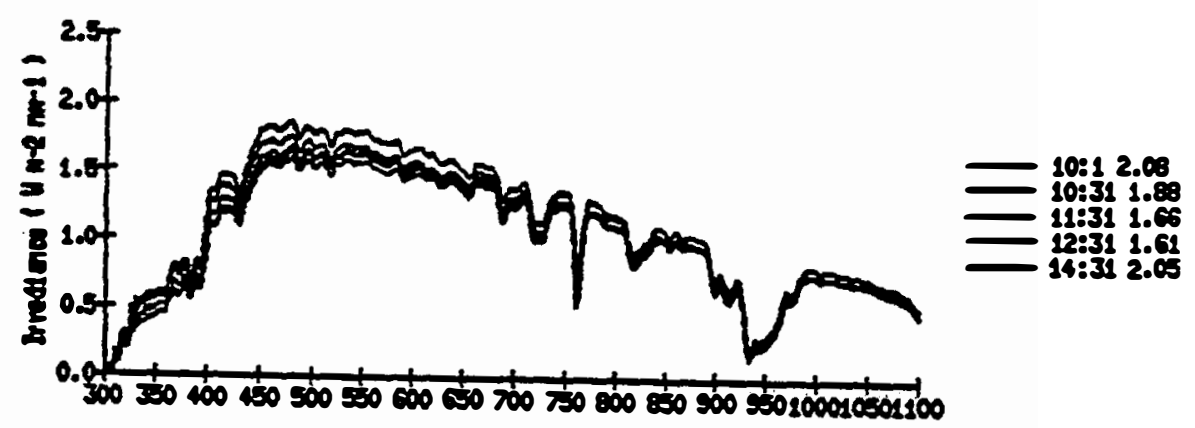

(tivlesth (m) 


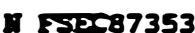

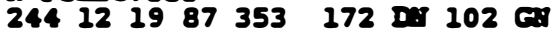

Partis cloudy

S11des: $0929 \mathrm{G}$

$1028 \mathrm{G}$

$1128 \mathrm{G}$

$1228 \mathrm{G}$

$1328 \mathrm{G}$

$1427 \mathrm{G}$

$1527 \mathrm{G}$

$1627 \mathrm{G}$

$\operatorname{cise} 5$

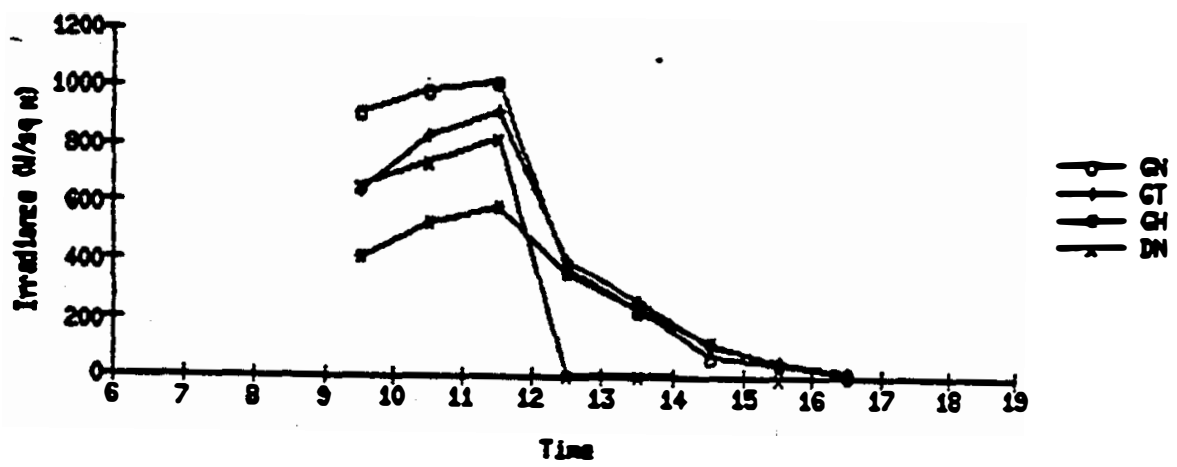

Fis

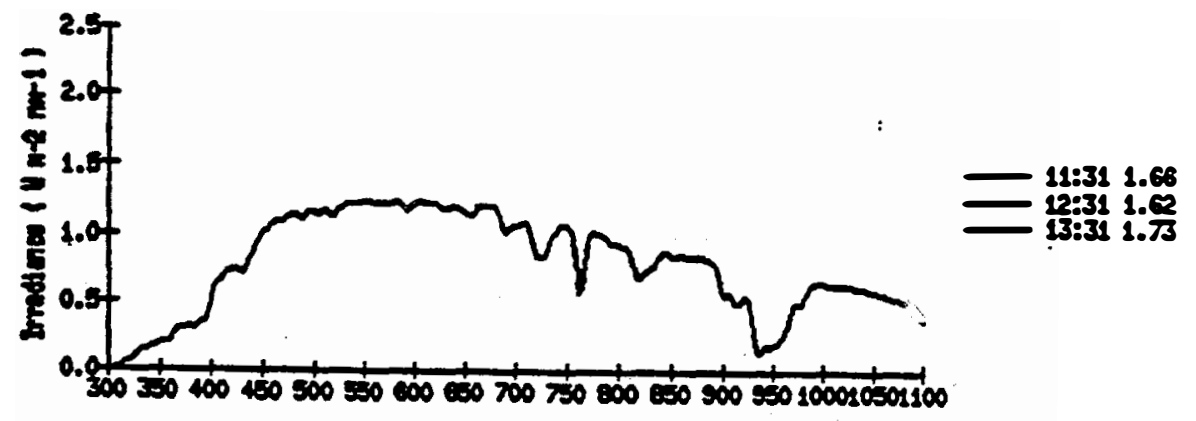

trelareth (m)

Fise 303 os

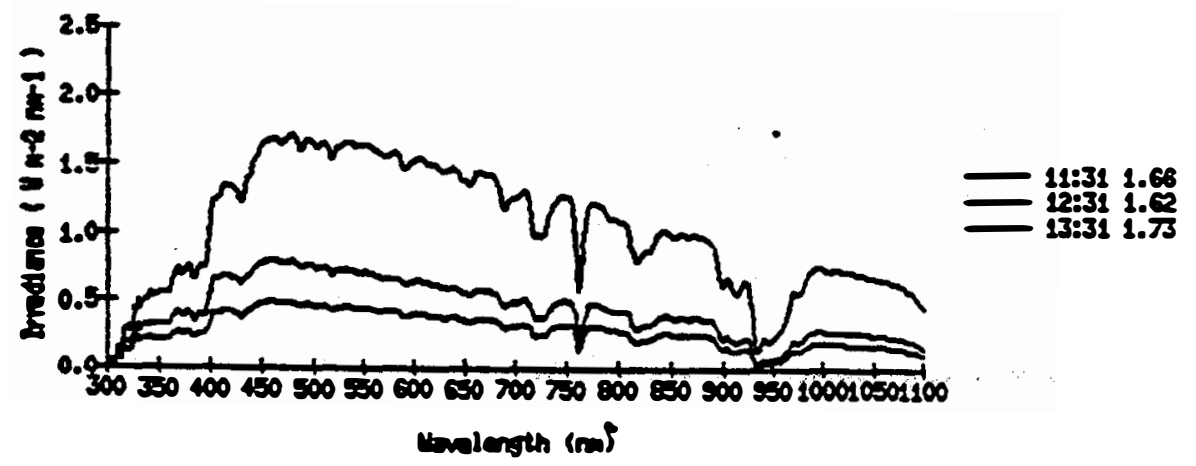




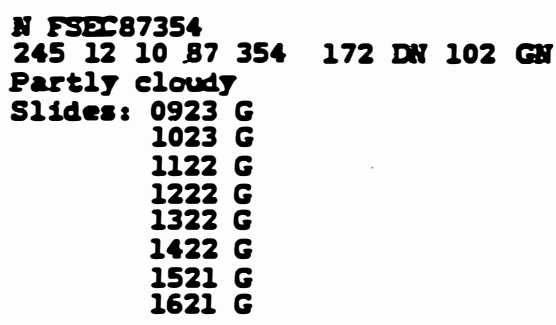

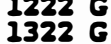

$1422 \mathrm{G}$

$1521 G$

$1621 \mathrm{G}$

Fsec 354

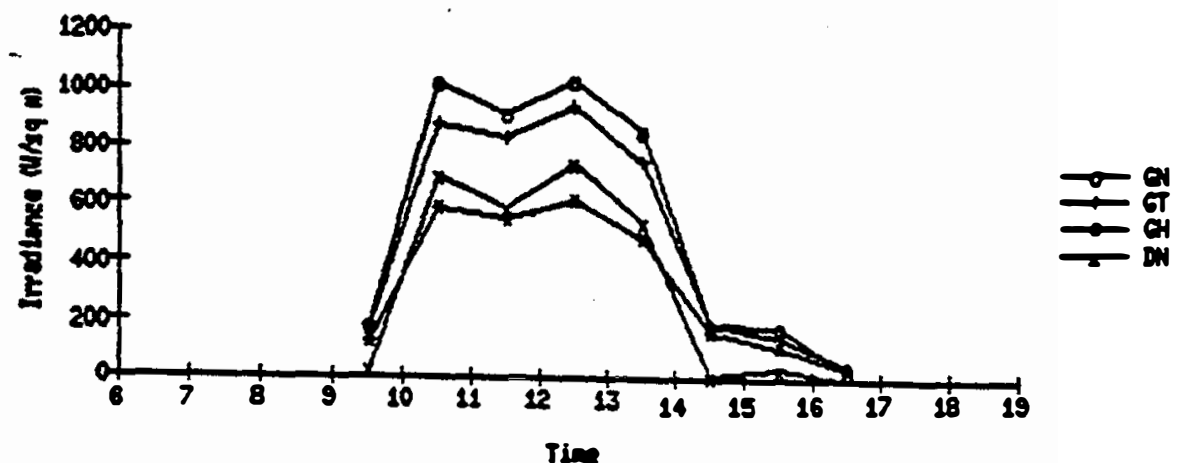

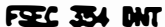

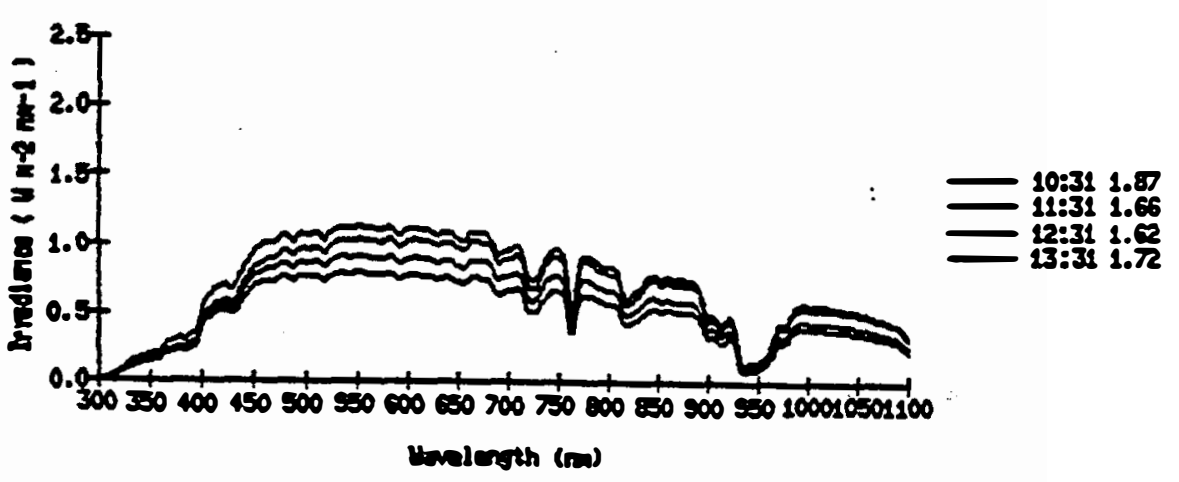

Fas 3ed as

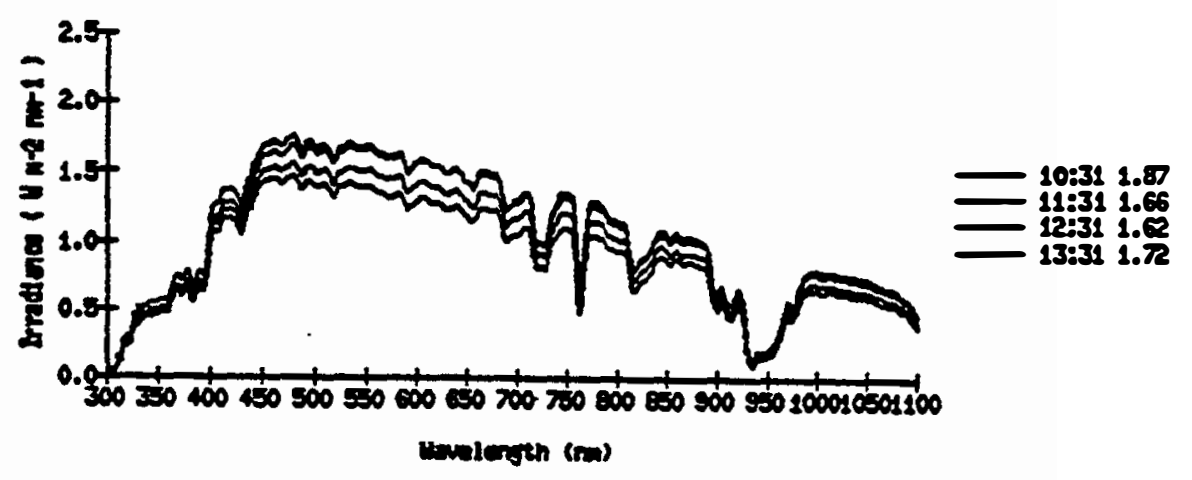




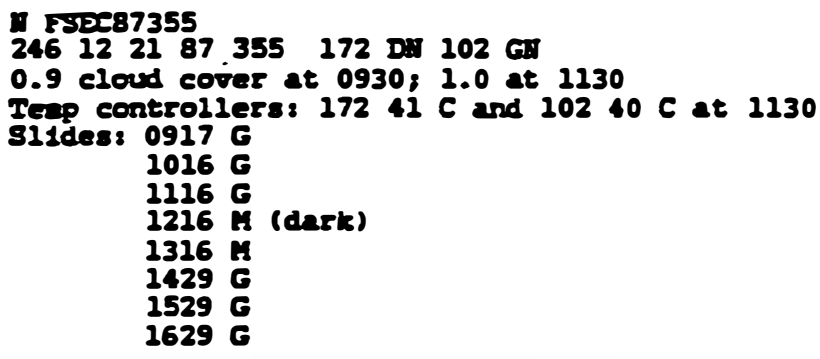

$1316 \mathrm{H}$

$1429 \mathrm{G}$

$1529 \mathrm{G}$

1629 G

Faי

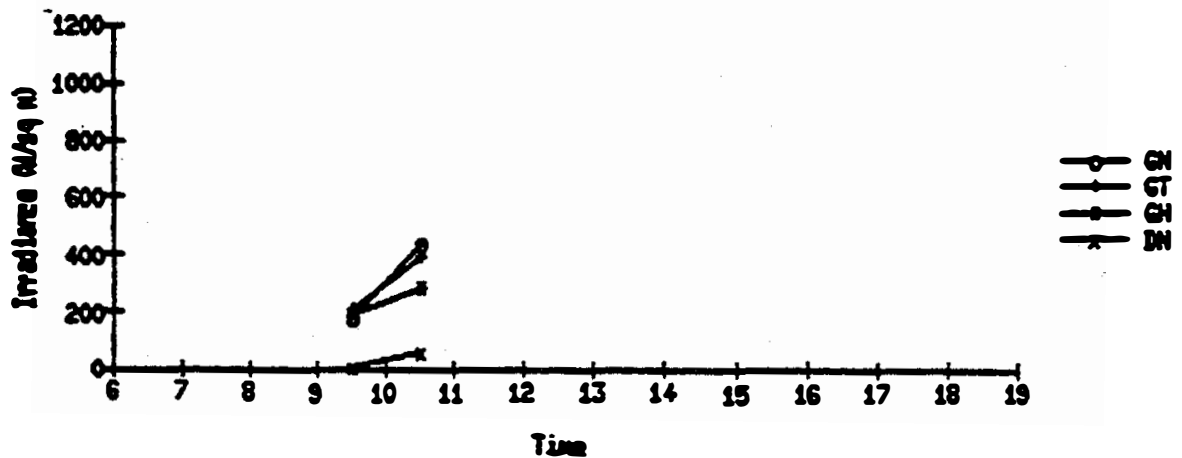

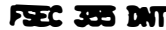

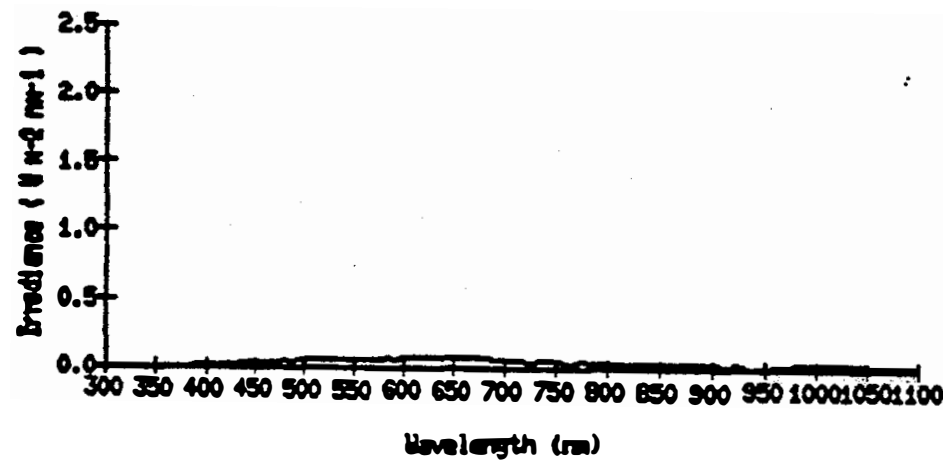

rase os

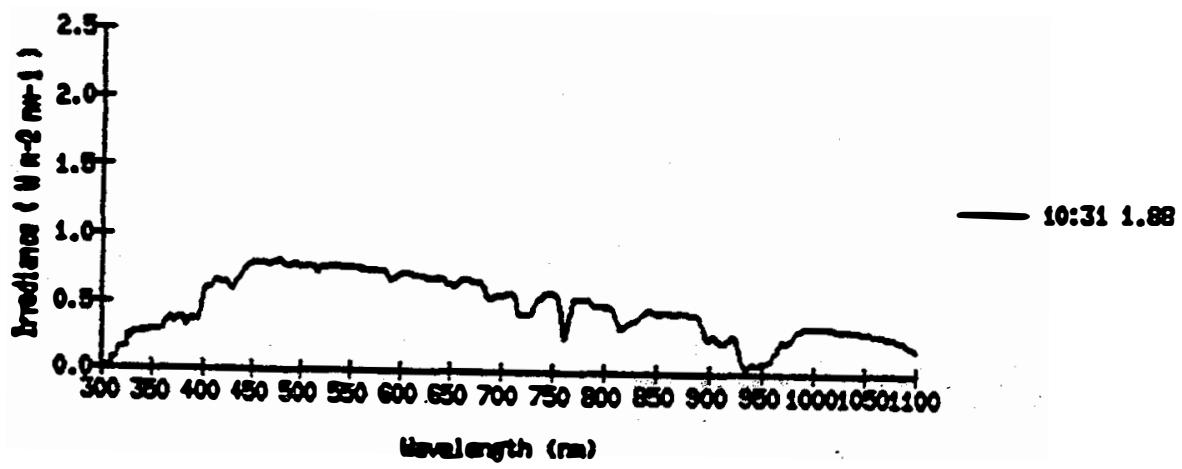


a FSEC 87356

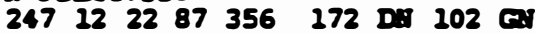

0.1 cloud cover at $0830 ; 0.6$ at $1130 ; 0.9$ at 1630

Tenp controllers: $172 \quad 102$

083039 C 39 C

$163040 \mathrm{C} 40 \mathrm{C}$

Slidesz $0924 G$

$2024 \mathrm{G}$

$1223 \mathrm{G}$

$1223 \mathrm{G}$

$1323 G$

$1422 \mathrm{G}$

1522 G

$1622 G$

Fise 350

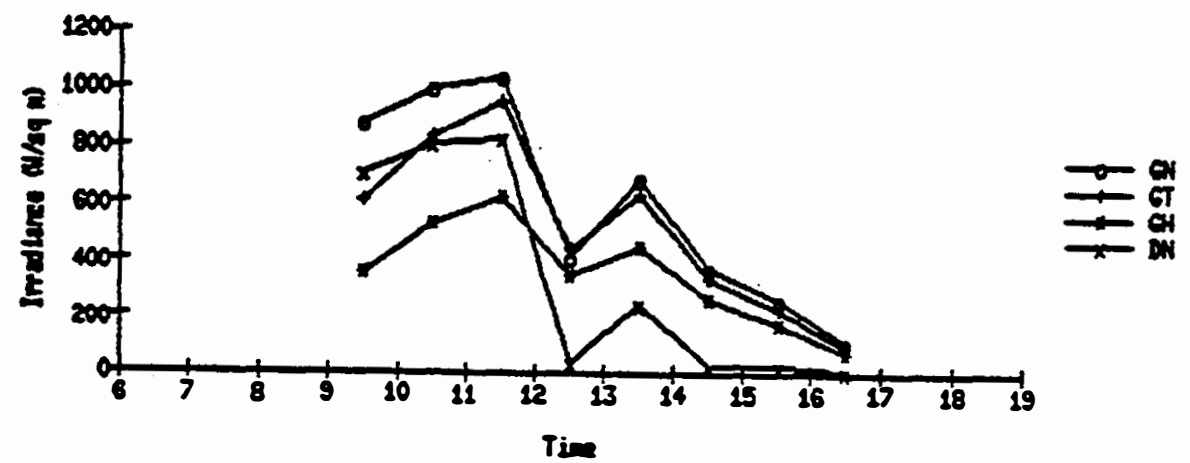

FEב 305 औT

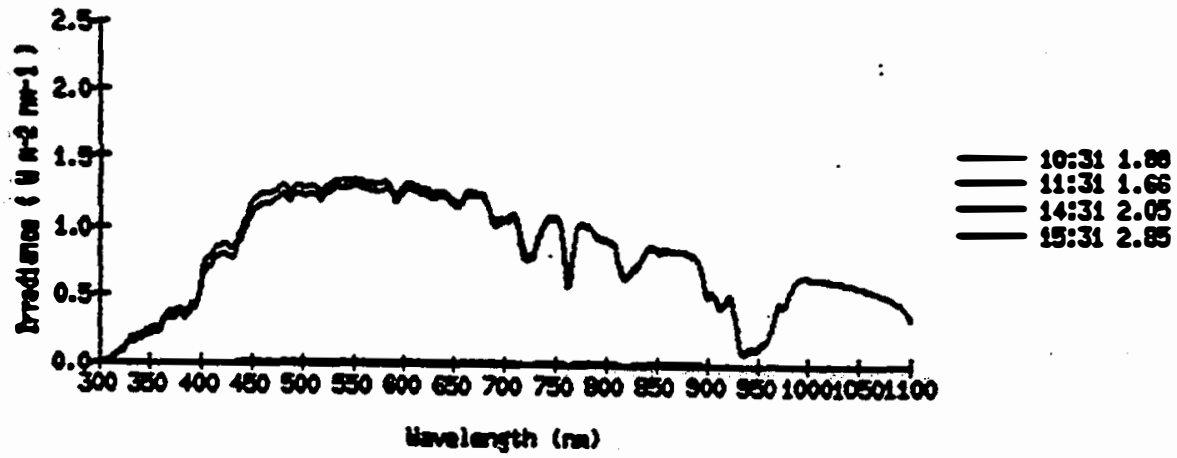

Fazt 350 Os

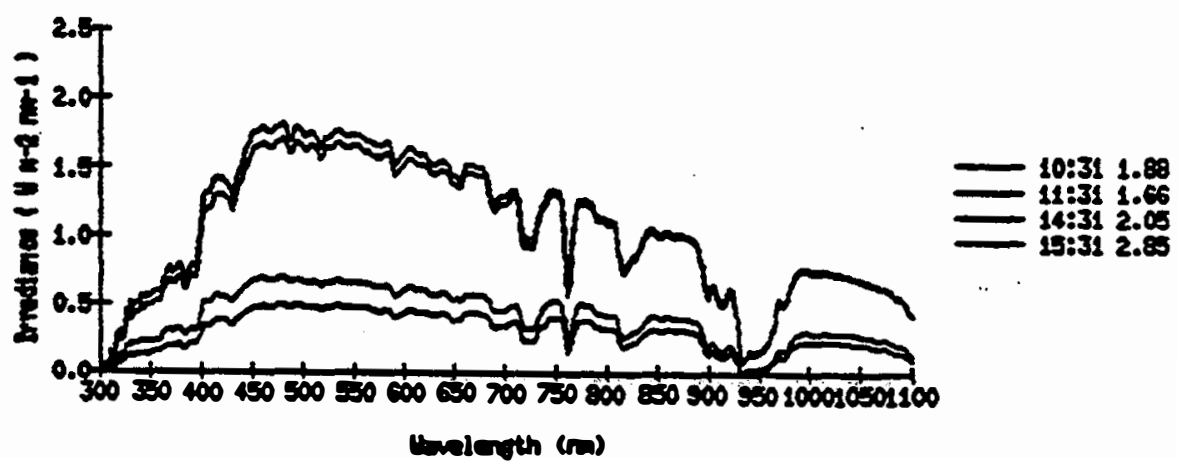




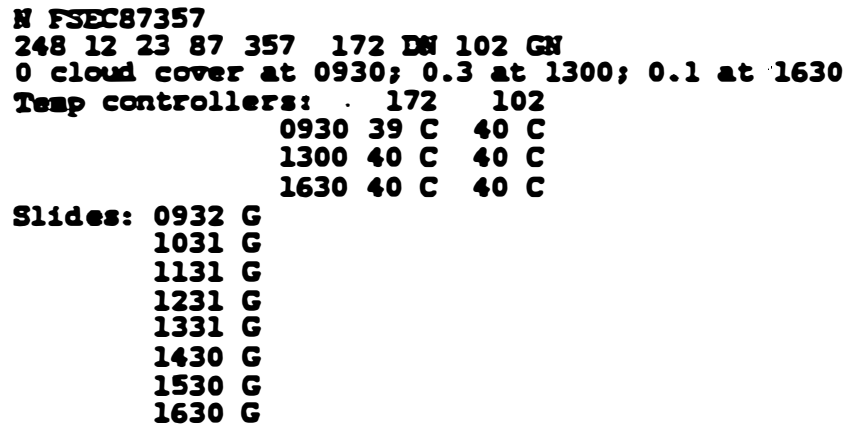

75x6

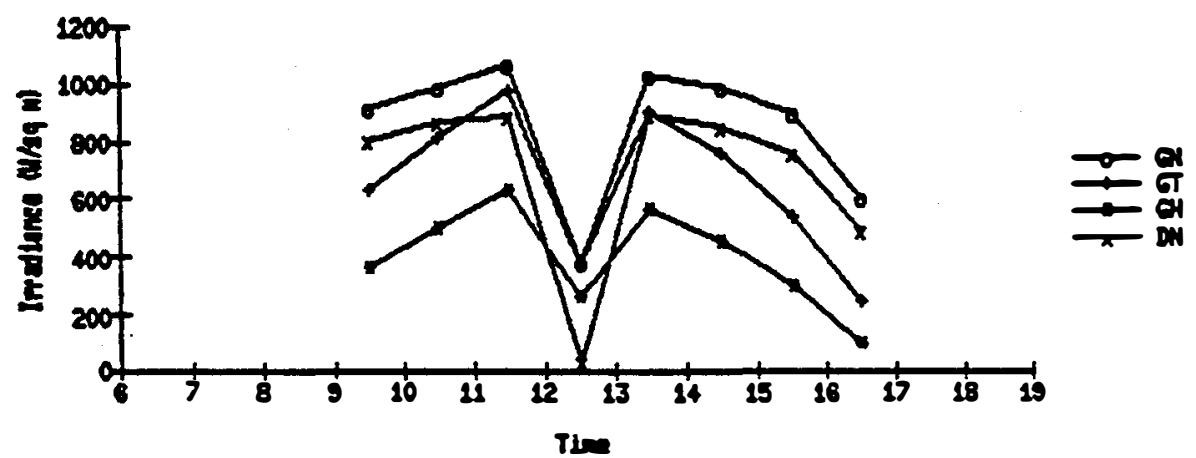

תבים

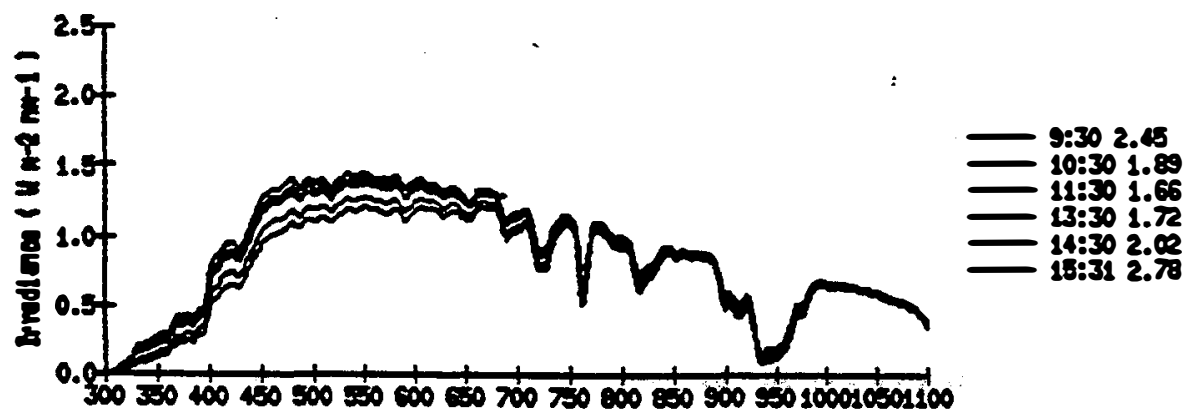

Heviersth (n)

5x

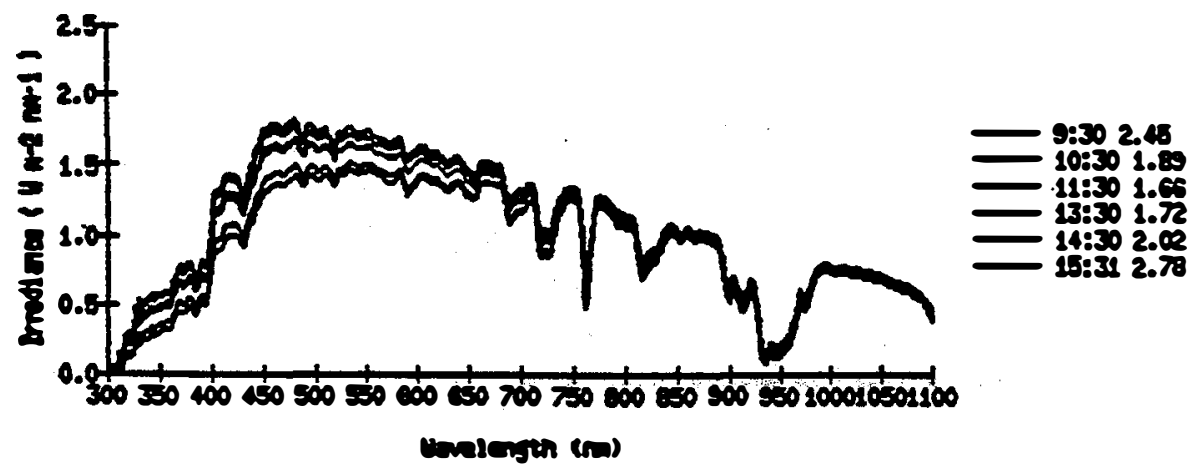


म) गSख्य87358

$\begin{array}{lllllllll}249 & 12 & 24 & 87 & 358 & 172 & \text { Dis } & 102 & \mathrm{GH}\end{array}$

0.3 cloud cover at $0930 ; 1.0$ at 1030

Tenp controllers: 172 and $10240 \mathrm{C}$ at 0930

Slides: $0924 \mathrm{G}$

$1024 \mathrm{G}$

$1224 \mathrm{G}$

$1223 \mathrm{G}$

$1323 \mathrm{G}$

$1423 \mathrm{G}$

$1522 \mathrm{G}$ (enter drops)

$1622 \mathrm{G}$ (veter drops)

$\operatorname{Fac} 3$

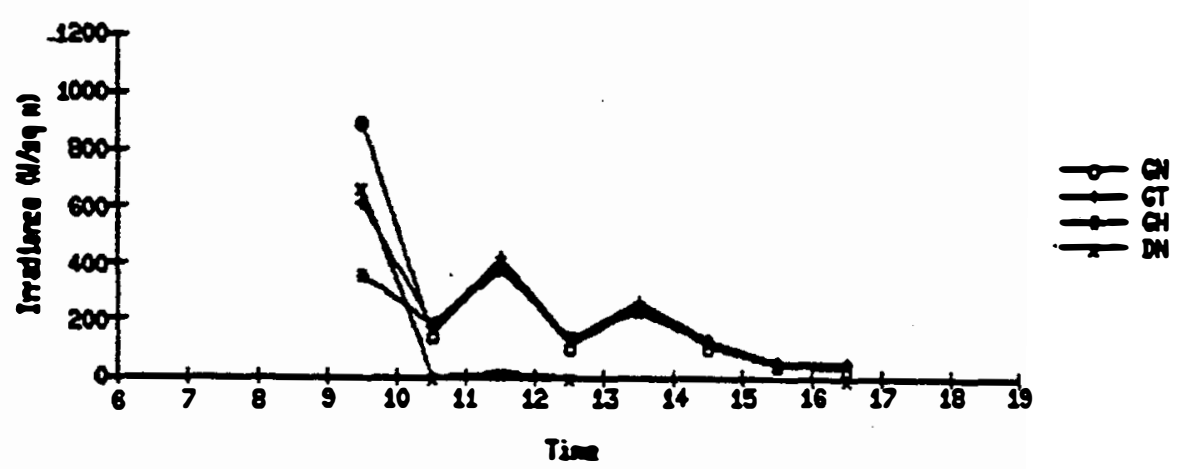

FEC 30000

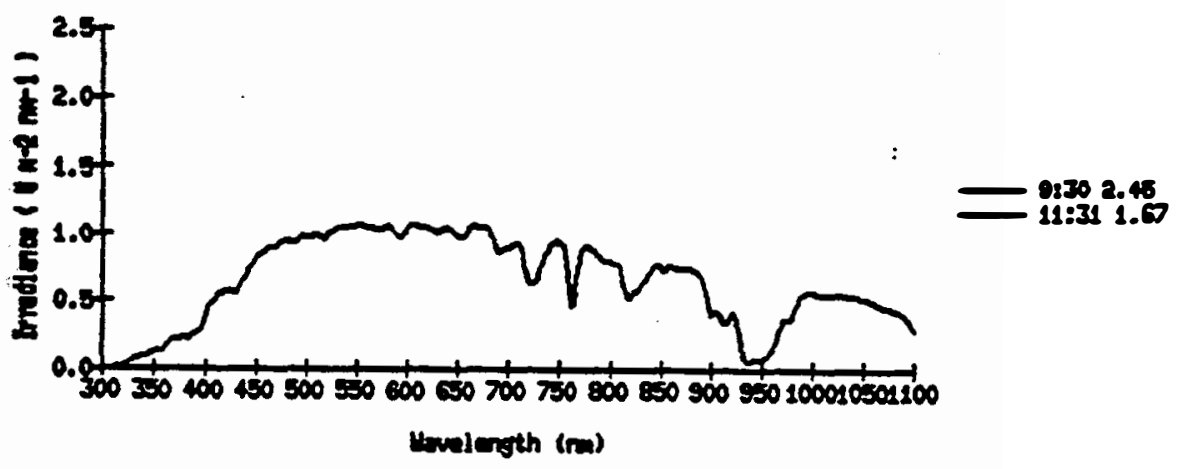

PEAc 300 as

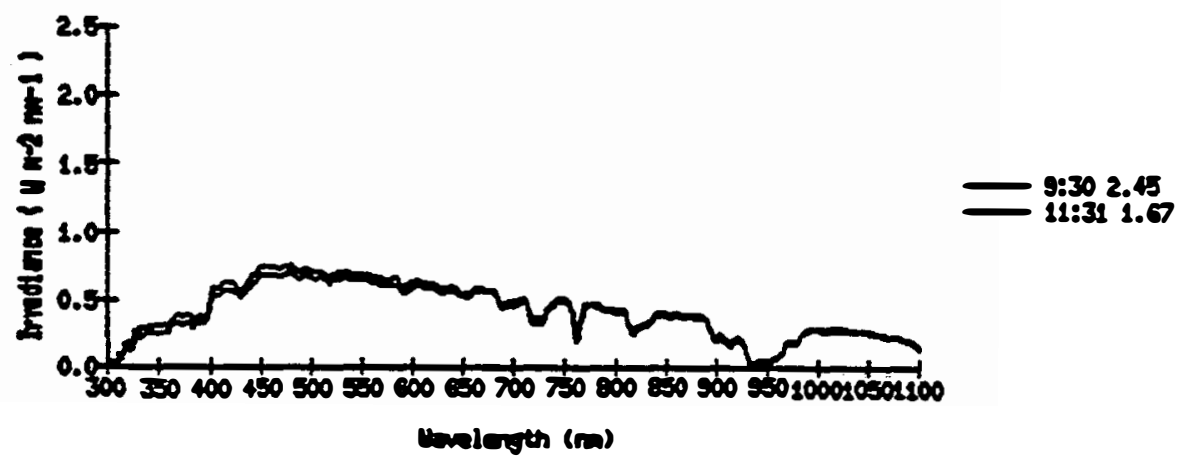




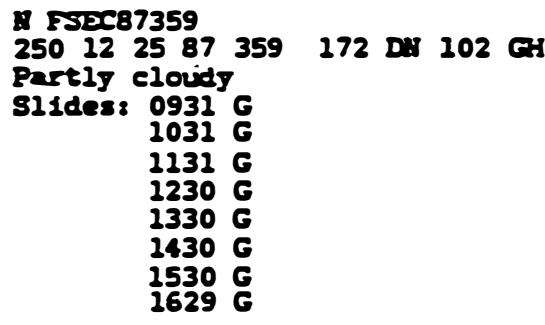

Psese 300

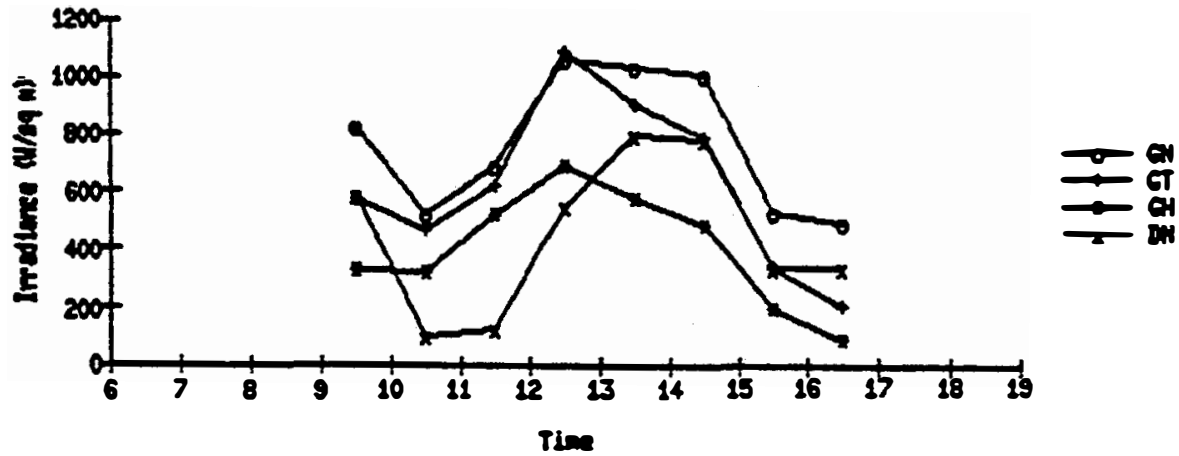

PEבC

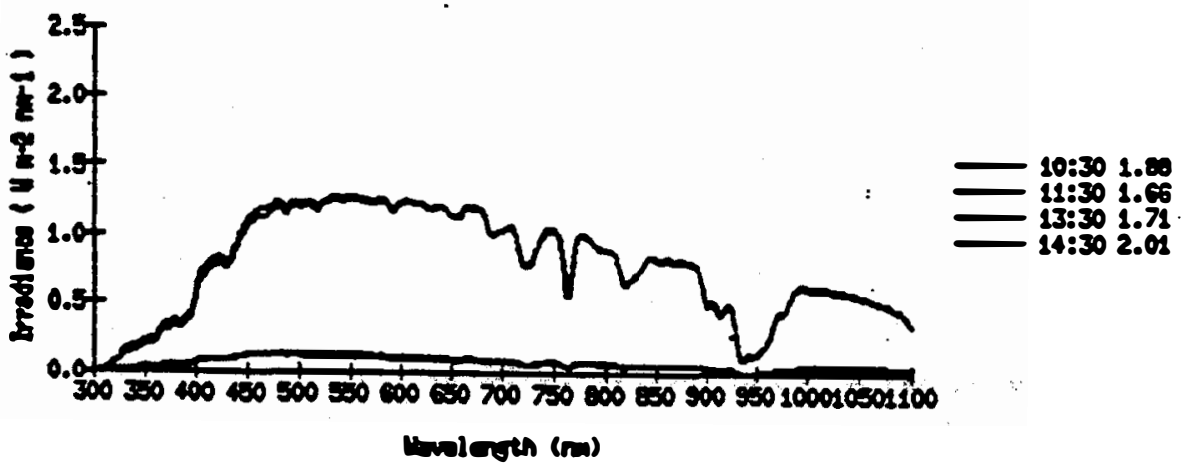

pise 0 Cos

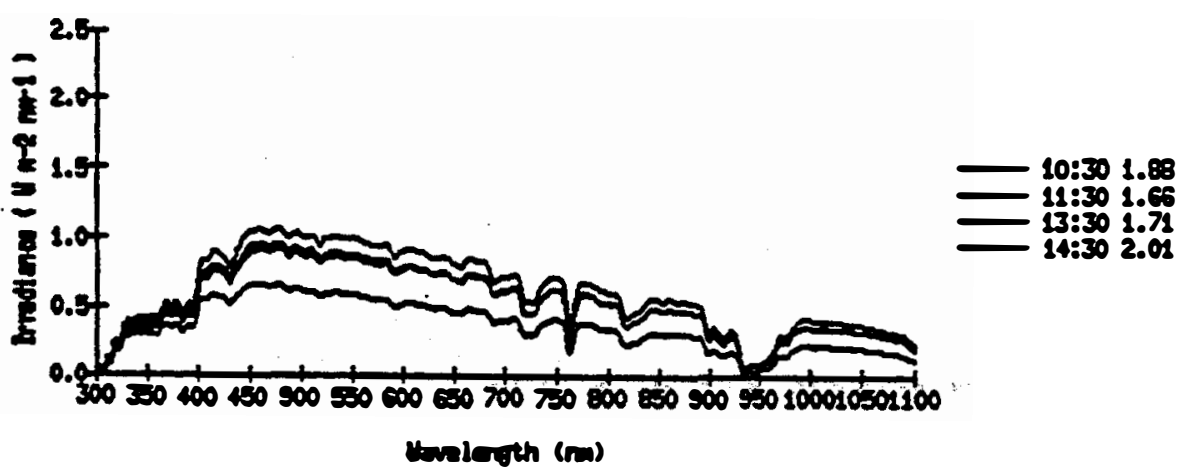




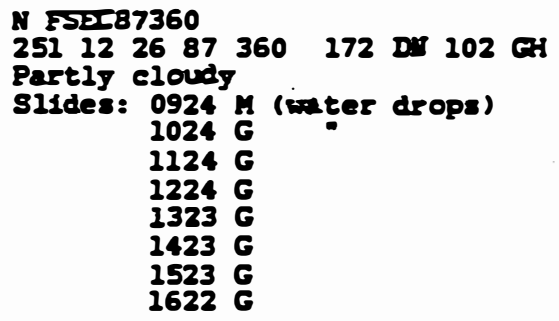

FSEC 300

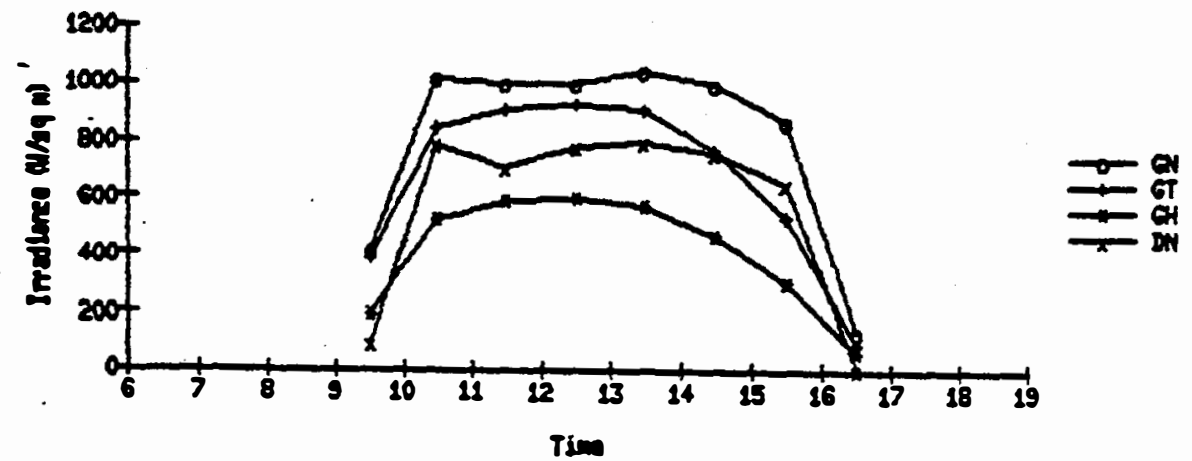

Fsec $300 \mathrm{MT}$

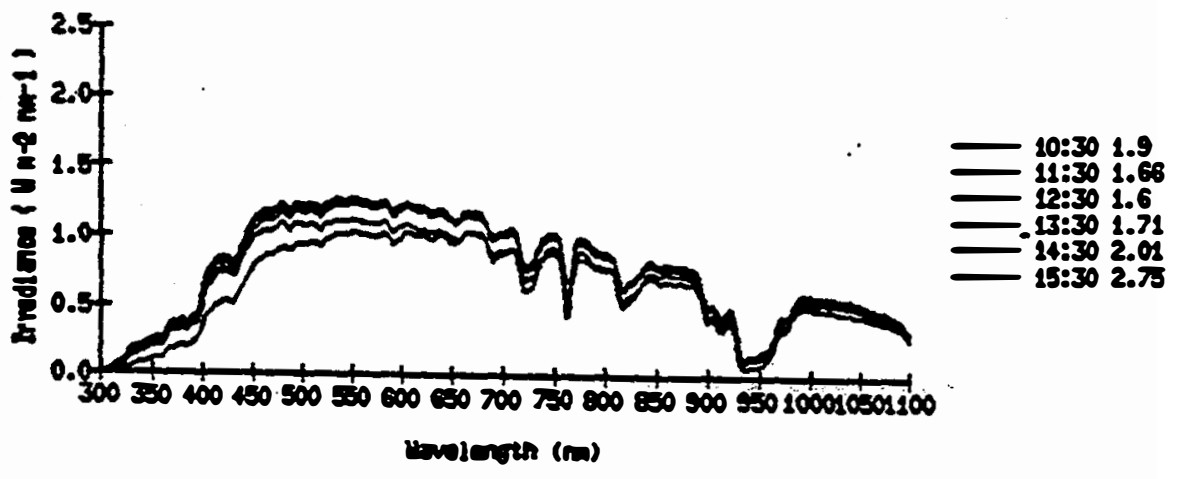

rex $300=18$

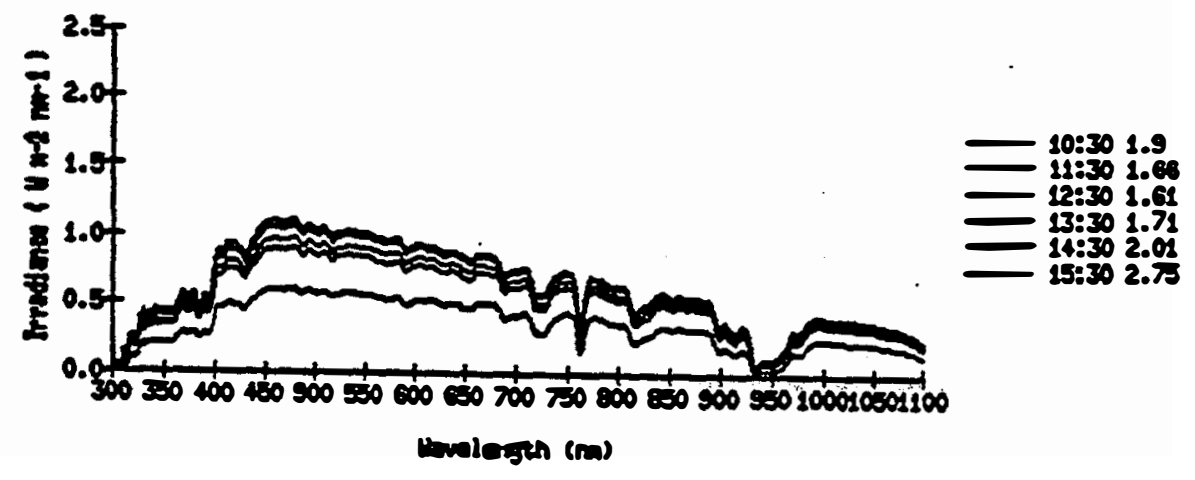




\section{SEPI *}

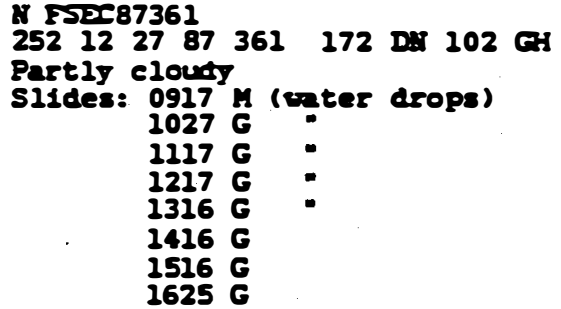

vax ens dow $1228 / 29$

FEבC 361

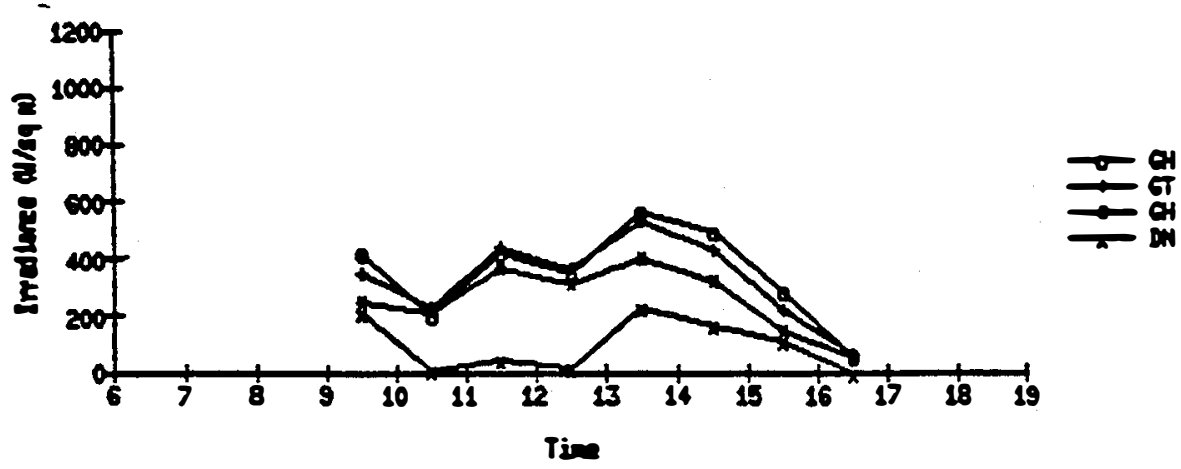

PEבC 361 BNT

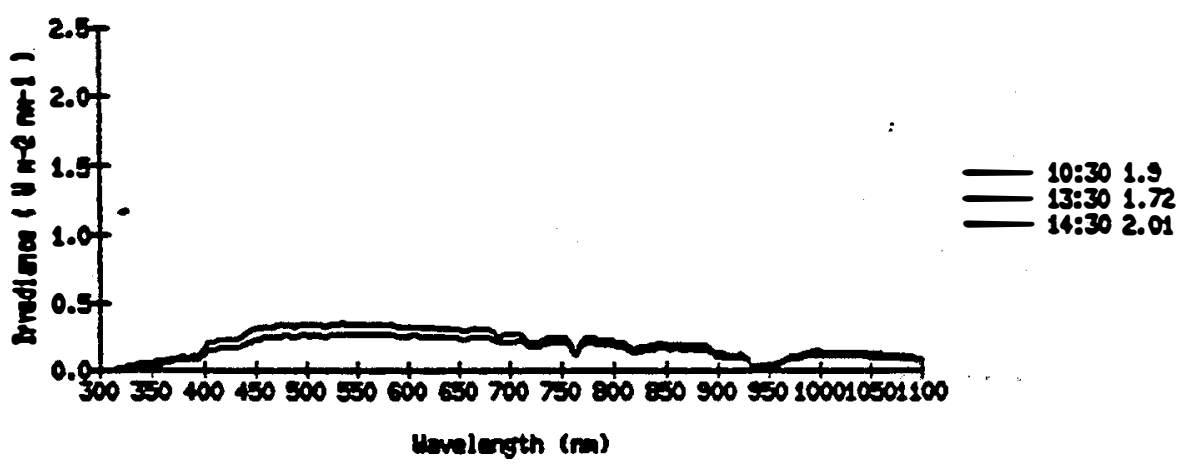

PISC 361 ars

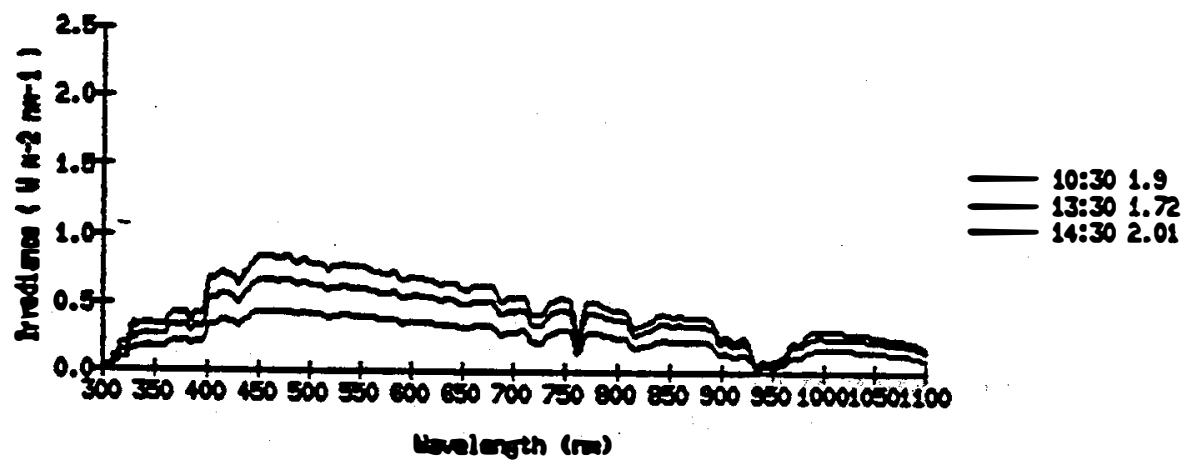


A FSP 87364

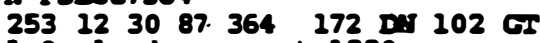

1.0 cloud cover at 1330

Tenp controllers $17238 \mathrm{C}$ and $10239 \mathrm{C}$ at 1330

Ho slides

FSEC 364

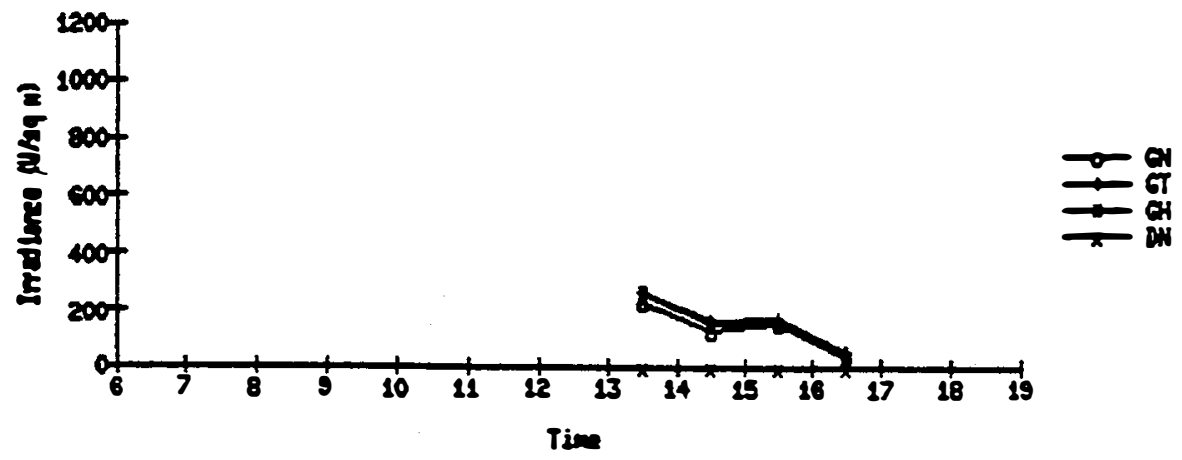

Fsec 354 unt

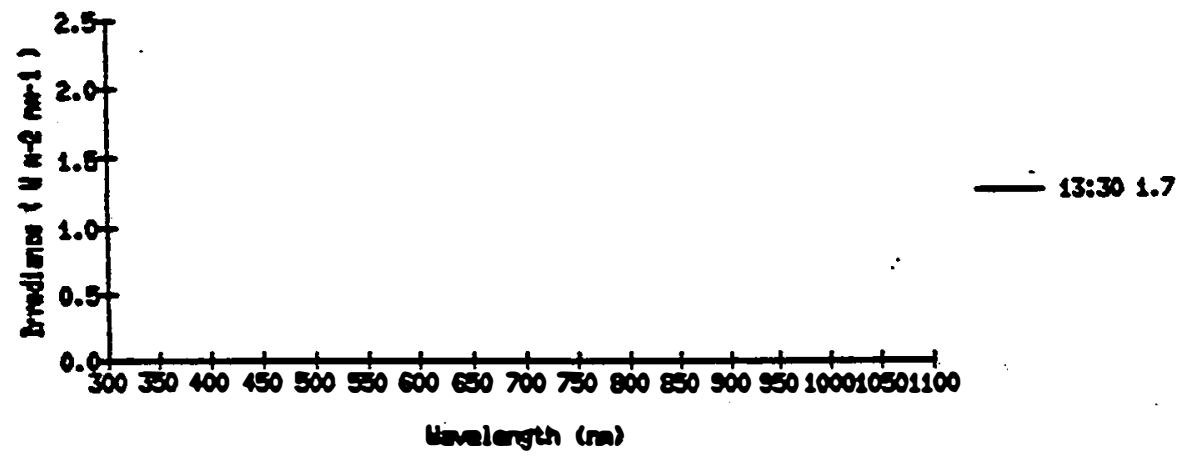

Fise se4 GT

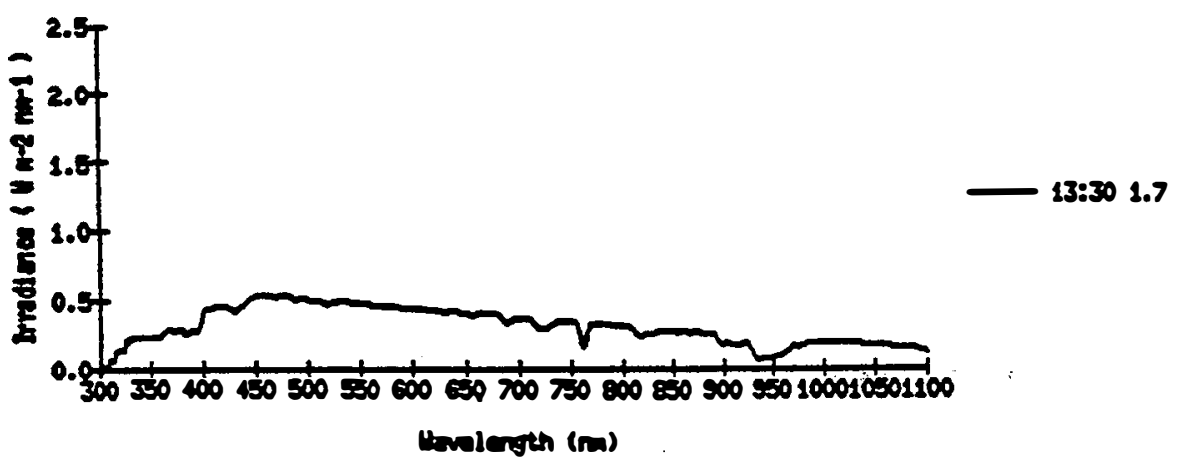


D. FSP87365

$\begin{array}{lllllllll}254 & 12 & 31 & 87 & 365 & 172 & 0.3 & 102 & \text { GI }\end{array}$

Cloudy: overcast

Ho slides

psese 350

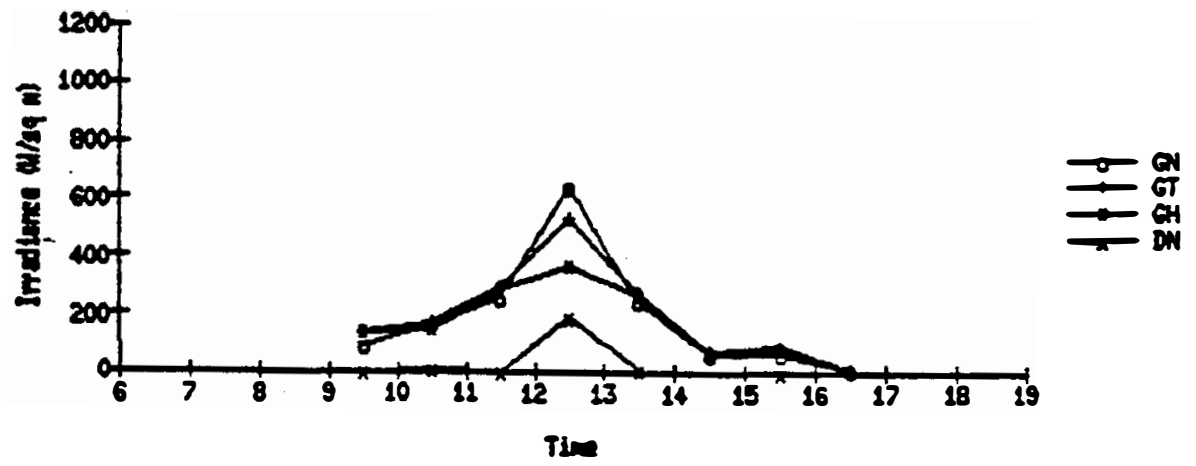

PSEC 3ES BNT

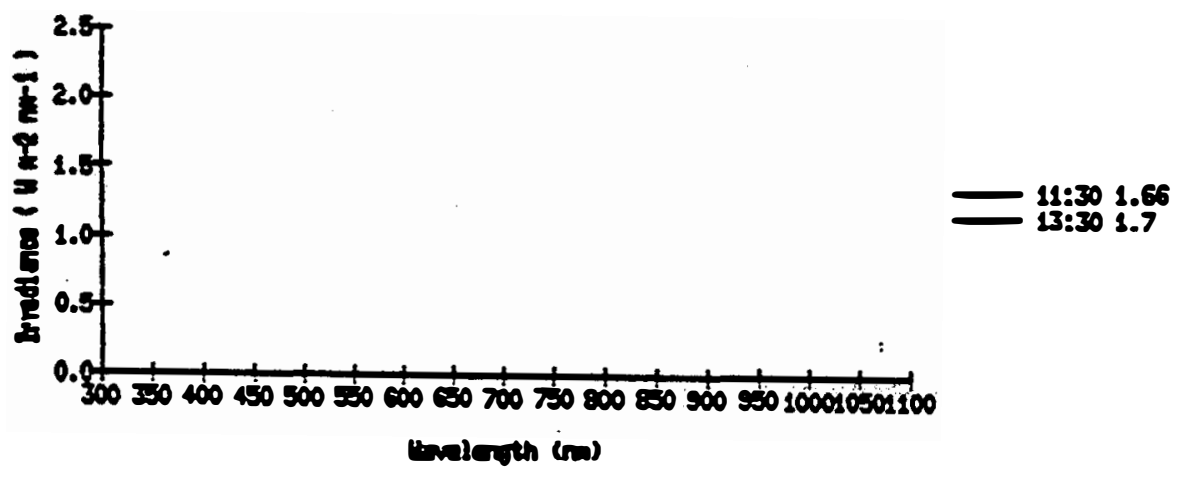

PIS TS GS

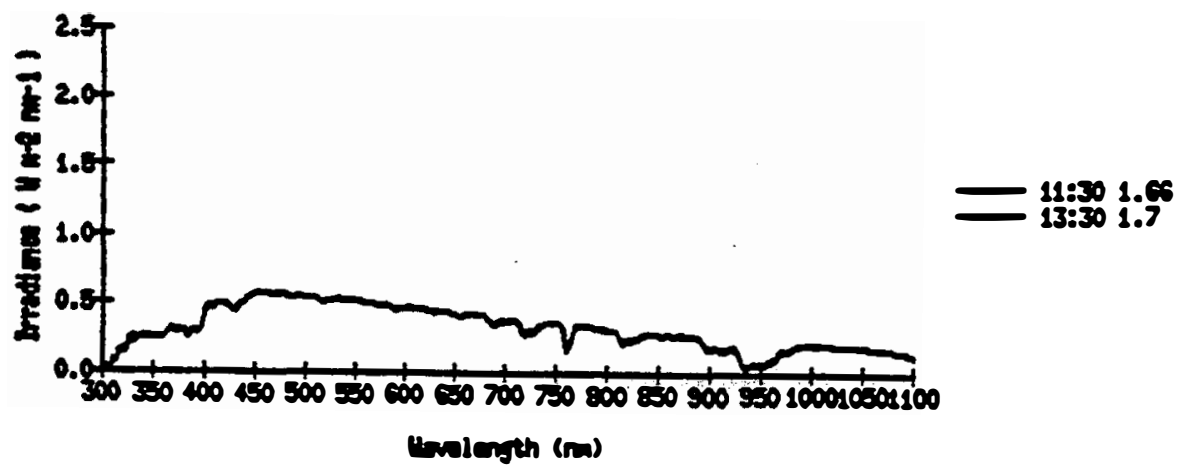




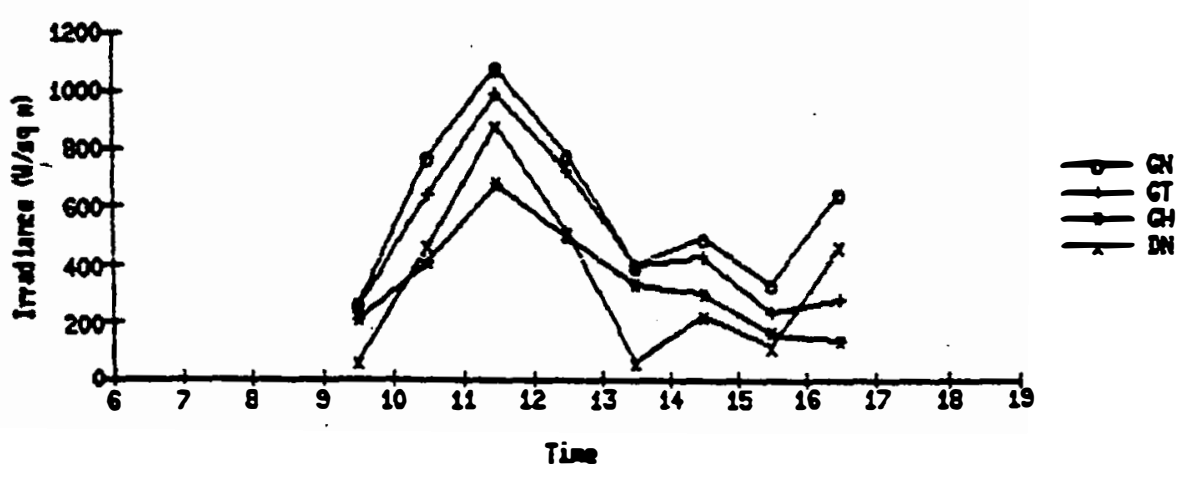

F्ञ 1 ENT

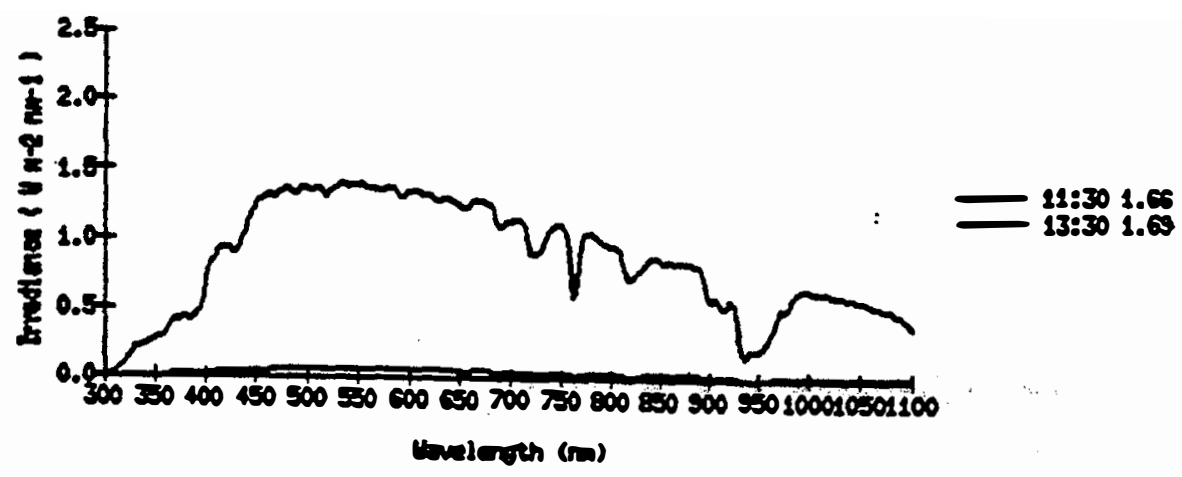

P5:5 1 GS

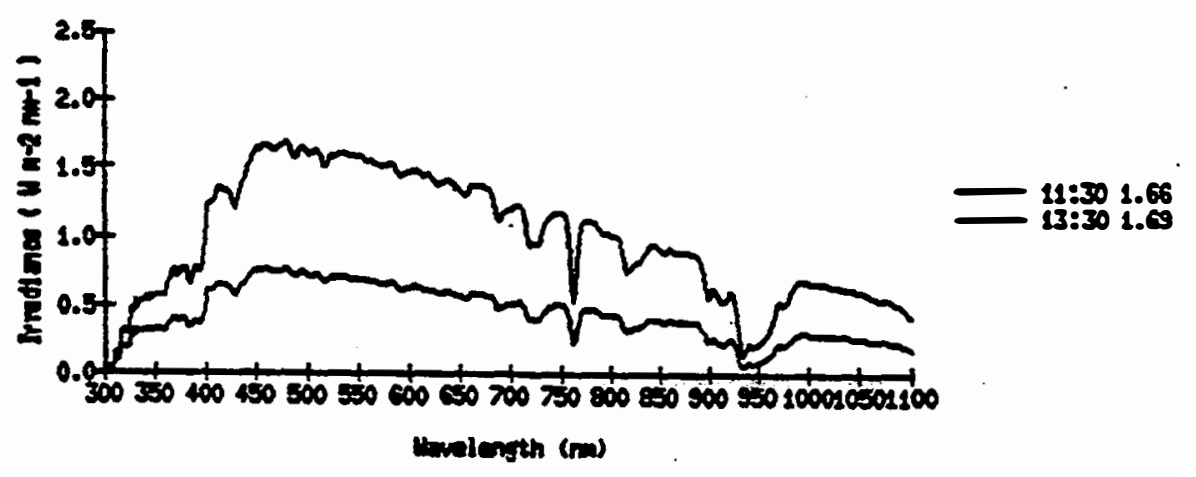


1 FSב88002

$256010288002 \quad 172$ DE 102 GT

Mostly clouds

Ho slides

Fsec 2

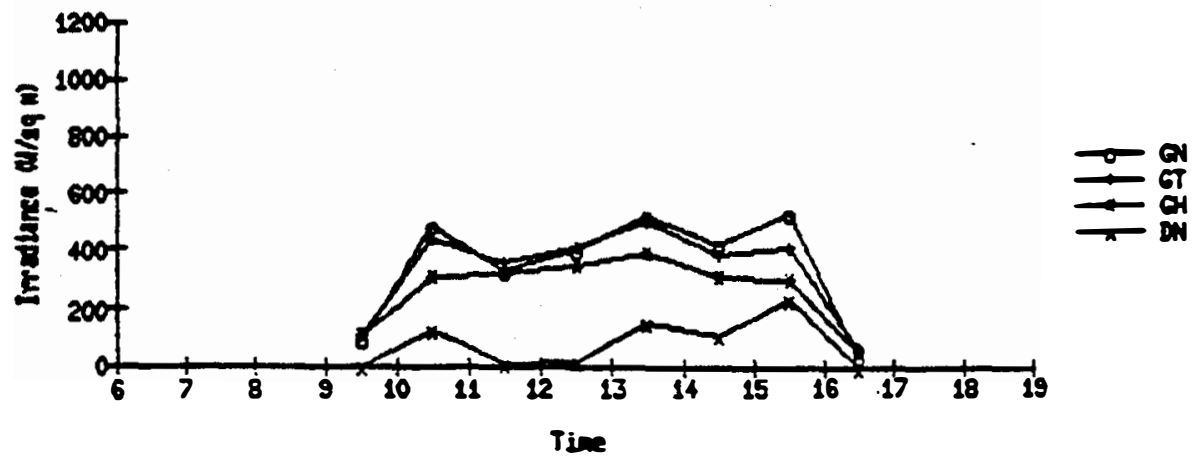

F⿻1 $2 \mathrm{~m}$

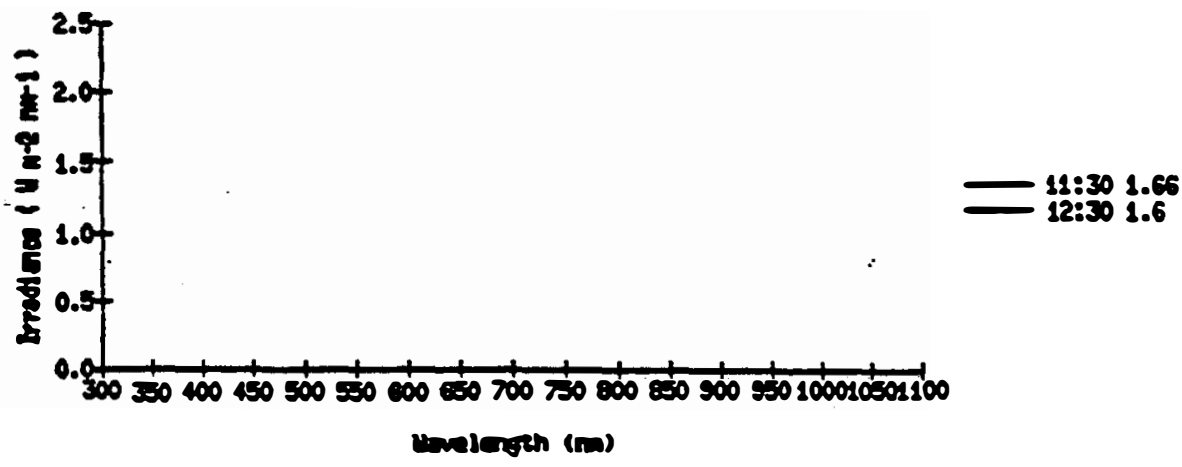

PSEC 2 GTS

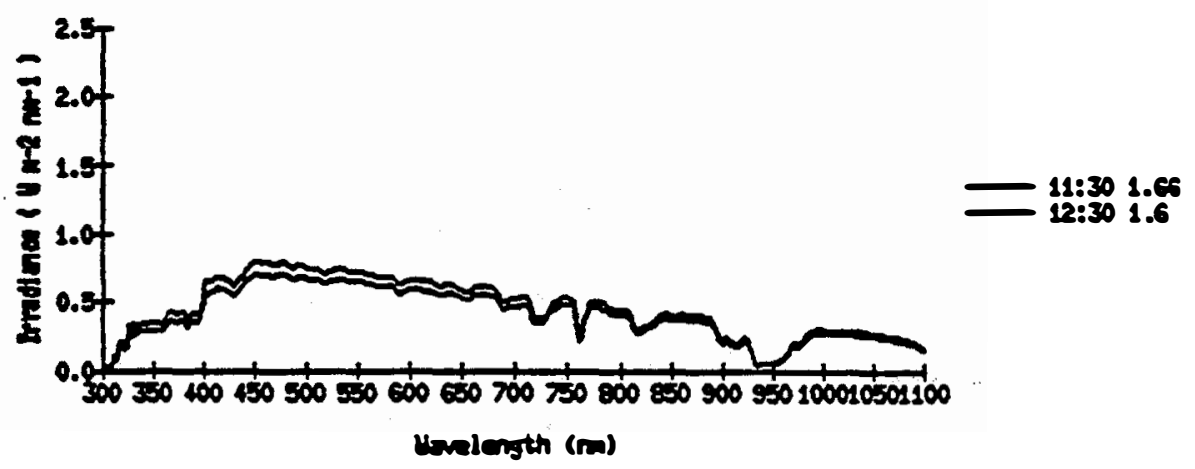


1. P5288003

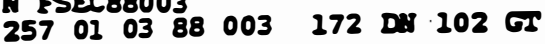

Clord5: óvercast

Ho slides

FEE 3

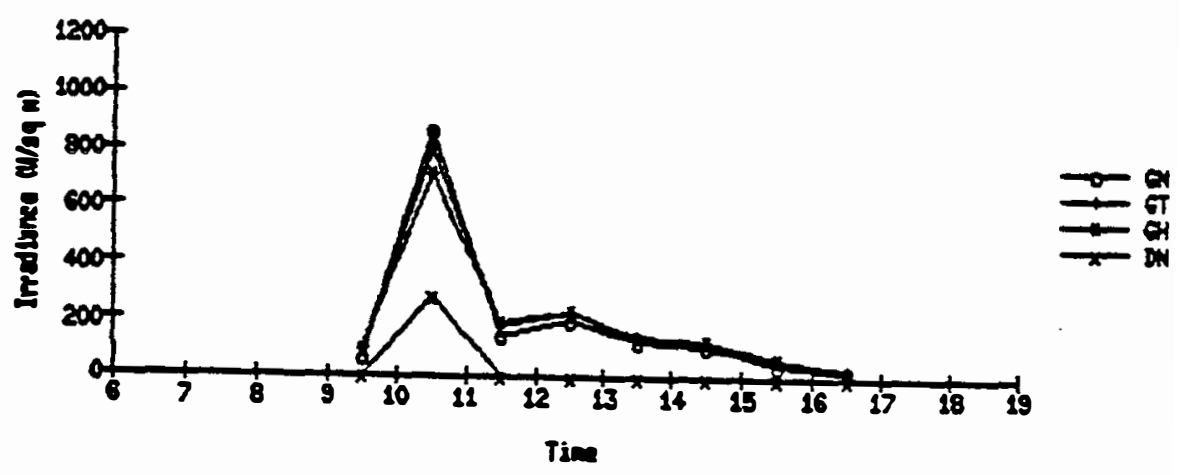

FEבC $3 \mathrm{BNT}$

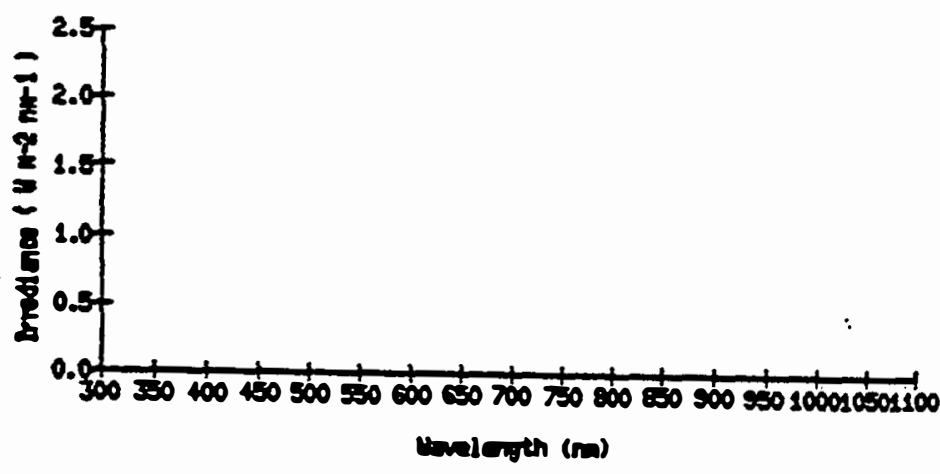

Fase 3 GTS

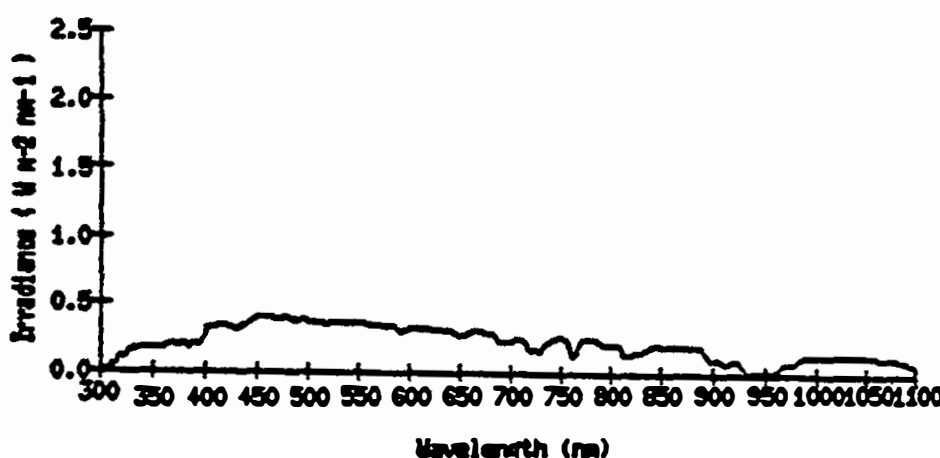

$11: 301.60$ 
SEPI *

4 ए:12088004

258010488.004172 D $102 \sigma$

1.0 cloud cover at 0830,1230 and 1630

Tep controllers: $17238 \mathrm{C}$ and $10239 \mathrm{C}$ at 0830

s11des: $1511 \mathrm{G}$

$1612 \mathrm{G}$

Bo spectra

FIS6 4

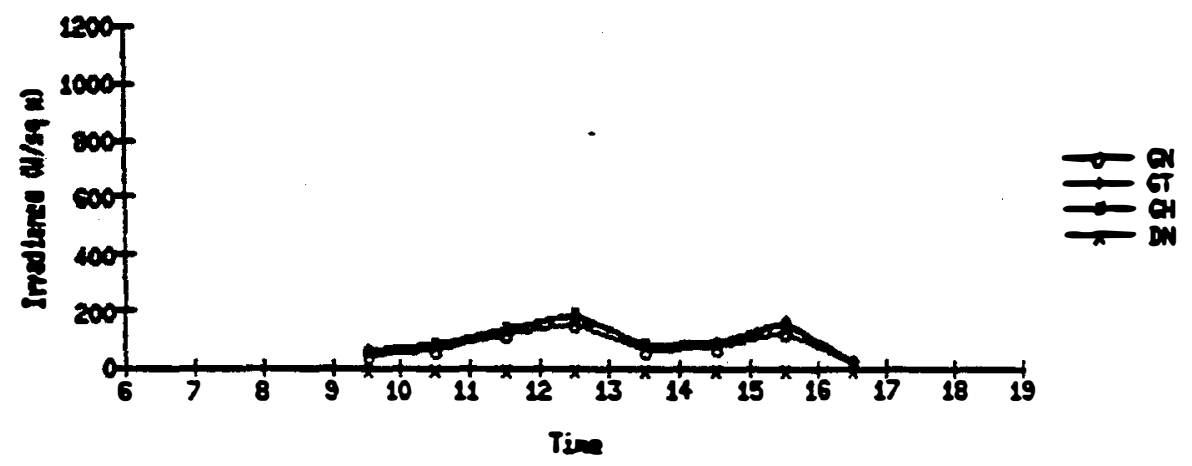




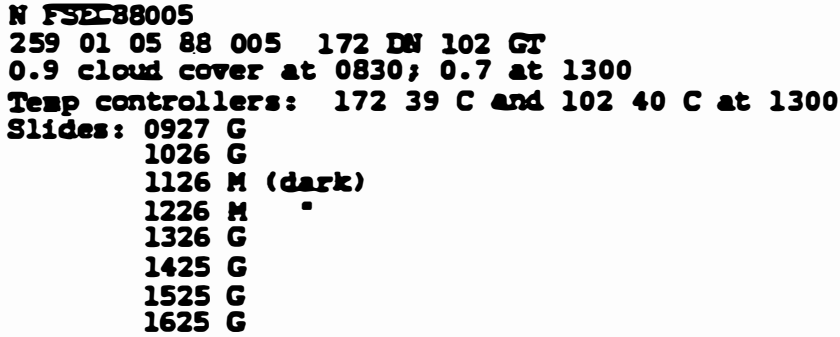

Fas 5

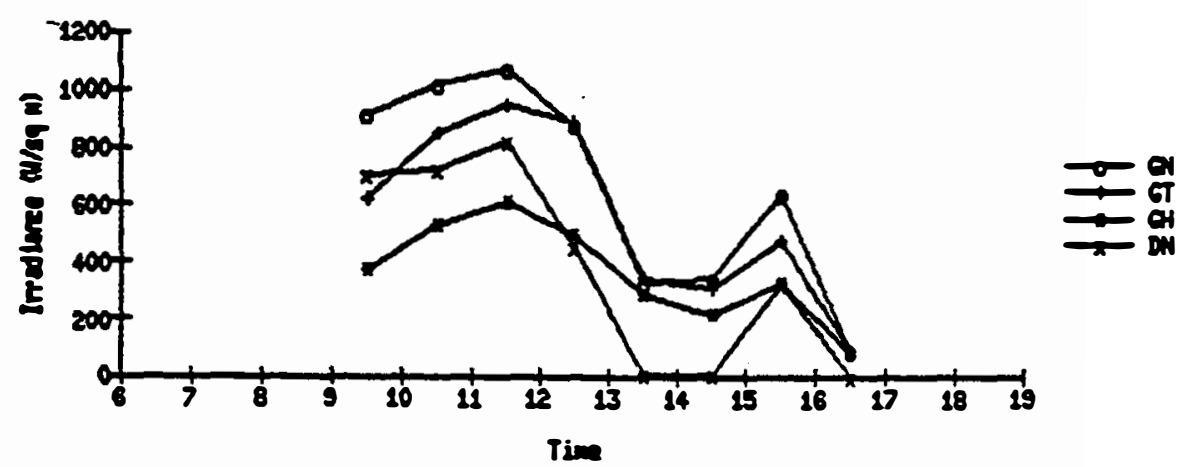

FEבC 5 DN

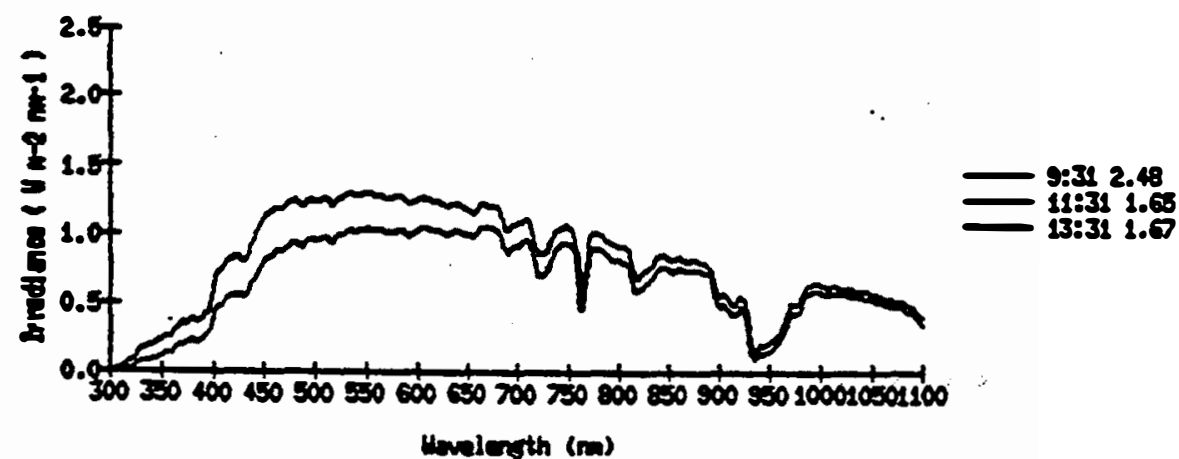

Fast 5 GTS

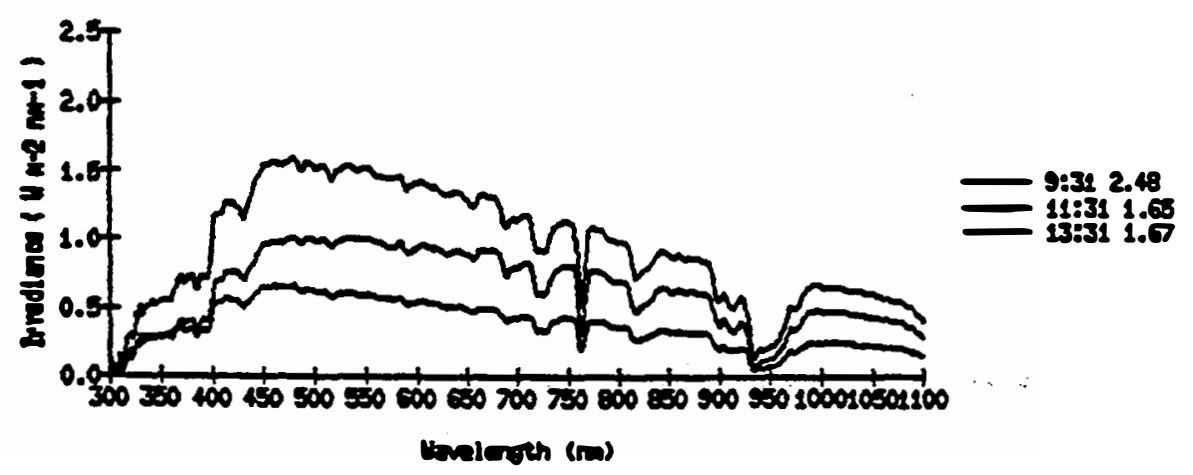




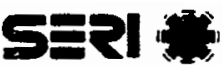

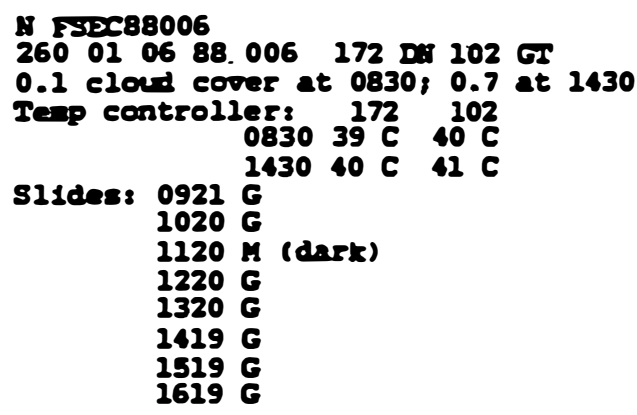

FSEC 6

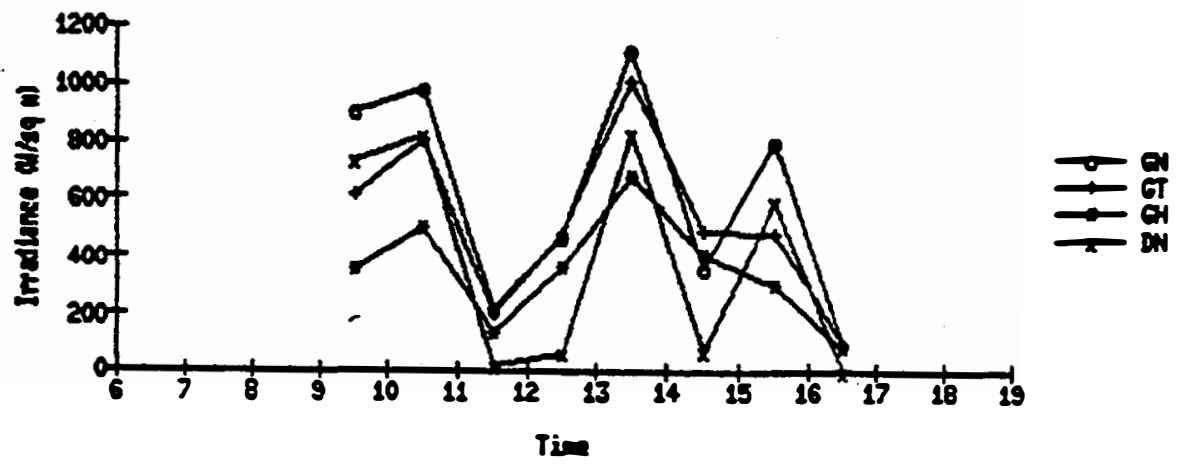

T2T 68

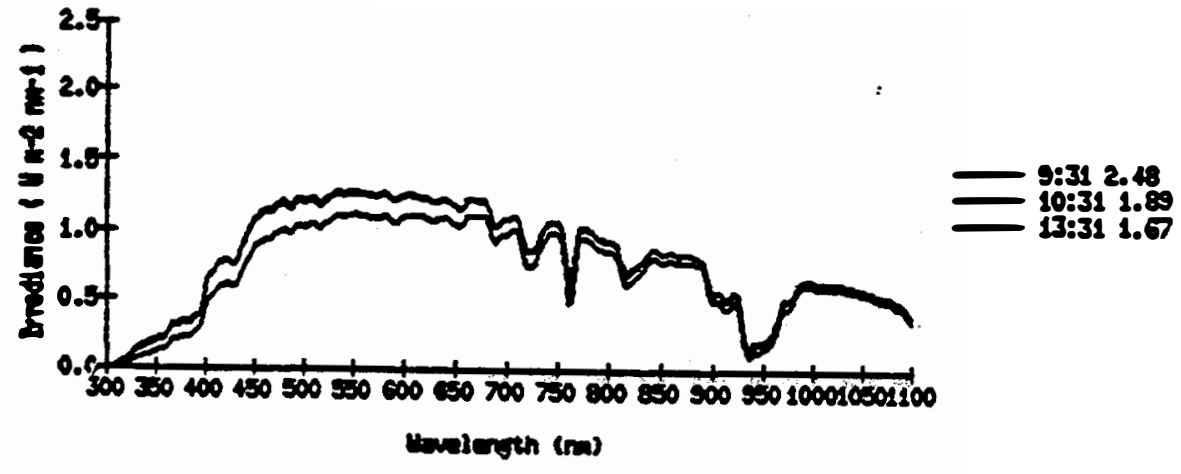

$\operatorname{PrEx} 6$ TTS

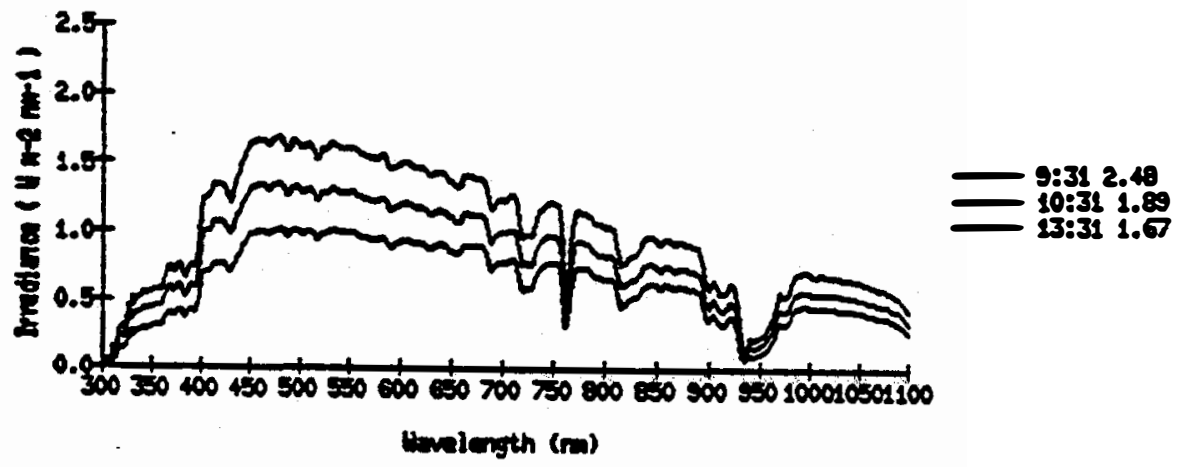


45208012

$26101 \quad 3288 \quad 012 \quad 172 \quad$ Ds $102 \mathrm{~GB}$

Clear untll later afternoon

PSPs changed for channels 2 and 4; new cal for 1 and 5 : Old

2 (GS) 18273539.29 (APF. 87) $24185 F 3$

1 (DIS) 23943566.08 (Dec. 86) sare 6.16 (Dec. 87)

5 (\$1 382631.8 (Sep. 86) sane 31.38 (Dec. 87)

ISR 172 aV drift errors at 0932,1031 , and 1331

Slides: $0927 \mathrm{M}$ (condensation)

$1027 \mathrm{G}$

$1127 \mathrm{G}$

$1227 \mathrm{G}$

$1326 \mathrm{G}$

$1426 \mathrm{G}$

1526 G

$2625 \mathrm{G}$

Brief spikes in OV in Dy spectrun at 1031

FisC 12

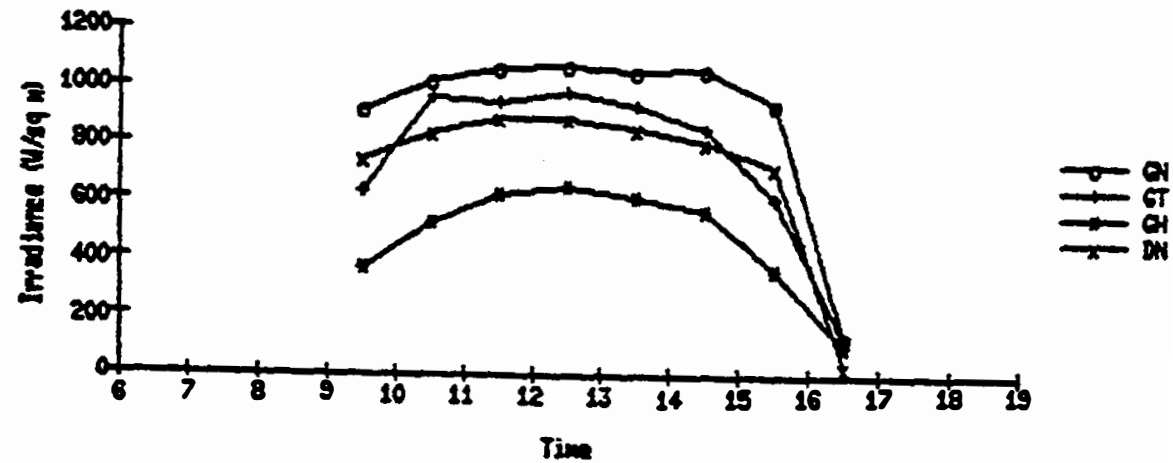

PEEC 12 DT

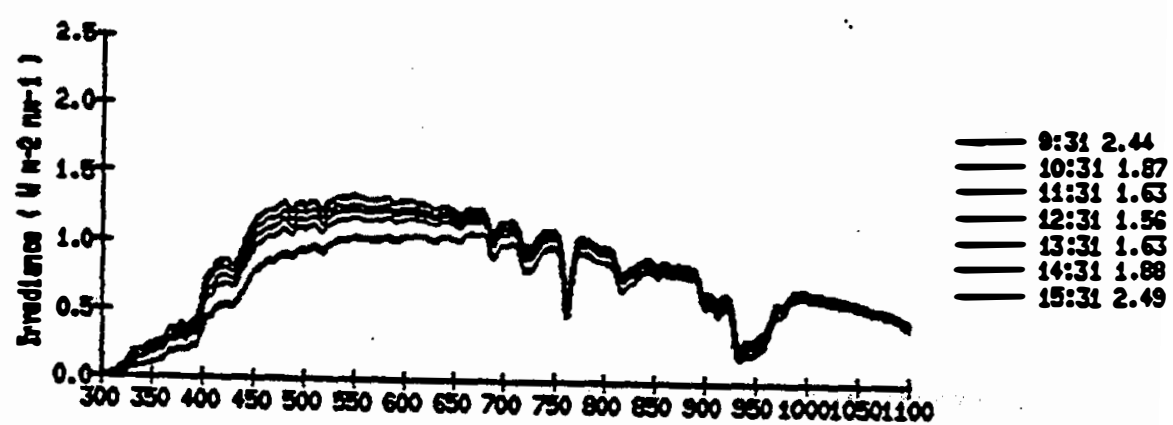

Hevelereth (ma)

$\operatorname{Frc} 12$ as

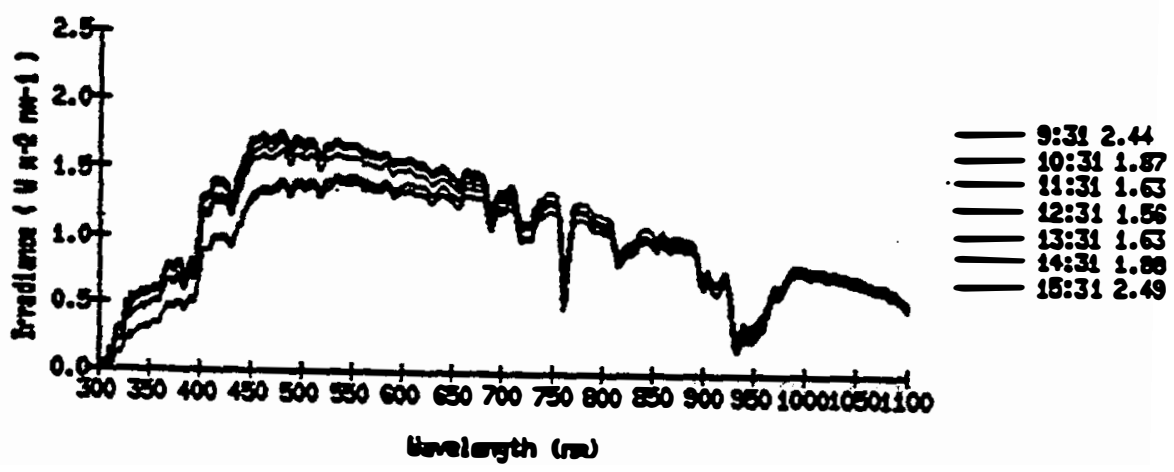




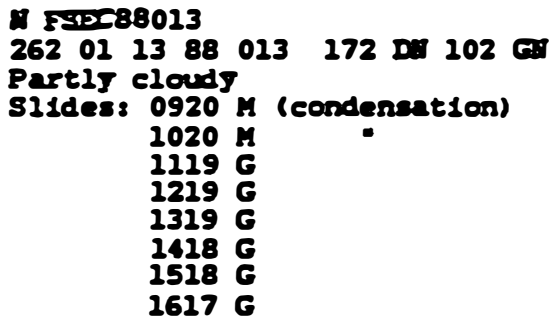

Fise 13

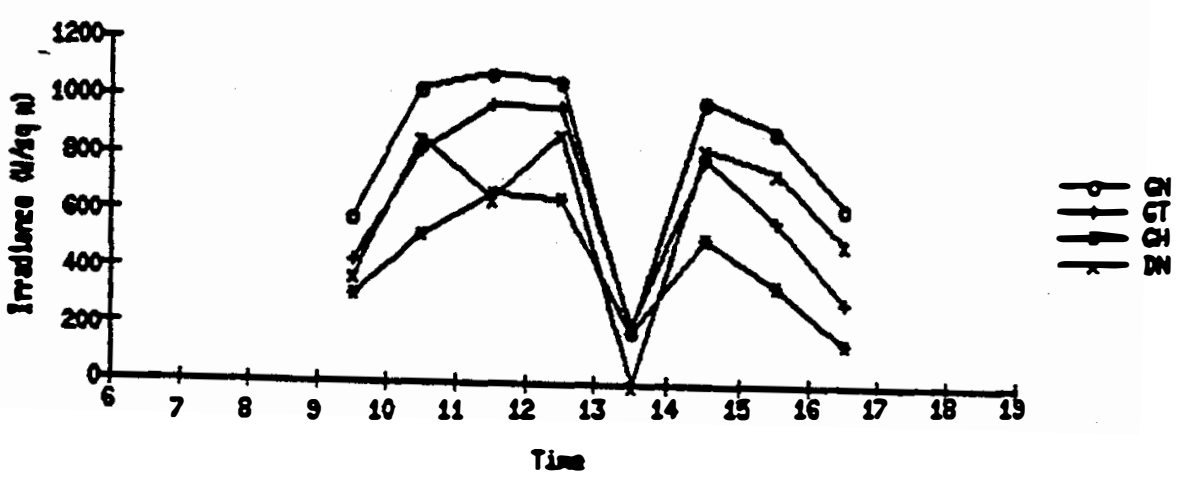

Fise 23 ant

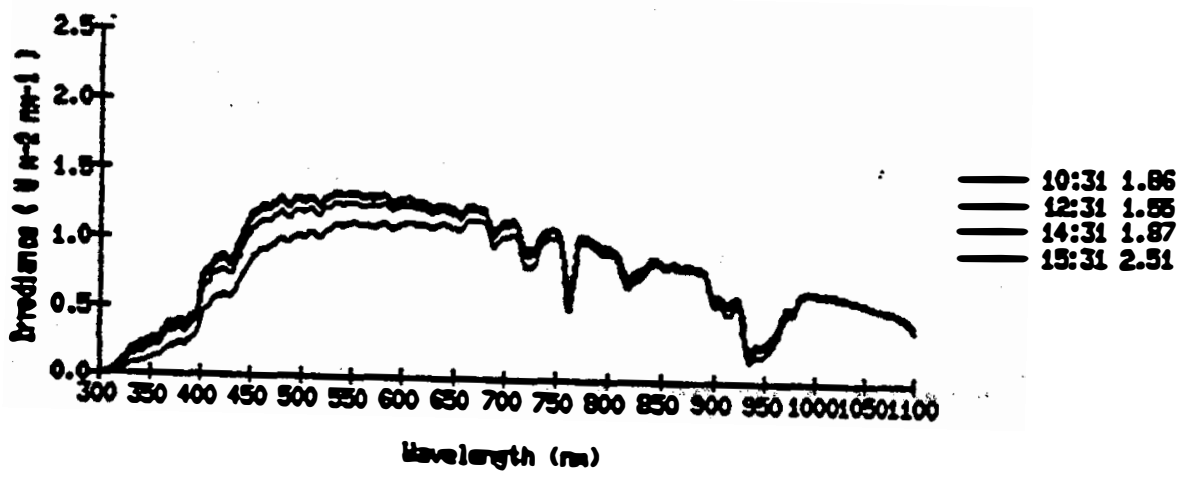

Fise 23 Os

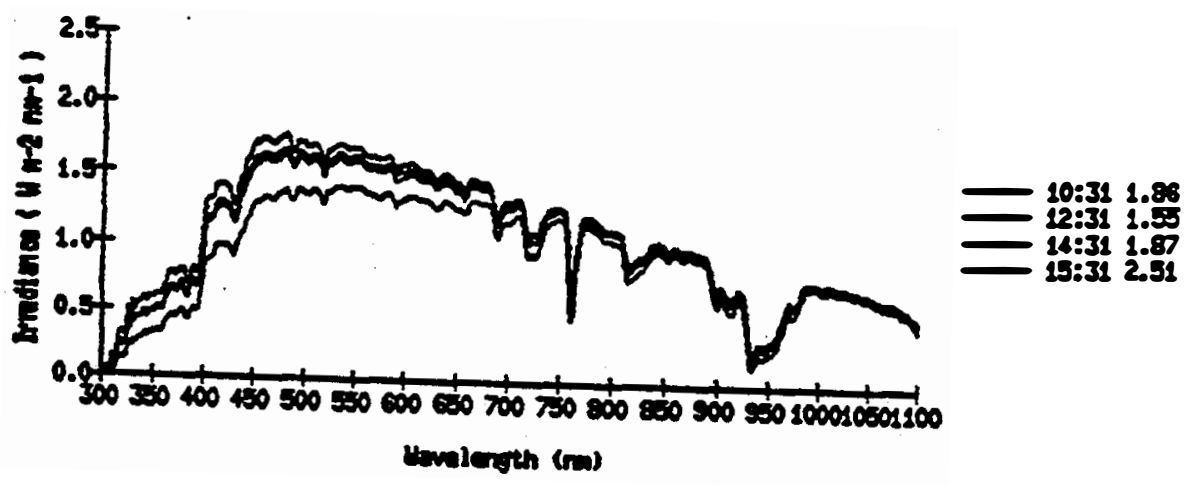


A FSEC88014

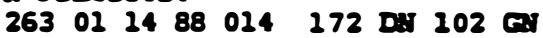

1.0 cloud cover at 0930 and 1230

Tenp controllers: $17238 \mathrm{C}$ and $10240 \mathrm{C}$ at 1230

Slides: $0913 \mathrm{G}$

$1013 \mathrm{G}$

$1233 \mathrm{G}$

$1233 \mathrm{G}$

$1333 \mathrm{G}$

$1432 \mathrm{G}$

$1532 \mathrm{G}$

$1632 \mathrm{G}$

Ho spectra

Fsec 14

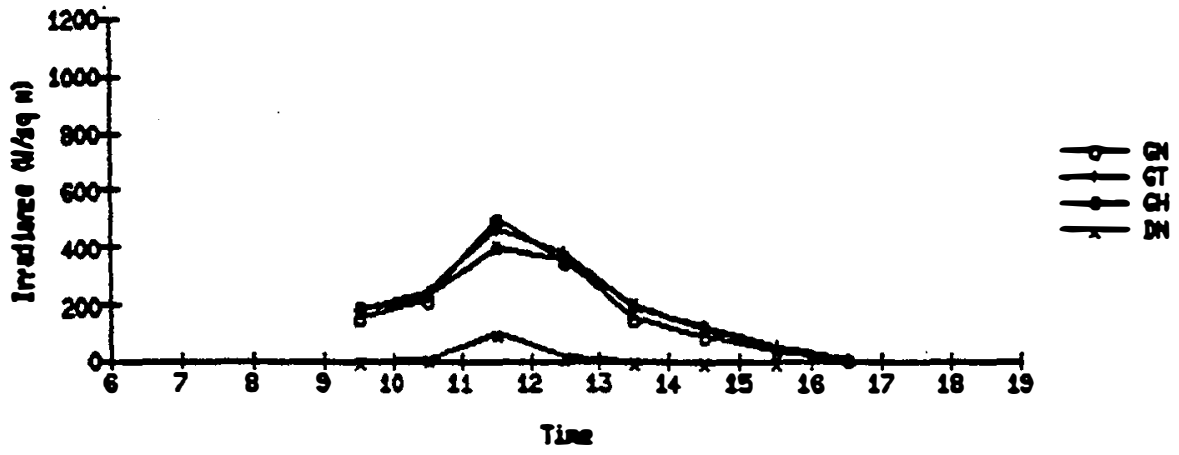


म FSPC88015

$\begin{array}{llllllll}264 & 01 & 15 & 88 & 015 & 172 & \mathrm{DW} & 102 \mathrm{CH}\end{array}$

0.9 cloud cover at 0930; 1.0 at 1530

Ieap controllers: $17238 \mathrm{C}$ and $10239 \mathrm{C}$ at 0930

Slides: $0929 \mathrm{G}$ (weter drops)

$1029 \mathrm{G}$

1128 A (dark)

$1228 \mathrm{G}$

$1328 \mathrm{G}$

$1428 \mathrm{G}$

$1528 \mathrm{G}$

$1627 \mathrm{G}$

rise 25

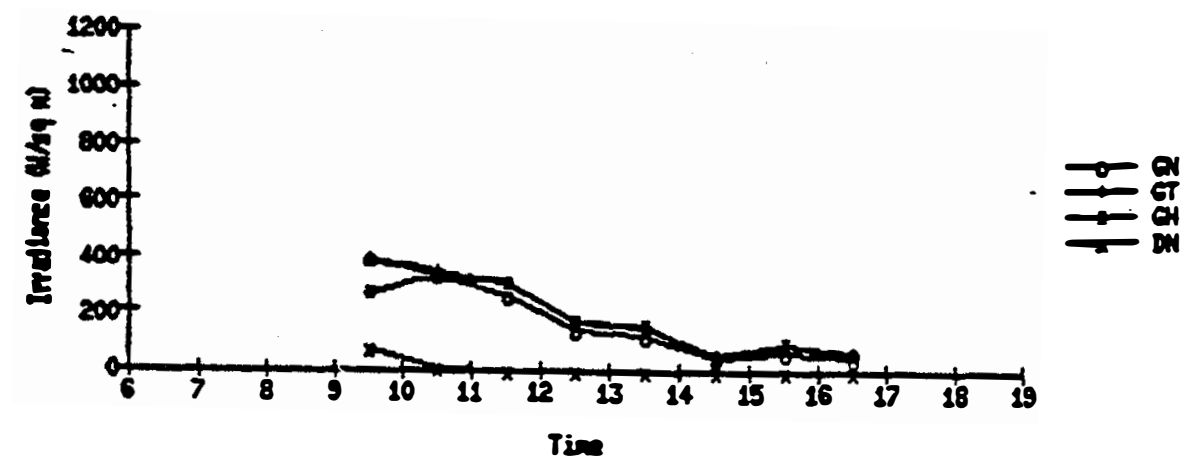

$\operatorname{rsec} 15 \mathrm{art}$

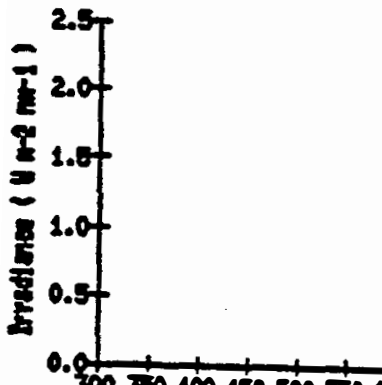

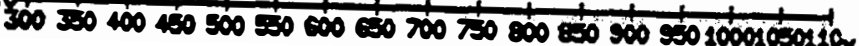

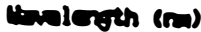

Fise 15 as

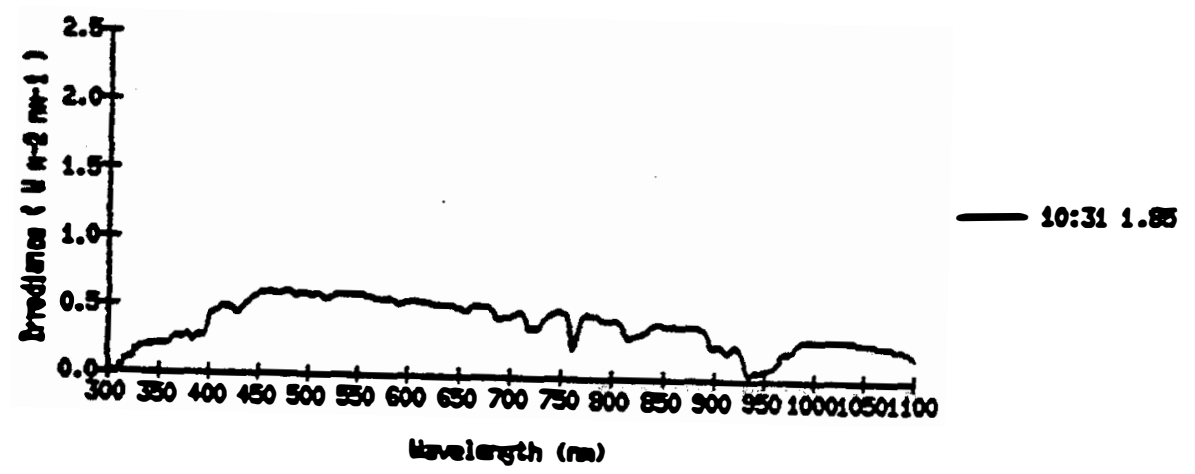


月 FSP88016

$\begin{array}{lllllllll}265 & 01 & 16 & 88 & 016 & 172 & \text { DW } & 102 & \text { GN }\end{array}$

Partly cloudy

ISR 172 nV drift error at 1132

Slides: $0925 \mathrm{G}$

$1025 \mathrm{G}$

1125 H (dark)

$1225 \mathrm{M}$ :

$1325 \mathrm{G}$

$1424 \mathrm{G}$

$1524 G$

$1624 \mathrm{G}$

FSEC 16

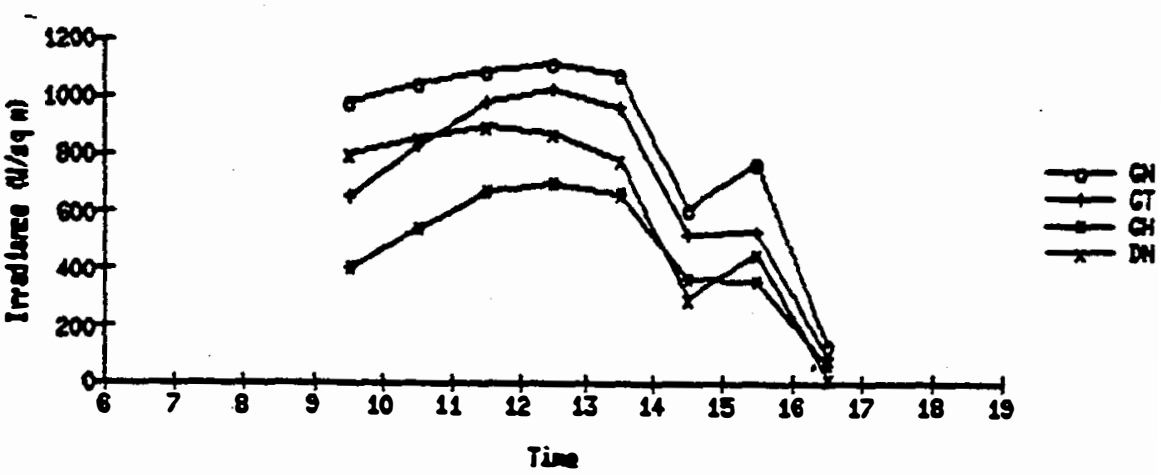

FIC 16 DNT

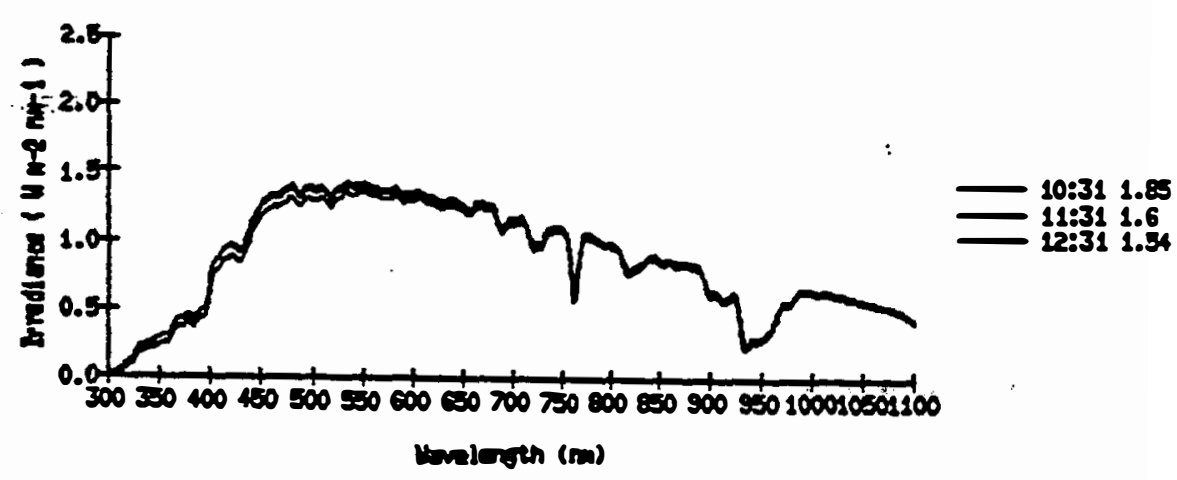

Fare 26 as

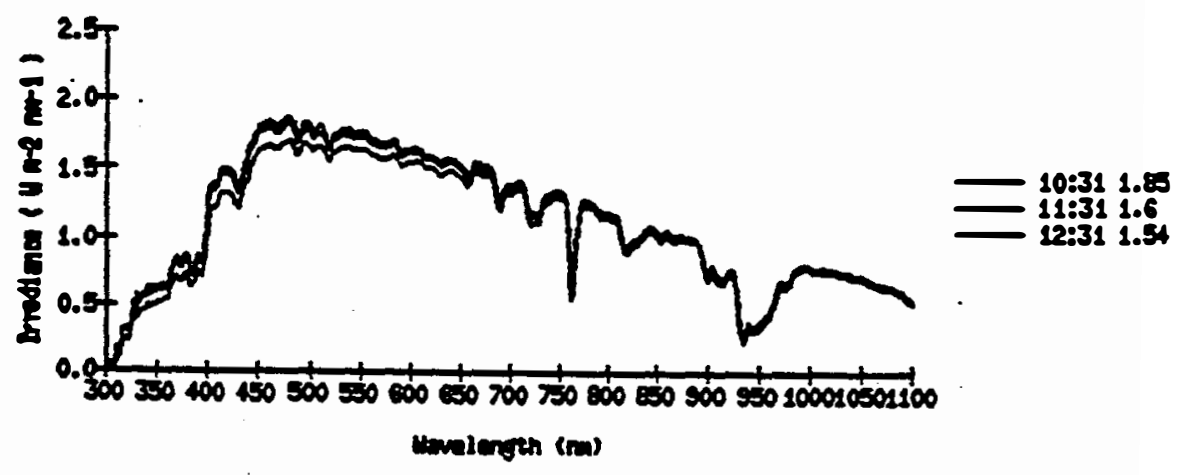




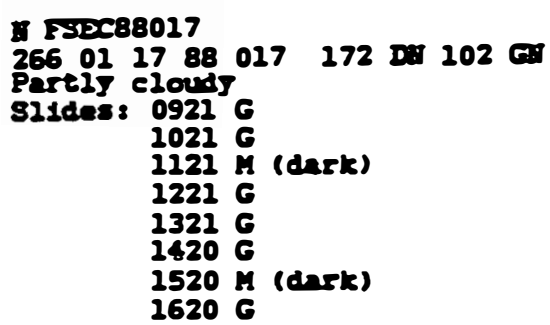

PSEC 17

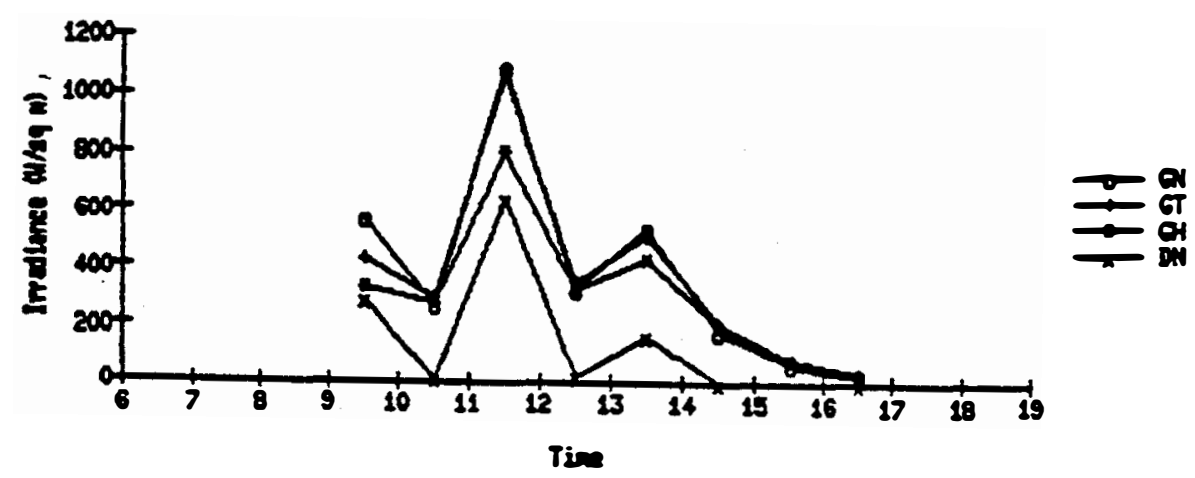

FEC 17 काT

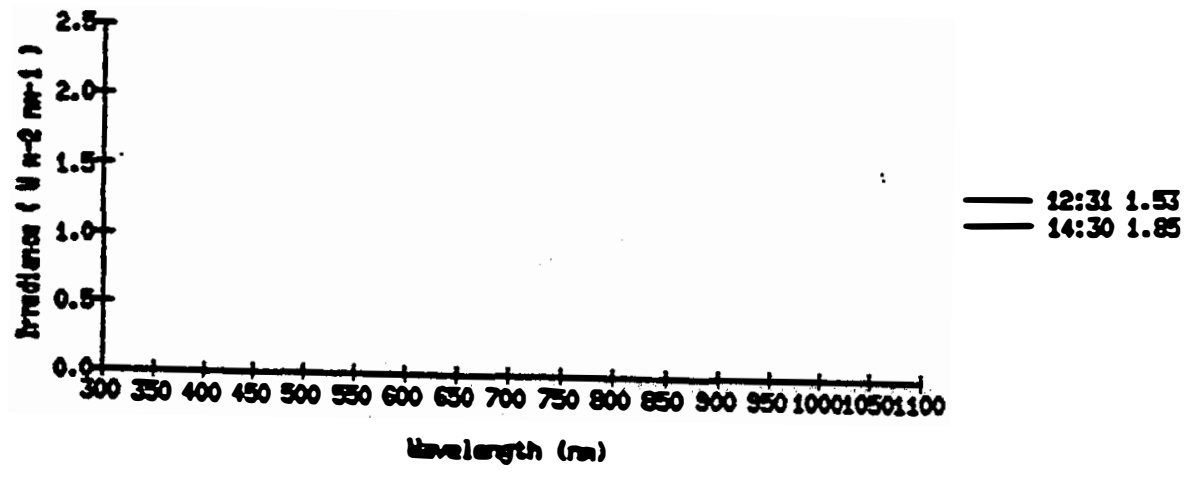

$\operatorname{PSEC} 1700$

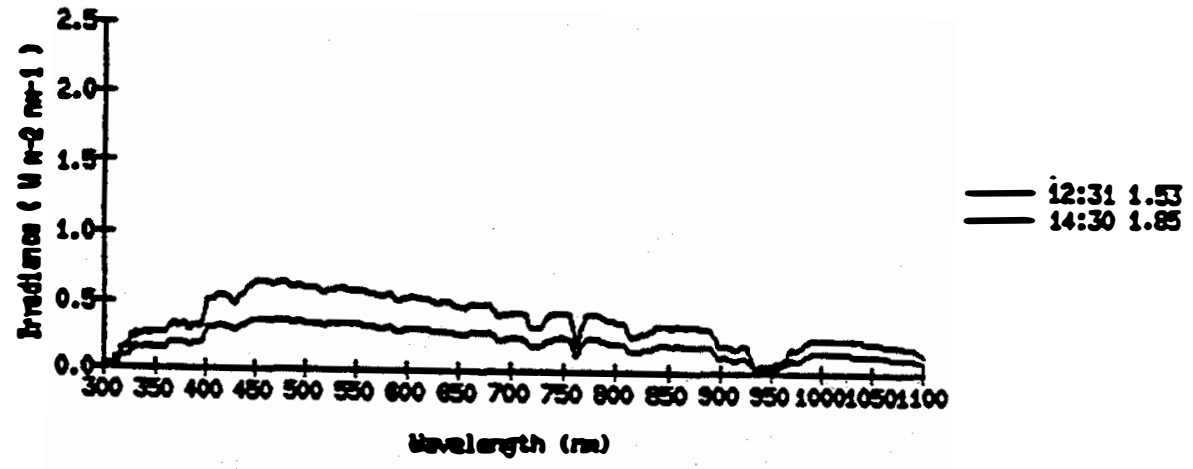




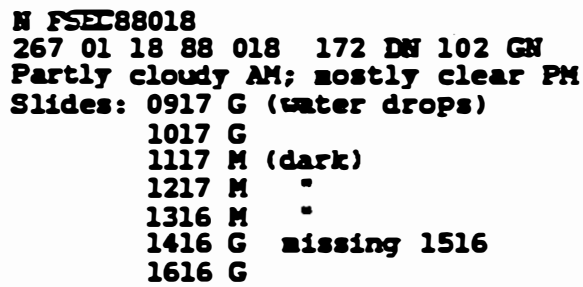

T5ES 28

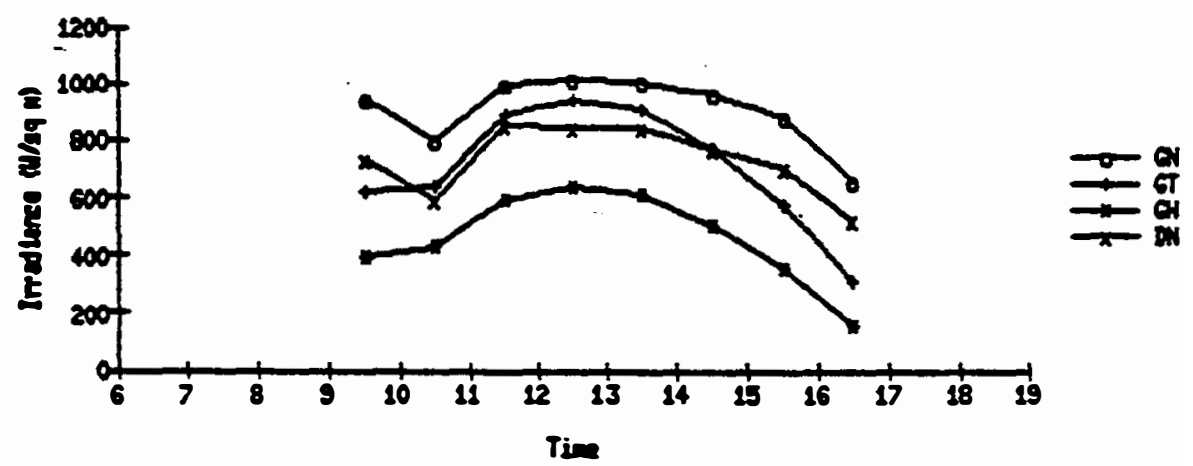

Fsec $19 \mathrm{BT}$

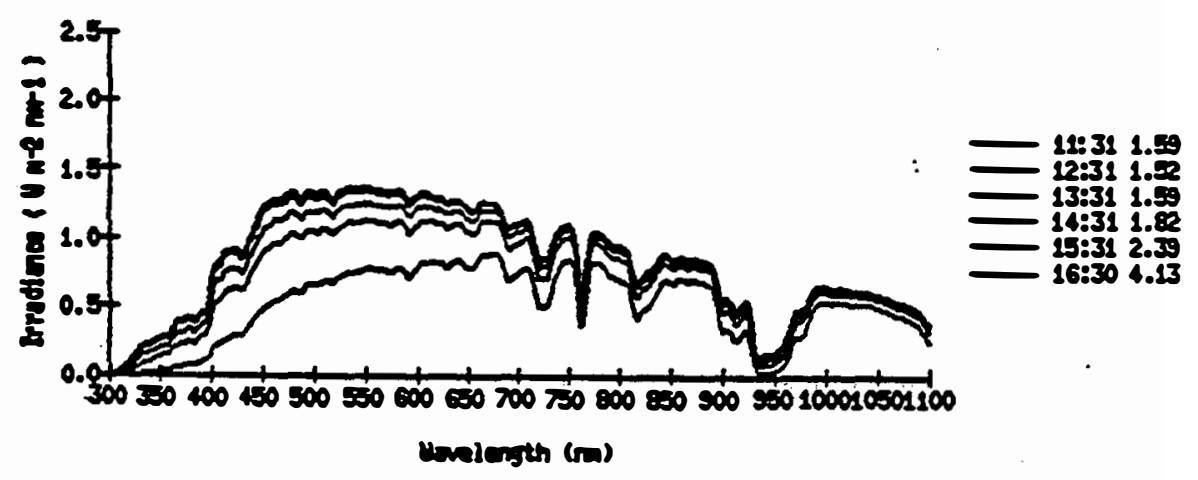

Fsec 18 as

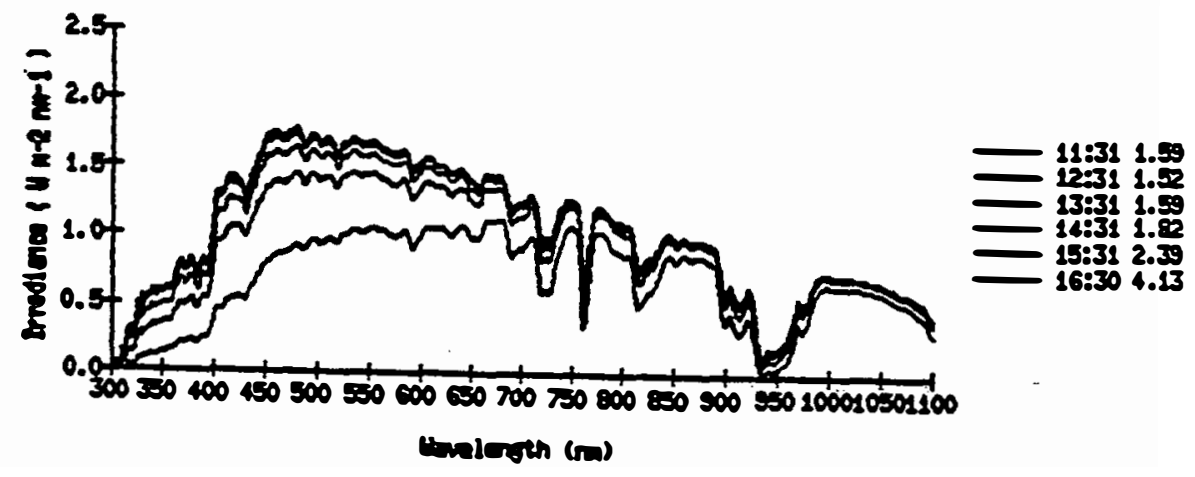


FSP88019

$268021988019272 \mathrm{DH} 202 \mathrm{GH}$

0.3 cloud cover at $0830 ; 0.4$ at $1230 ; 0.3$ at 1630

Terp controllers: $17241 \mathrm{C}$ and $10240 \mathrm{C}$ at 1230

Slides: $0916 \mathrm{G}$ (condensation)

$1016 \mathrm{G}$

$2126 \mathrm{G}$

$2216 \mathrm{~A}$ (dark)

$1316 \mathrm{H}$

$1416 \mathrm{G}$

$1516 \mathrm{G}$

$1616 \mathrm{G}$

FEXC 19

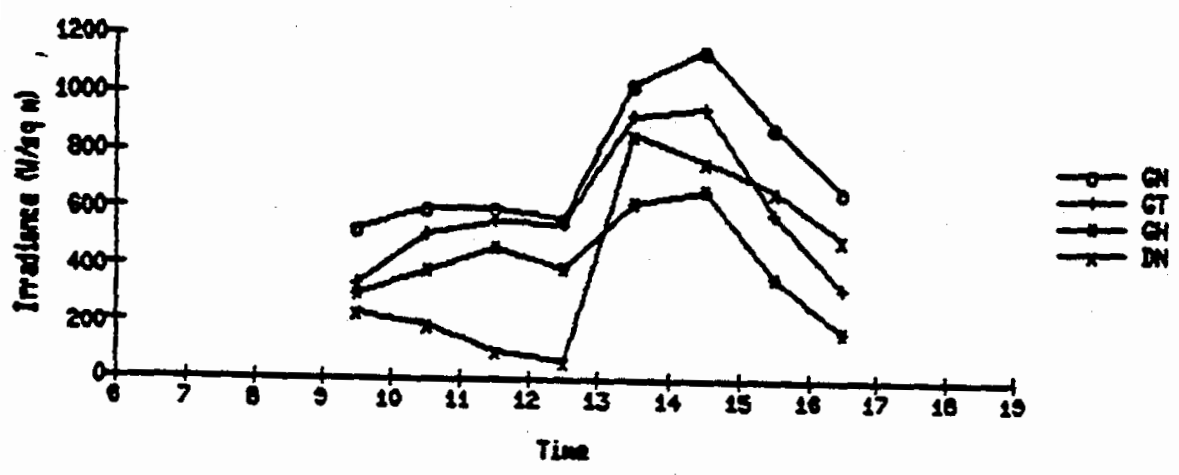

$\max 29$ an

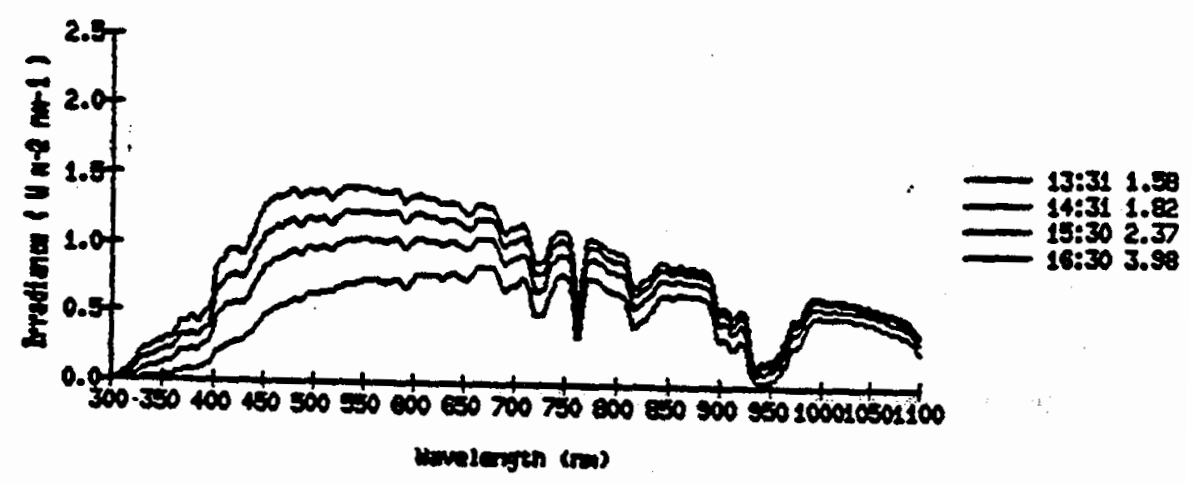

Fsec 19 as

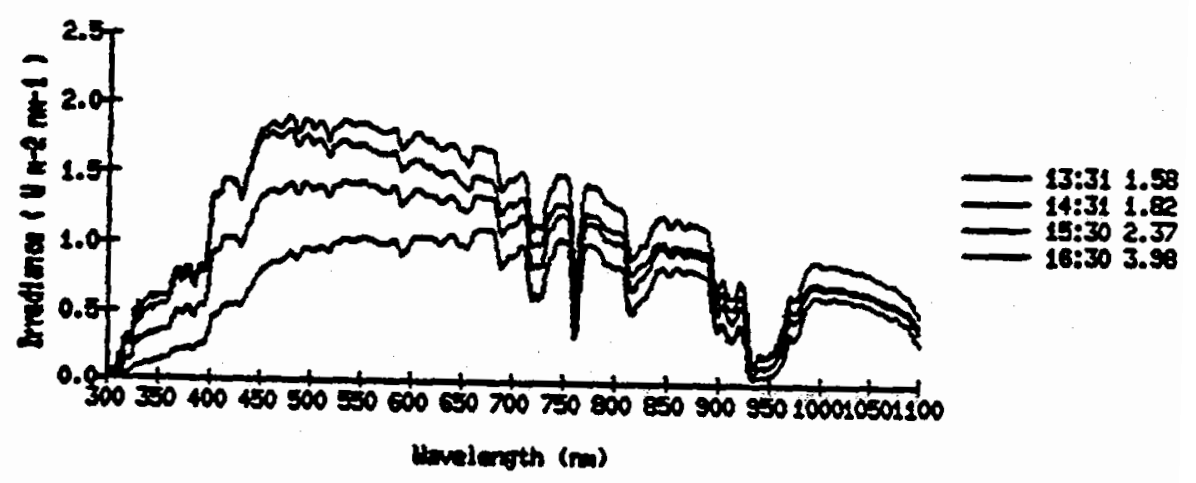


A. FST 88020

$\begin{array}{lllllllll}269 & 01 & 20 & 88 & 020 & 172 & \text { DN } & 102 & \text { GX }\end{array}$

0.9 cloud cover at 0830; 0.8 at 1230 and 1530

Teep controllers: $172 \quad 102$

083040 C 39 C

S11des: $0915 \mathrm{G}$

$1034 \mathrm{G}$

$1133 \mathrm{G}$

$1233 \mathrm{G}$

$1332 \mathrm{G}$

$.1432 \mathrm{G}$

1532 G

$1631 \mathrm{G}$

$\operatorname{PSEC} 20$

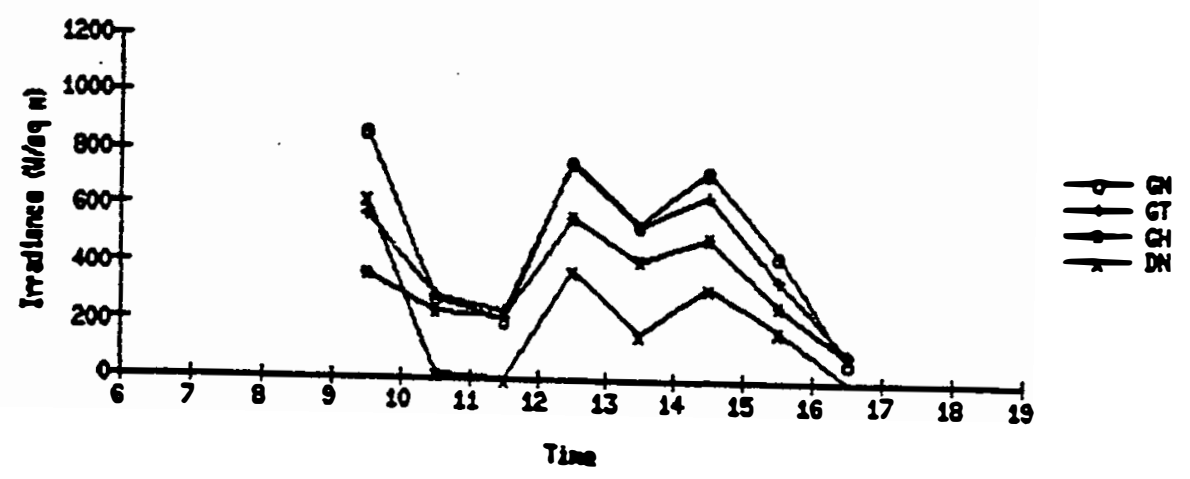

FEEC $20 \mathrm{BNT}$

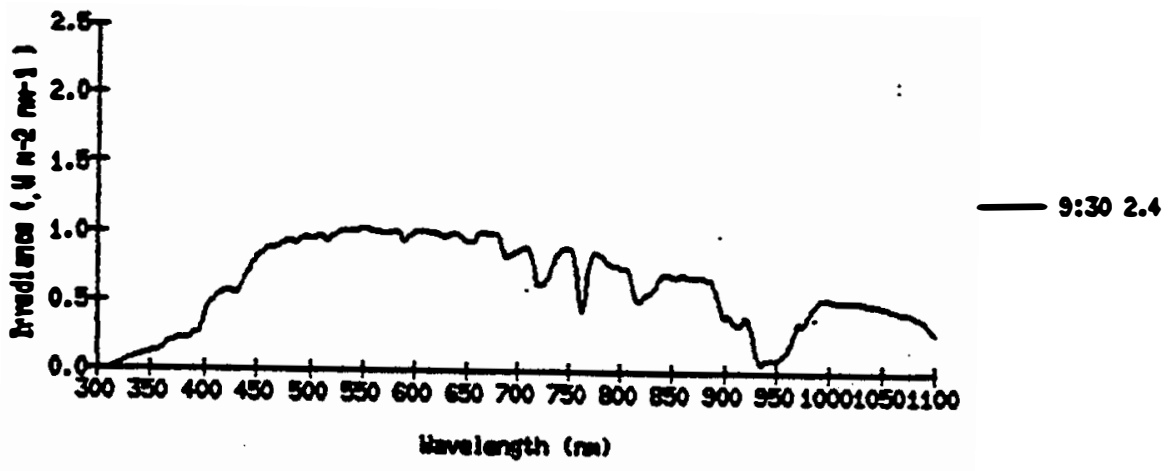

$\operatorname{Fsc} 20$ as

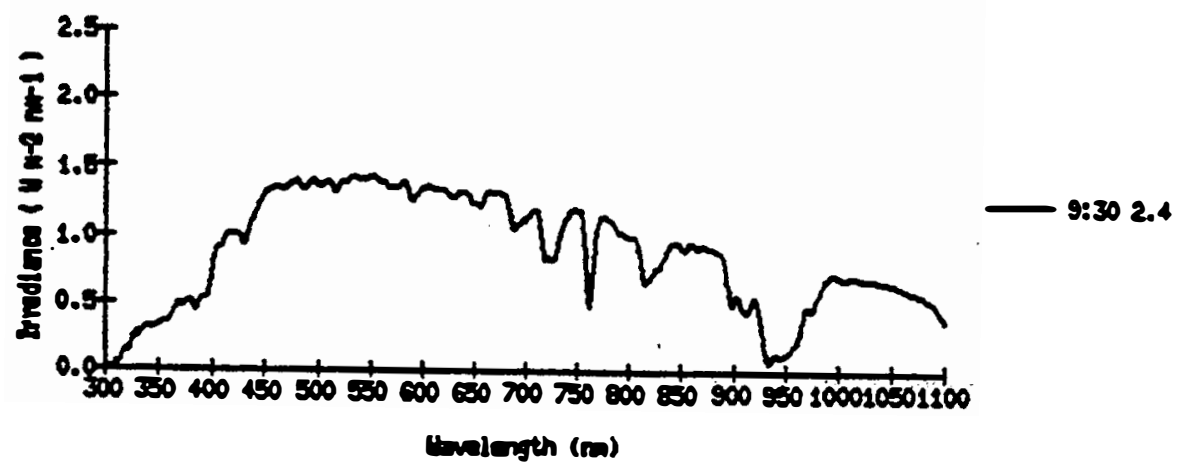



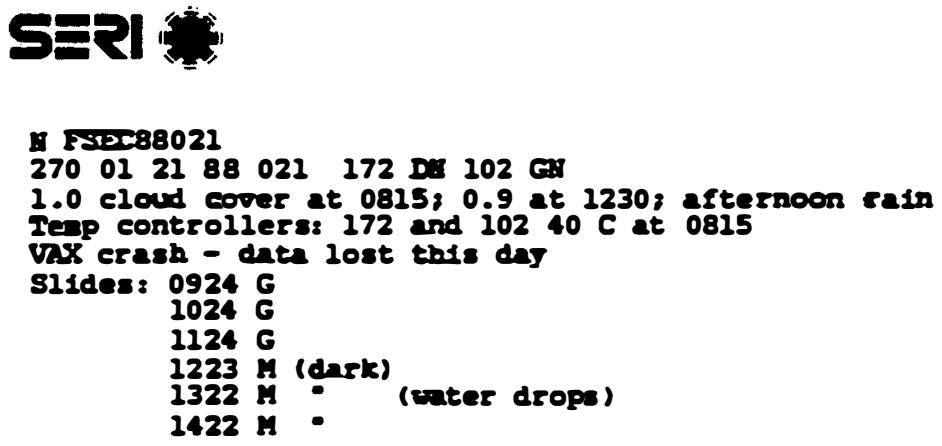

Ho spectra 
1. FSPC88022

$2710122.88022 \quad 172$ DI $102 \mathrm{GI}$

0 cloud cover at 0930 and $1230,0.8$ at 1630

Slides: $0908 \mathrm{G}$

$1033 \mathrm{G}$

1132 (dart)

$1232 \mathrm{M}$ -

$1331 \mathrm{M}$

$1431 \mathrm{G}$

$1530 \mathrm{G}$

$1630 \mathrm{G}$

$\operatorname{FsEC} 22$

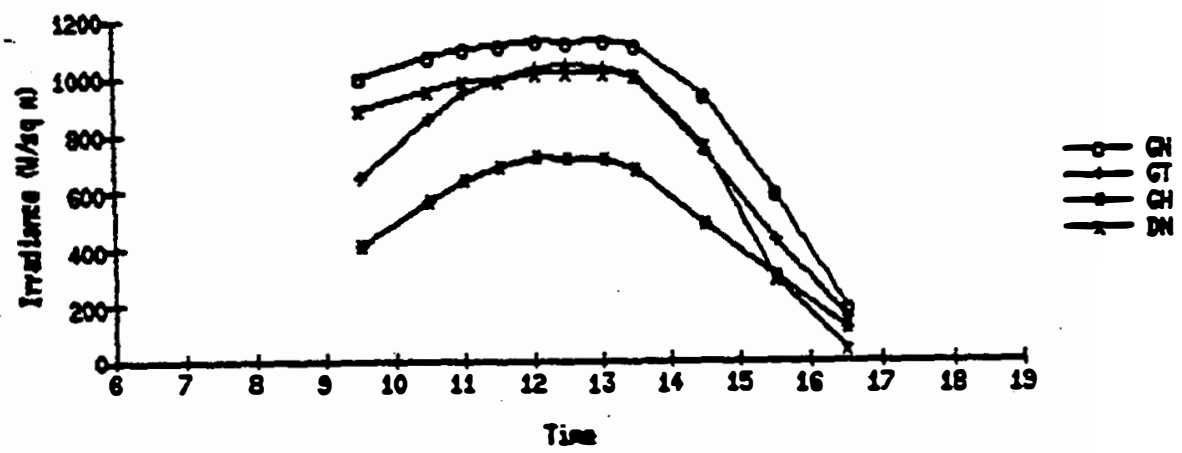

FSEC 22 MT

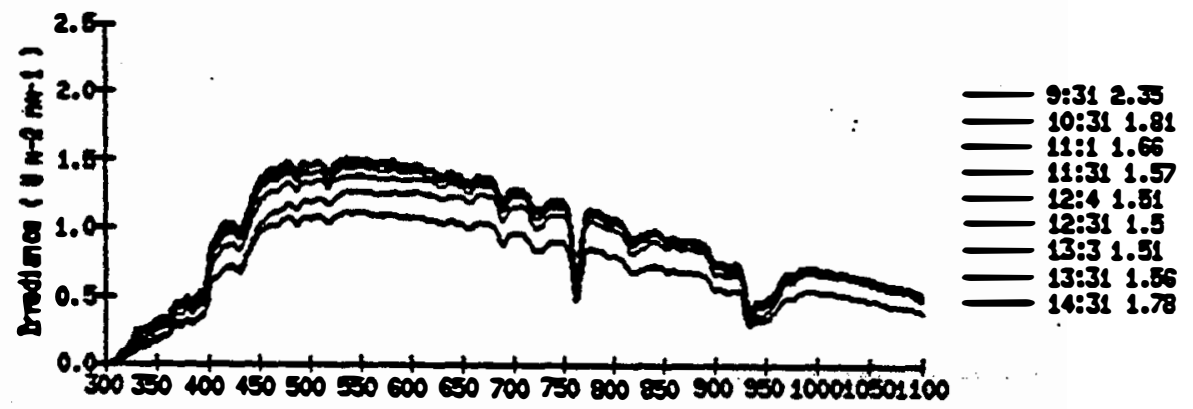

(toveresh (m)

$\operatorname{Psec} 22$ as

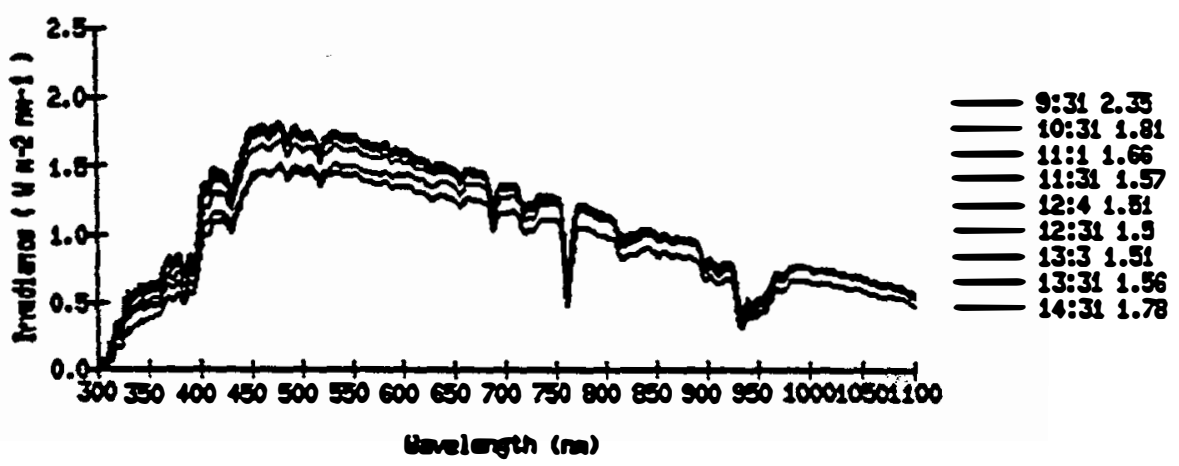




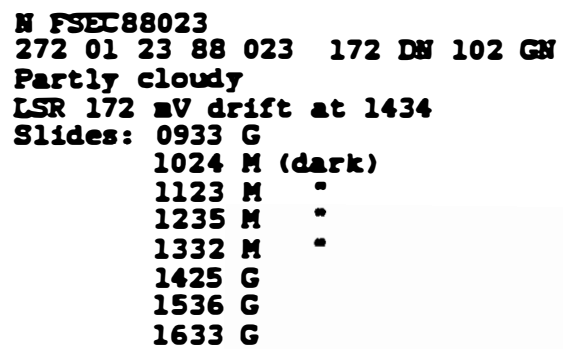

$\operatorname{Psec} 23$

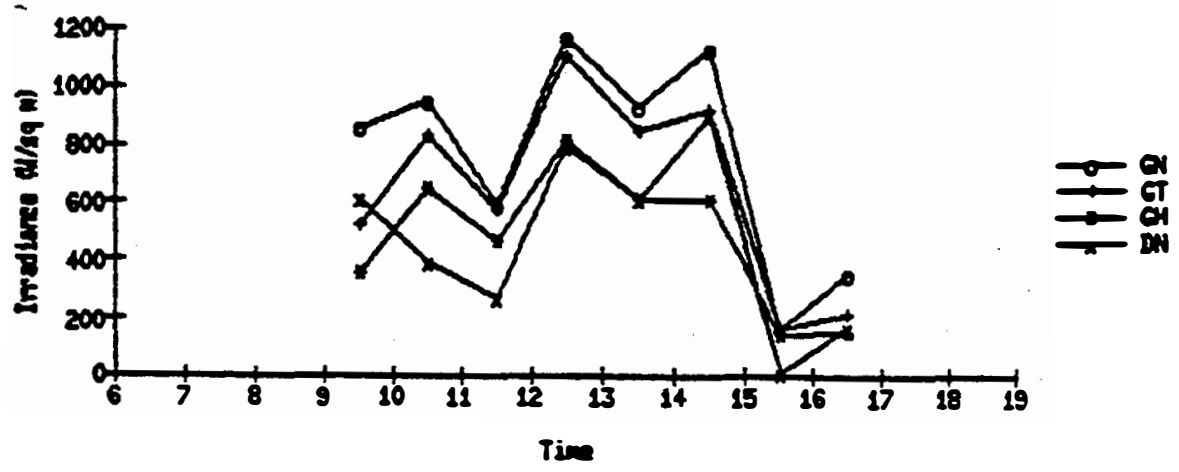

FISE $23 \mathrm{DT}$

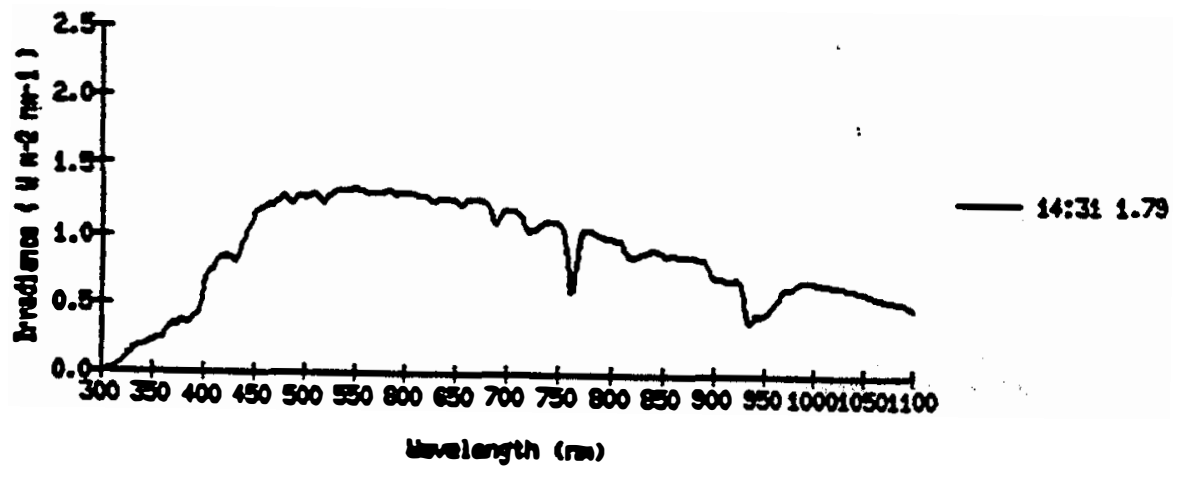

Fsec 23 as

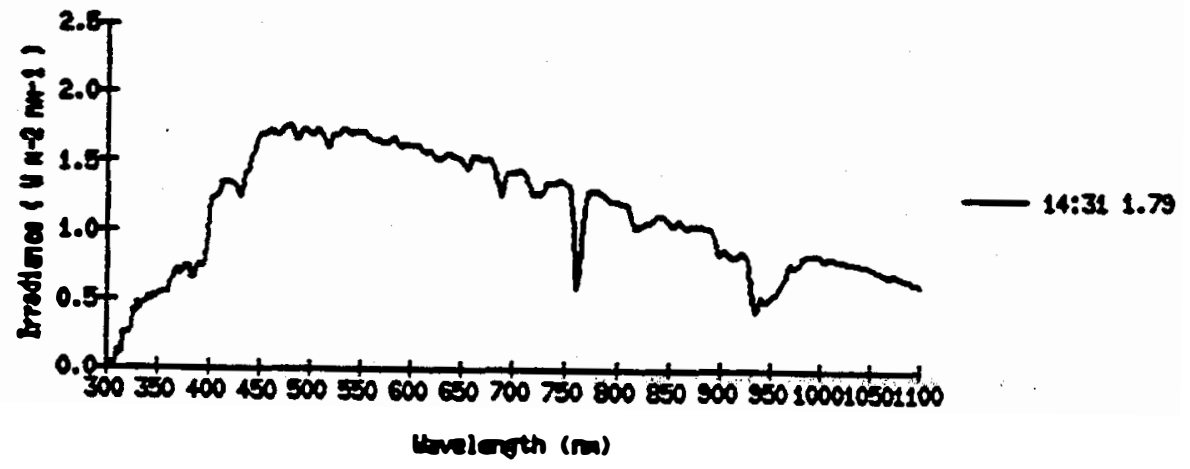


I. FTDE8024

$\begin{array}{lllllllll}273 & 01 & 24 & 88 & 024 & 172 & \text { D. } & 202 & \text { GS }\end{array}$

Overeast: rair

slides: $0932 \mathrm{G}$

$1042 \mathrm{G}$

$1134 \mathrm{G}$

$1235 \mathrm{G}$

$1335 \mathrm{G}$

$1427 \mathrm{H}$ (dark)

$1537 \mathrm{G}$

$1634 \mathrm{G}$

Fise 24

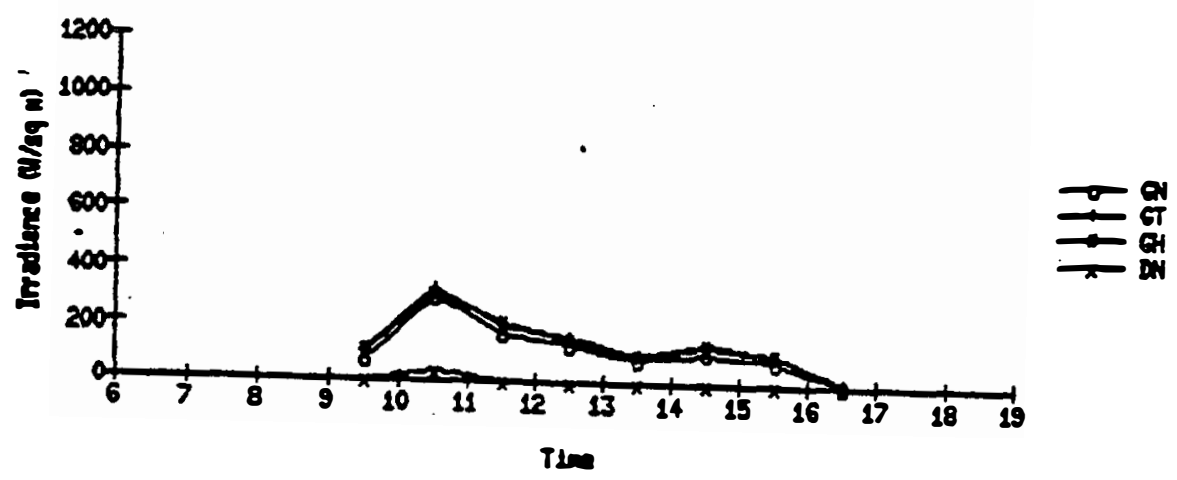

FESC 24 DNT

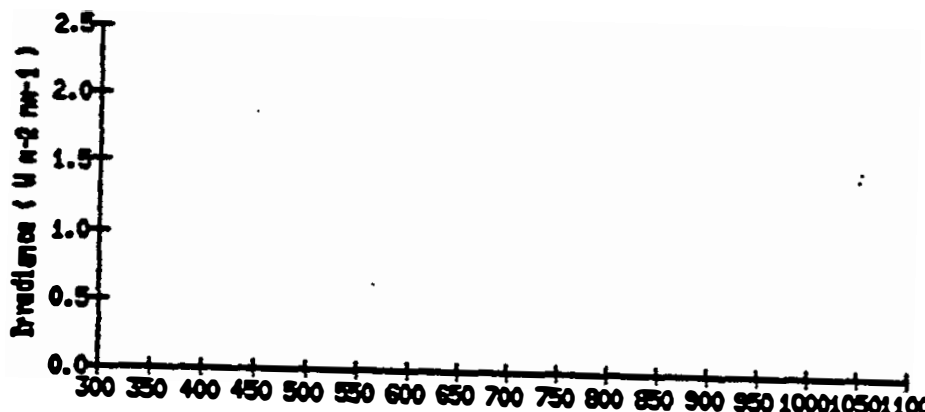

$12: 312.56$

Helath (m)

Fris 24 os

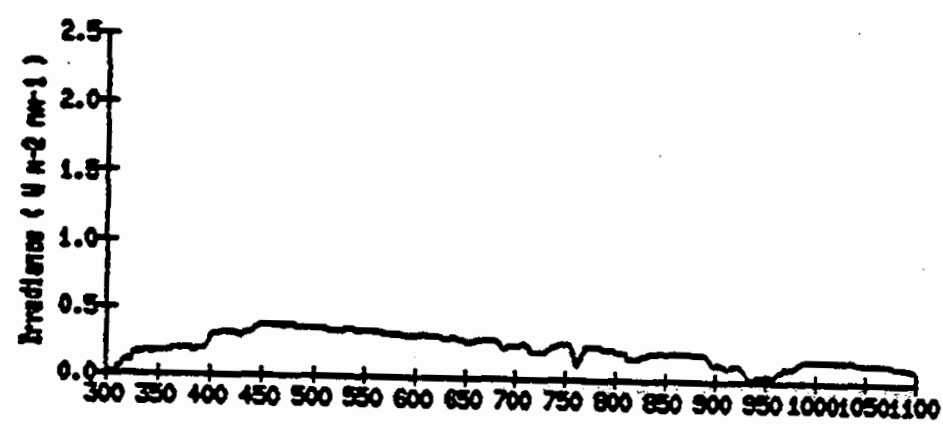

uncleath (m) 
$=213$

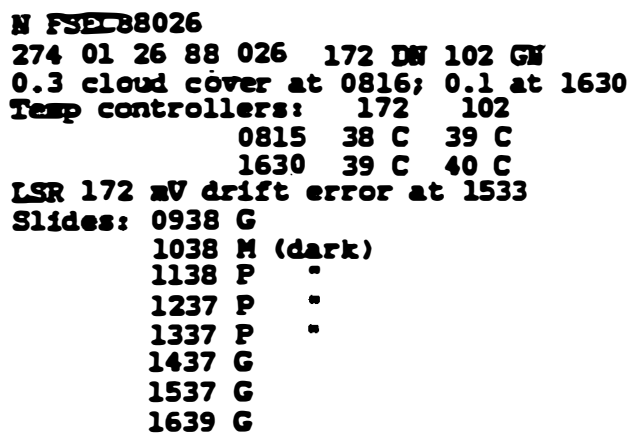

Fise 26

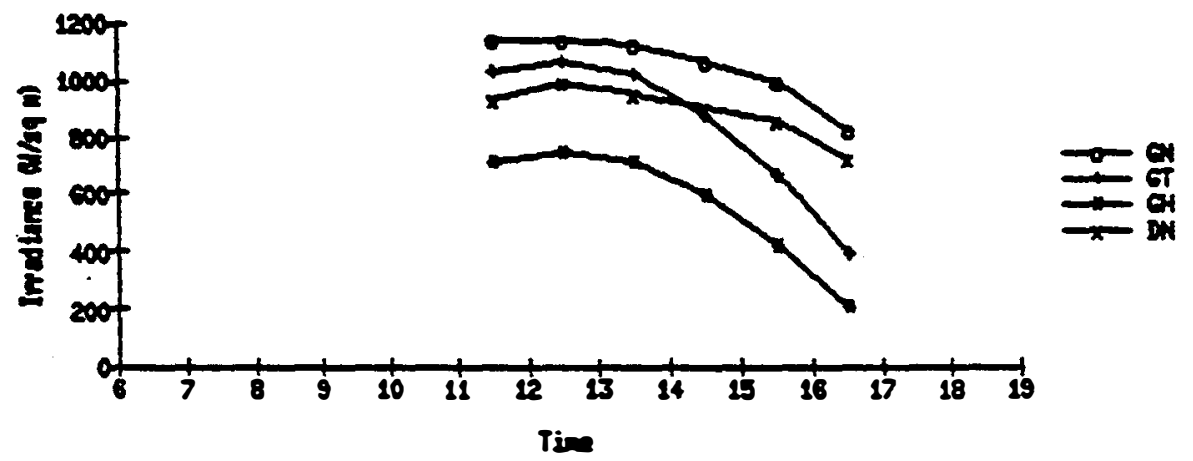

FבSC 26 BT

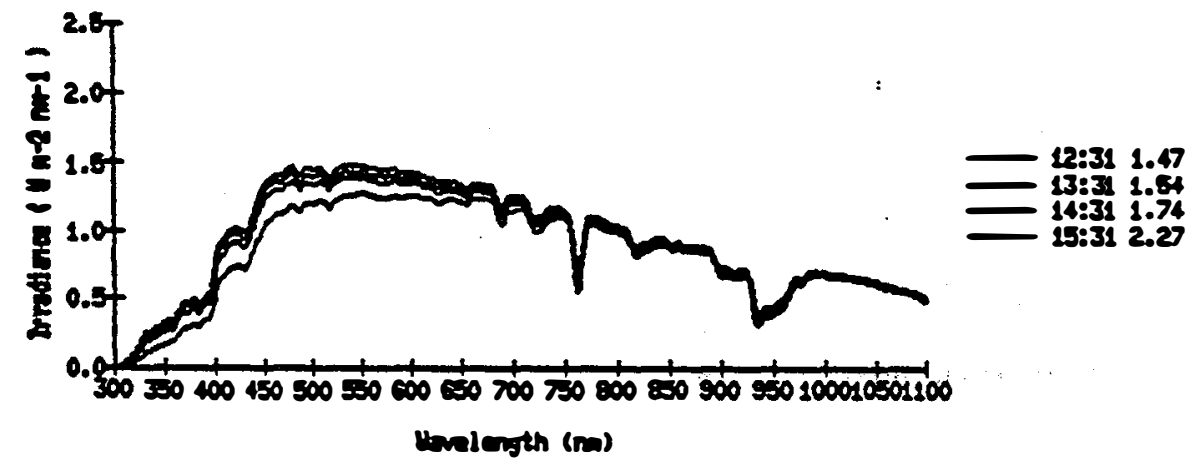

Fise 26 os

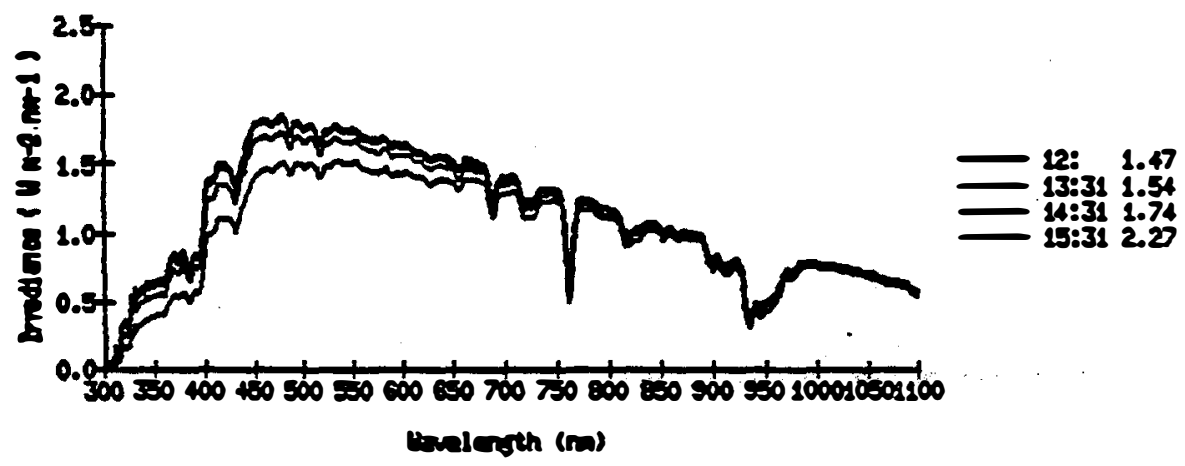


1. FSP88027

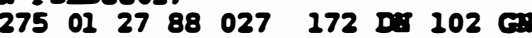

0.1 cloud-cover at $0830 ; 0$ at 1200

Tenp controllers: $17238 \mathrm{C}$ and $10240 \mathrm{C}$ at 1200

slides: $0935 \mathrm{G}$

$1034 \mathrm{G}$

$2134 M$ (dark)

$1234 \mathrm{P}$ :

$1334 \mathrm{P}$

$1433 G$

$1533 G$

$1633 \mathrm{G}$

FIs 20

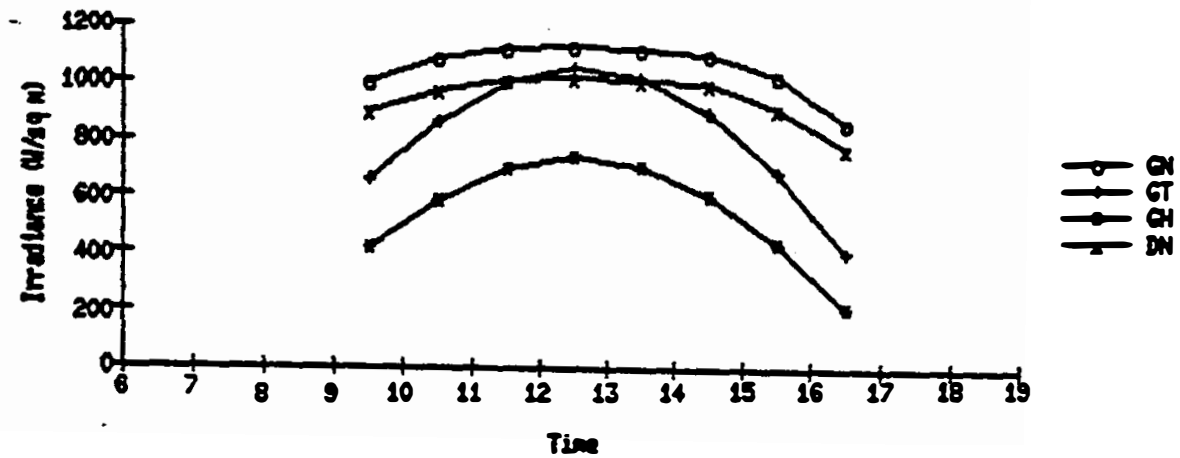

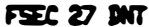

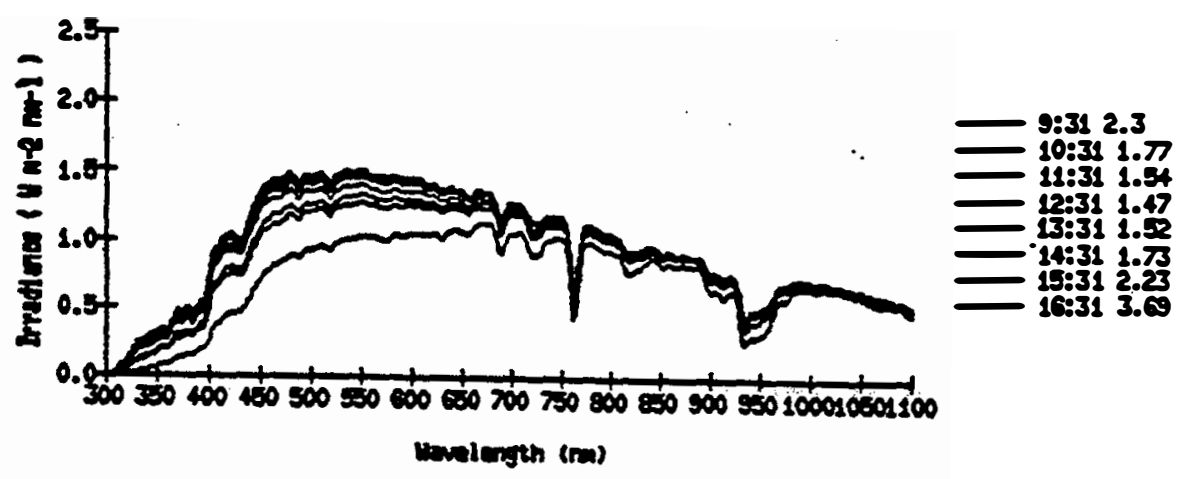

FEsc 27 as

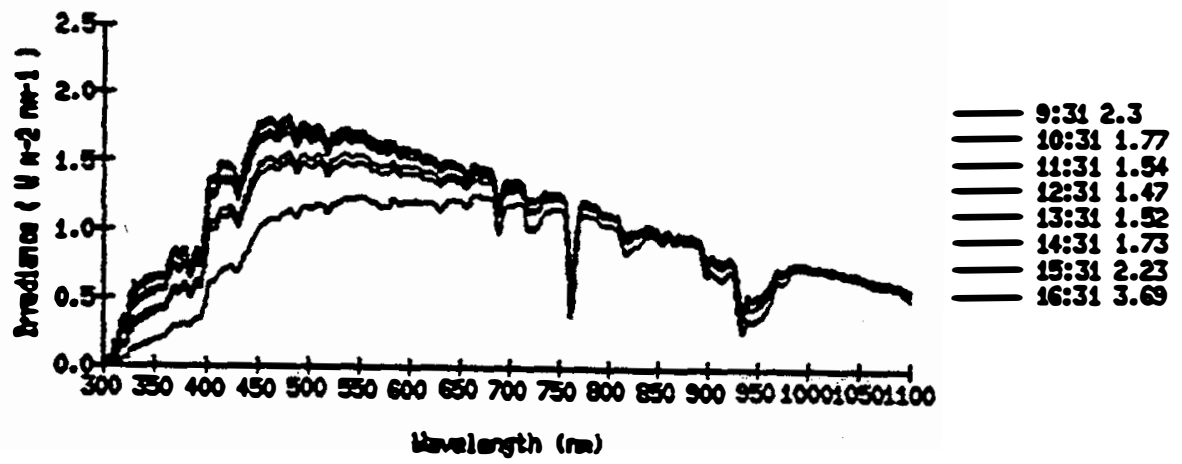




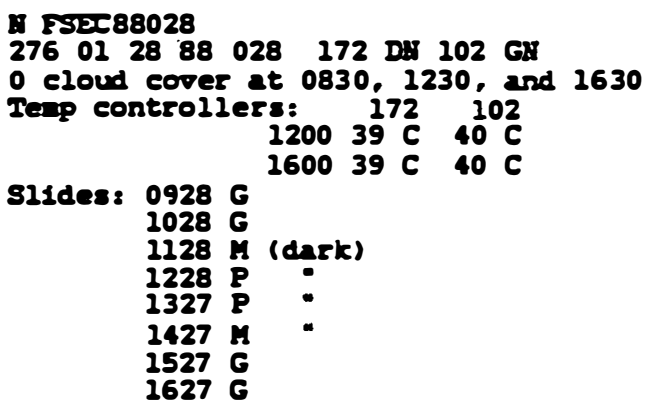

Fesc 28

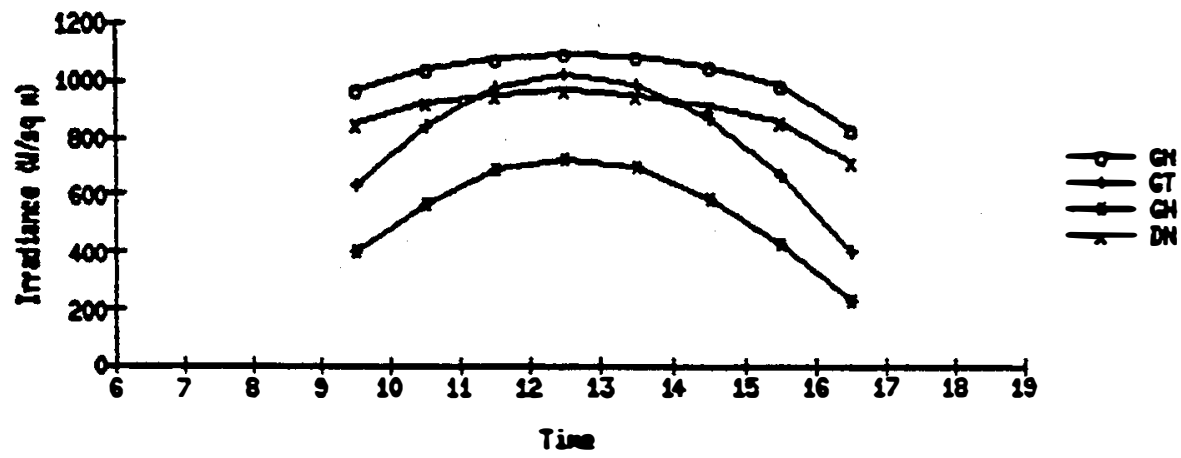

FEבC $28 \mathrm{mT}$

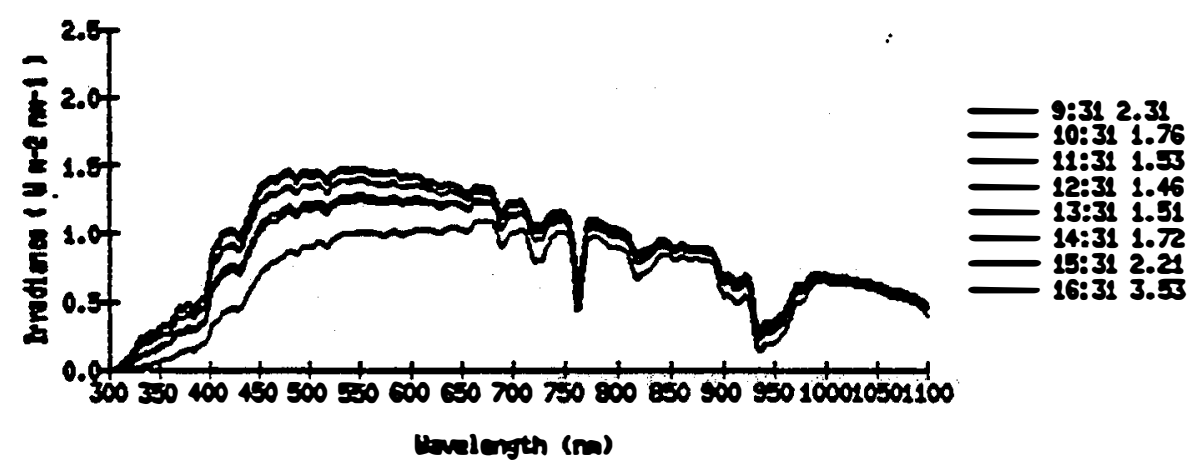

FEs 2805

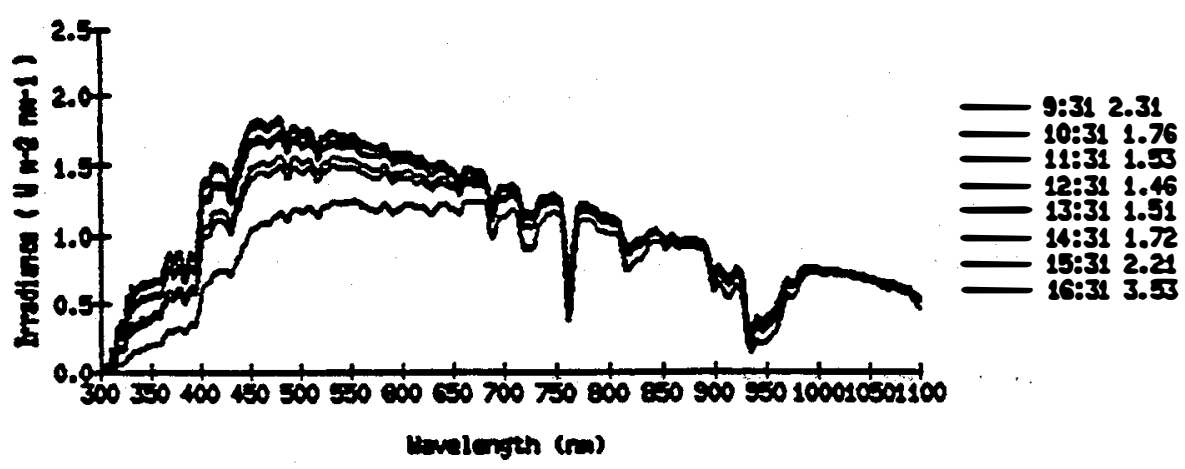




\section{SEP}

P TS2088029

$\begin{array}{lllllllll}277 & 01 & 29 & 88 & 029 & 172 & \mathrm{DM} & 102 & \mathrm{GH}\end{array}$

0.9 cloud cover at 1330: 0.7 at 1530

Terp controllers: $172 \quad 102$

311des: $0927 \mathrm{G} 3040 \mathrm{C} 41 \mathrm{C}$

S11des: 0921 G

$1120 \mathrm{G}$

$1220 \mathrm{G}$

1320 M (dark)

$1420 \mathrm{G}$

$1519 \mathrm{G}$

$1619 \mathrm{G}$

Spectral Integrals on GH are vers close to braodband all daf

P्I 20

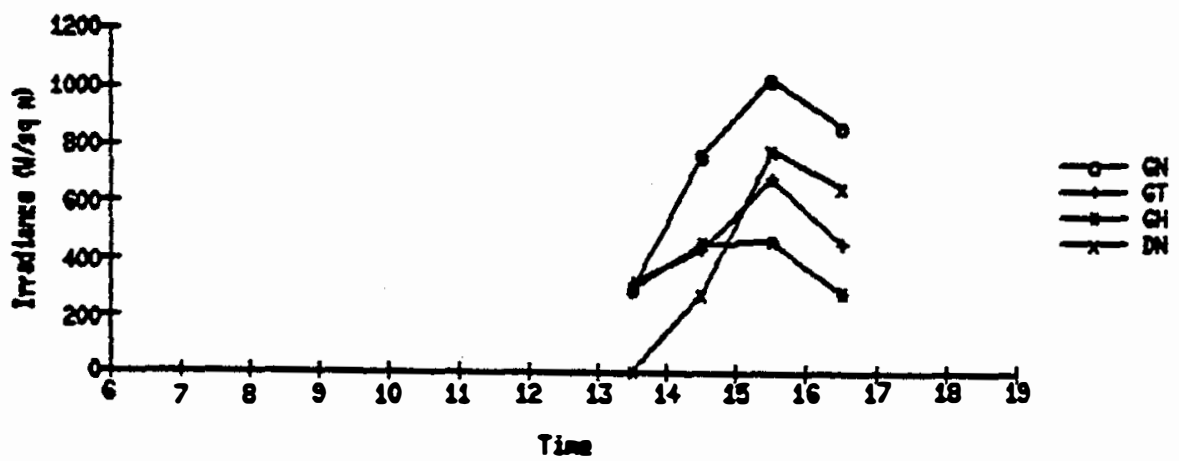

FISE 20 BNT

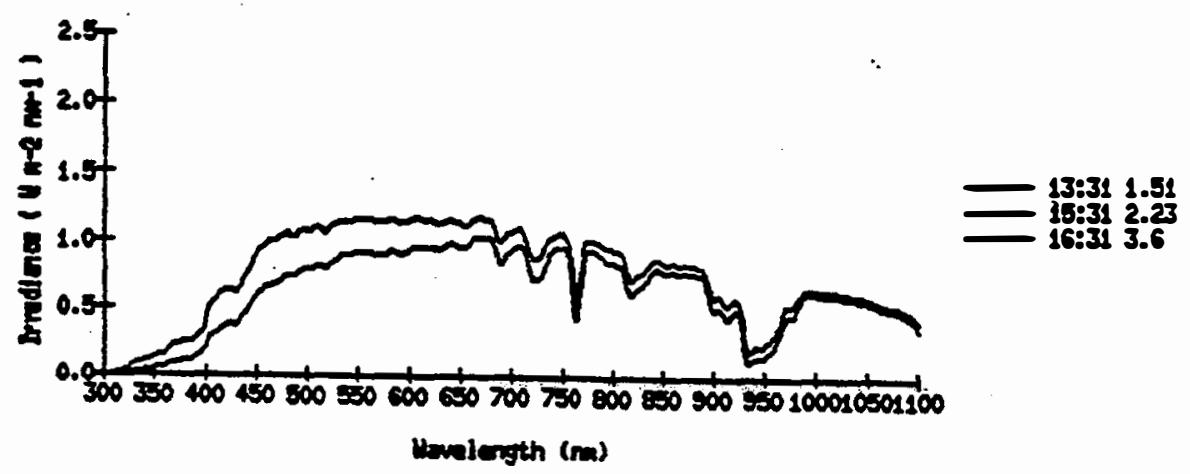

Fise a 00

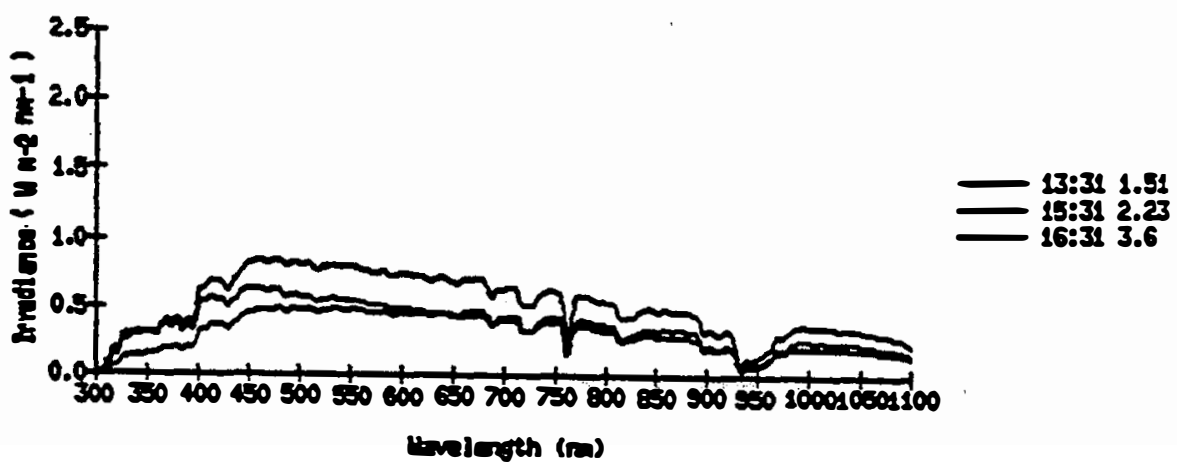


A. FSस 88030

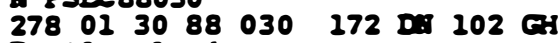

Partly cloudy

slides: 0919 H (dark)

$1019 \mathrm{G}$

1119 G

$1219 G$

$\operatorname{Psec} 30$

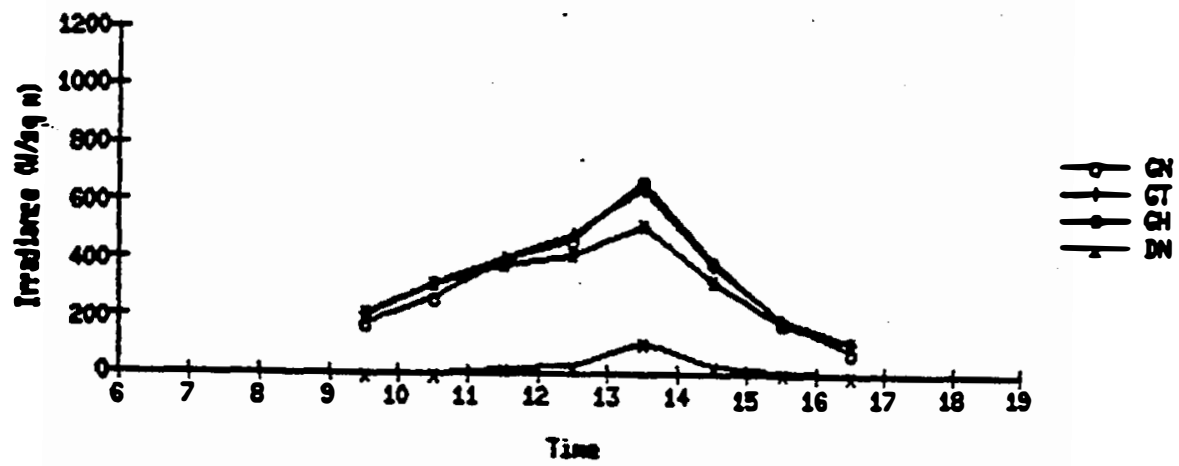

Fesc $30 \mathrm{nT}$

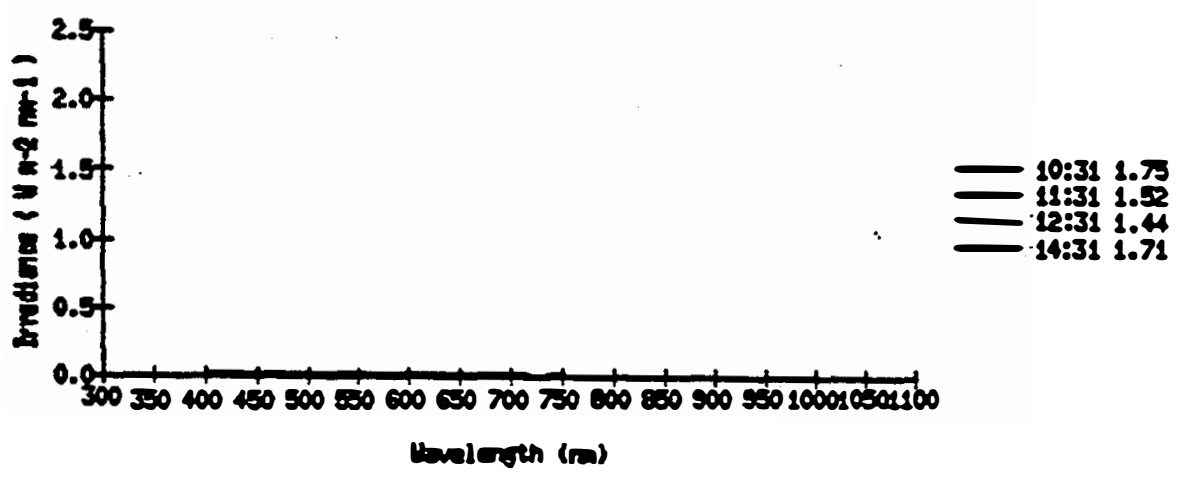

FEEC 30 AS

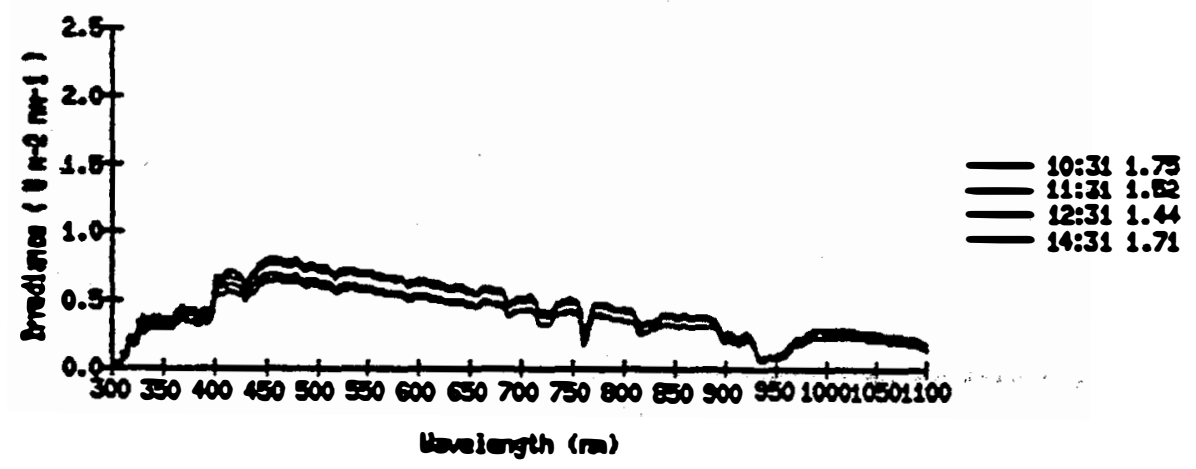


4. FST88031

$\begin{array}{llllllllll}279 & 01 & 31 & 88 & 031 & 172 & \mathrm{DA} & 102 & \mathrm{GH}\end{array}$

Partiy eloidy and elearing

Ho slides

$\operatorname{cosec} 34$

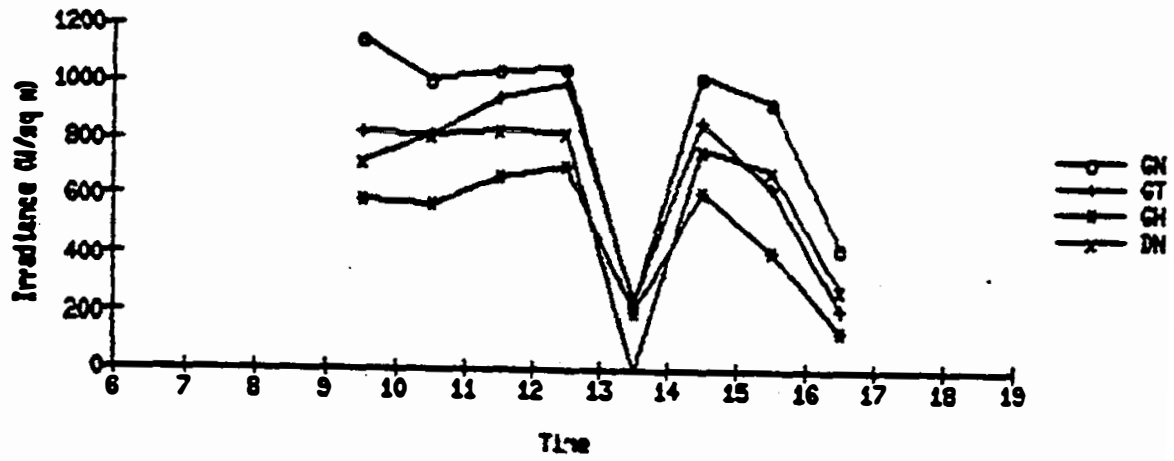

FEse $31 \mathrm{mT}$

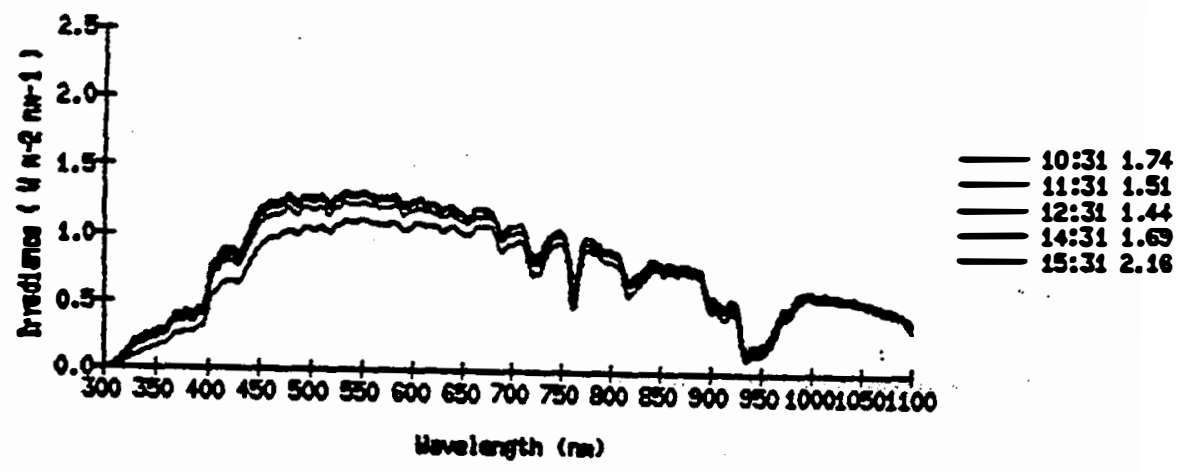

Fise 34 os

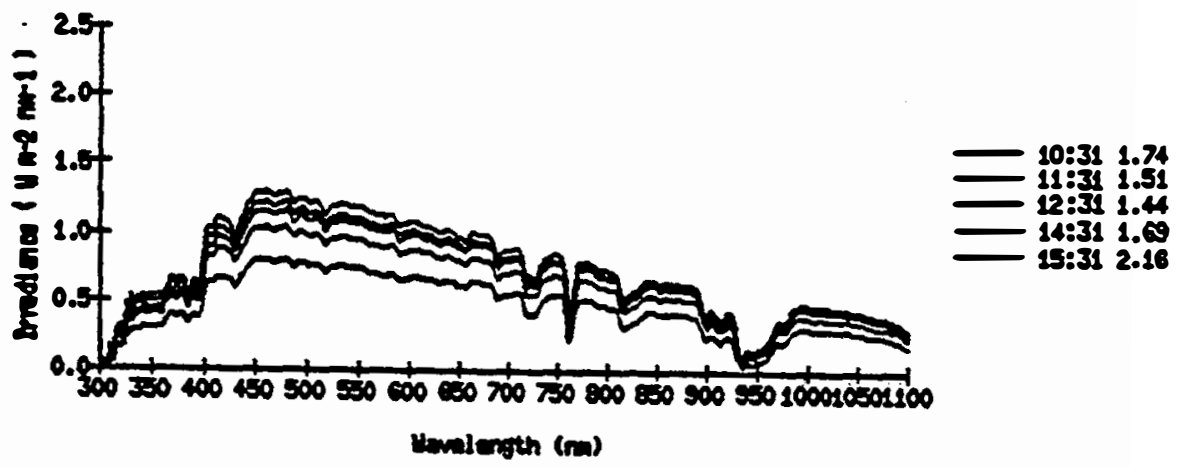


PST 88032

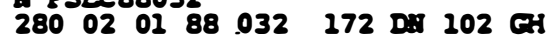

0.4 cloud cover at $1130 ; 0.3$ at 2430 , Fith base

Tep controllers: $172 \quad 102$

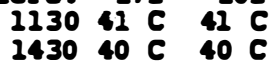

ISR $172 \mathrm{av}$ drift errors at 0931 and 1030

Slides: $1456 \mathrm{G}$

$1556 \mathrm{G}$

$-\infty-\infty$

$1656 \mathrm{G}$

Brief spikes in OV in Da spectra at 0931 and 1031

$\operatorname{cosec} 32$

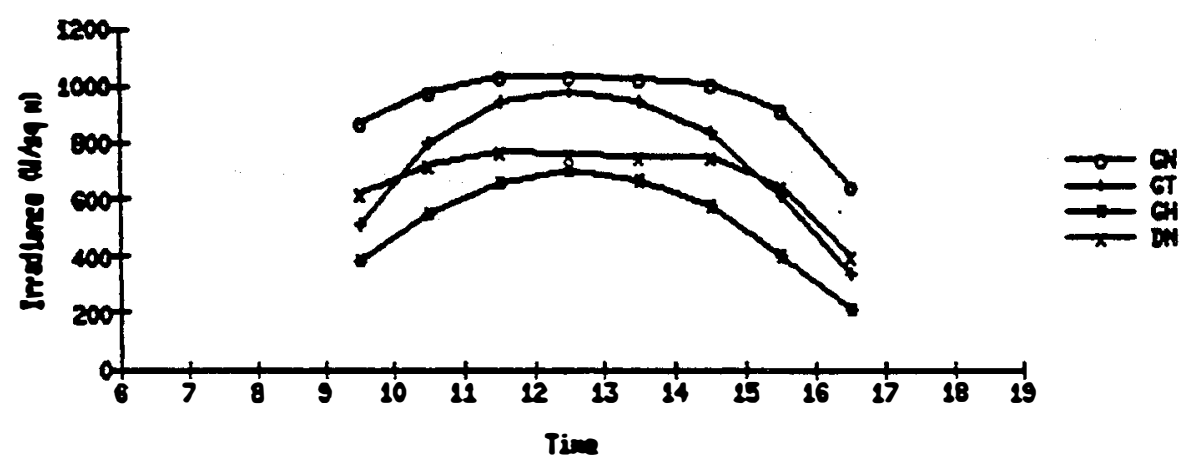

PESC 32 ant

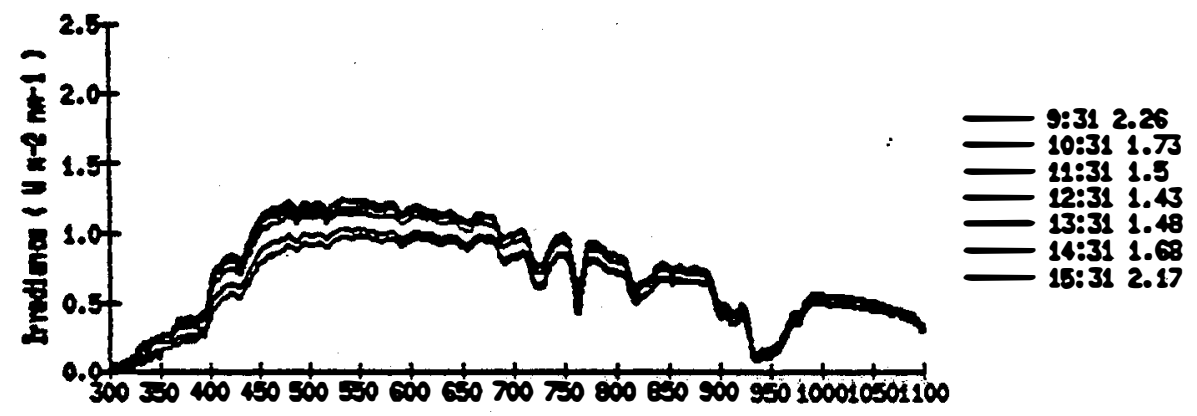

venlength (m)

$\operatorname{Pex} 32$ as

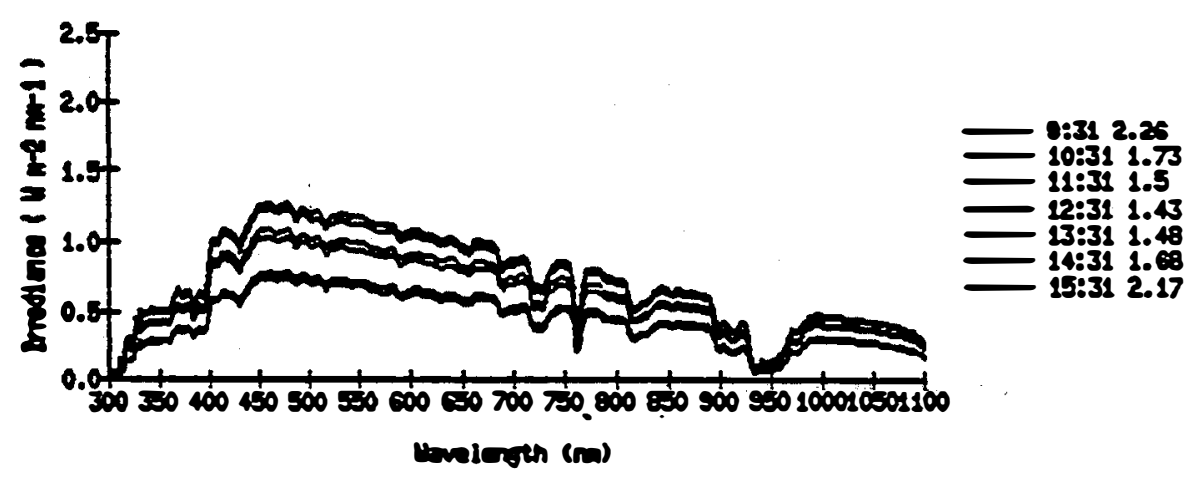




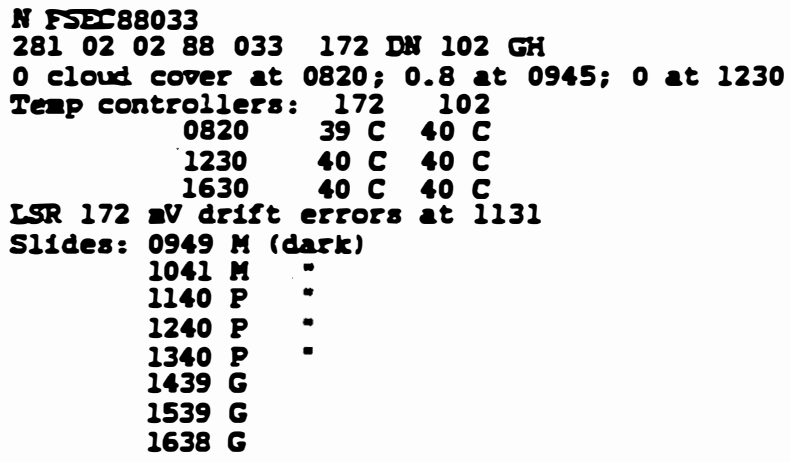

Verg sall spuke in ov in DN spectron at 1131

$\operatorname{FEEC~} 3$

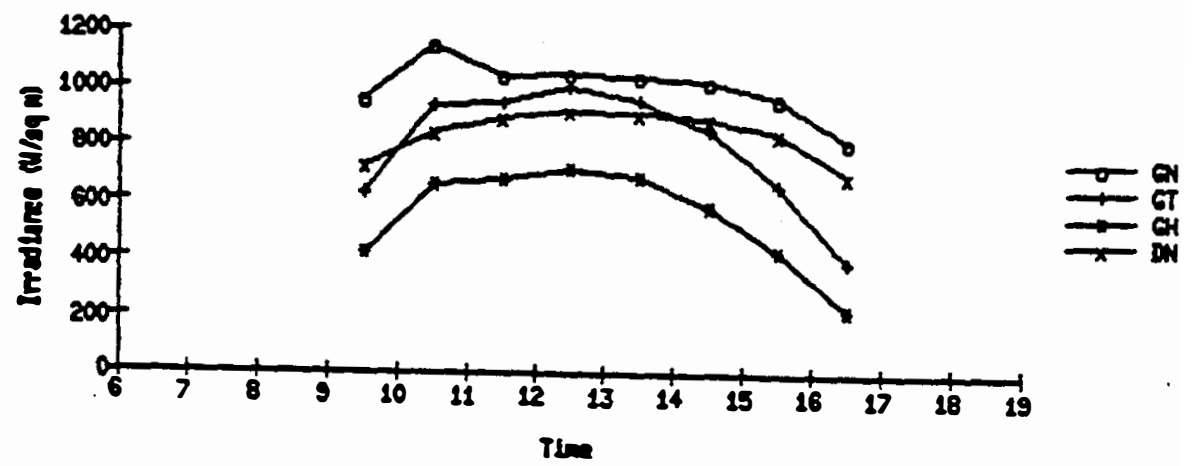

$\cos \pi \mathrm{BT}$

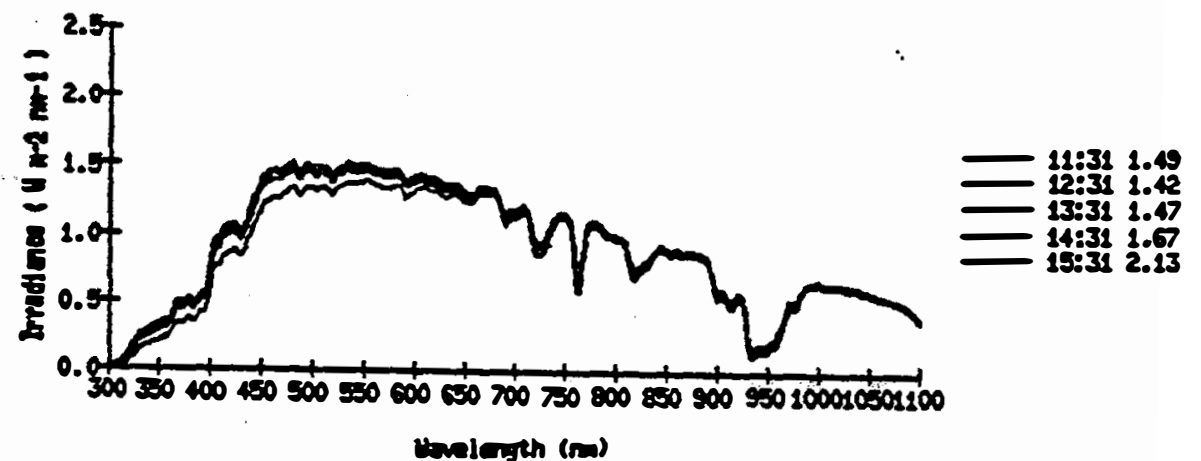

Fasc 38 as

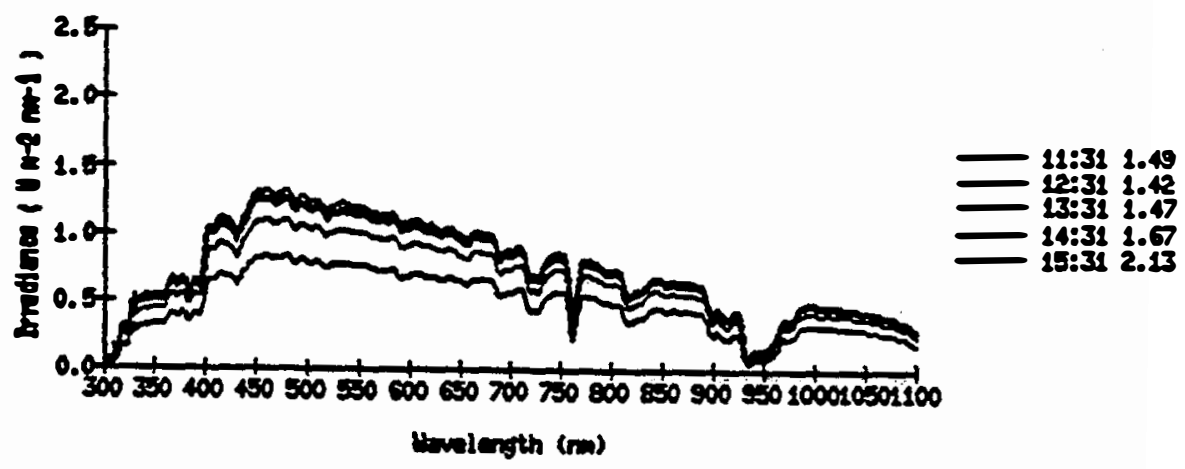


E FST्र88034

$\begin{array}{llllllllll}282 & 02 & 03 & 88 & 034 & 172 & \mathrm{Dm} & 102 & \mathrm{GH}\end{array}$

0.5 cloud cover at $0830 ; 0.6$ at $1230 ; 0.5$ at 1600

Ienp controllersi $172 \quad 102$

$0830 \quad 39 \mathrm{C} \quad 39 \mathrm{C}$

ISR 172 a drift errors at 1331

slidess 0931 A (enter drops)

$1031 \mathrm{~K}$ (dark)

$1130 \mathrm{G}$

$1229 P$ (dark)

$1329 \mathrm{P}$ :

1429

$1528 \mathrm{G}$

$1626 \mathrm{G}$

Fesc 34

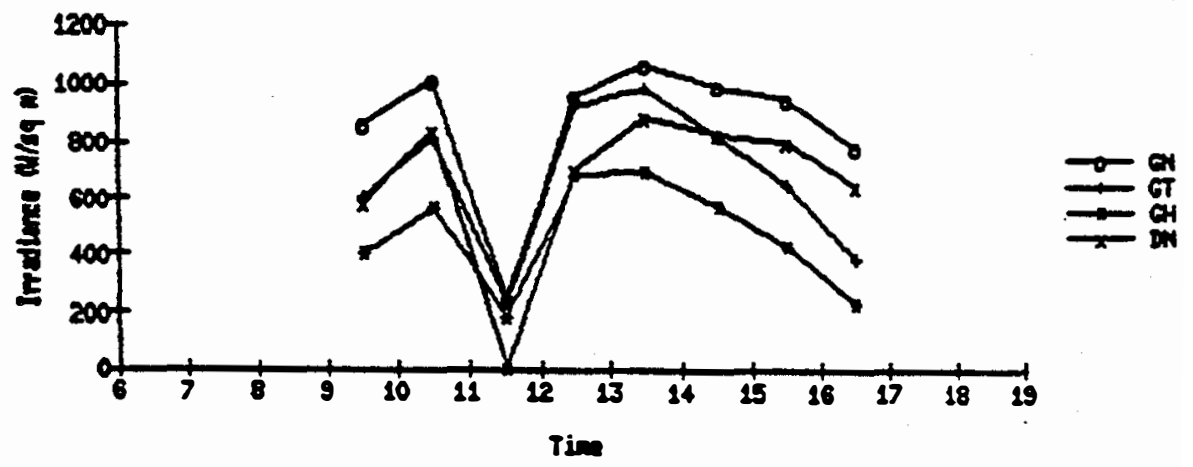

Fese at an

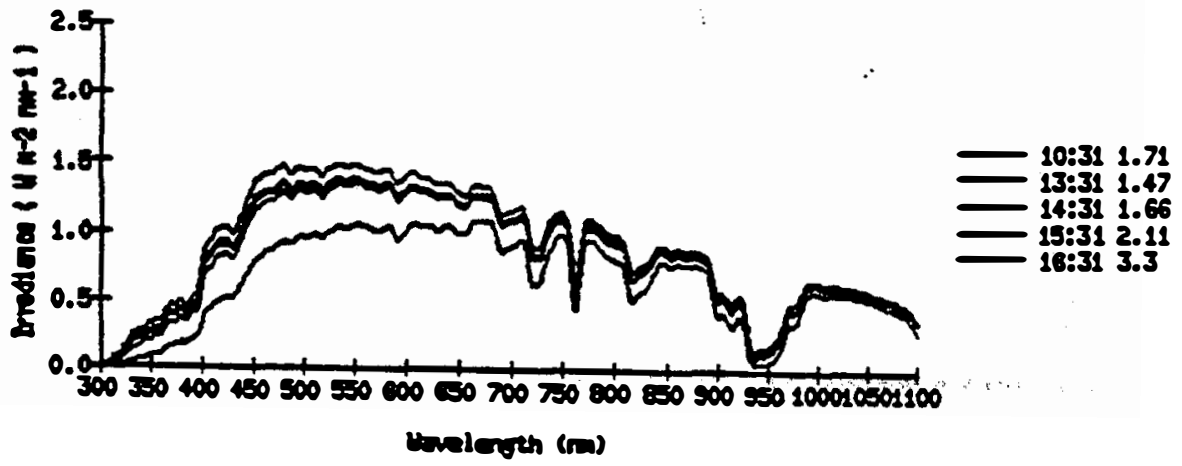

pres 34 os

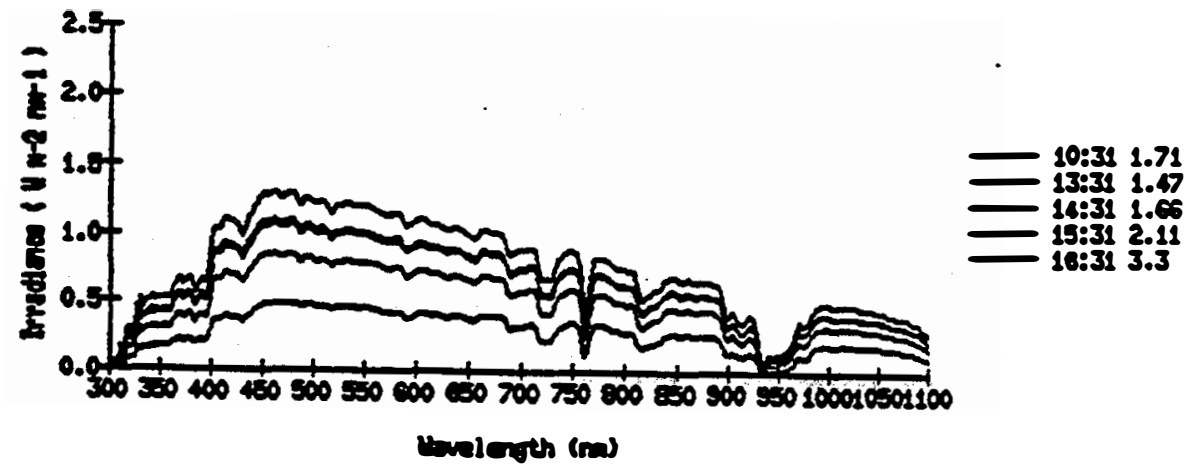




\section{SEPI}

a F512088035

$\begin{array}{llllllllll}283 & 02 & 04 & 88 & 035 & 172 & \mathrm{DA} & 102 \mathrm{GH}\end{array}$

0 cloud cover at $0815 ; 0.4$ at $1230 ; 0.1$ at 1700

Terp controllers: 172.102

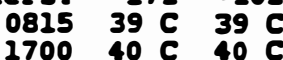

ISR 172 av drift errors at 0931, 1031, and 1233

Slides: 0929 il (weter drops)

1029 G

1128 M (dark)

$1228 \mathrm{P}$

$1328 \mathrm{P}$

$1427 \mathrm{G}$

$1527 \mathrm{G}$

$1626 \mathrm{G}$

Verg brief spike in UV in Dis spectra at 0931

Missing data in-Dis spectrun at 1031; noise in WV

$\operatorname{PsC} 3$

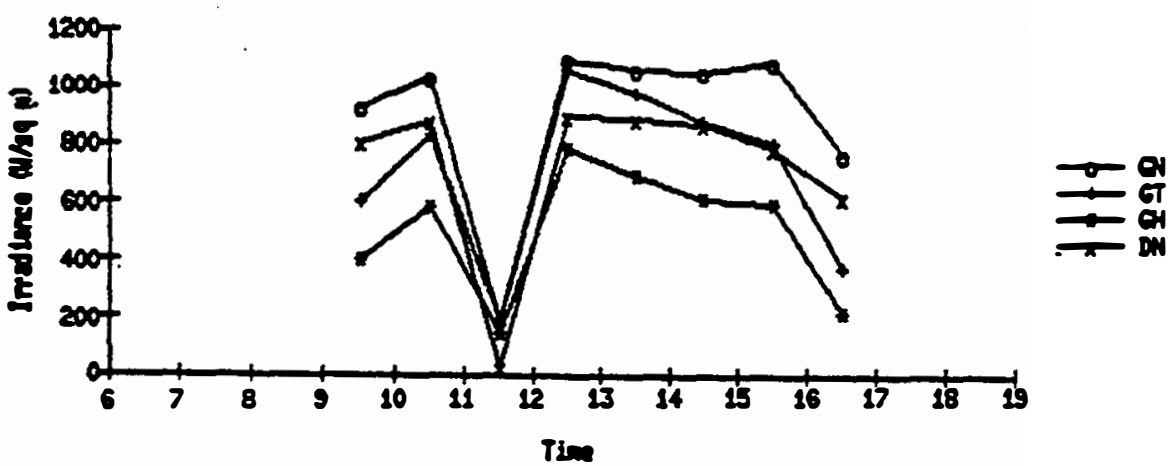

FEב $35 \mathrm{BT}$

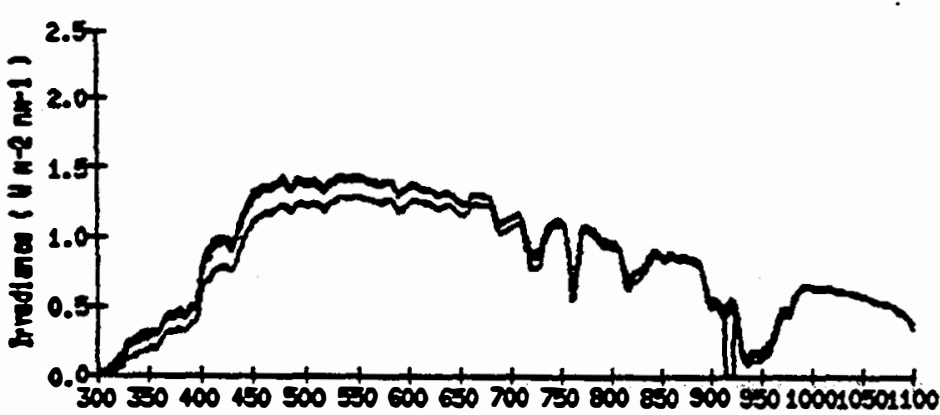

(belesth (m)

$\operatorname{cis} 306$

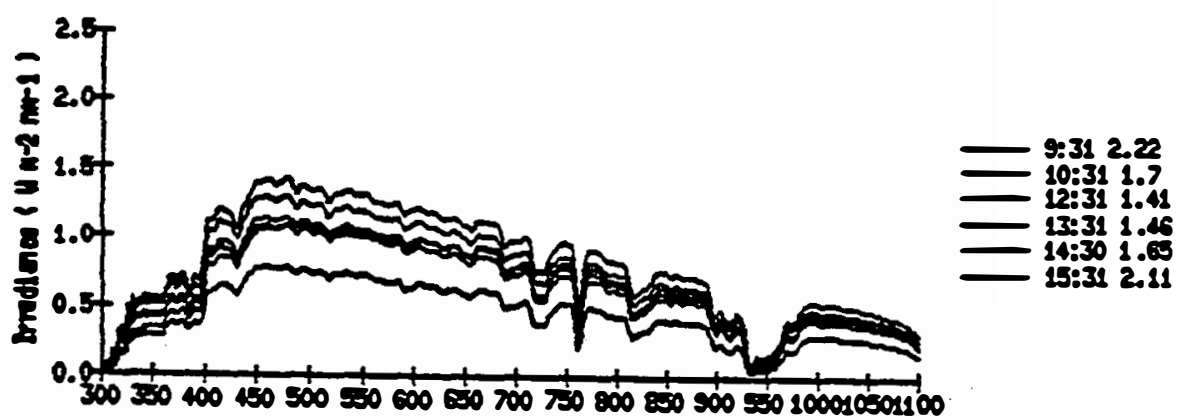

tureserth (m) 


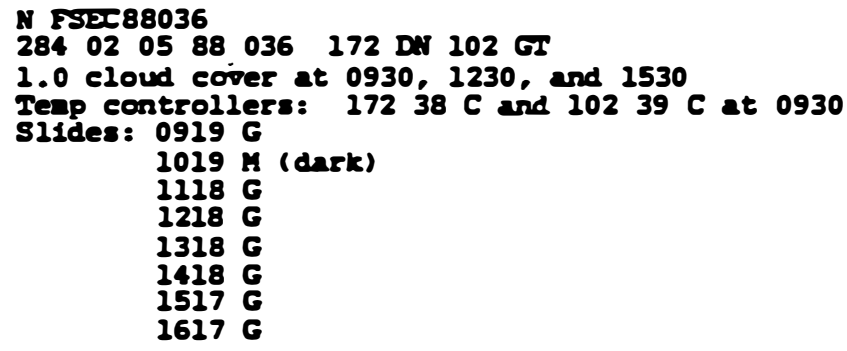

FSEC 36

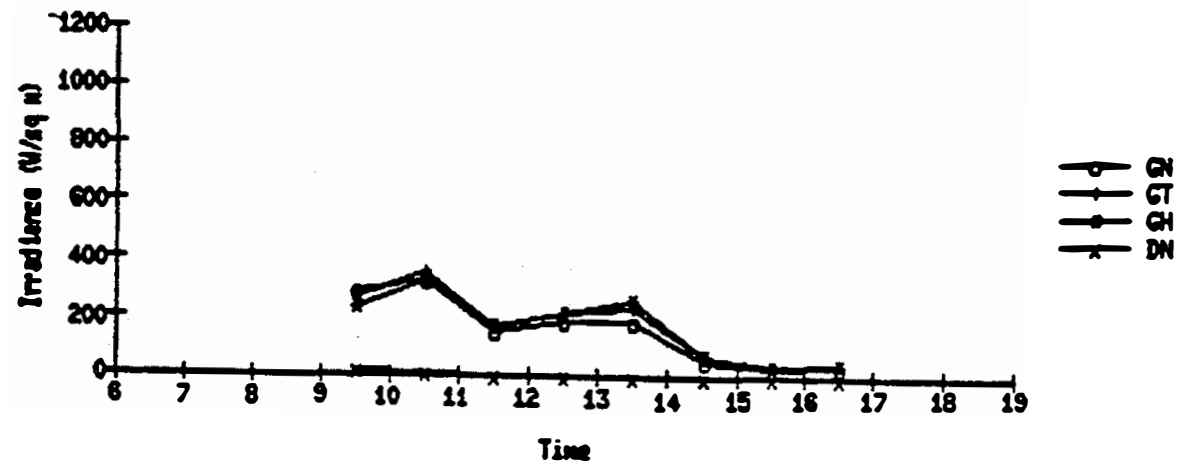

$\operatorname{Paxc} 35 \mathrm{art}$

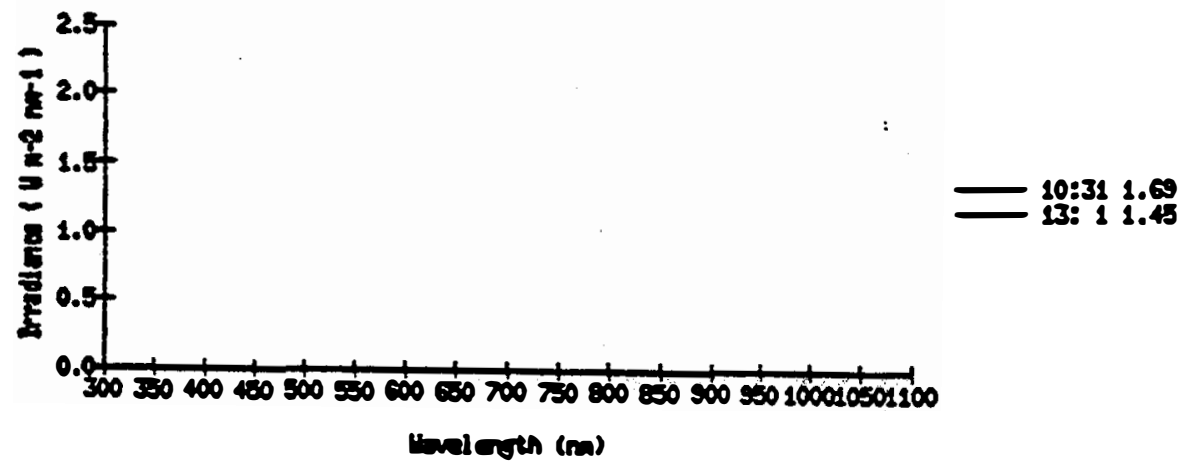

$\operatorname{Psec} 36$ GTS

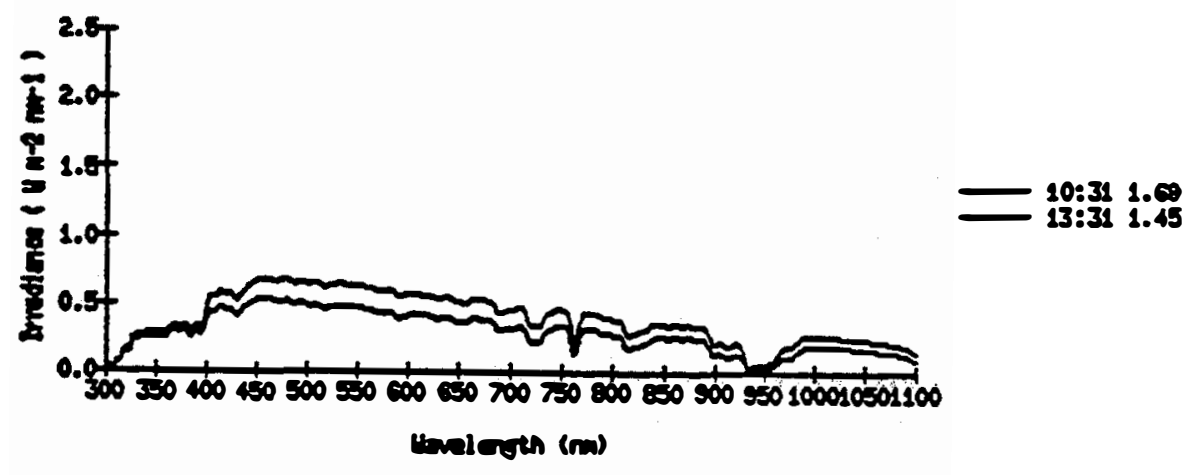


TR-3513B

SEP *

4)

$\begin{array}{llllllll}285 & 02 & 06 & 88 & 037 & 172 & \text { DN } & 102\end{array}$

overcest: risla (enter dropos)

$1012 \mathrm{G}$

$1112 \mathrm{G}$

1212

$1311 P$ (black)

$1411 P$

15116

1612 G

Ho specto

Five 9

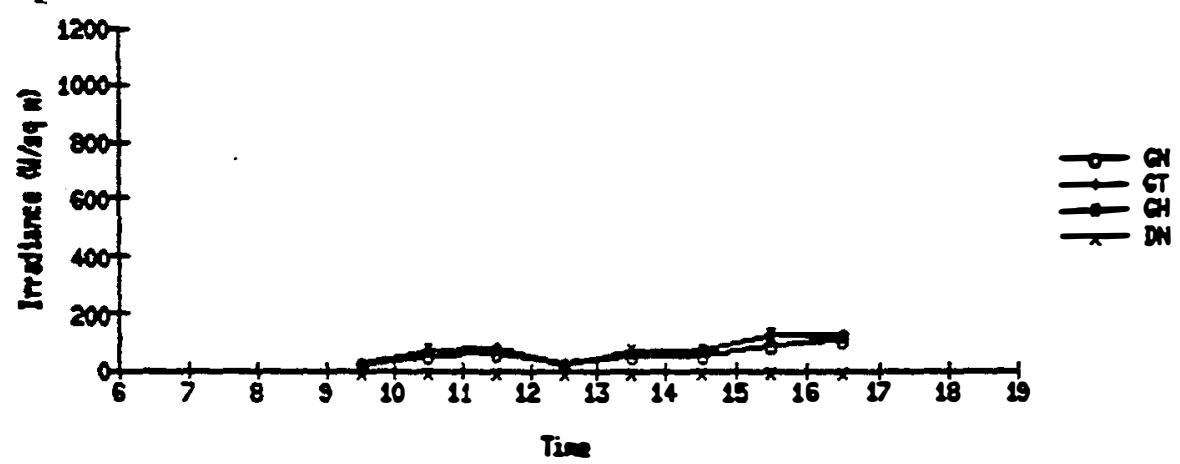




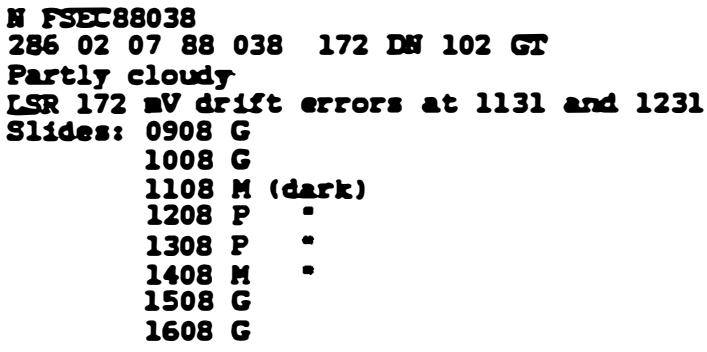

$\operatorname{PEx} 38$

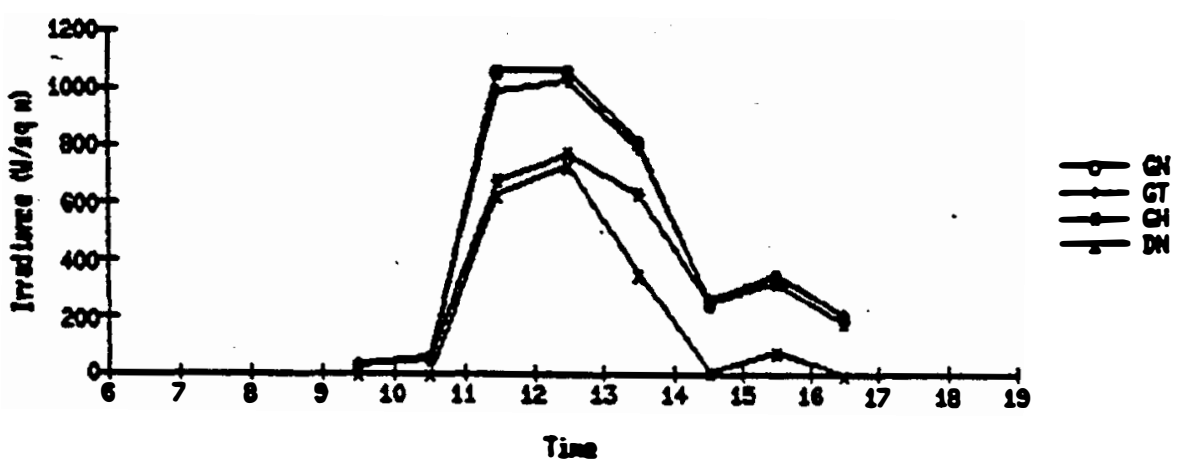

FISE 38 BN

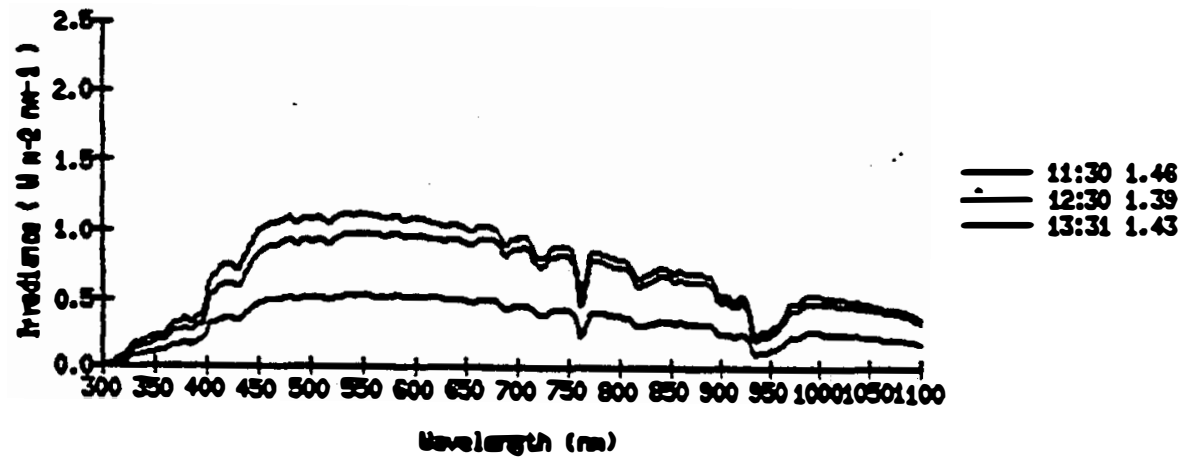

FEבC 38 GTS

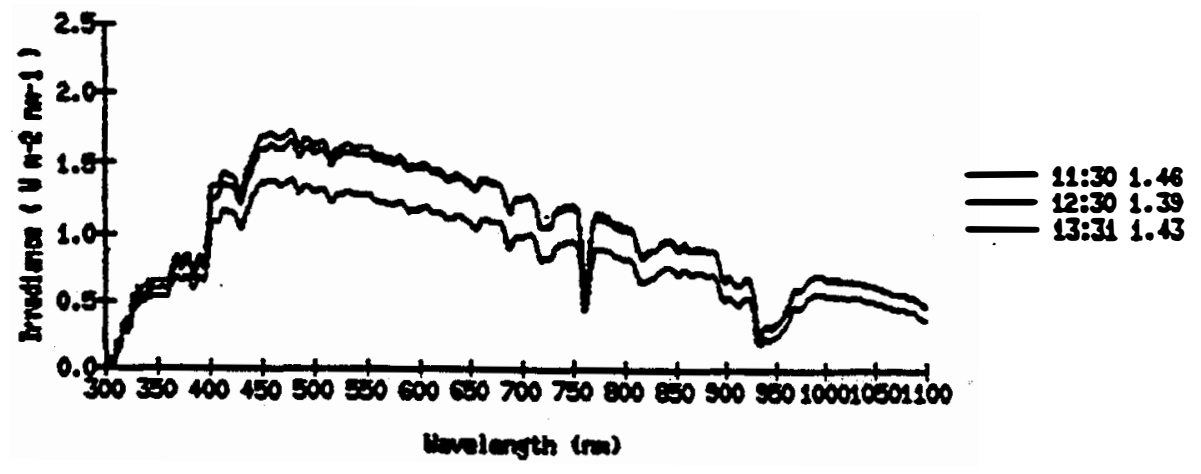




\section{SEPI}

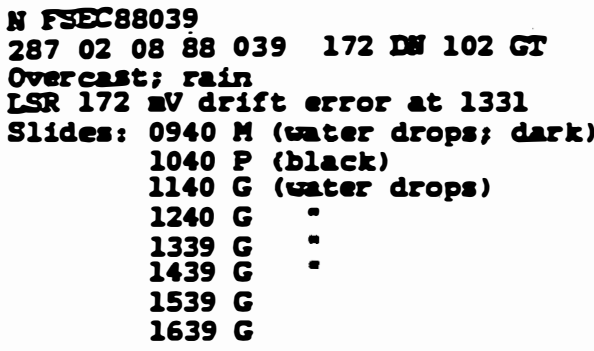

1539 G

$1639 \mathrm{G}$

an spectren bad at 1331 (no direct bear)

$F \leq 3039$

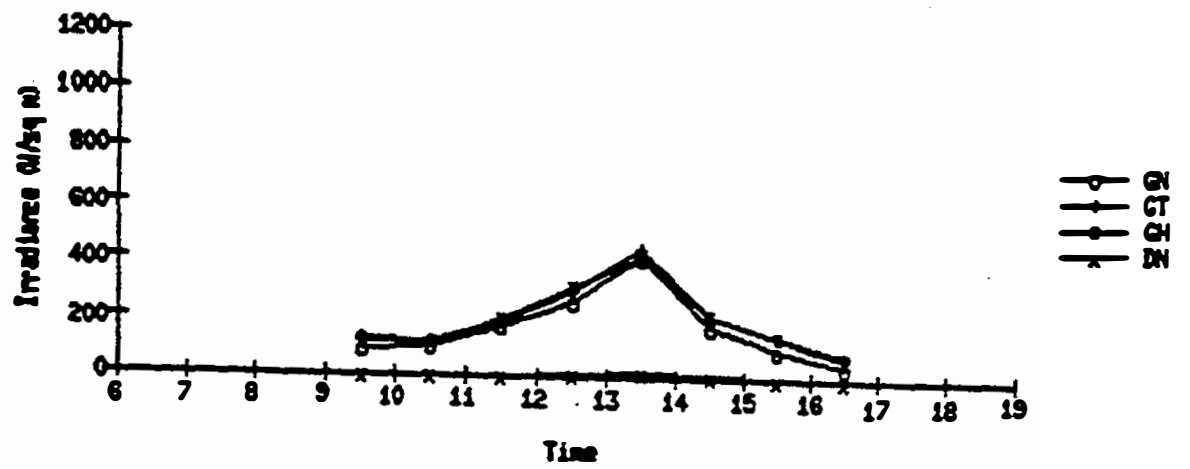

FEsc 30 an

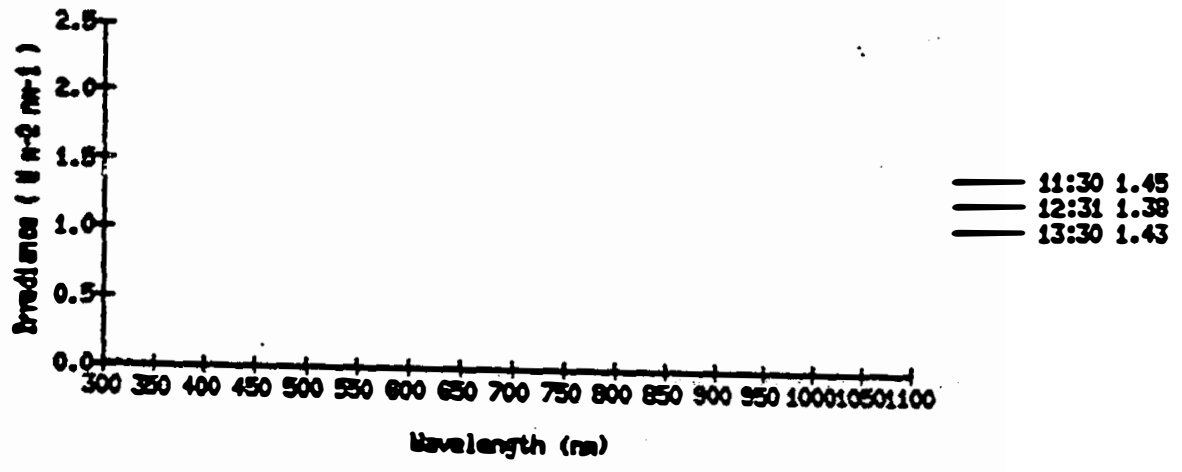

Psic 30 G5

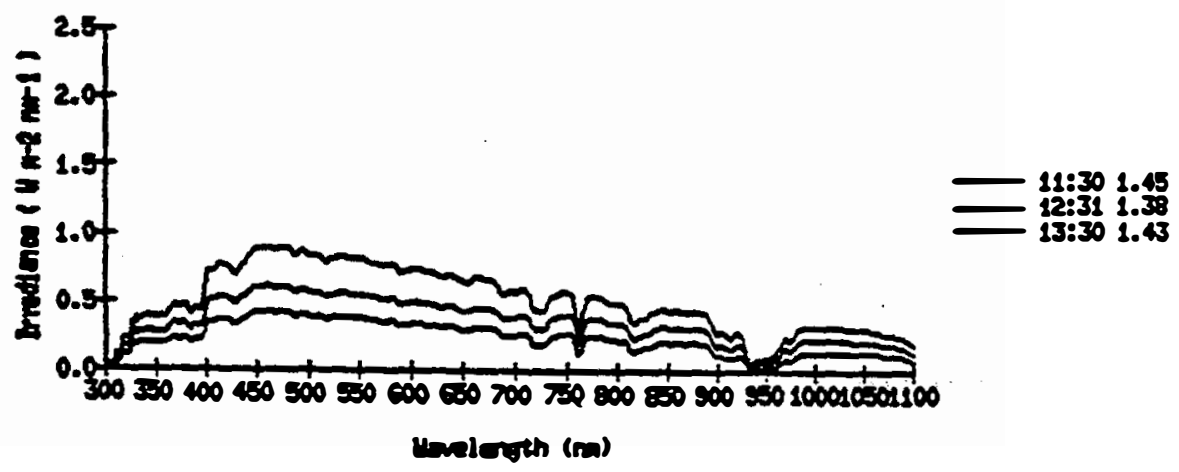


FST 88040

$\begin{array}{llllllllll}288 & 02 & 09 & 88 & 040 & 172 & \mathrm{Dm} & 102 & \mathrm{GT}\end{array}$

1.0 cloud corer at 0830,1230 , and 1630

Tenp controllers: $172 \quad 102$

083038 C $39 \mathrm{C}$

$\begin{array}{lllll}1230 & 38 & \mathrm{C} & 39 & \mathrm{C}\end{array}$

S11des: $0933 \mathrm{G}$

$1033 \mathrm{G}$

1233 H (dark)

$2233 \mathrm{P}$

$1432 M$ (dark)

$1532 \mathrm{G}$

$1632 \mathrm{G}$

$\operatorname{resc} 40$

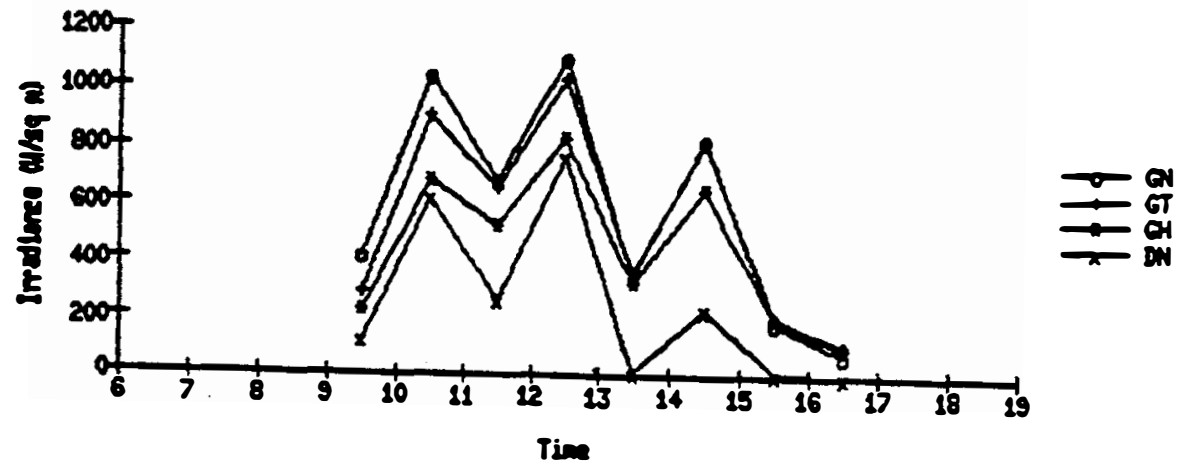

TEEC $40 \mathrm{mT}$

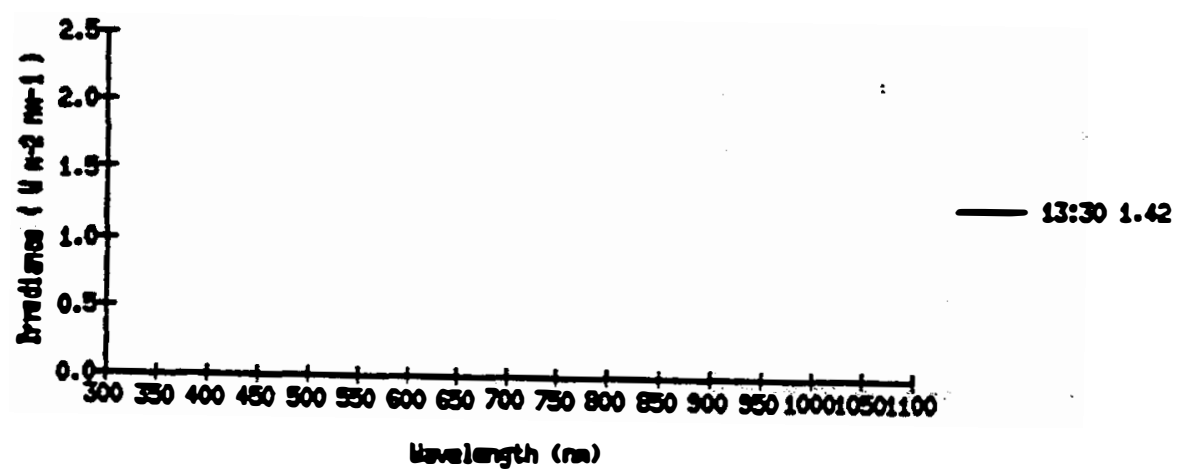

FSEC 40 GTS

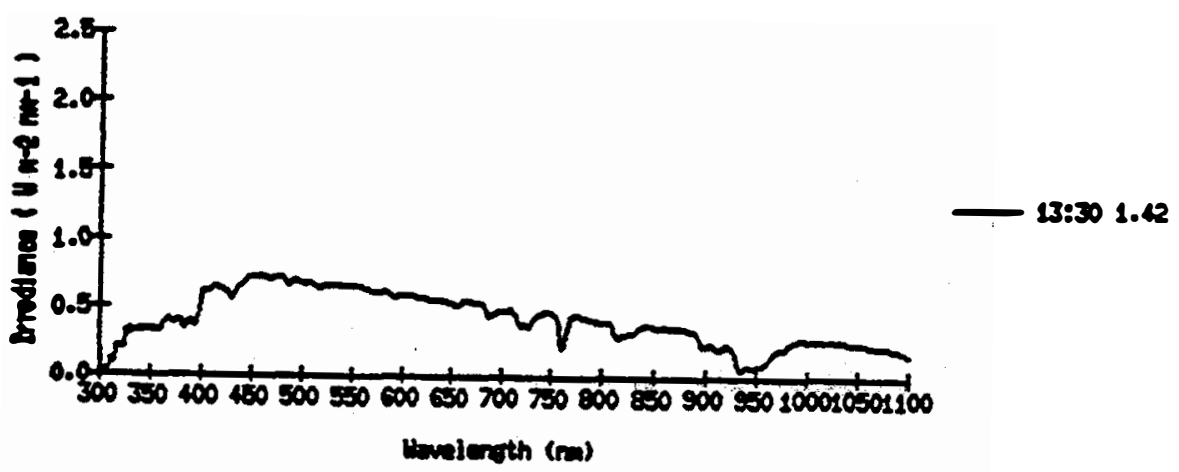


4. 35268041

$289021088041 \quad 172$ DI 102 Gr

0.7 cloud cover at $0830 ; 0.8$ at $1200 ; 1.0$ at 1700

ISR 172 av drift error at 0932

Slides: 0926 M (dark)

$1026 \mathrm{M}$ -

$2126 \mathrm{~A}:$

$1226 \mathrm{H}:$

1325

$1425 \mathrm{G}$

$1525 \mathrm{G}$

$1625 \mathrm{G}$

$\operatorname{FscC} 41$

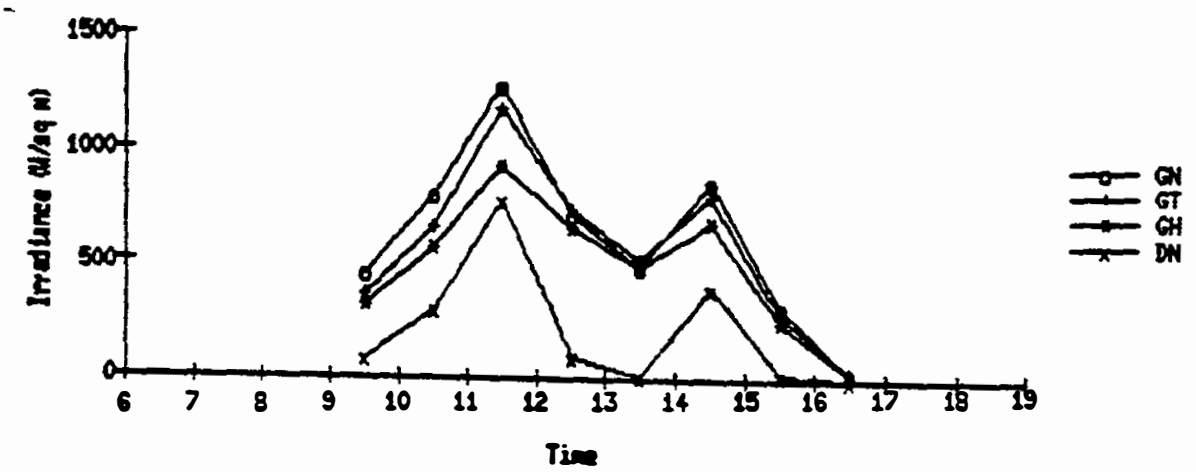

FEC 41 DT

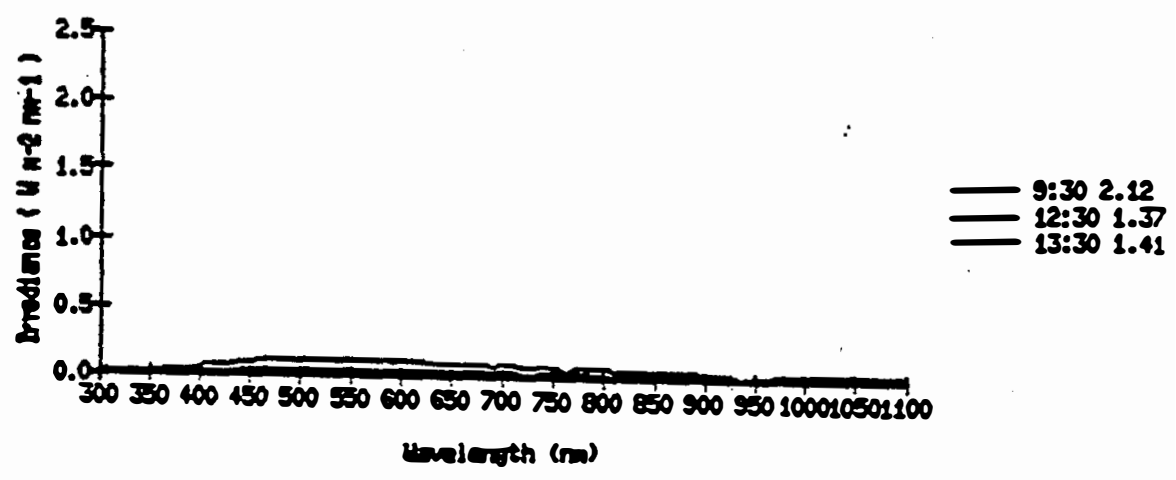

FES 42 GT

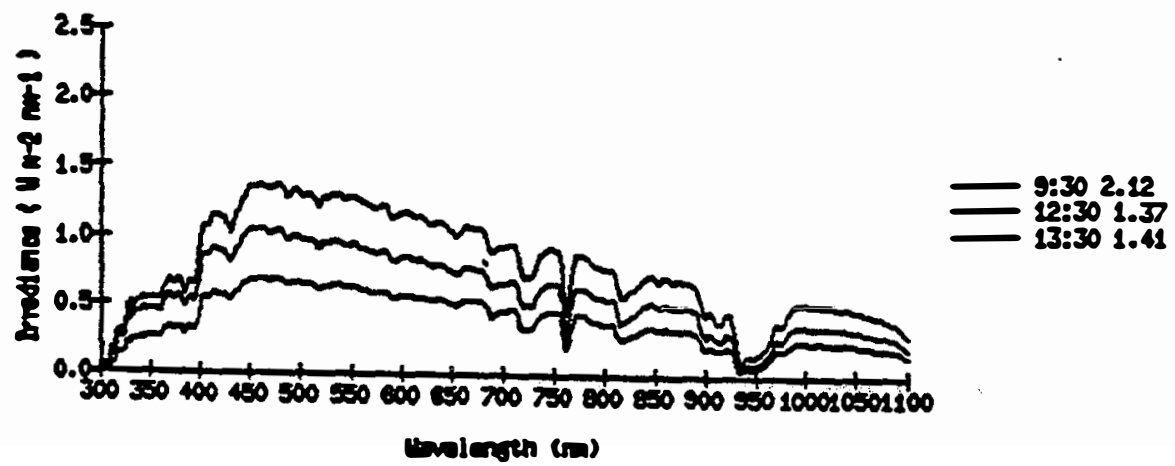



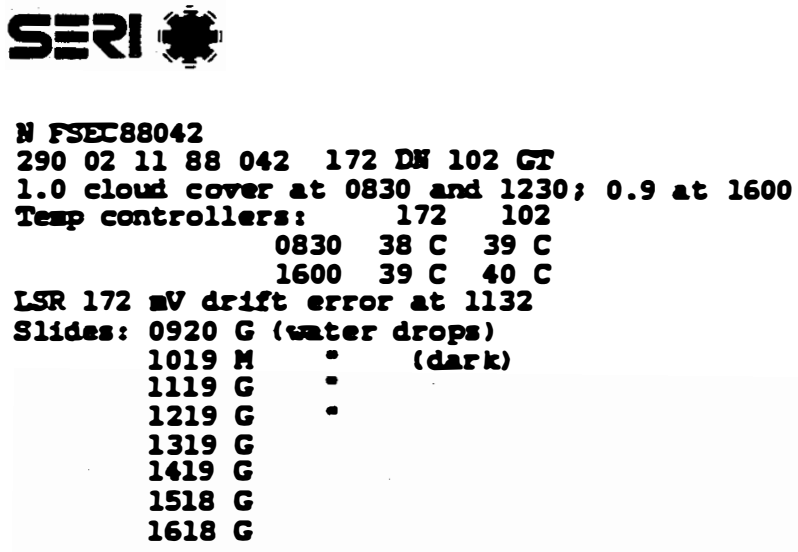

Dil spectru at 1132 is bed (no direct bear)

$\operatorname{csec} 42$

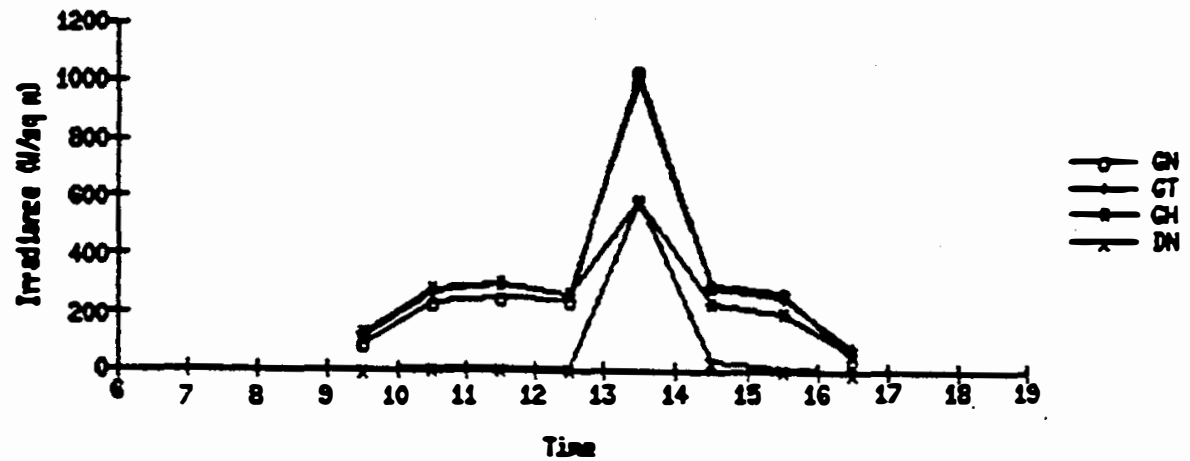

FEx 42 mT

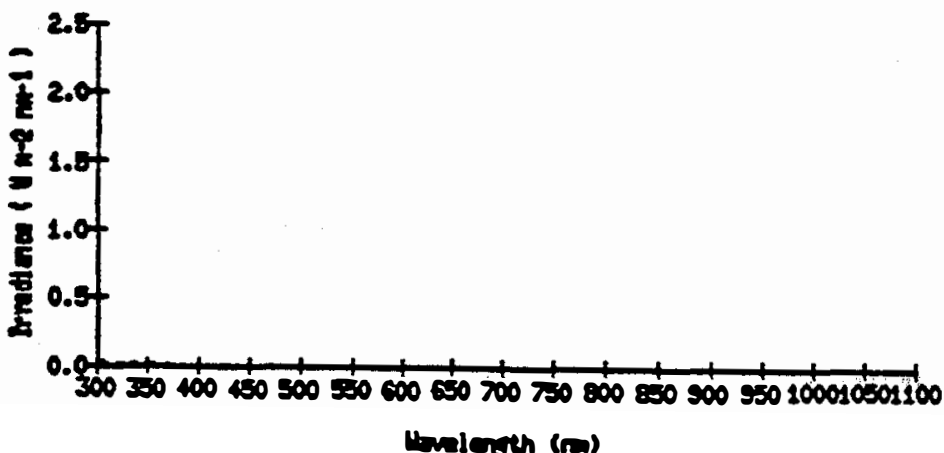

FIE 42 ETS

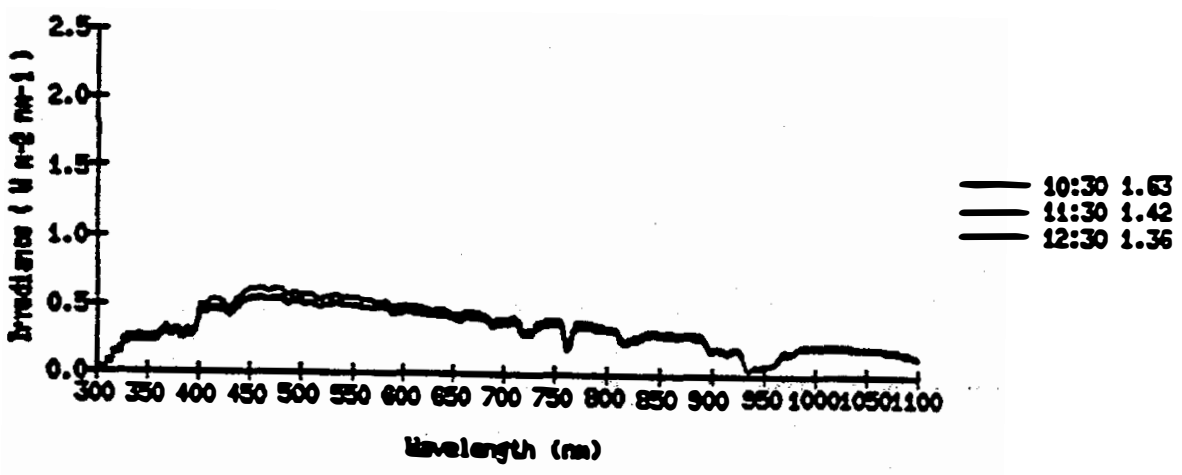


A FST 88043

$\begin{array}{lllllllll}291 & 02 & 12 & 88 & 043 & 172 & D & 102 & G T\end{array}$

1.0 cloud cover at 0830 ; 0.2 at 1400

Texp controllers: $172 \quad 102$

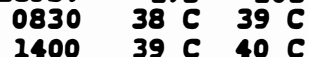

s11des: 0932 it (cordensat1on)

1032 I (dark)

$2132 \mathrm{P}:$

$1232 \mathrm{M}:$

$1431 \mathrm{M}$.

$1531 \mathrm{G}$

$1631 \mathrm{G}$

$\operatorname{Psec} 43$

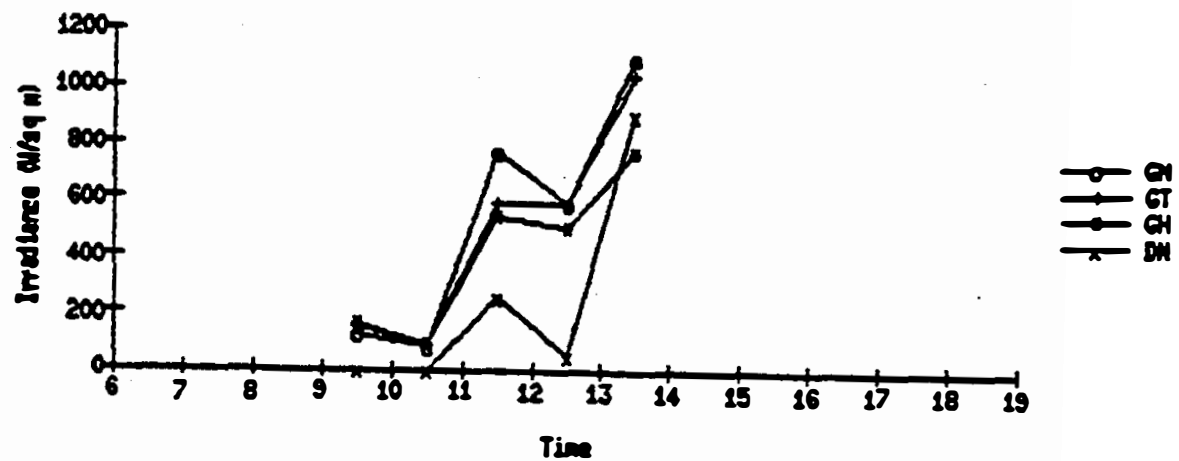

Fsec 43 ant

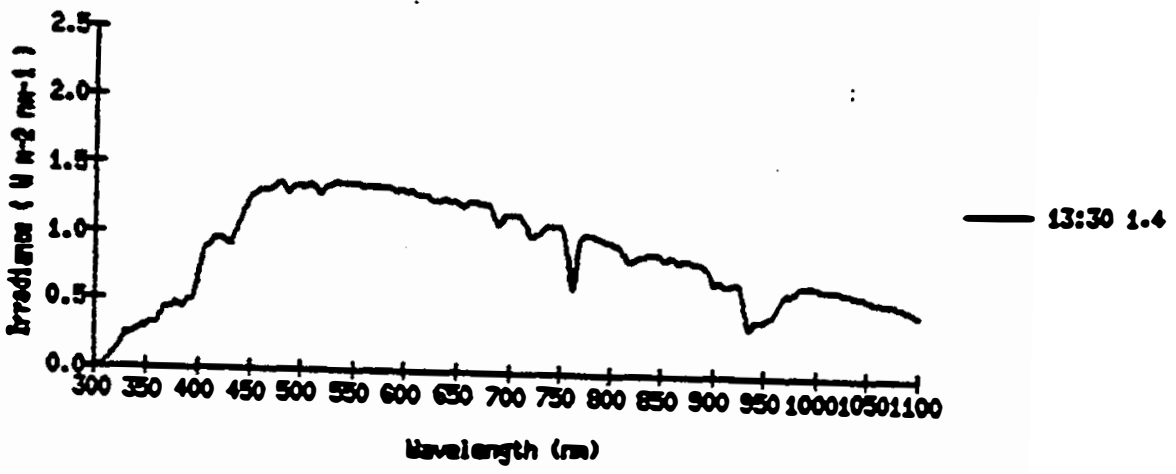

FIEC 43 GTS

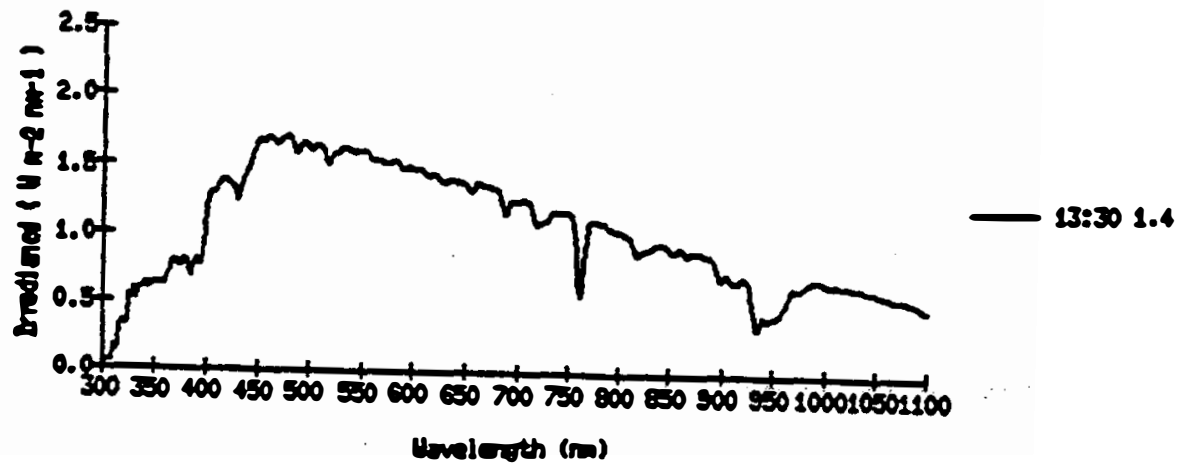


7 FIX 88044

$\begin{array}{llllllll}292 & 12 & 13.88 & 044 & 172 & \text { DA } & 102 & \text { GI }\end{array}$

Clear

ISR 172 and $102 \mathrm{aV}$ drift errors at 0837

Slides: $0826 \mathrm{G}$

$0926 \mathrm{G}$

$1026 \mathrm{G}$

$1226 P$ (dark)

$1226 P$ :

$1326 \mathrm{P}$

$1525 \mathrm{G}$

$1625 \mathrm{G}$

$\operatorname{PSEC} 44$

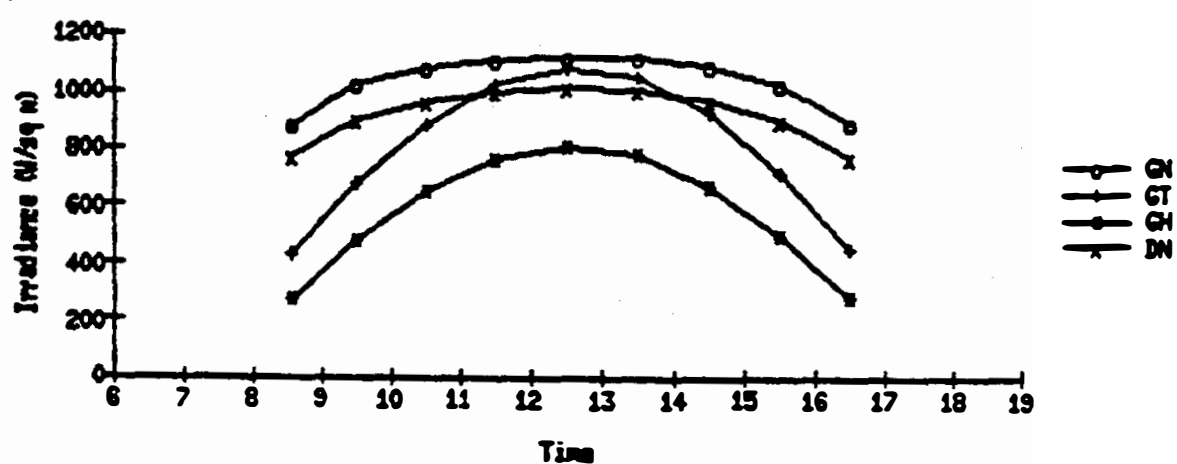

Fas $4 \mathrm{DT}$

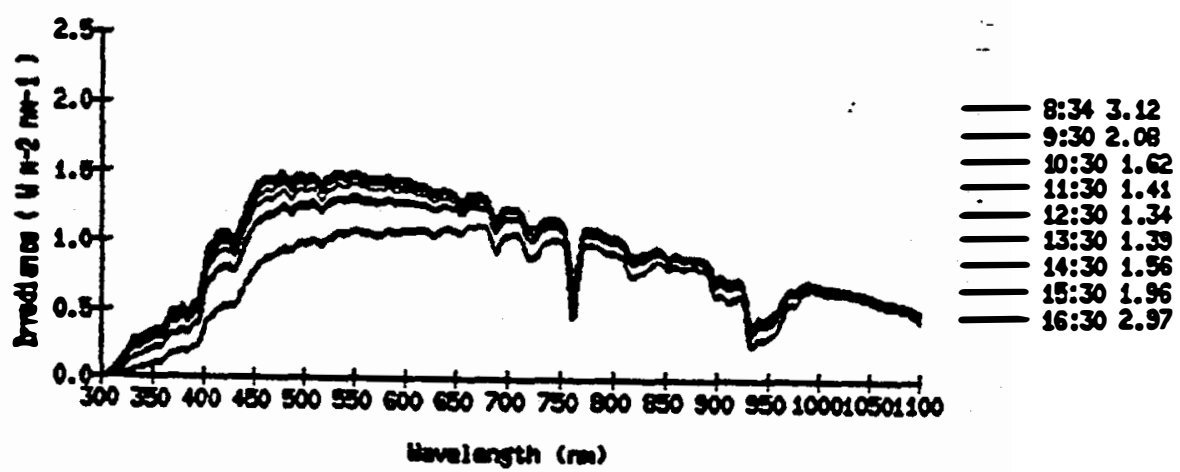

FIX 44 GTS

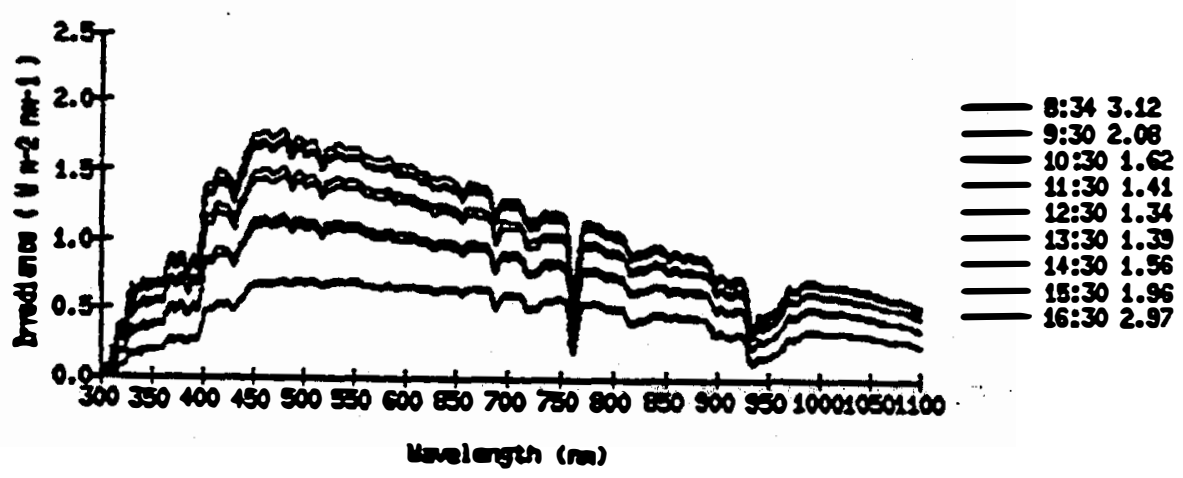




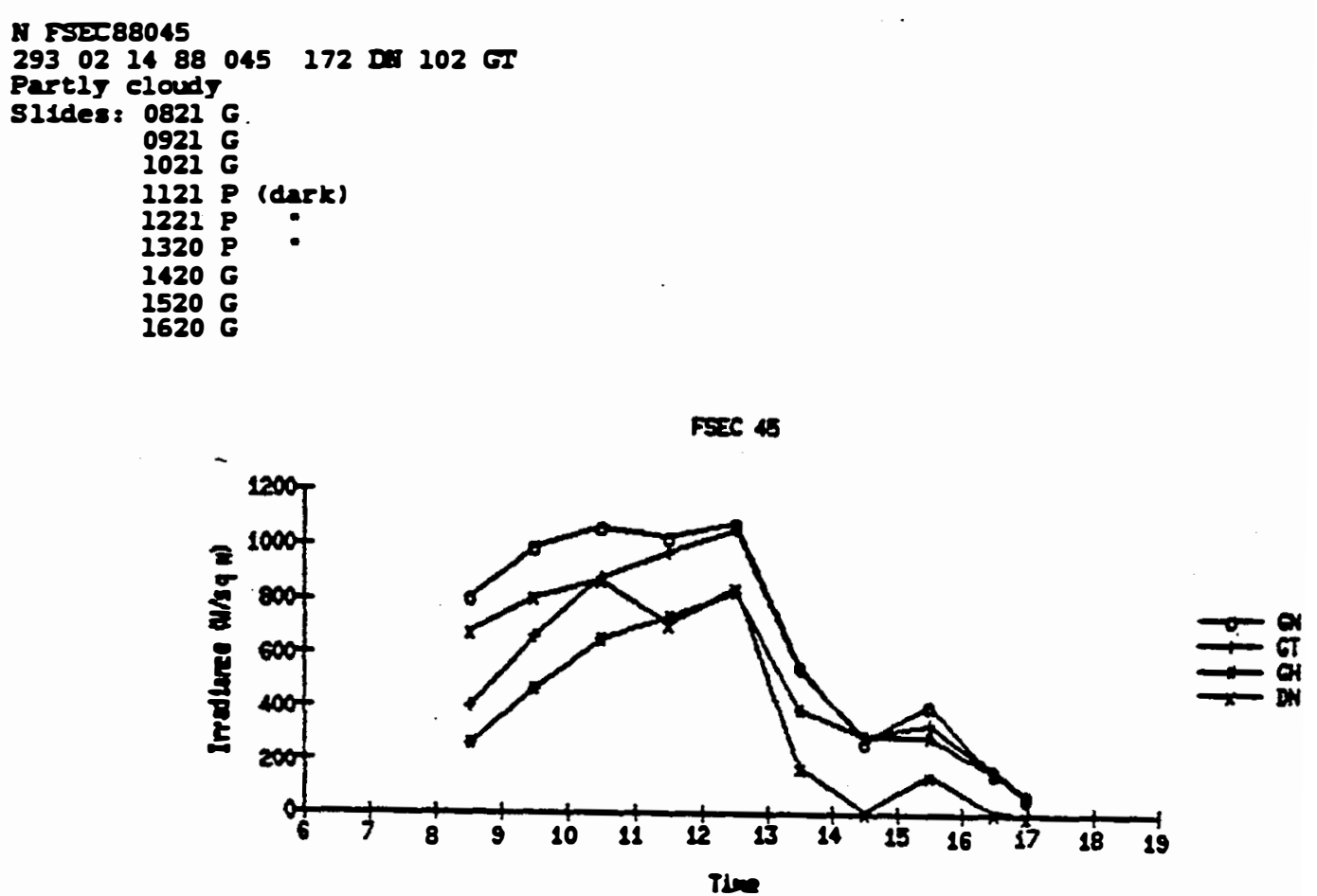

PSEC 45 INT

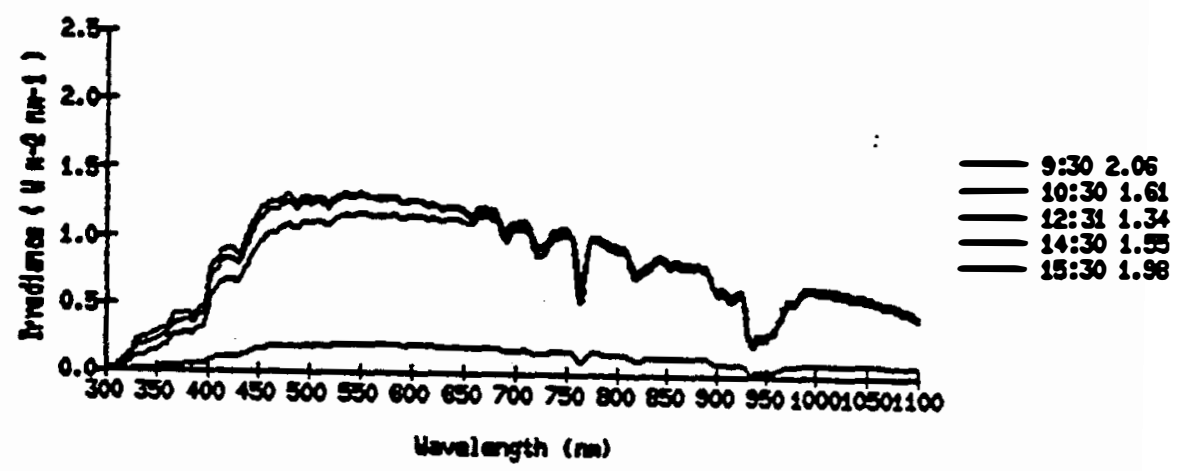

PSEC 45 GT

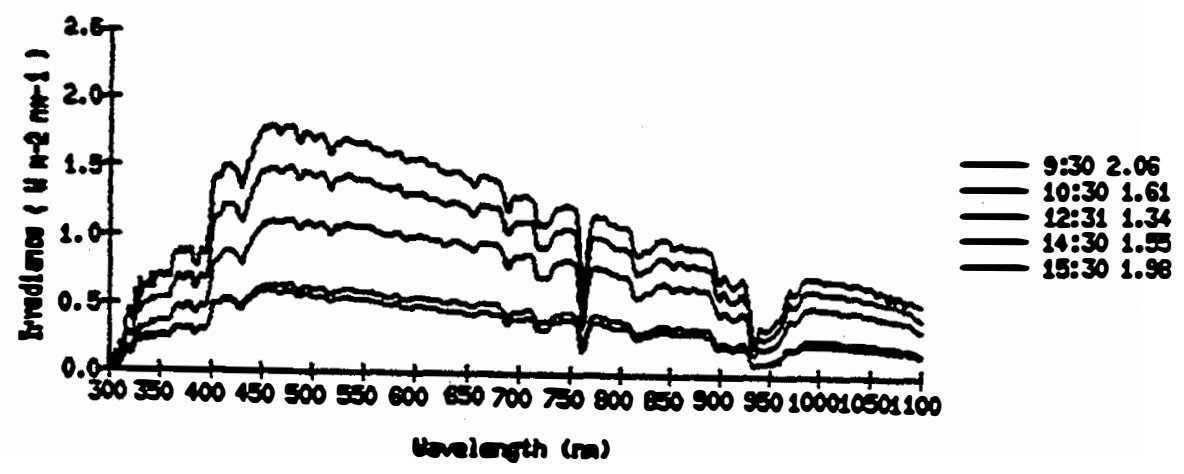


A FST्C88047

$\begin{array}{llllllllll}294 & 02 & 16 & 88 & 047 & 172 & 04 & 102 & \text { Gs }\end{array}$

0 cloud corer at 1100 and 1630

Tenp controllers $\quad 172 \quad 102$

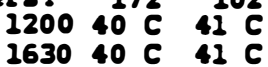

Do slides

F5e 47

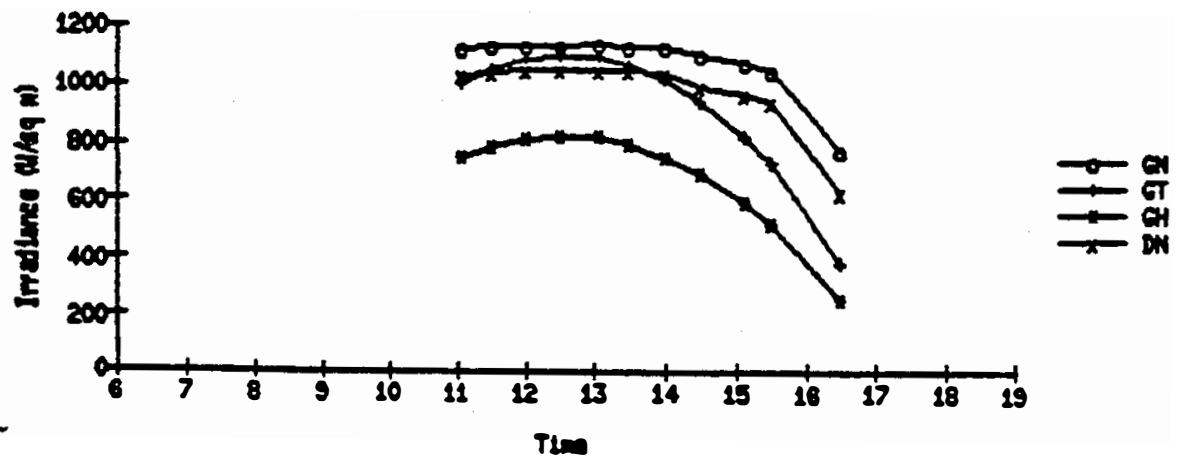

$\operatorname{csec} 67$ ant

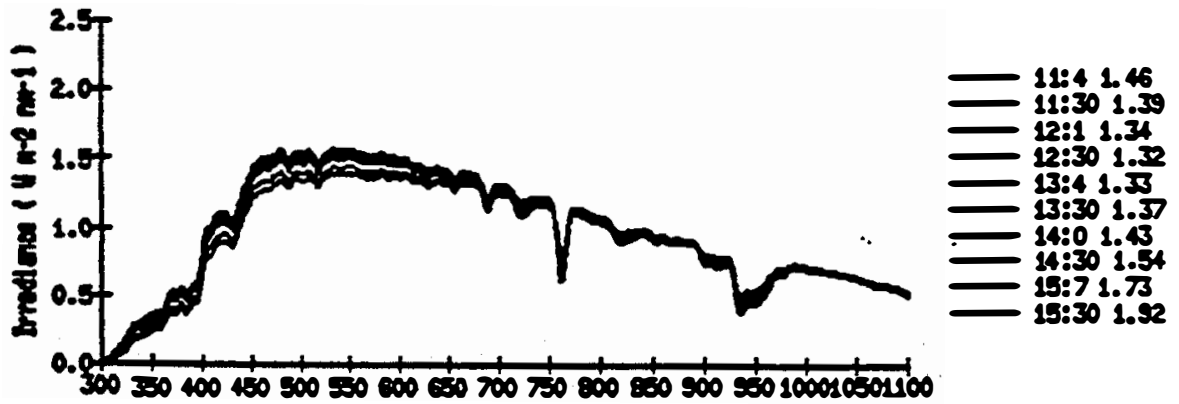

valoligth (m)

$\operatorname{sisc} 40$ os

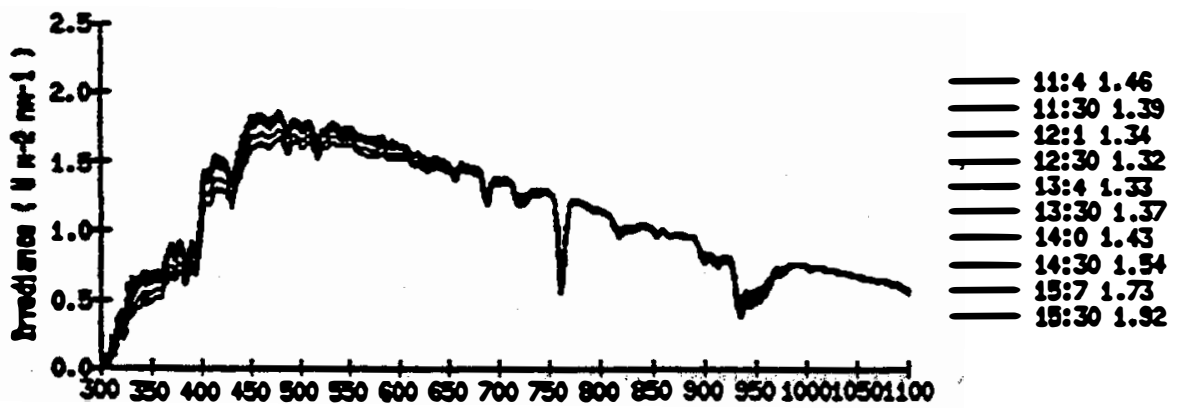

vaclergth (ra) 
ASTC88048

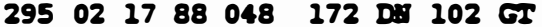

0.1 cloud cover at 0830 and $1230 ; 0.6$ at 1630

Tenp controllers: $172 \quad 102$

$123039 \mathrm{C} \quad 40 \mathrm{C}$

S11des: 1333 P (dark)

$1433 \mathrm{~K}$

$1532 \mathrm{G}$

$1632 \mathrm{G}$

Fise 48

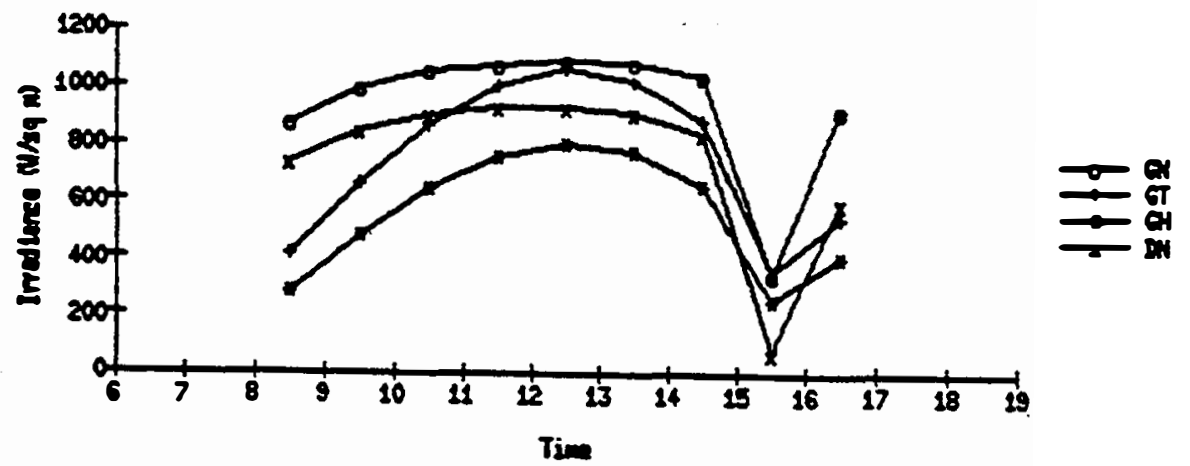

FIEC 48 DNT

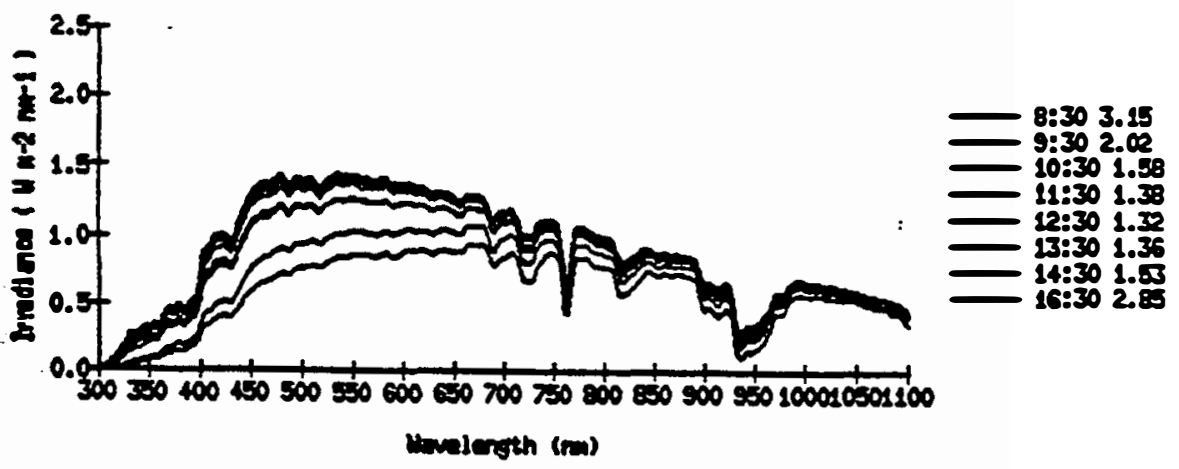

Fise 48 Os

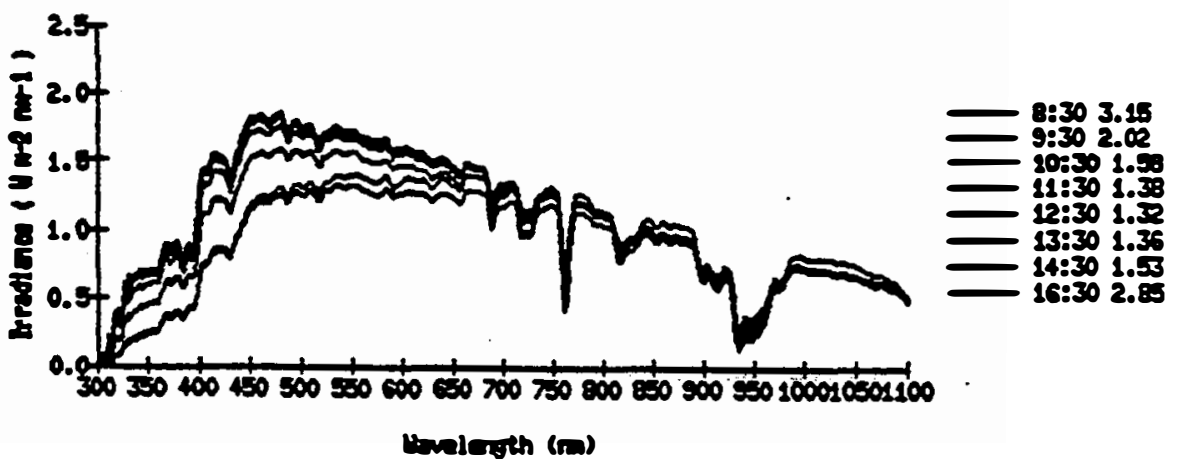




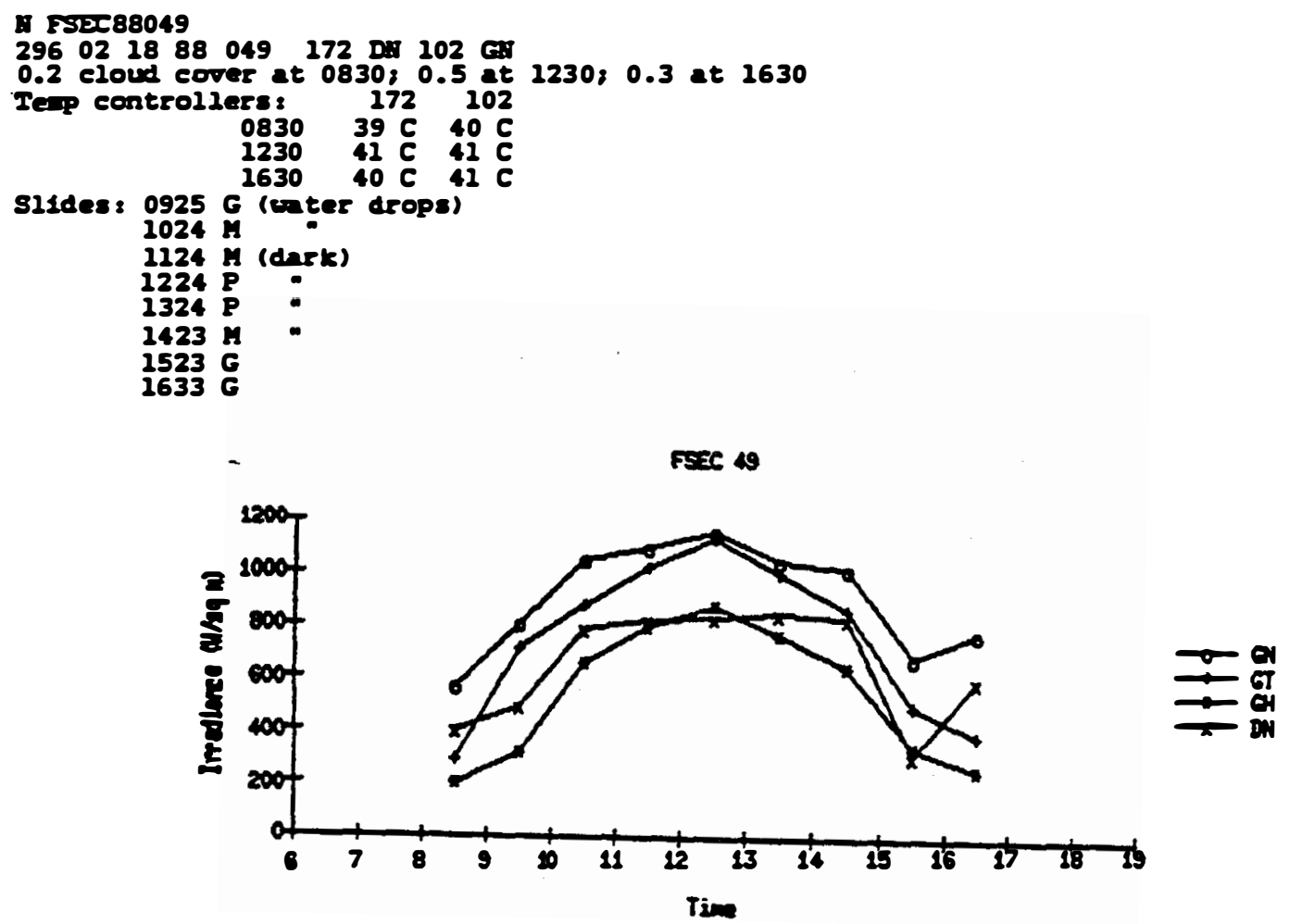

FEC 10 BN

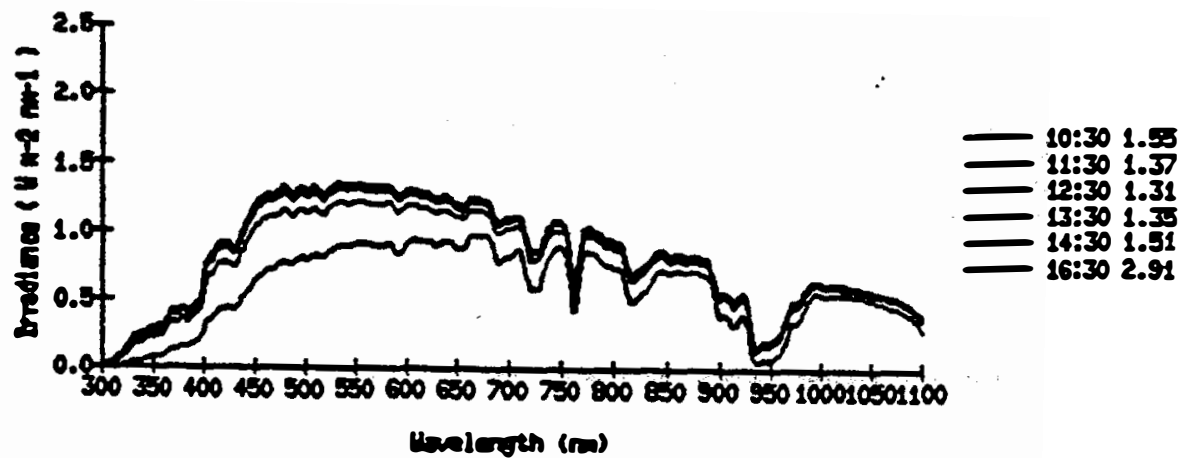

Fasc of as

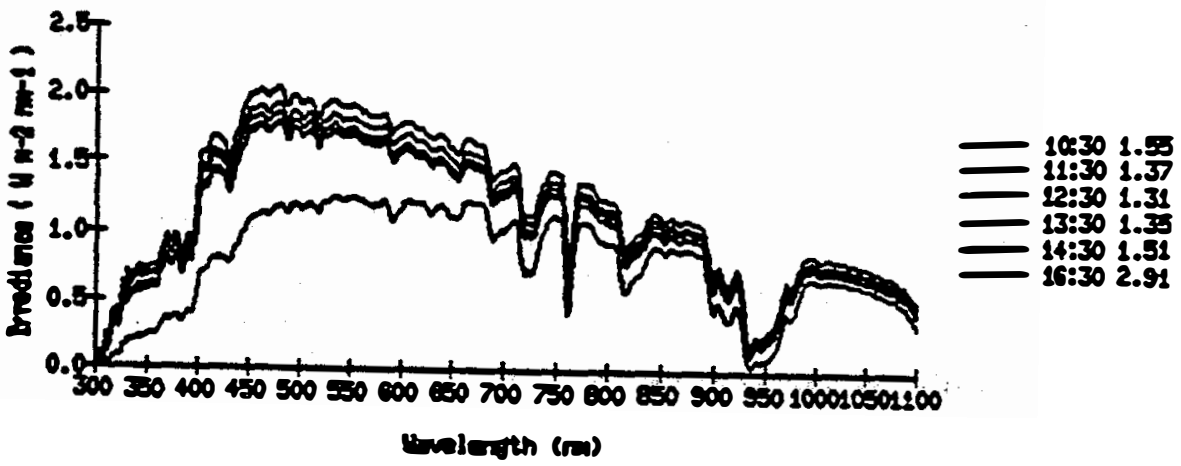


1) FSP्C88050

$\begin{array}{lllllllll}297 & 02 & 19 & 88 & 050 & 172 & \text { D: } & 102 & \mathrm{GI}\end{array}$

1.0 cloud corer at 083080.8 at 130080.9 at 1630

Iep controllers: $172 \quad 102$

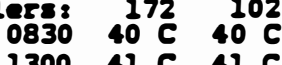

$163041 \mathrm{C} 40 \mathrm{C}$

sl1dess $0820 \mathrm{G}$

$0933 \mathrm{H}$ (dark)

$1020 \mathrm{G}$

$1119 \mathrm{G}$

$1219 P$ (dark)

$1319 \mathrm{P}$

$1418 \mathrm{G}$

$1518 \mathrm{G}$

$1634 \mathrm{G}$

vis doin on 22088

$\operatorname{Pres} 50$

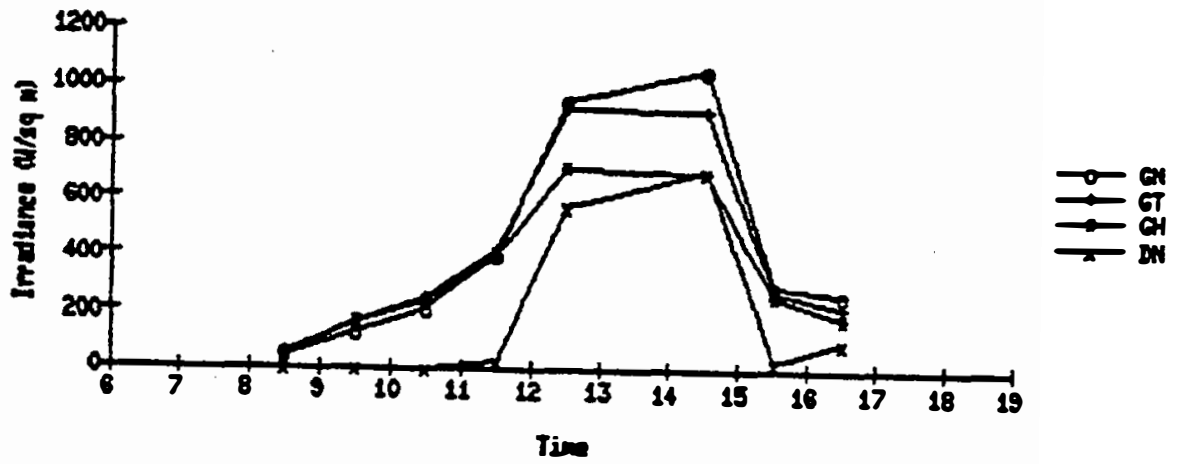

$\operatorname{cosec} 00 \mathrm{dx}$

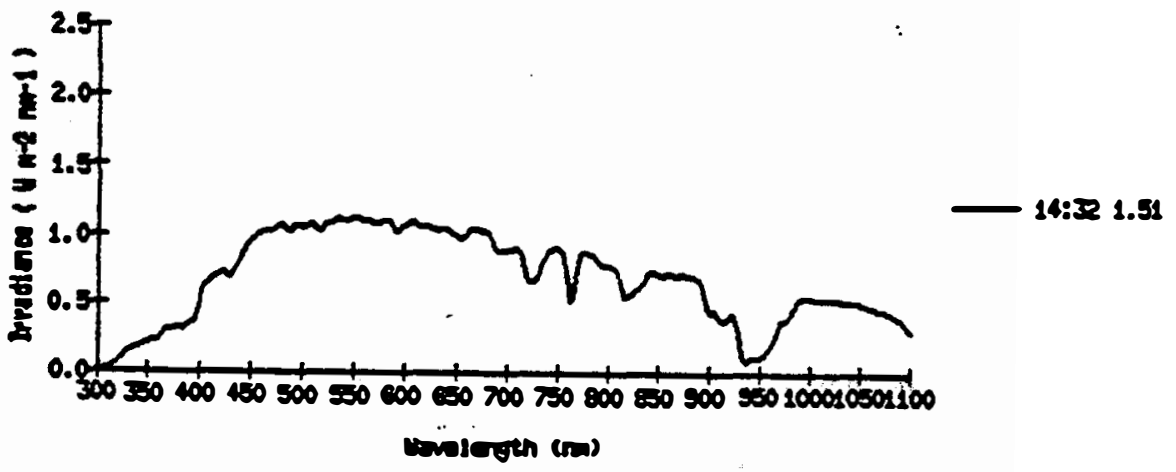

PIEC 50 OS

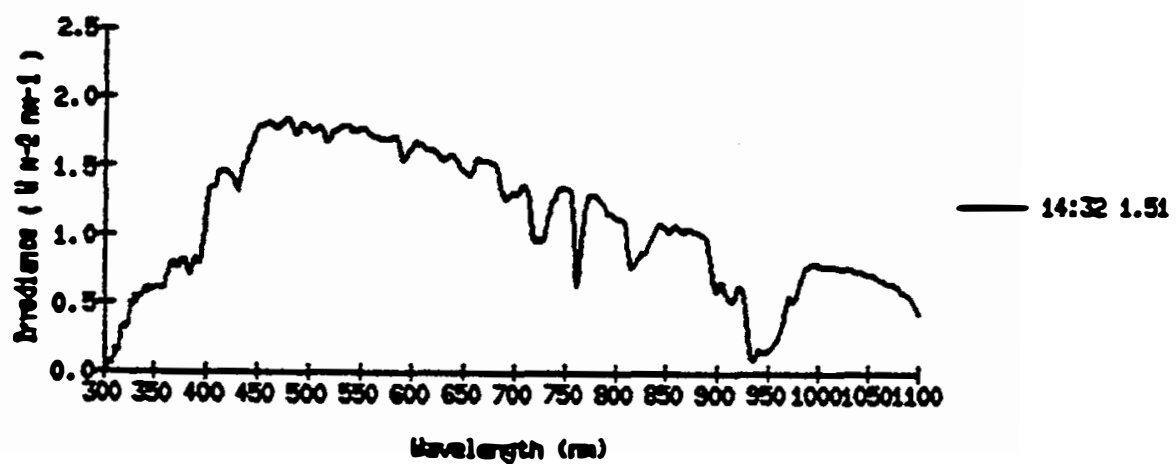


$\Rightarrow=011$

1. 55088052

$\begin{array}{llllllllll}298 & 02 & 21 & 88 & 052 & 172 & \mathrm{DA} & 102 & \mathrm{G}\end{array}$

overcast to partiy cloudy

Yo slides

FSE $x$

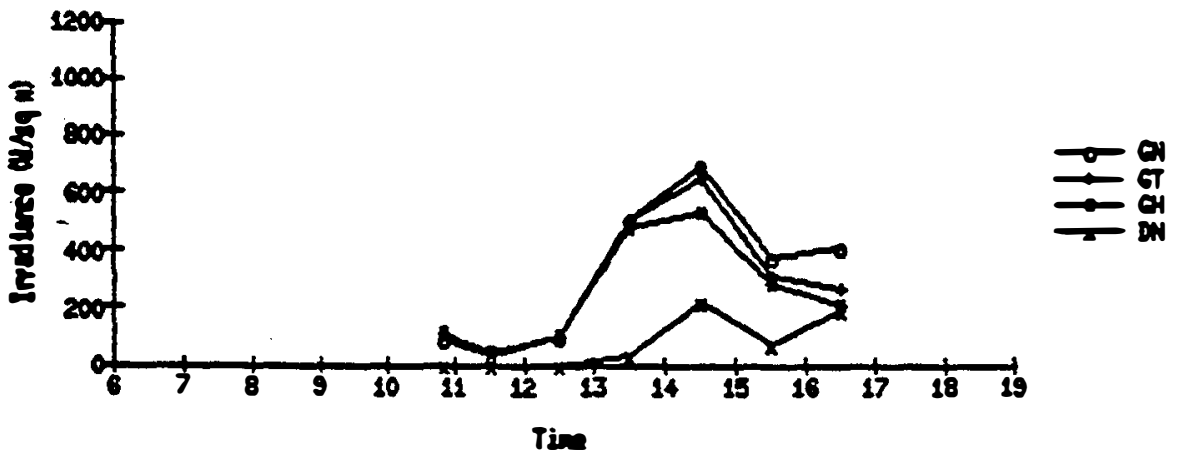

FIEC ER DNT

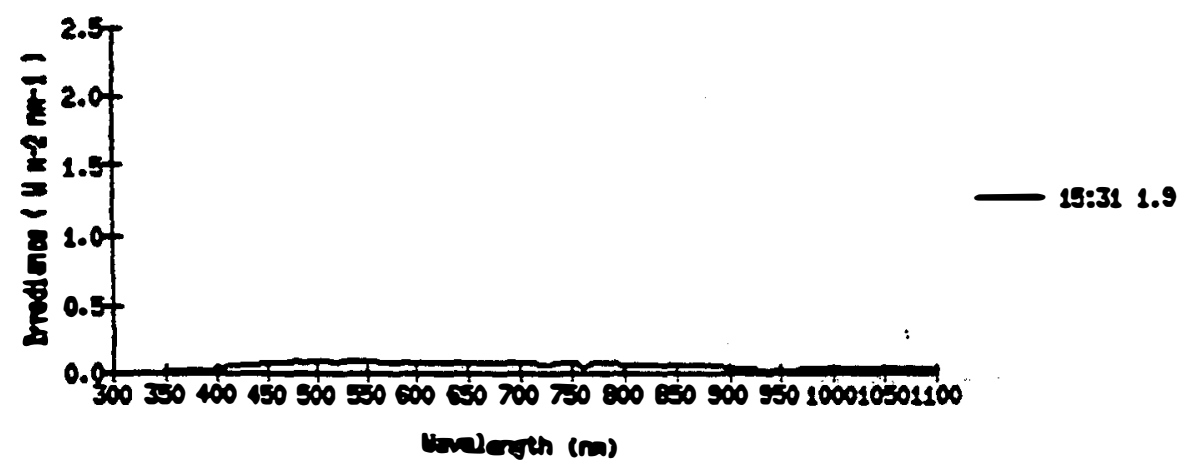

Psec as as

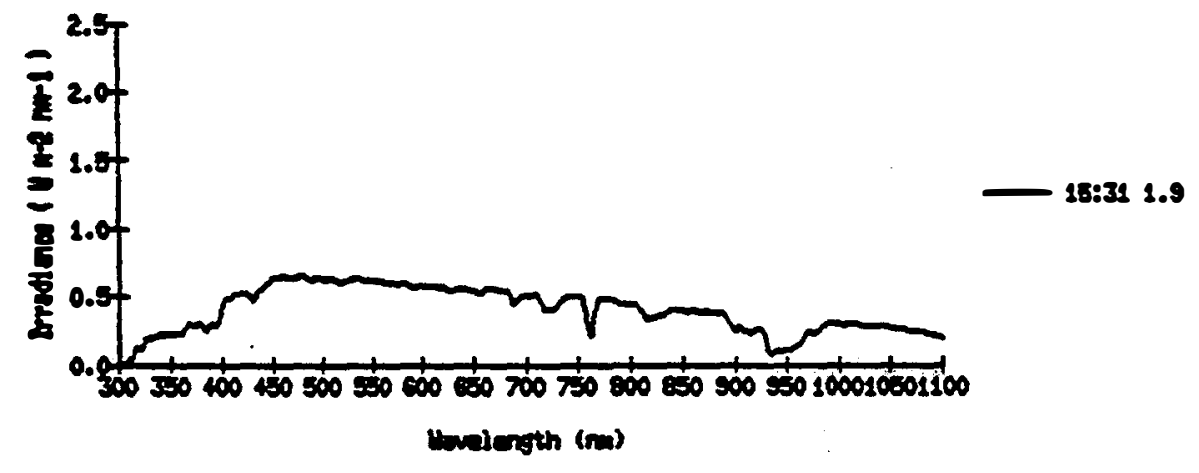




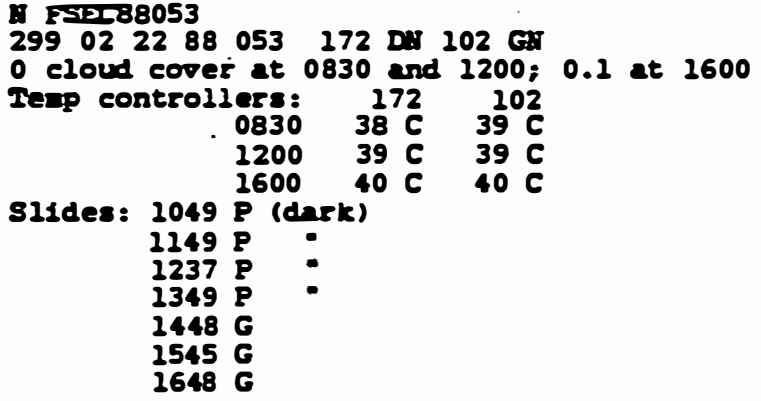

$F=53$

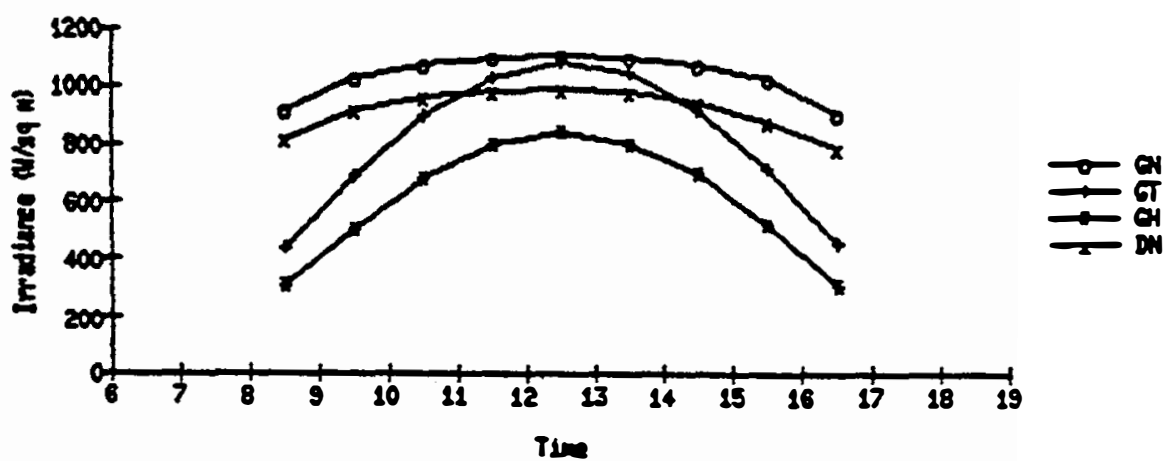

FSEC 53 DIT

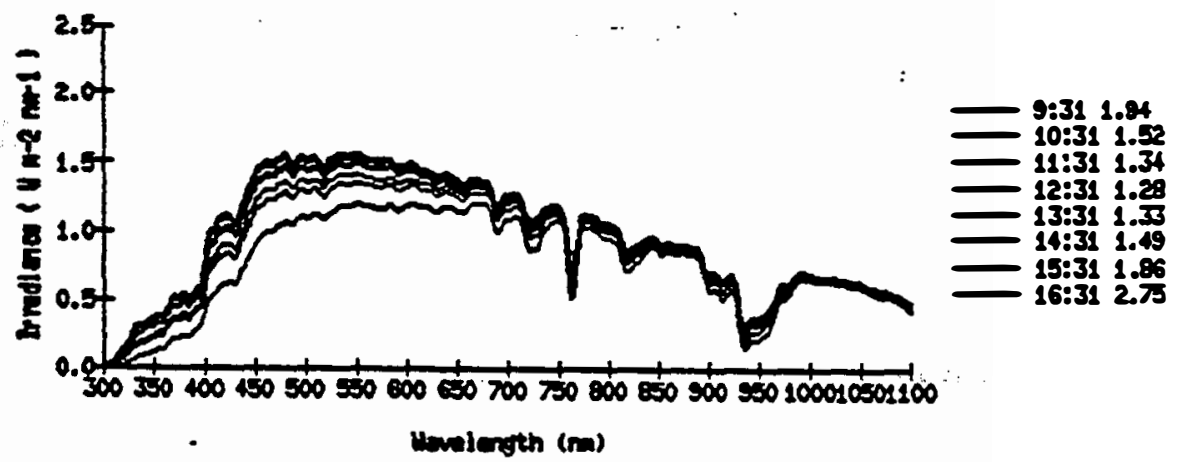

$\operatorname{resc} 50$

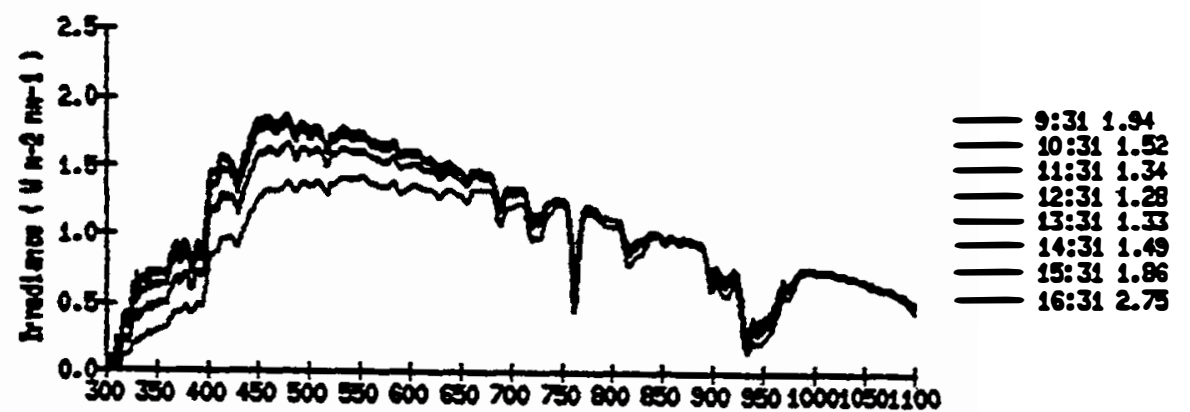

Uaviength (m) 


\section{SEP *}

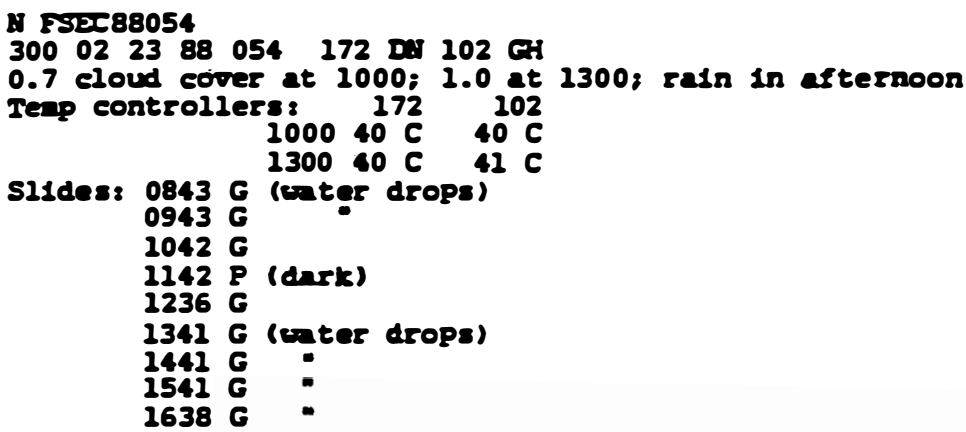

Fsse s4

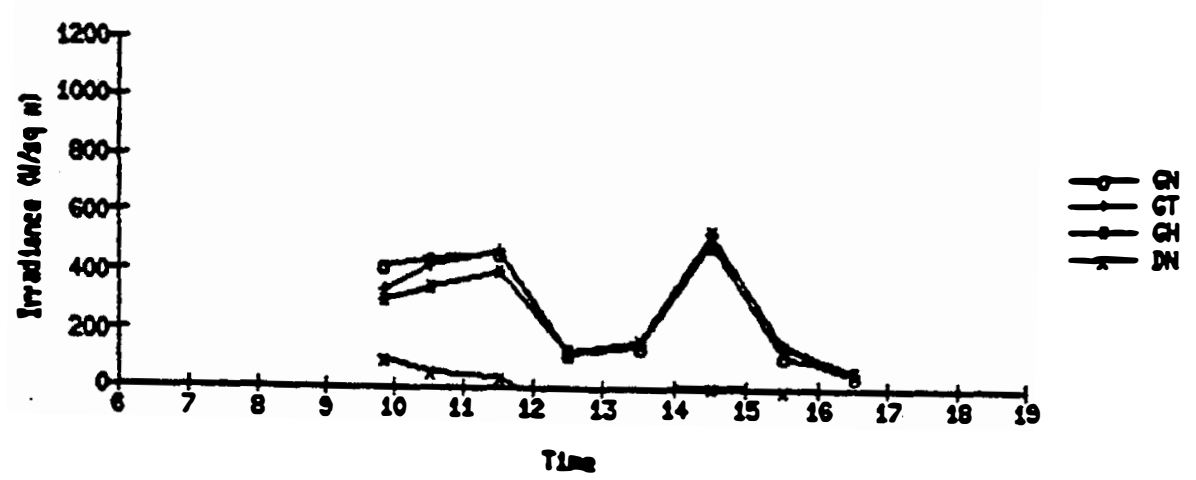

FES SA

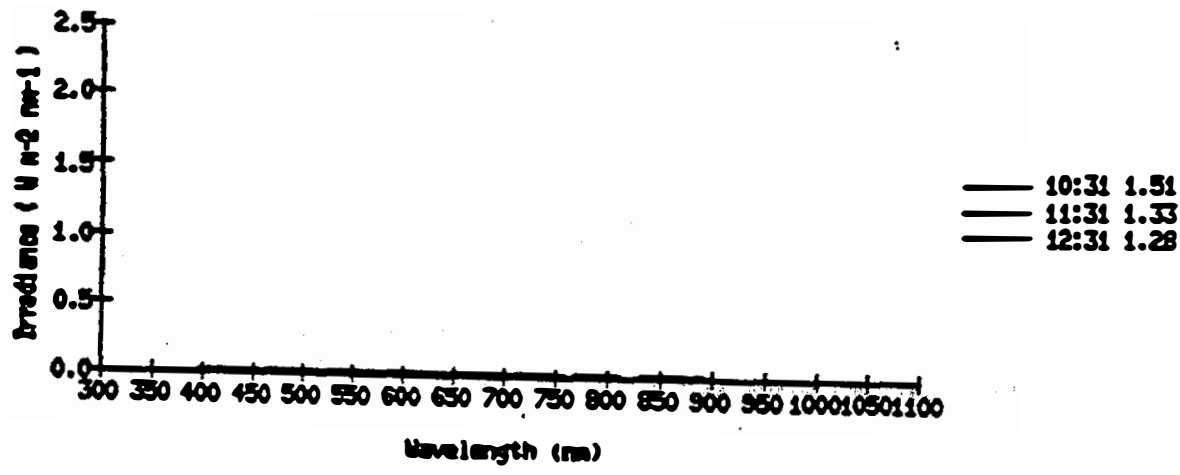

PSET 54 95

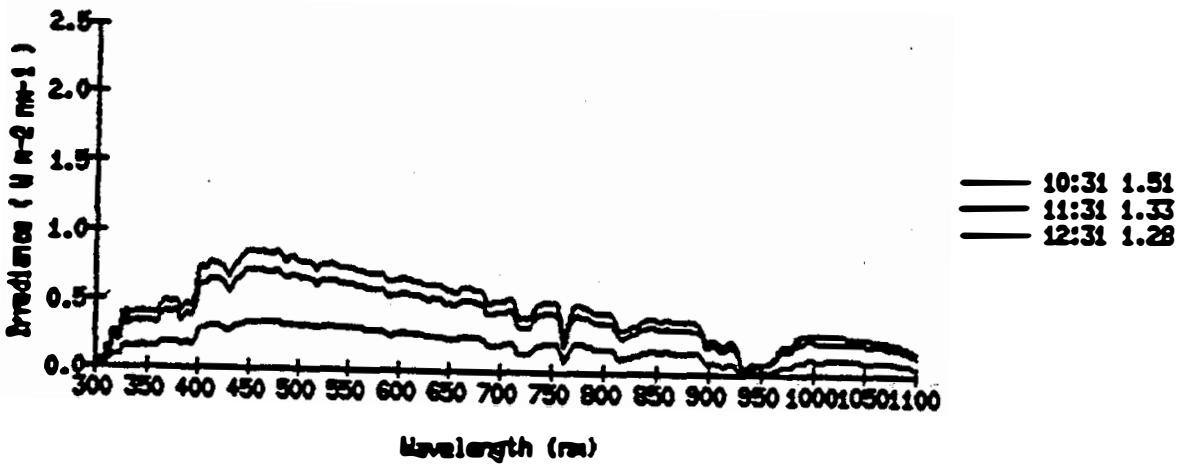


B. 5208055

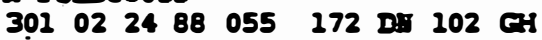

1.0 eloud cover at $1000 ; 0.1$ at 1530

Teap controllers: $172 \quad 102$

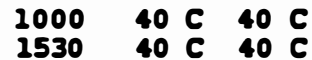

Ho slldes

FEsc as

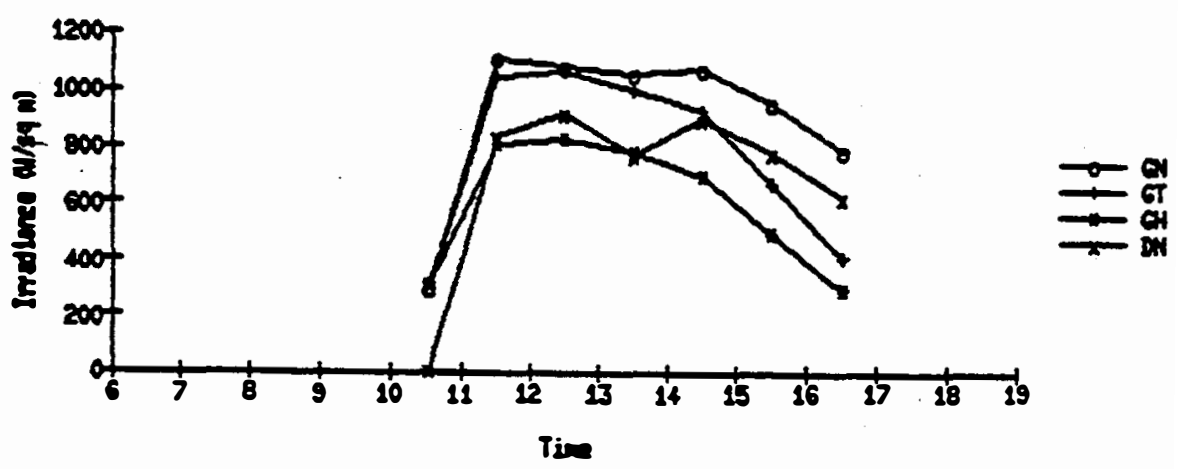

Fic

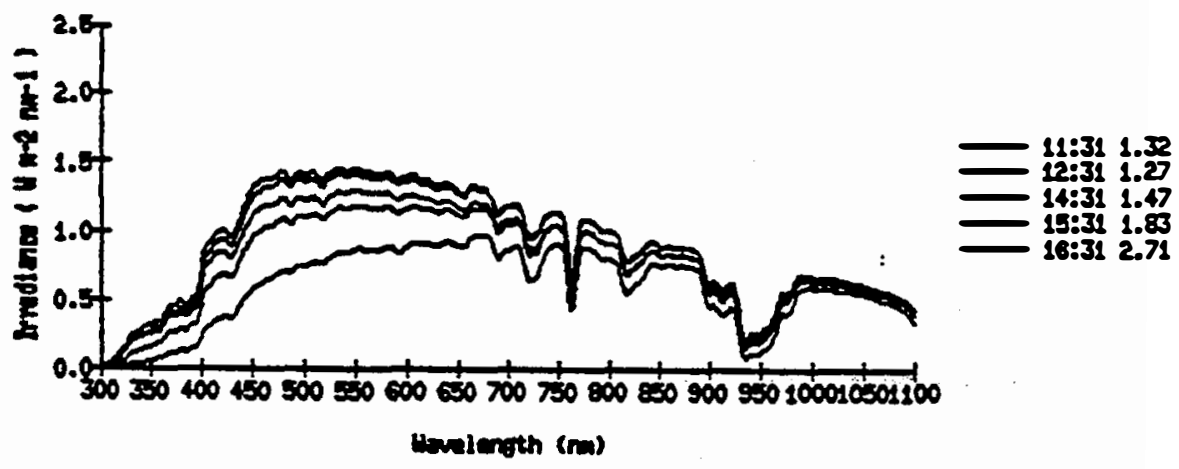

Fis 508

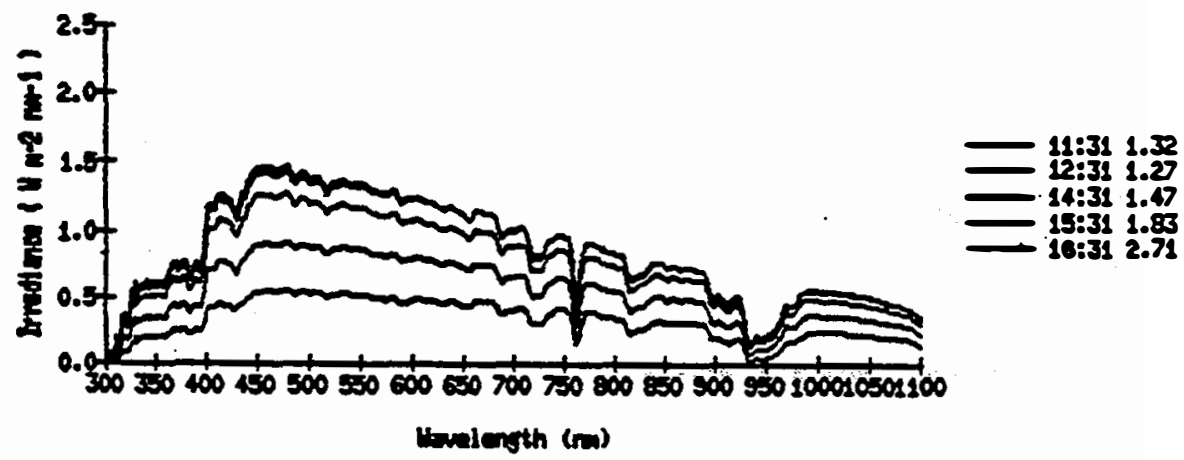




\section{SEPI *}

T. TS288056

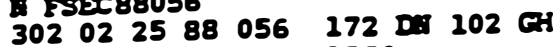

clens at 0815 and 1230

rop control1ers 172

$\begin{array}{lll}0815 & 38 \mathrm{C} & 102\end{array}$

$1230 \quad 39 \mathrm{C} 40 \mathrm{C}$

$160039 \mathrm{C} 40 \mathrm{C}$

Ho slides

Tases

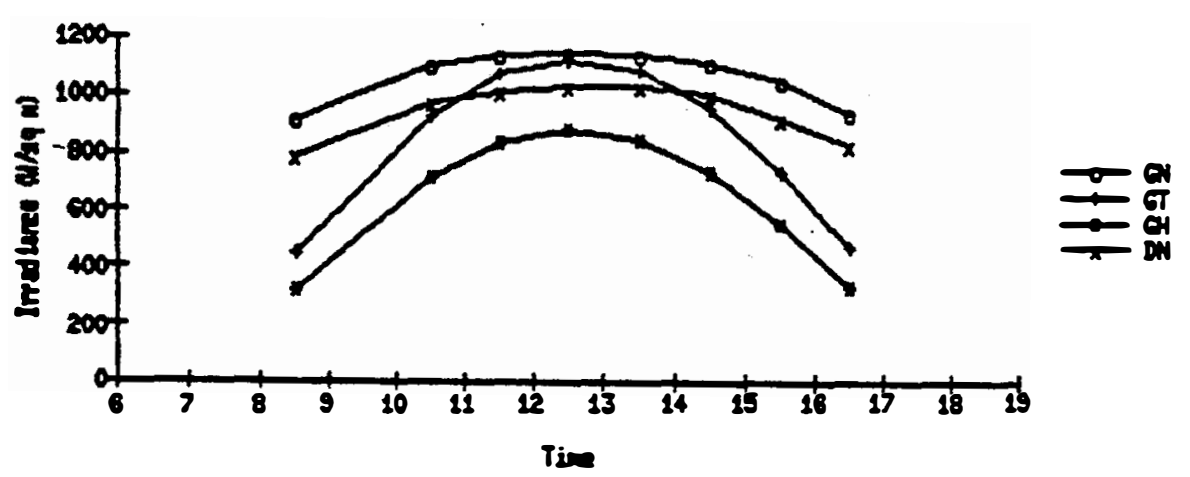

PSEC 05 ent

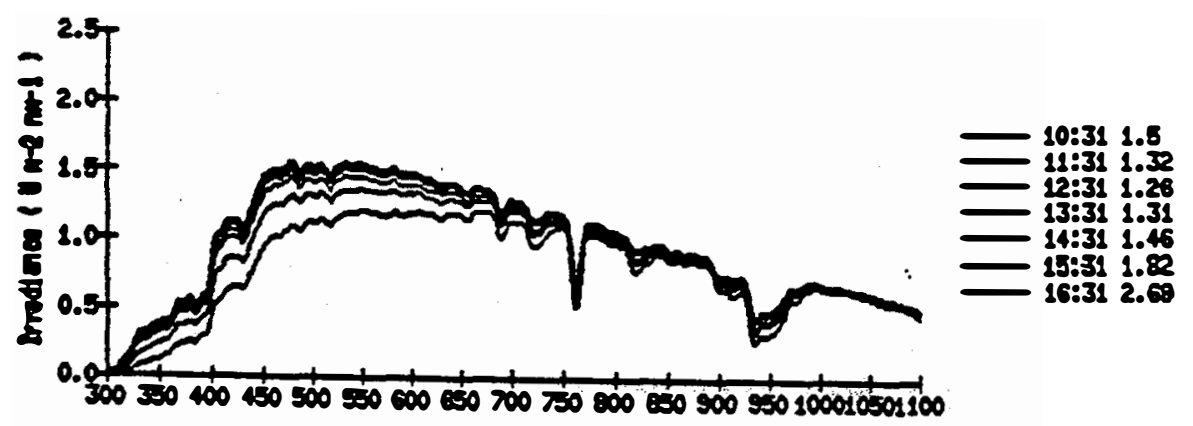

Holerith (m)

Psx6 so os

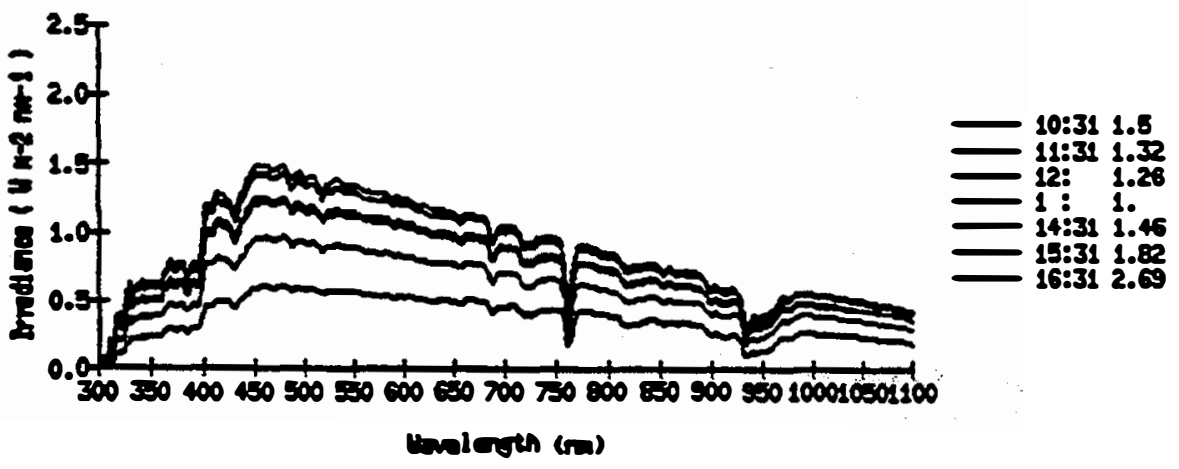




\section{SEP * *}

A 75288057

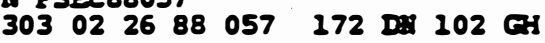

0 cloud corer at 0815: 0.1 at 1300 and 1630

Temp controllers: $172 \quad 102$

081538 C 39 C

$1300 \quad 39$ C 40 C

Ho slides

$1630 \quad 39$ C 40 C

FSEC $\Omega$

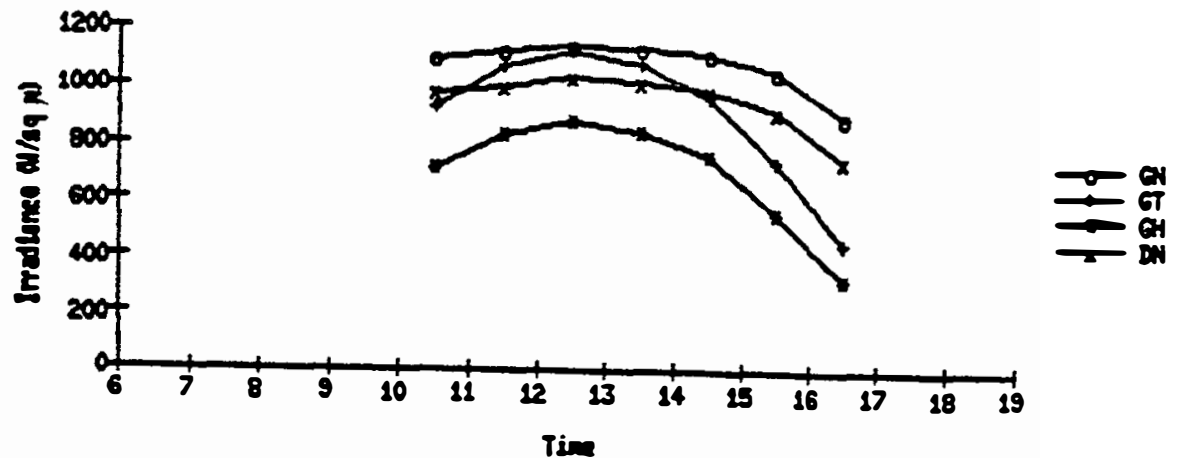

FEב $87 \mathrm{mT}$

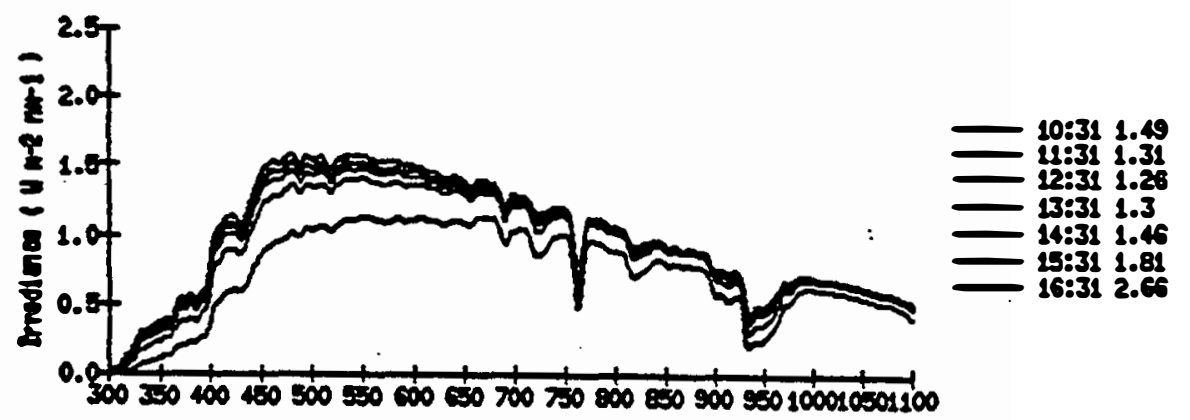

Howiergth (m)

Fise 57 as

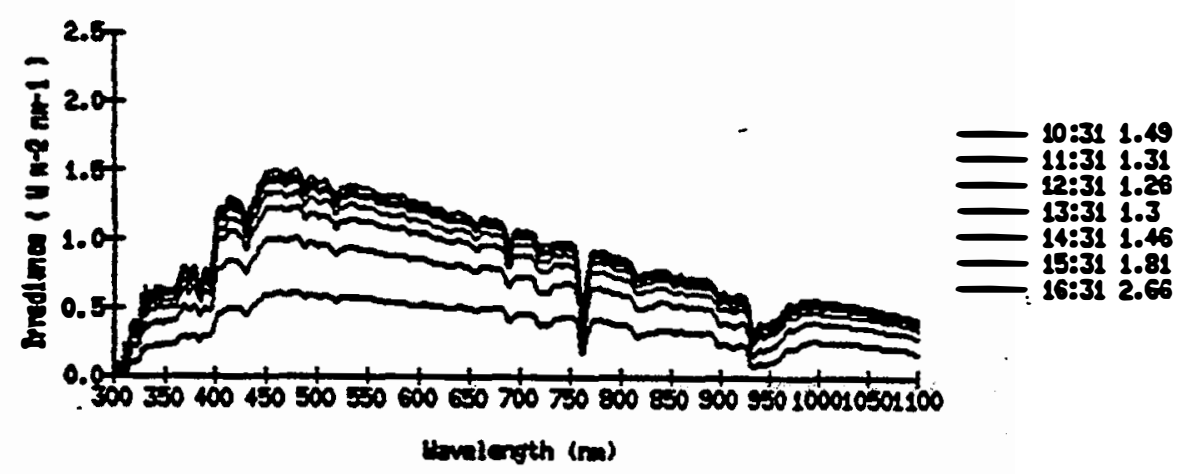




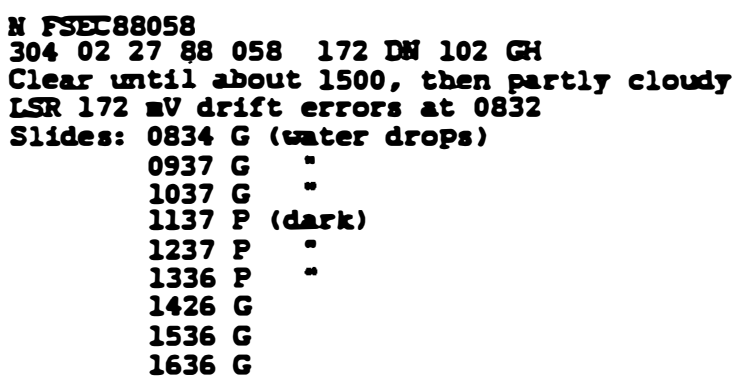

$\operatorname{Fsc} 5$

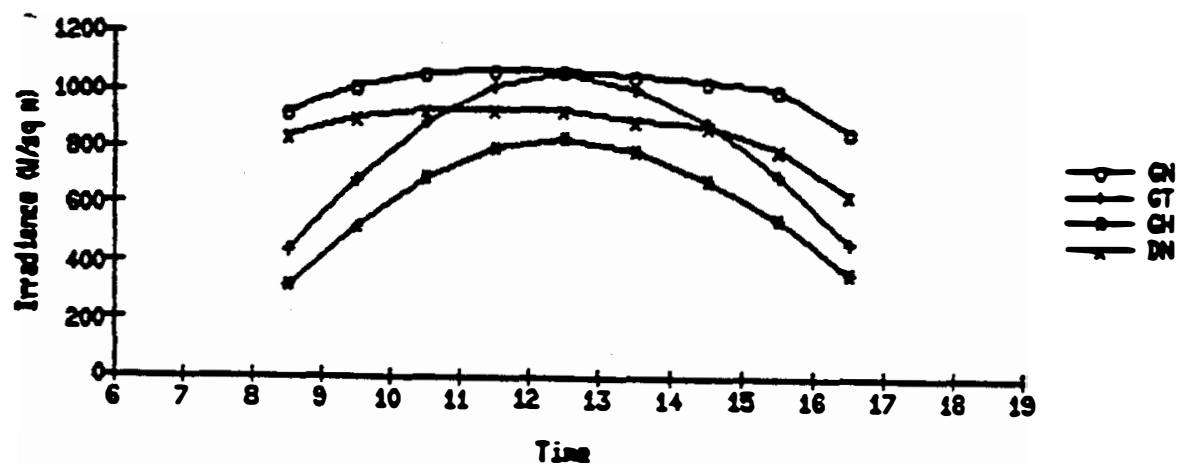

FIE $58 \mathrm{BNT}$

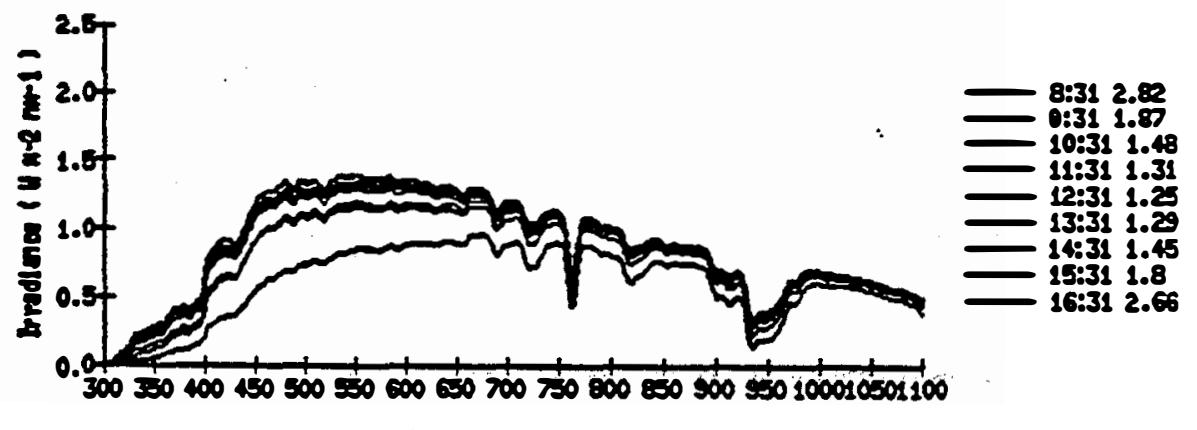

Henerath (ra)

roy 50 os

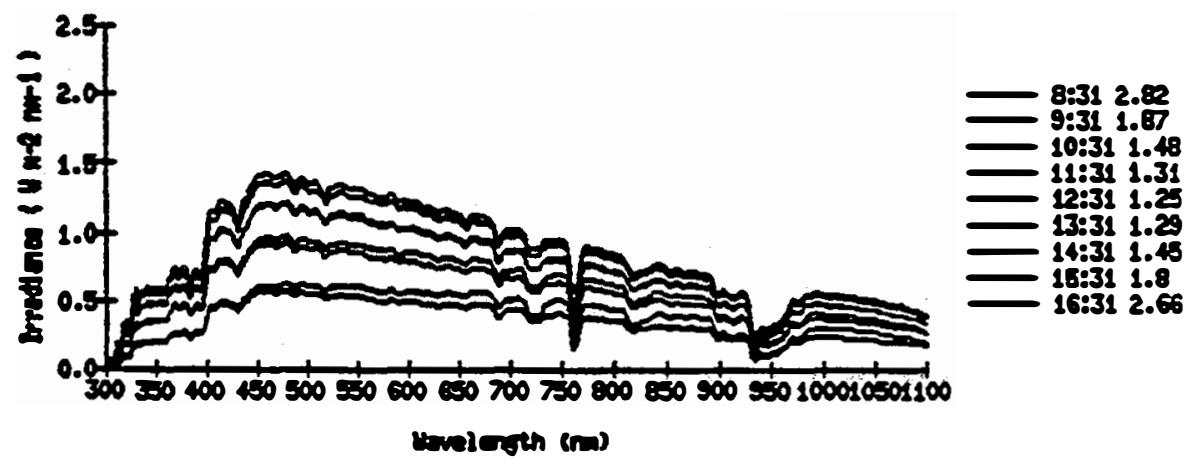


7 FSEC88059

30502 28.88 $059 \quad 272$ DN 202 어

Clear

ISR $172 \mathrm{av}$ drift errors at 0932

Sl1des: $0833 \mathrm{G}$

$0933 \mathrm{G}$

$1033 \mathrm{M}$ (dark)

$1133 \mathrm{P}$.

$1233 \mathrm{P}$

$1333 \mathrm{P}$

$1433 \mathrm{H}$

$1533 \mathrm{G}$

$1632 \mathrm{G}$

1732 G

Fise 3

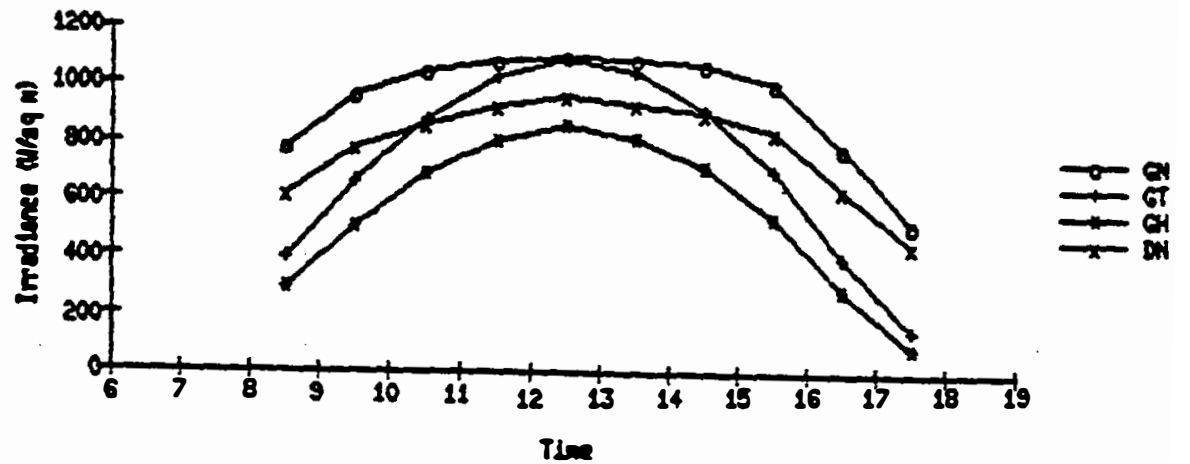

FEE SD DN

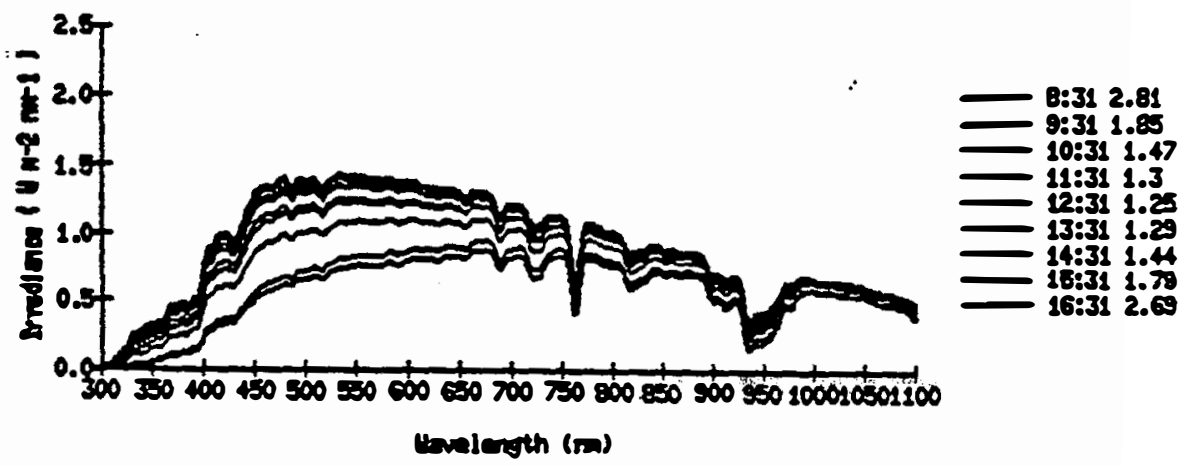

Fase as

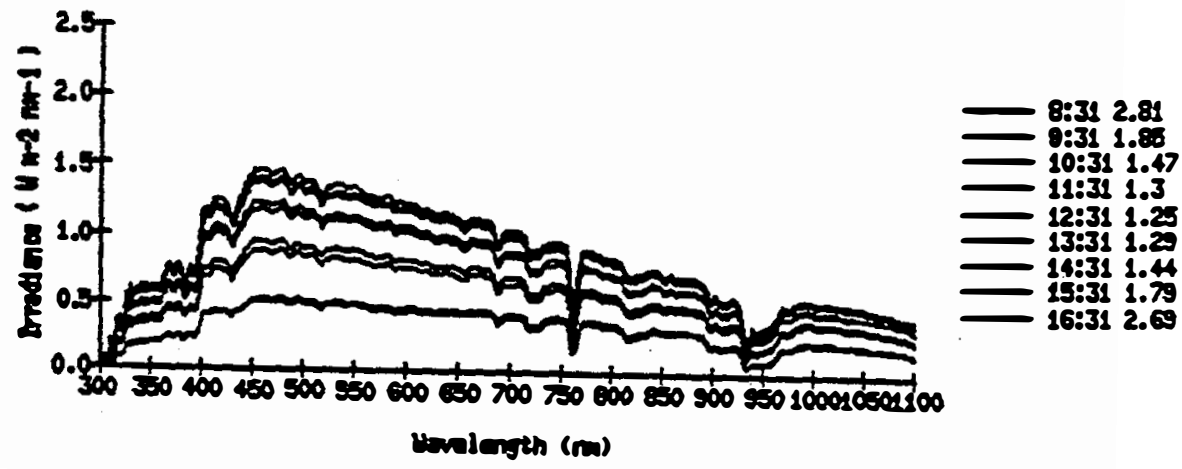


4 ISTDE8060

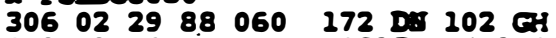

0.1 cloud cover at 0815 and 1400

Terp controllersi $172 \quad 102$

0815 40 C

$160040 \mathrm{C} 40 \mathrm{C}$

ISR 172 a drift errors at 0932 and 1032

slides: $0830 \mathrm{G}$

FSEC 0

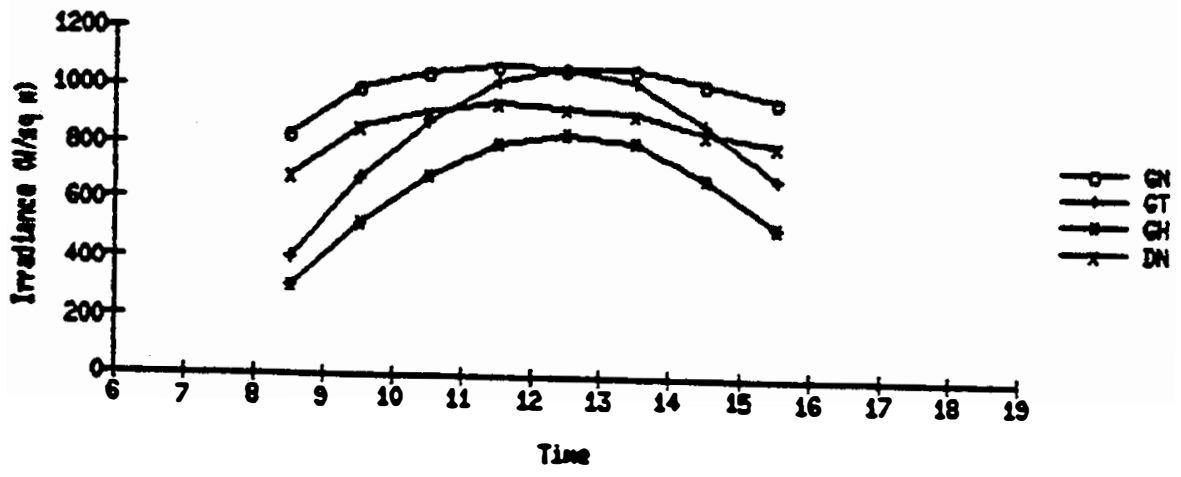

Fsese on axt

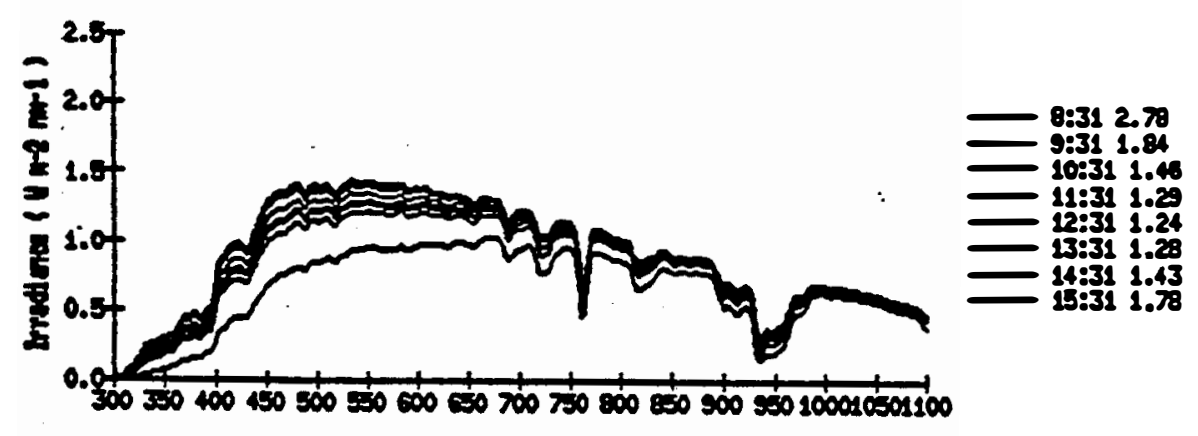

lavierith (n)

Fase $\infty$ as

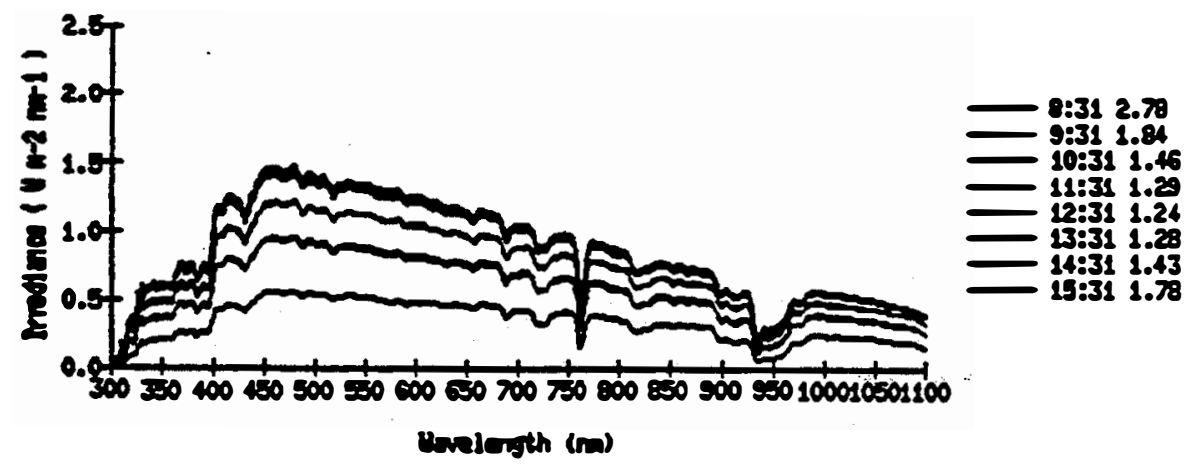




\section{SEP}

1. F5x88061

$\begin{array}{lllllllllll}307 & 03 & 01.88 & 061 & 172 & \mathrm{DA} & 102 & \mathrm{GL}\end{array}$

0.8 cloud cover at 0815; 0 at 1000,1230 , and 1615

Texp controllers: $172 \quad 102$

$\begin{array}{lll}0815 & 39 \mathrm{C} & 40 \mathrm{C} \\ 1230 & 40 \mathrm{C} & 40 \mathrm{C}\end{array}$

$161540 \mathrm{C} 40 \mathrm{C}$

ISR 172 av drift error at 1031

ISR $102 \mathrm{aV}$ drift error at 1531

Ho slides

-

Appenrs to be very brief apike in of in $\mathrm{DA}$ spectrm at 1031

ov in GI spectre at 1531 is bad

pses a

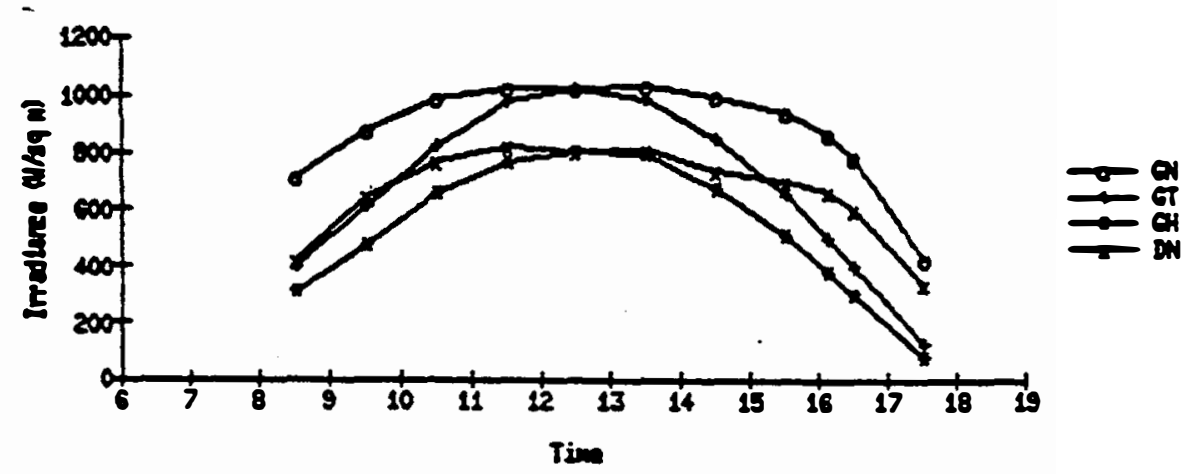

Pase a AT

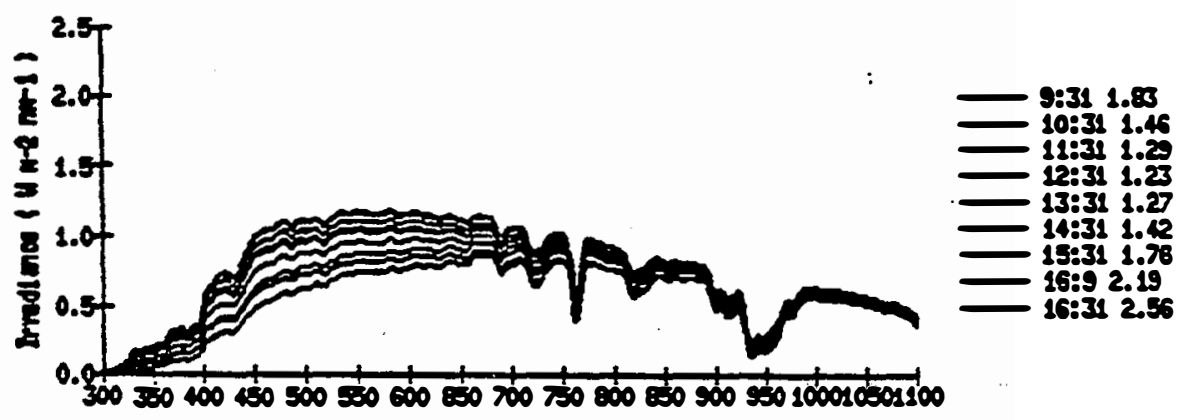

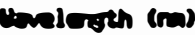

Fise QI GIS

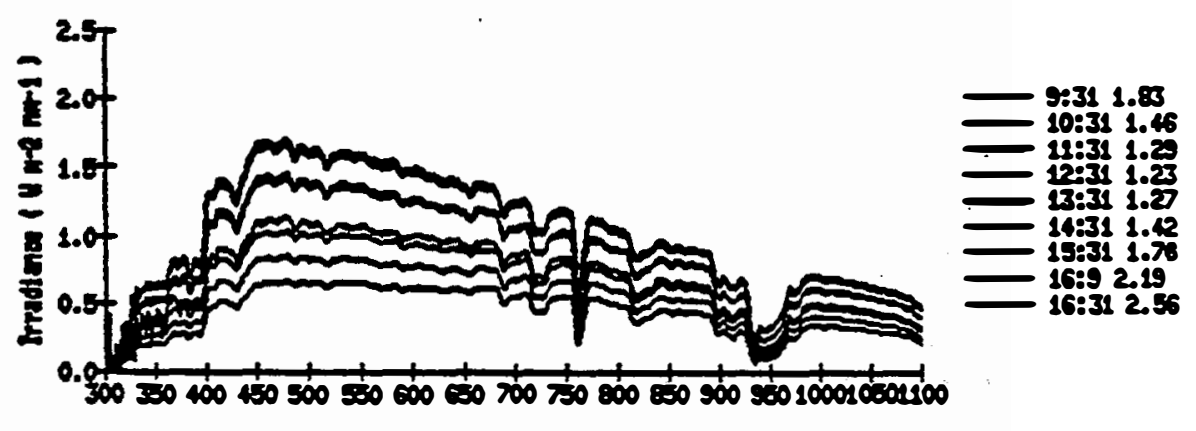

Hendergth (na) 


\section{SEP $*$}

1.

$\begin{array}{llllllllll}308 & 03 & 02 & 88 & 062 & 172 & \mathrm{DA} & 102 & \mathrm{GL}\end{array}$

0.5 cloud corer (mze) at 0830; 0.8 (baze) at 1250 ; 0.6 (baze) 1600

Terp controller: $172 \quad 102$

$\begin{array}{lll}01125: & 172 & 102 \\ 0830 & 40 \mathrm{C} & 40 \mathrm{C} \\ 1250 & 41 \mathrm{C} & 41\end{array}$

$160041 \mathrm{C} \quad 41 \mathrm{C}$

ISR 172 ov drift errors at 0931 and 1031

Ho slddes

spikes in ov in Da spectrum at 0931 and 1031

T25e $\approx$

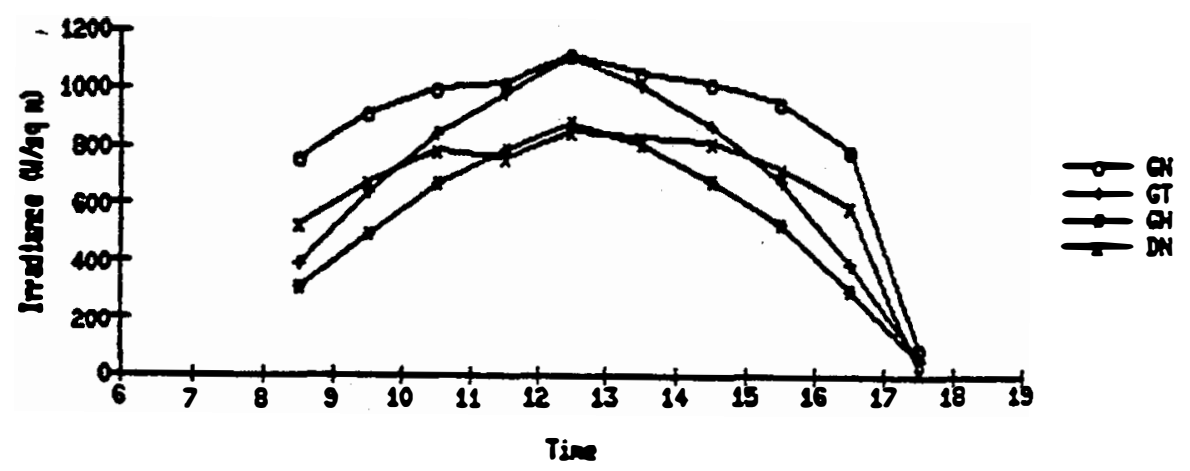

FSEC Q MT

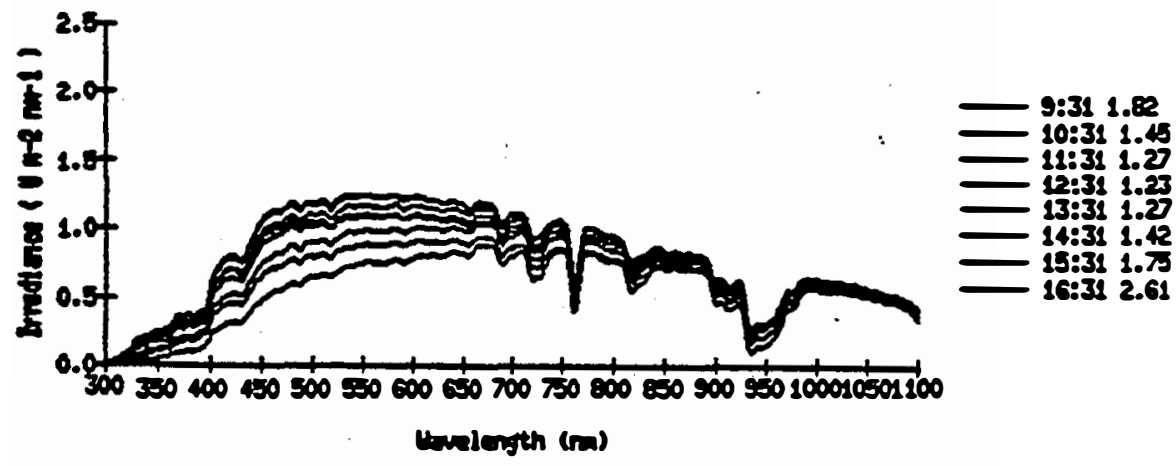

FIC $\propto$ GT

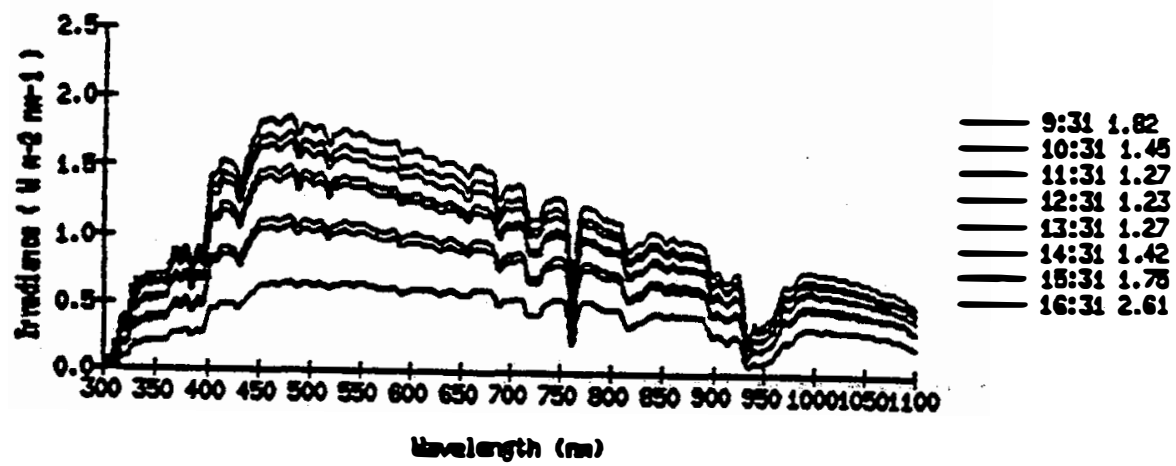




\section{SEN *}

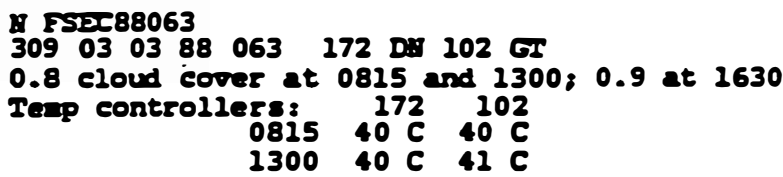

Fege $x$

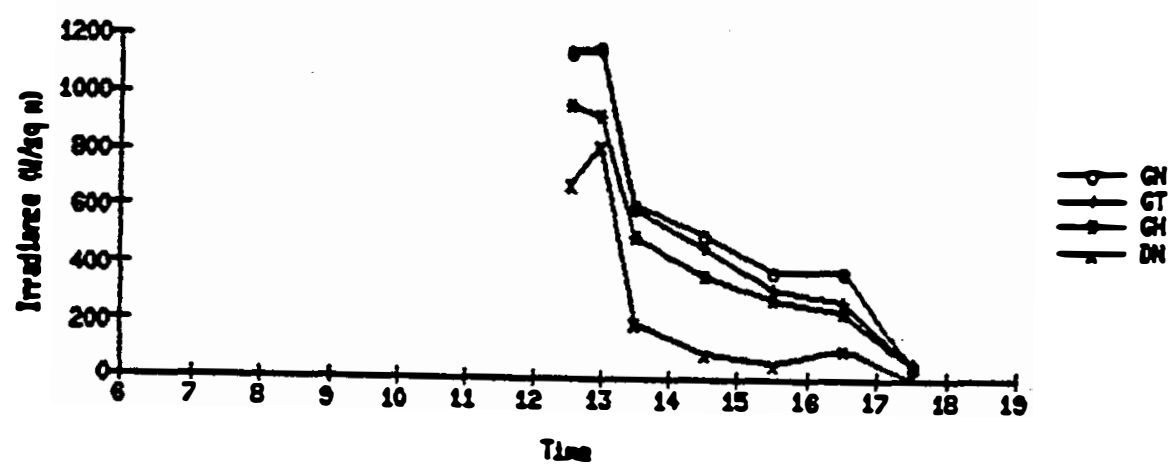

FIEC CB DNT

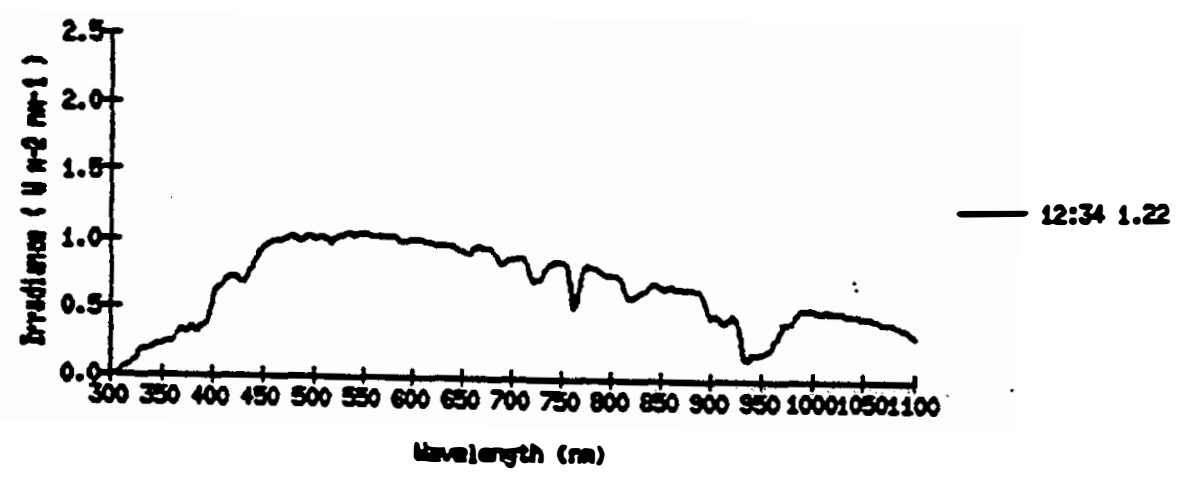

FSE 1365

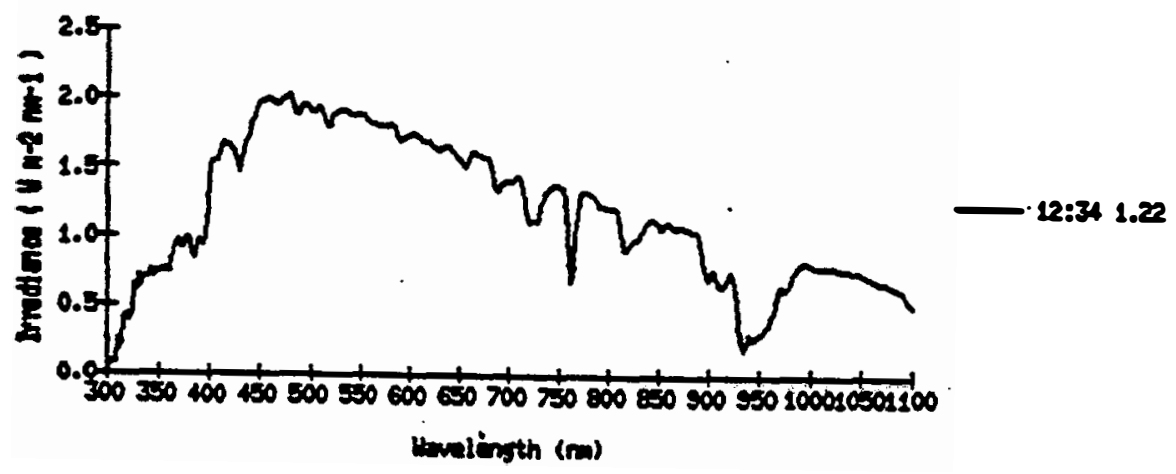


$\Rightarrow=0$

BST 88064

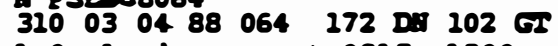

1.0 cloud cover at 0815, 1300, and 1530

Terp controllers $40 \mathrm{C}$ at 0815

jo slides

Bo spectra

Fsec 64

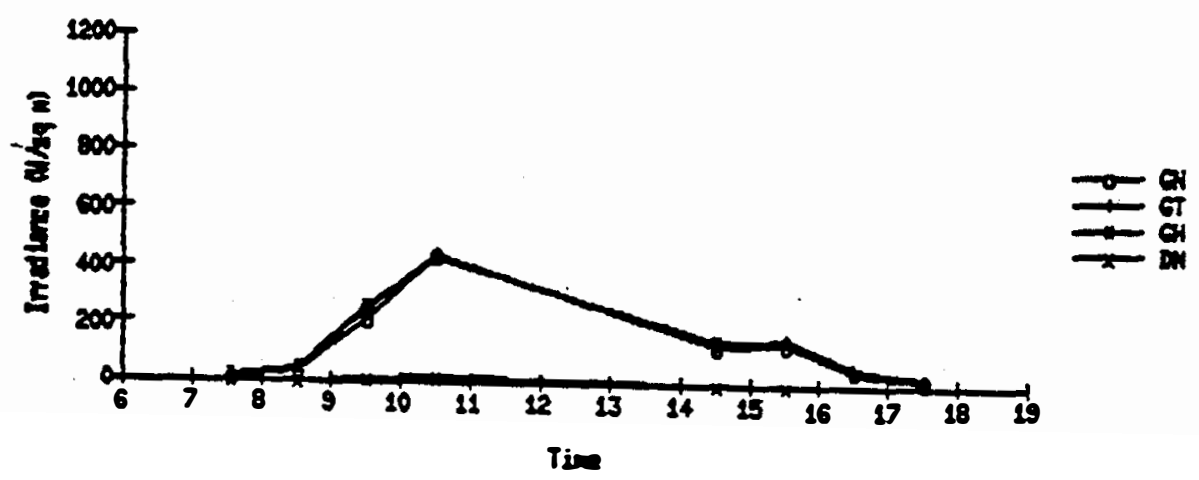


SEPI * *

1. FSPC88065

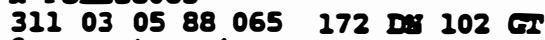

Orercast; rals

Ho slides

Bo spectra

$\operatorname{rsec} 6$

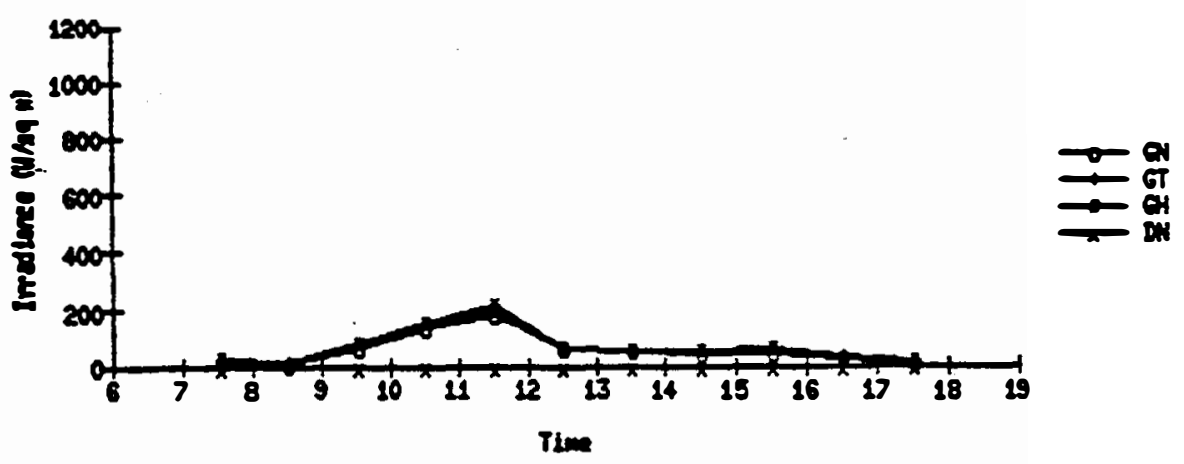


- $=01$ "

FST288066

$\begin{array}{llllllll}31203 \quad 06 & 88 & 066 & 172 & \text { DEI GI }\end{array}$

Overeast; rain

Ho sldas

Ho spetra

Fise 6

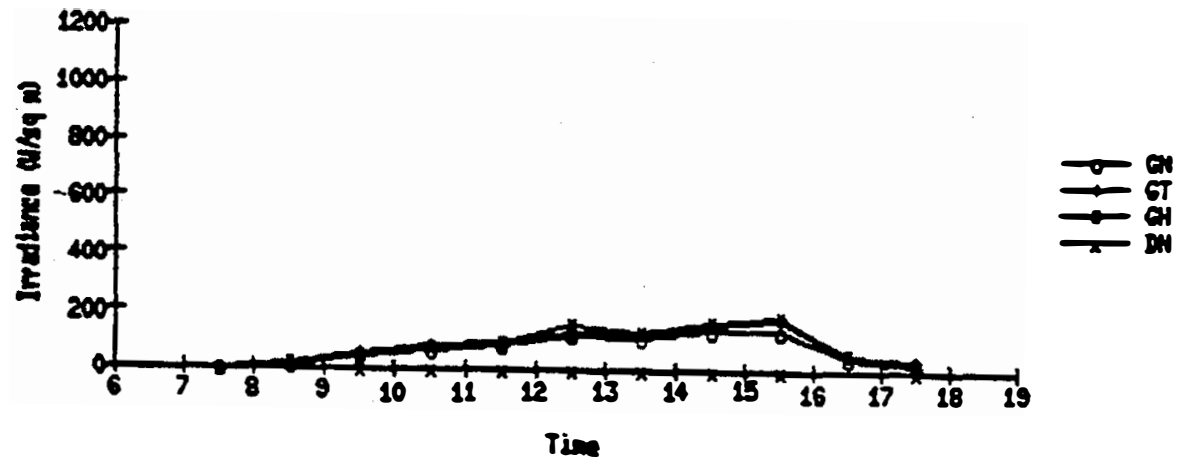


I.

$\begin{array}{lllllllll}313 & 0307 & 88 & 067 & 172 & \text { D. } & 102 & \text { GS }\end{array}$

1.0 cloud cover at $0815 ; 0.9$ at $1200 ; 0.5$ at 1630

Teap controllers: $172 \quad 102$

081539 C 40 C

$120040 \mathrm{C} 40 \mathrm{C}$

Ho slides

FseC $\boldsymbol{\nabla}$

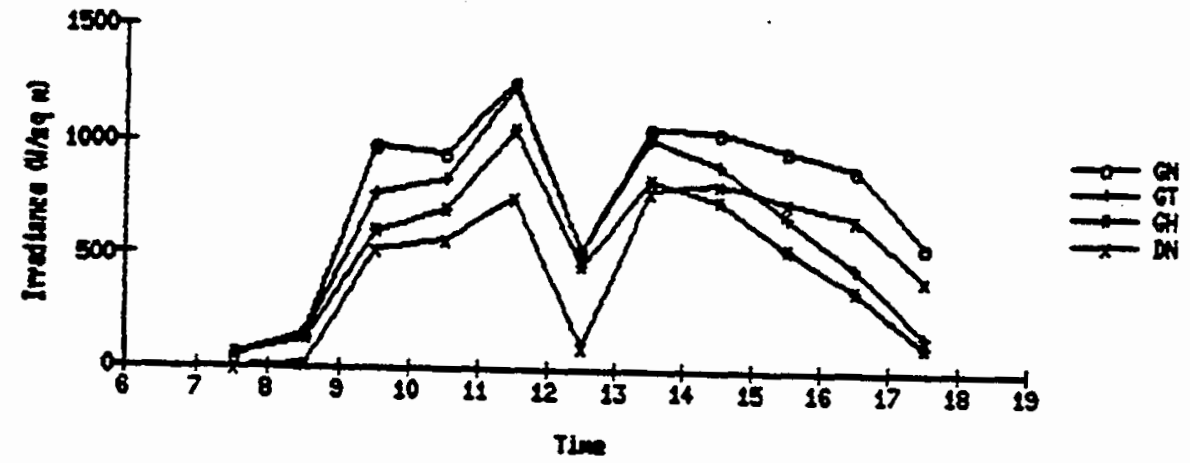

FEEC $\varnothing$ DIT

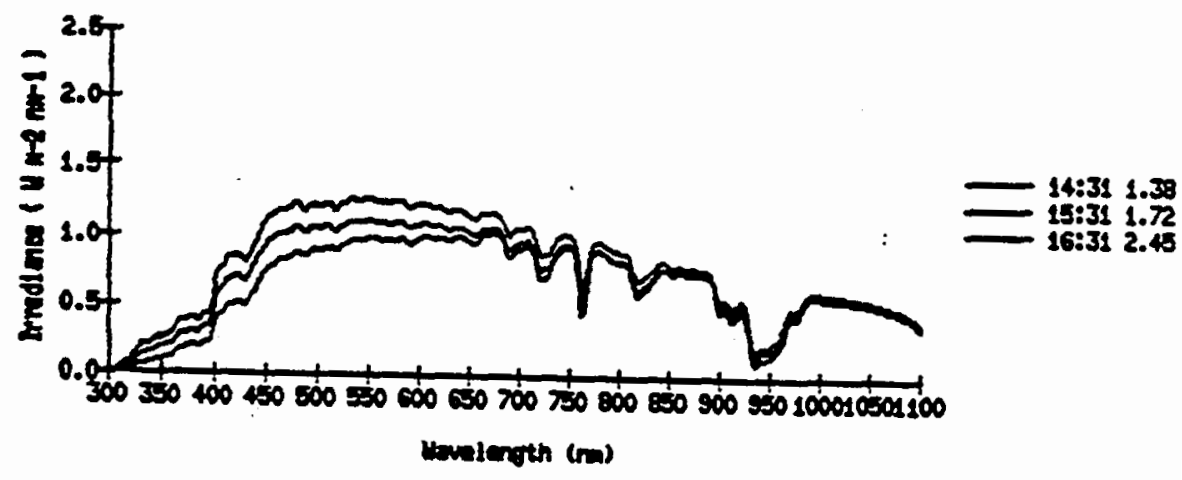

$\operatorname{Pses} \triangle \mathrm{GT}$

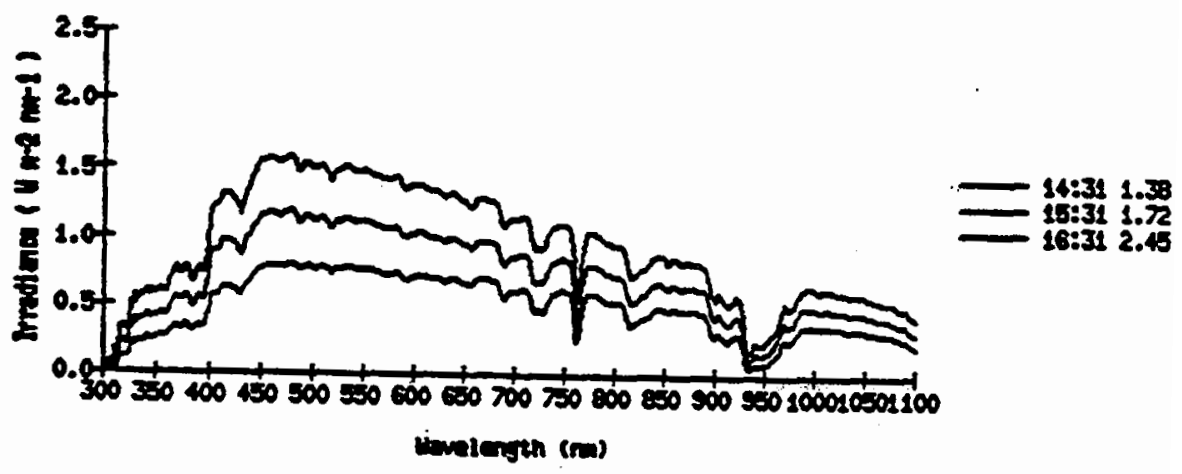


1. ए:T588068

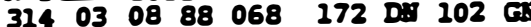

0.3 eloud cover at $1130 ; 0.6$ at 1630

repp controllers: $172 \quad 102$ $\begin{array}{lll}1130 & 11 & \mathrm{C} \\ 1630 & 40 \mathrm{C}\end{array}$

Ho sldes

PIS68

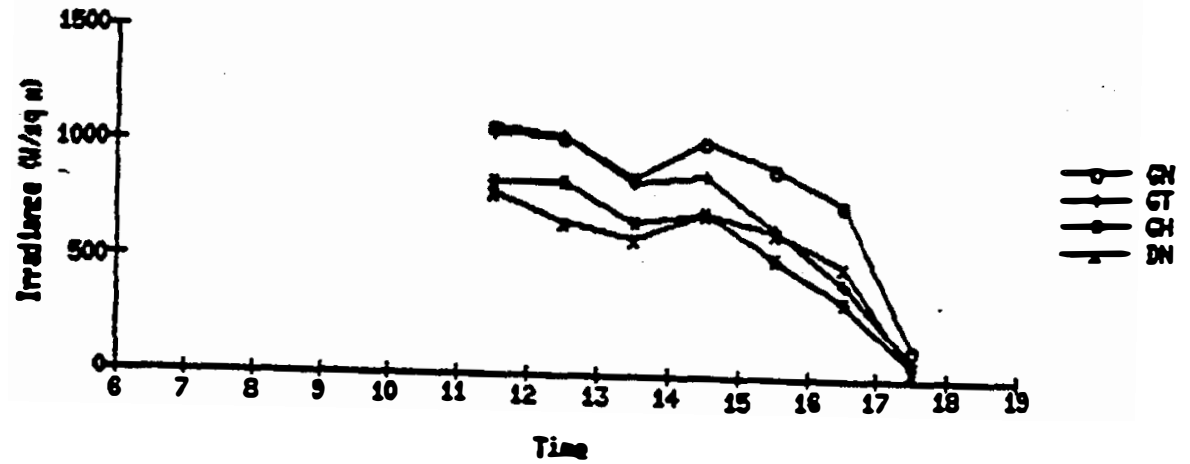

Fise $69 \mathrm{~min}$

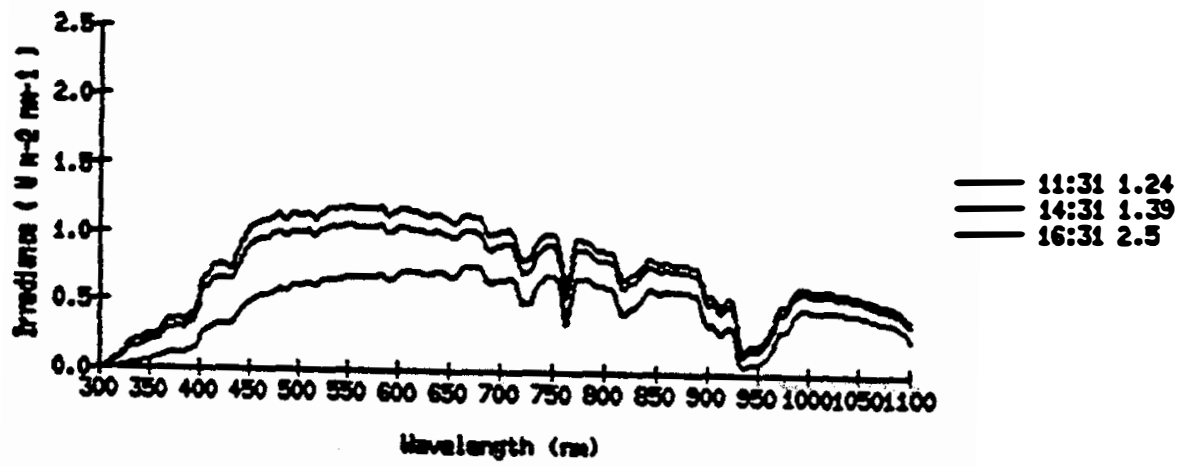

FEבE $\infty$ OS

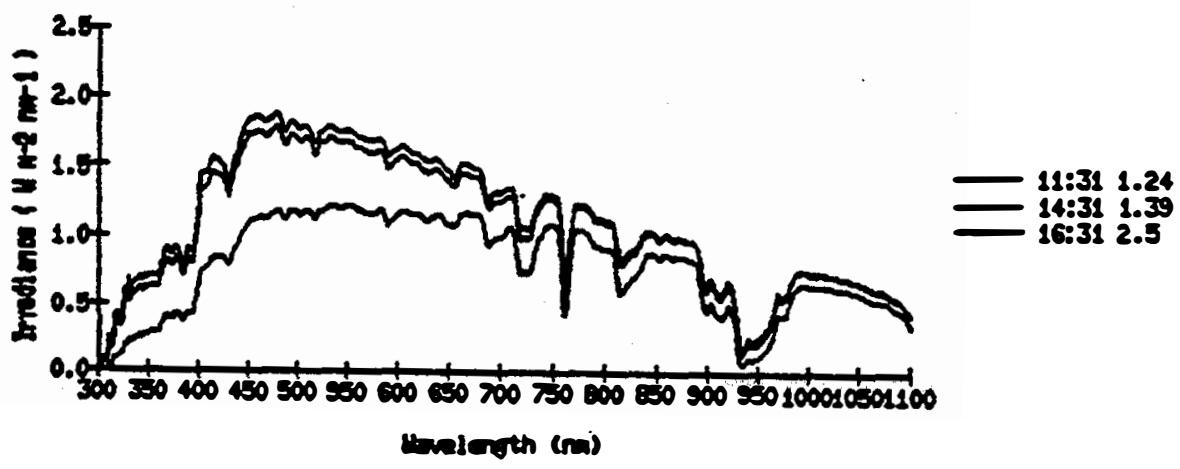


1. 521288069

$\begin{array}{lllllllll}315 & 03 & 09 & 88 & 069 & 172 & \mathrm{DH} & 102 & \mathrm{GM}\end{array}$

1.0 cloud cover at 0815, 1230, and 1630

Iep controllers $40 \mathrm{C}$ at 0815

Ho slides

Ho epecta

FEC

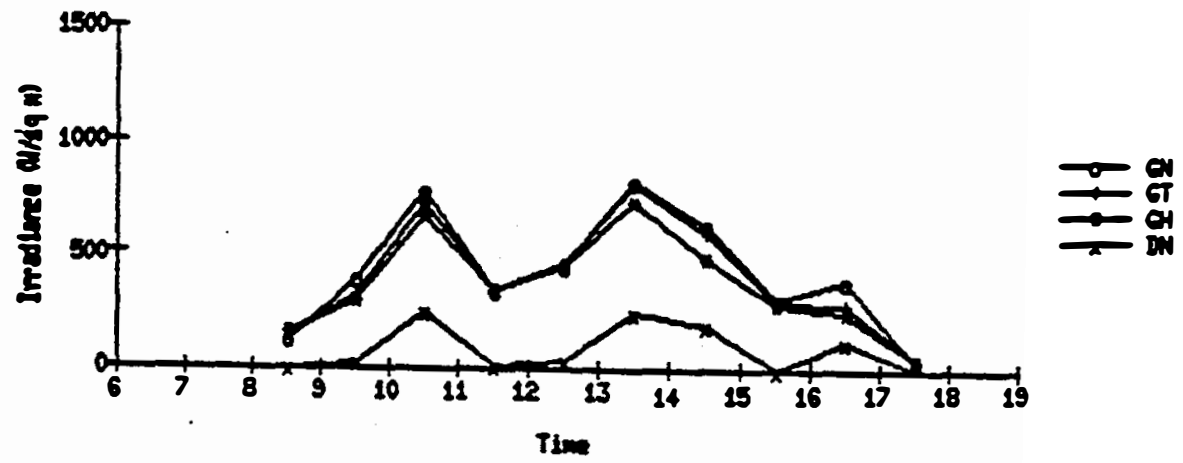


PSX 88070

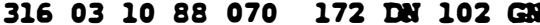

1.0 cloud cover at $0815 ; 0.8$ at $1130 ; 0.7$ at 1630

Tenp controllers: $172 \quad 102$ 081539 C 39 C

113039 C 39 C

ISR $172=V$ drsft erfor to 1331

Ho slides

$\operatorname{rsec} 70$

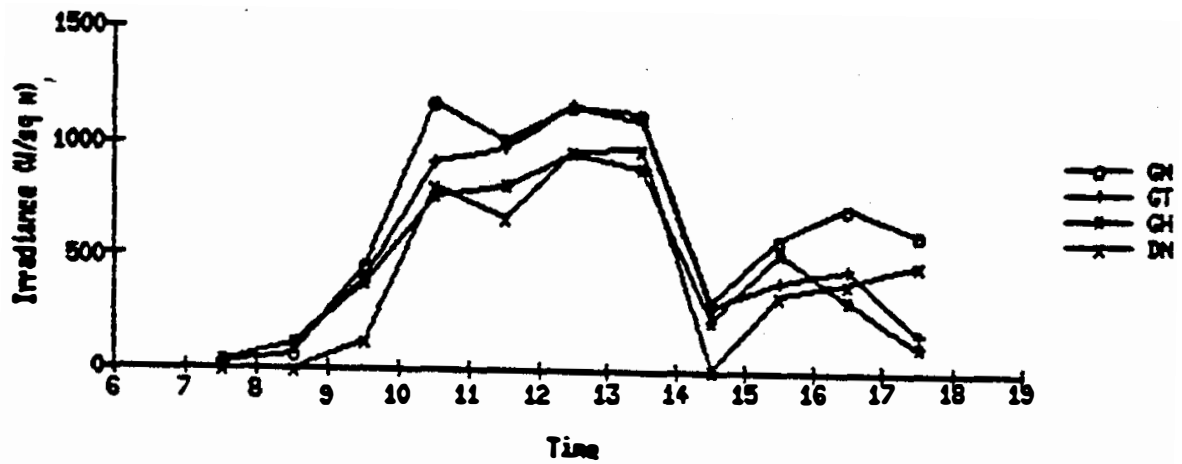

$\operatorname{PExC} 70 \mathrm{mit}$

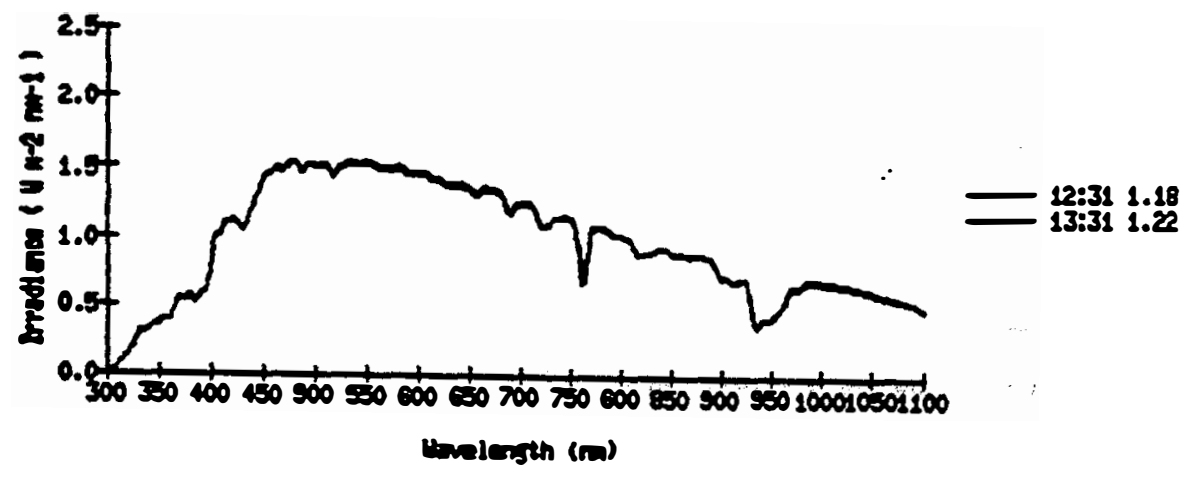

Pesc 70 os

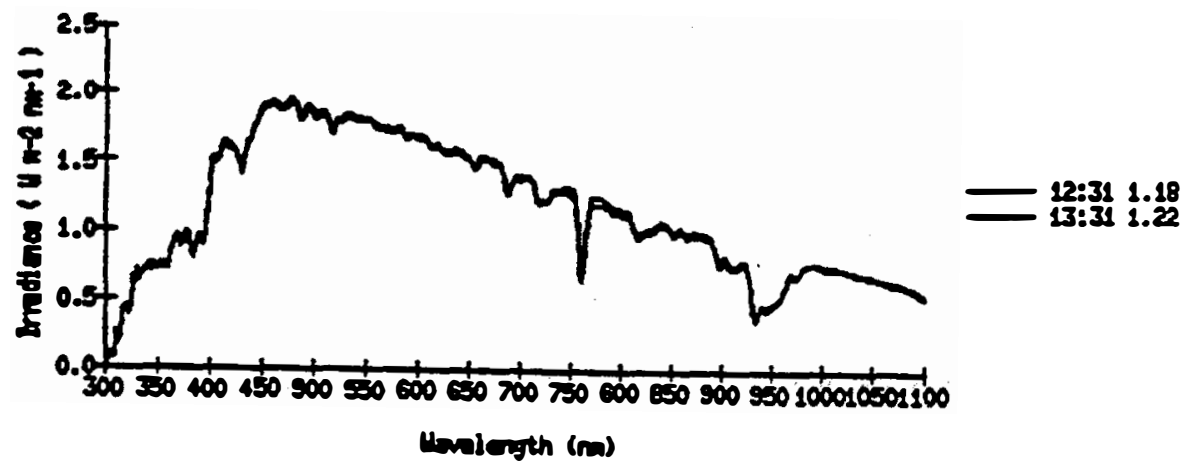


ए52088072

$\begin{array}{llllllll}318 & 03 & 12 & 88 & 072 & 172 & 02 & 0 .\end{array}$

Pertly cloudy Mi nostly clear PM

ISR $172 \mathrm{aV}$ drfft errors at 0931 and 1033

Ho slldes

In peetra at 0931 and 1033 are and (no direct been)

PIST

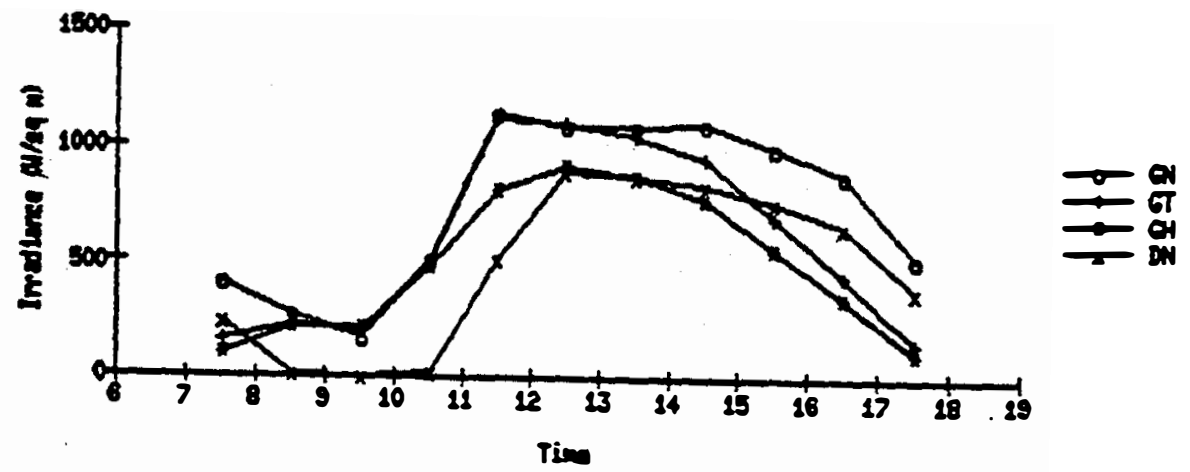

FSE 72 ant

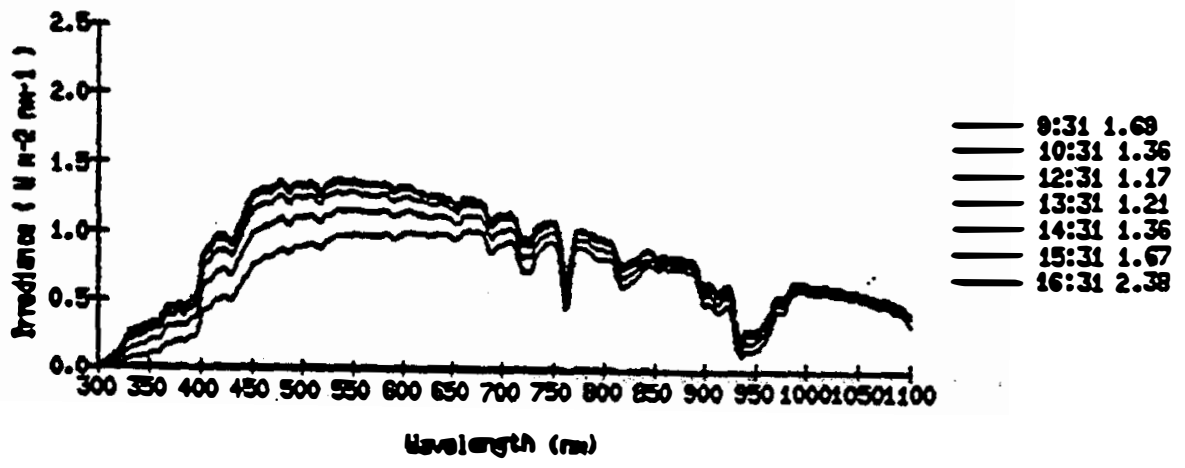

PISC $\pi 2$ OS

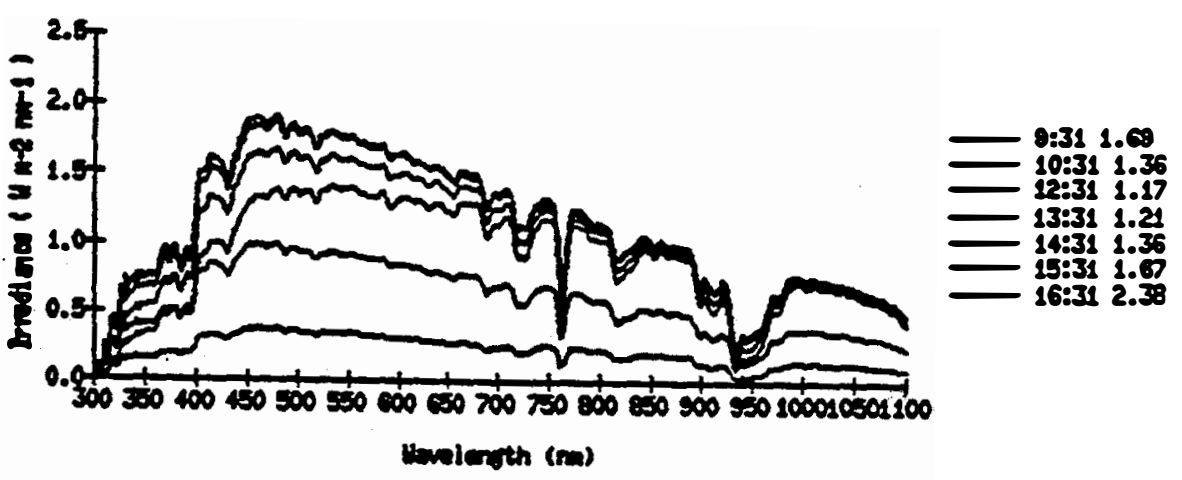




\section{$\rightarrow=01$ "}

A FSP 88073

$\begin{array}{lllllll}319 & 03 & 13 & 88 & 073 & 172 & 02\end{array}$

Partl clouds to overcast elth raln

ISR $172 \mathrm{av}$ drift error at 1433

Ho slides

- -c-

Dy spectron at 1433 $1 \mathrm{~s}$ bad (no drect bean)

PEAC 73

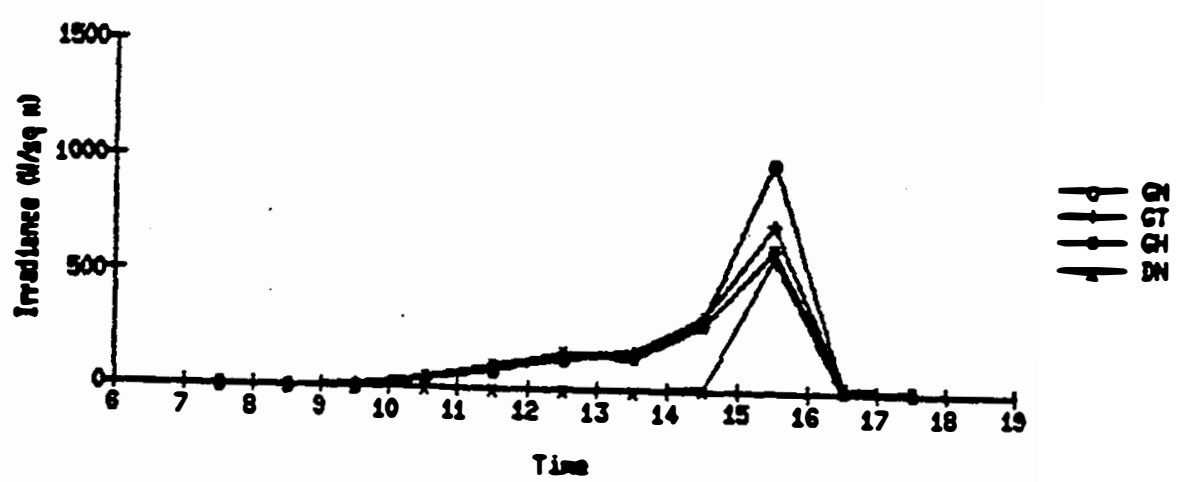

Fax 73 in

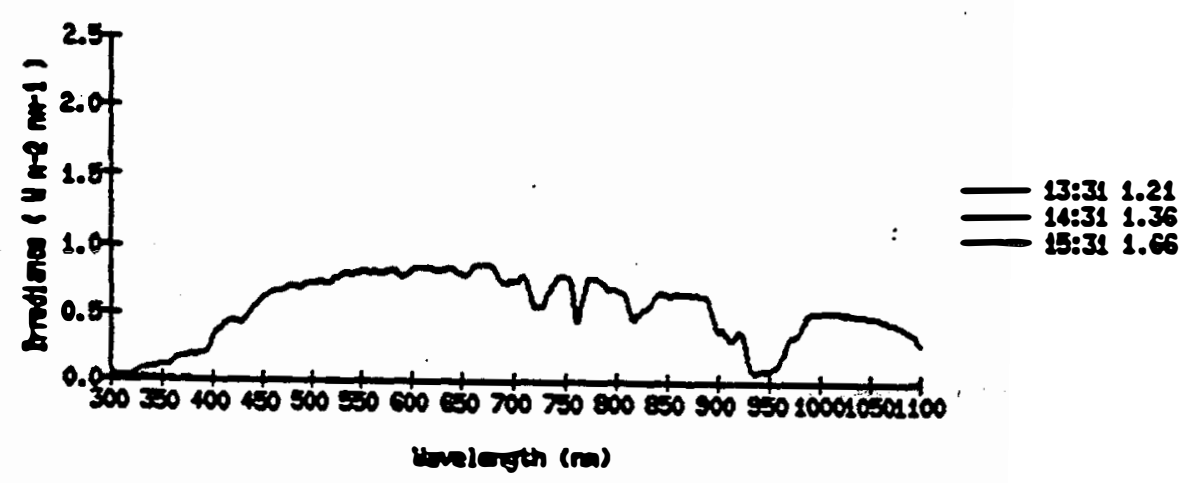

FEEC 73 OS

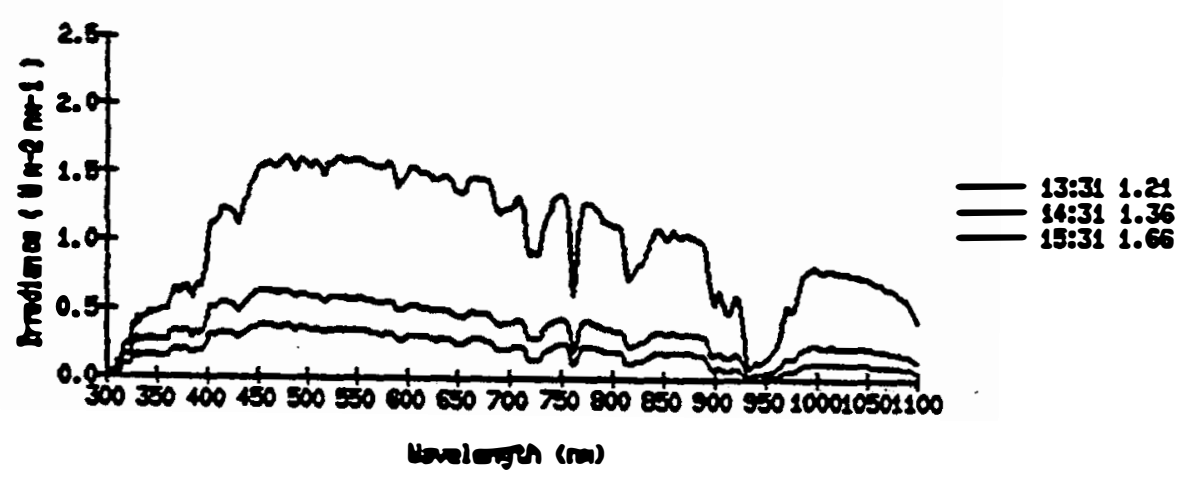




\section{SEN \%}

N F5x888074

$\begin{array}{lllllllll}320 & 03 & 14 & 88 & 074 & 172 & \text { DI } & 102 & \mathrm{GH}\end{array}$

0.7 cloud cover at $0815 ; 0$ at 1000,1230, and 1630

Ierp controllers: $172 \quad 102$

$\begin{array}{lllll}0815 & 39 & \mathrm{C} & 39 & \mathrm{C}\end{array}$

$1230 \quad 40 \mathrm{C} 39 \mathrm{C}$

$163040 \mathrm{C} 40 \mathrm{C}$

ISR $172 \mathrm{av}$ drift error at 1231

No slides

FEC 74

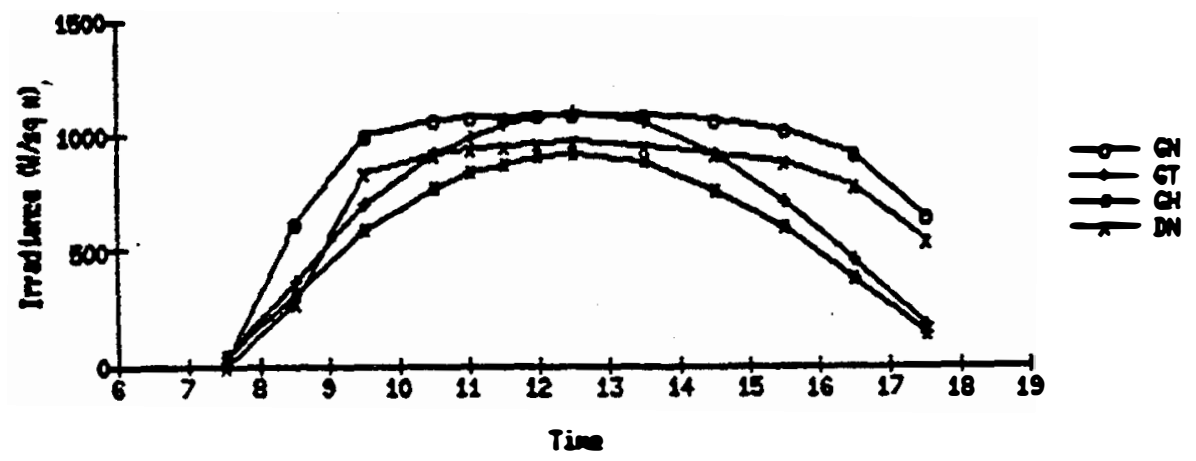

Fese 74 DN

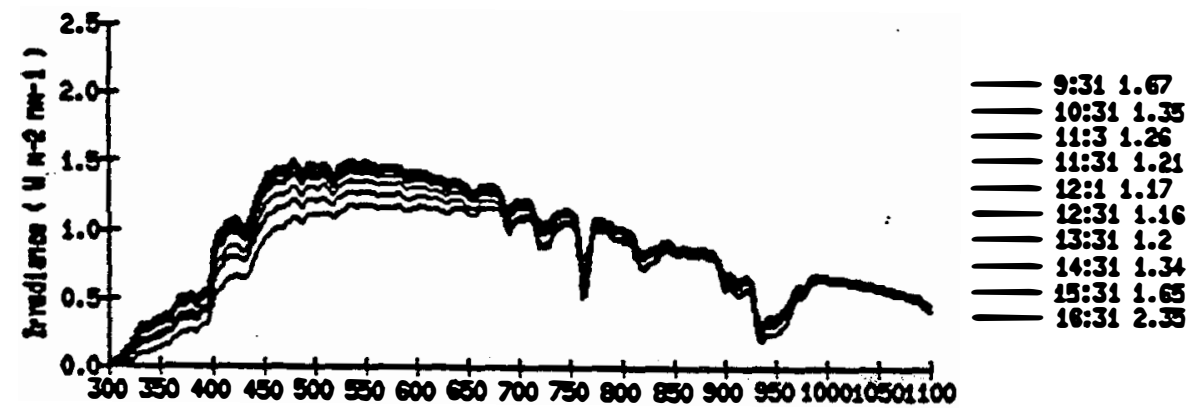

Conlength (m)

FEe 7405

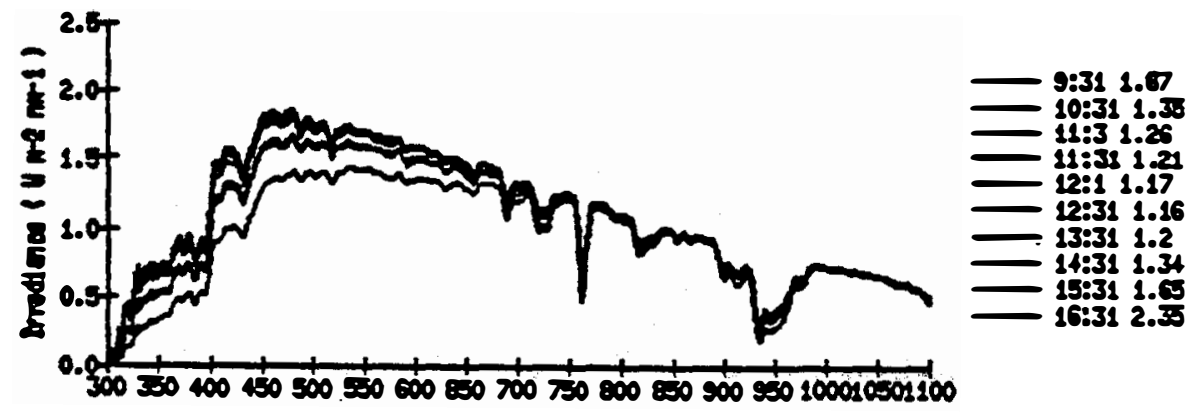

Nolosth (m) 
1. FSP 88075

$\begin{array}{lllllllll}321 & 03 & 15 & 88 & 075 & 172 & \mathrm{DA} & 102 & \mathrm{Cm}\end{array}$

0 cloud cover at 0800, 1300, and 1630

Terp controllers: $172 \quad 102$

$0800 \quad 38$ C 38 C

$1300 \quad 39$ C 40 C

$163039 \mathrm{C} 40 \mathrm{C}$

No slides

$\operatorname{rsec} \pi$

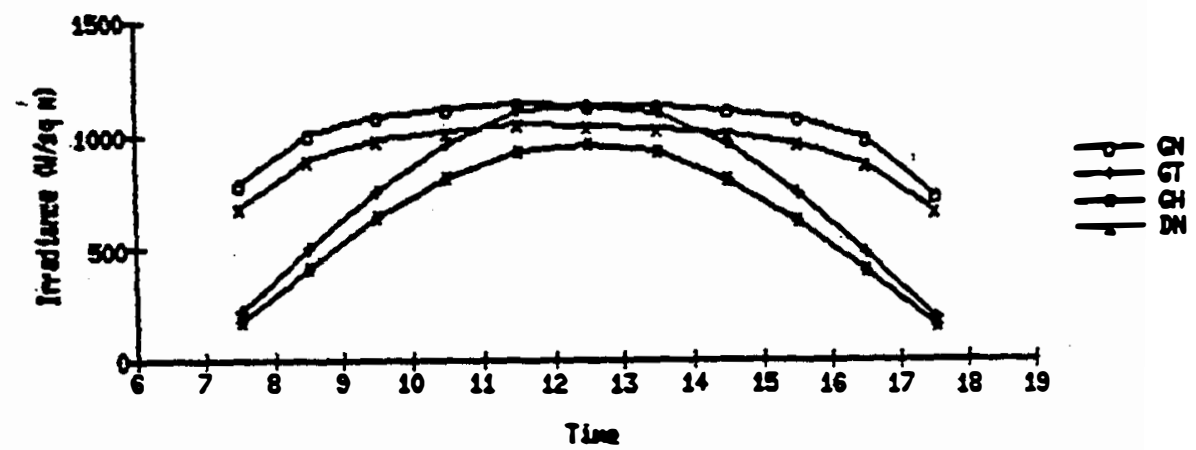

Fsec 75 ant

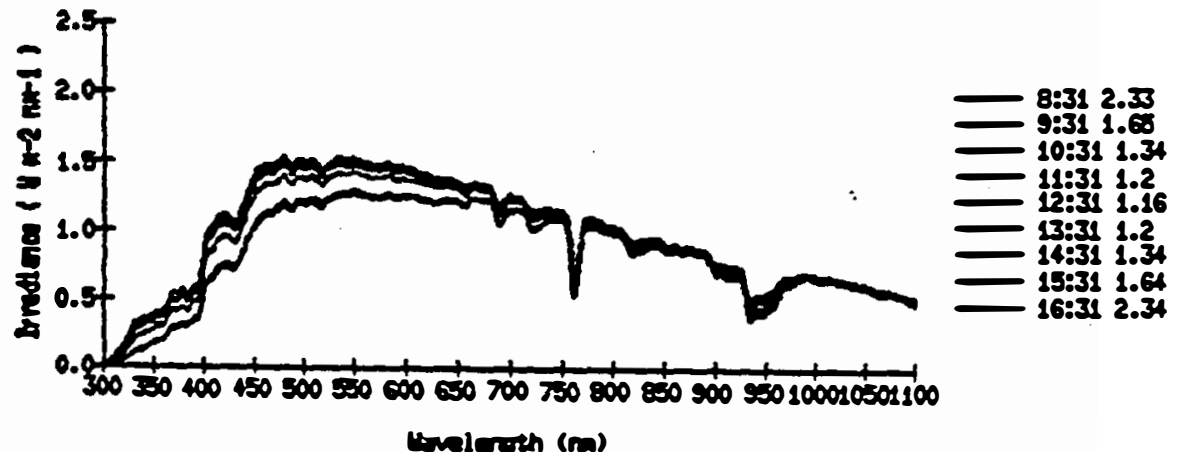

$\operatorname{rsec} \pi$ as

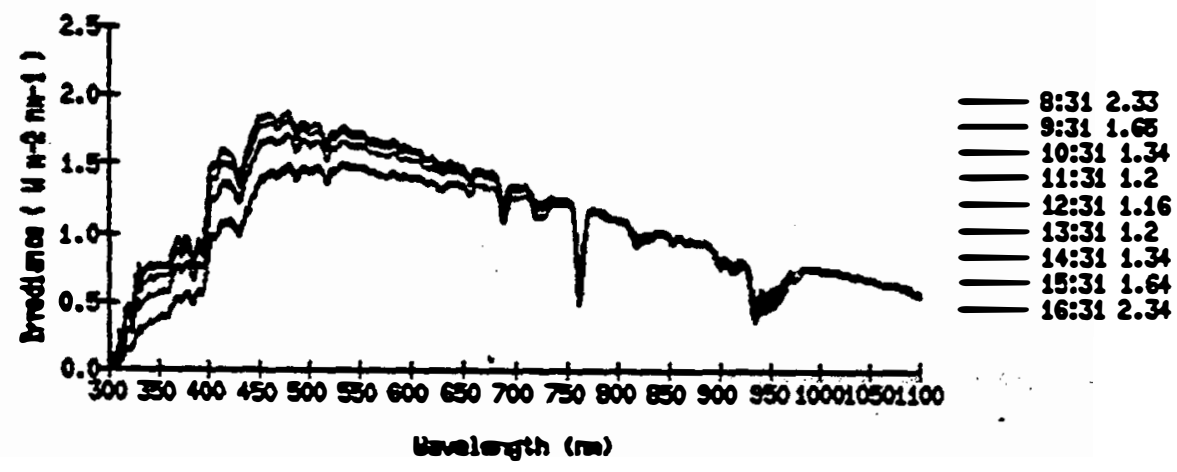


A FST88076

$\begin{array}{lllllll}322 & 03 \quad 16 & 88 & 076 \quad 172 \quad \text { DI } & 102 \quad \mathrm{GP}\end{array}$

0 cloud cover until 1400 , then partls clouds

IsR 172 av drift error at 1331

$\operatorname{Fsec} x$

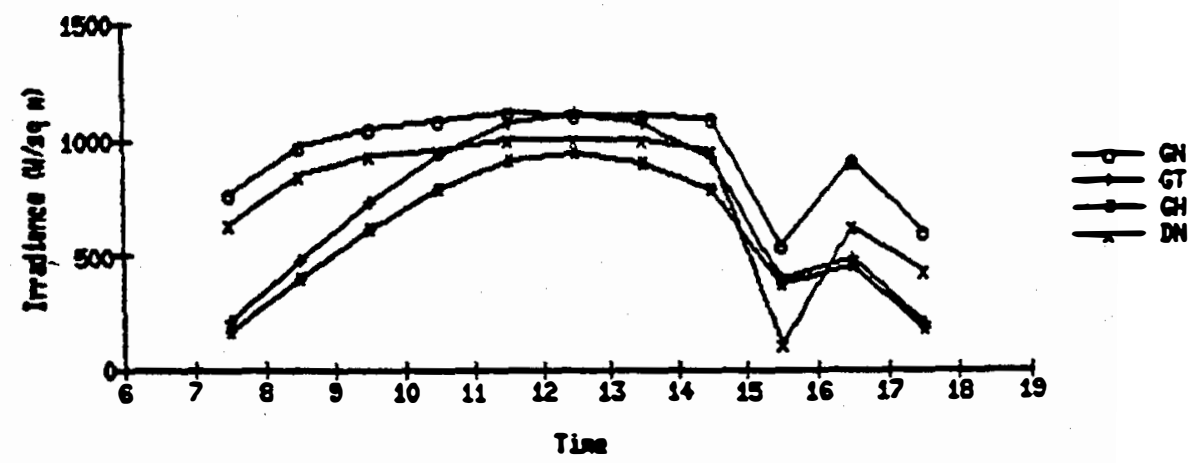

Fsec $\pi$ an

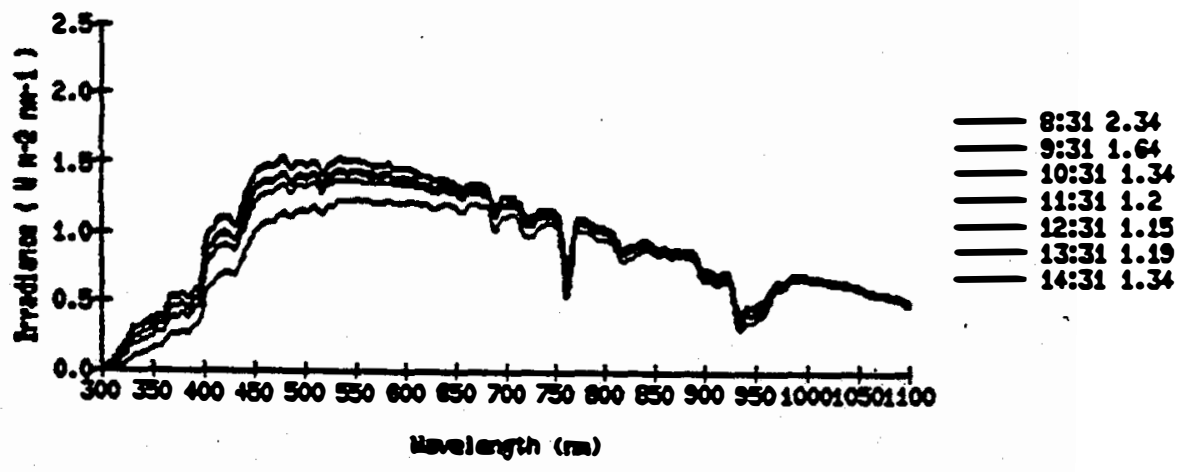

$\operatorname{rsse} x$ as

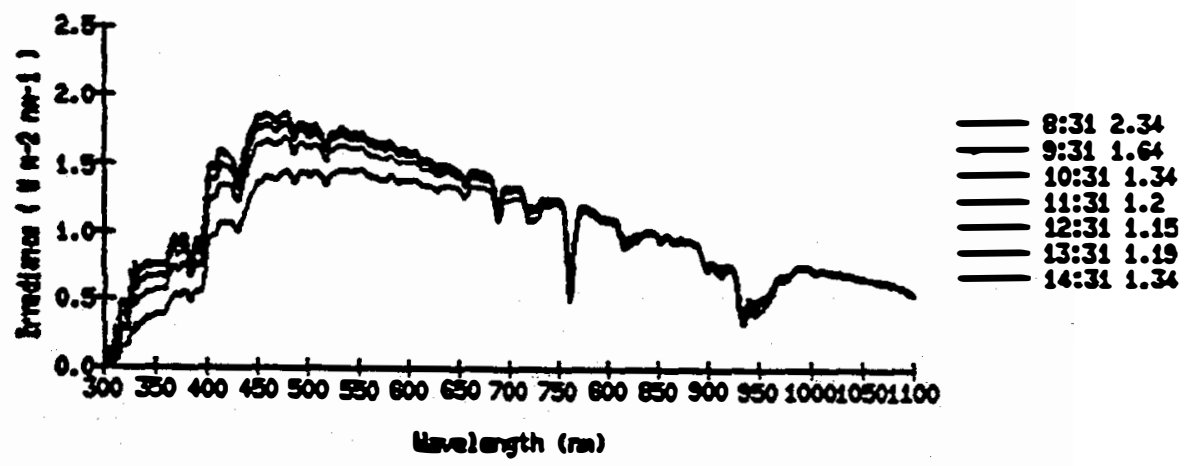


1. 75258077

$\begin{array}{lllllllll}323 & 03 & 17 & 88 & 077 & 172 & \mathrm{DA} & 102 & \mathrm{CH}\end{array}$

Ore GI spectre. then GH

0 eloud cover at 0730

Do slides

$\begin{array}{llllllllll}324 & 03 & 17 & 88 & 077 & 172 & \mathrm{DO} & 102 & \mathrm{GH}\end{array}$

0 cloud corer at 1530

Ienp controllers $40 \mathrm{C}$ at 1530

Ho elsdes

$\operatorname{Fsc} \pi$

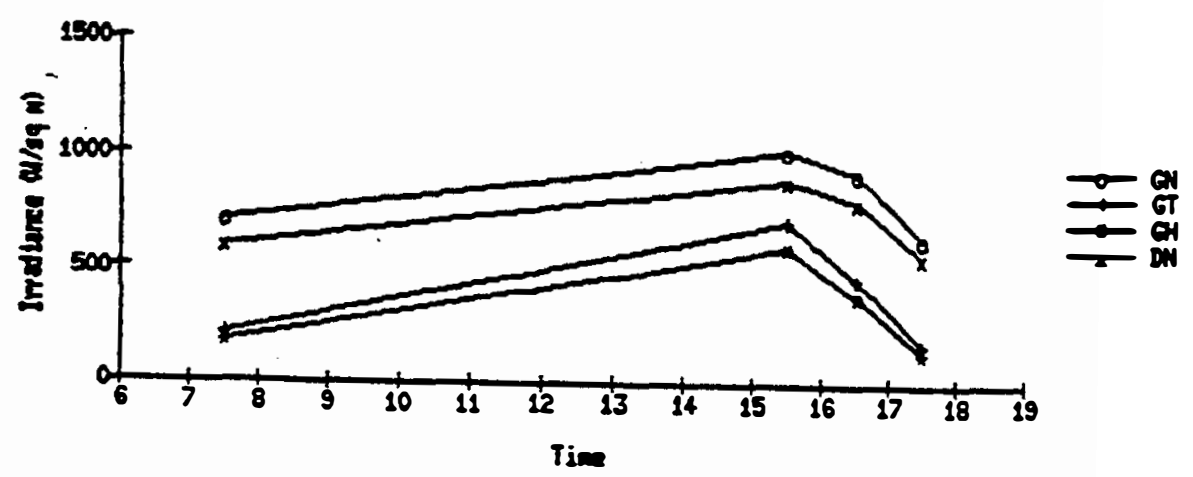

FEEC 77 ow

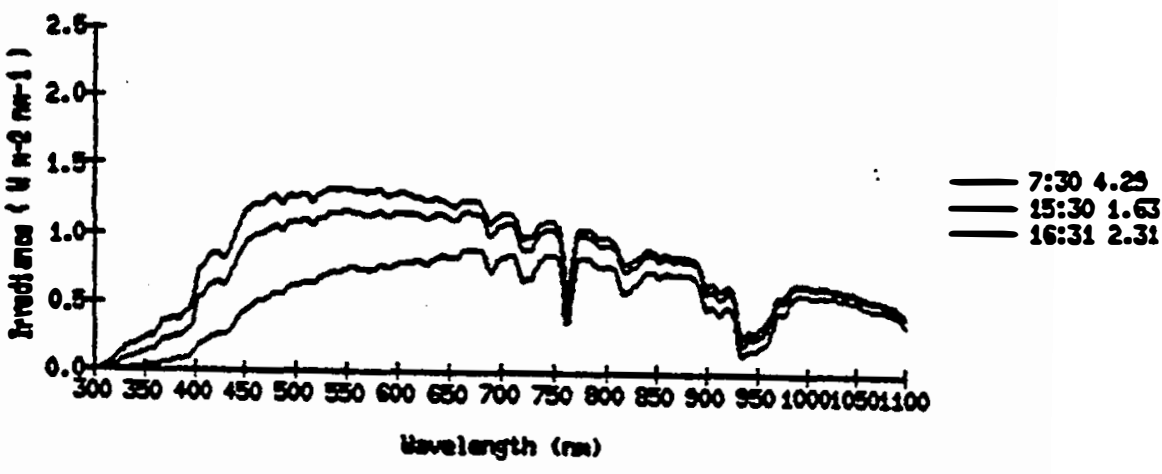

Fise 77 as

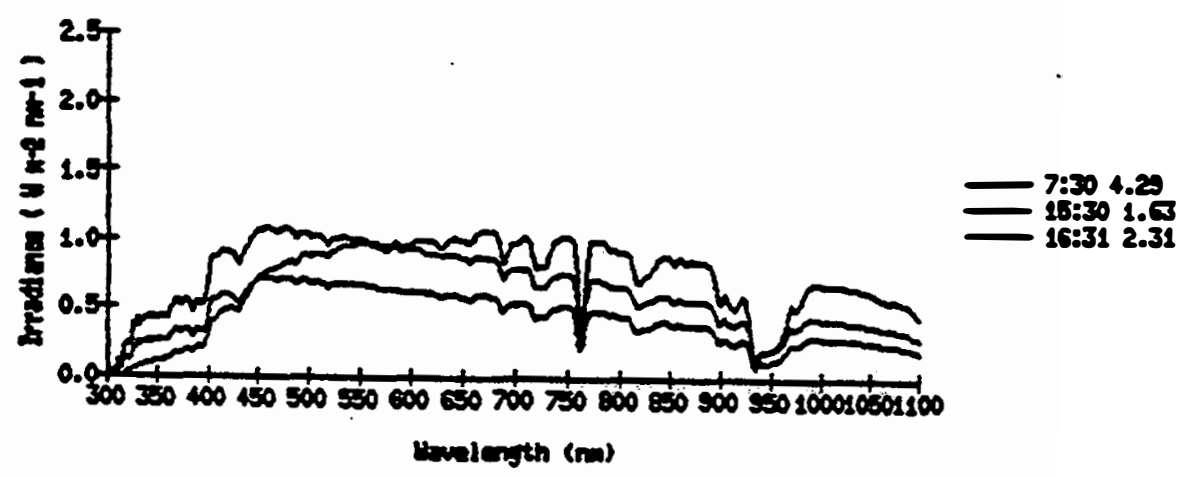




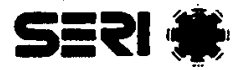

DSTE8078

$\begin{array}{llllllll}325 & 03 & 18 & 88 & 078 & 172 & 102 & \mathrm{GH}\end{array}$

1.0 cloud cover at 0815. 1300, and 1630

Teip controllers: $17239 \mathrm{C}$ and $10240 \mathrm{C}$ at 0815

Ho slldes

FEEC 78

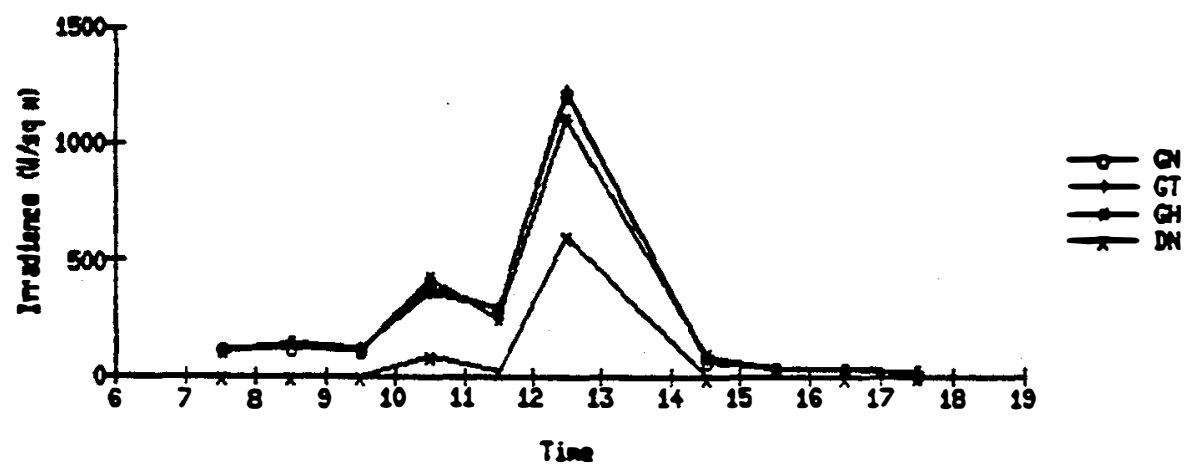

FEEC $78 \mathrm{mT}$

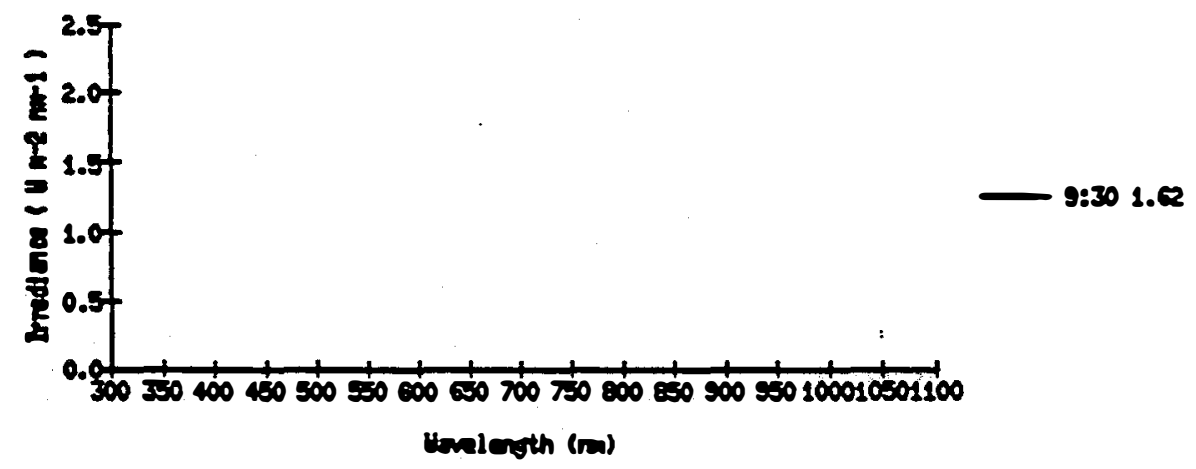

FEse 7806

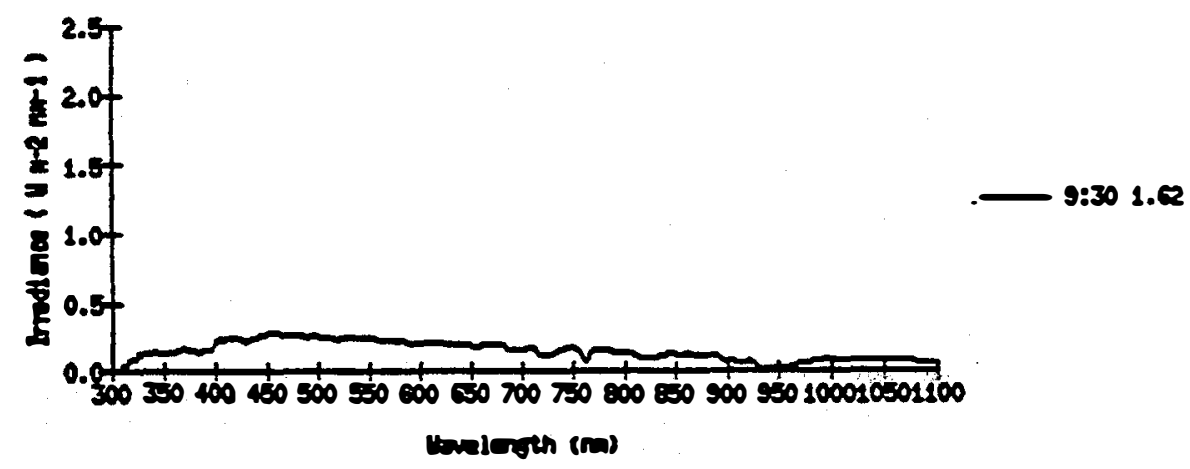


月 $52 \times 88079$

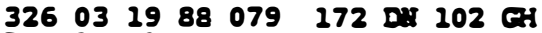

Partly cloudy

Bo slides

spectral integral is nuch lover than broadband for all Dis spectra

PSEC T9

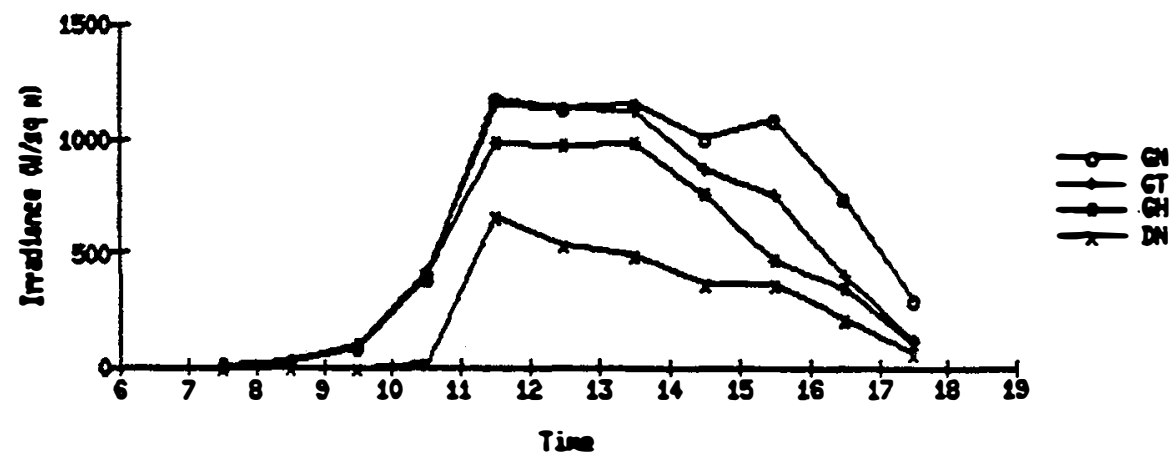

Fese 70 ant

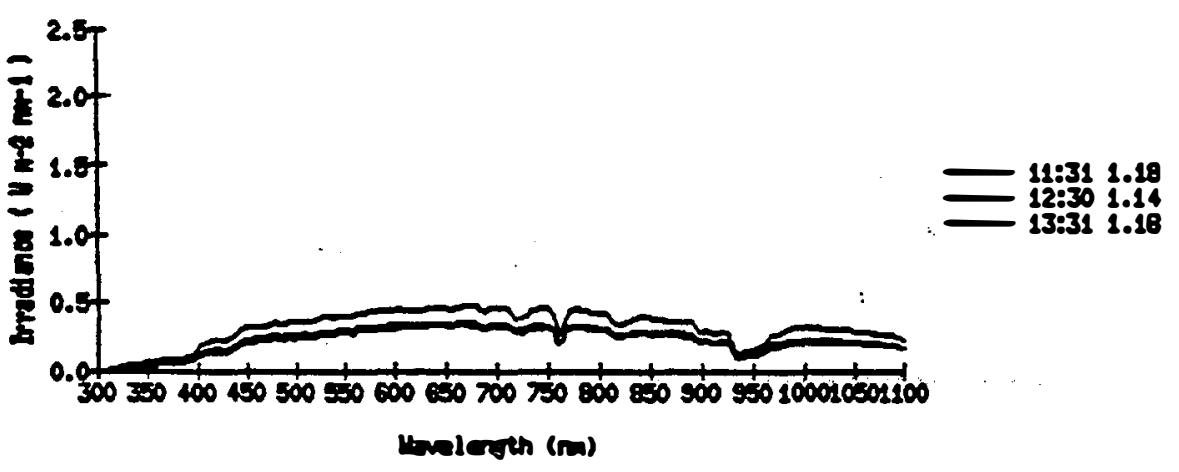

$\operatorname{PSEC} 79$ AS

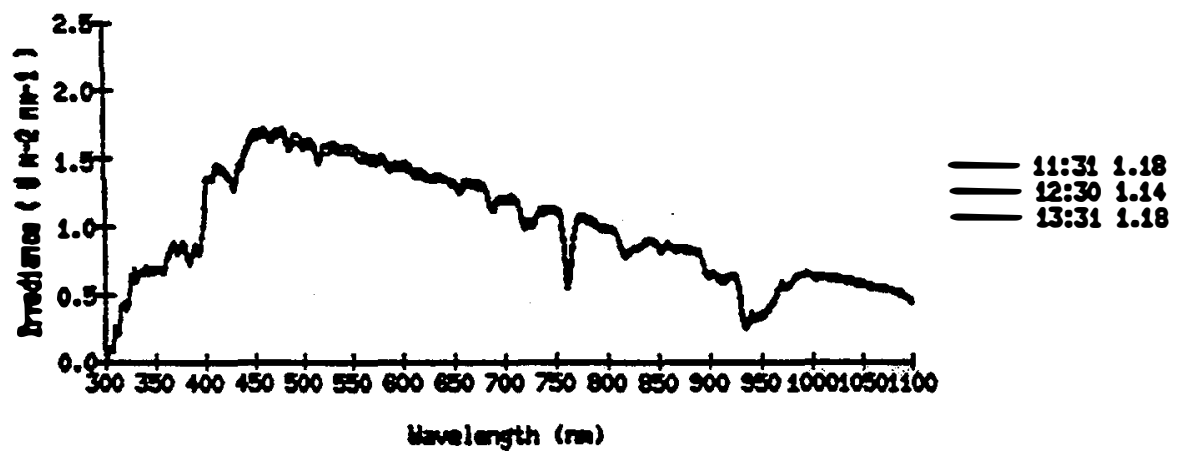


F

$327032088080 \quad 172$ D: 102 G

Partly clouds Ays clear PH

Bo slides

Spectral 1ntegral for Dir is too 100 for 1231, 1331, 1431, 1531, and 1631

Fise 00

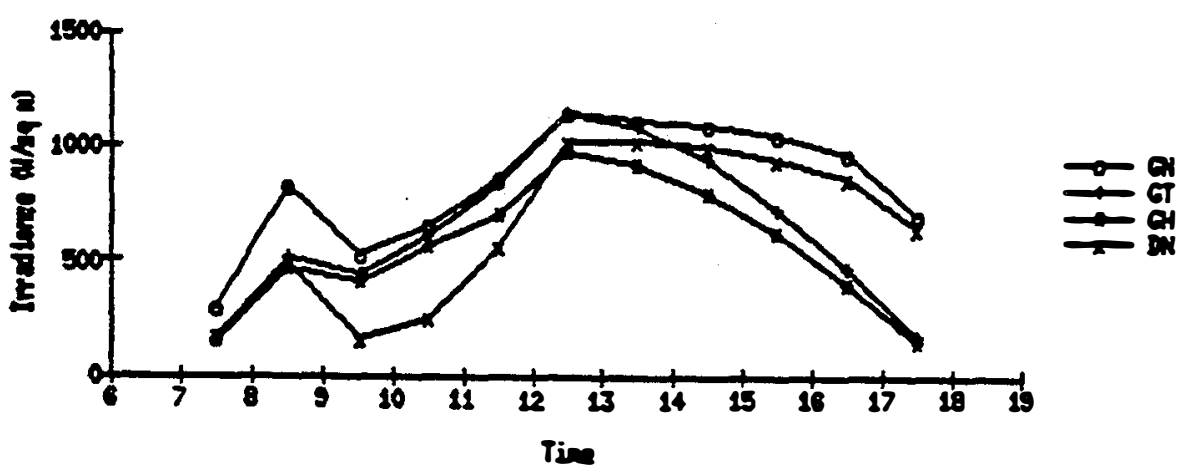

PEEC $80 \mathrm{DT}$

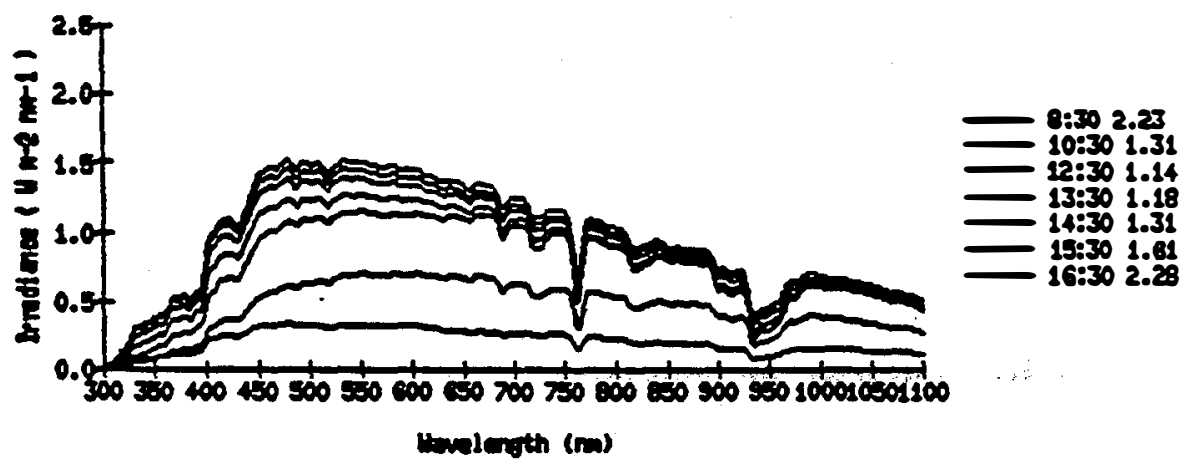

$\sin 0000$

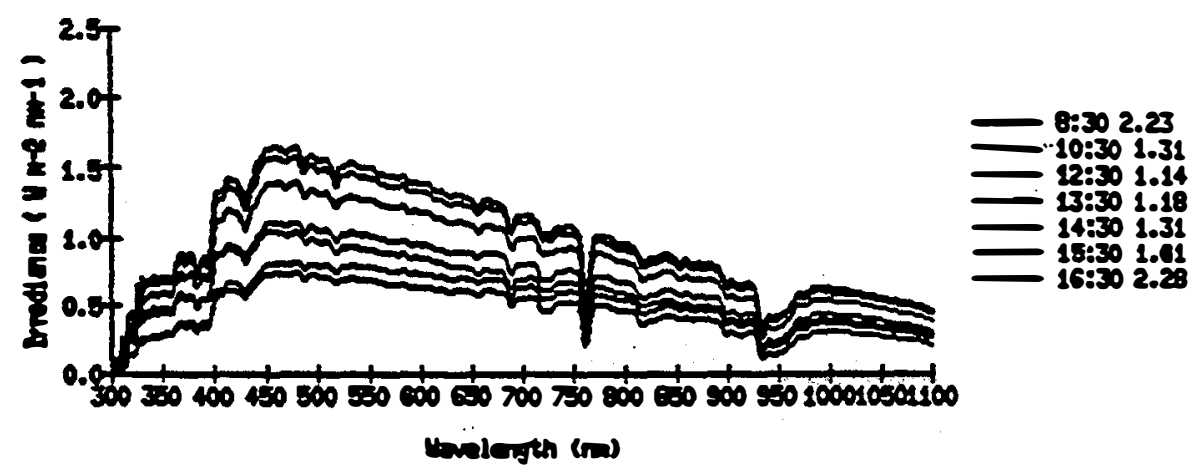


4 FIx-88081

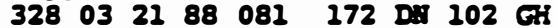

0 cloud cover at 0800,1200 , and 1600

Tep controllers: $172 \quad 102$ 080039 C $39 \mathrm{C}$ $120040 \mathrm{C} 40 \mathrm{C}$ $160040 \mathrm{C} 40 \mathrm{C}$

ISR $102 \mathrm{aV}$ drdft error at 0734

ISR 172 a drift error at 1131

Ho slides

Hear-IR in GH spectrm at 0734 is nolsy

Spectral Integral for Dil 18 too 100 for 1031 and 1131

P्בC 89

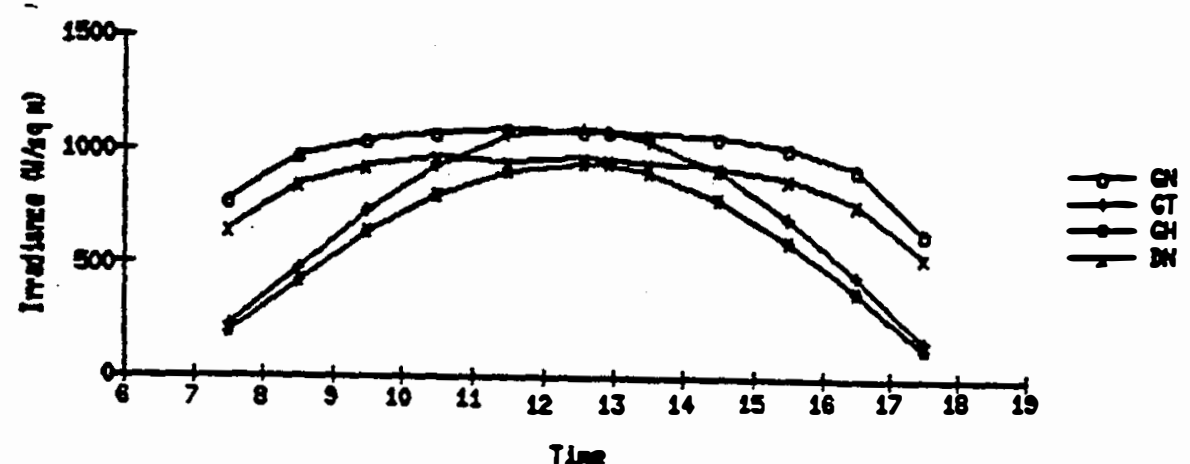

PF्בC 81 int

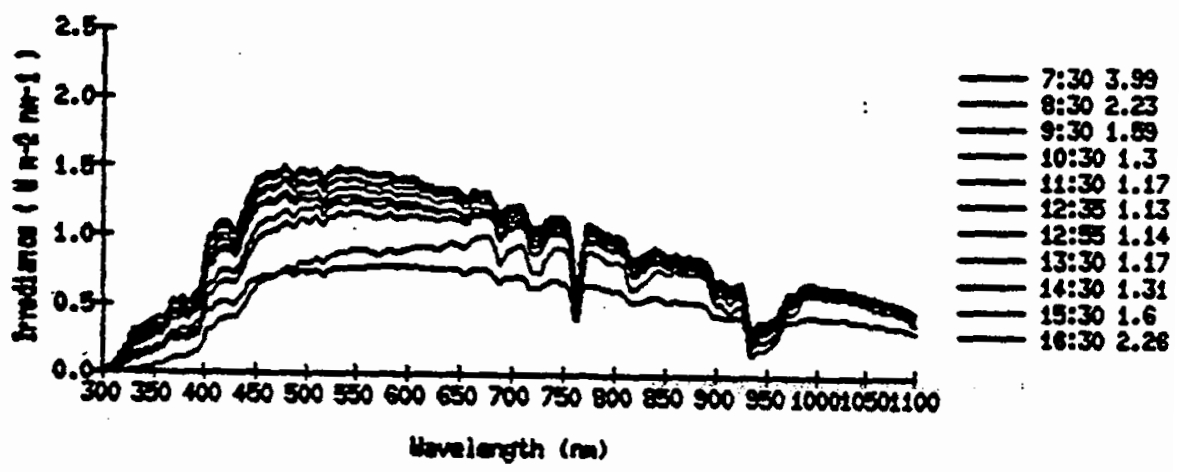

Fis 81 as
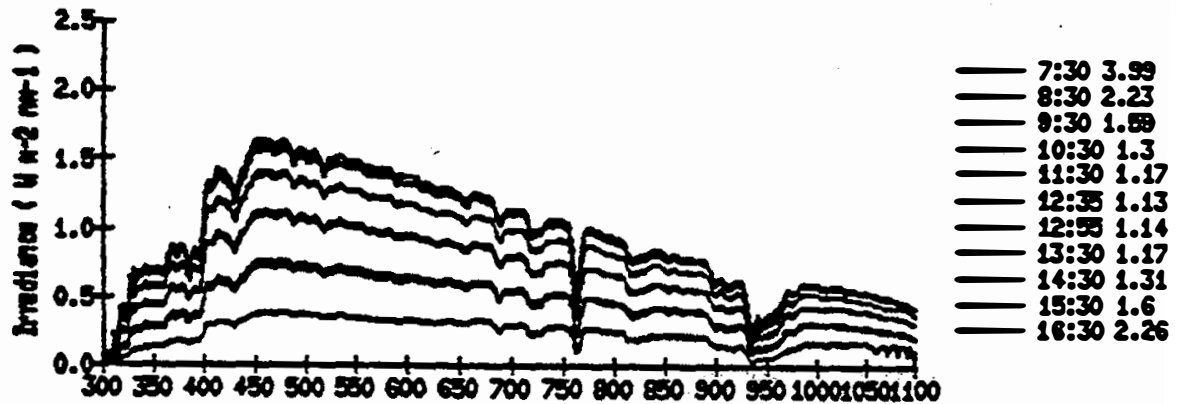

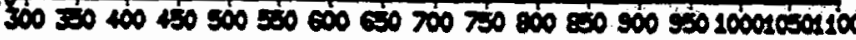

toularoth (m) 
-

$\begin{array}{lllllllll}329 & 03 & 22 & 88 & 082 & 172 & \mathrm{OH} & 102 & \mathrm{FH}\end{array}$

0 eloud cover at $0815 ; 0.8$ at 1600

rap controllersi $172 \quad 102$

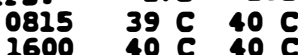

Bo slides

Fsex 82

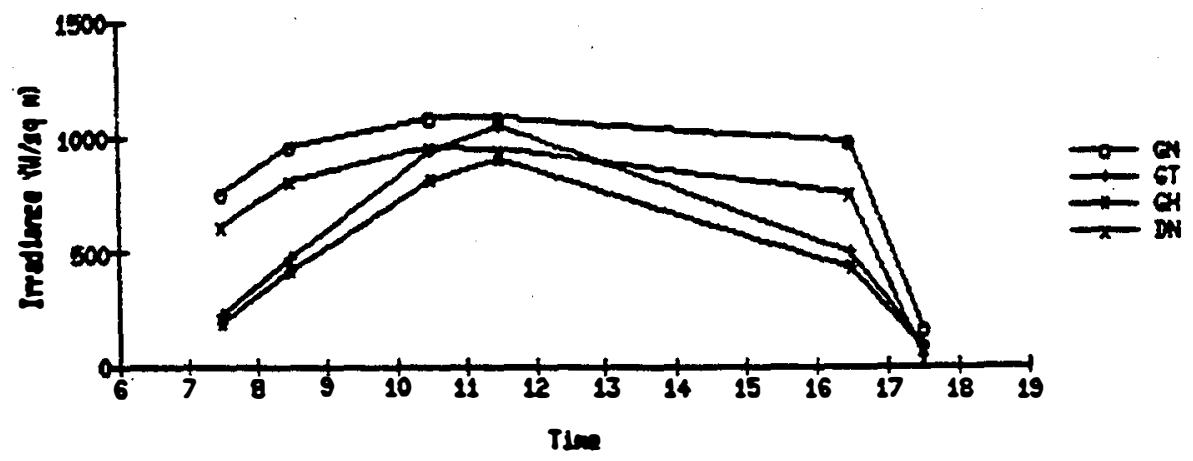

pasc an

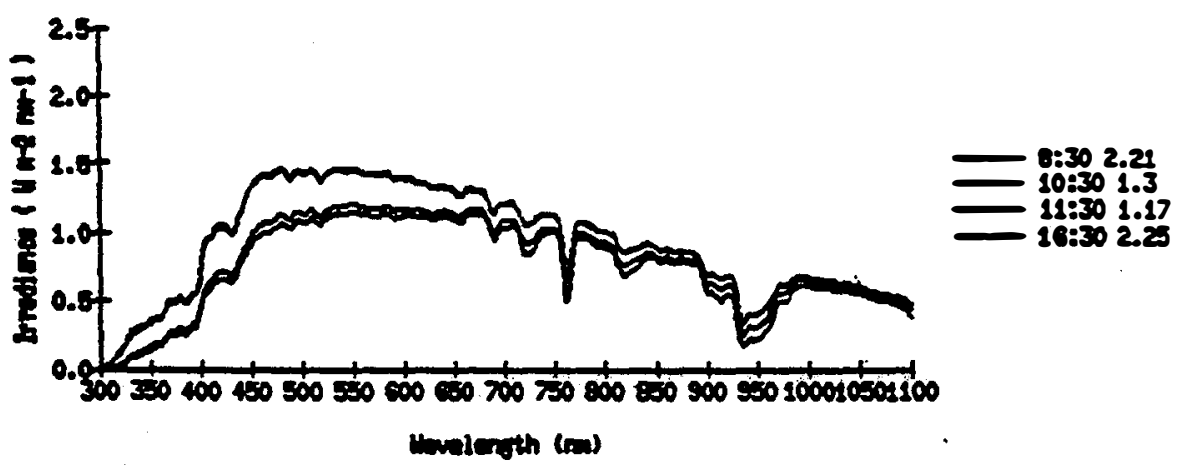

Fa्य 80 OS

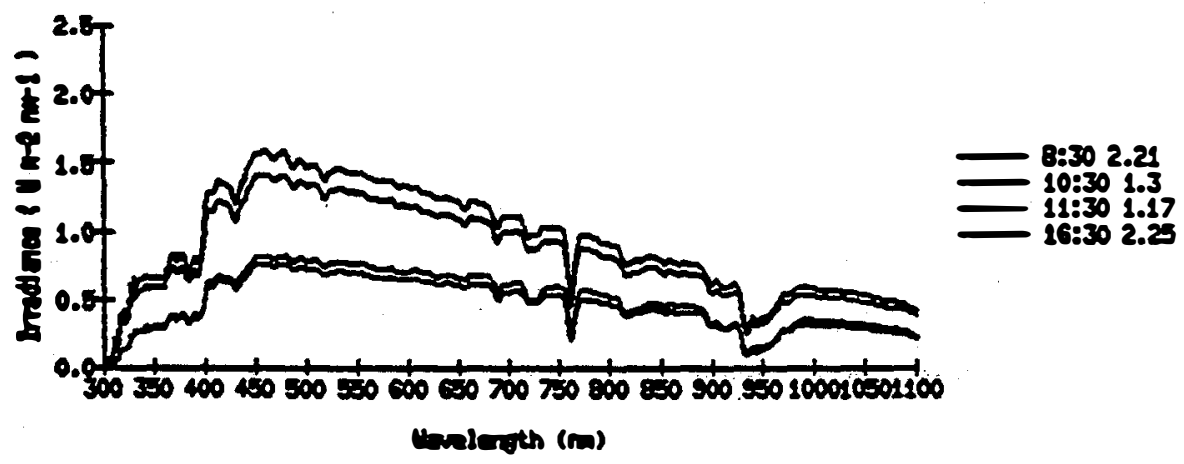


A FST 88083

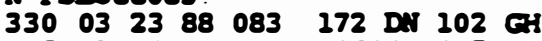

0.3 eloud cover at $0800,0.5$ at 1130

Tepp controllers: $172 \quad 102$ $\begin{array}{lll}0800 & 40 \mathrm{C} & 40 \mathrm{C} \\ 1130 & 40 \mathrm{C} & 40 \mathrm{C}\end{array}$

Tracking error of $2-3 \mathrm{deg}$ at 0800 , reset

May be tractedng errors in 0730 data and 0930 data

Trackers reset at 1030

Mo slides

FSEC 83

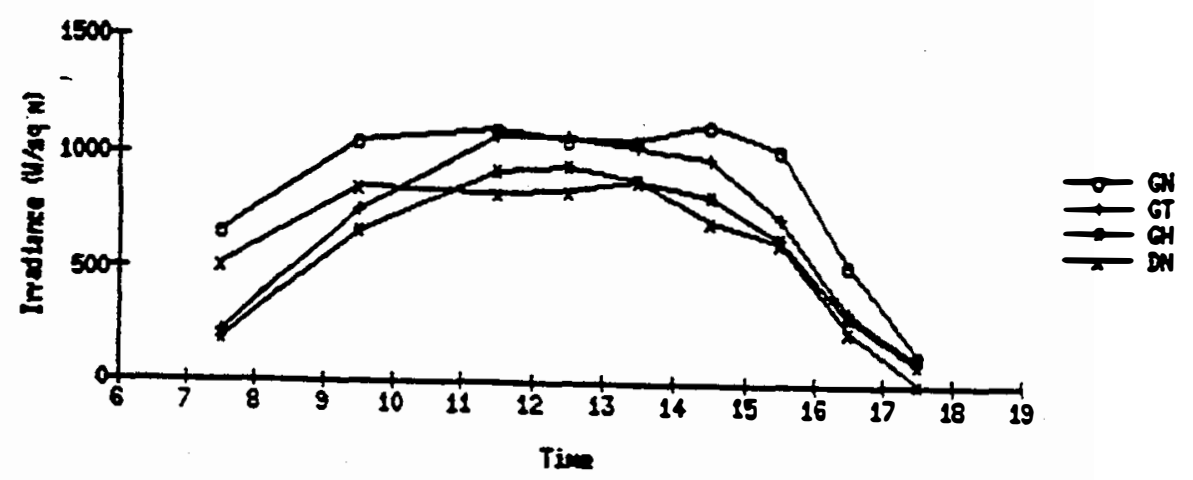

FEEC $83 \mathrm{~min}$

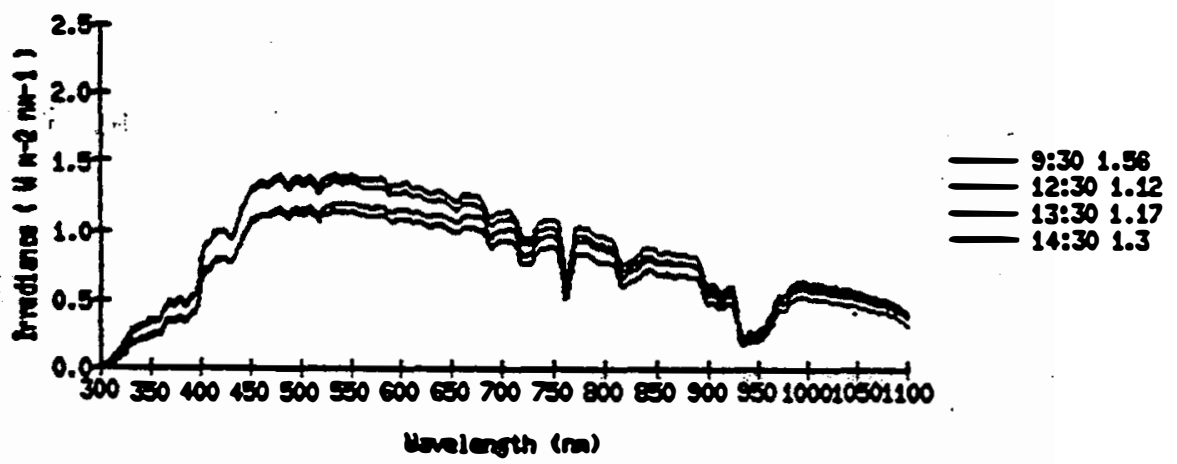

$\operatorname{serceses}$

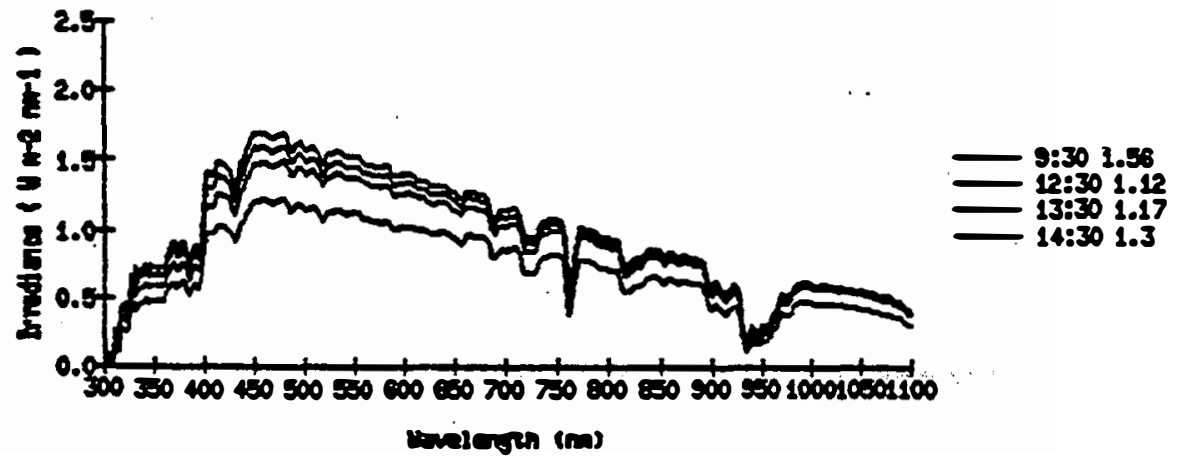




\section{TS088084}

$331032488084 \quad 172$ DI 102 GT

0.1 cloud cover whth haze at $1130 ; 0.7$ (mae) at 1600

Temp controllers: $172 \quad 102$

$\begin{array}{lll}582 & 172 & 102 \\ 1230 & 41 \mathrm{C} & 40 \mathrm{C} \\ 1600 & 40 \mathrm{C} & 40 \mathrm{C}\end{array}$

Bo slides

FIS 84

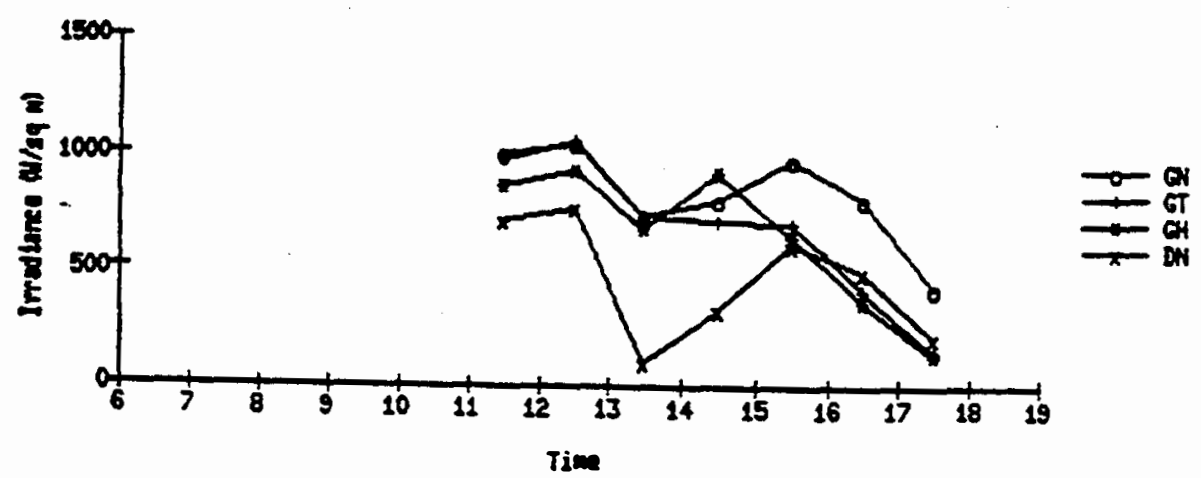

FISC 84 ONT

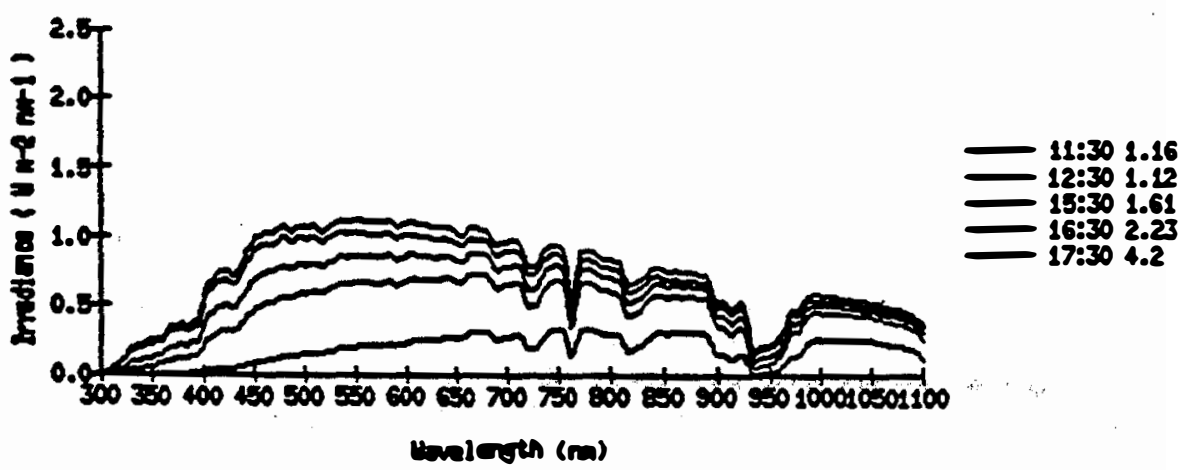

FIE 84 CTS

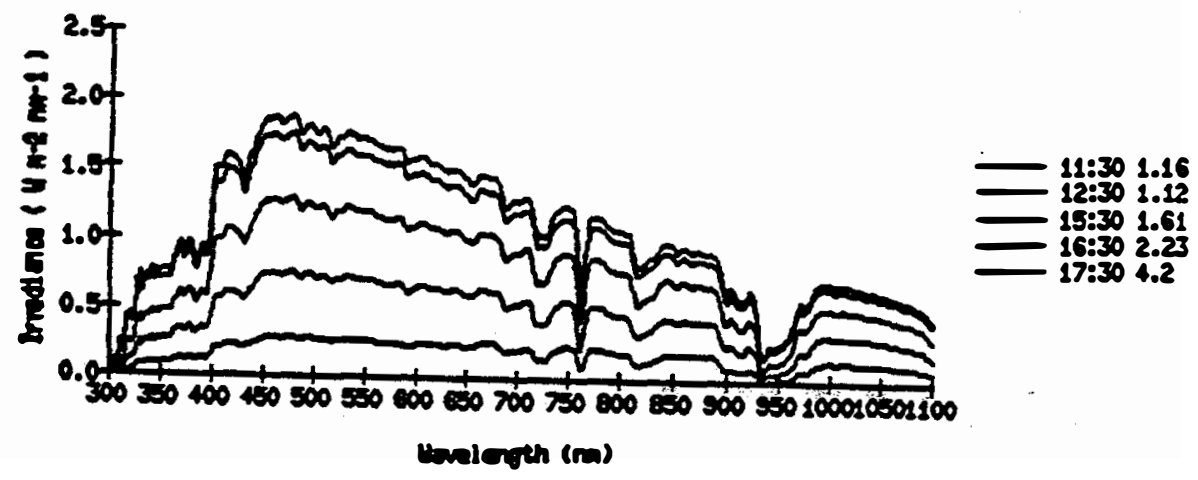




\section{SEP $*$}

4 ए52588085

$\begin{array}{llllllllll}332 & 03 & 25 & 88 & 085 & 172 & \mathrm{DA} & 102 & \sigma_{1}\end{array}$

0.6 eloud corer at 0845: 0.2 at 1340

Ienp controllers: $172 \quad 102$ $084540 \mathrm{C} 40 \mathrm{C}$

Traeking dater bad prior to 1430

Reset trackers at 1340

Sl1des: $1233 \mathrm{M}$ (dark)

$1332 \mathrm{P}$ -

$1432 P$ -

$1532 \mathrm{~K}$

$1632 \mathrm{G}$

$1732 \mathrm{G}$

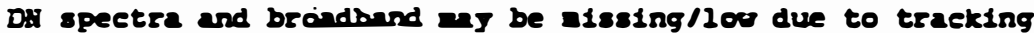
on exss day

Bad DI (spectzal and brandinad) at 0931 and 1031

TSE ES

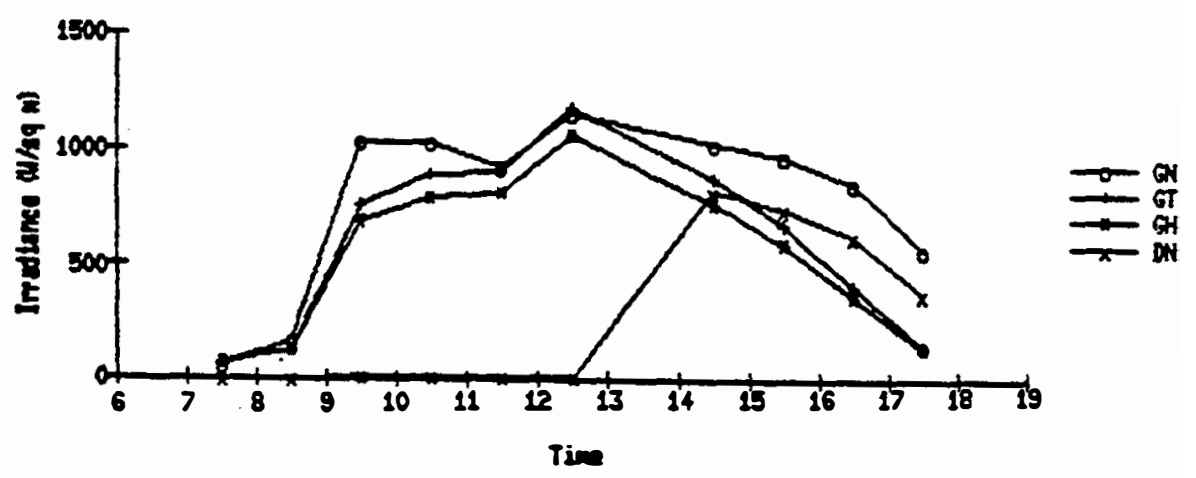

Pिese 8

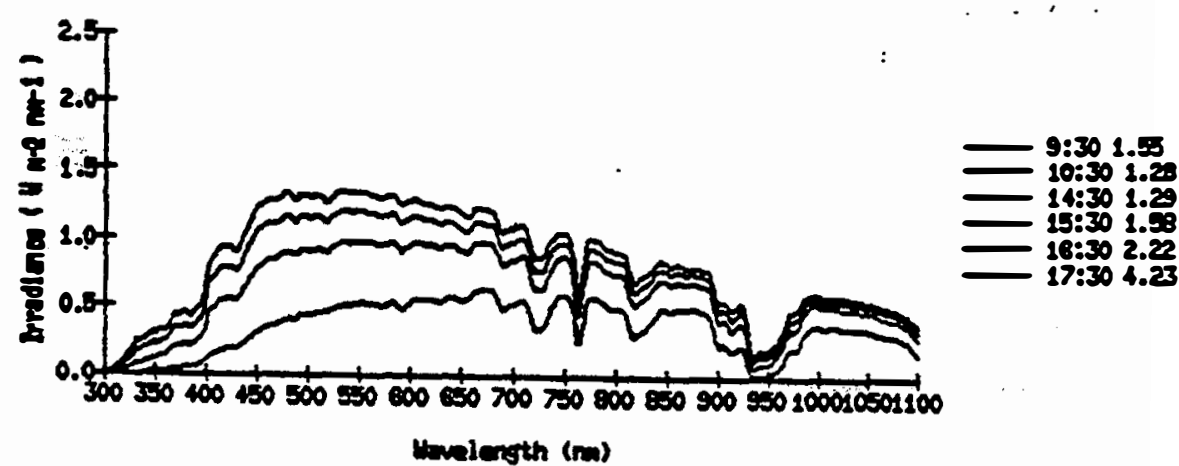

PSEC $80 \mathrm{GT}$

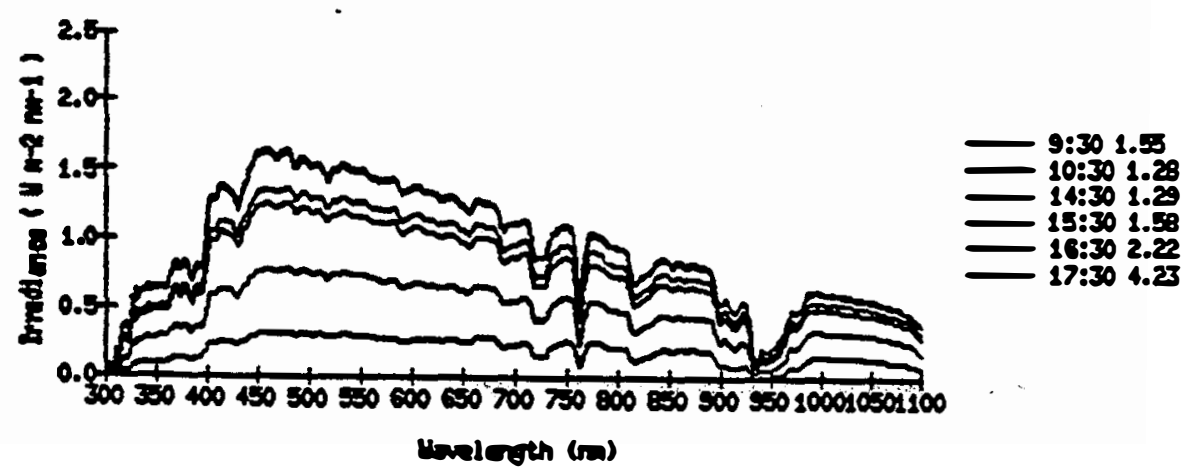


FSEC88086

$\begin{array}{llllll}33303 \quad 2688 & 086 \quad 172 \text { DU } & 102 & \text { GI }\end{array}$

Tracking errors; tracking data are bad

slides: 0732 A (condensation)

0828

$0930 \mathrm{H}$

1028 P (dark)

$1128 \mathrm{P}$.

$1228 \mathrm{P}=$

$1327 \mathrm{P}:$

$2527 \mathrm{G}$

1627 G

Din spectra and broadband are aissing/lo0 due to tracking errors on this day

Bad Dis date (spectral and brondband) all day

PSEC 86

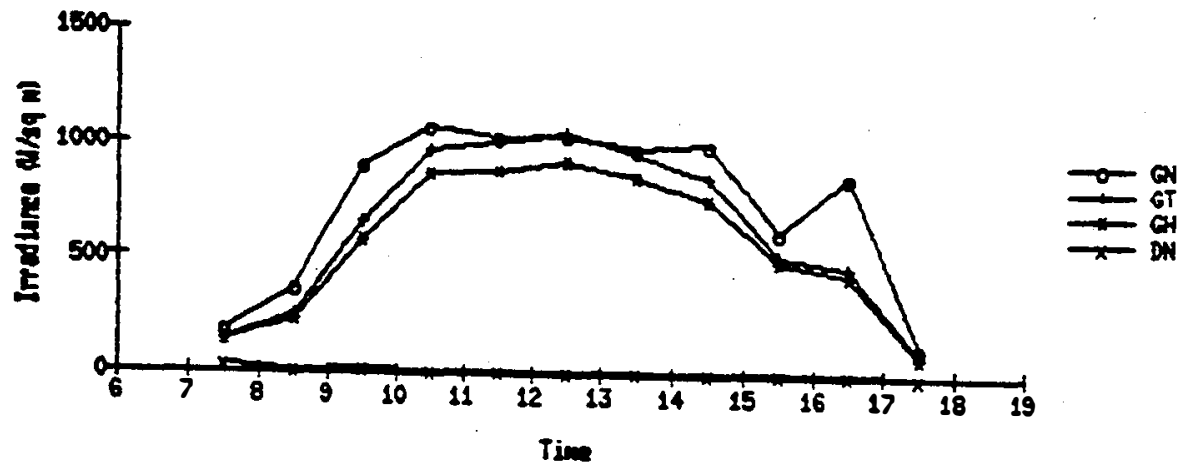

FEב $86 \mathrm{DAT}$

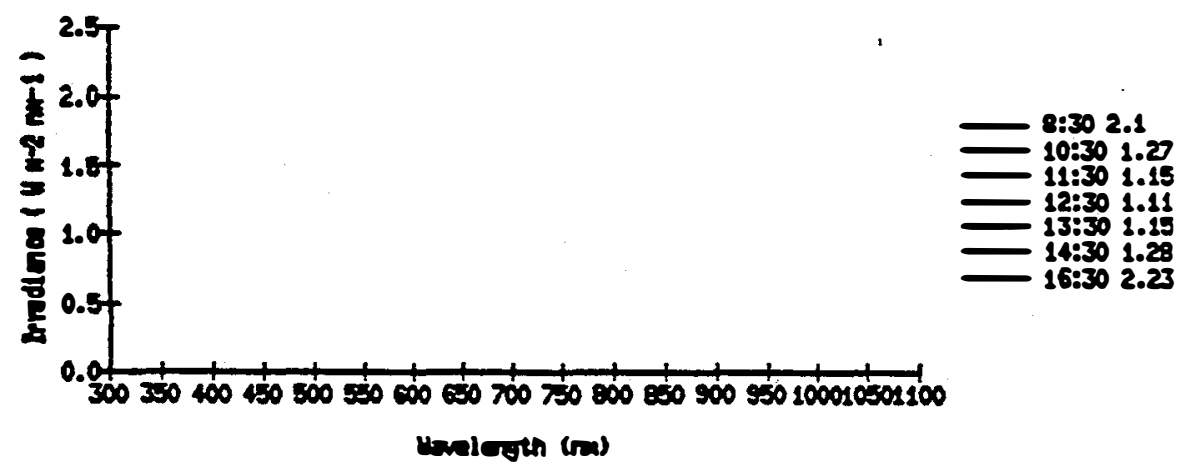

TISE CS GS

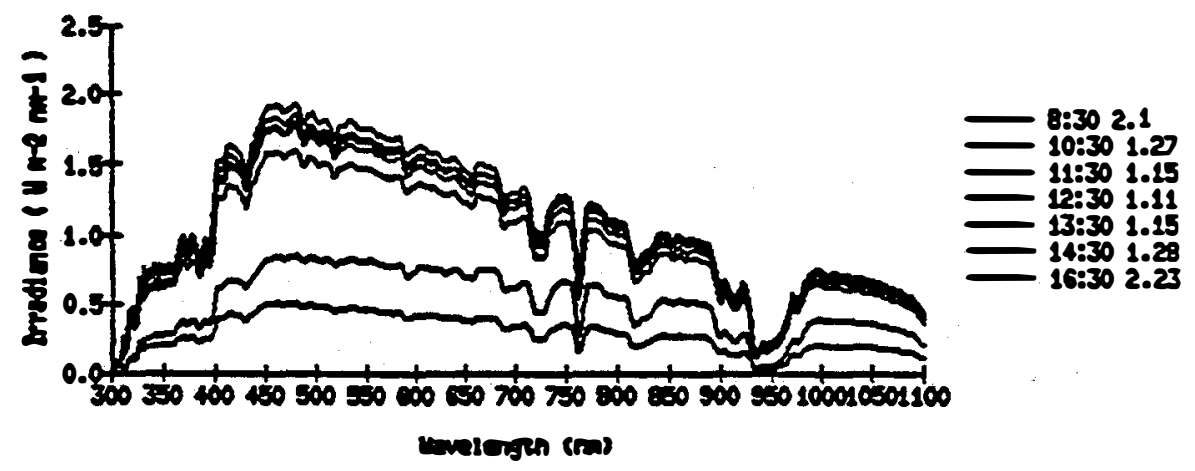


N TSx 88087

$\begin{array}{lllllll}334 & 03 \quad 2788 & 087 & 172 & \text { DI } & 102 & \text { GT }\end{array}$

No data for this day

sildes: 0724 M (enter drops)

0824 " " (dark)

$0929 \mathrm{P} \quad-\quad$

$1124 \mathrm{H}$ -

$1228 \mathrm{P}$
$1331 \mathrm{P}$

1423

$1523 \mathrm{G}$

$1623 \mathrm{G}$

1723 G

Bo dara 


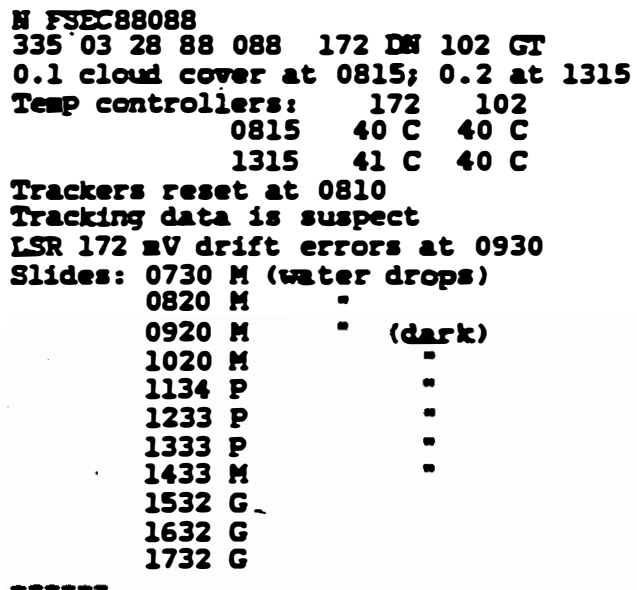

Dy spectru and bronding assing 1032 due to tracker

Fase 88

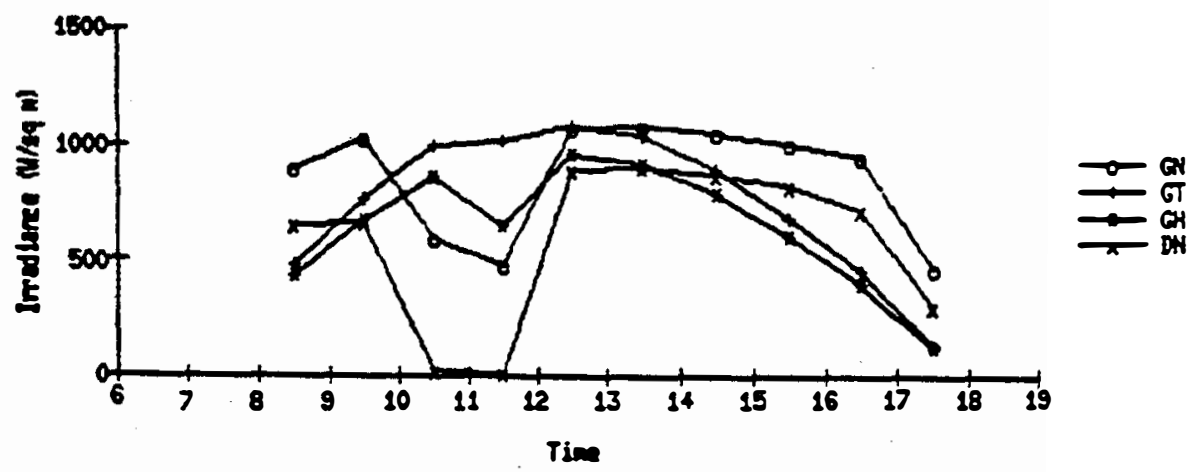

F्בS $88 \mathrm{BNT}$

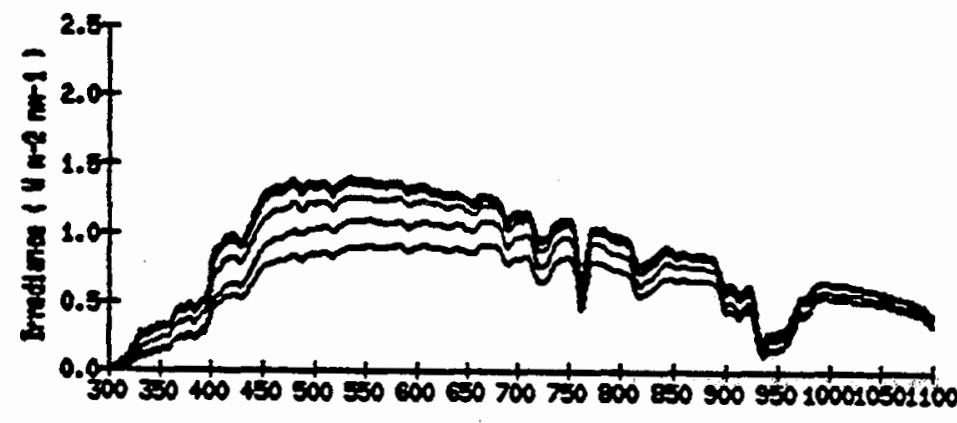

Hevelength (m)

FESC 88 GTS

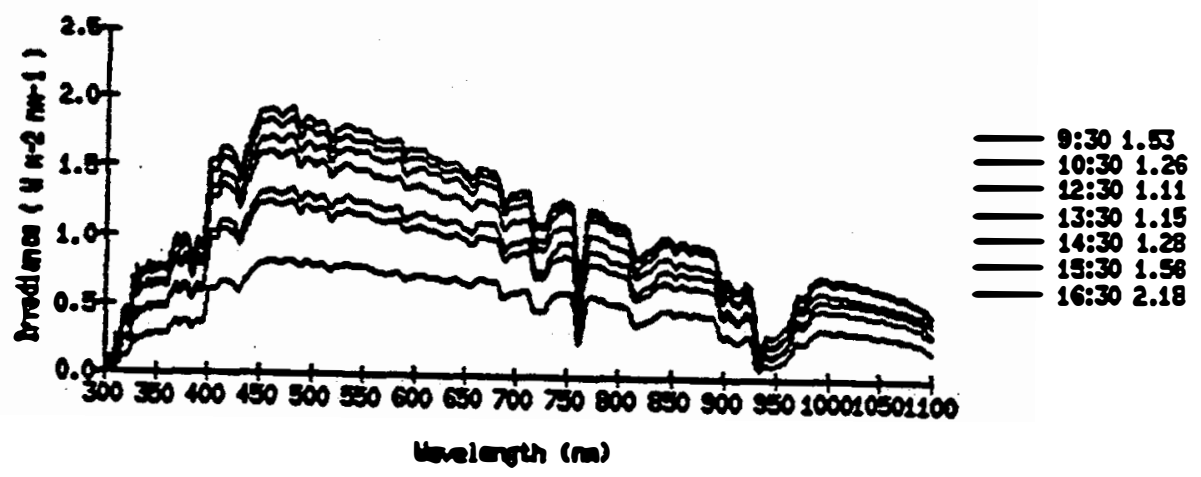




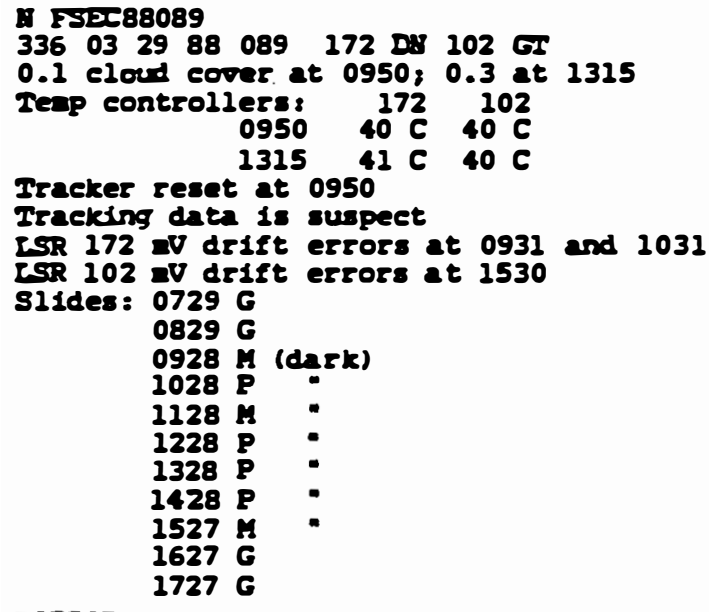

Tracker errors - spectra bad at 0830 and 0931

GI spection noiss in ov and near-IR at 1530
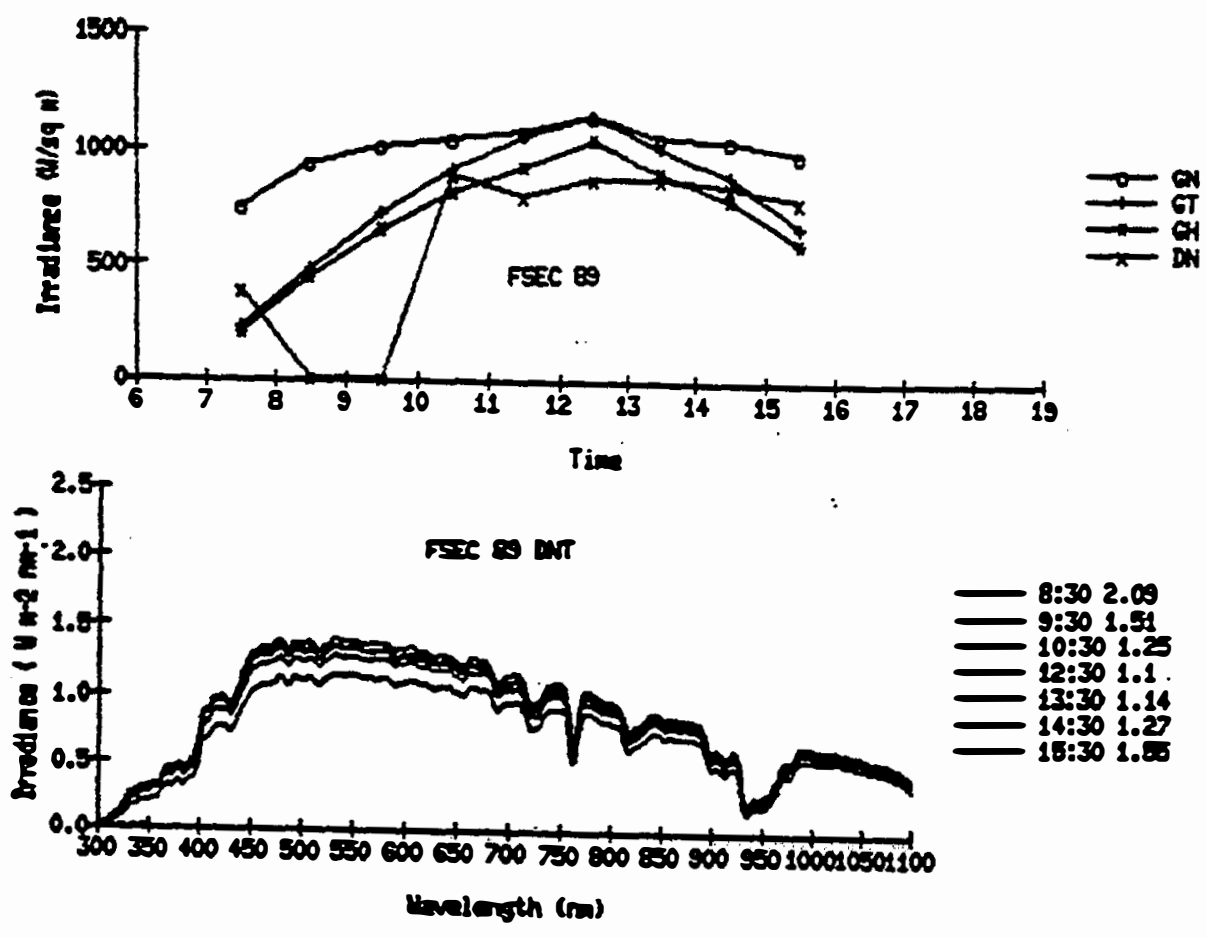

FISE 89 GTS

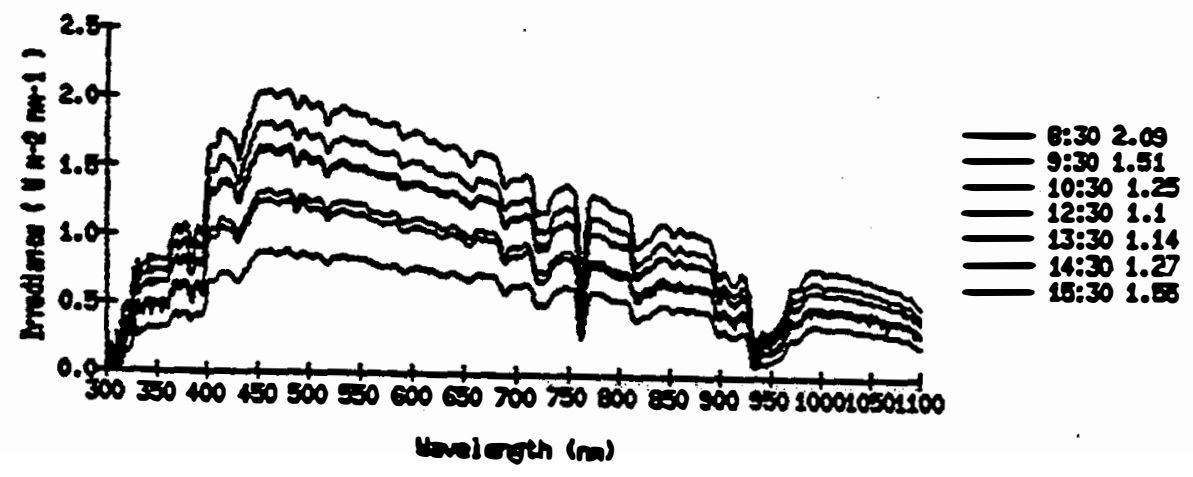


N FSX 88090

$\begin{array}{lllllllll}337 & 03 & 30 & 88 & 090 & 172 & \mathrm{DP} & 102 & \mathrm{GT}\end{array}$

0.1 cloud cover at 0800

Tenp controllers: 172 and $10240 \mathrm{C}$ at 0800

Iracking data is suspect in MHz PH data probnbly $0 . \mathrm{I}$.

Slides: $0729 \mathrm{G}$

$0824 G$

0924 I (dark)

$1024 \mathrm{P}$

$1130 \mathrm{P}$

$1230 \mathrm{P}$

$1330 \mathrm{P}$

$1529 G$

$1629 \mathrm{G}$

Dis data bad'et 0930 and 1030

rese so
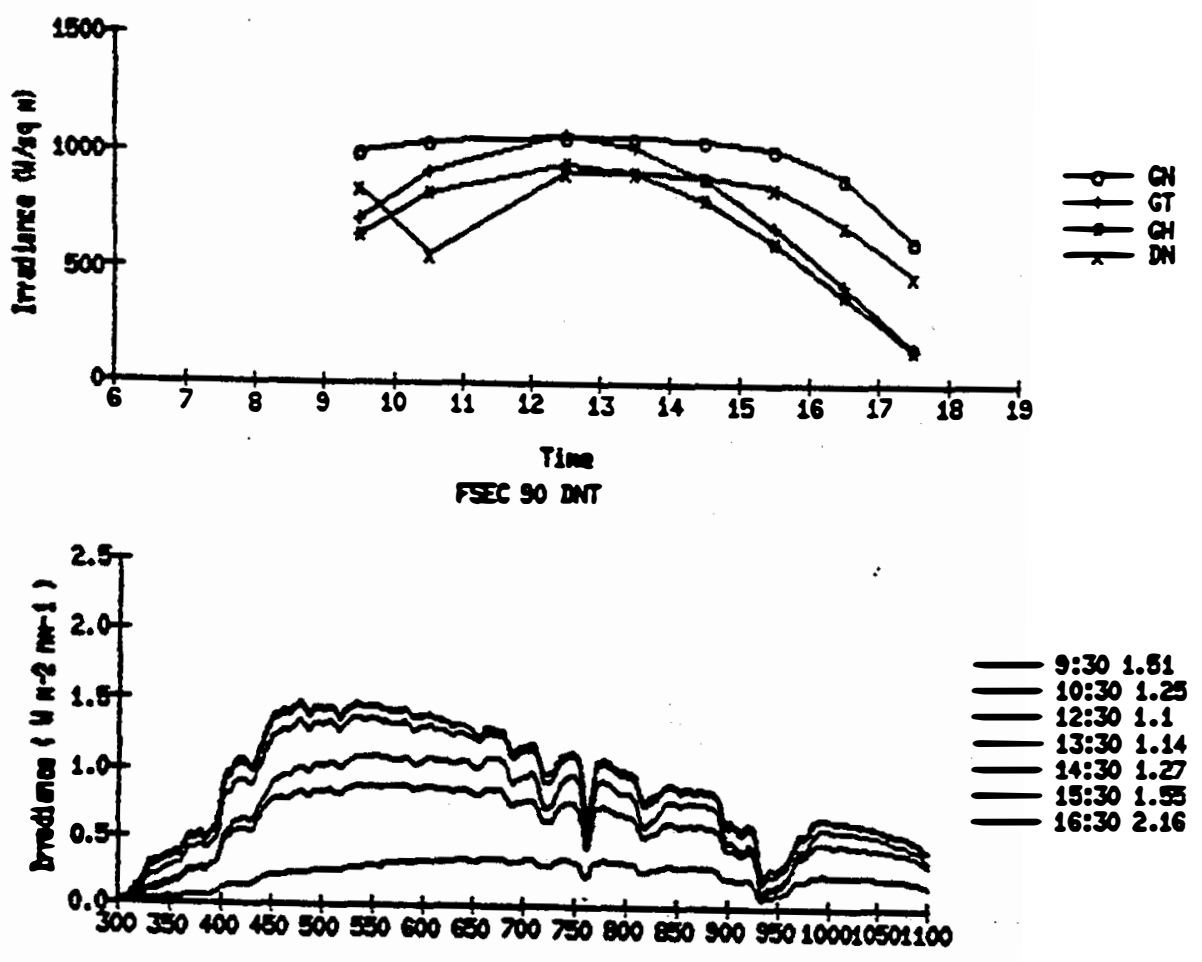

Howlergth (n)

FIS 90 GTS

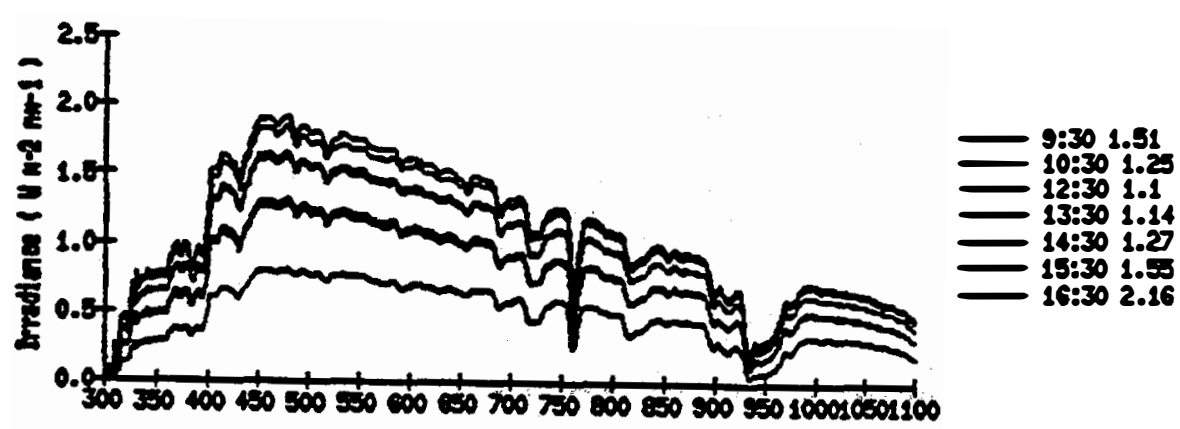

Howiogth (m) 
4. TST 88091

$\begin{array}{llllllllll}338 & 03 & 31 & 88 & 091 & 172 & \text { DI } & 102 & G T\end{array}$

0.25 cloud cover at 0815

Temp controllers: 172 and $10240 \mathrm{C}$ at 0815

Trackers reset at 0820

Iracteing data is suspect

Slides: $0726 \mathrm{G}$

$0826 \mathrm{G}$

0926 M (dark)

$1026 \mathrm{P}$

$1126 \mathrm{P}$

$1227 \mathrm{P}$.

Dis data bad at 1030 and 1532

FIE 91

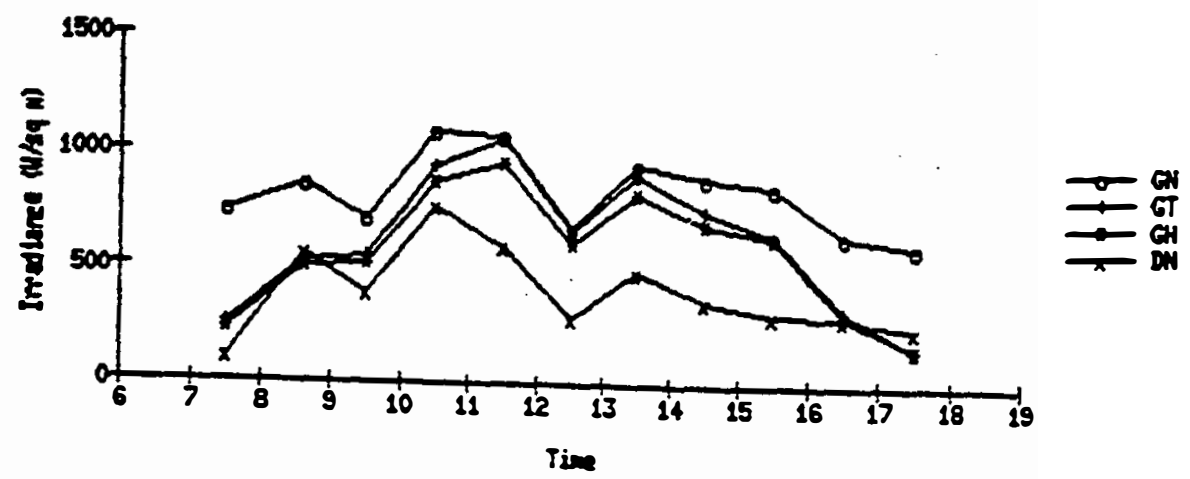

FSEC 91 an

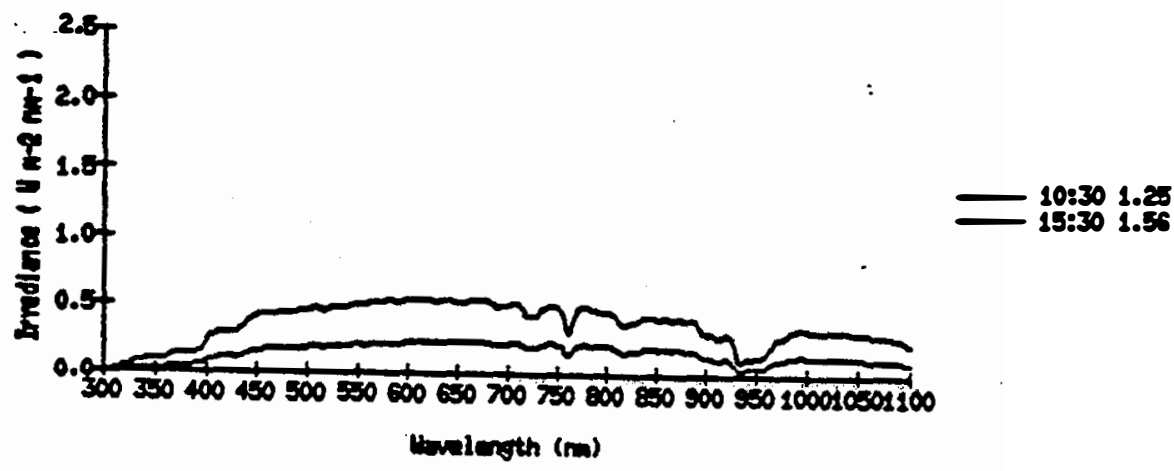

FIE I1 TIS

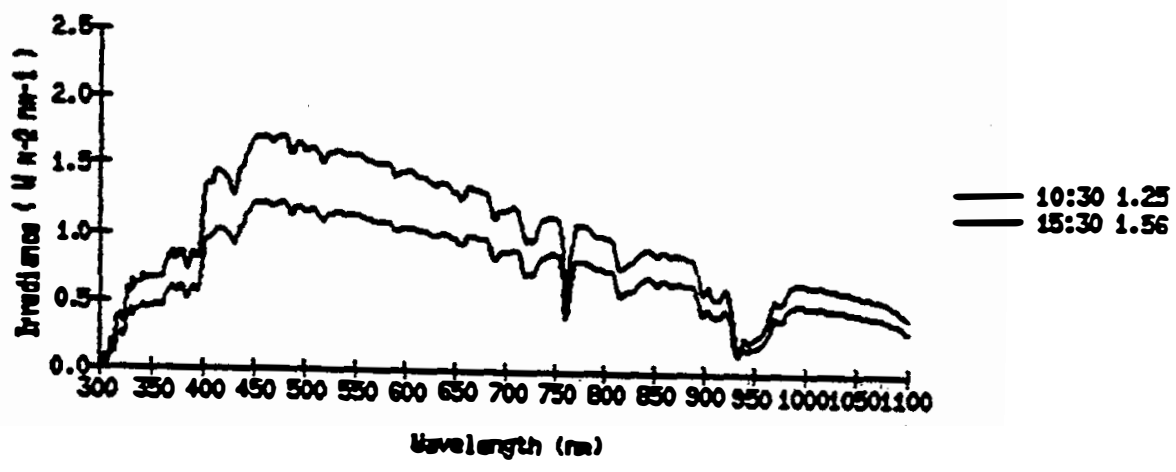


PSTE88092

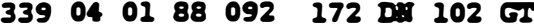

0.4 cloud cover at 0810

Tenp controllers: 172 and $10240 \mathrm{C}$ at 0810

Reset tracters at 0940

Tracking data suspect in $\mathrm{M}$

ISR $172=V$ drift errors at 1230

Ho slides

Di date are bad at 0730 and 1230

Fese 2

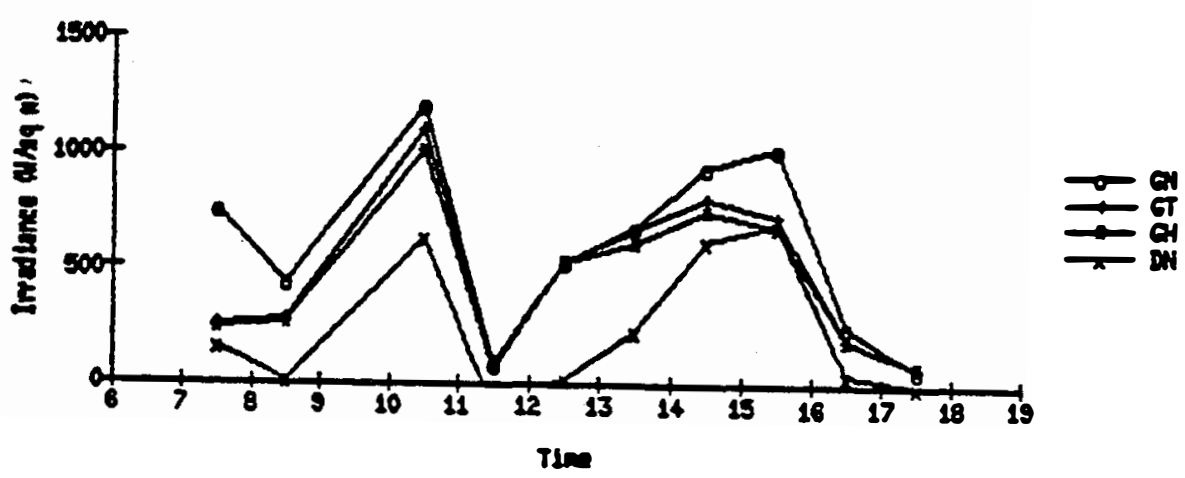

TEx 28 ant

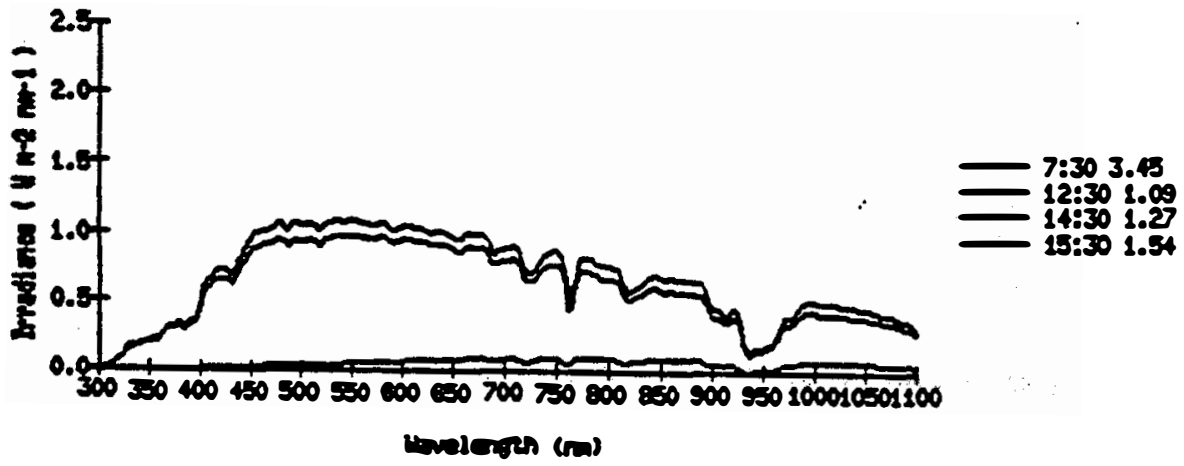

FEב 20 GS

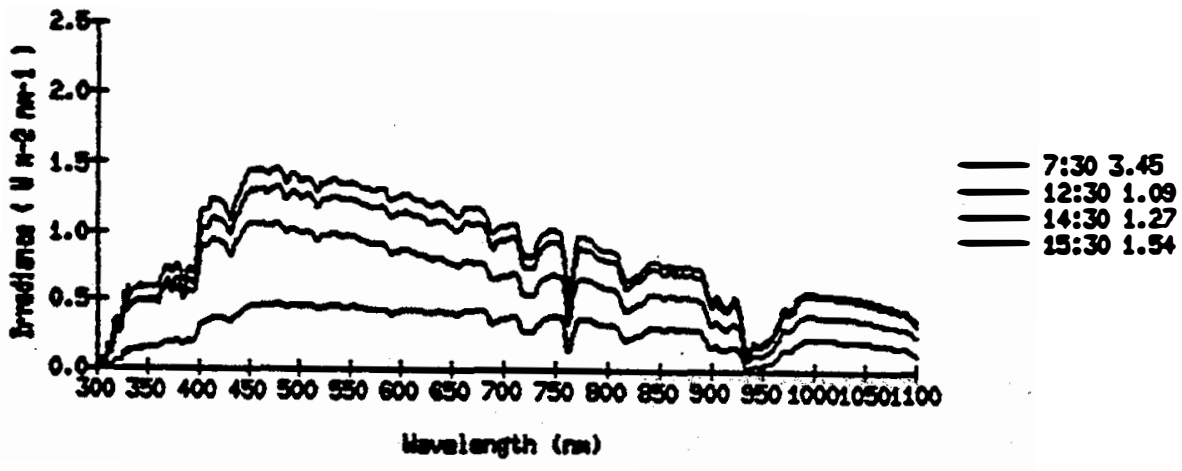


A. FSTC88093

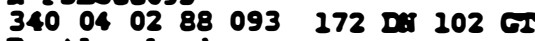

Partly cloudy

ISR 172 a drift errors at 0830 and 0930

Ho slides

(-)-

Verg brief apike in OV in DA spectra at 0830 and 0930

$\operatorname{PSEC} 33$

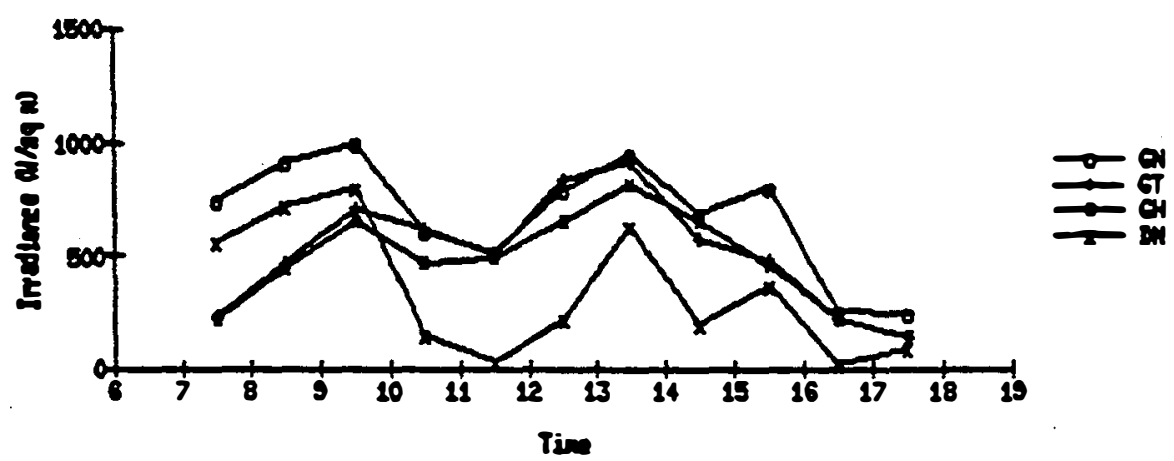

FEse 93 ant

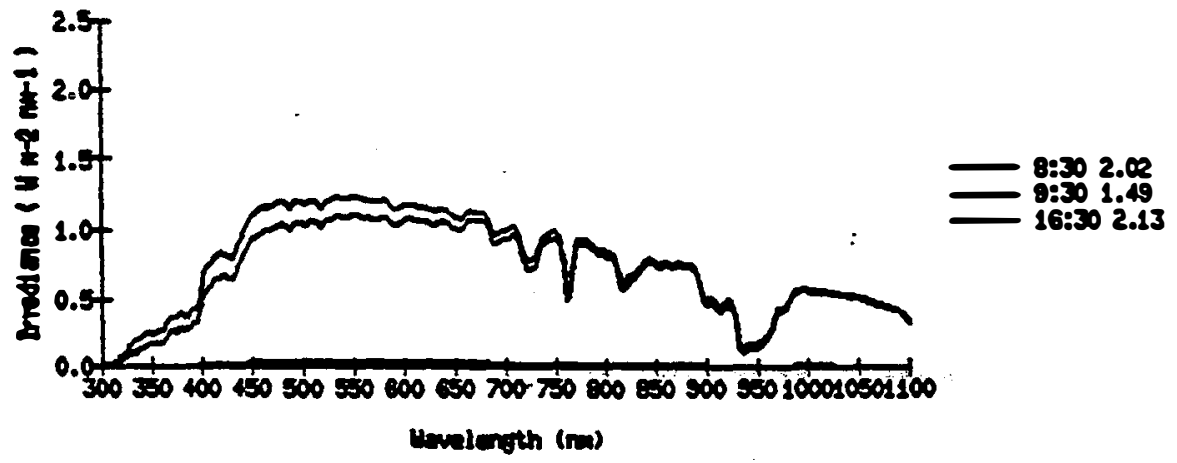

Prse 23 Gts

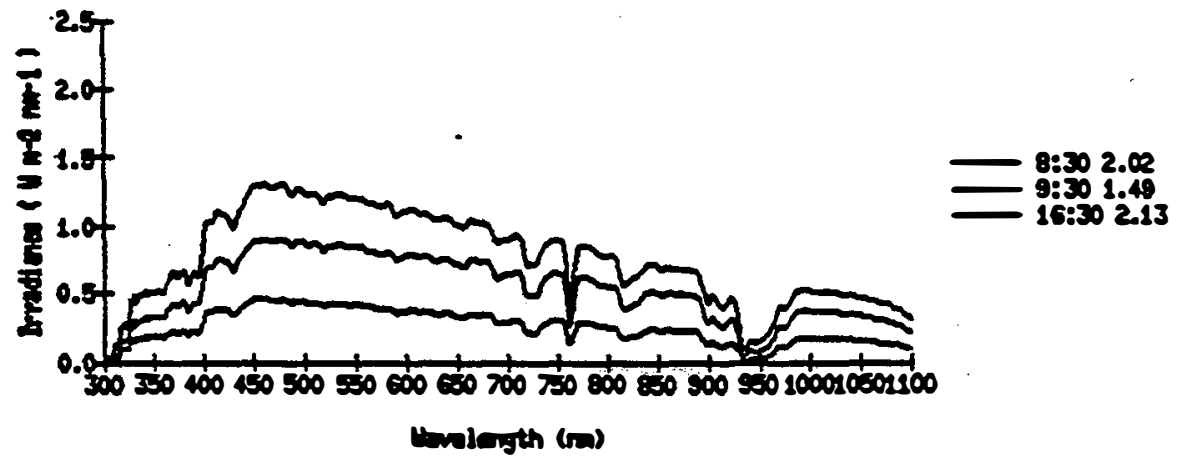




\section{$=01$}

P FST288094

$341040388.094 \quad 172$ D: 202 Gr

Partl clouds

rgR 172 ov drift errors at 0830, 1030, and 1130

Ho slides

Spikes in of in 0 spectra at 0733 and 0830

FSEC 9

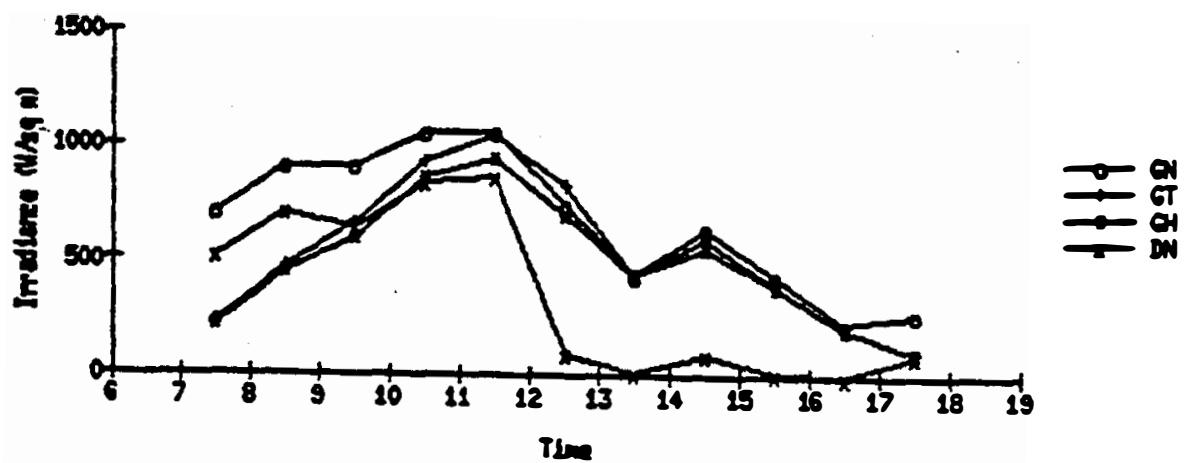

$\operatorname{Fsec} 94 \mathrm{bu}$

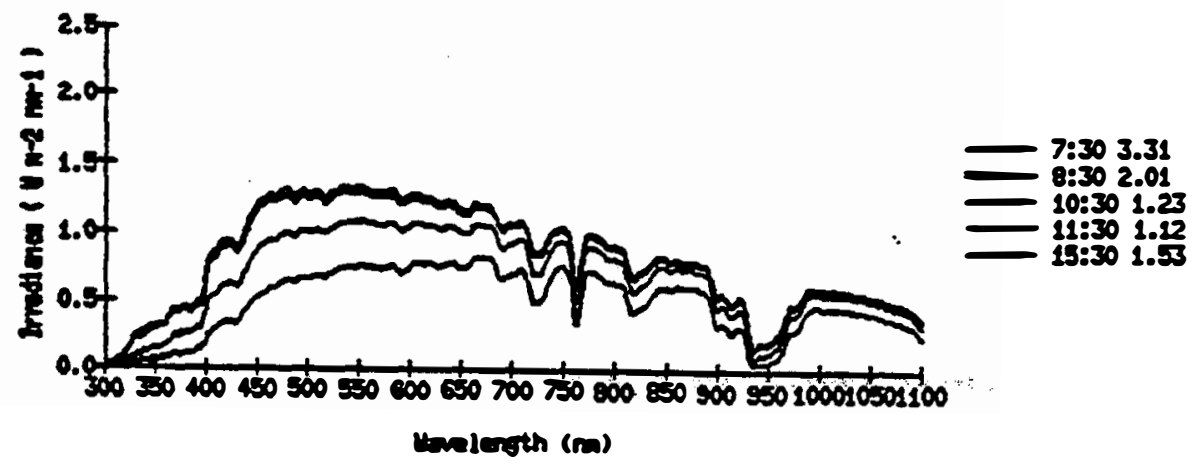

Fese at GT

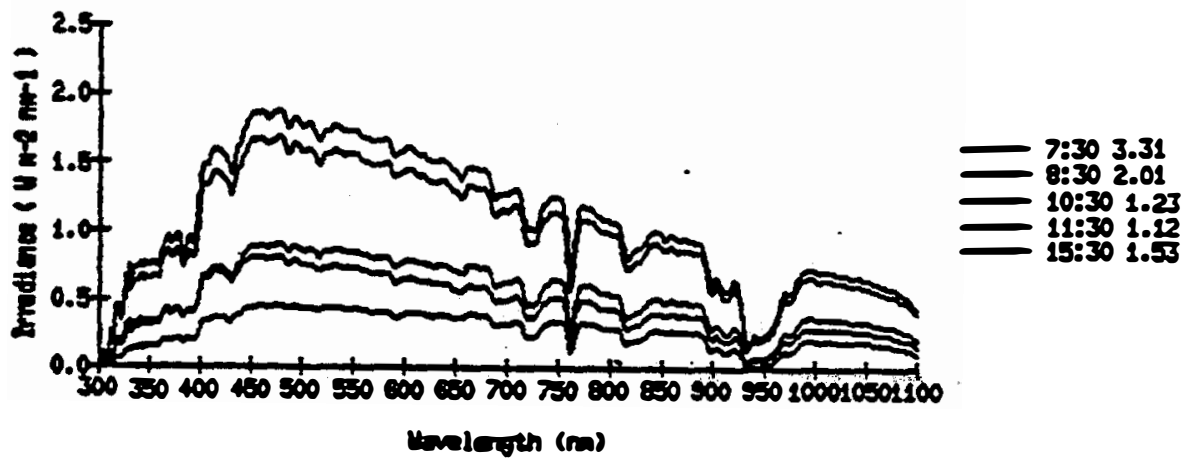


A 75288095

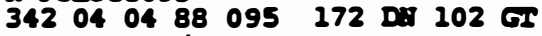

0.9 eloud cover at 0900

Texp controllers: 172 and $10240 \mathrm{C}$ at 0900

ISR 172 av drift errors at 1230

Ho slides

Pise go

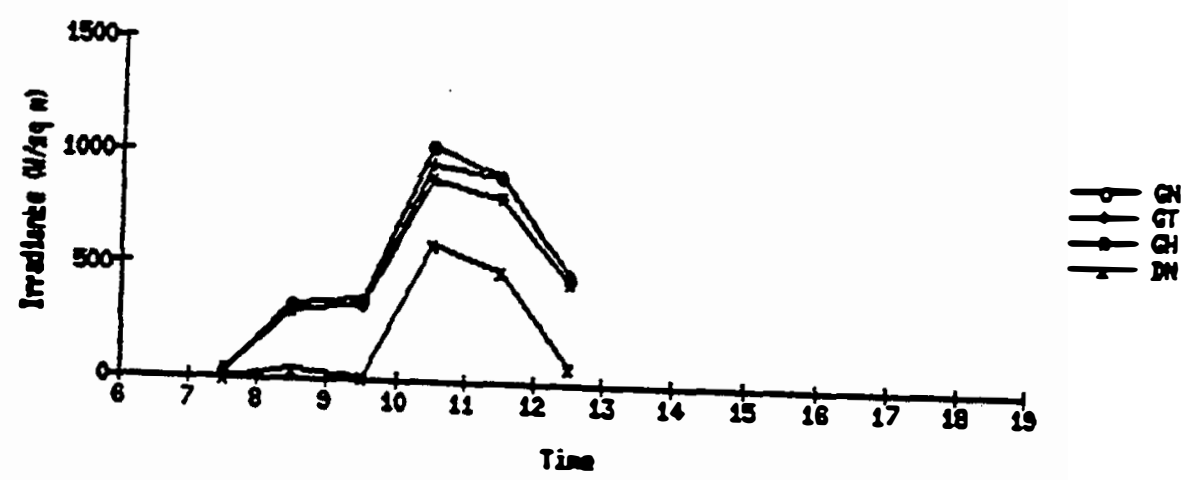

F्בse $808 \pi$

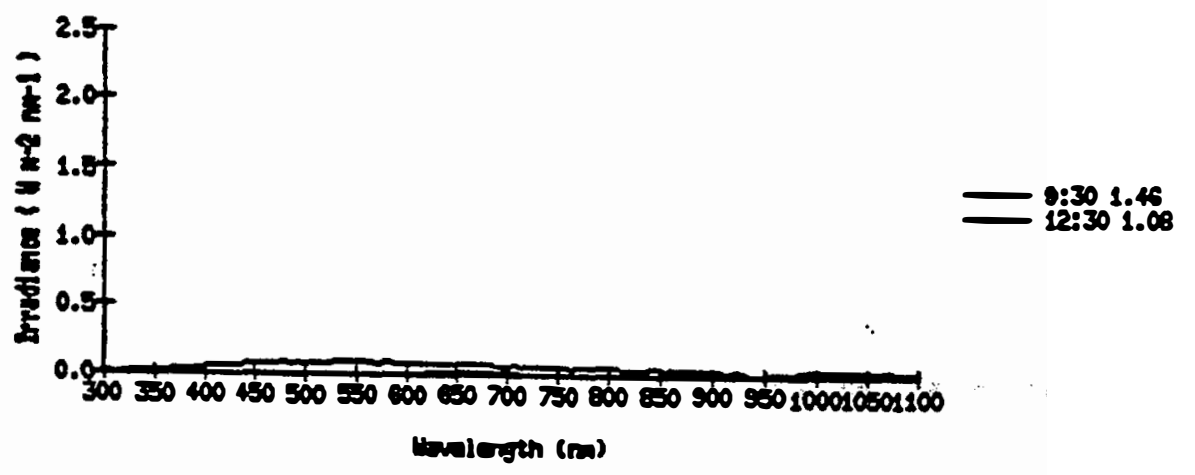

FEE SO GTS

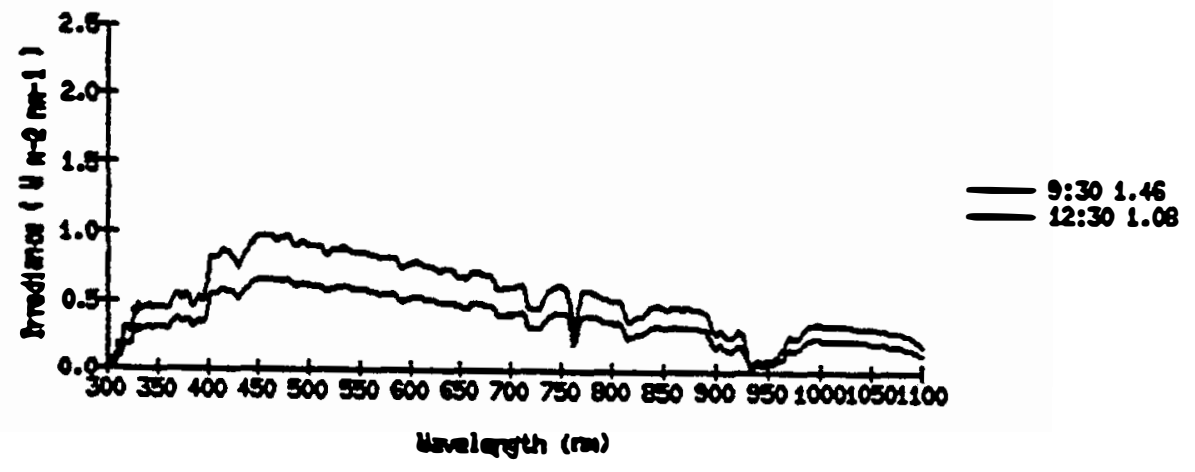


3.2 Pacific Gas and Electric Company, San Ramon, Calif. 
N. PCES87110

$042087310 \quad 274$ GV

Clear

Problez vith Matrix NIP and II-COR pyranoneter:

NIP aligment off; fixed 4-21

Prianoneter out of range at 850 th-2; changed roltage range 4-2l

Eppley and Matrix HIP tracker out of aligment; corrected 4-2l

No photos

-

NIP date are bad

PEI 110

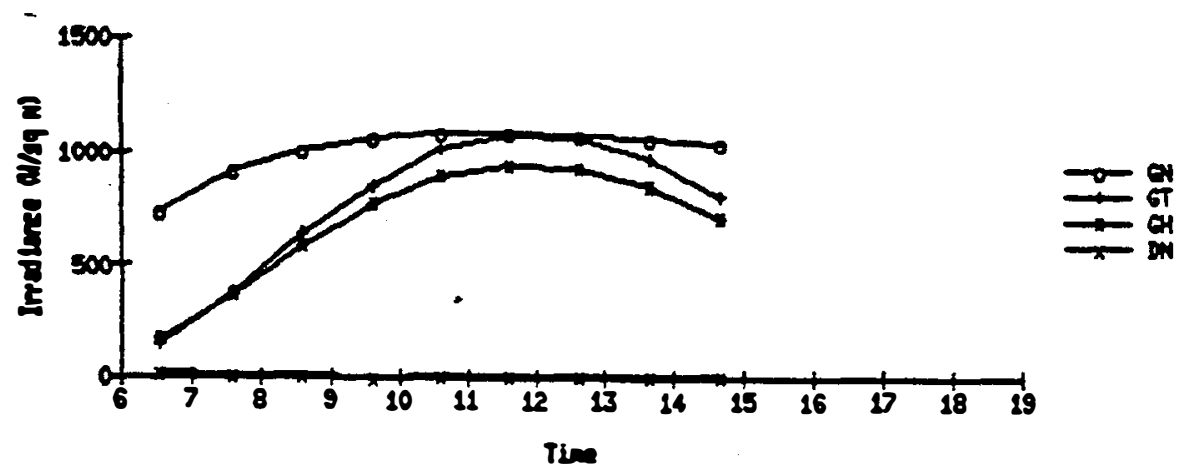

PAE 220 OD

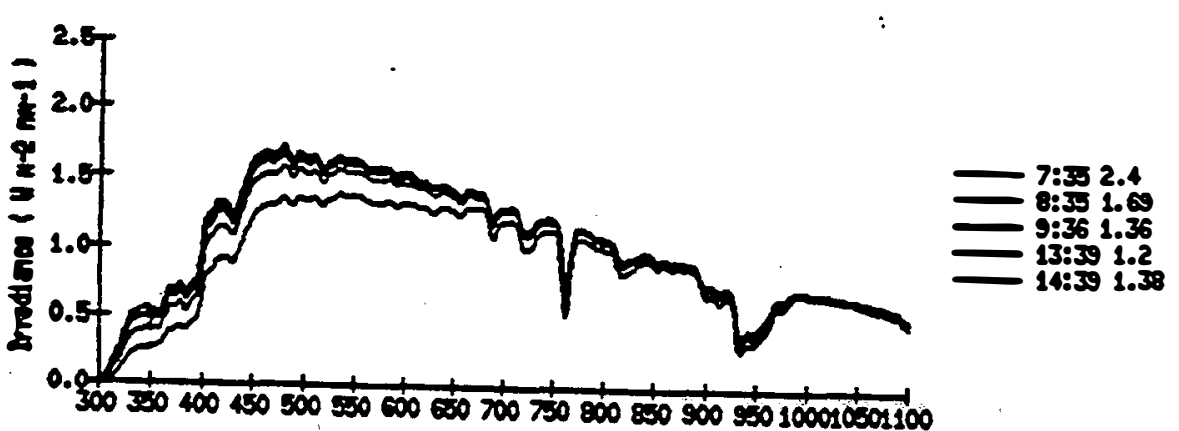

unlarth (ra) 
Pers87111

042187 III $174 \mathrm{Gr}$

Clear; sone haze

MIP aligment and prranoneter voltage fired

Power fafled about 0950; restarted at about 1020

No photos

PEE 111

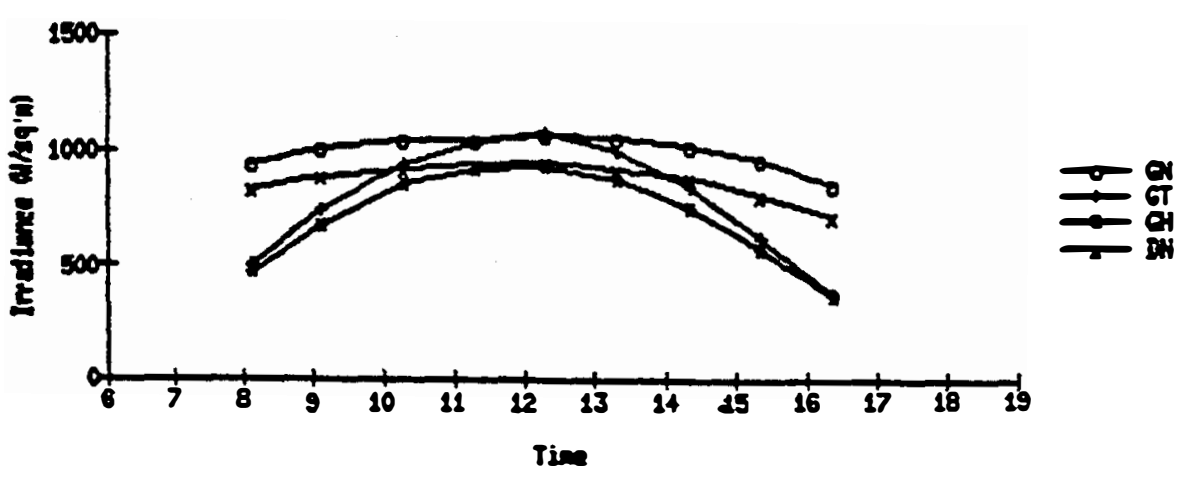

PAE 11100

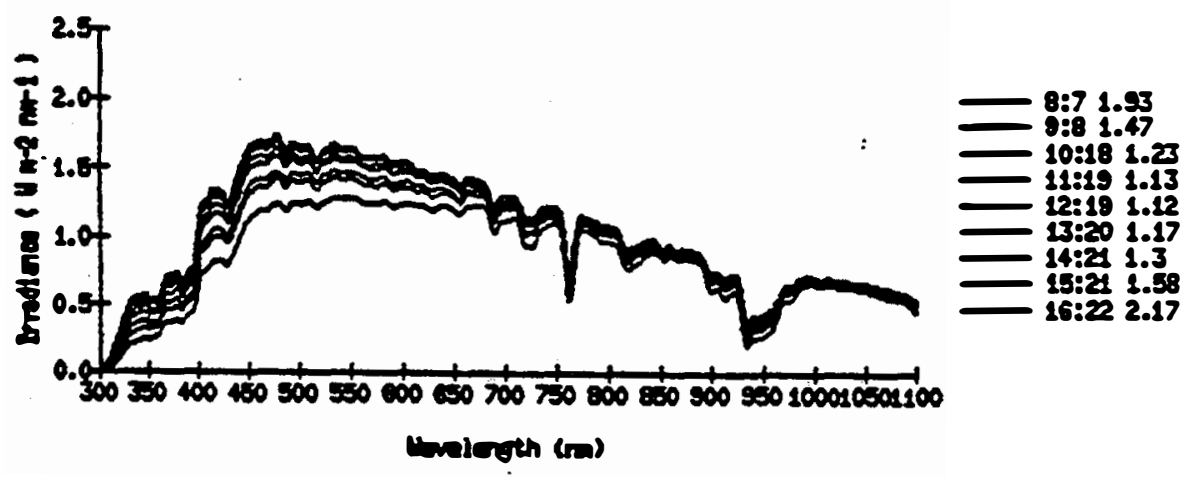




\section{SEPl}

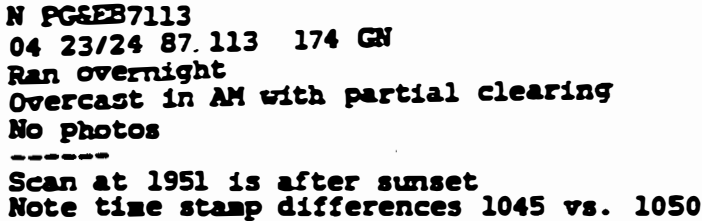

PEE 113

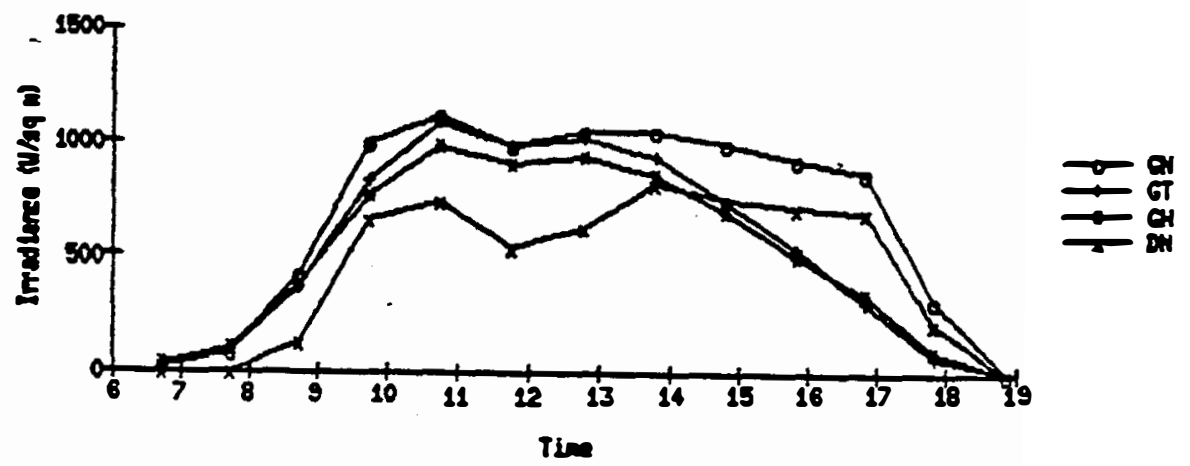

pate 123 as

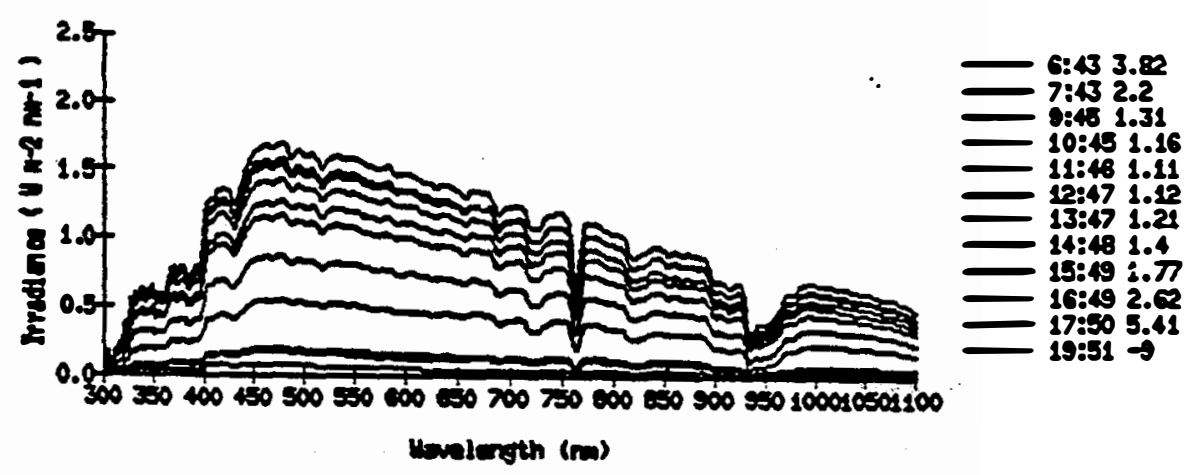




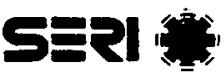

A Perx37124

see 87123

Note tine stenp differences 0759 vi. 0804

PEE 114

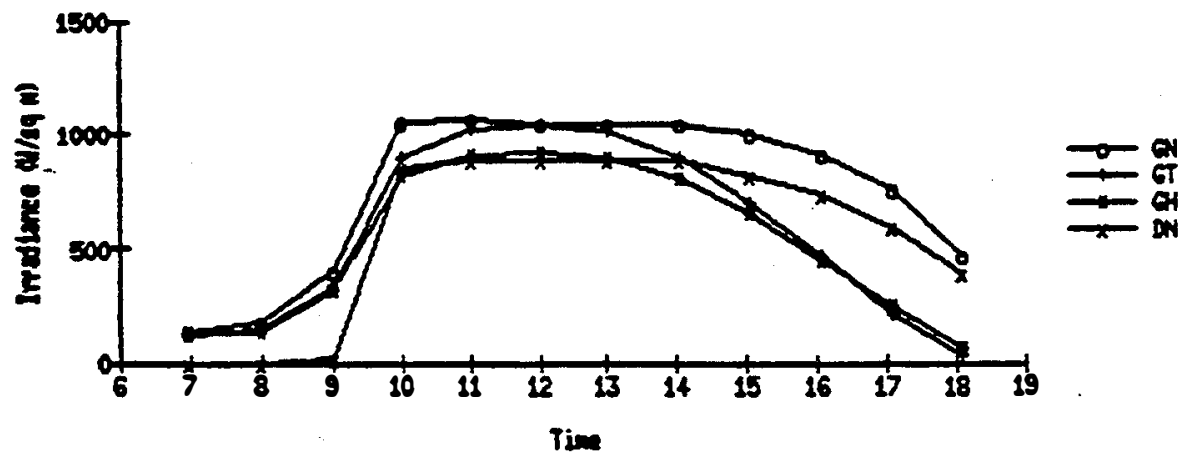

pare 114 OS

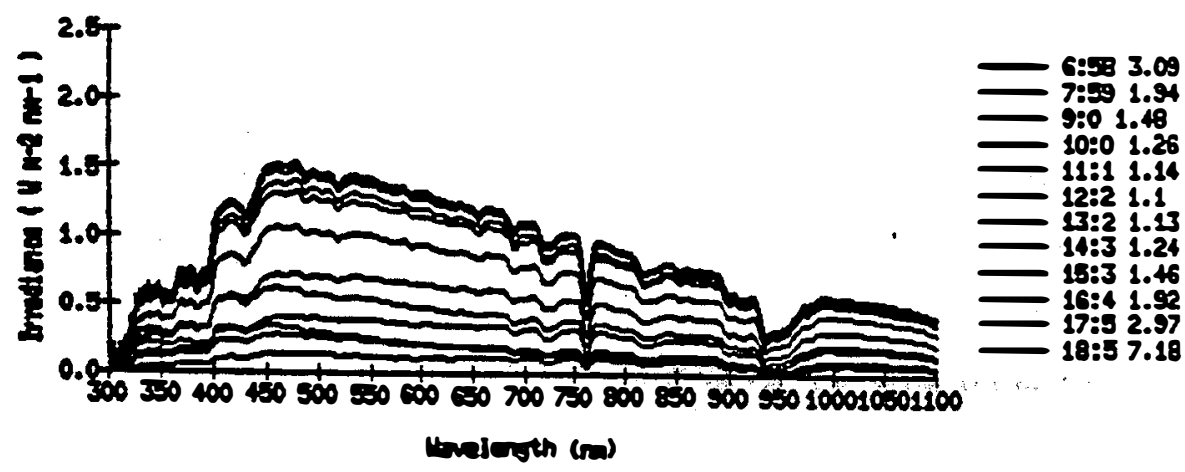




\section{SEP1 $\%$}

N PGes587133

$\begin{array}{llllll}05 & 13 & 87 & 133 & 174 & G L\end{array}$

Internttent high clouds

Ho photos

Spectral Integrals nearly ateb brondband values

PeE 23

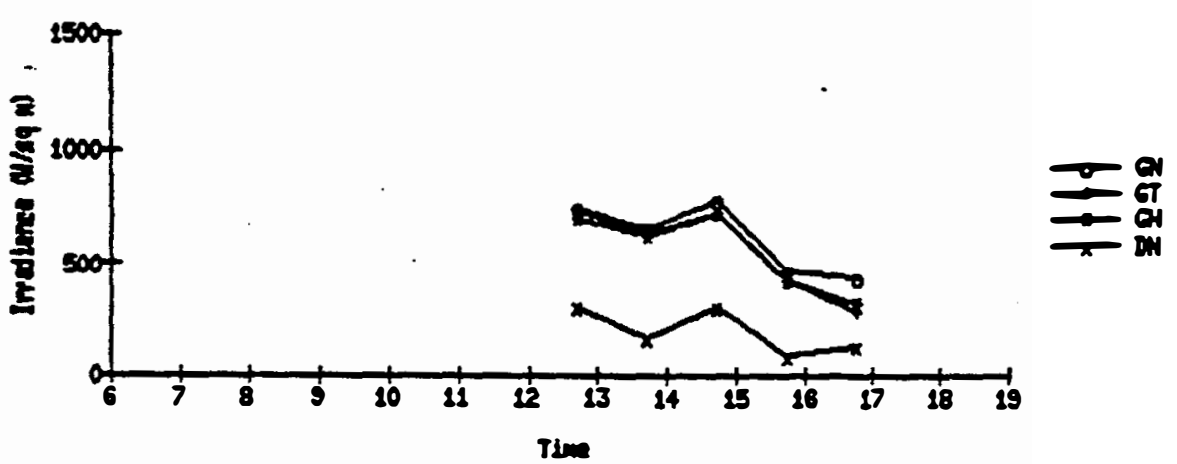

PAS 233 GTS

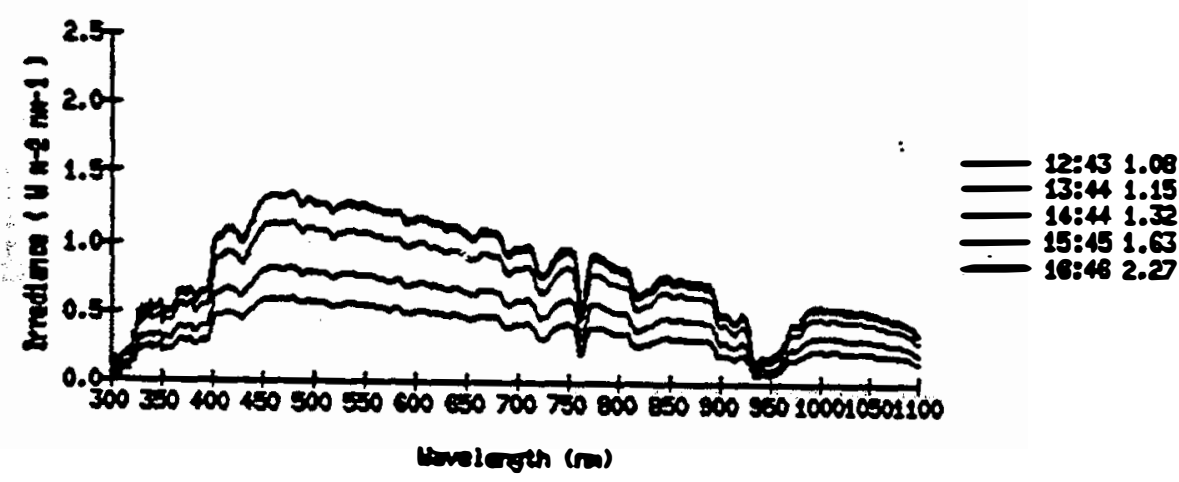


N FCES887134

$\begin{array}{lllll}05 & 14 & 87 & 134 & 174 \\ G L\end{array}$

High clouds

lio photos

PCE 134

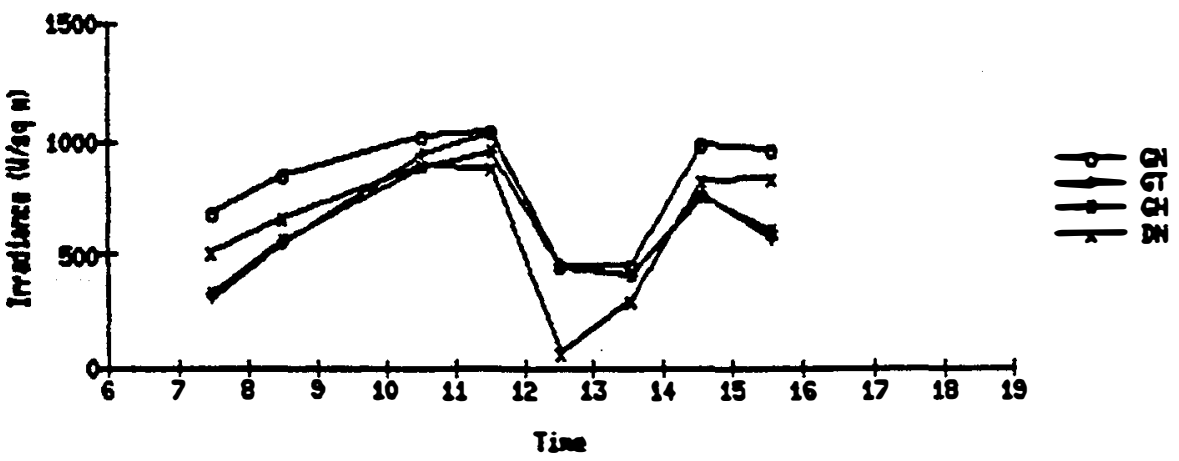

pans ize ets

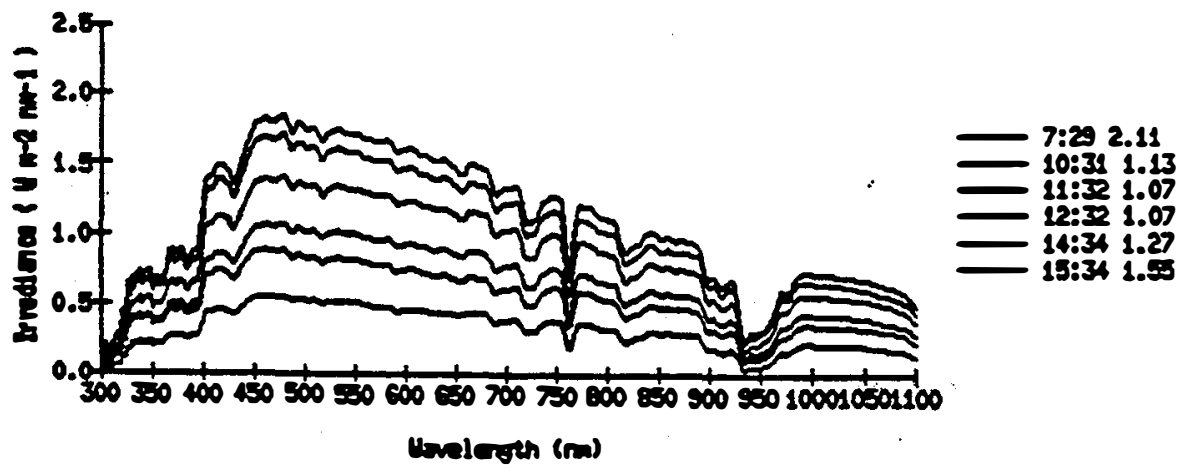




\section{SEPI 눙}

1. PGe.587135

$05 \quad \begin{array}{llll}05 & 8735 \quad 174 & \mathrm{GN}\end{array}$

Partial high clouds

No photos

PEE 15

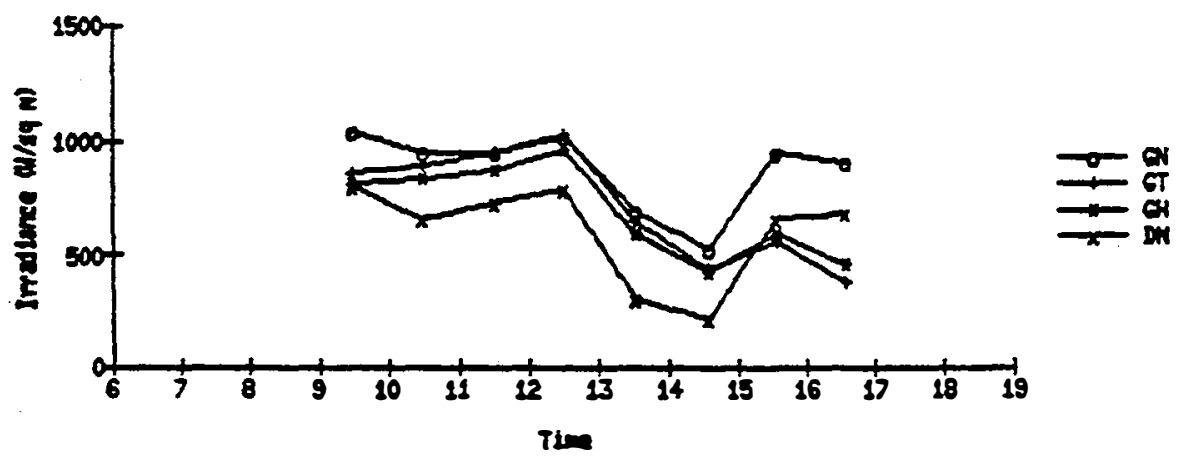

PaE 13000

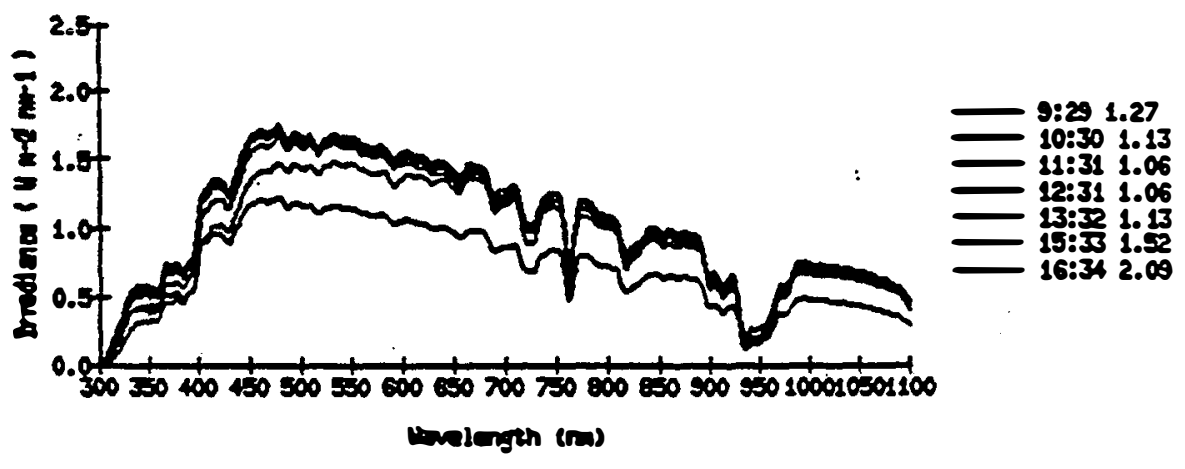




\section{틀}

$$
\begin{aligned}
& \text { A PCe587141 } \\
& 052187141274 \text { GI } \\
& \text { No } 109 \text { sbeet } \\
& \text { Ose spectre - GI }
\end{aligned}
$$

PeE 141

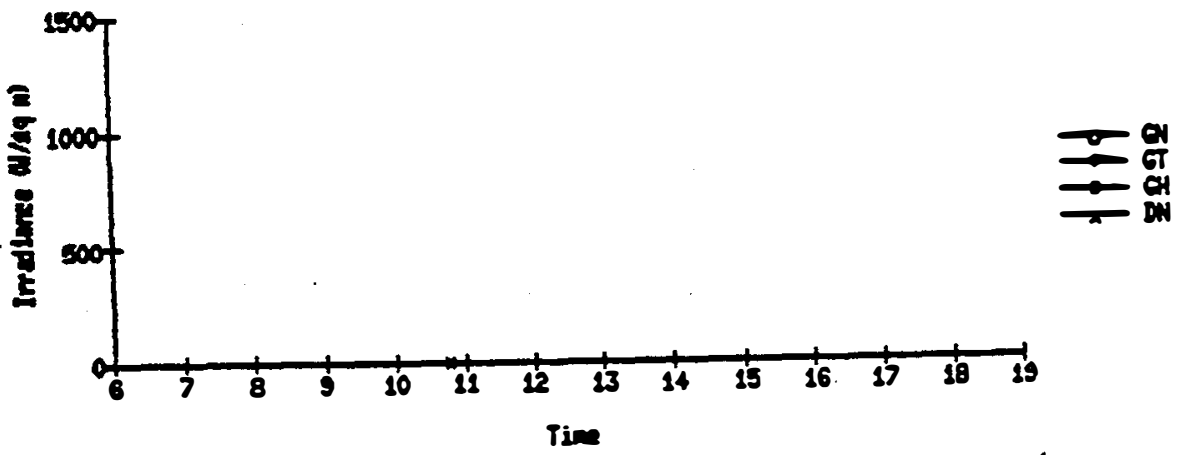

POE 141 GTS

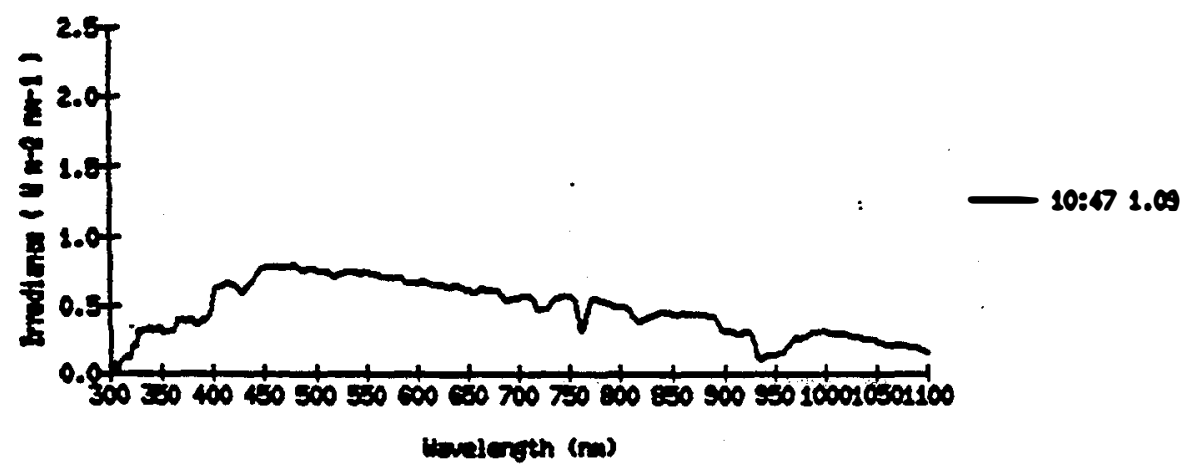


A Pers87142

$\begin{array}{lllll}05 & 22 & 87 & 142 & 174\end{array}$

Rartly cloudy Mi clear PM

Ho photos

Tist

different at 1151 and 1252

Spectral integral is larger than brondband at 1756

PeE 142

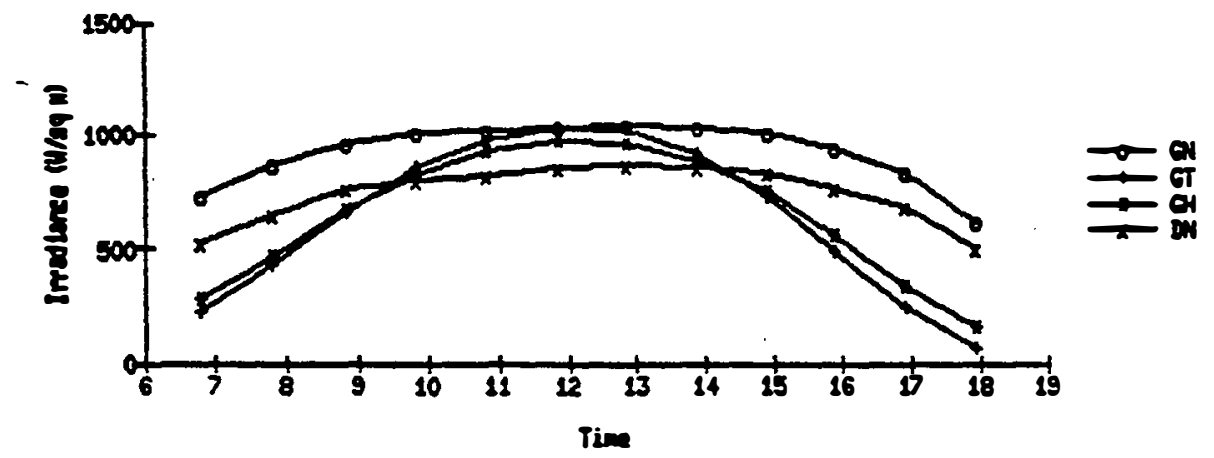

pere 142 GTS
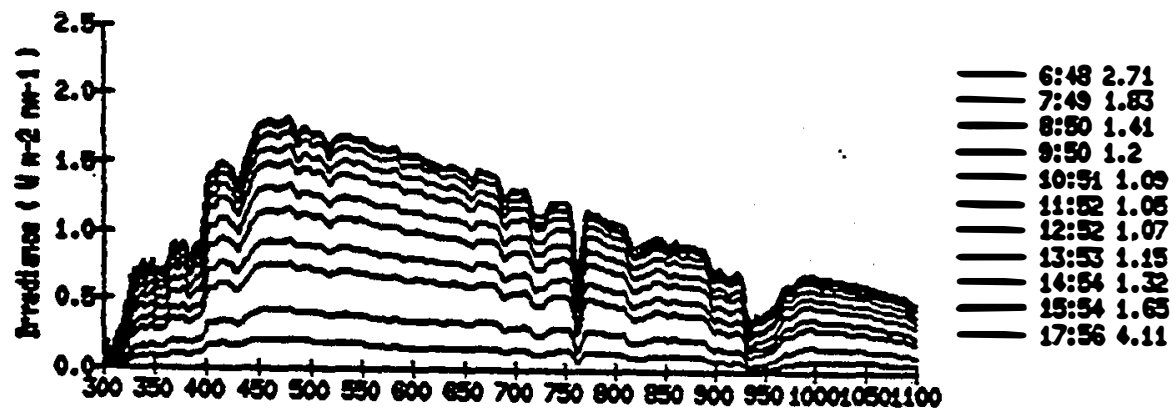

linalergth (n) 


\section{SEP}

4 PCED87148

$\begin{array}{llllll}05 & 28 & 87 & 148 & 274 & \text { DA }\end{array}$

Partly cloudy. clearing about 1000; hazy in late afternoon

No photos

Note tine seanp differences 1601 os. 1606

PEE 148

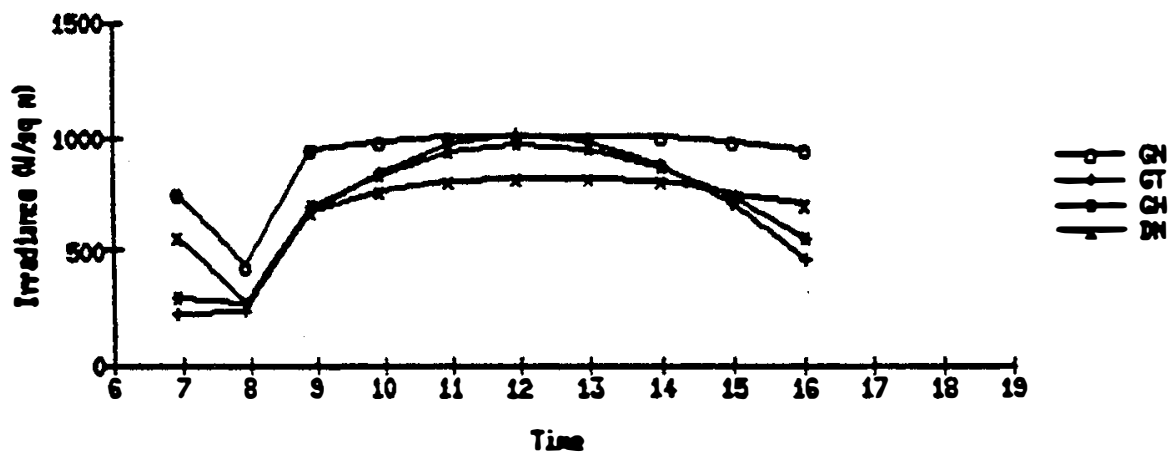

PaE 240 BNT

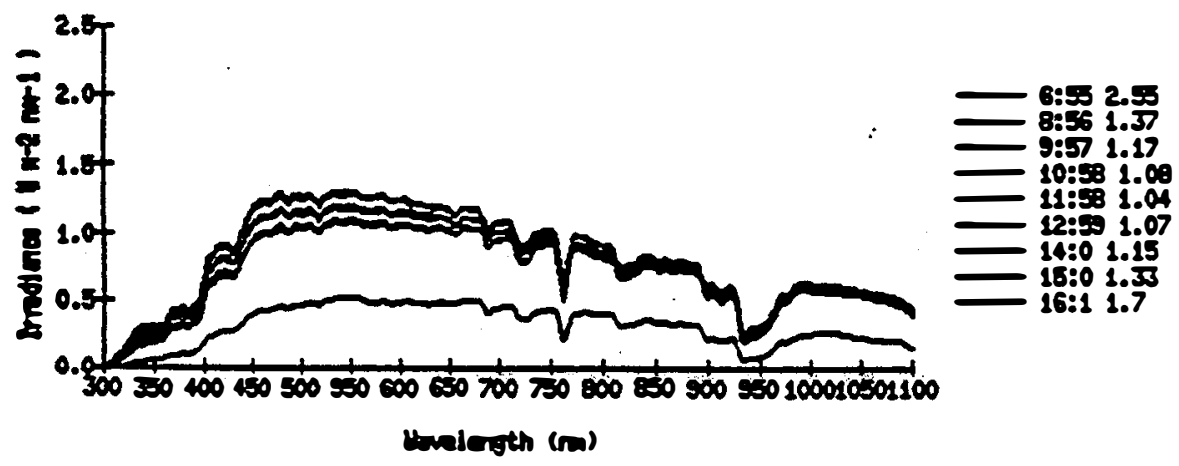


N Pres87153

$06 \quad 02 \quad 87 \quad 153 \quad 174$ GN

Cleari bot $50 \mathrm{C}$ with tenp controller off: anbient , $38 \mathrm{C}$

pee 253

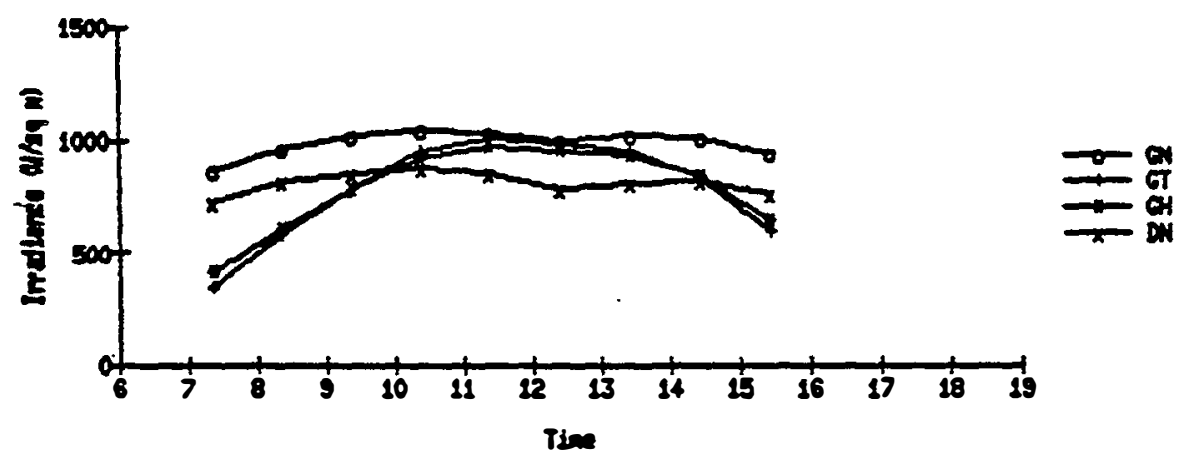

pare La

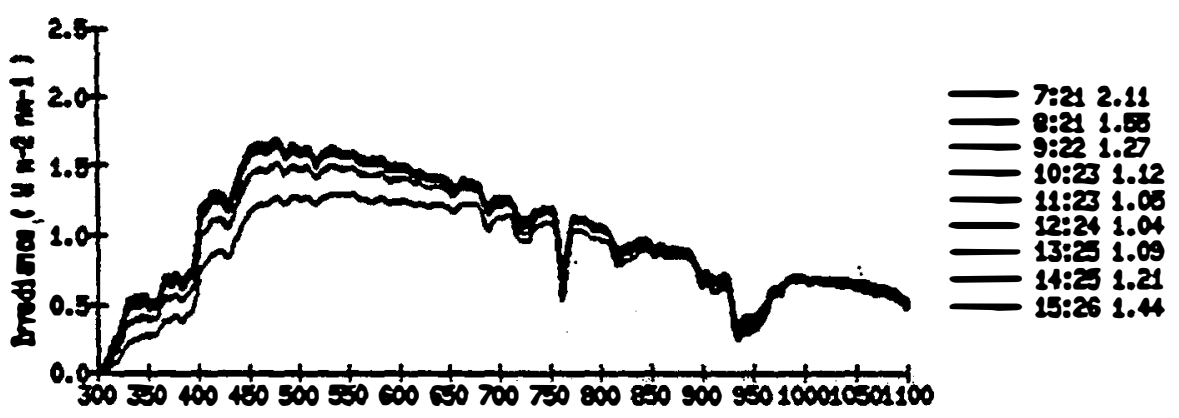

linglath (ra) 
A PCSग्र 1262

$\begin{array}{llllll}06 & 11 & 87 & 162 & 174 & \mathrm{EH}\end{array}$

Cloudy AM: clear. expdy PM

Red dust cap not renoved fron integrating sphere

Io photos

lio spectral data 
N PCED87163

$061287163 \quad 174$ GI

Clear: hot

Teap controller: $46 \mathrm{C}$ at 1330 : I(anblent) $39 \mathrm{C}$ i no foll cover

Dis/SN different at 0729

PEE 103

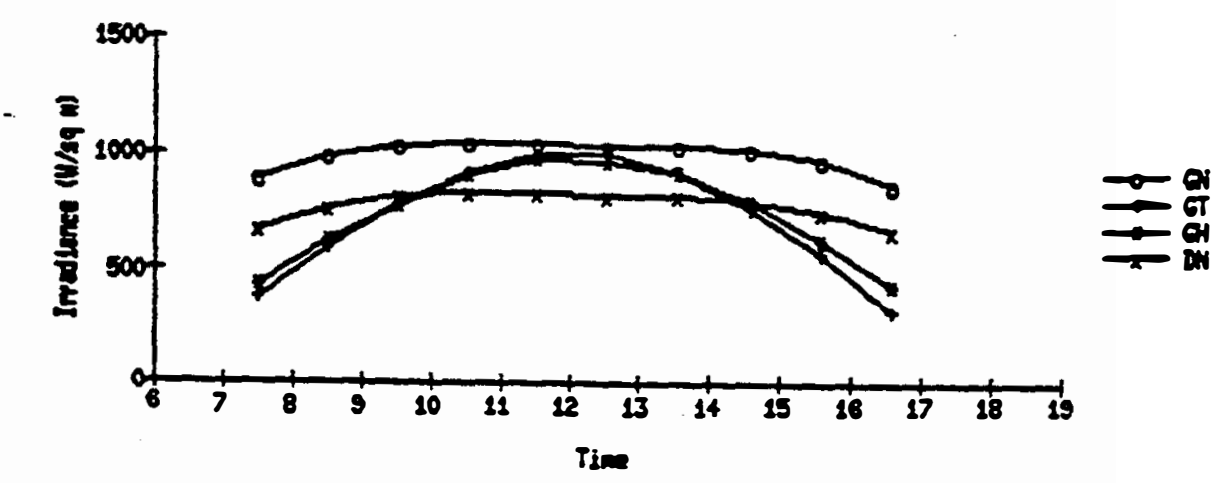

PATE 103 GIS

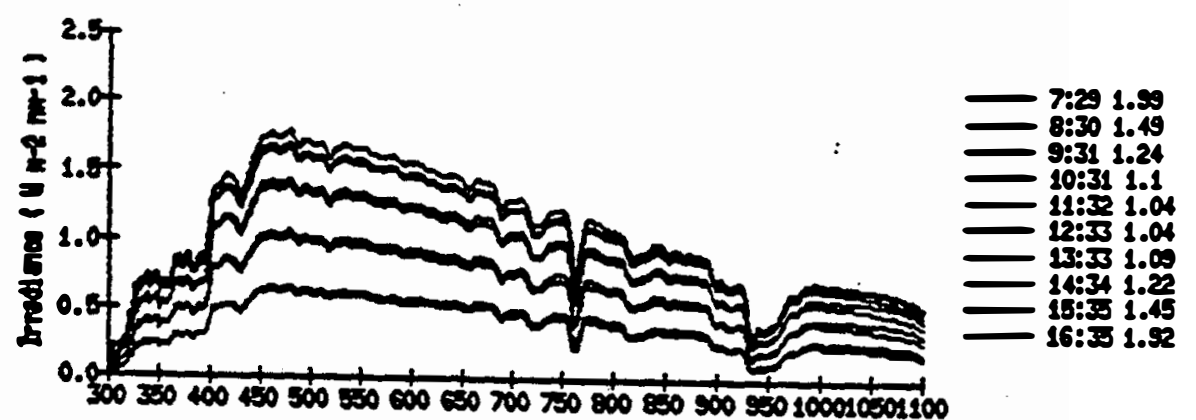

vevelongth (m) 


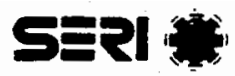

A. PC5.

$06 \quad 1587166 \quad 274$ GT

Partly cloudy M

No fOIl sinde on LSR

No pbotos

Note tine stanp differences 0748 vs. 0753

PEE 105

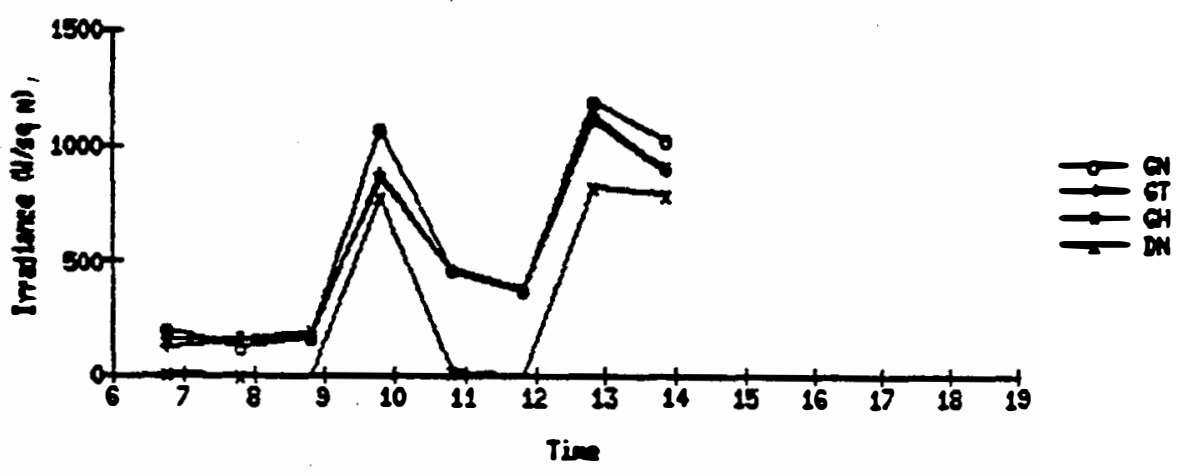

PAE 160 GTS

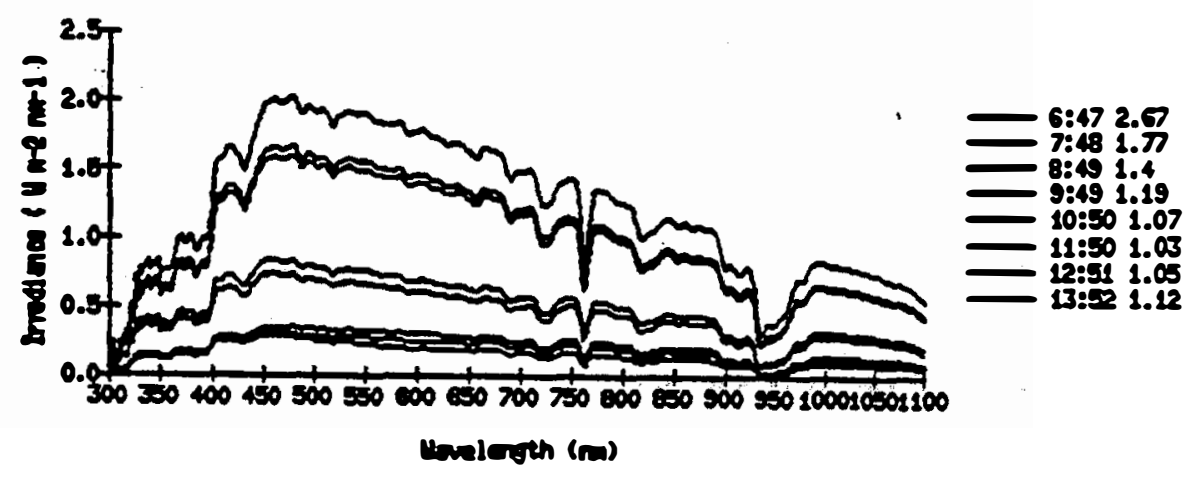




\section{SEP *}

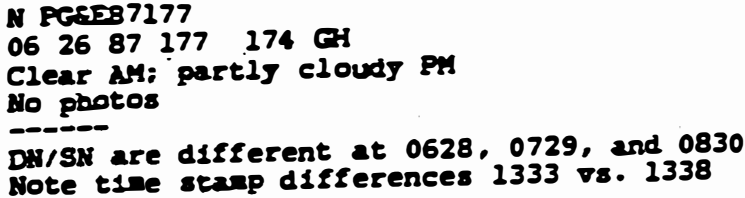

pes 177

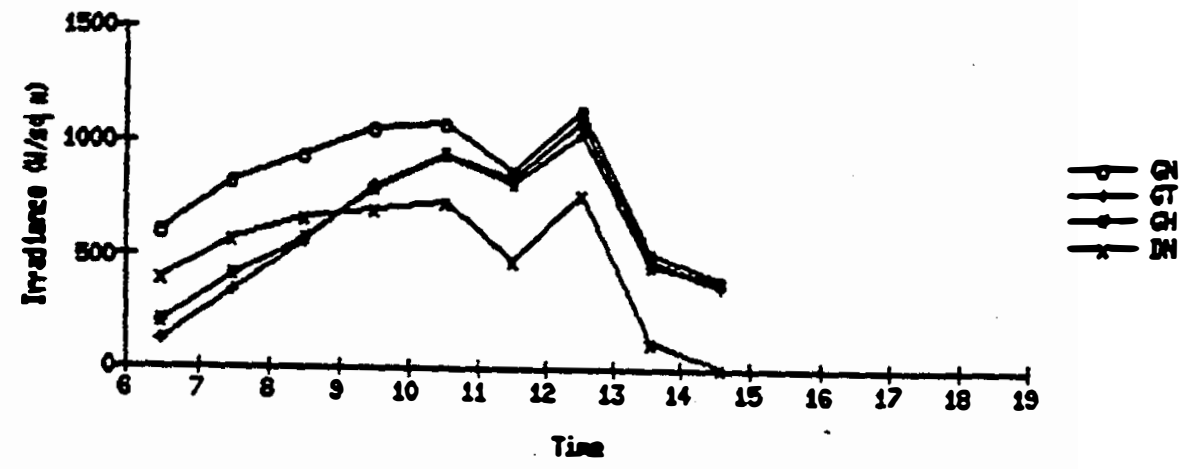

PEב 1706

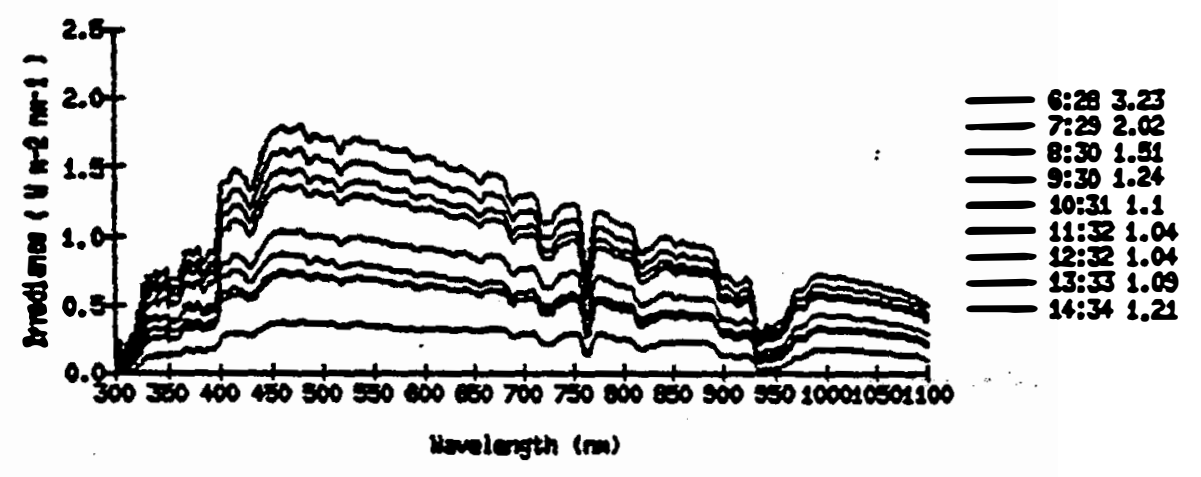


N PCES37183

$070287.183 \quad 174$ GI

Cloudy NH; clear by 1000

No paotos

Sw/DY are different at 0648, 0749, 0850, 1253, and 1353

PEE 183

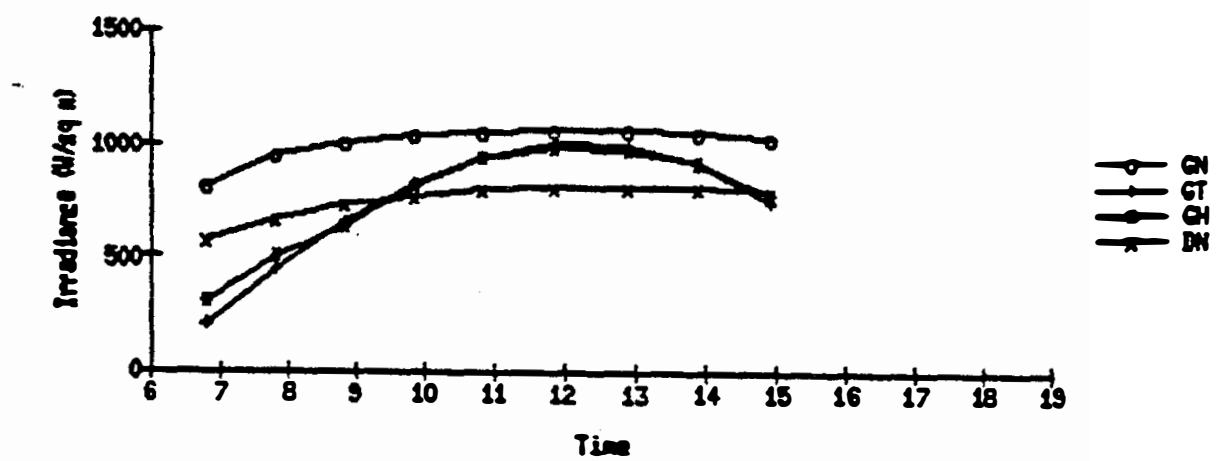

Pane 183 GTS

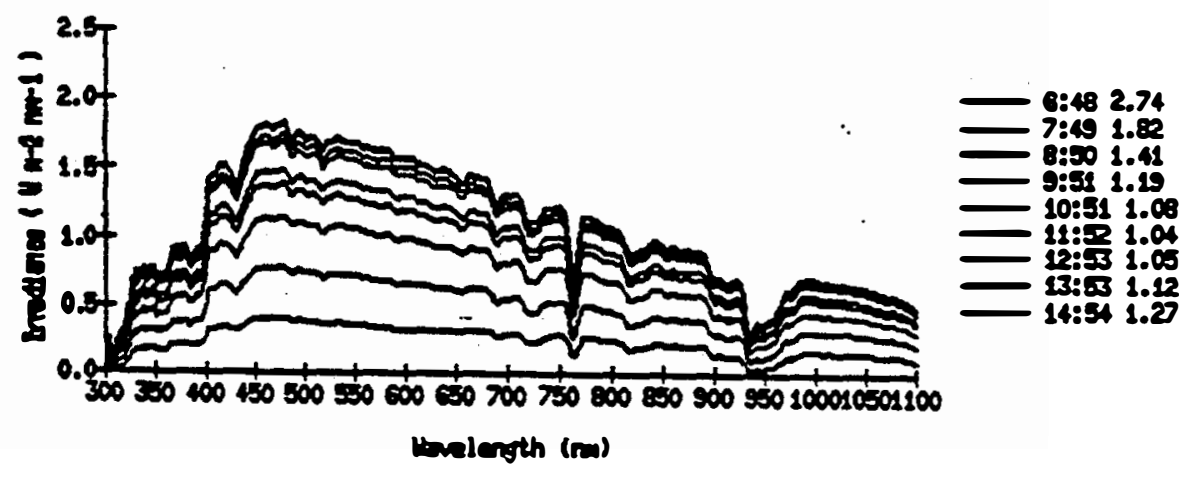


N PCSDB7188

$\begin{array}{lllll}07 \quad 07 \quad 87 & 188 & 174 & \mathrm{GH}\end{array}$

Clear: varn

No photos

SH/DN different at 0615 and 0716

SH/GH errors at 1722 and 1823 ; also spectral integral ) brandinad

PEE 188

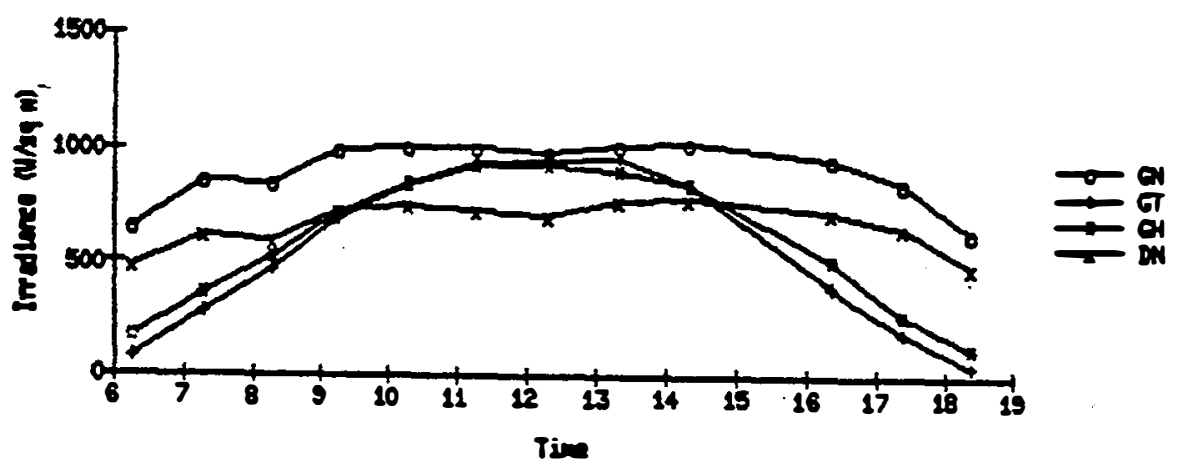

PAe 188 as

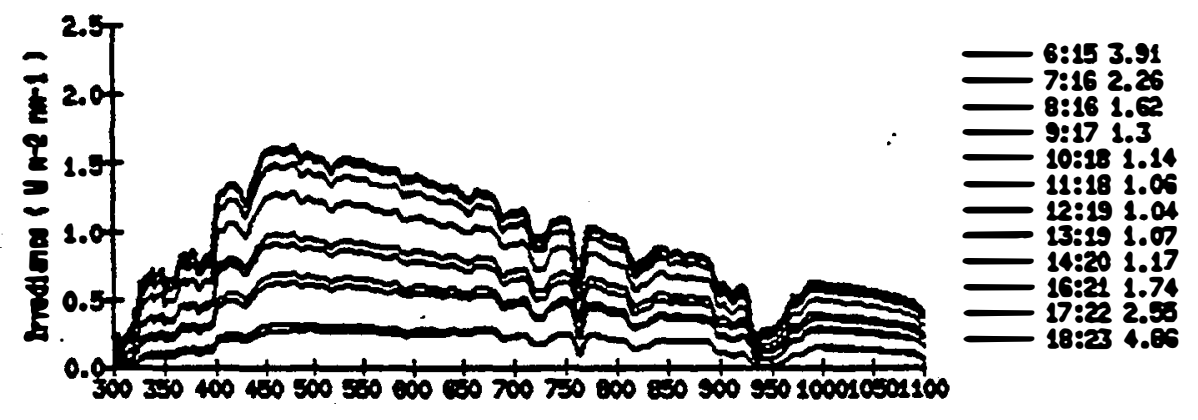


A Pes587198

$\begin{array}{lllll}07 & 1787 & 198 \quad 174 & G\end{array}$

(Note $\log$ sheet shors 07 16)

Cloudy NA

Tracker any be off by a feu degrees at tines

Iracker wind stound at 1300

No photos

PeE 208

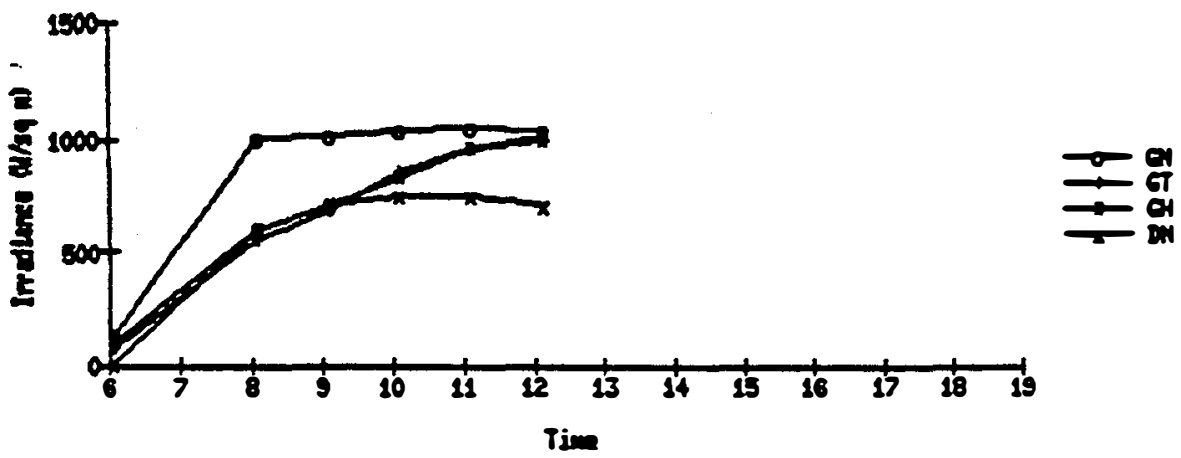

Pate 198 on

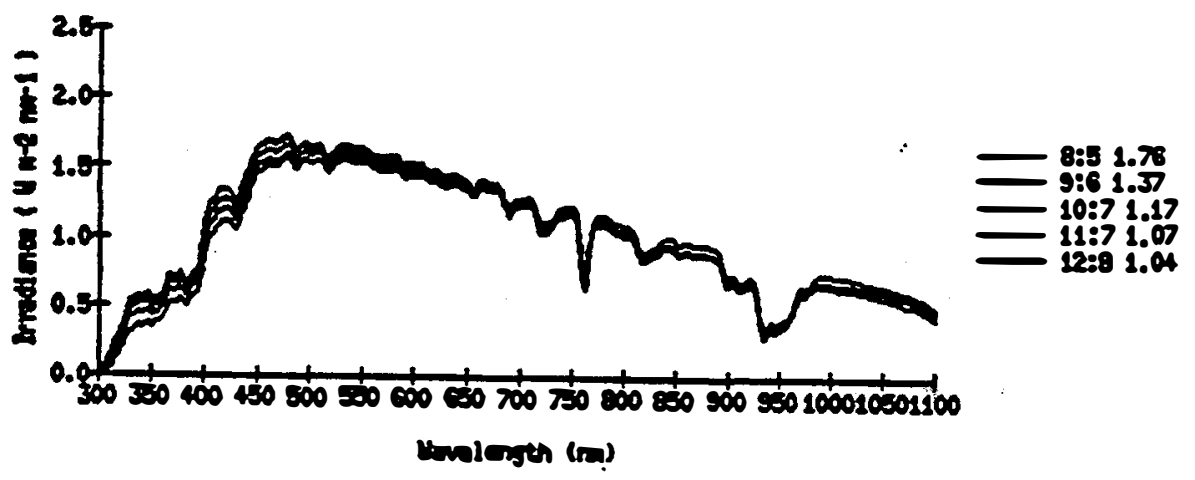


Pesp87202

$072287202 \quad 174$ GN

Partis cloudy AM

Tracker accuracy poor ( $+1-5$ deg); very poor in afternoon; bad

betveen 1300 and 1700; last scan at 1727 should be good

Ho photos

Tracker

errors: data bad at 1320 and 1622

Spectral integral = brondhnd at 1521; appears unstable

GH/SH errors at 1729

Note time stanp differences 1320 v8. 1325

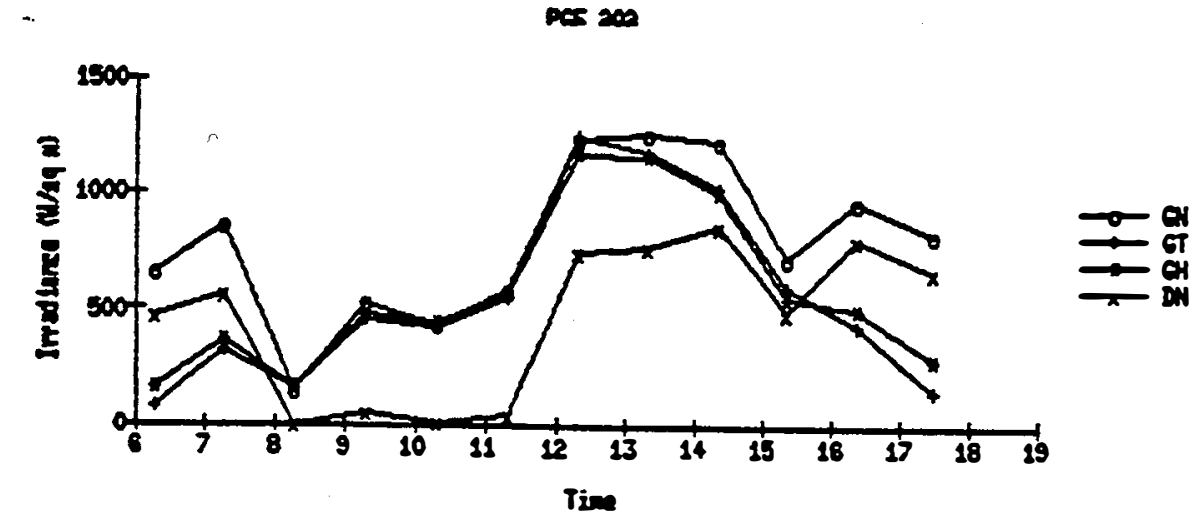

pare ando

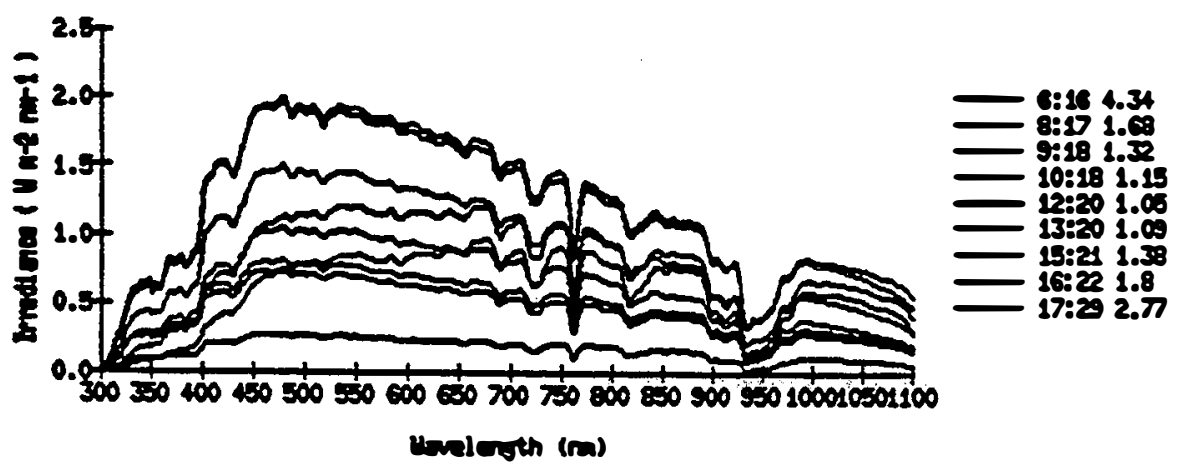


N Per587212

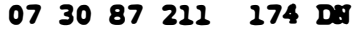

Partly cloudy in $\mathrm{M}$

Iracher functioning properly

Ho photos

Note 91 uIP consistently 200 in-2 below Epples AIP

Pez 21

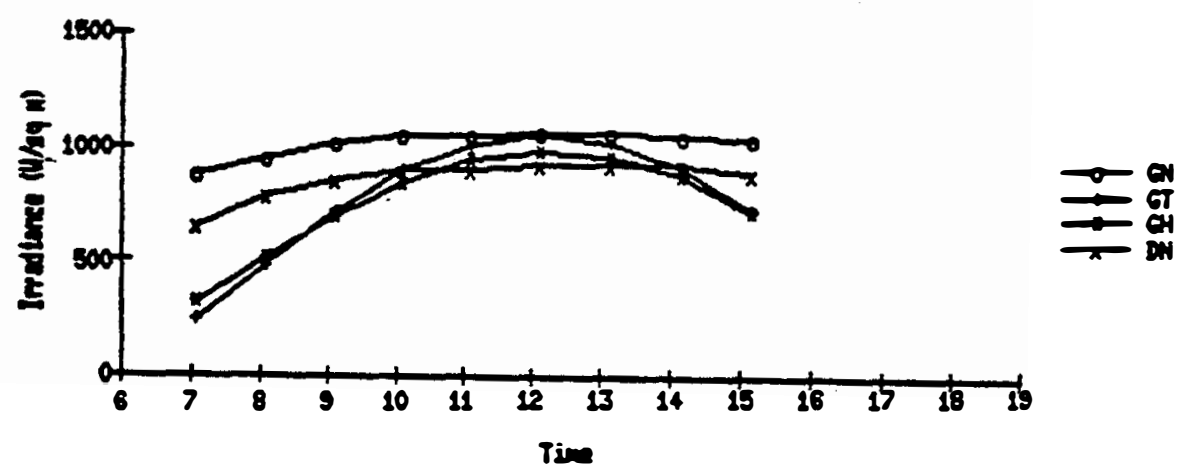

PEE 241 BiT

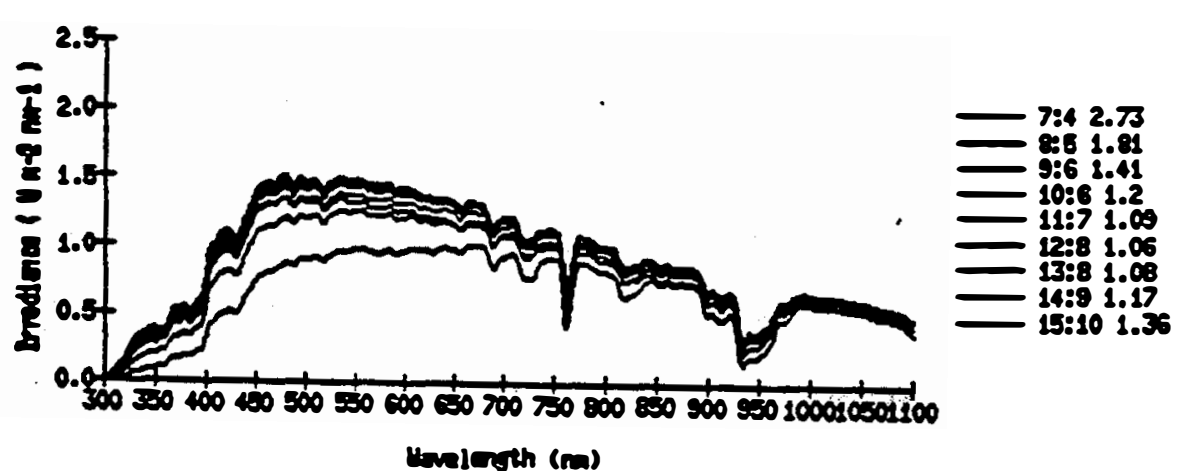

lumlath (m) 
4 Pespr7218

$\begin{array}{lllll}08 & 06 \quad 87 & 218 & 174 & \mathrm{DA}\end{array}$

Ho $\log$ sheet

Ho photos

SI AIP appears to be too 100 throughout day

ISR DN and HIP much different at 0655

Hote tine stamp differences 1659 78. 1704

PeE 218

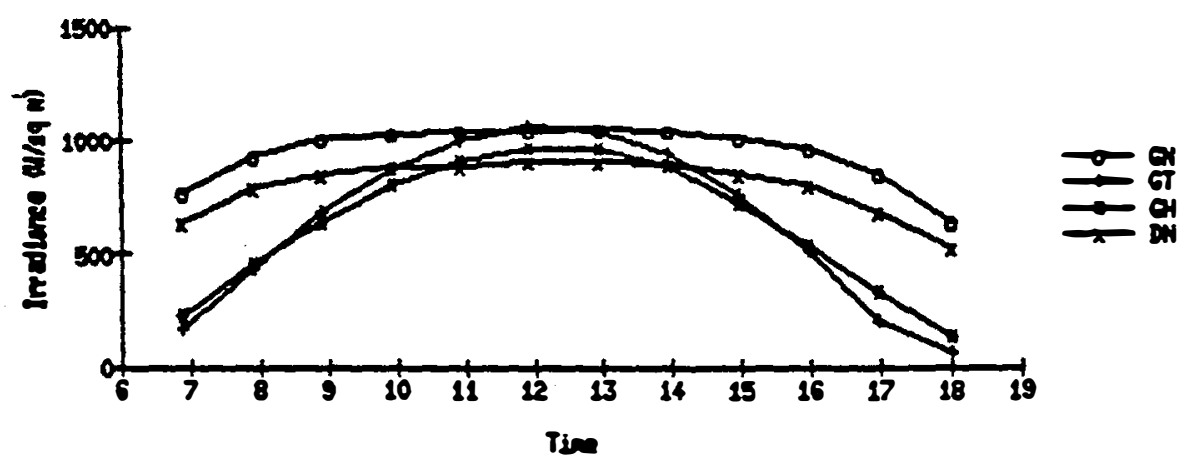

PEe 218 unt

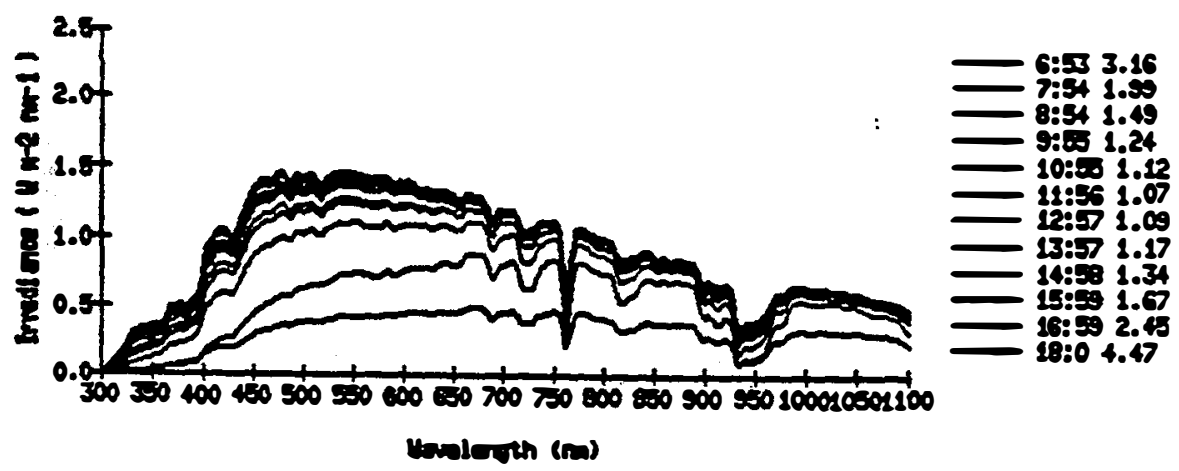


N ECST37328

$\begin{array}{llllll}11 & 24 & 87 & 328 & 174 & \mathrm{CH}\end{array}$

Cold: bigh clouds: overcast by noon

hind speed changed to $\mathrm{al}$

Fhotos (but 4 different sets indicate 11-24; cannot distinguish)

$-\infty-\infty$

S1 IIP appears to be too low throughout the dag

PEE 30

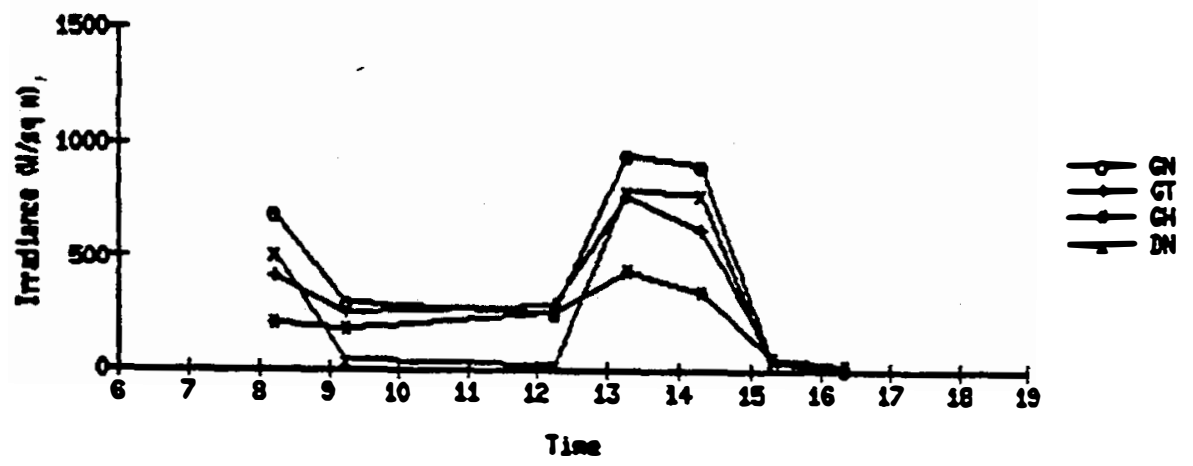

pare 328 as

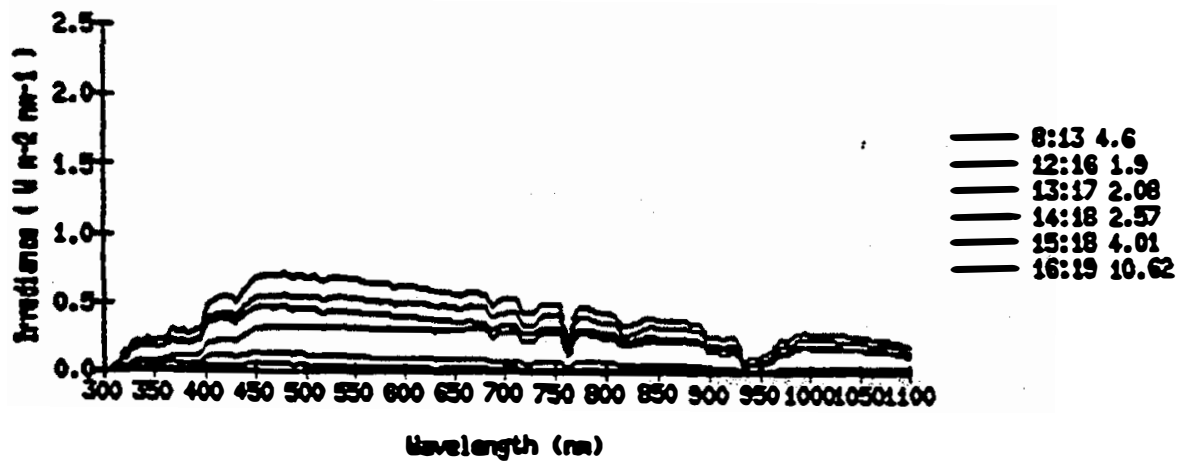


N Pes 237329

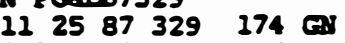

Cold: elndy: sone bigh clouds

Photos indicated, but none with this date stanp

ilind speed in a/s

Flrst run aborted: photo invalid

S1 IIP appears to be too low through 1253

spectrom at 0948 is bad

Data at 1656, 2755, and 1856 are after sunset

PAS 3

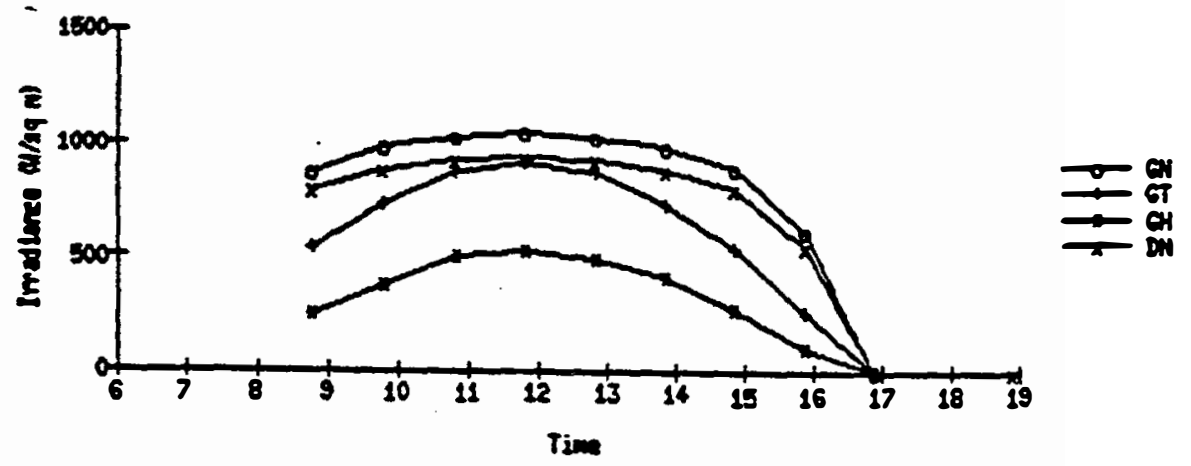

PaE 300

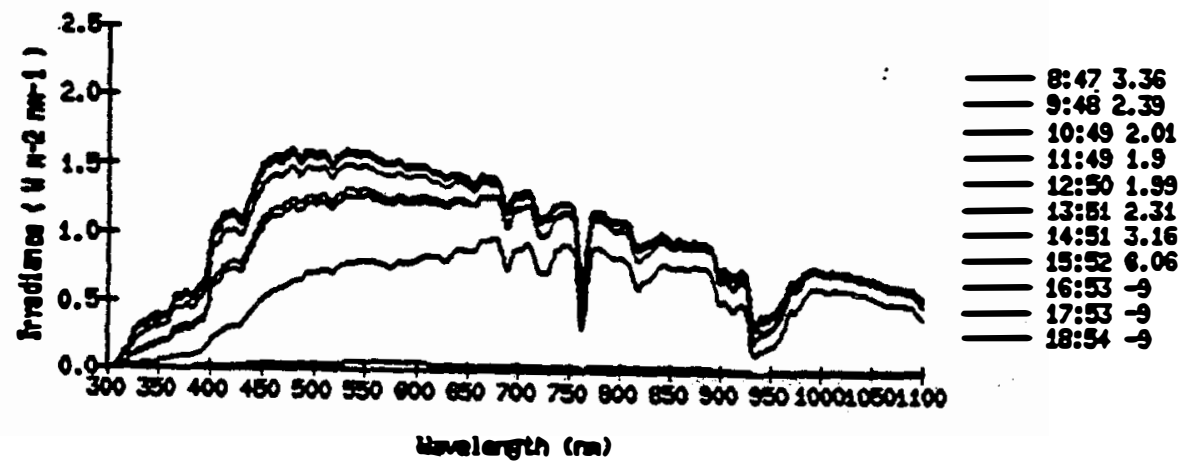


A Peer587330

$\begin{array}{lllll}11 & 26 & 87 & 330 & 174\end{array}$

Ho 109 sheet

Data at 0603. 0703, and 0804 before sunrise

Appears that ISR data 18 bad all day; doesn't follow broadband

Si IIP data are too low

pee 300

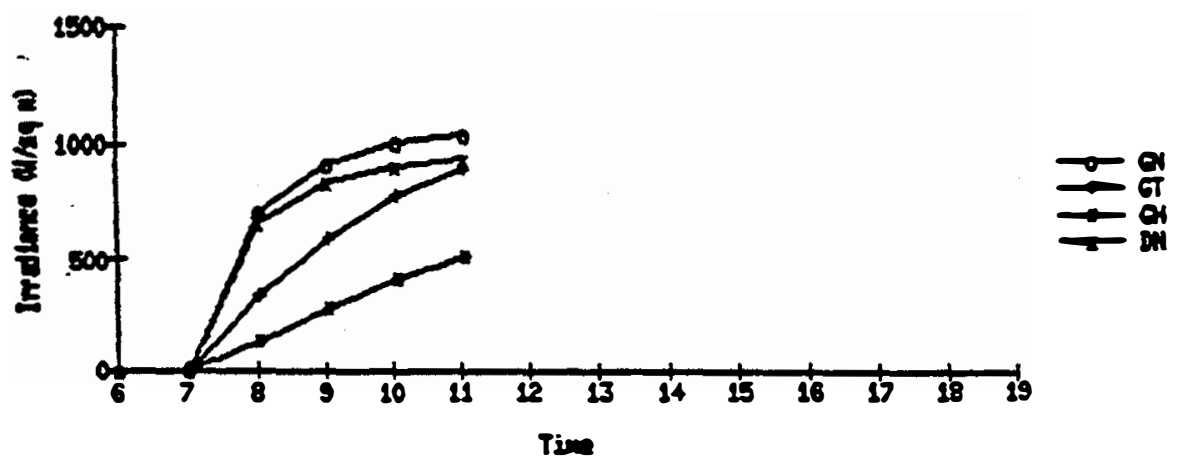

pare 300

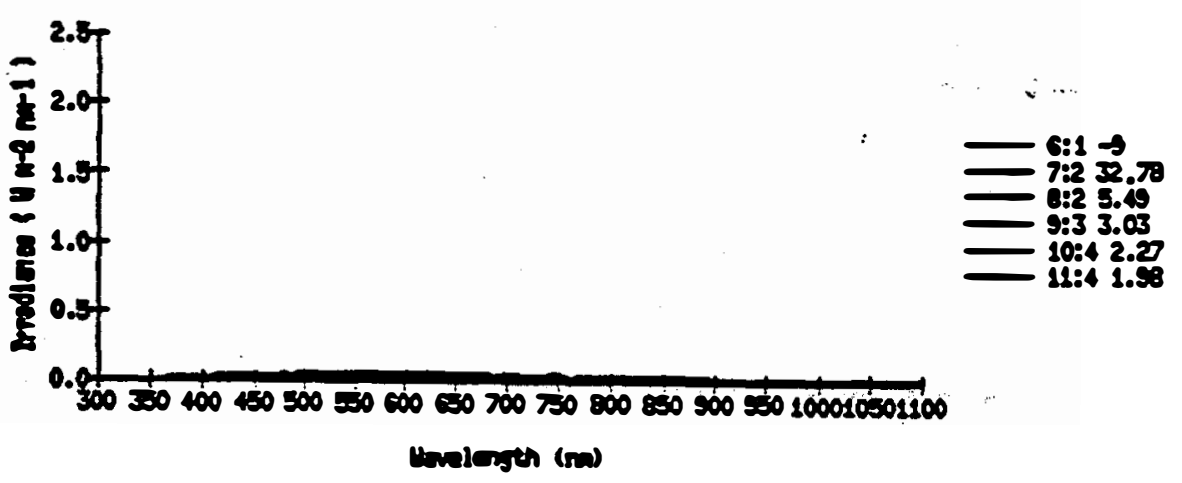




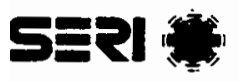

N PCs588019

$01 \quad 1988.019 \quad 174$ GI

First scan at $28 \mathrm{C}$

Clear: cold

FI and GH PSP and WIP ehanged betreen 1600 and 1630; constants cbanged

Photos 1ndicated, but none with this dnte stanp

PEE 19

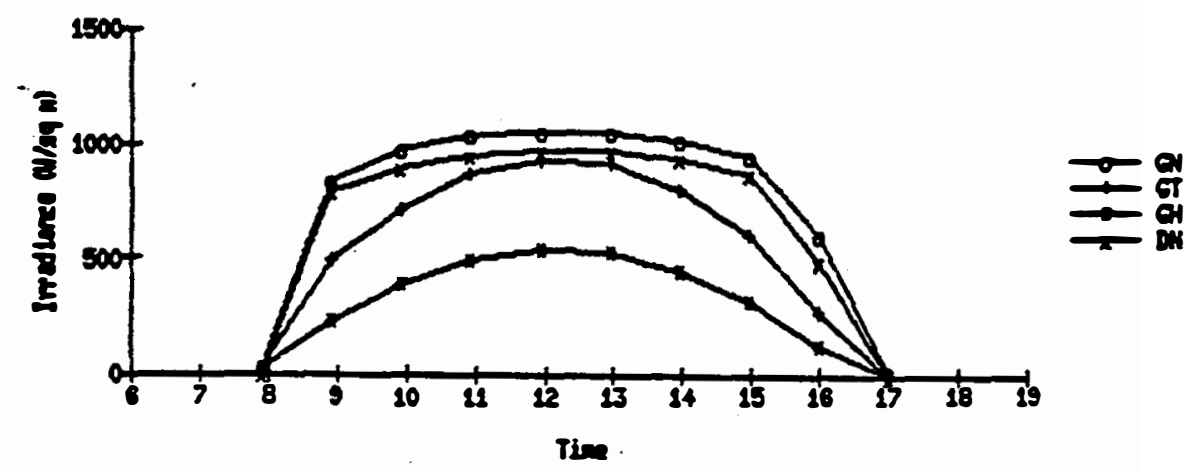

pare is GTS

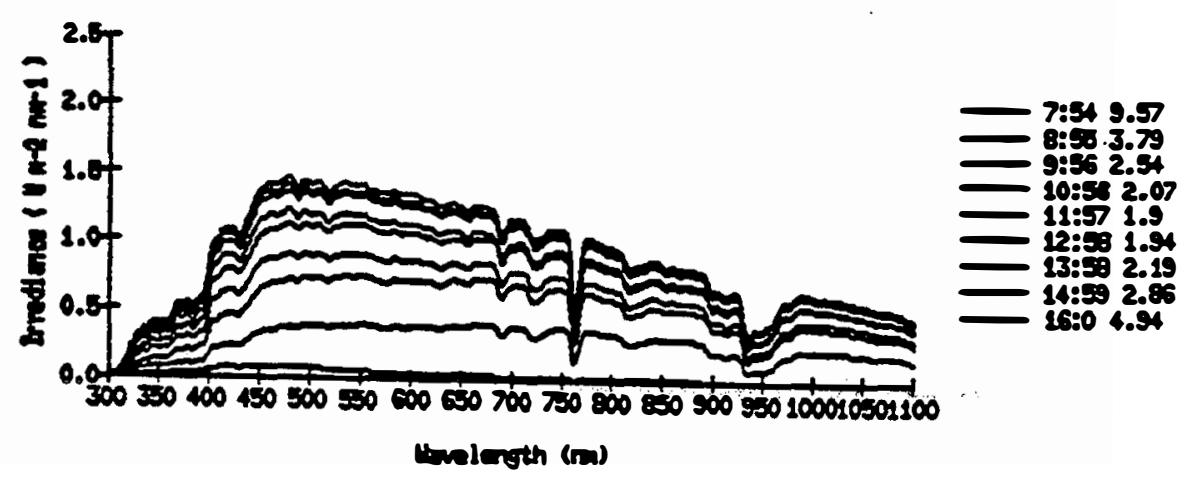


N Per588020

$012088 \quad 020 \quad 174$ GH

Clear (sone baze): cool

Pbotos indicated, but none with thds date stanp

-

Si MIP data look bad throughout the day

Date at 1722 and 1823 are after sunset

PeE 20

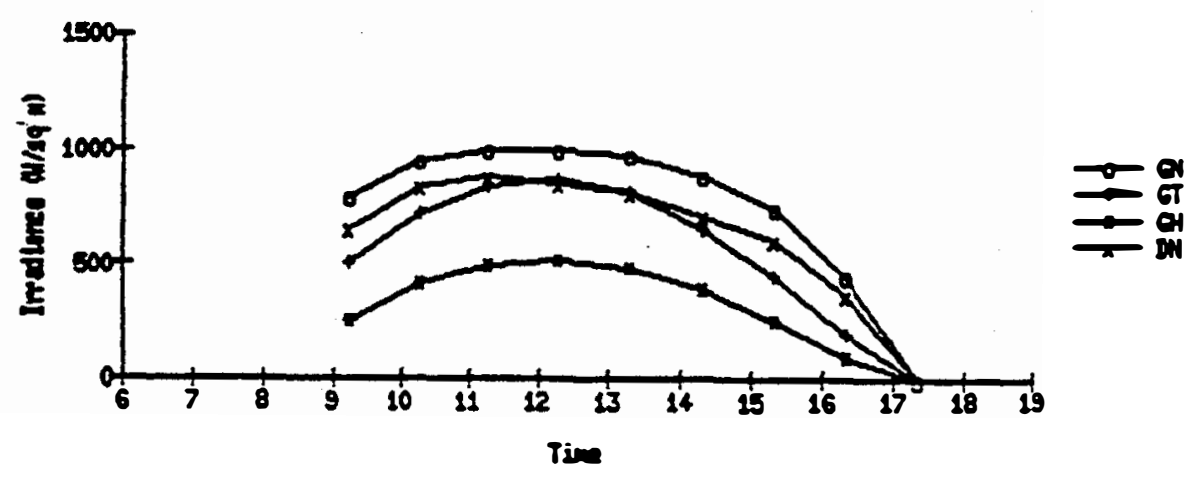

PAE 20 OS

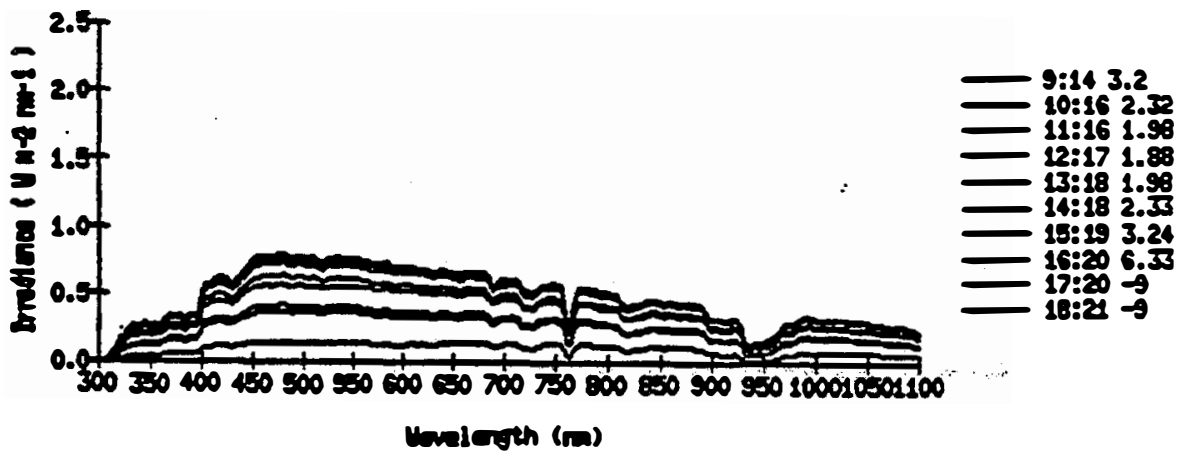


N Pes588021

$\begin{array}{lllllll}01 & 20 & 88 & 021 & 174 & \mathrm{DS}\end{array}$

Clear and cold: sone baze; late afternoon high vinds

Pbotos indicated. but none erth this date stanp

-

S1 MIP data look bad

Ho spectrum at 0803; too early

PeE a

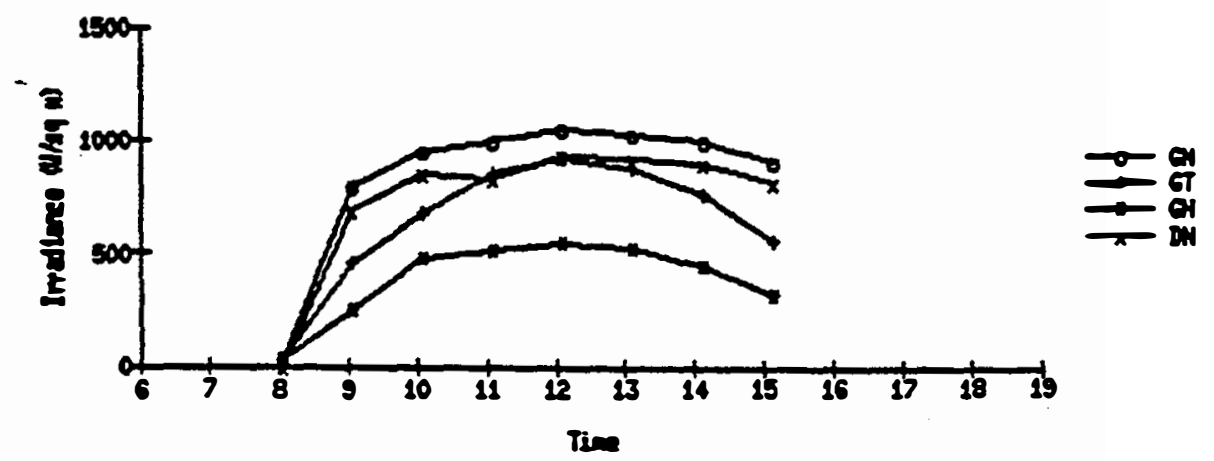

Pare a But

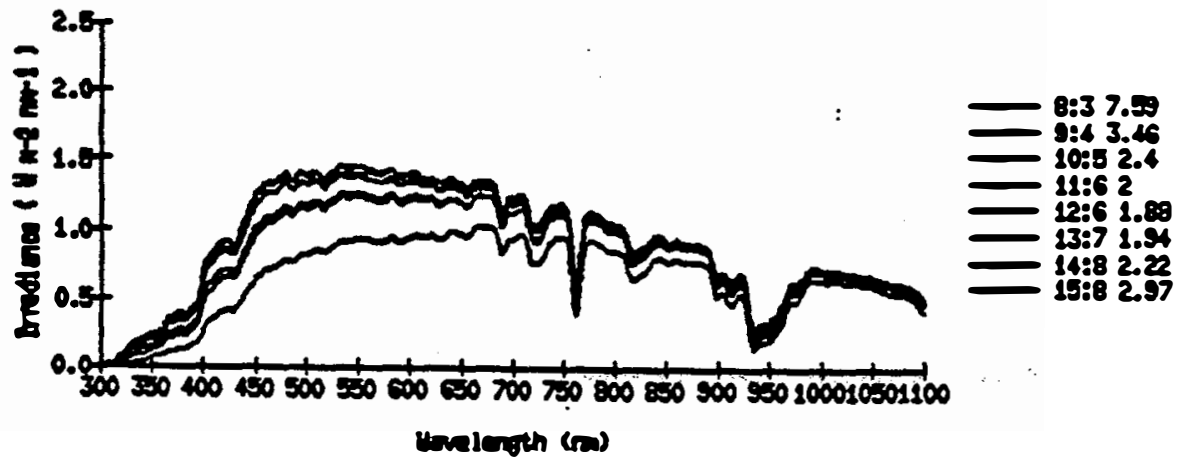




\section{SEP *}

1. perDa8022

$012288 \quad 022$

Hans and caln

$174 \mathrm{GS}$

Fhotos indleated, but none esth this date stang

(2)

S1 RIP den look bad

PEE 22

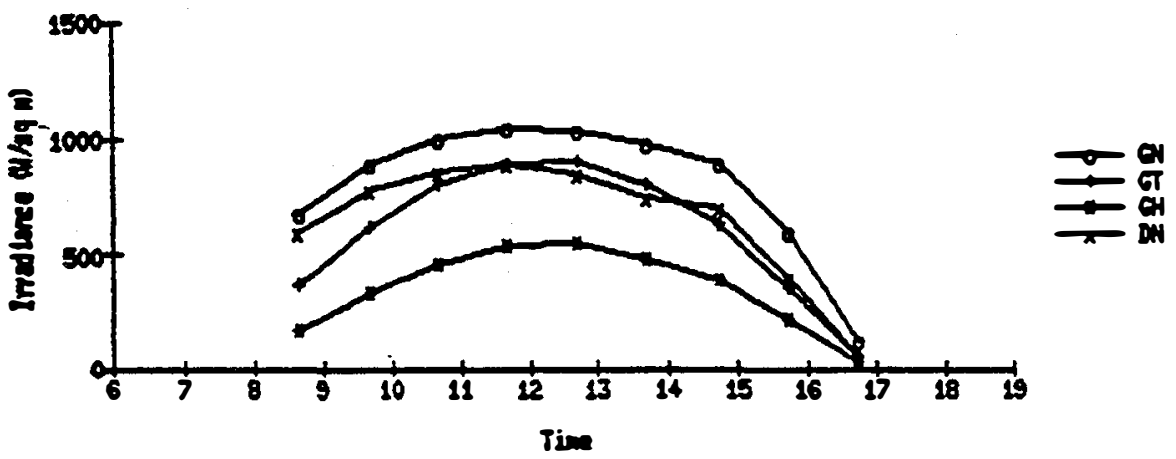

pere 2000

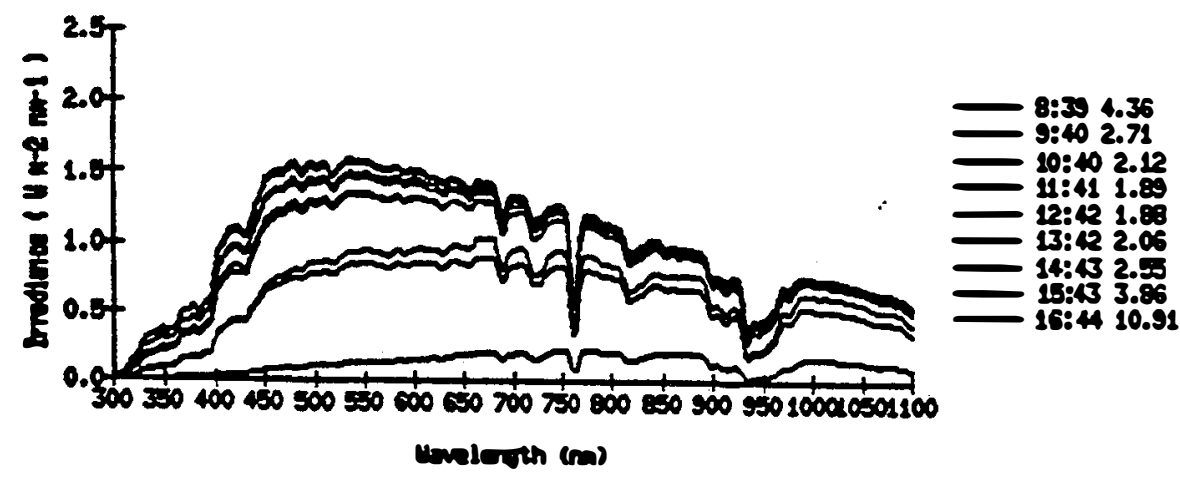


iv Peer288023

$012388023 \quad 174 \quad \mathrm{CH}$

Haz: cool

Photos indicated, but date stanp uncertain

Dater-

Date at 1708 are at sumset

S1 IIP date look bad

Spectrm at 1708 verg late in dags questionule

Pes 23

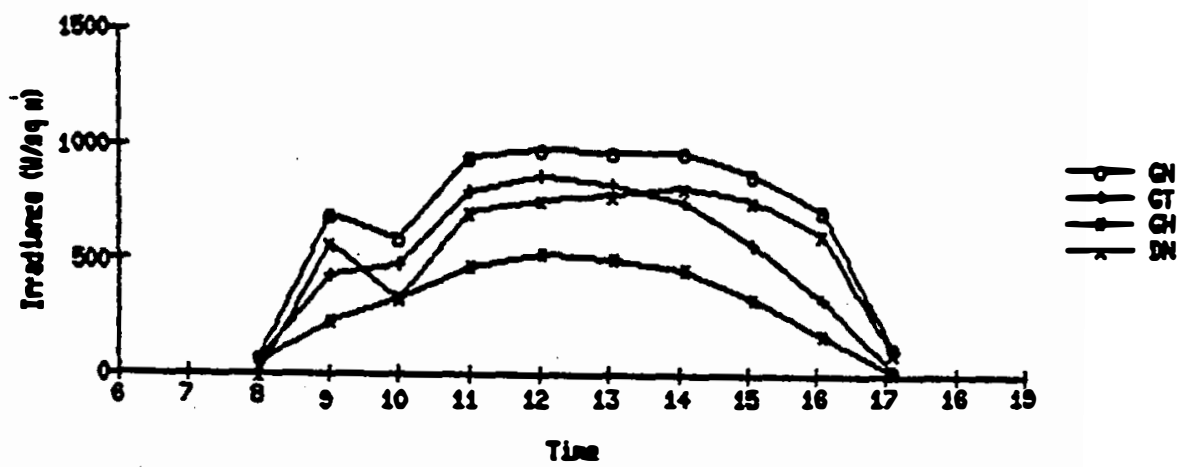

pexe 23 as

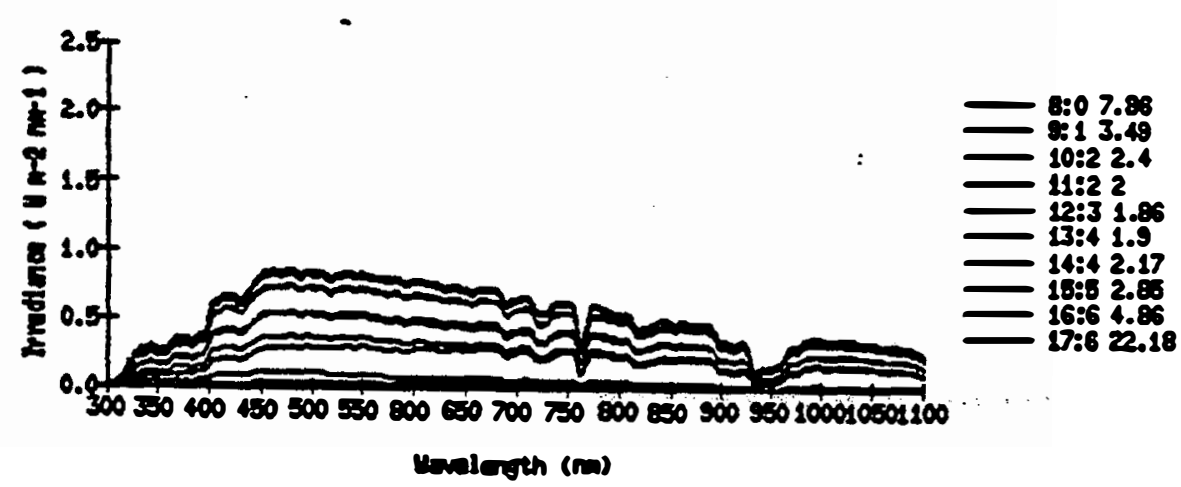


N PGe588024

$01 \quad 2488 \quad 024 \quad 174$ GI

Clear

Error stopped date collections restarted at 1220

Fhotos indicated. but none with this date stanp

Si WIP date look bad nost of the day

PEE 24

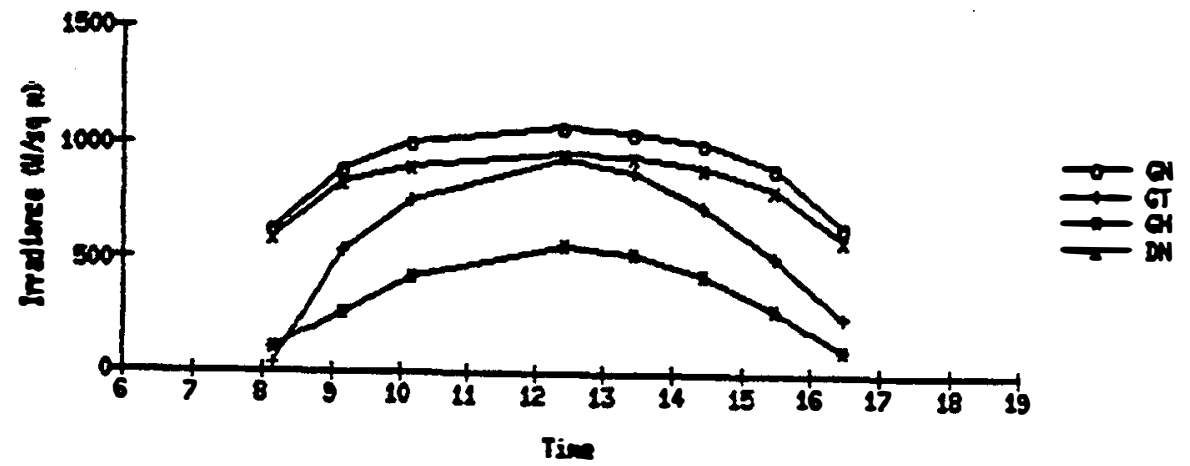

Pars 2400

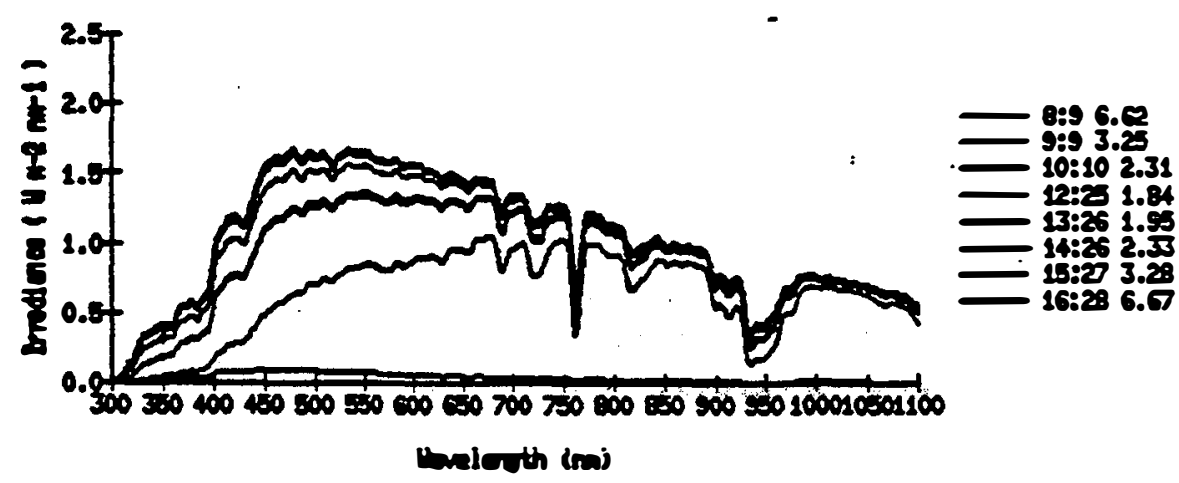


N PCES88025

$012588025 \quad 174 \quad F I$

Light 100

PSP and IIP constants changed in the HP85 files before starting

Pwotos indicated, but none with this date stanp

si IIP date look bad wost of the day

PeE ₹

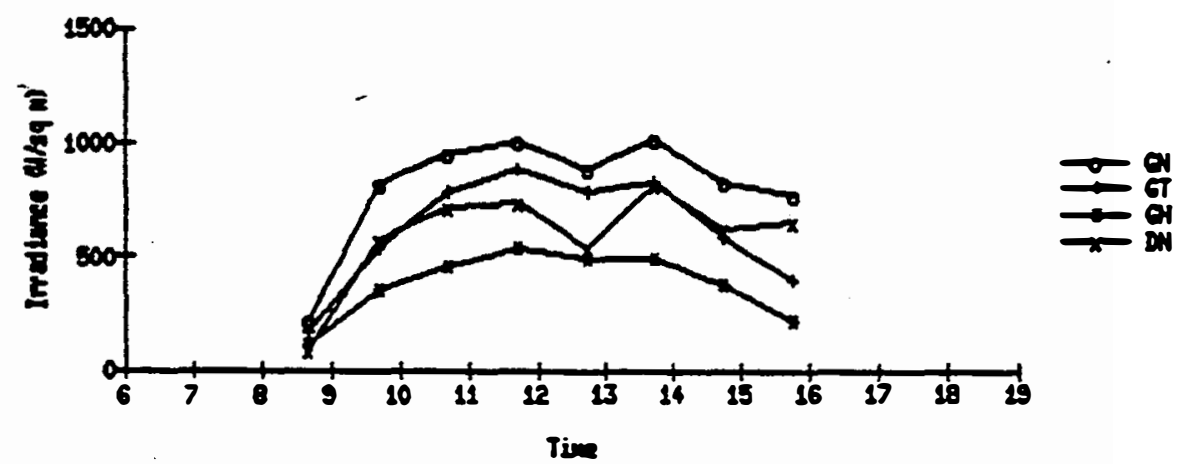

PAE 20 GTS

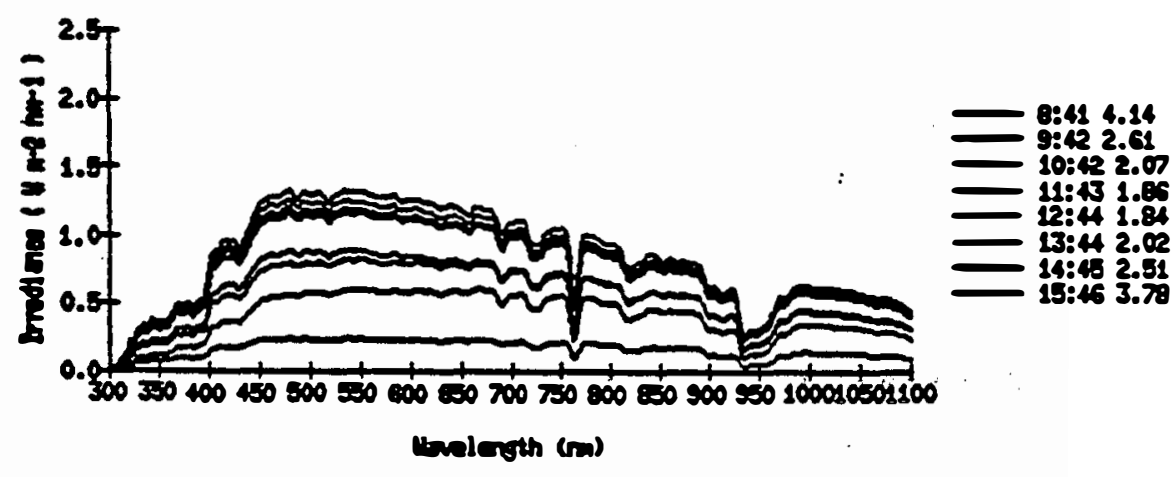


N PCes38040

$020988040 \quad 174 \quad \mathrm{DN}$

No wind speed or albedo PSP

Clear and cool

Photos indicated, but none with this date stanp

-crus

S1 HIP deta look bad on nost scans

Data at 0740 too early: quest1onble; no spectrn

PEE 40

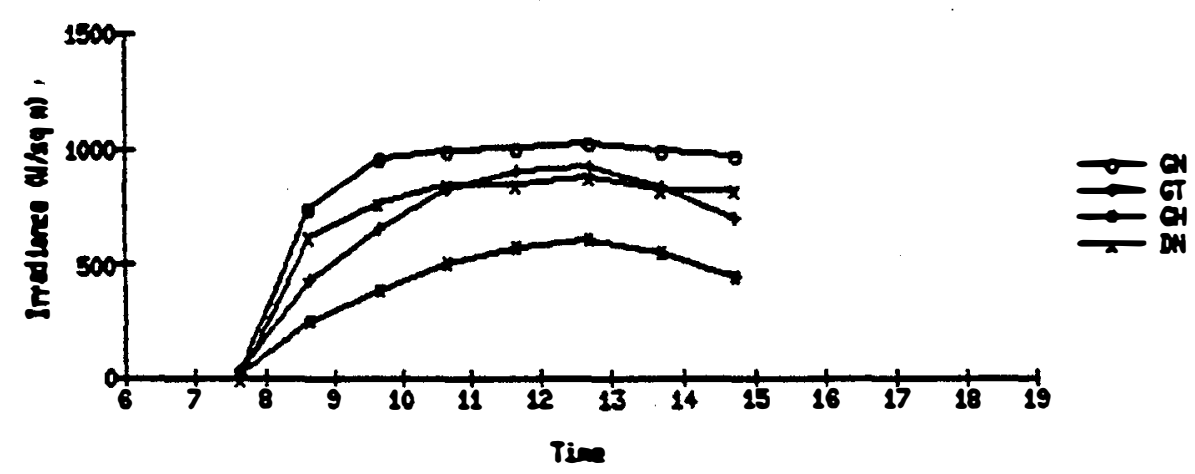

PER $40 \mathrm{BNT}$

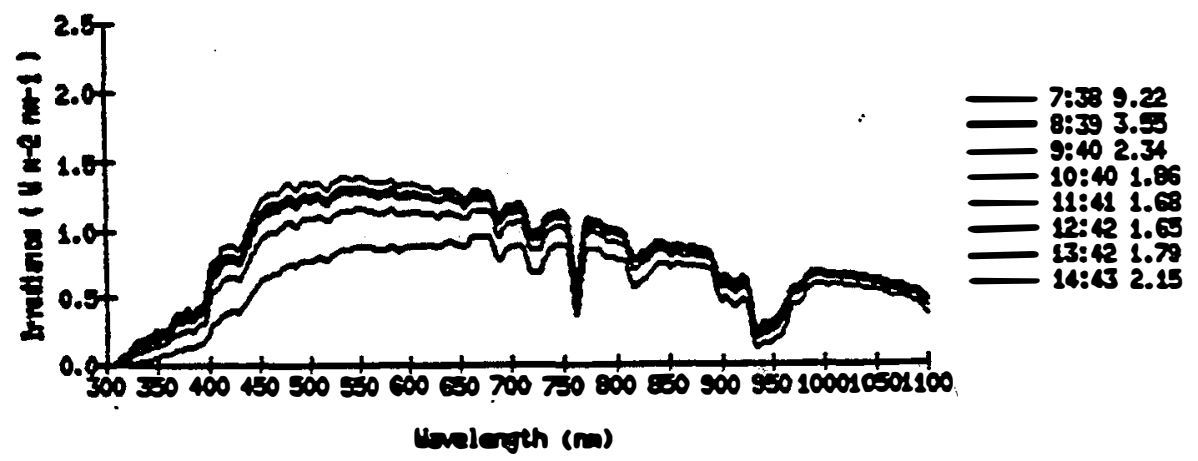


N FESBB8041

$\begin{array}{lllllll}02 & 10 & 88 & 041 & 174 & \text { DI }\end{array}$

Ho end speed or albedo PSP; sind speed added at 1345

Gi PSP tracker control replaced at 1345

Cleari can: crindy

Fhotos indreated, but none olth lbis date stanp

sic-or

s1 uIP date look bad nost of the day

pes 41

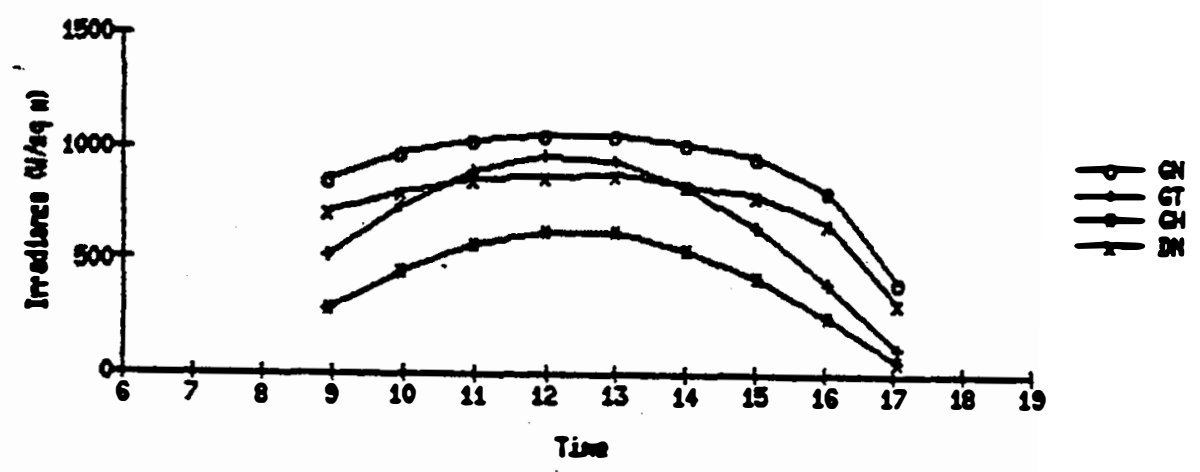

Pees at an

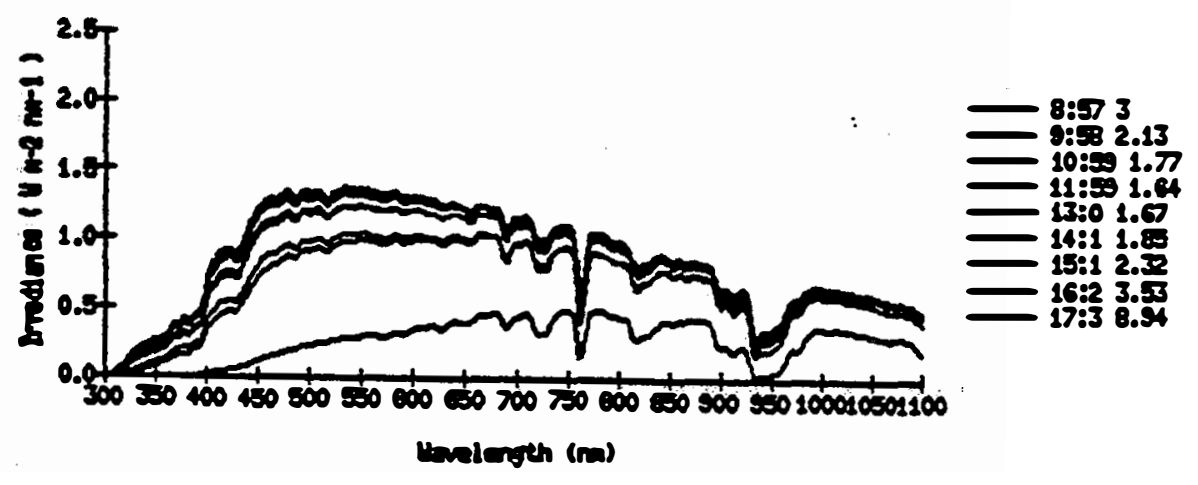


4 PCe 588042

$02 \quad 1188042 \quad 174 \quad \mathrm{DW}$

Ho alsedo PSP

Clear: cool

Photos indicated, but none with this date stamp

S1 IIP data look bad at 0800, 0901, and 1001

Hote time seamp differences 0751 v8. 0800

PaE 22

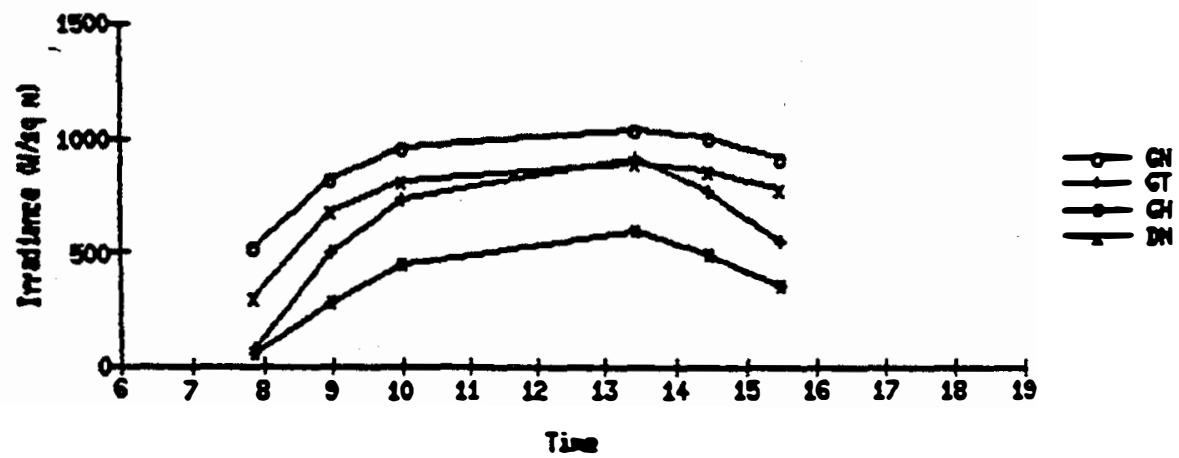

$\operatorname{mex} 42 \mathrm{Bm}$

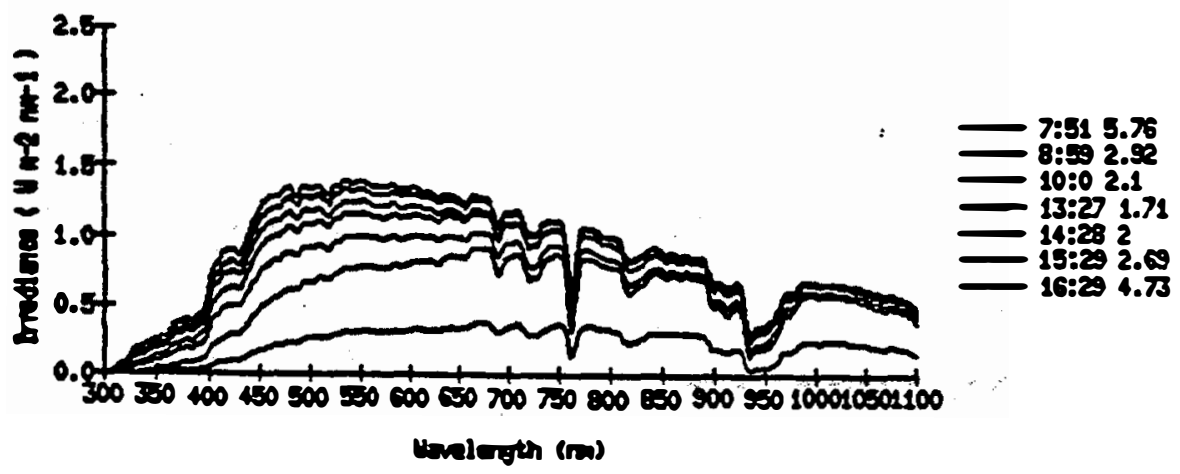


3.3 Solar Energy Research Institute, Golden, Colo. 
4 STRI 87328

$1 1 \longdiv { 2 4 } 8 7 3 2 8 \quad 1 5 8$

1050: Breezy; no brown cloud Fislble; hazy "ublte" to south and vest; Hountans just of sible

ixtellient atmospheric stability

Tenp controller at $40 \mathrm{C}$

1108:

1120: Tenp controller at $40 \mathrm{C}$

1223:

1149:

1201:

1222:

1224:

12268

Slides for ell spectra except diffuse

$-\infty-\infty-\infty$

87328 A: 1052 Da by Itself

87328 B: 1121 D:

C: 1122 GI

D: 1125 DF

87328 E: 2151 D:

F: $\quad$ :

$87328 \mathrm{~Hz} 1227 \mathrm{D}$

I\& $1230 \mathrm{GH}$

J? 1231 DF

23013

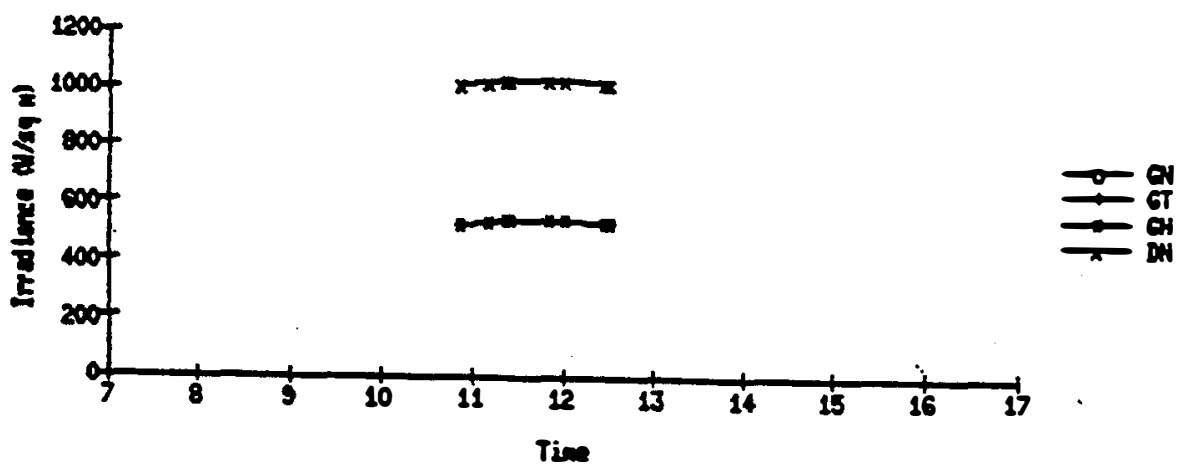


Sביב 3
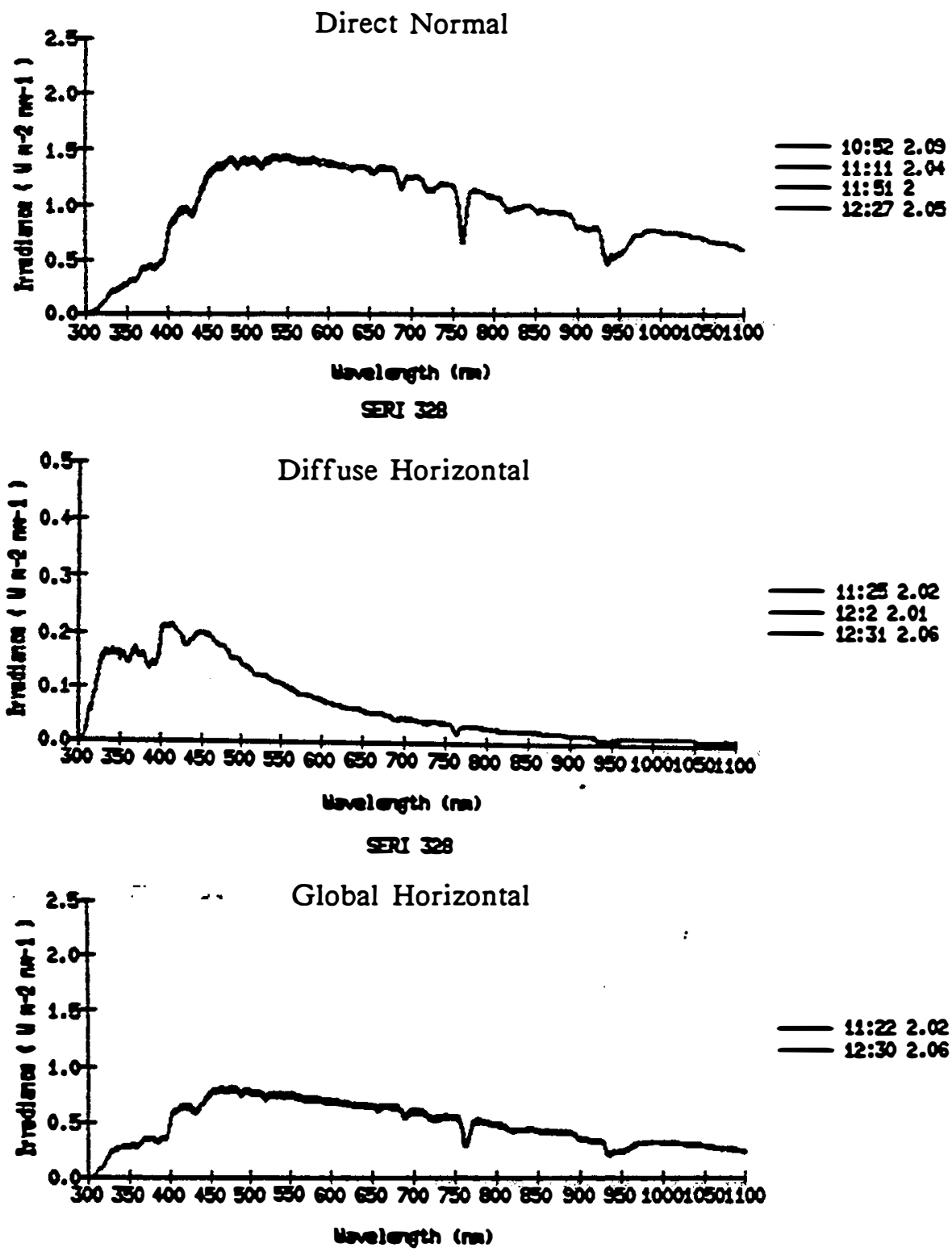

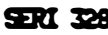

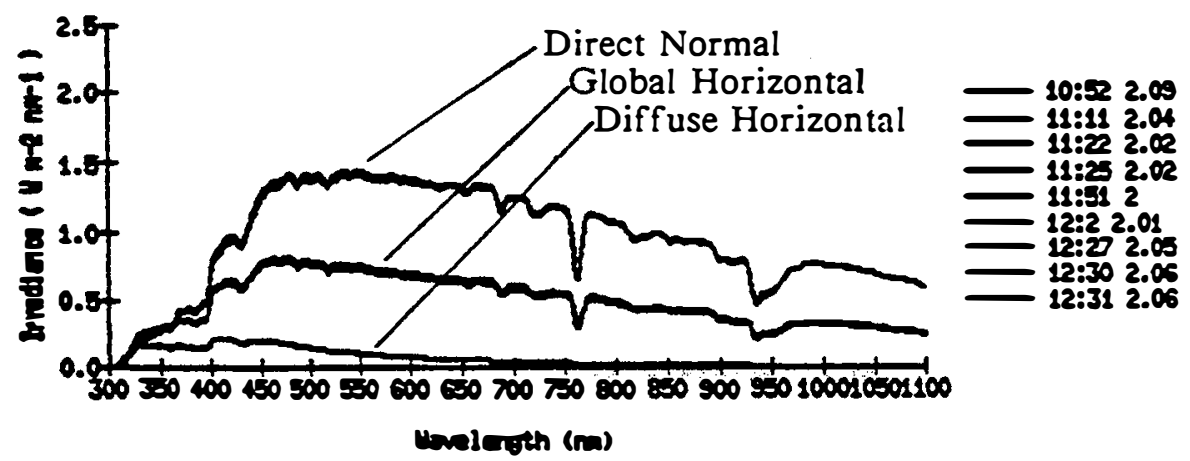


STRI87334

$1130 \quad 87334 \quad 158$

1219: Snov on ground and hill to vest (1ncreased global)

1224:

1229: $1 / 10 \mathrm{Cs}$ cloud cover on south horizon; pover plant cooling tover plune at abour 5 deg elevation

1252: Contra1ls do not persist

1258: Tenp controller $40 \mathrm{C}$

$1258+:$

1321: Pover plant plune (light) building belov sun above veter vapor pluareloud

l/10 Cs cloud cover on south horizon

1325:

1326:

1410: Tenp controller $40 \mathrm{C}$

1414:

1416:

1436:

1438:

1441:

1504:

1506:

1509: Sun belou porer line for series of three scans

Slides for all spectre. Including diffuse

$87334 \quad A_{8} 1222 \mathrm{DA}$

B\& 1227 GH

C: 1229 DF

87334 D: $1254 \mathrm{DA}$

shade disk slides ( 2 ea) $18 \& 19$ (bave only 3 )

E: $1258 \mathrm{GH}$

F: 1259 DF

$87334 \mathrm{G}: 1321 \mathrm{DA}$

H: $1324 \mathrm{FH}$

Is 1326 DF slides $23 \& 24$ vith cand; canere sertched to auto on 23

$87334 \mathrm{~J}: 1410 \mathrm{DA}$

$\mathrm{X}=1413 \mathrm{GH}$

Is $1814 \mathrm{DF}$

$87334 \mathrm{H}: 1435 \mathrm{DA}$

H: 1438 GH

O: $1440 \mathrm{DF}$

$87334 \mathrm{P}: 1503 \mathrm{DA}$

O: 1506 GH

R: 1508 DF nissing one data point; added -99 to end of scan

picture $\$ 33$ nissing

$\sin 3 \pi$

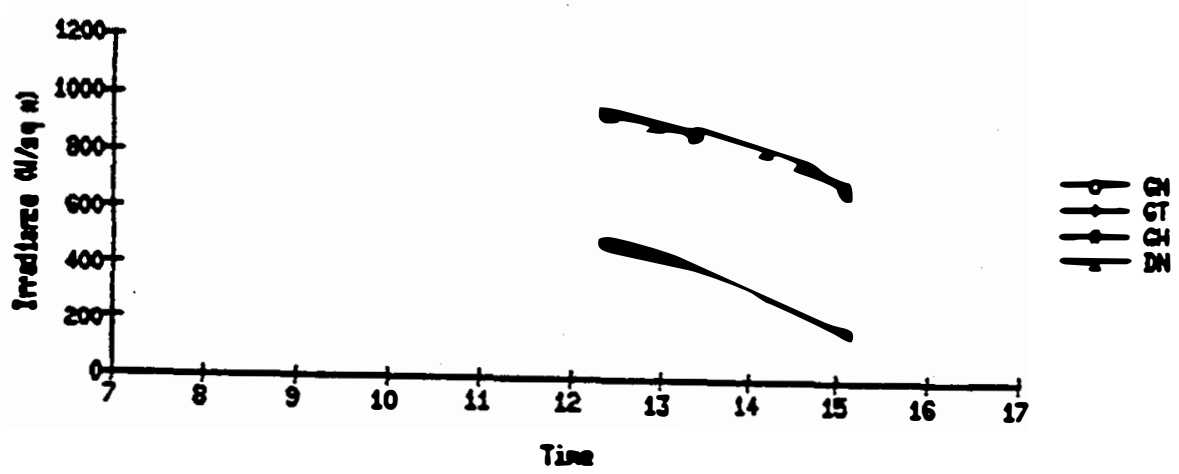


ज्या 34
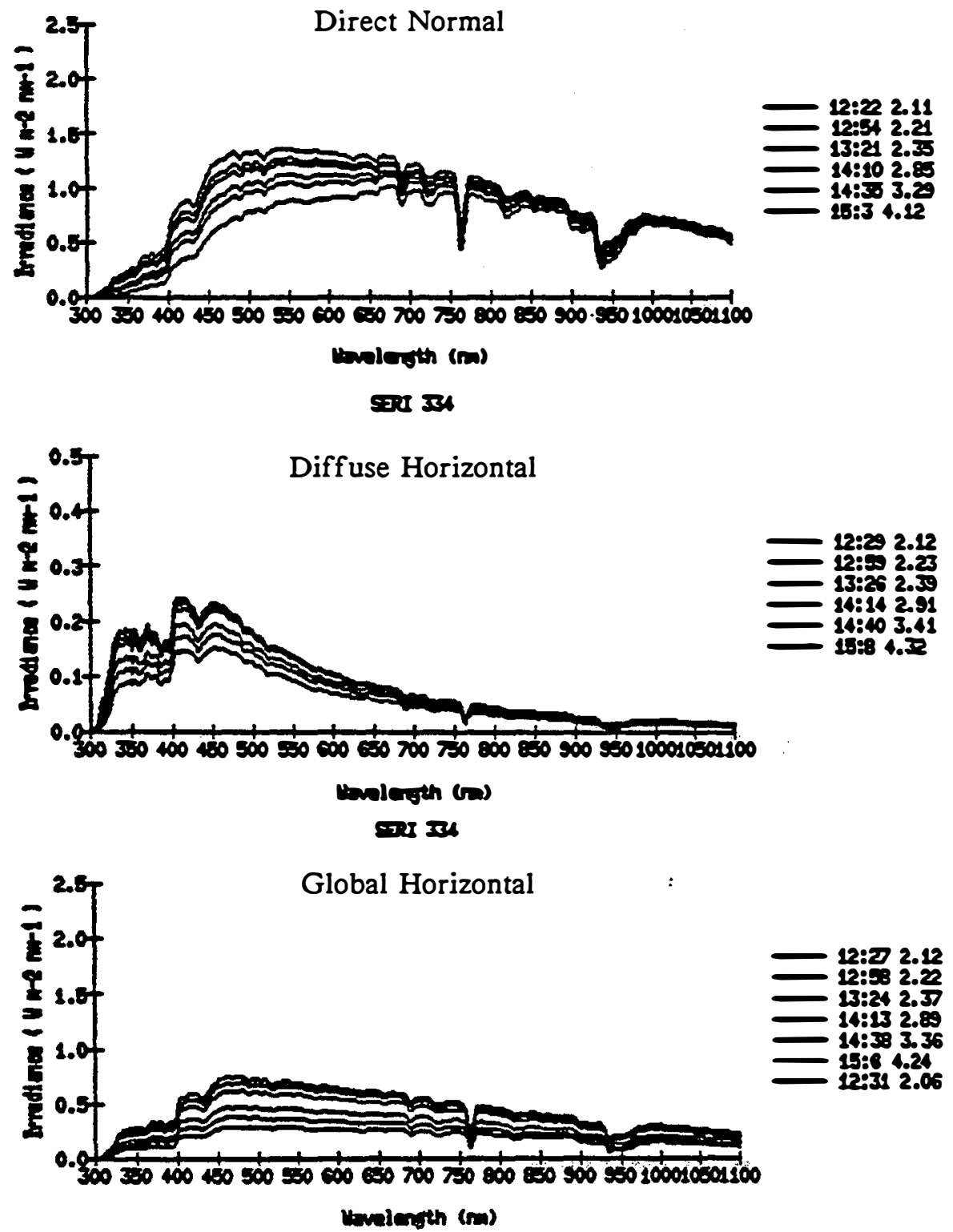

E्या 34

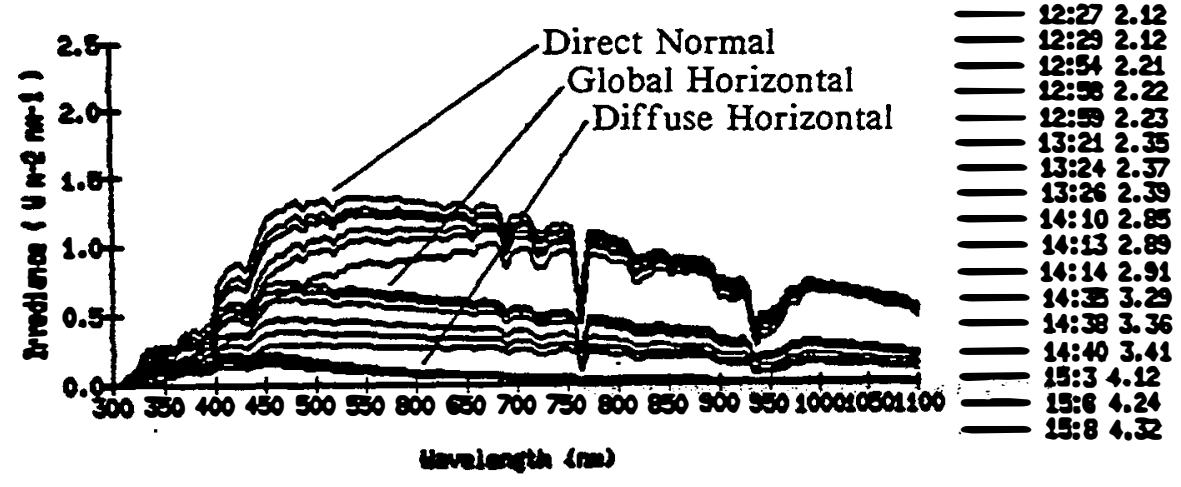


N STRI87335

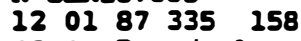

0840: Power plant cooling tower clouds abscure bank building downtown Tenp controller at $40 \mathrm{C}$

0840: Power plant cooling tower clouds obscure bank building downtown Tenp controller at $40 \mathrm{C}$

0911: Power plant plue visible belor sun and to rest Hinds increasing to $4-6 \mathrm{~m} / \mathrm{s}$

Tepp controller $39 \mathrm{C}$; set point $40 \mathrm{C}$ (after diffuse spectrus)

0942: Contralls do not persist

Sun in porer plant =joud (band) but drops to vest

Tenp controller at $40 \mathrm{C}$

1014: 1-2/10 As cloud corer building to vest

Tenp controller at $40 \mathrm{C}$

1113: Snelled sulfur, possibly fron refineries Teap controller at $40 \mathrm{C}$

(salil anount of noise in near-IR on diffuse spectrun at 1117

1250: Clouds dissipating; < $<1 / 10$ to vest; contralls lasting longer

Winds aloft chopping out sections (reve fore)

Tenp controller 40-41 C on direct spectru

Slides for all spectra, including diffuse

$87335 \mathrm{~A}: 0840 \mathrm{DP}$

B: 0845 GH

C: 0847 DF

87335 D: 0911 D.

E: $0913 \mathrm{GH}$

F: 0916 DF

15 a drift

$87335 \mathrm{G}: 0941 \mathrm{DI}$

$\mathrm{Hz} 0944 \mathrm{GH}$

I: $0946 \mathrm{DF}$

$87335 \mathrm{~J}: 1013 \mathrm{DH}$

$\mathrm{K}=1016 \mathrm{GH}$

L: $1019 \mathrm{DF}$

av drift

$3=v$ drift

$87335 \mathrm{M}: 1113 \mathrm{D}$

A: 1115 GH

O: 1117 DF

P: 1119 DF

$87335 \mathrm{Q}$ : $1151 \mathrm{DI} 18 \mathrm{aV}$ drift; one spectrun - nenory full

كיכ

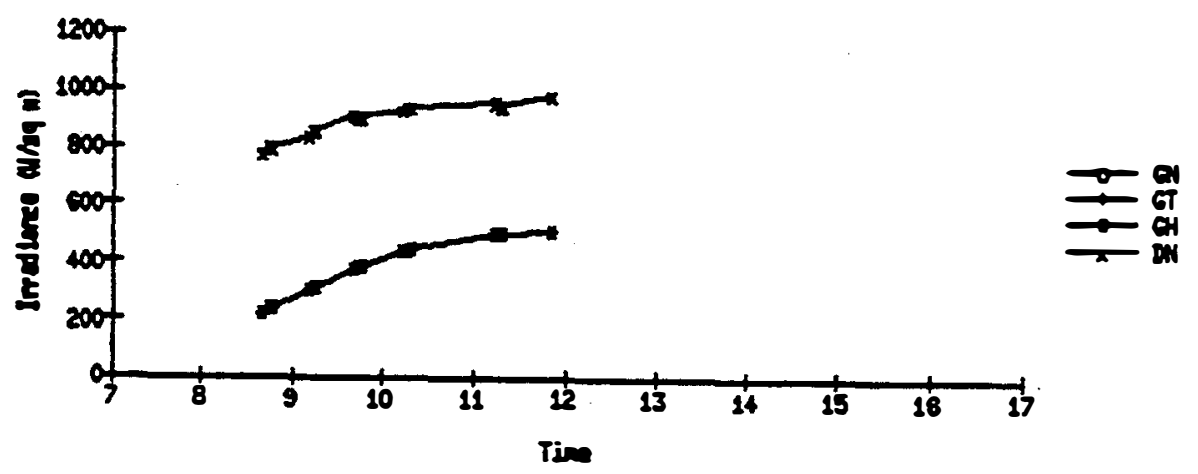


ज्यर
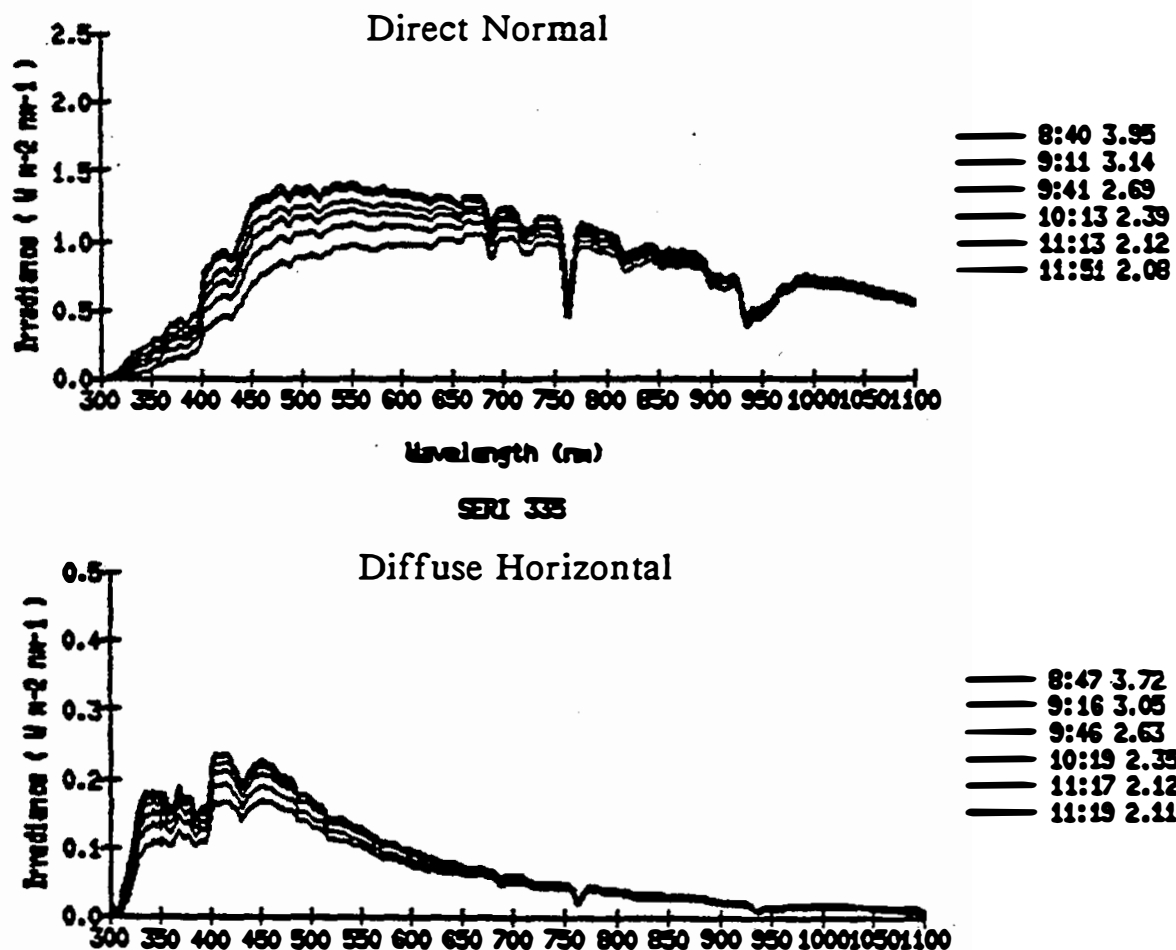

bolargth (m)

ज्या 30

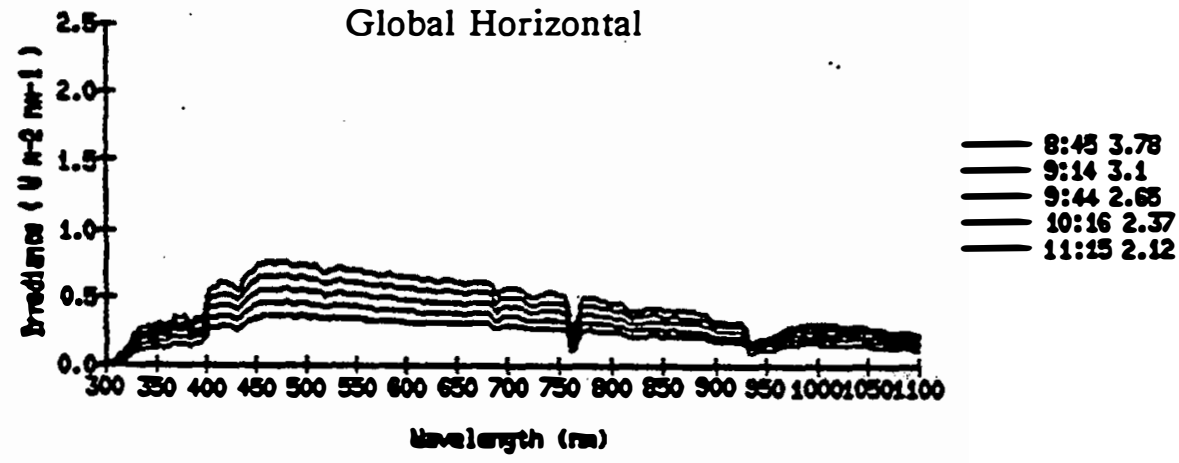

sed 305

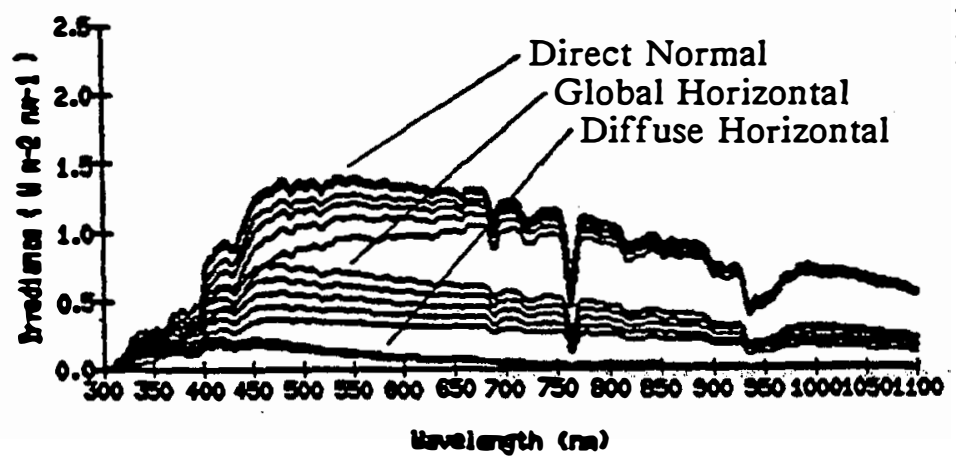

$8: 403.5$

$8: 453.70$

8:47 3.72

$9: 113.14$

$9: 143.1$

$9: 163.05$

9:16 3.05

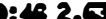

$10: 132.30$

10:16 2.37

10:19 2.35

$11: 232.12$

$11: 152.12$

$11: 172.12$

$11: 192.11$

$11: 192.11$
$11: 512.08$

vinelerth (m) 

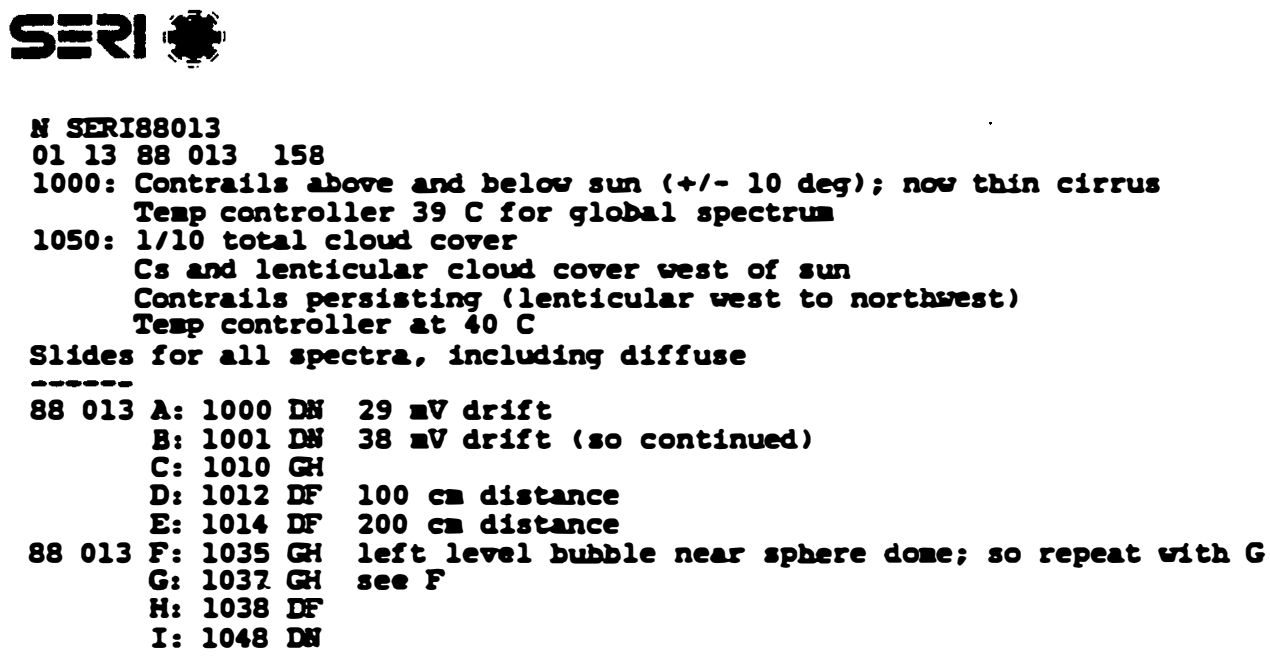

$\operatorname{sed} 13$

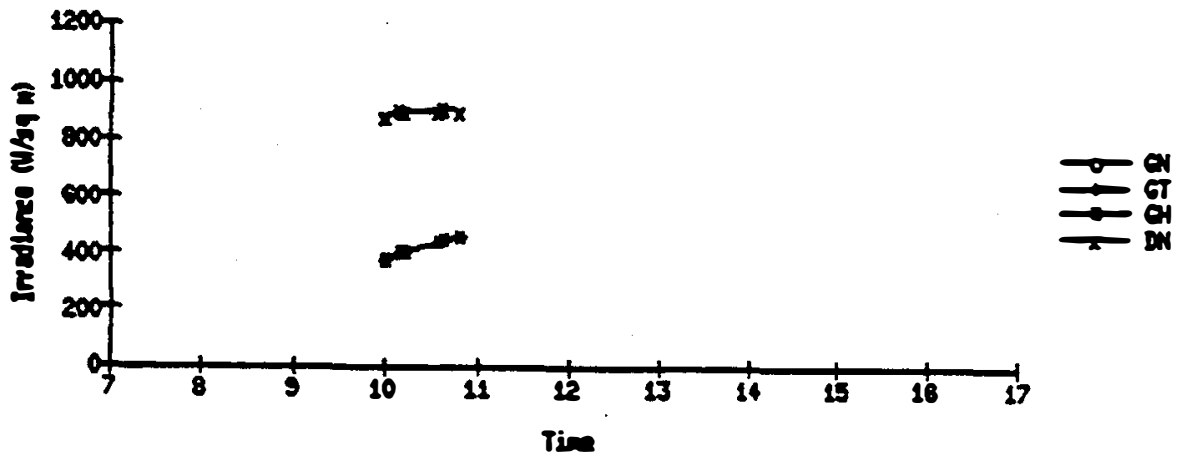


S्गया 13

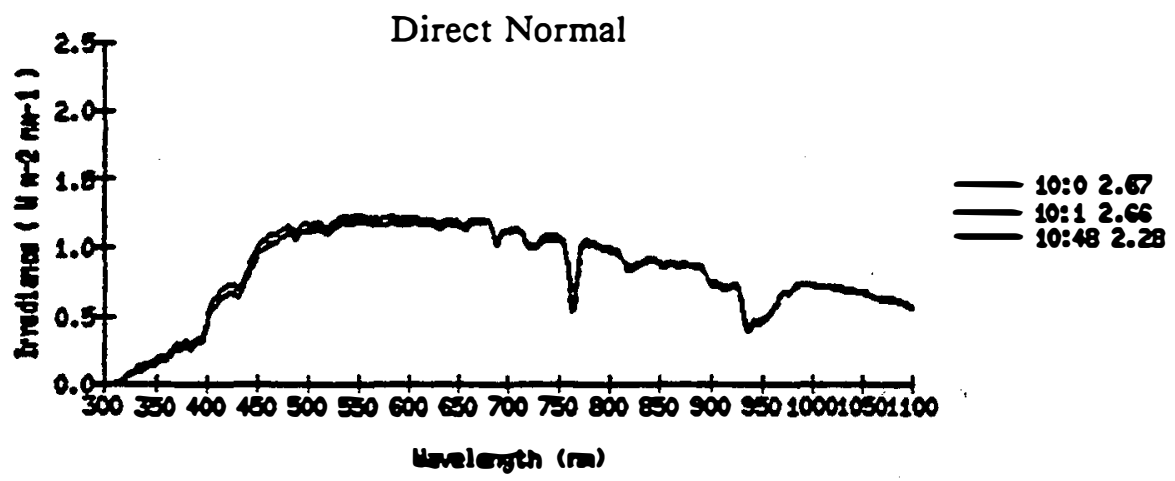

STRI 13

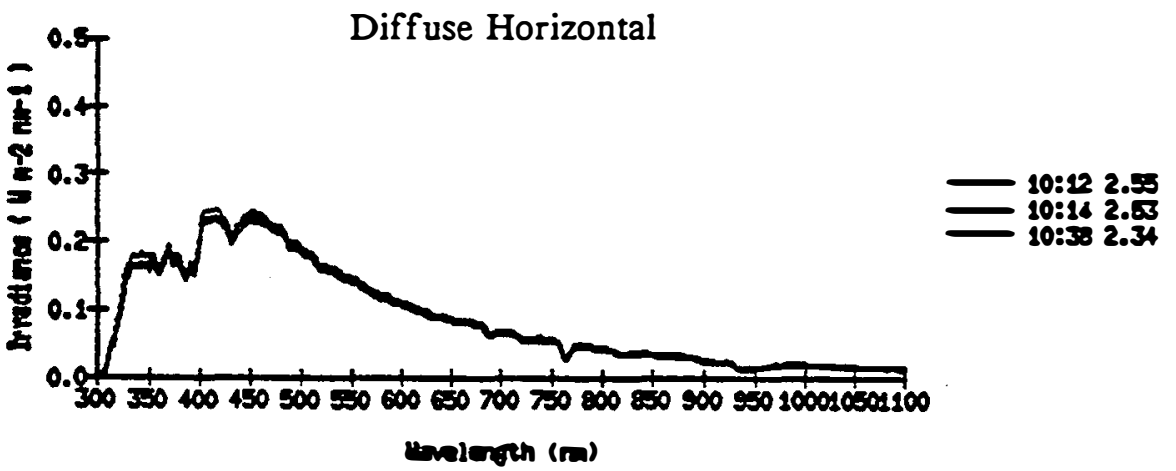

Sग्गI 13

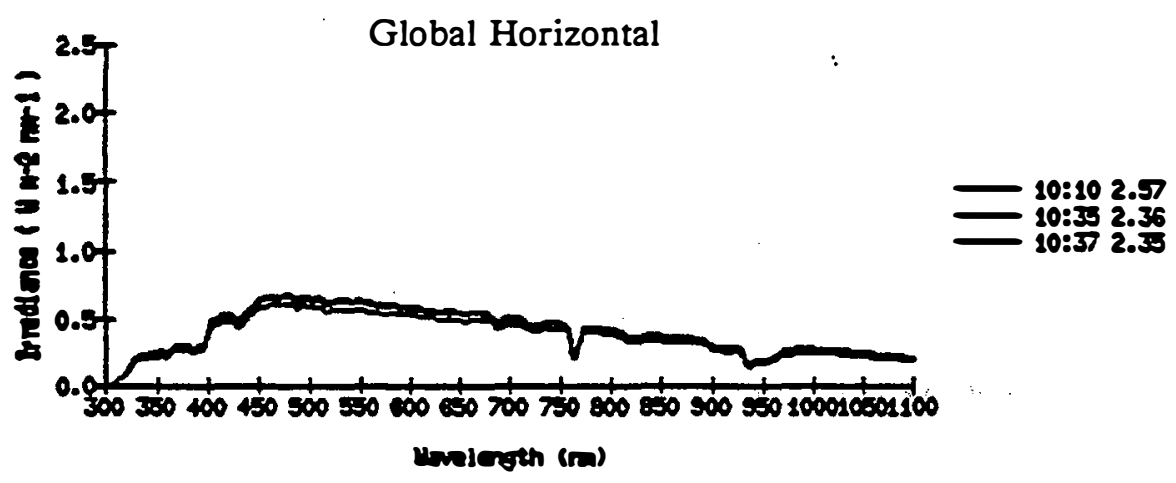

S्ञगI 13

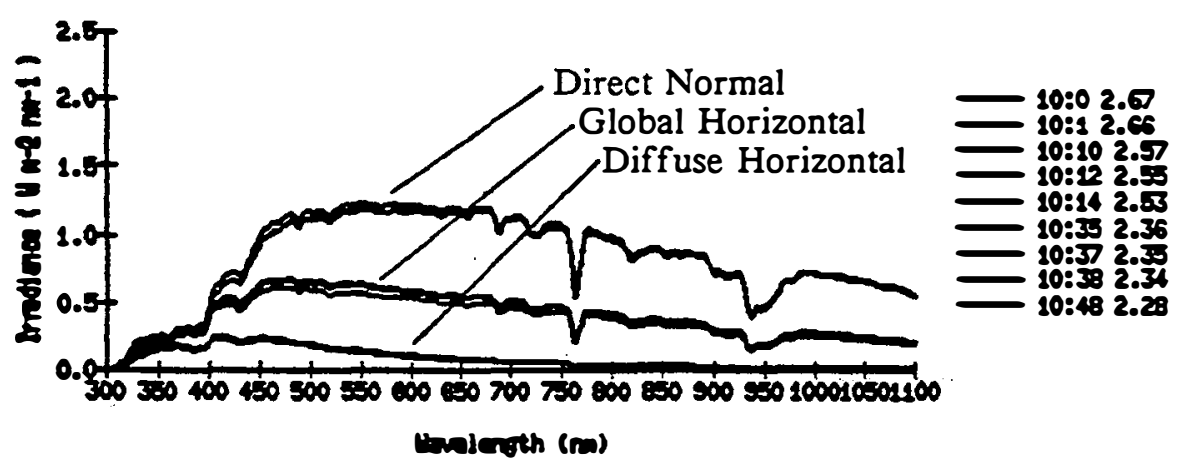




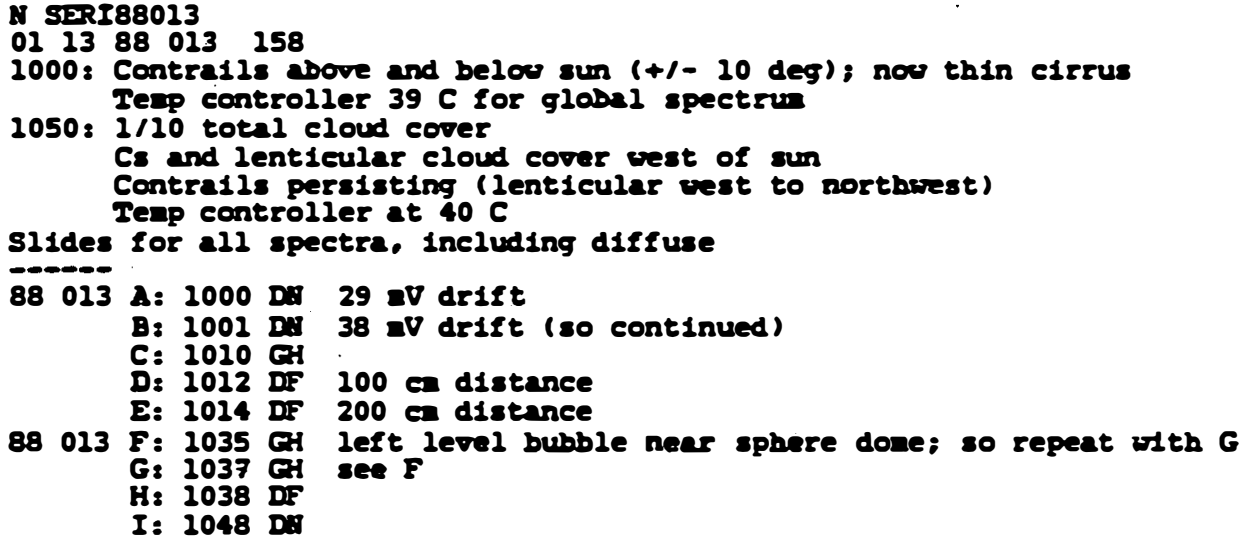

$\operatorname{sen} 13$

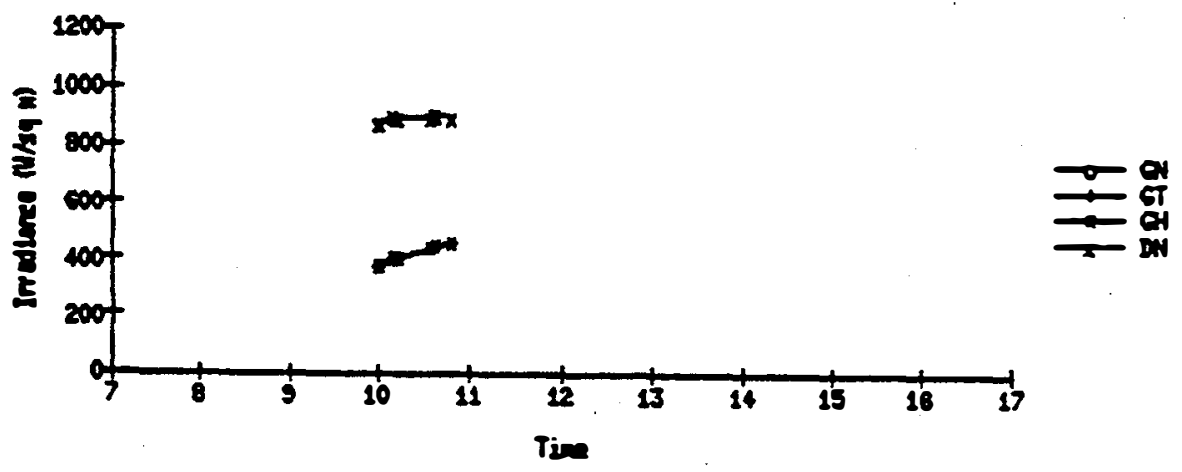


S2RI 23

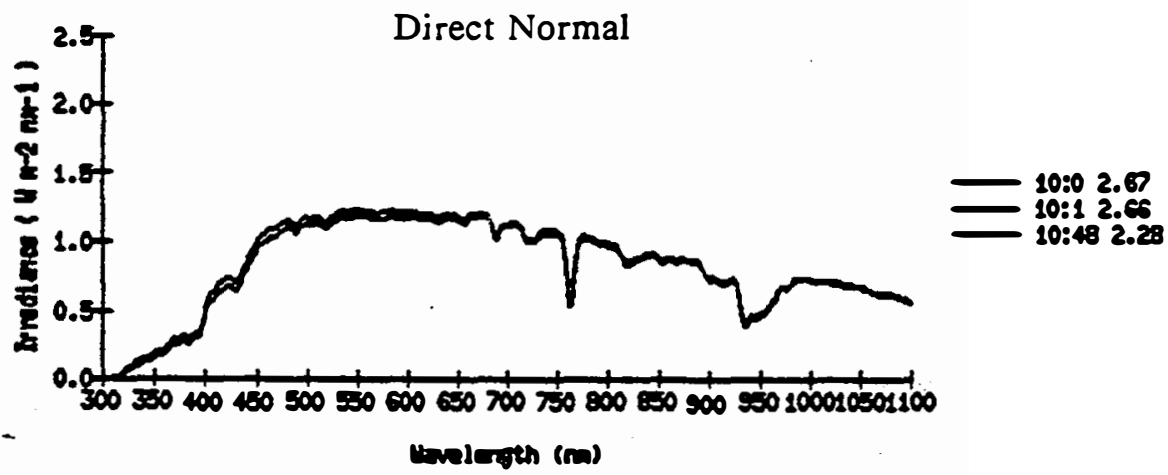

S्ञगय 13

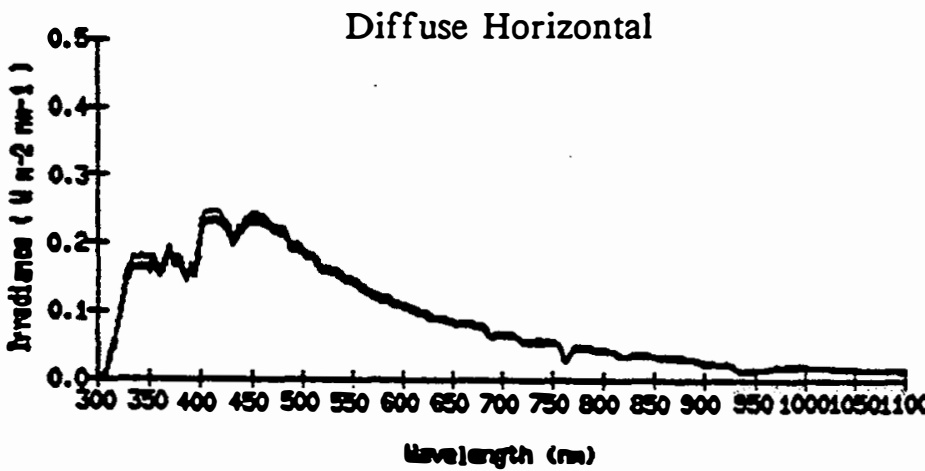

S्यरI 23

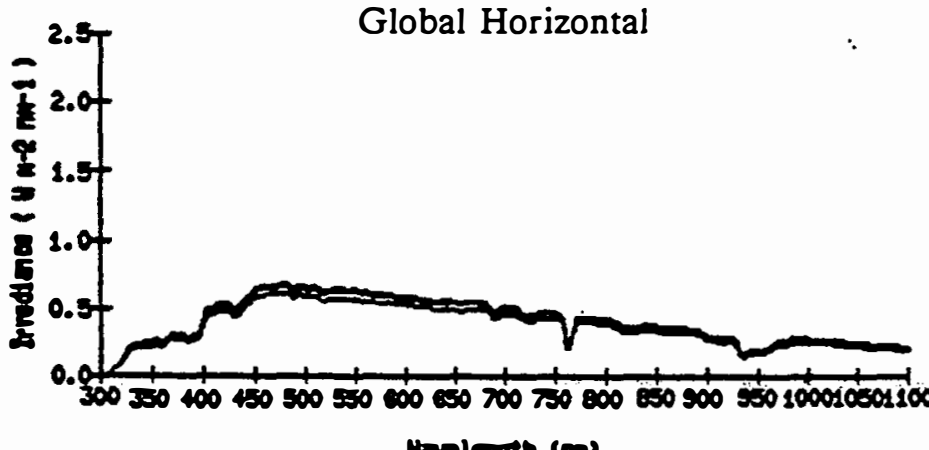

S्यरा 13

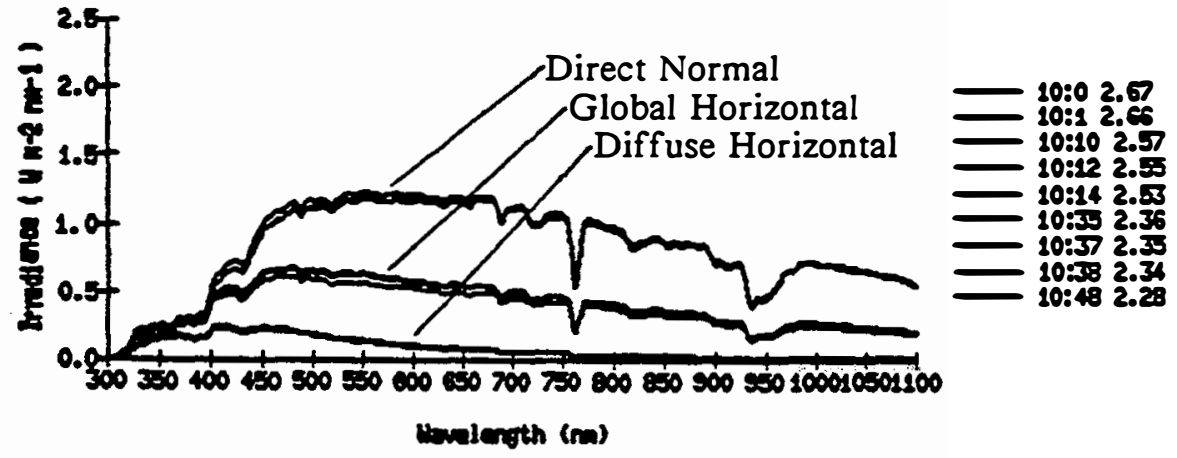




\section{SEP1 *}

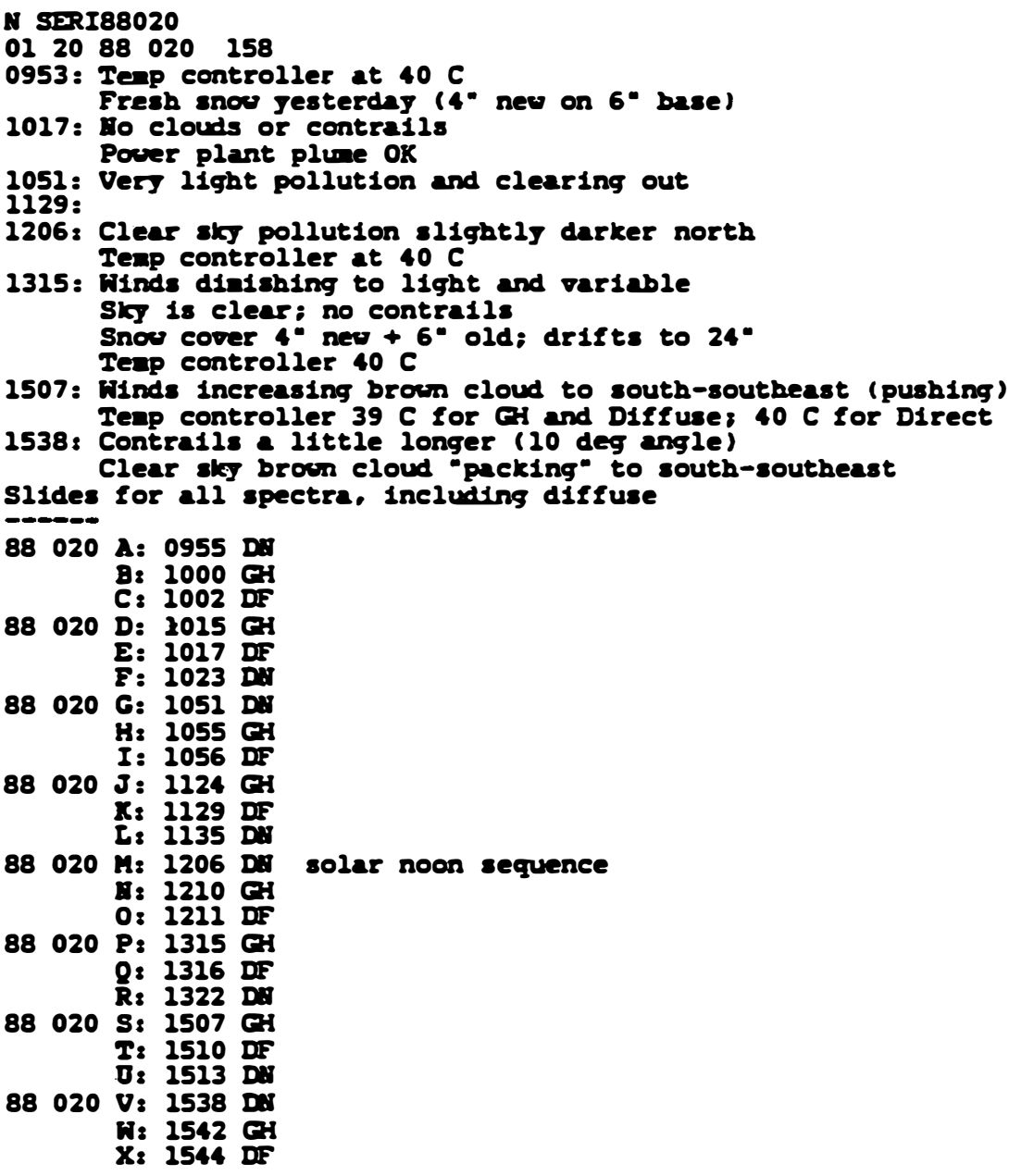

20

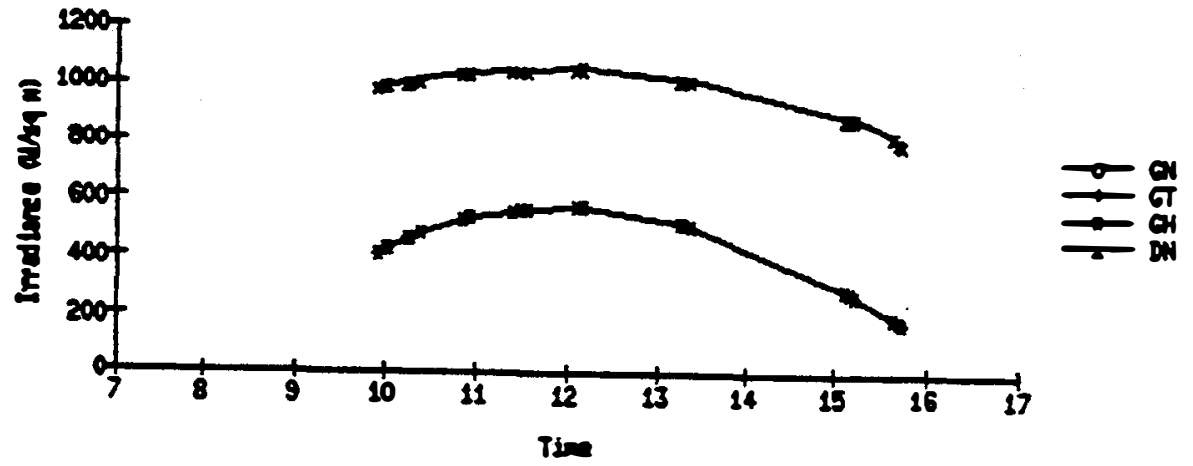


20

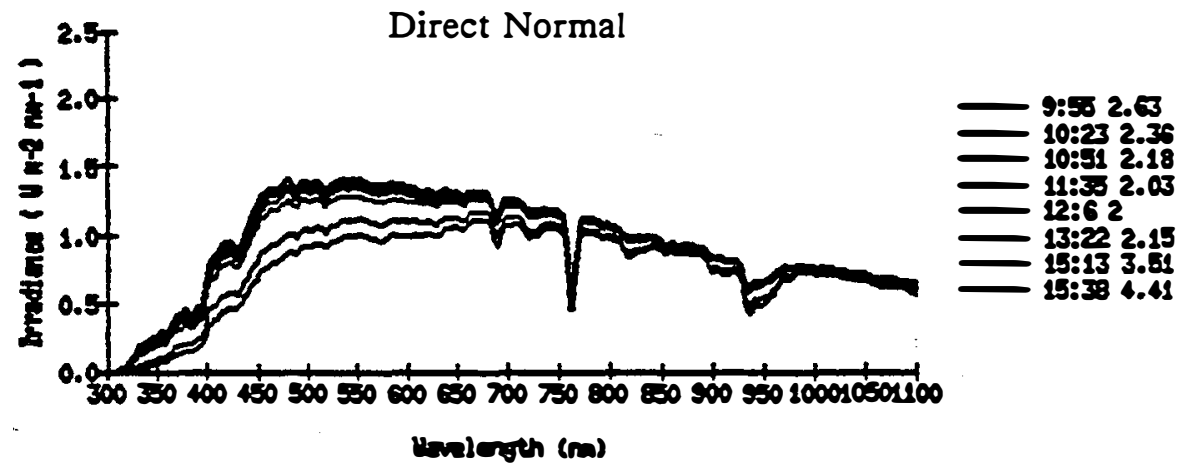

ב20

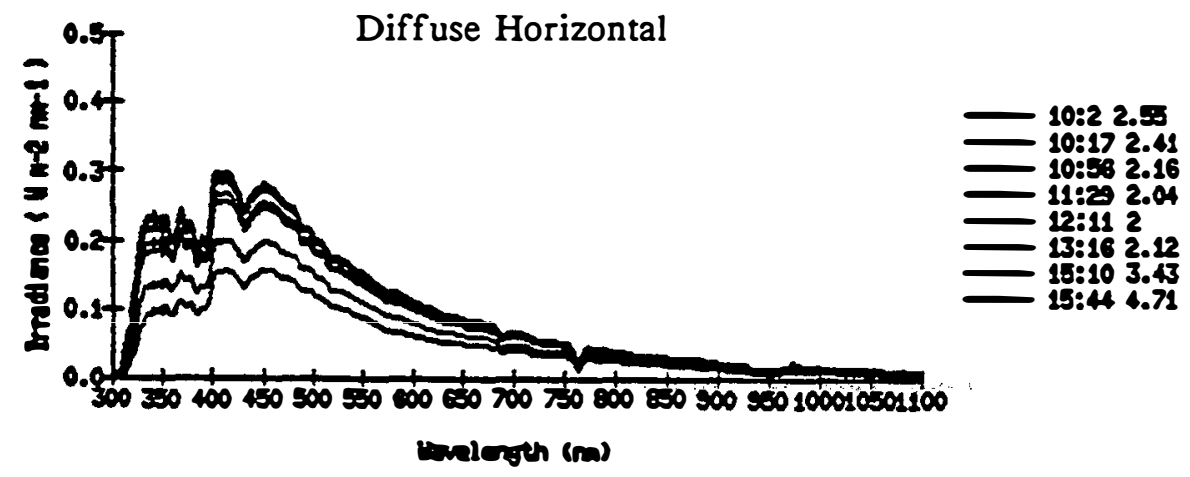

घ्या 20

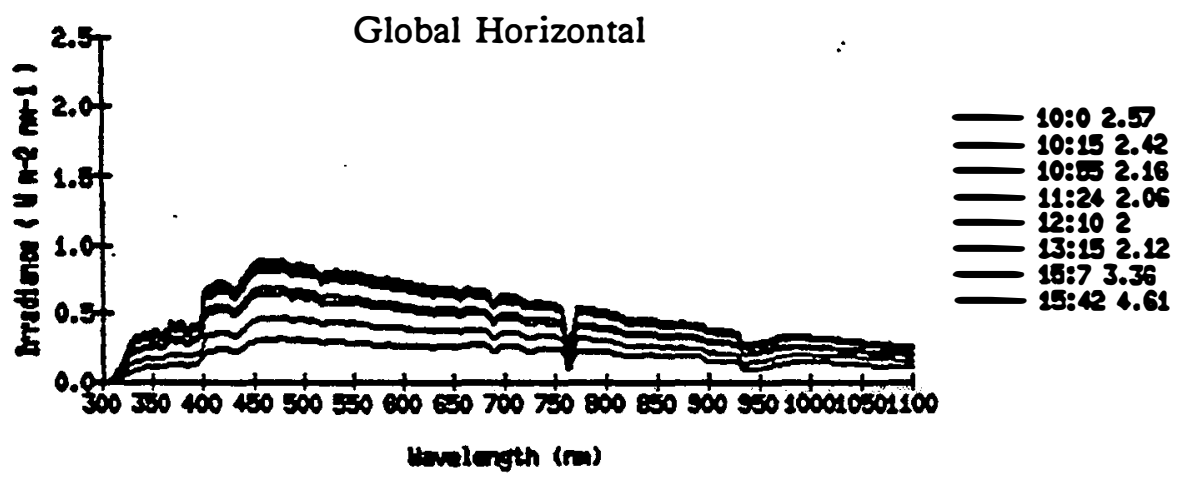


STRI88026

$012688026 \quad 158$

0916 : Hoderate brocn cloud; no clouds

Contrails not persisting

Temp controller 40 C for Di: 39 C for GH: 41 C for DIF

0945, Temp controller $40 \mathrm{C}$

Contrails across sun for diffuse with done at 0957; none for diffuse spectrun at 0958

1010s Winds shifted 180 (nov out of vest): pourer plant plume now across bank buliding downtown Contrails persisting; but linger near east Tenp controller $40 \mathrm{C}$

1043: Snou cover 85-90 percent; 1 to 10* drifts Pover plant pluie heading east $1 / 3$ distance between horizon and sun (white color) Contralls nou dissipating

1121: Teap controller at $40 \mathrm{C}$

1204: Clouds dereloping to west and southerest; \& $1 / 10$ As in linear bunds parallel to mountains Contrails persist depending on altitude of alrcraft Teap controller at $40 \mathrm{C}$

1241. Winds decreasing and nov out of south-southeast Teap controller at $40 \mathrm{C}$

1401: Teep controller at $40 \mathrm{C}$

1438: Wind has blown cloud to nountains Pouer plant plune rises to belos sun Still ( $1 / 10$ As to west and southerest (lenticular) Teap controller $40 \mathrm{C}$

slides for all spectra. Including diffuse

$88026 \mathrm{~A}: 0916 \mathrm{DA}$ B: 0921 GH C: 0922 DF

$88026 \mathrm{D}: 0945 \mathrm{GH}$ E! 0947 DF F: 0952 D. G8 $0957 \mathrm{GH}$ H: 0958 GH

$88026 \mathrm{I}: 1010 \mathrm{DB}$

GH wth Teflon dones contrail across sun J: 1013 G $\mathrm{C}_{8} 1014$ DF

$88026 \mathrm{I}: 1043 \mathrm{GH}$ $\mathrm{M} 21045 \mathrm{DF}$ 月: 1049 D.

$880260: 2121 \mathrm{DA}$ P: 1125 E. Q: 1127 DF

$88026 \mathrm{R}: 1204 \mathrm{GH}$ G w wh Teflon dones no contrall S: 1206 DF T: 1209 D: Oะ $1217 \mathrm{GH}$ O. $1218 \mathrm{DF}$

$88026 \mathrm{~K}$ K: $1241 \mathrm{D}$

odd plot - probably bad spectru

GH ertb Teflon done DF vitb Teflon done

$X: 1245$ GH

Y: 1246 DF

Z: 1252 FH

M: 1253 DF

88026 BB: $1401 \mathrm{DB}$

CC: 1409 타

DD: 1410 DF

88026 EE: $1438 \mathrm{DA}$

FF: 1443 FH GG: 1445 DF

GH estb Teflon done

DF with Teflon done

$23=v d r i f t$

2972 25

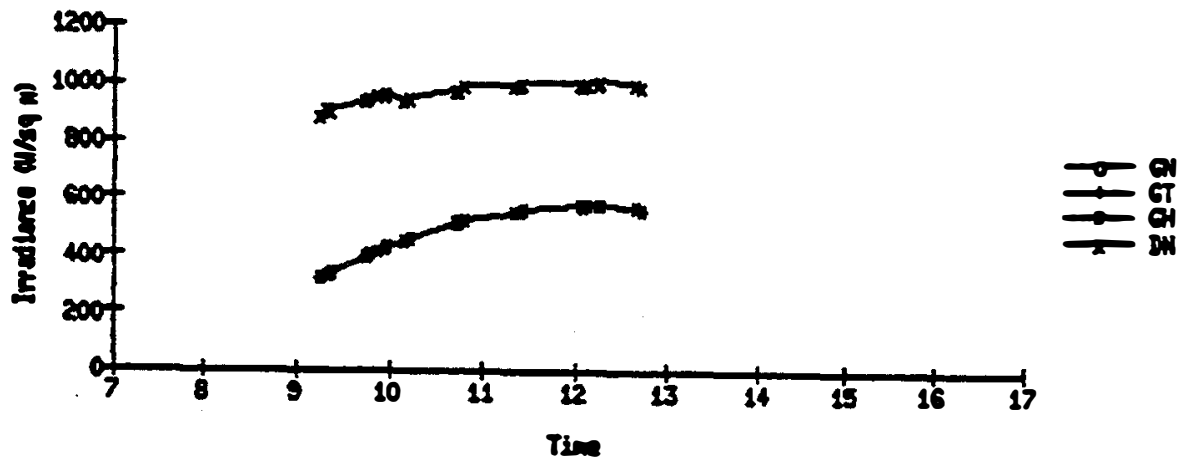


S्यग 26
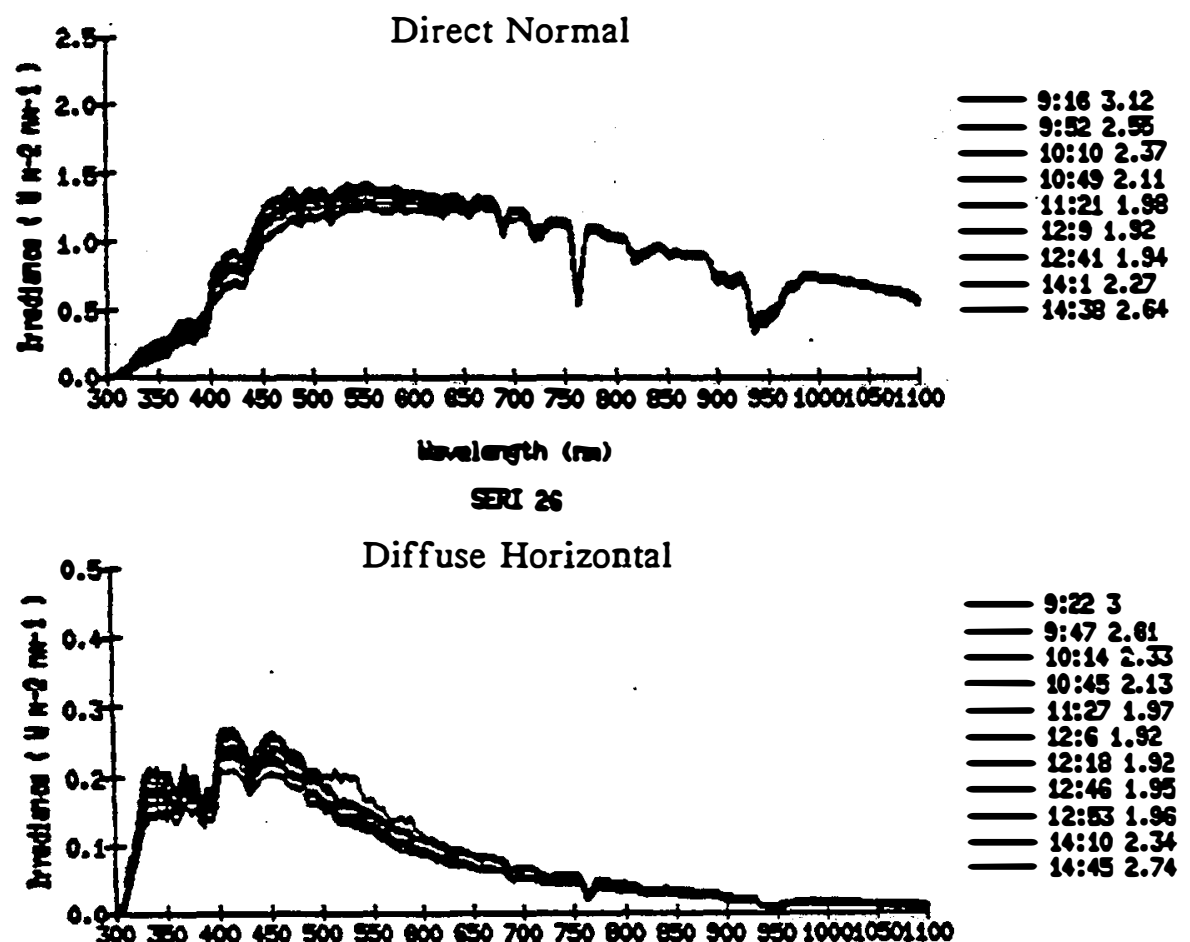

underth (m)

S्ביב 25

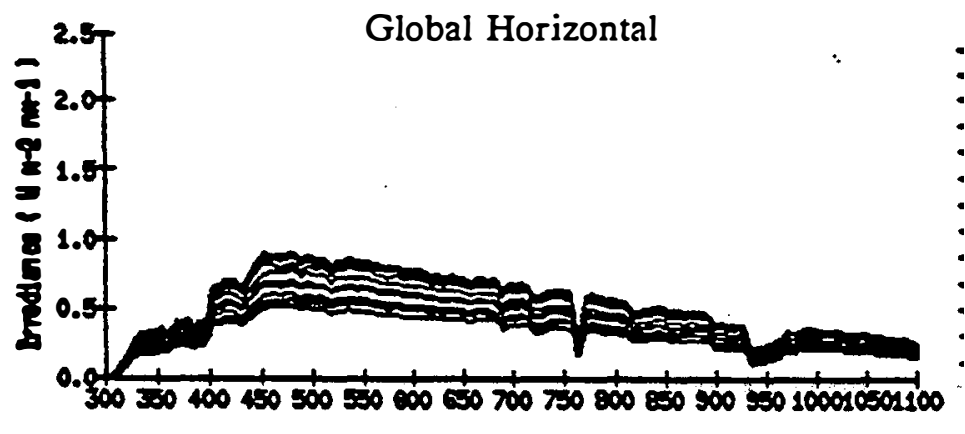

Hanlersth (m) 


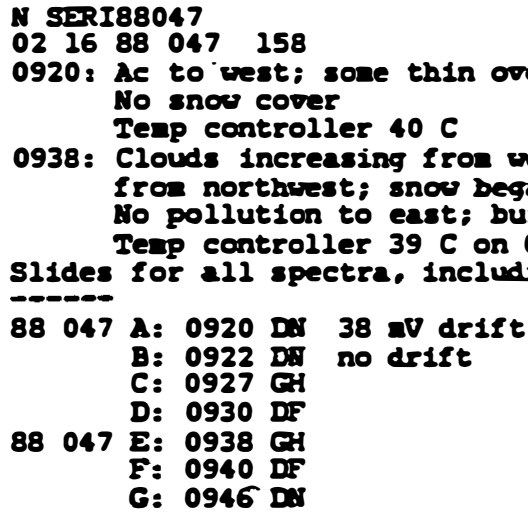

88047 A: 0920 Da 38 av drift

B: $0922 \mathrm{DI}$ no drift

C: $0927 \mathrm{G}$

D: $0930 \mathrm{DF}$

88047 E: $0938 \mathrm{GH}$

F: 0940 DIF

G: 0946 Dis

S201 47

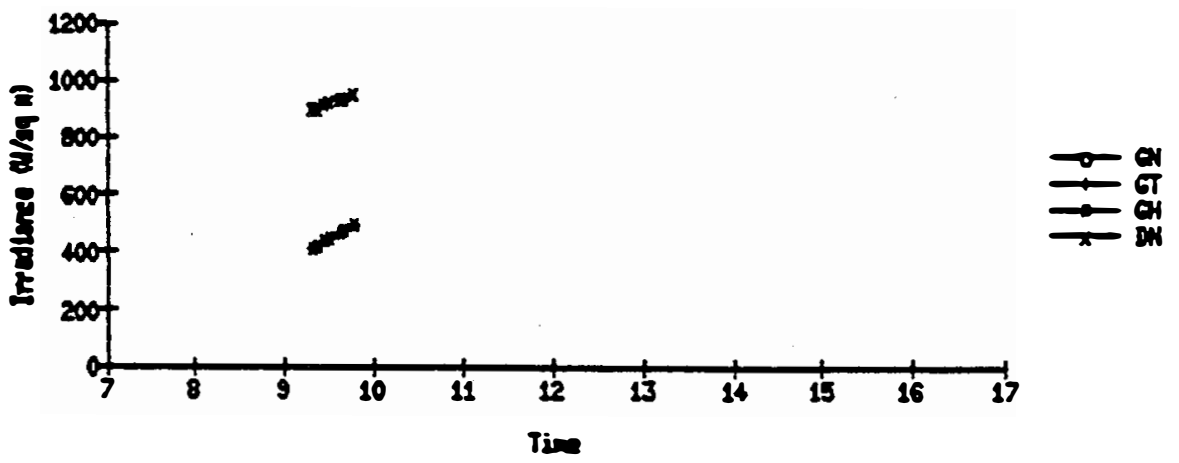


TR-3513B

\section{SEN}

S्यर्य 47
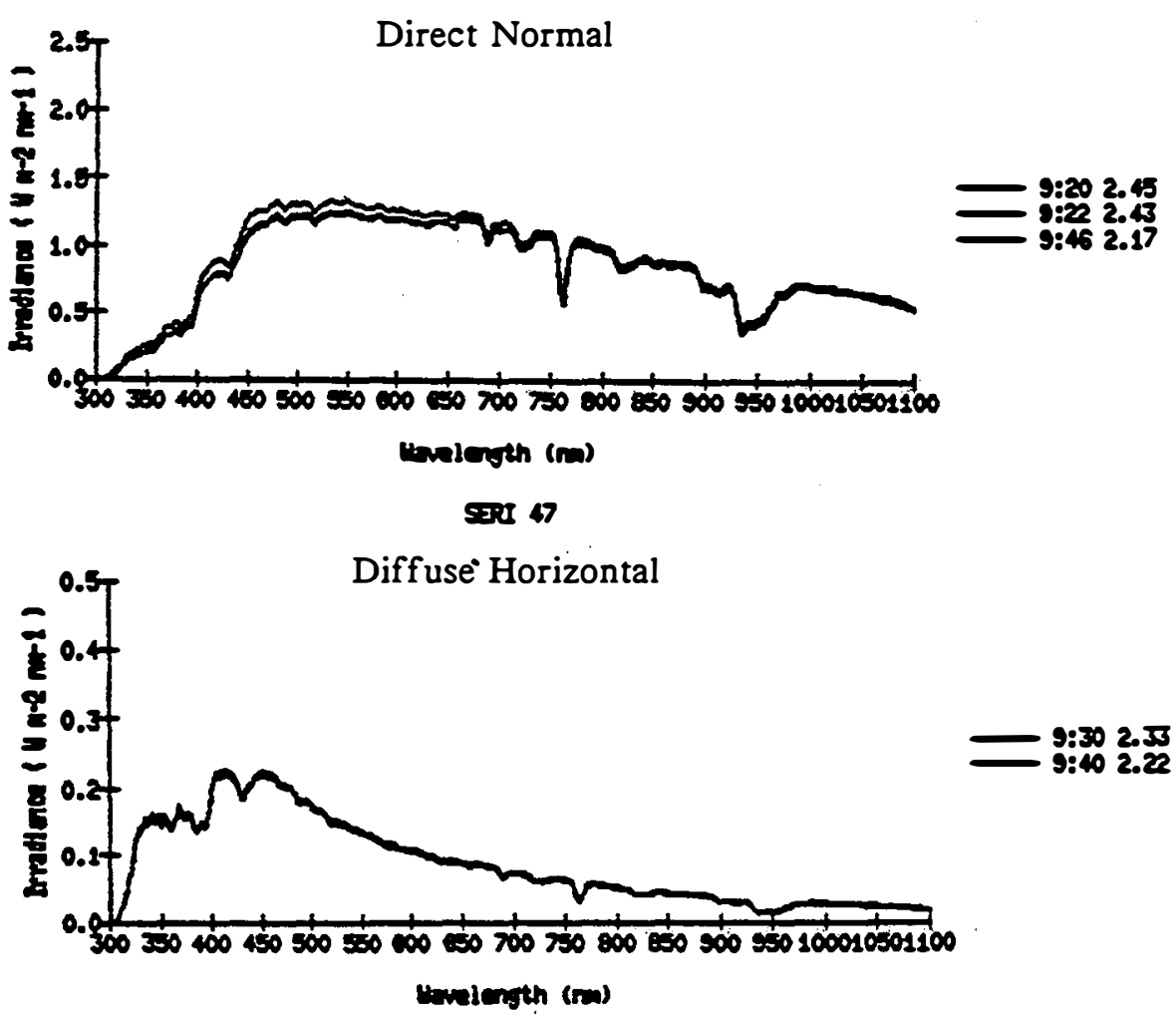

S्यश्र 47

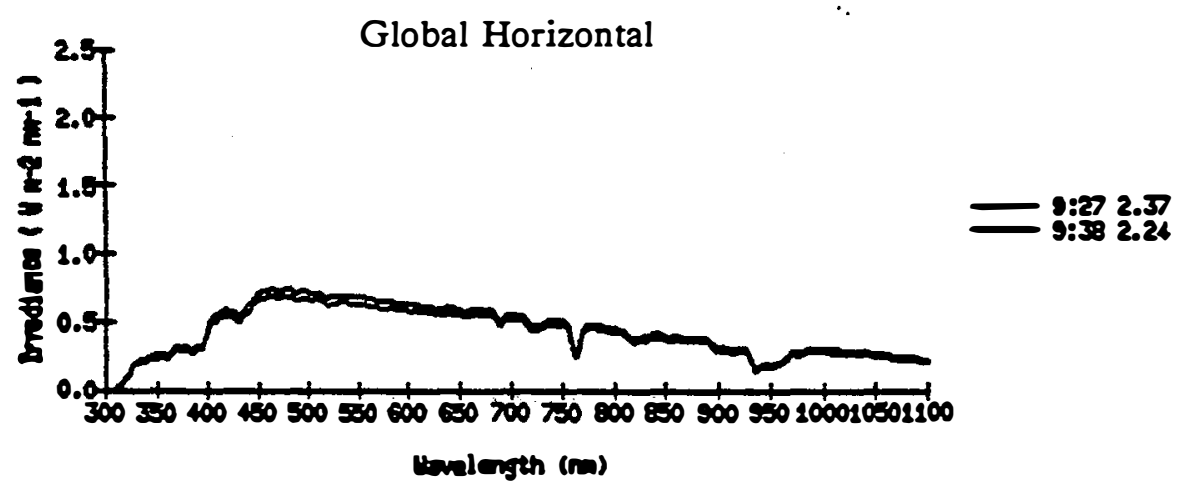




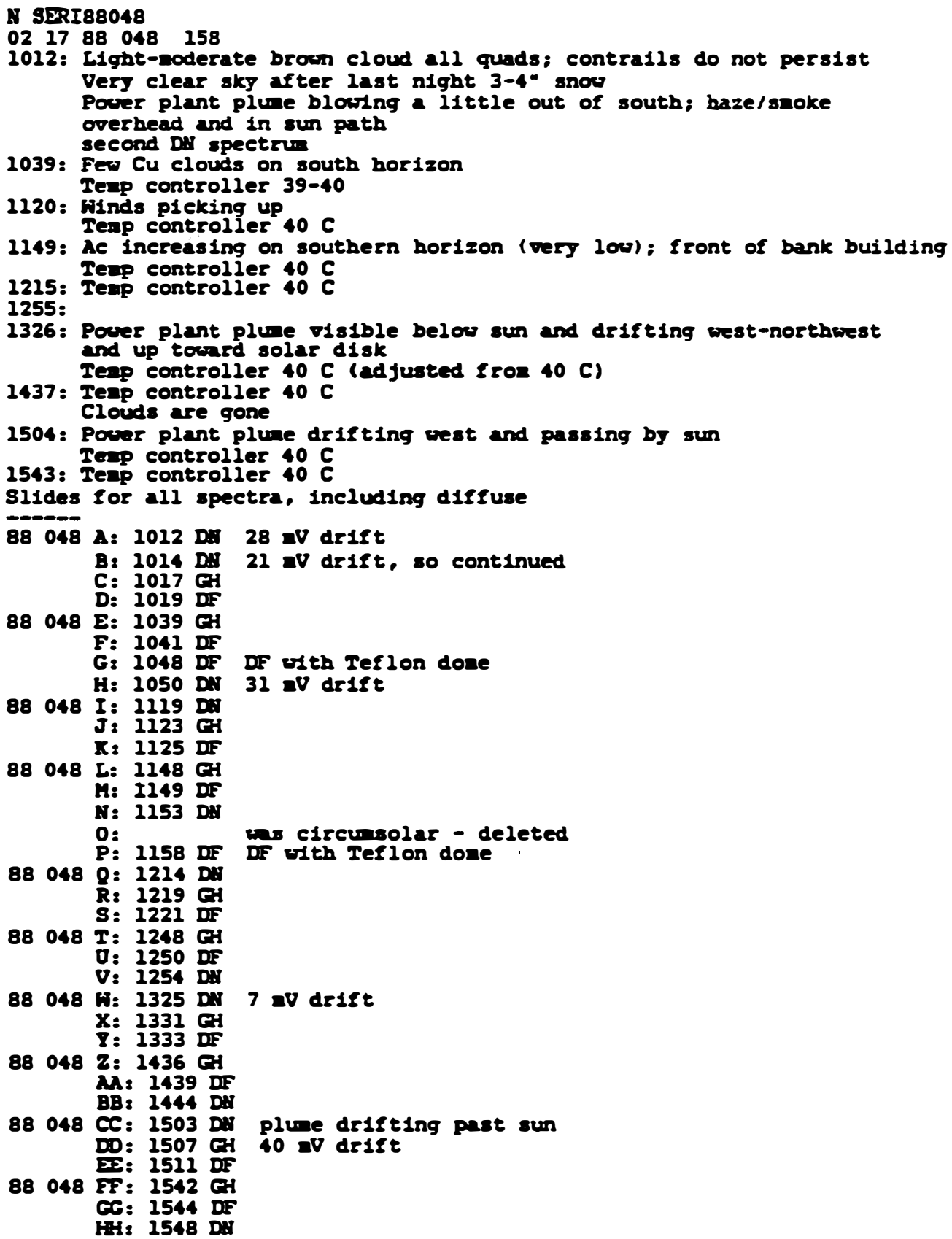

1149: Ac increasing on southern horizon (verg lov): front of bank building Temp controlier $40 \mathrm{C}$

1504: Power plant plune drifting west and passing by sun Terp controlier $40 \mathrm{C}$

1543: Tenp controller $40 \mathrm{C}$

Slides for all spectra, Including diffuse

$-0-0-1$

88048 A: $1012 \mathrm{Da} \quad 28 \mathrm{aV}$ drift

Bs 1014 DUI 21 aV drift. so continued

C: $1017 \mathrm{EH}$

D: 1019 DF

88048 E: $1039 \mathrm{GH}$

F: 1041 DF

G: 1048 DF DF with Teflon done

$88048 \mathrm{I}: 1119 \mathrm{DE}$

$31=0$ drift

J: 1123 G

$\mathrm{K}_{2} 1125$ DF

$88048 \mathrm{~L}=1148 \mathrm{G}$ H: I149 DF N: 1153 DA

$0:$

P: 1158 DF

88048 0: 1214 D:

R: 1219 G

S: 1221 DF

$88048 \mathrm{~T}: 1248 \mathrm{GH}$

O: 1250 DF

$V: 1254$ D.

$88048 \mathrm{NF} 1325 \mathrm{DN}$

$\mathrm{X}=1331 \mathrm{G}$

I: 1333 DF

$88048 \mathrm{Z}: 1436 \mathrm{GH}$ MA 1439 DF BB: 1444 DA

88048 CC: 1503 D. DD: 1507 GH Ex: 1511 DF

wo circunsolar - deleted

DF vith Tefion done

$7 \mathrm{ad}$ drift

plue drifting past sun

$40=0$ drift

88048 FF: 1542 G

CG: 1544 DF

FH: $1548 \mathrm{DA}$

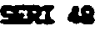

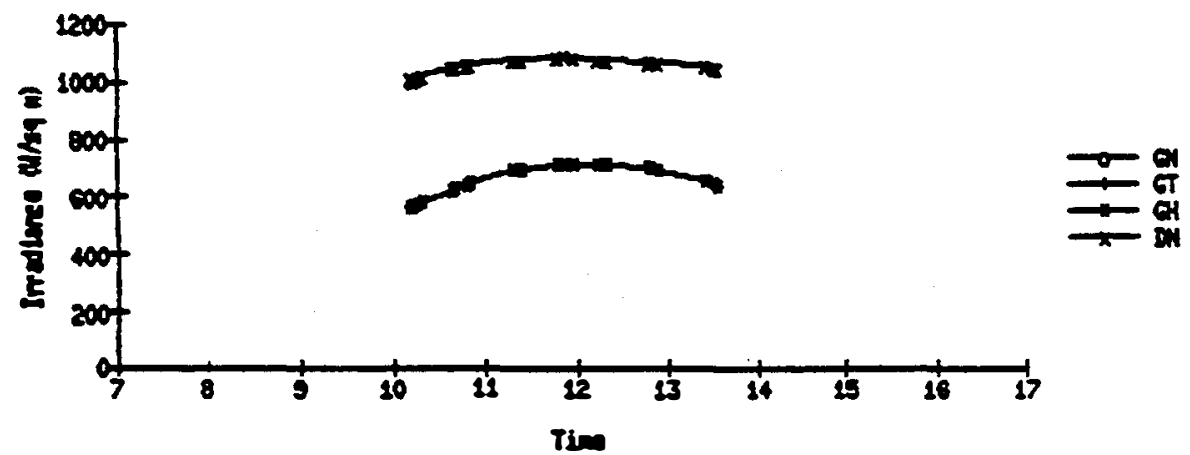


E्या 48
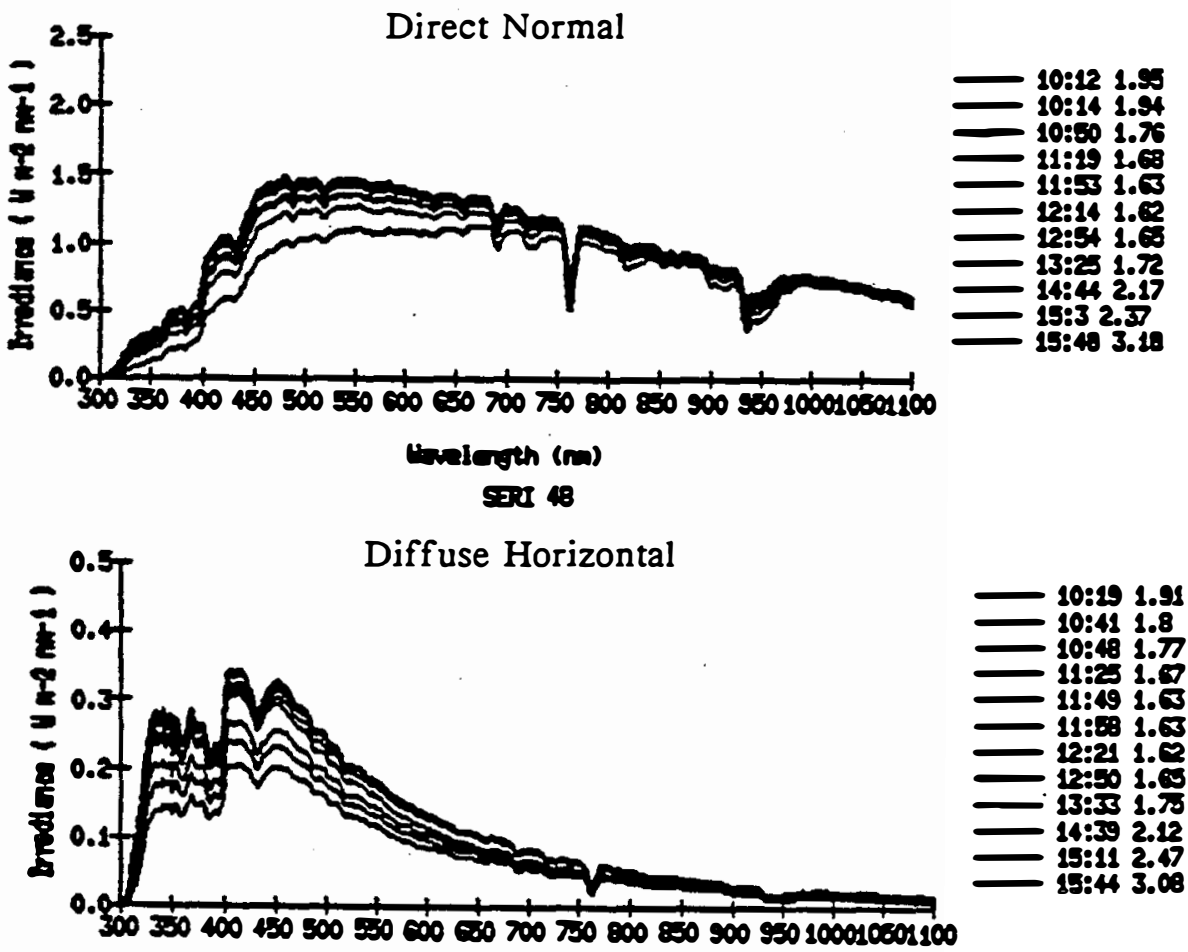

thesth (m)

ज्यरा 48

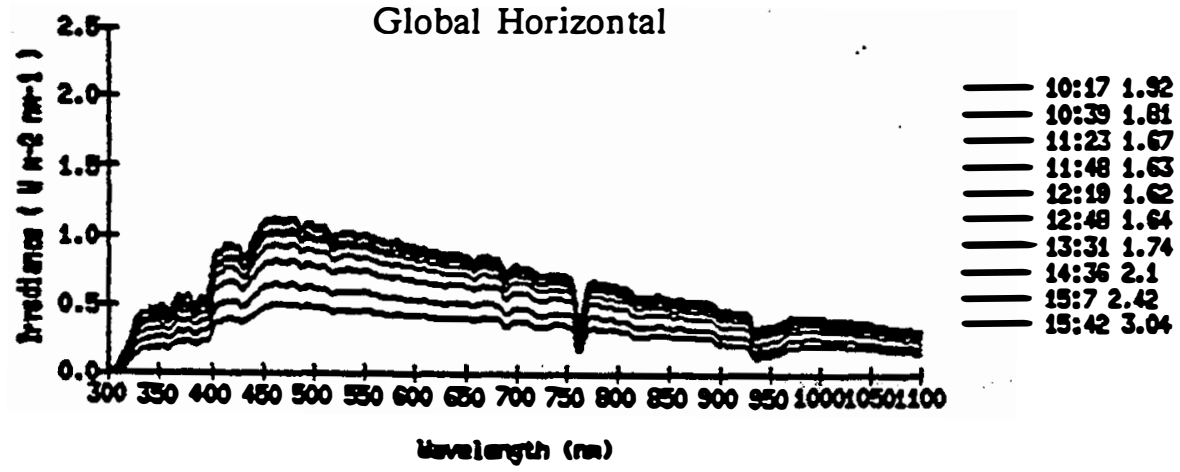


STRI 88055

022488055158

0803: Contri1ls pers1st; 5-6/10 thin C1 clouds

Pover plant plue drifting east

Teap controller $39 \mathrm{C}$

Tenp controller $40 \mathrm{C}$ on nert spectrm

GH spectrun at 0811 looks odd

0821: Tenp controller $39 \mathrm{C}$ on GH spectrms $41 \mathrm{C}$ on DA spectrum

0845: Tenp controller $40 \mathrm{C}$

0914: Tenp controller 39-40 C

1012: Porer plant plue now vertical; rinds shlfted to 90 deg east at $1 \mathrm{~m} / \mathrm{s}$

Contralls persisting across sk

slides for spectra beginning at about 1000, 1ncluding diffuse

88055 A: $0805 \mathrm{DN}$ C1rrus

B: 0811 어 $27 \mathrm{~m}$ drift; no185 spectruns c1rrus

C: $0813-$ DF Cirrus

88055 D: $0823 \mathrm{GH}$ C1rzus

E: 0825 DF Cirrus

F. 0829 DV C15rus

$88055 \mathrm{Gs} 0847 \mathrm{DA} 23 \mathrm{aV}$ drift: C1rrus

Hz 0851 GH C1rrus

Is $0853 \mathrm{DF}$ C15rus

$88055 J_{8} 0904$ GH Cirrus

$X_{8} 0906$ DF Cirrus

I: 0911 Di C1rrus

88055 M: 1015 DA $17 \mathrm{NV}$ drift: Cirrus

B: 1019 GH Cirrus

0 : 1021 DF C1rrus

P:

ces elreunsolar

ST2

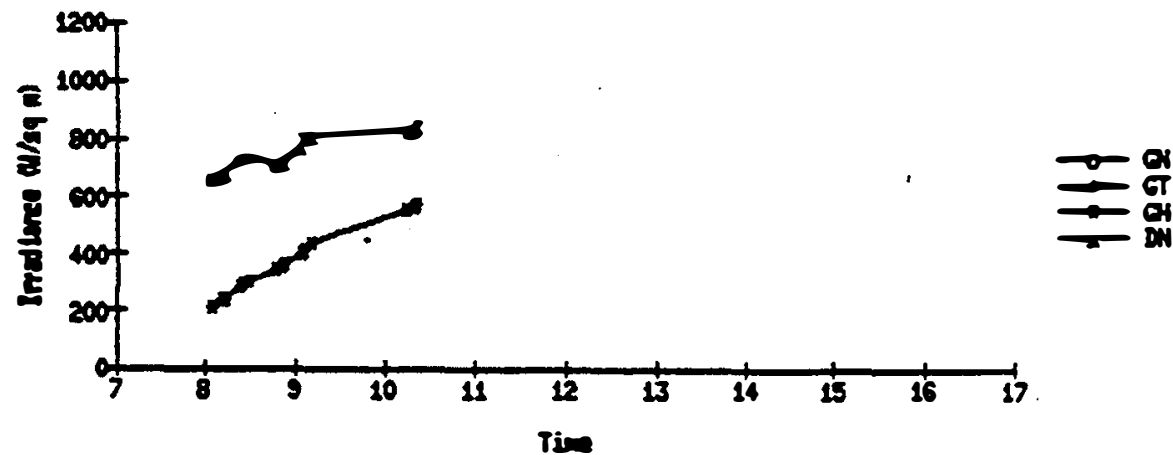


SगI 50

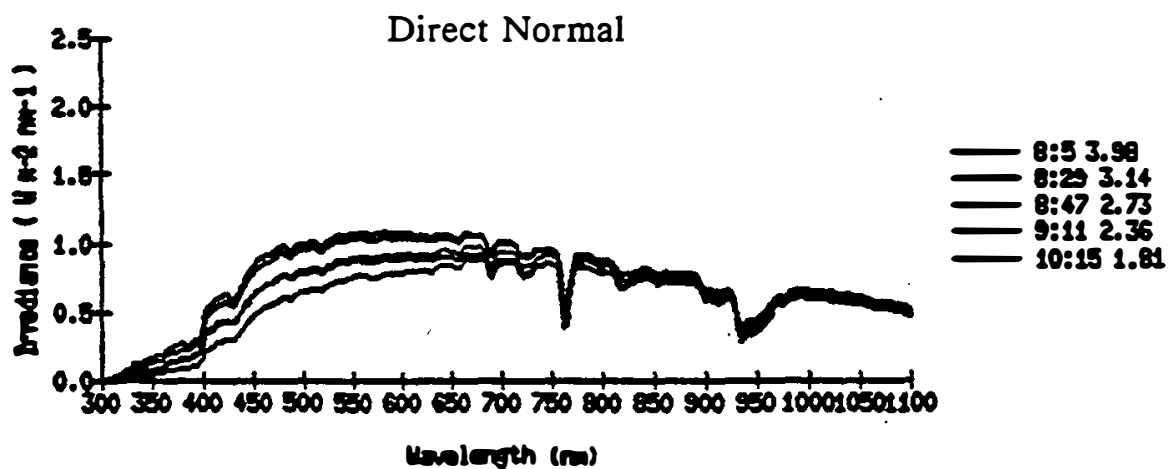

ड्यर 50

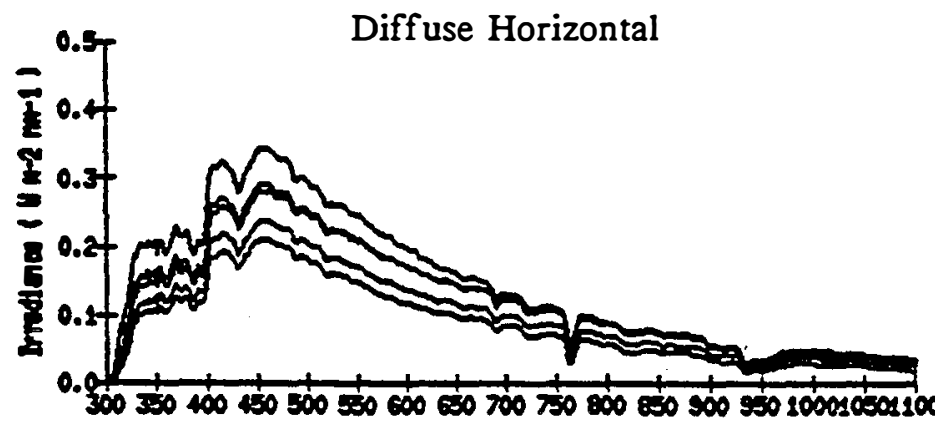

voluth (m)

S्या

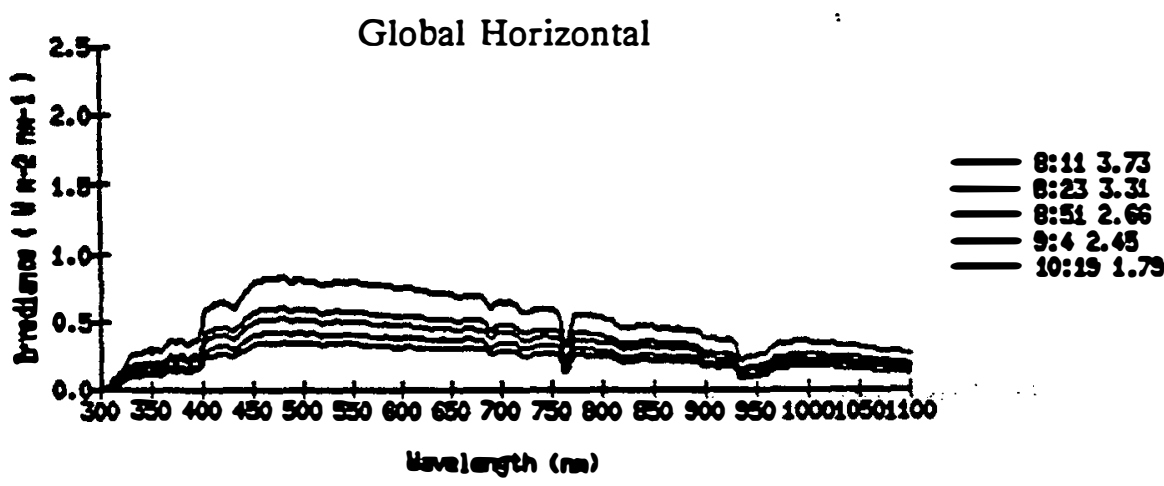




\section{SEP *}

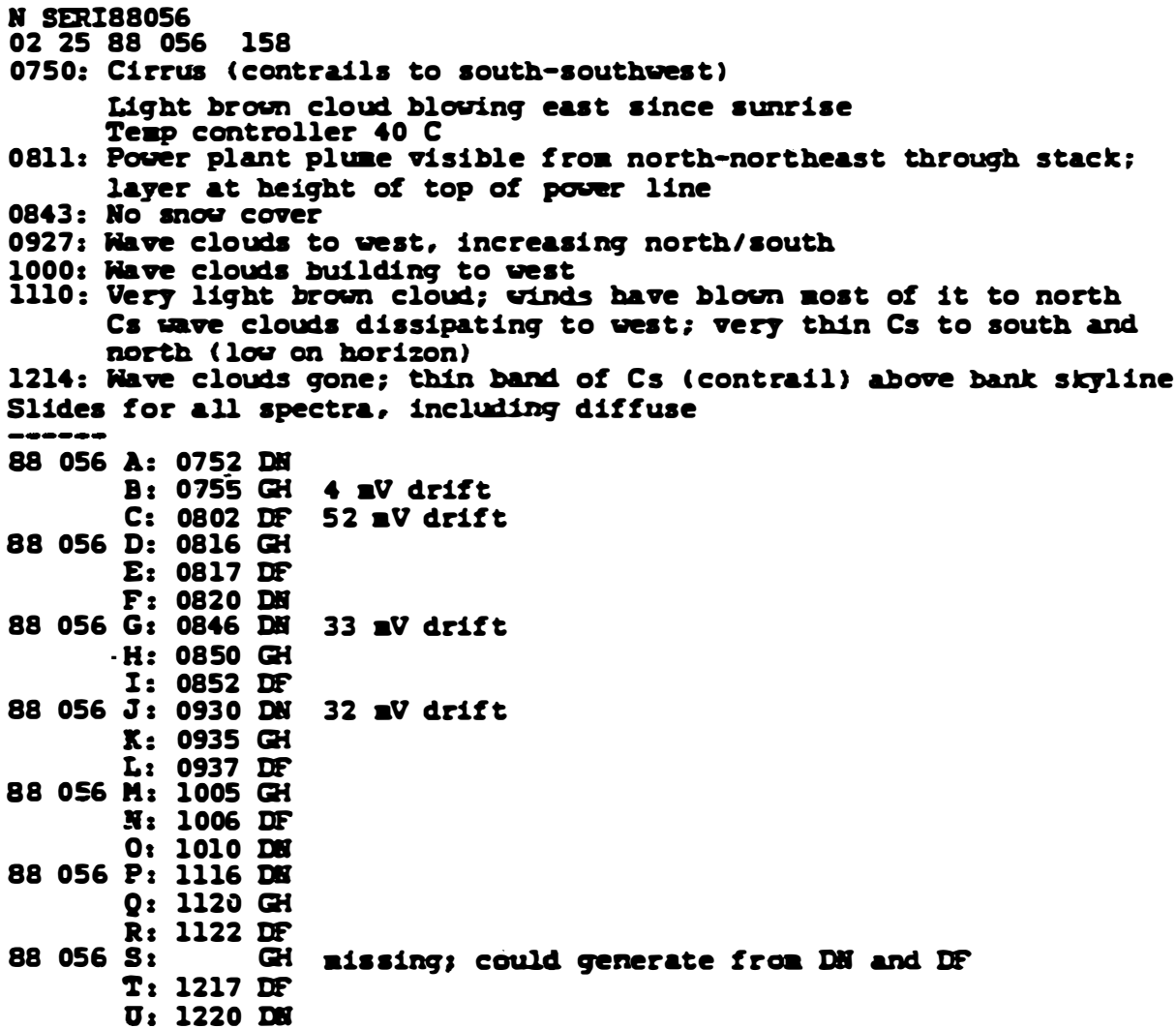

STR 50

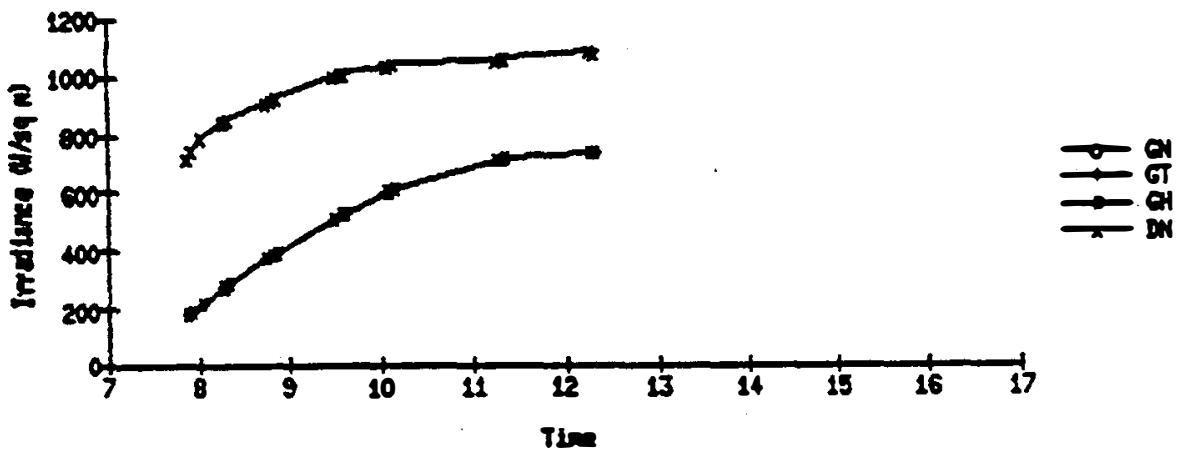


5206
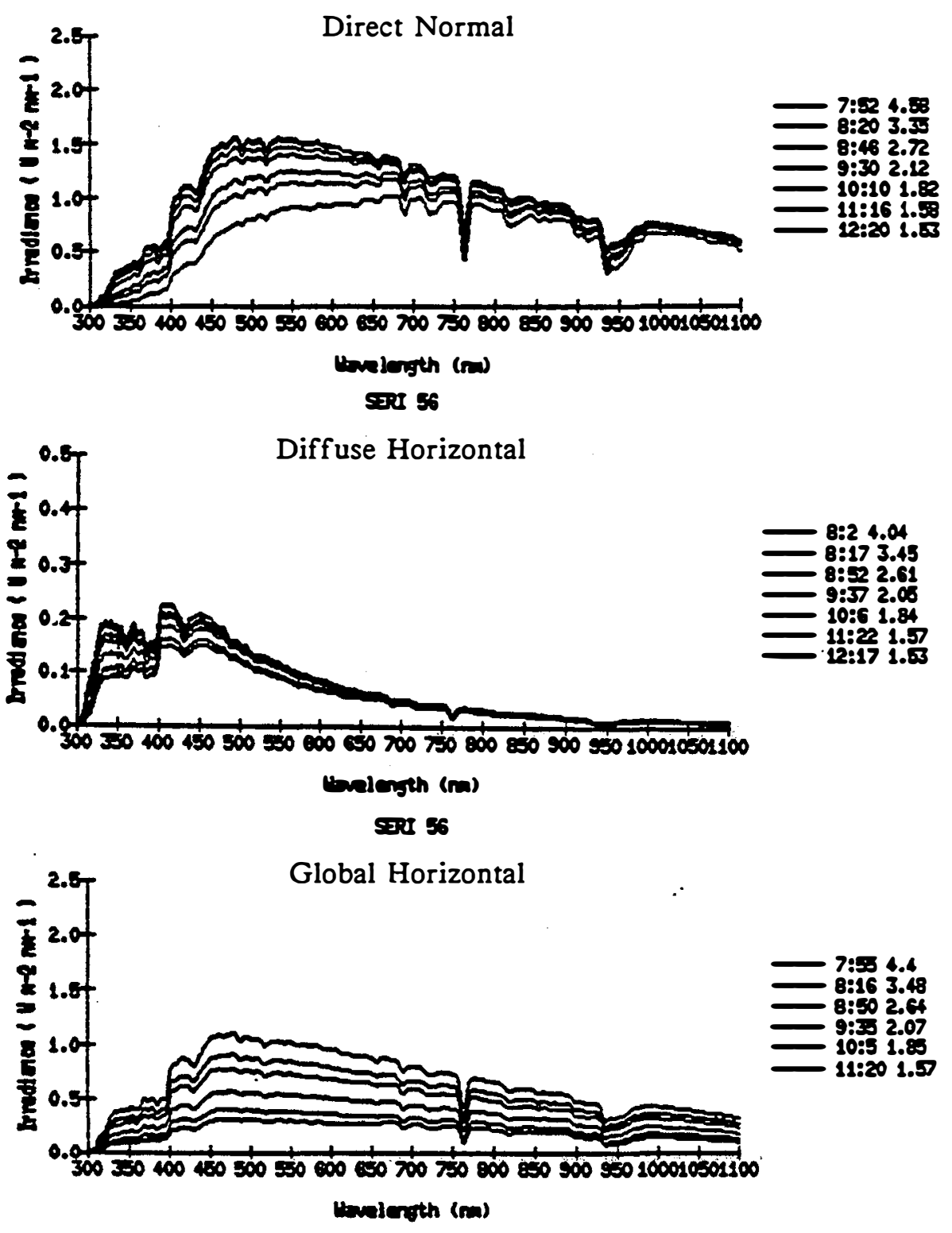


\begin{tabular}{|c|c|c|c|}
\hline $\begin{array}{l}\text { Document Control } \\
\text { Page }\end{array}$ & $\begin{array}{l}1 . \quad \text { Repor No. } \\
\text { SERI/TR-215-3513B }\end{array}$ & $\begin{array}{l}\text { Accession No. } \\
\text { DE89009448 }\end{array}$ & 3. Recipient's Accession No. \\
\hline \multicolumn{3}{|l|}{ 4. Title and Subtitle } & $\begin{array}{l}\text { 5. Publication Date } \\
\text { January } 1990\end{array}$ \\
\hline \multicolumn{3}{|c|}{$\begin{array}{l}\text { Spectral Solar Radiation Data Base Documentation, } \\
\text { Volume II }\end{array}$} & 6. \\
\hline \multicolumn{3}{|l|}{ 7. Author(s) } & 8. Pertorming Organization Rept. No. \\
\hline \multirow{2}{*}{\multicolumn{3}{|c|}{ 9. Pertorming Organization Name and Address }} & 10. Project/Task/Work Unit No. \\
\hline & & & $\begin{array}{l}\text { 11. Contract (C) or Grant (G) No. } \\
\text { (C) } \\
\text { (G) }\end{array}$ \\
\hline \multirow{2}{*}{\multicolumn{3}{|c|}{$\begin{array}{l}\text { 12. Sponsoring Organization Name and Address } \\
\text { Solar Energy Research Institute } \\
1617 \text { Cole Boulevard } \\
\text { Golden, Colorado } 80401-3393\end{array}$}} & $\begin{array}{l}\text { 13. Type of Report \& Period Covered } \\
\text { Technical Report }\end{array}$ \\
\hline & & & 14. \\
\hline
\end{tabular}

15. Supplementary Notes

16. Abstract (Limit: 200 words)

The Solar Energy Research Institute (SERI), Electric Power Research Institute, Florida Solar Energy Center, and Pacific Gas and Electric Company cooperated to produce a spectral solar radiation data base representing a range of atmospheric conditions. These data will help to characterize the natural variability in the spectral (color) content of outdoor solar radiation so that the sensitivity of spectrally selective solar devices (such as photovoltaics) to these variations can be studied quantitatively. Volume I of this report documents the history, approach content, and format of the data base; Volume II contains graphs and field notes for each of the spectral data sets. The data reside on magnetic tape at SERI.

17. Document Analysis

a. Descriptors Spectral solar radiation; broadband solar radiation ; meteorological data ; spectroradiometers

b. Identifiers/Open-Ended Terms

c. UC Categories

$$
233,234
$$

18. Availability Statement

National Technical Information Service

U.S. Department of Commerce

5285 Port Eoyal Road

Springfield, Virginia 22161
19. No. of Pages

410

20. Price

A18 